\title{
Olive Germplasm - Italian Catalogue of Olive Varieties
}

Innocenzo Muzzalupo

Additional information is available at the end of the chapter

http://dx.doi.org/10.5772/51719

\section{Introduction}

It is of great importance to evaluate and characterize the existing genetic diversity of the crop species, mainly for those, such as the case of olive, which still have a well preserved great cultivar patrimony, in spite of the disturbance of the environments where they are cultivated. This issue is of particular importance in areas where a number of varieties show adaptation to the difficult local environmental conditions.

The genetic patrimony of the Mediterranean Basin's olive trees are very rich and is characterised by an abundance of varieties. Based on estimates by the FAO Plant Production and Protection Division Olive Germplasm (FAO, 2010), the world's olive germplasm contains more than 2.629 different varieties, with many local varieties and ecotypes contributing to this richness.

The problem of olive germplasm classification is not only complicated by the richness of its genetic patrimony, but also by the absence of reference standards and by the confusion regarding the cultivar names, with numerous cases of homonymy (one denomination for several genotypes) and synonymy (one genotype with several denominations).

The Italian olive germplasm is estimated to include about 800 cultivars, most of them landraces vegetatively propagated at a farm level since ancient times. The number is probably underestimated because of the scarce information on minor local varieties widespread in the different olive growing areas. The study of these less-common cultivars is important because they may have traits not considered important in the past but necessary to meet the challenges of modern olive growing. Low vigour, resistance to low temperatures, salinity tolerance, adaptability to low pruning systems, late ripening and fatty-acid content are important traits for olive or olive oil quality. Additionally, 
morphological characters are sometime correlated or associated with disease susceptibility and can be used as markers in breeding.

The largest olive collection (accounting for 17 percent of the total olive trees with more than 500 varieties) is held by Agricultural Research Council - Olive growing and oil industry research centre (CRA-OLI) in Italy, followed by the collections of the Centro de Investigación y Formación Agroalimentaria Córdoba (CIFACOR) in Spain. The systematic collection of Italian olive varieties for deposit into specific catalogue fields began in Italy in the 1980s. A similar international collection was begun in 1997 by CRA-OLI of Rende, Italy. Collection entailed the following steps: a survey of the territory, individuation, basic characterization, and introduction into the gene bank field. Material identified by other international scientific institutions (International Treaty on Plant Genetic Resources for Food and Agriculture - Plant Genetic Resources RGV-FAO Projects) was also included. To date, roughly 500 varieties have been introduced into the CRA-OLI collection, and this list has been published (web site http://apps3.fao.org/wiews/olive/oliv.jsp). The goal of such collections is to safeguard all cultivars, and particularly the minor ones, to avoid a loss in genetic diversity and to offer an interesting genetic basis for breeding programs. Knowledge of genetic diversity in a crop species is fundamental to its improvement. A variety of molecular, chemical and morphological descriptors are used to characterize the genetic diversity among and within crop species.

Morphological and biological characters have been widely used for descriptive purposes and are commonly used to distinguish olive cultivars. Agronomic characterization also allowed the classification of different olive cultivars. Different molecular markers have recently been used to characterize and distinguish the olive cultivars.

Management of the CRA-OLI collection includes a description of its genetic diversity for a reliable characterization of all accessions since several cases of mislabelling, homonymy and synonymy could exist.

In the present work, we used morphological characterization and molecular markers and to characterize all accessions present in the CRA-OLI collection, to build a first molecular and morphological data-base and to analyze the genetic relationships between cultivars.

We used SSR markers for genotyping the complete collection of the olive germplasm. The experimental approach was based on using the parameters as recommended by International Olive Council (COI) for characterization and the bio-agronomic observations concerned the morphological characteristic of the trees, leaves, fruit and inflorescence and the flowering period. The fruits were examined for their morphology and oil composition and for endocarp characteristics.

Over 200 elaiographic cards with colour photos, graphs and tables and with full details relating to the identification the olive varieties growing in the CRA-OLI olive germplasm collection in Calabria, Italy were reported in annex. Additional information about agronomic behaviour of the plants, and the organoleptic oil values, as determined by a panel test, were also recorded. 


\section{Materials and methods}

The passport data of the olive cultivars has permitted the unequivocal classification and location of all identified genotypes. The information provided in the passport data has the aim of integrating the morphological, agronomic, and molecular data of the olive genetic resources; this information has been complemented by the photographic documentation of each accession recovered.

The elaiographical cards were made up according to the International Union for the Protection of New Varieties of Plants - UPOV method and the biometric and morphological parameters evaluated for each of the accessions were as follows: trees, leaves; inflorescences; fruits and stones.

The agronomic characteristics have been evaluated on the observations of $4-5$ trees over a period of 4-5 years. Additional information about cold and agronomic behaviour of the plants, and the organoleptic oil values, as determined by a panel test, were also recorded. All the olives varieties analysed were growing in the CRA-OLI olive germplasm collection at Mirto-Crosia (Cosenza, Italy).

\subsection{Morphological characterization}

The systematic utilization of descriptive morphological characters of the tree and various tree organs has enabled the characterization and discriminatory identification of varieties. The methodology used for describing the olive biodiversity recovered has considered a set of 24 morphological characters (tree: 3 characters; leaf: 3; inflorescence: 2; fruit: 8; endocarp: 8).

\subsubsection{Characters of the tree}

Three qualitative characters (vigour, growth habit, and canopy-density) are considered.

\subsubsection{Plant vigour}

This refers to both the size of the tree and the intrinsic ability of the scaffold branches and shoots to grow in length and width. It is divided into the following categories:

a. Weak. Tree whose growth is modest even under optimal agronomic conditions. When mature, the trunk and the area projected by the canopy of the tree are distinctly less than what is expected of a specimen of this species.

b. Medium. Tree which, in each area and when normal cultural practices areapplied, displays the average development expected of an olive tree.

c. Strong. Tree which, in each area and when normal cultural practices areapplied, displays strong growth, marked trunk and canopy development in terms of both height and volume, and vigorous, long branches. 


\subsubsection{Growth habit}

This character describes the natural distribution of the scaffold branches and shoots before there is interference from the training shape adopted and when vigour exerts little influence. Growth habit is divided into three categories (Figure 1):

a. Drooping. Natural growth habit that can be characterized by plagiotropic branching, i.e. by shoots and limbs which are small in diameter and bend downwards from the outset.

b. Spreading. Characterized by initial orthotropic branching. The weight of the canopy and/or of the crop subsequently forces the limb to bend down and turn in the direction in which the greatest amount of light and space is available. The canopy thus becomes hemispherical in shape (even when the olive has several trunks, they always remain quite distinct from each other).

c. Erect. Habit characteristic of certain cultivars whose branches tend to grow vertically and have a strong apical dominance. The tree acquires a fairly pronounced conical shape which becomes cylindrical on reaching maturity. As a rule, cultivars which have an erect growth habit are also vigorous although there are some major exceptions.

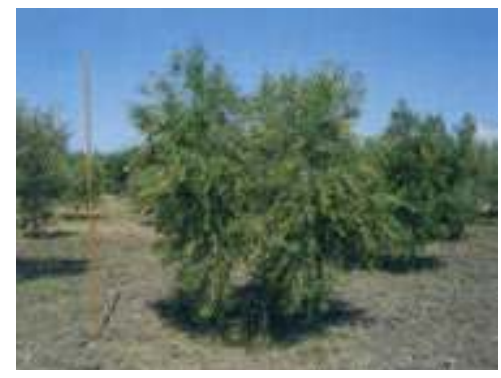

$a$

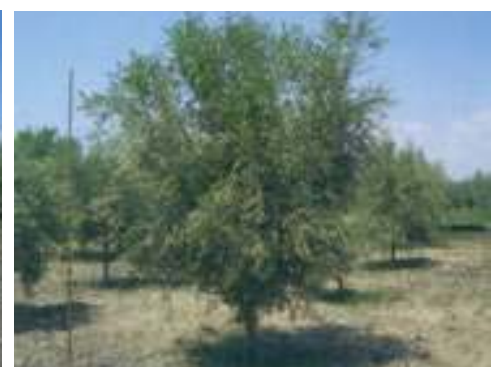

$b$

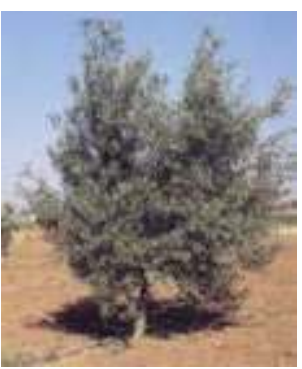

c

Figure 1. Categories of growth habit of the olive trees

\subsubsection{Growth habit}

Indicates the extent of canopy vegetation and can be measured by the possibility of light penetration. Result of the interaction between the length of the internodes, the number and vigour of the shoots and the size of the leaves. It is classified into three categories:

a. Sparse. This is normally associated with fast-growing cultivars with long internodes. From any point "space" is observed through which light can penetrate.

b. Medium. Density typical of the species. Vegetation is abundant but internode length and growth always leave internal space which produce a shading effect.

c. Dense. This is characteristic of cultivars with short internodes, abundant branching and heavy foliage. The canopy displays a compact surface, the inner section of which is shaded.

\subsubsection{Characters of the leaf}

Three quantitative characters (length, width, and shape) are considered. Observed in samples of about 100 adult leaves and taken from the middle section of 8-10 one year old shoots chosen from the most representative shoots on the south facing side of the tree at shoulder level. 


\subsubsection{Blade length}

Observed in samples of about 100 adult leaves taken from the middle section of 8-10 one year old shoots chosen from the most representative shoots on the south facing side of the tree at shoulder level.

Blade length (L) is classified into three categories:
a. Short
$(<$ at $5 \mathrm{~cm})$
b. Medium
(from 5 to $7 \mathrm{~cm}$ )
c. Long
$(>$ at $7 \mathrm{~cm})$

\subsubsection{Blade width}

Observed in samples of about 100 adult leaves taken from the middle section of 8-10 one year old shoots chosen from the most representative shoots on the south facing side of the tree at shoulder level. Blade width $(\mathrm{W})$ is classified into three categories:
a. Narrow
$(<$ at $1 \mathrm{~cm})$
b. Medium
(from 1 to $1.5 \mathrm{~cm}$ )
c. Broad
$(>1.5 \mathrm{~cm})$

\subsubsection{Shape}

This is determined by the ratio between the length and width. Shape is divided into three categories:

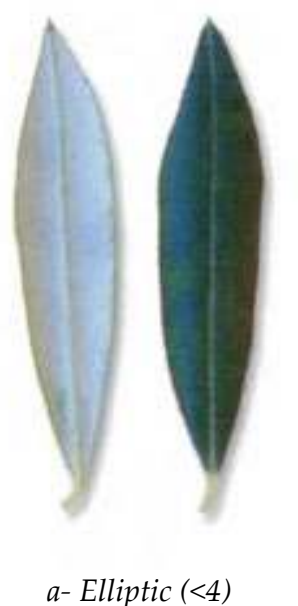

a-Elliptic $(<4)$

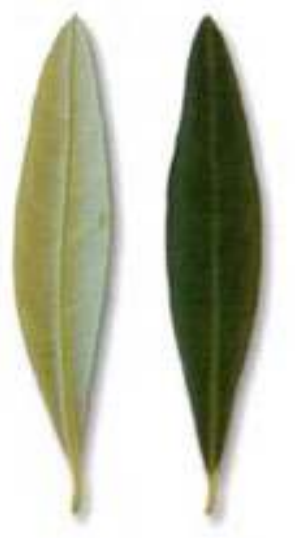

b-Elliptic-Lanceolate $(4 \leftrightarrow 6)$
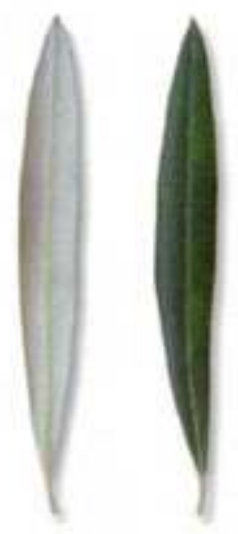

c-Lanceolate (>6)

\subsubsection{Characters of the inflorescence}

Two quantitative characters (length and number of flowers for inflorescence) are considered (Figure 2). 


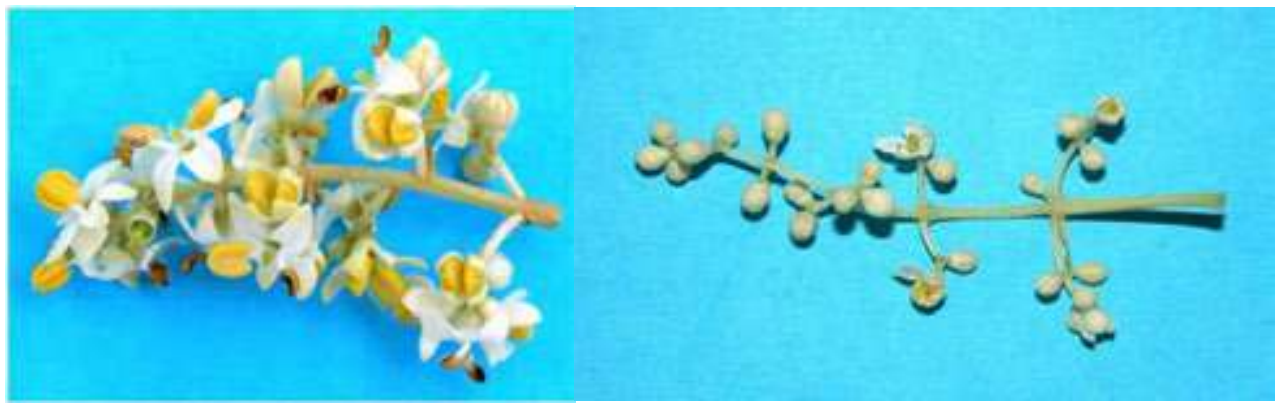

Figure 2. Two different inflorescences

\subsubsection{Length}

Observed in samples of about 100 inflorescence at bud stage, taken from the middle section of 8-10 fruiting shoots chosen from the most representative shoots on the south facing side of the tree. It is classified into three categories:
a. Short
(< at $2.5 \mathrm{~cm})$
b. Medium
(from 2.5 to $3.5 \mathrm{~cm}$ )
c. Long
$(>$ at $35 \mathrm{~cm}$ )

\subsubsection{Number of flowers for inflorescence}

Observed in samples of about 100 inflorescence at bud stage, taken from the middle section of 8-10 fruiting shoots chosen from the most representative shoots on the south facing side of the tree. It is classified into three categories:
a. Low
(< at 18 flowers)
b. Medium
(from 18 to 25 flowers)
c. High
(> 25 flowers)

\subsubsection{Characters of the fruits}

Two quantitative characters (weight and shape) and six qualitative characters (symmetry, position of maximum transverse diameter, apex, base, nipple, and lenticels) are considered. Some characters refer to two positions:

Position $A$ in which the fruit generally displays the greatest asymmetry when held by either and between the index finger and thumb.

Position $B$ is reached by turning $90^{\circ}$ from position $A$.

\subsubsection{Weight}

Determined in a sample of 100 fruits taken from the middle section of fruiting shoots chosen from the most representative shoots on the south facing side of the tree. It is classified into four categories: 

a. Low
(< at $2 \mathrm{~g})$
b. Medium
(from 2 to $4 \mathrm{~g}$ )
c. High
(from 4 to $6 \mathrm{~g}$ )
d. Very high
(> at 6)

\subsubsection{Shape (position A)}

Ratio between the length and width of fruits. Determined in a sample of 100 fruits taken from the middle section of fruiting shoots chosen from the most representative shoots on the south facing side of the tree. Shape is classified into three categories:

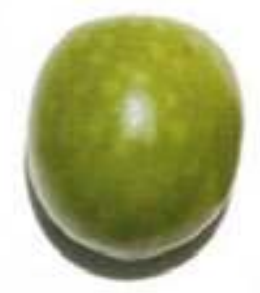

a-Spherical $(<1.25)$

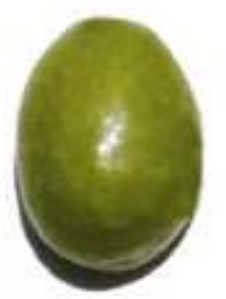

b- Ovoid $(1.25 \leftrightarrow 1.45)$

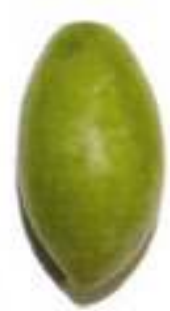

c- Elongated (>1.45)

\subsubsection{Symmetry (position A)}

Extent to which the two longitudinal halves match. Determined in a sample of 100 fruits taken from the middle section of fruiting shoots chosen from the most representative shoots on the south facing side of the tree. It is classified into three categories:

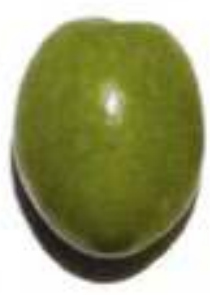

a- Symmetric

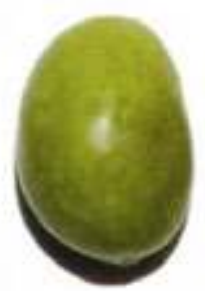

b-Slightly asymmetric

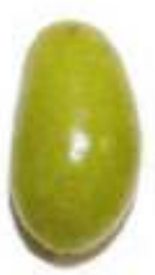

c-Asymmetric

\subsubsection{Position of maximum transverse diameter (position B)}

According to its location. Determined in a sample of 100 fruits taken from the middle section of fruiting shoots chosen from the most representative shoots on the south facing side of the tree. It is classified into three categories: 


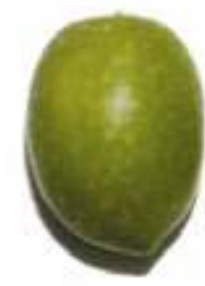

a- Towards base

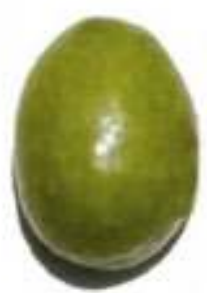

b-Central

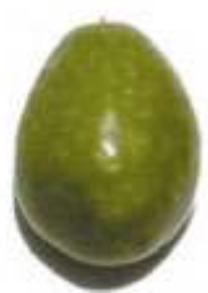

c- Towards apex

\subsubsection{Apex (position A)}

Determined in a sample of 100 fruits taken from the middle section of fruiting shoots chosen from the most representative shoots on the south facing side of the tree. It is classified into two categories:

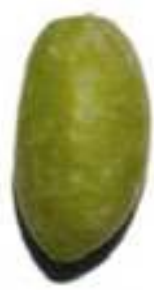

a-Pointed

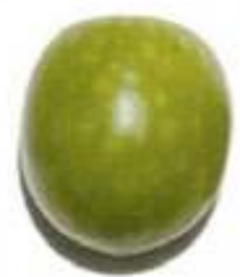

$b$ - Rounded

\subsubsection{Base (position A)}

Determined in a sample of 100 fruits taken from the middle section of fruiting shoots chosen from the most representative shoots on the south facing side of the tree. It is classified into two categories:

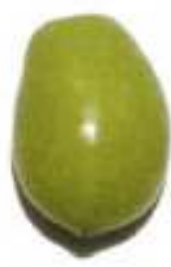

a- Truncate

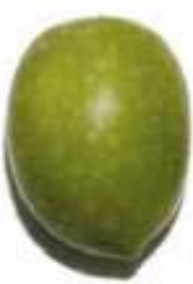

$b$ - Rounded 


\subsubsection{Nipple}

Determined in a sample of 100 fruits taken from the middle section of fruiting shoots chosen from the most representative shoots on the south facing side of the tree. This characteristic of the tip of the fruit style is:

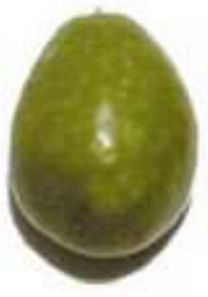

a-Absent

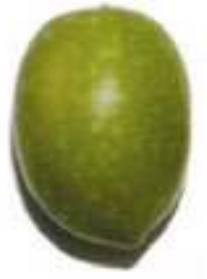

b-Tenuous

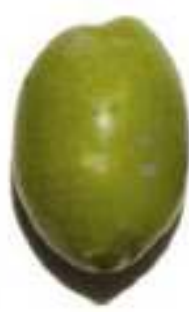

c-Obvious

\subsubsection{Lenticelles}

When the fruit is still green.Determined in a sample of 100 fruits taken from the middle section of fruiting shoots chosen from the most representative shoots on the south facing side of the tree. It is classified into the following categories:
a. few or many
b. small or large

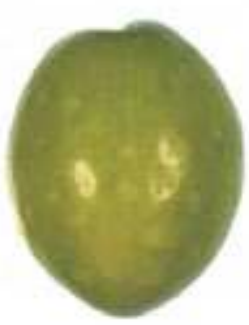

a- Few and large

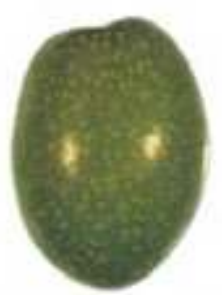

b-Many and small

\subsubsection{Characters of the endocarp (stone)}

The endocarp is the internal, woody part ofthe fruit that encloses the seed. Three quantitative characters (weight, shape, and number of grooves) and five qualitative characters (symmetry, position of maximum transverse diameter, apex, base, and surface) are considered. These are evaluated in the afore-mentioned sample of 100 fruits. As in the case of the fruit, some characters also refer to two positions. Position $A$ is normally the position of maximum asymmetry and is that in which the carpel suture faces the observer. Position $B$ is reached by turning $90^{\circ}$ from position $A$. 


\subsubsection{Weight}

The weight is evaluated on the afore-mentioned sample of 100 fruits. It is classified into four categories:
a. Low
(< at $0.3 \mathrm{~g})$
b. Medium
(from 0.3 to $0.45 \mathrm{~g}$ )
c. High
(from 0.45 to $0.7 \mathrm{~g}$ )
d. Very high
(> at 0.7 )

\subsubsection{Shape}

Ratio between the length and width of endocarps. Shape is classified into four categories:

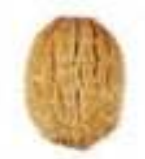

a-Spherical

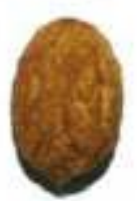

b- Ovoid

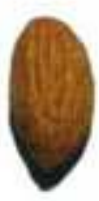

c- Elliptic

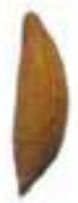

d-Elongated

\subsubsection{Symmetry (position A)}

Extent to which the two longitudinal halves match. It is classified into three categories:

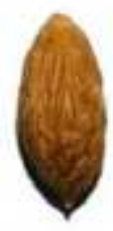

a-Symmetric

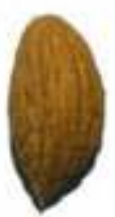

b-Slightly asymmetric

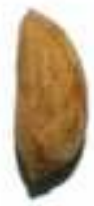

c-Asymmetric

2.1.5.4. Position of maximum transverse diameter (position B)

According to its location. It is classified into three categories:

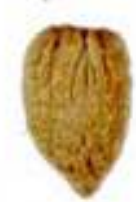

a- Towards base

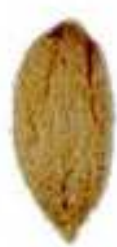

b-Central apex

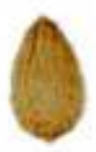

c- Towards apex 


\subsubsection{Apex (position A)}

The apex is classified into two categories:

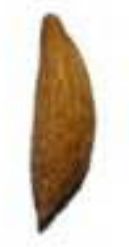

a- Pointed

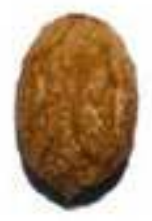

$b$-Rounded

\subsubsection{Base (position A)}

The base is classified into three categories:

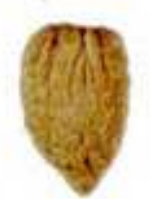

a- Truncate

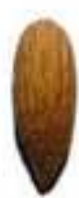

$b$ - Pointed

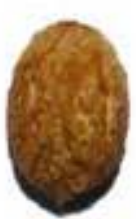

c-Rounded

\subsubsection{Surface (position A)}

Based on depth and abundance of fibrovascular bundles. It is classified into three categories:

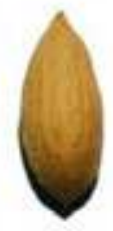

a-Smooth

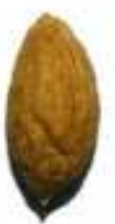

b-Rugose

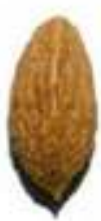

c-Scabrous

\subsubsection{Number of grooves}

Number of grooves that can be seen from the stalk insertion point. It is classified into three categories:
a. Low
$(<$ at 7$)$
b. Medium
(from 7 at 10)
c. High
$(>$ at 10$)$ 


\subsection{Pomological characterization and oil quality}

To complete the description of the varieties recovered a methodology has also been adopted for the secondary characterization (pomological and oil quality) of the varieties held in the CRA-OLI collections; this characterization had the aim of providing reliable data on the pomological value of the varieties identified and a common method has been used, the object being to unify the criteria for evaluating these parameters. This will help to minimize the differences that the use of different methods of study could cause in the characterization of world germplasm.

\subsubsection{Average fresh weight of fruit}

This parameter is calculated from 100 olives harvested at the black stage of maturation. The following categories have been established.
a. Very low
$(<$ at $2 \mathrm{~g})$
b. Low
(from 2 at $4 \mathrm{~g}$ )
c. Medium
(from 4 at $6 \mathrm{~g}$ )
d. High
(from 6 at $8 \mathrm{~g}$ )
e. Very high
$(>$ at $8 \mathrm{~g})$

\subsubsection{Average fresh weight of stone}

This parameter is calculated from 100 stones obtained from the fruit and after removing the flesh. The following categories have been established.
a. Very low
(< at $0.2 \mathrm{~g})$
b. Low
(from 0.2 at $0.4 \mathrm{~g}$ )
c. Medium
(from 0.4 at $0.6 \mathrm{~g}$ )
d. High
(from 0.6 at $0.8 \mathrm{~g}$ )
e. Very high
(> at $0.8 \mathrm{~g}$ )

\subsubsection{Flesh/Stone ratio of the fruit}

This parameter is calculated from the fruit and stone weight data obtained from samples. The following categories have been established.
a. Low
(< at 5.0)
b. Medium
(from 5.0 at 7.5)
c. High
(from 7.5 at 10.0)
d. Very high
(> at 10.0)

\subsubsection{Percentage of oil in the fruit}

This parameter is calculated from $0.3 \mathrm{~kg}$ paste olive samples that was milled for $1 \mathrm{~min}$ (standardized time to obtain a homogeneous sample and to minimize heating of the olive 
paste) in a small hammer mill. For oil content determination, the sample was analyzed using a Fourier Transform Near-InfraRed instrument (MPA Multi Purpose AnalyzerFT-NIR spectrometer system (Bruker Optics, Milan,Italy), according to Bendini et al., 2007. The following categories have been established.
a. Very low
(< at $30 \%)$
b. Low
(from 30 at $40 \%$ )
c. Medium
(from 40 at $50 \%$ )
d. High
(from 50 at $60 \%$ )
e. Very high
$(>$ at $60 \%)$

\subsubsection{Olive oil extraction}

For each sample $5 \mathrm{~kg}$ of olives were picked from five trees grown in the CRA-OLI germplasm collection, and then milled in a laboratory scale hammer mill (Oliomio, Toscana Enologica Mori, Tavernelle Val di Pesa, Italy). After 30 minutes of malaxation at room temperature the oil was separated by centrifugation in the same operative conditions (Figure 3). Then the oil was filtered and stored in the dark at $8{ }^{\circ} \mathrm{C}$ until analysis.

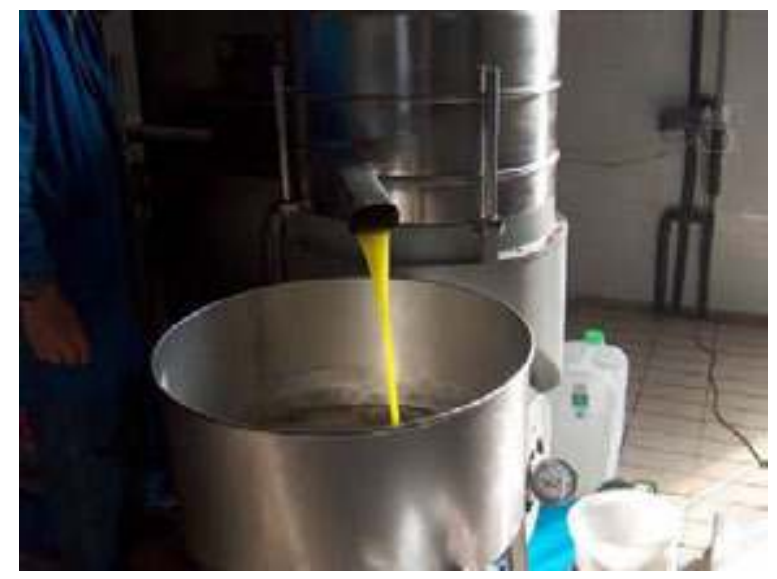

Figure 3. Decanter and disc stack centrifuges

\subsubsection{Oil determinations}

The quality of the oil has been determined according to its fatty acid composition (Muzzalupo et al., 2011). Expressed as a percentage and determined according to the official method of European Community (Commission Regulation No 61/2011).

\subsubsection{Sensory analysis}

Quantitative descriptive analysis was carried out by a total of 9 assessors, members of staff of the CRA-OLI of Rende (CS), using the procedure reported in a later chapter ("sensory 
properties of virgin olive oils"). The assessors had experience in Qualitative Data Analysis QDA (Stone and Sidel, 2004) and virgin olive oil sensory evaluation in accordance with the current UE Regulation (N. 61/2011).

Virgin olive oil samples (15 g) were served to the assessors in clear glass tumblers (100 mL), covered with watch glasses at room temperature (approximately $28{ }^{\circ} \mathrm{C}$ ). The samples were presented in duplicate, in balanced order to each assessor.

For a description of the virgin olive oils, the following terms were used: yellow, yellowgreen, intense green, musk-green to describe the virgin olive oil colour; fruity, cut-grass, floral, hay-like, almond, apple, and artichoke for the virgin olive oil olfactory profile; pungent, bitter, greasy, and sweet to define the virgin olive oil taste notes.

The intensity of those sensations was graded using a line scale and thus converted into a numerical score by measuring the position of the placed mark along a $10 \mathrm{~cm}$ line. The results were calculated as the medianamong assessor sensory scores.

\subsection{Molecular markers}

The total genomic DNA was isolated from fresh, young leaves following a CTAB protocol originally developed by Murry and Tompson (1980) and further modified by Muzzalupo and Perri (2002). The olive trees were genotyped at 11 nuclear microsatellite loci, selected among those available in literature and proved to be suitable for the characterization and
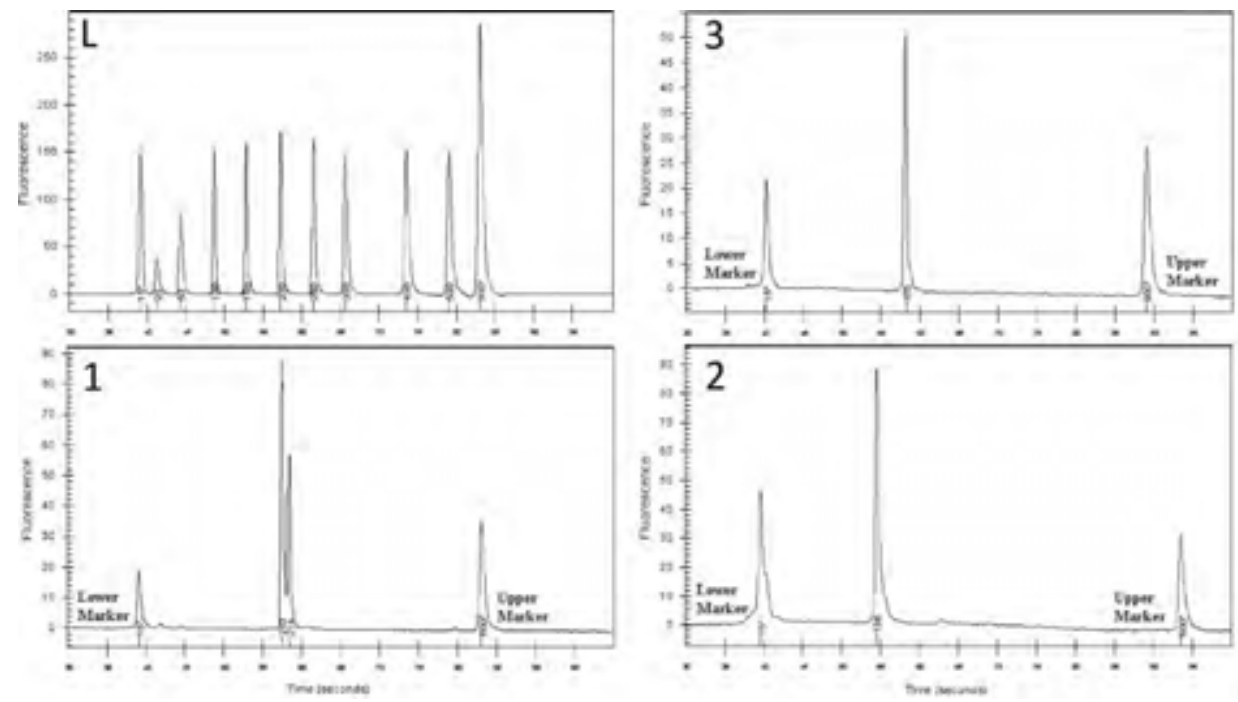

Figure 4. SSR profile of olive cultivar. The electropherograms were obtained using the 2100 Bioanalyzer running DNA 500 LabChips. DNA extracted from leaf. GAPU71A (1), UDO03 (2), UDO 39 (3), and Ladder $(\mathbf{L})$. The horizontal axis represents migration time of DNA fragments in seconds, and the vertical axis represents fluorescence. Upper and lower peaks in the electropherogram represent the internal DNA markers at 15 and $600 \mathrm{bp}$, which were used to size and quantify the PCR products 
identification of olive varieties in previous papers (Baldoni et al., 2009; Muzzalupo et al., 2009). The loci used were four (GAPU59, GAPU71A, GAPU71B and GAPU103A) among those described by Carriero et al. (2002) five (UDO01, UDO03, UDO12, UDO28 and UDO39) among those described by Cipriani et al. (2002) and two (DCA9 and DCA18) among those described by Sefc et al. (2000). Four loci of the selected markers (GAPU71B, GAPU103A, DCA9 and DCA18) were chosen from the common list reported by Baldoni et al. (2009) for use in a future comparison of data, while others (Gapu59, GAPU71A, UDO01, UDO03, UDO12, UDO28 and UDO39) were chosen as in other recent studies they were found to be very suitable for Italian intra-cultivars characterization and for characterization of olive germplasm collections (Muzzalupo et al., 2009; Muzzalupo et al., 2010). Electrophoresis and detection of PCR products were carried out according to Muzzalupo et al.(2007).

\section{Conclusion}

The morphological and molecular characterizations (elaiographic cards) are efficient for olive germplasm management, including the characterisation of varieties and the establishment of relationships between cultivars in the CRA-OLI olive collection. Beyond this identification, we constructed a data base that can be used to make a reference collection of Italian olive germplasm by comparing the morphological and molecular pattern of each identified varieties with samples from different areas.

Introducing new accessions by prospecting in different Mediterranean areas is currently in progress. The choice of which new varieties enter into the CRA-OLI collection can now take into account our results, both by avoiding duplicates and also by maximising genetic diversity. 


\section{Author details}

Innocenzo Muzzalupo

Agricultural Research Council - Olive Growing and Oil Industry Research Centre, Rende (CS), Italy

\section{Acknowledgement}

The elaiographic cards annex has been possible thanks to the contribution of time, energy and expertise by many individuals of CRA-OLI. The author would like to take this opportunity to acknowledge their generosity. The author thank the Projects: CERTOLIO, GERMOLI, OLIOPIU' and RGV-FAO for financial support.

\section{Annex - Elaiographic Cards}

Many CRA-OLI staff members and consultants have provided specific contributions to annexes:

- Alessandrino Maria, Boccuti Alfonso, Briccoli-Bati Caterina, Ciliberti Agostino, Cruceli Giuseppe, Godino Gianluca, Iannotta Nino, Lombardo Nicola, Madeo Alfreo, Muzzalupo Innocenzo, Pellegrino Massimiliano, Toscano Pietro, Turco Domenico, and Zaffina Francesco who contributed to the collection field;

- Alessandrino Maria, Boccuti Alfonso, Ciliberti Agostino, Cruceli Giuseppe, Godino Gianluca, Lombardo Nicola, Madeo Alfreo, Muzzalupo Innocenzo, Pellegrino Massimiliano, Turco Domenico, and Zaffina Francesco who contributed to the morphobioagronomic analysis;

- Fiorita Antonio, Longo Domenico, Parise Attilio, Ripoli Antonio, and Tucci Paolo who contributed to the extractionof monovarietal olive oil;

- Benincasa Cinzia, Muzzalupo Innocenzo, Parise Attilio, Patarino Alba, Pellegrino Massimiliano, and Perri Enzo who contributed to the biochemical analysis;

- Benincasa Cinzia, Godino Gianluca, Miranda Osvaldo, Muzzalupo Innocenzo, Pellegrino Massimiliano, Perri Enzo, Ripoli Antonio, Rizzuti Biagio, and Zaffina Francesco who contributed to the sensory analysis of monovarietal olive oil;

- Muzzalupo Innocenzo and Pellegrino Massimiliano who contributed to the molecular analysis;

- Alessandrino Maria and Muzzalupo Innocenzo who supported the preparation of design of the elaiografic card.

- Alessandrino Maria, Gianluca Godino, and Muzzalupo Innocenzo who provided photos of the olive varieties. 


\section{List of olive varieties}

\begin{tabular}{|c|c|c|c|c|c|c|c|}
\hline 1. & Abunara & 51. & Feglina & 101. & Nocellara etnea & 151. & Razzo \\
\hline 2. & Arnasca & 52. & Fosco & 102. & Nocellara messinese & 152. & Razzola \\
\hline 3. & Ascolana dura & 53. & Frangivento & 103. & Nociara & 153. & Riminino \\
\hline 4. & Ascolana semitenera & 54. & Frantoio & 104. & Nolca & 154. & Ritonnella \\
\hline 5. & Ascolana tenera & 55. & Gaggiolo & 105. & Nostrale di Fiano Romano & 155. & Rizzitella \\
\hline 6. & Augellina & 56. & Gentile di Chieti & 106. & Nostrale di Rigali & 156. & Romanella molisana \\
\hline 7. & Aurina & 57. & Gentile di Larino & 107. & Nostrana di Brisighella & 157. & Rosciola Coltodino \\
\hline 8. & Bianchera & 58. & Gentile nera di Colletorto & 108. & Ogliara & 158. & Rosciola di Rotello \\
\hline 9. & Borgese & 59. & Geracese & 109. & Ogliarola barese & 159. & Rosciola di Venafro \\
\hline 10. & Borgiona & 60. & Giarfara & 110. & Ogliarola del Vulture & 160. & Rosciola \\
\hline 11. & Bosana & 61. & Giarraffa & 111. & Ogliarola messinese & 161. & Rotondella di Sanza \\
\hline 12. & Brandofino & 62. & Giusta & 112. & Ogliastro grande & 162. & Salvia \\
\hline 13. & Buscionetto & 63. & Gnagnaro & 113. & Olivago & 163. & Sammartinara \\
\hline 14. & Cacaredda & 64. & Grappolo & 114. & Olivastra seggianese & 164. & Sammartinenga \\
\hline 15. & Calatina & 65. & Grignan & 115. & Olivastro di Bucchianico & 165. & San Benedetto \\
\hline 16. & Canino & 66. & Grossa di Cassano & 116. & Olivastro frentano & 166. & San Francesco \\
\hline 17. & Carboncella & 67. & Grossa di Spagna & 117. & Olivo da olio & 167. & Santa Caterina \\
\hline 18. & Carbonchia & 68. & Grossa di Venafro & 118. & Olivo da salare & 168. & Santa Maria \\
\hline 19. & Cariasina & 69. & Grossale & 119. & Olivo della Madonna & 169. & Sant'Agatese \\
\hline 20. & Carmelitana & 70. & I 77 & 120. & Orbetana & 170. & Sant'Agostino \\
\hline 21. & Carolea & 71. & Imperiale & 121. & Ortice & 171. & Santomauro \\
\hline 22. & Carpinetana & 72. & Intosso & 122. & Ortolana & 172. & Sargano di Fermo \\
\hline 23. & Castiglionese & 73. & Itrana & 123. & Ottobratica & 173. & Sargano di San Benedetto \\
\hline 24. & Castricianella rapparina & 74. & Laurina & 124. & Ottobrina & 174. & Scarpetta \\
\hline 25. & Cavalieri & 75. & Lavagnina & 125. & Paesana bianca & 175. & Semidana \\
\hline 26. & Cazzinicchio & 76. & Leccino & 126. & Paesana nera & 176. & Sessana \\
\hline 27. & Cellina di Nardò & 77. & Lezze & 127. & Palmarola & 177. & Simona \\
\hline 28. & Cellina di Rotello & 78. & Lumiaru & 128. & Pasola & 178. & Sinopolese \\
\hline 29. & Cerasuola & 79. & Maiatica di Ferrandina & 129. & Passulunara & 179. & Sirole \\
\hline 30. & Ciciariello & 80. & Marina & 130. & Pennulara & 180. & Sperone di gallo \\
\hline 31. & Cima di Melfi & 81. & Marzio & 131. & Peranzana & 181. & Termite di Bitetto \\
\hline 32. & Colombina & 82. & Mignola & 132. & Perciasacchi & 182. & Terza grande \\
\hline 33. & Coratina & 83. & Mignolo & 133. & Piangente & 183. & Tombarello \\
\hline 34. & Corneglia & 84. & Minna di vacca & 134. & Piantone di Falerone & 184. & Tonda di Alife \\
\hline 35. & Cornia & 85. & Monaca & 135. & Piantone di Mogliano & 185. & Tonda di Filadelfia \\
\hline 36. & Corniola & 86. & Mora & 136. & Pidicuddara & 186. & Tonda di Filogaso \\
\hline 37. & Corniolo & 87. & Moraiolo "T. Corsini" & 137. & Pignola & 187. & Tonda di Strongoli \\
\hline 38. & Coroncina & 88. & Moraiolo & 138. & Pisciottana & 188. & Tonda dolce di Partanna \\
\hline 39. & Corsicana da olio & 89. & Morchiaio & 139. & Pizzè' carroga & 189. & Tonda iblea \\
\hline 40. & Crognalegna & 90. & Moresca & 140. & Pizzutella & 190. & Tondina \\
\hline 41. & Cucca & 91. & Morinello & 141. & Posola & 191. & Tortiglione \\
\hline 42. & Dolce Agogia & 92. & Nasitana frutto grosso & 142. & Posolella & 192. & Toscanina \\
\hline 43. & Dolce d'Andria & 93. & Nebba & 143. & Provenzale & 193. & Tunnulidda \\
\hline 44. & Dolce di Cassano & 94. & Nebbia & 144. & Puntella & 194. & Vaddara \\
\hline 45. & Dolce di Rossano & 95. & Nebbio di Chieti & 145. & Racioppa & 195. & Vallanella \\
\hline 46. & Dritta & 96. & Nebbio di Pescara & 146. & Racioppella & 196. & Verdello \\
\hline 47. & Erbano & 97. & Negrera & 147. & Raggiola & 197. & Vocio \\
\hline 48. & Faresana & 98. & Nera di Cantinella & 148. & Raja sabina & 198. & Zaituna \\
\hline 49. & Favarol & 99. & Nera di Gonnos & 149. & Ravece & 199. & Zimbimbo \\
\hline 50. & Fecciaro & 100. & Nocellara del Belice & 150. & Raza & 200. & Zinzifarica \\
\hline
\end{tabular}




\section{References}

Baldoni, L.; Cultrera, G.; Mariotti, R.; Ricciolini, C.; Arcioni, S.; Vendramin, G.; Buonamici, A.; Porceddu, A.; Sarri, V.; Ojeda, M.; Trujillo, I.; Rallo, L.; Belaj, A.; Perri, E.; Muzzalupo, I.; Salimonti, A.; Casagrande, A.; Lain, O.; Messina, R. \& Testolin, R. (2009). A common protocol for olive genotyping using microsatellite DNA: a consensus list of markers. Molecular Breeding, Vol.24, pp. 213-231, ISSN 1380-3743.

Bendini, A.; Cerretani, L.; Di Virgilio, F.; Belloni, P.; Lercker, G. \& Gallina Toschi, T. (2007). In-process monitoring in industrial olive mill by means of near infrared spectroscopy (FT-NIR).European Journal of Lipid Science and Technology, Vol.109, pp. 498-504, ISSN 0308-8146.

Carriero, F.; Fontanazza, G.; Cellini, F. \& Giorio, G. (2002). Identification of simple sequence repeats (SSRs) in olive (Olea europaea L).Theoretical and Applied Genetics, Vol.104, pp. 301307, ISSN 0040-5752.

Cipriani, G., Marazzo, M.; Marconi, R.; Cimato, A. \& Testolin, R. (2002). Microsatellite markers isolated in olive (Olea europaeaL.) are suitable for individual fingerprinting and reveal polymorphism within ancient cultivars. Theoretical and Applied Genetics, Vol.104, pp. 223-228, ISSN 0040-5752.

COI (1997). Méthodologie pour la characterisation des varétés d'oliviers. Projet sur la conservation, characterisation collecte et utilisation des resources génétiques de l'olivier. Communauté Européenne. Conseil Oléicole International, ISSN 0255-9978.

E.U. Off. J. Eur. Communities. (2011). Regulation No 61/11 amending Regulation (EEC) No $2568 / 91$ on the characteristics of olive oil and olive-pomace oil and on the relevant methods of analysis modifies the CEE n. 2568/91 on olive oils and pomace olive oils characteristics and relative analysis methods.Official Journal of the European Union .L 23/1, ISSN 1725-258X.

Murry, M.G. \& Tompson, W.F. (1980).Rapid isolation of high molecular weight plant DNA.Nucleic Acids Res. Vol.8, pp. 4321-4325, ISSN 0305-1048.

Muzzalupo, I. \& Perri, E. (2002).Recovery and characterization of DNA from virgin olive oil.European Food Research and Technology, Vol.214, pp. 528-531, ISSN 1438-2377.

Muzzalupo, I.; Chiappetta, A.; Benincasa, C. \& Perri, E. (2010).Intra cultivar variability of three major olive cultivars grown in different areas of central-southern Italy and studied using microsatellite markers.Scientia Horticulturae, Vol.126, pp. 324-329, ISSN 0304-4238.

Muzzalupo, I.; Pellegrino, M. \& Perri, E. (2007). Detection of DNA in virgin olive oils extracted from destoned fruits. European Food Research and Technology, Vol.224, pp. 469475, ISSN 1438-2377.

Muzzalupo, I.; Stefanizzi, F. \& Perri, E. (2009). Evaluation of olives cultivated in Southern Italy by SSR Markers. HortScience Vol.44, pp. 582-588, ISSN 0018-5345.

Sefc, KM.; Lopes, MS.; Mendonca, D.; Rodrigues dos Santos, M. \& Laimer da Camara Machado, A. (2000).Identification of microsatellite loci in olive (Olea europaea L.) and 
their characterization in Italian and Iberian olive trees.Molecular Ecology, Vol.9, pp. 11711173, ISSN 0962-1083.

Stone, H. \&Sidel, J.; (2004).Sensory evaluation practices. Food Science and Technology, International Series, ISBN 0-12-672690-6. 



\section{"Abunara"}

(synonymy: Marsalesa, Marsalese, Marsalisa, Nuciddara, etc.)

Areal distribution or origin area: Sicilia

Flesh/pit weight ratio: medium $(7,18 \pm 0,85)$

Oil content (\%): medium $(44,06 \pm 3,11)$

Purpose: table

\section{Morphological characters}

Tree characters

Vigour: strong

Growth habit: spreading-erect

Canopy-density: dense

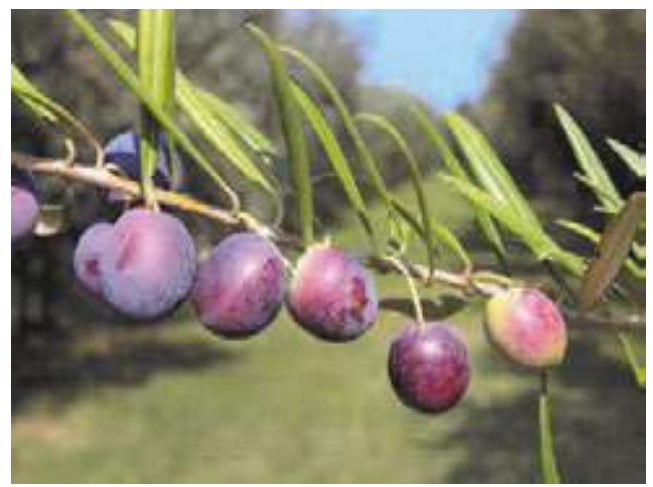

\section{Leaf characters}

Blade length $(\mathrm{cm})$ : medium $(6,36 \pm 0,54)$

Blade width $(\mathrm{cm})$ : medium $(1,46 \pm 0,12)$

Shape (length/width): elliptic-lanceolate

\section{Inflorescence characters}

Inflorescence length (cm): medium $(2,62 \pm 0,88)$

Number of flowers: low $(16,31 \pm 1,66)$

\section{Fruit characters}

Fresh weight of 100 fruits (g): very high $(6,28 \pm 0,45)$

Shape (length/width): spherical

Symmetry: slightly asymmetric

Position of maximum transverse diameter:

central

Apex: rounded

Base: rounded

Niplle: absent

Lenticels: few and large

\section{Pit characters}

Weight of 100 pits (g): very high $(0,77 \pm 0,03)$

Shape (length/width): elliptic

Mucron: obvious

Symmetry: slightly asymmetric

Position of maximum transverse diameter:

towards apex

Apex: rounded

Base: rounded

Surface: scabrous

Number of grooves: medium
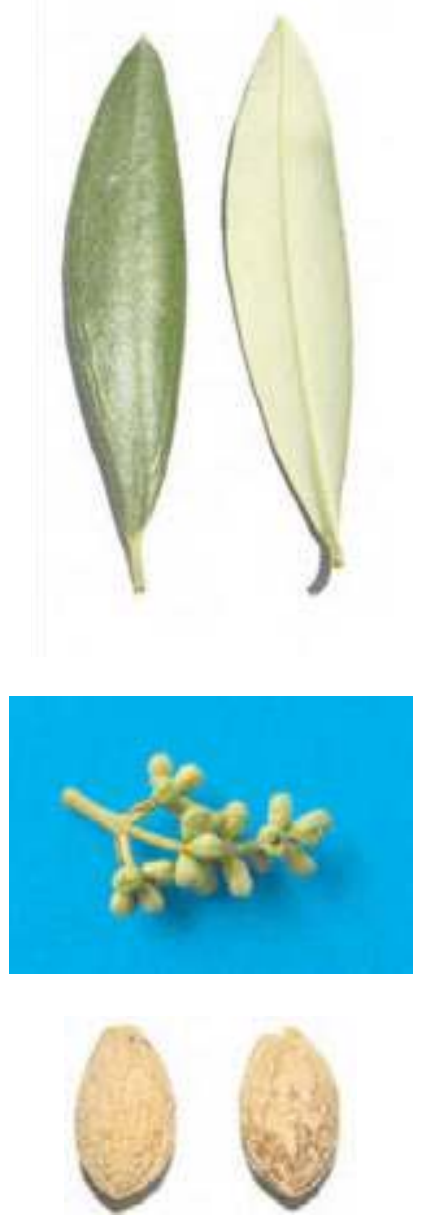


\section{Biochemical Characters}

\section{Fatty Acid Composition}

Table 1. Average values (express in $\% \pm$ standard deviations) of the fatty acids methyl esters and nutritional ratios obtained from single cultivar olive oils.

\begin{tabular}{|c|c|c|c|c|c|}
\hline Myristic acid & $0,01 \pm 0,00$ & Linoleic acid $(\omega 6)$ & $8,42 \pm 0,14$ & Lignoceric acid & $0,03 \pm 0,01$ \\
\hline Palmitic acid & $13,26 \pm 0,25$ & Linolenic acid $(\omega 3)$ & $0,75 \pm 0,03$ & & \\
\hline Palmitoleic acid & $1,30 \pm 1,13$ & Arachic acid & $0,26 \pm 0,05$ & Unsat./satured & $5,55 \pm 0,14$ \\
\hline Stearic acid & $1,62 \pm 0,10$ & Eicosenoic acid & $0,02 \pm 0,00$ & $\omega 6 / \omega 3$ & $11,23 \pm 0,67$ \\
\hline Oleic acid & $73,13 \pm 0,47$ & Behenic acid & $0,07 \pm 0,01$ & & \\
\hline
\end{tabular}

\section{Organoleptic oil values}

Sensory Analysis (Panel test)

Comment: fruity medium-light, with taste of almond. Balanced taste sensation with medium-light bitter and spicy. Medium-light fluidity.

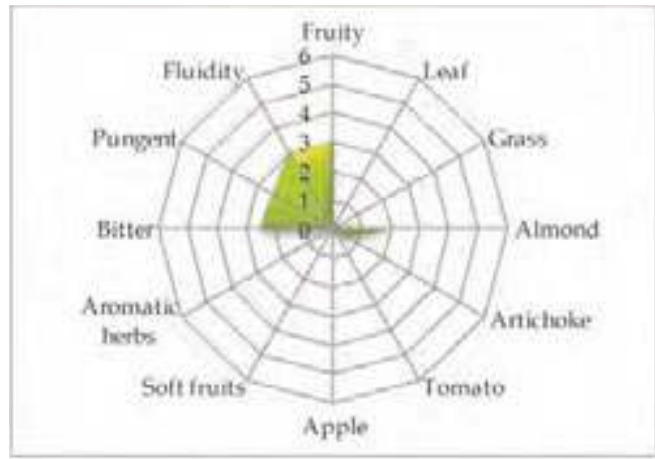

\section{Molecular Markers}

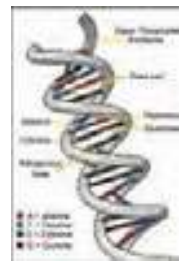

Table 2. Microsatellites (SSR) loci analyzed.

For each locus the allele size (expressed in base pairs) is reported.

\begin{tabular}{|c|c|c|c|c|c|}
\hline DCA09 & DCA18 & GAPU59 & GAPU71A & $G U P A 71 B$ & GAPU103A \\
\hline $162-162$ & $179-181$ & $208-212$ & $210-210$ & $124-124$ & $159-170$ \\
\hline UDO01 & UDO03 & UDO12 & UDO28 & UDO39 & \\
\hline $144-144$ & $143-143$ & $177-177$ & $182-182$ & $108-108$ & \\
\hline
\end{tabular}

\section{References:}

1 - Bottari V., Spina P. In: Le varietà di olivo coltivate in Sicilia, Ann. Sper. Agr. (1953), 7: pp. 937-1004.

2 - Muzzalupo I., Stefanizzi F., Perri E. HortScience, (2009), 44: pp. 582-588. 


\section{"Arnasca"}

(synonymy: Pignola di Arnasco)

Areal distribution or origin area: Liguria

Flesh/pit weight ratio: medium $(5,12 \pm 1,13)$

Oil content (\%): medium $(41,79 \pm 1,16)$

Purpose: oil

\section{Morphological characters}

Tree characters

Vigour: medium-weak

Growth habit: drooping

Canopy-density: dense

\section{Leaf characters}

Blade length (cm): medium $(6,38 \pm 0,56)$

Blade width $(\mathrm{cm})$ : medium $(\mathbf{1}, \mathbf{4 2} \pm \mathbf{0 , 1 1})$

Shape (length/width): elliptic-lanceolate

\section{Inflorescence characters}

Inflorescence length (cm): short $(2,20 \pm 1,35)$

Number of flowers: medium $(19,76 \pm 2,96)$

\section{Fruit characters}

Fresh weight of 100 fruits $(\mathrm{g})$ : medium $(2,09 \pm 0,52)$

Shape (length/width): ovoid

Symmetry: slightly

Position of maximum transverse diameter:

central

Apex: rounded

Base: rounded

Niplle: absent

Lenticels: few and small

\section{Pit characters}

Weight of 100 pits (g): medium $(0,34 \pm \mathbf{0 , 0 5})$

Shape (length/width): ovoid

Mucron: obvious

Symmetry: slightly asymmetric

Position of maximum transverse diameter:

central

Apex: rounded

Base: rounded

Surface: rugose

Number of grooves: low
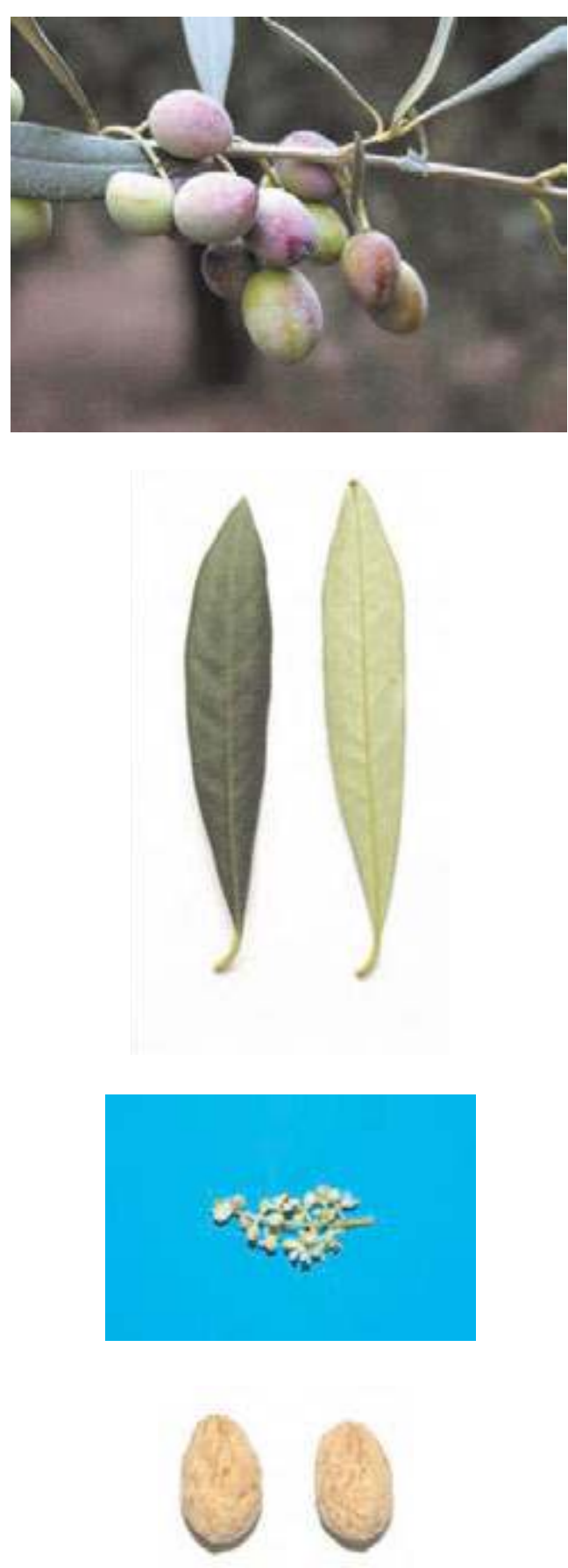


\section{Biochemical Characters}

\section{Fatty Acid Composition}

Table 1. Average values (express in $\% \pm$ standard deviations) of the fatty acids methyl esters and nutritional ratios obtained from single cultivar olive oils.

\begin{tabular}{|c|c|c|c|c|c|}
\hline Myristic acid & $0,01 \pm 0,01$ & Linoleic acid $(\omega 6)$ & $7,28 \pm 0,01$ & Lignoceric acid & $0,06 \pm 0,03$ \\
\hline Palmitic acid & $13,08 \pm 0,17$ & Linolenic acid $(\omega 3)$ & $0,50 \pm 0,04$ & & \\
\hline Palmitoleic acid & $1,47 \pm 0,00$ & Arachic acid & $0,31 \pm 0,16$ & Unsat./satured & $5,39 \pm 0,03$ \\
\hline Stearic acid & $2,04 \pm 0,11$ & Eicosenoic acid & $0,33 \pm 0,05$ & $\omega 6 / \omega 3$ & $14,59 \pm 1,24$ \\
\hline Oleic acid & $73,52 \pm 0,52$ & Behenic acid & $0,11 \pm 0,01$ & & \\
\hline
\end{tabular}

\section{Organoleptic oil values}

Sensory Analysis (Panel test)

Comment: fruity medium, with hints of artichoke and almond. Balanced in flavours, with hints of bitter and spicy medium intensity. Medium fluidity.

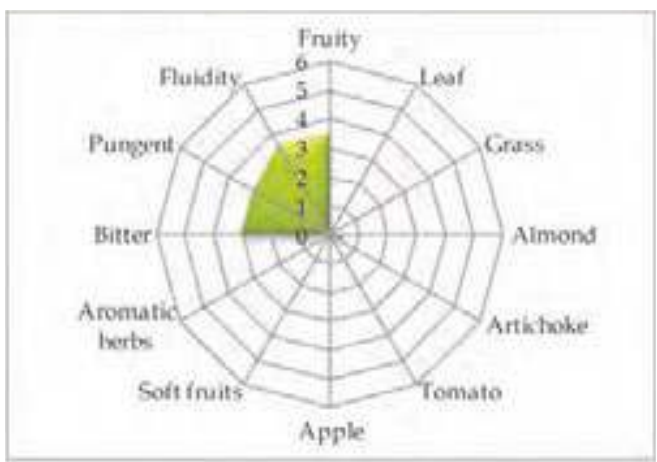

\section{Molecular Markers}

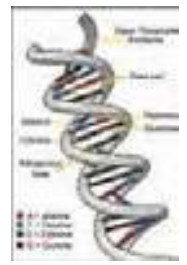

Table 2. Microsatellites (SSR) loci analyzed.

For each locus the allele size (expressed in base pairs) is reported.

\begin{tabular}{|c|c|c|c|c|c|}
\hline DCA09 & DCA18 & GAPU59 & GAPU71A & $G U P A 71 B$ & GAPU103A \\
\hline $162-176$ & $177-179$ & $212-212$ & $214-218$ & $130-144$ & $170-184$ \\
\hline UDO01 & UDO03 & UDO12 & UDO28 & UDO39 & \\
\hline $140-140$ & $135-135$ & $164-177$ & $161-205$ & $213-232$ & \\
\hline
\end{tabular}

\section{References:}

1 - Cimato A., Cantini C., Sani G. In: Atti $4^{\circ}$ Convegno Nazionale sulla Biodiversità, Alghero (2000), pp. 497-500.

2 - Muzzalupo I., Lombardo N., Salimonti A., et al. Adv. Hort. Sci. (2008), 22(2): pp. 142-148. 


\section{"Ascolana dura"}

(synonymy: Ascolana semidura, Gentile, Oliva di San Francesco, etc.)

Areal distribution or origin area: Marche

Flesh/pit weight ratio: medium $(6,79 \pm 0,24)$

Oil content (\%): medium $(46,31 \pm 0,13)$

Purpose: table

\section{Morphological characters}

Tree characters

Vigour: medium

Growth habit: spreading

Canopy-density: medium-dense

\section{Leaf characters}

Blade length $(\mathrm{cm})$ : medium $(6,19 \pm 0,37)$

Blade width $(\mathrm{cm})$ : medium $(\mathbf{1 , 1 8} \pm \mathbf{0 , 1 0})$

Shape (length/width): elliptic-lanceolate

\section{Inflorescence characters}

Inflorescence length (cm): medium $(2,87 \pm 0,50)$

Number of flowers: low $(\mathbf{1 4}, 72 \pm 0,52)$

\section{Fruit characters}

Fresh weight of 100 fruits $(\mathrm{g})$ : high $(\mathbf{5 , 1 5} \pm \mathbf{0 , 6 0})$

Shape (length/width): spherical

Simmetry: slightly asymmetric

Position of maximum transverse diameter:

central

Apex: rounded

Base: truncate

Niplle: tenuous

Lenticels: many and small

\section{Pit characters}

Weight of 100 pits (g): high $(0,67 \pm 0,09)$

Shape (length/width): ovoid

Mucron: obvious

Simmetry: slightly asymmetric

Position of maximum transverse diameter:

central

Apex: rounded

Base: rounded

Surface: rugose

Number of grooves: medium
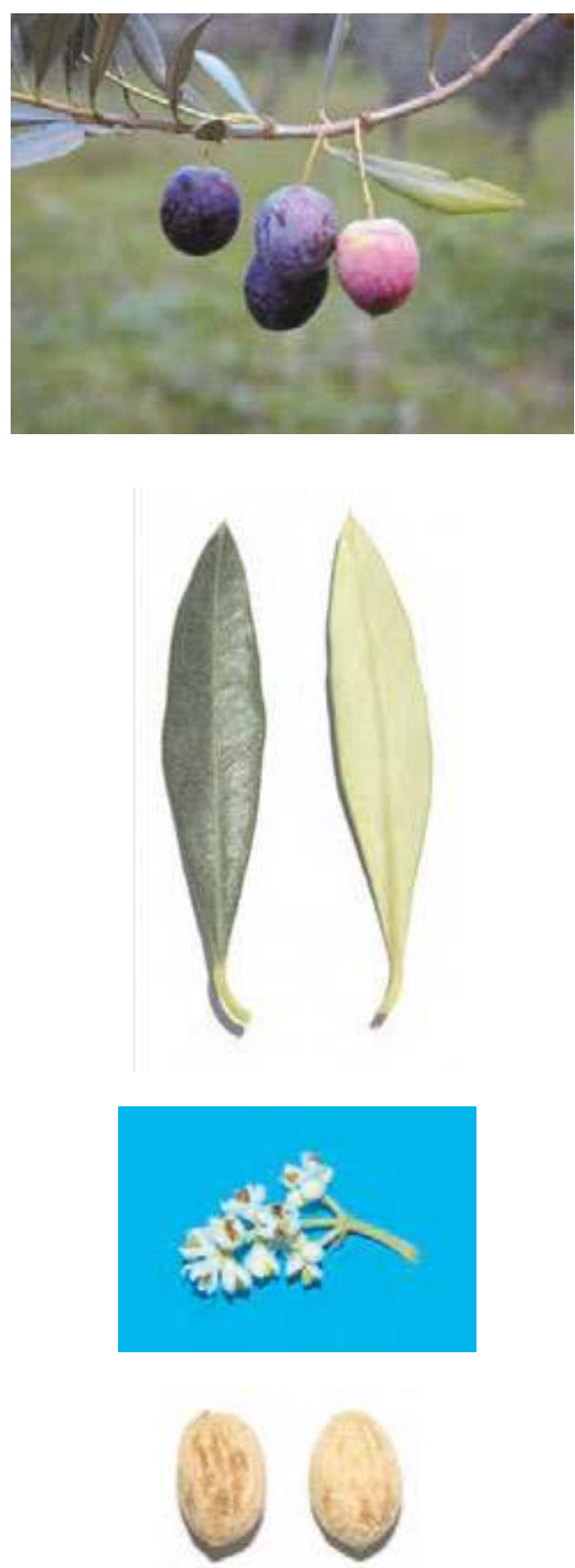


\section{Biochemical Characters}

\section{Fatty Acid Composition}

Table 1. Average values (express in $\% \pm$ standard deviations) of the fatty acids methyl esters and nutritional ratios obtained from single cultivar olive oils.

\begin{tabular}{|c|c|c|c|c|c|}
\hline Myristic acid & $0,01 \pm 0,00$ & Linoleic acid $(\omega 6)$ & $10,92 \pm 0,04$ & Lignoceric acid & $0,05 \pm 0,01$ \\
\hline Palmitic acid & $15,05 \pm 0,02$ & Linolenic acid $(\omega 3)$ & $0,78 \pm 0,01$ & & \\
\hline Palmitoleic acid & $3,07 \pm 0,04$ & Arachic acid & $0,19 \pm 0,01$ & Unsat./satured & $4,76 \pm 0,03$ \\
\hline ea & $1,64 \pm 0,04$ & Eicosenoic acid & $0,02 \pm 0,00$ & $\omega 6 / \omega 3$ & $14,00 \pm 0,30$ \\
\hline leic acid & $65,48 \pm 0,52$ & Behenic acid & $0,07 \pm 0,01$ & & \\
\hline
\end{tabular}

\section{Organoleptic oil values}

Sensory Analysis (Panel test)

Comment: fruity medium-high, with good sensation of grass, artichoke and tomato. Balanced in flavours, with hints of bitter and spicy medium-high intensity. Medium-high fluidity.

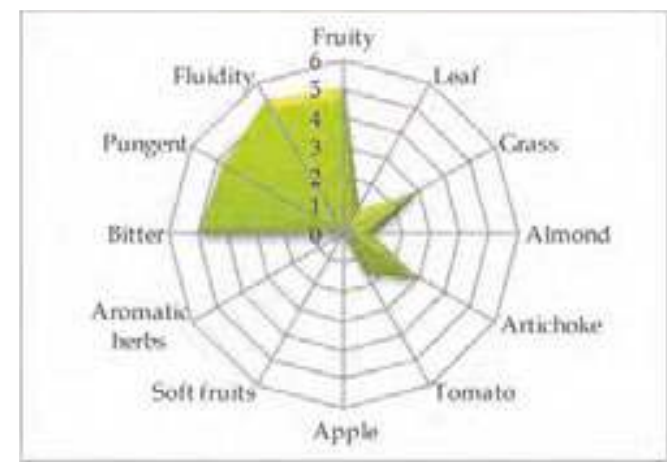

\section{Molecular Markers}

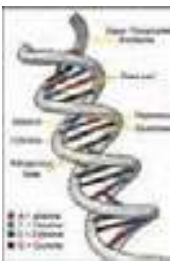

Table 2. Microsatellites (SSR) loci analyzed.

For each locus the allele size (expressed in base pairs) is reported.

\begin{tabular}{|c|c|c|c|c|c|}
\hline DCA09 & DCA18 & GAPU59 & GAPU71A & $G U P A 71 B$ & GAPU103A \\
\hline $198-206$ & $177-181$ & $214-222$ & $214-224$ & $124-130$ & $136-157$ \\
\hline UDO01 & UDO03 & UDO12 & UDO28 & UDO39 & \\
\hline $140-140$ & $143-143$ & $166-166$ & $182-210$ & $200-200$ & \\
\hline
\end{tabular}

\section{References:}

1 - Pannelli G., Alfei B., Santinelli A. In: Varietà di olivo nelle Marche, ASSAM (2001), pp. 13-16.

2 - Muzzalupo I., Lombardo N., Salimonti A., et al. Adv. Hort. Sci. (2008), 22(2): pp. 142-148. 


\section{“Ascolana semitenera "}

Areal distribution or origin area: Marche

Flesh/pit weight ratio: medium $(5,91 \pm 0,21)$

Oil content (\%): medium $(45,27 \pm 1,48)$

Purpose: table

\section{Morphological characters}

Tree characters

Vigour: medium

Growth habit: spreading-erect

Canopy-density: medium

\section{Leaf characters}

Blade length $(\mathrm{cm})$ : medium $(6,20 \pm 0,34)$

Blade width $(\mathrm{cm})$ : medium $(\mathbf{1 , 2 0} \pm \mathbf{0 , 0 1})$

Shape (length/width): elliptic-lanceolate

\section{Inflorescence characters}

Inflorescence length ( $\mathrm{cm})$ : long $(3,54 \pm 2,20)$

Number of flowers: medium $(\mathbf{1 8 , 8 1} \pm 0,53)$

\section{Fruit characters}

Fresh weight of 100 fruits $(\mathrm{g})$ : high $(\mathbf{4}, 74 \pm \mathbf{0 , 3 3})$

Shape (length/width): spherical

Symmetry: slightly asymmetric

Position of maximum transverse diameter:

central

Apex: rounded

Base: truncate

Niplle: tenuous

Lenticels: many and small

\section{Pit characters}

Weight of 100 pits (g): high $(\mathbf{0 , 6 8} \pm \mathbf{0 , 0 8})$

Shape (length/width): ovoid

Mucron: obvious

Symmetry: slightly asymmetric

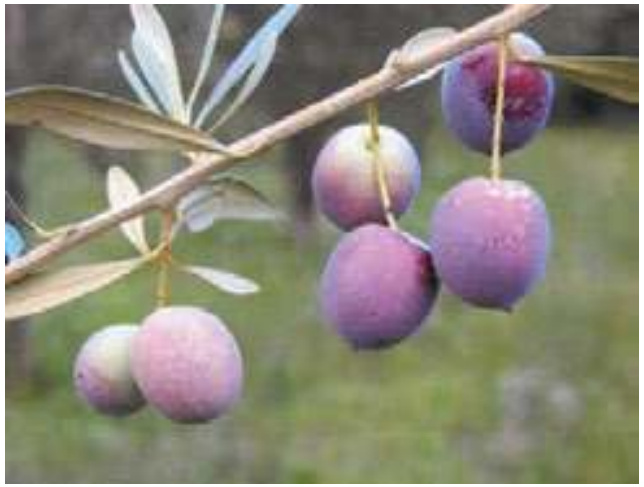

Position of maximum transverse diameter:

central

Apex: rounded

Base: rounded

Surface: rugose

Number of grooves: medium
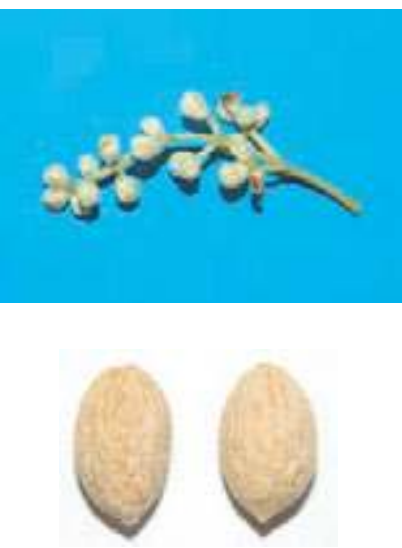


\section{Biochemical Characters}

\section{Fatty Acid Composition}

Table 1. Average values (express in $\% \pm$ standard deviations) of the fatty acids methyl esters and nutritional ratios obtained from single cultivar olive oils.

$\begin{array}{llllll}\text { Myristic acid } & \mathbf{0 , 0 1} \pm \mathbf{0 , 0 0} & \text { Linoleic acid }(\omega 6) & \mathbf{9 , 7 7} \pm \mathbf{0 , 0 9} & \text { Lignoceric acid } & \mathbf{0 , 0 4} \pm \mathbf{0 , 0 1} \\ \text { Palmitic acid } & \mathbf{1 2 , 1 3} \pm \mathbf{0 , 2 4} & \text { Linolenic acid }(\omega 3) \mathbf{0 , 7 9} \pm \mathbf{0 , 0 2} & & \\ \text { Palmitoleic acid } & \mathbf{3 , 5 2} \pm \mathbf{0 , 2 2} & \text { Arachic acid } & \mathbf{0 , 2 2} \pm \mathbf{0 , 0 6} & \text { Unsat./satured } & \mathbf{5 , 9 3} \pm \mathbf{0 , 0 9} \\ \text { Stearic acid } & \mathbf{1 , 6 1} \pm \mathbf{0 , 1 1} & \text { Eicosenoic acid } & \mathbf{0 , 0 3} \pm \mathbf{0 , 0 1} & \omega 6 / \omega 3 & \mathbf{1 2 , 3 5} \pm \mathbf{0 , 5 0} \\ \text { Oleic acid } & \mathbf{6 8 , 5 9} \pm \mathbf{0 , 1 0} & \text { Behenic acid } & \mathbf{0 , 0 8} \pm \mathbf{0 , 0 0} & & \end{array}$

\section{Organoleptic oil values}

Sensory Analysis (Panel test)

Comment: fruity medium-high, with good sensation of grass, artichoke and tomato. Balanced in flavors, with hints of bitter and spicy medium-high intensity. Medium-high fluidity.

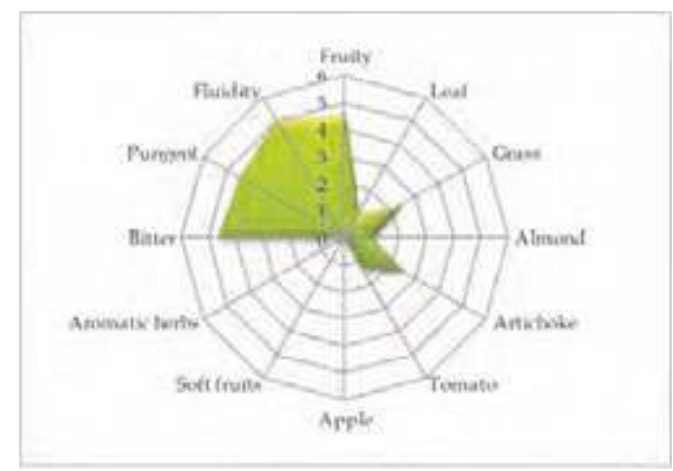

\section{Molecular Markers}

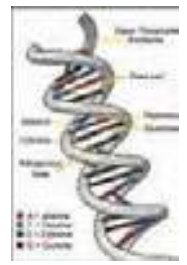

Table 2. Microsatellites (SSR) loci analyzed.

For each locus the allele size (expressed in base pairs) is reported.

$\begin{array}{cccccc}D C A 09 & \text { DCA18 } & \text { GAPU59 } & \text { GAPU71A } & \text { GUPA71B } & \text { GAPU103A } \\ \mathbf{1 9 8} \mathbf{- 2 0 6} & \mathbf{1 7 7 - \mathbf { 1 8 1 }} & \mathbf{2 1 4} \mathbf{- 2 2 2} & \mathbf{2 1 4} \mathbf{- 2 2 4} & \mathbf{1 2 4} \mathbf{- 1 3 0} & \mathbf{1 3 6} \mathbf{- 1 5 7} \\ \text { UDOO1 } & \text { UDOO3 } & \text { UDO12 } & \text { UDO28 } & \text { UDO39 } & \\ \mathbf{1 4 0 - \mathbf { 1 4 0 }} & \mathbf{1 3 5 - \mathbf { 1 3 5 }} & \mathbf{1 6 6 - \mathbf { 1 6 6 }} & \mathbf{1 8 2} \mathbf{- 2 1 0} & \mathbf{2 0 0 - \mathbf { 2 3 2 }} & \end{array}$

\section{References:}

1 - Jacoboni N., Fontanazza G. In: Le Cultivar, Ed. Reda l'Olivo (1981), pp. 9-52.

2 - Muzzalupo I., Lombardo N., Salimonti A., et al. Adv. Hort. Sci. (2008), 22(2): pp. 142-148. 


\section{"Ascolana tenera"}

(synonymy: Noce, Oliva noce, Oliva grossa, etc.)

Areal distribution or origin area: Marche

Flesh/pit weight ratio: high $(\mathbf{9 , 3 0} \pm \mathbf{0 , 1 6})$

Oil content (\%): medium $(43,22 \pm 1,96)$

Purpose: table

\section{Morphological characters}

Tree characters

Vigour: medium-strong

Growth habit: spreading-erect

Canopy-density: medium-dense

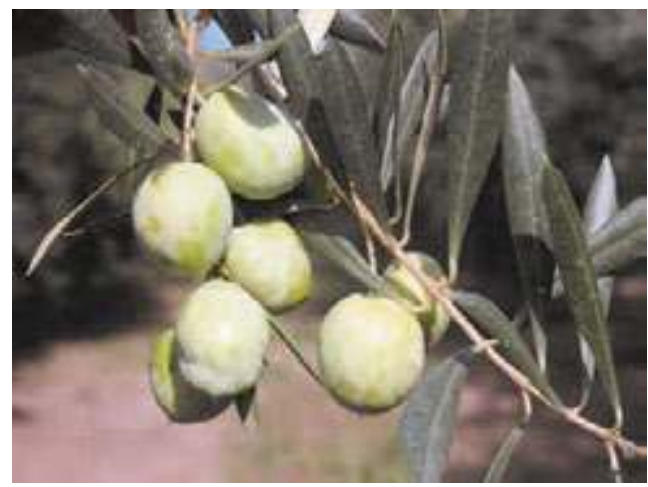

\section{Leaf characters}

Blade length (cm): medium $(5,79 \pm 0,53)$

Blade width $(\mathrm{cm})$ : medium $(1,42 \pm 0,19)$

Shape (length/width): elliptic-lanceolate

\section{Inflorescence characters}

Inflorescence length (cm): short $(2,49 \pm 1,86)$

Number of flowers: low $(16,76 \pm 1,01)$

\section{Fruit characters}

Fresh weight of 100 fruits (g): very high $(7,04 \pm 1,27)$

Shape (length/width): ovoid

Symmetry: slightly asymmetric

Position of maximum transverse diameter:

central

Apex: rounded

Base: rounded

Niplle: tenuous

Lenticels: few and small

\section{Pit characters}

Weight of 100 pits (g): high $(\mathbf{0 , 6 6} \pm \mathbf{0 , 1 4})$

Shape (length/width): elliptic

Mucron: obvious

Symmetry: symmetric

Position of maximum transverse diameter:

towards apex

Apex: pointed

Base: pointed

Surface: rugose

Number of grooves: high
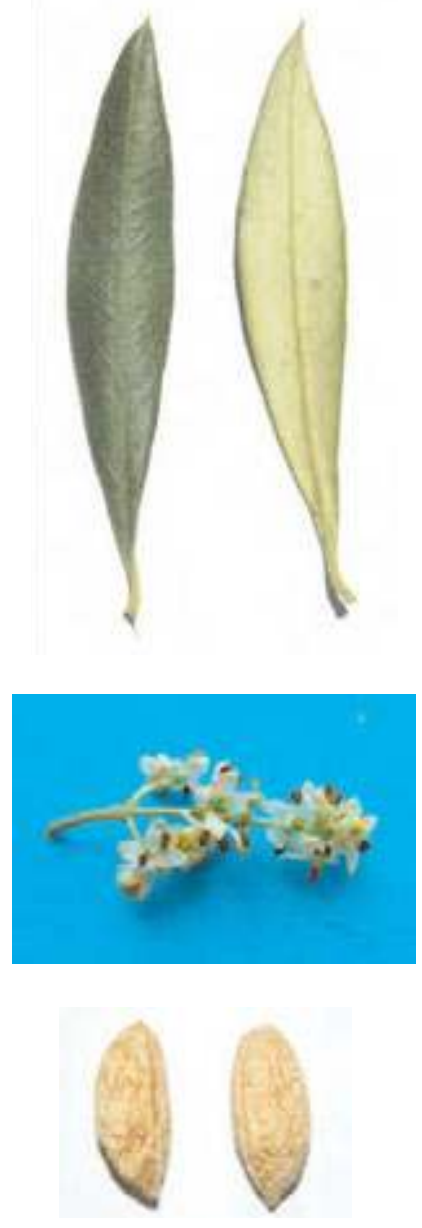


\section{Biochemical Characters}

\section{Fatty Acid Composition}

Table 1. Average values (express in $\% \pm$ standard deviations) of the fatty acids methyl esters and nutritional ratios obtained from single cultivar olive oils.

$\begin{array}{llllll}\text { Myristic acid } & \mathbf{0 , 0 1} \pm \mathbf{0 , 0 0} & \text { Linoleic acid }(\omega 6) & \mathbf{4 , 9 8} \pm \mathbf{0 , 6 4} & \text { Lignoceric acid } & \mathbf{0 , 0 7 \pm 0 , 0 1} \\ \text { Palmitic acid } & \mathbf{1 0 , 6 5 \pm \mathbf { 1 , 0 0 }} & \text { Linolenic acid }(\omega 3) & \mathbf{0 , 6 6} \pm \mathbf{0 , 0 4} & & \\ \text { Palmitoleic acid } & \mathbf{1 , 5 1} \pm \mathbf{0 , 1 6} & \text { Arachic acid } & \mathbf{0 , 0 3} \pm \mathbf{0 , 0 1} & \text { Unsat./satured } & \mathbf{6 , 8 1} \pm \mathbf{0 , 8 2} \\ \text { Stearic acid } & \mathbf{1 , 7 9} \pm \mathbf{0 , 1 6} & \text { Eicosenoic acid } & \mathbf{0 , 0 2} \pm \mathbf{0 , 0 0} & \omega 6 / \omega 3 & \mathbf{7 , 5 5} \pm \mathbf{0 , 1 5} \\ \text { Oleic acid } & \mathbf{7 8 , 9 4 \pm \mathbf { 1 , 0 6 }} & \text { Behenic acid } & \mathbf{0 , 0 6} \pm \mathbf{0 , 0 3} & & \end{array}$

\section{Organoleptic oil values}

Sensory Analysis (Panel test)

Comment: medium fruity, with good sensation of grass, less almond and artichoke. Balanced taste sensation, with bitter and spicy medium. Medium fluidity.

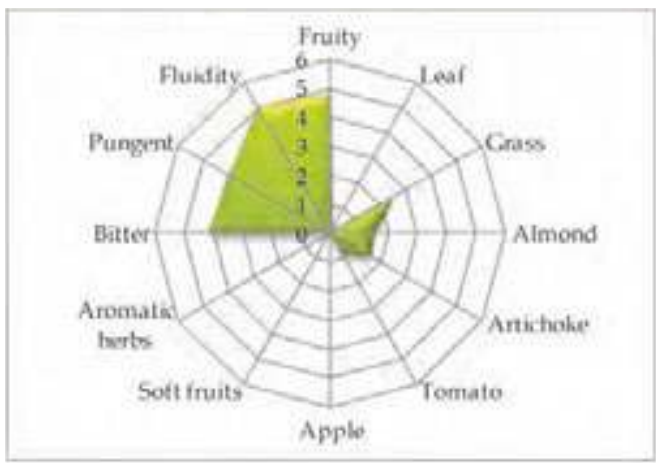

\section{Molecular Markers}

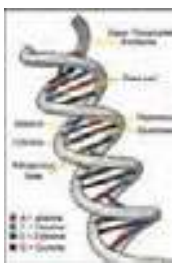

Table 2. Microsatellites (SSR) loci analyzed.

For each locus the allele size (expressed in base pairs) is reported.

\begin{tabular}{|c|c|c|c|c|c|}
\hline DCA09 & DCA18 & GAPU59 & GAPU71A & $G U P A 71 B$ & GAPU103A \\
\hline $198-206$ & $177-179$ & $214-222$ & $214-224$ & $126-144$ & $136-184$ \\
\hline UDO01 & UDO03 & UDO12 & UDO28 & UDO39 & \\
\hline $140-140$ & $143-143$ & $166-166$ & $150-161$ & $200-200$ & \\
\hline
\end{tabular}

\section{References:}

1 - Pannelli G., Alfei B., Santinelli A. In: Varietà di olivo nelle Marche, ASSAM (2001), pp. 17-20.

2 - Various authors. In: Catalogo Nazionale delle Varietà di Olivo, University of Bari (in press), ISBN 978-88-8879397-9. 


\section{"Augellina"}

(synonymy: Agnellina, Cellina, etc.)

Areal distribution or origin area: Basilicata

Flesh/pit weight ratio: high $(7,82 \pm 0,82)$

Oil content (\%): medium $(41,14 \pm 2,43)$

Purpose: dual purpose

\section{Morphological characters}

Tree characters

Vigour: medium-strong

Growth habit: spreading

Canopy-density: dense

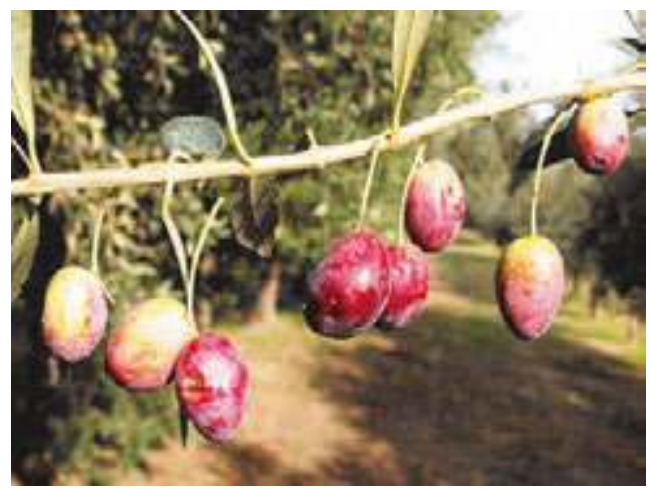

\section{Leaf characters}

Blade length (cm): medium $(6,84 \pm 0,55)$

Blade width (cm): broad $(\mathbf{1}, 55 \pm \mathbf{0 , 1 6})$

Shape (length/width): elliptic-lanceolate

\section{Inflorescence characters}

Inflorescence length $(\mathrm{cm})$ : long $(3,65 \pm 1,76)$

Number of flowers: medium $(20,83 \pm 4,39)$

\section{Fruit characters}

Fresh weight of 100 fruits (g): high $(\mathbf{4 , 6 4} \pm \mathbf{0 , 2 9}$ )

Shape (length/width): ovoid

Symmetry: slightly asymmetric

Position of maximum transverse diameter:

central

Apex: rounded

Base: rounded

Niplle: absent

Lenticels: many and small

\section{Pit characters}

Weight of 100 pits (g): high $(0,64 \pm 0,05)$

Shape (length/width): elliptic

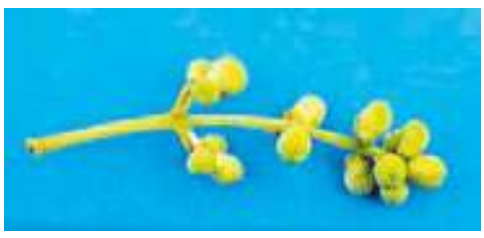

Mucron: absent

Symmetry: slightly asymmetric

Position of maximum transverse diameter:

central

Apex: pointed

Base: pointed

Surface: rugose

Number of grooves: high

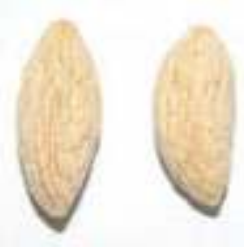




\section{Biochemical Characters}

\section{Fatty Acid Composition}

Table 1. Average values (express in $\% \pm$ standard deviations) of the fatty acids methyl esters and nutritional ratios obtained from single cultivar olive oils.

$\begin{array}{llllll}\text { Myristic acid } & \mathbf{0 , 0 1} \pm \mathbf{0 , 0 1} & \text { Linoleic acid }(\omega 6) & \mathbf{8 , 7 9} \pm \mathbf{0 , 7 0} & \text { Lignoceric acid } & \mathbf{0 , 0 7 \pm 0 , 0 2} \\ \text { Palmitic acid } & \mathbf{1 5 , 1 7} \pm \mathbf{0 , 6 1} & \text { Linolenic acid }(\omega 3) \mathbf{0 , 8 1} \pm \mathbf{0 , 0 8} & & \\ \text { Palmitoleic acid } & \mathbf{1 , 2 5} \pm \mathbf{0 , 0 6} & \text { Arachic acid } & \mathbf{0 , 2 4} \pm \mathbf{0 , 0 4} & \text { Unsat./satured } & \mathbf{4 , 9 3} \pm \mathbf{0 , 1 8} \\ \text { Stearic acid } & \mathbf{1 , 4 6} \pm \mathbf{0 , 0 4} & \text { Eicosenoic acid } & \mathbf{0 , 2 4} \pm \mathbf{0 , 1 5} & \omega 6 / \omega 3 & \mathbf{1 0 , 8 5} \pm \mathbf{0 , 1 0} \\ \text { Oleic acid } & \mathbf{7 1 , 6 6 \pm 0 , 6 9} & \text { Behenic acid } & \mathbf{0 , 1 0} \pm \mathbf{0 , 0 3} & & \end{array}$

\section{Organoleptic oil values}

Sensory Analysis (Panel test)

Comment: fruity medium, with hints of soft fruits and read sensations of almond and grass. Balanced taste sensation with a medium-high bitter and spicy. Medium-high fluidity.

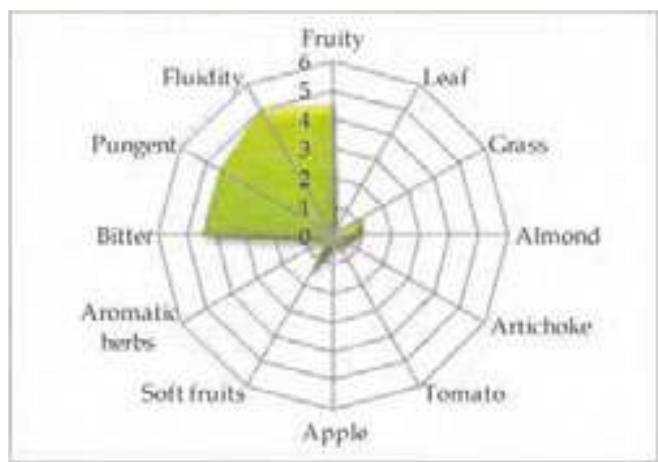

\section{Molecular Markers}

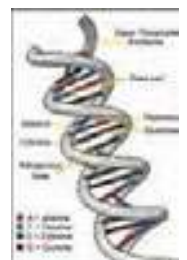

Table 2. Microsatellites (SSR) loci analyzed. For each locus the allele size (expressed in base pairs) is reported.

$\begin{array}{cccccc}D C A 09 & \text { DCA18 } & \text { GAPU59 } & \text { GAPU71A } & \text { GUPA71B } & \text { GAPU103A } \\ \mathbf{1 9 4 - \mathbf { 1 9 8 }} & \mathbf{1 7 7 - \mathbf { 1 8 1 }} & \mathbf{2 0 8 - \mathbf { 2 0 8 }} & \mathbf{2 1 0 - \mathbf { 2 1 0 }} & \mathbf{1 2 4 - \mathbf { 1 2 6 }} & \mathbf{1 5 7 - \mathbf { 2 0 3 }} \\ \text { UDO01 } & \text { UDO03 } & \text { UDO12 } & \text { UDO28 } & \text { UDO39 } & \\ \mathbf{1 4 0 - \mathbf { 1 4 0 }} & \mathbf{1 4 3 - \mathbf { 1 4 3 }} & \mathbf{1 6 4 - \mathbf { 1 6 6 }} & \mathbf{1 5 0 - \mathbf { 1 5 0 }} & \mathbf{2 0 5 - \mathbf { 2 0 5 }} & \end{array}$

\section{References:}

1 - Rotundo A., Marone E. In: Il germoplasma olivicolo lucano, Olita-Potenza (2002), pp. 53-65.

2 - Rotundo A., Perri E.,Muzzalupo I., et al. In: Il germoplasma olivicolo meridionale, (2012 in press).

3 - Muzzalupo I., Stefanizzi F., Perri E. HortScience (2009), 44: pp. 582-588. 


\section{"Aurina "}

(synonymy: Aurina di Venafro, Liciniana, Oliva di Venafro, etc.)

Areal distribution or origin area: Molise

Flesh/pit weight ratio: low $(3,92 \pm 0,49)$

Oil content (\%): medium $(42,50 \pm 1,69)$

Purpose: oil

\section{Morphological characters}

Tree characters

Vigour: medium-weak

Growth habit: erect

Canopy-density: dense

\section{Leaf characters}

Blade length $(\mathrm{cm})$ : medium $(6,02 \pm 0,61)$

Blade width $(\mathrm{cm})$ : broad $(\mathbf{1}, \mathbf{6 2} \pm \mathbf{0 , 2 6})$

Shape (length/width): elliptic

\section{Inflorescence characters}

Inflorescence length $(\mathrm{cm})$ : medium $(2,69 \pm 1,11)$

Number of flowers: medium $(21,28 \pm 2,72)$

\section{Fruit characters}

Fresh weight of 100 fruits $(\mathrm{g})$ : low $(\mathbf{1}, \mathbf{2 8} \pm \mathbf{0 , 1 5})$

Shape (length/width): ovoid

Symmetry: slightly asymmetric

Position of maximum transverse diameter: central

Apex: rounded

Base: truncate

Niplle: absent

Lenticels: many and small

\section{Pit characters}

Weight of 100 pits $(\mathrm{g})$ : low $(\mathbf{0 , 2 6} \pm \mathbf{0 , 0 1})$

Shape (length/width): ovoid

Mucron: obvious

Symmetry: slightly asymmetric

Position of maximum transverse diameter:

central

Apex: rounded

Base: rounded

Surface: smooth

Number of grooves: medium
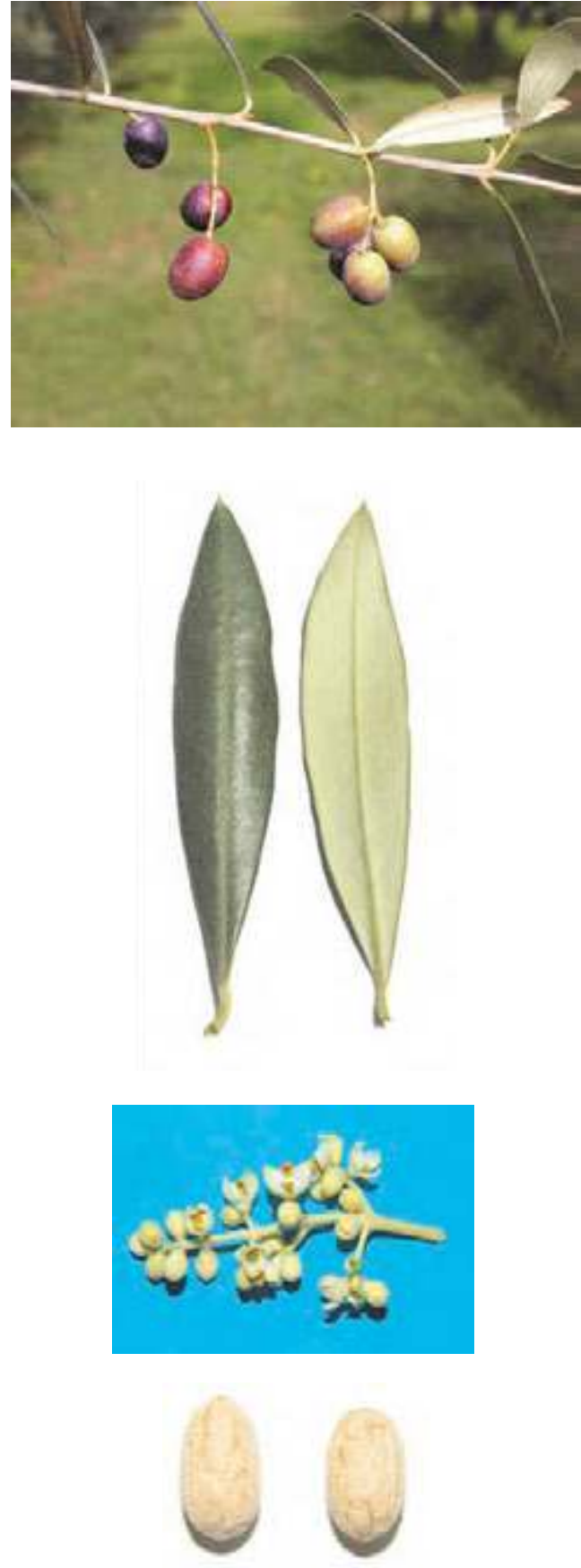


\section{Biochemical Characters}

\section{Fatty Acid Composition}

Table 1. Average values (express in $\% \pm$ standard deviations) of the fatty acids methyl esters and nutritional ratios obtained from single cultivar olive oils.

$\begin{array}{llllll}\text { Myristic acid } & \mathbf{0 , 0 1} \pm \mathbf{0 , 0 0} & \text { Linoleic acid }(\omega 6) & \mathbf{7 , 9 2} \pm \mathbf{0 , 6 2} & \text { Lignoceric acid } & \mathbf{0 , 0 3} \pm \mathbf{0 , 0 1} \\ \text { Palmitic acid } & \mathbf{1 6 , 0 3} \pm \mathbf{1 , 2 2} & \text { Linolenic acid }(\omega 3) \mathbf{0 , 8 0} \pm \mathbf{0 , 0 5} & & \\ \text { Palmitoleic acid } & \mathbf{1 , 9 0} \pm \mathbf{0 , 2 4} & \text { Arachic acid } & \mathbf{0 , 2 8} \pm \mathbf{0 , 2 1} & \text { Unsat./satured } & \mathbf{4 , 4 1} \pm \mathbf{0 , 3 9} \\ \text { Stearic acid } & \mathbf{2 , 0 0} \pm \mathbf{0 , 2 1} & \text { Eicosenoic acid } & \mathbf{0 , 0 2} \pm \mathbf{0 , 0 0} & \omega 6 / \omega 3 & \mathbf{9 , 9 8} \pm \mathbf{1 , 4 5} \\ \text { Oleic acid } & \mathbf{6 9 , 2 7} \pm \mathbf{1 , 1 6} & \text { Behenic acid } & \mathbf{0 , 0 7} \pm \mathbf{0 , 0 1} & & \end{array}$

\section{Organoleptic oil values}

Sensory Analysis (Panel test)

Comment: fruity medium, with hints of almond and read sensations of grass and artichoke. Balanced taste sensation with a medium bitter and spicy. Medium fluidity.

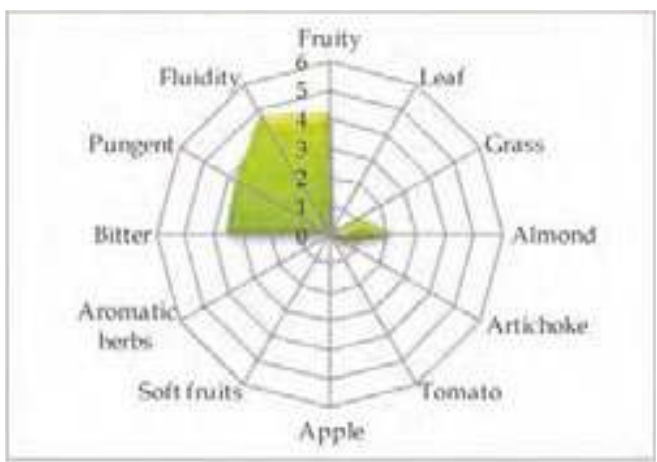

\section{Molecular Markers}

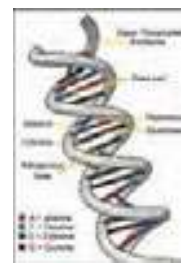

Table 2. Microsatellites (SSR) loci analyzed.

For each locus the allele size (expressed in base pairs) is reported.

$\begin{array}{cccccc}D C A 09 & \text { DCA18 } & \text { GAPU59 } & \text { GAPU71A } & \text { GUPA71B } & \text { GAPU103A } \\ \mathbf{1 6 2} \mathbf{- 2 0 6} & \mathbf{1 7 3 - \mathbf { 1 7 5 }} & \mathbf{2 2 2} \mathbf{- 2 2 2} & \mathbf{2 1 0 - \mathbf { 2 1 4 }} & \mathbf{1 2 4} \mathbf{- 1 4 4} & \mathbf{1 5 9 - \mathbf { 1 7 0 }} \\ \text { UDOO1 } & \text { UDO03 } & \text { UDO12 } & \text { UDO28 } & \text { UDO39 } & \\ \mathbf{1 4 4 - \mathbf { 1 4 4 }} & \mathbf{1 5 0 - \mathbf { 1 5 0 }} & \mathbf{1 7 7 - \mathbf { 1 7 7 }} & \mathbf{1 5 4 - \mathbf { 2 0 5 }} & \mathbf{2 1 3 - \mathbf { 2 1 3 }} & \end{array}$

\section{References:}

1 - Macrì T., Picone G., La Porta G. In : Olivo e Olio, (1988), 5: pp. 34-41.

2 - Various authors. In: Catalogo Nazionale delle Varietà di Olivo, University of Bari (in press), ISBN 978-88-8879397-9. 


\section{"Bianchera"}

(synonymy: Belica, Bellica, Bianca istriana, Biancara, etc.)

Areal distribution or origin area: Friuli

Flesh/ pit weight ratio: very high $(\mathbf{8 , 1 0} \pm \mathbf{1 , 0 0})$

Oil content (\%): medium $(\mathbf{5 0 , 2 1} \pm 0,84)$

Purpose: oil

\section{Morphological characters}

Tree characters

Vigour: medium

Growth habit: spreading-erect

Canopy-density: medium

\section{Leaf characters}

Blade length (cm): short $(4,58 \pm 0,49)$

Blade width (cm): medium $(1,25 \pm 0,17)$

Shape (length/width): elliptic-lanceolate

\section{Inflorescence characters}

Inflorescence length (cm): short $(2,47 \pm 0,26)$

Number of flowers: low $(\mathbf{1 4}, \mathbf{8 3} \pm 0, \mathbf{9 8})$

\section{Fruit characters}

Fresh weight of 100 fruits $(\mathrm{g})$ : medium $(3,37 \pm 0,08)$

Shape (length/width): ovoid

Symmetry: slightly asymmetric

Position of maximum transverse diameter:

central

Apex: rounded

Base: truncate

Niplle: tenuous

Lenticels: many and small

\section{Pit characters}

Weight of 100 pits (g): medium $(\mathbf{0 , 4 1} \pm \mathbf{0 , 0 7})$

Shape (length/width): elliptic

Mucron: absent

Symmetry: slightly asymmetric

Position of maximum transverse diameter:

towards apex

Apex: rounded

Base: pointed

Surface: scabrous

Number of grooves: medium
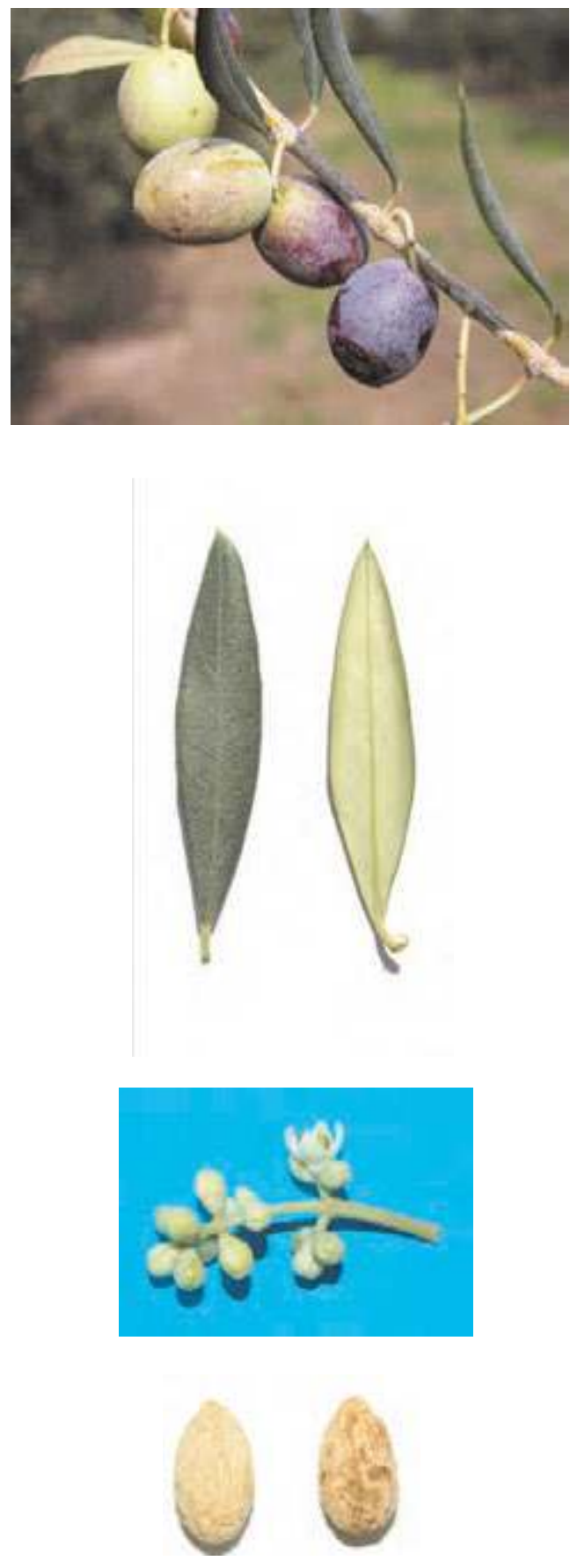


\section{Biochemical Characters}

\section{Fatty Acid Composition}

Table 1. Average values (express in $\% \pm$ standard deviations) of the fatty acids methyl esters and nutritional ratios obtained from single cultivar olive oils.

$\begin{array}{llllll}\text { Myristic acid } & \mathbf{0 , 0 1} \pm \mathbf{0 , 0 0} & \text { Linoleic acid }(\omega 6) & \mathbf{8 , 2 6} \pm \mathbf{0 , 0 1} & \text { Lignoceric acid } & \mathbf{0 , 0 5} \pm \mathbf{0 , 0 2} \\ \text { Palmitic acid } & \mathbf{1 1 , 4 7} \pm \mathbf{0 , 7 3} & \text { Linolenic acid }(\omega 3) \mathbf{0 , 9 6} \pm \mathbf{0 , 0 3} & & \\ \text { Palmitoleic acid } & \mathbf{1 , 1 8} \pm \mathbf{0 , 4 3} & \text { Arachic acid } & \mathbf{0 , 3 0} \pm \mathbf{0 , 0 0} & \text { Unsat./satured } & \mathbf{6 , 1 3} \pm \mathbf{0 , 1 1} \\ \text { Stearic acid } & \mathbf{2 , 1 4} \pm \mathbf{0 , 8 4} & \text { Eicosenoic acid } & \mathbf{0 , 0 2} \pm \mathbf{0 , 0 0} & \omega 6 / \omega 3 & \mathbf{8 , 6 1} \pm \mathbf{0 , 2 4} \\ \text { Oleic acid } & \mathbf{7 4 , 6 0 \pm 0 , 6 6} & \text { Behenic acid } & \mathbf{0 , 1 0} \pm \mathbf{0 , 0 4} & & \end{array}$

\section{Organoleptic oil values}

Sensory Analysis (Panel test)

Comment: fruity medium-high, with good sensation of grass, artichoke and almond. Balanced in flavours, with hints of bitter and spicy medium-high intensity. Medium-high fluidity.

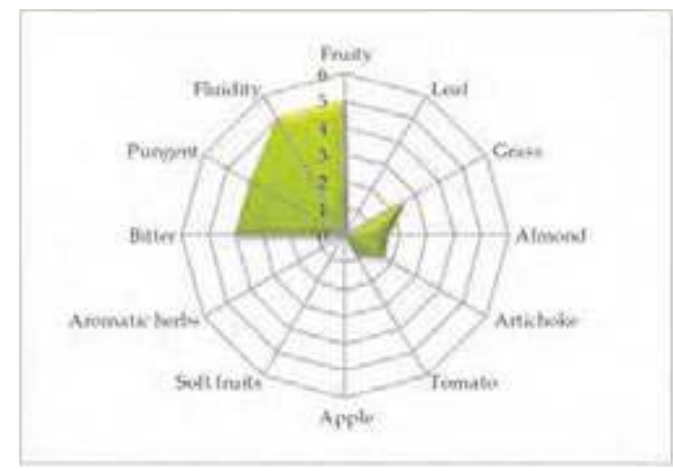

\section{Molecular Markers}

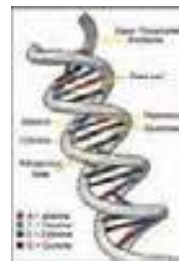

Table 2. Microsatellites (SSR) loci analyzed.

For each locus the allele size (expressed in base pairs) is reported.

\begin{tabular}{|c|c|c|c|c|c|}
\hline DCA09 & DCA18 & GAPU59 & GAPU71A & $G U P A 71 B$ & GAPU103A \\
\hline $182-182$ & $177-185$ & $214-214$ & $214-224$ & $130-130$ & $136-157$ \\
\hline UDO01 & UDO03 & UDO12 & UDO28 & UDO39 & \\
\hline $140-140$ & $150-150$ & $166-193$ & $182-205$ & $213-232$ & \\
\hline
\end{tabular}

\section{References:}

1 - Consiglio Oleicolo Internazionale. In: Catalogo Mondiale delle Varietà di Olivo, COI (2003).

2 - Muzzalupo I., Lombardo N., Salimonti A., et al. Adv. Hort. Sci. (2008), 22(2): pp. 142-148. 


\section{“Borgese "}

Areal distribution or origin area: Calabria

Flesh/pit weight ratio: medium $(6,90 \pm 1,07)$

Oil content (\%): medium $(49,13 \pm 2,04)$

Purpose: dual purpose

\section{Morphological characters}

Tree characters

Vigour: medium-strong

Growth habit: spreading

Canopy-density: dense

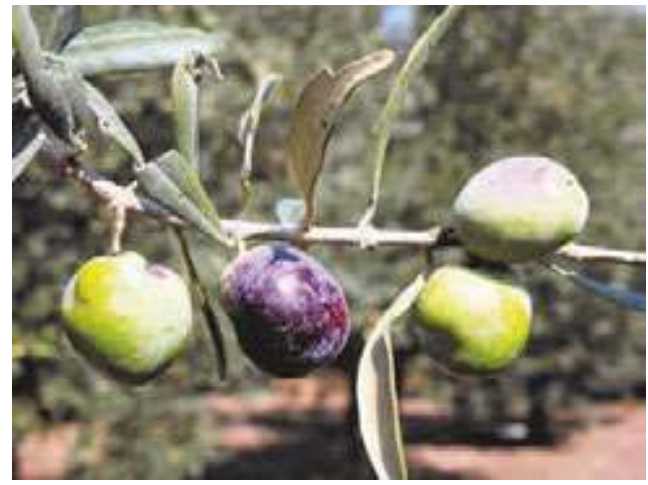

\section{Leaf characters}

Blade length $(\mathrm{cm})$ : long $(7,53 \pm 0,71)$

Blade width (cm): broad $(1,68 \pm 0,09)$

Shape (length/width): elliptic-lanceolate

\section{Inflorescence characters}

Inflorescence length $(\mathrm{cm})$ : short $(2,19 \pm 3,20)$

Number of flowers: medium $(6,90 \pm 1,07)$

\section{Fruit characters}

Fresh weight of 100 fruits $(\mathrm{g})$ : very high $(\mathbf{6 , 1 3} \pm \mathbf{1 , 6 9 )}$

Shape (length/width): ovoid

Symmetry: slightly asymmetric

Position of maximum transverse diameter:

central

Apex: rounded

Base: truncate

Niplle: absent

Lenticels: many and small

\section{Pit characters}

Weight of 100 pits (g): high $(\mathbf{0 , 6 1} \pm \mathbf{0 , 1 0})$

Shape (length/width): elliptic

Mucron: obvious

Symmetry: slightly asymmetric

Position of maximum transverse diameter:

central

Apex: pointed

Base: rounded

Surface: rugose

Number of grooves: low
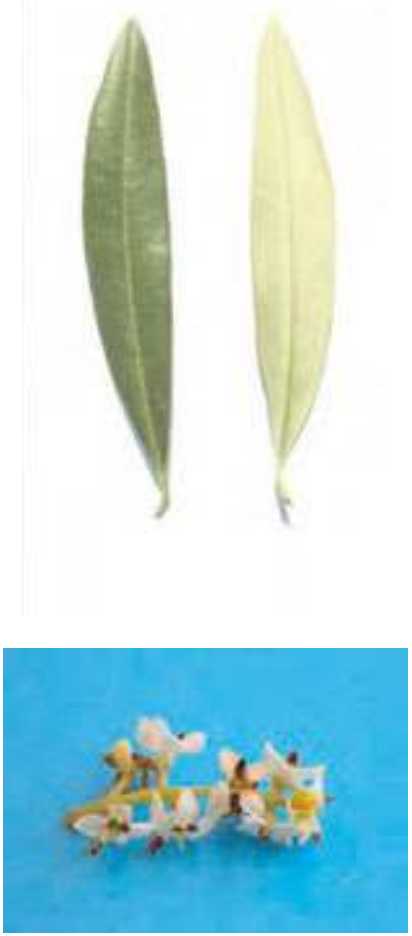


\section{Biochemical Characters}

\section{Fatty Acid Composition}

Table 1. Average values (express in $\% \pm$ standard deviations) of the fatty acids methyl esters and nutritional ratios obtained from single cultivar olive oils.

\begin{tabular}{|c|c|c|c|c|c|}
\hline Myristic acid & $0,01 \pm 0,01$ & Linoleic acid $(\omega 6)$ & $7,81 \pm 0,93$ & Lignoceric acid & $0,05 \pm 0,04$ \\
\hline Palmitic acid & $8,57 \pm 0,15$ & Linolenic acid $(\omega 3)$ & $0,80 \pm 0,31$ & & \\
\hline Palmitoleic acid & $0,45 \pm 0,03$ & Arachic acid & $0,35 \pm 0,07$ & Unsat./satured & $7,93 \pm 0,30$ \\
\hline Stearic acid & $2,33 \pm 0,21$ & Eicosenoic acid & $0,14 \pm 0,21$ & $\omega 6 / \omega 3$ & $11,58 \pm 7,00$ \\
\hline Oleic acid & $79,28 \pm 0,84$ & Behenic acid & $0,10 \pm 0,04$ & & \\
\hline
\end{tabular}

\section{Organoleptic oil values}

Sensory Analysis (Panel test)

Comment: fruity medium-high, with hints of almond and tomato, read sensations of grass and leaves. Balanced taste sensation with a light bitter and spicy. Medium fluidity.

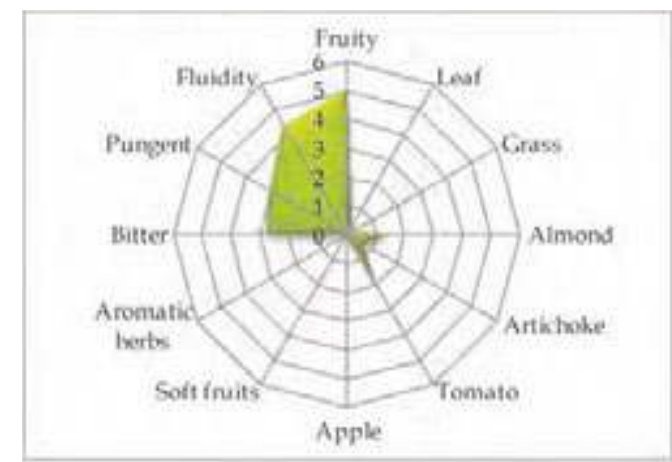

\section{Molecular Markers}

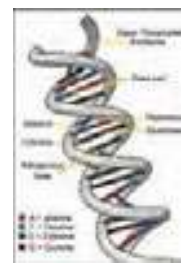

Table 2. Microsatellites (SSR) loci analyzed.

For each locus the allele size (expressed in base pairs) is reported.

\begin{tabular}{|c|c|c|c|c|c|}
\hline$D C A 09$ & $D C A 18$ & GAPU59 & GAPU71A & GUPA71B & GAPU103A \\
\hline $162-198$ & $179-179$ & $212-222$ & $214-214$ & $130-144$ & $150-184$ \\
\hline UDO01 & UDO03 & UDO12 & UDO28 & UDO39 & \\
\hline $144-144$ & $143-143$ & $166-193$ & $143-205$ & $200-220$ & \\
\hline
\end{tabular}

\section{References:}

1 - Lombardo N., Perri E., Muzzalupo I., et al. In: Il germoplasma olivicolo calabrese, Ist. Sper. Olivic.(2003), pp. 19.

2 - Perri E., Mazzotti F., Muzzalupo I., et al. In: Relazione attività, CO.R.ASS.OL. (2003).

3 - Muzzalupo I., Stefanizzi F., Perri E. HortScience (2009), 44: pp. 582-588. 


\section{“Borgiona "}

(synonymy: Basciona, Borgiana, Borsciona, Morcona, etc.)

Areal distribution or origin area: Umbria

Flesh/pit weight ratio: medium $(\mathbf{6}, \mathbf{4 1} \pm \mathbf{0 , 6 8})$

Oil content (\%): medium $(45,46 \pm 0,73)$

Purpose: oil

\section{Morphological characters}

Tree characters

Vigour: medium-weak

Growth habit: spreading

Canopy-density: medium-dense

\section{Leaf characters}

Blade length (cm): medium $(5,25 \pm 0,53)$

Blade width $(\mathrm{cm})$ : medium $(1,18 \pm 0,14)$

Shape (length/width): elliptic-lanceolate

\section{Inflorescence characters}

Inflorescence length (cm): long $(3,74 \pm 1,65)$

Number of flowers: medium $(\mathbf{1 8 , 0 4} \pm \mathbf{2 , 6 9})$

\section{Fruit characters}

Fresh weight of 100 fruits $(\mathrm{g})$ : medium $(3,52 \pm 0,35)$

Shape (length/width): spherical

Symmetry: slightly asymmetric

Position of maximum transverse diameter:

central

Apex: rounded

Base: rounded

Niplle: tenuous

Lenticels: many and small

\section{Pit characters}

Weight of 100 pits (g): high $(\mathbf{0 , 4 8} \pm \mathbf{0 , 0 5})$

Shape (length/width): ovoid

Mucron: obvious

Symmetry: slightly asymmetric

Position of maximum transverse diameter:

\section{towards apex}

Apex: rounded

Base: rounded

Surface: rugose

Number of grooves: medium
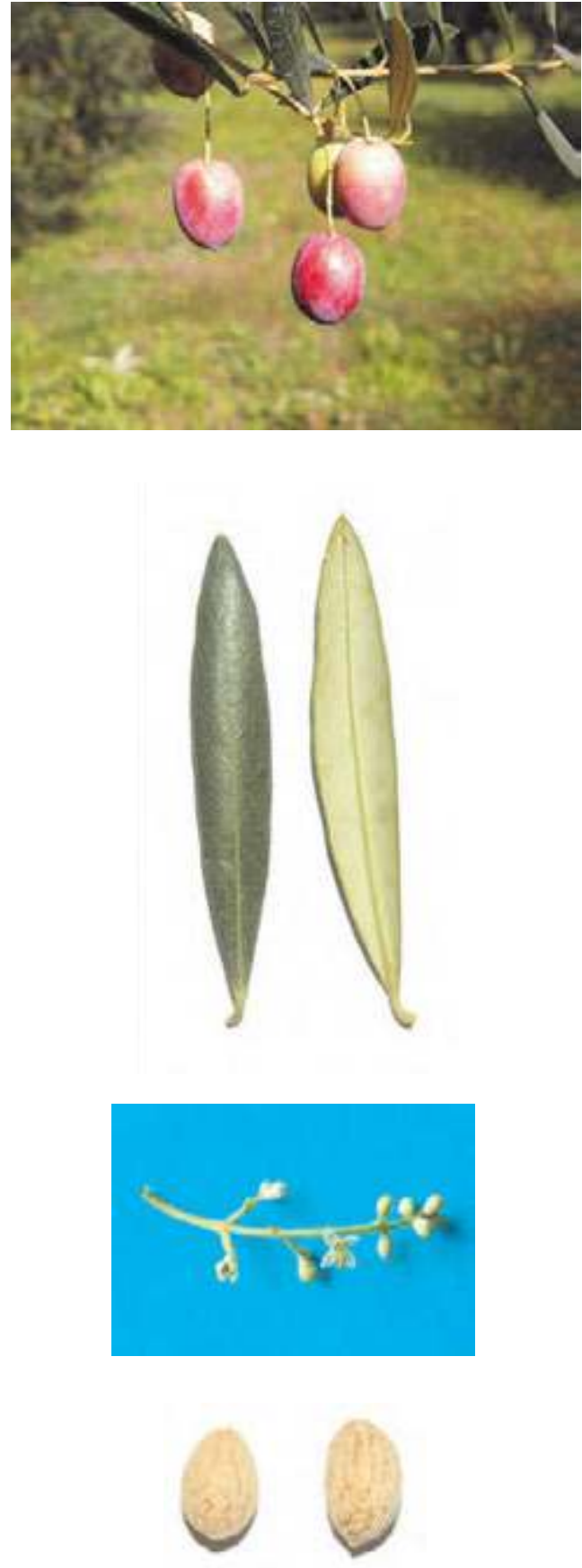


\section{Biochemical Characters}

\section{Fatty Acid Composition}

Table 1. Average values (express in $\% \pm$ standard deviations) of the fatty acids methyl esters and nutritional ratios obtained from single cultivar olive oils.

$\begin{array}{llllll}\text { Myristic acid } & \mathbf{0 , 0 1} \pm \mathbf{0 , 0 0} & \text { Linoleic acid }(\omega 6) & \mathbf{7 , 5 6} \pm \mathbf{0 , 0 5} & \text { Lignoceric acid } & \mathbf{0 , 0 2} \pm \mathbf{0 , 0 0} \\ \text { Palmitic acid } & \mathbf{1 1 , 0 5 \pm 0 , 0 7} & \text { Linolenic acid }(\omega 3) \mathbf{0 , 9 4} \pm \mathbf{0 , 0 6} & & \\ \text { Palmitoleic acid } & \mathbf{1 , 0 1} \pm \mathbf{0 , 3 2} & \text { Arachic acid } & \mathbf{0 , 2 8} \pm \mathbf{0 , 0 6} & \text { Unsat./satured } & \mathbf{6 , 2 7} \pm \mathbf{0 , 1 7} \\ \text { Stearic acid } & \mathbf{2 , 3 6} \pm \mathbf{0 , 3 9} & \text { Eicosenoic acid } & \mathbf{0 , 0 3} \pm \mathbf{0 , 0 2} & \omega 6 / \omega 3 & \mathbf{8 , 1 0} \pm \mathbf{0 , 5 0} \\ \text { Oleic acid } & \mathbf{7 5 , 5 7 \pm \mathbf { 0 , 7 6 }} & \text { Behenic acid } & \mathbf{0 , 0 7} \pm \mathbf{0 , 0 1} & & \end{array}$

\section{Organoleptic oil values}

Sensory Analysis (Panel test)

Comment: fruity medium, with hints of almond and artichoke. Balanced taste sensation with a medium-light bitter and spicy. Medium-light fluidity.

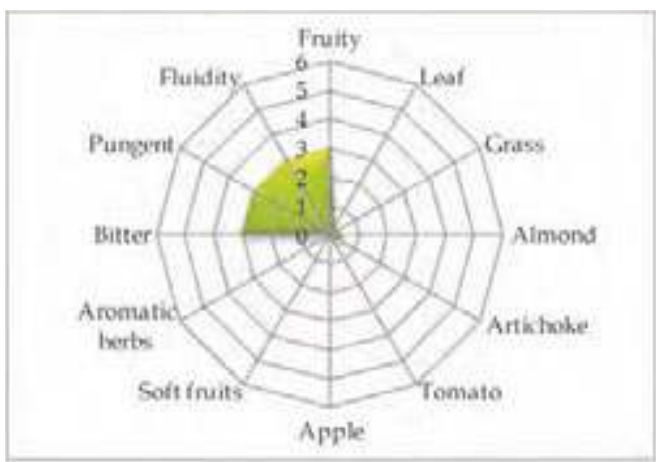

\section{Molecular Markers}

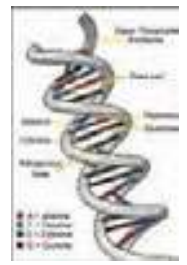

Table 2. Microsatellites (SSR) loci analyzed.

For each locus the allele size (expressed in base pairs) is reported.

$\begin{array}{cccccc}D C A 09 & D C A 18 & \text { GAPU59 } & \text { GAPU71A } & \text { GUPA71B } & \text { GAPU103A } \\ \mathbf{1 6 6 - 1 9 8} & \mathbf{1 7 7 - \mathbf { 1 8 1 }} & \mathbf{2 1 4 - \mathbf { 2 2 2 }} & \mathbf{2 1 4} \mathbf{- 2 2 4} & \mathbf{1 3 0 - \mathbf { 1 4 4 }} & \mathbf{1 5 7 - \mathbf { 1 5 7 }} \\ \text { UDOO1 } & \text { UDO03 } & \text { UDO12 } & \text { UDO28 } & \text { UDO39 } & \\ \mathbf{1 4 0 - \mathbf { 1 4 0 }} & \mathbf{1 4 3 - \mathbf { 1 4 3 }} & \mathbf{1 6 6 - \mathbf { 1 9 3 }} & \mathbf{1 5 0 - \mathbf { 2 1 0 }} & \mathbf{2 0 0 - \mathbf { 2 0 0 }} & \end{array}$

\section{References:}

1 - Pannelli G., Alfei B., D’ Ambrosio A., et al. In: Varietà di olivo in Umbria, Ed. Pliniana (2000), pp. 1-6.

2 - Various authors. In: Catalogo Nazionale delle Varietà di Olivo, University of Bari (in press), ISBN 978-88-8879397-9. 


\section{"Bosana "}

(synonymy: Bosana a scopa, Bosana pendula, Olia de ozzu, etc.)

Areal distribution or origin area: Sardegna

Flesh/pit weight ratio: medium $(5,29 \pm 0,23)$

Oil content (\%): medium $(40,99 \pm 1,96)$

Purpose: oil

\section{Morphological characters}

Tree characters

Vigour: medium-weak

Growth habit: spreading

Canopy-density: medium

\section{Leaf characters}

Blade length $(\mathrm{cm})$ : medium $(6,38 \pm 0,29)$

Blade width $(\mathrm{cm})$ : broad $(\mathbf{1}, \mathbf{6 1} \pm \mathbf{0 , 1 5})$

Shape (length/width): elliptic

\section{Inflorescence characters}

Inflorescence length $(\mathrm{cm})$ : medium $(2,81 \pm 1,51)$

Number of flowers: medium $(20,32 \pm 1,26)$

\section{Fruit characters}

Fresh weight of 100 fruits $(\mathrm{g})$ : medium $(3,19 \pm 0,25)$

Shape (length/width): spherical

Symmetry: slightly asymmetric

Position of maximum transverse diameter:

central

Apex: rounded

Base: rounded

Niplle: absent

Lenticels: few and small

\section{Pit characters}

Weight of 100 pits (g): high $(\mathbf{5 , 2 9} \pm \mathbf{0 , 2 3})$

Shape (length/width): ovoid

Mucron: obvious

Symmetry: symmetric

Position of maximum transverse diameter:

\section{towards apex}

Apex: rounded

Base: pointed

Surface: smooth

Number of grooves: medium
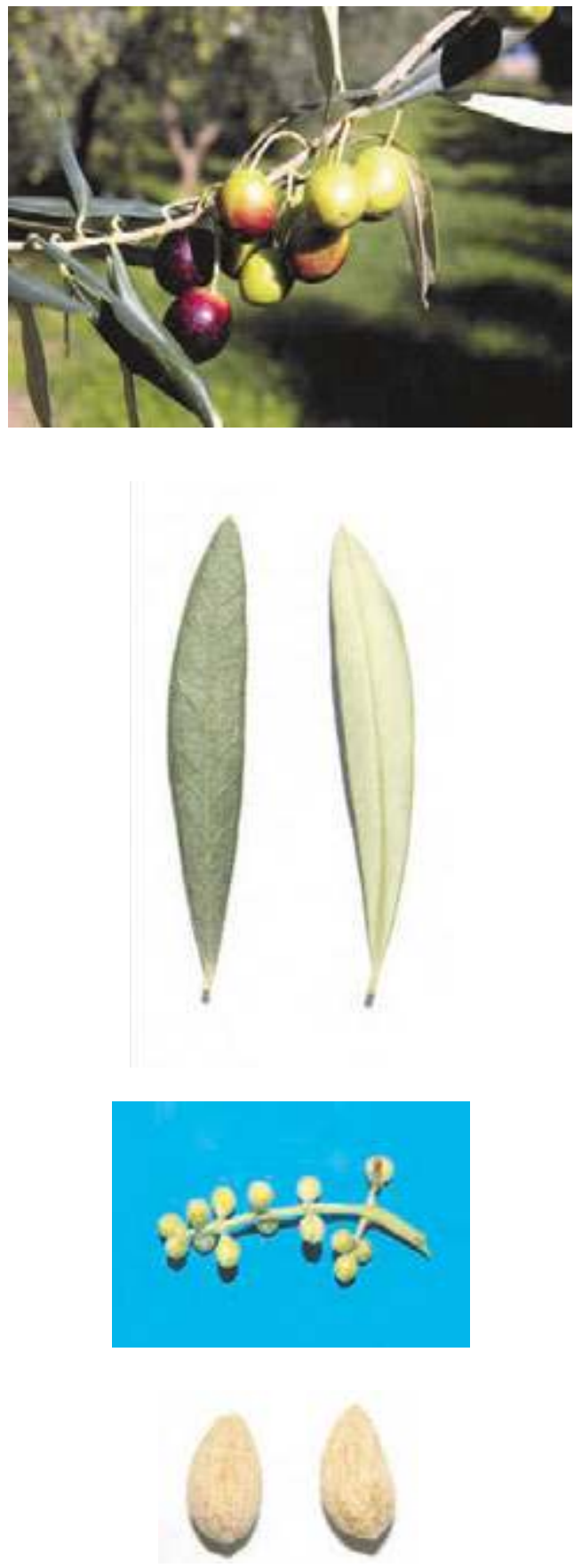


\section{Biochemical Characters}

\section{Fatty Acid Composition}

Table 1. Average values (express in $\% \pm$ standard deviations) of the fatty acids methyl esters and nutritional ratios obtained from single cultivar olive oils.

$\begin{array}{llllll}\text { Myristic acid } & \mathbf{0 , 0 2} \pm \mathbf{0 , 0 0} & \text { Linoleic acid }(\omega 6) & \mathbf{6 , 3 4} \pm \mathbf{1 , 9 4} & \text { Lignoceric acid } & \mathbf{0 , 0 3} \pm \mathbf{0 , 0 1} \\ \text { Palmitic acid } & \mathbf{9 , 7 1} \pm \mathbf{1 , 4 6} & \text { Linolenic acid }(\omega 3) \mathbf{0 , 8 9} \pm \mathbf{0 , 0 3} & & \\ \text { Palmitoleic acid } & \mathbf{0 , 4 3} \pm \mathbf{0 , 0 7} & \text { Arachic acid } & \mathbf{0 , 2 6} \pm \mathbf{0 , 0 2} & \text { Unsat./satured } & \mathbf{7 , 1 4} \pm \mathbf{0 , 9 9} \\ \text { Stearic acid } & \mathbf{2 , 4 5} \pm \mathbf{0 , 1 3} & \text { Eicosenoic acid } & \mathbf{0 , 0 8} \pm \mathbf{0 , 0 4} & \omega 6 / \omega 3 & \mathbf{7 , 0 7} \pm \mathbf{1 , 9 4} \\ \text { Oleic acid } & \mathbf{7 9 , 4 3 \pm \mathbf { 0 , 1 8 }} & \text { Behenic acid } & \mathbf{0 , 0 8} \pm \mathbf{0 , 0 1} & & \end{array}$

\section{Organoleptic oil values}

Sensory Analysis (Panel test)

Comment: medium fruity, with good sensation of grass, less almond and artichoke. Balanced taste sensation, with bitter and spicy medium. Medium fluidity.

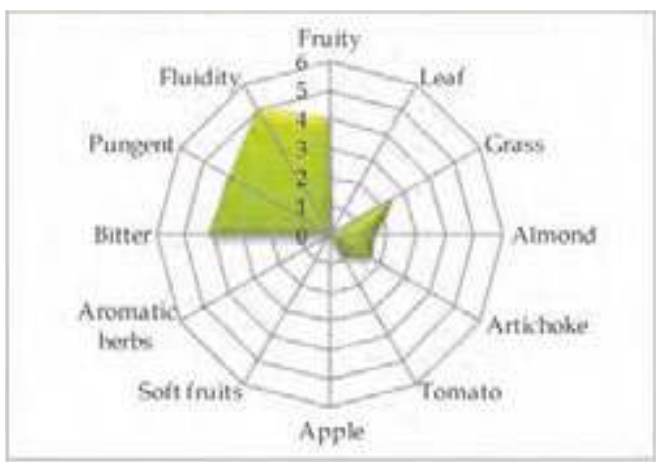

\section{Molecular Markers}

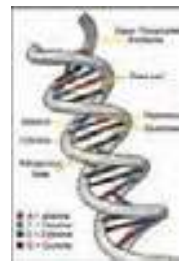

Table 2. Microsatellites (SSR) loci analyzed.

For each locus the allele size (expressed in base pairs) is reported.

\begin{tabular}{|c|c|c|c|c|c|}
\hline$D C A 09$ & $D C A 18$ & GAPU59 & GAPU71A & GUPA71B & GAPU103A \\
\hline $194-198$ & $181-185$ & $214-222$ & $214-218$ & $130-144$ & $170-184$ \\
\hline UDO01 & UDO03 & UDO12 & UDO28 & UDO39 & \\
\hline $144-150$ & $135-135$ & $177-193$ & $161-205$ & $205-205$ & \\
\hline
\end{tabular}

\section{References:}

1 - Various authors. In: Catalogo Nazionale delle Varietà di Olivo . University of Bari (in press), ISBN 978-88-8879397-9.

2 - Muzzalupo I., Stefanizzi F., Perri E. HortScience (2009), 44: pp. 582-588. 


\section{"Brandofino"}

(synonymy: Mantonica, Nostrale, Randazzese, Randazzisa, etc.)

Areal distribution or origin area: Sicilia

Flesh/ pit weight ratio: very high $(\mathbf{1 4 , 3 3} \pm \mathbf{0 , 6 0})$

Oil content (\%): medium $(49,24 \pm 2,49)$

Purpose: dual purpose

\section{Morphological characters}

Tree characters

Vigour: strong

Growth habit: spreading-erect

Canopy-density: dense

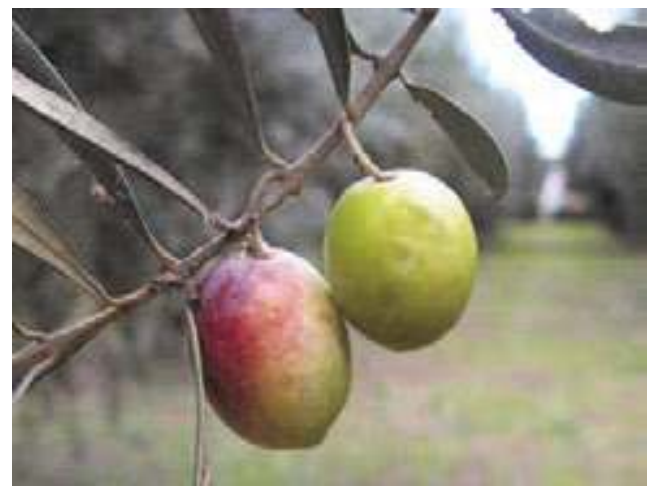

\section{Leaf characters}

Blade length $(\mathrm{cm})$ : medium $(5,71 \pm 0,46)$

Blade width $(\mathrm{cm})$ : medium $(1,42 \pm 0,19)$

Shape (length/width): elliptic-lanceolate

\section{Inflorescence characters}

Inflorescence length (cm): short $(2,37 \pm 0,87)$

Number of flowers: low $(\mathbf{1 2 , 4 7} \pm \mathbf{2 , 3 7 )}$

\section{Fruit characters}

Fresh weight of 100 fruits (g): very high $(7,92 \pm 1,40)$

Shape (length/width): ovoid

Symmetry: asymmetric

Position of maximum transverse diameter:

central

Apex: rounded

Base: truncate

Niplle: tenuous

Lenticels: many and large

\section{Pit characters}

Weight of 100 pits (g): high $(0,60 \pm 0,03)$

Shape (length/width): elliptic

Mucron: absent

Symmetry: symmetric

Position of maximum transverse diameter:

towards apex

Apex: rounded

Base: rounded

Surface: rugose

Number of grooves: high
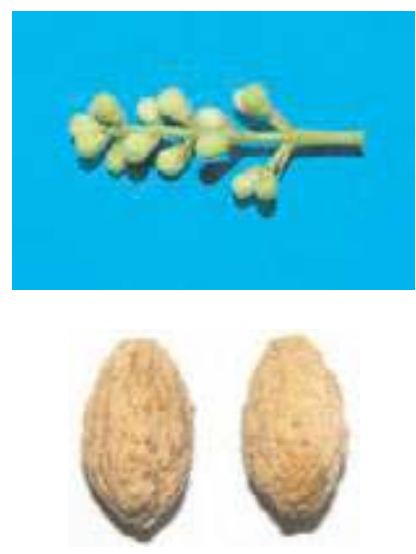


\section{Biochemical Characters}

\section{Fatty Acid Composition}

Table 1. Average values (express in $\% \pm$ standard deviations) of the fatty acids methyl esters and nutritional ratios obtained from single cultivar olive oils.

\begin{tabular}{|c|c|c|c|c|c|}
\hline Myristic acid & $0,01 \pm 0,00$ & Linoleic acid $(\omega 6)$ & $10,47 \pm 0,05$ & Lignoceric acid & $0,03 \pm 0,00$ \\
\hline Palmitic acid & $12,71 \pm 1,00$ & Linolenic acid $(\omega 3)$ & $0,91 \pm 0,03$ & & \\
\hline Palmitoleic acid & $1,33 \pm 0,65$ & Arachic acid & $0,21 \pm 0,05$ & Unsat./satured & $5,87 \pm 0,67$ \\
\hline teari & $1,54 \pm 0,32$ & Eicosenoic acid & $0,03 \pm 0,01$ & $\omega 6 / \omega 3$ & $11,51 \pm 0,42$ \\
\hline leic acid & $71,32 \pm 2,67$ & Behenic acid & $0,06 \pm 0,01$ & & \\
\hline
\end{tabular}

\section{Organoleptic oil values}

Sensory Analysis (Panel test)

Comment: fruity medium-light, with taste of almond, read sensations of tomato and aromatic herbs. Balanced taste sensation with medium bitter and spicy. Medium fluidity.

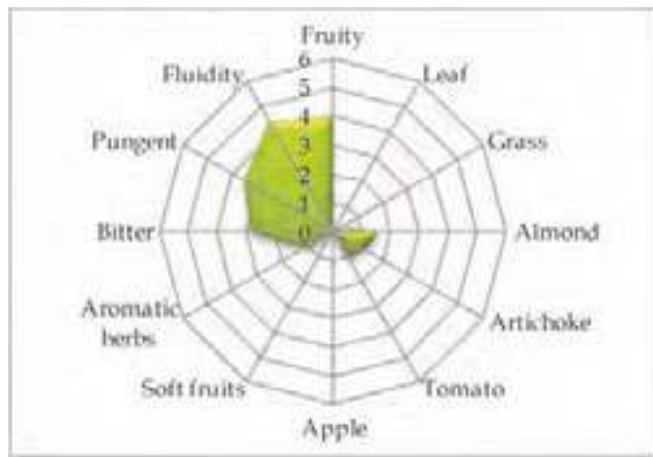

\section{Molecular Markers}

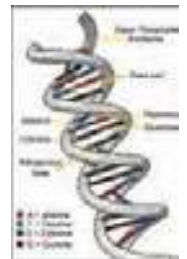

Table 2. Microsatellites (SSR) loci analyzed. For each locus the allele size (expressed in base pairs) is reported.

$\begin{array}{cccccc}D C A 09 & D C A 18 & \text { GAPU59 } & \text { GAPU71A } & \text { GUPA71B } & \text { GAPU103A } \\ \mathbf{1 7 6 - \mathbf { 2 0 6 }} & \mathbf{1 7 9 - \mathbf { 1 7 9 }} & \mathbf{2 0 8 - \mathbf { 2 1 2 }} & \mathbf{2 1 0 - \mathbf { 2 1 4 }} & \mathbf{1 2 6 - \mathbf { 1 4 4 }} & \mathbf{1 3 6} \mathbf{- 1 5 9} \\ \text { UDOO1 } & \text { UDO03 } & \text { UDO12 } & \text { UDO28 } & \text { UDO39 } & \\ \mathbf{1 4 4 - \mathbf { 1 4 4 }} & \mathbf{1 5 0 - \mathbf { 1 5 0 }} & \mathbf{1 6 6 - \mathbf { 1 9 3 }} & \mathbf{1 4 3 - \mathbf { 1 4 3 }} & \mathbf{2 1 3 - \mathbf { 2 3 2 }} & \end{array}$

\section{References:}

1 - Caruso T., Cartabellotta D., Motisi A., et all. In: Cultivar di olivo siciliane, Università degli Studi di Palermo (2007), pp. 82-86.

2 - Muzzalupo I., Stefanizzi F., Perri E. HortScience (2009), 44: pp. 582-588. 


\section{"Buscionetto"}

(synonymy: Biancolilla, Caltabellottese, Ianculidda, Oliva di Caltabellotta, etc.)

Areal distribution or origin area: Sicilia

Flesh/pit weight ratio: high $(7,03 \pm 0,74)$

Oil content (\%): medium $(49,66 \pm 2,79)$

Purpose: oil

\section{Morphological characters}

Tree characters

Vigour: medium

Growth habit: spreading

Canopy-density: dense

\section{Leaf characters}

Blade length (cm): short $(3,44 \pm 0,38)$

Blade width $(\mathrm{cm})$ : medium $(\mathbf{1 , 3 8} \pm 0,20)$

Shape (length/width): elliptic - lanceolate

\section{Inflorescence characters}

Inflorescence length $(\mathrm{cm})$ : short $(2,06 \pm 0,68)$

Number of flowers: low $(\mathbf{1 2 , 9 0} \pm 2,93)$

\section{Fruit characters}

Fresh weight of 100 fruits $(\mathrm{g})$ : medium $(\mathbf{3 , 9 1} \pm \mathbf{1 , 2 3})$

Shape (length/width): ovoid

Symmetry: slightly asymmetric

Position of maximum transverse diameter: central

Apex: rounded

Base: truncate

Niplle: tenuous

Lenticels: few and large

\section{Pit characters}

Weight of 100 pits (g): high $(7,03 \pm 0,74)$

Shape (length/width): elliptic

Mucron: absent

Symmetry: slightly asymmetric

Position of maximum transverse diameter:

central

Apex: pointed

Base: rounded

Surface: rugose

Number of grooves: low
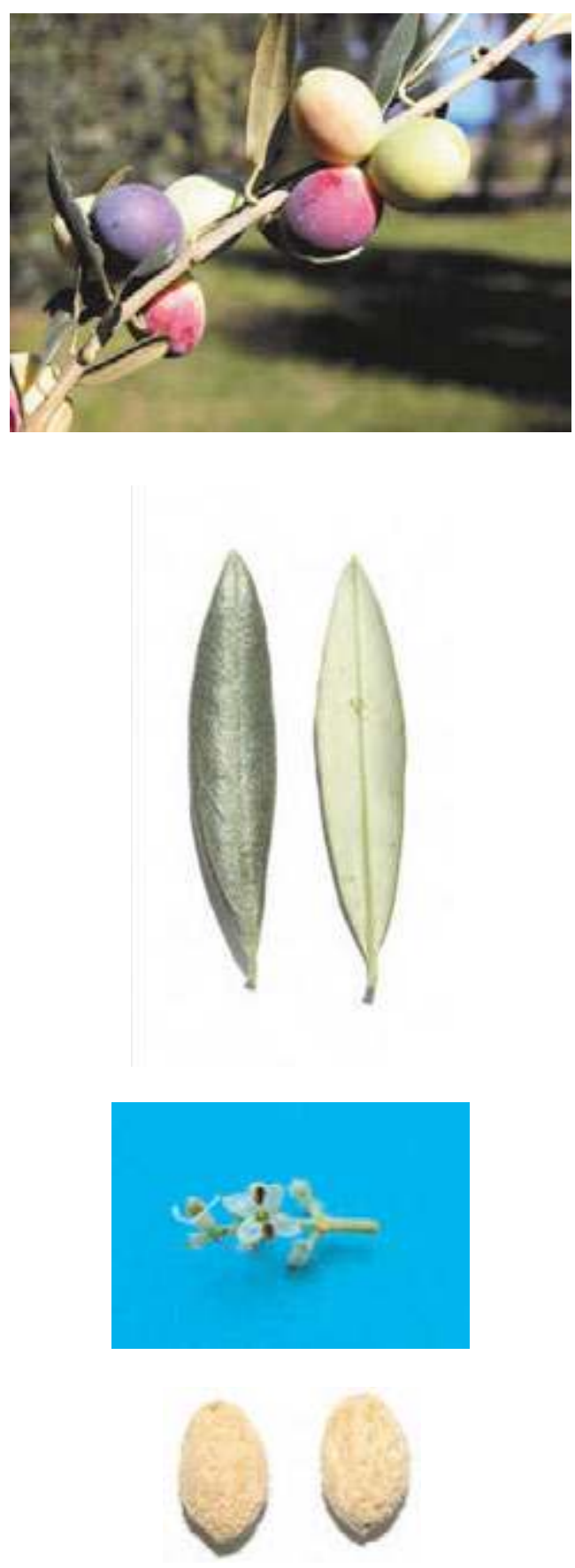


\section{Biochemical Characters}

\section{Fatty Acid Composition}

Table 1. Average values (express in $\% \pm$ standard deviations) of the fatty acids methyl esters and nutritional ratios obtained from single cultivar olive oils.

$\begin{array}{llllll}\text { Myristic acid } & \mathbf{0 , 0 1} \pm \mathbf{0 , 0 0} & \text { Linoleic acid }(\omega 6) & \mathbf{6 , 6 5} \pm \mathbf{0 , 6 3} & \text { Lignoceric acid } & \mathbf{0 , 0 5} \pm \mathbf{0 , 0 3} \\ \text { Palmitic acid } & \mathbf{1 2 , 9 3} \pm \mathbf{0 , 0 8} & \text { Linolenic acid }(\omega 3) \mathbf{0 , 8 2} \pm \mathbf{0 , 0 4} & & \\ \text { Palmitoleic acid } & \mathbf{1 , 0 7} \pm \mathbf{0 , 4 9} & \text { Arachic acid } & \mathbf{0 , 2 1} \pm \mathbf{0 , 0 3} & \text { Unsat./satured } & \mathbf{5 , 6 4 \pm 0 , 2 4} \\ \text { Stearic acid } & \mathbf{1 , 8 0} \pm \mathbf{0 , 5 3} & \text { Eicosenoic acid } & \mathbf{0 , 0 4} \pm \mathbf{0 , 0 1} & \omega 6 / \omega 3 & \mathbf{8 , 2 5} \pm \mathbf{6 , 0 5} \\ \text { Oleic acid } & \mathbf{7 5 , 4 6 \pm \mathbf { 1 , 2 0 }} & \text { Behenic acid } & \mathbf{0 , 0 6} \pm \mathbf{0 , 0 3} & & \end{array}$

\section{Organoleptic oil values}

Sensory Analysis (Panel test)

Comment: fruity medium-high, with hints of almond and tomato, read sensations of grass and leaves. Balanced taste sensation with a light bitter and spicy. Medium fluidity.

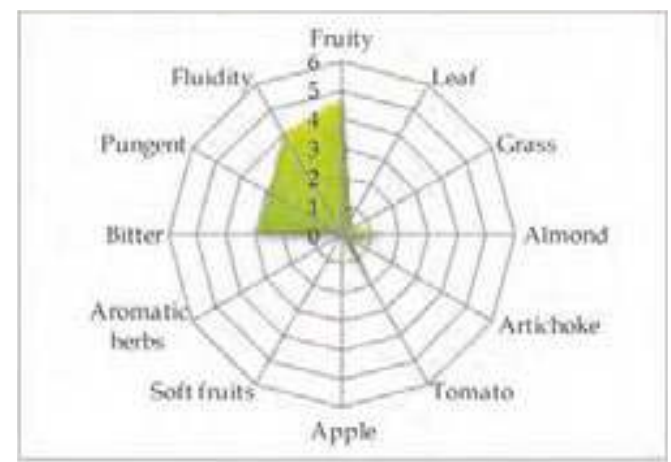

\section{Molecular Markers}

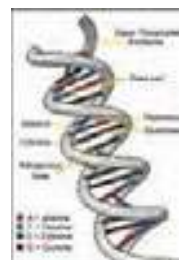

Table 2. Microsatellites (SSR) loci analyzed. For each locus the allele size (expressed in base pairs) is reported.

$\begin{array}{cccccc}D C A 09 & D C A 18 & \text { GAPU59 } & \text { GAPU71A } & \text { GUPA71B } & \text { GAPU103A } \\ \mathbf{1 7 6 - \mathbf { 1 9 8 }} & \mathbf{1 8 1 - \mathbf { 1 8 1 }} & \mathbf{2 1 2} \mathbf{- 2 1 2} & \mathbf{2 1 0 - \mathbf { 2 1 4 }} & \mathbf{1 2 4} \mathbf{- 1 2 6} & \mathbf{1 3 6} \mathbf{- 1 5 0} \\ \text { UDOO1 } & \text { UDOO3 } & \text { UDO12 } & \text { UDO28 } & \text { UDO39 } & \\ \mathbf{1 4 0 - \mathbf { 1 4 0 }} & \mathbf{1 5 0 - \mathbf { 1 5 0 }} & \mathbf{1 6 6 - \mathbf { 1 9 3 }} & \mathbf{1 5 4 - \mathbf { 1 5 4 }} & \mathbf{2 1 3 - \mathbf { 2 1 3 }} & \end{array}$

\section{References:}

1 - Caruso T., Cartabellotta D., Motisi A., et all. In: Cultivar di olivo siciliane, Università degli Studi di Palermo (2007), pp. 29-34.

2 - Muzzalupo I., Stefanizzi F., Perri E. HortScience (2009), 44: pp. 582-588. 


\section{"Cacaredda "}

Areal distribution or origin area: Sicilia

Flesh/pit weight ratio: medium $(6,57 \pm 0,50)$

Oil content (\%): medium $(48,89 \pm 1,18)$

Purpose: oil

\section{Morphological characters}

Tree characters

Vigour: medium

Growth habit: spreading-erect

Canopy-density: medium-dense

\section{Leaf characters}

Blade length $(\mathrm{cm})$ : medium $(5,48 \pm 0,39)$

Blade width $(\mathrm{cm})$ : medium $(\mathbf{1}, 35 \pm \mathbf{0 , 1 2})$

Shape (length/width): elliptic-lanceolate

\section{Inflorescence characters}

Inflorescence length $(\mathrm{cm})$ : medium $(2,73 \pm 1,01)$

Number of flowers: low $(\mathbf{1 4 , 0 9} \pm 0,39)$

\section{Fruit characters}

Fresh weight of 100 fruits $(\mathrm{g})$ : medium $(3,74 \pm 0,11)$

Shape (length/width): spherical

Symmetry: symmetric

Position of maximum transverse diameter:

central

Apex: rounded

Base: rounded

Niplle: absent

Lenticels: few and small

\section{Pit characters}

Weight of 100 pits (g): high $(\mathbf{0 , 5 0} \pm \mathbf{0 , 0 5})$

Shape (length/width): ovoid

Mucron: obvious

Simmetry: symmetric

Position of maximum transverse diameter:

towards apex

Apex: rounded

Base: rounded

Surface: smooth

Number of grooves: medium
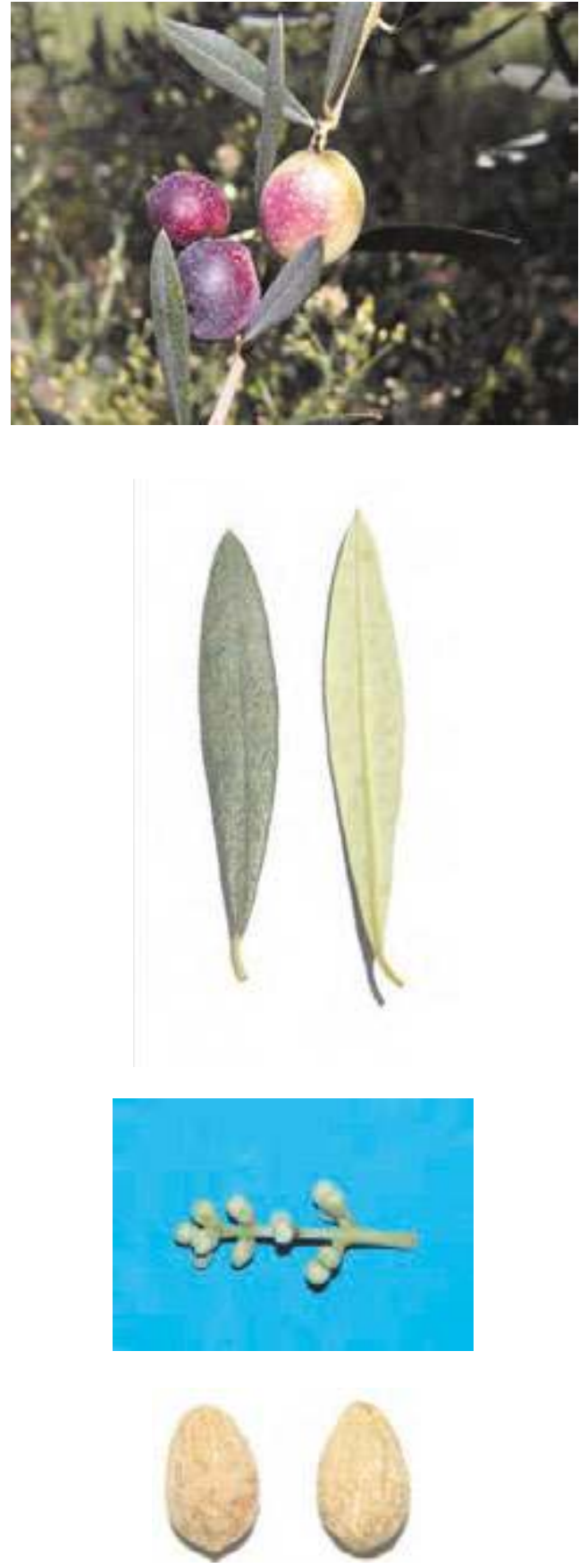


\section{Biochemical Characters}

\section{Fatty Acid Composition}

Table 1. Average values (express in $\% \pm$ standard deviations) of the fatty acids methyl esters and nutritional ratios obtained from single cultivar olive oils.

\begin{tabular}{|c|c|c|c|c|c|}
\hline Myristic acid & $0,01 \pm 0,00$ & Linoleic acid $(\omega 6)$ & $10,01 \pm 1,21$ & Lignoceric acid & $0,03 \pm 0,00$ \\
\hline Palmitic acid & $7,58 \pm 0,03$ & Linolenic acid $(\omega 3)$ & $0,89 \pm 0,06$ & & \\
\hline Palmitoleic acid & $0,59 \pm 0,24$ & Arachic acid & $0,21 \pm 0,00$ & Unsat./satured & $9,80 \pm 0,09$ \\
\hline Stearic acid & $1,44 \pm 0,08$ & Eicosenoic acid & $0,02 \pm 0,00$ & $\omega 6 / \omega 3$ & $11,27 \pm 0,62$ \\
\hline Oleic acid & $78,99 \pm 1,55$ & Behenic acid & $0,06 \pm 0,02$ & & \\
\hline
\end{tabular}

\section{Organoleptic oil values}

Sensory Analysis (Panel test)

Comment: fruity medium, with taste of grass, read sensations of almond, artichoke and leaves. Balanced taste sensation with medium bitter and spicy. Medium fluidity.

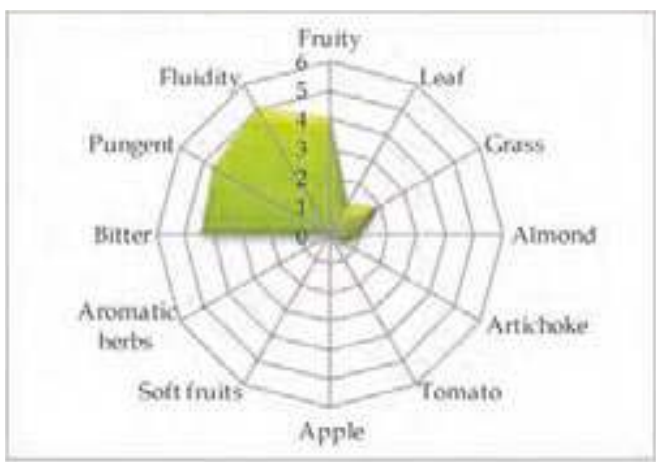

\section{Molecular Markers}

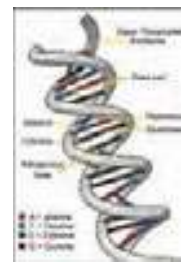

Table 2. Microsatellites (SSR) loci analyzed.

For each locus the allele size (expressed in base pairs) is reported.

$\begin{array}{cccccc}D C A 09 & D C A 18 & \text { GAPU59 } & \text { GAPU71A } & \text { GUPA71B } & \text { GAPU103A } \\ \mathbf{1 7 6 - \mathbf { 2 0 6 }} & \mathbf{1 8 1 - \mathbf { 1 8 1 }} & \mathbf{2 0 8 - \mathbf { 2 1 2 }} & \mathbf{2 1 0 - \mathbf { 2 1 0 }} & \mathbf{1 2 4} \mathbf{- 1 4 4} & \mathbf{1 5 9} \mathbf{- 1 8 4} \\ \text { UDOO1 } & \text { UDO03 } & \text { UDO12 } & \text { UDO28 } & \text { UDO39 } & \\ \mathbf{1 4 4 - \mathbf { 1 4 4 }} & \mathbf{1 4 3 - \mathbf { 1 4 3 }} & \mathbf{1 6 6 - \mathbf { 1 9 3 }} & \mathbf{1 8 2} \mathbf{- 1 8 2} & \mathbf{2 0 5} \mathbf{- 2 0 5} & \end{array}$

\section{References:}

1 - Tucci A., Mule' R., Fodale A. S. In: Indagini sul germoplasma olivicolo in Sicilia, Sviluppo Agricolo (1995), 1/2: pp. 55-64.

2 - Muzzalupo I., Lombardo N., Salimonti A., et al. Adv. Hort. Sci. 2008, 22(2): pp. 142-148. 


\section{"Calatina "}

(synonymy: Galatina, Montagna, Montagna Calatina, etc.)

Areal distribution or origin area: Sicilia

Flesh/pit weight ratio: medium $(5,35 \pm 0,42)$

Oil content (\%): medium $(45,39 \pm 2,10)$

Purpose: oil

\section{Morphological characters}

Tree characters

Vigour: medium-weak

Growth habit: spreading

Canopy-density: medium-dense

\section{Leaf characters}

Blade length $(\mathrm{cm})$ : medium $(5,26 \pm 0,16)$

Blade width $(\mathrm{cm})$ : medium $(1,27 \pm 0,14)$

Shape (length/width): elliptic - lanceolate

\section{Inflorescence characters}

Inflorescence length $(\mathrm{cm})$ : medium $(3,12 \pm 0,02)$

Number of flowers: medium $(23,25 \pm 2,52)$

\section{Fruit characters}

Fresh weight of 100 fruits $(\mathrm{g})$ : medium $(2,54 \pm 0,82)$

Shape (length/width): elongated

Symmetry: asymmetric

Position of maximum transverse diameter:

central

Apex: pointed

Base: rounded

Niplle: absent

Lenticels: many and small

\section{Pit characters}

Weight of 100 pits (g): medium $(\mathbf{0 , 4 0} \pm \mathbf{0 , 1 1})$

Shape (length/width): elongated

Mucron: absent

Symmetry: slightly asymmetric

Position of maximum transverse diameter:

central

Apex: pointed

Base: rounded

Surface: scabrous

Number of grooves: medium
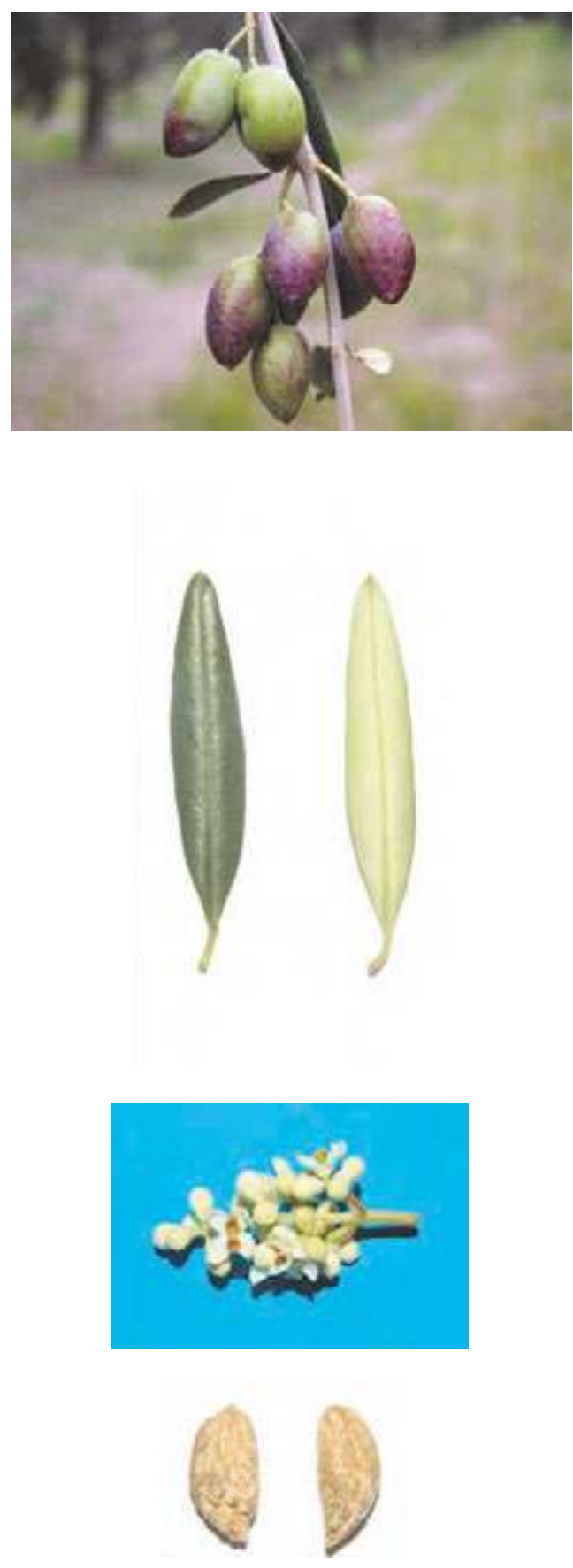


\section{Biochemical Characters}

\section{Fatty Acid Composition}

Table 1. Average values (express in $\% \pm$ standard deviations) of the fatty acids methyl esters and nutritional ratios obtained from single cultivar olive oils.

$\begin{array}{llllll}\text { Myristic acid } & \mathbf{0 , 0 1} \pm \mathbf{0 , 0 1} & \text { Linoleic acid }(\omega 6) & \mathbf{8 , 4 0} \pm \mathbf{0 , 0 1} & \text { Lignoceric acid } & \mathbf{0 , 0 7 \pm 0 , 0 1} \\ \text { Palmitic acid } & \mathbf{9 , 2 7} \pm \mathbf{0 , 0 2} & \text { Linolenic acid }(\omega 3) \mathbf{0 , 7 1} \pm \mathbf{0 , 0 3} & & \\ \text { Palmitoleic acid } & \mathbf{0 , 4 1} \pm \mathbf{0 , 0 0} & \text { Arachic acid } & \mathbf{0 , 2 0} \pm \mathbf{0 , 0 3} & \text { Unsat./satured } & \mathbf{7 , 5 6} \pm \mathbf{0 , 0 3} \\ \text { Stearic acid } & \mathbf{2 , 1 4} \pm \mathbf{0 , 0 1} & \text { Eicosenoic acid } & \mathbf{0 , 3 6} \pm \mathbf{0 , 0 2} & \omega 6 / \omega 3 & \mathbf{1 1 , 8 5} \pm \mathbf{0 , 4 4} \\ \text { Oleic acid } & \mathbf{7 7 , 9 1} \pm \mathbf{0 , 0 7} & \text { Behenic acid } & \mathbf{0 , 0 7} \pm \mathbf{0 , 0 2} & & \end{array}$

\section{Organoleptic oil values}

Sensory Analysis (Panel test)

Comment: fruity medium, with taste of almond and tomato, read sensations of aromatic herbs, grass and leaves. Balanced taste sensation with medium bitter and spicy. Medium fluidity.

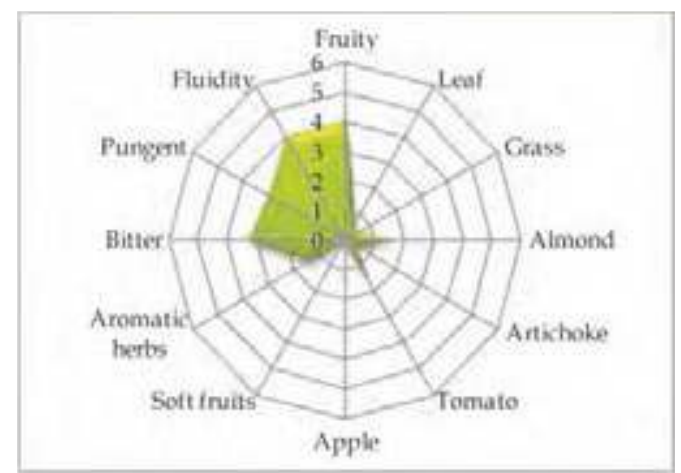

\section{Molecular Markers}

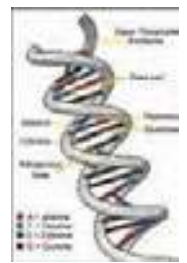

Table 2. Microsatellites (SSR) loci analyzed.

For each locus the allele size (expressed in base pairs) is reported.

$\begin{array}{cccccc}D C A 09 & \text { DCA18 } & \text { GAPU59 } & \text { GAPU71A } & \text { GUPA71B } & \text { GAPU103A } \\ \mathbf{1 8 2} \mathbf{- 1 8 2} & \mathbf{1 7 5 - \mathbf { 1 7 9 }} & \mathbf{2 1 2} \mathbf{- 2 2 2} & \mathbf{2 1 0 - \mathbf { 2 1 4 }} & \mathbf{1 2 4} \mathbf{- 1 2 6} & \mathbf{1 3 6} \mathbf{- 1 3 6} \\ \text { UDOO1 } & \text { UDOO3 } & \text { UDO12 } & \text { UDO28 } & \text { UDO39 } & \\ \mathbf{1 4 4 - \mathbf { 1 4 4 }} & \mathbf{1 5 0 - \mathbf { 1 5 0 }} & \mathbf{1 7 7 - \mathbf { 1 7 7 }} & \mathbf{1 4 3 - \mathbf { 1 8 2 }} & \mathbf{2 0 5 - \mathbf { 2 0 5 }} & \end{array}$

\section{References:}

1 - Caruso T., Cartabellotta D., Motisi A., et all. In: Cultivar di olivo siciliane, Università degli Studi di Palermo (2007), pp. 88-92.

2 - Muzzalupo I., Lombardo N., Salimonti A., et al. Adv. Hort. Sci. (2008), 22(2): pp. 142-148. 


\section{"Canino"}

(synonymy: Caninese, Canino blasi,Cornino, Montignoso, etc.)

Areal distribution or origin area: Lazio

Flesh/pit weight ratio: low $(\mathbf{4 , 2 2} \pm \mathbf{0 , 0 7})$

Oil content (\%): medium $(45,75 \pm 2,77)$

Purpose: oil

\section{Morphological characters}

Tree characters

Vigour: medium-strong

Growth habit: erect-spreading

Canopy-density: medium

\section{Leaf characters}

Blade length $(\mathrm{cm})$ : medium $(5,77 \pm 0,44)$

Blade width $(\mathrm{cm})$ : medium $(\mathbf{1}, 21 \pm 0,13)$

Shape (length/width): elliptic-lanceolate

\section{Inflorescence characters}

Inflorescence length $(\mathrm{cm})$ : medium $(2,70 \pm 1,00)$

Number of flowers: low $(15,81 \pm 3,01)$

\section{Fruit characters}

Fresh weight of 100 fruits (g): low $(\mathbf{1}, \mathbf{2 6} \pm \mathbf{0 , 1 3})$

Shape (length/width): ovoid

Symmetry: slightly asymmetric

Position of maximum transverse diameter:

central

Apex: rounded

Base: truncate

Niplle: absent

Lenticels: many and small

\section{Pit characters}

Weight of 100 pits $(\mathrm{g})$ : low $(\mathbf{0 , 2 5} \pm \mathbf{0 , 0 2})$

Shape (length/width): ovoid

Mucron: tenuous

Symmetry: slightly asymmetric

Position of maximum transverse diameter:

towards apex

Apex: rounded

Base: rounded

Surface: smooth

Number of grooves: low
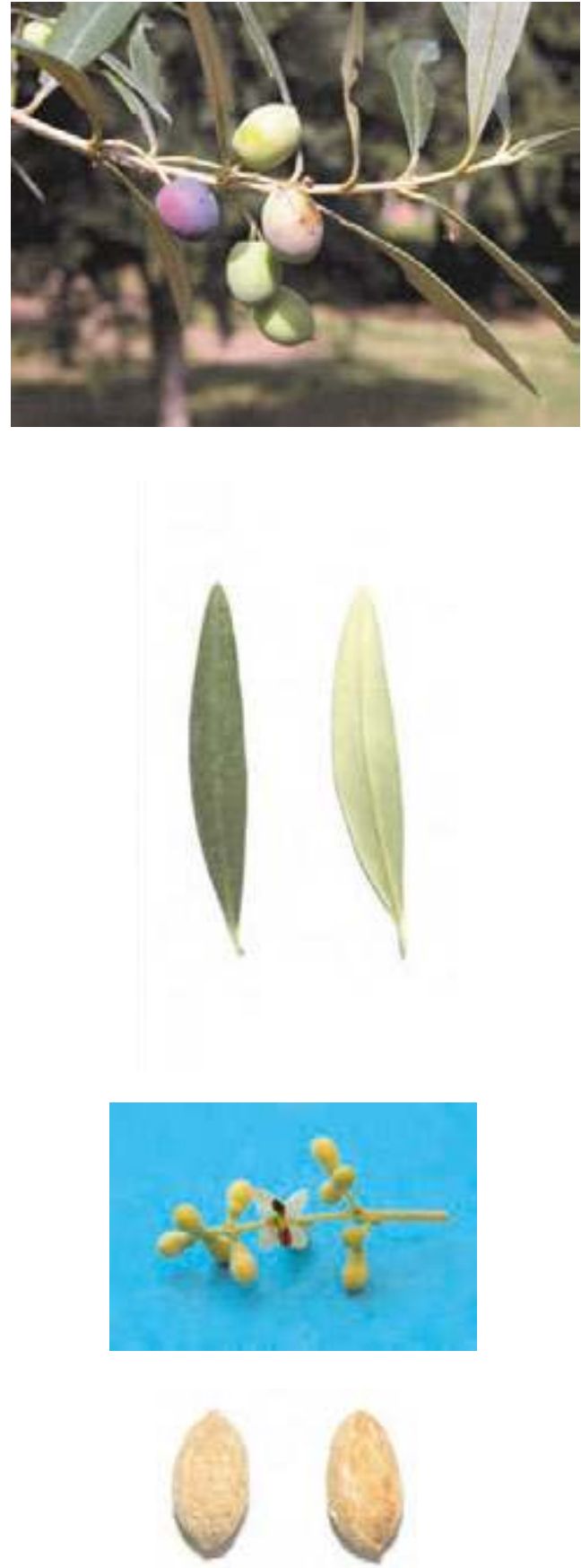


\section{Biochemical Characters}

\section{Fatty Acid Composition}

Table 1. Average values (express in $\% \pm$ standard deviations) of the fatty acids methyl esters and nutritional ratios obtained from single cultivar olive oils.

\begin{tabular}{|c|c|c|c|c|c|}
\hline Myristic acid & $0,01 \pm 0,00$ & Linoleic acid $(\omega 6)$ & $7,81 \pm 0,89$ & Lignoceric acid & $0,05 \pm 0,03$ \\
\hline Palmitic acid & $13,52 \pm 1,75$ & Linolenic acid $(\omega 3)$ & $0,84 \pm 0,08$ & & \\
\hline Palmitoleic acid & $1,18 \pm 0,31$ & Arachic acid & $0,37 \pm 0,13$ & Unsat./satured & $5,26 \pm 0,60$ \\
\hline Stearic acid & $2,10 \pm 0,38$ & Eicosenoic acid & $0,84 \pm 0,08$ & $\omega 6 / \omega 3$ & $9,38 \pm 1,40$ \\
\hline Oleic acid & $72,94 \pm 1,71$ & Behenic acid & $0,11 \pm 0,04$ & & \\
\hline
\end{tabular}

\section{Organoleptic oil values}

Sensory Analysis (Panel test)

Comment: fruity medium, with hints of almond and read sensations of grass and artichoke. Balanced taste sensation with a medium bitter and spicy. Medium-high fluidity.

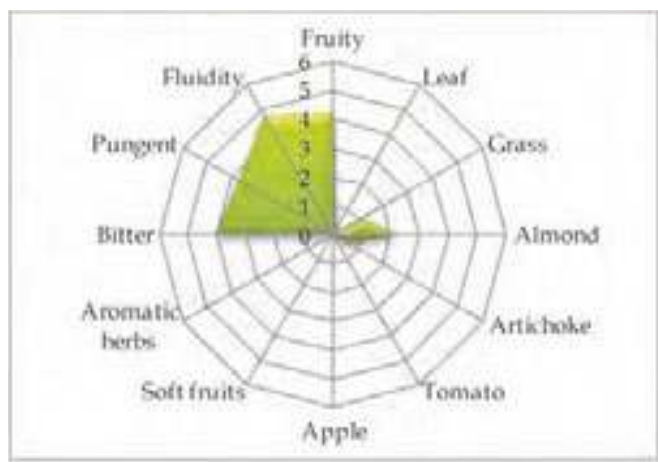

\section{Molecular Markers}

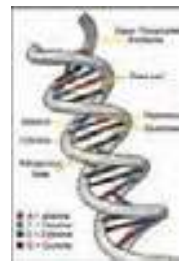

Table 2. Microsatellites (SSR) loci analyzed.

For each locus the allele size (expressed in base pairs) is reported.

\begin{tabular}{|c|c|c|c|c|c|}
\hline DCA09 & $D C A 18$ & GAPU59 & GAPU71A & GUPA71B & GAPU103A \\
\hline $172-182$ & $173-189$ & $212-222$ & $214-221$ & $126-144$ & $159-159$ \\
\hline UDO01 & UDO03 & UDO12 & UDO28 & UDO39 & \\
\hline $150-150$ & $135-135$ & $166-182$ & $143-154$ & $213-232$ & \\
\hline
\end{tabular}

\section{References:}

1 - Various authors. In: Catalogo Nazionale delle Varietà di Olivo . University of Bari (in press), ISBN 978-88-8879397-9.

2 - Muzzalupo I., Lombardo N., Salimonti A., et al. Adv. Hort. Sci. (2008), 22(2): pp. 142-148. 


\section{"Carboncella"}

(synonymy: Carboncella Pianacce A, Carboncella Pianacce B, Carboncella Sabina, etc.)

Areal distribution or origin area: Lazio

Flesh/pit weight ratio: medium $(5,30 \pm 1,26)$

Oil content (\%): medium $(48,19 \pm 2,81)$

Purpose: oil

\section{Morphological characters}

Tree characters

Vigour: medium

Growth habit: spreading

Canopy-density: medium-dense

\section{Leaf characters}

Blade length $(\mathrm{cm})$ : medium $(5,23 \pm 0,31)$

Blade width (cm): medium $(1,45 \pm 0,15)$

Shape (length/width): elliptic

\section{Inflorescence characters}

Inflorescence length $(\mathrm{cm})$ : medium $(2,71 \pm 5,02)$

Number of flowers: medium $(1,60 \pm 2,84)$

\section{Fruit characters}

Fresh weight of 100 fruits (g): medium $(2,05 \pm 0,40)$

Shape (length/width): ovoid

Symmetry: slightly asymmetric

Position of maximum transverse diameter:

central

Apex: rounded

Base: truncate

Niplle: tenuous

Lenticels: many and small

\section{Pit characters}

Weight of 100 pits $(\mathrm{g})$ : medium $(\mathbf{0 , 3 4} \pm \mathbf{0 , 1 3})$

Shape (length/width): ovoid

Mucron: obvious

Siymmetry: slightly asymmetric

Position of maximum transverse diameter:

towards apex

Apex: rounded

Base: rounded

Surface: smooth

Number of grooves: medium
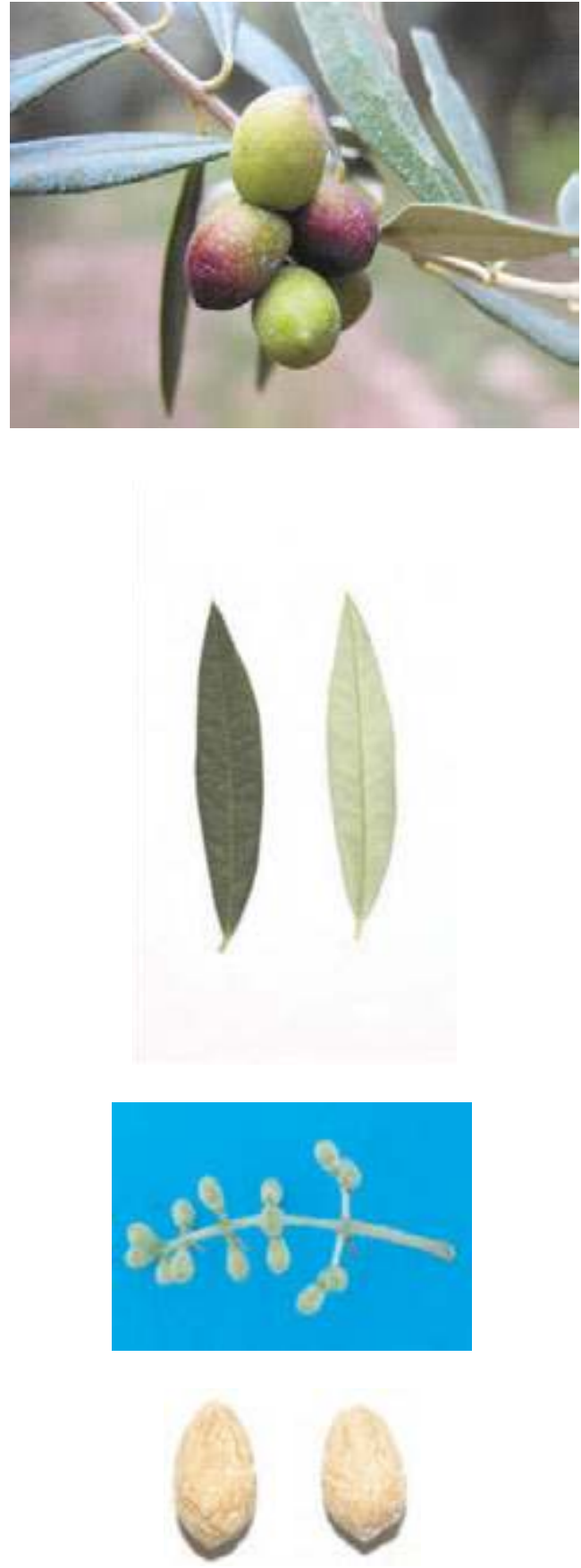


\section{Biochemical Characters}

\section{Fatty Acid Composition}

Table 1. Average values (express in $\% \pm$ standard deviations) of the fatty acids methyl esters and nutritional ratios obtained from single cultivar olive oils.

\begin{tabular}{|c|c|c|c|c|c|}
\hline Myristic acid & $0,01 \pm 0,01$ & Linoleic acid $(\omega 6)$ & $10,11 \pm 0,93$ & Lignoceric acid & $0,06 \pm 0,01$ \\
\hline Palmitic acid & $13,02 \pm 1,23$ & Linolenic acid $(\omega 3)$ & $0,84 \pm 0,05$ & & \\
\hline Palmitoleic acid & $1,38 \pm 0,02$ & Arachic acid & $0,49 \pm 0,04$ & Unsat./satured & $5,36 \pm 0,53$ \\
\hline teari & $2,16 \pm 0,03$ & Eicosenoic acid & $0,40 \pm 0,01$ & $\omega 6 / \omega 3$ & $12,16 \pm 1,84$ \\
\hline leic acid & $70,12 \pm 0,38$ & Behenic acid & $0,14 \pm 0,02$ & & \\
\hline
\end{tabular}

\section{Organoleptic oil values}

Sensory Analysis (Panel test)

Comment: fruity medium-high, with hints of almond and read sensations of grass and artichoke. Balanced taste sensation with a medium bitter and spicy. Medium fluidity.

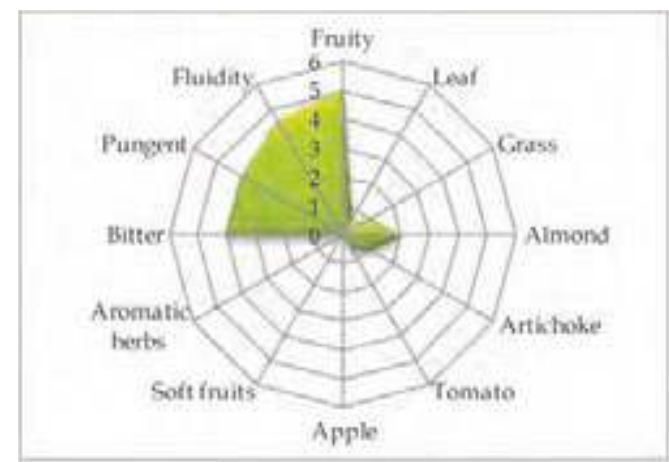

\section{Molecular Markers}

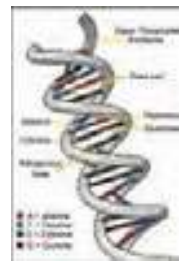

Table 2. Microsatellites (SSR) loci analyzed.

For each locus the allele size (expressed in base pairs) is reported.

\begin{tabular}{|c|c|c|c|c|c|}
\hline DCA09 & DCA18 & GAPU59 & GAPU71A & $G U P A 71 B$ & GAPU103A \\
\hline $166-194$ & $177-181$ & $208-212$ & $214-224$ & $124-144$ & $170-184$ \\
\hline UDO01 & UDO03 & UDO12 & UDO28 & UDO39 & \\
\hline $144-144$ & $150-150$ & $166-193$ & $182-210$ & $213-213$ & \\
\hline
\end{tabular}

\section{References:}

1 - Parlati M.V., Pandolfi S. In: Catalogo delle principali varietà di olivo del Lazio, Ist. Sper. Olivic. (2003), pp. 25-26. 2 - Various authors. In: Catalogo Nazionale delle Varietà di Olivo. University of Bari (in press), ISBN 978-88-8879397-9. 


\section{"Carbonchia"}

(synonymy: Carboncella, Carbonella, etc.)

Areal distribution or origin area: Abruzzo

Flesh/pit weight ratio: medium $(6,08 \pm 0,38)$

Oil content (\%): medium $(44,63 \pm 1,50)$

Purpose: oil

\section{Morphological characters}

Tree characters

Vigour: medium

Growth habit: erect-spreading

Canopy-density: medium

\section{Leaf characters}

Blade length (cm): medium 5,28 $\pm 0,59$ )

Blade width $(\mathrm{cm})$ : medium $(1,42 \pm 0,22)$

Shape (length/width): elliptic

\section{Inflorescence characters}

Inflorescence length (cm): short $(2,42 \pm 0,80)$

Number of flowers: low $(\mathbf{1 2}, \mathbf{2 5} \pm \mathbf{1}, \mathbf{0 0})$

\section{Fruit characters}

Fresh weight of 100 fruits (g): medium $(2,02 \pm 0,17)$

Shape (length/width): spherical

Symmetry: slightly asymmetric

Position of maximum transverse diameter:

central

Apex: rounded

Base: truncate

Niplle: absent

Lenticels: many and small

\section{Pit characters}

Weight of 100 pits (g): low $(0,29 \pm 0,04)$

Shape (length/width): ovoid

Mucron: obvious

Symmetry: symmetric

Position of maximum transverse diameter:

central

Apex: rounded

Base: rounded

Surface: smooth

Number of grooves: high
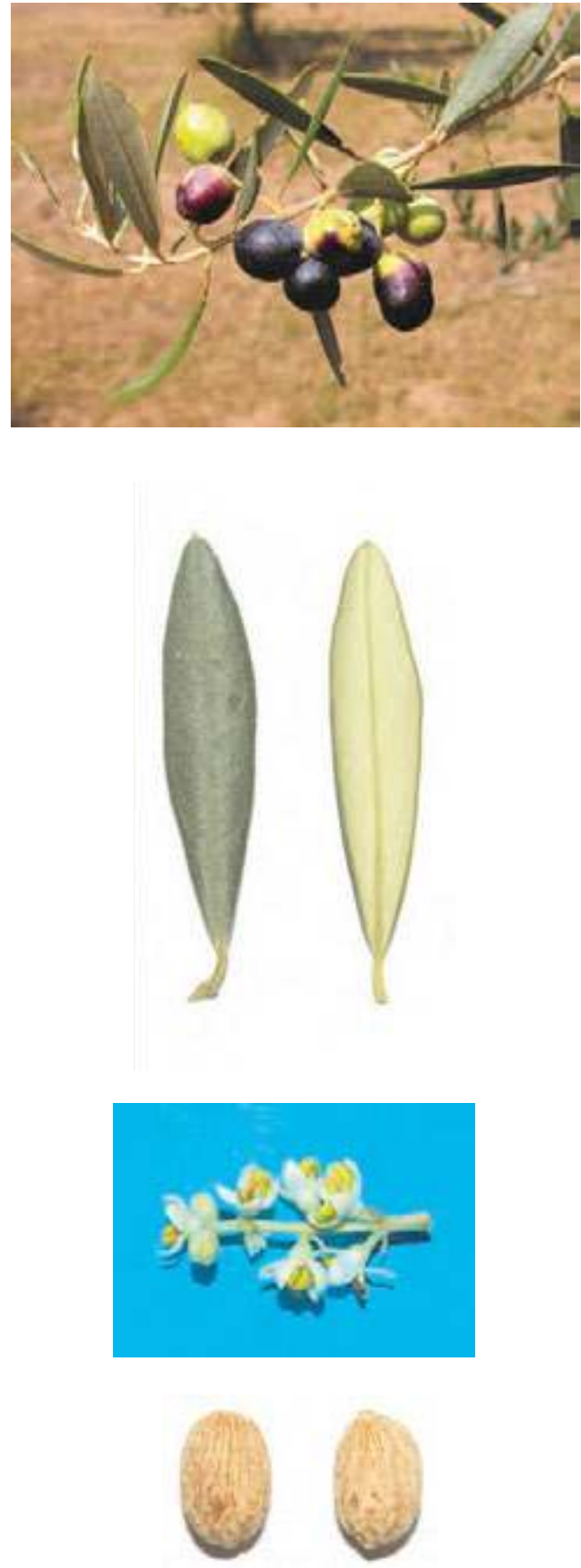


\section{Biochemical Characters}

\section{Fatty Acid Composition}

Table 1. Average values (express in $\% \pm$ standard deviations) of the fatty acids methyl esters and nutritional ratios obtained from single cultivar olive oils.

\begin{tabular}{|c|c|c|c|c|c|}
\hline Myristic acid & $0,01 \pm 0,00$ & Linoleic acid $(\omega 6)$ & $15,88 \pm 0,25$ & Lignoceric acid & $0,03 \pm 0,01$ \\
\hline Palmitic acid & $16,00 \pm 0,35$ & Linolenic acid $(\omega 3)$ & $0,84 \pm 0,05$ & & \\
\hline Palmitoleic acid & $2,65 \pm 0,05$ & Arachic acid & $0,29 \pm 0,03$ & Unsat./satured & $4,40 \pm 0,10$ \\
\hline teari & $1,85 \pm 0,08$ & Eicosenoic acid & $0,02 \pm 0,01$ & $\omega 6 / \omega 3$ & $19,04 \pm 1,44$ \\
\hline leic acid & $59,86 \pm 0,13$ & Behenic acid & $0,07 \pm 0,01$ & & \\
\hline
\end{tabular}

\section{Organoleptic oil values}

Sensory Analysis (Panel test)

Comment: fruity medium, with hints of almond, read sensations of grass and artichoke. Balanced taste sensation with a medium bitter and spicy. Medium-high fluidity.

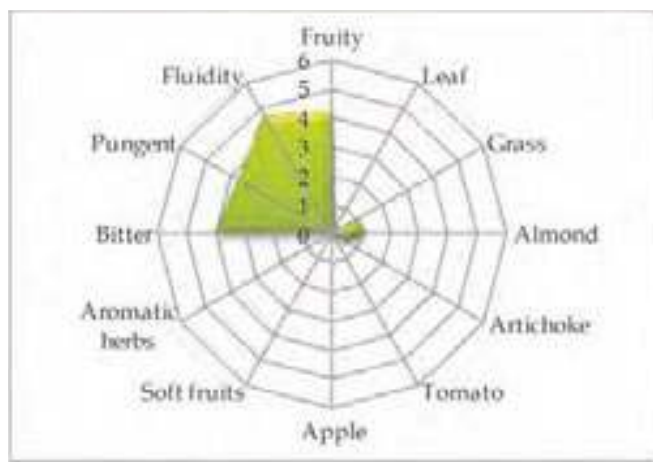

\section{Molecular Markers}

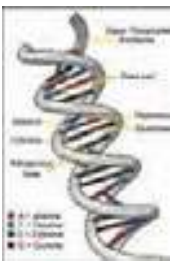

Table 2. Microsatellites (SSR) loci analyzed.

For each locus the allele size (expressed in base pairs) is reported.

$\begin{array}{cccccc}D C A 09 & \text { DCA18 } & \text { GAPU59 } & \text { GAPU71A } & \text { GUPA71B } & \text { GAPU103A } \\ \mathbf{1 9 8} \mathbf{- 2 1 0} & \mathbf{1 8 1} \mathbf{- 1 8 5} & \mathbf{2 1 2} \mathbf{- 2 1 8} & \mathbf{2 1 4 - \mathbf { 2 1 4 }} & \mathbf{1 2 4 - \mathbf { 1 2 4 }} & \mathbf{1 5 0 - \mathbf { 1 7 0 }} \\ \text { UDOO1 } & \text { UDO03 } & \text { UDO12 } & \text { UDO28 } & \text { UDO39 } & \\ \mathbf{1 4 0 - \mathbf { 1 4 0 }} & \mathbf{1 5 0 - \mathbf { 1 5 0 }} & \mathbf{1 7 7 - \mathbf { 1 9 3 }} & \mathbf{1 5 4 - \mathbf { 2 0 5 }} & \mathbf{2 1 3 - \mathbf { 2 1 3 }} & \end{array}$

\section{References:}

1 - Pietrangeli E., Russo A., In: Olivi D’Abruzzo, Grafiche di Prinzio (1997), pp. 28-29.

2 - Muzzalupo I., Lombardo N., Salimonti A., et al. Adv. Hort. Sci. (2008), 22(2): pp. 142-148. 


\section{"Cariasina"}

(synonymy: Cariascinu, Cariasina di Dorgali, Cerasina, Ciriegia, etc.)

Areal distribution or origin area: Sardegna

Flesh/pit weight ratio: medium $(6,46 \pm 1,11)$

Oil content (\%): low $(41,48 \pm 0,08)$

Purpose: dual purpose

\section{Morphological characters}

Tree characters

Vigour: medium-weak

Growth habit: spreading-erect

Canopy-density: medium

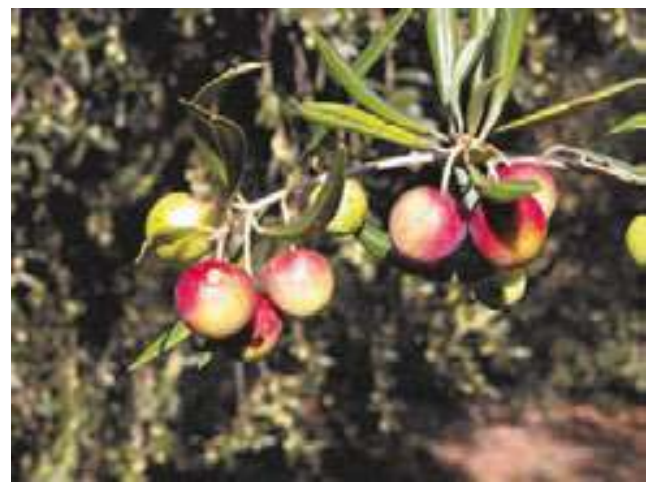

\section{Leaf characters}

Blade length $(\mathrm{cm})$ : long $(7,18 \pm 0,72)$

Blade width (cm): broad $(\mathbf{1}, 57 \pm 0,21)$

Shape (length/width): elliptic-lanceolate

\section{Inflorescence characters}

Inflorescence length $(\mathrm{cm})$ : medium $(2,96 \pm 0,24)$

Number of flowers: low $(\mathbf{1 5 , 6 2 \pm 1 , 0 2 )}$

\section{Fruit characters}

Fresh weight of 100 fruits (g): medium $(3,87 \pm 1,60)$

Shape (length/width): spherical

Symmetry: symmetric

Position of maximum transverse diameter:

central

Apex: rounded

Base: truncate

Niplle:absent

Lenticels: few and large

\section{Pit characters}

Weight of 100 pits (g): high $(\mathbf{0 , 5 1} \pm \mathbf{0 , 1 7})$

Shape (length/width): ovoid

Mucron: absent

Symmetry: slightly asymmetric

Position of maximum transverse diameter:

\section{towards base}

Apex: pointed

Base: truncate

Surface: rugose

Number of grooves: medium
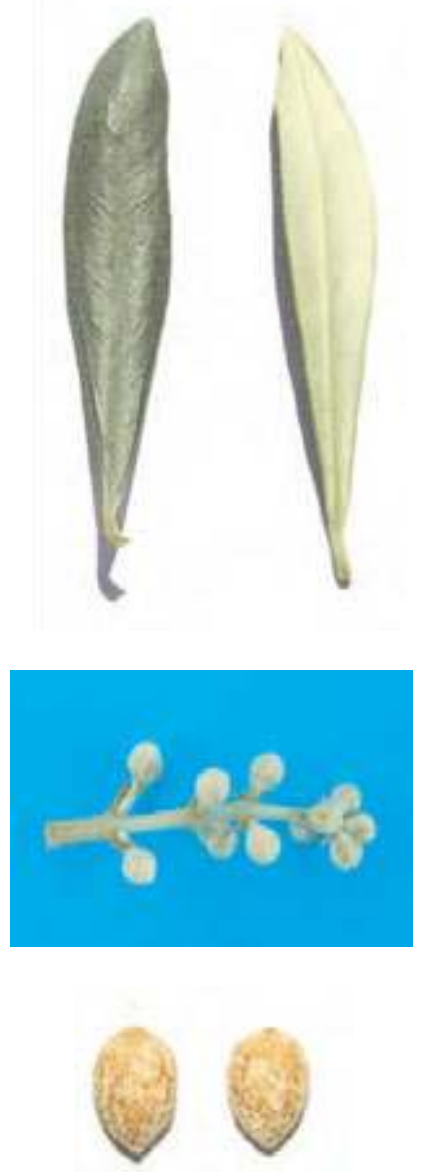


\section{Biochemical Characters}

\section{Fatty Acid Composition}

Table 1. Average values (express in $\% \pm$ standard deviations) of the fatty acids methyl esters and nutritional ratios obtained from single cultivar olive oils.

\begin{tabular}{|c|c|c|c|c|c|}
\hline Myristic acid & $0,01 \pm 0,01$ & Linoleic acid $(\omega 6)$ & $7,81 \pm 0,85$ & Lignoceric acid & $0,02 \pm 0,01$ \\
\hline Palmitic acid & $12,32 \pm 0,47$ & Linolenic acid $(\omega 3)$ & $0,80 \pm 0,07$ & & \\
\hline Palmitoleic acid & $0,92 \pm 0,10$ & Arachic acid & $0,25 \pm 0,06$ & Unsat./satured & $5,94 \pm 0,29$ \\
\hline Stearic acid & $1,83 \pm 0,05$ & Eicosenoic acid & $0,03 \pm 0,01$ & $\omega 6 / \omega 3$ & $9,74 \pm 0,18$ \\
\hline Oleic acid & $75,28 \pm 1,53$ & Behenic acid & $0,06 \pm 0,01$ & & \\
\hline
\end{tabular}

\section{Organoleptic oil values}

Sensory Analysis (Panel test)

Comment: fruity medium, with hints of grass, almond and artichoke. Balanced taste sensation with a medium-light bitter and spicy. Medium-light fluidity.

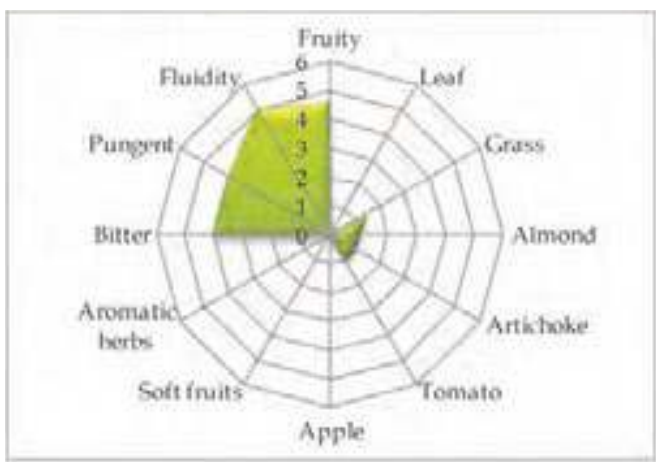

\section{Molecular Markers}

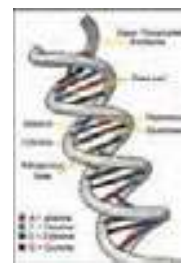

Table 2. Microsatellites (SSR) loci analyzed.

For each locus the allele size (expressed in base pairs) is reported.

\begin{tabular}{|c|c|c|c|c|c|}
\hline$D C A 09$ & $D C A 18$ & GAPU59 & GAPU71A & GUPA71B & GAPU103A \\
\hline $162-172$ & $179-181$ & $214-214$ & $214-218$ & $130-144$ & $136-184$ \\
\hline UDO01 & UDO03 & UDO12 & UDO28 & UDO39 & \\
\hline $144-150$ & $143-143$ & $166-193$ & $150-161$ & $205-205$ & \\
\hline
\end{tabular}

\section{References:}

1 - Bandino G., Sedda P., Moro C., et al. In: Atti $4^{\circ}$ Convegno Nazionale sulla Biodiversità, Alghero (2000), pp 243246.

2 - Muzzalupo I., Stefanizzi F., Perri E. HortScience (2009), 44: pp. 582-588. 


\section{"Carmelitana "}

Areal distribution or origin area: Puglia

Flesh/pit weight ratio: medium $(5,47 \pm 0,01)$

Oil content (\%): medium $(47,94 \pm 2,47)$

Purpose: table

\section{Morphological characters}

Tree characters

Vigour: medium

Growth habit: spreading-erect

Canopy-density: medium-dense

\section{Leaf characters}

Blade length $(\mathrm{cm})$ : medium $(5,23 \pm 0,52)$

Blade width (cm): broad $(\mathbf{1}, 54 \pm 0,16)$

Shape (length/width): elliptic

\section{Inflorescence characters}

Inflorescence length $(\mathrm{cm})$ : long $(3,56 \pm 0,74)$

Number of flowers: medium $(15,90 \pm 1,36)$

\section{Fruit characters}

Fresh weight of 100 fruits $(\mathrm{g})$ : high $(\mathbf{4}, \mathbf{6 0} \pm \mathbf{0 , 8 2})$

Shape (length/width): elongated

Symmetry: slightly asymmetric

Position of maximum transverse diameter:

central

Apex: pointed

Base: truncate

Niplle: obvious

Lenticels: few and large

\section{Pit characters}

Weight of 100 pits $(\mathrm{g})$ : very high $(\mathbf{0 , 7 1} \pm \mathbf{0 , 0 8})$

Shape (length/width): elongated

Mucron: obvious

Symmetry: asymmetric

Position of maximum transverse diameter:

\section{towards apex}

Apex: pointed

Base: pointed

Surface: rugose

Number of grooves: medium
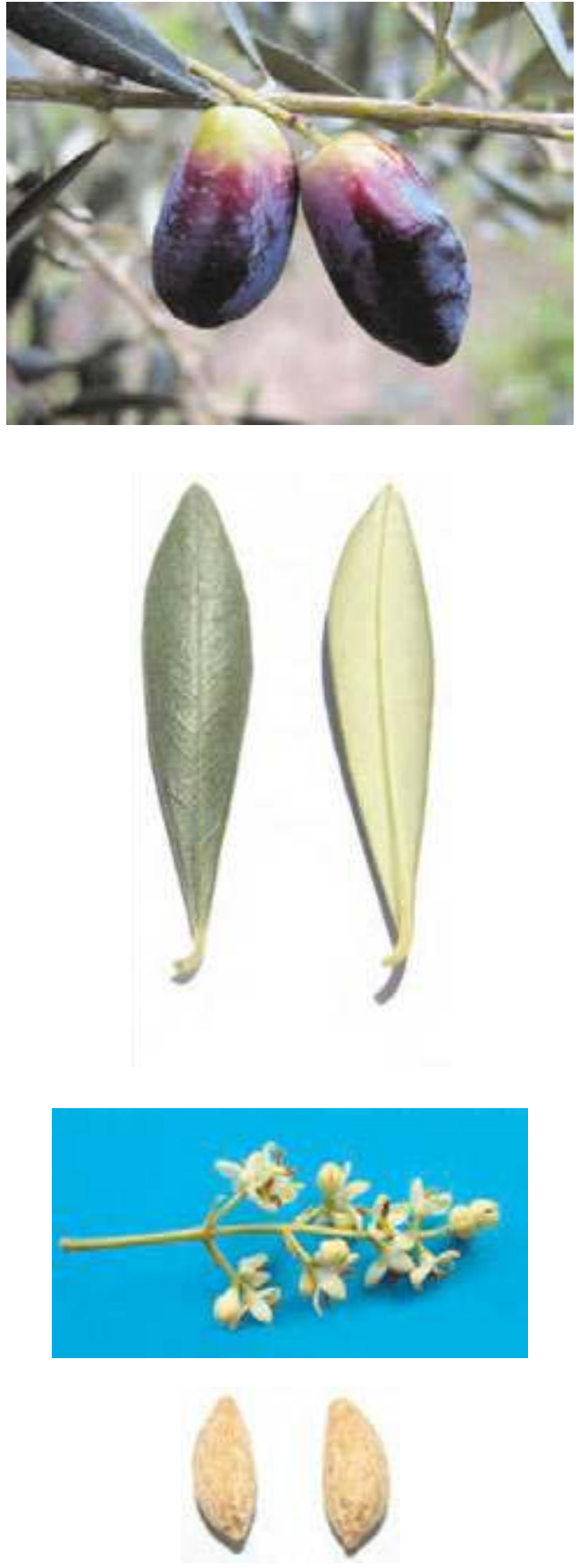


\section{Biochemical Characters}

\section{Fatty Acid Composition}

Table 1. Average values (express in $\% \pm$ standard deviations) of the fatty acids methyl esters and nutritional ratios obtained from single cultivar olive oils.

\begin{tabular}{|c|c|c|c|c|c|}
\hline Myristic acid & $0,01 \pm 0,01$ & Linoleic acid $(\omega 6)$ & $11,55 \pm 0,10$ & Lignoceric acid & $0,07 \pm 0,01$ \\
\hline Palmitic acid & $14,87 \pm 1,29$ & Linolenic acid $(\omega 3)$ & $0,46 \pm 0,08$ & & \\
\hline Palmitoleic acid & $1,72 \pm 0,34$ & Arachic acid & $0,52 \pm 0,05$ & Unsat./satured & $4,74 \pm 0,35$ \\
\hline tearic acid & $1,91 \pm 0,03$ & Eicosenoic acid & $0,41 \pm 0,02$ & $\omega 6 / \omega 3$ & $25,21 \pm 4,05$ \\
\hline leic acid & $66,83 \pm 0,38$ & Behenic acid & $0,14 \pm 0,06$ & & \\
\hline
\end{tabular}

\section{Organoleptic oil values}

Sensory Analysis (Panel test)

Comment: fruity medium-high, with hints of almond, read sensations of grass, soft fruits, and artichoke. Balanced taste sensation with a medium bitter and spicy. Medium-high fluidity.

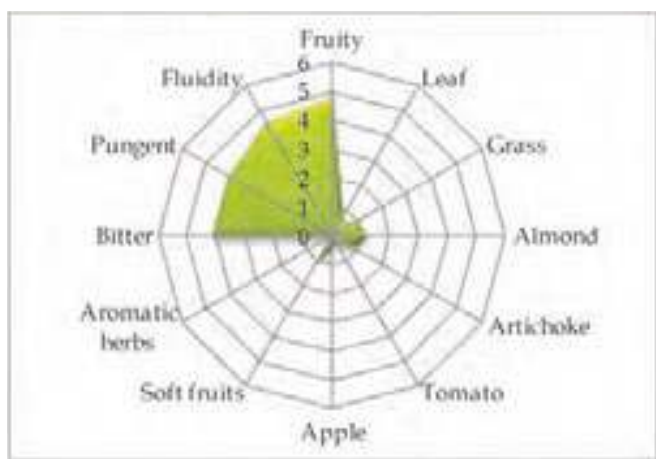

\section{Molecular Markers}

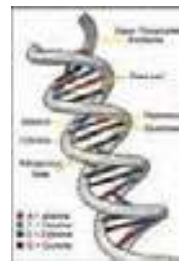

Table 2. Microsatellites (SSR) loci analyzed.

For each locus the allele size (expressed in base pairs) is reported.

$\begin{array}{cccccc}D C A 09 & \text { DCA18 } & \text { GAPU59 } & \text { GAPU71A } & \text { GUPA71B } & \text { GAPU103A } \\ \mathbf{1 9 8} \mathbf{- 1 9 8} & \mathbf{1 7 9 - \mathbf { 1 8 1 }} & \mathbf{2 0 8 - \mathbf { 2 0 8 }} & \mathbf{2 1 4} \mathbf{- 2 2 4} & \mathbf{1 2 4} \mathbf{- 1 2 6} & \mathbf{1 3 6} \mathbf{- 1 5 9} \\ \text { UDOO1 } & \text { UDOO3 } & \text { UDO12 } & \text { UDO28 } & \text { UDO39 } & \\ \mathbf{1 4 4 - \mathbf { 1 4 4 }} & \mathbf{1 4 3 - \mathbf { 1 4 3 }} & \mathbf{1 6 6 - 1 9 3} & \mathbf{1 8 2} \mathbf{- 1 8 2} & \mathbf{1 8 5 - \mathbf { 1 8 5 }} & \end{array}$

\section{References:}

1 - Lombardo N., Perri E., Muzzalupo I., et al. In: Contributo alla caratterizzazione del germoplasma olivicolo pugliese, Ist. Sper. Olivic. (2004), pp. 29-32.

2 - Muzzalupo I., Stefanizzi F., Perri E. HortScience (2009), 44: pp. 582-588. 


\section{"Carolea"}

(synonymy: Becco di Corvo, Borgese, Catanzarese, Cumignana, Olivona, etc.)

Areal distribution or origin area: Calabria

Flesh/ pit weight ratio: high $(7,66 \pm 1,16)$

Oil content (\%): hig $(\mathbf{5 1 , 4 4} \pm \mathbf{1 , 5 2})$

Purpose: dual purpose

\section{Morphological characters}

Tree characters

Vigour: medium

Growth habit: erect

Canopy-density: medium

\section{Leaf characters}

Blade length $(\mathrm{cm})$ : medium $(6,46 \pm 0,83)$

Blade width $(\mathrm{cm})$ : medium $(1,34 \pm 0,14)$

Shape (length/width): elliptic-lanceolate

\section{Inflorescence characters}

Inflorescence length $(\mathrm{cm})$ : medium $(2,56 \pm 3,63)$

Number of flowers: low $(\mathbf{1 3}, \mathbf{2 0} \pm \mathbf{4 , 3 0})$

\section{Fruit characters}

Fresh weight of 100 fruits $(\mathrm{g})$ : medium $(\mathbf{5 , 4 1} \pm \mathbf{1 , 5 1})$

Shape (length/width): ovoid

Symmetry: asymmetric

Position of maximum transverse diameter:

central

Apex: rounded

Base: rounded

Nipple: obvious

Lenticels: few and large

\section{Pit characters}

Weight of 100 pits (g): high $(\mathbf{0 , 6 2} \pm \mathbf{0 , 1 5})$

Shape (length/width): elliptic

Mucron: obvious

Symmetry: asymmetric

Position of maximum transverse diameter:

central

Apex: rounded

Base: pointed

Surface: rugose

Number of grooves: medium
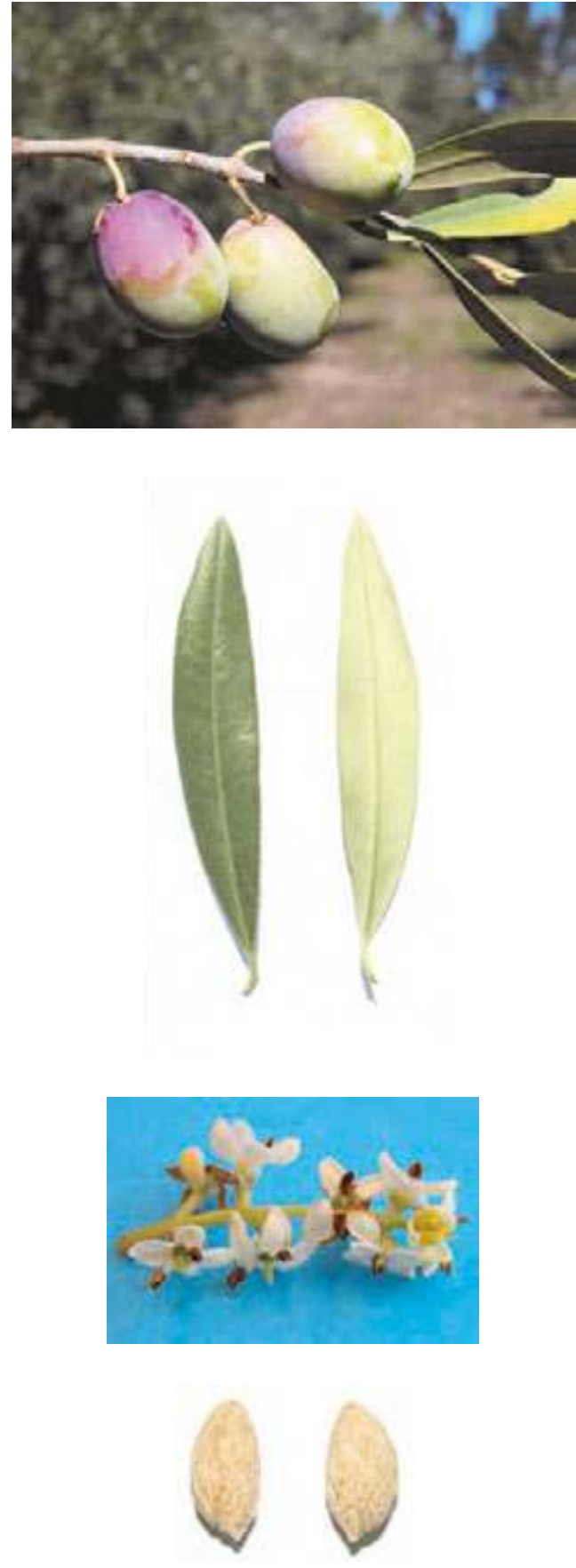


\section{Biochemical Characters}

\section{Fatty Acid Composition}

Table 1. Average values (express in $\% \pm$ standard deviations) of the fatty acids methyl esters and nutritional ratios obtained from single cultivar olive oils.

$\begin{array}{llllll}\text { Myristic acid } & \mathbf{0 , 0 1} \pm \mathbf{0 , 0 1} & \text { Linoleic acid }(\omega 6) & \mathbf{6 , 0 4} \pm \mathbf{0 , 3 5} & \text { Lignoceric acid } & \mathbf{0 , 0 8} \pm \mathbf{0 , 0 3} \\ \text { Palmitic acid } & \mathbf{1 4 , 8 3} \pm \mathbf{0 , 9 1} & \text { Linolenic acid }(\omega 3) & \mathbf{0 , 4 6} \pm \mathbf{0 , 1 6} & & \\ \text { Palmitoleic acid } & \mathbf{1 , 7 0 \pm 0 , 2 2} & \text { Arachic acid } & \mathbf{0 , 4 0} \pm \mathbf{0 , 0 8} & \text { Unsat./satured } & \mathbf{4 , 5 9 \pm 0 , 2 6} \\ \text { Stearic acid } & \mathbf{2 , 8 1} \pm \mathbf{0 , 4 6} & \text { Eicosenoic acid } & \mathbf{0 , 2 0} \pm \mathbf{0 , 1 2} & \omega 6 / \omega 3 & \mathbf{1 4 . 0 9} \pm \mathbf{3 , 8 5} \\ \text { Oleic acid } & \mathbf{7 2 , 8 5 \pm 0 , 7 0} & \text { Behenic acid } & \mathbf{0 , 1 2} \pm \mathbf{0 , 0 2} & & \end{array}$

\section{Organoleptic oil values}

Sensory Analysis (Panel test)

Comment: medium-high fruity, with good sensation of grass, less almond and artichoke. Balanced taste sensation, with bitter and spicy medium. Medium-high fluidity.

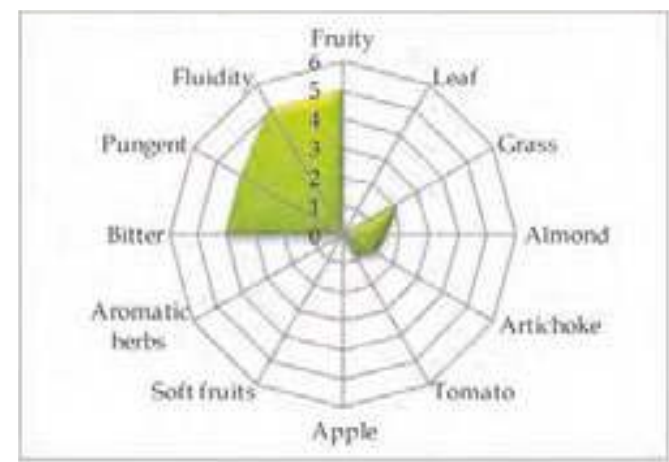

\section{Molecular Markers}

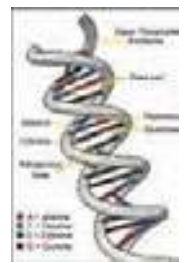

Table 2. Microsatellites (SSR) loci analyzed.

For each locus the allele size (expressed in base pairs) is reported.

\begin{tabular}{|c|c|c|c|c|c|}
\hline$D C A 09$ & $D C A 18$ & GAPU59 & GAPU71A & GUPA71B & GAPU103A \\
\hline $162-198$ & $179-185$ & $212-222$ & $214-214$ & $121-130$ & $136-170$ \\
\hline UDO01 & UDO03 & UDO12 & UDO28 & UDO39 & \\
\hline $144-144$ & $143-143$ & $166-193$ & $154-205$ & $200-200$ & \\
\hline
\end{tabular}

\section{References:}

1 - Lombardo N., Perri E., Muzzalupo I., et al. In: Il germoplasma olivicolo calabrese, Ist. Sper. Olivic.(2003), pp: 3-4.

2 - Perri E., Mazzotti F., Muzzalupo I., et al. In: Relazione attività, CO.R.ASS.OL. (2003).

3 - Muzzalupo I., Chiappetta A., Benincasa C., et al. Sci Hortic-Amsterdam, (2010), 126: pp. 324-329. 


\section{“Carpinetana"}

(synonymy: Femminina, Nebbio di montagna, Pizzutella, Posolella, etc.)

Areal distribution or origin area: Abruzzo

Flesh/pit weight ratio: medium $(5,50 \pm 0,27)$

Oil content (\%): medium $(46,26 \pm 1,37)$

Purpose: oil

\section{Morphological characters}

Tree characters

Vigour: weak

Growth habit: spreading-erect

Canopy-density: medium

\section{Leaf characters}

Blade length $(\mathrm{cm})$ : medium $(6,02 \pm 0,30)$

Blade width $(\mathrm{cm})$ : medium $(\mathbf{1}, 46 \pm \mathbf{0 , 1 2})$

Shape (length/width): elliptic-lanceolate

\section{Inflorescence characters}

Inflorescence length $(\mathrm{cm})$ : medium $(2,95 \pm 0,18)$

Number of flowers: medium $(20,09 \pm 1,82)$

\section{Fruit characters}

Fresh weight of 100 fruits $(\mathrm{g})$ : medium $(3,57 \pm 0,37)$

Shape (length/width): spherical

Symmetry: symmetric

Position of maximum transverse diameter:

central

Apex: rounded

Base: truncate

Niplle: absent

Lenticels: few and small

\section{Pit characters}

Weight of 100 pits (g): medium $(0,55 \pm 0,04)$

Shape (length/width): ovoid

Mucron: absent

Symmetry: symmetric

Position of maximum transverse diameter:

towards apex

Apex: rounded

Base: rounded

Surface: smooth

Number of grooves: medium
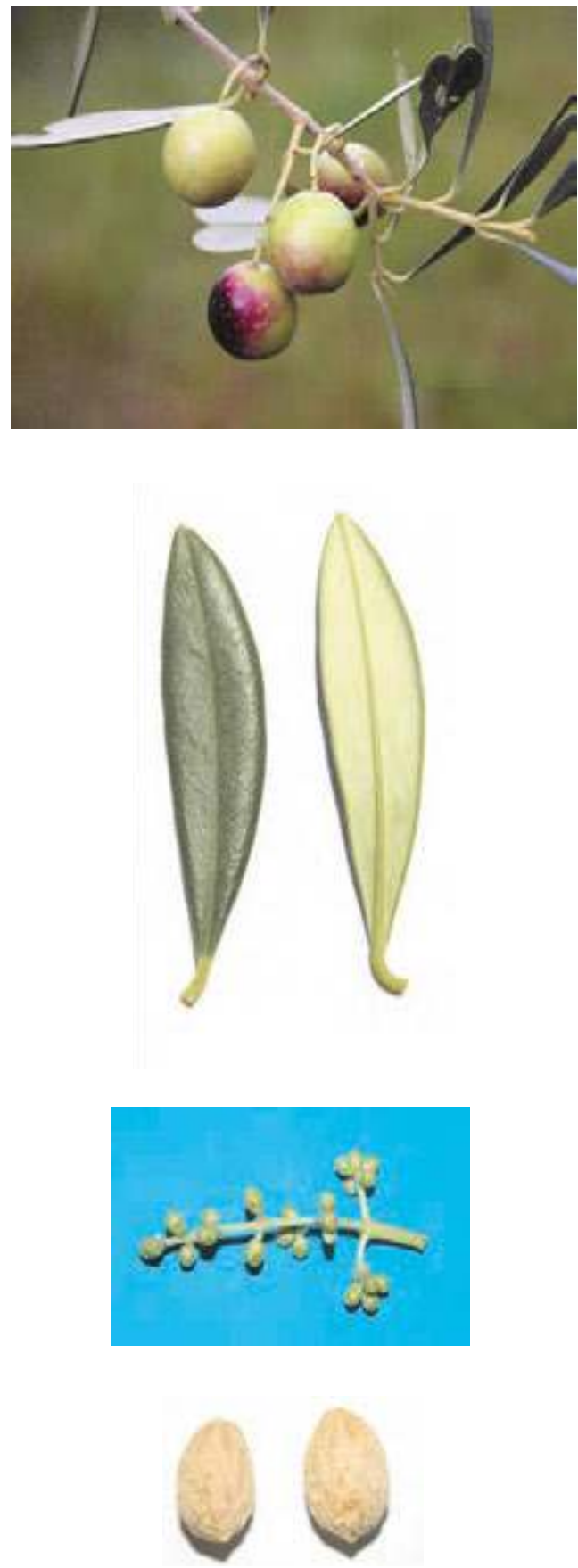


\section{Biochemical Characters}

\section{Fatty Acid Composition}

Table 1. Average values (express in $\% \pm$ standard deviations) of the fatty acids methyl esters and nutritional ratios obtained from single cultivar olive oils.

\begin{tabular}{|c|c|c|c|c|c|}
\hline Myristic acid & $0,01 \pm 0,00$ & Linoleic acid $(\omega 6)$ & $11,75 \pm 1,04$ & Lignoceric acid & $0,03 \pm 0,01$ \\
\hline Palmitic acid & $11,64 \pm 1,91$ & Linolenic acid $(\omega 3)$ & $0,93 \pm 0,02$ & & \\
\hline Palmitoleic acid & $0,74 \pm 0,42$ & Arachic acid & $0,32 \pm 0,13$ & Unsat./satured & $6,26 \pm 0,87$ \\
\hline tearic acid & $1,94 \pm 0,21$ & Eicosenoic acid & $0,02 \pm 0,00$ & $\omega 6 / \omega 3$ & $12,57 \pm 0,82$ \\
\hline leic acid & $72,14 \pm 0,26$ & Behenic acid & $0,08 \pm 0,01$ & & \\
\hline
\end{tabular}

\section{Organoleptic oil values}

Sensory Analysis (Panel test)

Comment: fruity medium-hight, with good sensation of grass, artichoke and almond. Balanced in flavours, with hints of bitter and spicy medium-high intensity. Medium-high fluidity.

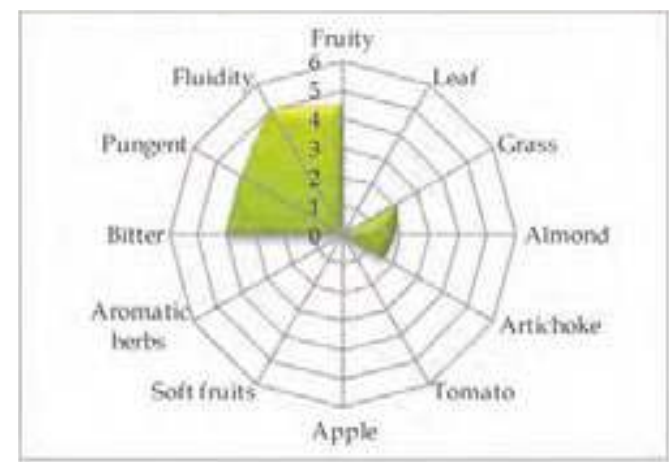

\section{Molecular Markers}

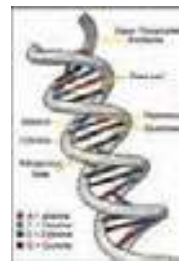

Table 2. Microsatellites (SSR) loci analyzed.

For each locus the allele size (expressed in base pairs) is reported.

\begin{tabular}{|c|c|c|c|c|c|}
\hline DCA09 & DCA18 & GAPU59 & GAPU71A & $G U P A 71 B$ & GAPU103A \\
\hline $166-206$ & $177-181$ & $212-222$ & $214-214$ & $126-144$ & $184-184$ \\
\hline UDO01 & UDO03 & UDO12 & UDO28 & UDO39 & \\
\hline $144-144$ & $150-150$ & $177-193$ & $154-210$ & $205-205$ & \\
\hline
\end{tabular}

\section{References:}

1 - Pietrangeli E., Russo A., In: Olivi D'Abruzzo, Grafiche di Prinzio (1997), pp. 30-31.

2 - Muzzalupo I., Salimonti A., Caravita M. A., et al. Adv. Hort. Sci. (2008), 22(2): pp. 129-135. 


\section{"Castiglionese"}

(synonymy: Dritta, Oliva di San Francesco)

Areal distribution or origin area: Abruzzo

Flesh/pit weight ratio: medium $(5,88 \pm 0,30)$

Oil content (\%): medium $(40,21 \pm 1,21)$

Purpose: oil

\section{Morphological characters}

Tree characters

Vigour: medium-strong

Growth habit: spreading-erect

Canopy-density: medium

\section{Leaf characters}

Blade length (cm): medium $(5,54 \pm 0,54)$

Blade width $(\mathrm{cm})$ : medium $(1,30 \pm 0,16)$

Shape (length/width): elliptic - lanceolate

\section{Inflorescence characters}

Inflorescence length (cm): medium $(2,57 \pm 0,58)$

Number of flowers: medium $(\mathbf{1 8 , 8 6} \pm 3,38)$

\section{Fruit characters}

Fresh weight of 100 fruits $(\mathrm{g})$ : medium $(2,09 \pm 0,09)$

Shape (length/width): ovoid

Symmetry: slightly asymmetric

Position of maximum transverse diameter:

central

Apex: rounded

Base: truncate

Niplle: tenuous

Lenticels: many and small

\section{Pit characters}

Weight of 100 pits (g): medium $(0,31 \pm 0,03)$

Shape (length/width): elliptic

Mucron: obvious

Symmetry: symmetric

Position of maximum transverse diameter:

central

Apex: pointed

Base: rounded

Surface: rugose

Number of grooves: high
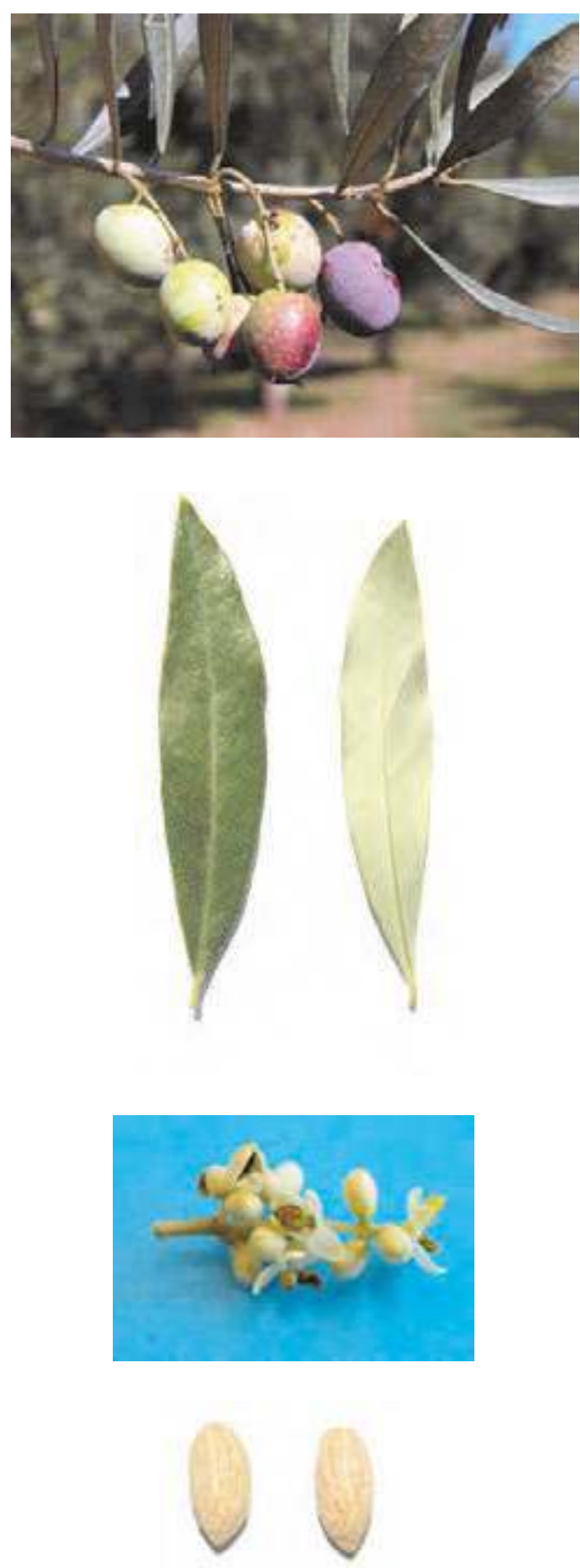


\section{Biochemical Characters}

\section{Fatty Acid Composition}

Table 1. Average values (express in $\% \pm$ standard deviations) of the fatty acids methyl esters and nutritional ratios obtained from single cultivar olive oils.

\begin{tabular}{|c|c|c|c|c|c|}
\hline Myristic acid & $0,01 \pm 0,00$ & Linoleic acid $(\omega 6)$ & $11,25 \pm 0,38$ & Lignoceric acid & $0,05 \pm 0,03$ \\
\hline Palmitic acid & $0,02 \pm 0,01$ & Linolenic acid $(\omega 3)$ & $0,80 \pm 0,09$ & & \\
\hline Palmitoleic acid & $1,51 \pm 0,64$ & Arachic acid & $0,30 \pm 0,14$ & Unsat./satured & $5,20 \pm 0,01$ \\
\hline teari & $1,62 \pm 0,04$ & Eicosenoic acid & $0,44 \pm 0,06$ & $\omega 6 / \omega 3$ & $14,27 \pm 2,13$ \\
\hline leic acid & $69,03 \pm 0,24$ & Behenic acid & $0,10 \pm 0,00$ & & \\
\hline
\end{tabular}

\section{Organoleptic oil values}

Sensory Analysis (Panel test)

Comment: fruity medium-high, with hints of grass and artichoke, read sensations of almond. Balanced taste sensation with a medium-high bitter and spicy. Medium-high fluidity.

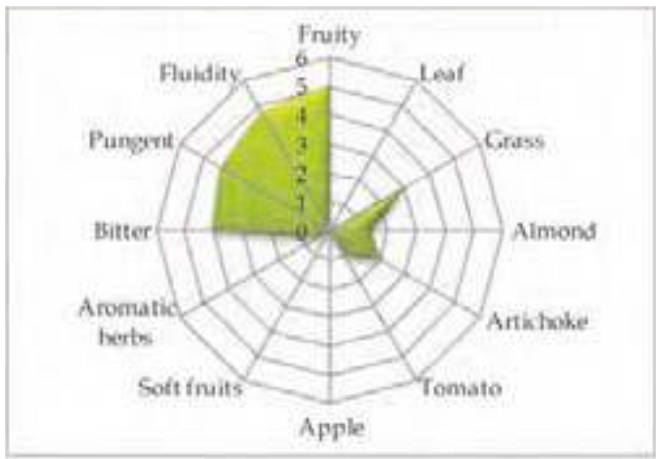

\section{Molecular Markers}

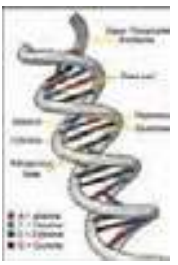

Table 2. Microsatellites (SSR) loci analyzed.

For each locus the allele size (expressed in base pairs) is reported.

$\begin{array}{cccccc}D C A 09 & \text { DCA18 } & \text { GAPU59 } & \text { GAPU71A } & \text { GUPA71B } & \text { GAPU103A } \\ \mathbf{1 9 8} \mathbf{- 2 0 6} & \mathbf{1 6 3 - \mathbf { 1 8 1 }} & \mathbf{2 0 8 - \mathbf { 2 1 2 }} & \mathbf{2 1 0 - \mathbf { 2 1 4 }} & \mathbf{1 2 6 - \mathbf { 1 2 6 }} & \mathbf{1 5 9 - \mathbf { 1 8 4 }} \\ \text { UDOO1 } & \text { UDOO3 } & \text { UDO12 } & \text { UDO28 } & \text { UDO39 } & \\ \mathbf{1 4 4 - \mathbf { 1 4 4 }} & \mathbf{1 5 0 - \mathbf { 1 5 0 }} & \mathbf{1 6 6 - \mathbf { 1 9 3 }} & \mathbf{1 5 4 - \mathbf { 2 1 0 }} & \mathbf{2 0 5 - \mathbf { 2 3 2 }} & \end{array}$

\section{References:}

1 - Pietrangeli E., Russo A., In: Olivi D'Abruzzo, Grafiche di Prinzio (1997), pp. 32-33.

2 - Muzzalupo I., Salimonti A., Caravita M. A., et al. Adv. Hort. Sci. (2008), 22(2): pp. 129-135. 


\section{“Castricianella Rapparina"}

Areal distribution or origin area: Sicilia

Flesh/pit weight ratio: medium $(6,66 \pm 3,76)$

Oil content (\%): medium $(46,10 \pm 1,80)$

Purpose: oil

\section{Morphological characters}

Tree characters

Vigour: medium-strong

Growth habit: erect-spreading

Canopy-density: medium-dense

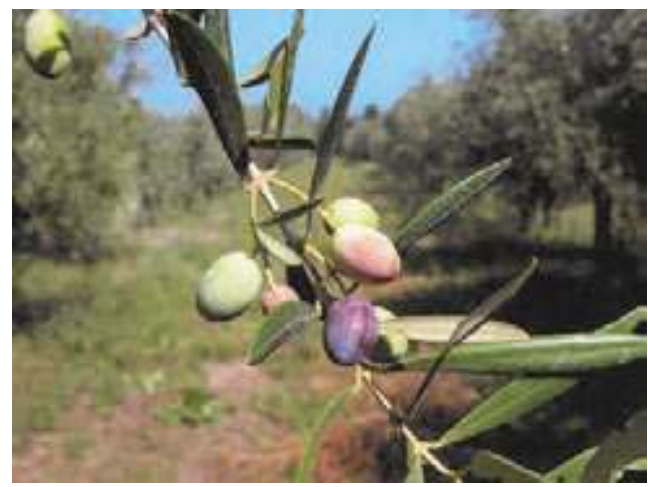

\section{Leaf characters}

Blade length $(\mathrm{cm})$ : medium $(6,30 \pm 0,55)$

Blade width $(\mathrm{cm})$ : medium $(\mathbf{1 , 2 7} \pm \mathbf{0 , 1 2})$

Shape (length/width): elliptic-lanceolate

\section{Inflorescence characters}

Inflorescence length $(\mathrm{cm})$ : medium $(2,94 \pm 0,90)$

Number of flowers: medium $(21,32 \pm 1,63)$

\section{Fruit characters}

Fresh weight of 100 fruits $(\mathrm{g})$ : medium $(2,20 \pm 0,90)$

Shape (length/width): ovoid

Symmetry: slightly asymmetric

Position of maximum transverse diameter:

central

Apex: rounded

Base: truncate

Niplle: tenuous

Lenticels: many and small

\section{Pit characters}

Weight of 100 pits (g): medium $(0,30 \pm 0,03)$

Shape (length/width): elliptic

Mucron: obvious

Symmetry: symmetric

Position of maximum transverse diameter:

central

Apex: rounded

Base: rounded

Surface: smooth

Number of grooves: low
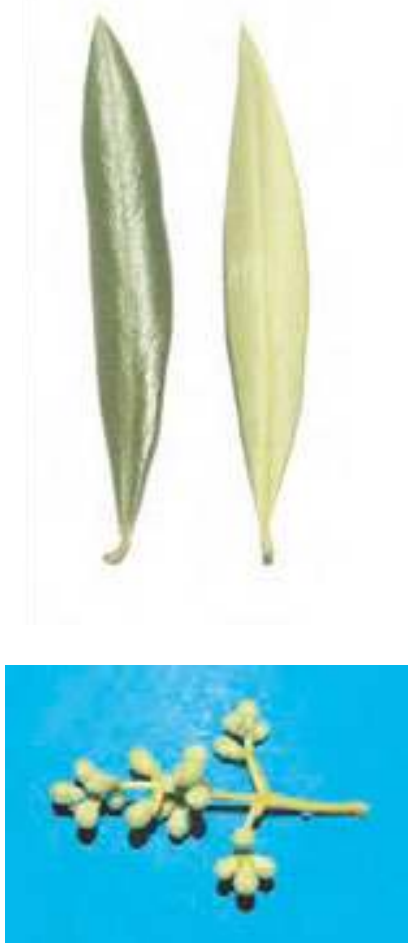


\section{Biochemical Characters}

\section{Fatty Acid Composition}

Table 1. Average values (express in $\% \pm$ standard deviations) of the fatty acids methyl esters and nutritional ratios obtained from single cultivar olive oils.

\begin{tabular}{|c|c|c|c|c|c|}
\hline Myristic acid & $0,02 \pm 0,01$ & Linoleic acid $(\omega 6)$ & $10,14 \pm 0,15$ & Lignoceric acid & $0,02 \pm 0,01$ \\
\hline Palmitic acid & $13,44 \pm 0,77$ & Linolenic acid $(\omega 3)$ & $0,62 \pm 0,07$ & & \\
\hline Palmitoleic acid & $1,45 \pm 0,02$ & Arachic acid & $0,24 \pm 0,03$ & Unsat./satured & $4,74 \pm 0,10$ \\
\hline teari & $3,53 \pm 0,51$ & Eicosenoic acid & $0,03 \pm 0,01$ & $\omega 6 / \omega 3$ & $16,46 \pm 2,16$ \\
\hline leic acid & $68,87 \pm 0,35$ & Behenic acid & $0,06 \pm 0,01$ & & \\
\hline
\end{tabular}

\section{Organoleptic oil values}

Sensory Analysis (Panel test)

Comment: fruity medium-high, with hints of almond and read sensations of grass and artichoke. Balanced taste sensation with a medium-high bitter and spicy. Medium-high fluidity.

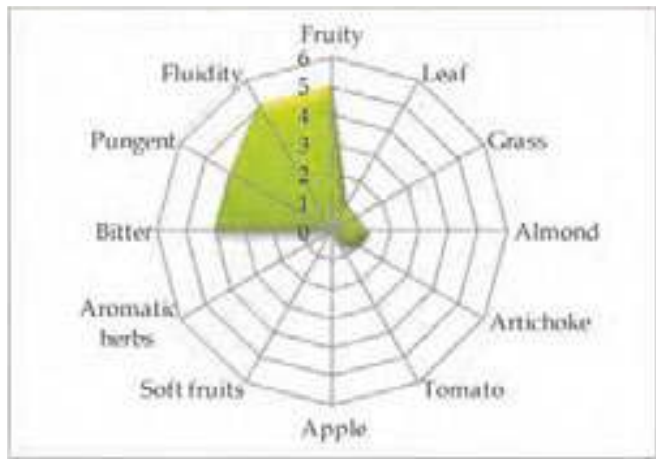

\section{Molecular Markers}

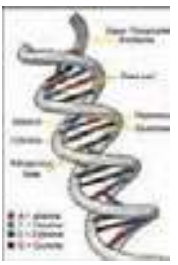

Table 2. Microsatellites (SSR) loci analyzed.

For each locus the allele size (expressed in base pairs) is reported.

$\begin{array}{cccccc}D C A 09 & D C A 18 & \text { GAPU59 } & \text { GAPU71A } & \text { GUPA71B } & \text { GAPU103A } \\ \mathbf{1 9 8 - \mathbf { 2 0 6 }} & \mathbf{1 7 9 - \mathbf { 1 7 9 }} & \mathbf{2 0 8 - \mathbf { 2 2 2 }} & \mathbf{2 1 0 - \mathbf { 2 1 4 }} & \mathbf{1 2 4} \mathbf{- 1 3 0} & \mathbf{1 5 9} \mathbf{- 1 7 0} \\ \text { UDOO1 } & \text { UDO03 } & \text { UDO12 } & \text { UDO28 } & \text { UDO39 } & \\ \mathbf{1 4 4 - \mathbf { 1 4 4 }} & \mathbf{1 5 0 - \mathbf { 1 5 0 }} & \mathbf{1 7 7 - \mathbf { 1 7 7 }} & \mathbf{1 5 4 - \mathbf { 2 0 5 }} & \mathbf{1 7 0 - \mathbf { 2 1 3 }} & \end{array}$

\section{References:}

1 - Lombardo N. In: Le risorse genetiche vegetali presso gli IRSA, MIPAF (2001), 1: pp. 361-405.

2 - Muzzalupo I., Stefanizzi F., Perri E. HortScience (2009), 44: 582-588. 


\section{"Cavalieri "}

Areal distribution or origin area: Sicilia

Flesh/pit weight ratio: medium $(7,06 \pm 1,59)$

Oil content (\%): medium $(48,12 \pm 1,37)$

Purpose: oil

\section{Morphological characters}

Tree characters

Vigour: weak

Growth habit: spreading-drooping

Canopy-density: medium-dense

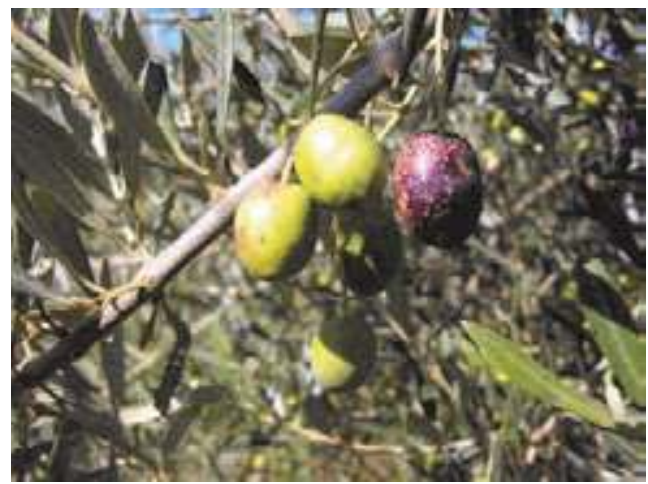

\section{Leaf characters}

Blade length $(\mathrm{cm})$ : medium $(5,66 \pm 0,44)$

Blade width $(\mathrm{cm})$ : narrow $(0,99 \pm 0,12)$

Shape (length/width): elliptic - lanceolate

\section{Inflorescence characters}

Inflorescence length $(\mathrm{cm})$ : medium $(2,72 \pm 1,33)$

Number of flowers: medium $(\mathbf{1 8 , 0 4} \pm \mathbf{2 , 6 9})$

\section{Fruit characters}

Fresh weight of 100 fruits $(\mathrm{g})$ : medium $(2,71 \pm 0,26)$

Shape (length/width): ovoid

Symmetry: slightly asymmetric

Position of maximum transverse diameter:

central

Apex: rounded

Base: truncate

Niplle: absent

Lenticels: many and small

\section{Pit characters}

Weight of 100 pits (g): medium $(0,34 \pm \mathbf{0 , 0 3})$

Shape (length/width): elliptic

Mucron: tenuous

Symmetry: slightly asymmetric

Position of maximum transverse diameter:

towards apex

Apex: rounded

Base: rounded

Surface: smooth

Number of grooves: low
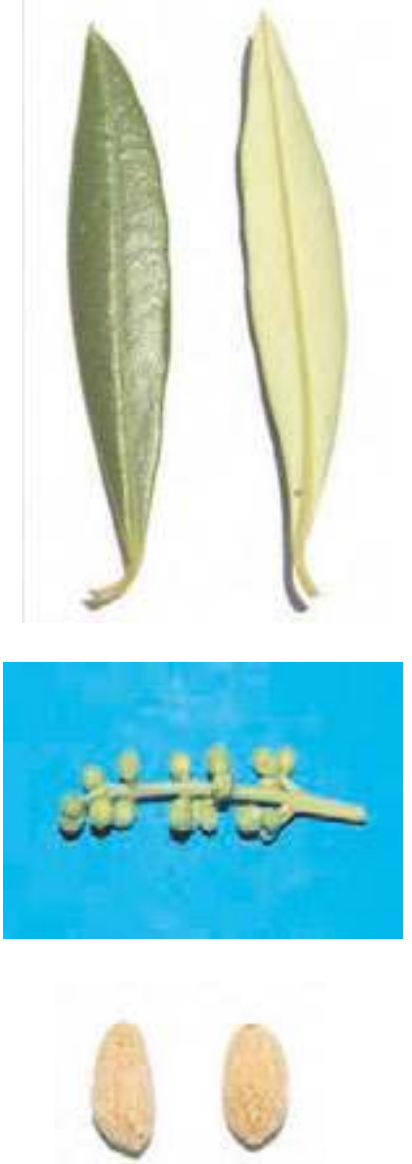


\section{Biochemical Characters}

\section{Fatty Acid Composition}

Table 1. Average values (express in $\% \pm$ standard deviations) of the fatty acids methyl esters and nutritional ratios obtained from single cultivar olive oils.

$\begin{array}{llllll}\text { Myristic acid } & \mathbf{0 , 0 1} \pm \mathbf{0 , 0 0} & \text { Linoleic acid }(\omega 6) & \mathbf{8 , 6 5} \pm \mathbf{0 , 7 1} & \text { Lignoceric acid } & \mathbf{0 , 0 4 \pm 0 , 0 1} \\ \text { Palmitic acid } & \mathbf{1 2 , 6 0 \pm 0 , 8 6} & \text { Linolenic acid }(\omega 3) \mathbf{0 , 8 9} \pm \mathbf{0 , 1 0} & & \\ \text { Palmitoleic acid } & \mathbf{1 , 7 8} \pm \mathbf{0 , 2 2} & \text { Arachic acid } & \mathbf{0 , 3 0} \pm \mathbf{0 , 0 1} & \text { Unsat./satured } & \mathbf{5 , 7 1 \pm 0 , 4 1} \\ \text { Stearic acid } & \mathbf{1 , 9 1} \pm \mathbf{0 , 0 7} & \text { Eicosenoic acid } & \mathbf{0 , 0 2} \pm \mathbf{0 , 0 1} & \omega 6 / \omega 3 & \mathbf{9 , 7 6} \pm \mathbf{0 , 3 3} \\ \text { Oleic acid } & \mathbf{7 2 , 1 3} \pm \mathbf{0 , 2 2} & \text { Behenic acid } & \mathbf{0 , 0 5} \pm \mathbf{0 , 0 5} & & \end{array}$

\section{Organoleptic oil values}

Sensory Analysis (Panel test)

Comment: fruity medium, with taste of almond. Balanced taste sensation with medium-light bitter and spicy. Medium fluidity.

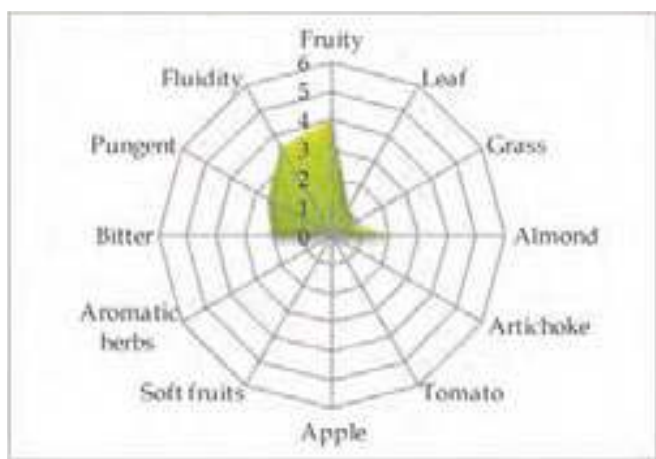

\section{Molecular Markers}

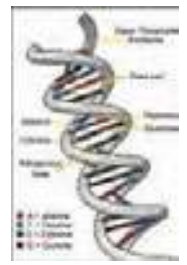

Table 2. Microsatellites (SSR) loci analyzed.

For each locus the allele size (expressed in base pairs) is reported.

$\begin{array}{cccccc}D C A 09 & \text { DCA18 } & \text { GAPU59 } & \text { GAPU71A } & \text { GUPA71B } & \text { GAPU103A } \\ \mathbf{1 6 2} \mathbf{- 1 6 2} & \mathbf{1 7 7 - \mathbf { 1 8 5 }} & \mathbf{2 1 2} \mathbf{- 2 2 2} & \mathbf{2 1 0 - \mathbf { 2 1 0 }} & \mathbf{1 2 4} \mathbf{- 1 4 4} & \mathbf{1 5 9} \mathbf{- 1 5 9} \\ \text { UDOO1 } & \text { UDOO3 } & \text { UDO12 } & \text { UDO28 } & \text { UDO39 } & \\ \mathbf{1 4 4 - \mathbf { 1 4 4 }} & \mathbf{1 5 0 - \mathbf { 1 5 0 }} & \mathbf{1 7 7 - \mathbf { 1 9 3 }} & \mathbf{1 5 4} \mathbf{- 1 5 4} & \mathbf{1 0 8 - 1 0 8} & \end{array}$

\section{References:}

1 - Caruso T., Cartabellotta D., Motisi A., et all. In: Cultivar di olivo siciliane, Università degli Studi di Palermo (2007), pp. 146-150.

2 - Muzzalupo I., Stefanizzi F., Perri E. HortScience (2009), 44: pp. 582-588. 


\section{"Cazzinicchio"}

Areal distribution or origin area: Puglia

Flesh/pit weight ratio: medium $(5,69 \pm 0,05)$

Oil content (\%): medium $(43,83 \pm 0,88)$

Purpose: dual purpose

\section{Morphological characters}

Tree characters

Vigour: medium

Growth habit: spreading

Canopy-density: dense

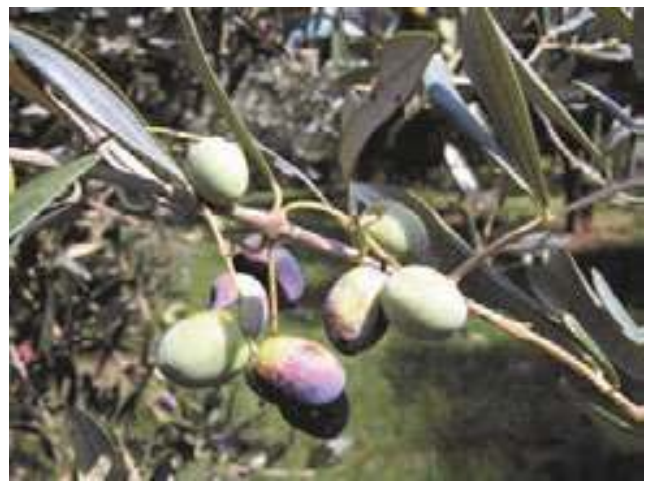

\section{Leaf characters}

Blade length $(\mathrm{cm})$ : medium $(6,22 \pm 1,66)$

Blade width $(\mathrm{cm})$ : broad $(\mathbf{1}, \mathbf{6 6} \pm \mathbf{0 , 1 8})$

Shape (length/width): elliptic

\section{Inflorescence characters}

Inflorescence length $(\mathrm{cm})$ : medium $(3,36 \pm 0,67)$

Number of flowers: medium $(18,69 \pm 3,71)$

\section{Fruit characters}

Fresh weight of 100 fruits $(\mathrm{g})$ : medium $(3,34 \pm 0,55)$

Shape (length/width): ovoid

Symmetry: slightly asymmetric

Position of maximum transverse diameter:

central

Apex: rounded

Base: truncate

Niplle: absent

Lenticels: many and large

\section{Pit characters}

Weight of 100 pits (g): medium $(0,42 \pm 0,05)$

Shape (length/width): ovoid

Mucron: obvious

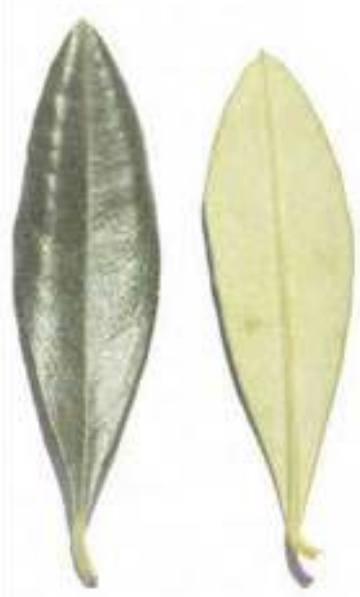

Symmetry: slightly asymmetric

Position of maximum transverse diameter:

central

Apex: rounded

Base: rounded

Surface: rugose

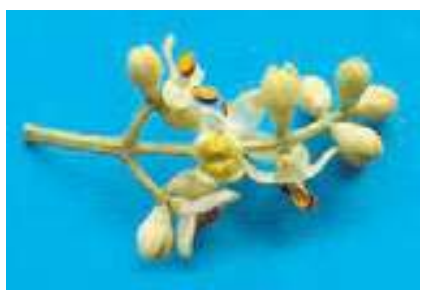

Number of grooves: low

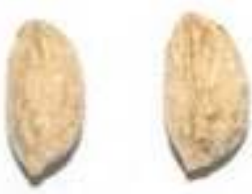




\section{Biochemical Characters}

\section{Fatty Acid Composition}

Table 1. Average values (express in $\% \pm$ standard deviations) of the fatty acids methyl esters and nutritional ratios obtained from single cultivar olive oils.

\begin{tabular}{|c|c|c|c|c|c|}
\hline Myristic acid & $0,01 \pm 0,01$ & Linoleic acid $(\omega 6)$ & $11,87 \pm 1,25$ & Lignoceric acid & $0,06 \pm 0,00$ \\
\hline Palmitic acid & $12,50 \pm 1,97$ & Linolenic acid $(\omega 3)$ & $0,93 \pm 0,06$ & & \\
\hline Palmitoleic acid & $3,14 \pm 0,30$ & Arachic acid & $0,52 \pm 0,45$ & Unsat./satured & $5,89 \pm 0,86$ \\
\hline earic acid & $1,40 \pm 0,03$ & Eicosenoic acid & $0,35 \pm 0,08$ & $\omega 6 / \omega 3$ & $12,75 \pm 0,57$ \\
\hline leic acid & $66,35 \pm 1,98$ & Behenic acid & $0,11 \pm 0,02$ & & \\
\hline
\end{tabular}

\section{Organoleptic oil values}

Sensory Analysis (Panel test)

Comment: fruity medium, with hints of almond and read sensations of grass and artichoke. Balanced taste sensation with a medium bitter and spicy. Medium fluidity.

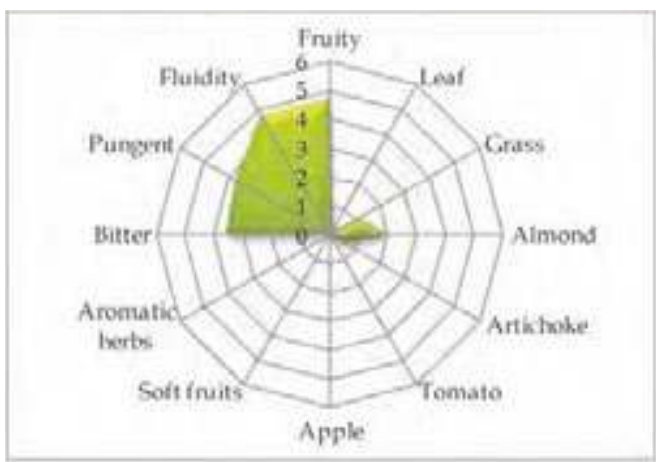

\section{Molecular Markers}

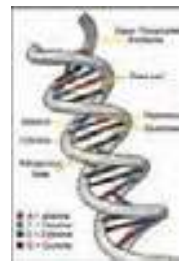

Table 2. Microsatellites (SSR) loci analyzed.

For each locus the allele size (expressed in base pairs) is reported.

$\begin{array}{cccccc}D C A 09 & \text { DCA } 18 & \text { GAPU59 } & \text { GAPU71A } & \text { GUPA71B } & \text { GAPU103A } \\ \mathbf{1 8 2} \mathbf{- 1 9 8} & \mathbf{1 8 1} \mathbf{- 1 8 1} & \mathbf{2 1 2} \mathbf{- 2 1 2} & \mathbf{2 1 0 - \mathbf { 2 1 4 }} & \mathbf{1 2 4 - \mathbf { 1 2 6 }} & \mathbf{1 5 9} \mathbf{- 1 5 9} \\ \text { UDOO1 } & \text { UDO03 } & \text { UDO12 } & \text { UDO28 } & \text { UDO39 } & \\ \mathbf{1 4 0} \mathbf{- 1 4 0} & \mathbf{1 4 3} \mathbf{- 1 4 3} & \mathbf{1 6 6 - 1 9 3} & \mathbf{1 4 3 - \mathbf { 2 0 5 }} & \mathbf{1 0 8 - \mathbf { 2 0 5 }} & \end{array}$

\section{References:}

1 - Lombardo N., Perri E., Muzzalupo I., et al. In: Contributo alla caratterizzazione del germoplasma olivicolo pugliese, Istituto Sperimentale per l'Olivicoltura (2004), pp. 33-36.

2 - Muzzalupo I., Stefanizzi F., Perri E. HortScience (2009), 44: pp. 582-588. 


\section{“Cellina di Nardo" "}

(synonymy: Cafarella, Leccese, Oliva di Nardo', Saracena, Vosciola, etc.)

Areal distribution or origin area: Puglia

Flesh/pit weight ratio: low $(3,34 \pm 0,01)$

Oil content (\%): low $(36,23 \pm 1,55)$

Purpose: oil

\section{Morphological characters}

Tree characters

Vigour: strong

Growth habit: erect-spreading

Canopy-density: medium-dense

\section{Leaf characters}

Blade length $(\mathrm{cm})$ : medium $(5,36 \pm 0,56)$

Blade width (cm): broad $(\mathbf{1}, 60 \pm \mathbf{0 , 2 5})$

Shape (length/width): elliptic

\section{Inflorescence characters}

Inflorescence length $(\mathrm{cm})$ : medium $(3,40 \pm 0,72)$

Number of flowers: medium $(\mathbf{1 7 , 8 3} \pm \mathbf{2 , 5 2})$

\section{Fruit characters}

Fresh weight of 100 fruits $(\mathrm{g})$ : low $(1,39 \pm 0,35)$

Shape (length/width): ovoid

Symmetry: symmetric

Position of maximum transverse diameter:

central

Apex: rounded

Base: rounded

Niplle: tenuous

Lenticels: few and small

\section{Pit characters}

Weight of 100 pits (g): medium $(0,32 \pm 0,06)$

Shape (length/width): elliptic

Mucron: obvious

Symmetry: symmetric

Position of maximum transverse diameter:

central

Apex: pointed

Base: pointed

Surface: rugose

Number of grooves: medium
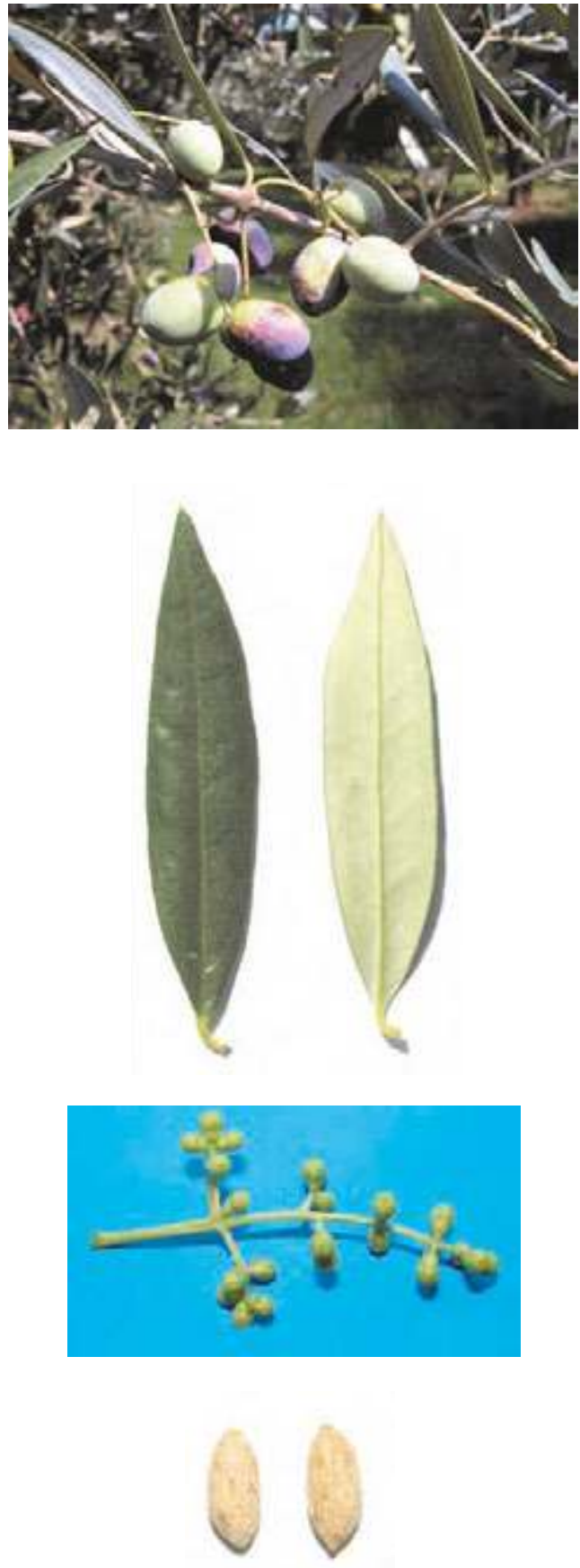


\section{Biochemical Characters}

\section{Fatty Acid Composition}

Table 1. Average values (express in $\% \pm$ standard deviations) of the fatty acids methyl esters and nutritional ratios obtained from single cultivar olive oils.

\begin{tabular}{|c|c|c|c|c|c|}
\hline Myristic acid & $0,01 \pm 0,00$ & Linoleic acid $(\omega 6)$ & $10,36 \pm 1,04$ & Lignoceric acid & $0,04 \pm 0,02$ \\
\hline Palmitic acid & $12,96 \pm 1,22$ & Linolenic acid $(\omega 3)$ & $0,91 \pm 0,09$ & & \\
\hline Palmitoleic acid & $1,53 \pm 0,13$ & Arachic acid & $0,30 \pm 0,06$ & Unsat./satured & $5,49 \pm 0,40$ \\
\hline teari & $2,04 \pm 0,32$ & Eicosenoic acid & $0,14 \pm 0,14$ & $\omega 6 / \omega 3$ & $11,41 \pm 0,23$ \\
\hline leic acid & $70,22 \pm 2,01$ & Behenic acid & $0,07 \pm 0,03$ & & \\
\hline
\end{tabular}

\section{Organoleptic oil values}

Sensory Analysis (Panel test)

Comment: fruity medium, with hints of soft fruits and read sensations of almond, grass and artichoke. Balanced taste sensation with a medium-high bitter and spicy. Mediumhigh.

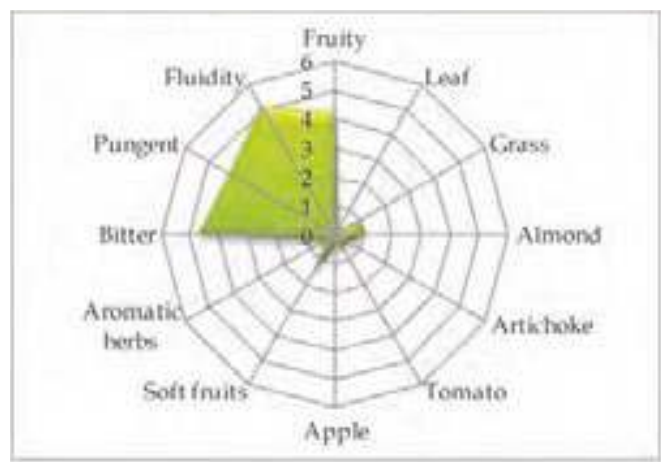

\section{Molecular Markers}

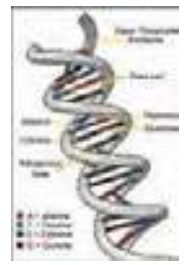

Table 2. Microsatellites (SSR) loci analyzed.

For each locus the allele size (expressed in base pairs) is reported.

$\begin{array}{cccccc}D C A 09 & D C A 18 & \text { GAPU59 } & \text { GAPU71A } & \text { GUPA71B } & \text { GAPU103A } \\ \mathbf{1 7 6 - \mathbf { 1 9 8 }} & \mathbf{1 7 7 - \mathbf { 1 7 7 }} & \mathbf{2 0 8 - \mathbf { 2 2 2 }} & \mathbf{2 1 4} \mathbf{- 2 2 8} & \mathbf{1 2 4} \mathbf{- 1 2 6} & \mathbf{1 7 0 - \mathbf { 1 7 0 }} \\ \text { UDOO1 } & \text { UDOO3 } & \text { UDO12 } & \text { UDO28 } & \text { UDO39 } & \\ \mathbf{1 4 4 - \mathbf { 1 4 4 }} & \mathbf{1 5 0 - \mathbf { 1 5 0 }} & \mathbf{1 6 6 - \mathbf { 1 6 6 }} & \mathbf{1 8 2} \mathbf{- 2 0 5} & \mathbf{1 0 8 - \mathbf { 2 1 3 }} & \end{array}$

\section{References:}

1 - Lombardo N., Perri E., Muzzalupo I., et al. In: Contributo alla caratterizzazione del germoplasmaolivicolo pugliese, Ist. Sper. Olivic. (2004), pp. 41-44.

2 - Muzzalupo I., Stefanizzi F., Perri E. HortScience (2009), 44: pp. 582-588. 


\section{"Cellina di Rotello"}

Areal distribution or origin area: Molise

Flesh/pit weight ratio: low $(3,05 \pm 0,18)$

Oil content (\%): low $(37,04 \pm 1,32)$

Purpose: oil

\section{Morphological characters}

Tree characters

Vigour: medium-strong

Growth habit: spreading

Canopy-density: medium-sparse

\section{Leaf characters}

Blade length $(\mathrm{cm})$ : medium $(5,59 \pm 0,45)$

Blade width $(\mathrm{cm})$ : broad $(\mathbf{1}, 58 \pm \mathbf{0 , 1 1})$

Shape (length/width): elliptic

\section{Inflorescence characters}

Inflorescence length $(\mathrm{cm})$ : long $(3,76 \pm 0,99)$

Number of flowers: medium $(18,49 \pm 1,85)$

\section{Fruit characters}

Fresh weight of 100 fruits $(\mathrm{g})$ : medium $(2,49 \pm 0,11)$

Shape (length/width): ovoid

Symmetry: slightly asymmetric

Position of maximum transverse diameter: central

Apex: rounded

Base: rounded

Niplle: absent

Lenticels: many and small

\section{Pit characters}

Weight of 100 pits (g): high $(\mathbf{0 , 6 1} \pm \mathbf{0 , 0 1})$

Shape (length/width): ovoid

Mucron: tenuous

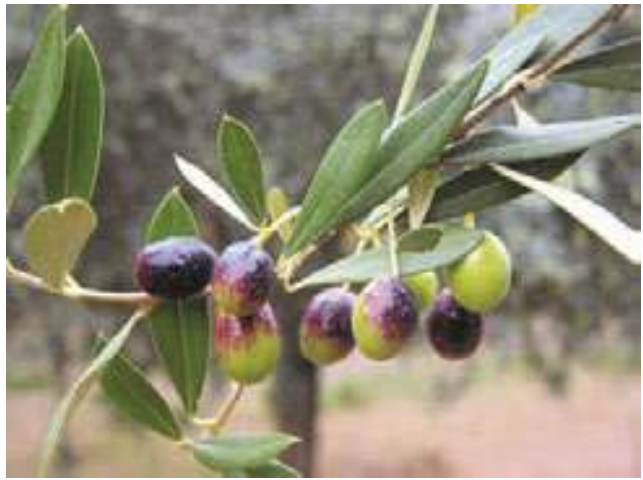

Symmetry: asymmetric

Position of maximum transverse diameter:

towards apex

Apex: rounded

Base: pointed

Surface: rugose

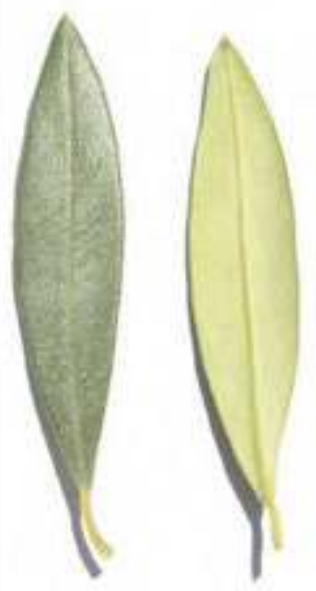

Number of grooves: medium
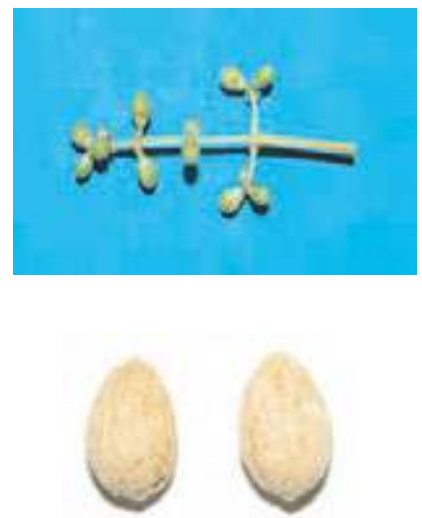


\section{Biochemical Characters}

\section{Fatty Acid Composition}

Table 1. Average values (express in $\% \pm$ standard deviations) of the fatty acids methyl esters and nutritional ratios obtained from single cultivar olive oils.

\begin{tabular}{|c|c|c|c|c|c|}
\hline Myristic acid & $0,01 \pm 0,00$ & Linoleic acid $(\omega 6)$ & $13,89 \pm 1,06$ & Lignoceric acid & $0,03 \pm 0,00$ \\
\hline Palmitic acid & $15,94 \pm 1,15$ & Linolenic acid $(\omega 3)$ & $0,97 \pm 0,05$ & & \\
\hline Palmitoleic acid & $1,61 \pm 0,03$ & Arachic acid & $0,26 \pm 0,04$ & Unsat./satured & $4,50 \pm 0,33$ \\
\hline tearic acid & $1,77 \pm 0,01$ & Eicosenoic acid & $0,03 \pm 0,02$ & $\omega 6 / \omega 3$ & $14,25 \pm 0,40$ \\
\hline leic acid & $63,82 \pm 0,63$ & Behenic acid & $0,07 \pm 0,01$ & & \\
\hline
\end{tabular}

\section{Organoleptic oil values}

Sensory Analysis (Panel test)

Comment: fruity medium, with hints of soft fruits and read sensations of almond and tomato. Balanced taste sensation with a medium bitter and spicy. Medium-high fluidity.

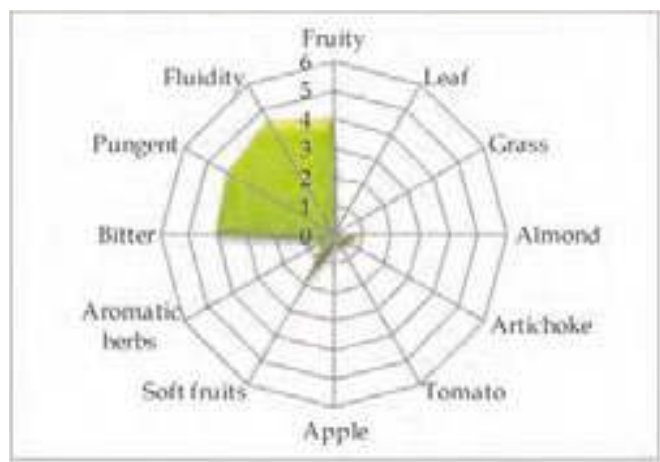

\section{Molecular Markers}

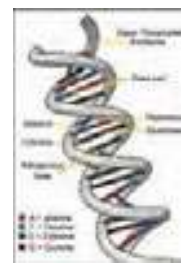

Table 2. Microsatellites (SSR) loci analyzed.

For each locus the allele size (expressed in base pairs) is reported.

$\begin{array}{cccccc}D C A 09 & \text { DCA18 } & \text { GAPU59 } & \text { GAPU71A } & \text { GUPA71B } & \text { GAPU103A } \\ \mathbf{1 9 4 - \mathbf { 2 0 6 }} & \mathbf{1 7 7 - \mathbf { 1 8 1 }} & \mathbf{2 2 2} \mathbf{- 2 2 2} & \mathbf{2 1 0 - \mathbf { 2 1 4 }} & \mathbf{1 2 4} \mathbf{- 1 2 6} & \mathbf{1 5 0 - \mathbf { 1 8 4 }} \\ \text { UDOO1 } & \text { UDOO3 } & \text { UDO12 } & \text { UDO28 } & \text { UDO39 } & \\ \mathbf{1 4 0 - \mathbf { 1 4 0 }} & \mathbf{2 0 2 - \mathbf { 2 0 2 }} & \mathbf{1 7 7 - \mathbf { 1 9 3 }} & \mathbf{1 5 4 - \mathbf { 2 1 0 }} & \mathbf{1 7 0 - \mathbf { 2 1 3 }} & \end{array}$

\section{References:}

1 - Ente regionale di sviluppo agricolo per il Molise. In: Il germoplasma dell'olivo nel Molise, Quaderno divulgativo dell'ERSA (2000), n 5 .

2 - Muzzalupo I., Lombardo N., Salimonti A., et al. Adv. Hort. Sci. (2008), 22(2): pp. 129-135. 


\section{"Cerasuola"}

(synonymy: Allora, Cerasara, Grappusa, Nocellara, Ogliarola, Ugghiara, etc.)

Areal distribution or origin area: Sicilia

Flesh/pit weight ratio: high $(8,27 \pm 0,20)$

Oil content (\%): medium $(47,89 \pm 1,15)$

Purpose: oil

\section{Morphological characters}

Tree characters

Vigour: medium

Growth habit: spreading-erect

Canopy-density: medium

\section{Leaf characters}

Blade length $(\mathrm{cm})$ : medium $(6,13 \pm 0,64)$

Blade width $(\mathrm{cm})$ : medium $(1,48 \pm 0,16)$

Shape (length/width): elliptic - lanceolate

\section{Inflorescence characters}

Inflorescence length (cm): short $(2,26 \pm 0,57)$

Number of flowers: low $(16,28 \pm 1,45)$

\section{Fruit characters}

Fresh weight of 100 fruits $(\mathrm{g})$ : medium $(3,10 \pm 0,16)$

Shape (length/width): spherical

Symmetry: slightly asymmetric

Position of maximum transverse diameter:

central

Apex: rounded

Base: rounded

Niplle: absent

Lenticels: many and small

\section{Pit characters}

Weight of 100 pits (g): medium $(0,35 \pm 0,00)$

Shape (length/width): ovoid

Mucron: absent

Symmetry: symmetric

Position of maximum transverse diameter:

towards apex

Apex: rounded

Base: rounded

Surface: rugose

Number of grooves: high
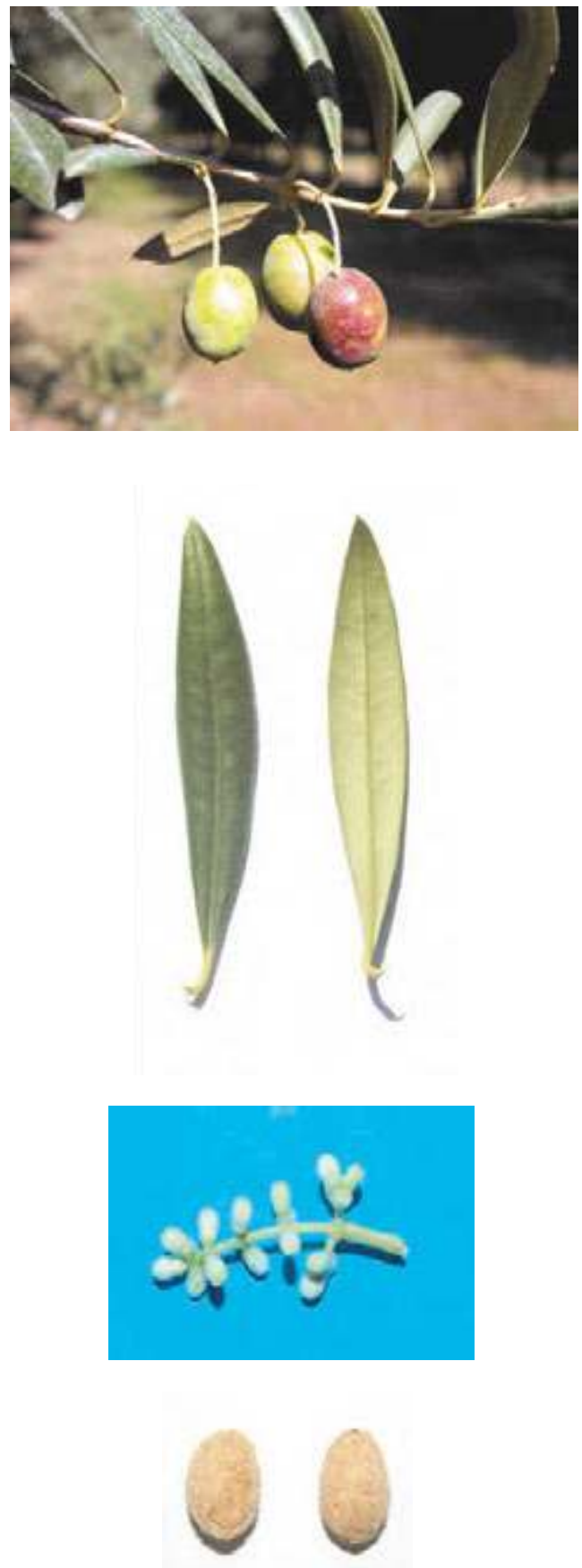


\section{Biochemical Characters}

\section{Fatty Acid Composition}

Table 1. Average values (express in $\% \pm$ standard deviations) of the fatty acids methyl esters and nutritional ratios obtained from single cultivar olive oils.

\begin{tabular}{|c|c|c|c|c|c|}
\hline Myristic acid & $0,01 \pm 0,00$ & Linoleic acid $(\omega 6)$ & $10,83 \pm 0,31$ & Lignoceric acid & $0,03 \pm 0,01$ \\
\hline Palmitic acid & $10,60 \pm 2,01$ & Linolenic acid $(\omega 3)$ & $0,99 \pm 0,12$ & & \\
\hline Palmitoleic acid & $0,54 \pm 0,30$ & Arachic acid & $0,28 \pm 0,01$ & Unsat./satured & $6,77 \pm 1,24$ \\
\hline acid & $2,13 \pm 0,03$ & Eicosenoic acid & $0,02 \pm 0,00$ & $\omega 6 / \omega 3$ & $10,99 \pm 1,63$ \\
\hline leic acid & $73,92 \pm 2,76$ & Behenic acid & $0,08 \pm 0,00$ & & \\
\hline
\end{tabular}

\section{Organoleptic oil values}

Sensory Analysis (Panel test)

Comment: fruity high, with taste of artichoke and tomato, read sensations of grass and leaves. Balanced taste sensation with a intense bitter and spicy. Medium fluidity.

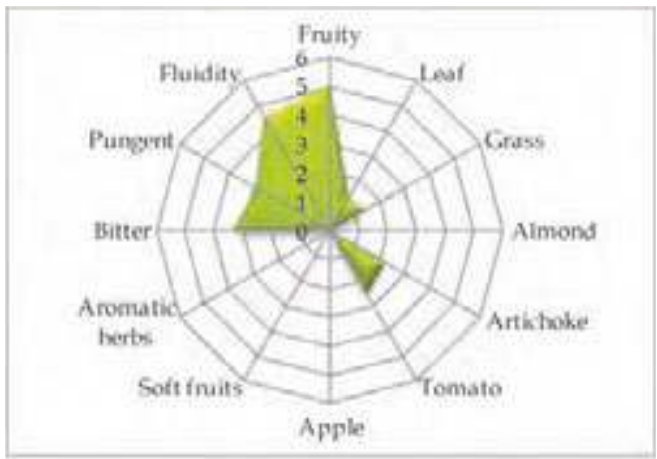

\section{Molecular Markers}

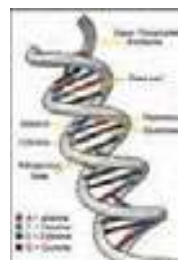

Table 2. Microsatellites (SSR) loci analyzed. For each locus the allele size (expressed in base pairs) is reported.

$\begin{array}{cccccc}D C A 09 & D C A 18 & \text { GAPU59 } & \text { GAPU71A } & \text { GUPA71B } & \text { GAPU103A } \\ \mathbf{1 7 6 - \mathbf { 2 0 6 }} & \mathbf{1 7 7 - \mathbf { 1 8 1 }} & \mathbf{2 0 8 - \mathbf { 2 1 2 }} & \mathbf{2 1 0 - \mathbf { 2 1 4 }} & \mathbf{1 2 4} \mathbf{- 1 4 4} & \mathbf{1 5 0 - \mathbf { 1 8 4 }} \\ \text { UDOO1 } & \text { UDO03 } & \text { UDO12 } & \text { UDO28 } & \text { UDO39 } & \\ \mathbf{1 4 4 - \mathbf { 1 4 4 }} & \mathbf{1 5 0 - \mathbf { 1 5 0 }} & \mathbf{1 6 6 - \mathbf { 1 6 6 }} & \mathbf{1 5 4} \mathbf{- 1 5 4} & \mathbf{2 1 3 - \mathbf { 2 1 3 }} & \end{array}$

\section{References:}

1 - Caruso T., Cartabellotta D., Motisi A., et all. In: Cultivar di olivo siciliane, Università di Palermo (2007), pp. 3640.

2 - Muzzalupo I., Stefanizzi F., Perri E. HortScience (2009), 44: pp. 582-588. 


\section{"Ciciariello"}

Areal distribution or origin area: Calabria Flesh/pit weight ratio: low $(4,18 \pm 0,52)$ Oil content (\%): medium $(44,94 \pm 3,51)$ Purpose: oil

\section{Morphological characters}

Tree characters

Vigour: medium-strong

Growth habit: spreading-erect

Canopy-density: medium-dense

\section{Leaf characters}

Blade length $(\mathrm{cm})$ : medium $(5,16 \pm 0,46)$

Blade width $(\mathrm{cm})$ : medium $(\mathbf{1}, 16 \pm 0,17)$

Shape (length/width): elliptic-lanceolate

\section{Inflorescence characters}

Inflorescence length ( $\mathrm{cm})$ : long $(3,64 \pm 0,26)$

Number of flowers: medium $(20,39 \pm 2,77)$

\section{Fruit characters}

Fresh weight of 100 fruits $(\mathrm{g})$ : low $(\mathbf{1}, \mathbf{8 6} \pm \mathbf{0 , 4 6})$

Shape (length/width): ovoid

Symmetry: slightly asymmetric

Position of maximum transverse diameter: central

Apex: rounded

Base: truncate

Niplle: tenuous

Lenticels: many and small

\section{Pit characters}

Weight of 100 pits (g): medium $(0,36 \pm 0,07)$

Shape (length/width): ovoid

Mucron: tenuous

Symmetry: symmetric

Position of maximum transverse diameter: central

Apex: rounded

Base: rounded

Surface: rugose

Number of grooves: medium
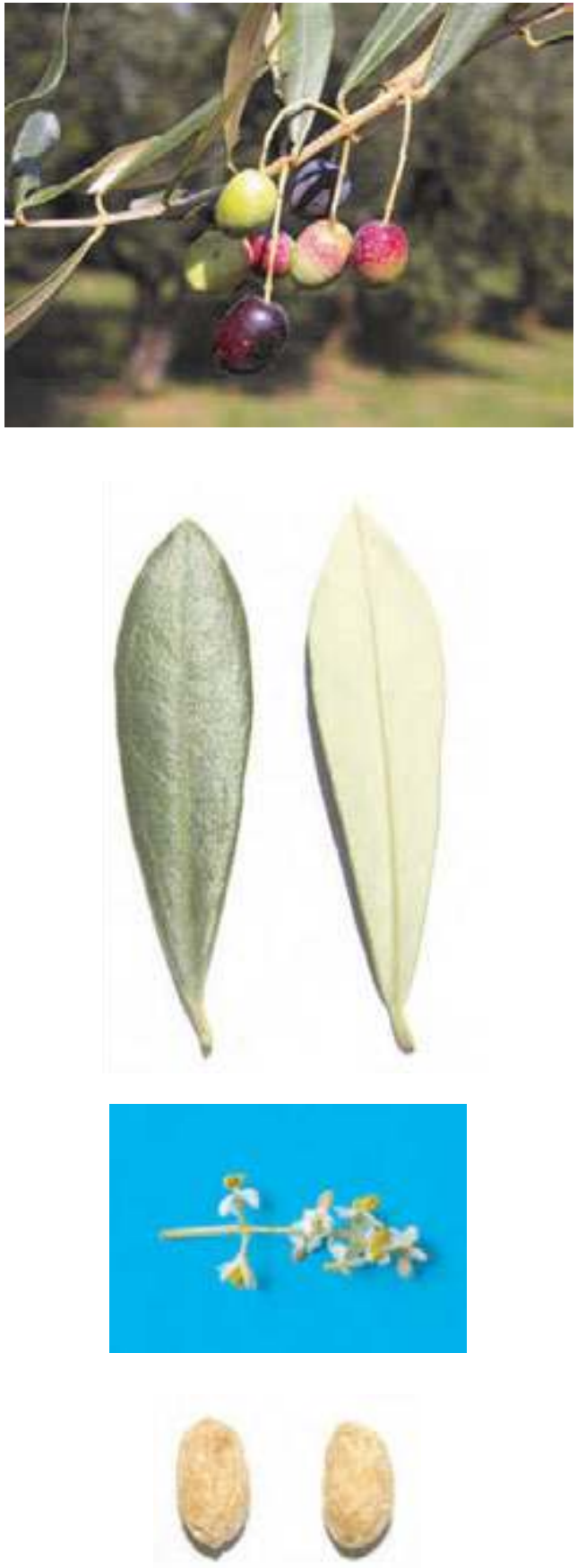


\section{Biochemical Characters}

\section{Fatty Acid Composition}

Table 1. Average values (express in $\% \pm$ standard deviations) of the fatty acids methyl esters and nutritional ratios obtained from single cultivar olive oils.

$\begin{array}{llllll}\text { Myristic acid } & \mathbf{0 , 0 1} \pm \mathbf{0 , 0 1} & \text { Linoleic acid }(\omega 6) & \mathbf{5 , 6 1} \pm \mathbf{0 , 6 6} & \text { Lignoceric acid } & \mathbf{0 , 0 5} \pm \mathbf{0 , 0 3} \\ \text { Palmitic acid } & \mathbf{1 1 , 9 9} \pm \mathbf{0 , 6 4} & \text { Linolenic acid }(\omega 3) \mathbf{0 , 7 0 \pm 0 , 0 5} & & \\ \text { Palmitoleic acid } & \mathbf{1 , 0 0 \pm \mathbf { 0 , 2 0 }} & \text { Arachic acid } & \mathbf{0 , 4 1} \pm \mathbf{0 , 0 5} & \text { Unsat./satured } & \mathbf{5 , 7 4 \pm 0 , 2 9} \\ \text { Stearic acid } & \mathbf{2 , 4 0 \pm \mathbf { 0 , 1 3 }} & \text { Eicosenoic acid } & \mathbf{0 , 1 5} \pm \mathbf{0 , 1 6} & \omega 6 / \omega 3 & \mathbf{7 , 9 1} \pm \mathbf{0 , 9 8} \\ \text { Oleic acid } & \mathbf{7 6 , 8 4} \pm \mathbf{1 , 3 9} & \text { Behenic acid } & \mathbf{0 , 1 2} \pm \mathbf{0 , 0 4} & & \end{array}$

\section{Organoleptic oil values}

Sensory Analysis (Panel test)

Comment: fruity medium, with read sensations of almond, grass and artichoke. Balanced taste sensation with a medium-high bitter and spicy. Medium-high fluidity.

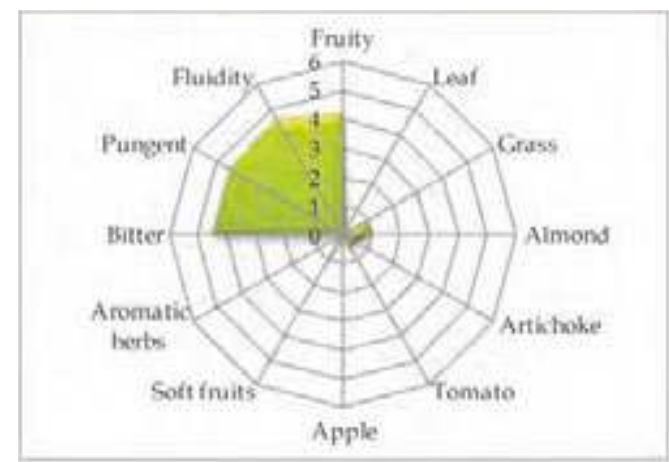

\section{Molecular Markers}

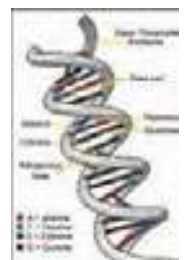

Table 2. Microsatellites (SSR) loci analyzed. For each locus the allele size (expressed in base pairs) is reported.

$\begin{array}{cccccc}D C A 09 & \text { DCA18 } & \text { GAPU59 } & \text { GAPU71A } & \text { GUPA71B } & \text { GAPU103A } \\ \mathbf{1 9 8} \mathbf{- 1 9 8} & \mathbf{1 7 7 - \mathbf { 1 7 7 }} & \mathbf{2 1 2} \mathbf{- 2 1 2} & \mathbf{2 1 2} \mathbf{- 2 1 2} & \mathbf{1 2 4 - \mathbf { 1 2 4 }} & \mathbf{1 5 9 - \mathbf { 1 7 0 }} \\ \text { UDOO1 } & \text { UDOO3 } & \text { UDO12 } & \text { UDO28 } & \text { UDO39 } & \\ \mathbf{1 4 4 - \mathbf { 1 4 4 }} & \mathbf{1 4 3 - \mathbf { 1 4 3 }} & \mathbf{1 7 7 - \mathbf { 1 9 3 }} & \mathbf{1 4 3 - \mathbf { 1 4 3 }} & \mathbf{2 0 5 - \mathbf { 2 0 5 }} & \end{array}$

\section{References:}

1 - Lombardo N., Perri E., Muzzalupo I., et al. In: Il germoplasma olivicolo calabrese, Ist. Sper. Olivic.(2003), pp: 20.

2 - Perri E., Mazzotti F., Muzzalupo I., et al. In: Relazione attività, CO.R.ASS.OL. (2003).

3 - Muzzalupo I., Stefanizzi F., Perri E. HortScience (2009), 44: pp. 582-588. 


\section{"Cima di Melfi "}

Areal distribution or origin area: Basilicata

Flesh/pit weight ratio: medium $(5,55 \pm 1,14)$

Oil content (\%): medium $(42,84 \pm 2,01)$

Purpose: oil

\section{Morphological characters}

Tree characters

Vigour: medium-strong

Growth habit: spreading

Canopy-density: dense

\section{Leaf characters}

Blade length $(\mathrm{cm})$ : medium $(6,08 \pm 0,59)$

Blade width $(\mathrm{cm})$ : medium $(1,49 \pm 0,17)$

Shape (length/width): elliptic-lanceolate

\section{Inflorescence characters}

Inflorescence length $(\mathrm{cm})$ : medium $(3,24 \pm 0,53)$

Number of flowers: low $(\mathbf{1 4 , 5 3} \pm \mathbf{2 , 3 5})$

\section{Fruit characters}

Fresh weight of 100 fruits $(\mathrm{g})$ : medium $(2,60 \pm 0,33)$

Shape (length/width): ovoid

Symmetry: slightly asymmetric

Position of maximum transverse diameter:

central

Apex: rounded

Base: rounded

Niplle: tenuous

Lenticels: many and small

\section{Pit characters}

Weight of 100 pits (g): medium $(\mathbf{0 , 4 2} \pm \mathbf{0 , 0 8})$

Shape (length/width): elliptic

Mucron: obvious

Symmetry: slightly asymmetric

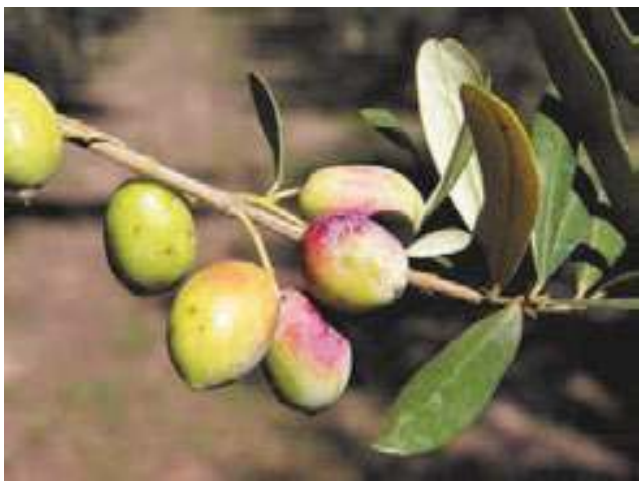

Position of maximum transverse diameter:

\section{towards apex}

Apex: rounded

Base: pointed

Surface: rugose

Number of grooves: medium
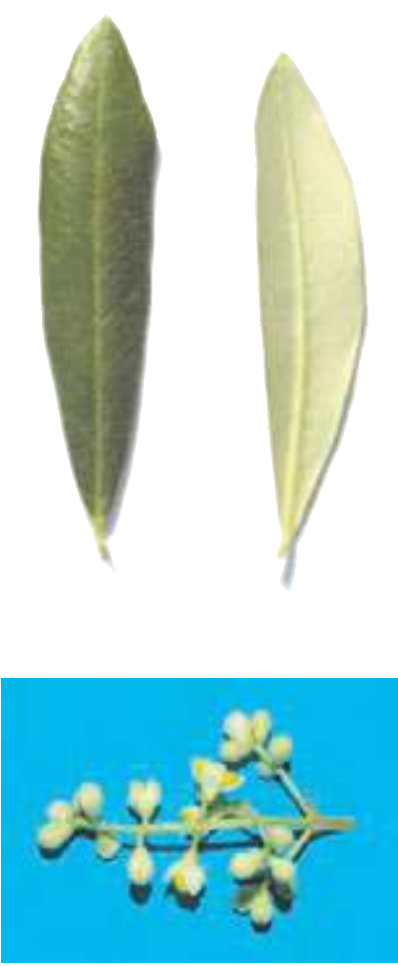


\section{Biochemical Characters}

\section{Fatty Acid Composition}

Table 1. Average values (express in $\% \pm$ standard deviations) of the fatty acids methyl esters and nutritional ratios obtained from single cultivar olive oils.

$\begin{array}{llllll}\text { Myristic acid } & \mathbf{0 , 0 2} \pm \mathbf{0 , 0 1} & \text { Linoleic acid }(\omega 6) & \mathbf{7 , 0 6} \pm \mathbf{0 , 0 1} & \text { Lignoceric acid } & \mathbf{0 , 0 6} \pm \mathbf{0 , 0 2} \\ \text { Palmitic acid } & \mathbf{1 2 , 2 5} \pm \mathbf{1 , 1 9} & \text { Linolenic acid }(\omega 3) \mathbf{0 , 7 6} \pm \mathbf{0 , 0 0} & & \\ \text { Palmitoleic acid } & \mathbf{0 , 5 3} \pm \mathbf{0 , 4 6} & \text { Arachic acid } & \mathbf{0 , 3 5} \pm \mathbf{0 , 1 4} & \text { Unsat./satured } & \mathbf{5 , 7 0 \pm 0 , 3 6} \\ \text { Stearic acid } & \mathbf{2 , 1 7} \pm \mathbf{0 , 4 6} & \text { Eicosenoic acid } & \mathbf{0 , 2 8} \pm \mathbf{0 , 1 5} & \omega 6 / \omega 3 & \mathbf{9 , 2 9} \pm \mathbf{1 , 2 0} \\ \text { Oleic acid } & \mathbf{7 6 , 1 5} \pm \mathbf{0 , 8 2} & \text { Behenic acid } & \mathbf{0 , 0 9} \pm \mathbf{0 , 0 3} & & \end{array}$

\section{Organoleptic oil values}

Sensory Analysis (Panel test)

Comment: fruity medium - light, with hints of almond and read sensations of grass and artichoke. Balanced taste sensation with a medium - light bitter and spicy. Medium fluidity.

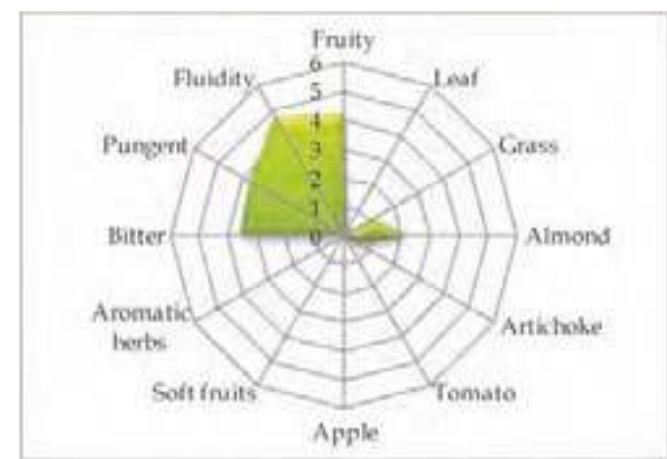

\section{Molecular Markers}

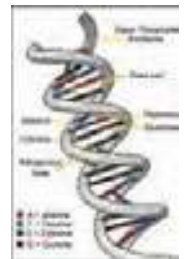

Table 2. Microsatellites (SSR) loci analyzed. For each locus the allele size (expressed in base pairs) is reported.

$\begin{array}{cccccc}D C A 09 & D C A 18 & \text { GAPU59 } & \text { GAPU71A } & \text { GUPA71B } & \text { GAPU103A } \\ \mathbf{1 9 4 - \mathbf { 1 9 4 }} & \mathbf{1 8 1} \mathbf{- 1 8 1} & \mathbf{2 1 2} \mathbf{- 2 2 2} & \mathbf{2 1 0 - \mathbf { 2 2 4 }} & \mathbf{1 2 4} \mathbf{- 1 4 4} & \mathbf{1 7 0 - \mathbf { 1 7 0 }} \\ \text { UDOO1 } & \text { UDOO3 } & \text { UDO12 } & \text { UDO28 } & \text { UDO39 } & \\ \mathbf{1 4 4 - \mathbf { 1 4 4 }} & \mathbf{1 5 0 - \mathbf { 1 5 0 }} & \mathbf{1 6 6 - \mathbf { 1 9 3 }} & \mathbf{1 5 4 - \mathbf { 2 1 0 }} & \mathbf{1 7 0 - \mathbf { 2 1 3 }} & \end{array}$

\section{References:}

1 - Rotundo A., Marone E. In: Il germoplasma olivicolo lucano, Olita-Potenza (2002), pp. 99-102.

2 - Rotundo A., Perri E.,Muzzalupo I., et al. In: Il germoplasma olivicolo meridionale, (2012 in press).

3 - Muzzalupo I., Stefanizzi F., Perri E. HortScience (2009), 44: pp. 582-588. 


\section{"Colombina"}

(synonymy: Colombina 17, Colombina lunga.)

Areal distribution or origin area: Emilia

Flesh/pit weight ratio: medium $(5,60 \pm \mathbf{0 , 4 3})$

Oil content (\%): low $(35,63 \pm 0,99)$

Purpose: oil

\section{Morphological characters}

Tree characters

Vigour: medium-strong

Growth habit: spreading

Canopy-density: dense

\section{Leaf characters}

Blade length (cm): medium $(5,78 \pm 0,46)$

Blade width $(\mathrm{cm})$ : medium $(\mathbf{1}, 40 \pm 0,17)$

Shape (length/width): elliptic-lanceolate

\section{Inflorescence characters}

Inflorescence length $(\mathrm{cm})$ : medium $(3,12 \pm 1,13)$

Number of flowers: medium $(18,97 \pm 1,19)$

\section{Fruit characters}

Fresh weight of 100 fruits (g): medium $(2,05 \pm 0,25)$

Shape (length/width): ovoid

Symmetry: slightly asymmetric

Position of maximum transverse diameter:

towards base

Apex: rounded

Base: truncate

Niplle: absent

Lenticels: many and small

\section{Pit characters}

Weight of 100 pits (g): medium $(0,31 \pm \mathbf{0 , 0 2})$

Shape (length/width): elongated

Mucron: obvious

Symmetry: slightly asymmetric

Position of maximum transverse diameter:

towards base

Apex: pointed

Base: rounded

Surface: rugose

Number of grooves: medium
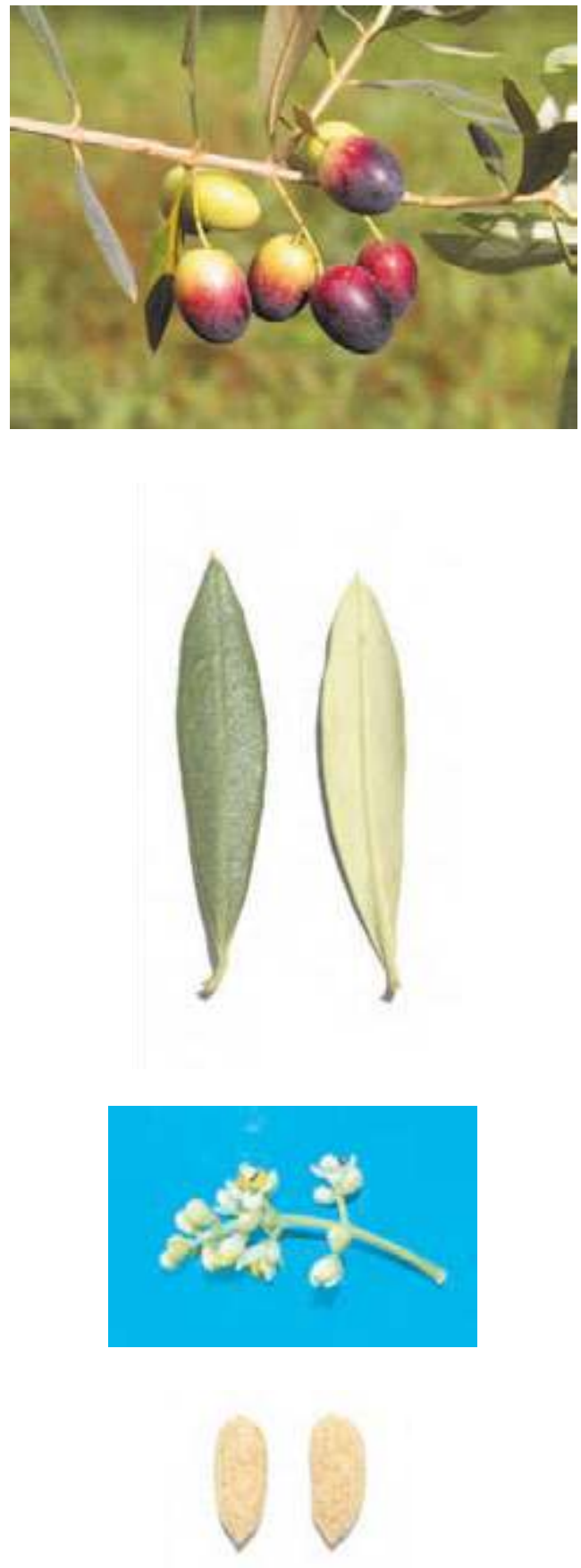


\section{Biochemical Characters}

\section{Fatty Acid Composition}

Table 1. Average values (express in $\% \pm$ standard deviations) of the fatty acids methyl esters and nutritional ratios obtained from single cultivar olive oils.

\begin{tabular}{|c|c|c|c|c|c|}
\hline Myristic acid & $0,01 \pm 0,01$ & Linoleic acid $(\omega 6)$ & $10,38 \pm 0,13$ & Lignoceric acid & $0,04 \pm 0,02$ \\
\hline Palmitic acid & $10,73 \pm 0,35$ & Linolenic acid $(\omega 3)$ & $0,96 \pm 0,02$ & & \\
\hline Palmitoleic acid & $0,39 \pm 0,09$ & Arachic acid & $0,26 \pm 0,04$ & Unsat./satured & $6,84 \pm 0,18$ \\
\hline tearic & $1,76 \pm 0,17$ & Eicosenoic acid & $0,28 \pm 0,22$ & $\omega 6 / \omega 3$ & $10,81 \pm 0,15$ \\
\hline leic acid & $74,85 \pm 0,68$ & Behenic acid & $0,09 \pm 0,04$ & & \\
\hline
\end{tabular}

\section{Organoleptic oil values}

Sensory Analysis (Panel test)

Comment: fruity medium-high, with hints of almond and artichoke, read sensations of grass. Balanced taste sensation with a medium bitter and spicy. Medium-high fluidity.

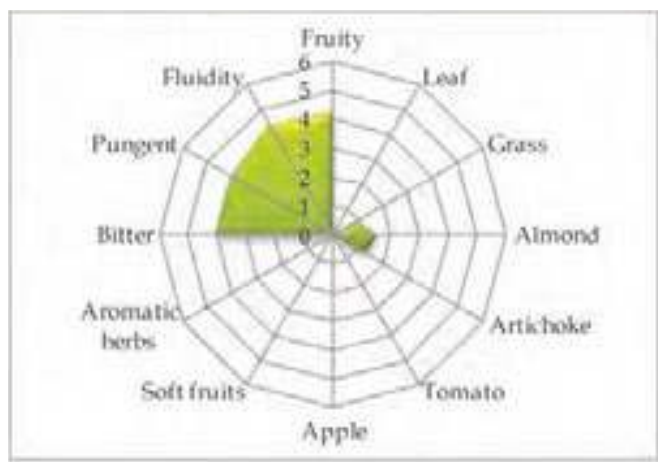

\section{Molecular Markers}

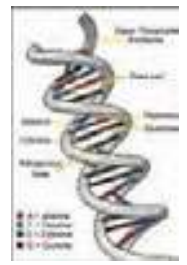

Table 2. Microsatellites (SSR) loci analyzed.

For each locus the allele size (expressed in base pairs) is reported.

$\begin{array}{cccccc}D C A 09 & D C A 18 & \text { GAPU59 } & \text { GAPU71A } & \text { GUPA71B } & \text { GAPU103A } \\ \mathbf{1 7 2} \mathbf{- 2 0 6} & \mathbf{1 7 9 - \mathbf { 1 7 9 }} & \mathbf{2 1 2} \mathbf{- 2 2 2} & \mathbf{2 1 8 - \mathbf { 2 2 4 }} & \mathbf{1 4 4 - \mathbf { 1 4 4 }} & \mathbf{1 8 4 - \mathbf { 2 0 3 }} \\ \text { UDO01 } & \text { UDOO3 } & \text { UDO12 } & \text { UDO28 } & \text { UDO39 } & \\ \mathbf{1 5 0 - \mathbf { 1 5 0 }} & \mathbf{1 4 3 - \mathbf { 1 4 3 }} & \mathbf{1 6 6 - \mathbf { 1 9 3 }} & \mathbf{1 8 2} \mathbf{- 1 8 2} & \mathbf{2 1 3 - \mathbf { 2 1 3 }} & \end{array}$

\section{References:}

1 - Cristoferi G., Rotondi A., Magli M. In: Il germoplasma dell'olivo in Emilia Romagna, ISTEA CNR (1997).

2 - Muzzalupo I., Lombardo N., Salimonti A., et al. Adv. Hort. Sci. (2008), 22(2): pp. 142-148. 


\section{"Coratina"}

(synonymy: Cima di Corato, Olivo a grappoli, Racemo, Racioppa, etc.)

Areal distribution or origin area: Puglia

Flesh/ pit weight ratio: medium $(3,95 \pm 0,05)$

Oil content (\%): high $\mathbf{( 5 0 , 4 8 \pm 2 , 8 1 )}$

Purpose: oil

\section{Morphological characters}

Tree characters

Vigour: medium

Growth habit: spreading

Canopy-density: medium

\section{Leaf characters}

Blade length $(\mathrm{cm})$ : medium $(6,24 \pm 0,59)$

Blade width $(\mathrm{cm})$ : medium $(1,33 \pm 0,20)$

Shape (length/width): elliptic-lanceolate

\section{Inflorescence characters}

Inflorescence length $(\mathrm{cm})$ : medium $(3,04 \pm 0,59)$

Number of flowers: medium $(\mathbf{1 7 , 1 9} \pm 1,75)$

\section{Fruit characters}

Fresh weight of 100 fruits $(\mathrm{g})$ : medium $(3,05 \pm 0,60)$

Shape (length/width): ovoid

Symmetry: slightly asymmetric

Position of maximum transverse diameter:

central

Apex: rounded

Base: rounded

Niplle: absent

Lenticels: many and small

\section{Pit characters}

Weight of 100 pits (g): high $(0,63 \pm 0,08)$

Shape (length/width): elliptic

Mucron: obvious

Symmetry: asymmetric

Position of maximum transverse diameter:

\section{towards base}

Apex: pointed

Base: rounded

Surface: rugose

Number of grooves: high
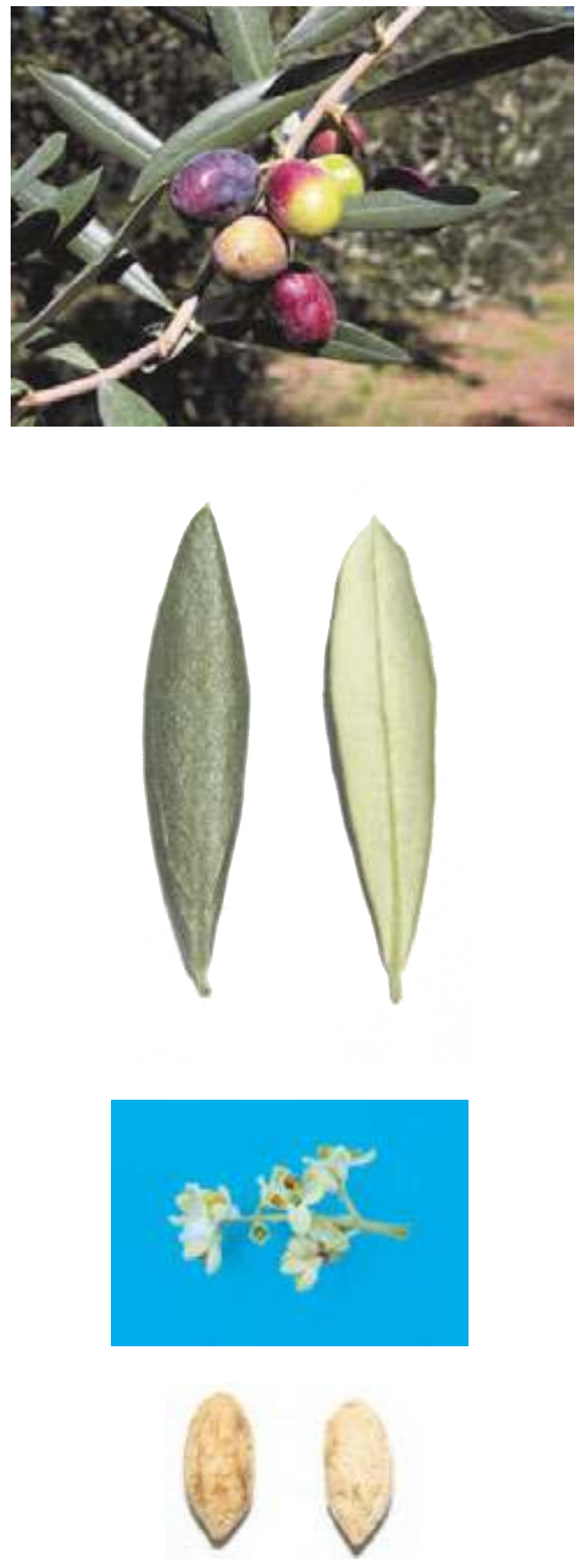


\section{Biochemical Characters}

\section{Fatty Acid Composition}

Table 1. Average values (express in $\% \pm$ standard deviations) of the fatty acids methyl esters and nutritional ratios obtained from single cultivar olive oils.

$\begin{array}{llllll}\text { Myristic acid } & \mathbf{0 , 0 1} \pm \mathbf{0 , 0 0} & \text { Linoleic acid }(\omega 6) & \mathbf{7 , 7 2} \pm \mathbf{1 , 6 0} & \text { Lignoceric acid } & \mathbf{0 , 0 5} \pm \mathbf{0 , 0 3} \\ \text { Palmitic acid } & \mathbf{1 0 , 8 8} \pm \mathbf{2 , 2 6} & \text { Linolenic acid }(\omega 3) \mathbf{0 , 8 1} \pm \mathbf{0 , 1 1} & & \\ \text { Palmitoleic acid } & \mathbf{0 , 5 5} \pm \mathbf{0 , 2 9} & \text { Arachic acid } & \mathbf{0 , 3 4} \pm \mathbf{0 , 1 0} & \text { Unsat./satured } & \mathbf{6 , 7 2} \pm \mathbf{1 , 3 2} \\ \text { Stearic acid } & \mathbf{2 , 0 8} \pm \mathbf{0 , 3 3} & \text { Eicosenoic acid } & \mathbf{0 , 2 1} \pm \mathbf{0 , 1 8} & \omega 6 / \omega 3 & \mathbf{9 , 6 7 \pm \mathbf { 1 , 1 5 }} \\ \text { Oleic acid } & \mathbf{7 7 , 1 4} \pm \mathbf{0 , 0 8} & \text { Behenic acid } & \mathbf{0 , 1 1} \pm \mathbf{0 , 0 4} & & \end{array}$

\section{Organoleptic oil values}

Sensory Analysis (Panel test)

Comment: fruity medium-high, with hints of almond and read sensations of grass and artichoke. Balanced taste sensation with a medium-high bitter and spicy. Medium-high fluidity.

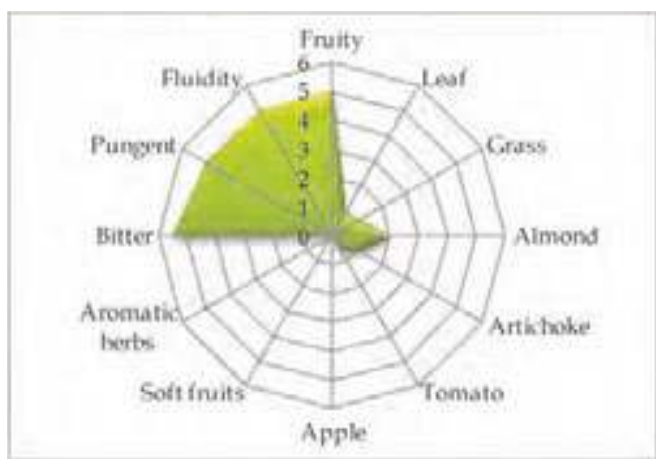

\section{Molecular Markers}

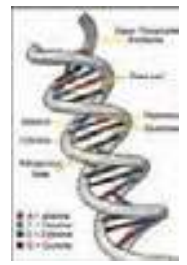

Table 2. Microsatellites (SSR) loci analyzed.

For each locus the allele size (expressed in base pairs) is reported.

\begin{tabular}{|c|c|c|c|c|c|}
\hline$D C A 09$ & $D C A 18$ & GAPU59 & GAPU71A & GUPA71B & GAPU103A \\
\hline $172-194$ & $181-181$ & $212-212$ & $210-224$ & $124-144$ & $136-170$ \\
\hline UDO01 & UDO03 & UDO12 & UDO28 & UDO39 & \\
\hline $140-140$ & $135-166$ & $166-193$ & $143-210$ & $170-170$ & \\
\hline
\end{tabular}

\section{References:}

1 - Lombardo N., Perri E., Muzzalupo I., et al. In: Contributo alla caratterizzazione del germoplasma olivicolo pugliese, Istituto Sperimentale per l'Olivicoltura, (2004), pp. 45-48.

2 - Muzzalupo I., Stefanizzi F., Perri E. HortScience (2009), 44: pp. 582-588. 


\section{"Corneglia "}

Areal distribution or origin area: Campania

Flesh/ pit weight ratio: low $(4,83 \pm 0,65)$

Oil content (\%): medium $(42,95 \pm 0,29)$

Purpose: oil

\section{Morphological characters}

Tree characters

Vigour: weak

Growth habit: spreading

Canopy-density: medium-sparse

\section{Leaf characters}

Blade length ( $\mathrm{cm})$ : medium $(5,72 \pm 0,43)$

Blade width $(\mathrm{cm})$ : medium $(1,47 \pm 0,13)$

Shape (length/width): elliptic

\section{Inflorescence characters}

Inflorescence length $(\mathrm{cm})$ : medium $(3,12 \pm 1,56)$

Number of flowers: low $(16,31 \pm 1,54)$

\section{Fruit characters}

Fresh weight of 100 fruits $(\mathrm{g})$ : medium $(3,27 \pm 0,12)$

Shape (length/width): elongated

Symmetry: asymmetric

Position of maximum transverse diameter:

central

Apex: rounded

Base: rounded

Niplle: absent

Lenticels: many and small

\section{Pit characters}

Weight of 100 pits (g): high $(\mathbf{0 , 5 7} \pm \mathbf{0 , 0 5})$

Shape (length/width): elongated

Mucron: obvious

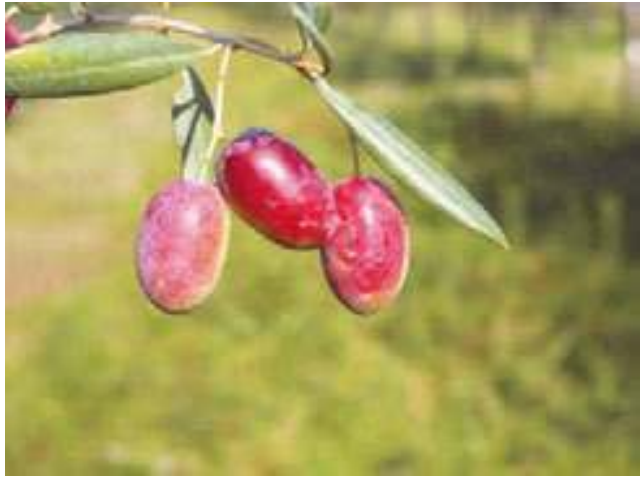

Symmetry: slightly asymmetric

Position of maximum transverse diameter:

central

Apex: rounded

Base: rounded

Surface: rugose

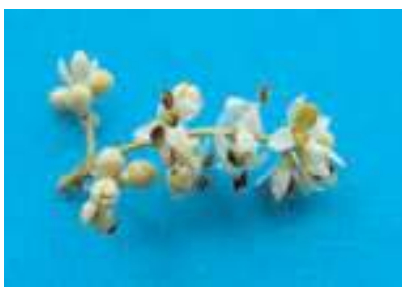

Number of grooves: medium 


\section{Biochemical Characters}

\section{Fatty Acid Composition}

Table 1. Average values (express in $\% \pm$ standard deviations) of the fatty acids methyl esters and nutritional ratios obtained from single cultivar olive oils.

\begin{tabular}{|c|c|c|c|c|c|}
\hline Myristic acid & $0,01 \pm 0,00$ & Linoleic acid $(\omega 6)$ & $8,83 \pm 0,55$ & Lignoceric acid & $0,07 \pm 0,03$ \\
\hline Palmitic acid & $14,42 \pm 0,25$ & Linolenic acid $(\omega 3)$ & $0,77 \pm 0,05$ & & \\
\hline Palmitoleic acid & $1,45 \pm 0,17$ & Arachic acid & $0,49 \pm 0,06$ & Unsat./satured & $4,45 \pm 0,01$ \\
\hline Stearic acid & $3,29 \pm 0,21$ & Eicosenoic acid & $0,11 \pm 0,14$ & $\omega 6 / \omega 3$ & $11,50 \pm 0,04$ \\
\hline Oleic acid & $69,09 \pm 0,52$ & Behenic acid & $0,13 \pm 0,03$ & & \\
\hline
\end{tabular}

\section{Organoleptic oil values}

Sensory Analysis (Panel test)

Comment: fruity medium, with read sensations of almond and leaves. Balanced taste sensation with a medium bitter and spicy. Medium fluidity.

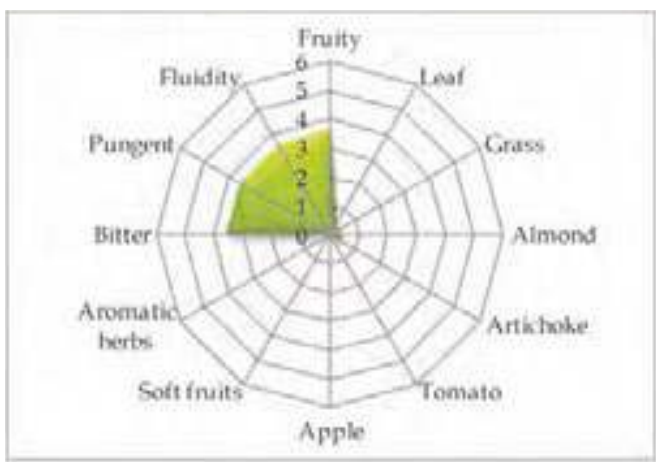

\section{Molecular Markers}

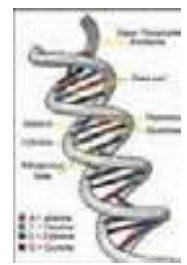

Table 2. Microsatellites (SSR) loci analyzed.

For each locus the allele size (expressed in base pairs) is reported.

\begin{tabular}{|c|c|c|c|c|c|}
\hline DCA09 & DCA18 & GAPU59 & GAPU71A & $G U P A 71 B$ & GAPU103A \\
\hline $172-198$ & $181-181$ & $218-222$ & $214-224$ & $126-126$ & $136-170$ \\
\hline UDO01 & UDO03 & UDO12 & UDO28 & UDO39 & \\
\hline $140-140$ & $143-143$ & $166-177$ & $154-182$ & $213-220$ & \\
\hline
\end{tabular}

\section{References:}

1 - Pugliano G., Flaminio G., Pugliano M.L., et al. In: La risorsa genetica dell'olivo in Campania, SE. S.I.R.C.A. Ed. Napoli, (2000).

2 - Muzzalupo I., Stefanizzi F., Perri E. HortScience (2009), 44: pp. 582-588. 


\section{"Cornia "}

(synonymy: Corniola)

Areal distribution or origin area: Campania

Flesh/pit weight ratio: low $(3,06 \pm 0,05)$

Oil content (\%): medium $(42,70 \pm 0,13)$

Purpose: oil

\section{Morphological characters}

Tree characters

Vigour: medium-strong

Growth habit: spreading

Canopy-density: medium-sparse

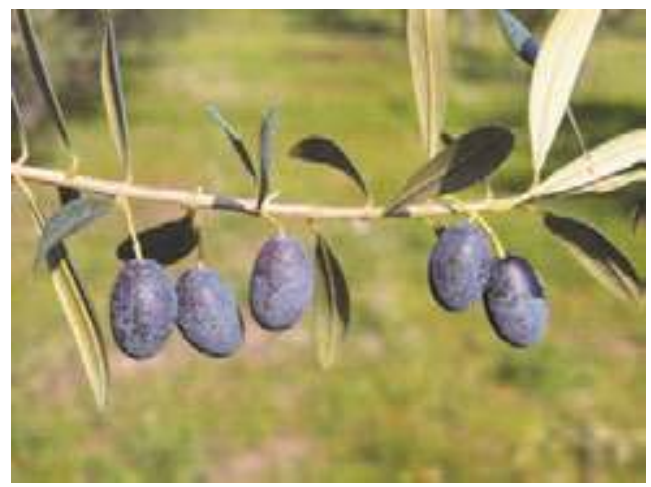

\section{Leaf characters}

Blade length $(\mathrm{cm})$ : medium $(6,48 \pm 0,56)$

Blade width $(\mathrm{cm})$ : medium $(\mathbf{1}, \mathbf{4 2} \pm 0,17)$

Shape (length/width): elliptic-lanceolate

\section{Inflorescence characters}

Inflorescence length $(\mathrm{cm})$ : medium $(2,80 \pm 2,50)$

Number of flowers: low $(10,34 \pm 1,32)$

\section{Fruit characters}

Fresh weight of 100 fruits (g): medium $(2,19 \pm 0,38)$

Shape (length/width): elongated

Symmetry: symmetric

Position of maximum transverse diameter:

towards apex

Apex: rounded

Base: rounded

Niplle: basente

Lenticels: many and small

\section{Pit characters}

Weight of 100 pits (g): medium $(\mathbf{0 , 4 1} \pm \mathbf{0 , 0 9})$

Shape (length/width): elliptic

Mucron: tenuous

Symmetry: symmetric

Position of maximum transverse diameter:

central

Apex: rounded

Base: rounded

Surface: rugose

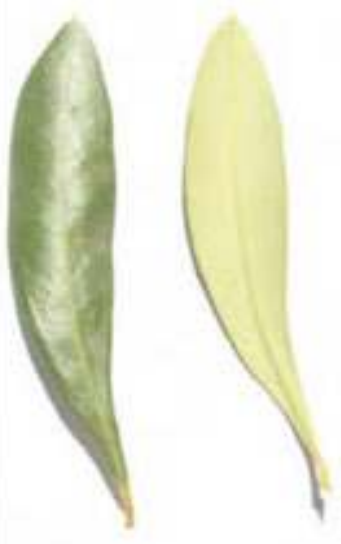

Number of grooves: medium
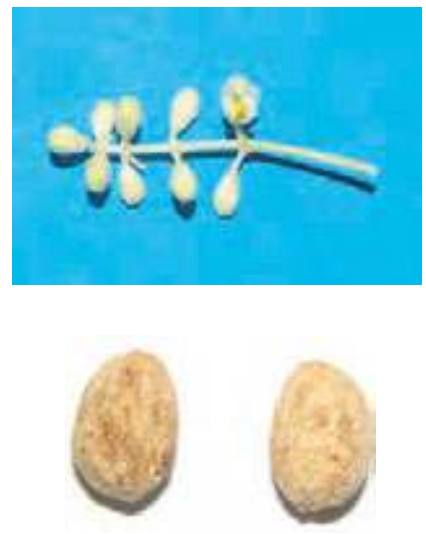


\section{Biochemical Characters}

\section{Fatty Acid Composition}

Table 1. Average values (express in $\% \pm$ standard deviations) of the fatty acids methyl esters and nutritional ratios obtained from single cultivar olive oils.

$\begin{array}{llllll}\text { Myristic acid } & \mathbf{0 , 0 1} \pm \mathbf{0 , 0 1} & \text { Linoleic acid }(\omega 6) & \mathbf{3 , 8 1} \pm \mathbf{0 , 4 0} & \text { Lignoceric acid } & \mathbf{0 , 0 5} \pm \mathbf{0 , 0 2} \\ \text { Palmitic acid } & \mathbf{1 1 , 8 9} \pm \mathbf{0 , 5 6} & \text { Linolenic acid }(\omega 3) \mathbf{0 , 8 9} \pm \mathbf{0 , 0 6} & & \\ \text { Palmitoleic acid } & \mathbf{1 , 5 4} \pm \mathbf{0 , 2 2} & \text { Arachic acid } & \mathbf{0 , 3 5} \pm \mathbf{0 , 1 9} & \text { Unsat./satured } & \mathbf{6 , 2 4} \pm \mathbf{0 , 2 9} \\ \text { Stearic acid } & \mathbf{1 , 5 0} \pm \mathbf{0 , 1 9} & \text { Eicosenoic acid } & \mathbf{0 , 2 5} \pm \mathbf{0 , 2 0} & \omega 6 / \omega 3 & \mathbf{4 , 2 9} \pm \mathbf{0 , 2 7} \\ \text { Oleic acid } & \mathbf{7 8 , 3 2} \pm \mathbf{1 , 2 6} & \text { Behenic acid } & \mathbf{0 , 0 8} \pm \mathbf{0 , 0 2} & & \end{array}$

\section{Organoleptic oil values}

Sensory Analysis (Panel test)

Comment: fruity medium, with hints of almond and read sensations of grass and artichoke. Balanced taste sensation with a medium bitter and spicy. Medium fluidity.

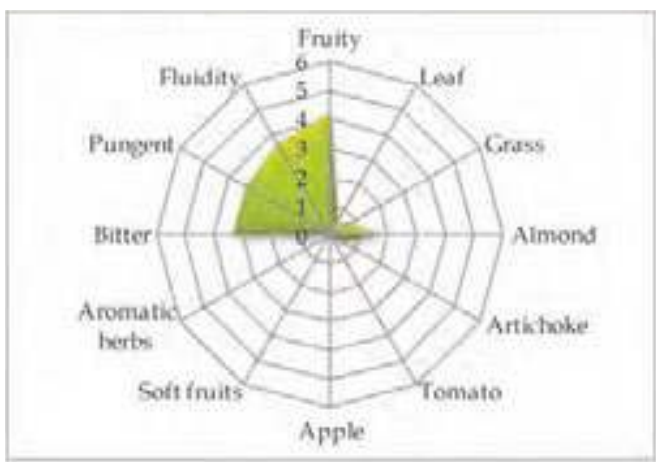

\section{Molecular Markers}

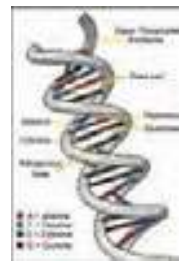

Table 2. Microsatellites (SSR) loci analyzed.

For each locus the allele size (expressed in base pairs) is reported.

$\begin{array}{cccccc}D C A 09 & \text { DCA18 } & \text { GAPU59 } & \text { GAPU71A } & \text { GUPA71B } & \text { GAPU103A } \\ \mathbf{1 9 8} \mathbf{- 1 9 8} & \mathbf{1 8 1} \mathbf{- 1 8 1} & \mathbf{2 1 4 - \mathbf { 2 1 8 }} & \mathbf{2 1 8 - \mathbf { 2 1 8 }} & \mathbf{1 3 0 - \mathbf { 1 3 4 }} & \mathbf{1 8 4} \mathbf{- 1 9 7} \\ \text { UDOO1 } & \text { UDO03 } & \text { UDO12 } & \text { UDO28 } & \text { UDO39 } & \\ \mathbf{1 4 0 - \mathbf { 1 4 0 }} & \mathbf{1 4 3 - \mathbf { 1 4 3 }} & \mathbf{1 6 4 - \mathbf { 1 7 7 }} & \mathbf{1 4 3 - \mathbf { 1 5 4 }} & \mathbf{1 6 4 - \mathbf { 2 1 3 }} & \end{array}$

\section{References:}

1 - Various authors. In: Catalogo Nazionale delle Varietà di Olivo . University of Bari (in press), ISBN 978-88-8879397-9.

2 - Muzzalupo I., Stefanizzi F., Perri E. HortScience (2009), 44: pp. 582-588. 


\section{"Corniola "}

(synonymy: Cornale, Corniola Villapiana, Farisana, etc.)

Areal distribution or origin area: Calabria

Flesh/pit weight ratio: medium $(5,65 \pm 0,50)$

Oil content (\%): medium $(44,12 \pm 1,25)$

Purpose: oil

\section{Morphological characters}

Tree characters

Vigour: medium-strong

Growth habit: spreading-erect

Canopy-density: medium-dense

\section{Leaf characters}

Blade length (cm): medium $(5,25 \pm 0,43)$

Blade width $(\mathrm{cm})$ : medium $(\mathbf{1 , 2 8} \pm 0,09)$

Shape (blade length/width): elliptic-lanceolate

\section{Inflorescence characters}

Inflorescence length $(\mathrm{cm})$ : medium $(2,52 \pm 1,22)$

Number of flowers: medium $(\mathbf{1 8 , 2 4} \pm \mathbf{1 , 9 6 )}$

\section{Fruit characters}

Fresh weight of 100 fruits $(\mathrm{g})$ : medium $(2,83 \pm \mathbf{0 , 2 8})$

Shape (length/width): elongated

Position of maximum transverse diameter:

towards apex

Symmetry: asymmetric

Apex: rounded

Base: rounded

Nipple: tenuous

Lenticels: few and large

\section{Pit characters}

Weight of 100 pits (g): medium $(0,42 \pm 0,07)$

Shape (length/width): elongated

Mucron: obvious

Symmetry: slightly asymmetric

Position of maximum transverse diameter:

towards apex

Apex: pointed

Base: pointed

Surface: smooth

Number of grooves: medium
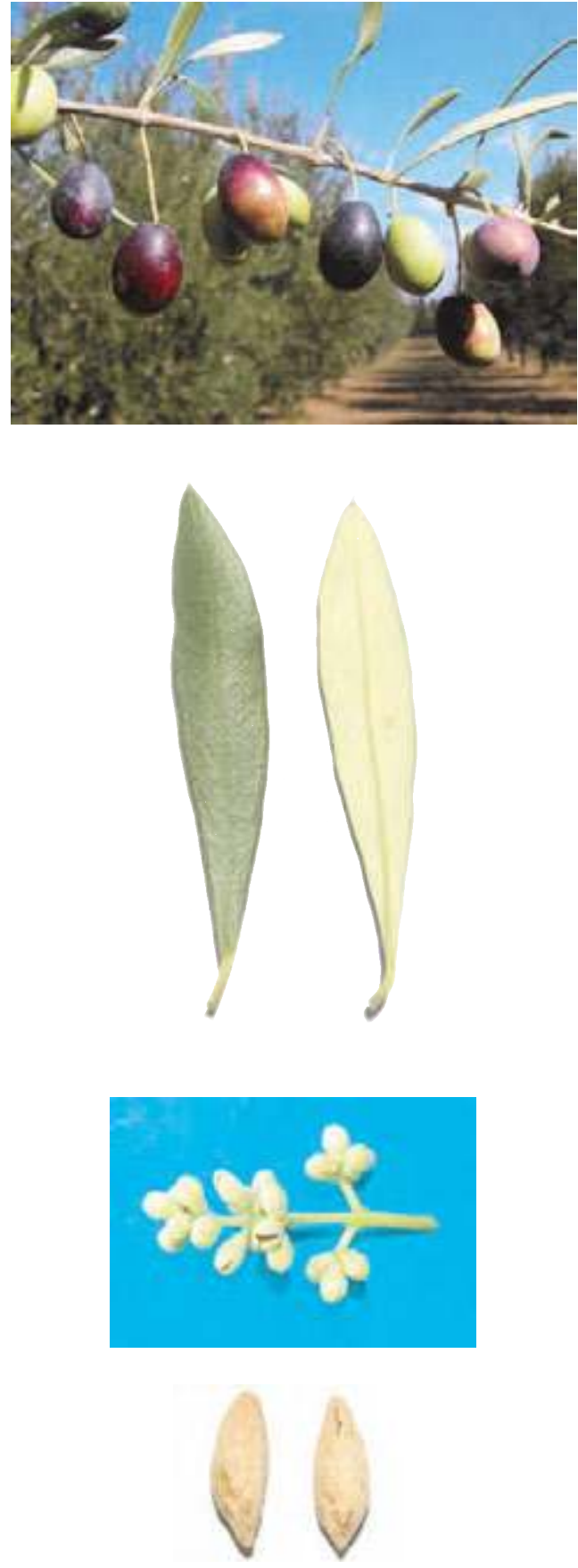


\section{Biochemical Characters}

\section{Fatty Acid Composition}

Table 1. Average values (express in $\% \pm$ standard deviations) of the fatty acids methyl esters and nutritional ratios obtained from single cultivar olive oils.

\begin{tabular}{|c|c|c|c|c|c|}
\hline Myristic acid & $0,00 \pm 0.00$ & Linoleic acid $(\omega 6)$ & $18,40 \pm 1,46$ & Lignoceric acid & $0,08 \pm 0,01$ \\
\hline Palmitic acid & $20,52 \pm 1,14$ & Linolenic acid $(\omega 3)$ & $0,80 \pm 0,07$ & & \\
\hline almitoleic acid & $2,79 \pm 0,48$ & Arachic acid & $0,37 \pm 0,06$ & Unsat./satured & $3,34 \pm 0,19$ \\
\hline earic acid & $2,25 \pm 0,13$ & Eicosenoic acid & $0,23 \pm 0,05$ & $\omega 6 / \omega 3$ & $23,26 \pm 3,85$ \\
\hline leic acid & $55,65 \pm 2,79$ & Behenic acid & $0,11 \pm 0,02$ & & \\
\hline
\end{tabular}

\section{Organoleptic oil values}

Sensory Analysis (Panel test)

Comment: fruity medium, with hints of almond. Balanced taste sensation with a medium-light bitter and medium-high spicy. Medium fluidity.

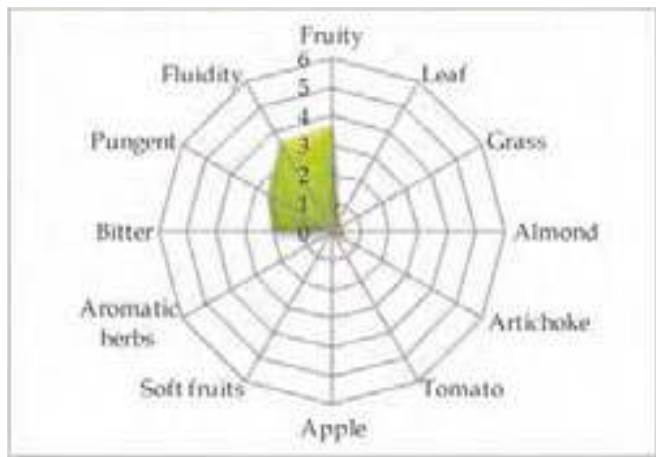

\section{Molecular Markers}

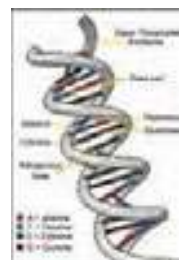

Table 2. Microsatellites (SSR) loci analyzed. For each locus the allele size (expressed in base pairs) is reported.

$\begin{array}{cccccc}D C A 09 & D C A 18 & \text { GAPU59 } & \text { GAPU71A } & \text { GUPA71B } & \text { GAPU103A } \\ \mathbf{1 7 2} \mathbf{- 1 8 2} & \mathbf{1 7 7 - \mathbf { 1 7 9 }} & \mathbf{2 0 8 - \mathbf { 2 1 2 }} & \mathbf{2 1 2} \mathbf{- 2 1 2} & \mathbf{1 2 4} \mathbf{- 1 4 4} & \mathbf{1 7 0 - \mathbf { 1 8 4 }} \\ \text { UDOO1 } & \text { UDO03 } & \text { UDO12 } & \text { UDO28 } & \text { UDO39 } & \\ \mathbf{1 4 4 - \mathbf { 1 4 4 }} & \mathbf{1 4 3 - \mathbf { 1 4 3 }} & \mathbf{1 7 7 - \mathbf { 1 9 3 }} & \mathbf{1 4 3 - \mathbf { 2 0 5 }} & \mathbf{2 0 0 - \mathbf { 2 0 0 }} & \end{array}$

\section{References:}

1 - Lombardo N., Perri E., Muzzalupo I., et al. In: Il germoplasma olivicolo calabrese, Ist. Sper. Olivic.(2003), pp: 20.

2 - Perri E., Mazzotti F., Muzzalupo I., et al. In: Relazione attività, CO.R.ASS.OL. (2003).

3 - Muzzalupo I., Stefanizzi F., Perri E. HortScience (2009), 44: 582-588. 


\section{"Corniolo"}

(synonymy: Corgniola, Corgnolo, Coriola, Cornigliola, etc.)

Areal distribution or origin area: Umbria

Flesh/pit weight ratio: medium $(5,33 \pm 1,17)$

Oil content (\%): medium $(43,61 \pm 0,88)$

Purpose: oil

\section{Morphological characters}

Tree characters

Vigour: medium-weak

Growth habit: spreading-erect

Canopy-density: medium

\section{Leaf characters}

Blade length (cm): medium $(6,04 \pm 0,46)$

Blade width $(\mathrm{cm})$ : medium $(1,42 \pm 0,16)$

Shape (length/width): elliptic-lanceolate

\section{Inflorescence characters}

Inflorescence length (cm): long $(4,05 \pm 1,08)$

Number of flowers: high $(\mathbf{3 0 , 2 4} \pm \mathbf{1 , 4 4})$

\section{Fruit characters}

Fresh weight of 100 fruits $(\mathrm{g})$ : medium $(2,23 \pm 0,35)$

Shape (length/width): spherical

Symmetry: slightly asymmetric

Position of maximum transverse diameter:

towards base

Apex: rounded

Base: truncate

Niplle: absent

Lenticels: many and small

\section{Pit characters}

Weight of 100 pits (g): medium $(0,36 \pm 0,05)$

Shape (length/width): ovoid

Mucron: absent

Symmetry: symmetric

Position of maximum transverse diameter:

central

Apex: rounded

Base: rounded

Surface: rugose

Number of grooves: medium
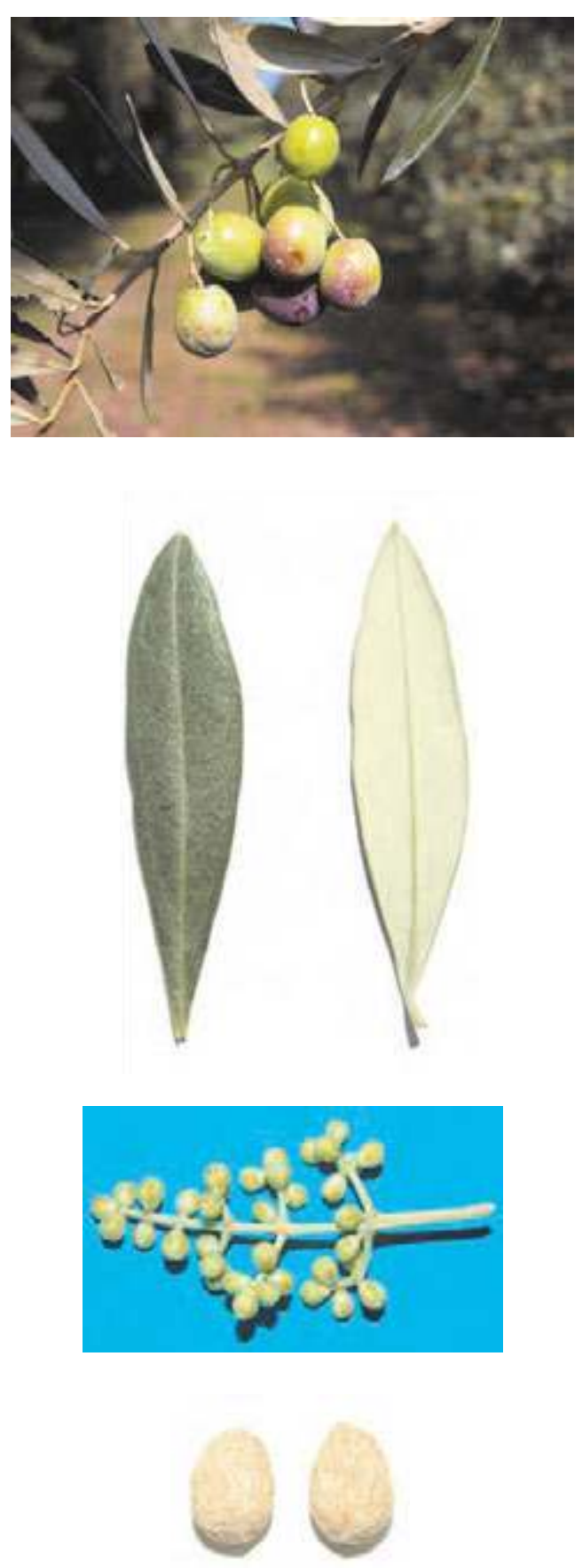


\section{Biochemical Characters}

\section{Fatty Acid Composition}

Table 1. Average values (express in $\% \pm$ standard deviations) of the fatty acids methyl esters and nutritional ratios obtained from single cultivar olive oils.

\begin{tabular}{|c|c|c|c|c|c|}
\hline Myristic acid & $0,01 \pm 0,01$ & Linoleic acid $(\omega 6)$ & $10,19 \pm 0,29$ & Lignoceric acid & $0,04 \pm 0,03$ \\
\hline Palmitic acid & $14,85 \pm 0,85$ & Linolenic acid $(\omega 3)$ & $0,37 \pm 0,02$ & & \\
\hline Palmitoleic acid & $1,09 \pm 0,26$ & Arachic acid & $0,43 \pm 0,28$ & Unsat./satured & $4,87 \pm 0,15$ \\
\hline tear & $1,78 \pm 0,32$ & Eicosenoic acid & $0,19 \pm 0,25$ & $\omega 6 / \omega 3$ & $27,95 \pm 0,82$ \\
\hline leic acid & $70,09 \pm 0,90$ & Behenic acid & $0,08 \pm 0,04$ & & \\
\hline
\end{tabular}

\section{Organoleptic oil values}

Sensory Analysis (Panel test)

Comment: fruity medium, with hints of almond, leaf, and artichoke. Balanced taste sensation with a medium bitter and mediumhigh spicy. Medium fluidity.

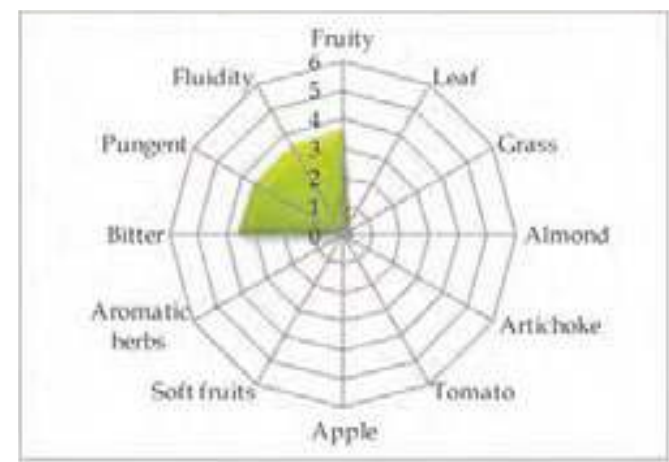

\section{Molecular Markers}

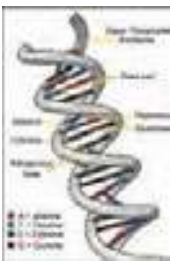

Table 2. Microsatellites (SSR) loci analyzed.

For each locus the allele size (expressed in base pairs) is reported.

$\begin{array}{cccccc}D C A 09 & \text { DCA18 } & \text { GAPU59 } & \text { GAPU71A } & \text { GUPA71B } & \text { GAPU103A } \\ \mathbf{1 8 2} \mathbf{- 2 0 6} & \mathbf{1 7 9 - \mathbf { 1 8 1 }} & \mathbf{2 1 4 - \mathbf { 2 2 2 }} & \mathbf{2 1 4 - \mathbf { 2 1 4 }} & \mathbf{1 3 0 - \mathbf { 1 4 4 }} & \mathbf{1 5 0 - \mathbf { 1 5 7 }} \\ \text { UDOO1 } & \text { UDO03 } & \text { UDO12 } & \text { UDO28 } & \text { UDO39 } & \\ \mathbf{1 4 0 - \mathbf { 1 4 0 }} & \mathbf{1 3 5 - \mathbf { 1 4 3 }} & \mathbf{1 7 7 - \mathbf { 1 9 3 }} & \mathbf{1 6 1 - \mathbf { 2 0 5 }} & \mathbf{2 0 5 - 2 0 5} & \end{array}$

\section{References:}

1 - Lombardo N. In: Le risorse genetiche vegetali presso gli IRSA, MIPAF (2001), 1: pp. 361-405

2 - Muzzalupo I., Stefanizzi F., Bucci C., et al. In Acta Italus Hortus, (2011), 1: 138 -140. 


\section{"Coroncina"}

(synonymy: Corallina, Corona, Coronella, ect.)

Areal distribution or origin area: Marche

Flesh/pit weight ratio: medium $(5,57 \pm 0,33)$

Oil content (\%): medium $(44,34 \pm 1,96)$

Purpose: oil

\section{Morphological characters}

Tree characters

Vigour: weak

Growth habit: spreading

Canopy-density: medium-sparse

\section{Leaf characters}

Blade length (cm): medium $(5,88 \pm 0,47)$

Blade width $(\mathrm{cm})$ : medium $(\mathbf{1}, \mathbf{4 4} \pm \mathbf{0 , 1 2})$

Shape (length/width): elliptic-lanceolate

\section{Inflorescence characters}

Inflorescence length $(\mathrm{cm})$ : medium $(3,23 \pm 1,41)$

Number of flowers: medium $(24,79 \pm 0,88)$

\section{Fruit characters}

Fresh weight of 100 fruits $(\mathrm{g})$ : medium $(3,01 \pm 0,19)$

Shape (length/width): spherical

Symmetry: symmetric

Position of maximum transverse diameter:

central

Apex: rounded

Base: rounded

Niplle: absent

Lenticels: few and small

\section{Pit characters}

Weight of 100 pits (g): high $(0,52 \pm \mathbf{0 , 0 6})$

Shape (length/width): ovoid

Mucron: obvious

Symmetry: symmetric

Position of maximum transverse diameter:

towards apex

Apex: rounded

Base: pointed

Surface: rugose

Number of grooves: medium
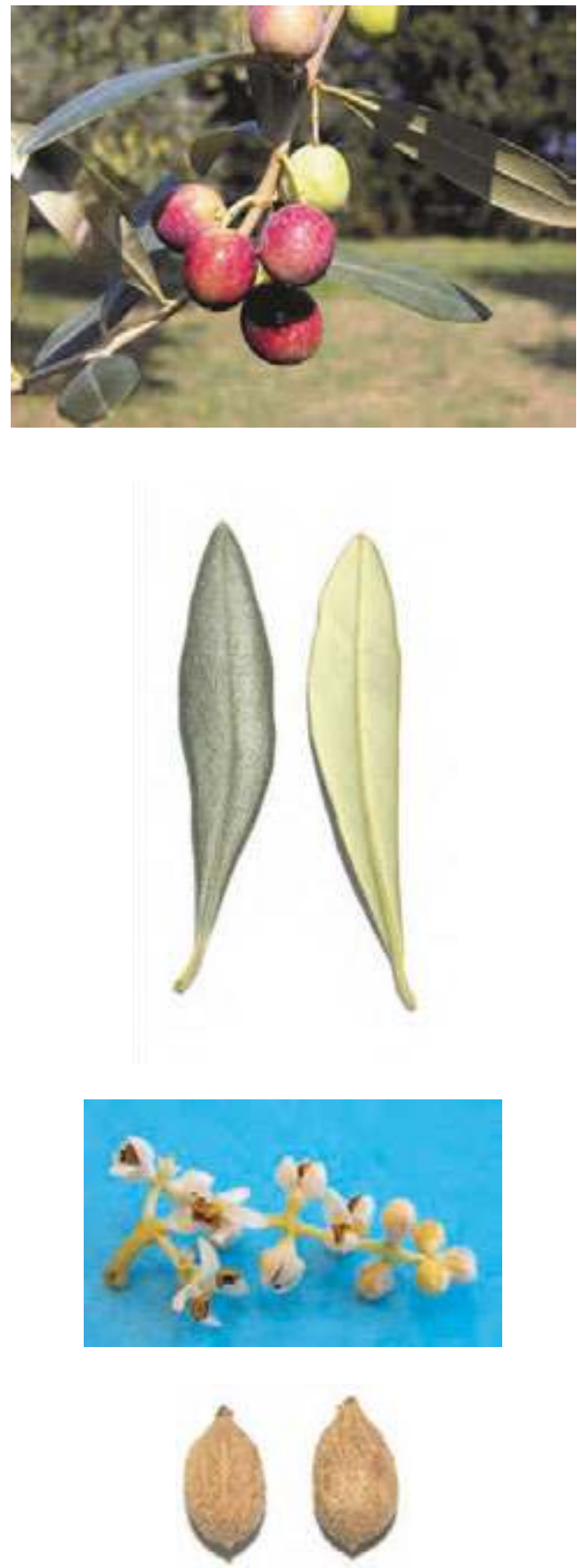


\section{Biochemical Characters}

\section{Fatty Acid Composition}

Table 1. Average values (express in $\% \pm$ standard deviations) of the fatty acids methyl esters and nutritional ratios obtained from single cultivar olive oils.

\begin{tabular}{|c|c|c|c|c|c|}
\hline Myristic acid & $0,00 \pm 0,00$ & Linoleic acid $(\omega 6)$ & $9,89 \pm 0,10$ & Lignoceric acid & $0,06 \pm 0,05$ \\
\hline Palmitic acid & $14,09 \pm 0,17$ & Linolenic acid $(\omega 3)$ & $0,91 \pm 0,03$ & & \\
\hline Palmitoleic acid & $0,92 \pm 0,02$ & Arachic acid & $0,40 \pm 0,23$ & Unsat./satured & $4,93 \pm 0,12$ \\
\hline Stearic acid & $2,25 \pm 0,30$ & Eicosenoic acid & $0,21 \pm 0,26$ & $\omega 6 / \omega 3$ & $10,84 \pm 0,49$ \\
\hline Oleic acid & $70,06 \pm 0,71$ & Behenic acid & $0,12 \pm 0,08$ & & \\
\hline
\end{tabular}

\section{Organoleptic oil values}

Sensory Analysis (Panel test)

Comment: fruity medium, with hints of grass, almond and artichoke. Balanced taste sensation with a medium-light bitter and spicy. Medium-light fluidity.

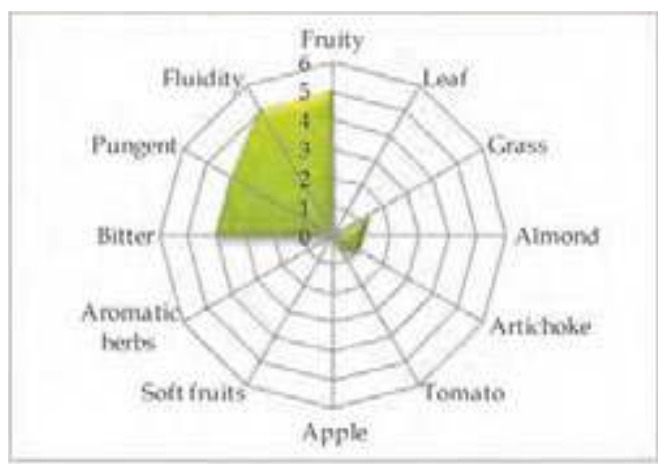

\section{Molecular Markers}

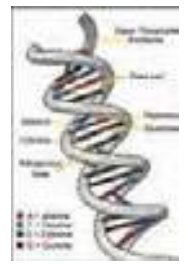

Table 2. Microsatellites (SSR) loci analyzed.

For each locus the allele size (expressed in base pairs) is reported.

\begin{tabular}{|c|c|c|c|c|c|}
\hline DCA09 & DCA18 & GAPU59 & GAPU71A & $G U P A 71 B$ & GAPU103A \\
\hline $172-194$ & $181-185$ & $212-222$ & $212-214$ & $130-144$ & $170-184$ \\
\hline UDO01 & UDO03 & UDO12 & UDO28 & UDO39 & \\
\hline $150-150$ & $135-135$ & $177-193$ & $161-182$ & $200-200$ & \\
\hline
\end{tabular}

\section{References:}

1 - Pannelli G., Alfei B., Santinelli A. In: Varietà di olivo nelle Marche. ASSAM (2001), pp. 37-40.

2 - Various authors. In: Catalogo Nazionale delle Varietà di Olivo . University of Bari (in press), ISBN 978-88-8879397-9. 


\section{"Corsicana da olio"}

Areal distribution or origin area: Sardegna

Flesh/pit weight ratio: low $(4,29 \pm 0,08)$

Oil content (\%): medium $(44,35 \pm 3,34)$

Purpose: oil

\section{Morphological characters}

Tree characters

Vigour: medium-strong

Growth habit: spreadinf-erect

Canopy-density: dense

\section{Leaf characters}

Blade length (cm): medium $(5,02 \pm 0,46)$

Blade width $(\mathrm{cm})$ : medium $(\mathbf{1}, \mathbf{4 4} \pm \mathbf{0 , 2 0})$

Shape (length/width): elliptic

\section{Inflorescence characters}

Inflorescence length (cm): long $(3,55 \pm 0,43)$

Number of flowers: low $(17,55 \pm 1,72)$

\section{Fruit characters}

Fresh weight of 100 fruits $(\mathrm{g})$ : medium $(2,65 \pm 0,06)$

Shape (length/width): ovoid

Symmetry: slightly asymmetric

Position of maximum transverse diameter:

central

Apex: rounded

Base: rounded

Niplle: absent

Lenticels: many and large

\section{Pit characters}

Weight of 100 pits (g): high $(\mathbf{0 , 5 1} \pm \mathbf{0 , 0 1})$

Shape (length/width): elliptic

Mucron: obvious

Symmetry: slightly asymmetric

Position of maximum transverse diameter:

towards apex

Apex: rounded

Base: rounded

Surface: smooth

Number of grooves: low
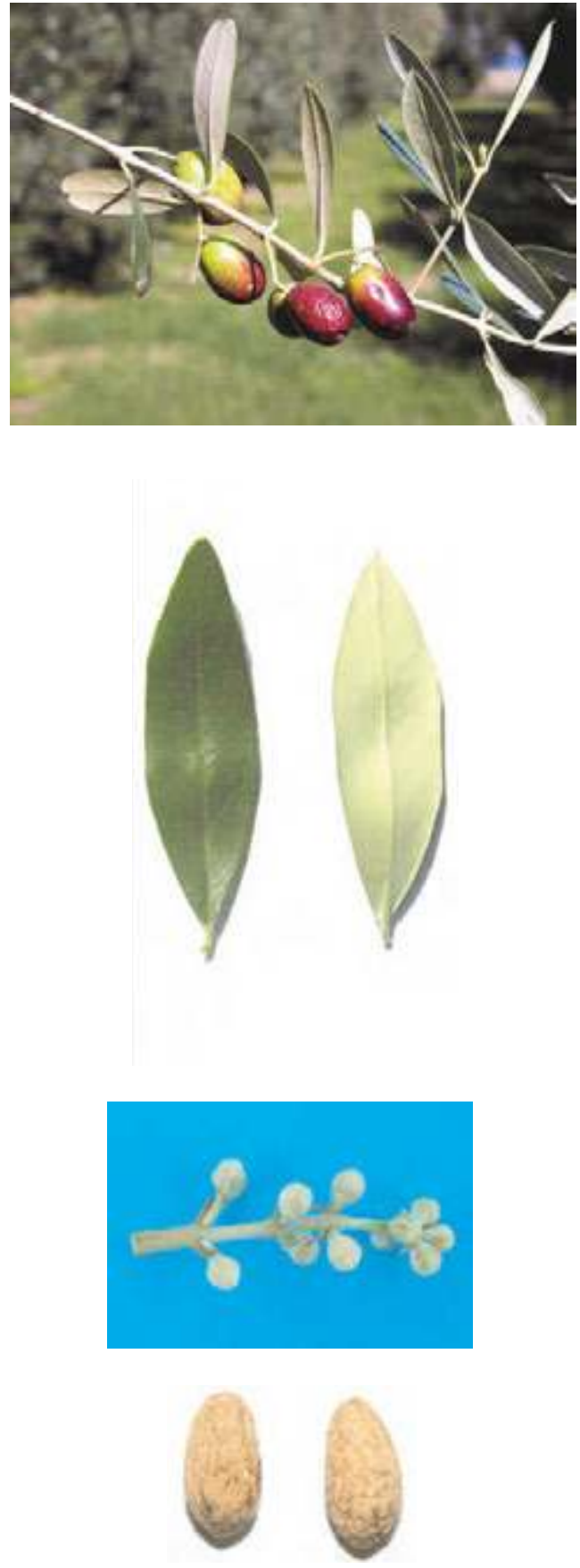


\section{Biochemical Characters}

\section{Fatty Acid Composition}

Table 1. Average values (express in $\% \pm$ standard deviations) of the fatty acids methyl esters and nutritional ratios obtained from single cultivar olive oils.

$\begin{array}{llllll}\text { Myristic acid } & \mathbf{0 , 0 1} \pm \mathbf{0 , 0 1} & \text { Linoleic acid }(\omega 6) & \mathbf{9 , 5 8} \pm \mathbf{0 , 3 6} & \text { Lignoceric acid } & \mathbf{0 , 0 2} \pm \mathbf{0 , 0 1} \\ \text { Palmitic acid } & \mathbf{1 2 , 9 2} \pm \mathbf{0 , 1 5} & \text { Linolenic acid }(\omega 3) \mathbf{0 , 5 6} \pm \mathbf{0 , 2 3} & & \\ \text { Palmitoleic acid } & \mathbf{0 , 7 0 \pm 0 , 0 3} & \text { Arachic acid } & \mathbf{0 , 2 5} \pm \mathbf{0 , 0 6} & \text { Unsat./satured } & \mathbf{5 , 2 6} \pm \mathbf{0 , 0 5} \\ \text { Stearic acid } & \mathbf{2 , 8 3} \pm \mathbf{0 , 0 9} & \text { Eicosenoic acid } & \mathbf{0 , 0 2} \pm \mathbf{0 , 0 1} & \omega 6 / \omega 3 & \mathbf{1 2 , 7 6 \pm 0 , 2 1} \\ \text { Oleic acid } & \mathbf{7 2 , 3 8} \pm \mathbf{0 , 3 3} & \text { Behenic acid } & \mathbf{0 , 0 6} \pm \mathbf{0 , 0 3} & & \end{array}$

\section{Organoleptic oil values}

Sensory Analysis (Panel test)

Comment: fruity medium, with taste of almond, read sensations of artichoke and aromatic herbs. Balanced taste sensation with medium bitter and spicy. Medium fluidity.

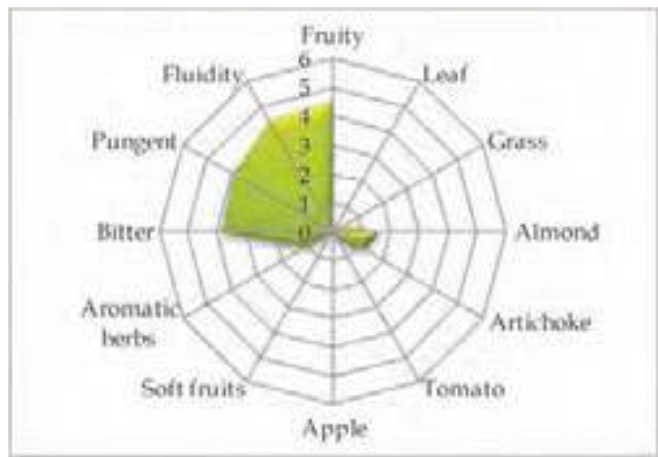

\section{Molecular Markers}

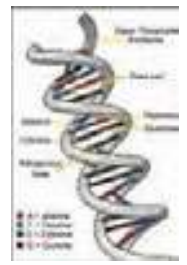

Table 2. Microsatellites (SSR) loci analyzed.

For each locus the allele size (expressed in base pairs) is reported.

$\begin{array}{cccccc}D C A 09 & \text { DCA18 } & \text { GAPU59 } & \text { GAPU71A } & \text { GUPA71B } & \text { GAPU103A } \\ \mathbf{1 8 2} \mathbf{- 1 9 8} & \mathbf{1 8 1} \mathbf{- 1 8 5} & \mathbf{2 1 2} \mathbf{- 2 2 2} & \mathbf{2 1 4} \mathbf{- 2 2 4} & \mathbf{1 2 6 - 1 4 4} & \mathbf{1 5 9 - \mathbf { 1 8 4 }} \\ \text { UDOO1 } & \text { UDOO3 } & \text { UDO12 } & \text { UDO28 } & \text { UDO39 } & \\ \mathbf{1 4 4 - \mathbf { 1 5 0 }} & \mathbf{1 4 3 - \mathbf { 1 4 3 }} & \mathbf{1 7 7 - \mathbf { 1 8 2 }} & \mathbf{1 8 2} \mathbf{- 2 1 0} & \mathbf{1 8 5 - \mathbf { 1 8 5 }} & \end{array}$

\section{References:}

1 - Bandino G., Sedda P., Moro C., et al. In: Atti Convegno Nazionale Biodiversità, Ed. Delfino (2000), pp. 243-246.

2 - Muzzalupo I., Stefanizzi F., Perri E. HortScience (2009), 44: pp. 582-588. 


\section{“Crognalegna”}

Areal distribution or origin area: Abruzzo

Flesh/pit weight ratio: low $(4,84 \pm 0,09)$

Oil content (\%): medium $(44,16 \pm 2,09)$

Purpose: dual purpose

\section{Morphological characters}

Tree characters

Vigour: medium-weak

Growth habit: spreading-erect

Canopy-density: medium-dense

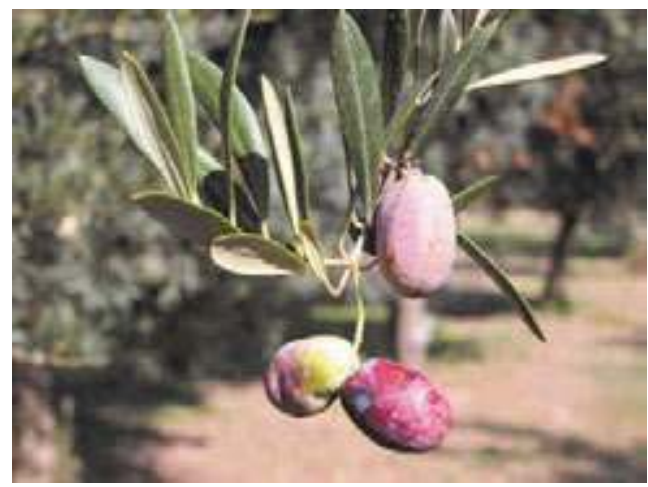

\section{Leaf characters}

Blade length (cm): short $(4,45 \pm 0,38)$

Blade width $(\mathrm{cm})$ : medium $(\mathbf{1}, 16 \pm 0,14)$

Shape (length/width): elliptic

\section{Inflorescence characters}

Inflorescence length $(\mathrm{cm})$ : medium $(3,06 \pm 0,97)$

Number of flowers: medium $(16,54 \pm 2,23)$

\section{Fruit characters}

Fresh weight of 100 fruits $(\mathrm{g})$ : medium $(2,37 \pm 0,61)$

Shape (length/width): ovoid

Symmetry: slightly asymmetric

Position of maximum transverse diameter:

central

Apex: rounded

Base: truncate

Niplle: absent

Lenticels: many and small

\section{Pit characters}

Weight of 100 pits (g): medium $(\mathbf{0 , 4 2} \pm \mathbf{0 , 1 3})$

Shape (length/width): elliptic

Mucron: obvious

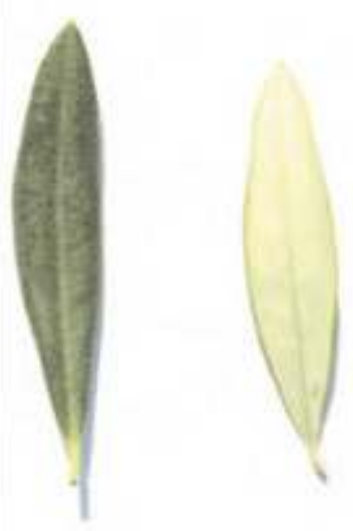

Simmetry: slightly asymmetric

Position of maximum transverse diameter:

towards apex

Apex: rounded

Base: pointed

Surface: smooth

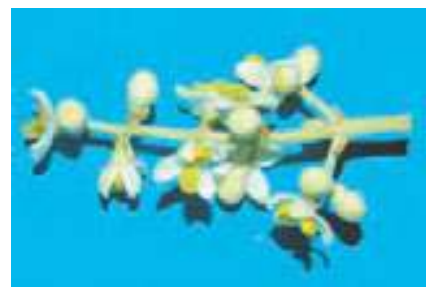

Number of grooves: high 


\section{Biochemical Characters}

\section{Fatty Acid Composition}

Table 1. Average values (express in $\% \pm$ standard deviations) of the fatty acids methyl esters and nutritional ratios obtained from single cultivar olive oils.

\begin{tabular}{|c|c|c|c|c|c|}
\hline Myristic acid & $0,01 \pm 0,01$ & Linoleic acid $(\omega 6)$ & $12,38 \pm 0,42$ & Lignoceric acid & $0,03 \pm 0,00$ \\
\hline Palmitic acid & $12,46 \pm 0,23$ & Linolenic acid $(\omega 3)$ & $0,63 \pm 0,04$ & & \\
\hline Palmitoleic acid & $1,12 \pm 0,17$ & Arachic acid & $0,10 \pm 0,03$ & Unsat./satured & $5,89 \pm 0,09$ \\
\hline tearic & $1,85 \pm 0,09$ & Eicosenoic acid & $0,04 \pm 0,01$ & $\omega 6 / \omega 3$ & $19,65 \pm 0,59$ \\
\hline leic acid & $70,60 \pm 0,35$ & Behenic acid & $0,02 \pm 0,00$ & & \\
\hline
\end{tabular}

\section{Organoleptic oil values}

Sensory Analysis (Panel test)

Comment: fruity medium-light, with taste of artichoke and aromatic herbs, read sensations of almond. Balanced taste sensation with medium-light bitter and spicy. Medium -high.

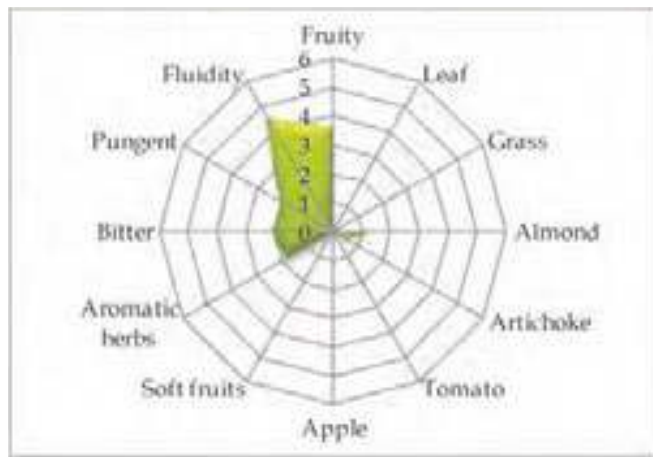

\section{Molecular Markers}

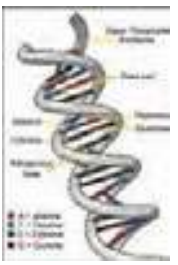

Table 2. Microsatellites (SSR) loci analyzed.

For each locus the allele size (expressed in base pairs) is reported.

$\begin{array}{cccccc}D C A 09 & \text { DCA18 } & \text { GAPU59 } & \text { GAPU71A } & \text { GUPA71B } & \text { GAPU103A } \\ \mathbf{1 7 6 - 1 9 8} & \mathbf{1 7 9 - \mathbf { 1 8 1 }} & \mathbf{2 1 4} \mathbf{- 2 2 2} & \mathbf{2 1 8 - 2 2 4} & \mathbf{1 2 4} \mathbf{- 1 4 4} & \mathbf{1 5 7 - \mathbf { 1 7 0 }} \\ \text { UDOO1 } & \text { UDO03 } & \text { UDO12 } & \text { UDO28 } & \text { UDO39 } & \\ \mathbf{1 5 0 - \mathbf { 1 5 0 }} & \mathbf{1 5 0 - \mathbf { 1 5 0 }} & \mathbf{1 7 7 - \mathbf { 1 8 2 }} & \mathbf{1 8 2} \mathbf{- 2 1 0} & \mathbf{1 6 4 - \mathbf { 1 6 4 }} & \end{array}$

\section{References:}

1 - Pietrangeli E., Russo A., In: Olivi D’Abruzzo, Grafiche di Prinzio (1997), pp. 32-33.

2 - Muzzalupo I., Lombardo N., Salimonti A., et al. Adv. Hort. Sci. (2008), 22(2): pp. 142-148. 


\section{"Cucca"}

(synonymy: Cucco, Oliva da indolcire, Olivone etc.)

Areal distribution or origin area: Toscana

Flesh/pit weight ratio: low $(\mathbf{4}, \mathbf{2 2} \pm \mathbf{0 , 4 0 )}$

Oil content (\%): low $(37,51 \pm 0,08)$

Purpose: table

\section{Morphological characters}

Tree characters

Vigour: medium-strong

Growth habit: spreading

Canopy-density: medium-dense

\section{Leaf characters}

Blade length $(\mathrm{cm})$ : medium $(6,13 \pm 0,82)$

Blade width $(\mathrm{cm})$ : medium $(\mathbf{1 , 2 3} \pm \mathbf{0 , 1 7})$

Shape (length/width): elliptic

\section{Inflorescence characters}

Inflorescence length (cm): medium $(2,93 \pm 2,81)$

Number of flowers: medium $(\mathbf{1 7}, 95 \pm \mathbf{2 , 1 0})$

\section{Fruit characters}

Fresh weight of 100 fruits $(\mathrm{g})$ : medium $(2,51 \pm 0,29)$

Shape (length/width): ovoid

Symmetry: slightly asymmetric

Position of maximum transverse diameter:

central

Apex: pointed

Base: truncate

Niplle: absent

Lenticels: many and small

\section{Pit characters}

Weight of 100 pits (g): medium $(\mathbf{0 , 4 5} \pm \mathbf{0 , 1 3})$

Shape (length/width): elliptic

Mucron: obvious

Symmetry: asymmetric

Position of maximum transverse diameter:

central

Apex: rounded

Base: rounded

Surface: rugose

Number of grooves: medium
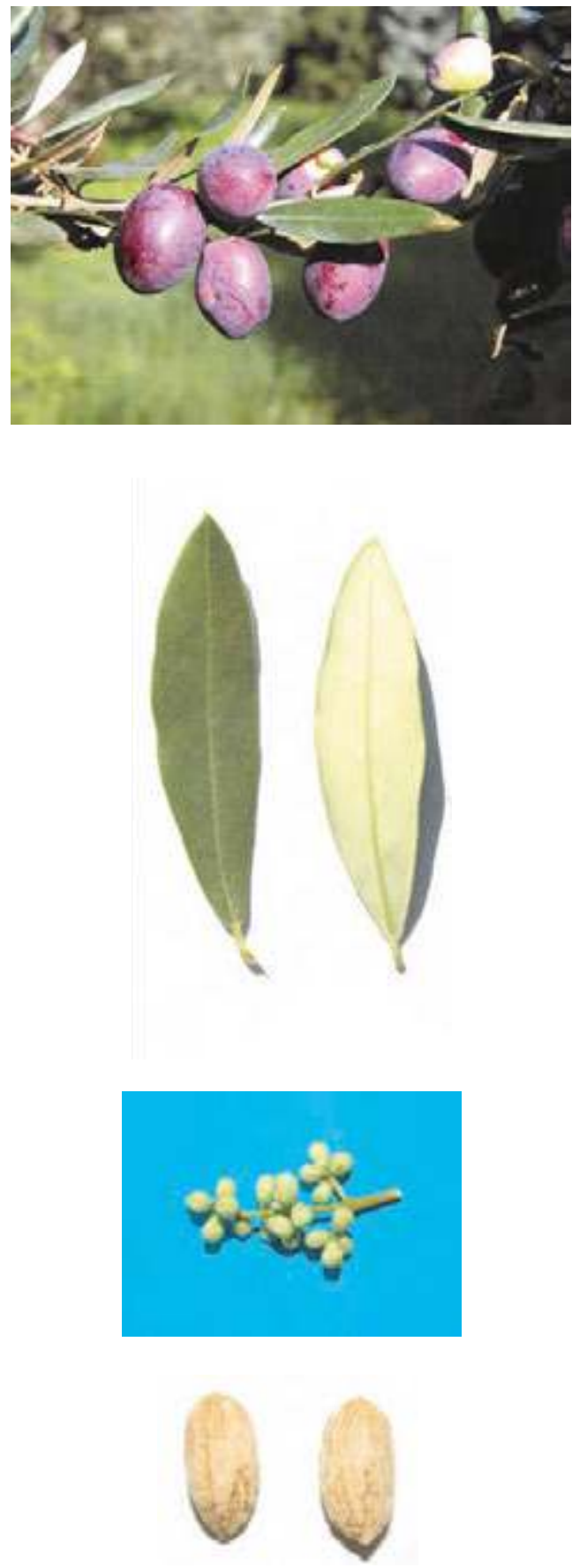


\section{Biochemical Characters}

\section{Fatty Acid Composition}

Table 1. Average values (express in $\% \pm$ standard deviations) of the fatty acids methyl esters and nutritional ratios obtained from single cultivar olive oils.

$\begin{array}{llllll}\text { Myristic acid } & \mathbf{0 , 0 1} \pm \mathbf{0 , 0 0} & \text { Linoleic acid }(\omega 6) & \mathbf{4 , 1 6} \pm \mathbf{0 , 2 6} & \text { Lignoceric acid } & \mathbf{0 , 0 5} \pm \mathbf{0 , 0 1} \\ \text { Palmitic acid } & \mathbf{1 1 , 7 3} \pm \mathbf{0 , 3 4} & \text { Linolenic acid }(\omega 3) & \mathbf{0 , 9 9} \pm \mathbf{0 , 0 1} & & \\ \text { Palmitoleic acid } & \mathbf{1 , 6 7} \pm \mathbf{0 , 0 7} & \text { Arachic acid } & \mathbf{0 , 3 2} \pm \mathbf{0 , 0 0} & \text { Unsat./satured } & \mathbf{6 , 3 2} \pm \mathbf{0 , 1 6} \\ \text { Stearic acid } & \mathbf{1 , 6 6} \pm \mathbf{0 , 0 6} & \text { Eicosenoic acid } & \mathbf{0 , 0 3} \pm \mathbf{0 , 0 0} & \omega 6 / \omega 3 & \mathbf{4 , 1 8} \pm \mathbf{0 , 2 3} \\ \text { Oleic acid } & \mathbf{8 0 , 1 5} \pm \mathbf{0 , 0 4} & \text { Behenic acid } & \mathbf{0 , 0 9} \pm \mathbf{0 , 0 0} & & \end{array}$

\section{Organoleptic oil values}

Sensory Analysis (Panel test)

Comment: fruity medium-high, with hints of artichoke and read sensations of grass and almond. Balanced taste sensation with a medium-high bitter and spicy. Medium-high fluidity.

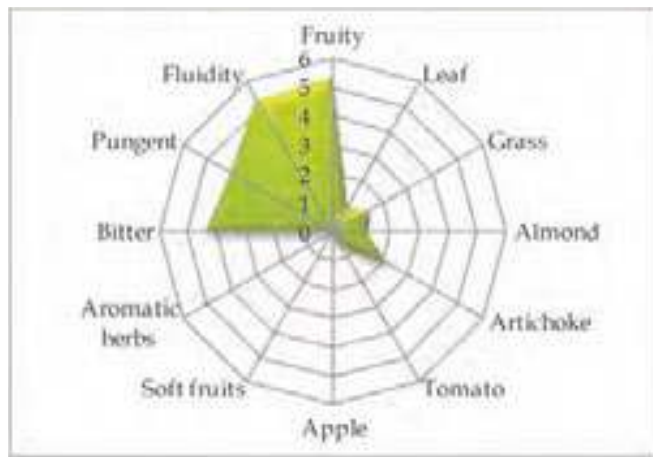

\section{Molecular Markers}

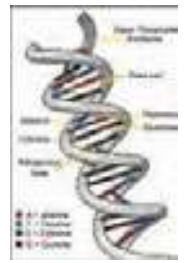

Table 2. Microsatellites (SSR) loci analyzed.

For each locus the allele size (expressed in base pairs) is reported.

\begin{tabular}{|c|c|c|c|c|c|}
\hline DCA09 & DCA18 & GAPU59 & GAPU71A & $G U P A 71 B$ & GAPU103A \\
\hline $162-198$ & $179-181$ & $208-212$ & $214-214$ & $124-144$ & $170-170$ \\
\hline UDO01 & UDO03 & UDO12 & UDO28 & UDO39 & \\
\hline $144-144$ & $150-150$ & $177-193$ & $182-182$ & $213-213$ & \\
\hline
\end{tabular}

\section{References:}

1 - Cimato A., Cantini C., Sani G. In: L'olivo in Toscana: il germoplasma autoctono, Ed. ARSIA (2001).

2 - Muzzalupo I., Lombardo N., Salimonti A., et al. Adv. Hort. Sci. (2008), 22(2): pp. 142-148. 


\section{“Dolce Agogia "}

(synonymy: Agogia, Dolce, Nerello, Olivella.)

Areal distribution or origin area: Umbria

Flesh/pit weight ratio: low $(4,98 \pm 0,06)$

Oil content (\%): medium $(44,30 \pm 1,80)$

Purpose: oil

\section{Morphological characters}

Tree characters

Vigour: medium

Growth habit: erect

Canopy-density: medium

\section{Leaf characters}

Blade length $(\mathrm{cm})$ : medium $(5,95 \pm 0,55)$

Blade width $(\mathrm{cm})$ : medium $(\mathbf{1}, \mathbf{2 1} \pm \mathbf{0 , 1 0})$

Shape (length/width): elliptic-lanceolate

\section{Inflorescence characters}

Inflorescence length $(\mathrm{cm})$ : medium $(2,90 \pm 1,50)$

Number of flowers: low $(15,36 \pm 1,30)$

\section{Fruit characters}

Fresh weight of 100 fruits $(\mathrm{g})$ : medium $(2,12 \pm 0,28)$

Shape (length/width): spherical

Symmetry: slightly asymmetric

Position of maximum transverse diameter:

central

Apex: rounded

Base: rounded

Niplle: absent

Lenticels: many and small

\section{Pit characters}

Weight of 100 pits (g): medium $(\mathbf{0 , 3 2} \pm \mathbf{0 , 0 1})$

Shape (length/width): ovoid

Mucron: obvious

Symmetry: symmetric

Position of maximum transverse diameter:

central

Apex: rounded

Base: rounded

Surface: rugose

Number of grooves: high
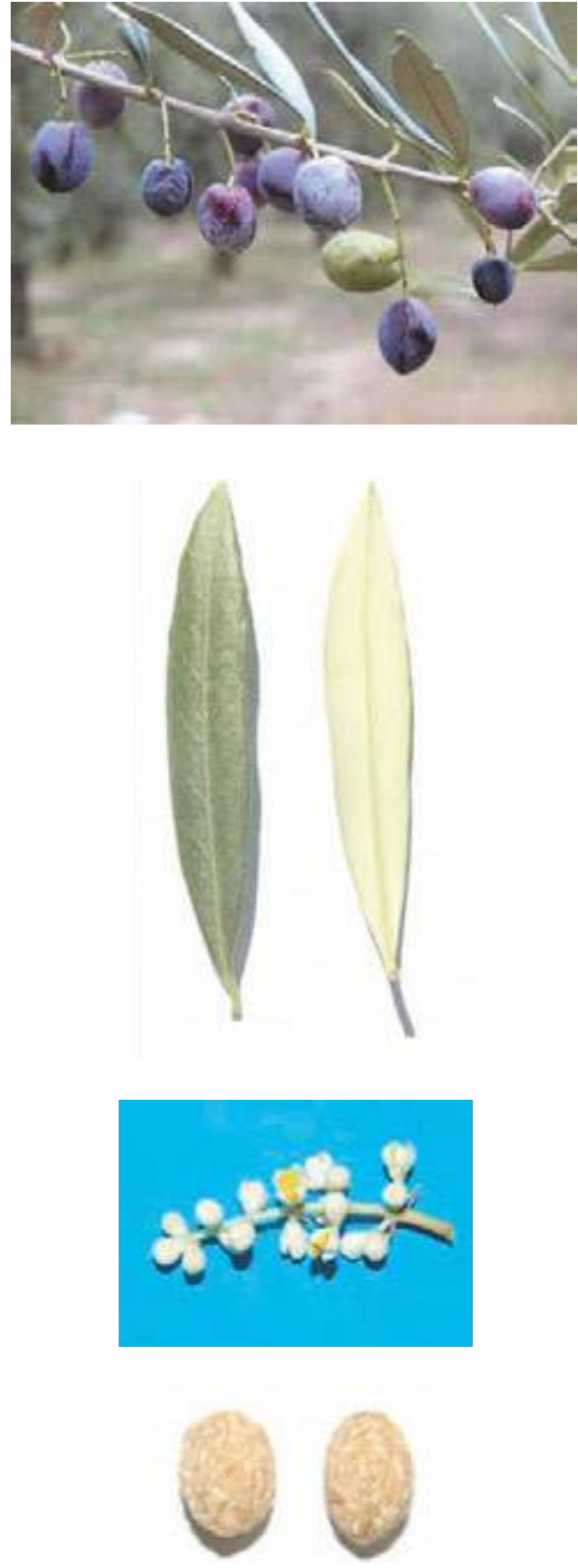


\section{Biochemical Characters}

\section{Fatty Acid Composition}

Table 1. Average values (express in $\% \pm$ standard deviations) of the fatty acids methyl esters and nutritional ratios obtained from single cultivar olive oils.

$\begin{array}{llllll}\text { Myristic acid } & \mathbf{0 , 0 0} \pm \mathbf{0 , 0 0} & \text { Linoleic acid }(\omega 6) & \mathbf{7 , 2 6} \pm \mathbf{0 , 2 6} & \text { Lignoceric acid } & \mathbf{0 , 0 5} \pm \mathbf{0 , 0 2} \\ \text { Palmitic acid } & \mathbf{1 2 , 3 3 \pm 0 , 3 3} & \text { Linolenic acid }(\omega 3) \mathbf{0 , 7 7} \pm \mathbf{0 , 0 3} & & \\ \text { Palmitoleic acid } & \mathbf{1 , 8 4} \pm \mathbf{0 , 2 7} & \text { Arachic acid } & \mathbf{0 , 2 7} \pm \mathbf{0 , 0 2} & \text { Unsat./satured } & \mathbf{5 , 8 2} \pm \mathbf{0 , 0 9} \\ \text { Stearic acid } & \mathbf{1 , 8 2} \pm \mathbf{0 , 3 9} & \text { Eicosenoic acid } & \mathbf{0 , 1 0} \pm \mathbf{0 , 1 2} & \omega 6 / \omega 3 & \mathbf{9 , 4 3} \pm \mathbf{0 , 0 2} \\ \text { Oleic acid } & \mathbf{7 3 , 5 5 \pm \mathbf { 1 , 3 1 }} & \text { Behenic acid } & \mathbf{0 , 0 8} \pm \mathbf{0 , 0 2} & & \end{array}$

\section{Organoleptic oil values}

Sensory Analysis (Panel test)

Comment: fruity medium, with hints of almond and leaves. Balanced taste sensation with a medium bitter and medium-light spicy. Medium fluidity.

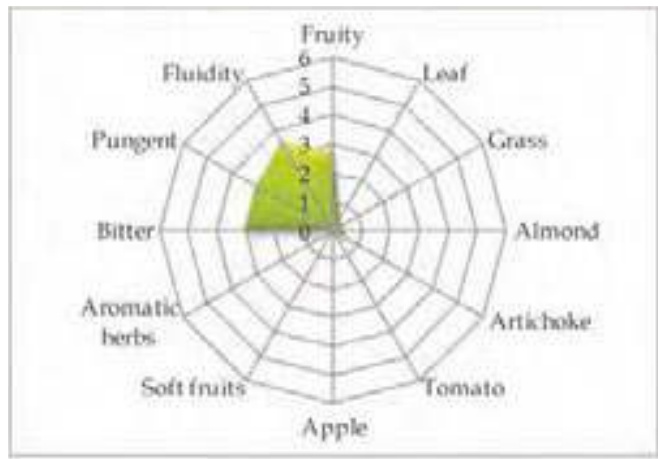

\section{Molecular Markers}

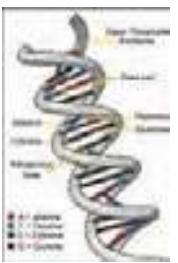

Table 2. Microsatellites (SSR) loci analyzed.

For each locus the allele size (expressed in base pairs) is reported.

$\begin{array}{cccccc}D C A 09 & \text { DCA18 } & \text { GAPU59 } & \text { GAPU71A } & \text { GUPA71B } & \text { GAPU103A } \\ \mathbf{1 7 2} \mathbf{- 1 8 2} & \mathbf{1 7 9 - \mathbf { 1 8 1 }} & \mathbf{2 1 2} \mathbf{- 2 1 8} & \mathbf{2 1 8 - \mathbf { 2 1 8 }} & \mathbf{1 2 4 - \mathbf { 1 2 4 }} & \mathbf{1 8 4 - \mathbf { 1 9 7 }} \\ \text { UDOO1 } & \text { UDO03 } & \text { UDO12 } & \text { UDO28 } & \text { UDO39 } & \\ \mathbf{1 4 0 - \mathbf { 1 4 0 }} & \mathbf{1 4 3 - \mathbf { 1 4 3 }} & \mathbf{1 7 7 - \mathbf { 1 9 3 }} & \mathbf{1 4 3 - \mathbf { 1 4 3 }} & \mathbf{1 8 5 - \mathbf { 2 1 3 }} & \end{array}$

\section{References:}

1 - Pannelli G., Alfei B., Rosati S., et al. In: Varietà di olivo in Umbria. Ed. Pliniana (2000), pp.13-18.

2 - Various authors. In: Catalogo Nazionale delle Varietà di Olivo . University of Bari (in press), ISBN 978-88-8879397-9. 


\section{"Dolce d'Andria"}

Areal distribution or origin area: Puglia

Flesh/pit weight ratio: medium $(6,62 \pm 1,29)$

Oil content (\%): low $(36,97 \pm 0,08)$

Purpose: dual purpose

\section{Morphological characters}

Tree characters

Vigour: medium-strong

Growth habit: spreading

Canopy-density: dense

\section{Leaf characters}

Blade length $(\mathrm{cm})$ : medium $(5,76 \pm 0,73)$

Blade width $(\mathrm{cm})$ : medium $(\mathbf{1}, 49 \pm 0,32)$

Shape (length/width): elliptic

\section{Inflorescence characters}

Inflorescence length $(\mathrm{cm})$ : long $(3,60 \pm 2,40)$

Number of flowers: low $(\mathbf{1 5 , 2 0 \pm 0 , 7 8 )}$

\section{Fruit characters}

Fresh weight of 100 fruits $(\mathrm{g})$ : medium $(3,86 \pm 0,33)$

Shape (length/width): spherical

Symmetry: slightly asymmetric

Position of maximum transverse diameter:

central

Apex: rounded

Base: truncate

Niplle: tenuous

Lenticels: many and small

\section{Pit characters}

Weight of 100 pits (g): high $(\mathbf{0 , 5 2} \pm \mathbf{0 , 1 3})$

Shape (length/width): ovoid

Mucron: obvious

Symmetry: slightly asymmetric

Position of maximum transverse diameter:

central

Apex: rounded

Base: rounded

Surface: rugose

Number of grooves: low
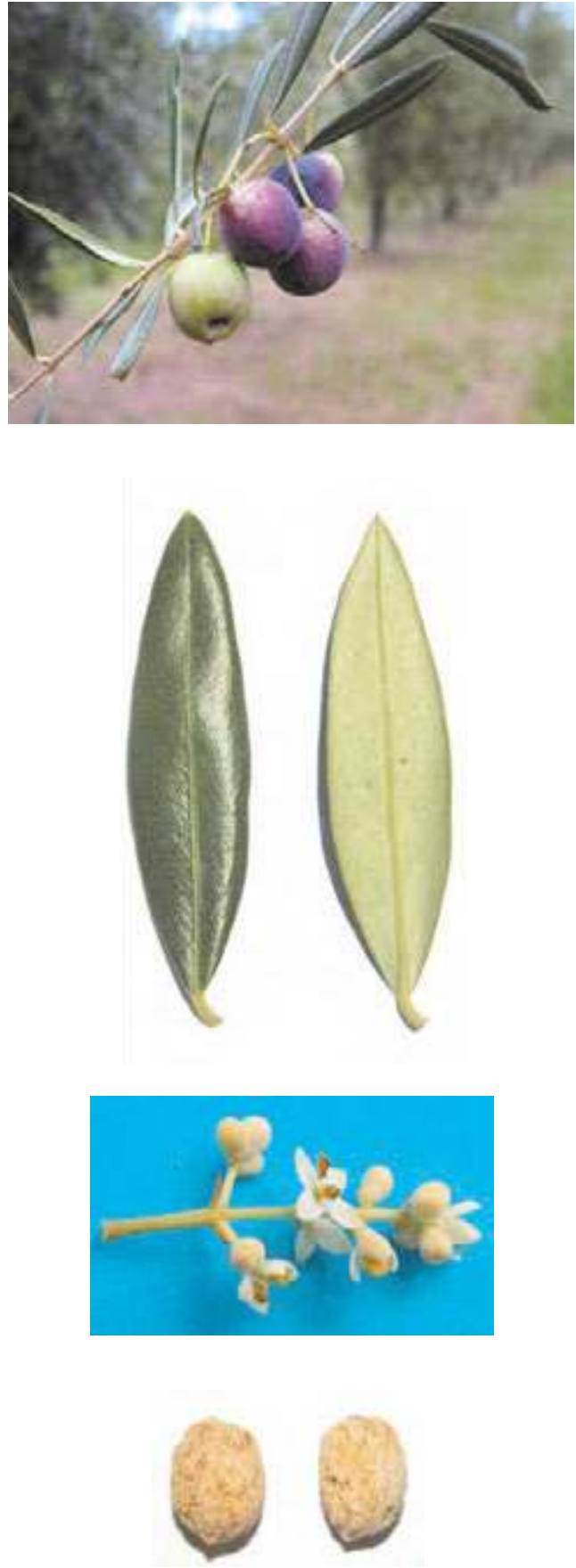


\section{Biochemical Characters}

\section{Fatty Acid Composition}

Table 1. Average values (express in $\% \pm$ standard deviations) of the fatty acids methyl esters and nutritional ratios obtained from single cultivar olive oils.

\begin{tabular}{|c|c|c|c|c|c|}
\hline Myristic acid & $0,01 \pm 0,01$ & Linoleic acid $(\omega 6)$ & $10,69 \pm 0,57$ & Lignoceric acid & $0,08 \pm 0,08$ \\
\hline Palmitic acid & $17,92 \pm 1,15$ & Linolenic acid $(\omega 3)$ & $0,93 \pm 0,07$ & & \\
\hline Palmitoleic acid & $5,44 \pm 0,08$ & Arachic acid & $0,21 \pm 0,11$ & Unsat./satured & $3,87 \pm 0,38$ \\
\hline tearic & $1,24 \pm 0,10$ & Eicosenoic acid & $0,18 \pm 0,22$ & $\omega 6 / \omega 3$ & $11,52 \pm 0,23$ \\
\hline leic acid & $57,70 \pm 2,24$ & Behenic acid & $0,10 \pm 0,06$ & & \\
\hline
\end{tabular}

\section{Organoleptic oil values}

Sensory Analysis (Panel test)

Comment: fruity medium, with hints of almond and leaves. Balanced taste sensation with a medium bitter and medium-light spicy. Medium fluidity.

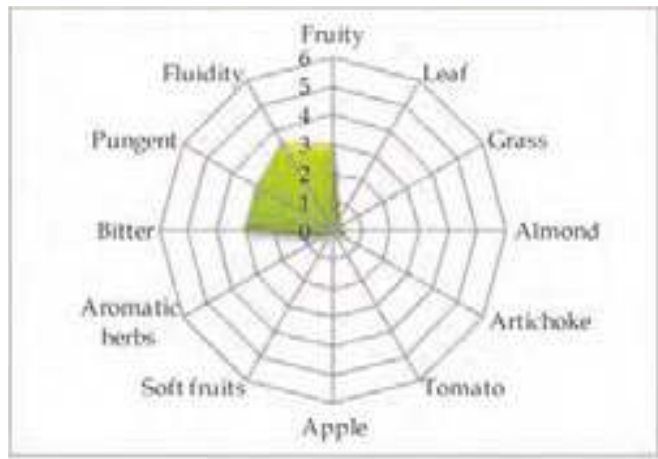

\section{Molecular Markers}

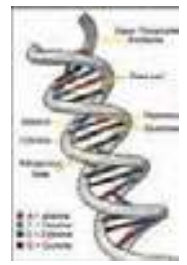

Table 2. Microsatellites (SSR) loci analyzed.

For each locus the allele size (expressed in base pairs) is reported.

$\begin{array}{cccccc}D C A 09 & \text { DCA18 } & \text { GAPU59 } & \text { GAPU71A } & \text { GUPA71B } & \text { GAPU103A } \\ \mathbf{1 7 2} \mathbf{- 1 9 4} & \mathbf{1 7 7 - \mathbf { 1 7 7 }} & \mathbf{2 0 8 - \mathbf { 2 1 8 }} & \mathbf{2 1 4} \mathbf{- 2 1 4} & \mathbf{1 2 4} \mathbf{- 1 4 4} & \mathbf{1 5 0 - \mathbf { 1 5 7 }} \\ \text { UDOO1 } & \text { UDOO3 } & \text { UDO12 } & \text { UDO28 } & \text { UDO39 } & \\ \mathbf{1 4 4 - \mathbf { 1 4 4 }} & \mathbf{1 4 3 - \mathbf { 1 4 3 }} & \mathbf{1 7 7 - \mathbf { 1 8 2 }} & \mathbf{1 4 3 - \mathbf { 2 0 5 }} & \mathbf{1 0 8 - \mathbf { 1 7 0 }} & \end{array}$

\section{References:}

1 - Russo G. In: Atti Convegno Nazionale di Olivicoltura, Ist. Sper. Olivic. Spoleto (2002), pp. 423-426.

2 - Muzzalupo I., Stefanizzi F., Perri E. HortScience (2009), 44: pp. 582-588. 


\section{"Dolce di Cassano"}

Areal distribution or origin area: Puglia

Flesh/pit weight ratio: medium $(5,18 \pm 0,03)$

Oil content (\%): medium $(42,33 \pm 1,71)$

Purpose: oil

\section{Morphological characters}

Tree characters

Vigour: medium

Growth habit: spreading

Canopy-density: medium

\section{Leaf characters}

Blade length ( $\mathrm{cm})$ : medium $(5,63 \pm 0,55)$

Blade width (cm): broad $(\mathbf{1}, 87 \pm \mathbf{0 , 2 5})$

Shape (length/width): elliptic

\section{Inflorescence characters}

Inflorescence length (cm): short $(1,98 \pm 0,34)$

Number of flowers: low $(\mathbf{1 1}, 86 \pm 0,91)$

\section{Fruit characters}

Fresh weight of 100 fruits (g): high $(4,50 \pm 0,63)$

Shape (length/width): spherical

Symmetry: slightly asymmetric

Position of maximum transverse diameter:

central

Apex: rounded

Base: truncate

Niplle: absent

Lenticels: few and large

\section{Pit characters}

Weight of 100 pits (g): very hygh $(0,73 \pm 0,08)$

Shape (length/width): ovoid

Mucron: absent

Symmetry: slightly asymmetric

Position of maximum transverse diameter:

central

Apex: rounded

Base: rounded

Surface: rugose

Number of grooves: medium
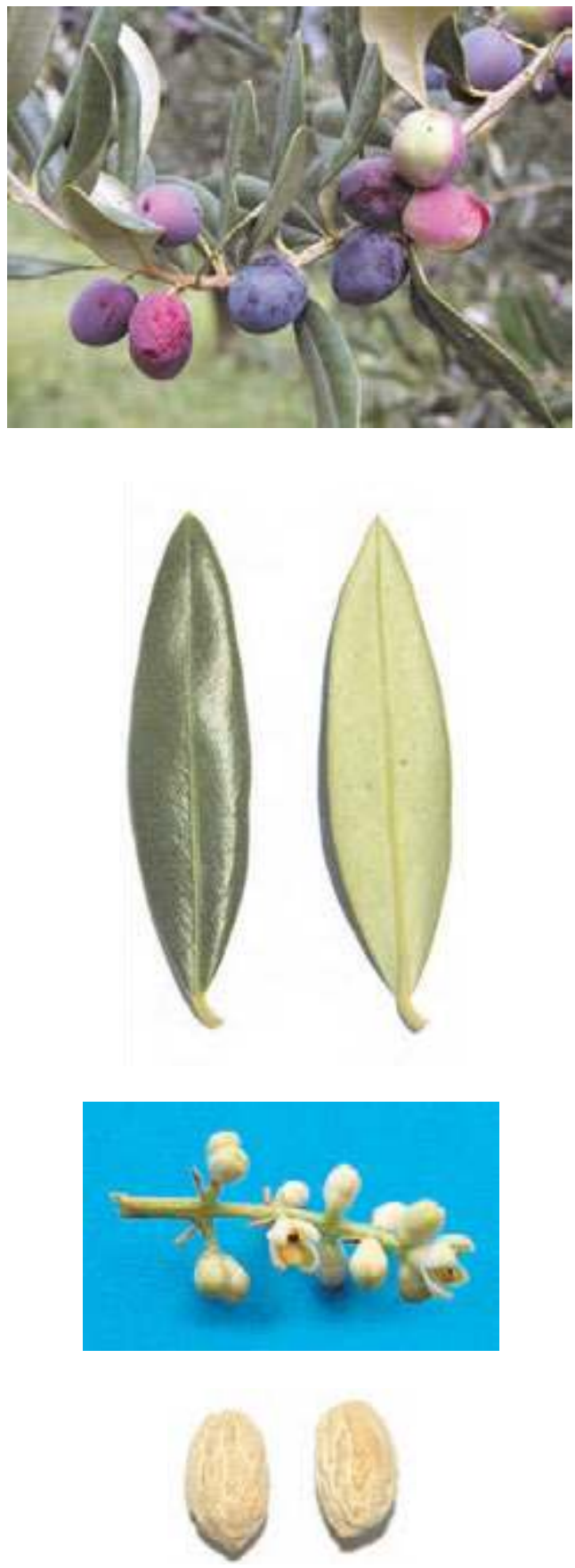


\section{Biochemical Characters}

\section{Fatty Acid Composition}

Table 1. Average values (express in $\% \pm$ standard deviations) of the fatty acids methyl esters and nutritional ratios obtained from single cultivar olive oils.

\begin{tabular}{|c|c|c|c|c|c|}
\hline Myristic acid & $0,01 \pm 0,01$ & Linoleic acid $(\omega 6)$ & $14,35 \pm 1,15$ & Lignoceric acid & $0,01 \pm 0,00$ \\
\hline Palmitic acid & $9,47 \pm 1,69$ & Linolenic acid $(\omega 3)$ & $0,67 \pm 0,03$ & & \\
\hline Palmitoleic acid & $0,60 \pm 0,05$ & Arachic acid & $0,08 \pm 0,09$ & Unsat./satured & $8,54 \pm 1,32$ \\
\hline teari & $0,95 \pm 0,23$ & Eicosenoic acid & $0,02 \pm 0,01$ & $\omega 6 / \omega 3$ & $21,47 \pm 2,63$ \\
\hline leic acid & $73,13 \pm 2,46$ & Behenic acid & $0,03 \pm 0,01$ & & \\
\hline
\end{tabular}

\section{Organoleptic oil values}

Sensory Analysis (Panel test)

Comment: fruity medium, with read sensations of almond and artichoke. Balanced taste sensation with a medium bitter and spicy. Medium fluidity.

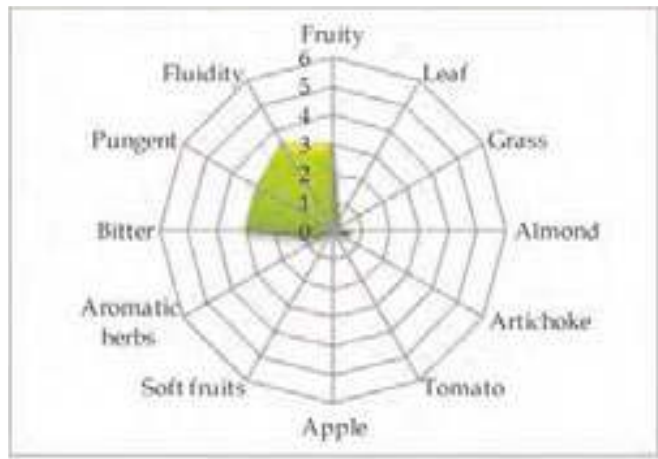

\section{Molecular Markers}

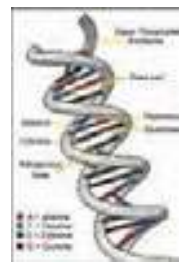

Table 2. Microsatellites (SSR) loci analyzed.

For each locus the allele size (expressed in base pairs) is reported.

$\begin{array}{cccccc}D C A 09 & \text { DCA18 } & \text { GAPU59 } & \text { GAPU71A } & \text { GUPA71B } & \text { GAPU103A } \\ \mathbf{1 6 2} \mathbf{- 1 9 8} & \mathbf{1 8 1 - \mathbf { 1 8 1 }} & \mathbf{2 0 8 - \mathbf { 2 0 8 }} & \mathbf{2 1 4 - \mathbf { 2 2 4 }} & \mathbf{1 3 0 - \mathbf { 1 3 0 }} & \mathbf{1 5 0 - \mathbf { 1 5 7 }} \\ \text { UDOO1 } & \text { UDO03 } & \text { UDO12 } & \text { UDO28 } & \text { UDO39 } & \\ \mathbf{1 4 4 - \mathbf { 1 4 4 }} & \mathbf{1 4 3 - \mathbf { 1 4 3 }} & \mathbf{1 7 7 - \mathbf { 1 7 7 }} & \mathbf{1 8 2} \mathbf{- 2 0 5} & \mathbf{1 0 8} \mathbf{- 1 7 0} & \end{array}$

\section{References:}

1 - Lombardo N., Perri E., Muzzalupo I., et al. In: Contributo alla caratterizzazione del germoplasma olivicolo pugliese, Ist. Sper. Olivic. (2004), pp. 49-52.

2 - Muzzalupo I., Stefanizzi F., Perri E. HortScience (2009), 44: pp. 582-588. 


\section{“Dolce di Rossano"}

(synonymy: Dolce, Rossanese, etc.)

Areal distribution or origin area: Calabria

Flesh/pit weight ratio: high $(5,20 \pm 0,29)$

Oil content (\%): medium $(48,50 \pm 0,53)$

Purpose: oil

\section{Morphological characters}

Tree characters

Vigour: medium-strong

Growth habit: spreading-erect

Canopy-density: medium-dense

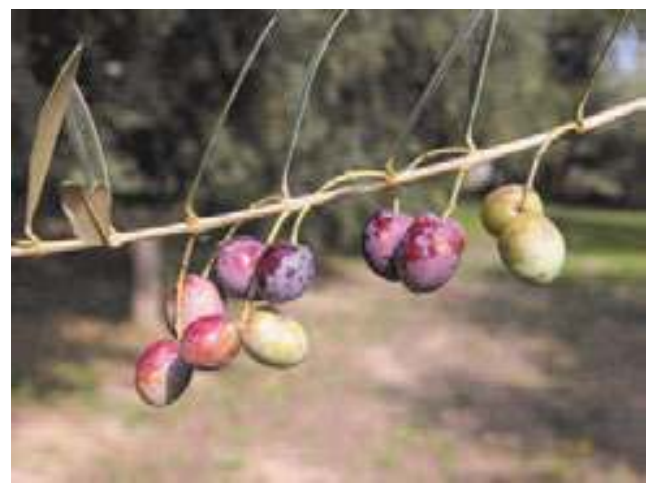

\section{Leaf characters}

Blade length $(\mathrm{cm})$ : medium $(5,41 \pm 0,54)$

Blade width $(\mathrm{cm})$ : medium $(1,37 \pm 0,17)$

Shape (length/width): elliptic

\section{Inflorescence characters}

Inflorescence length $(\mathrm{cm})$ : medium $(2,98 \pm 1,78)$

Number of flowers: medium $(22,11 \pm 5,65)$

\section{Fruit characters}

Fresh weight of 100 fruits $(\mathrm{g})$ : low $(1,93 \pm 0,33)$

Shape (length/width): spherical

Symmetry: slightly asymmetric

Position of maximum transverse diameter:

central

Apex: rounded

Base: truncate

Niplle: tenuous

Lenticels: many and small

\section{Pit characters}

Weight of 100 pits (g): medium $(0,30 \pm 0,04)$

Shape (length/width): elliptic

Mucron: obvious

Symmetry: symmetric

Position of maximum transverse diameter:

central

Apex: pointed

Base: rounded

Surface: rugose

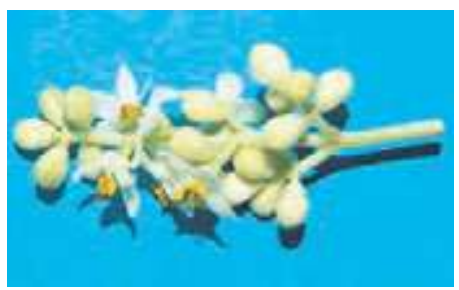

Number of grooves: medium 


\section{Biochemical Characters}

\section{Fatty Acid Composition}

Table 1. Average values (express in $\% \pm$ standard deviations) of the fatty acids methyl esters and nutritional ratios obtained from single cultivar olive oils.

$\begin{array}{llllll}\text { Myristic acid } & \mathbf{0 , 0 1} \pm \mathbf{0 , 0 1} & \text { Linoleic acid }(\omega 6) & \mathbf{8 , 3 2} \pm \mathbf{1 , 6 6} & \text { Lignoceric acid } & \mathbf{0 , 0 6} \pm \mathbf{0 , 0 3} \\ \text { Palmitic acid } & \mathbf{1 5 , 3 3} \pm \mathbf{1 , 7 7} & \text { Linolenic acid }(\omega 3) & \mathbf{0 , 6 9} \pm \mathbf{0 , 1 8} & & \\ \text { Palmitoleic acid } & \mathbf{1 , 5 0} \pm \mathbf{0 , 4 4} & \text { Arachic acid } & \mathbf{0 , 3 2} \pm \mathbf{0 , 0 8} & \text { Unsat./satured } & \mathbf{4 , 6 9} \pm \mathbf{0 , 8 6} \\ \text { Stearic acid } & \mathbf{2 , 0 8} \pm \mathbf{0 , 3 2} & \text { Eicosenoic acid } & \mathbf{0 , 1 5} \pm \mathbf{0 , 1 2} & \omega 6 / \omega 3 & \mathbf{1 2 , 9 9} \pm \mathbf{3 , 9 2} \\ \text { Oleic acid } & \mathbf{7 1 , 2 0 \pm 3 , 0 6} & \text { Behenic acid } & \mathbf{0 , 0 9} \pm \mathbf{0 , 0 3} & & \end{array}$

\section{Organoleptic oil values}

Sensory Analysis (Panel test)

Comment: fruity medium-high, with hints of almond and read sensations of grass and artichoke. Balanced taste sensation with a medium bitter and spicy. Medium fluidity.

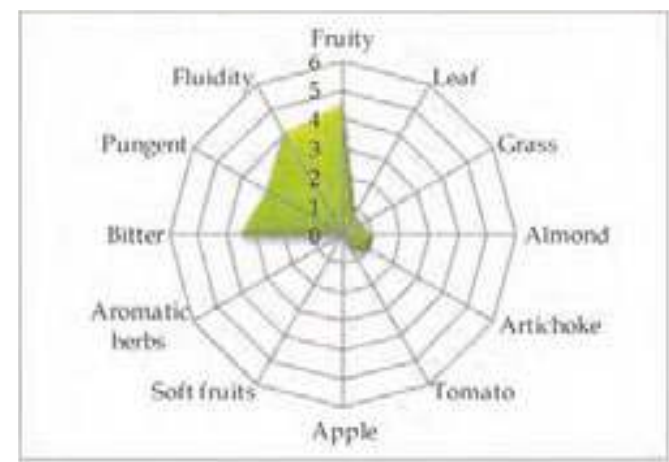

\section{Molecular Markers}

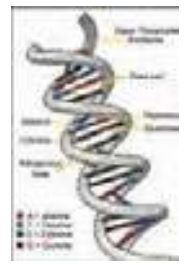

Table 2. Microsatellites (SSR) loci analyzed.

For each locus the allele size (expressed in base pairs) is reported.

$\begin{array}{cccccc}D C A 09 & \text { DCA18 } & \text { GAPU59 } & \text { GAPU71A } & \text { GUPA71B } & \text { GAPU103A } \\ \mathbf{1 7 2} \mathbf{- 1 8 2} & \mathbf{1 7 7 - \mathbf { 1 7 9 }} & \mathbf{2 1 2} \mathbf{- 2 1 2} & \mathbf{2 1 4} \mathbf{- 2 1 4} & \mathbf{1 3 0 - \mathbf { 1 4 4 }} & \mathbf{1 5 0 - \mathbf { 1 5 9 }} \\ \text { UDOO1 } & \text { UDO03 } & \text { UDO12 } & \text { UDO28 } & \text { UDO39 } & \\ \mathbf{1 4 4 - \mathbf { 1 4 4 }} & \mathbf{1 4 3 - \mathbf { 1 4 3 }} & \mathbf{1 7 7 - \mathbf { 1 7 7 }} & \mathbf{1 5 4 - \mathbf { 1 8 2 }} & \mathbf{2 0 5 - \mathbf { 2 0 5 }} & \end{array}$

\section{References:}

1 - Lombardo N., Perri E., Muzzalupo I., et al. In: Il germoplasma olivicolo calabrese, Ist. Sper. Olivic.(2003), pp. 5-6.

2 - Perri E., Mazzotti F., Muzzalupo I., et al. In: Relazione attività, CO.R.ASS.OL. (2003).

3 - Muzzalupo I., Stefanizzi F., Perri E. HortScience (2009), 44: pp. 582-588. 


\section{“Dritta”}

(synonymy: Dolce San Felice, Dritta di Loreto, Dritta di Moscufo, San Felice, etc.)

Areal distribution or origin area: Abruzzo

Flesh/pit weight ratio: high $(7,77 \pm 0,44)$

Oil content (\%): medium $(47,49 \pm 3,03)$

Purpose: oil

\section{Morphological characters}

Tree characters

Vigour: medium-strong

Growth habit: spreding

Canopy-density: dense

\section{Leaf characters}

Blade length $(\mathrm{cm})$ : medium $(5,60 \pm 0,63)$

Blade width $(\mathrm{cm})$ : medium $(\mathbf{1}, 35 \pm \mathbf{0 , 1 3})$

Shape (length/width): elliptic-lanceolate

\section{Inflorescence characters}

Inflorescence length $(\mathrm{cm})$ : medium $(2,95 \pm 0,73)$

Number of flowers: low $(\mathbf{1 6 , 1 4} \pm \mathbf{1 , 1 8})$

\section{Fruit characters}

Fresh weight of 100 fruits $(\mathrm{g})$ : medium $(\mathbf{2 , 2 7} \pm \mathbf{0 , 0 4})$

Shape (length/width): ovoid

Symmetry: slightly asymmetric

Position of maximum transverse diameter:

central

Apex: rounded

Base: rounded

Niplle: tenuous

Lenticels: many and small

\section{Pit characters}

Weight of 100 pits (g): medium $(0,31 \pm 0,07)$

Shape (length/width): elliptic

Mucron: obvious

Symmetry: symmetric

Position of maximum transverse diameter:

\section{towards apex}

Apex: rounded

Base: pointed

Surface: rugose

Number of grooves: medium
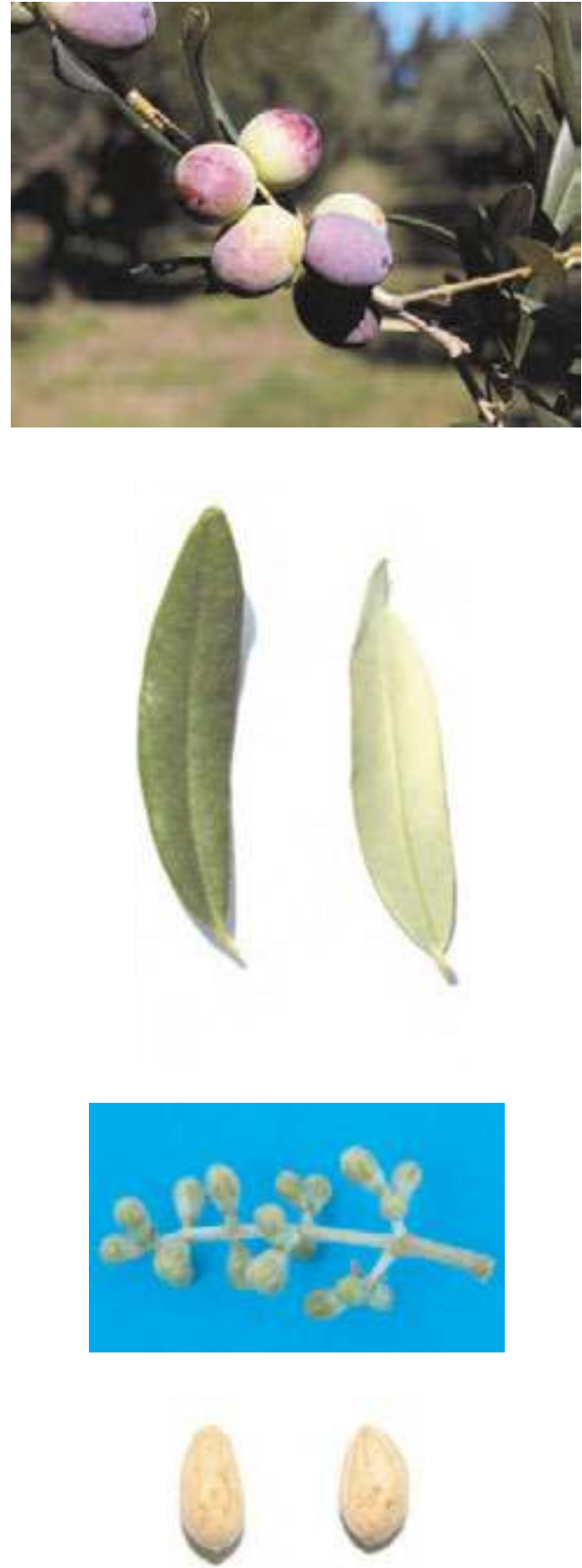


\section{Biochemical Characters}

\section{Fatty Acid Composition}

Table 1. Average values (express in $\% \pm$ standard deviations) of the fatty acids methyl esters and nutritional ratios obtained from single cultivar olive oils.

\begin{tabular}{|c|c|c|c|c|c|}
\hline Myristic acid & $0,01 \pm 0,00$ & Linoleic acid $(\omega 6)$ & $9,26 \pm 0,06$ & Lignoceric acid & $0,07 \pm 0,08$ \\
\hline Palmitic acid & $13,88 \pm 1,19$ & Linolenic acid $(\omega 3)$ & $0,64 \pm 0,02$ & & \\
\hline Palmitoleic acid & $1,12 \pm 0,03$ & Arachic acid & $0,03 \pm 0,03$ & Unsat./satured & $5,13 \pm 0,26$ \\
\hline Stearic acid & $2,61 \pm 0,03$ & Eicosenoic acid & $0,08 \pm 0,09$ & $\omega 6 / \omega 3$ & $14,17 \pm 0,59$ \\
\hline Oleic acid & $71,61 \pm 0,03$ & Behenic acid & $0,09 \pm 0,11$ & & \\
\hline
\end{tabular}

\section{Organoleptic oil values}

Sensory Analysis (Panel test)

Comment: fruity medium-high, with hints of grass and artichoke, read sensations of almond. Balanced taste sensation with a medium bitter and spicy. Medium fluidity.

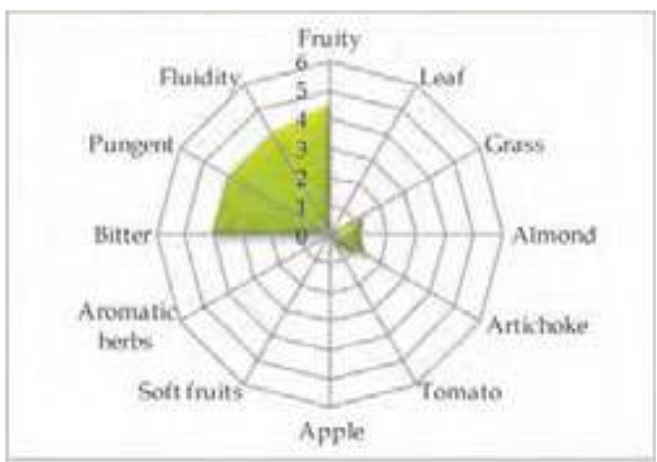

\section{Molecular Markers}

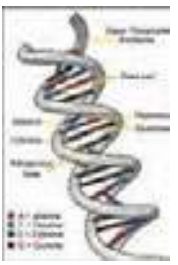

Table 2. Microsatellites (SSR) loci analyzed.

For each locus the allele size (expressed in base pairs) is reported.

\begin{tabular}{|c|c|c|c|c|c|}
\hline DCA09 & DCA18 & GAPU59 & GAPU71A & $G U P A 71 B$ & GAPU103A \\
\hline $172-176$ & $169-179$ & $208-212$ & $214-214$ & $124-130$ & $159-170$ \\
\hline UDO01 & UDO03 & UDO12 & UDO28 & UDO39 & \\
\hline $140-140$ & $143-143$ & $166-193$ & $182-182$ & $220-220$ & \\
\hline
\end{tabular}

\section{References:}

1 - Pietrangeli E., Russo A., In: Olivi D’Abruzzo, Grafiche di Prinzio (1997), pp. 38-39.

2 - Muzzalupo I., Lombardo N., Salimonti A., et al. Adv. Hort. Sci. (2008), 22(2): pp. 142-148. 


\section{"Erbano"}

(synonymy: Ebanu, Erbanu.)

Areal distribution or origin area: Sicilia

Flesh/pit weight ratio: low $(3,73 \pm 0,10)$

Oil content (\%): low $(41,36 \pm 0,04)$

Purpose: oil

\section{Morphological characters}

Tree characters

Vigour: medium-weak

Growth habit: spreading

Canopy-density: medium-dense

\section{Leaf characters}

Blade length $(\mathrm{cm})$ : medium $(5,72 \pm 0,64)$

Blade width $(\mathrm{cm})$ : medium $(\mathbf{1}, \mathbf{3 1} \pm \mathbf{0 , 2 0})$

Shape (length/width): elliptic-lanceolate

\section{Inflorescence characters}

Inflorescence length $(\mathrm{cm})$ : medium $(2,57 \pm 1,83)$

Number of flowers: low $(17,66 \pm 2,40)$

\section{Fruit characters}

Fresh weight of 100 fruits $(\mathrm{g})$ : low $(\mathbf{1}, \mathbf{9 7} \pm \mathbf{0 , 2 2})$

Shape (length/width): ovoid

Symmetry: slightly asymmetric

Position of maximum transverse diameter:

towards apex

Apex: pointed

Base: truncate

Niplle: absent

Lenticels: many and small

\section{Pit characters}

Weight of 100 pits (g): medium $(0,40 \pm 0,01)$

Shape (length/width): elliptic

Mucron: obvious

Symmetry: slightly asymmetric

Position of maximum transverse diameter:

towards apex

Apex: rounded

Base: rounded

Surface: smooth

Number of grooves: medium
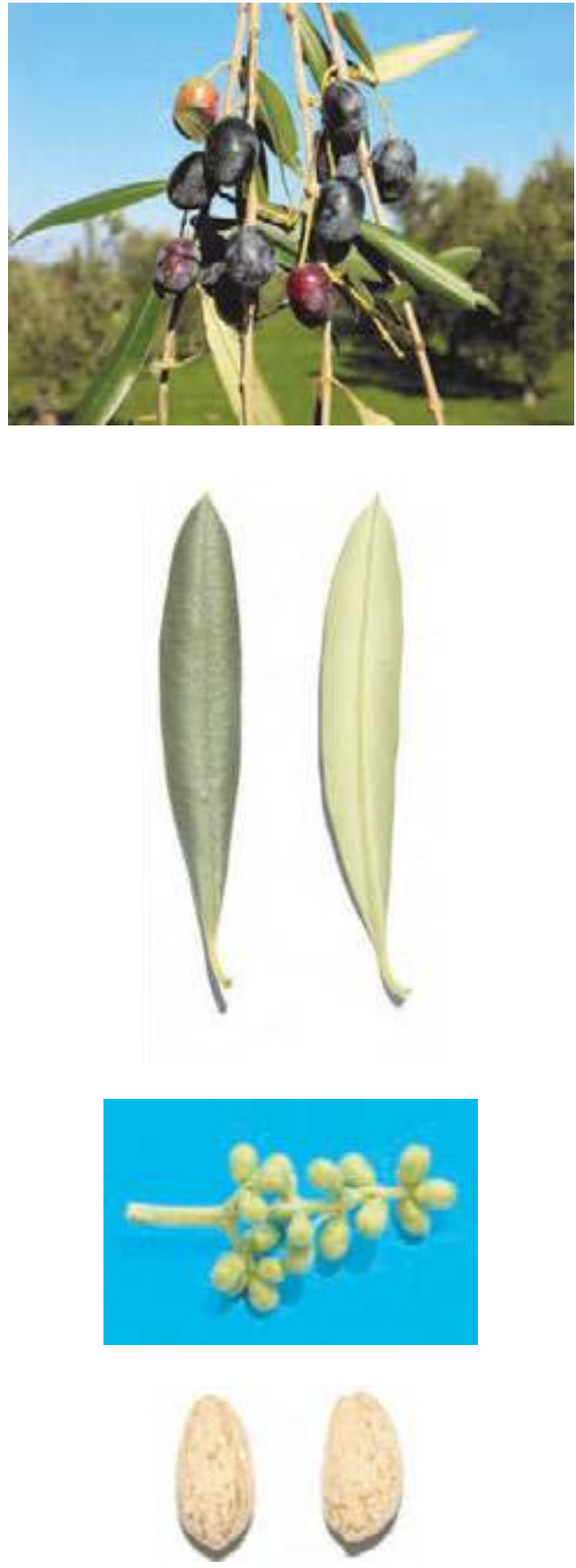


\section{Biochemical Characters}

\section{Fatty Acid Composition}

Table 1. Average values (express in $\% \pm$ standard deviations) of the fatty acids methyl esters and nutritional ratios obtained from single cultivar olive oils.

\begin{tabular}{|c|c|c|c|c|c|}
\hline Myristic acid & $0,01 \pm 0,00$ & Linoleic acid $(\omega 6)$ & $73,04 \pm 0,08$ & Lignoceric acid & $0,06 \pm 0,02$ \\
\hline Palmitic acid & $11,67 \pm 0,45$ & Linolenic acid $(\omega 3)$ & $9,15 \pm 0,25$ & & \\
\hline Palmitoleic acid & $1,25 \pm 0,01$ & Arachic acid & $0,82 \pm 0,08$ & Unsat./satured & $6,08 \pm 0,23$ \\
\hline Stearic acid & $1,95 \pm 0,08$ & Eicosenoic acid & $0,31 \pm 0,08$ & $\omega 6 / \omega 3$ & $11,23 \pm 1,47$ \\
\hline Oleic acid & \pm & Behenic acid & $0,37 \pm 0,04$ & & \\
\hline
\end{tabular}

\section{Organoleptic oil values}

Sensory Analysis (Panel test)

Comment: fruity medium-high, with taste of almond and artichoke, with read sensations of aromatic herbs. Balanced taste sensation with medium bitter and spicy. Medium-high.

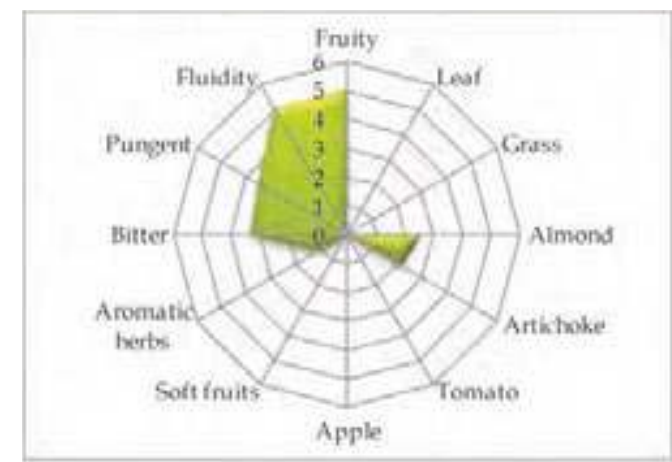

\section{Molecular Markers}

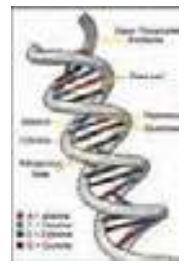

Table 2. Microsatellites (SSR) loci analyzed.

For each locus the allele size (expressed in base pairs) is reported.

$\begin{array}{cccccc}D C A 09 & \text { DCA18 } & \text { GAPU59 } & \text { GAPU71A } & \text { GUPA71B } & \text { GAPU103A } \\ \mathbf{1 7 2} \mathbf{- 1 8 2} & \mathbf{1 7 3 - 1 7 7} & \mathbf{2 1 2} \mathbf{- 2 1 2} & \mathbf{2 1 0 - \mathbf { 2 1 2 }} & \mathbf{1 2 4} \mathbf{- 1 4 4} & \mathbf{1 5 9 - \mathbf { 1 5 9 }} \\ \text { UDOO1 } & \text { UDOO3 } & \text { UDO12 } & \text { UDO28 } & \text { UDO39 } & \\ \mathbf{1 4 0 - \mathbf { 1 4 0 }} & \mathbf{1 5 0 - \mathbf { 1 5 0 }} & \mathbf{1 7 7 - \mathbf { 1 9 3 }} & \mathbf{1 8 2} \mathbf{- 1 8 2} & \mathbf{1 0 8 - \mathbf { 1 0 8 }} & \end{array}$

\section{References:}

1 - Caruso T., Cartabellotta D., Motisi A., et all. In: Cultivar di olivo siciliane, Università degli Studi di Palermo (2007), pp. 152-156.

2 - Muzzalupo I., Stefanizzi F., Perri E. HortScience (2009), 44: pp. 582-588. 


\section{“Faresana "}

(synonymy: Baresana, Cellara, Farasano, Pierasana, Varesana etc.)

Areal distribution or origin area: Basilicata

Flesh/pit weight ratio: medium $(5,59 \pm 0,28)$

Oil content (\%): medium $(40,87 \pm 1,49)$

Purpose: dual purpose

\section{Morphological characters}

Tree characters

Vigour: medium

Growth habit: spreading

Canopy-density: medium-dense

\section{Leaf characters}

Blade length $(\mathrm{cm})$ : medium $(6,44 \pm 0,50)$

Blade width (cm): medium $(1,45 \pm 0,17)$

Shape (length/width): elliptic-lanceolate

\section{Inflorescence characters}

Inflorescence length (cm): long $(4,41 \pm 1,70)$

Number of flowers: medium $(21,79 \pm 1,64)$

\section{Fruit characters}

Fresh weight of 100 fruits $(\mathrm{g})$ : high $(\mathbf{4 , 6 4} \pm \mathbf{1 , 0 4})$

Shape (length/width): ovoid

Symmetry: symmetric

Position of maximum transverse diameter: central

Apex: rounded

Base: truncate

Niplle: absent

Lenticels: large and few

\section{Pit characters}

Weight of 100 pits (g): high $(0,59 \pm 0,05)$

Shape (length/width): elliptic

Mucron: absent

Symmetry: slightly asymmetric

Position of maximum transverse diameter:

central

Apex: pointed

Base: rounded

Surface: scabrous

Number of grooves: medium
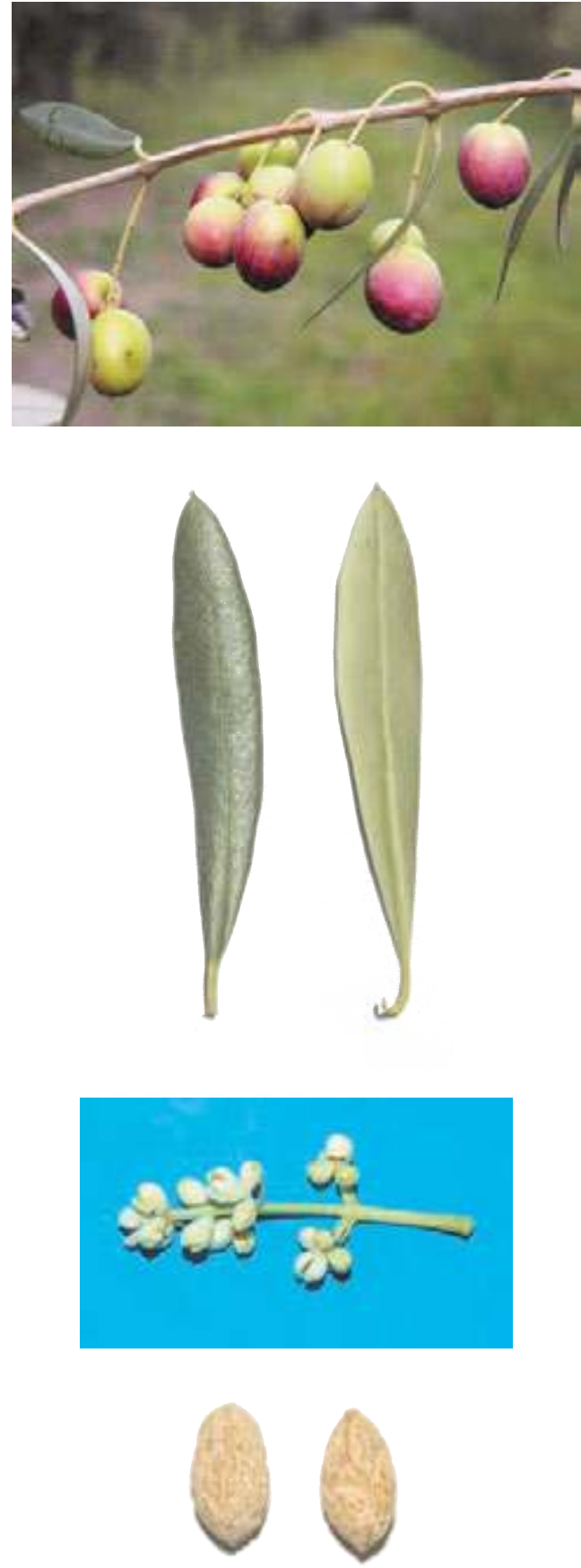


\section{Biochemical Characters}

\section{Fatty Acid Composition}

Table 1. Average values (express in $\% \pm$ standard deviations) of the fatty acids methyl esters and nutritional ratios obtained from single cultivar olive oils.

$\begin{array}{llllll}\text { Myristic acid } & \mathbf{0 , 0 1} \pm \mathbf{0 , 0 1} & \text { Linoleic acid }(\omega 6) & \mathbf{8 , 8 6} \pm \mathbf{2 , 5 8} & \text { Lignoceric acid } & \mathbf{0 , 0 4} \pm \mathbf{0 , 0 2} \\ \text { Palmitic acid } & \mathbf{1 0 , 4 0 \pm 0 , 3 0} & \text { Linolenic acid }(\omega 3) & \mathbf{0 , 9 6} \pm \mathbf{0 , 0 9} & & \\ \text { Palmitoleic acid } & \mathbf{0 , 6 3} \pm \mathbf{0 , 0 5} & \text { Arachic acid } & \mathbf{0 , 0 7} \pm \mathbf{0 , 0 2} & \text { Unsat./satured } & \mathbf{6 , 9 8} \pm \mathbf{0 , 0 6} \\ \text { Stearic acid } & \mathbf{1 , 7 8} \pm \mathbf{0 , 2 0} & \text { Eicosenoic acid } & \mathbf{0 , 1 7} \pm \mathbf{0 , 2 2} & \omega 6 / \omega 3 & \mathbf{9 , 3 8} \pm \mathbf{3 , 5 3} \\ \text { Oleic acid } & \mathbf{7 5 , 6 8 \pm 2 , 3 5} & \text { Behenic acid } & \mathbf{0 , 0 7} \pm \mathbf{0 , 0 2} & & \end{array}$

\section{Organoleptic oil values}

Sensory Analysis (Panel test)

Comment: fruity medium-high, with hints of almond and read sensations of grass and artichoke. Balanced taste sensation with a medium-high bitter and spicy. Medium-high fluidity.

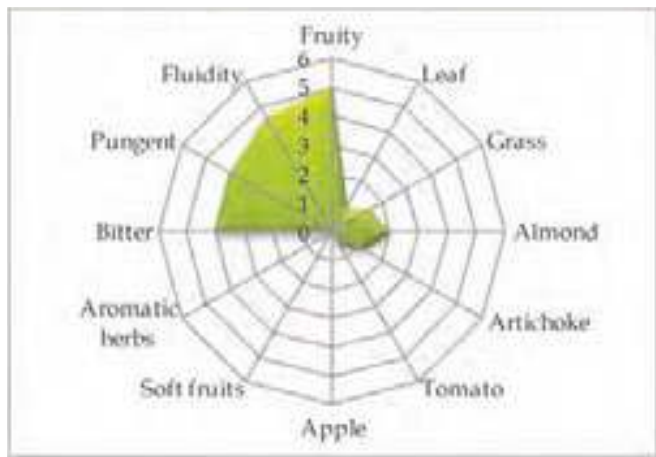

\section{Molecular Markers}

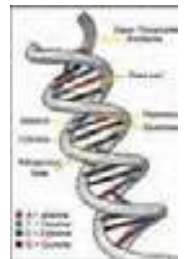

Table 2. Microsatellites (SSR) loci analyzed. For each locus the allele size (expressed in base pairs) is reported.

$\begin{array}{cccccr}D C A 09 & \text { DCA18 } & \text { GAPU59 } & \text { GAPU71A } & \text { GUPA71B } & \text { GAPU103A } \\ \mathbf{1 8 2} \mathbf{- 1 8 2} & \mathbf{1 7 9 - \mathbf { 1 8 1 }} & \mathbf{2 1 2} \mathbf{- 2 1 2} & \mathbf{2 1 4} \mathbf{- 2 1 4} & \mathbf{1 2 4} \mathbf{- 1 2 6} & \mathbf{1 5 9 - \mathbf { 1 1 8 4 }} \\ \text { UDOO1 } & \text { UDOO3 } & \text { UDO12 } & \text { UDO28 } & \text { UDO39 } & \\ \mathbf{1 5 0} \mathbf{- 1 5 0} & \mathbf{1 4 3 - \mathbf { 1 4 3 }} & \mathbf{1 6 6 - \mathbf { 1 7 7 }} & \mathbf{1 5 0 - \mathbf { 1 5 0 }} & \mathbf{2 0 5 - \mathbf { 2 0 5 }} & \end{array}$

\section{References:}

1 - Rotundo A., Marone E. In: Il germoplasma olivicolo lucano, Olita-Potenza (2002), pp. 81-84.

2 - Rotundo A., Perri E.,Muzzalupo I., et al. In: Il germoplasma olivicolo meridionale, (2012 in press).

3 - Muzzalupo I., Stefanizzi F., Perri E. HortScience (2009), 44: pp. 582-588. 


\section{“Favarol"}

(synonymy: Favar, Favera, Perlarol, etc.)

Areal distribution or origin area: Veneto

Flesh/pit weight ratio: low $(4,95 \pm 0,75)$

Oil content (\%): medium $(49,05 \pm 1,07)$

Purpose: oil

\section{Morphological characters}

Tree characters

Vigour: medium-weak

Growth habit: erect-spreading

Canopy-density: medium-dense

\section{Leaf characters}

Blade length (cm): short $(4,71 \pm 0,25)$

Blade width $(\mathrm{cm})$ : medium $(\mathbf{1}, \mathbf{4 0} \pm \mathbf{0 , 1 1})$

Shape (length/width): elliptic

\section{Inflorescence characters}

Inflorescence length (cm): medium $(3,39 \pm 0,41)$

Number of flowers: medium $(23,17 \pm 1,45)$

\section{Fruit characters}

Fresh weight of 100 fruits $(\mathrm{g})$ : medium $(\mathbf{2 , 2 0 \pm 0 , 3 3 )}$

Shape (length/width): spherical

Symmetry: slightly asymmetric

Position of maximum transverse diameter:

central

Apex: rounded

Base: rounded

Niplle: tenuous

Lenticels: many and small

\section{Pit characters}

Weight of 100 pits (g): medium $(0,37 \pm 0,03$ )

Shape (length/width): ovoid

Mucron: obvious

Symmetry: slightly asymmetric

Position of maximum transverse diameter:

towards apex

Apex: rounded

Base: rounded

Surface: smooth

Number of grooves: medium
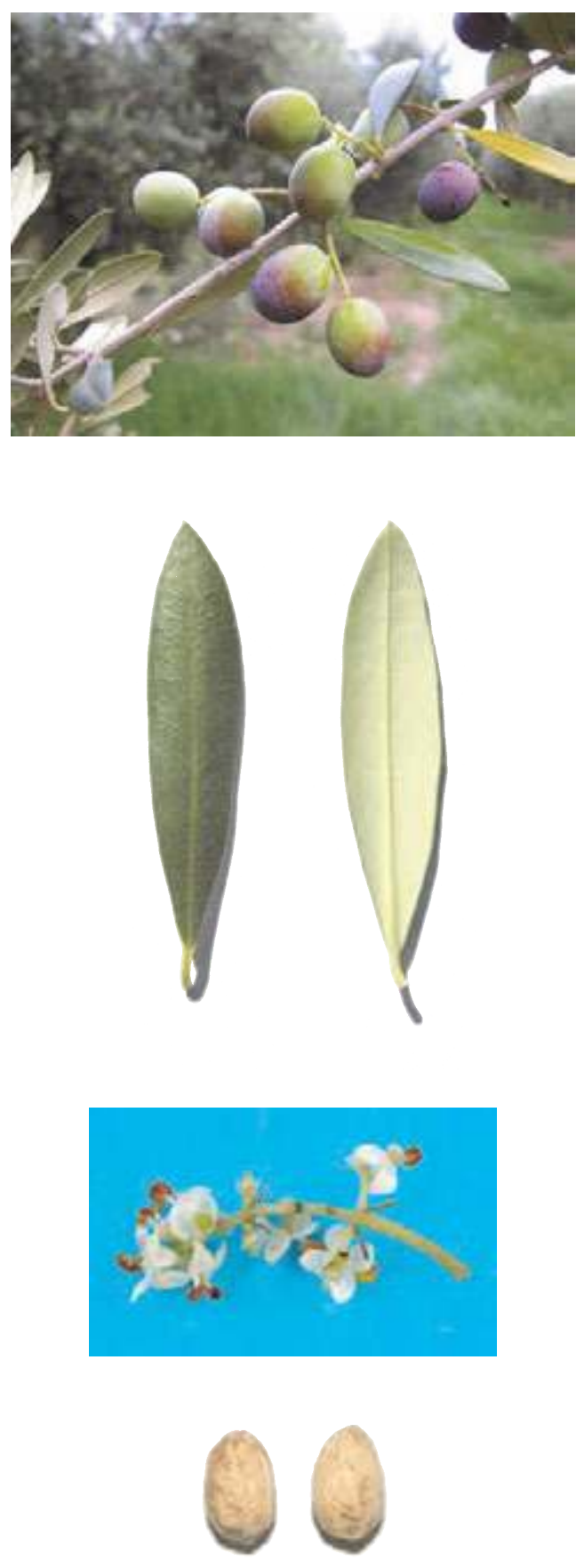


\section{Biochemical Characters}

\section{Fatty Acid Composition}

Table 1. Average values (express in $\% \pm$ standard deviations) of the fatty acids methyl esters and nutritional ratios obtained from single cultivar olive oils.

$\begin{array}{llllll}\text { Myristic acid } & \mathbf{0 , 0 1} \pm \mathbf{0 , 0 0} & \text { Linoleic acid }(\omega 6) & \mathbf{9 , 3 6} \pm \mathbf{0 , 9 9} & \text { Lignoceric acid } & \mathbf{0 , 0 3} \pm \mathbf{0 , 0 0} \\ \text { Palmitic acid } & \mathbf{1 3 , 0 2} \pm \mathbf{0 , 8 9} & \text { Linolenic acid }(\omega 3) \mathbf{0 , 7 6} \pm \mathbf{0 , 0 5} & & \\ \text { Palmitoleic acid } & \mathbf{0 , 9 4 \pm 0 , 2 0} & \text { Arachic acid } & \mathbf{0 , 2 9} \pm \mathbf{0 , 0 5} & \text { Unsat./satured } & \mathbf{5 , 4 0 \pm 0 , 5 3} \\ \text { Stearic acid } & \mathbf{2 , 3 0 \pm 0 , 4 5} & \text { Eicosenoic acid } & \mathbf{0 , 0 2} \pm \mathbf{0 , 0 1} & \omega 6 / \omega 3 & \mathbf{1 2 , 4 2} \pm \mathbf{2 , 1 8} \\ \text { Oleic acid } & \mathbf{7 2 , 3 0 \pm \mathbf { 1 , 7 3 }} & \text { Behenic acid } & \mathbf{0 , 0 8} \pm \mathbf{0 , 0 1} & & \end{array}$

\section{Organoleptic oil values}

Sensory Analysis (Panel test)

Comment: fruity medium, with read sensations of artichoke and almond. Balanced in flavors, with hints of bitter and spicy medium intensity. Medium fluidity.

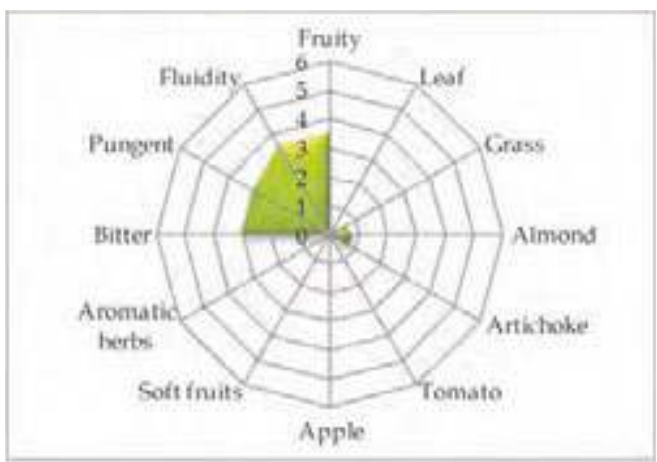

\section{Molecular Markers}

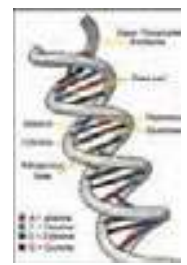

Table 2. Microsatellites (SSR) loci analyzed.

For each locus the allele size (expressed in base pairs) is reported.

$\begin{array}{cccccc}D C A 09 & \text { DCA18 } & \text { GAPU59 } & \text { GAPU71A } & \text { GUPA71B } & \text { GAPU103A } \\ \mathbf{1 8 2} \mathbf{- 2 0 6} & \mathbf{1 7 7 - \mathbf { 1 7 7 }} & \mathbf{2 0 8 - \mathbf { 2 0 8 }} & \mathbf{2 1 4 - \mathbf { 2 1 4 }} & \mathbf{1 2 6 - \mathbf { 1 4 4 }} & \mathbf{1 5 0 - \mathbf { 1 5 7 }} \\ \text { UDOO1 } & \text { UDO03 } & \text { UDO12 } & \text { UDO28 } & \text { UDO39 } & \\ \mathbf{1 5 0 - \mathbf { 1 5 0 }} & \mathbf{1 4 3 - \mathbf { 1 4 3 }} & \mathbf{1 6 6 - \mathbf { 1 7 7 }} & \mathbf{1 5 4 - \mathbf { 2 1 0 }} & \mathbf{1 8 5 - \mathbf { 1 8 5 }} & \end{array}$

\section{References:}

1 - Carocci Buzi C. In: Annali Istituto Sperimentale per l'Olivicoltura e l'Oleificio, Ed. Bracco Imperia (1965), pp. 1-31. 2 - Muzzalupo I., Lombardo N., Salimonti A., et al. Adv. Hort. Sci. (2008), 22(2): pp. 142-148. 


\section{"Fecciaro"}

(synonymy: Pallottona, Raggio bastardo, etc.)

Areal distribution or origin area: Umbria

Flesh/pit weight ratio: medium $(5,62 \pm 0,63)$

Oil content (\%): low $(31,95 \pm 0,08)$

Purpose: oil

\section{Morphological characters}

Tree characters

Vigour: medium-strong

Growth habit: spreading-erect

Canopy-density: medium

\section{Leaf characters}

Blade length (cm): medium $(6,18 \pm 0,45)$

Blade width (cm): broad $(\mathbf{1}, 62 \pm 0,16)$

Shape (length/width): elliptic

\section{Inflorescence characters}

Inflorescence length (cm): long $(5,04 \pm 1,72)$

Number of flowers: high $(32,78 \pm 1,55)$

\section{Fruit characters}

Fresh weight of 100 fruits (g): high $(\mathbf{4 , 1 9} \pm 0,59)$

Shape (length/width): ovoid

Symmetry: symmetric

Position of maximum transverse diameter:

central

Apex: rounded

Base: truncate

Niplle: tenuous

Lenticels: many and small

\section{Pit characters}

Weight of 100 pits (g): high $(\mathbf{0 , 6 4} \pm \mathbf{0 , 1 2})$

Shape (length/width): ovoid

Mucron: obvious

Symmetry: symmetric

Position of maximum transverse diameter:

towards apex

Apex: rounded

Base: rounded

Surface: rugose

Number of grooves: medium
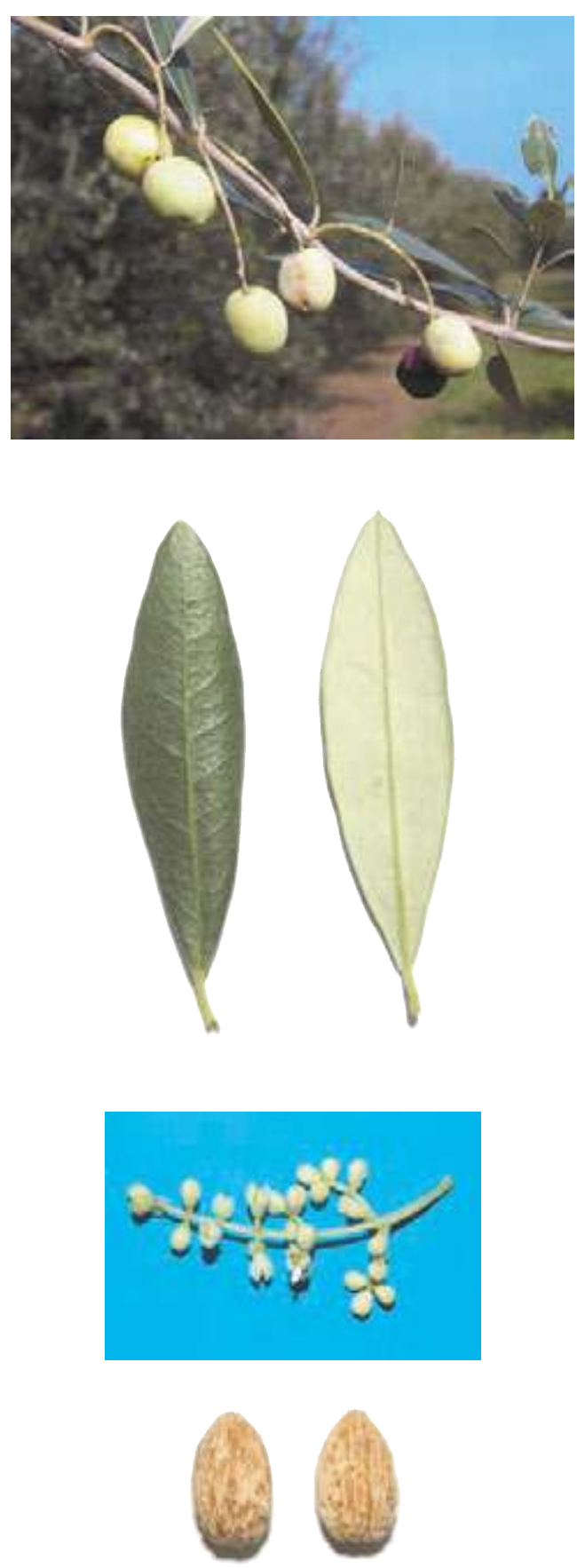


\section{Biochemical Characters}

\section{Fatty Acid Composition}

Table 1. Average values (express in $\% \pm$ standard deviations) of the fatty acids methyl esters and nutritional ratios obtained from single cultivar olive oils.

\begin{tabular}{|c|c|c|c|c|c|}
\hline Myristic acid & $0,01 \pm 0,00$ & Linoleic acid $(\omega 6)$ & $11,33 \pm 0,10$ & Lignoceric acid & $0,02 \pm 0,01$ \\
\hline Palmitic acid & $14,15 \pm 0,07$ & Linolenic acid $(\omega 3)$ & $0,85 \pm 0,03$ & & \\
\hline Palmitoleic acid & $0,97 \pm 0,00$ & Arachic acid & $0,21 \pm 0,03$ & Unsat./satured & $5,12 \pm 0,03$ \\
\hline earic & $1,94 \pm 0,04$ & Eicosenoic acid & $0,03 \pm 0,00$ & $\omega 6 / \omega 3$ & $13,33 \pm 0,55$ \\
\hline leic acid & $69,84 \pm 0,37$ & Behenic acid & $0,04 \pm 0,00$ & & \\
\hline
\end{tabular}

\section{Organoleptic oil values}

Sensory Analysis (Panel test)

Comment: fruity medium-high, with hints of almond and read sensations of grass and artichoke. Balanced taste sensation with a medium-high bitter and spicy. Medium-high fluidity.

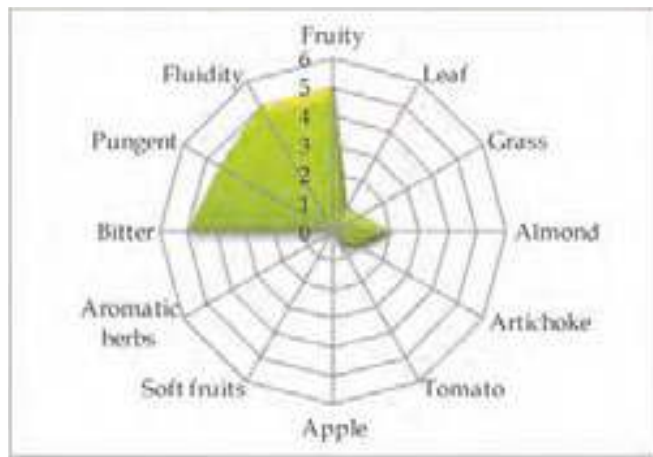

\section{Molecular Markers}

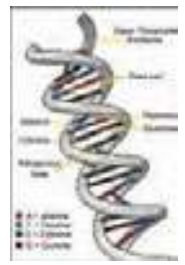

Table 2. Microsatellites (SSR) loci analyzed.

For each locus the allele size (expressed in base pairs) is reported.

\begin{tabular}{|c|c|c|c|c|c|}
\hline$D C A 09$ & $D C A 18$ & GAPU59 & GAPU71A & GUPA71B & GAPU103A \\
\hline $176-198$ & $179-181$ & $214-222$ & $214-221$ & $124-130$ & $159-159$ \\
\hline UDO01 & UDO03 & UDO12 & UDO28 & UDO39 & \\
\hline $140-140$ & $143-143$ & $166-177$ & $143-182$ & $205-205$ & \\
\hline
\end{tabular}

\section{References:}

1 - Pannelli G., Alfei B., D’ Ambrosio A., et al. In: Varietà di olivo in Umbria, Ed. Pliniana (2000), pp.79-84.

2 - Muzzalupo I., Lombardo N., Salimonti A., et al. Adv. Hort. Sci. (2008), 22(2): pp. 142-148. 


\section{“Fosco"}

(synonymy: Carbonella, Morellino, Morello, Nostrale, Oliva Tonda, etc.)

Areal distribution or origin area: Lazio

Flesh/pit weight ratio: medium $(5,00 \pm 0,15)$

Oil content (\%): medium $(48,31 \pm 1,23)$

Purpose: oil

\section{Morphological characters}

Tree characters

Vigour: medium-strong

Growth habit: spreading

Canopy-density: medium

\section{Leaf characters}

Blade length $(\mathrm{cm})$ : medium $(5,74 \pm 0,49)$

Blade width $(\mathrm{cm})$ : medium $(\mathbf{1}, 37 \pm \mathbf{0 , 1 1})$

Shape (length/width): elliptic-lanceolate

\section{Inflorescence characters}

Inflorescence length $(\mathrm{cm})$ : long $(3,86 \pm 0,71)$

Number of flowers: medium $(24,59 \pm 2,31)$

\section{Fruit characters}

Fresh weight of 100 fruits (g): low $(\mathbf{1}, \mathbf{6 2} \pm \mathbf{0 , 0 3})$

Shape (length/width): spherical

Symmetry: slightly asymmetric

Position of maximum transverse diameter: central

Apex: rounded

Base: truncate

Niplle: absent

Lenticels: many and small

\section{Pit characters}

Weight of 100 pits $(\mathrm{g})$ : low $(\mathbf{0 , 2 9} \pm \mathbf{0 , 0 1})$

Shape (length/width): ovoid

Mucron: absent

Symmetry: slightly asymmetric

Position of maximum transverse diameter:

central

Apex: rounded

Base: rounded

Surface: smooth

Number of grooves: medium
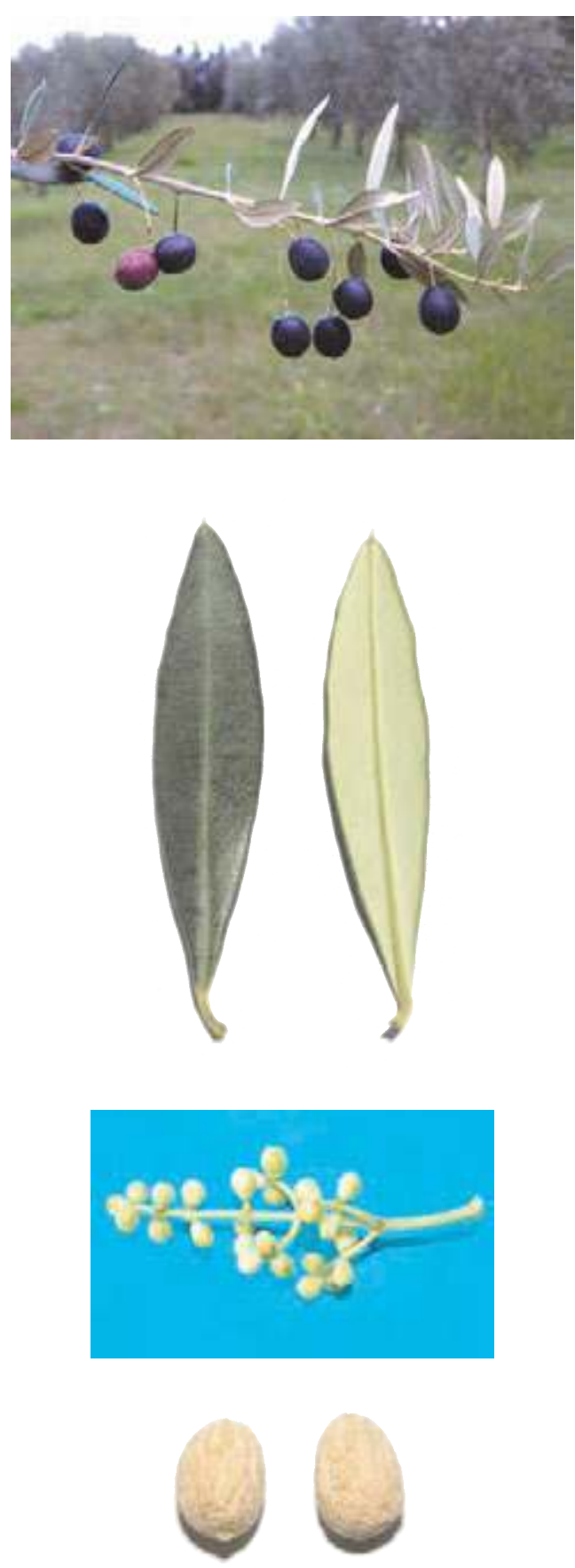


\section{Biochemical Characters}

\section{Fatty Acid Composition}

Table 1. Average values (express in $\% \pm$ standard deviations) of the fatty acids methyl esters and nutritional ratios obtained from single cultivar olive oils.

$\begin{array}{llllll}\text { Myristic acid } & \mathbf{0 , 0 1} \pm \mathbf{0 , 0 0} & \text { Linoleic acid }(\omega 6) & \mathbf{9 , 8 3} \pm \mathbf{1 , 7 0} & \text { Lignoceric acid } & \mathbf{0 , 0 2} \pm \mathbf{0 , 0 0} \\ \text { Palmitic acid } & \mathbf{1 4 , 9 8} \pm \mathbf{0 , 1 7} & \text { Linolenic acid }(\omega 3) \mathbf{0 , 8 3} \pm \mathbf{0 , 1 2} & & \\ \text { Palmitoleic acid } & \mathbf{1 , 4 0 \pm 0 , 1 0} & \text { Arachic acid } & \mathbf{0 , 2 2} \pm \mathbf{0 , 0 1} & \text { Unsat./satured } & \mathbf{4 , 9 0} \pm \mathbf{0 , 1 2} \\ \text { Stearic acid } & \mathbf{1 , 6 0} \pm \mathbf{0 , 1 3} & \text { Eicosenoic acid } & \mathbf{0 , 0 2} \pm \mathbf{0 , 0 1} & \omega 6 / \omega 3 & \mathbf{1 1 , 8 7} \pm \mathbf{0 , 3 7} \\ \text { Oleic acid } & \mathbf{6 9 , 8 2} \pm \mathbf{1 , 4 3} & \text { Behenic acid } & \mathbf{0 , 0 6} \pm \mathbf{0 , 0 0} & & \end{array}$

\section{Organoleptic oil values}

Sensory Analysis (Panel test)

Comment: fruity medium-high, with hints of almond and read sensations of grass and artichoke. Balanced taste sensation with a medium bitter and spicy. Medium-high fluidity.

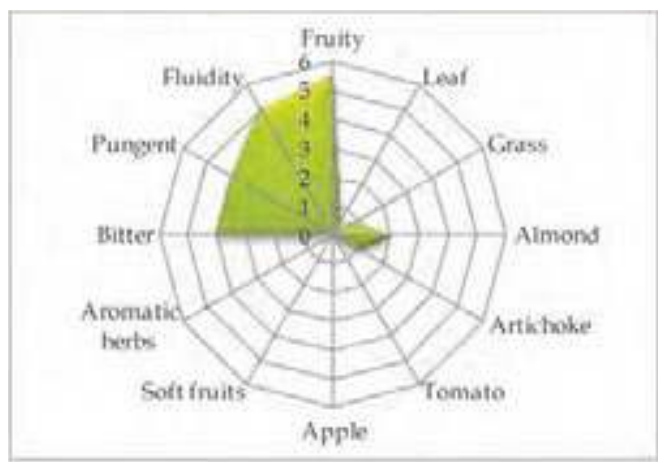

\section{Molecular Markers}

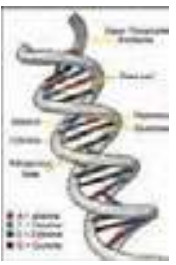

Table 2. Microsatellites (SSR) loci analyzed.

For each locus the allele size (expressed in base pairs) is reported.

$\begin{array}{cccccc}D C A 09 & \text { DCA18 } & \text { GAPU59 } & \text { GAPU71A } & \text { GUPA71B } & \text { GAPU103A } \\ \mathbf{1 7 2} \mathbf{- 1 9 4} & \mathbf{1 7 9 - \mathbf { 1 8 1 }} & \mathbf{2 0 8 - \mathbf { 2 1 2 }} & \mathbf{2 1 4} \mathbf{- 2 2 4} & \mathbf{1 2 4} \mathbf{- 1 4 4} & \mathbf{1 5 9} \mathbf{- \mathbf { 1 7 0 }} \\ \text { UDOO1 } & \text { UDOO3 } & \text { UDO12 } & \text { UDO28 } & \text { UDO39 } & \\ \mathbf{1 4 4 - \mathbf { 1 4 4 }} & \mathbf{1 5 0 - \mathbf { 1 5 0 }} & \mathbf{1 7 7 - \mathbf { 1 7 7 }} & \mathbf{1 8 2} \mathbf{- 2 1 0} & \mathbf{2 0 5 - \mathbf { 2 0 5 }} & \end{array}$

\section{References:}

1 - Consiglio Oleicolo Internazionale. In: Catalogo Mondiale delle Varietà di Olivo, COI (2003).

2 - Muzzalupo I., Stefanizzi F., Bucci C., et al. In Acta Italus Hortus, (2011), 1: 138 -140. 


\section{"Frangivento"}

(synonymy: Cipressino, Franjivento, Olivo Cipressino, Olivo di Pietrafitta, etc.)

Areal distribution or origin area: Puglia

Flesh/pit weight ratio: medium $(5,80 \pm 0,81)$

Oil content (\%): medium $(42,19 \pm 1,23)$

Purpose: oil

\section{Morphological characters}

Tree characters

Vigour: strong

Growth habit: erect

Canopy-density: dense

\section{Leaf characters}

Blade length $(\mathrm{cm})$ : medium $(6,32 \pm 0,56)$

Blade width $(\mathrm{cm})$ : medium $(\mathbf{1}, 22 \pm 0,13)$

Shape (length/width): elliptic - lanceolate

\section{Inflorescence characters}

Inflorescence length $(\mathrm{cm})$ : long $(4,36 \pm 0,76)$

Number of flowers: high $(32,90 \pm 5,01)$

\section{Fruit characters}

Fresh weight of 100 fruits $(\mathrm{g})$ : medium $(\mathbf{2 , 1 2} \pm \mathbf{0 , 4 2})$

Shape (length/width): spherical

Symmetry: symmetric

Position of maximum transverse diameter:

central

Apex: rounded

Base: truncate

Niplle: absent

Lenticels: many and small

\section{Pit characters}

Weight of 100 pits (g): medium $(0,34 \pm 0,05)$

Shape (length/width): ovoid

Mucron: obvious

Symmetry: symmetric

Position of maximum transverse diameter:

central

Apex: rounded

Base: rounded

Surface: smooth

Number of grooves: low
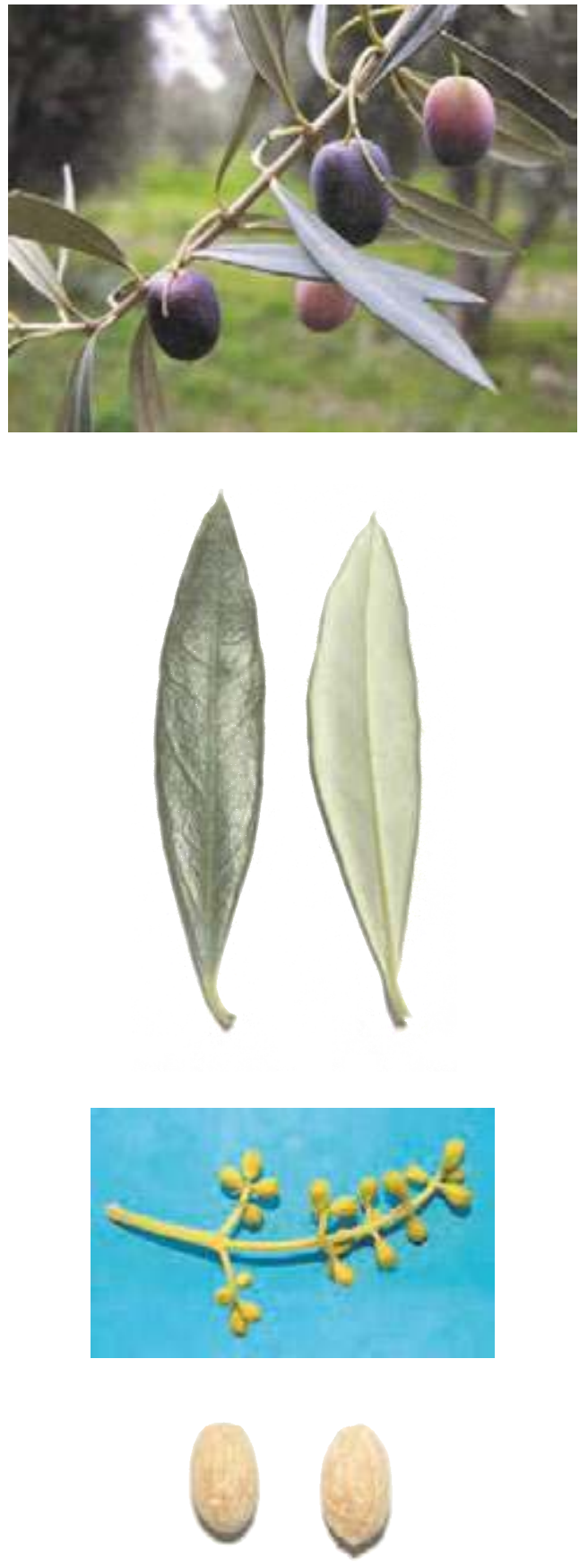


\section{Biochemical Characters}

\section{Fatty Acid Composition}

Table 1. Average values (express in $\% \pm$ standard deviations) of the fatty acids methyl esters and nutritional ratios obtained from single cultivar olive oils.

\begin{tabular}{|c|c|c|c|c|c|}
\hline Myristic acid & $0,01 \pm 0,00$ & Linoleic acid $(\omega 6)$ & $12,22 \pm 0,02$ & Lignoceric acid & $0,02 \pm 0,01$ \\
\hline Palmitic acid & $0,01 \pm 0,01$ & Linolenic acid $(\omega 3)$ & $0,31 \pm 0,04$ & & \\
\hline Palmitoleic acid & $15,00 \pm 0,15$ & Arachic acid & $0,27 \pm 0,02$ & Unsat./satured & $4,71 \pm 0,49$ \\
\hline earic acid & $2,06 \pm 0,04$ & Eicosenoic acid & $0,01 \pm 0,00$ & $\omega 6 / \omega 3$ & $39,42 \pm 0,08$ \\
\hline leic acid & $66,37 \pm 0,09$ & Behenic acid & $0,05 \pm 0,03$ & & \\
\hline
\end{tabular}

\section{Organoleptic oil values}

Sensory Analysis (Panel test)

Comment: fruity medium-high, with hints of almond and read sensations of grass and artichoke. Balanced taste sensation with a medium-high bitter and spicy. Medium-high fluidity.

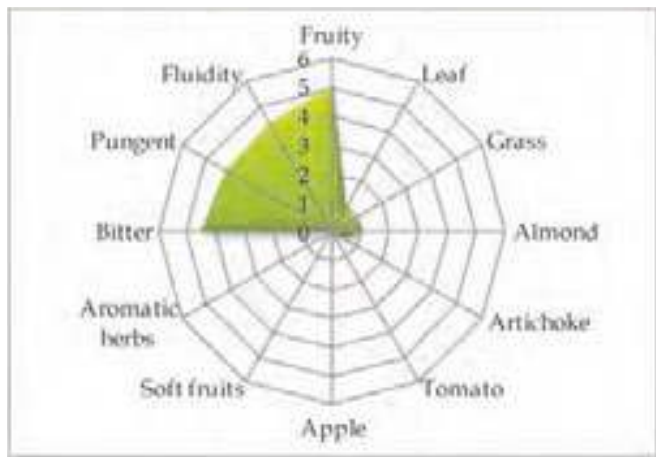

\section{Molecular Markers}

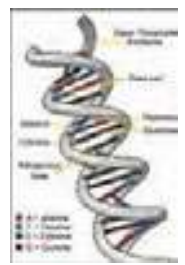

Table 2. Microsatellites (SSR) loci analyzed.

For each locus the allele size (expressed in base pairs) is reported.

\begin{tabular}{|c|c|c|c|c|c|}
\hline DCA09 & DCA18 & GAPU59 & GAPU71A & GUPA71B & GAPU103A \\
\hline $162-176$ & $179-185$ & $208-208$ & $214-214$ & $124-130$ & $159-170$ \\
\hline UDO01 & UDO03 & UDO12 & UDO28 & UDO39 & \\
\hline $144-144$ & $135-166$ & $177-177$ & $154-205$ & $205-232$ & \\
\hline
\end{tabular}

\section{References:}

1 - Lombardo N., Perri E., Muzzalupo I., et al. In: Contributo alla caratterizzazione del germoplasma olivicolo pugliese, Istituto Sperimentale per l'Olivicoltura (2004), pp. 53-56.

2 - Muzzalupo I., Stefanizzi F., Perri E. HortScience (2009), 44: pp. 582-588. 


\section{"Frangivento"}

(synonymy: Cipressino, Franjivento, Olivo Cipressino, Olivo di Pietrafitta, etc.)

Areal distribution or origin area: Puglia

Flesh/pit weight ratio: medium $(5,80 \pm 0,81)$

Oil content (\%): medium $(42,19 \pm 1,23)$

Purpose: oil

\section{Morphological characters}

Tree characters

Vigour: strong

Growth habit: erect

Canopy-density: dense

\section{Leaf characters}

Blade length $(\mathrm{cm})$ : medium $(6,32 \pm 0,56)$

Blade width $(\mathrm{cm})$ : medium $(\mathbf{1}, 22 \pm 0,13)$

Shape (length/width): elliptic - lanceolate

\section{Inflorescence characters}

Inflorescence length $(\mathrm{cm})$ : long $(4,36 \pm 0,76)$

Number of flowers: high $(32,90 \pm 5,01)$

\section{Fruit characters}

Fresh weight of 100 fruits $(\mathrm{g})$ : medium $(\mathbf{2 , 1 2} \pm \mathbf{0 , 4 2})$

Shape (length/width): spherical

Symmetry: symmetric

Position of maximum transverse diameter:

central

Apex: rounded

Base: truncate

Niplle: absent

Lenticels: many and small

\section{Pit characters}

Weight of 100 pits (g): medium $(0,34 \pm 0,05)$

Shape (length/width): ovoid

Mucron: obvious

Symmetry: symmetric

Position of maximum transverse diameter:

central

Apex: rounded

Base: rounded

Surface: smooth

Number of grooves: low
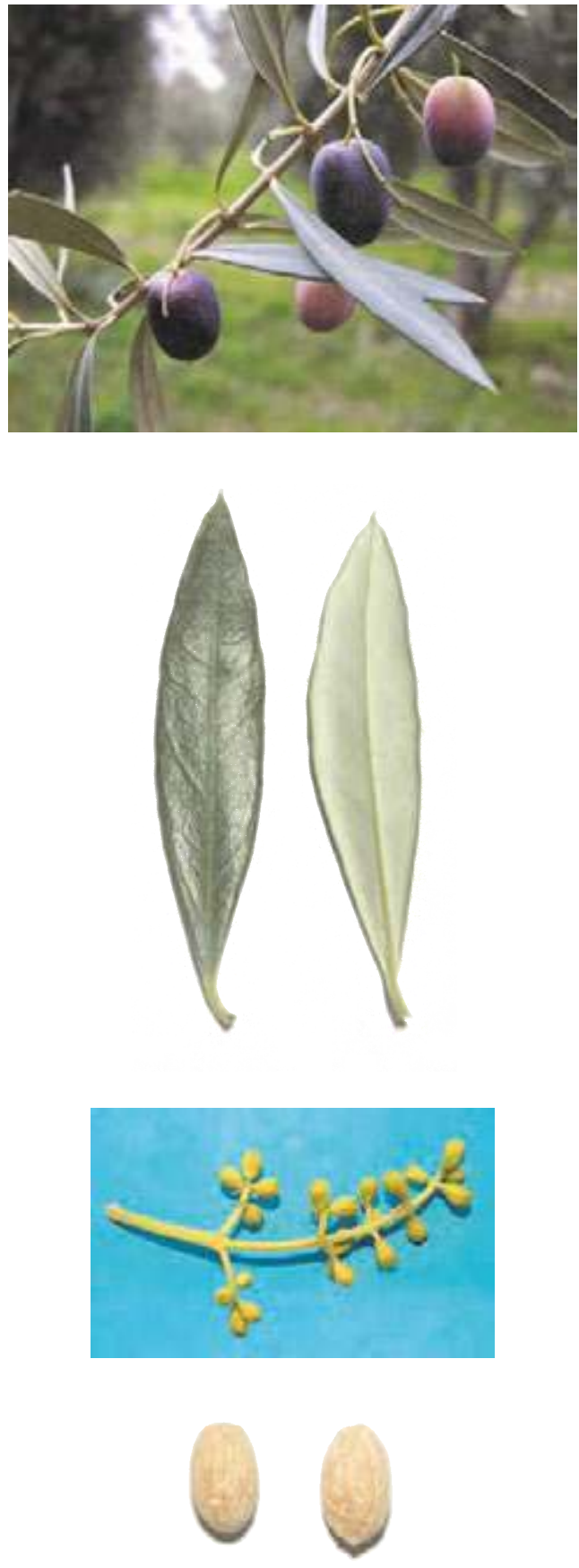


\section{Biochemical Characters}

\section{Fatty Acid Composition}

Table 1. Average values (express in $\% \pm$ standard deviations) of the fatty acids methyl esters and nutritional ratios obtained from single cultivar olive oils.

\begin{tabular}{|c|c|c|c|c|c|}
\hline Myristic acid & $0,01 \pm 0,00$ & Linoleic acid $(\omega 6)$ & $12,22 \pm 0,02$ & Lignoceric acid & $0,02 \pm 0,01$ \\
\hline Palmitic acid & $0,01 \pm 0,01$ & Linolenic acid ( $\omega 3)$ & $0,31 \pm 0,04$ & & \\
\hline Palmitoleic acid & $15,00 \pm 0,15$ & Arachic acid & $0,27 \pm 0,02$ & Unsat./satured & $4,71 \pm 0,49$ \\
\hline tearic acid & $2,06 \pm 0,04$ & Eicosenoic acid & $0,01 \pm 0,00$ & $\omega 6 / \omega 3$ & $39,42 \pm 0,08$ \\
\hline Oleic acid & $66,37 \pm 0,09$ & Behenic acid & $0,05 \pm 0,03$ & & \\
\hline
\end{tabular}

\section{Organoleptic oil values}

Sensory Analysis (Panel test)

Comment: fruity medium-high, with hints of almond and read sensations of grass and artichoke. Balanced taste sensation with a medium-high bitter and spicy. Medium-high fluidity.

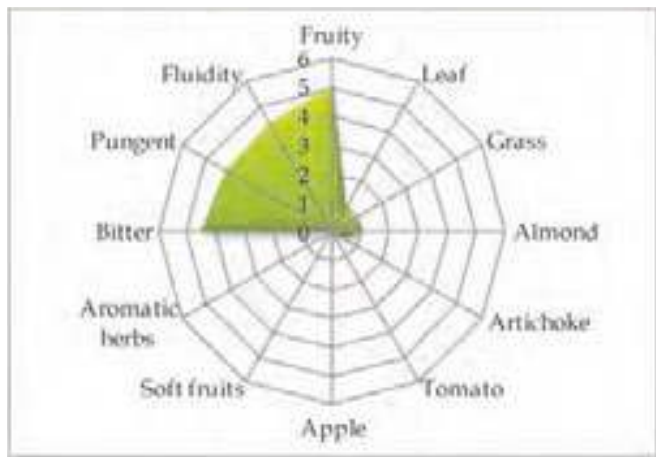

\section{Molecular Markers}

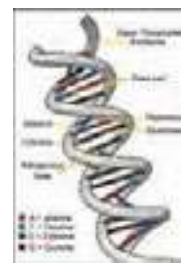

Table 2. Microsatellites (SSR) loci analyzed.

For each locus the allele size (expressed in base pairs) is reported.

\begin{tabular}{|c|c|c|c|c|c|}
\hline DCA09 & DCA18 & GAPU59 & GAPU71A & GUPA71B & GAPU103A \\
\hline $162-176$ & $179-185$ & $208-208$ & $214-214$ & $124-130$ & $159-170$ \\
\hline UDO01 & UDO03 & UDO12 & UDO28 & UDO39 & \\
\hline $144-144$ & $135-166$ & $177-177$ & $154-205$ & $205-232$ & \\
\hline
\end{tabular}

\section{References:}

1 - Lombardo N., Perri E., Muzzalupo I., et al. In: Contributo alla caratterizzazione del germoplasma olivicolo pugliese, Istituto Sperimentale per l'Olivicoltura (2004), pp. 53-56.

2 - Muzzalupo I., Stefanizzi F., Perri E. HortScience (2009), 44: pp. 582-588. 


\section{"Frantoio"}

(synonymy: Comune, Correggiolo, Gentile, Larcianese, Razzo, etc.)

Areal distribution or origin area: Toscana

Flesh/pit weight ratio: medium $(5,92 \pm 1,12)$

Oil content (\%): medium $(44,87 \pm 2,89)$

Purpose: oil

\section{Morphological characters}

Tree characters

Vigour: medium-strong

Growth habit: spreading-erect

Canopy-density: medium-dense

\section{Leaf characters}

Blade length $(\mathrm{cm})$ : medium $(6,15 \pm 0,54)$

Blade width (cm): broad $(\mathbf{1}, 58 \pm \mathbf{0 , 2 2})$

Shape (length/width): elliptic

\section{Inflorescence characters}

Inflorescence length $(\mathrm{cm})$ : long $(3,67 \pm 0,70)$

Number of flowers: medium $(19,16 \pm 2,80)$

\section{Fruit characters}

Fresh weight of 100 fruits $(\mathrm{g})$ : medium $(2,56 \pm 0,40)$

Shape (length/width): ovoid

Symmetry: slightly asymmetric

Position of maximum transverse diameter:

central

Apex: rounded

Base: rounded

Niplle: absent

Lenticels: many and small

\section{Pit characters}

Weight of 100 pits (g): medium $(0,42 \pm 0,05)$

Shape (length/width): elliptic

Mucron: obvious

Symmetry: symmetric

Position of maximum transverse diameter:

central

Apex: pointed

Base: pointed

Surface: rugose

Number of grooves: medium
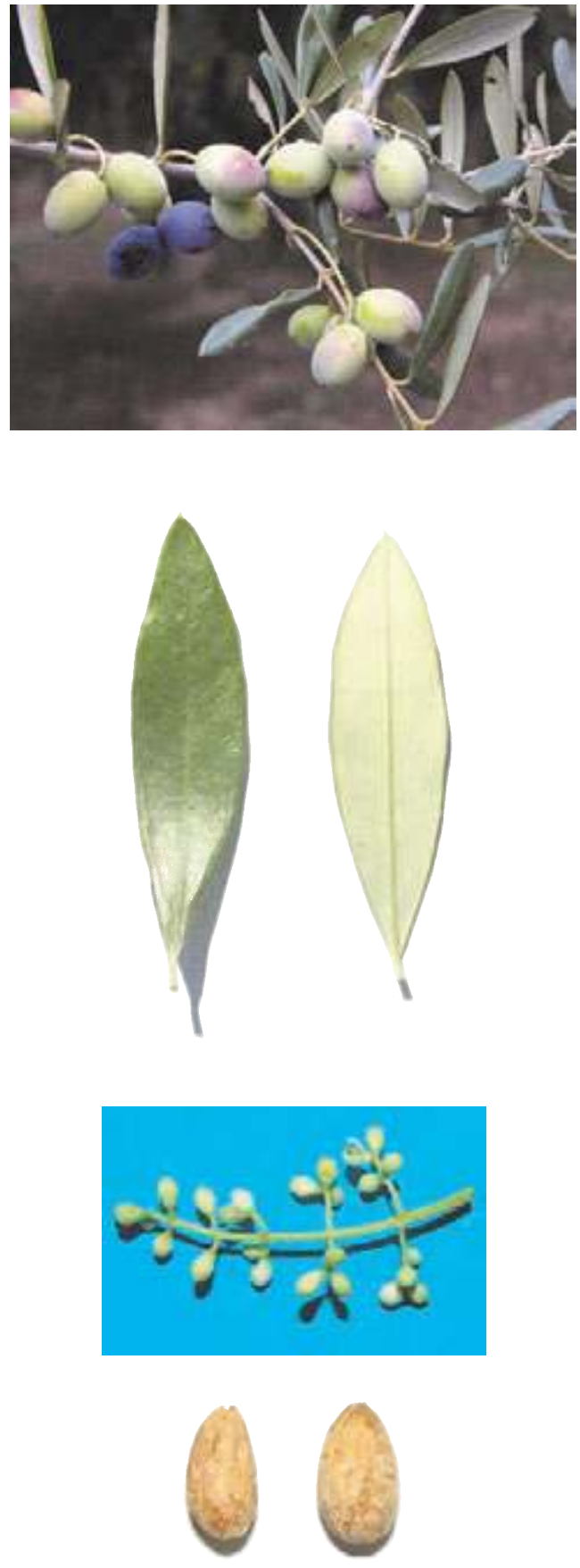


\section{Biochemical Characters}

\section{Fatty Acid Composition}

Table 1. Average values (express in $\% \pm$ standard deviations) of the fatty acids methyl esters and nutritional ratios obtained from single cultivar olive oils.

$\begin{array}{llllll}\text { Myristic acid } & \mathbf{0 , 0 1} \pm \mathbf{0 , 0 1} & \text { Linoleic acid }(\omega 6) & \mathbf{6 , 7 0} \pm \mathbf{1 , 5 8} & \text { Lignoceric acid } & \mathbf{0 , 0 3} \pm \mathbf{0 , 0 2} \\ \text { Palmitic acid } & \mathbf{1 3 , 3 9 \pm \mathbf { 1 , 6 7 }} & \text { Linolenic acid }(\omega 3) & \mathbf{0 , 7 9} \pm \mathbf{0 , 0 5} & & \\ \text { Palmitoleic acid } & \mathbf{1 , 0 3} \pm \mathbf{0 , 3 0} & \text { Arachic acid } & \mathbf{0 , 3 3} \pm \mathbf{0 , 1 2} & \text { Unsat./satured } & \mathbf{5 , 4 2} \pm \mathbf{0 , 5 8} \\ \text { Stearic acid } & \mathbf{1 , 9 0 \pm 0 , 1 1} & \text { Eicosenoic acid } & \mathbf{0 , 1 1} \pm \mathbf{0 , 1 7} & \omega 6 / \omega 3 & \mathbf{8 , 4 9} \pm \mathbf{1 , 9 6} \\ \text { Oleic acid } & \mathbf{7 4 , 8 3 \pm \mathbf { 2 , 2 8 }} & \text { Behenic acid } & \mathbf{0 , 0 8} \pm \mathbf{0 , 0 2} & & \end{array}$

\section{Organoleptic oil values}

Sensory Analysis (Panel test)

Comment: fruity medium-high, with hints of almond and read sensations of grass and artichoke. Balanced taste sensation with a medium-high bitter and spicy. Medium-high fluidity.

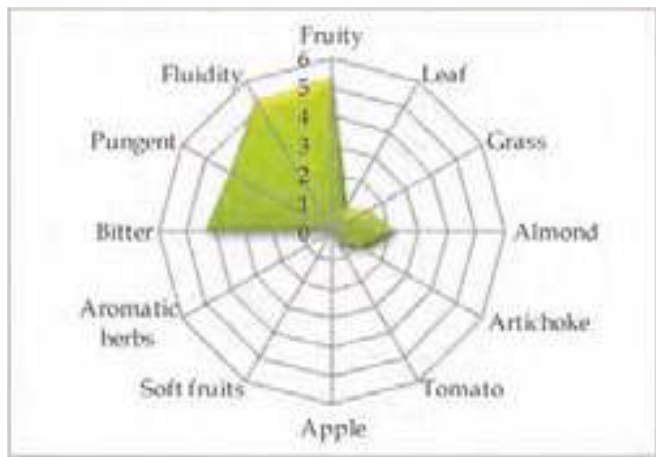

\section{Molecular Markers}

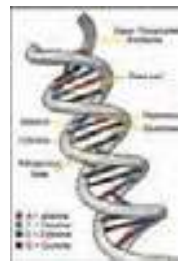

Table 2. Microsatellites (SSR) loci analyzed.

For each locus the allele size (expressed in base pairs) is reported.

\begin{tabular}{|c|c|c|c|c|c|}
\hline DCA09 & DCA18 & GAPU59 & GAPU71A & GUPA71B & GAPU103A \\
\hline $172-194$ & $179-181$ & $208-212$ & $214-224$ & $124-144$ & $159-179$ \\
\hline UDO01 & UDO03 & UDO12 & UDO28 & UDO39 & \\
\hline $144-144$ & $143-143$ & $177-182$ & $182-205$ & $205-205$ & \\
\hline
\end{tabular}

\section{References:}

1 - Pannelli G., Alfei B., D’ Ambrosio A., et al. In: Varietà di olivo in Umbria, Ed. Pliniana (2000), pp. 19-24.

2 - Various authors. In: Catalogo Nazionale delle Varietà di Olivo . University of Bari (in press), ISBN 978-88-8879397-9. 


\section{“Gaggiolo"}

(synonymy: Cornella, Corno, Crignola, Giaggiola, Lunga, etc.)

Areal distribution or origin area: Lombardia

Flesh/pit weight ratio: low $(4,32 \pm 0,08)$

Oil content (\%): medium $(44,50 \pm 0,43)$

Purpose: oil

\section{Morphological characters}

Tree characters

Vigour: strong

Growth habit: spreading-erect

Canopy-density: dense

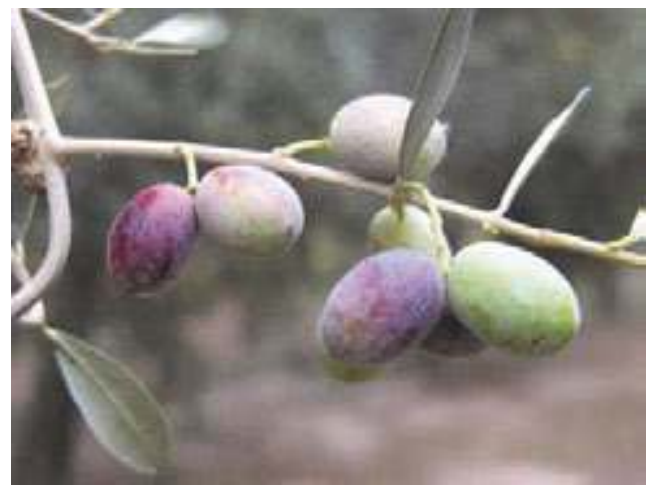

\section{Leaf characters}

Blade length $(\mathrm{cm})$ : medium $(6,05 \pm 0,47)$

Blade width (cm): broad $(\mathbf{1}, \mathbf{5 2} \pm \mathbf{0 , 2 0})$

Shape (length/width): elliptic

\section{Inflorescence characters}

Inflorescence length $(\mathrm{cm})$ : medium $(3,40 \pm 0,78)$

Number of flowers: medium $(\mathbf{1 8 , 5 1} \pm \mathbf{1 , 8 2})$

\section{Fruit characters}

Fresh weight of 100 fruits $(\mathrm{g})$ : medium $(2,43 \pm 0,17)$

Shape (length/width): ovoid

Symmetry: slightly asymmetric

Position of maximum transverse diameter:

central

Apex: rounded

Base: rounded

Niplle: tenuous

Lenticels: many and large

\section{Pit characters}

Weight of 100 pits (g): high $(\mathbf{0 , 4 8} \pm \mathbf{0 , 0 6})$

Shape (length/width): elliptic

Mucron: obvious

Symmetry: symmetric

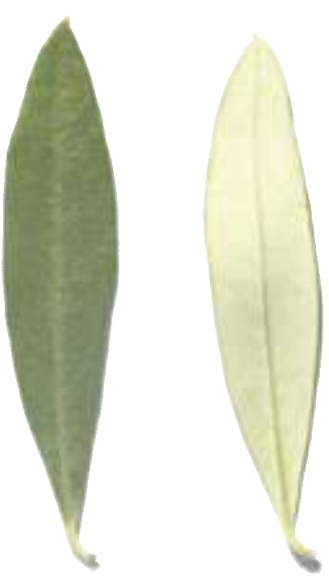

Position of maximum transverse diameter:

towards apex

Apex: rounded

Base: pointed

Surface: rugose

Number of grooves: medium
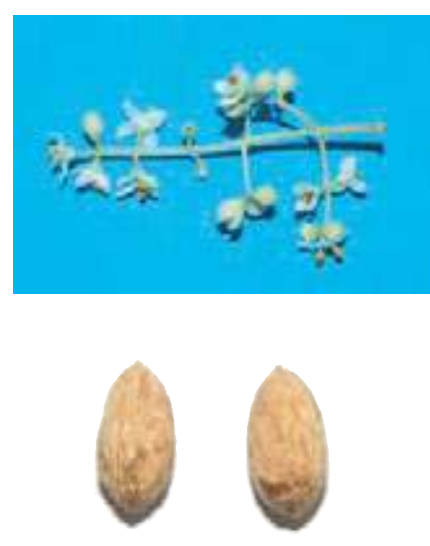


\section{Biochemical Characters}

\section{Fatty Acid Composition}

Table 1. Average values (express in $\% \pm$ standard deviations) of the fatty acids methyl esters and nutritional ratios obtained from single cultivar olive oils.

$\begin{array}{llllll}\text { Myristic acid } & \mathbf{0 , 0 1} \pm \mathbf{0 , 0 0} & \text { Linoleic acid }(\omega 6) & \mathbf{6 , 9 3} \pm \mathbf{0 , 9 2} & \text { Lignoceric acid } & \mathbf{0 , 0 3} \pm \mathbf{0 , 0 1} \\ \text { Palmitic acid } & \mathbf{1 2 , 0 3} \pm \mathbf{0 , 5 3} & \text { Linolenic acid }(\omega 3) \mathbf{0 , 6 3} \pm \mathbf{0 , 0 9} & & \\ \text { Palmitoleic acid } & \mathbf{1 , 2 2} \pm \mathbf{0 , 0 2} & \text { Arachic acid } & \mathbf{0 , 2 8} \pm \mathbf{0 , 0 5} & \text { Unsat./satured } & \mathbf{5 , 9 0 \pm 0 , 4 2} \\ \text { Stearic acid } & \mathbf{2 , 1 5} \pm \mathbf{0 , 2 4} & \text { Eicosenoic acid } & \mathbf{0 , 0 1} \pm \mathbf{0 , 0 0} & \omega 6 / \omega 3 & \mathbf{1 1 , 0 8} \pm \mathbf{0 , 1 4} \\ \text { Oleic acid } & \mathbf{7 5 , 8 0 \pm \mathbf { 0 , 2 3 }} & \text { Behenic acid } & \mathbf{0 , 0 7} \pm \mathbf{0 , 0 2} & & \end{array}$

\section{Organoleptic oil values}

Sensory Analysis (Panel test)

Comment: fruity medium-high, with hints of almond and read sensations of grass and artichoke. Balanced taste sensation with a medium-high bitter and spicy. Medium-high fluidity.

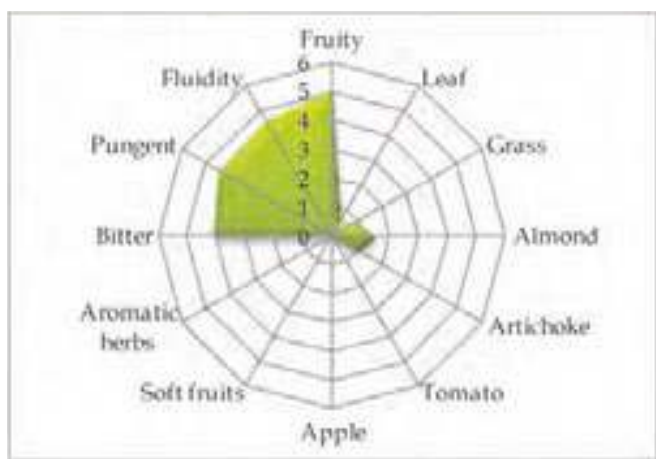

\section{Molecular Markers}

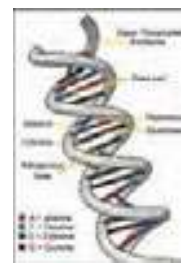

Table 2. Microsatellites (SSR) loci analyzed.

For each locus the allele size (expressed in base pairs) is reported.

$\begin{array}{cccccc}D C A 09 & \text { DCA18 } & \text { GAPU59 } & \text { GAPU71A } & \text { GUPA71B } & \text { GAPU103A } \\ \mathbf{1 8 2} \mathbf{- 2 0 6} & \mathbf{1 7 9 - \mathbf { 1 7 9 }} & \mathbf{2 1 4 - \mathbf { 2 2 2 }} & \mathbf{2 1 4 - \mathbf { 2 2 4 }} & \mathbf{1 2 6 - \mathbf { 1 4 4 }} & \mathbf{1 5 9 - \mathbf { 1 7 0 }} \\ \text { UDOO1 } & \text { UDO03 } & \text { UDO12 } & \text { UDO28 } & \text { UDO39 } & \\ \mathbf{1 5 0 - \mathbf { 1 5 0 }} & \mathbf{1 4 3 - \mathbf { 1 4 3 }} & \mathbf{1 7 7 - \mathbf { 1 8 2 }} & \mathbf{1 8 2} \mathbf{- 2 0 5} & \mathbf{1 8 5 - \mathbf { 1 8 5 }} & \end{array}$

\section{References:}

1 - Carocci Buzi C. In: Annali Istituto Sperimentale per l'Olivicoltura e l'Oleificio, Ed. Bracco (1965), pp. 1-31.

2 - Muzzalupo I., Lombardo N., Salimonti A., et al. Adv. Hort. Sci. (2008), 22(2): pp. 142-148. 


\section{"Gentile di Chieti "}

(synonymy: Gentile, Nostrana, Olivastro.)

Areal distribution or origin area: Abruzzo

Flesh/pit weight ratio: medium $(5,30 \pm 0,25)$

Oil content (\%): medium $(41,98 \pm 2,15)$

Purpose: oil

\section{Morphological characters}

Tree characters

Vigour: medium-weak

Growth habit: erect-spreading

Canopy-density: medium

\section{Leaf characters}

Blade length (cm): medium $(5,53 \pm 0,57)$

Blade width $(\mathrm{cm})$ : medium $(1,48 \pm 0,23)$

Shape (length/width): elliptic

\section{Inflorescence characters}

Inflorescence length (cm): long $(3,93 \pm 2,88)$

Number of flowers: medium $(19,16 \pm 2,07)$

\section{Fruit characters}

Fresh weight of 100 fruits $(\mathrm{g})$ : medium $(2,61 \pm 0,44)$

Shape (length/width): spherical

Symmetry: symmetric

Position of maximum transverse diameter:

central

Apex: rounded

Base: rounded

Niplle: absent

Lenticels: few and small

\section{Pit characters}

Weight of 100 pits (g): high $(\mathbf{0 , 9} \pm \mathbf{0 , 0 0})$

Shape (length/width): ovoid

Mucron: absent

Symmetry: symmetric

Position of maximum transverse diameter:

towards apex

Apex: rounded

Base: pointed

Surface: rugose

Number of grooves: high
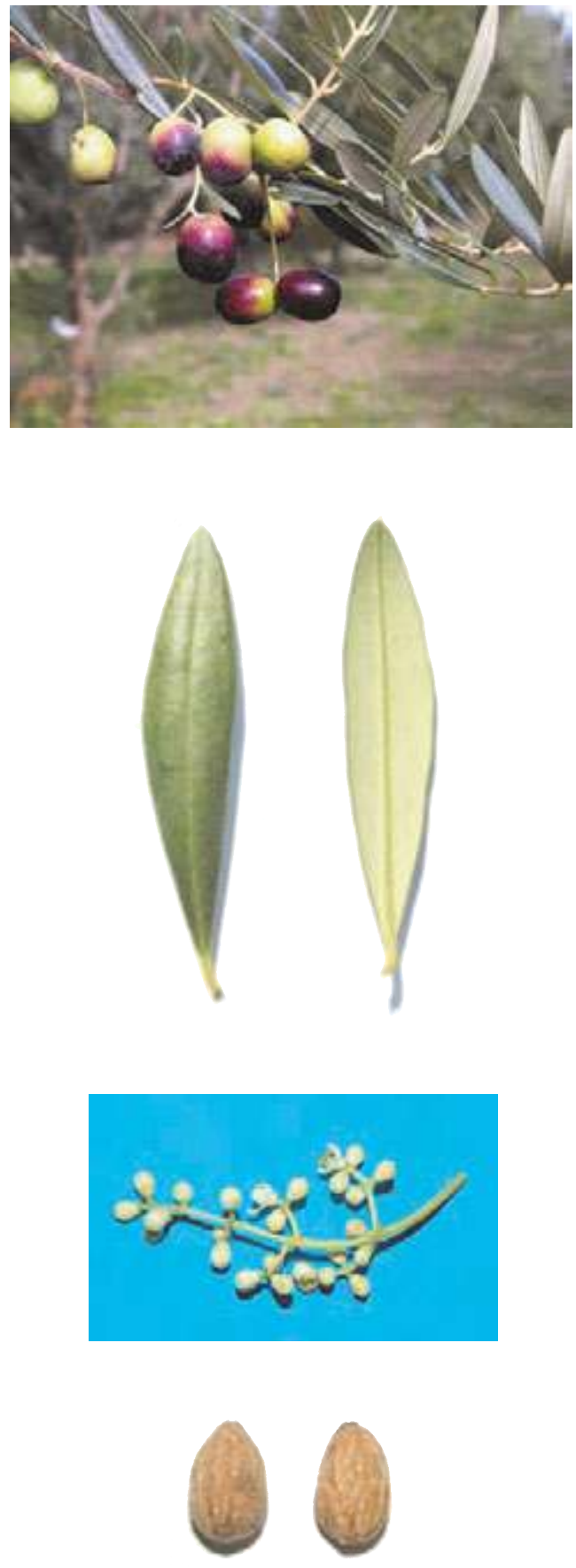


\section{Biochemical Characters}

\section{Fatty Acid Composition}

Table 1. Average values (express in $\% \pm$ standard deviations) of the fatty acids methyl esters and nutritional ratios obtained from single cultivar olive oils.

$\begin{array}{llllll}\text { Myristic acid } & \mathbf{0 , 0 1} \pm \mathbf{0 , 0 0} & \text { Linoleic acid }(\omega 6) & \mathbf{1 6 , 0 1} \pm \mathbf{0 , 1 1} & \text { Lignoceric acid } & \mathbf{0 , 0 3} \pm \mathbf{0 , 0 1} \\ \text { Palmitic acid } & \mathbf{1 5 , 6 1 \pm 0 , 4 9} & \text { Linolenic acid }(\omega 3) \mathbf{0 , 8 3} \pm \mathbf{0 , 0 4} & & \\ \text { Palmitoleic acid } & \mathbf{1 , 4 2} \pm \mathbf{0 , 0 4} & \text { Arachic acid } & \mathbf{0 , 3 2} \pm \mathbf{0 , 0 3} & \text { Unsat./satured } & \mathbf{4 , 4 7} \pm \mathbf{0 , 1 6} \\ \text { Stearic acid } & \mathbf{2 , 2 1} \pm \mathbf{0 , 0 4} & \text { Eicosenoic acid } & \mathbf{0 , 0 2} \pm 0,00 & \omega 6 / \omega 3 & \mathbf{1 9 , 3 5} \pm \mathbf{1 , 1 7} \\ \text { Oleic acid } & \mathbf{6 2 , 2 0 \pm 0 , 5 7} & \text { Behenic acid } & \mathbf{0 , 0 7} \pm \mathbf{0 , 0 1} & & \end{array}$

\section{Organoleptic oil values}

Sensory Analysis (Panel test)

Comment: fruity medium - light, with hints of almond and read sensations of grass and artichoke. Balanced taste sensation with a medium - light bitter and spicy. Medium fluidity.

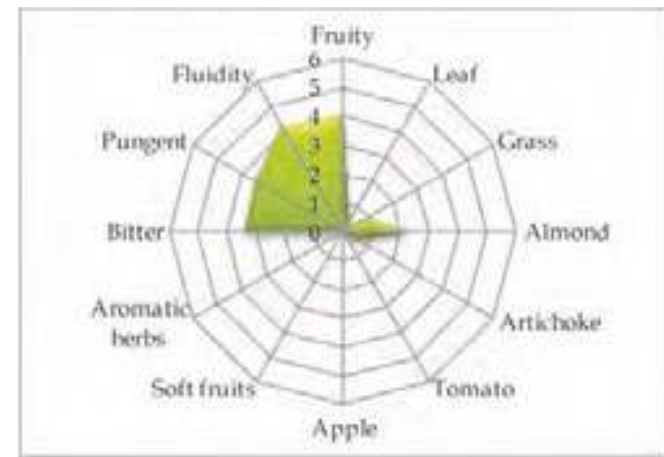

\section{Molecular Markers}

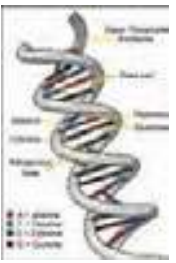

Table 2. Microsatellites (SSR) loci analyzed.

For each locus the allele size (expressed in base pairs) is reported.

$\begin{array}{cccccc}D C A 09 & \text { DCA18 } & \text { GAPU59 } & \text { GAPU71A } & \text { GUPA71B } & \text { GAPU103A } \\ \mathbf{1 7 2} \mathbf{- 1 7 2} & \mathbf{1 6 9 - \mathbf { 1 7 9 }} & \mathbf{2 0 8 - \mathbf { 2 1 2 }} & \mathbf{2 1 0 - \mathbf { 2 1 4 }} & \mathbf{1 2 4 - \mathbf { 1 3 0 }} & \mathbf{1 7 0 - \mathbf { 1 7 0 }} \\ \text { UDOO1 } & \text { UDOO3 } & \text { UDO12 } & \text { UDO28 } & \text { UDO39 } & \\ \mathbf{1 4 0 - \mathbf { 1 4 0 }} & \mathbf{1 4 3 - \mathbf { 1 4 3 }} & \mathbf{1 6 6 - \mathbf { 1 9 3 }} & \mathbf{1 5 4 - \mathbf { 2 0 5 }} & \mathbf{2 1 3 - \mathbf { 2 1 3 }} & \end{array}$

\section{References:}

1 - Pietrangeli E., Russo A., In: Olivi D’Abruzzo, Grafiche di Prinzio (1997), pp. 42-43.

2 - Muzzalupo I., Lombardo N., Salimonti A., et al. Adv. Hort. Sci. (2008), 22(2): pp. 142-148. 


\section{"Gentile di Larino"}

(synonymy: Gentile di Trivento, Olivacchione, etc.)

Areal distribution or origin area: Molise

Flesh/pit weight ratio: medium $(\mathbf{6 , 4 5} \pm \mathbf{0 , 3 6})$

Oil content (\%): medium $(44,75 \pm 0,77)$

Purpose: oil

\section{Morphological characters}

Tree characters

Vigour: medium

Growth habit: spreading

Canopy-density: medium-sparse

\section{Leaf characters}

Blade length $(\mathrm{cm})$ : medium $(5,49 \pm 0,56)$

Blade width $(\mathrm{cm})$ : medium $(1,17 \pm 0,16)$

Shape (length/width): elliptic - lanceolate

\section{Inflorescence characters}

Inflorescence length $(\mathrm{cm})$ : medium $(3,21 \pm 2,49)$

Number of flowers: low $(\mathbf{1 7}, \mathbf{1 6} \pm 0,73)$

\section{Fruit characters}

Fresh weight of 100 fruits $(\mathrm{g})$ : medium $(2,31 \pm 0,10)$

Shape (length/width): ovoid

Symmetry: asymmetric

Position of maximum transverse diameter:

central

Apex: rounded

Base: truncate

Niplle: tenuous

Lenticels: many and small

\section{Pit characters}

Weight of 100 pits (g): medium $(0,31 \pm 0,03$ )

Shape (length/width): ovoid

Mucron: obvious

Symmetry: slightly asymmetric

Position of maximum transverse diameter:

towards apex

Apex: rounded

Base: pointed

Surface: rugose

Number of grooves: low
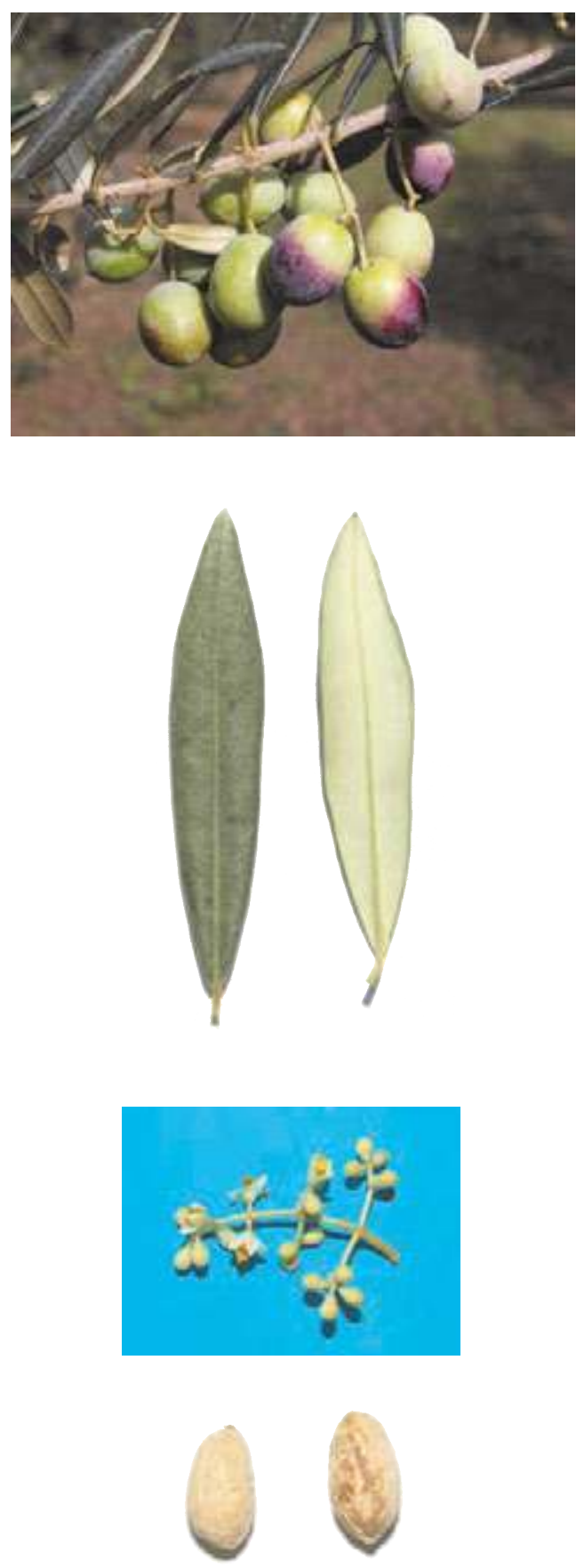


\section{Biochemical Characters}

\section{Fatty Acid Composition}

Table 1. Average values (express in $\% \pm$ standard deviations) of the fatty acids methyl esters and nutritional ratios obtained from single cultivar olive oils.

$\begin{array}{llllll}\text { Myristic acid } & \mathbf{0 , 0 1} \pm \mathbf{0 , 0 0} & \text { Linoleic acid }(\omega 6) & \mathbf{9 , 4 6} \pm \mathbf{2 , 1 4} & \text { Lignoceric acid } & \mathbf{0 , 0 3} \pm \mathbf{0 , 0 0} \\ \text { Palmitic acid } & \mathbf{1 2 , 3 0 \pm \mathbf { 1 , 4 9 }} & \text { Linolenic acid }(\omega 3) \mathbf{1 , 0 3} \pm \mathbf{0 , 3 6} & & \\ \text { Palmitoleic acid } & \mathbf{0 , 6 3} \pm \mathbf{0 , 4 1} & \text { Arachic acid } & \mathbf{0 , 3 3} \pm \mathbf{0 , 0 9} & \text { Unsat./satured } & \mathbf{5 , 7 7} \pm \mathbf{0 , 9 3} \\ \text { Stearic acid } & \mathbf{2 , 3 5 \pm 0 , 5 3} & \text { Eicosenoic acid } & \mathbf{0 , 0 2} \pm \mathbf{0 , 0 0} & \omega 6 / \omega 3 & \mathbf{9 , 4 4} \pm \mathbf{1 , 2 5} \\ \text { Oleic acid } & \mathbf{7 3 , 5 9 \pm 0 , 0 7} & \text { Behenic acid } & \mathbf{0 , 0 8} \pm \mathbf{0 , 0 1} & & \end{array}$

\section{Organoleptic oil values}

Sensory Analysis (Panel test)

Comment: fruity medium-high, with hints of almond and read sensations of grass and artichoke. Balanced taste sensation with a medium-high bitter and spicy. Medium-high fluidity.

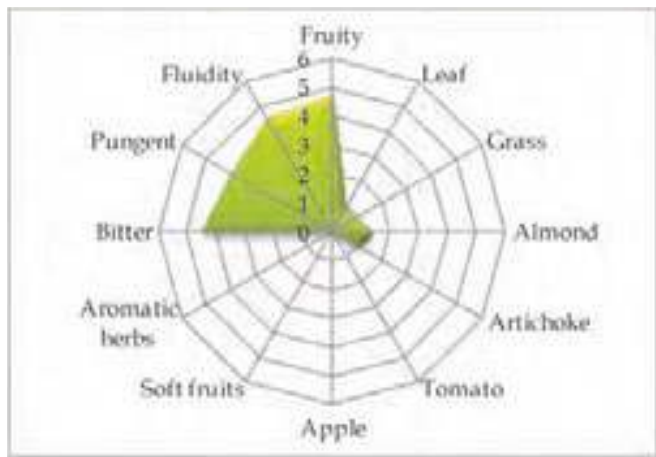

\section{Molecular Markers}

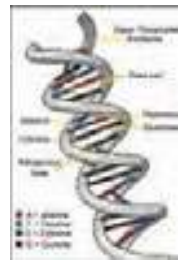

Table 2. Microsatellites (SSR) loci analyzed.

For each locus the allele size (expressed in base pairs) is reported.

$\begin{array}{cccccc}D C A 09 & \text { DCA18 } & \text { GAPU59 } & \text { GAPU71A } & \text { GUPA71B } & \text { GAPU103A } \\ \mathbf{1 7 2} \mathbf{- 1 9 4} & \mathbf{1 6 9 - \mathbf { 1 7 9 }} & \mathbf{2 0 8 - \mathbf { 2 1 2 }} & \mathbf{2 1 4} \mathbf{- 2 1 4} & \mathbf{1 2 4} \mathbf{- 1 3 0} & \mathbf{1 5 0 - \mathbf { 1 8 4 }} \\ \text { UDOO1 } & \text { UDOO3 } & \text { UDO12 } & \text { UDO28 } & \text { UDO39 } & \\ \mathbf{1 4 0 - \mathbf { 1 4 0 }} & \mathbf{1 5 0 - \mathbf { 1 5 0 }} & \mathbf{1 6 6 - \mathbf { 1 9 3 }} & \mathbf{1 8 2} \mathbf{- 1 8 2} & \mathbf{2 1 3 - \mathbf { 2 1 3 }} & \end{array}$

\section{References:}

1 - Various authors. In: Catalogo Nazionale delle Varietà di Olivo, University of Bari (in press), ISBN 978-88-8879397-9.

2 - Muzzalupo I., Lombardo N., Salimonti A., et al. Adv. Hort. Sci. (2008), 22(2): pp. 142-148. 


\section{"Gentile nera di Colletorto"}

(synonymy: Colletortese, Gentile di Colletorto, Noccioluta, Oliva nera di Colletorto, etc.)

Areal distribution or origin area: Molise

Flesh/pit weight ratio: low $(4,35 \pm 0,11)$

Oil content (\%): medium $(43,56 \pm 1,26)$

Purpose: oil

\section{Morphological characters}

Tree characters

Vigour: medium-weak

Growth habit: spreading

Canopy-density: medium

\section{Leaf characters}

Blade length $(\mathrm{cm})$ : medium $(5,35 \pm 0,45)$

Blade width $(\mathrm{cm})$ : medium $(\mathbf{1 , 1 0} \pm \mathbf{0 , 1 2})$

Shape (length/width): elliptic-lanceolate

\section{Inflorescence characters}

Inflorescence length $(\mathrm{cm})$ : short $(2,34 \pm 0,75)$

Number of flowers: low $(\mathbf{1 3 , 0 8} \pm 0,00)$

\section{Fruit characters}

Fresh weight of 100 fruits $(\mathrm{g})$ : medium $(2,66 \pm 0,08)$

Shape (length/width): ovoid

Symmetry: slightly asymmetric

Position of maximum transverse diameter:

central

Apex: rounded

Base: truncate

Niplle: tenuous

Lenticels: few and small

\section{Pit characters}

Weight of 100 pits (g): high $(\mathbf{0 , 5 0} \pm \mathbf{0 , 0 1})$

Shape (length/width): ovoid

Mucron: obvious

Symmetry: slightly asymmetric

Position of maximum transverse diameter:

towards apex

Apex: rounded

Base: rounded

Surface: smooth

Number of grooves: medium
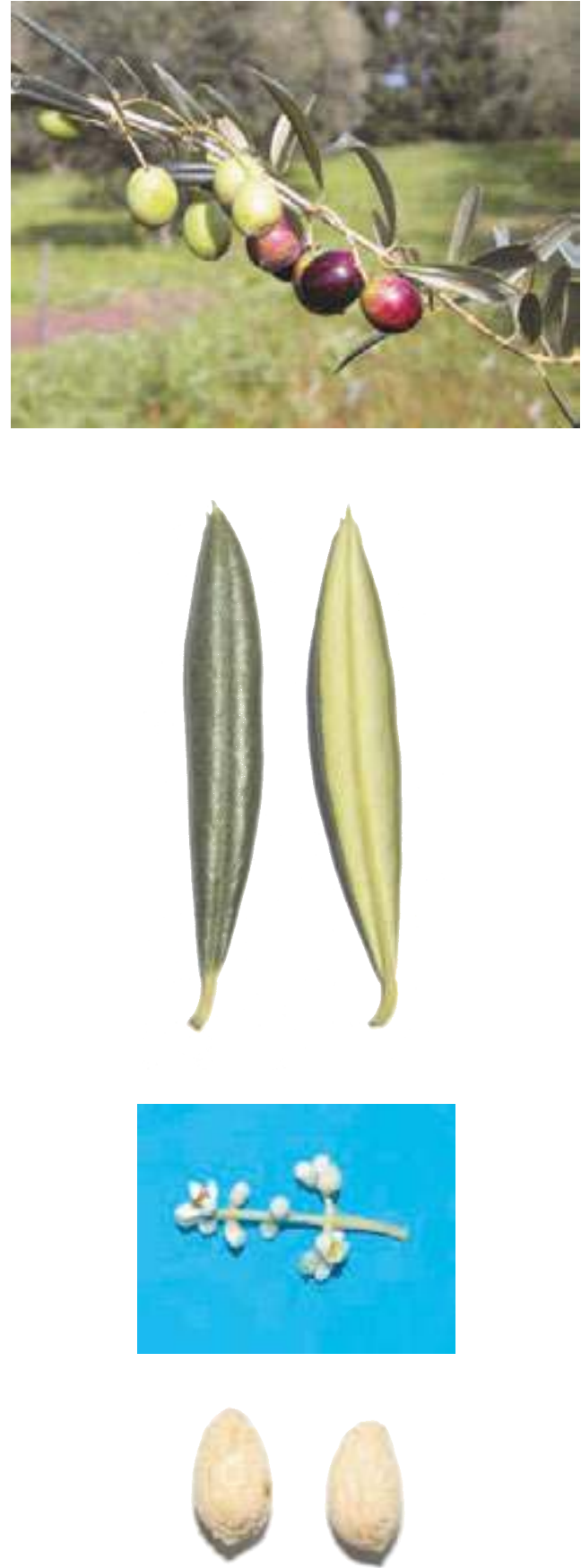


\section{Biochemical Characters}

\section{Fatty Acid Composition}

Table 1. Average values (express in $\% \pm$ standard deviations) of the fatty acids methyl esters and nutritional ratios obtained from single cultivar olive oils.

\begin{tabular}{|c|c|c|c|c|c|}
\hline Myristic acid & $0,02 \pm 0,00$ & Linoleic acid $(\omega 6)$ & $10,36 \pm 0,39$ & Lignoceric acid & $0,04 \pm 0,01$ \\
\hline Palmitic acid & $15,20 \pm 1,33$ & Linolenic acid $(\omega 3)$ & $0,86 \pm 0,07$ & & \\
\hline Palmitoleic acid & $2,48 \pm 0,30$ & Arachic acid & $0,28 \pm 0,07$ & Unsat./satured & $4,37 \pm 0,52$ \\
\hline teari & $2,83 \pm 0,52$ & Eicosenoic acid & $0,02 \pm 0,01$ & $\omega 6 / \omega 3$ & $12,04 \pm 0,57$ \\
\hline leic acid & $65,34 \pm 0,66$ & Behenic acid & $0,05 \pm 0,00$ & & \\
\hline
\end{tabular}

\section{Organoleptic oil values}

Sensory Analysis (Panel test)

Comment: fruity medium-high, with hints of almond and read sensations of grass and artichoke. Balanced taste sensation with a medium-high bitter and spicy. Medium-high fluidity.

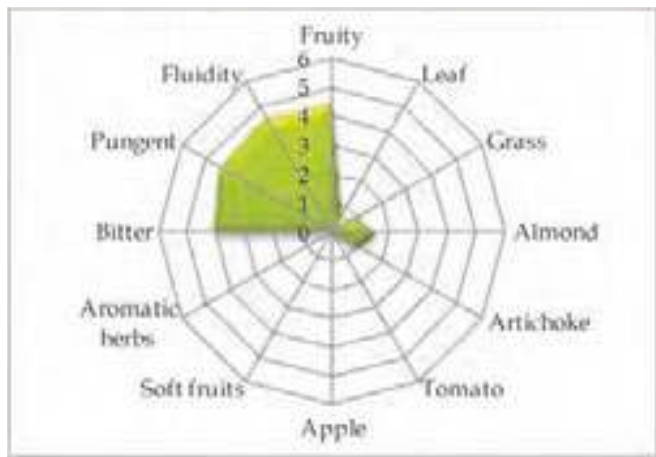

\section{Molecular Markers}

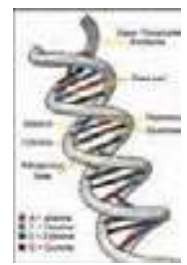

Table 2. Microsatellites (SSR) loci analyzed.

For each locus the allele size (expressed in base pairs) is reported.

$\begin{array}{cccccc}D C A 09 & \text { DCA18 } & \text { GAPU59 } & \text { GAPU71A } & \text { GUPA71B } & \text { GAPU103A } \\ \mathbf{1 9 8} \mathbf{- 2 1 0} & \mathbf{1 7 7 - \mathbf { 1 7 9 }} & \mathbf{2 0 8 - \mathbf { 2 1 2 }} & \mathbf{2 1 4} \mathbf{- 2 1 4} & \mathbf{1 2 4} \mathbf{- 1 2 6} & \mathbf{1 3 6} \mathbf{- 1 7 0} \\ \text { UDOO1 } & \text { UDO03 } & \text { UDO12 } & \text { UDO28 } & \text { UDO39 } & \\ \mathbf{1 4 0 - \mathbf { 1 4 0 }} & \mathbf{1 3 5 - \mathbf { 1 3 5 }} & \mathbf{1 7 7 - \mathbf { 1 9 3 }} & \mathbf{1 5 4 - \mathbf { 2 0 5 }} & \mathbf{2 1 3 - \mathbf { 2 1 3 }} & \end{array}$

\section{References:}

1 - Various authors. In: Catalogo Nazionale delle Varietà di Olivo, University of Bari (in press), ISBN 978-88-8879397-9.

2 - Muzzalupo I., Lombardo N., Salimonti A., et al. Adv. Hort. Sci. (2008), 22(2): pp. 142-148. 


\section{"Geracese "}

(synonymy: Dolce di Gerace, Mammolese, Oliva grossa, Oliva di Bianco, Paesana, etc.)

Areal distribution or origin area: Calabria Flesh/ pit weight ratio: high $(8,35 \pm 0,60)$ Oil content (\%): medium $(47,02 \pm 0,28)$

Purpose: dual purpose

\section{Morphological characters}

Tree characters

Vigour: medium-strong

Growth habit: spreading

Canopy-density: medium-dense

\section{Leaf characters}

Blade length $(\mathrm{cm})$ : medium $(5,62 \pm 0,57)$

Blade width $(\mathrm{cm})$ : medium $(\mathbf{1 , 2 8} \pm 0,01)$

Shape (length/width): elliptic - lanceolate

\section{Inflorescence characters}

Inflorescence length $(\mathrm{cm})$ : short $(2,21 \pm 1,78)$

Number of flowers: low $(\mathbf{1 2 , 5 8} \pm \mathbf{1 , 3 8})$

\section{Fruit characters}

Fresh weight of 100 fruits $(\mathrm{g})$ : medium $(2,74 \pm 0,33)$

Shape (length/width): ovoid

Symmetry: slightly asymmetric

Position of maximum transverse diameter:

central

Apex: rounded

Base: rounded

Niplle: obvious

Lenticels: many and small

\section{Pit characters}

Weight of 100 pits (g): medium $(\mathbf{0 , 4 3} \pm \mathbf{0 , 1 5})$

Shape (length/width): ovoid

Mucron: obvious

Symmetry: slightly asymmetric

Position of maximum transverse diameter:

towards apex

Apex: rounded

Base: rounded

Surface: rugose

Number of grooves: medium
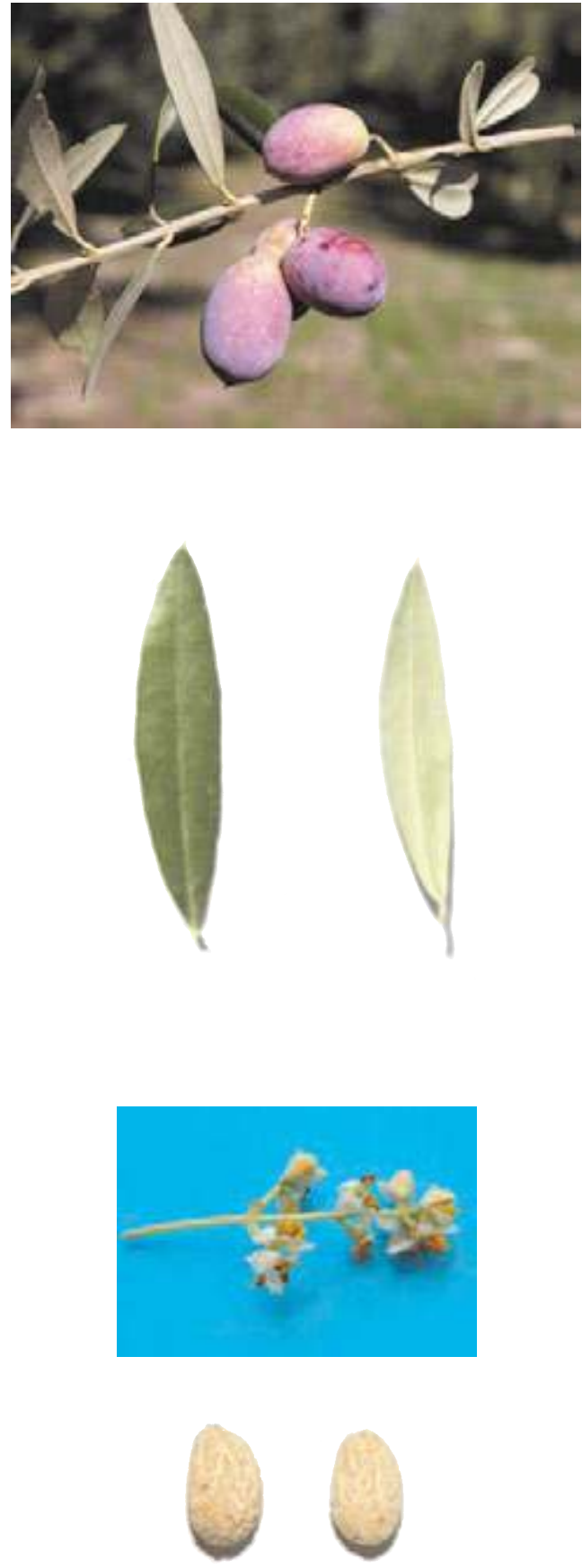


\section{Biochemical Characters}

\section{Fatty Acid Composition}

Table 1. Average values (express in $\% \pm$ standard deviations) of the fatty acids methyl esters and nutritional ratios obtained from single cultivar olive oils.

$\begin{array}{llllll}\text { Myristic acid } & \mathbf{0 , 0 1} \pm \mathbf{0 , 0 1} & \text { Linoleic acid }(\omega 6) & \mathbf{8 , 6 3} \pm \mathbf{0 , 0 2} & \text { Lignoceric acid } & \mathbf{0 , 0 5} \pm \mathbf{0 , 0 5} \\ \text { Palmitic acid } & \mathbf{1 4 , 0 9} \pm \mathbf{0 , 0 1} & \text { Linolenic acid }(\omega 3) \mathbf{0 , 7 5} \pm \mathbf{0 , 1 1} & & \\ \text { Palmitoleic acid } & \mathbf{1 , 2 6} \pm \mathbf{0 , 1 2} & \text { Arachic acid } & \mathbf{0 , 3 6} \pm \mathbf{0 , 1 4} & \text { Unsat./satured } & \mathbf{5 , 0 1} \pm \mathbf{0 , 1 3} \\ \text { Stearic acid } & \mathbf{2 , 0 4} \pm \mathbf{0 , 1 5} & \text { Eicosenoic acid } & \mathbf{0 , 1 9} \pm \mathbf{0 , 2 3} & \omega 6 / \omega 3 & \mathbf{1 1 , 6 9 \pm \mathbf { 1 , 7 6 }} \\ \text { Oleic acid } & \mathbf{7 1 , 3 3} \pm \mathbf{0 , 7 4} & \text { Behenic acid } & \mathbf{0 , 1 1} \pm \mathbf{0 , 0 6} & & \end{array}$

\section{Organoleptic oil values}

Sensory Analysis (Panel test)

Comment: fruity medium-high, with hints of leaves and grass, pleasant sensations of artichoke and tomato. Balanced taste sensation with a medium-high bitter and spicy. Medium-high fluidity.

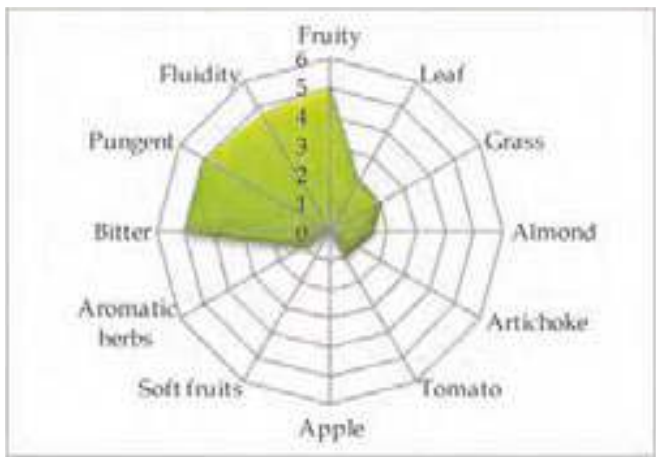

\section{Molecular Markers}

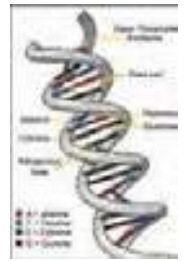

Table 2. Microsatellites (SSR) loci analyzed.

For each locus the allele size (expressed in base pairs) is reported.

$\begin{array}{cccccc}D C A 09 & D C A 18 & \text { GAPU59 } & \text { GAPU71A } & \text { GUPA71B } & \text { GAPU103A } \\ \mathbf{1 8 2} \mathbf{- 1 9 8} & \mathbf{1 7 9 - \mathbf { 1 8 1 }} & \mathbf{2 1 2} \mathbf{- 2 1 2} & \mathbf{2 1 2} \mathbf{- 2 1 2} & \mathbf{1 2 6 - \mathbf { 1 4 4 }} & \mathbf{1 5 0 - \mathbf { 1 7 0 }} \\ \text { UDOO1 } & \text { UDOO3 } & \text { UDO12 } & \text { UDO28 } & \text { UDO39 } & \\ \mathbf{1 4 0 - \mathbf { 1 5 0 }} & \mathbf{1 4 3 - \mathbf { 1 4 3 }} & \mathbf{1 6 6 - \mathbf { 1 7 7 }} & \mathbf{1 4 3 - \mathbf { 1 8 2 }} & \mathbf{2 0 0 - \mathbf { 2 0 0 }} & \end{array}$

\section{References:}

1 - Lombardo N., Perri E., Muzzalupo I., et al. In: Il germoplasma olivicolo Calabrese, Ist. Sper. Olivic.(2003), pp: 9.

2 - Perri E., Mazzotti F., Muzzalupo I., et al. In: Relazione attività CO.R.ASS.OL. (2003).

3 - Muzzalupo I., Stefanizzi F., Perri E. HortScience (2009), 44: pp. 582-588. 


\section{“Giarfara "}

Areal distribution or origin area: Sicilia

Flesh/pit weight ratio: high $(\mathbf{8}, \mathbf{4 3} \pm \mathbf{1 , 4 2})$

Oil content (\%): high $(\mathbf{5 3}, \mathbf{4 8} \pm \mathbf{0 , 7 0})$

Purpose: table

\section{Morphological characters}

Tree characters

Vigour: weak

Growth habit: spreading

Canopy-density: medium

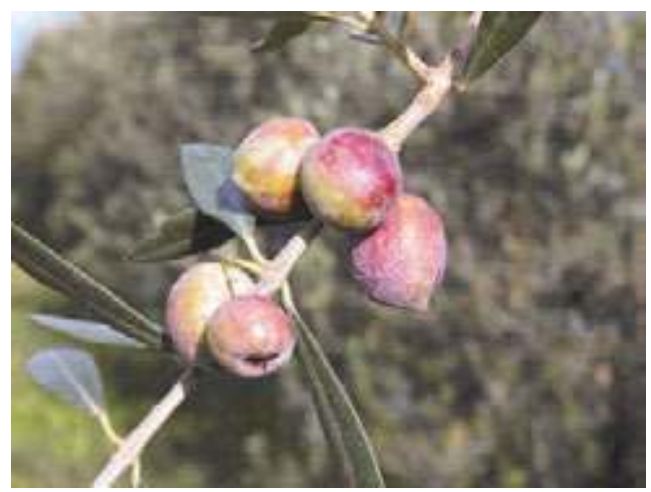

\section{Leaf characters}

Blade length $(\mathrm{cm})$ : medium $(5,97 \pm 0,48)$

Blade width $(\mathrm{cm})$ : broad $(\mathbf{1}, 67 \pm 0,17)$

Shape (length/width): elliptic

\section{Inflorescence characters}

Inflorescence length $(\mathrm{cm})$ : medium $(3,00 \pm 0,24)$

Number of flowers: low $(\mathbf{1 4 , 0 4} \pm 0,89)$

\section{Fruit characters}

Fresh weight of 100 fruits (g): high $(5,37 \pm 0,44)$

Shape (length/width): spherical

Symmetry: slightly asymmetric

Position of maximum transverse diameter:

towards base

Apex: rounded

Base: rounded

Niplle: tenuous

Lenticels: few and small

\section{Pit characters}

Weight of 100 pits (g): high $(0,57 \pm 0,05)$

Shape (length/width): ovoid

Mucron: obvious

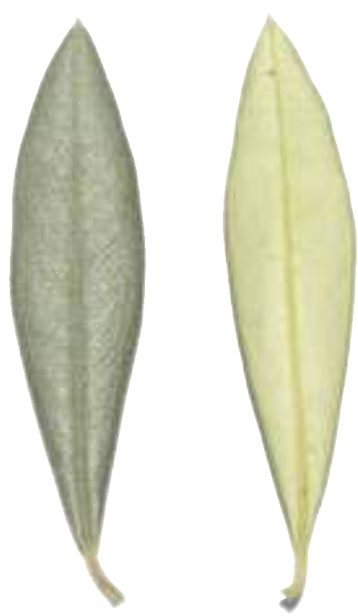

Symmetry: slightly asymmetric

Position of maximum transverse diameter:

towards base

Apex: rounded

Base: truncate

Surface: scabrous

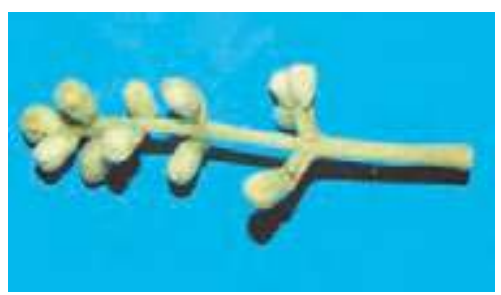

Number of grooves: medium 


\section{Biochemical Characters}

\section{Fatty Acid Composition}

Table 1. Average values (express in $\% \pm$ standard deviations) of the fatty acids methyl esters and nutritional ratios obtained from single cultivar olive oils.

\begin{tabular}{|c|c|c|c|c|c|}
\hline Myristic acid & $0,01 \pm 0,00$ & Linoleic acid $(\omega 6)$ & $6,34 \pm 1,28$ & Lignoceric acid & $0,03 \pm 0,00$ \\
\hline Palmitic acid & $11,11 \pm 1,54$ & Linolenic acid $(\omega 3)$ & $0,82 \pm 0,05$ & & \\
\hline Palmitoleic acid & $0,80 \pm 0,23$ & Arachic acid & $0,26 \pm 0,04$ & Unsat./satured & $6,37 \pm 0,60$ \\
\hline Stearic acid & $2,23 \pm 0,45$ & Eicosenoic acid & $0,02 \pm 0,00$ & $\omega 6 / \omega 3$ & $7,68 \pm 1,09$ \\
\hline Oleic acid & $77,71 \pm 0,51$ & Behenic acid & $0,06 \pm 0,01$ & & \\
\hline
\end{tabular}

\section{Organoleptic oil values}

Sensory Analysis (Panel test)

Comment: fruity medium-high, with hints of grass and tomato, read sensations of almond and artichoke. Balanced taste sensation with a medium-high bitter and spicy. Mediumhigh.

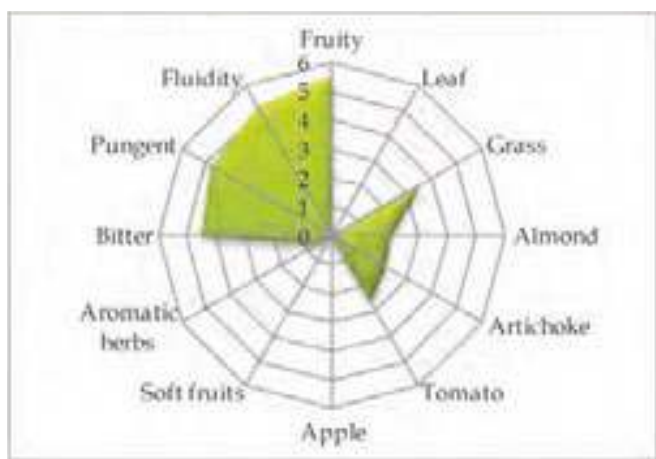

\section{Molecular Markers}

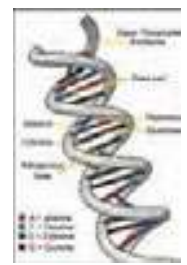

Table 2. Microsatellites (SSR) loci analyzed.

For each locus the allele size (expressed in base pairs) is reported.

\begin{tabular}{|c|c|c|c|c|c|}
\hline$D C A 09$ & $D C A 18$ & GAPU59 & GAPU71A & GUPA71B & GAPU103A \\
\hline $176-182$ & $179-179$ & $212-212$ & $214-214$ & $144-144$ & $157-184$ \\
\hline UDO01 & UDO03 & UDO12 & UDO28 & UDO39 & \\
\hline $140-140$ & $150-150$ & $166-193$ & $182-182$ & $108-108$ & \\
\hline
\end{tabular}

\section{References:}

1 - Bottari V., Spina P. In: Le varietà di olivo coltivate in Sicilia, Ann. Sper. Agr. (1953), 7: pp. 937-1004.

2 - Muzzalupo I., Lombardo N., Salimonti A., et al. Adv. Hort. Sci. (2008), 22(2): pp. 142-148. 


\section{"Giarraffa "}

(synonymy: Becco di Corvo, Cefalutana, Giarrafara, Giarraffedda, Mammona, etc.)

Areal distribution or origin area: Sicilia

Flesh/pit weight ratio: high $(\mathbf{9}, 28 \pm 1,65)$

Oil content (\%): medium $(46,69 \pm 1,44)$

Purpose: table

\section{Morphological characters}

Tree characters

Vigour: medium

Growth habit: spreading

Canopy-density: medium-dense

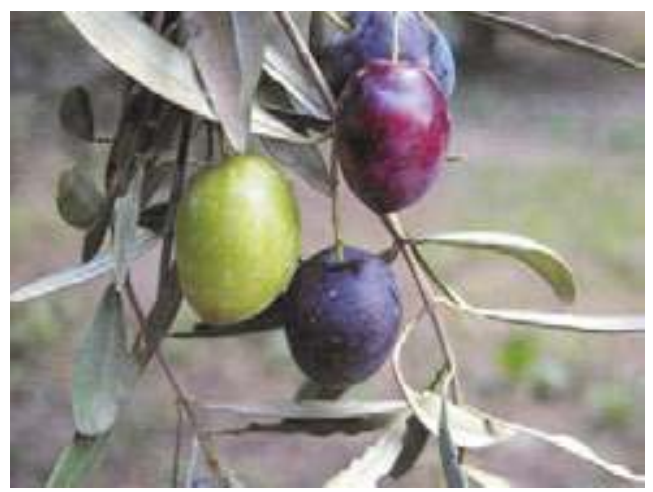

\section{Leaf characters}

Blade length (cm): medium $(6,38 \pm 0,59)$

Blade width $(\mathrm{cm})$ : medium $(1,34 \pm 0,26)$

Shape (length/width): elliptic-lanceolate

\section{Inflorescence characters}

Inflorescence length $(\mathrm{cm})$ : medium $(2,56 \pm 1,19)$

Number of flowers: low $(\mathbf{1 5 , 2 8} \pm \mathbf{1}, \mathbf{5 7})$

\section{Fruit characters}

Fresh weight of 100 fruits (g): very high $(8,25 \pm 1,66)$

Shape (length/width): ovoid

Symmetry: slightly asymmetric

Position of maximum transverse diameter:

towards base

Apex: rounded

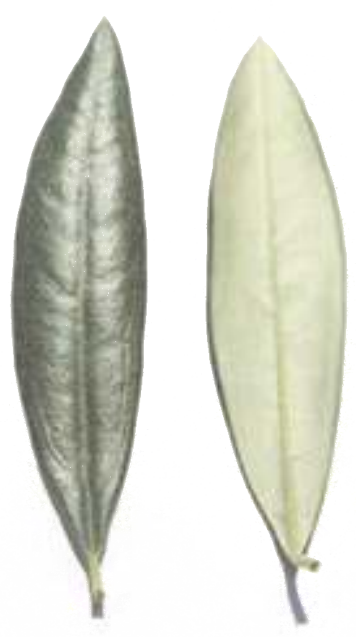

Base: truncate

Niplle: absent

Lenticels: few and large

\section{Pit characters}

Weight of 100 pits (g): very high $(0,82 \pm 0,20)$

Shape (length/width): elliptic

Mucron: obious

Symmetry: slightly asymmetric

Position of maximum transverse diameter:

central

Apex: rounded

Base: rounded

Surface: scabrous

Number of grooves: high
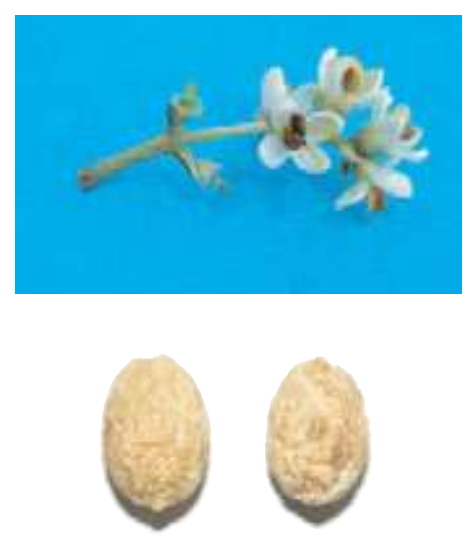


\section{Biochemical Characters}

\section{Fatty Acid Composition}

Table 1. Average values (express in $\% \pm$ standard deviations) of the fatty acids methyl esters and nutritional ratios obtained from single cultivar olive oils.

\begin{tabular}{|c|c|c|c|c|c|}
\hline Myristic acid & $0,00 \pm 0,00$ & Linoleic acid $(\omega 6)$ & $19,05 \pm 0,21$ & Lignoceric acid & $0,06 \pm 0,02$ \\
\hline Palmitic acid & $12,90 \pm 0,44$ & Linolenic acid $(\omega 3)$ & $0,87 \pm 0,04$ & & \\
\hline Palmitoleic acid & $1,09 \pm 0,05$ & Arachic acid & $1,12 \pm 0,05$ & Unsat./satured & $5,54 \pm 0,26$ \\
\hline ea & $1,86 \pm 0,10$ & Eicosenoic acid & $0,36 \pm 0,06$ & $\omega 6 / \omega 3$ & $22,05 \pm 1,15$ \\
\hline leic acid & $61,61 \pm 0,76$ & Behenic acid & $0,08 \pm 0,00$ & & \\
\hline
\end{tabular}

\section{Organoleptic oil values}

Sensory Analysis (Panel test)

Comment: fruity medium-light, with taste of almond, read sensations of grass and leaves. Balanced taste sensation with medium-light bitter and spicy. Medium-high fluidity.

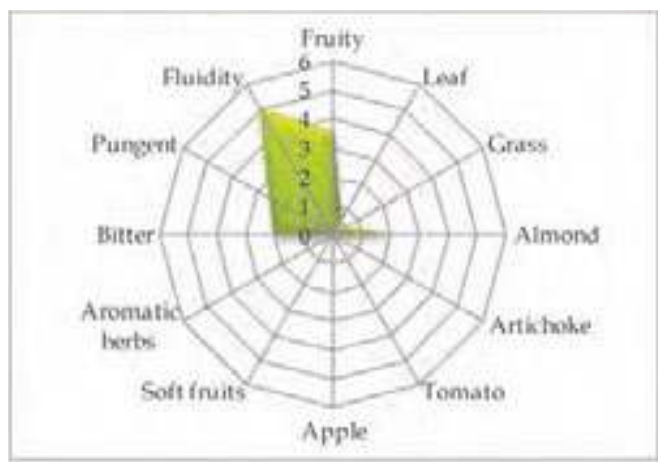

\section{Molecular Markers}

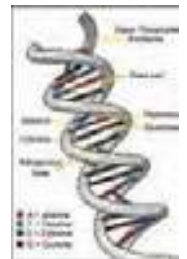

Table 2. Microsatellites (SSR) loci analyzed. For each locus the allele size (expressed in base pairs) is reported.

$\begin{array}{cccccc}D C A 09 & D C A 18 & \text { GAPU59 } & \text { GAPU71A } & \text { GUPA71B } & \text { GAPU103A } \\ \mathbf{1 6 2} \mathbf{- 1 9 8} & \mathbf{1 8 1} \mathbf{- 1 8 5} & \mathbf{2 1 2} \mathbf{- 2 1 2} & \mathbf{2 1 0 - \mathbf { 2 1 4 }} & \mathbf{1 2 4} \mathbf{- 1 4 4} & \mathbf{1 3 6} \mathbf{- 1 5 9} \\ \text { UDOO1 } & \text { UDO03 } & \text { UDO12 } & \text { UDO28 } & \text { UDO39 } & \\ \mathbf{1 4 4 - \mathbf { 1 4 4 }} & \mathbf{1 5 0 - \mathbf { 1 5 0 }} & \mathbf{1 6 6 - \mathbf { 1 6 6 }} & \mathbf{1 4 3 - \mathbf { 1 8 2 }} & \mathbf{2 1 3 - \mathbf { 2 1 3 }} & \end{array}$

\section{References:}

1 - Caruso T., Cartabellotta D., Motisi A., et all. In: Cultivar di olivo siciliane, Università degli Studi di Palermo (2007), pp. 100-104.

2 - Muzzalupo I., Stefanizzi F., Perri E. HortScience (2009), 44: pp. 582-588. 


\section{"Giusta "}

(synonymy: Justa, Mezza oliva, Oliva tonda, Rizza, etc.)

Areal distribution or origin area: Basilicata

Flesh/pit weight ratio: medium $(6,94 \pm 1,61)$

Oil content (\%): low $(41,59 \pm 2,66)$

Purpose: dual purpose

\section{Morphological characters}

Tree characters

Vigour: medium-strong

Growth habit: erect-spreading

Canopy-density: medium-dense

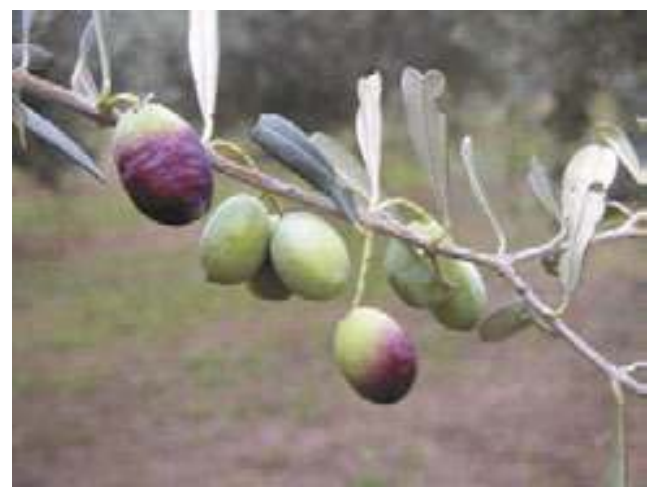

\section{Leaf characters}

Blade length $(\mathrm{cm})$ : medium $(5,98 \pm 0,45)$

Blade width $(\mathrm{cm})$ : broad $(\mathbf{1}, 54 \pm 0,15)$

Shape (length/width): elliptic

\section{Inflorescence characters}

Inflorescence length $(\mathrm{cm})$ : medium $(3,04 \pm 1,23)$

Number of flowers: low $(\mathbf{1 4 , 3 4} \pm \mathbf{1 , 8 6})$

\section{Fruit characters}

Fresh weight of 100 fruits (g): medium $(3,72 \pm 0,06)$

Shape (length/width): ovoid

Symmetry: slightly asymmetric

Position of maximum transverse diameter:

central

Apex: rounded

Base: rounded

Niplle: obvious

Lenticels: many and small

\section{Pit characters}

Weight of 100 pits (g): high $(\mathbf{0 , 4 8} \pm \mathbf{0 1 1})$

Shape (length/width): elliptic

Mucron: obvious

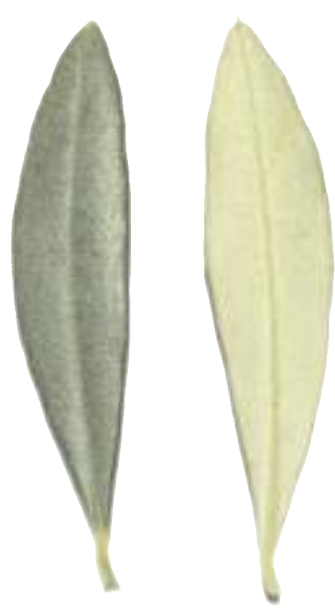

Symmetry: slightly asymmetric

Position of maximum transverse diameter:

towards apex

Apex: rounded

Base: pointed

Surface: rugose

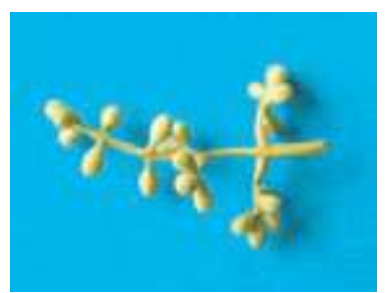

Number of grooves: medium 


\section{Biochemical Characters}

\section{Fatty Acid Composition}

Table 1. Average values (express in $\% \pm$ standard deviations) of the fatty acids methyl esters and nutritional ratios obtained from single cultivar olive oils.

\begin{tabular}{|c|c|c|c|c|c|}
\hline Myristic acid & $0,01 \pm 0,00$ & Linoleic acid $(\omega 6)$ & $18,04 \pm 0,25$ & Lignoceric acid & $0,03 \pm 0,00$ \\
\hline Palmitic acid & $13,86 \pm 0,44$ & Linolenic acid $(\omega 3)$ & $0,94 \pm 0,02$ & & \\
\hline Palmitoleic acid & $0,85 \pm 0,02$ & Arachic acid & $0,06 \pm 0,34$ & Unsat./satured & $4,86 \pm 0,08$ \\
\hline teari & $2,75 \pm 0,19$ & Eicosenoic acid & $0,02 \pm 0,00$ & $\omega 6 / \omega 3$ & $19,16 \pm 0,24$ \\
\hline leic acid & $61,86 \pm 0,45$ & Behenic acid & $0,08 \pm 0,01$ & & \\
\hline
\end{tabular}

\section{Organoleptic oil values}

Sensory Analysis (Panel test)

Comment: fruity medium-high, with hints of almond and read sensations of grass and artichoke. Balanced taste sensation with a medium-high bitter and spicy. Medium-high fluidity.

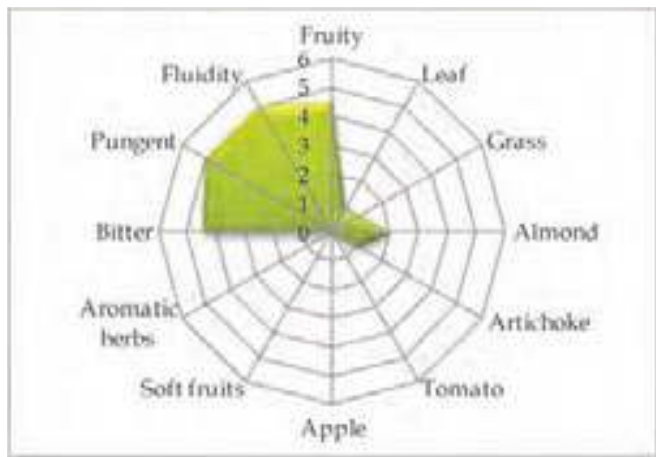

\section{Molecular Markers}

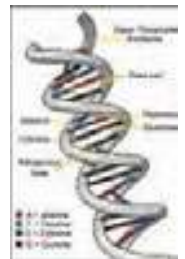

Table 2. Microsatellites (SSR) loci analyzed. For each locus the allele size (expressed in base pairs) is reported.

$\begin{array}{cccccc}D C A 09 & D C A 18 & \text { GAPU59 } & \text { GAPU71A } & \text { GUPA71B } & \text { GAPU103A } \\ \mathbf{1 6 2} \mathbf{- 1 9 8} & \mathbf{1 7 7 - \mathbf { 1 7 9 }} & \mathbf{2 1 4} \mathbf{- 2 2 2} & \mathbf{2 1 4 - \mathbf { 2 1 4 }} & \mathbf{1 2 6 - \mathbf { 1 4 4 }} & \mathbf{1 7 0 - \mathbf { 1 8 4 }} \\ \text { UDO01 } & \text { UDOO3 } & \text { UDO12 } & \text { UDO28 } & \text { UDO39 } & \\ \mathbf{1 4 4 - \mathbf { 1 4 4 }} & \mathbf{1 4 3 - \mathbf { 1 4 3 }} & \mathbf{1 7 7 - \mathbf { 1 7 7 }} & \mathbf{1 8 2} \mathbf{- 1 8 2} & \mathbf{2 0 5 - \mathbf { 2 0 5 }} & \end{array}$

\section{References:}

1 - Rotundo A., Marone E. In: Il germoplasma olivicolo lucano, Olita-Potenza (2002), pp. 57-60.

2 - Rotundo A., Perri E.,Muzzalupo I., et al. In: Il germoplasma olivicolo meridionale, (2012 in press).

3 - Muzzalupo I., Stefanizzi F., Perri E. HortScience (2009), 44: pp. 582-588. 


\section{“Gnagnaro"}

(synonymy: Gragnaro)

Areal distribution or origin area: Molise

Flesh/pit weight ratio: low $(\mathbf{1}, 71 \pm \mathbf{0 , 0 6})$

Oil content (\%): low $(36,14 \pm 1,56)$

Purpose: oil

\section{Morphological characters}

Tree characters

Vigour: medium-weak

Growth habit: spreading-erect

Canopy-density: medium

\section{Leaf characters}

Blade length (cm): short $(4,98 \pm 0,43)$

Blade width $(\mathrm{cm})$ : medium $(1,34 \pm 0,18)$

Shape (length/width): elliptic

\section{Inflorescence characters}

Inflorescence length $(\mathrm{cm})$ : medium $(3,21 \pm 1,85)$

Number of flowers: low $(19,47 \pm 1,42)$

\section{Fruit characters}

Fresh weight of 100 fruits $(\mathrm{g})$ : low $(0,59 \pm 0,01)$

Shape (length/width): elongated

Symmetry: asymmetric

Position of maximum transverse diameter:

central

Apex: pointed

Base: rounded

Niplle: tenuous

Lenticels: many and small

\section{Pit characters}

Weight of 100 pits (g): low $(0,22 \pm 0,01)$

Shape (length/width): elliptic

Mucron: obvious

Symmetry: asymmetric

Position of maximum transverse diameter:

central

Apex: pointed

Base: rounded

Surface: smooth

Number of grooves: low
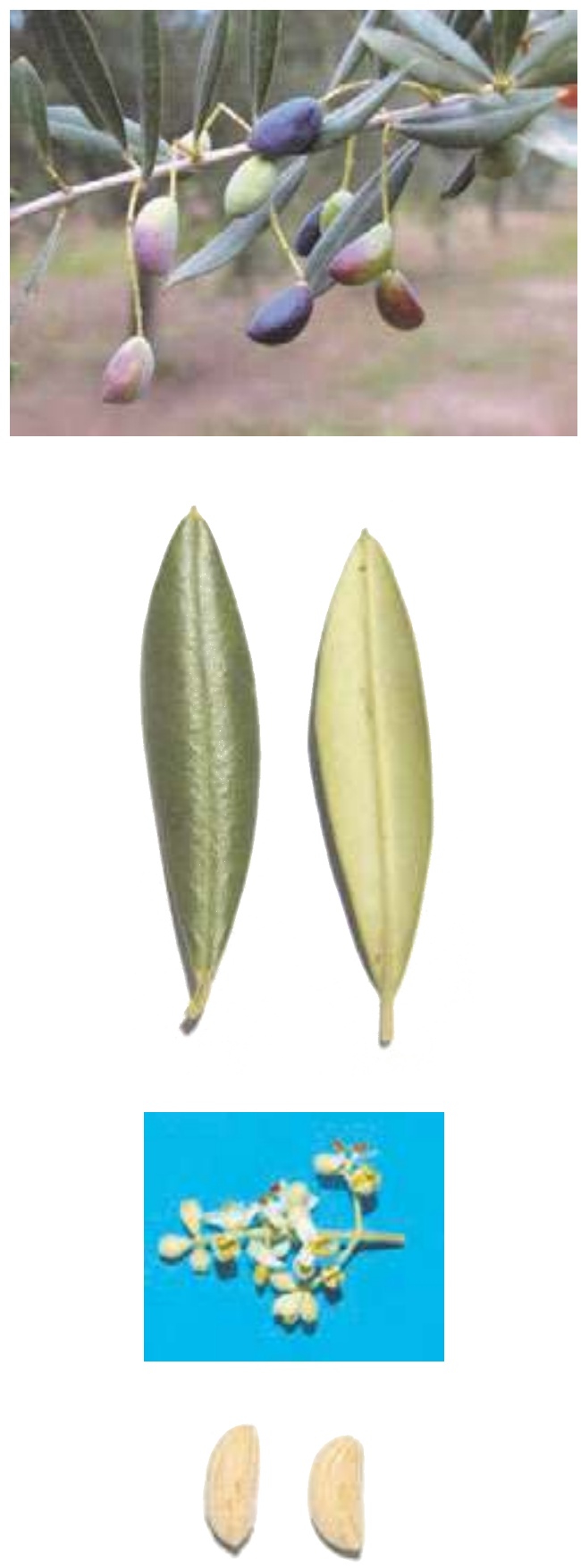


\section{Biochemical Characters}

\section{Fatty Acid Composition}

Table 1. Average values (express in $\% \pm$ standard deviations) of the fatty acids methyl esters and nutritional ratios obtained from single cultivar olive oils.

\begin{tabular}{|c|c|c|c|c|c|}
\hline Myristic acid & $0,02 \pm 0,01$ & Linoleic acid $(\omega 6)$ & $16,40 \pm 2,45$ & Lignoceric acid & $0,04 \pm 0,03$ \\
\hline Palmitic acid & $13,78 \pm 1,53$ & Linolenic acid $(\omega 3)$ & $0,95 \pm 0,07$ & & \\
\hline Palmitoleic acid & $1,10 \pm 0,03$ & Arachic acid & $0,24 \pm 0,05$ & Unsat./satured & $5,14 \pm 0,62$ \\
\hline teari & $2,20 \pm 0,14$ & Eicosenoic acid & $0,15 \pm 0,22$ & $\omega 6 / \omega 3$ & $17,40 \pm 3,01$ \\
\hline leic acid & $63,94 \pm 1,90$ & Behenic acid & $0,08 \pm 0,02$ & & \\
\hline
\end{tabular}

\section{Organoleptic oil values}

Sensory Analysis (Panel test)

Comment: fruity medium-high, with hints of almond and read sensations of grass and artichoke. Balanced taste sensation with a medium bitter and spicy. Medium fluidity.

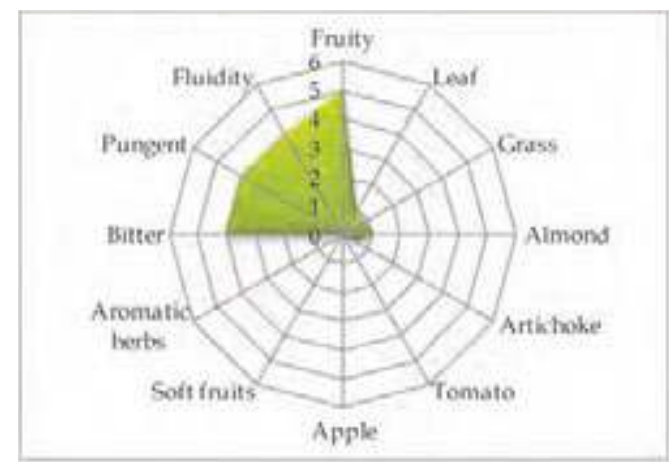

\section{Molecular Markers}

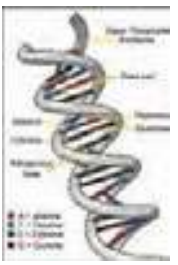

Table 2. Microsatellites (SSR) loci analyzed.

For each locus the allele size (expressed in base pairs) is reported.

$\begin{array}{cccccc}D C A 09 & D C A 18 & \text { GAPU59 } & \text { GAPU71A } & \text { GUPA71B } & \text { GAPU103A } \\ \mathbf{1 6 2} \mathbf{- 2 0 6} & \mathbf{1 7 5 - \mathbf { 1 7 5 }} & \mathbf{2 1 4 - \mathbf { 2 1 4 }} & \mathbf{2 1 0 - \mathbf { 2 1 4 }} & \mathbf{1 2 4 - \mathbf { 1 4 4 }} & \mathbf{1 7 0 - \mathbf { 1 7 0 }} \\ \text { UDOO1 } & \text { UDOO3 } & \text { UDO12 } & \text { UDO28 } & \text { UDO39 } & \\ \mathbf{1 4 4 - \mathbf { 1 4 4 }} & \mathbf{1 5 0 - \mathbf { 1 8 2 }} & \mathbf{1 6 6 - \mathbf { 1 6 6 }} & \mathbf{1 5 4} \mathbf{- 1 8 2} & \mathbf{2 2 0} \mathbf{- 2 4 3} & \end{array}$

\section{References:}

1 - Macrì T., Picone G., La Porta G. In : Olivo e Olio, (1988), 5: pp. 34-41.

2 - Muzzalupo I., Salimonti A., Caravita M. A., et al. Adv. Hort. Sci. (2008), 22(2): pp. 129-135. 


\section{“Grappolo"}

(synonymy: Frantoie a grappoli)

Areal distribution or origin area: Toscana

Flesh/pit weight ratio: medium $(6,01 \pm 0,47)$

Oil content (\%): medium $(47,30 \pm 1,64)$

Purpose: oil

\section{Morphological characters}

Tree characters

Vigour: medium

Growth habit: spreading

Canopy-density: medium-dense

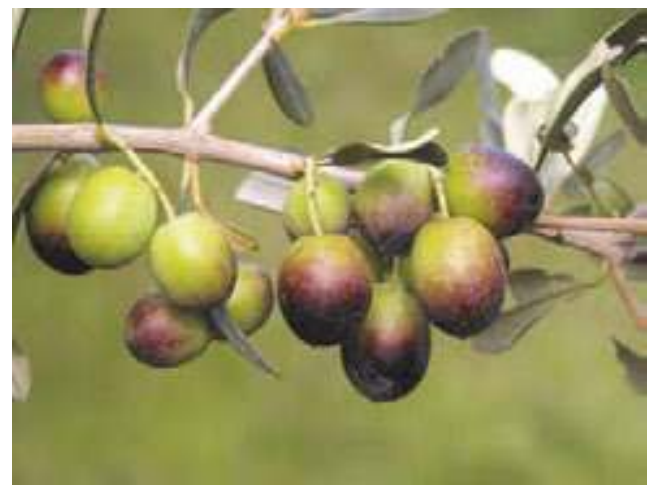

\section{Leaf characters}

Blade length $(\mathrm{cm})$ : medium $(5,60 \pm 0,46)$

Blade width $(\mathrm{cm})$ : broad $(\mathbf{1}, 59 \pm \mathbf{0 , 1 2})$

Shape (length/width): elliptic

\section{Inflorescence characters}

Inflorescence length $(\mathrm{cm})$ : medium $(2,95 \pm 0,35)$

Number of flowers: low $(17,73 \pm 1,82)$

\section{Fruit characters}

Fresh weight of 100 fruits $(\mathrm{g})$ : medium $(2,59 \pm 0,06)$

Shape (length/width): spherical

Symmetry: symmetric

Position of maximum transverse diameter:

central

Apex: rounded

Base: truncate

Niplle: absent

Lenticels: many and small

\section{Pit characters}

Weight of 100 pits (g): medium $(0,37 \pm 0,02)$

Shape (length/width): ovoid

Mucron: obvious

Symmetry: slightly asymmetric

Position of maximum transverse diameter:

\section{towards apex}

Apex: rounded

Base: rounded

Surface: smooth

Number of grooves: medium
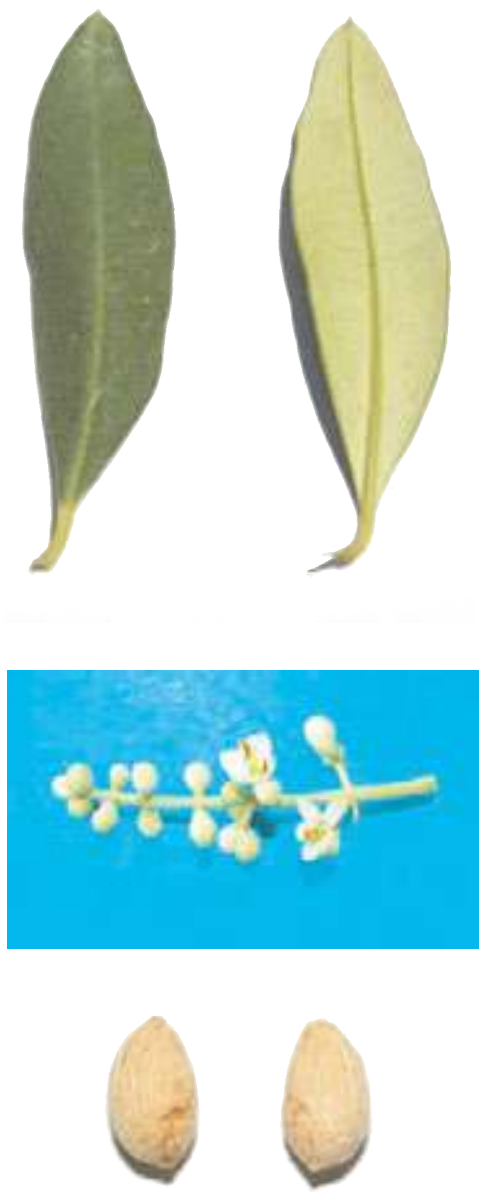


\section{Biochemical Characters}

\section{Fatty Acid Composition}

Table 1. Average values (express in $\% \pm$ standard deviations) of the fatty acids methyl esters and nutritional ratios obtained from single cultivar olive oils.

$\begin{array}{llllll}\text { Myristic acid } & \mathbf{0 , 0 1} \pm \mathbf{0 , 0 1} & \text { Linoleic acid }(\omega 6) & \mathbf{6 , 4 0} \pm \mathbf{0 , 7 2} & \text { Lignoceric acid } & \mathbf{0 , 0 4} \pm \mathbf{0 , 0 3} \\ \text { Palmitic acid } & \mathbf{1 0 , 4 8} \pm \mathbf{0 , 3 1} & \text { Linolenic acid }(\omega 3) & \mathbf{0 , 9 7} \pm \mathbf{0 , 0 2} & & \\ \text { Palmitoleic acid } & \mathbf{0 , 4 7} \pm \mathbf{0 , 1 5} & \text { Arachic acid } & \mathbf{0 , 3 7} \pm \mathbf{0 , 1 7} & \text { Unsat./satured } & \mathbf{6 , 7 7} \pm \mathbf{0 , 1 5} \\ \text { Stearic acid } & \mathbf{2 , 1 4} \pm \mathbf{0 , 0 3} & \text { Eicosenoic acid } & \mathbf{0 , 1 5} \pm \mathbf{0 , 1 9} & \omega 6 / \omega 3 & \mathbf{6 , 5 9} \pm \mathbf{0 , 8 4} \\ \text { Oleic acid } & \mathbf{7 8 , 7 1 \pm 0 , 8 5} & \text { Behenic acid } & \mathbf{0 , 0 7} \pm \mathbf{0 , 0 1} & & \end{array}$

\section{Organoleptic oil values}

Sensory Analysis (Panel test)

Comment: fruity medium - light, with hints of artichoke and almond. Balanced in flavours, with hints of bitter and spicy medium intensity, medium fluidity.

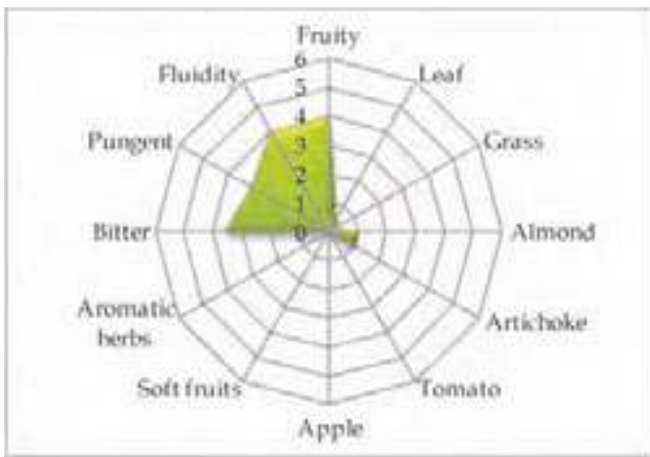

\section{Molecular Markers}

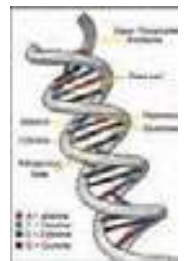

Table 2. Microsatellites (SSR) loci analyzed.

For each locus the allele size (expressed in base pairs) is reported.

$\begin{array}{cccccc}D C A 09 & \text { DCA } 18 & \text { GAPU59 } & \text { GAPU71A } & \text { GUPA71B } & \text { GAPU103A } \\ \mathbf{1 8 2} \mathbf{- 2 0 6} & \mathbf{1 8 1} \mathbf{- 1 8 1} & \mathbf{2 0 8 - \mathbf { 2 1 8 }} & \mathbf{2 1 0 - \mathbf { 2 1 8 }} & \mathbf{1 2 4} \mathbf{- 1 4 4} & \mathbf{1 7 0 - \mathbf { 1 8 4 }} \\ \text { UDOO1 } & \text { UDOO3 } & \text { UDO12 } & \text { UDO28 } & \text { UDO39 } & \\ \mathbf{1 5 0} \mathbf{- 1 5 0} & \mathbf{1 5 0} \mathbf{- 1 5 0} & \mathbf{1 5 6} \mathbf{- 1 8 2} & \mathbf{1 8 2} \mathbf{- 1 8 2} & \mathbf{2 2 0} \mathbf{- 2 2 0} & \end{array}$

\section{References:}

1 - Cimato A., Cantini C., Sani G., In: L'olivo in Toscana: il germoplasma autoctono, Ed. ARSIA (2001).

2 - Various authors. In: Catalogo Nazionale delle Varietà di Olivo, University of Bari (in press), ISBN 978-88-88793-

97-9. 


\section{"Grignan"}

(synonymy: Brescian, Gargnano, Gregnara, etc.)

Areal distribution or origin area: Veneto

Flesh/pit weight ratio: low $(4,90 \pm 1,25)$

Oil content (\%): medium $(40,00 \pm 0,62)$

Purpose: oil

\section{Morphological characters}

Tree characters

Vigour: medium

Growth habit: erect-spreading

Canopy-density: medium

\section{Leaf characters}

Blade length $(\mathrm{cm})$ : medium $(5,66 \pm 0,59)$

Blade width $(\mathrm{cm})$ : medium $(1,41 \pm 0,19)$

Shape (length/width): elliptic-lanceolate

\section{Inflorescence characters}

Inflorescence length $(\mathrm{cm})$ : medium $(2,77 \pm 0,21)$

Number of flowers: low $(\mathbf{1 4}, 58 \pm 0,67)$

\section{Fruit characters}

Fresh weight of 100 fruits $(\mathrm{g})$ : medium $(3,31 \pm 0,06)$

Shape (length/width): spherical

Symmetry: slightly asymmetric

Position of maximum transverse diameter:

central

Apex: rounded

Base: truncate

Niplle: tenuous

Lenticels: few and small

\section{Pit characters}

Weight of 100 pits (g): high $(\mathbf{0 , 6 0} \pm \mathbf{0 , 1 5})$

Shape (length/width): ovoid

Mucron: obvious

Symmetry: slightly asymmetric

Position of maximum transverse diameter:

central

Apex: rounded

Base: rounded

Surface: scabrous

Number of grooves: high
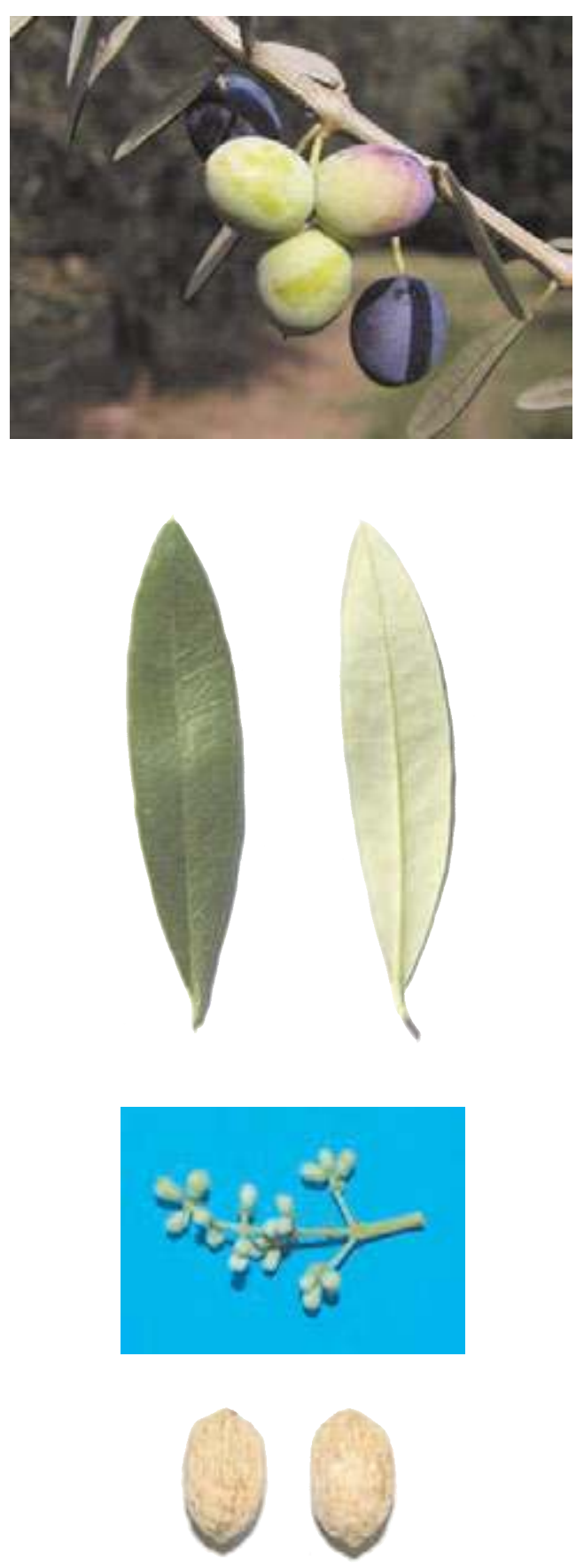


\section{Biochemical Characters}

\section{Fatty Acid Composition}

Table 1. Average values (express in $\% \pm$ standard deviations) of the fatty acids methyl esters and nutritional ratios obtained from single cultivar olive oils.

$\begin{array}{llllll}\text { Myristic acid } & \mathbf{0 , 0 2} \pm \mathbf{0 , 0 0} & \text { Linoleic acid }(\omega 6) & \mathbf{7 , 1 8} \pm \mathbf{1 , 0 7} & \text { Lignoceric acid } & \mathbf{0 , 0 3} \pm \mathbf{0 , 0 0} \\ \text { Palmitic acid } & \mathbf{1 1 , 5 4} \pm \mathbf{0 , 4 4} & \text { Linolenic acid }(\omega 3) \mathbf{0 , 9 0} \pm \mathbf{0 , 0 2} & & \\ \text { Palmitoleic acid } & \mathbf{0 , 9 5} \pm \mathbf{0 , 0 9} & \text { Arachic acid } & \mathbf{0 , 2 8} \pm \mathbf{0 , 0 1} & \text { Unsat./satured } & \mathbf{6 , 1 4} \pm \mathbf{0 , 1 5} \\ \text { Stearic acid } & \mathbf{2 , 1 9} \pm \mathbf{0 , 0 5} & \text { Eicosenoic acid } & \mathbf{0 , 0 2} \pm \mathbf{0 , 0 0} & \omega 6 / \omega 3 & \mathbf{7 , 9 7} \pm \mathbf{1 , 0 3} \\ \text { Oleic acid } & \mathbf{7 6 , 2 1} \pm \mathbf{1 , 4 9} & \text { Behenic acid } & \mathbf{0 , 0 6} \pm \mathbf{0 , 0 1} & & \end{array}$

\section{Organoleptic oil values}

Sensory Analysis (Panel test)

Comment: fruity medium-high, with hints of almond and read sensations of grass and artichoke. Balanced taste sensation with a medium-high bitter and spicy. Medium-high fluidity.

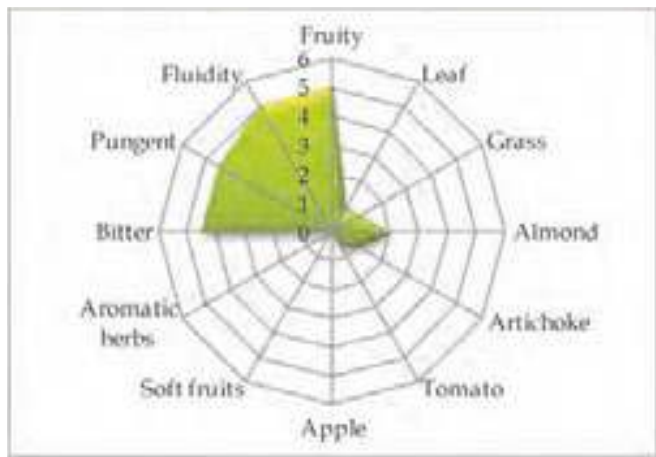

\section{Molecular Markers}

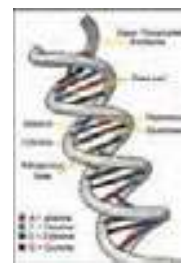

Table 2. Microsatellites (SSR) loci analyzed.

For each locus the allele size (expressed in base pairs) is reported.

$\begin{array}{cccccc}D C A 09 & \text { DCA18 } & \text { GAPU59 } & \text { GAPU71A } & \text { GUPA71B } & \text { GAPU103A } \\ \mathbf{1 9 4 - \mathbf { 1 9 8 }} & \mathbf{1 7 7 - \mathbf { 1 8 5 }} & \mathbf{2 1 2} \mathbf{- 2 1 2} & \mathbf{2 1 2} \mathbf{- 2 2 1} & \mathbf{1 2 4 - \mathbf { 1 3 0 }} & \mathbf{1 5 7 - \mathbf { 1 5 7 }} \\ \text { UDOO1 } & \text { UDO03 } & \text { UDO12 } & \text { UDO28 } & \text { UDO39 } & \\ \mathbf{1 4 0 - \mathbf { 1 4 0 }} & \mathbf{1 5 0 - \mathbf { 1 5 0 }} & \mathbf{1 6 6 - \mathbf { 1 6 6 }} & \mathbf{1 8 2} \mathbf{- 2 1 0} & \mathbf{2 1 3 - \mathbf { 2 2 0 }} & \end{array}$

\section{References:}

1 - Consiglio Oleicolo Internazionale. In: Catalogo Mondiale delle Varietà di Olivo, COI (2003).

2 - Muzzalupo I., Lombardo N., Salimonti A., et al. Adv. Hort. Sci. (2008), 22(2): pp. 142-148. 


\section{"Grossa di Cassano"}

(synonymy: Cassanese, Precoce di Cassano.)

Areal distribution or origin area: Calabria

Flesh/ pit weight ratio: high $(7,62 \pm 0,98)$

Oil content (\%): low $(36,56 \pm 3,14)$

Purpose: dual purpose

\section{Morphological characters}

Tree characters

Vigour: medium-strong

Growth habit: spreading

Canopy-density: medium-dense

\section{Leaf characters}

Blade length (cm): medium $(6,37 \pm 0,51)$

Blade width (cm): broad $(\mathbf{1}, 79 \pm 0,20)$

Shape (blade length/width): elliptic

\section{Inflorescence characters}

Inflorescence length $(\mathrm{cm})$ : medium $(3,27 \pm 1,27)$

Number of flowers: medium $(22,07 \pm 4,54)$

\section{Fruit characters}

Fresh weight of 100 fruits $(\mathrm{g})$ : medium $(3,86 \pm 0,26)$

Shape (length/width): ovoid

Symmetry: slightly asymmetrical

Position of maximum transverse diameter:

central

Apex: rounded

Base: rounded

Nipple: obvious

Lenticels: many and small

\section{Pit characters}

Weight of 100 pits (g): high $(\mathbf{0 , 5 2} \pm \mathbf{0 , 0 1})$

Shape (length/width): elliptic

Mucron: obvious

Symmetry: slightly asymmetrical

Position of maximum transverse diameter:

central

Apex: rounded

Base: rounded

Surface: rugose

Number of grooves: medium
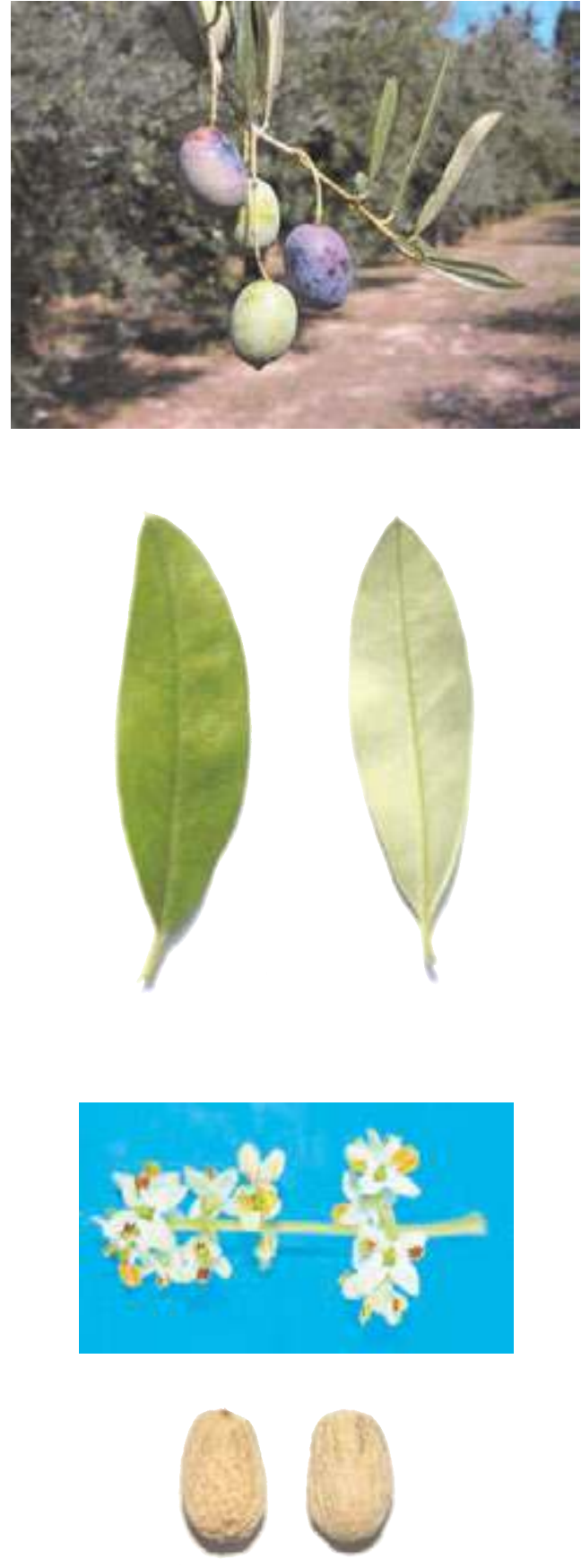


\section{Biochemical Characters}

\section{Fatty Acid Composition}

Table 1. Average values (express in $\% \pm$ standard deviations) of the fatty acids methyl esters and nutritional ratios obtained from single cultivar olive oils.

$\begin{array}{llllll}\text { Myristic acid } & \mathbf{0 , 0 1} \pm \mathbf{0 , 0 1} & \text { Linoleic acid }(\omega 6) & \mathbf{9 , 8 0} \pm \mathbf{1 , 7 7} & \text { Lignoceric acid } & \mathbf{0 , 0 5} \pm \mathbf{0 , 0 3} \\ \text { Palmitic acid } & \mathbf{1 2 , 9 2} \pm \mathbf{2 , 1 8} & \text { Linolenic acid }(\omega 3) \mathbf{0 , 9 4} \pm \mathbf{0 , 0 9} & & \\ \text { Palmitoleic acid } & \mathbf{0 , 7 2} \pm \mathbf{0 , 1 4} & \text { Arachic acid } & \mathbf{0 , 0 7} \pm \mathbf{0 , 0 2} & \text { Unsat./satured } & \mathbf{5 , 6 7 \pm 0 , 9 2} \\ \text { Stearic acid } & \mathbf{1 , 9 9} \pm \mathbf{0 , 2 1} & \text { Eicosenoic acid } & \mathbf{0 , 1 0} \pm \mathbf{0 , 1 2} & \omega 6 / \omega 3 & \mathbf{1 0 , 6 9 \pm 3 , 0 9} \\ \text { Oleic acid } & \mathbf{7 2 , 4 8} \pm 3,09 & \text { Behenic acid } & \mathbf{0 , 0 7} \pm 0,02 & & \end{array}$

\section{Organoleptic oil values}

Sensory Analysis (Panel test)

Comment: fruity medium-high, with hints of almond, and read sensations of artichoke and grass. Balanced taste sensation with a medium bitter and spicy. Medium-high fluidity.

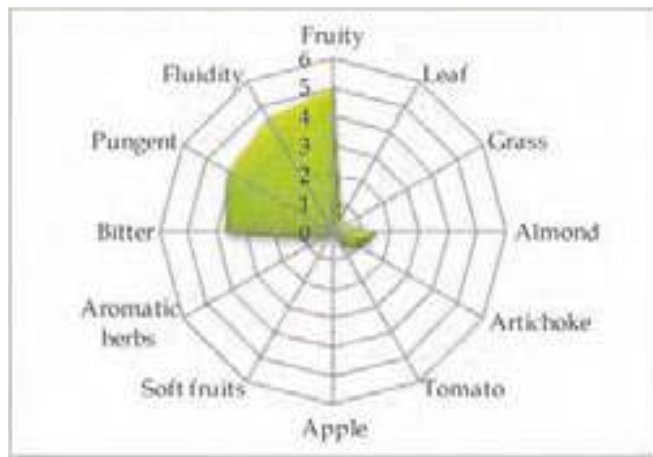

\section{Molecular Markers}

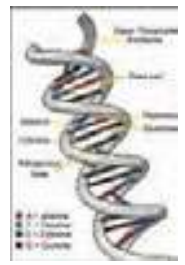

Table 2. Microsatellites (SSR) loci analyzed.

For each locus the allele size (expressed in base pairs) is reported.

$\begin{array}{cccccc}D C A 09 & \text { DCA } 18 & \text { GAPU59 } & \text { GAPU71A } & \text { GUPA71B } & \text { GAPU103A } \\ \mathbf{1 6 2} \mathbf{- 1 8 2} & \mathbf{1 7 9 - \mathbf { 1 7 9 }} & \mathbf{2 0 8 - \mathbf { 2 1 8 }} & \mathbf{2 1 0 - \mathbf { 2 1 4 }} & \mathbf{1 2 4 - \mathbf { 1 4 4 }} & \mathbf{1 3 6} \mathbf{- 1 5 9} \\ \text { UDO01 } & \text { UDO03 } & \text { UDO12 } & \text { UDO28 } & \text { UDO39 } & \\ \mathbf{1 4 0} \mathbf{- 1 4 0} & \mathbf{1 4 3} \mathbf{- 1 4 3} & \mathbf{1 6 6 - \mathbf { 1 7 7 }} & \mathbf{1 8 2} \mathbf{- 1 8 2} & \mathbf{2 0 5 - \mathbf { 2 3 2 }} & \end{array}$

\section{References:}

1 - Lombardo N., Perri E., Muzzalupo I., et al. In: Il germoplasma olivicolo Calabrese, Ist. Sper. Olivic. (2003), pp: 7-8.

2 - Perri E., Mazzotti F., Muzzalupo I., et al. In: Relazione attività CO.R.ASS.OL. (2003).

3 - Muzzalupo I., Stefanizzi F., Perri E. HortScience (2009), 44: pp. 582-588. 


\section{"Grossa di Spanga "}

(synonymy: Bella della Daunia, Bella di Cerignola, Manna, Oliva a prugna, etc.)

Areal distribution or origin area: Puglia

Flesh/pit weight ratio: high $(8,40 \pm 0,21)$

Oil content (\%): medium $(45,63 \pm 1,75)$

Purpose: table

\section{Morphological characters}

Tree characters

Vigour: weak

Growth habit: spreading

Canopy-density: medium-dense

\section{Leaf characters}

Blade length $(\mathrm{cm})$ : long $(7,07 \pm 0,56)$

Blade width $(\mathrm{cm})$ : medium $(1,45 \pm 0,20)$

Shape (length/width): elliptic-lanceolate

\section{Inflorescence characters}

Inflorescence length (cm): medium $(2,93 \pm 0,53)$

Number of flowers: low $(13.91 \pm 1,79)$

\section{Fruit characters}

Fresh weight of 100 fruits (g): very high $(9,07 \pm 1,83$ )

Shape (length/width): elongated

Symmetry: slightly asymmetric

Position of maximum transverse diameter:

towards apex

Apex: rounded

Base: rounded

Niplle: tenuous

Lenticels: many and large

\section{Pit characters}

Weight of 100 pits (g): very high $(0,98 \pm 0,15)$

Shape (length/width): elongated

Mucron: absent

Symmetry: asymmetric

Position of maximum transverse diameter:

towards apex

Apex: pointed

Base: pointed

Surface: rugose

Number of grooves: high
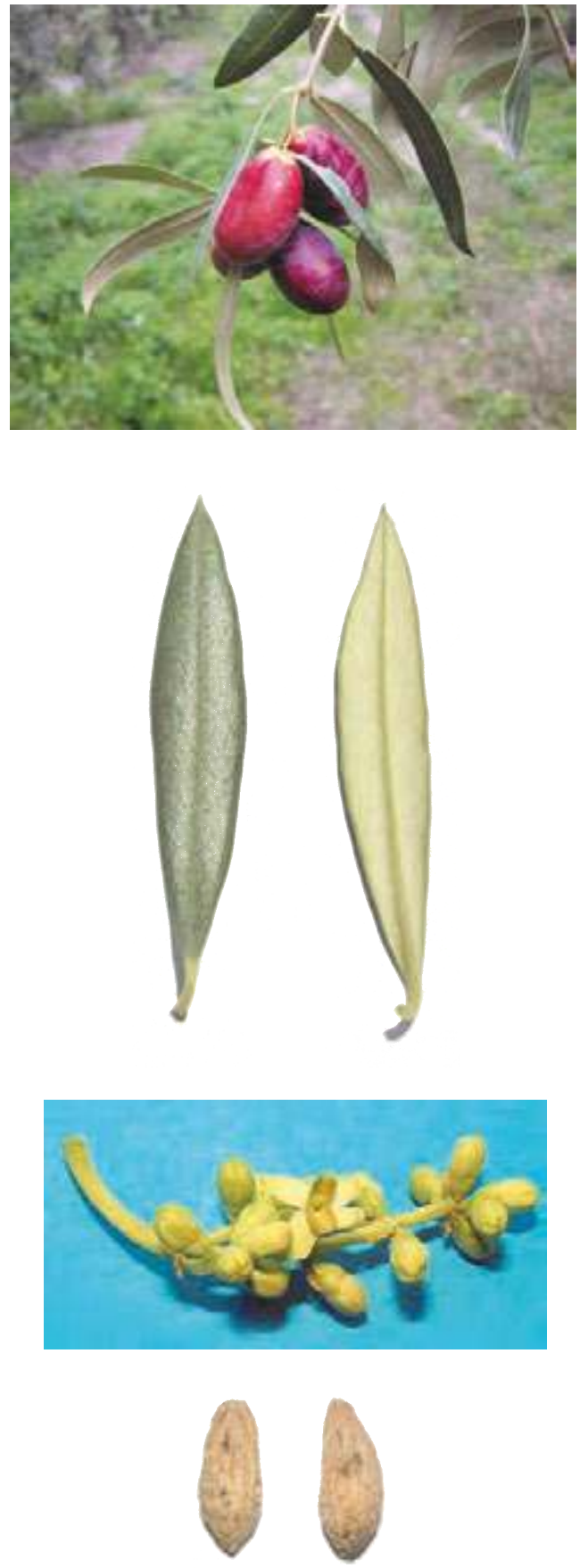


\section{Biochemical Characters}

\section{Fatty Acid Composition}

Table 1. Average values (express in $\% \pm$ standard deviations) of the fatty acids methyl esters and nutritional ratios obtained from single cultivar olive oils.

$\begin{array}{llllll}\text { Myristic acid } & \mathbf{0 , 0 1} \pm \mathbf{0 , 0 1} & \text { Linoleic acid }(\omega 6) & \mathbf{8 , 6 1} \pm \mathbf{0 , 1 2} & \text { Lignoceric acid } & \mathbf{0 , 0 4} \pm \mathbf{0 , 0 3} \\ \text { Palmitic acid } & \mathbf{1 4 , 2 9} \pm \mathbf{0 , 6 7} & \text { Linolenic acid }(\omega 3) \mathbf{0 , 8 3} \pm \mathbf{0 , 2 3} & & \\ \text { Palmitoleic acid } & \mathbf{0 , 8 6} \pm \mathbf{0 , 0 5} & \text { Arachic acid } & \mathbf{0 , 3 0} \pm \mathbf{0 , 1 4} & \text { Unsat./satured } & \mathbf{4 , 7 9} \pm \mathbf{0 , 6 8} \\ \text { Stearic acid } & \mathbf{2 , 7 1} \pm \mathbf{1 , 2 8} & \text { Eicosenoic acid } & \mathbf{0 , 1 2} \pm \mathbf{0 , 1 4} & \omega 6 / \omega 3 & \mathbf{1 0 , 7 6} \pm \mathbf{2 , 7 9} \\ \text { Oleic acid } & \mathbf{7 1 , 3 1} \pm \mathbf{1 , 6 8} & \text { Behenic acid } & \mathbf{0 , 0 8} \pm \mathbf{0 , 0 3} & & \end{array}$

\section{Organoleptic oil values}

Sensory Analysis (Panel test)

Comment: medium-high fruity, with good sensation of grass, less almond and artichoke. Balanced taste sensation, with bitter and spicy medium. Medium-high fluidity.

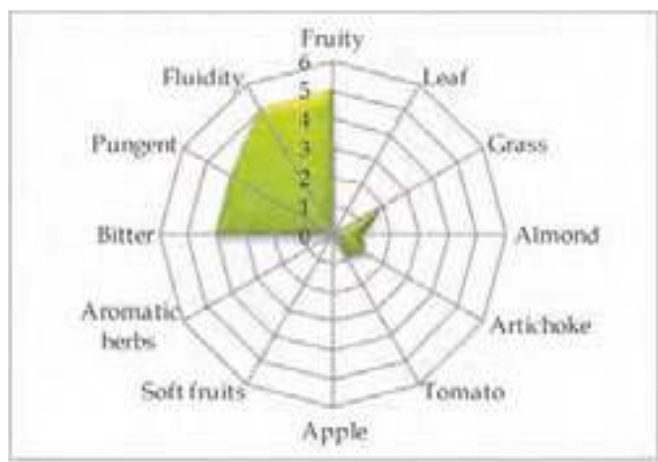

\section{Molecular Markers}

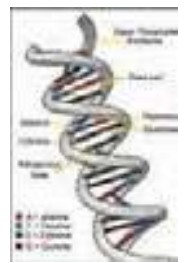

Table 2. Microsatellites (SSR) loci analyzed.

For each locus the allele size (expressed in base pairs) is reported.

$\begin{array}{cccccc}D C A 09 & \text { DCA } 18 & \text { GAPU59 } & \text { GAPU71A } & \text { GUPA71B } & \text { GAPU103A } \\ \mathbf{1 6 6 - \mathbf { 1 7 2 }} & \mathbf{1 7 7 - \mathbf { 1 7 7 }} & \mathbf{2 0 8 - \mathbf { 2 0 8 }} & \mathbf{2 1 0 - \mathbf { 2 1 4 }} & \mathbf{1 2 4 - \mathbf { 1 4 4 }} & \mathbf{1 3 6} \mathbf{- 1 8 4} \\ \text { UDO01 } & \text { UDO03 } & \text { UDO12 } & \text { UDO28 } & \text { UDO39 } & \\ \mathbf{1 4 4} \mathbf{- 1 4 4} & \mathbf{1 4 3} \mathbf{- 1 4 3} & \mathbf{1 6 6 - \mathbf { 1 6 6 }} & \mathbf{1 4 3} \mathbf{- 2 4 5} & \mathbf{1 0 8} \mathbf{- 1 0 8} & \end{array}$

\section{References:}

1 - Lombardo N., Perri E., Muzzalupo I., et al. In: Contributo alla caratterizzazione del germoplasma olivicolo pugliese, Ist. Sper. Olivic. (2004), pp. 25-28.

2 - Muzzalupo I., Stefanizzi F., Perri E. HortScience (2009), 44: pp. 582-588. 


\section{"Grossa di Venafro"}

Areal distribution or origin area: Molise

Flesh/pit weight ratio: medium $(6,40 \pm 0,33)$

Oil content (\%): medium $(46,92 \pm 1,03)$

Purpose: oil

\section{Morphological characters}

Tree characters

Vigour: medium-strong

Growth habit: spreading-erect

Canopy-density: medium-sparse

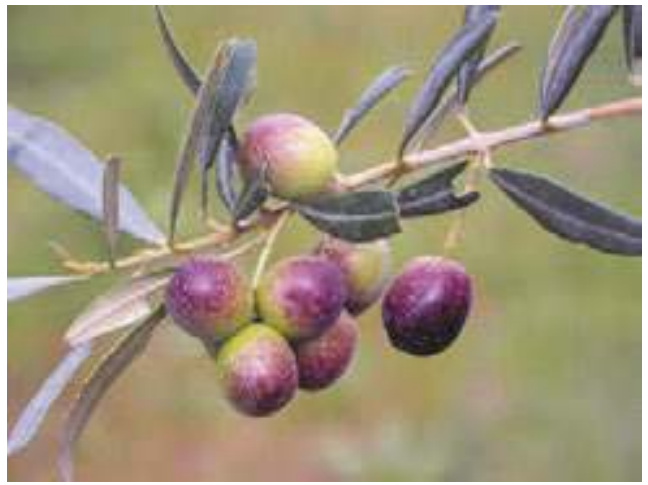

\section{Leaf characters}

Blade length $(\mathrm{cm})$ : medium $(5,41 \pm 0,48)$

Blade width $(\mathrm{cm})$ : medium $(\mathbf{1 , 3 1} \pm 0,17)$

Shape (length/width): elliptic - lanceolate

\section{Inflorescence characters}

Inflorescence length $(\mathrm{cm})$ : long $(3,69 \pm 0,54)$

Number of flowers: high $(2,78 \pm 2,27)$

\section{Fruit characters}

Fresh weight of 100 fruits $(\mathrm{g})$ : medium $(\mathbf{2 , 1 0} \pm \mathbf{0 , 0 2})$

Shape (length/width): spherical

Symmetry: slightly asymmetric

Position of maximum transverse diameter:

central

Apex: rounded

Base: truncate

Niplle: absent

Lenticels: many and small

\section{Pit characters}

Weight of 100 pits (g): low $(\mathbf{0 , 2 8} \pm \mathbf{0 , 0 1})$

Shape (length/width): ovoid

Mucron: tenuous

Symmetry: slightly asymmetric

Position of maximum transverse diameter:

central

Apex: rounded

Base: rounded

Surface: rugose

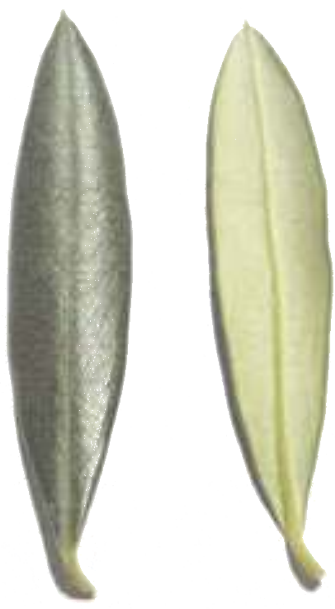

Number of grooves: medium
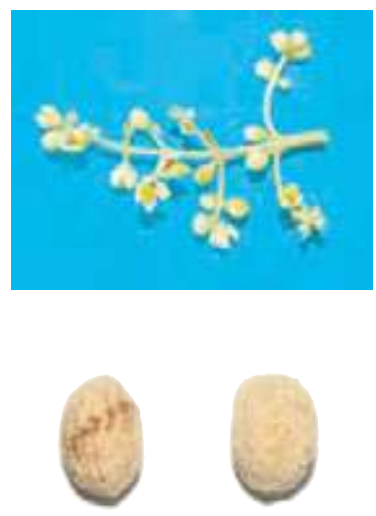


\section{Biochemical Characters}

\section{Fatty Acid Composition}

Table 1. Average values (express in $\% \pm$ standard deviations) of the fatty acids methyl esters and nutritional ratios obtained from single cultivar olive oils.

$\begin{array}{llllll}\text { Myristic acid } & \mathbf{0 , 0 1} \pm \mathbf{0 , 0 0} & \text { Linoleic acid }(\omega 6) & \mathbf{8 , 8 2} \pm \mathbf{0 , 6 7} & \text { Lignoceric acid } & \mathbf{0 , 0 2} \pm \mathbf{0 , 0 0} \\ \text { Palmitic acid } & \mathbf{1 5 , 2 4} \pm \mathbf{0 , 3 6} & \text { Linolenic acid }(\omega 3) \mathbf{0 , 8 7} \pm \mathbf{0 , 0 4} & & \\ \text { Palmitoleic acid } & \mathbf{1 , 3 5} \pm \mathbf{0 , 0 2} & \text { Arachic acid } & \mathbf{0 , 2 4} \pm \mathbf{0 , 0 0} & \text { Unsat./satured } & \mathbf{4 , 8 0} \pm \mathbf{0 , 0 7} \\ \text { Stearic acid } & \mathbf{1 , 6 4} \pm \mathbf{0 , 1 4} & \text { Eicosenoic acid } & \mathbf{0 , 0 2} \pm \mathbf{0 , 0 0} & \omega 6 / \omega 3 & \mathbf{1 0 , 0 8} \pm \mathbf{0 , 3 5} \\ \text { Oleic acid } & \mathbf{7 0 , 6 3 \pm 0 , 4 8} & \text { Behenic acid } & \mathbf{0 , 0 6} \pm \mathbf{0 , 0 0} & & \end{array}$

\section{Organoleptic oil values}

Sensory Analysis (Panel test)

Comment: medium fruity, with good sensation of grass, less almond and artichoke. Balanced taste sensation, with bitter and spicy medium. Medium fluidity.

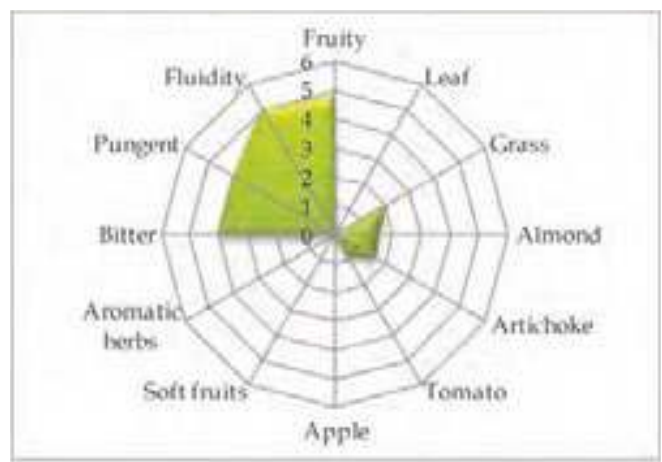

\section{Molecular Markers}

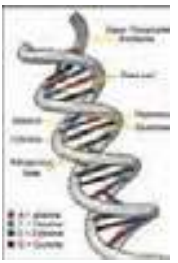

Table 2. Microsatellites (SSR) loci analyzed.

For each locus the allele size (expressed in base pairs) is reported.

\begin{tabular}{|c|c|c|c|c|c|}
\hline$D C A 09$ & $D C A 18$ & GAPU59 & GAPU71A & GUPA71B & GAPU103A \\
\hline $162-204$ & $177-177$ & $212-222$ & $214-214$ & $124-130$ & $150-150$ \\
\hline UDO01 & UDO03 & UDO12 & UDO28 & UDO39 & \\
\hline $144-144$ & $150-150$ & $177-193$ & $154-205$ & $213-213$ & \\
\hline
\end{tabular}

\section{References:}

1 - Consiglio Oleicolo Internazionale. In: Catalogo Mondiale delle Varietà di Olivo, COI (2003).

2 - Muzzalupo I., Lombardo N., Salimonti A., et al. Adv. Hort. Sci. (2008), 22(2): pp. 142-148. 


\section{"Grossale "}

(synonymy: Provenzale, Provenzana, Ursale, etc.)

Areal distribution or origin area: Campania

Flesh/pit weight ratio: medium $(6,82 \pm 0,49)$

Oil content (\%): medium $(42,43 \pm 0,72)$

Purpose: oil

\section{Morphological characters}

Tree characters

Vigour: medium-weak

Growth habit: spreading

Canopy-density: medium

\section{Leaf characters}

Blade length $(\mathrm{cm})$ : medium $(5,26 \pm 0,44)$

Blade width $(\mathrm{cm})$ : medium $(1,06 \pm 0,09)$

Shape (length/width): elliptic-lanceolate

\section{Inflorescence characters}

Inflorescence length $(\mathrm{cm})$ : medium $(2,87 \pm 1,46)$

Number of flowers: medium $(\mathbf{1 8 , 2 2} \pm \mathbf{1 , 5 4})$

\section{Fruit characters}

Fresh weight of 100 fruits (g): medium $(2,73 \pm 0,01)$

Shape (length/width): spherical

Symmetry: symmetric

Position of maximum transverse diameter:

towards base

Apex: rounded

Base: rounded

Niplle: absent

Lenticels: many and small

\section{Pit characters}

Weight of 100 pits (g): medium $(0,35 \pm 0,02)$

Shape (length/width): ovoid

Mucron: absent

Symmetry: slightly asymmetric

Position of maximum transverse diameter:

central

Apex: rounded

Base: rounded

Surface: rugose

Number of grooves: medium
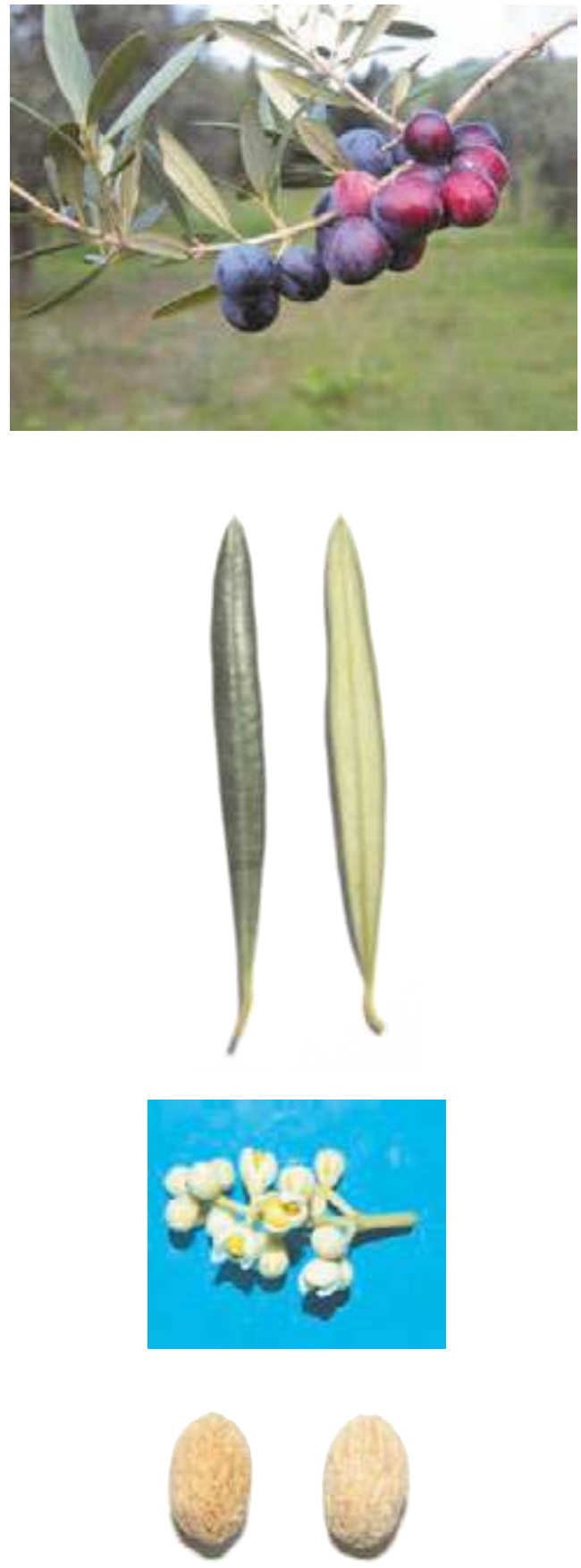


\section{Biochemical Characters}

\section{Fatty Acid Composition}

Table 1. Average values (express in $\% \pm$ standard deviations) of the fatty acids methyl esters and nutritional ratios obtained from single cultivar olive oils.

$\begin{array}{lllll}\text { Myristic acid } & \mathbf{0 , 0 1} \pm \mathbf{0 , 0 0} \quad \text { Linoleic acid }(\omega 6) & \mathbf{1 2 , 0 3} \pm \mathbf{1 , 1 0} \quad \text { Lignoceric acid } & \mathbf{0 , 0 2} \pm \mathbf{0 , 0 1}\end{array}$

Palmitic acid $16,20 \pm 0,70 \quad$ Linolenic acid $(\omega 3) 0,95 \pm 0,04$

Palmitoleic acid

$1,36 \pm 0,01$

Arachic acid

$0,16 \pm 0,03$

Unsat./satured

$4,52 \pm 0,23$

Stearic acid

$1,60 \pm 0,06$

Eicosenoic acid

$0,06 \pm 0,00$

$\omega 6 / \omega 3$

$12,72 \pm 1,74$

Oleic acid

$66,47 \pm 0,32$

Behenic acid

$0,03 \pm 0,01$

\section{Organoleptic oil values}

Sensory Analysis (Panel test)

Comment: medium fruity, with good sensation of grass, less almond and artichoke. Balanced taste sensation, with bitter and spicy medium. Medium fluidity.

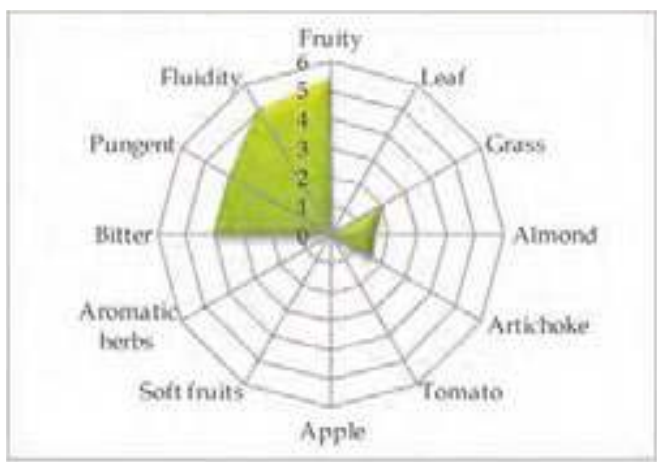

\section{Molecular Markers}

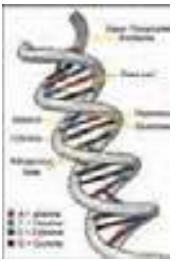

Table 2. Microsatellites (SSR) loci analyzed.

For each locus the allele size (expressed in base pairs) is reported.

\begin{tabular}{|c|c|c|c|c|c|}
\hline DCA09 & DCA18 & GAPU59 & GAPU71A & GUPA71B & GAPU103A \\
\hline $194-198$ & 179 - 181 & $214-214$ & $214-214$ & $126-144$ & 157 - 159 \\
\hline UDO01 & UDO03 & UDO12 & UDO28 & UDO39 & \\
\hline $144-144$ & $143-150$ & $166-166$ & $143-143$ & $205-239$ & \\
\hline
\end{tabular}

\section{References:}

1 - Pugliano G., Flaminio G., Pugliano M.L., et al. In: La risorsa genetica dell'olivo in Campania, SE. S.I.R.C.A. Ed. Napoli, (2000).

2 - Muzzalupo I., Lombardo N., Salimonti A., et al. Adv. Hort. Sci. (2008), 22(2): pp. 142-148. 


\section{“ I $77 "$}

Areal distribution or origin area: Umbria

Flesh/pit weight ratio: medium $(5,02 \pm 0,02)$

Oil content (\%): medium $(48,36 \pm 0,10)$

Purpose: oil

\section{Morphological characters}

Tree characters

Vigour: medium-weak

Growth habit: spreading

Canopy-density: medium

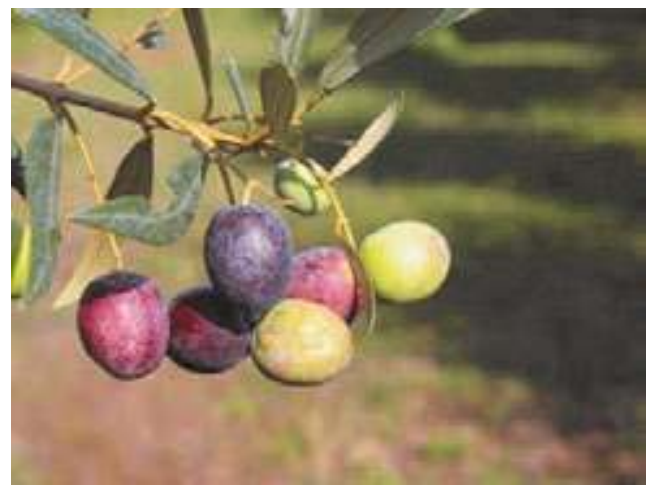

\section{Leaf characters}

Blade length $(\mathrm{cm})$ : medium $(6,82 \pm 0,64)$

Blade width $(\mathrm{cm})$ : medium $(1,38 \pm 0,18)$

Shape (length/width): elliptic lanceolate

\section{Inflorescence characters}

Inflorescence length $(\mathrm{cm})$ : medium $(3,62 \pm 0,68)$

Number of flowers: medium $(20,89 \pm 2,38)$

\section{Fruit characters}

Fresh weight of 100 fruits $(\mathrm{g})$ : medium $(3,73 \pm 0,23)$

Shape (length/width): spherical

Symmetry: slightly asymmetric

Position of maximum transverse diameter:

central

Apex: rounded

Base: truncate

Niplle: tenuous

Lenticels: many and small

\section{Pit characters}

Weight of 100 pits (g): high $(\mathbf{0 , 6 2} \pm \mathbf{0 , 0 4})$

Shape (length/width): ovoid

Mucron: obvious

Symmetry: slightly asymmetric

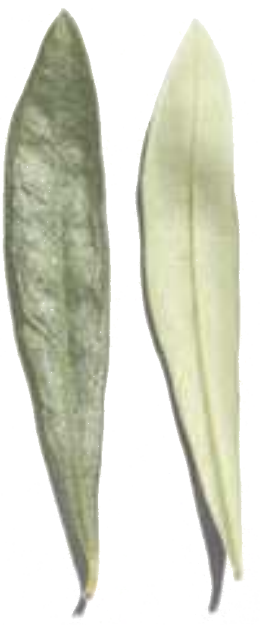

Position of maximum transverse diameter:

towards apex

Apex: rounded

Base: rounded

Surface: rugose

Number of grooves: medium
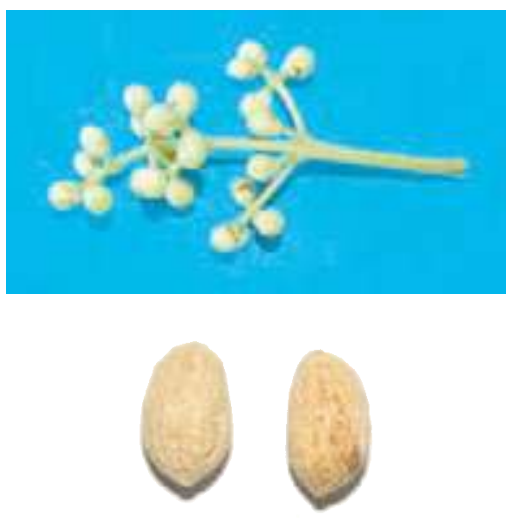


\section{Biochemical Characters}

\section{Fatty Acid Composition}

Table 1. Average values (express in $\% \pm$ standard deviations) of the fatty acids methyl esters and nutritional ratios obtained from single cultivar olive oils.

\begin{tabular}{|c|c|c|c|c|c|}
\hline Myristic acid & $0,01 \pm 0,01$ & Linoleic acid $(\omega 6)$ & $9,28 \pm 0,04$ & Lignoceric acid & $0,05 \pm 0,02$ \\
\hline Palmitic acid & $10,09 \pm 0,83$ & Linolenic acid $(\omega 3)$ & $0,92 \pm 0,04$ & & \\
\hline Palmitoleic acid & $0,86 \pm 0,43$ & Arachic acid & $0,28 \pm 0,07$ & Unsat./satured & $7,32 \pm 0,53$ \\
\hline ea & $1,63 \pm 0,03$ & Eicosenoic acid & $0,04 \pm 0,01$ & $\omega 6 / \omega 3$ & $10,14 \pm 0,44$ \\
\hline leic acid & $76,10 \pm 0,26$ & Behenic acid & $0,09 \pm 0,02$ & & \\
\hline
\end{tabular}

\section{Organoleptic oil values}

Sensory Analysis (Panel test)

Comment: fruity medium-high, with hints of almond and read sensations of grass and artichoke. Balanced taste sensation with a medium-high bitter and spicy. Medium-high fluidity.

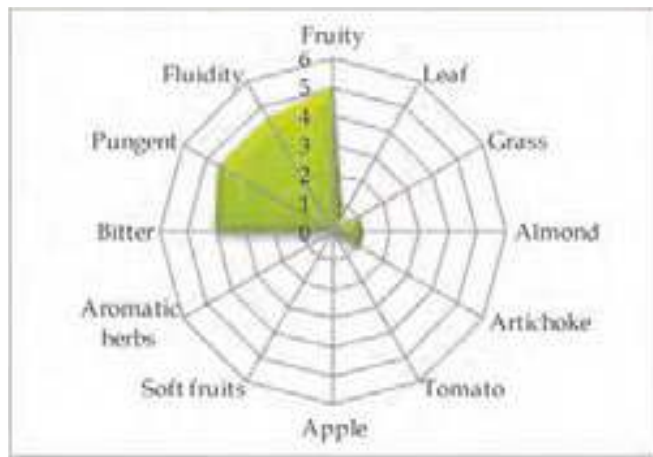

\section{Molecular Markers}

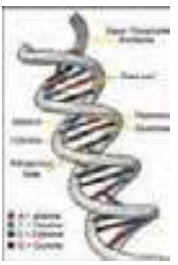

Table 2. Microsatellites (SSR) loci analyzed.

For each locus the allele size (expressed in base pairs) is reported.

\begin{tabular}{|c|c|c|c|c|c|}
\hline$D C A 09$ & $D C A 18$ & GAPU59 & GAPU71A & GUPA71B & GAPU103A \\
\hline $198-206$ & $179-179$ & $218-222$ & $218-218$ & $124-130$ & $150-157$ \\
\hline UDO01 & UDO03 & UDO12 & UDO28 & UDO39 & \\
\hline $140-140$ & $135-135$ & $166-177$ & $154-205$ & $205-205$ & \\
\hline
\end{tabular}

\section{References:}

1 - Lombardo N. In: Le risorse genetiche vegetali presso gli IRSA, MIPAF (2001), 1: pp. 361-405

2 - Muzzalupo I., Stefanizzi F., Bucci C., et al. In Acta Italus Hortus, (2011), 1: 138 -140. 


\section{“Imperiale"}

Areal distribution or origin area: Calabria

Flesh/ pit weight ratio: very high $(\mathbf{1 2 , 8 2} \pm \mathbf{1 , 1 4})$

Oil content (\%): high $\mathbf{( 5 0 , 6 5 \pm 0 , 5 0 )}$

Purpose: dual purpose

\section{Morphological characters}

Tree characters

Vigour: medium-strong

Growth habit: erect-spreading

Canopy-density: medium

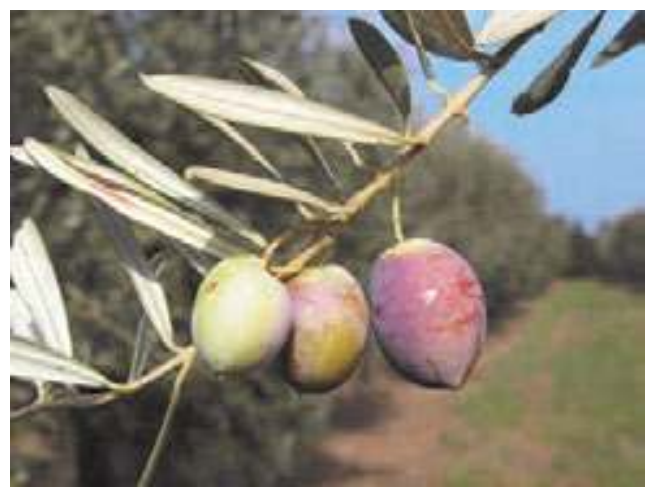

\section{Leaf characters}

Blade length $(\mathrm{cm})$ : medium $(5,51 \pm 0,69)$

Blade width (cm): medium $(1,19 \pm 0,19)$

Shape (length/width): elliptic-lanceolate

\section{Inflorescence characters}

Inflorescence length (cm): short $(2,43 \pm 0,90)$

Number of flowers: low $(16,43 \pm 2,43)$

\section{Fruit characters}

Fresh weight of 100 fruits (g): very high $(9,38 \pm 0,63)$

Shape (length/width): ovoid

Symmetry: slightly asymmetric

Position of maximum transverse diameter:

central

Apex: rounded

Base: truncate

Niplle: obvious

Lenticels: many and large

\section{Pit characters}

Weight of 100 pits (g): high $(\mathbf{0 , 6 8} \pm \mathbf{0 , 0 8})$

Shape (length/width): elliptic

Mucron: obvious

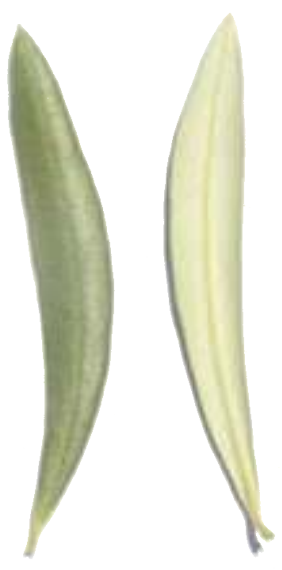

Symmetry: slightly asymmetric

Position of maximum transverse diameter:

towards apex

Apex: rounded

Base: pointed

Surface: scabrous

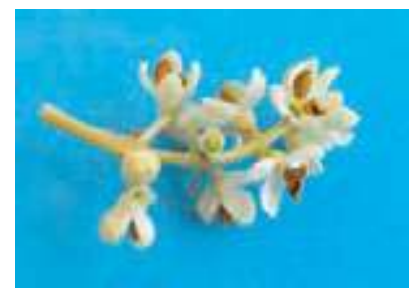

Number of grooves: high 


\section{Biochemical Characters}

\section{Fatty Acid Composition}

Table 1. Average values (express in $\% \pm$ standard deviations) of the fatty acids methyl esters and nutritional ratios obtained from single cultivar olive oils.

\begin{tabular}{|c|c|c|c|c|c|}
\hline Myristic acid & $0,01 \pm 0,01$ & Linoleic acid $(\omega 6)$ & $10,63 \pm 1,53$ & Lignoceric acid & $0,07 \pm 0,03$ \\
\hline Palmitic acid & $10,93 \pm 1,75$ & Linolenic acid $(\omega 3)$ & $0,92 \pm 0,04$ & & \\
\hline Palmitoleic acid & $0,95 \pm 0,31$ & Arachic acid & $0,40 \pm 0,11$ & Unsat./satured & $6,16 \pm 0,75$ \\
\hline tearic acid & $2,67 \pm 0,21$ & Eicosenoic acid & $0,21 \pm 0,21$ & $\omega 6 / \omega 3$ & $11,49 \pm 1,26$ \\
\hline leic acid & $72,07 \pm 1,53$ & Behenic acid & $0,12 \pm 0,06$ & & \\
\hline
\end{tabular}

\section{Organoleptic oil values}

Sensory Analysis (Panel test)

Comment: fruity medium-high, with good sensation of grass, artichoke and tomato. Balanced in flavours, with hints of bitter and spicy medium-high intensity. Medium-high fluidity.

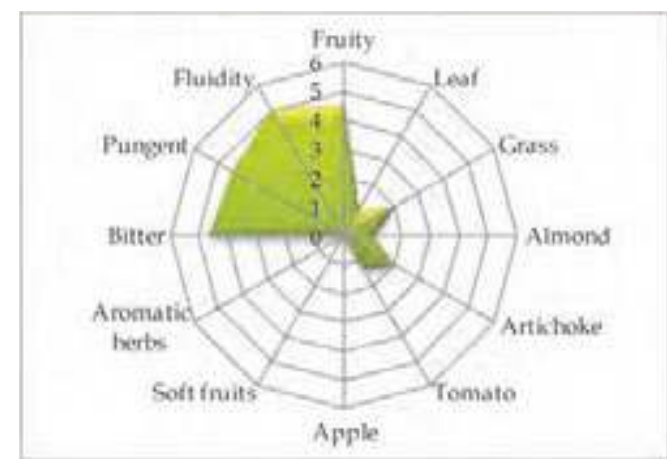

\section{Molecular Markers}

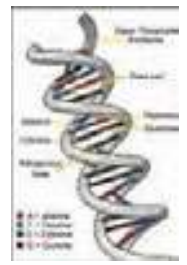

Table 2. Microsatellites (SSR) loci analyzed.

For each locus the allele size (expressed in base pairs) is reported.

\begin{tabular}{|c|c|c|c|c|c|}
\hline$D C A 09$ & $D C A 18$ & GAPU59 & GAPU71A & GUPA71B & GAPU103A \\
\hline $182-198$ & $177-185$ & $208-212$ & $210-250$ & $130-144$ & $159-184$ \\
\hline UDO01 & UDO03 & UDO12 & UDO28 & UDO39 & \\
\hline $144-144$ & $150-150$ & $166-193$ & $143-143$ & $213-232$ & \\
\hline
\end{tabular}

\section{References:}

1 - Lombardo N., Perri E., Muzzalupo I., et al. In: Il germoplasma olivicolo Calabrese, Ist. Sper. Olivic. (2003), pp: 21.

2 - Perri E., Mazzotti F., Muzzalupo I., et al. In: Relazione attività CO.R.ASS.OL. (2003).

3 - Muzzalupo I., Stefanizzi F., Perri E. HortScience (2009), 44: pp. 582-588. 


\section{"Intosso"}

(synonymy: Grossa, Indossa, Olivo da cuccare, etc.)

Areal distribution or origin area: Abruzzo

Flesh/pit weight ratio: high $(\mathbf{9 , 1 8} \pm \mathbf{0 , 2 6})$

Oil content (\%): medium $(42,80 \pm 0,25)$

Purpose: dual purpose

\section{Morphological characters}

Tree characters

Vigour: medium-weak

Growth habit: spreading

Canopy-density: medium

\section{Leaf characters}

Blade length $(\mathrm{cm})$ : medium $(5,62 \pm 0,63)$

Blade width $(\mathrm{cm})$ : medium $(\mathbf{1}, \mathbf{4 6} \pm \mathbf{0 , 1 7})$

Shape (length/width): elliptic

\section{Inflorescence characters}

Inflorescence length $(\mathrm{cm})$ : medium $(2,93 \pm 0,43)$

Number of flowers: medium $(19,37 \pm 3,71)$

\section{Fruit characters}

Fresh weight of 100 fruits (g): medium $(4,36 \pm 0,37)$

Shape (length/width): ovoid

Symmetry: slightly asymmetric

Position of maximum transverse diameter:

central

Apex: rounded

Base: truncate

Niplle: tenuous

Lenticels: many and small

\section{Pit characters}

Weight of 100 pits (g): medium $(0, \mathbf{4} \pm \mathbf{0 , 0 1}$ )

Shape (length/width): elliptic

Mucron: obvious

Symmetry: slightly asymmetric

Position of maximum transverse diameter:

towards apex

Apex: pointed

Base: pointed

Surface: rugose

Number of grooves: high
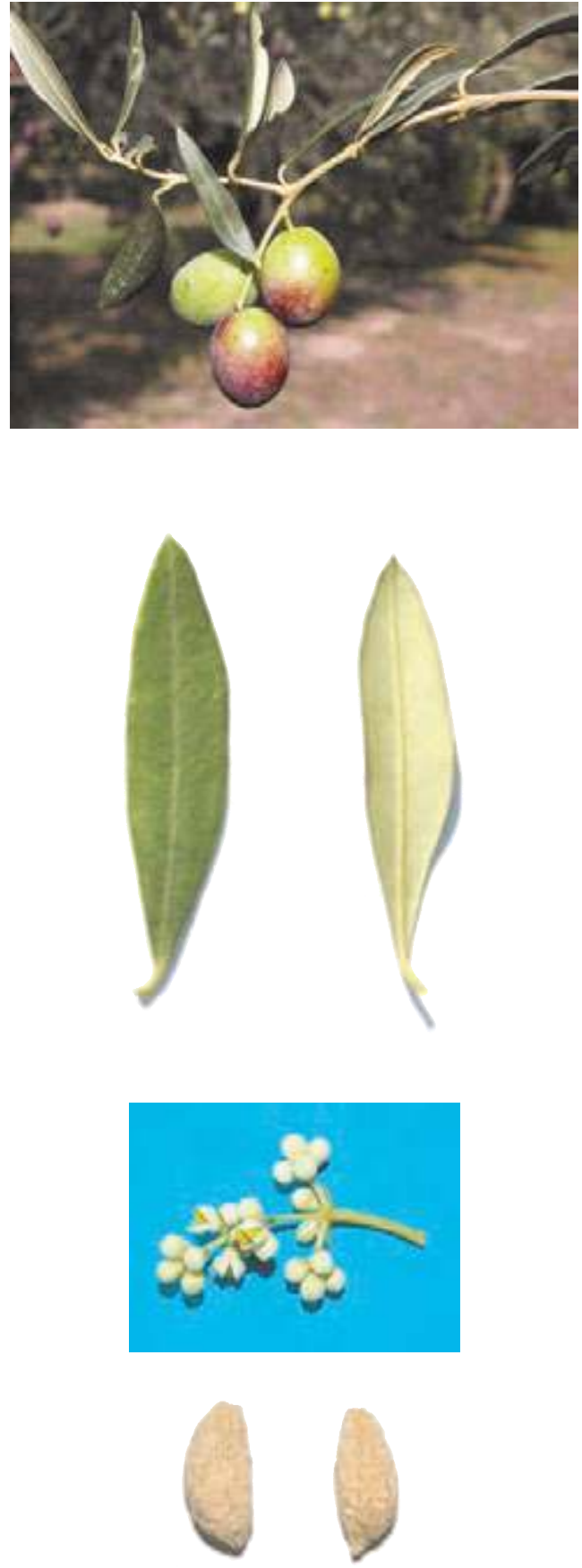


\section{Biochemical Characters}

\section{Fatty Acid Composition}

Table 1. Average values (express in $\% \pm$ standard deviations) of the fatty acids methyl esters and nutritional ratios obtained from single cultivar olive oils.

$\begin{array}{llllll}\text { Myristic acid } & \mathbf{0 , 0 1} \pm \mathbf{0 , 0 1} & \text { Linoleic acid }(\omega 6) & \mathbf{9 , 6 2} \pm \mathbf{1 , 6 5} & \text { Lignoceric acid } & \mathbf{0 , 0 4} \pm \mathbf{0 , 0 1} \\ \text { Palmitic acid } & \mathbf{1 0 , 1 2} \pm \mathbf{0 , 1 6} & \text { Linolenic acid }(\omega 3) \mathbf{0 , 8 6} \pm \mathbf{0 , 0 4} & & \\ \text { Palmitoleic acid } & \mathbf{0 , 5 1} \pm \mathbf{0 , 0 8} & \text { Arachic acid } & \mathbf{0 , 4 9} \pm \mathbf{0 , 2 1} & \text { Unsat./satured } & \mathbf{6 , 6 4 \pm 0 , 3 9} \\ \text { Stearic acid } & \mathbf{2 , 6 6} \pm \mathbf{0 , 3 3} & \text { Eicosenoic acid } & \mathbf{0 , 0 2} \pm \mathbf{0 , 0 0} & \omega 6 / \omega 3 & \mathbf{1 1 , 1 5} \pm \mathbf{1 , 3 7} \\ \text { Oleic acid } & \mathbf{7 5 , 1 8} \pm \mathbf{0 , 4 9} & \text { Behenic acid } & \mathbf{0 , 0 8} \pm \mathbf{0 , 0 1} & & \end{array}$

\section{Organoleptic oil values}

Sensory Analysis (Panel test)

Comment: fruity medium-high, with good sensation of grass, artichoke and tomato. Balanced in flavours, with hints of bitter and spicy medium-high intensity. Medium-high fluidity.

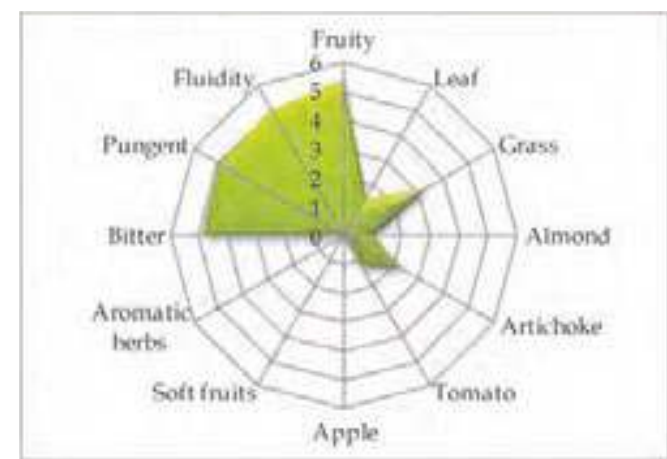

\section{Molecular Markers}

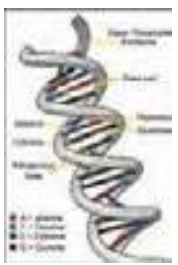

Table 2. Microsatellites (SSR) loci analyzed.

For each locus the allele size (expressed in base pairs) is reported.

$\begin{array}{cccccc}D C A 09 & \text { DCA18 } & \text { GAPU59 } & \text { GAPU71A } & \text { GUPA71B } & \text { GAPU103A } \\ \mathbf{1 6 2 - \mathbf { 2 1 0 }} & \mathbf{1 7 9 - \mathbf { 1 8 1 }} & \mathbf{2 1 2} \mathbf{- 2 2 2} & \mathbf{2 1 0 - \mathbf { 2 1 4 }} & \mathbf{1 2 4 - \mathbf { 1 3 0 }} & \mathbf{1 5 9 - \mathbf { 1 8 4 }} \\ \text { UDOO1 } & \text { UDOO3 } & \text { UDO12 } & \text { UDO28 } & \text { UDO39 } & \\ \mathbf{1 4 0 - \mathbf { 1 4 0 }} & \mathbf{1 6 6 - \mathbf { 1 6 6 }} & \mathbf{1 6 6 - \mathbf { 1 6 6 }} & \mathbf{1 5 4 - \mathbf { 2 0 5 }} & \mathbf{2 0 5 - \mathbf { 2 0 5 }} & \end{array}$

\section{References:}

1 - Pietrangeli E., Russo A., In: Olivi D'Abruzzo, Grafiche di Prinzio (1997), pp. 44-45.

2 - Muzzalupo I., Salimonti A., Caravita M. A., et al. Adv. Hort. Sci. (2008), 22(2): pp. 129-135. 


\section{“Itrana"}

(synonymy: Aitana, Cicerone, Gaetana, Oliva di Gaeta, Oliva di Itri, Trana, etc.)

Areal distribution or origin area: Lazio

Flesh/pit weight ratio: low $(4,59 \pm 1,17)$

Oil content (\%): medium $(44,93 \pm 2,43)$

Purpose: dual purpose

\section{Morphological characters}

Tree characters

Vigour: medium-strong

Growth habit: spreading

Canopy-density: medium-dense

\section{Leaf characters}

Blade length $(\mathrm{cm})$ : medium $(5,80 \pm 0,93)$

Blade width $(\mathrm{cm})$ : medium $(\mathbf{1}, 40 \pm \mathbf{0 , 2 2})$

Shape (length/width): elliptic-lanceolate

\section{Inflorescence characters}

Inflorescence length $(\mathrm{cm})$ : medium $(2,98 \pm 0,97)$

Number of flowers: low $(16,790,93)$

\section{Fruit characters}

Fresh weight of 100 fruits $(\mathrm{g})$ : medium $(3,03 \pm 1,12)$

Shape (length/width): spherical

Symmetry: slightly asymmetric

Position of maximum transverse diameter:

central

Apex: rounded

Base: rounded

Niplle: tenuous

Lenticels: many and small

\section{Pit characters}

Weight of 100 pits (g): high $(\mathbf{0 , 5 1} \pm \mathbf{0 , 1 8})$

Shape (length/width): ovoid

Mucron: obvious

Symmetry: slightly asymmetric

Position of maximum transverse diameter:

central

Apex: rounded

Base: pointed

Surface: rugose

Number of grooves: medium
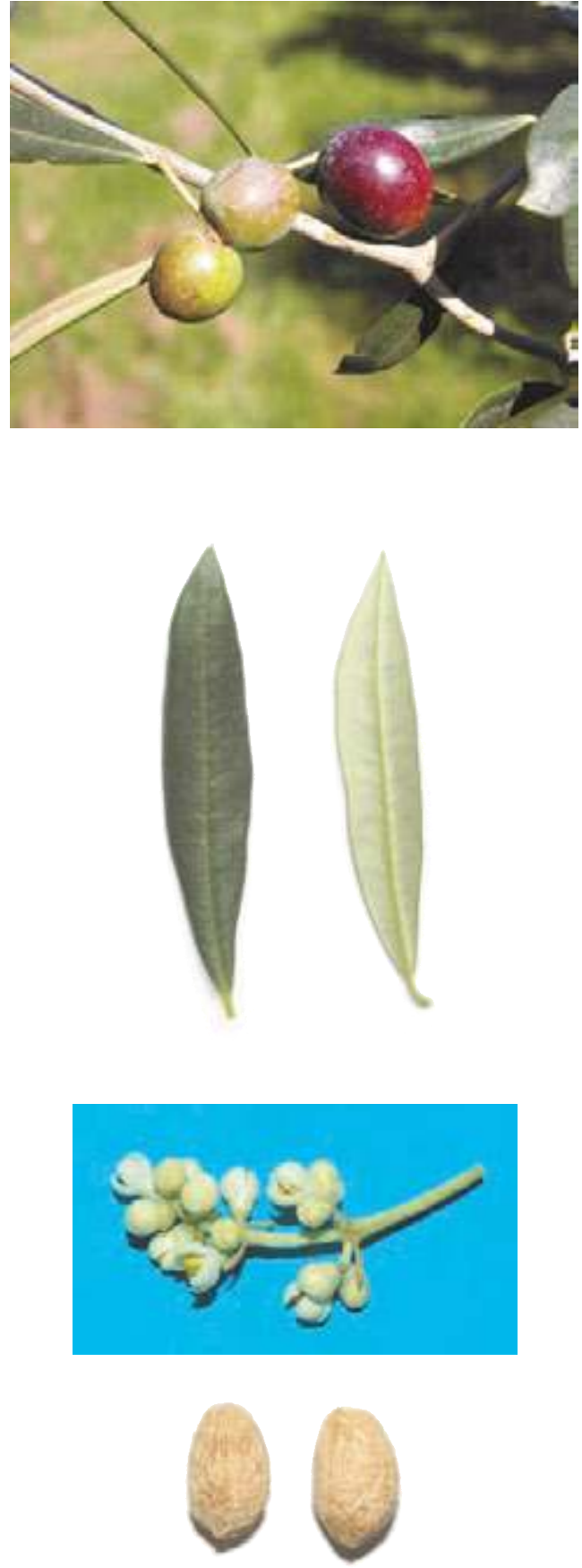


\section{Biochemical Characters}

\section{Fatty Acid Composition}

Table 1. Average values (express in $\% \pm$ standard deviations) of the fatty acids methyl esters and nutritional ratios obtained from single cultivar olive oils.

$\begin{array}{llllll}\text { Myristic acid } & \mathbf{0 , 0 1} \pm \mathbf{0 , 0 0} & \text { Linoleic acid }(\omega 6) & \mathbf{6 , 2 0} \pm \mathbf{0 , 7 2} & \text { Lignoceric acid } & \mathbf{0 , 0 2} \pm \mathbf{0 , 0 0} \\ \text { Palmitic acid } & \mathbf{1 3 , 4 8} \pm \mathbf{1 , 8 7} & \text { Linolenic acid }(\omega 3) & \mathbf{0 , 8 4} \pm \mathbf{0 , 0 1} & & \\ \text { Palmitoleic acid } & \mathbf{1 , 1 9} \pm \mathbf{0 , 0 6} & \text { Arachic acid } & \mathbf{0 , 2 2} \pm \mathbf{0 , 0 0} & \text { Unsat./satured } & \mathbf{5 , 5 7 \pm 0 , 7 7} \\ \text { Stearic acid } & \mathbf{1 , 5 5} \pm \mathbf{0 , 0 5} & \text { Eicosenoic acid } & \mathbf{0 , 0 3} \pm \mathbf{0 , 0 1} & \omega 6 / \omega 3 & \mathbf{7 , 4 0 \pm 0 , 7 2} \\ \text { Oleic acid } & \mathbf{7 5 , 4 1 \pm 0 , 7 7} & \text { Behenic acid } & \mathbf{0 , 0 5} \pm \mathbf{0 , 0 0} & & \end{array}$

\section{Organoleptic oil values}

Sensory Analysis (Panel test)

Comment: fruity medium-high, with hints of grass and tomato, read sensations of almond and artichoke. Balanced taste sensation with a medium-high bitter and spicy. Mediumhigh.

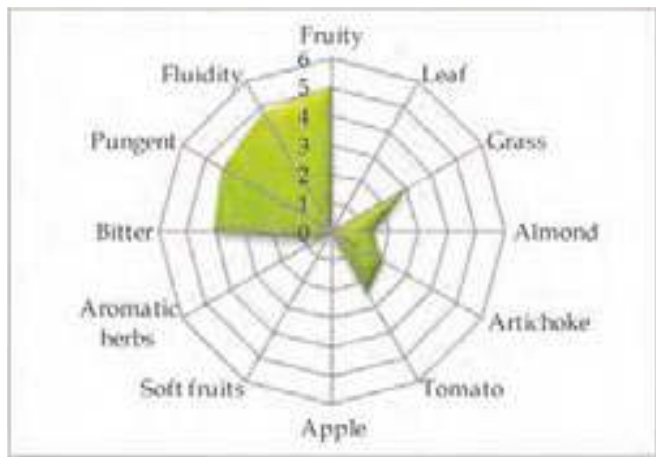

\section{Molecular Markers}

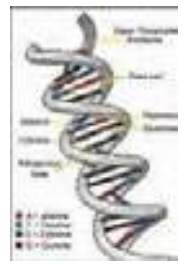

Table 2. Microsatellites (SSR) loci analyzed.

For each locus the allele size (expressed in base pairs) is reported.

\begin{tabular}{|c|c|c|c|c|c|}
\hline DCA09 & DCA18 & GAPU59 & GAPU71A & GUPA71B & GAPU103A \\
\hline $182-198$ & 179 - 181 & $214-218$ & $214-224$ & $130-144$ & 150 - 170 \\
\hline UDO01 & UDO03 & UDO12 & UDO28 & UDO39 & \\
\hline $150-150$ & $143-143$ & $166-177$ & $150-161$ & $220-220$ & \\
\hline
\end{tabular}

\section{References:}

1 - Parlati M.V., Pandolfi S. In: Catalogo delle principali varietà di olivo del Lazio, Ist. Sper. Olivic. (2003), pp. 15-16. 2 - Various authors. In: Catalogo Nazionale delle Varietà di Olivo, University of Bari (in press), ISBN 978-88-8879397-9. 


\section{“Laurina"}

(synonymy: Carbonella, Laura, Mignola, etc.)

Areal distribution or origin area: Marche

Flesh/pit weight ratio: medium $(\mathbf{7 , 2 4} \pm \mathbf{1 , 2 0})$

Oil content (\%): medium $(40,51 \pm 0,09)$

Purpose: oil

\section{Morphological characters}

Tree characters

Vigour: medium

Growth habit: spreading

Canopy-density: medium-dense

\section{Leaf characters}

Blade length $(\mathrm{cm})$ : medium $(6,15 \pm 0,71)$

Blade width (cm): medium $(\mathbf{1 , 3 1} \pm 0,15)$

Shape (length/width): elliptic-lanceolate

\section{Inflorescence characters}

Inflorescence length (cm): long $(4,45 \pm 0,01)$

Number of flowers: high $(\mathbf{2 5 , 4 3 \pm 0 , 2 7 )}$

\section{Fruit characters}

Fresh weight of 100 fruits $(\mathrm{g})$ : low $(\mathbf{1}, \mathbf{2 8} \pm \mathbf{0 , 0 2})$

Shape (length/width): ovoid

Symmetry: asymmetric

Position of maximum transverse diameter:

central

Apex: pointed

Base: truncate

Niplle: obvious

Lenticels: few and small

\section{Pit characters}

Weight of 100 pits (g): low $(\mathbf{0 , 1 8} \pm \mathbf{0 , 0 2})$

Shape (length/width): elliptic

Mucron: tenuous

Symmetry: slightly asymmetric

Position of maximum transverse diameter:

central

Apex: pointed

Base: pointed

Surface: rugose

Number of grooves: low
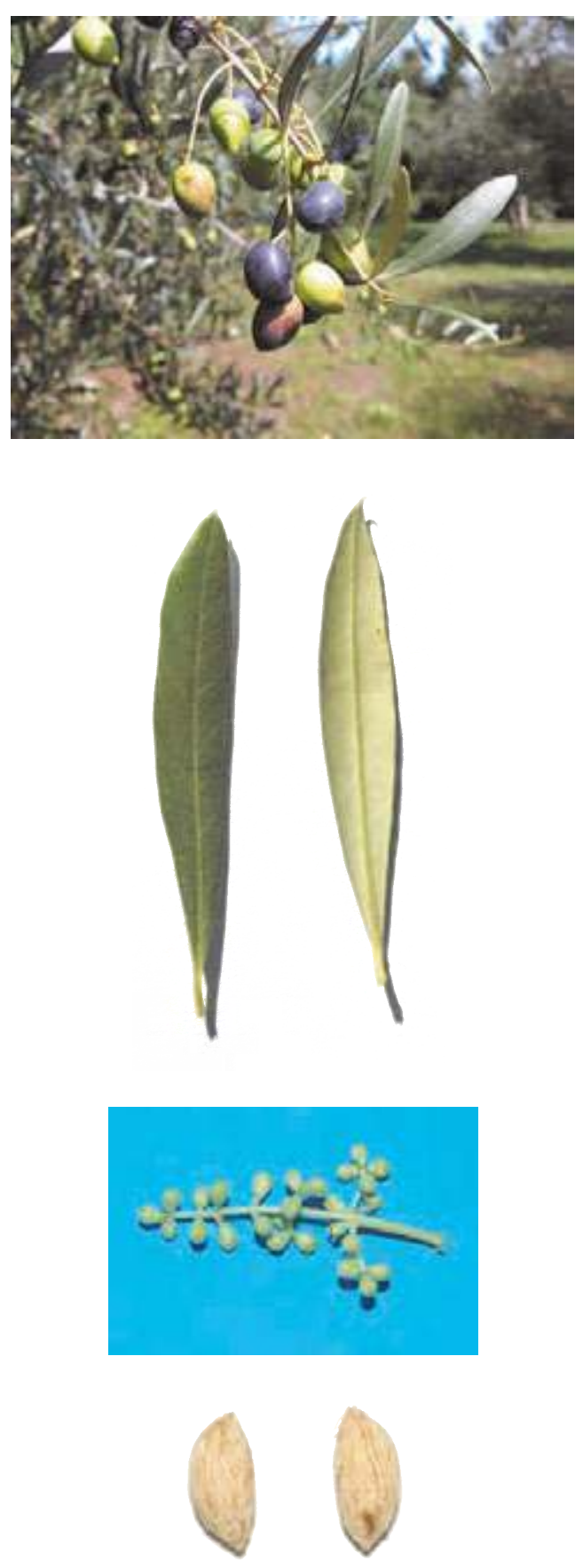


\section{Biochemical Characters}

\section{Fatty Acid Composition}

Table 1. Average values (express in $\% \pm$ standard deviations) of the fatty acids methyl esters and nutritional ratios obtained from single cultivar olive oils.

$\begin{array}{lllll}\text { Myristic acid } \quad 0,00 \pm 0,00 & \text { Linoleic acid }(\omega 6) & \mathbf{1 0 , 5 4} \pm \mathbf{0 , 4 1} \quad \text { Lignoceric acid } \quad 0,03 \pm 0,01\end{array}$

Palmitic acid $13,95 \pm 0,93 \quad$ Linolenic acid $(\omega 3)$ 0,84 $\pm 0,07$

Palmitoleic acid

$$
2,59 \pm 0,28
$$

Arachic acid

$0,24 \pm 0,04$

Unsat./satured

$5,21 \pm 0,30$

Stearic acid

$1,60 \pm 0,08$

Eicosenoic acid

$0,02 \pm 0,01$

Oleic acid

$67,76 \pm 0,48$

Behenic acid

$0,07 \pm 0,01$

\section{Organoleptic oil values}

Sensory Analysis (Panel test)

Comment: fruity medium - light, with hints of artichoke and almond. Balanced in flavours, with hints of bitter and spicy medium intensity, medium fluidity.

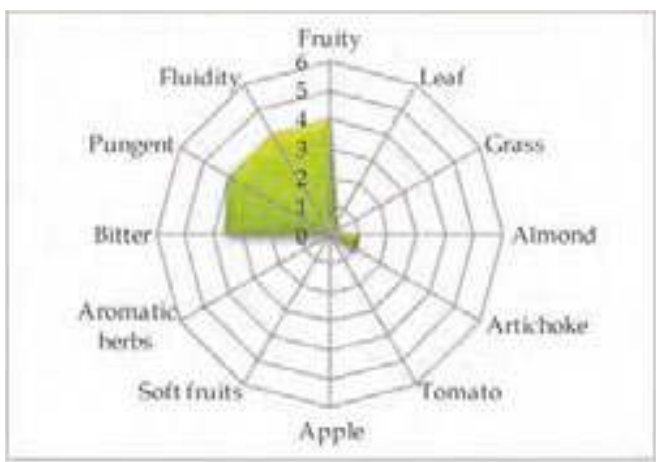

\section{Molecular Markers}

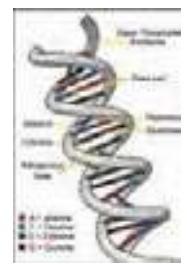

Table 2. Microsatellites (SSR) loci analyzed.

For each locus the allele size (expressed in base pairs) is reported.

$\begin{array}{cccccc}D C A 09 & \text { DCA18 } & \text { GAPU59 } & \text { GAPU71A } & \text { GUPA71B } & \text { GAPU103A } \\ \mathbf{1 7 2} \mathbf{- 1 7 2} & \mathbf{1 7 7 - \mathbf { 1 8 5 }} & \mathbf{2 1 4 - \mathbf { 2 1 4 }} & \mathbf{2 1 4} \mathbf{- 2 2 4} & \mathbf{1 2 4} \mathbf{- 1 4 4} & \mathbf{1 5 7 - \mathbf { 1 5 7 }} \\ \text { UDOO1 } & \text { UDO03 } & \text { UDO12 } & \text { UDO28 } & \text { UDO39 } & \\ \mathbf{1 4 4 - \mathbf { 1 4 4 }} & \mathbf{1 4 3 - \mathbf { 1 4 3 }} & \mathbf{1 7 7 - \mathbf { 1 9 3 }} & \mathbf{1 5 4 - \mathbf { 2 0 5 }} & \mathbf{2 0 0 - \mathbf { 2 0 0 }} & \end{array}$

\section{References:}

1 - Alfei B. In: olivo e Olio, (2003), 1: pp. 18-20.

2 - Various authors. In: Catalogo Nazionale delle Varietà di Olivo . University of Bari (in press), ISBN 978-88-8879397-9. 


\section{"Lavagnina"}

(synonymy: Olivastro, Olivo di Taggia, Olivo taggiasco, Pignola di Oneglia, etc.)

Areal distribution or origin area: Liguria

Flesh/pit weight ratio: low $(3,41 \pm 0,08)$

Oil content (\%): medium $(45,50 \pm 1,50)$

Purpose: oil

\section{Morphological characters}

Tree characters

Vigour: medium-strong

Growth habit: spreading

Canopy-density: medium

\section{Leaf characters}

Blade length ( $\mathrm{cm})$ : medium $(5,88 \pm 0,59)$

Blade width $(\mathrm{cm})$ : medium $(\mathbf{1}, 43 \pm \mathbf{0 , 1 4})$

Shape (length/width): elliptic-lanceolate

\section{Inflorescence characters}

Inflorescence length (cm): long $(3,78 \pm 1,99)$

Number of flowers: medium $(20,14 \pm 1,18)$

\section{Fruit characters}

Fresh weight of 100 fruits $(\mathrm{g})$ : medium $(2,28 \pm 0,21)$

Shape (length/width): ovoid

Symmetry: symmetric

Position of maximum transverse diameter: central

Apex: rounded

Base: rounded

Niplle: tenuous

Lenticels: many and small

\section{Pit characters}

Weight of 100 pits (g): high $(0,48 \pm 0,05)$

Shape (length/width): elliptic

Mucron: obvious

Symmetry: slightly asymmetric

Position of maximum transverse diameter:

\section{towards apex}

Apex: rounded

Base: rounded

Surface: rugose

Number of grooves: low
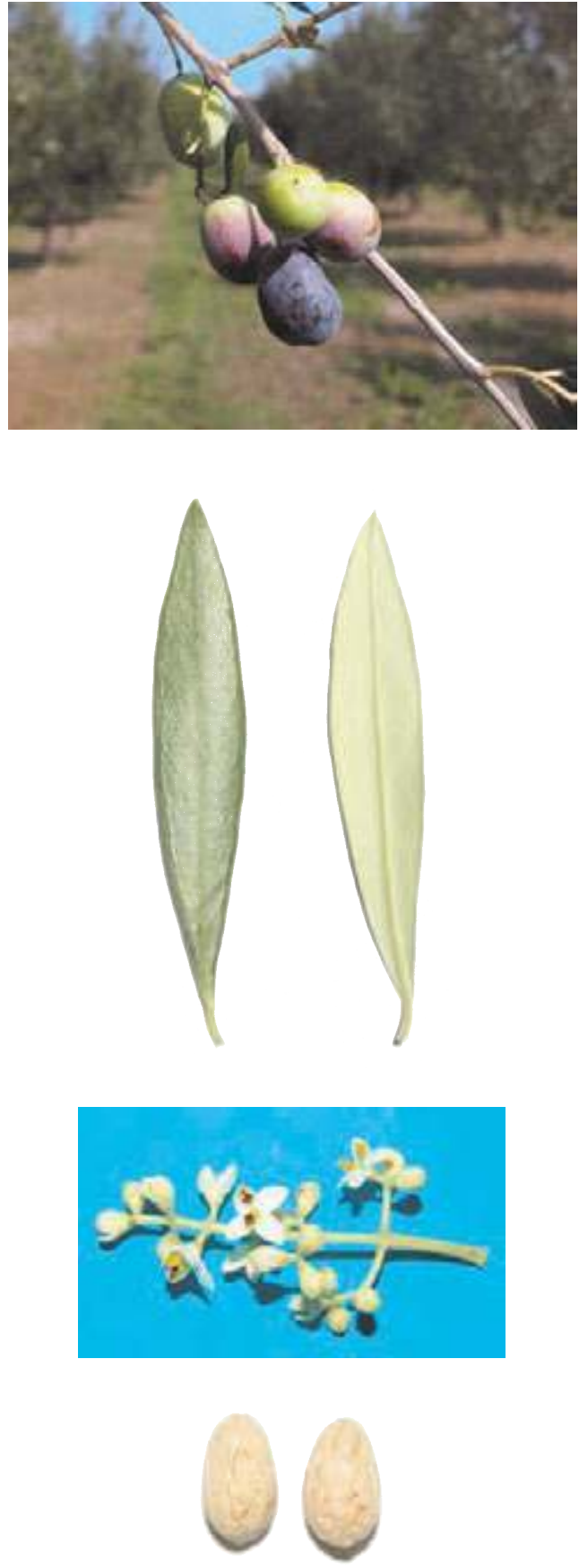


\section{Biochemical Characters}

\section{Fatty Acid Composition}

Table 1. Average values (express in $\% \pm$ standard deviations) of the fatty acids methyl esters and nutritional ratios obtained from single cultivar olive oils.

$\begin{array}{llllll}\text { Myristic acid } & \mathbf{0 , 0 1} \pm \mathbf{0 , 0 0} & \text { Linoleic acid }(\omega 6) & \mathbf{9 , 8 2} \pm \mathbf{0 , 5 3} & \text { Lignoceric acid } & \mathbf{0 , 0 2} \pm \mathbf{0 , 0 1} \\ \text { Palmitic acid } & \mathbf{1 3 , 6 8} \pm \mathbf{0 , 2 5} & \text { Linolenic acid }(\omega 3) \mathbf{0 , 8 4} \pm \mathbf{0 , 0 7} & & \\ \text { Palmitoleic acid } & \mathbf{1 , 5 7} \pm \mathbf{0 , 0 1} & \text { Arachic acid } & \mathbf{0 , 2 2} \pm \mathbf{0 , 0 2} & \text { Unsat./satured } & \mathbf{5 , 2 7} \pm \mathbf{0 , 1 1} \\ \text { Stearic acid } & \mathbf{1 , 9 2} \pm \mathbf{0 , 0 4} & \text { Eicosenoic acid } & \mathbf{0 , 0 2} \pm \mathbf{0 , 0 1} & \omega 6 / \omega 3 & \mathbf{1 1 , 6 5} \pm \mathbf{0 , 2 6} \\ \text { Oleic acid } & \mathbf{7 0 , 6 1} \pm \mathbf{0 , 4 6} & \text { Behenic acid } & \mathbf{0 , 0 5} \pm \mathbf{0 , 0 1} & & \end{array}$

\section{Organoleptic oil values}

Sensory Analysis (Panel test)

Comment: fruity medium - light, with hints of artichoke and almond. Balanced in flavours, with hints of bitter and spicy medium intensity, medium fluidity.

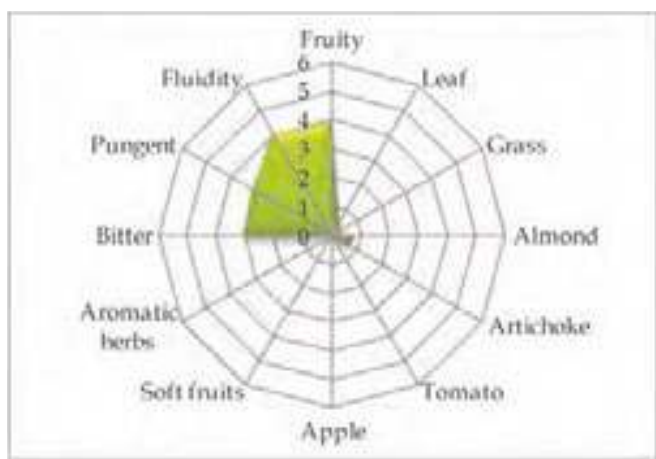

\section{Molecular Markers}

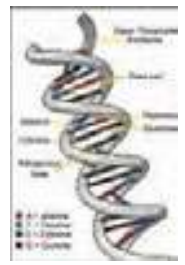

Table 2. Microsatellites (SSR) loci analyzed.

For each locus the allele size (expressed in base pairs) is reported.

$\begin{array}{cccccc}D C A 09 & \text { DCA18 } & \text { GAPU59 } & \text { GAPU71A } & \text { GUPA71B } & \text { GAPU103A } \\ \mathbf{1 7 2} \mathbf{- 1 7 6} & \mathbf{1 7 9 - \mathbf { 1 8 1 }} & \mathbf{2 1 2} \mathbf{- 2 1 2} & \mathbf{1 2 3} \mathbf{- 1 2 3} & \mathbf{2 1 0 - \mathbf { 2 1 4 }} & \mathbf{1 3 0 - \mathbf { 1 4 4 }} \\ \text { UDOO1 } & \text { UDOO3 } & \text { UDO12 } & \text { UDO28 } & \text { UDO39 } & \\ \mathbf{1 4 4 - \mathbf { 1 4 4 }} & \mathbf{1 5 0 - \mathbf { 1 8 2 }} & \mathbf{1 6 6 - \mathbf { 1 9 3 }} & \mathbf{1 4 3 - \mathbf { 2 0 5 }} & \mathbf{2 1 3 - \mathbf { 2 3 2 }} & \end{array}$

\section{References:}

1 - Cimato A., Cantini C., Sani G. In: Atti $4^{\circ}$ Convegno Nazionale sulla Biodiversità, Alghero (2000), pp. 497-500. 2 - Various authors. In: Catalogo Nazionale delle Varietà di Olivo. University of Bari (in press), ISBN 978-88-8879397-9. 


\section{“Leccino"}

(synonymy: Colombina, Leccino di Belmonte, Toscano, Verolana, etc.)

Areal distribution or origin area: Toscana

Flesh/pit weight ratio: low $(4,03 \pm 0,17)$

Oil content (\%): medium $(46,20 \pm 0,71)$

Purpose: oil

\section{Morphological characters}

Tree characters

Vigour: medium-strong

Growth habit: spreading

Canopy-density: dense

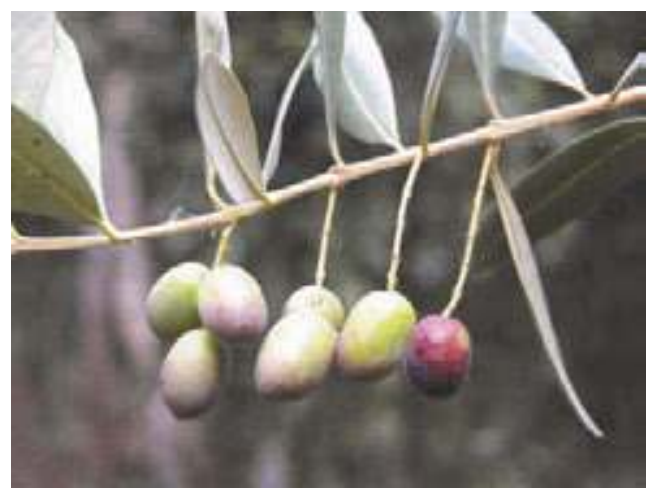

\section{Leaf characters}

Blade length (cm): medium $(5,10 \pm 0,39)$

Blade width $(\mathrm{cm})$ : broad $(\mathbf{1}, \mathbf{5 3} \pm \mathbf{0 , 1 3})$

Shape (length/width): elliptic

\section{Inflorescence characters}

Inflorescence length $(\mathrm{cm})$ : medium $(2,90 \pm 1,67)$

Number of flowers: low $(\mathbf{1 2}, \mathbf{8 3} \pm \mathbf{1}, \mathbf{9 1})$

\section{Fruit characters}

Fresh weight of 100 fruits $(\mathrm{g})$ : medium $(2,41 \pm 0,19)$

Shape (length/width): ovoid

Symmetry: slightly asymmetric

Position of maximum transverse diameter:

central

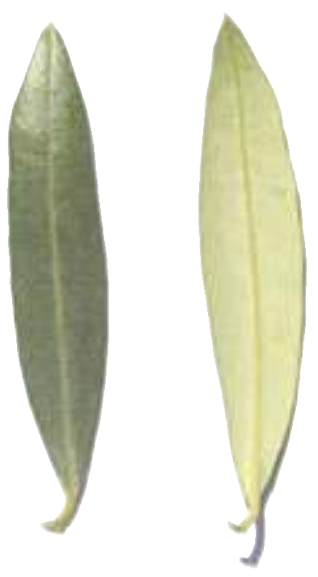

Apex: rounded

Base: rounded

Niplle: absent

Lenticels: many and small

\section{Pit characters}

Weight of 100 pits (g): high $(0,52 \pm \mathbf{0 , 0 6})$

Shape (length/width): elliptic

Mucron: obvious

Symmetry: asymmetric

Position of maximum transverse diameter:

central

Apex: rounded

Base: rounded

Surface: smooth

Number of grooves: high
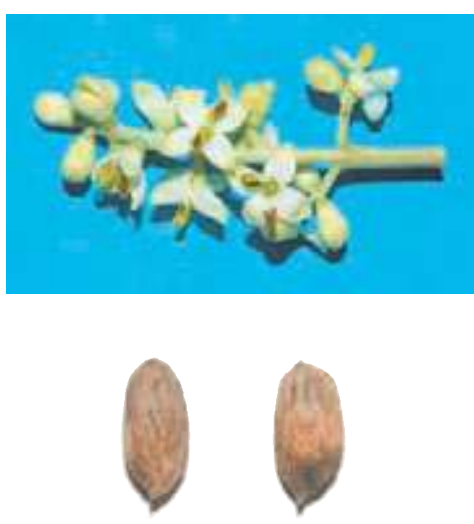


\section{Biochemical Characters}

\section{Fatty Acid Composition}

Table 1. Average values (express in $\% \pm$ standard deviations) of the fatty acids methyl esters and nutritional ratios obtained from single cultivar olive oils.

$\begin{array}{llllll}\text { Myristic acid } & \mathbf{0 , 0 1} \pm \mathbf{0 , 0 0} & \text { Linoleic acid }(\omega 6) & \mathbf{4 , 9 6} \pm \mathbf{0 , 7 4} & \text { Lignoceric acid } & \mathbf{0 , 0 3} \pm \mathbf{0 , 0 1} \\ \text { Palmitic acid } & \mathbf{1 2 , 9 8} \pm \mathbf{0 , 7 3} & \text { Linolenic acid }(\omega 3) & \mathbf{0 , 8 2} \pm \mathbf{0 , 0 8} & & \\ \text { Palmitoleic acid } & \mathbf{1 , 2 2} \pm \mathbf{0 , 1 9} & \text { Arachic acid } & \mathbf{0 , 2 7} \pm \mathbf{0 , 0 2} & \text { Unsat./satured } & \mathbf{5 , 5 6 \pm 0 , 2 4} \\ \text { Stearic acid } & \mathbf{1 , 8 9} \pm \mathbf{0 , 2 4} & \text { Eicosenoic acid } & \mathbf{0 , 0 6} \pm \mathbf{0 , 1 2} & \omega 6 / \omega 3 & \mathbf{6 , 0 9 \pm \mathbf { 1 , 2 1 }} \\ \text { Oleic acid } & \mathbf{7 6 , 4 7 \pm \mathbf { 1 , 2 4 }} & \text { Behenic acid } & \mathbf{0 , 0 7} \pm \mathbf{0 , 0 1} & & \end{array}$

\section{Organoleptic oil values}

Sensory Analysis (Panel test)

Comment: fruity medium-high, with hints of almond and read sensations of grass and artichoke. Balanced taste sensation with a medium bitter and spicy. Medium fluidity.

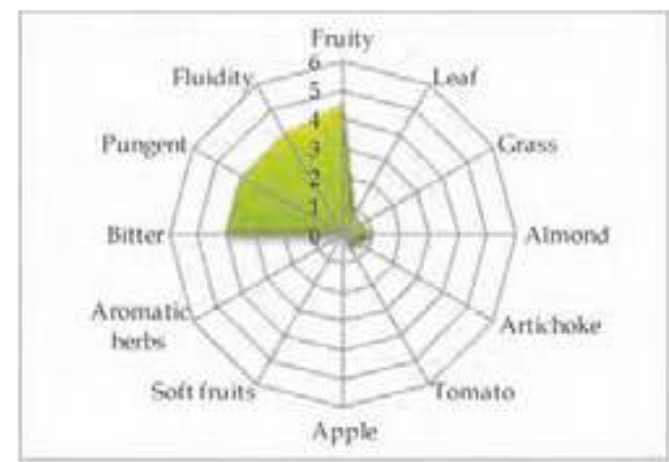

\section{Molecular Markers}

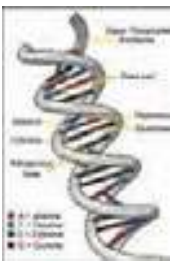

Table 2. Microsatellites (SSR) loci analyzed.

For each locus the allele size (expressed in base pairs) is reported.

$\begin{array}{cccccc}D C A 09 & \text { DCA18 } & \text { GAPU59 } & \text { GAPU71A } & \text { GUPA71B } & \text { GAPU103A } \\ \mathbf{1 6 2} \mathbf{- 2 0 6} & \mathbf{1 7 7 - \mathbf { 1 7 7 }} & \mathbf{2 1 2} \mathbf{- 2 2 2} & \mathbf{2 1 4 - \mathbf { 2 1 4 }} & \mathbf{1 2 4} \mathbf{- 1 3 0} & \mathbf{1 5 0 - \mathbf { 1 5 0 }} \\ \text { UDOO1 } & \text { UDOO3 } & \text { UDO12 } & \text { UDO28 } & \text { UDO39 } & \\ \mathbf{1 4 4 - \mathbf { 1 5 0 }} & \mathbf{1 4 3 - \mathbf { 1 4 3 }} & \mathbf{1 6 6 - 1 9 3} & \mathbf{1 5 4 - \mathbf { 2 0 5 }} & \mathbf{2 1 3 - \mathbf { 2 1 3 }} & \end{array}$

\section{References:}

1 - Pannelli G., Alfei B., Rosati S., et al. In: Varietà di olivo in Umbria. Ed. Pliniana (2000), pp.25-30.

2 - Muzzalupo I., Stefanizzi F., Salimonti A., et al. Sci. Agric. (2009), 66 (5): pp. 685-690. 


\section{“Lezze"}

(synonymy: Oliastro, Olivastro di Conversano.)

Areal distribution or origin area: Puglia

Flesh/pit weight ratio: low $(4,46 \pm 0,64)$

Oil content (\%): medium $(40,01 \pm 0,59)$

Purpose: oil

\section{Morphological characters}

Tree characters

Vigour: medium

Growth habit: spreading

Canopy-density: dense

\section{Leaf characters}

Blade length $(\mathrm{cm})$ : medium $(5,96 \pm 0,40)$

Blade width $(\mathrm{cm})$ : medium $(1,38 \pm 0,12)$

Shape (length/width): elliptic-lanceolate

\section{Inflorescence characters}

Inflorescence length $(\mathrm{cm})$ : medium $(2,99 \pm 0,56)$

Number of flowers: high $(\mathbf{2 2 , 8 4} \pm \mathbf{1}, \mathbf{9 7})$

\section{Fruit characters}

Fresh weight of 100 fruits $(\mathrm{g})$ : medium $(2,05 \pm 0,34)$

Shape (length/width): ovoid

Symmetry: slightly asymmetric

Position of maximum transverse diameter:

central

Apex: pointed

Base: rounded

Niplle: tenuous

Lenticels: many and small

\section{Pit characters}

Weight of 100 pits (g): medium $(\mathbf{0 , 4 1} \pm \mathbf{0 , 0 5})$

Shape (length/width): elliptic

Mucron: obvious

Symmetry: symmetric

Position of maximum transverse diameter:

central

Apex: rounded

Base: rounded

Surface: smooth

Number of grooves: low
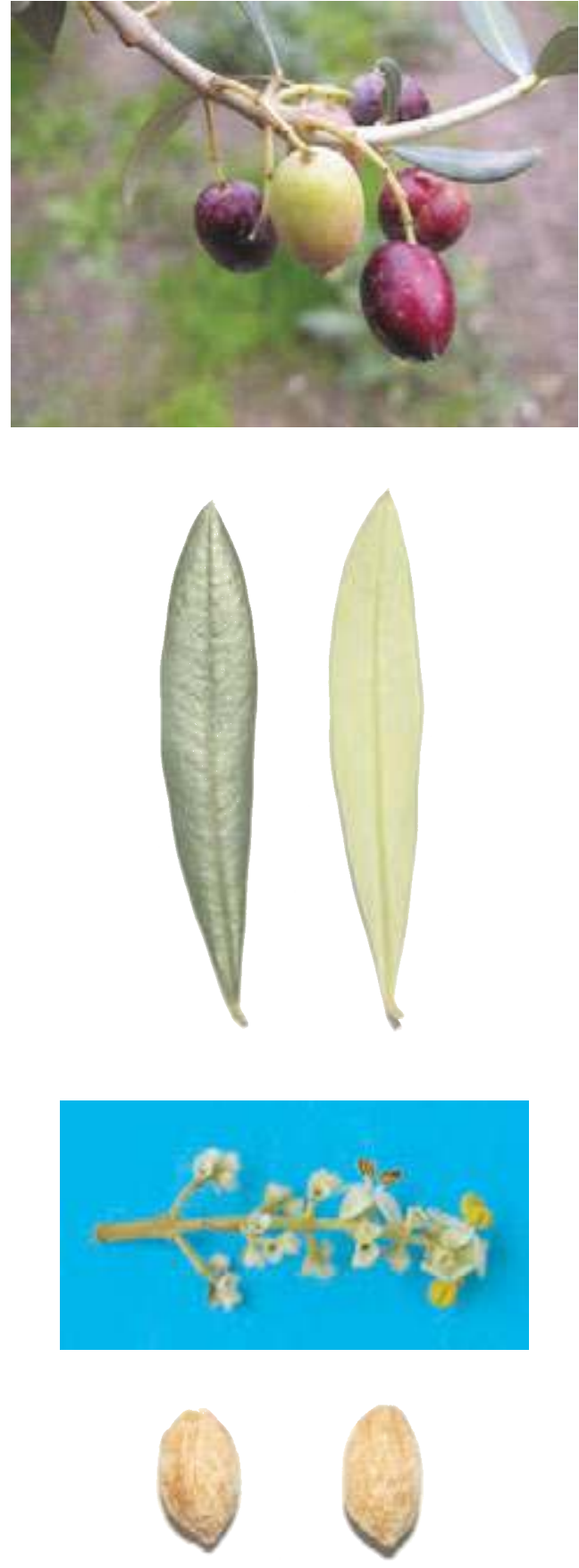


\section{Biochemical Characters}

\section{Fatty Acid Composition}

Table 1. Average values (express in $\% \pm$ standard deviations) of the fatty acids methyl esters and nutritional ratios obtained from single cultivar olive oils.

\begin{tabular}{|c|c|c|c|c|c|}
\hline Myristic acid & $0,01 \pm 0,00$ & Linoleic acid $(\omega 6)$ & $14,00 \pm 3,42$ & Lignoceric acid & $0,04 \pm 0,02$ \\
\hline Palmitic acid & $14,66 \pm 0,47$ & Linolenic acid $(\omega 3)$ & $0,82 \pm 0,20$ & & \\
\hline Palmitoleic acid & $1,54 \pm 0,05$ & Arachic acid & $0,26 \pm 0,06$ & Unsat./satured & $4,88 \pm 0,25$ \\
\hline tearic & $1,91 \pm 0,30$ & Eicosenoic acid & $0,18 \pm 0,20$ & $\omega 6 / \omega 3$ & $17,12 \pm 0,10$ \\
\hline leic acid & $65,20 \pm 2,79$ & Behenic acid & $0,07 \pm 0,03$ & & \\
\hline
\end{tabular}

\section{Organoleptic oil values}

Sensory Analysis (Panel test)

Comment: fruity medium, with hints of artichoke and almond. Balanced in flavours, with hints of bitter and spicy medium-light intensity. Medium fluidity.

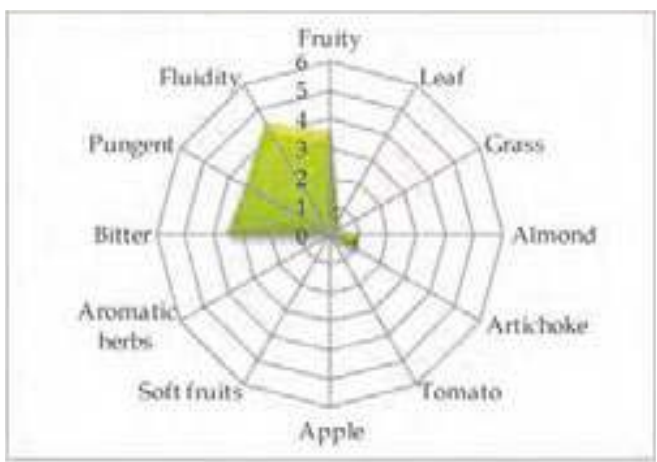

\section{Molecular Markers}

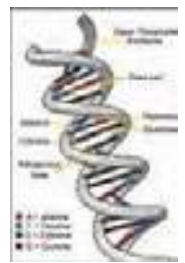

Table 2. Microsatellites (SSR) loci analyzed.

For each locus the allele size (expressed in base pairs) is reported.

$\begin{array}{cccccc}D C A 09 & \text { DCA18 } & \text { GAPU59 } & \text { GAPU71A } & \text { GUPA71B } & \text { GAPU103A } \\ \mathbf{1 7 2} \mathbf{- 1 9 4} & \mathbf{1 8 1} \mathbf{- 1 8 1} & \mathbf{2 0 8 - \mathbf { 2 1 8 }} & \mathbf{2 1 0 - \mathbf { 2 1 0 }} & \mathbf{1 2 4} \mathbf{- 1 4 4} & \mathbf{1 3 6} \mathbf{- 1 5 9} \\ \text { UDOO1 } & \text { UDO03 } & \text { UDO12 } & \text { UDO28 } & \text { UDO39 } & \\ \mathbf{1 4 0 - \mathbf { 1 4 0 }} & \mathbf{1 3 5 - \mathbf { 1 3 5 }} & \mathbf{1 7 7 - \mathbf { 1 8 2 }} & \mathbf{1 4 3 - \mathbf { 1 6 1 }} & \mathbf{1 6 4 - \mathbf { 1 8 5 }} & \end{array}$

\section{References:}

1 - Lombardo N., Perri E., Muzzalupo I., et al. In: Contributo alla caratterizzazione del germoplasma olivicolo pugliese, Istituto Sperimentale per l'Olivicoltura (2004), pp. 73-76.

2 - Muzzalupo I., Stefanizzi F., Perri E. HortScience (2009), 44: pp. 582-588. 


\section{"Lumiaru"}

(synonymy: Leminara, Luriaro, Luriara, etc.)

Areal distribution or origin area: Sicilia

Flesh/ pit weight ratio: high $(\mathbf{1 0 , 0 0} \pm \mathbf{1}, \mathbf{2 2})$

Oil content (\%): high $\mathbf{( 5 0 , 1 0 \pm 0 , 8 8 )}$

Purpose: oil

\section{Morphological characters}

Tree characters

Vigour: medium-weak

Growth habit: spreading

Canopy-density: medium-sparse

\section{Leaf characters}

Blade length (cm): medium $(6,35 \pm 0,70)$

Blade width (cm): medium $(\mathbf{1}, \mathbf{4 4} \pm \mathbf{0 , 2 0})$

Shape (length/width): elliptic-lanceolate

\section{Inflorescence characters}

Inflorescence length $(\mathrm{cm})$ : medium $(3,07 \pm 1,72)$

Number of flowers: medium $(\mathbf{1 8 , 2 2} \pm \mathbf{0 , 9 5})$

\section{Fruit characters}

Fresh weight of 100 fruits $(\mathrm{g})$ : high $(\mathbf{4 , 5 8} \pm \mathbf{0 , 3 9})$

Shape (length/width): spherical

Symmetry: slightly asymmetric

Position of maximum transverse diameter:

towards base

Apex: rounded

Base: truncate

Niplle: absent

Lenticels: few and large

\section{Pit characters}

Weight of 100 pits (g): high $(\mathbf{0 , 4 2} \pm \mathbf{0 , 0 8})$

Shape (length/width): elliptic

Mucron: obvious

Symmetry: slightly asymmetric

Position of maximum transverse diameter:

central

Apex: rounded

Base: pointed

Surface: scabrous

Number of grooves: medium
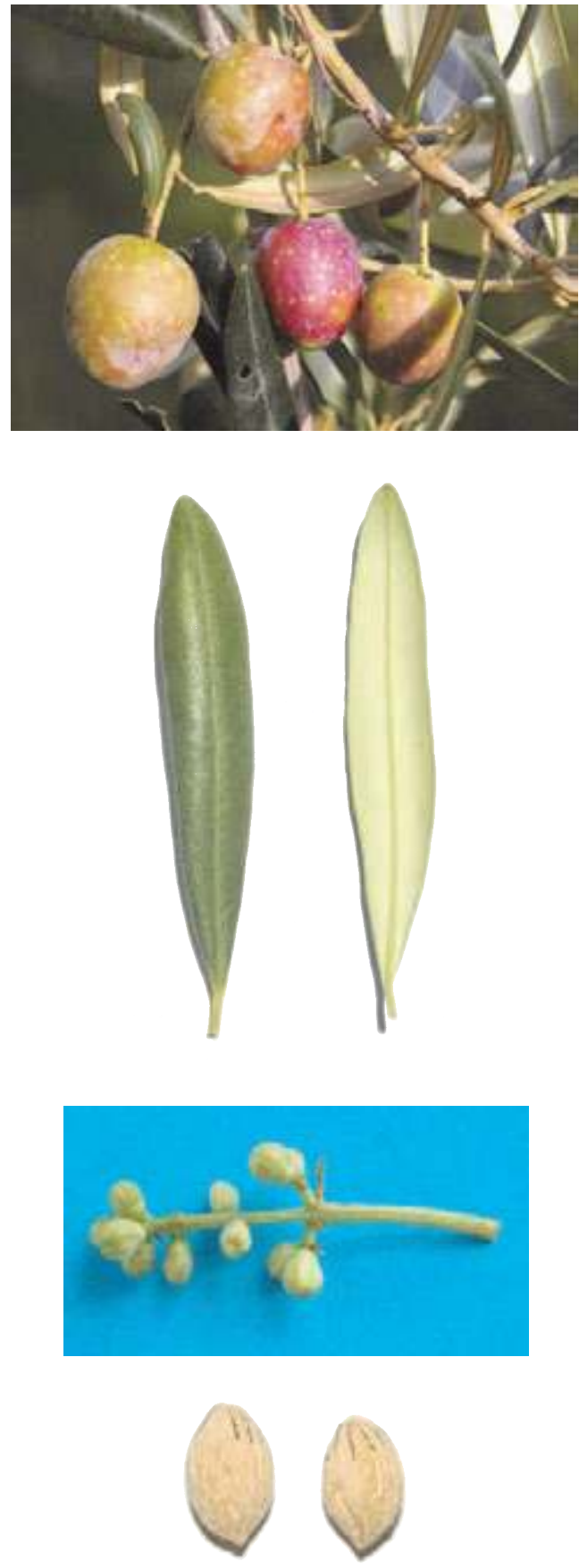


\section{Biochemical Characters}

\section{Fatty Acid Composition}

Table 1. Average values (express in $\% \pm$ standard deviations) of the fatty acids methyl esters and nutritional ratios obtained from single cultivar olive oils.

$\begin{array}{llllll}\text { Myristic acid } & \mathbf{0 , 0 2} \pm \mathbf{0 , 0 1} & \text { Linoleic acid }(\omega 6) & \mathbf{1 0 , 2 8} \pm \mathbf{0 , 0 1} & \text { Lignoceric acid } & \mathbf{0 , 0 2} \pm \mathbf{0 , 0 1} \\ \text { Palmitic acid } & \mathbf{1 5 , 1 0 \pm 0 , 4 2} & \text { Linolenic acid }(\omega 3) \mathbf{1 , 0 9} \pm \mathbf{0 , 0 2} & & \\ \text { Palmitoleic acid } & \mathbf{1 , 4 3} \pm \mathbf{0 , 0 0} & \text { Arachic acid } & \mathbf{0 , 2 5} \pm \mathbf{0 , 0 1} & \text { Unsat./satured } & \mathbf{4 , 8 3} \pm \mathbf{0 , 1 8} \\ \text { Stearic acid } & \mathbf{1 , 6 3} \pm \mathbf{0 , 0 9} & \text { Eicosenoic acid } & \mathbf{0 , 0 2} \pm \mathbf{0 , 0 1} & \omega 6 / \omega 3 & \mathbf{9 , 4 6} \pm \mathbf{0 , 2 3} \\ \text { Oleic acid } & \mathbf{6 8 , 7 6 \pm 0 , 5 5} & \text { Behenic acid } & \mathbf{0 , 0 6} \pm \mathbf{0 , 0 0} & & \end{array}$

\section{Organoleptic oil values}

Sensory Analysis (Panel test)

Comment: fruity medium-high, with taste of almond. Balanced taste sensation with medium bitter and spicy. Medium-high fluidity.

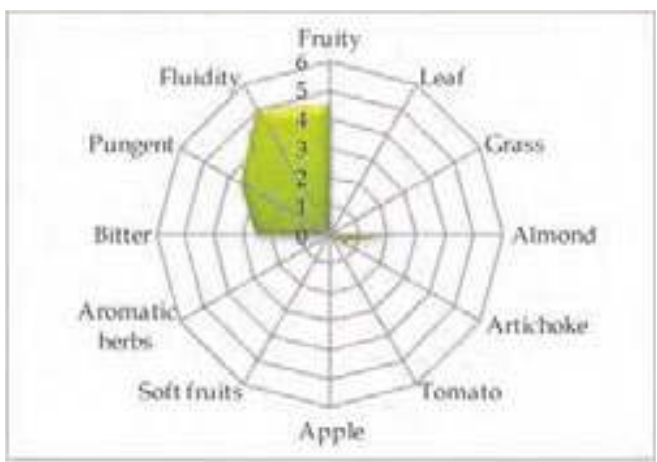

\section{Molecular Markers}

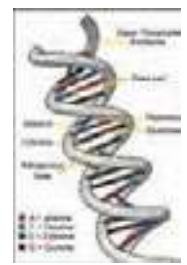

Table 2. Microsatellites (SSR) loci analyzed.

For each locus the allele size (expressed in base pairs) is reported.

$\begin{array}{cccccc}D C A 09 & \text { DCA18 } & \text { GAPU59 } & \text { GAPU71A } & \text { GUPA71B } & \text { GAPU103A } \\ \mathbf{1 7 6 - \mathbf { 2 0 6 }} & \mathbf{1 7 7 - \mathbf { 1 8 1 }} & \mathbf{2 0 8 - \mathbf { 2 1 2 }} & \mathbf{2 1 4} \mathbf{- 2 1 4} & \mathbf{1 2 4} \mathbf{- 1 4 4} & \mathbf{1 5 9 - \mathbf { 1 8 4 }} \\ \text { UDOO1 } & \text { UDOO3 } & \text { UDO12 } & \text { UDO28 } & \text { UDO39 } & \\ \mathbf{1 4 4 - \mathbf { 1 4 4 }} & \mathbf{1 4 3 - \mathbf { 1 4 3 }} & \mathbf{1 6 6 - 1 9 3} & \mathbf{1 3 3 - \mathbf { 1 8 2 }} & \mathbf{2 0 5 - \mathbf { 2 0 5 }} & \end{array}$

\section{References:}

1 - Caruso T., Cartabellotta D., Motisi A., et al. In: Cultivar di olivo siciliane, Università degli Studi di Palermo (2007), pp. 158-162.

2 - Muzzalupo I., Stefanizzi F., Perri E. HortScience (2009), 44: pp. 582-588. 


\section{"Maiatica di Ferrandina"}

(synonymy: Gentile di Ferrandina, Gentile, Maggiatica, Paesana, Oliva dolce, etc.)

Areal distribution or origin area: Basilicata

Flesh/pit weight ratio: high $(9,07 \pm 0,83)$

Oil content (\%): high $(\mathbf{5 2 , 2 4} \pm \mathbf{1 , 0 3})$

Purpose: dual purpose

\section{Morphological characters}

Tree characters

Vigour: medium-strong

Growth habit: spreding-erect

Canopy-density: medium-dense

\section{Leaf characters}

Blade length $(\mathrm{cm})$ : medium $(5,26 \pm 0,55)$

Blade width $(\mathrm{cm})$ : medium $(\mathbf{1 , 1 5} \pm \mathbf{0 , 1 0})$

Shape (length/width): elliptic-lanceolate

\section{Inflorescence characters}

Inflorescence length (cm): medium $(2,50 \pm 0,15)$

Number of flowers: low $(13,57 \pm 0,81)$

\section{Fruit characters}

Fresh weight of 100 fruits $(\mathrm{g})$ : medium $(3,72 \pm 0,87)$

Shape (length/width): ovoid

Symmetry: slightly asymmetric

Position of maximum transverse diameter:

central

Apex: rounded

Base: truncate

Niplle: absent

Lenticels: many and small

\section{Pit characters}

Weight of 100 pits (g): medium $(0,37 \pm 0,08)$

Shape (length/width): elliptic

Mucron: obvious

Symmetry: slightly asymmetric

Position of maximum transverse diameter:

towards apex

Apex: rounded

Base: pointed

Surface: smooth

Number of grooves: medium
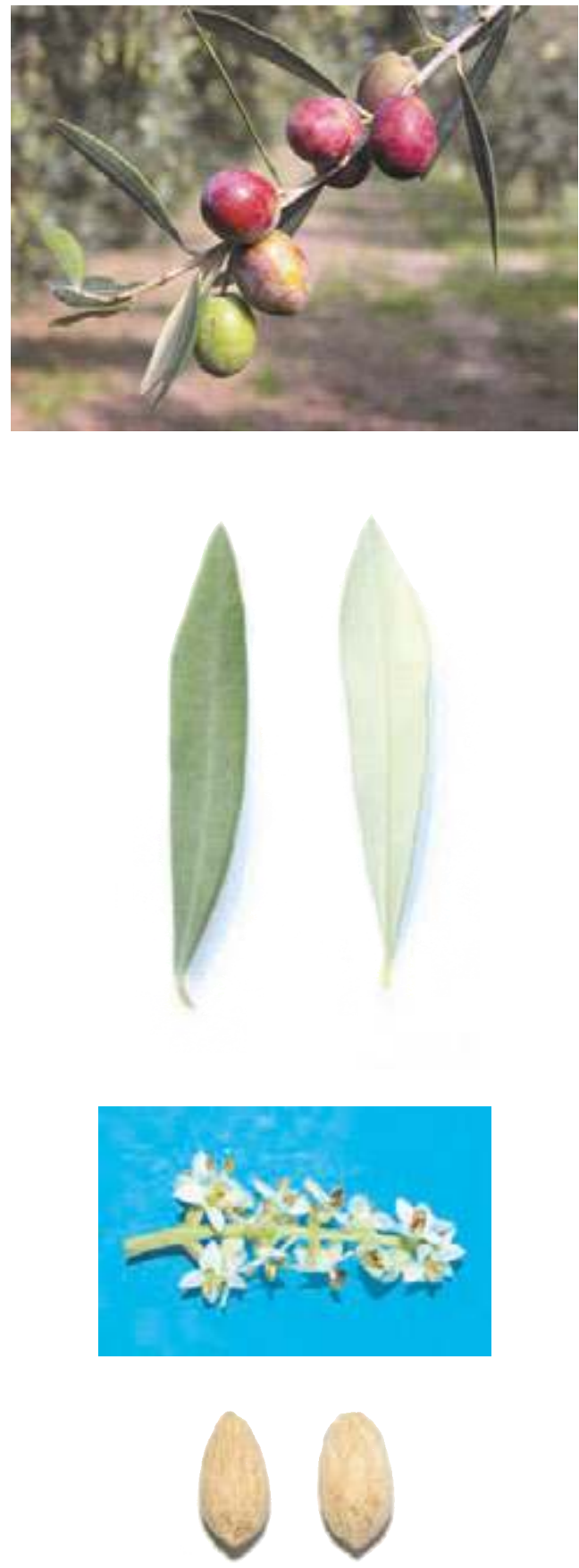


\section{Biochemical Characters}

\section{Fatty Acid Composition}

Table 1. Average values (express in $\% \pm$ standard deviations) of the fatty acids methyl esters and nutritional ratios obtained from single cultivar olive oils.

$\begin{array}{llllll}\text { Myristic acid } & \mathbf{0 , 0 1} \pm \mathbf{0 , 0 0} & \text { Linoleic acid }(\omega 6) & \mathbf{6 , 7 2} \pm \mathbf{1 , 5 9} & \text { Lignoceric acid } & \mathbf{0 , 0 5} \pm \mathbf{0 , 0 2} \\ \text { Palmitic acid } & \mathbf{1 0 , 5 3 \pm \mathbf { 1 , 9 6 }} & \text { Linolenic acid }(\omega 3) & \mathbf{0 , 8 0} \pm \mathbf{0 , 0 6} & & \\ \text { Palmitoleic acid } & \mathbf{0 , 8 8} \pm \mathbf{0 , 1 2} & \text { Arachic acid } & \mathbf{0 , 3 9} \pm \mathbf{0 , 1 3} & \text { Unsat./satured } & \mathbf{6 , 0 7 \pm 0 , 6 3} \\ \text { Stearic acid } & \mathbf{3 , 2 3} \pm \mathbf{0 , 6 7} & \text { Eicosenoic acid } & \mathbf{0 , 0 3} \pm \mathbf{0 , 0 1} & \omega 6 / \omega 3 & \mathbf{8 , 4 7} \pm \mathbf{2 , 1 0} \\ \text { Oleic acid } & \mathbf{7 6 , 1 5} \pm \mathbf{2 , 8 4} & \text { Behenic acid } & \mathbf{0 , 1 0} \pm \mathbf{0 , 0 1} & & \end{array}$

\section{Organoleptic oil values}

Sensory Analysis (Panel test)

Comment: fruity medium-high, with good sensation of grass, artichoke and almond. Balanced in flavours, with hints of bitter and spicy medium intensity. Medium-high fluidity.

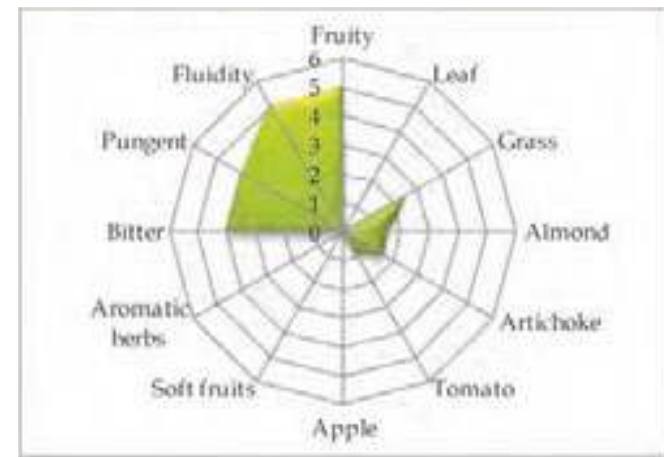

Molecular Markers

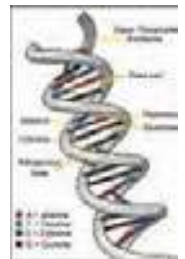

Table 2. Microsatellites (SSR) loci analyzed. For each locus the allele size (expressed in base pairs) is reported.

$\begin{array}{cccccc}D C A 09 & \text { DCA18 } & \text { GAPU59 } & \text { GAPU71A } & \text { GUPA71B } & \text { GAPU103A } \\ \mathbf{1 9 8} \mathbf{- 2 0 6} & \mathbf{1 7 7 - \mathbf { 1 7 9 }} & \mathbf{2 0 8 - \mathbf { 2 0 8 }} & \mathbf{2 1 4 - \mathbf { 2 1 4 }} & \mathbf{1 2 4 - \mathbf { 1 4 4 }} & \mathbf{1 7 0 - \mathbf { 1 8 4 }} \\ \text { UDOO1 } & \text { UDO03 } & \text { UDO12 } & \text { UDO28 } & \text { UDO39 } & \\ \mathbf{1 4 4 - \mathbf { 1 4 4 }} & \mathbf{1 4 3 - \mathbf { 1 4 3 }} & \mathbf{1 6 6 - \mathbf { 1 9 3 }} & \mathbf{1 8 2} \mathbf{- 1 8 2} & \mathbf{2 0 5 - 2 0 5} & \end{array}$

\section{References:}

1 - Rotundo A., Marone E. In: Il germoplasma olivicolo lucano, Olita - Potenza (2002), pp. 61-64.

2 - Rotundo A., Perri E., Muzzalupo I., et al. In: Il germoplasma olivicolo meridionale, (2012 in press).

3 - Muzzalupo I., Stefanizzi F., Perri E. HortScience (2009), 44: pp. 582-588. 


\section{“Marina "}

(synonymy: Marina Valcomino)

Areal distribution or origin area: Lazio

Flesh/pit weight ratio: medium $(5,02 \pm \mathbf{1 , 2 0})$

Oil content (\%): medium $(46,63 \pm 0,74)$

Purpose: olio

\section{Morphological characters}

Tree characters

Vigour: medium

Growth habit: spreading-erect

Canopy-density: medium

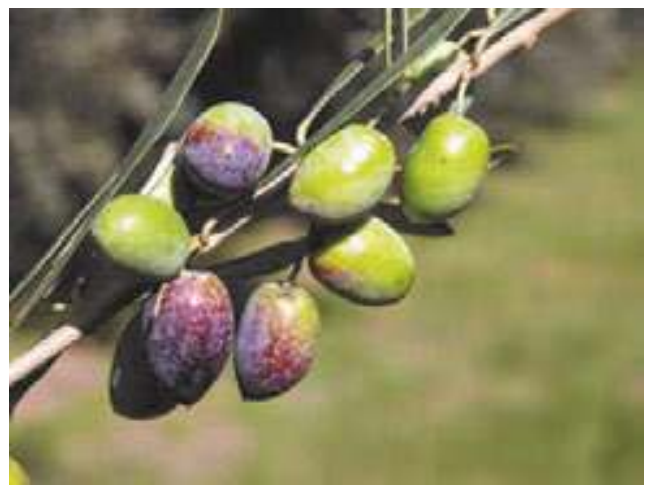

\section{Leaf characters}

Blade length $(\mathrm{cm})$ : medium $(6.26 \pm 0,42)$

Blade width $(\mathrm{cm})$ : medium $(\mathbf{1}, 29 \pm \mathbf{0 , 1 2})$

Shape (length/width): elliptic-lanceolate

\section{Inflorescence characters}

Inflorescence length $(\mathrm{cm})$ : medium $(2,65 \pm 0,26)$

Number of flowers: low $(\mathbf{1 4 , 8 5} \pm \mathbf{2 , 7 7})$

\section{Fruit characters}

Fresh weight of 100 fruits $(\mathrm{g})$ : medium $(2,64 \pm 0,42)$

Shape (length/width): elongated

Symmetry: asymmetric

Position of maximum transverse diameter:

towards base

Apex: pointed

Base: truncate

Niplle: absent

Lenticels: many and large

\section{Pit characters}

Weight of 100 pits (g): high $(0,49 \pm 0,08)$

Shape (length/width): elongated

Mucron: absent

Symmetry: symmetric

Position of maximum transverse diameter:

towards apex

Apex: pointed

Base: rounded

Surface: rugose

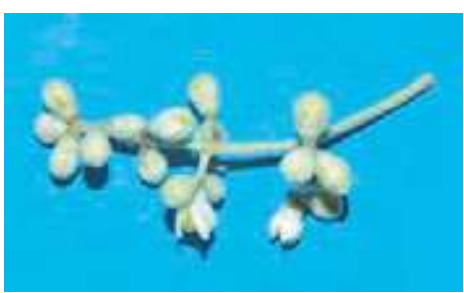

Number of grooves: high 


\section{Biochemical Characters}

\section{Fatty Acid Composition}

Table 1. Average values (express in $\% \pm$ standard deviations) of the fatty acids methyl esters and nutritional ratios obtained from single cultivar olive oils.

$\begin{array}{llllll}\text { Myristic acid } & \mathbf{0 , 0 2} \pm \mathbf{0 , 0 1} & \text { Linoleic acid }(\omega 6) & \mathbf{6 , 9 5} \pm \mathbf{0 , 2 9} & \text { Lignoceric acid } & \mathbf{0 , 0 8} \pm \mathbf{0 , 0 2} \\ \text { Palmitic acid } & \mathbf{1 2 , 5 3} \pm \mathbf{0 , 7 6} & \text { Linolenic acid }(\omega 3) \mathbf{0 , 7 6} \pm \mathbf{0 , 0 5} & & \\ \text { Palmitoleic acid } & \mathbf{2 , 0 8} \pm \mathbf{0 , 0 4} & \text { Arachic acid } & \mathbf{0 , 5 4} \pm \mathbf{0 , 0 4} & \text { Unsat./satured } & \mathbf{5 , 2 0 \pm 0 , 2 0} \\ \text { Stearic acid } & \mathbf{2 , 7 9} \pm \mathbf{0 , 2 7} & \text { Eicosenoic acid } & \mathbf{0 , 2 8} \pm \mathbf{0 , 0 1} & \omega 6 / \omega 3 & \mathbf{9 , 2 1} \pm \mathbf{0 , 2 2} \\ \text { Oleic acid } & \mathbf{7 7 , 0 2} \pm \mathbf{0 , 8 4} & \text { Behenic acid } & \mathbf{0 , 1 9} \pm \mathbf{0 , 0 1} & & \end{array}$

\section{Organoleptic oil values}

Sensory Analysis (Panel test)

Comment: fruity medium-high, with hints of almond, read sensations of grass, tomato and artichoke. Balanced taste sensation with a medium bitter and spicy. Medium fluidity.

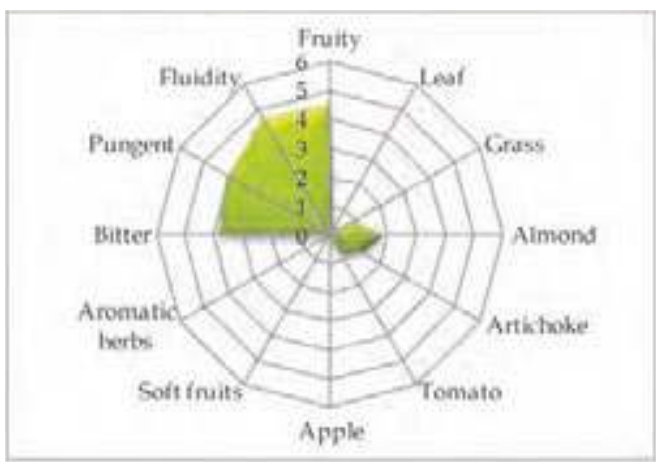

\section{Molecular Markers}

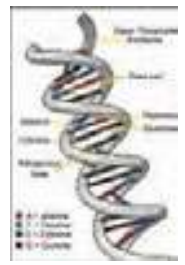

Table 2. Microsatellites (SSR) loci analyzed.

For each locus the allele size (expressed in base pairs) is reported.

$\begin{array}{cccccc}D C A 09 & \text { DCA18 } & \text { GAPU59 } & \text { GAPU71A } & \text { GUPA71B } & \text { GAPU103A } \\ \mathbf{1 6 2} \mathbf{- 1 9 8} & \mathbf{1 7 9 - \mathbf { 1 8 1 }} & \mathbf{2 1 2} \mathbf{- 2 2 2} & \mathbf{2 1 4 - \mathbf { 2 1 4 }} & \mathbf{1 2 6 - \mathbf { 1 2 6 }} & \mathbf{1 8 4} \mathbf{- 1 9 7} \\ \text { UDOO1 } & \text { UDOO3 } & \text { UDO12 } & \text { UDO28 } & \text { UDO39 } & \\ \mathbf{1 4 4 - \mathbf { 1 4 4 }} & \mathbf{1 3 5 - \mathbf { 1 3 5 }} & \mathbf{1 6 6 - 1 9 3} & \mathbf{1 5 4 - \mathbf { 1 5 4 }} & \mathbf{2 1 3 - 2 3 2} & \end{array}$

\section{References:}

1 - Parlati M.V., Pandolfi S. In: Catalogo delle principali varietà di olivo del Lazio, Ist. Sper. Olivic. (2003), pp. 39-40. 2 - Various authors. In: Catalogo Nazionale delle Varietà di Olivo, University of Bari (in press), ISBN 978-88-8879397-9. 


\section{“Marzio"}

Areal distribution or origin area: Toscana

Flesh/pit weight ratio: medium $(6,67 \pm 0,31)$

Oil content (\%): medium $(45,62 \pm 0,69)$

Purpose: oil

\section{Morphological characters}

Tree characters

Vigour: medium

Growth habit: spreading

Canopy-density: medium-dense

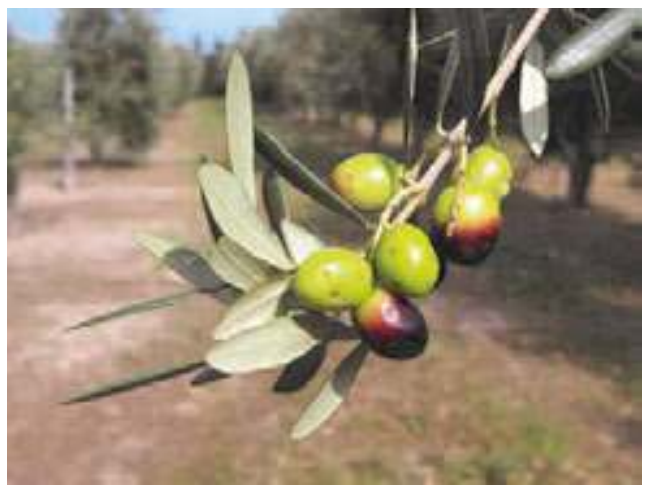

\section{Leaf characters}

Blade length $(\mathrm{cm})$ : medium $(5,34 \pm 0,39)$

Blade width $(\mathrm{cm})$ : medium $(\mathbf{1}, 31 \pm \mathbf{0 , 1 3})$

Shape (length/width): elliptic-lanceolate

\section{Inflorescence characters}

Inflorescence length $(\mathrm{cm})$ : medium $(2,57 \pm 0,53)$

Number of flowers: low $(16,95 \pm 1,70)$

\section{Fruit characters}

Fresh weight of 100 fruits $(\mathrm{g})$ : medium $(2,11 \pm 0,15)$

Shape (length/width): spherical

Symmetry: slightly asymmetric

Position of maximum transverse diameter:

central

Apex: rounded

Base: truncate

Niplle: absent

Lenticels: many and small

\section{Pit characters}

Weight of 100 pits $(\mathrm{g})$ : low $(\mathbf{0 , 2 8} \pm \mathbf{0 , 0 3})$

Shape (length/width): ovoid

Mucron: obvious

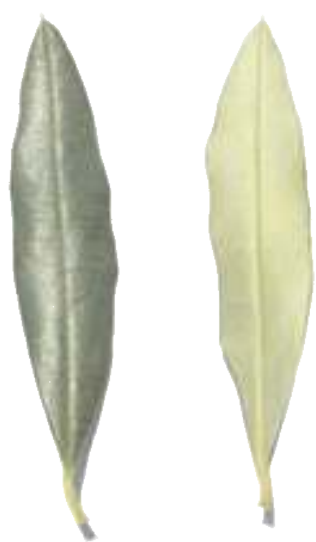

Symmetry: slightly asymmetric

Position of maximum transverse diameter:

central

Apex: rounded

Base: rounded

Surface: smooth

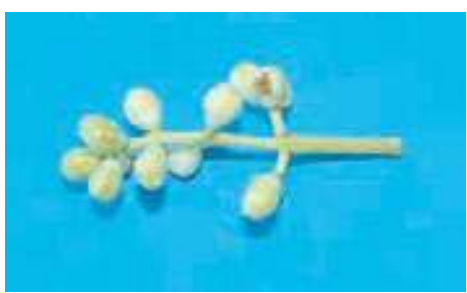

Number of grooves: low 


\section{Biochemical Characters}

\section{Fatty Acid Composition}

Table 1. Average values (express in $\% \pm$ standard deviations) of the fatty acids methyl esters and nutritional ratios obtained from single cultivar olive oils.

$\begin{array}{llllll}\text { Myristic acid } & \mathbf{0 , 0 1} \pm \mathbf{0 , 0 1} & \text { Linoleic acid }(\omega 6) & \mathbf{1 0 , 3 3} \pm \mathbf{0 , 5 1} & \text { Lignoceric acid } & \mathbf{0 , 0 3} \pm \mathbf{0 , 0 0} \\ \text { Palmitic acid } & \mathbf{1 2 , 0 0 \pm \mathbf { 1 , 2 0 }} & \text { Linolenic acid }(\omega 3) \mathbf{0 , 7 4} \pm \mathbf{0 , 0 5} & & \\ \text { Palmitoleic acid } & \mathbf{0 , 9 8} \pm \mathbf{0 , 1 3} & \text { Arachic acid } & \mathbf{0 , 4 8} \pm \mathbf{0 , 0 5} & \text { Unsat./satured } & \mathbf{5 , 7 7} \pm \mathbf{0 , 2 1} \\ \text { Stearic acid } & \mathbf{2 , 3 9} \pm \mathbf{0 , 7 7} & \text { Eicosenoic acid } & \mathbf{0 , 2 1} \pm \mathbf{0 , 0 2} & \omega 6 / \omega 3 & \mathbf{1 3 , 9 0 \pm 1 , 6 7} \\ \text { Oleic acid } & \mathbf{7 1 , 7 6 \pm 0 , 2 5} & \text { Behenic acid } & \mathbf{0 , 0 8} \pm \mathbf{0 , 0 0} & & \end{array}$

\section{Organoleptic oil values}

Sensory Analysis (Panel test)

Comment: medium fruity, with good sensation of almond and aromatic herbs, less grass and artichoke. Balanced taste sensation, with bitter and spicy medium. Medium-high fluidity.

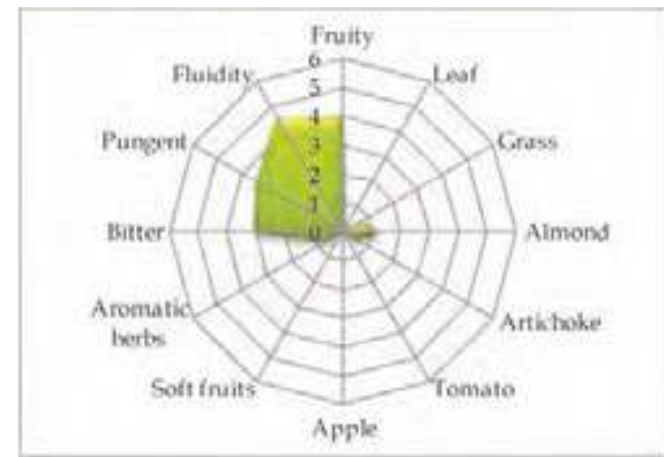

\section{Molecular Markers}

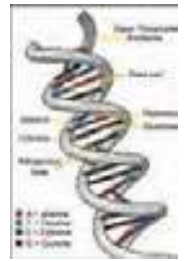

Table 2. Microsatellites (SSR) loci analyzed.

For each locus the allele size (expressed in base pairs) is reported.

$\begin{array}{cccccc}D C A 09 & \text { DCA18 } & \text { GAPU59 } & \text { GAPU71A } & \text { GUPA71B } & \text { GAPU103A } \\ \mathbf{1 6 2} \mathbf{- 1 9 8} & \mathbf{1 8 1} \mathbf{- 1 8 1} & \mathbf{2 0 8 - \mathbf { 2 1 8 }} & \mathbf{2 1 0 - \mathbf { 2 1 8 }} & \mathbf{1 3 0 - \mathbf { 1 3 0 }} & \mathbf{1 5 9 - \mathbf { 1 5 9 }} \\ \text { UDOO1 } & \text { UDOO3 } & \text { UDO12 } & \text { UDO28 } & \text { UDO39 } & \\ \mathbf{1 5 9 - \mathbf { 1 5 9 }} & \mathbf{1 5 0 - \mathbf { 1 5 0 }} & \mathbf{1 6 6 - \mathbf { 1 8 2 }} & \mathbf{1 8 2} \mathbf{- 2 0 5} & \mathbf{2 0 0 - \mathbf { 2 2 0 }} & \end{array}$

\section{References:}

1 - Cimato A., Cantini C., Sani G., In: L'olivo in Toscana: il germoplasma autoctono, Ed. ARSIA (2001).

2 - Various authors. In: Catalogo Nazionale delle Varietà di Olivo, University of Bari (in press), ISBN 978-88-8879397-9. 


\section{"Mignola"}

(synonymy: Carbonella, Laurina, Sargana, Sarganella, Suricina, etc.)

Areal distribution or origin area: Marche

Flesh/pit weight ratio: medium $(5,48 \pm 0,24)$

Oil content (\%): medium $(45,29 \pm 0,70)$

Purpose: oil

\section{Morphological characters}

Tree characters

Vigour: medium-strong

Growth habit: spreading-erect

Canopy-density: medium-dense

\section{Leaf characters}

Blade length $(\mathrm{cm})$ : medium $(6,60 \pm 0,58)$

Blade width (cm): broad $(\mathbf{1}, 55 \pm 0,15)$

Shape (length/width): elliptic-lanceolate

\section{Inflorescence characters}

Inflorescence length (cm): long $(4,04 \pm 1,39)$

Number of flowers: medium $(20,94 \pm 3,07)$

\section{Fruit characters}

Fresh weight of 100 fruits $(\mathrm{g})$ : low $(\mathbf{1}, 35 \pm 0,05)$

Shape (length/width): ovoid

Symmetry: asymmetric

Position of maximum transverse diameter:

central

Apex: pointed

Base: rounded

Niplle: absent

Lenticels: many and small

\section{Pit characters}

Weight of 100 pits (g): low $(\mathbf{0 , 2 1} \pm \mathbf{0 , 0 2}$ )

Shape (length/width): elliptic

Mucron: obvious

Symmetry: slightly asymmetric

Position of maximum transverse diameter:

central

Apex: pointed

Base: pointed

Surface: smooth

Number of grooves: high
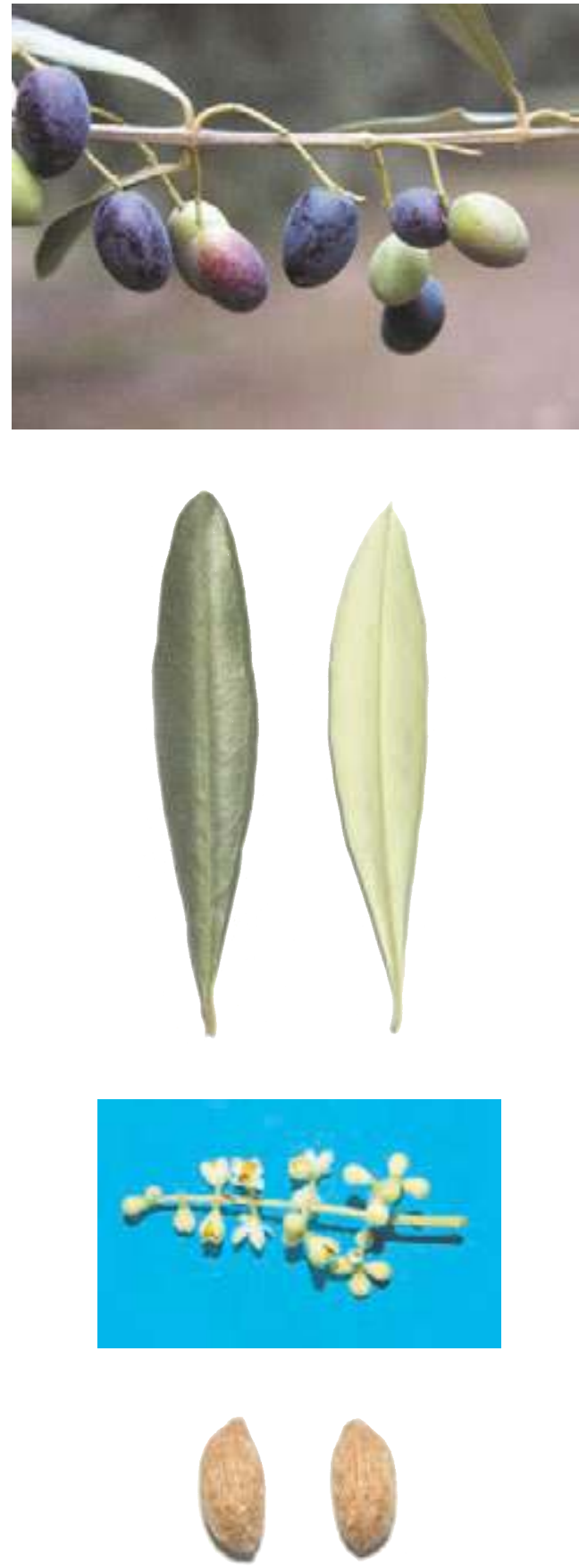


\section{Biochemical Characters}

\section{Fatty Acid Composition}

Table 1. Average values (express in $\% \pm$ standard deviations) of the fatty acids methyl esters and nutritional ratios obtained from single cultivar olive oils.

$\begin{array}{llllll}\text { Myristic acid } & \mathbf{0 , 0 1} \pm \mathbf{0 , 0 0} & \text { Linoleic acid }(\omega 6) & \mathbf{8 , 9 4} \pm \mathbf{0 , 4 7} & \text { Lignoceric acid } & \mathbf{0 , 0 5} \pm \mathbf{0 , 0 1} \\ \text { Palmitic acid } & \mathbf{1 3 , 3 3} \pm \mathbf{0 , 4 6} & \text { Linolenic acid }(\omega 3) \mathbf{0 , 4 6} \pm \mathbf{0 , 0 4} & & \\ \text { Palmitoleic acid } & \mathbf{1 , 9 3} \pm \mathbf{0 , 2 6} & \text { Arachic acid } & \mathbf{0 , 3 7} \pm \mathbf{0 , 1 6} & \text { Unsat./satured } & \mathbf{5 , 4 6} \pm \mathbf{0 , 3 9} \\ \text { Stearic acid } & \mathbf{1 , 7 0} \pm \mathbf{0 , 2 4} & \text { Eicosenoic acid } & \mathbf{0 , 2 7} \pm \mathbf{0 , 0 8} & \omega 6 / \omega 3 & \mathbf{1 9 , 6 6} \pm \mathbf{0 , 4 9} \\ \text { Oleic acid } & \mathbf{7 1 , 2 3} \pm \mathbf{2 , 3 3} & \text { Behenic acid } & \mathbf{0 , 0 6} \pm \mathbf{0 , 0 6} & & \end{array}$

\section{Organoleptic oil values}

Sensory Analysis (Panel test)

Comment: fruity medium, with hints of soft fruits and read sensations of almond, grass and artichoke. Balanced taste sensation with a medium-high bitter and spicy. Mediumhigh.

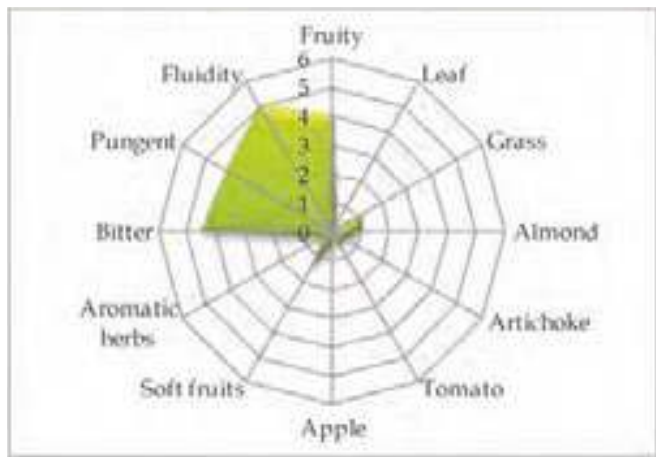

\section{Molecular Markers}

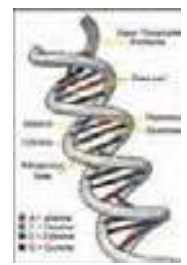

Table 2. Microsatellites (SSR) loci analyzed.

For each locus the allele size (expressed in base pairs) is reported.

$\begin{array}{cccccc}D C A 09 & \text { DCA18 } & \text { GAPU59 } & \text { GAPU71A } & \text { GUPA71B } & \text { GAPU103A } \\ \mathbf{1 7 2} \mathbf{- 1 7 2} & \mathbf{1 7 7 - \mathbf { 1 8 5 }} & \mathbf{2 1 4 - \mathbf { 2 1 8 }} & \mathbf{2 1 4 - \mathbf { 2 2 1 }} & \mathbf{1 2 6 - \mathbf { 1 4 4 }} & \mathbf{1 5 7 - \mathbf { 1 5 7 }} \\ \text { UDOO1 } & \text { UDO03 } & \text { UDO12 } & \text { UDO28 } & \text { UDO39 } & \\ \mathbf{1 4 4 - \mathbf { 1 4 4 }} & \mathbf{1 4 3 - \mathbf { 1 4 3 }} & \mathbf{1 7 7 - 1 9 3} & \mathbf{1 5 4 - \mathbf { 2 0 5 }} & \mathbf{2 0 0 - \mathbf { 2 0 0 }} & \end{array}$

\section{References:}

1 - Pannelli G., Alfei B., Santinelli A. In: Varietà di olivo nelle Marche. ASSAM (2001), pp. 65-68.

2 - Various authors. In: Catalogo Nazionale delle Varietà di Olivo, University of Bari (in press), ISBN 978-88-8879397-9. 


\section{“Mignolo"}

(synonymy: Gremignolo, Mignola, Minuto, Prugnolo, etc.)

Areal distribution or origin area: Toscana

Flesh/pit weight ratio: low $(2,67 \pm 0,98)$

Oil content (\%): medium $(44,17 \pm 0,71)$

Purpose: oil

\section{Morphological characters}

Tree characters

Vigour: strong

Growth habit: spreading

Canopy-density: medium-dense

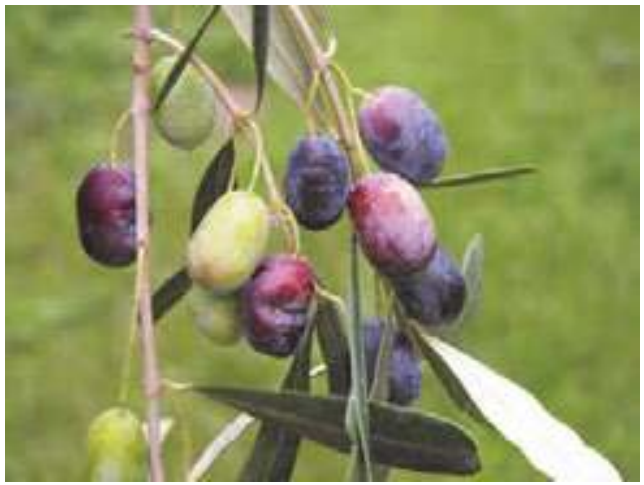

\section{Leaf characters}

Blade length $(\mathrm{cm})$ : medium $(5,69 \pm 0,75)$

Blade width $(\mathrm{cm})$ : medium $(\mathbf{1}, \mathbf{4 1} \pm \mathbf{0 , 2 1})$

Shape (length/width): elliptic lanceolate

\section{Inflorescence characters}

Inflorescence length (cm): long $(3,98 \pm 0,68)$

Number of flowers: medium $(19,04 \pm 1,19)$

\section{Fruit characters}

Fresh weight of 100 fruits $(\mathrm{g})$ : medium $(2,06 \pm 0,85)$

Shape (length/width): ovoid

Symmetry: slightly asymmetric

Position of maximum transverse diameter: central

Apex: rounded

Base: rounded

Niplle: absent

Lenticels: many and large

\section{Pit characters}

Weight of 100 pits (g): high $(0,55 \pm 0,09)$

Shape (length/width): elliptic

Mucron: obvious

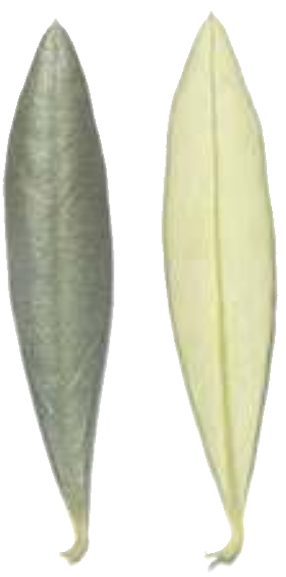

Symmetry: slightly asymmetric

Position of maximum transverse diameter:

\section{towards apex}

Apex: rounded

Base: rounded

Surface: rugose

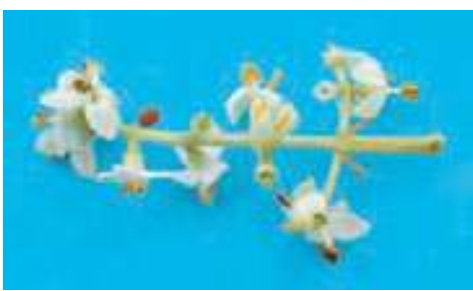

Number of grooves: low 


\section{Biochemical Characters}

\section{Fatty Acid Composition}

Table 1. Average values (express in $\% \pm$ standard deviations) of the fatty acids methyl esters and nutritional ratios obtained from single cultivar olive oils.

$\begin{array}{llllll}\text { Myristic acid } & \mathbf{0 , 0 1} \pm \mathbf{0 , 0 0} & \text { Linoleic acid }(\omega 6) & \mathbf{1 0 , 4 6} \pm \mathbf{0 , 6 5} & \text { Lignoceric acid } & \mathbf{0 , 0 2} \pm \mathbf{0 , 0 1} \\ \text { Palmitic acid } & \mathbf{1 0 , 1 8} \pm \mathbf{0 , 0 8} & \text { Linolenic acid }(\omega 3) \mathbf{0 , 9 1} \pm \mathbf{0 , 0 2} & & \\ \text { Palmitoleic acid } & \mathbf{1 , 2 1} \pm \mathbf{0 , 6 8} & \text { Arachic acid } & \mathbf{0 , 3 4} \pm \mathbf{0 , 0 3} & \text { Unsat./satured } & \mathbf{6 , 8 4} \pm \mathbf{0 , 4 9} \\ \text { Stearic acid } & \mathbf{2 , 3 4} \pm \mathbf{1 , 3 2} & \text { Eicosenoic acid } & \mathbf{0 , 0 3} \pm \mathbf{0 , 0 1} & \omega 6 / \omega 3 & \mathbf{1 1 , 5 4} \pm \mathbf{0 , 4 5} \\ \text { Oleic acid } & \mathbf{7 3 , 4 0 \pm 0 , 6 3} & \text { Behenic acid } & \mathbf{0 , 0 9} \pm \mathbf{0 , 0 0} & & \end{array}$

\section{Organoleptic oil values}

Sensory Analysis (Panel test)

Comment: fruity medium-high, with hints of almond and read sensations of grass and artichoke. Balanced taste sensation with a medium bitter and spicy. Medium fluidity.

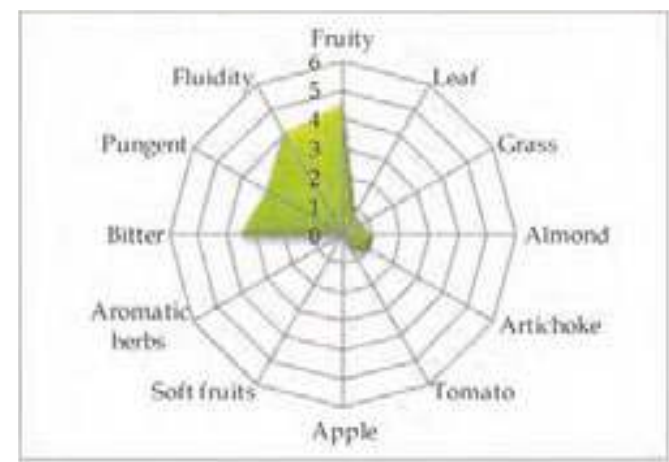

\section{Molecular Markers}

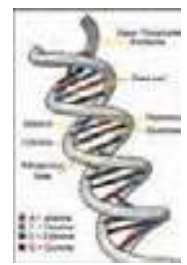

Table 2. Microsatellites (SSR) loci analyzed.

For each locus the allele size (expressed in base pairs) is reported.

$\begin{array}{cccccc}D C A 09 & \text { DCA18 } & \text { GAPU59 } & \text { GAPU71A } & \text { GUPA71B } & \text { GAPU103A } \\ \mathbf{1 8 2} \mathbf{- 2 0 6} & \mathbf{1 8 1} \mathbf{- 1 8 1} & \mathbf{2 0 8 - \mathbf { 2 1 0 }} & \mathbf{2 1 0 - \mathbf { 2 1 8 }} & \mathbf{1 2 4 - \mathbf { 1 4 4 }} & \mathbf{1 5 9 - \mathbf { 1 7 0 }} \\ \text { UDOO1 } & \text { UDOO3 } & \text { UDO12 } & \text { UDO28 } & \text { UDO39 } & \\ \mathbf{1 5 0 - \mathbf { 1 5 0 }} & \mathbf{1 5 0 - \mathbf { 1 5 0 }} & \mathbf{1 5 6 - \mathbf { 1 7 7 }} & \mathbf{1 8 2} \mathbf{- 2 0 5} & \mathbf{2 0 0 - \mathbf { 2 0 0 }} & \end{array}$

\section{References:}

1 - Cimato A., Cantini C., Sani G., In: L'olivo in Toscana: il germoplasma autoctono, Ed. ARSIA (2001).

2 - Various authors. In: Catalogo Nazionale delle Varietà di Olivo, University of Bari (in press), ISBN 978-88-8879397-9. 


\section{“Minna di vacca"}

Areal distribution or origin area: Sicilia

Flesh/ pit weight ratio: high $(\mathbf{9 , 2 4} \pm \mathbf{0 , 8 6})$

Oil content (\%): high $(\mathbf{5 3 , 4 8} \pm \mathbf{0 , 7 0})$

Purpose: table

\section{Morphological characters}

Tree characters

Vigour: medium

Growth habit: spreading

Canopy-density: medium-sparse

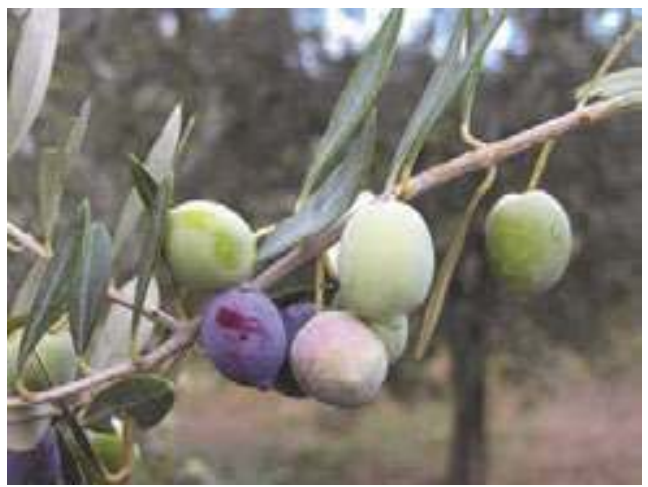

\section{Leaf characters}

Blade length $(\mathrm{cm})$ : medium $(5,08 \pm 0,36)$

Blade width $(\mathrm{cm})$ : medium $(\mathbf{1}, \mathbf{4 7} \pm \mathbf{0 , 1 5})$

Shape (length/width): elliptic

\section{Inflorescence characters}

Inflorescence length $(\mathrm{cm})$ : medium $(2,56 \pm 1,68)$

Number of flowers: low $(13,41 \pm 1,04)$

\section{Fruit characters}

Fresh weight of 100 fruits $(\mathrm{g})$ : high $(5,59 \pm 0,54)$

Shape (length/width): spherical

Symmetry: symmetric

Position of maximum transverse diameter:

central

Apex: rounded

Base: rounded

Niplle: obvious

Lenticels: many and small

\section{Pit characters}

Weight of 100 pits (g): high $(\mathbf{0 , 5 5} \pm \mathbf{0 , 0 1})$

Shape (length/width): ovoid

Mucron: obvious

Symmetry: slightly asymmetric

Position of maximum transverse diameter:

\section{towuards apex}

Apex: rounded

Base: rounded

Surface: scabrous

Number of grooves: medium
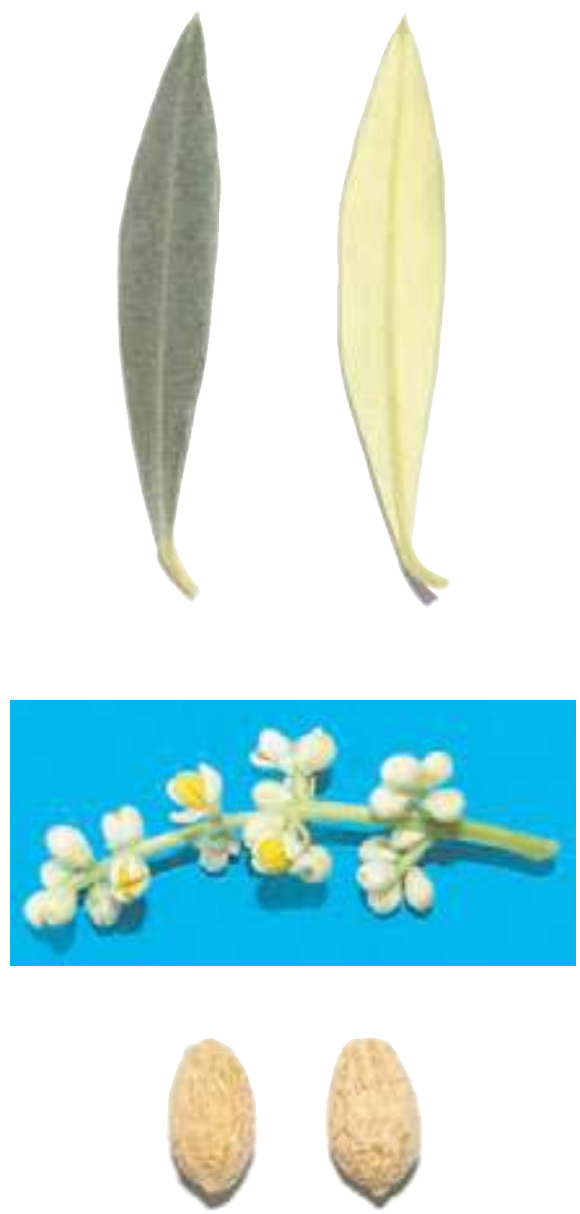


\section{Biochemical Characters}

\section{Fatty Acid Composition}

Table 1. Average values (express in $\% \pm$ standard deviations) of the fatty acids methyl esters and nutritional ratios obtained from single cultivar olive oils.

$\begin{array}{llllll}\text { Myristic acid } & \mathbf{0 , 0 2} \pm \mathbf{0 , 0 1} & \text { Linoleic acid }(\omega 6) & \mathbf{9 , 6 9} \pm \mathbf{0 , 7 2} & \text { Lignoceric acid } & \mathbf{0 , 0 4} \pm \mathbf{0 , 0 1} \\ \text { Palmitic acid } & \mathbf{1 1 , 2 4} \pm \mathbf{0 , 3 7} & \text { Linolenic acid }(\omega 3) & \mathbf{0 , 9 7} \pm \mathbf{0 , 0 1} & & \\ \text { Palmitoleic acid } & \mathbf{0 , 4 1} \pm \mathbf{0 , 0 4} & \text { Arachic acid } & \mathbf{0 , 2 2} \pm \mathbf{0 , 0 9} & \text { Unsat./satured } & \mathbf{6 , 5 7 \pm 0 , 2 2} \\ \text { Stearic acid } & \mathbf{1 , 7 7} \pm \mathbf{0 , 0 2} & \text { Eicosenoic acid } & \mathbf{0 , 0 3} \pm \mathbf{0 , 0 2} & \omega 6 / \omega 3 & \mathbf{9 , 9 9} \pm \mathbf{0 , 5 9} \\ \text { Oleic acid } & \mathbf{7 5 , 3 5 \pm \mathbf { 1 , 0 8 }} & \text { Behenic acid } & \mathbf{0 , 0 5} \pm \mathbf{0 , 0 1} & & \end{array}$

\section{Organoleptic oil values}

Sensory Analysis (Panel test)

Comment: fruity medium-high, with taste of artichoke and tomato, read sensations of almond. Balanced taste sensation with medium bitter and spicy. Medium-high fluidity.

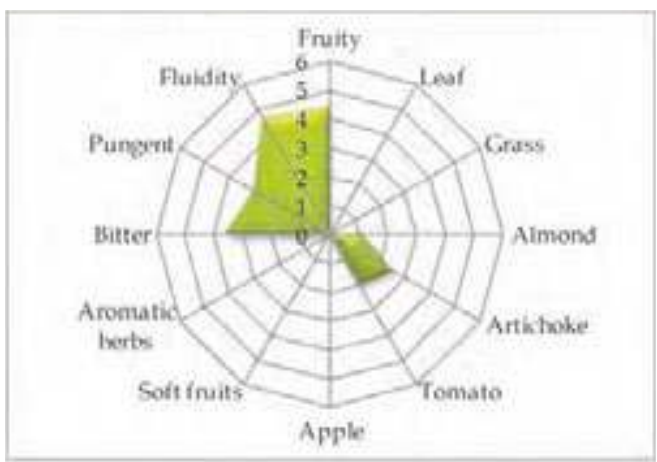

\section{Molecular Markers}

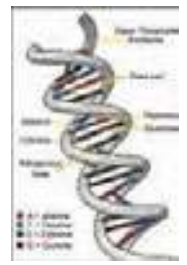

Table 2. Microsatellites (SSR) loci analyzed.

For each locus the allele size (expressed in base pairs) is reported.

$\begin{array}{cccccc}D C A 09 & \text { DCA18 } & \text { GAPU59 } & \text { GAPU71A } & \text { GUPA71B } & \text { GAPU103A } \\ \mathbf{1 7 6 - \mathbf { 2 0 6 }} & \mathbf{1 8 1} \mathbf{- 1 8 5} & \mathbf{2 0 8 - \mathbf { 2 1 2 }} & \mathbf{2 1 4 - \mathbf { 2 1 4 }} & \mathbf{1 2 4 - \mathbf { 1 3 0 }} & \mathbf{1 5 9 - \mathbf { 1 8 4 }} \\ \text { UDOO1 } & \text { UDO03 } & \text { UDO12 } & \text { UDO28 } & \text { UDO39 } & \\ \mathbf{1 4 0 - \mathbf { 1 4 0 }} & \mathbf{1 5 0 - \mathbf { 1 5 0 }} & \mathbf{1 6 6 - \mathbf { 1 9 3 }} & \mathbf{1 8 2} \mathbf{- 1 8 2} & \mathbf{1 0 8 - 1 0 8} & \end{array}$

\section{References:}

1 - Bottari V., Spina P. In: Le varietà di olivo coltivate in Sicilia, Ann. Sper. Agr. (1953), 7: pp. 937-1004.

2 - Muzzalupo I., Stefanizzi F., Perri E. HortScience (2009), 44: pp. 582-588. 


\section{"Monaca"}

(synonymy: Calabrise, Monaca, etc.)

Areal distribution or origin area: Sicilia

Flesh/pit weight ratio: medium $(5,20 \pm 1,09)$

Oil content (\%): medium $(40,74 \pm 0,93)$

Purpose: oil

\section{Morphological characters}

Tree characters

Vigour: strong

Growth habit: spreading-erect

Canopy-density: medium

\section{Leaf characters}

Blade length $(\mathrm{cm})$ : medium $(5,06 \pm 0,42)$

Blade width $(\mathrm{cm})$ : medium $(\mathbf{1 , 3 8} \pm \mathbf{0 , 1 3})$

Shape (length/width): elliptic

\section{Inflorescence characters}

Inflorescence length (cm): medium $(2,95 \pm 2,30)$

Number of flowers: high $(\mathbf{2 5 , 0 8} \pm \mathbf{1}, \mathbf{7 6})$

\section{Fruit characters}

Fresh weight of 100 fruits (g): medium $(3,38 \pm 0,02)$

Shape (length/width): ovoid

Symmetry: symmetric

Position of maximum transverse diameter:

central

Apex: rounded

Base: truncate

Niplle: tenuous

Lenticels: few and large

\section{Pit characters}

Weight of 100 pits (g): high $(0,55 \pm 0,06)$

Shape (length/width): elliptic

Mucron: obvious

Symmetry: symmetric

Position of maximum transverse diameter:

towards apex

Apex: pointed

Base: rounded

Surface: rugose

Number of grooves: low
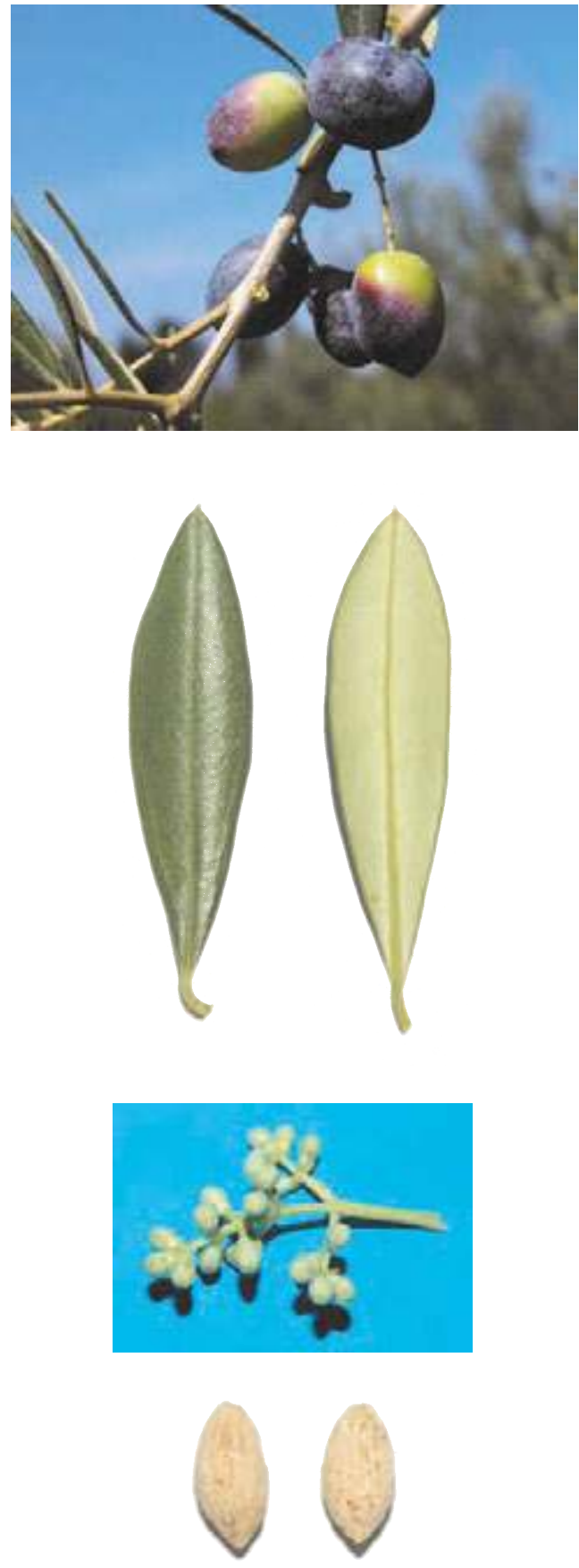


\section{Biochemical Characters}

\section{Fatty Acid Composition}

Table 1. Average values (express in $\% \pm$ standard deviations) of the fatty acids methyl esters and nutritional ratios obtained from single cultivar olive oils.

\begin{tabular}{|c|c|c|c|c|c|}
\hline Myristic acid & $0,01 \pm 0,00$ & Linoleic acid $(\omega 6)$ & $13,94 \pm 1,02$ & Lignoceric acid & $0,05 \pm 0,03$ \\
\hline Palmitic acid & $14,66 \pm 0,67$ & Linolenic acid $(\omega 3)$ & $0,76 \pm 0,03$ & & \\
\hline Palmitoleic acid & $3,03 \pm 0,10$ & Arachic acid & $0,30 \pm 0,04$ & Unsat./satured & $4,73 \pm 0,23$ \\
\hline ea & $1,96 \pm 0,05$ & Eicosenoic acid & $0,09 \pm 0,13$ & $\omega 6 / \omega 3$ & $18,35 \pm 0,81$ \\
\hline leic acid & $61,84 \pm 1,80$ & Behenic acid & $0,09 \pm 0,02$ & & \\
\hline
\end{tabular}

\section{Organoleptic oil values}

Sensory Analysis (Panel test)

Comment: fruity medium-light, with taste of aromatic herbs, read sensations of almond, grass and leaves. Balanced taste sensation with medium bitter and spicy. Medium fluidity.

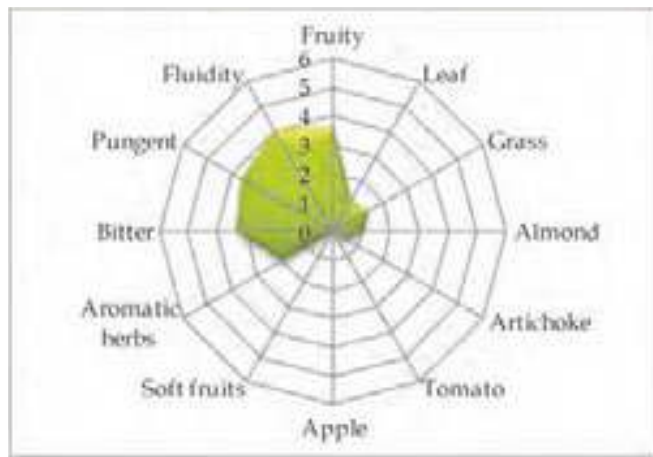

\section{Molecular Markers}

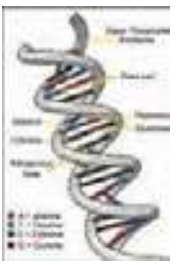

Table 2. Microsatellites (SSR) loci analyzed.

For each locus the allele size (expressed in base pairs) is reported.

$\begin{array}{cccccc}D C A 09 & \text { DCA18 } & \text { GAPU59 } & \text { GAPU71A } & \text { GUPA71B } & \text { GAPU103A } \\ \mathbf{1 7 6 - \mathbf { 2 0 6 }} & \mathbf{1 8 1 - \mathbf { 1 8 1 }} & \mathbf{2 0 8 - \mathbf { 2 1 2 }} & \mathbf{2 1 0 - \mathbf { 2 1 4 }} & \mathbf{1 2 4} \mathbf{- 1 4 4} & \mathbf{1 5 7 - \mathbf { 1 5 9 }} \\ \text { UDOO1 } & \text { UDOO3 } & \text { UDO12 } & \text { UDO28 } & \text { UDO39 } & \\ \mathbf{1 4 4 - \mathbf { 1 4 4 }} & \mathbf{1 5 0 - \mathbf { 1 5 0 }} & \mathbf{1 7 7 - \mathbf { 1 9 3 }} & \mathbf{1 5 4} \mathbf{- 1 8 2} & \mathbf{1 6 4 - \mathbf { 2 0 5 }} & \end{array}$

\section{References:}

1 - Lombardo N. In: Le risorse genetiche vegetali presso gli IRSA, Ed. MIPAF (2001), 1:pp. 361-405.

2 - Muzzalupo I., Stefanizzi F., Perri E. HortScience (2009), 44: pp. 582-588. 


\section{“Mora "}

Areal distribution or origin area: Puglia

Flesh/ pit weight ratio: high $(8,63 \pm 1,73)$

Oil content (\%): low $(37,70 \pm 1,46)$

Purpose: dual purpose

\section{Morphological characters}

Tree characters

Vigour: medium-strong

Growth habit: spreading

Canopy-density: dense

\section{Leaf characters}

Blade length $(\mathrm{cm})$ : medium $(6,64 \pm 0,54)$

Blade width $(\mathrm{cm})$ : broad $(\mathbf{1}, 83 \pm \mathbf{0 , 2 7})$

Shape (length/width): elliptic

\section{Inflorescence characters}

Inflorescence length $(\mathrm{cm})$ : medium $(2,34 \pm 1,71)$

Number of flowers: low $(13,07 \pm 1,17)$

\section{Fruit characters}

Fresh weight of 100 fruits $(\mathrm{g})$ : low $(4,750,76)$

Shape (length/width): ovoid

Symmetry: slightly asymmetric

Position of maximum transverse diameter: central

Apex: rounded

Base: rounded

Niplle: absent

Lenticels: many and small

\section{Pit characters}

Weight of 100 pits (g): high $(\mathbf{0 , 5 1} \pm \mathbf{0 , 1 5})$

Shape (length/width): elliptic

Mucron: absent

Symmetry: slightly asymmetric

Position of maximum transverse diameter:

towards apice

Apex: rounded

Base: rounded

Surface: rugose

Number of grooves: medium
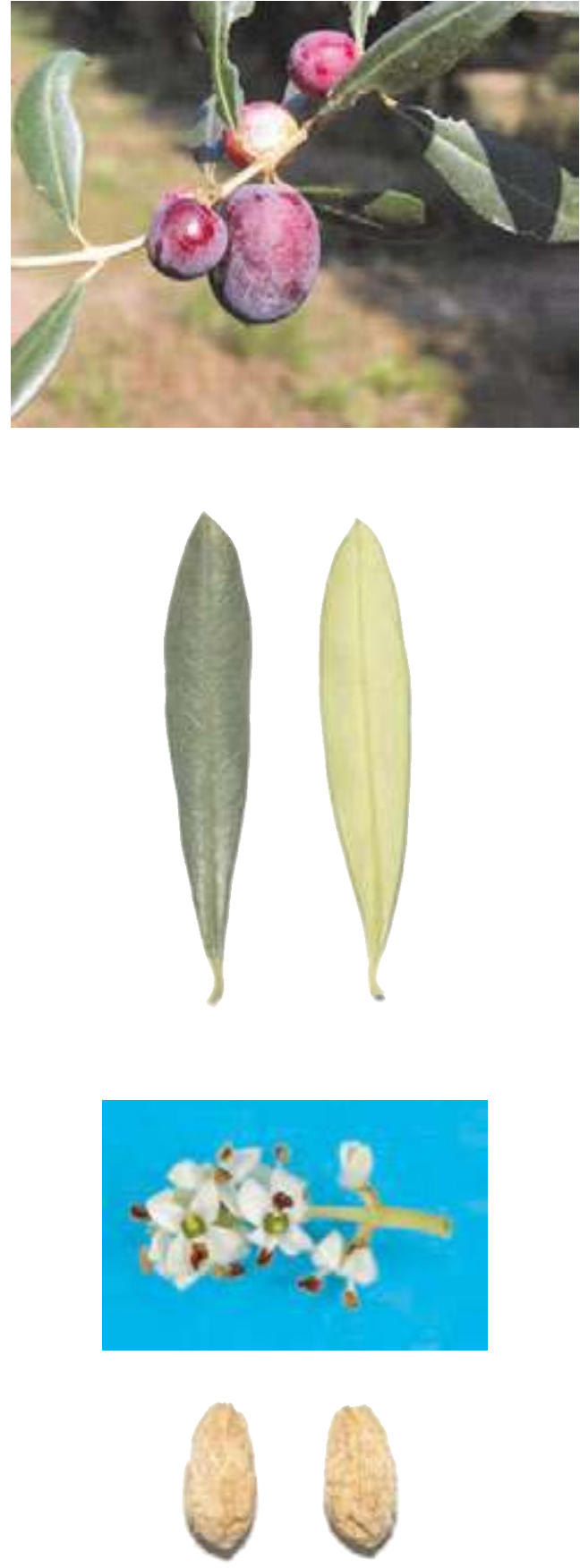


\section{Biochemical Characters}

\section{Fatty Acid Composition}

Table 1. Average values (express in $\% \pm$ standard deviations) of the fatty acids methyl esters and nutritional ratios obtained from single cultivar olive oils.

$\begin{array}{llllll}\text { Myristic acid } & \mathbf{0 , 0 1} \pm \mathbf{0 , 0 0} & \text { Linoleic acid }(\omega 6) & \mathbf{1 6 , 0 2} \pm \mathbf{1 , 7 7} & \text { Lignoceric acid } & \mathbf{0 , 0 3} \pm \mathbf{0 , 0 1} \\ \text { Palmitic acid } & \mathbf{1 4 , 0 5} \pm \mathbf{0 , 0 8} & \text { Linolenic acid }(\omega 3) \mathbf{1 , 2 9} \pm \mathbf{0 , 0 3} & & \\ \text { Palmitoleic acid } & \mathbf{2 , 3 4} \pm \mathbf{0 , 5 5} & \text { Arachic acid } & \mathbf{0 , 2 3} \pm \mathbf{0 , 0 2} & \text { Unsat./satured } & \mathbf{5 , 3 0 \pm 0 , 0 6} \\ \text { Stearic acid } & \mathbf{1 , 2 7} \pm \mathbf{0 , 0 4} & \text { Eicosenoic acid } & \mathbf{0 , 0 3} \pm \mathbf{0 , 0 0} & \omega 6 / \omega 3 & \mathbf{1 2 , 4 1} \pm \mathbf{1 , 0 5} \\ \text { Oleic acid } & \mathbf{6 2 , 5 1 \pm 2 , 9 3} & \text { Behenic acid } & \mathbf{0 , 0 7} \pm \mathbf{0 , 0 1} & & \end{array}$

\section{Organoleptic oil values}

Sensory Analysis (Panel test)

Comment: fruity medium-high, with hints of almond, read sensations of grass, tomato, and artichoke. Balanced taste sensation with a medium-high bitter and spicy. Mediumhigh fluidity.

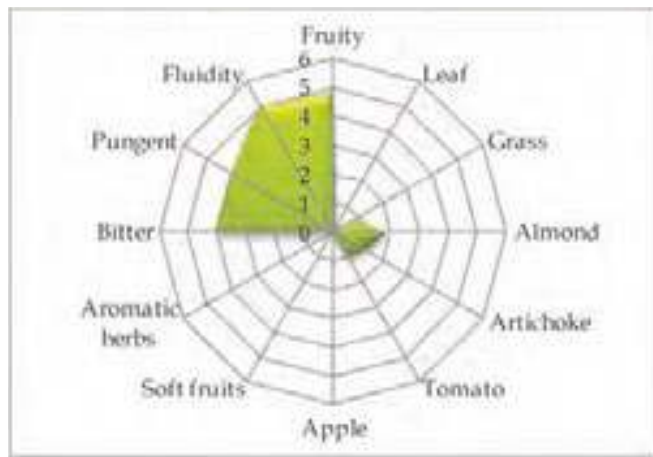

\section{Molecular Markers}

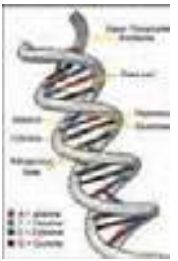

Table 2. Microsatellites (SSR) loci analyzed.

For each locus the allele size (expressed in base pairs) is reported.

$\begin{array}{cccccc}D C A 09 & \text { DCA18 } & \text { GAPU59 } & \text { GAPU71A } & \text { GUPA71B } & \text { GAPU103A } \\ \mathbf{1 6 2} \mathbf{- 1 8 2} & \mathbf{1 7 9 - \mathbf { 1 8 1 }} & \mathbf{2 0 8 - \mathbf { 2 1 2 }} & \mathbf{2 1 0 - \mathbf { 2 2 4 }} & \mathbf{1 2 4} \mathbf{- 1 2 6} & \mathbf{1 7 0 - \mathbf { 1 8 4 }} \\ \text { UDOO1 } & \text { UDO03 } & \text { UDO12 } & \text { UDO28 } & \text { UDO39 } & \\ \mathbf{1 4 4 - \mathbf { 1 4 4 }} & \mathbf{1 4 3 - \mathbf { 1 4 3 }} & \mathbf{1 6 6 - \mathbf { 1 9 3 }} & \mathbf{1 4 3 - \mathbf { 2 1 0 }} & \mathbf{1 0 8 - \mathbf { 1 6 4 }} & \end{array}$

\section{References:}

1 - Ferrara E., Lamparelli F. In: Atti Convegno l'Olivicoltura mediterranea, Ist, Sper, Olivic. (1995), pp. $133-141$. 3 - Muzzalupo I., Stefanizzi F., Perri E. HortScience (2009), 44: pp. 582-588. 


\section{“Moraiolo T. Corsini "}

Areal distribution or origin area: Toscana

Flesh/pit weight ratio: low $(4,43 \pm 0,88)$

Oil content (\%): medium $(39,07 \pm 1,70)$

Purpose: oil

\section{Morphological characters}

Tree characters

Vigour: medium

Growth habit: spreading-erect

Canopy-density: medium-sparse

\section{Leaf characters}

Blade length $(\mathrm{cm})$ : medium $(5,89 \pm 0,36)$

Blade width $(\mathrm{cm})$ : medium $(\mathbf{1}, 43 \pm \mathbf{0 , 1 0})$

Shape (length/width): elliptic-lanceolate

\section{Inflorescence characters}

Inflorescence length ( $\mathrm{cm})$ : long $(3,56 \pm 0,88)$

Number of flowers: medium $(\mathbf{2 4 , 8 0 \pm 0 , 9 7 )}$

\section{Fruit characters}

Fresh weight of 100 fruits $(\mathrm{g})$ : low $(\mathbf{1}, 90 \pm \mathbf{0 , 2 3})$

Shape (length/width): ovoid

Symmetry: slightly asymmetric

Position of maximum transverse diameter:

central

Apex: rounded

Base: truncate

Niplle: absent

Lenticels: many and small

\section{Pit characters}

Weight of 100 pits $(\mathrm{g})$ : medium $(0,36 \pm 0,04)$

Shape (length/width): ovoid

Mucron: obvious

Symmetry: slightly asymmetric

Position of maximum transverse diameter:

central

Apex: pointed

Base: rounded

Surface: rugose

Number of grooves: medium
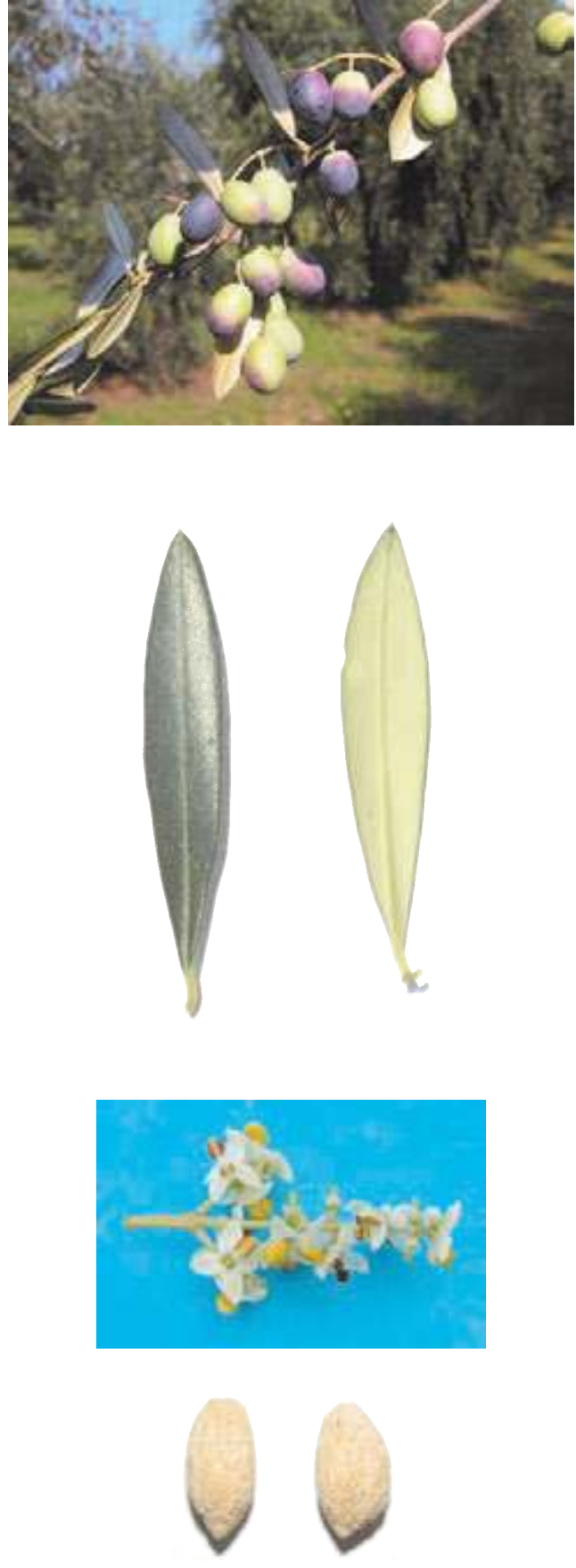


\section{Biochemical Characters}

\section{Fatty Acid Composition}

Table 1. Average values (express in $\% \pm$ standard deviations) of the fatty acids methyl esters and nutritional ratios obtained from single cultivar olive oils.

$\begin{array}{llllll}\text { Myristic acid } & \mathbf{0 , 0 1} \pm \mathbf{0 , 0 0} & \text { Linoleic acid }(\omega 6) & \mathbf{6 , 8 4} \pm \mathbf{0 , 7 3} & \text { Lignoceric acid } & \mathbf{0 , 0 9 \pm 0 , 1 2} \\ \text { Palmitic acid } & \mathbf{1 2 , 8 1} \pm \mathbf{0 , 5 0} & \text { Linolenic acid }(\omega 3) & \mathbf{0 , 8 5} \pm \mathbf{0 , 1 0} & & \\ \text { Palmitoleic acid } & \mathbf{0 , 8 8} \pm \mathbf{0 , 2 8} & \text { Arachic acid } & \mathbf{0 , 2 3} \pm \mathbf{0 , 1 0} & \text { Unsat./satured } & \mathbf{5 , 7 1} \pm \mathbf{0 , 3 1} \\ \text { Stearic acid } & \mathbf{1 , 7 7} \pm \mathbf{0 , 2 4} & \text { Eicosenoic acid } & \mathbf{0 , 0 4} \pm \mathbf{0 , 0 2} & \omega 6 / \omega 3 & \mathbf{8 , 0 5} \pm \mathbf{0 , 4 8} \\ \text { Oleic acid } & \mathbf{7 5 , 7 9 \pm 0 , 8 3} & \text { Behenic acid } & \mathbf{0 , 0 6} \pm \mathbf{0 , 0 2} & & \end{array}$

\section{Organoleptic oil values}

Sensory Analysis (Panel test)

Comment: fruity medium-high, with hints of almond, grass and artichoke. Balanced taste sensation with a medium bitter and spicy. Medium fluidity.

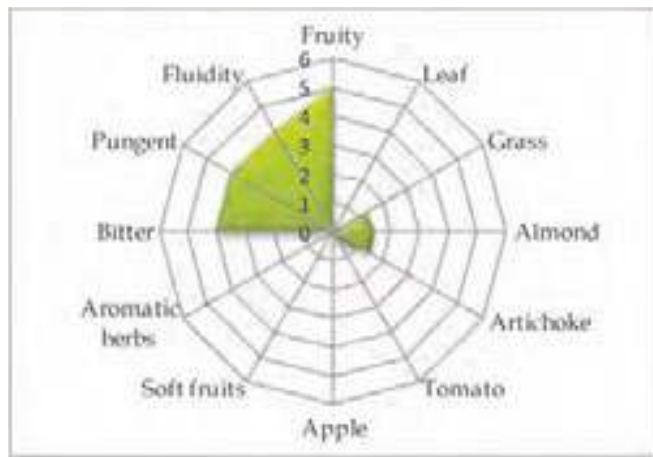

\section{Molecular Markers}

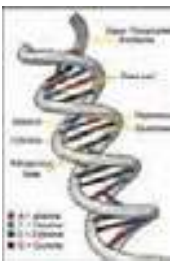

Table 2. Microsatellites (SSR) loci analyzed.

For each locus the allele size (expressed in base pairs) is reported.

\begin{tabular}{|c|c|c|c|c|c|}
\hline$D C A 09$ & $D C A 18$ & GAPU59 & GAPU71A & GUPA71B & GAPU103A \\
\hline $184-206$ & $179-185$ & $212-222$ & $214-214$ & $124-130$ & $150-157$ \\
\hline UDO01 & UDO03 & UDO12 & UDO28 & UDO39 & \\
\hline $144-144$ & $166-182$ & $177-193$ & $154-205$ & $213-220$ & \\
\hline
\end{tabular}

\section{References:}

1 - Lombardo N. In: Le risorse genetiche vegetali presso gli IRSA, MIPAF (2001), 1: pp. 361-405

2 - Muzzalupo I., Stefanizzi F., Bucci C., et al. In Acta Italus Hortus, (2011), 1: 138 -140. 


\section{“Moraiolo"}

(synonymy: Carboncella, Marinello, Morellino, Ogliolo, Oliva tonda, etc.)

Areal distribution or origin area: Toscana

Flesh/pit weight ratio: medium $(7,00 \pm 1,36)$

Oil content (\%): medium $(44,13 \pm 1,44)$

Purpose: oil

\section{Morphological characters}

Tree characters

Vigour: medium

Growth habit: spreading-erect

Canopy-density: medium

\section{Leaf characters}

Blade length $(\mathrm{cm})$ : medium $(5,29 \pm 0,64)$

Blade width $(\mathrm{cm})$ : medium $(\mathbf{1}, 26 \pm 0,16)$

Shape (length/width): elliptic-lanceolate

\section{Inflorescence characters}

Inflorescence length $(\mathrm{cm})$ : long $(3,51 \pm 0,70)$

Number of flowers: high $(\mathbf{2 6 , 4 9} \pm \mathbf{1 , 5 8})$

\section{Fruit characters}

Fresh weight of 100 fruits $(\mathrm{g})$ : medium $(2,36 \pm 0,34)$

Shape (length/width): spherical

Symmetry: slightly asymmetric

Position of maximum transverse diameter:

towards apex

Apex: rounded

Base: rounded

Niplle: absent

Lenticels: many and small

\section{Pit characters}

Weight of 100 pits $(\mathrm{g})$ : medium $(\mathbf{0 , 3 1} \pm \mathbf{0 , 0 1})$

Shape (length/width): ovoid

Mucron: absent

Symmetry: symmetric

Position of maximum transverse diameter:

central

Apex: rounded

Base: rounded

Surface: rugose

Number of grooves: high
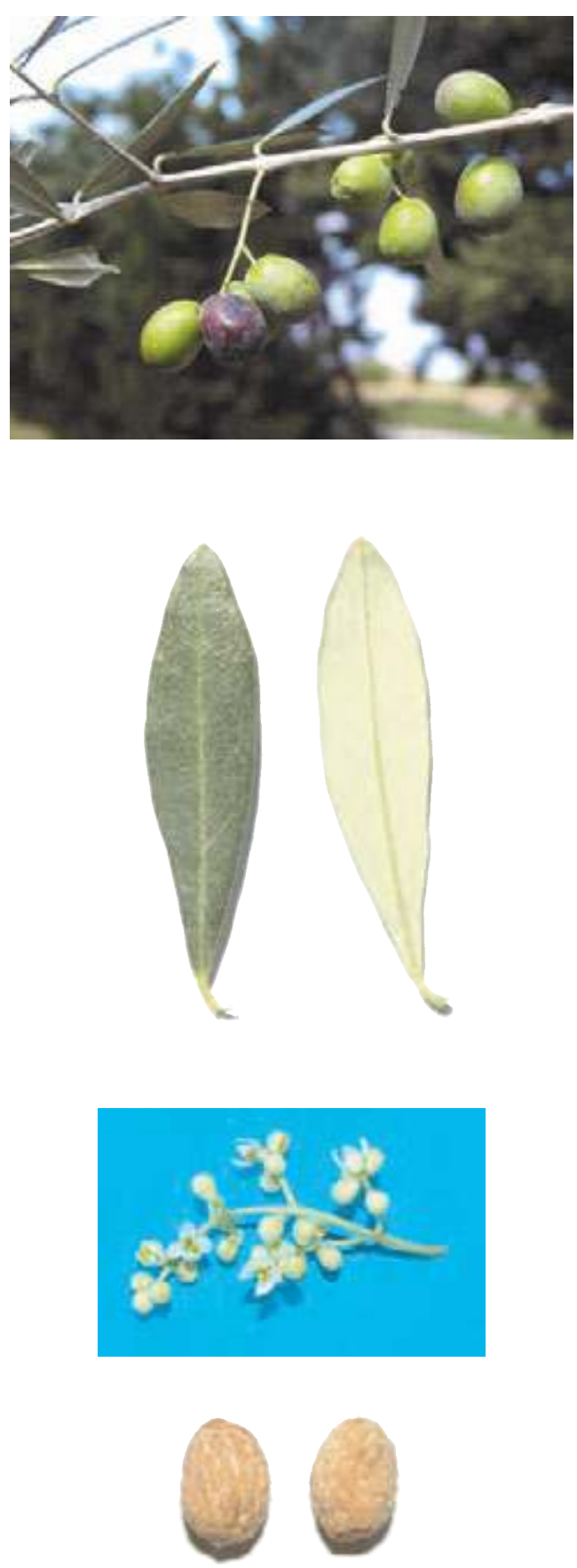


\section{Biochemical Characters}

\section{Fatty Acid Composition}

Table 1. Average values (express in $\% \pm$ standard deviations) of the fatty acids methyl esters and nutritional ratios obtained from single cultivar olive oils.

\begin{tabular}{|c|c|c|c|c|c|}
\hline Myri & $0,01 \pm 0,00$ & Linoleic acid $(\omega 6)$ & $9,55 \pm 0,17$ & Lignoceric acid & $0,03 \pm 0,00$ \\
\hline Palmitic acid & $14,47 \pm 1,31$ & Linolenic acid $(\omega 3)$ & $0,97 \pm 0,01$ & & \\
\hline almitoleic acid & $0,64 \pm 0,35$ & Arachic acid & $0,27 \pm 0,06$ & Unsat./satured & $4,90 \pm 0,1$ \\
\hline ear & $2,15 \pm 0,99$ & Eicosenoic acid & $0,02 \pm 0,01$ & $\omega 6 / \omega 3$ & $9,90 \pm 0,2$ \\
\hline leic acid & $70,74 \pm 1,09$ & Behenic acid & $0,07 \pm 0,02$ & & \\
\hline
\end{tabular}

\section{Organoleptic oil values}

Sensory Analysis (Panel test)

Comment: fruity medium-high, with hints of almond and read sensations of grass and artichoke. Balanced taste sensation with a medium bitter and spicy. Medium-high fluidity.

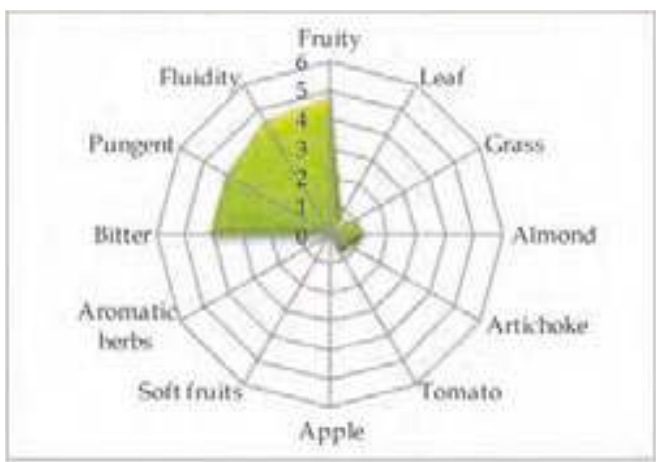

\section{Molecular Markers}

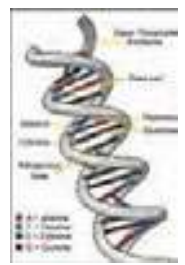

Table 2. Microsatellites (SSR) loci analyzed.

For each locus the allele size (expressed in base pairs) is reported.

$\begin{array}{cccccc}D C A 09 & \text { DCA18 } & \text { GAPU59 } & \text { GAPU71A } & \text { GUPA71B } & \text { GAPU103A } \\ \mathbf{1 8 4 - \mathbf { 2 1 6 }} & \mathbf{1 7 9 - \mathbf { 1 8 5 }} & \mathbf{2 1 2} \mathbf{- 2 2 2} & \mathbf{2 1 4} \mathbf{- 2 1 4} & \mathbf{1 2 4} \mathbf{- 1 2 4} & \mathbf{1 5 0 - \mathbf { 1 5 0 }} \\ \text { UDOO1 } & \text { UDOO3 } & \text { UDO12 } & \text { UDO28 } & \text { UDO39 } & \\ \mathbf{1 4 4 - \mathbf { 1 4 4 }} & \mathbf{1 8 2} \mathbf{- 1 8 2} & \mathbf{1 7 7 - \mathbf { 1 9 3 }} & \mathbf{1 5 4 - \mathbf { 2 0 5 }} & \mathbf{2 1 3 - \mathbf { 2 1 3 }} & \end{array}$

\section{References:}

1 - Pannelli G., Alfei B., D’ Ambrosio A., et al. In: Varietà di olivo in Umbria, Ed. Pliniana (2000), pp. 37-42.

2 - Muzzalupo I., Stefanizzi F., Salimonti A., et al. Sci. Agric. (2009), 66 (5): pp. 685-690. 


\section{"Morchiaio"}

(synonymy: Fecciaio, Maschio, Mezzolano, Morchiolo, Olivastro, etc.)

Areal distribution or origin area: Toscana

Flesh/pit weight ratio: high $(\mathbf{9 , 5 9} \pm 0,39)$

Oil content (\%): medium $(47,90 \pm 2,12)$

Purpose: oil

\section{Morphological characters}

Tree characters

Vigour: weak

Growth habit: erect-spreading

Canopy-density: medium

\section{Leaf characters}

Blade length $(\mathrm{cm})$ : medium $(5,98 \pm 0,40)$

Blade width $(\mathrm{cm})$ : medium $(\mathbf{1}, 21 \pm 0,12)$

Shape (length/width): elliptic-lanceolate

\section{Inflorescence characters}

Inflorescence length $(\mathrm{cm})$ : medium $(2,72 \pm 0,82)$

Number of flowers: medium $(\mathbf{1 8 , 1 4} \pm \mathbf{1 , 4 4})$

\section{Fruit characters}

Fresh weight of 100 fruits $(\mathrm{g})$ : medium $(3,87 \pm 0,05)$

Shape (length/width): ovoid

Symmetry: slightly asymmetric

Position of maximum transverse diameter:

central

Apex: pointed

Base: rounded

Niplle: absent

Lenticels: few and small

\section{Pit characters}

Weight of 100 pits (g): medium $(0,37 \pm 0,01)$

Shape (length/width): elongated

Mucron: absent

Symmetry: slightly asymmetric

Position of maximum transverse diameter:

central

Apex: pointed

Base: pointed

Surface: rugose

Number of grooves: low
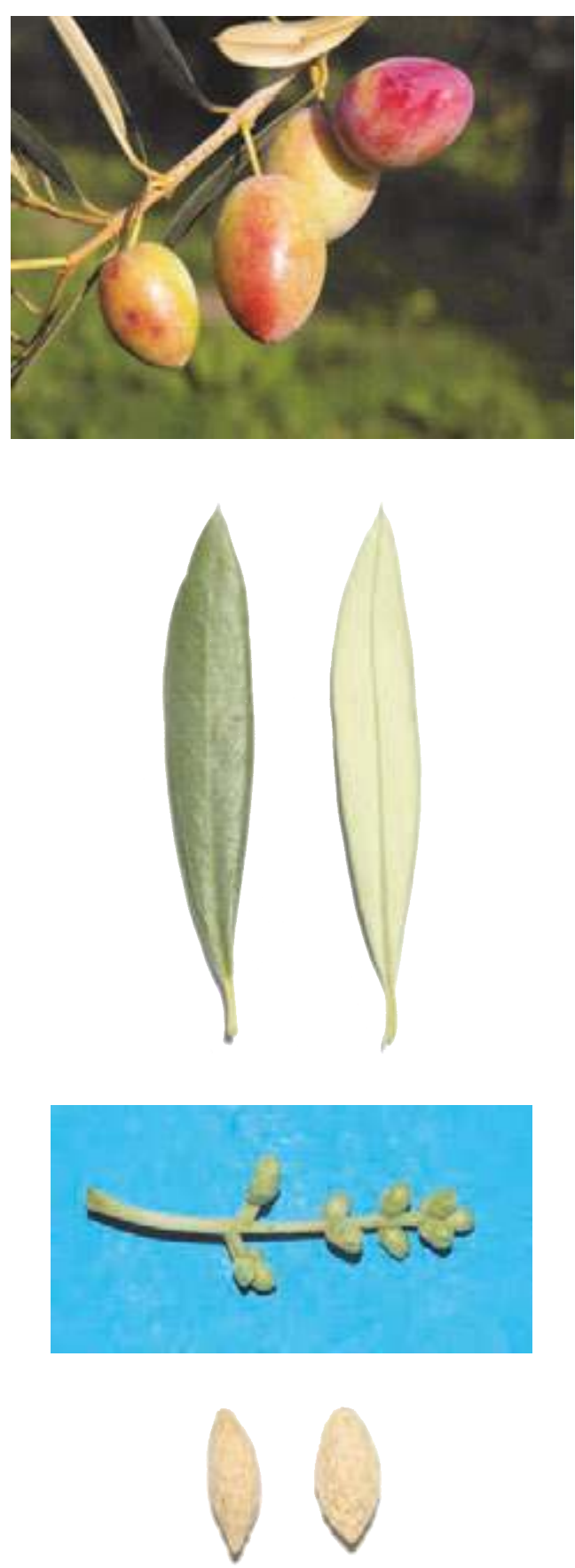


\section{Biochemical Characters}

\section{Fatty Acid Composition}

Table 1. Average values (express in $\% \pm$ standard deviations) of the fatty acids methyl esters and nutritional ratios obtained from single cultivar olive oils.

$\begin{array}{llllll}\text { Myristic acid } & \mathbf{0 , 0 1} \pm \mathbf{0 , 0 1} & \text { Linoleic acid }(\omega 6) & \mathbf{6 , 1 0} \pm \mathbf{0 , 1 7} & \text { Lignoceric acid } & \mathbf{0 , 0 6} \pm \mathbf{0 , 0 2} \\ \text { Palmitic acid } & \mathbf{1 3 , 8 2} \pm \mathbf{0 , 2 4} & \text { Linolenic acid }(\omega 3) & \mathbf{0 , 9 5} \pm \mathbf{0 , 0 3} & & \\ \text { Palmitoleic acid } & \mathbf{0 , 8 1} \pm \mathbf{0 , 0 1} & \text { Arachic acid } & \mathbf{0 , 3 1} \pm \mathbf{0 , 0 7} & \text { Unsat./satured } & \mathbf{5 , 1 6} \pm \mathbf{0 , 0 9} \\ \text { Stearic acid } & \mathbf{2 , 0 3} \pm \mathbf{0 , 0 4} & \text { Eicosenoic acid } & \mathbf{0 , 0 6} \pm \mathbf{0 , 0 1} & \omega 6 / \omega 3 & \mathbf{6 , 4 2} \pm \mathbf{0 , 0 0} \\ \text { Oleic acid } & \mathbf{7 5 , 2 5 \pm 0 , 7 0} & \text { Behenic acid } & \mathbf{0 , 1 1} \pm \mathbf{0 , 0 1} & & \end{array}$

\section{Organoleptic oil values}

Sensory Analysis (Panel test)

Comment: medium-high, with hints of almond and read sensations of grass and artichoke. Balanced taste sensation with a medium-high bitter and spicy. Medium-high fluidity.

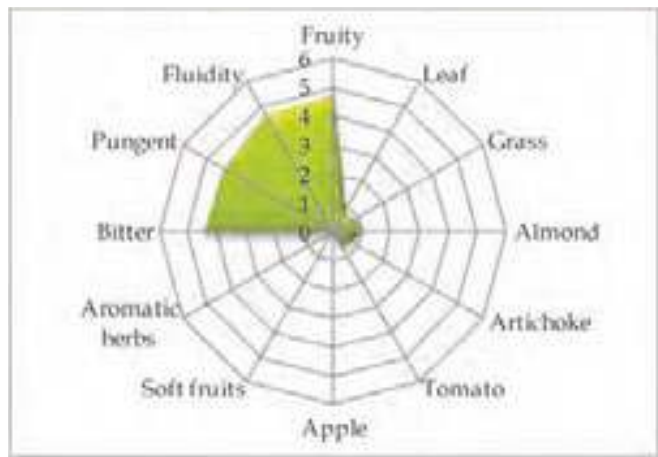

\section{Molecular Markers}

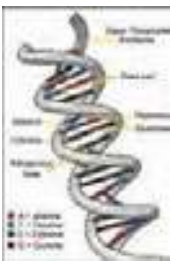

Table 2. Microsatellites (SSR) loci analyzed.

For each locus the allele size (expressed in base pairs) is reported.

\begin{tabular}{|c|c|c|c|c|c|}
\hline DCA09 & $D C A 18$ & GAPU59 & GAPU71A & $G U P A 71 B$ & GAPU103A \\
\hline $194-194$ & $177-181$ & $218-212$ & $212-212$ & $124-144$ & $150-157$ \\
\hline UDO01 & UDO03 & UDO12 & UDO28 & UDO39 & \\
\hline $140-140$ & $143-143$ & $156-177$ & $150-182$ & $108-108$ & \\
\hline
\end{tabular}

\section{References:}

1 - Cimato A., Cantini C., Sani G., In: L'olivo in Toscana: il germoplasma autoctono, Ed. ARSIA (2001).

2 - Muzzalupo I., Lombardo N., Salimonti A., et al. Adv. Hort. Sci. (2008), 22(2): pp. 142-148. 


\section{"Moresca"}

(synonymy: Aliva riali, Bianculidda, Catanisa, Marsalisa, Nucidda grossa, etc.)

Areal distribution or origin area: Sicilia

Flesh/pit weight ratio: medium $(6,58 \pm 1,71)$

Oil content (\%): medium $(47,54 \pm 0,79)$

Purpose: dual purpose

\section{Morphological characters}

Tree characters

Vigour: medium

Growth habit: spreading-erect

Canopy-density: medium

\section{Leaf characters}

Blade length $(\mathrm{cm})$ : medium $(5,56 \pm 0,63)$

Blade width $(\mathrm{cm})$ : medium $(1,46 \pm 0,22)$

Shape (length/width): elliptic

\section{Inflorescence characters}

Inflorescence length (cm): short $(2,34 \pm 0,93$ )

Number of flowers: low $(16,24 \pm 2,39)$

\section{Fruit characters}

Fresh weight of 100 fruits (g): high $(4,87 \pm 0,05)$

Shape (length/width): ovoid

Symmetry: slightly asymmetric

Position of maximum transverse diameter:

central

Apex: rounded

Base: truncate

Niplle: obvious

Lenticels: many and small

\section{Pit characters}

Weight of 100 pits (g): high $(\mathbf{0 , 6 6} \pm \mathbf{0 , 1 0})$

Shape (length/width): elliptic

Mucron: obvious

Symmetry: slightly asymmetric

Position of maximum transverse diameter:

central

Apex: pointed

Base: rounded

Surface: rugose

Number of grooves: medium
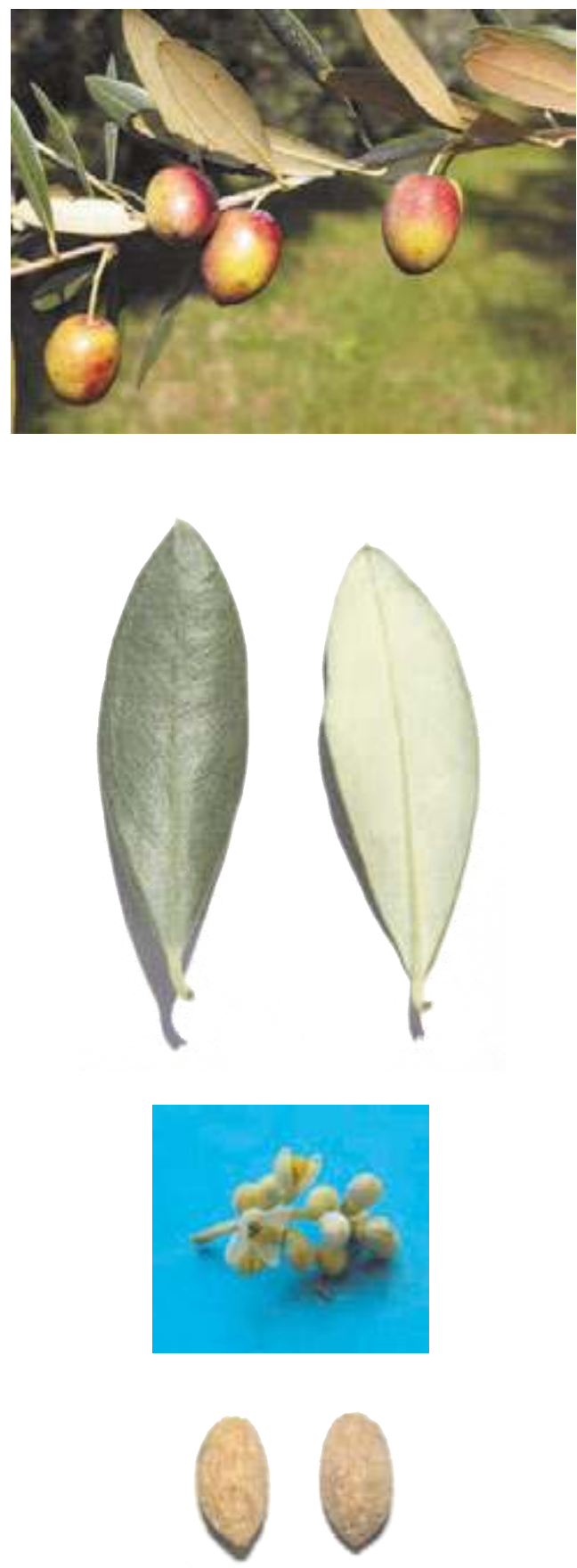


\section{Biochemical Characters}

\section{Fatty Acid Composition}

Table 1. Average values (express in $\% \pm$ standard deviations) of the fatty acids methyl esters and nutritional ratios obtained from single cultivar olive oils.

\begin{tabular}{|c|c|c|c|c|c|}
\hline Myristi & $0,01 \pm 0,00$ & Linoleic acid $(\omega 6)$ & $12,50 \pm 0,23$ & Lignoceric acid & $0,02 \pm 0,01$ \\
\hline Palmitic acid & $13,40 \pm 0,35$ & Linolenic acid $(\omega 3)$ & $0,87 \pm 0,13$ & & \\
\hline almitoleic acid & $2,22 \pm 0,06$ & Arachic acid & $0,22 \pm 0,02$ & Unsat./satured & $5,38 \pm 0,13$ \\
\hline$\cdot x^{-} \cdot 1$ & $1,78 \pm 0,06$ & Eicosenoic acid & $0,01 \pm 0,00$ & $\omega 6 / \omega 3$ & $14,56 \pm 1,95$ \\
\hline eic acid & $66,71 \pm 0,25$ & Behenic acid & $0,05 \pm 0,01$ & & \\
\hline
\end{tabular}

\section{Organoleptic oil values}

Sensory Analysis (Panel test)

Comment: fruity medium, with hints of artichoke, read sensations of tomato, grass and leaves. Balanced taste sensation with a medium-light bitter and spicy. Medium fluidity.

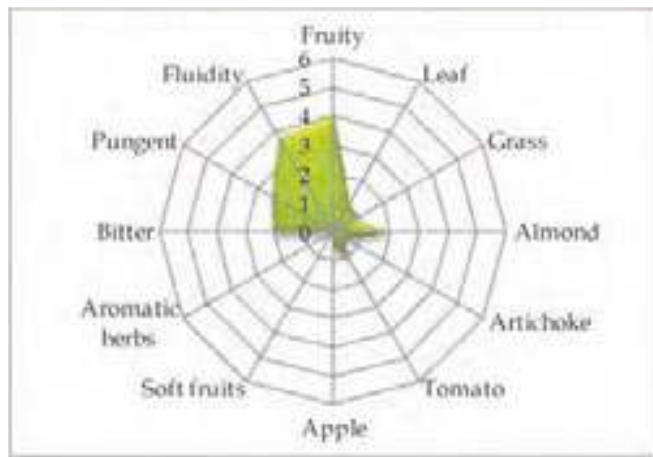

\section{Molecular Markers}

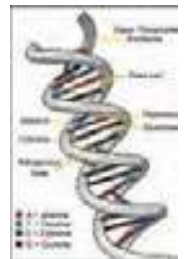

Table 2. Microsatellites (SSR) loci analyzed. For each locus the allele size (expressed in base pairs) is reported.

$\begin{array}{cccccc}\text { DCA09 } & \text { DCA18 } & \text { GAPU59 } & \text { GAPU71A } & \text { GUPA71B } & \text { GAPU103A } \\ \mathbf{1 8 2} \mathbf{- 1 9 8} & \mathbf{1 7 7 - \mathbf { 1 7 9 }} & \mathbf{2 0 8 - \mathbf { 2 1 2 }} & \mathbf{2 1 4 - \mathbf { 2 1 4 }} & \mathbf{1 2 4} \mathbf{- 1 4 4} & \mathbf{1 5 9} \mathbf{- 1 8 4} \\ \text { UDOO1 } & \text { UDOO3 } & \text { UDO12 } & \text { UDO28 } & \text { UDO39 } & \\ \mathbf{1 4 4 - \mathbf { 1 4 4 }} & \mathbf{1 6 6 - \mathbf { 2 0 2 }} & \mathbf{1 6 6 - \mathbf { 1 9 3 }} & \mathbf{1 4 3 - \mathbf { 1 8 2 }} & \mathbf{1 0 8 - \mathbf { 2 1 3 }} & \end{array}$

\section{References:}

1 - Caruso T., Cartabellotta D., Motisi A., et all. In: Cultivar di olivo siciliane, Università degli Studi di Palermo (2007), pp. 42-46.

2 - Muzzalupo I., Stefanizzi F., Perri E. HortScience (2009), 44: pp. 582-588. 


\section{"Morinello"}

(synonymy: Aurina, Arancino, Carbogna, More, Oriolo, etc.)

Areal distribution or origin area: Toscana

Flesh/pit weight ratio: low $(4,92 \pm 0,29)$

Oil content (\%): medium $(42,42 \pm 1,86)$

Purpose: oil

\section{Morphological characters}

Tree characters

Vigour: medium-strong

Growth habit: spreading-erect

Canopy-density: medium-sparse

\section{Leaf characters}

Blade length $(\mathrm{cm})$ : medium $(5,84 \pm 0,38)$

Blade width $(\mathrm{cm})$ : medium $(\mathbf{1 , 3 1} \pm \mathbf{0 , 1 6})$

Shape (length/width): elliptic-lanceolate

\section{Inflorescence characters}

Inflorescence length (cm): medium $(3,44 \pm 0,44)$

Number of flowers: high $(25,55 \pm 2,94$

\section{Fruit characters}

Fresh weight of 100 fruits $(\mathrm{g})$ : low $(\mathbf{1}, \mathbf{8 4} \pm \mathbf{0 , 0 1})$

Shape (length/width): spherical

Symmetry: symmetric

Position of maximum transverse diameter:

central

Apex: rounded

Base: truncate

Niplle: absent

Lenticels: many and small

\section{Pit characters}

Weight of 100 pits (g): medium $(\mathbf{0 , 3 2} \pm \mathbf{0 , 0 2})$

Shape (length/width): ovoid

Mucron: obvious

Symmetry: slightly asymmetric

Position of maximum transverse diameter:

central

Apex: rounded

Base: rounded

Surface: smooth

Number of grooves: medium
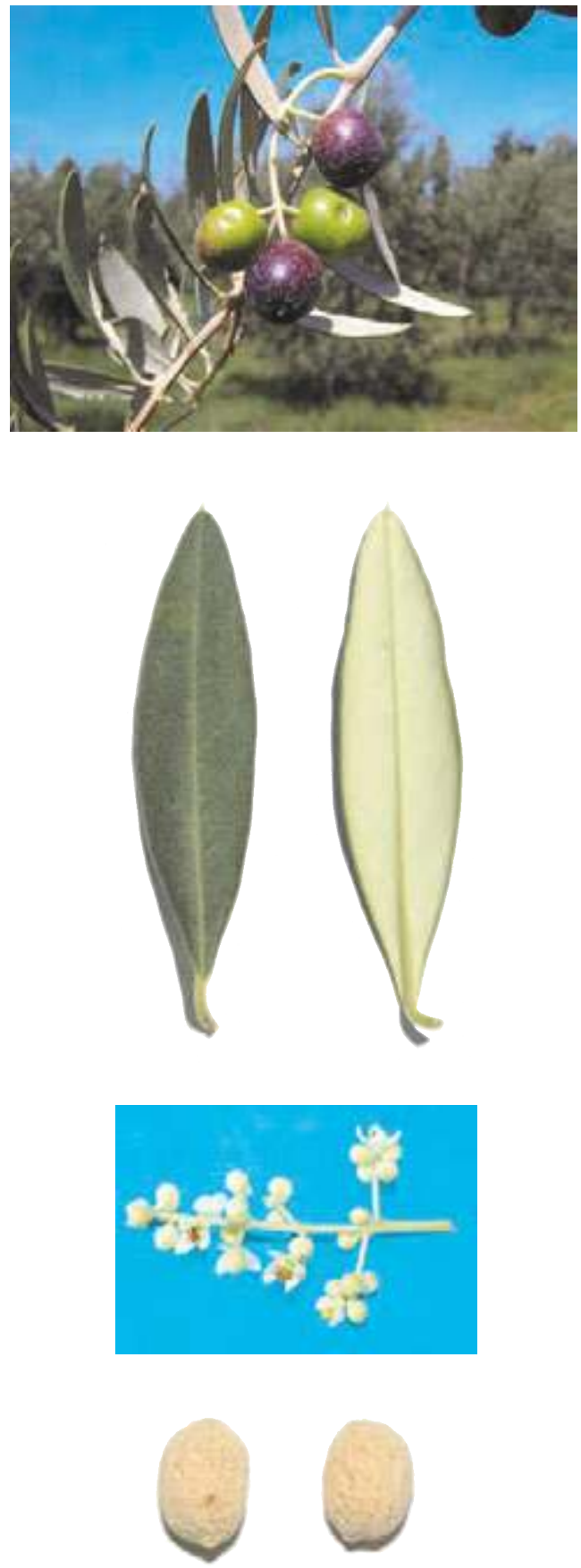


\section{Biochemical Characters}

\section{Fatty Acid Composition}

Table 1. Average values (express in $\% \pm$ standard deviations) of the fatty acids methyl esters and nutritional ratios obtained from single cultivar olive oils.

\begin{tabular}{|c|c|c|c|c|c|}
\hline Myristic acid & $0,01 \pm 0,00$ & Linoleic acid $(\omega 6)$ & $10,60 \pm 1,10$ & Lignoceric acid & $0,04 \pm 0,03$ \\
\hline Palmitic acid & $14,47 \pm 1,14$ & Linolenic acid $(\omega 3)$ & $0,93 \pm 0,03$ & & \\
\hline Palmitoleic acid & $1,30 \pm 0,15$ & Arachic acid & $0,33 \pm 0,13$ & Unsat./satured & $5,06 \pm 0,45$ \\
\hline tearic & $1,63 \pm 0,14$ & Eicosenoic acid & $0,02 \pm 0,01$ & $\omega 6 / \omega 3$ & $11,38 \pm 1,18$ \\
\hline leic acid & $69,44 \pm 1,57$ & Behenic acid & $0,09 \pm 0,03$ & & \\
\hline
\end{tabular}

\section{Organoleptic oil values}

Sensory Analysis (Panel test)

Comment: fruity medium, with taste of almond, grass and leaves, read sensations of soft fruits and aromatic herb. Balanced taste sensation with medium bitter and spicy. Medium fluidity.

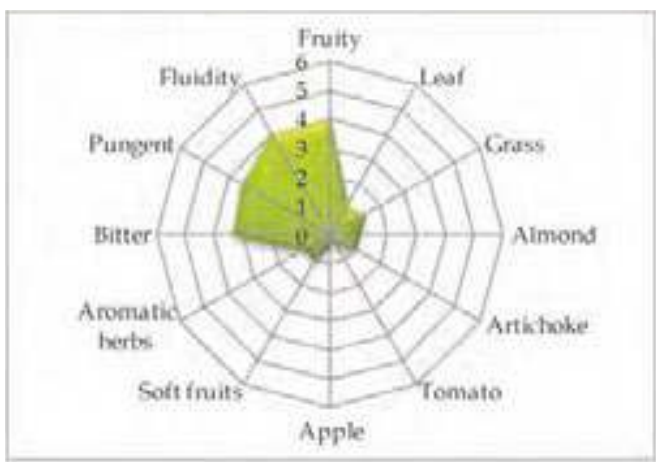

\section{Molecular Markers}

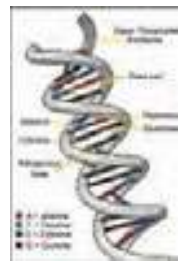

Table 2. Microsatellites (SSR) loci analyzed.

For each locus the allele size (expressed in base pairs) is reported.

$\begin{array}{cccccc}D C A 09 & \text { DCA18 } & \text { GAPU59 } & \text { GAPU71A } & \text { GUPA71B } & \text { GAPU103A } \\ \mathbf{1 8 2} \mathbf{- 2 0 6} & \mathbf{1 7 9 - \mathbf { 1 8 1 }} & \mathbf{2 1 2} \mathbf{- 2 1 4} & \mathbf{2 1 8 - \mathbf { 2 1 8 }} & \mathbf{1 3 0 - \mathbf { 1 4 4 }} & \mathbf{1 5 0 - \mathbf { 1 5 7 }} \\ \text { UDOO1 } & \text { UDO03 } & \text { UDO12 } & \text { UDO28 } & \text { UDO39 } & \\ \mathbf{1 4 0 - \mathbf { 1 4 0 }} & \mathbf{1 5 0 - \mathbf { 1 5 0 }} & \mathbf{1 7 7 - \mathbf { 1 9 3 }} & \mathbf{1 5 4 - \mathbf { 1 8 2 }} & \mathbf{2 0 0 - \mathbf { 2 0 0 }} & \end{array}$

\section{References:}

1 - Cimato A., Cantini C., Sani G., In: L'olivo in Toscana: il germoplasma autoctono, Ed. ARSIA (2001).

2 - Various authors. In: Catalogo Nazionale delle Varietà di Olivo, University of Bari (in press), ISBN 978-88-8879397-9. 


\section{“Nasitana Frutto Grosso"}

Areal distribution or origin area: Sicilia

Flesh/pit weight ratio: medium $(6,62 \pm 0,68)$

Oil content (\%): low $(35,53 \pm 0,40)$

Purpose: dual purpose

\section{Morphological characters}

Tree characters

Vigour: medium-weak

Growth habit: spreading-erect

Canopy-density: sparse

\section{Leaf characters}

Blade length $(\mathrm{cm})$ : medium $(6,28 \pm 0,47)$

Blade width $(\mathrm{cm})$ : medium $(\mathbf{1}, \mathbf{1 8} \pm \mathbf{0 , 1 3})$

Shape (length/width): elliptic-lanceolate

\section{Inflorescence characters}

Inflorescence length $(\mathrm{cm})$ : medium $(2,84 \pm 1,48)$

Number of flowers: low $(15,99 \pm 1,60)$

\section{Fruit characters}

Fresh weight of 100 fruits (g): medium $(3,44 \pm 0,41)$

Shape (length/width): ovoid

Symmetry: slightly asymmetric

Position of maximum transverse diameter:

towards apex

Apex: rounded

Base: truncate

Niplle: absent

Lenticels: many and small

\section{Pit characters}

Weight of 100 pits (g): medium-high $(0,45 \pm 0,06)$

Shape (length/width): ovoid

Mucron: obvious

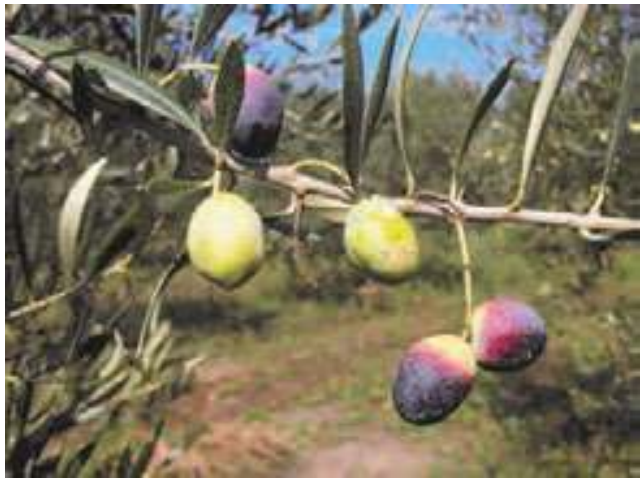

Symmetry: slightly asymmetric

Position of maximum transverse diameter:

towards apex

Apex: rounded

Base: pointed

Surface: rugose

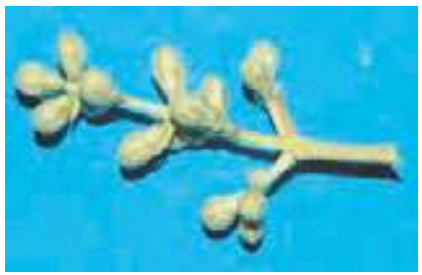

Number of grooves: medium 


\section{Biochemical Characters}

\section{Fatty Acid Composition}

Table 1. Average values (express in $\% \pm$ standard deviations) of the fatty acids methyl esters and nutritional ratios obtained from single cultivar olive oils.

\begin{tabular}{|c|c|c|c|c|c|}
\hline Myristic acid & $0,01 \pm 0,00$ & Linoleic acid $(\omega 6)$ & $11,50 \pm 1,71$ & Lignoceric acid & $0,03 \pm 0,01$ \\
\hline Palmitic acid & $14,59 \pm 0,27$ & Linolenic acid $(\omega 3)$ & $0,76 \pm 0,08$ & & \\
\hline Palmitoleic acid & $3,15 \pm 0,53$ & Arachic acid & $0,21 \pm 0,03$ & Unsat./satured & $4,84 \pm 0,08$ \\
\hline earic & $1,76 \pm 0,09$ & Eicosenoic acid & $0,02 \pm 0,00$ & $\omega 6 / \omega 3$ & $15,38 \pm 3,97$ \\
\hline leic acid & $64,56 \pm 0,85$ & Behenic acid & $0,07 \pm 0,01$ & & \\
\hline
\end{tabular}

\section{Organoleptic oil values}

Sensory Analysis (Panel test)

Comment: fruity medium, with taste of almond, grass and leaves, read sensations of soft fruits. Balanced taste sensation with medium bitter and spicy. Medium fluidity.

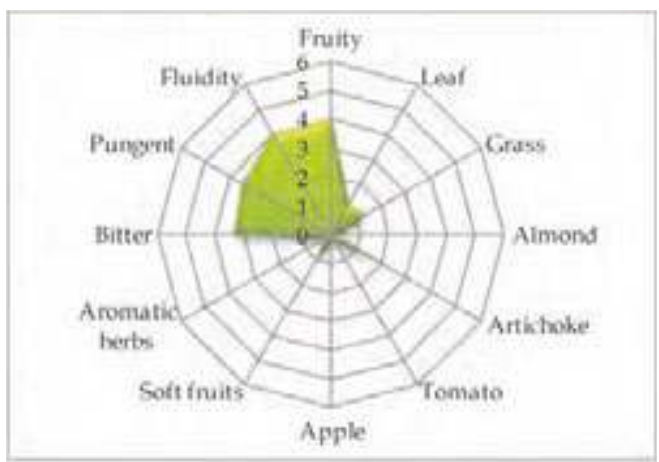

\section{Molecular Markers}

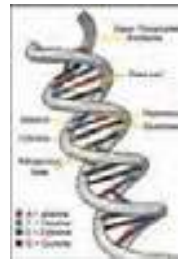

Table 2. Microsatellites (SSR) loci analyzed.

For each locus the allele size (expressed in base pairs) is reported.

$\begin{array}{cccccc}D C A 09 & \text { DCA18 } & \text { GAPU59 } & \text { GAPU71A } & \text { GUPA71B } & \text { GAPU103A } \\ \mathbf{1 6 2} \mathbf{- 1 9 8} & \mathbf{1 7 7 - \mathbf { 1 8 5 }} & \mathbf{2 0 8 - \mathbf { 2 1 2 }} & \mathbf{2 1 4 - \mathbf { 2 1 4 }} & \mathbf{1 2 4 - \mathbf { 1 3 0 }} & \mathbf{1 5 9 - \mathbf { 1 7 0 }} \\ \text { UDOO1 } & \text { UDO03 } & \text { UDO12 } & \text { UDO28 } & \text { UDO39 } & \\ \mathbf{1 4 4 - \mathbf { 1 4 4 }} & \mathbf{1 4 3 - \mathbf { 1 4 3 }} & \mathbf{1 6 6 - \mathbf { 1 9 3 }} & \mathbf{2 1 0 - \mathbf { 2 1 0 }} & \mathbf{2 0 5 - \mathbf { 2 0 5 }} & \end{array}$

\section{References:}

1 - Caruso T., Cartabellotta D., Motisi A., et all. In: Cultivar di olivo siciliane, Università degli Studi di Palermo (2007), pp. 164-168.

2 - Muzzalupo I., Stefanizzi F., Perri E. HortScience (2009), 44: pp. 582-588. 


\section{“Nebba"}

(synonymy: Anerba, Prunara, Tortella, Ugghiara, etc.)

Areal distribution or origin area: Sicilia

Flesh/pit weight ratio: high $(\mathbf{9 , 8 9} \pm 0,55)$

Oil content (\%): high $\mathbf{( 5 0 , 8 2 \pm \mathbf { 1 } , 8 8 )}$

Purpose: oil

\section{Morphological characters}

Tree characters

Vigour: weak

Growth habit: spreading-drooping

Canopy-density: medium

\section{Leaf characters}

Blade length $(\mathrm{cm})$ : long $(7,01 \pm 0,44)$

Blade width $(\mathrm{cm})$ : broad $(\mathbf{1}, 54 \pm 0,16)$

Shape (length/width): elliptic-lanceolate

\section{Inflorescence characters}

Inflorescence length $(\mathrm{cm})$ : medium $(3,12 \pm 1,96)$

Number of flowers: low $(15,79 \pm 1,76)$

\section{Fruit characters}

Fresh weight of 100 fruits (g): high $(5,62 \pm 1,03)$

Shape (length/width): spherical

Symmetry: symmetric

Position of maximum transverse diameter:

central

Apex: rounded

Base: truncate

Niplle: tenuous

Lenticels: few and large

\section{Pit characters}

Weight of 100 pits (g): medium $(0,37 \pm 0,15)$

Shape (length/width): ovoid

Mucron: obvious

Symmetry: slightly asymmetric

Position of maximum transverse diameter:

central

Apex: rounded

Base: rounded

Surface: rugose

Number of grooves: low
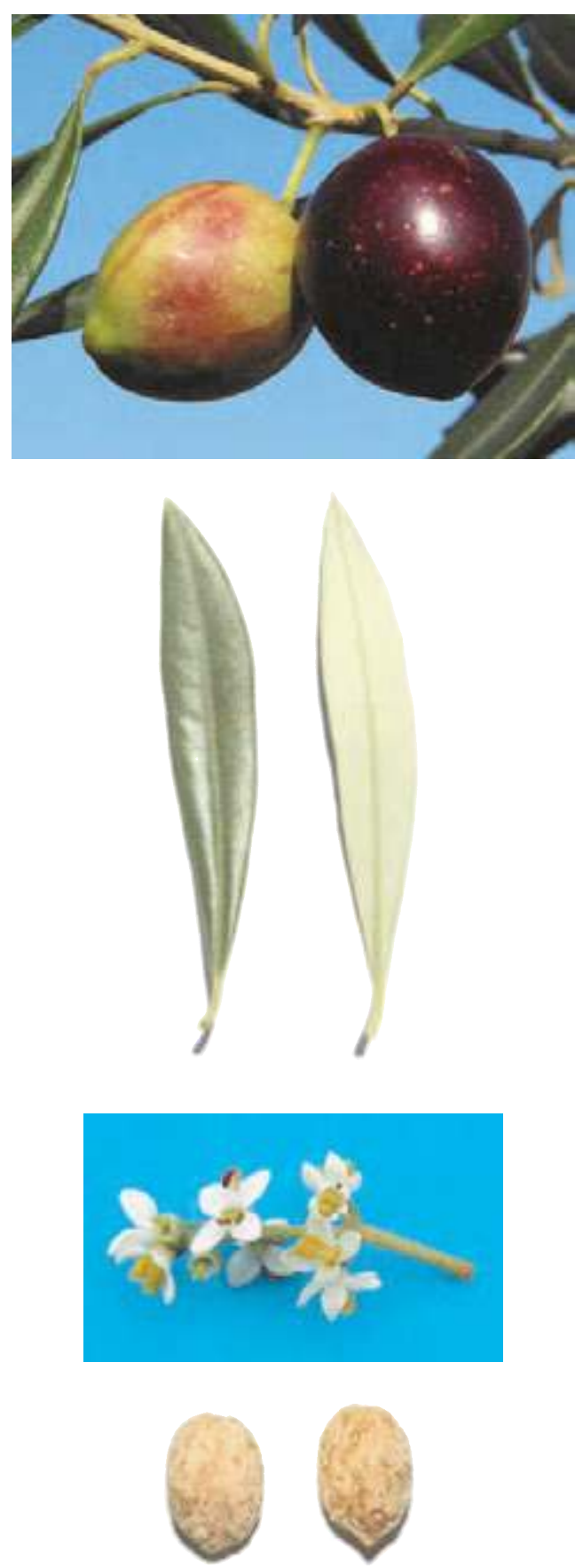


\section{Biochemical Characters}

\section{Fatty Acid Composition}

Table 1. Average values (express in $\% \pm$ standard deviations) of the fatty acids methyl esters and nutritional ratios obtained from single cultivar olive oils.

$\begin{array}{llllll}\text { Myristic acid } & \mathbf{0 , 0 1} \pm \mathbf{0 , 0 0} & \text { Linoleic acid }(\omega 6) & \mathbf{6 , 4 3} \pm \mathbf{1 , 0 7} & \text { Lignoceric acid } & \mathbf{0 , 0 6} \pm \mathbf{0 , 0 4} \\ \text { Palmitic acid } & \mathbf{1 1 , 4 7} \pm \mathbf{0 , 7 2} & \text { Linolenic acid }(\omega 3) & \mathbf{0 , 8 3} \pm \mathbf{0 , 1 4} & & \\ \text { Palmitoleic acid } & \mathbf{0 , 9 5} \pm \mathbf{0 , 4 0} & \text { Arachic acid } & \mathbf{0 , 3 7} \pm \mathbf{0 , 0 7} & \text { Unsat./satured } & \mathbf{6 , 0 9} \pm \mathbf{0 , 1 6} \\ \text { Stearic acid } & \mathbf{2 , 2 5} \pm \mathbf{0 , 2 9} & \text { Eicosenoic acid } & \mathbf{0 , 1 7} \pm \mathbf{0 , 2 1} & \omega 6 / \omega 3 & \mathbf{8 , 0 2} \pm \mathbf{2 , 6 7} \\ \text { Oleic acid } & \mathbf{7 6 , 6 1} \pm \mathbf{1 , 3 5} & \text { Behenic acid } & \mathbf{0 , 0 9} \pm \mathbf{0 , 0 3} & & \end{array}$

\section{Organoleptic oil values}

Sensory Analysis (Panel test)

Comment: fruity medium, with taste of tomato. Balanced taste sensation with medium bitter and spicy. Medium fluidity.

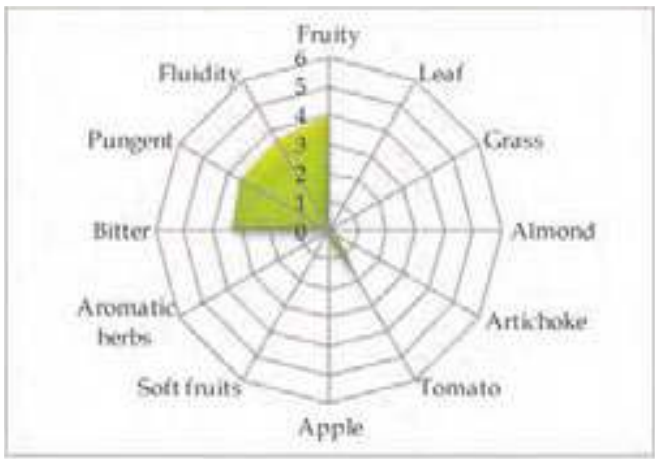

\section{Molecular Markers}

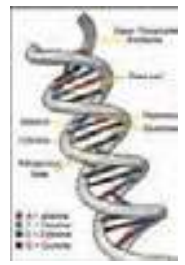

Table 2. Microsatellites (SSR) loci analyzed.

For each locus the allele size (expressed in base pairs) is reported.

$\begin{array}{cccccc}D C A 09 & \text { DCA18 } & \text { GAPU59 } & \text { GAPU71A } & \text { GUPA71B } & \text { GAPU103A } \\ \mathbf{1 7 6 - \mathbf { 2 0 6 }} & \mathbf{1 8 1} \mathbf{- 1 8 5} & \mathbf{2 0 8 - \mathbf { 2 0 8 }} & \mathbf{2 1 4 - \mathbf { 2 1 4 }} & \mathbf{1 4 4 - \mathbf { 1 4 4 }} & \mathbf{1 5 0 - \mathbf { 1 8 4 }} \\ \text { UDOO1 } & \text { UDO03 } & \text { UDO12 } & \text { UDO28 } & \text { UDO39 } & \\ \mathbf{1 4 0 - \mathbf { 1 4 0 }} & \mathbf{1 5 0 - \mathbf { 1 5 0 }} & \mathbf{1 6 6 - \mathbf { 1 9 3 }} & \mathbf{1 8 2} \mathbf{- 1 8 2} & \mathbf{2 0 5 - \mathbf { 2 0 5 }} & \end{array}$

\section{References:}

1 - Caruso T., Cartabellotta D., Motisi A., et all. In: Cultivar di olivo siciliane, Università degli Studi di Palermo (2007), pp. 169-174.

2 - Muzzalupo I., Stefanizzi F., Perri E. HortScience (2009), 44: pp. 582-588. 


\section{“Nebbia"}

(synonymy: Maglianese, Nebbia del Menocchia, Oliva cocia, etc.)

Areal distribution or origin area: Marche

Flesh/pit weight ratio: medium $(6,79 \pm 0,31)$

Oil content (\%): medium $(44,91 \pm 1,34)$

Purpose: oil

\section{Morphological characters}

Tree characters

Vigour: medium-strong

Growth habit: spreading

Canopy-density: dense

\section{Leaf characters}

Blade length (cm): short $(4,62 \pm 0,26)$

Blade width $(\mathrm{cm})$ : medium $(\mathbf{1 , 2 1} \pm \mathbf{0 , 1 0})$

Shape (length/width): elliptic

\section{Inflorescence characters}

Inflorescence length (cm): medium $(2,85 \pm 1,67)$

Number of flowers: low $(16,68 \pm 1,63)$

\section{Fruit characters}

Fresh weight of 100 fruits (g): medium $(2,44 \pm 0,26)$

Shape (length/width): ovoid

Symmetry: symmetric

Position of maximum transverse diameter:

central

Apex: rounded

Base: rounded

Niplle: absent

Lenticels: many and small

\section{Pit characters}

Weight of 100 pits (g): medium $(0,33 \pm 0,04)$

Shape (length/width): elliptic

Mucron: absent

Symmetry: slightly asymmetric

Position of maximum transverse diameter:

towards apex

Apex: pointed

Base: pointed

Surface: rugose

Number of grooves: medium
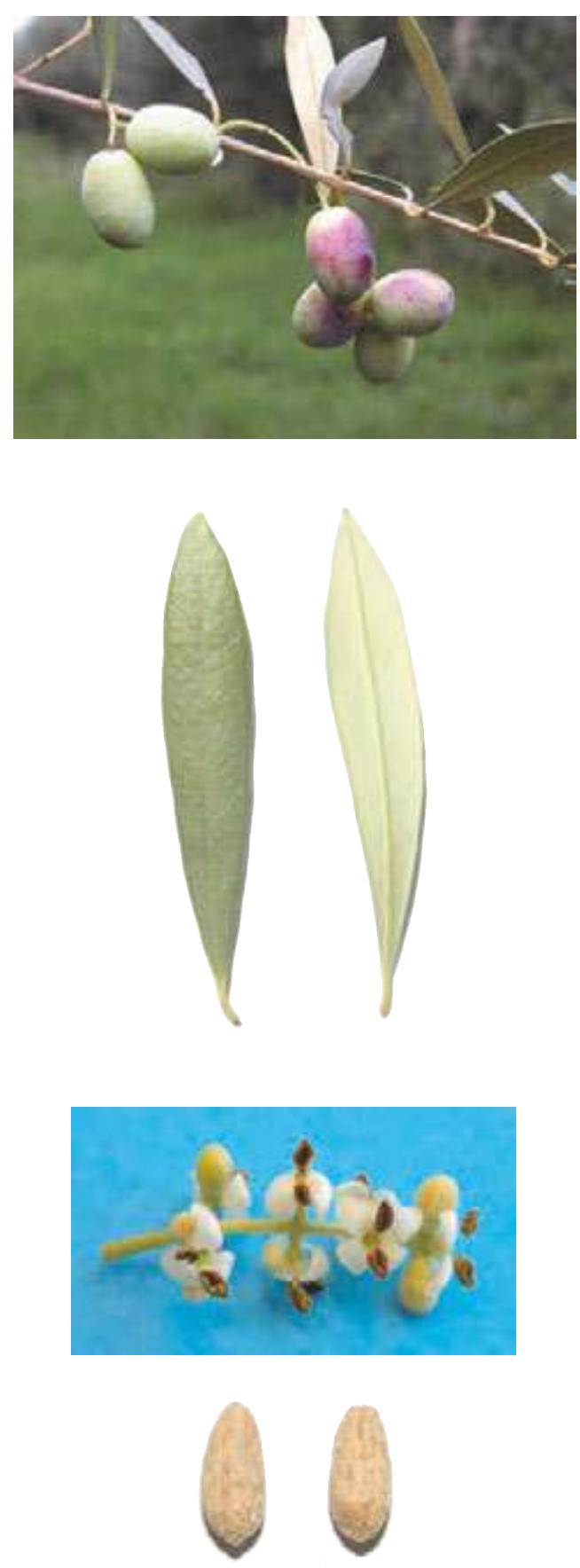


\section{Biochemical Characters}

\section{Fatty Acid Composition}

Table 1. Average values (express in $\% \pm$ standard deviations) of the fatty acids methyl esters and nutritional ratios obtained from single cultivar olive oils.

\begin{tabular}{|c|c|c|c|c|c|}
\hline Myristic acid & $0,01 \pm 0,00$ & Linoleic acid $(\omega 6)$ & $11,82 \pm 0,15$ & Lignoceric acid & $x 0,04 x \pm 0,01$ \\
\hline Palmitic acid & $12,20 \pm 0,06$ & Linolenic acid $(\omega 3)$ & $0,80 \pm 0,05$ & & \\
\hline almitoleic acid & $1,24 \pm 0,99$ & Arachic acid & $0,40 \pm 0,02$ & Unsat./satured & $4,94 \pm 0,35$ \\
\hline d & $4,24 \pm 1,25$ & Eicosenoic acid & $0,04 \pm 0,01$ & $\omega 6 / \omega 3$ & $14,74 \pm 0,78$ \\
\hline leic acid & $68,03 \pm 1,30$ & Behenic acid & $0,08 \pm 0,00$ & & \\
\hline
\end{tabular}

\section{Organoleptic oil values}

Sensory Analysis (Panel test)

Comment: fruity medium - light, with hints of almond and read sensations of grass and artichoke. Balanced taste sensation with a medium - light bitter and spicy. Medium fluidity.

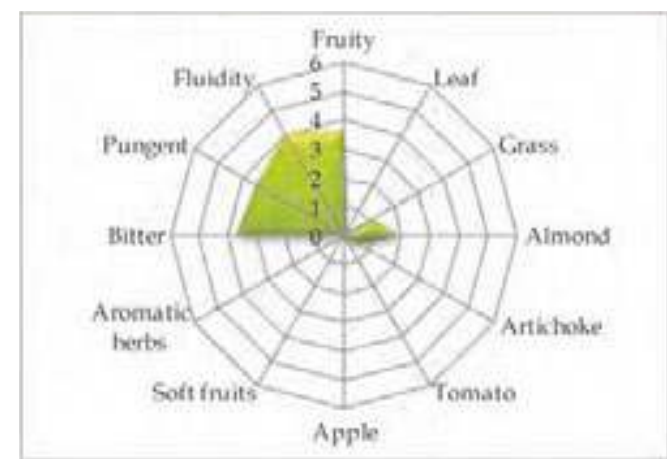

\section{Molecular Markers}

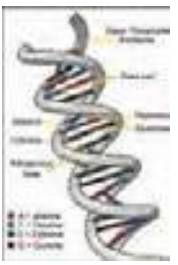

Table 2. Microsatellites (SSR) loci analyzed.

For each locus the allele size (expressed in base pairs) is reported.

$\begin{array}{cccccc}D C A 09 & \text { DCA18 } & \text { GAPU59 } & \text { GAPU71A } & \text { GUPA71B } & \text { GAPU103A } \\ \mathbf{1 7 2} \mathbf{- 2 0 6} & \mathbf{1 7 7 - \mathbf { 1 8 1 }} & \mathbf{2 0 8 - \mathbf { 2 1 2 }} & \mathbf{2 1 0 - \mathbf { 2 1 4 }} & \mathbf{1 2 4 - \mathbf { 1 3 0 }} & \mathbf{1 5 9 - \mathbf { 1 8 4 }} \\ \text { UDOO1 } & \text { UDOO3 } & \text { UDO12 } & \text { UDO28 } & \text { UDO39 } & \\ \mathbf{1 4 0 - \mathbf { 1 4 0 }} & \mathbf{1 3 5 - \mathbf { 1 3 5 }} & \mathbf{1 7 7 - \mathbf { 1 7 7 }} & \mathbf{1 5 4 - \mathbf { 2 1 0 }} & \mathbf{2 0 5 - \mathbf { 2 0 5 }} & \end{array}$

\section{References:}

1 - Pannelli G., Alfei B., Santinelli A. In: Varietà di olivo nelle Marche, (2001), ASSAM pp. 73-76.

2 - Muzzalupo I., Lombardo N., Salimonti A., et al. Adv. Hort. Sci. (2008), 22(2): pp. 142-148. 


\section{"Nebbio di Chieti "}

(synonymy: Comune, Gentile, Nebbia, Nebbio di Vasto, etc.)

Areal distribution or origin area: Abruzzo

Flesh/pit weight ratio: low $(\mathbf{4}, 25 \pm 0,13)$

Oil content (\%): medium $(40,73 \pm 2,28)$

Purpose: oil

\section{Morphological characters}

Tree characters

Vigour: medium-strong

Growth habit: erect- spreading

Canopy-density: medium

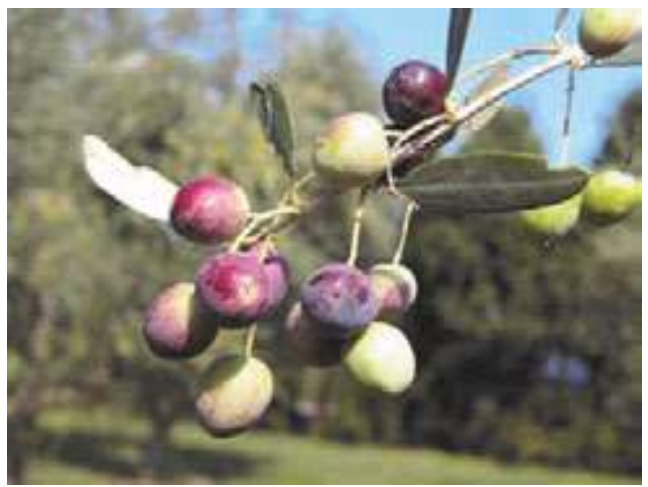

\section{Leaf characters}

Blade length $(\mathrm{cm})$ : medium $(5,20 \pm 0,23)$

Blade width $(\mathrm{cm})$ : medium $(\mathbf{1 , 3 0} \pm \mathbf{0 , 1 1})$

Shape (length/width): elliptic-lanceolate

\section{Inflorescence characters}

Inflorescence length $(\mathrm{cm})$ : medium $(3,40 \pm 2,05)$

Number of flowers: high $(25,06 \pm 1,80)$

\section{Fruit characters}

Fresh weight of 100 fruits $(\mathrm{g})$ : medium $(2,03 \pm 0,26)$

Shape (length/width): pherical

Symmetry: slightly asymmetric

Position of maximum transverse diameter:

central

Apex: rounded

Base: truncate

Niplle: absent

Lenticels: many and small

\section{Pit characters}

Weight of 100 pits (g): low $(0,22 \pm 0,07)$

Shape (length/width): ovoid

Mucron: absent

Symmetry: slightly asymmetric

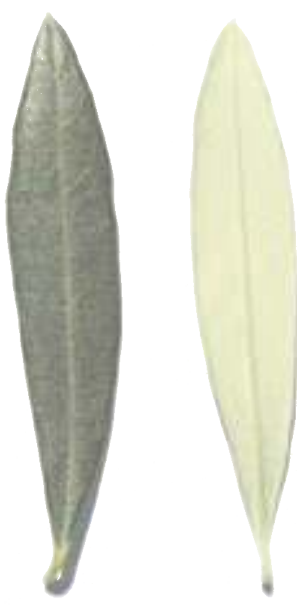

Position of maximum transverse diameter:

central

Apex: rounded

Base: rounded

Surface: rugose

Number of grooves: medium
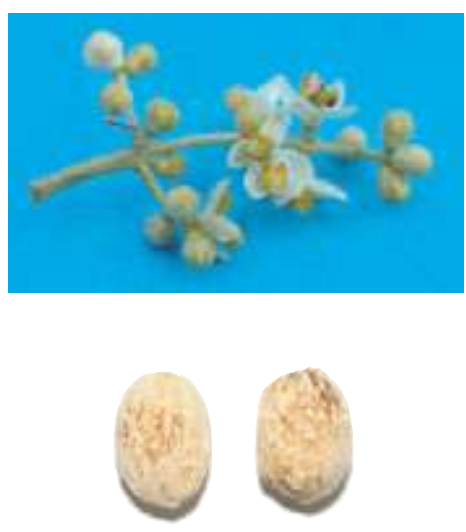


\section{Biochemical Characters}

\section{Fatty Acid Composition}

Table 1. Average values (express in $\% \pm$ standard deviations) of the fatty acids methyl esters and nutritional ratios obtained from single cultivar olive oils.

\begin{tabular}{|c|c|c|c|c|c|}
\hline Myristic acid & $0,01 \pm 0,00$ & Linoleic acid $(\omega 6)$ & $16,11 \pm 1,29$ & Lignoceric acid & $0,03 \pm 0,01$ \\
\hline Palmitic acid & $16,07 \pm 1,37$ & Linolenic acid $(\omega 3)$ & $0,74 \pm 0,06$ & & \\
\hline Palmitoleic acid & $1,18 \pm 0,02$ & Arachic acid & $0,24 \pm 0,04$ & Unsat./satured & $4,53 \pm 0,46$ \\
\hline earic acid & $1,63 \pm 0,09$ & Eicosenoic acid & $0,28 \pm 0,09$ & $\omega 6 / \omega 3$ & $21,92 \pm 0,15$ \\
\hline leic acid & $62,47 \pm 0,24$ & Behenic acid & $0,07 \pm 0,01$ & & \\
\hline
\end{tabular}

\section{Organoleptic oil values}

Sensory Analysis (Panel test)

Comment: fruity medium-high, with hints of almond and read sensations of grass, leaves, and artichoke. Balanced taste sensation with a medium-high bitter and spicy. Mediumhigh fluidity.

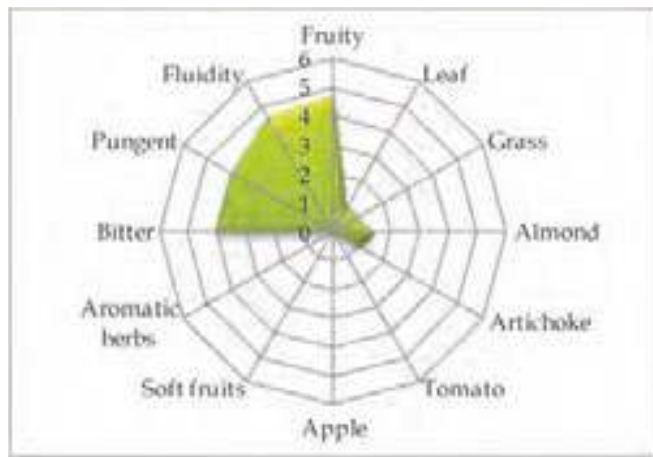

\section{Molecular Markers}

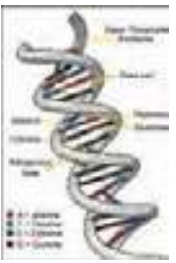

Table 2. Microsatellites (SSR) loci analyzed.

For each locus the allele size (expressed in base pairs) is reported.

\begin{tabular}{|c|c|c|c|c|c|}
\hline DCA09 & DCA18 & GAPU59 & GAPU71A & GUPA71B & GAPU103A \\
\hline $172-210$ & $175-179$ & $212-222$ & $214-214$ & $124-130$ & $159-159$ \\
\hline UDO01 & UDO03 & UDO12 & UDO28 & UDO39 & \\
\hline $144-144$ & $135-135$ & $177-193$ & $154-205$ & $213-213$ & \\
\hline
\end{tabular}

\section{References:}

1 - Pietrangeli E., Russo A., In: Olivi D'Abruzzo, Grafiche di Prinzio (1997), pp. 46-47.

2 - Muzzalupo I., Salimonti A., Caravita M. A., et al. Adv. Hort. Sci. (2008), 22(2): pp. 129-135. 


\section{“Nebbio di Pescara"}

(synonymy: Nebbio, Neja.)

Areal distribution or origin area: Abruzzo

Flesh/pit weight ratio: medium $(5,12 \pm 0,54)$

Oil content (\%): medium $(44,66 \pm 0,19)$

Purpose: oil

\section{Morphological characters}

Tree characters

Vigour: medium

Growth habit: erect

Canopy-density: medium-sparse

\section{Leaf characters}

Blade length $(\mathrm{cm})$ : short $(4,92 \pm 0,42)$

Blade width $(\mathrm{cm})$ : medium $(\mathbf{1 , 2 2} \pm \mathbf{0 , 1 7})$

Shape (length/width): elliptic-lanceolate

\section{Inflorescence characters}

Inflorescence length (cm): long $(4,03 \pm 0,16)$

Number of flowers: medium $(20,69 \pm 2,38)$

\section{Fruit characters}

Fresh weight of 100 fruits (g): medium $(2,13 \pm 0,02)$

Shape (length/width): ovoid

Symmetry: asymmetric

Position of maximum transverse diameter:

central

Apex: rounded

Base: rounded

Niplle: obvious

Lenticels: many and small

\section{Pit characters}

Weight of 100 pits (g): medium $(0,35 \pm 0,03$ )

Shape (length/width): ovoid

Mucron: obvious

Symmetry: slightly asymmetric

Position of maximum transverse diameter:

central

Apex: rounded

Base: rounded

Surface: smooth

Number of grooves: medium
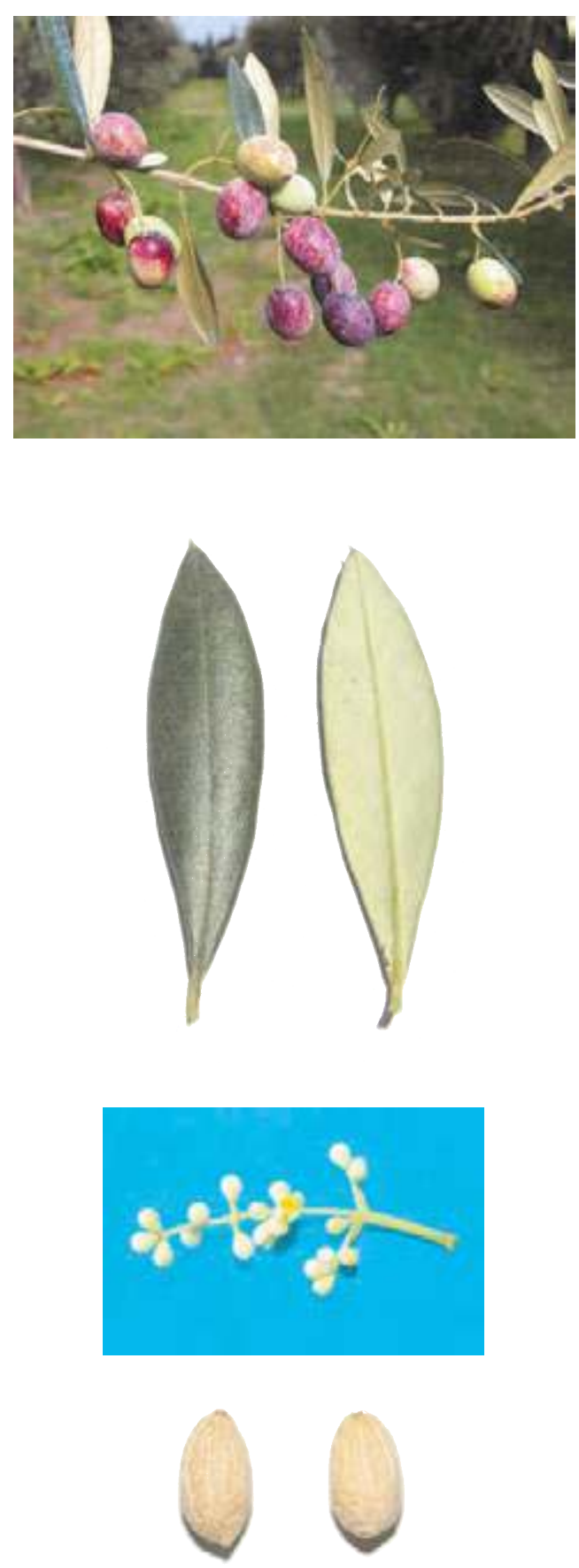


\section{Biochemical Characters}

\section{Fatty Acid Composition}

Table 1. Average values (express in $\% \pm$ standard deviations) of the fatty acids methyl esters and nutritional ratios obtained from single cultivar olive oils.

$\begin{array}{llllll}\text { Myristic acid } & \mathbf{0 , 0 1} \pm \mathbf{0 , 0 0} & \text { Linoleic acid }(\omega 6) & \mathbf{1 1 , 3 6} \pm \mathbf{0 , 0 7} & \text { Lignoceric acid } & \mathbf{0 , 0 2} \pm \mathbf{0 , 0 1} \\ \text { Palmitic acid } & \mathbf{1 3 , 9 7 \pm 0 , 0 2} & \text { Linolenic acid }(\omega 3) \mathbf{0 , 7 6} \pm \mathbf{0 , 0 3} & & \\ \text { Palmitoleic acid } & \mathbf{2 , 0 4} \pm \mathbf{0 , 0 7} & \text { Arachic acid } & \mathbf{0 , 2 3} \pm \mathbf{0 , 0 3} & \text { Unsat./satured } & \mathbf{5 , 1 5} \pm \mathbf{0 , 0 4} \\ \text { Stearic acid } & \mathbf{1 , 8 3} \pm \mathbf{0 , 0 3} & \text { Eicosenoic acid } & \mathbf{0 , 0 2} \pm 0,01 & \omega 6 / \omega 3 & \mathbf{1 5 , 0 1} \pm \mathbf{0 , 7 2} \\ \text { Oleic acid } & \mathbf{6 7 , 7 8 \pm 0 , 2 8} & \text { Behenic acid } & \mathbf{0 , 0 5} \pm \mathbf{0 , 0 1} & & \end{array}$

\section{Organoleptic oil values}

Sensory Analysis (Panel test)

Comment: fruity medium - light, with hints of almond and read sensations of grass and artichoke. Balanced taste sensation with a medium - light bitter and spicy. Medium fluidity.

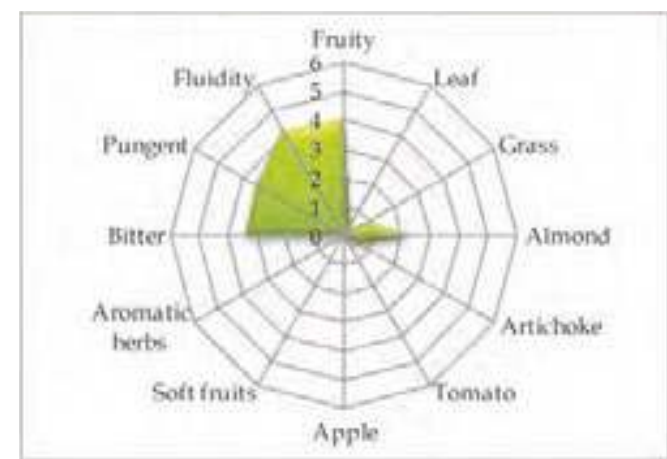

\section{Molecular Markers}

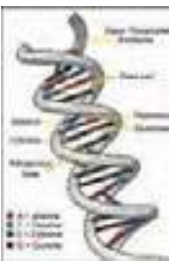

Table 2. Microsatellites (SSR) loci analyzed.

For each locus the allele size (expressed in base pairs) is reported.

$\begin{array}{cccccc}D C A 09 & \text { DCA18 } & \text { GAPU59 } & \text { GAPU71A } & \text { GUPA71B } & \text { GAPU103A } \\ \mathbf{1 7 2} \mathbf{- 1 8 2} & \mathbf{1 6 9 - \mathbf { 1 7 9 }} & \mathbf{2 0 8 - \mathbf { 2 0 8 }} & \mathbf{2 1 0 - \mathbf { 2 1 4 }} & \mathbf{1 2 4} \mathbf{- 1 3 0} & \mathbf{1 7 0 - \mathbf { 1 7 0 }} \\ \text { UDOO1 } & \text { UDOO3 } & \text { UDO12 } & \text { UDO28 } & \text { UDO39 } & \\ \mathbf{1 4 0 - \mathbf { 1 4 0 }} & \mathbf{1 6 6 - \mathbf { 1 6 6 }} & \mathbf{1 6 6 - \mathbf { 1 9 3 }} & \mathbf{1 5 4 - \mathbf { 2 1 0 }} & \mathbf{2 1 3 - \mathbf { 2 3 2 }} & \end{array}$

\section{References:}

1 - Pietrangeli E., Russo A., In: Olivi D’Abruzzo, Grafiche di Prinzio (1997), pp. 48-49.

2 - Muzzalupo I., Salimonti A., Caravita M. A., et al. Adv. Hort. Sci. (2008), 22(2): pp. 129-135. 


\section{“Negrera "}

Areal distribution or origin area: Liguria

Flesh/pit weight ratio: medium $(6,54 \pm 0,23)$

Oil content (\%): low $(38,04 \pm 0,08)$

Purpose: oil

\section{Morphological characters}

Tree characters

Vigour: weak

Growth habit: spreading

Canopy-density: medium-sparse

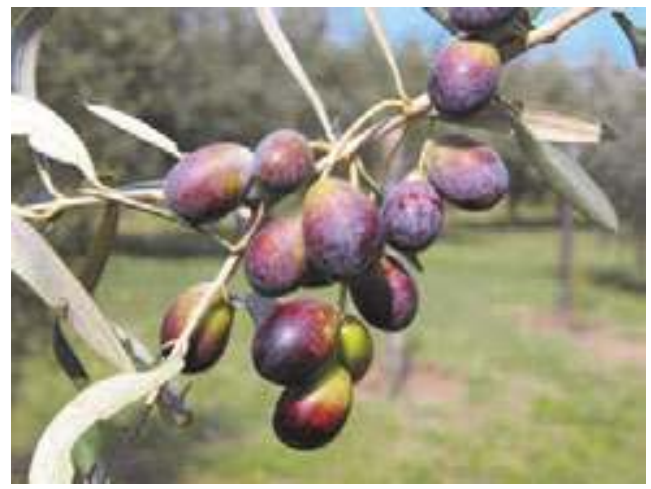

\section{Leaf characters}

Blade length $(\mathrm{cm})$ : medium $(5,18 \pm 0,43)$

Blade width $(\mathrm{cm})$ : medium $(1,00 \pm 0,09)$

Shape (length/width): elliptic-lanceolate

\section{Inflorescence characters}

Inflorescence length $(\mathrm{cm})$ : medium $(3,27 \pm 0,76)$

Number of flowers: medium $(22,83 \pm 0,33)$

\section{Fruit characters}

Fresh weight of 100 fruits $(\mathrm{g})$ : medium $(2,35 \pm 0,42)$

Shape (length/width): ovoid

Symmetry: slightly asymmetric

Position of maximum transverse diameter:

\section{towards apex}

Apex: rounded

Base: rounded

Niplle: absent

Lenticels: many and small

\section{Pit characters}

Weight of 100 pits $(\mathrm{g})$ : medium $(0,37 \pm \mathbf{0 , 0 4})$

Shape (length/width): elliptic

Mucron: absent

Symmetry: slightly asymmetric

Position of maximum transverse diameter:

\section{towards apex}

Apex: rounded

Base: rounded

Surface: rugose

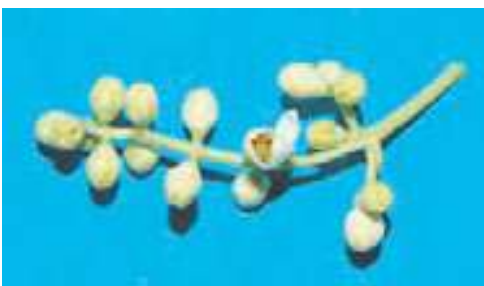

Number of grooves: low 


\section{Biochemical Characters}

\section{Fatty Acid Composition}

Table 1. Average values (express in $\% \pm$ standard deviations) of the fatty acids methyl esters and nutritional ratios obtained from single cultivar olive oils.

\begin{tabular}{|c|c|c|c|c|c|}
\hline Myristic acid & $0,01 \pm 0,00$ & Linoleic acid ( $\omega 6)$ & $7,65 \pm 0,47$ & Lignoceric acid & $0,02 \pm 0,01$ \\
\hline Palmitic acid & $14,86 \pm 0,18$ & Linolenic acid $(\omega 3)$ & $0,92 \pm 0,02$ & & \\
\hline Palmitoleic acid & $1,18 \pm 0,02$ & Arachic acid & $0,24 \pm 0,02$ & Unsat./satured & $5,03 \pm 0,05$ \\
\hline Stearic acid & $1,41 \pm 0,07$ & Eicosenoic acid & $0,02 \pm 0,00$ & $\omega 6 / \omega 3$ & $8,02 \pm 0,67$ \\
\hline Oleic acid & $72,80 \pm 0,22$ & Behenic acid & $0,06 \pm 0,01$ & & \\
\hline
\end{tabular}

\section{Organoleptic oil values}

Sensory Analysis (Panel test)

Comment: fruity medium, with hints of artichoke and almond. Balanced in flavours, with hints of bitter and spicy medium intensity, medium fluidity.

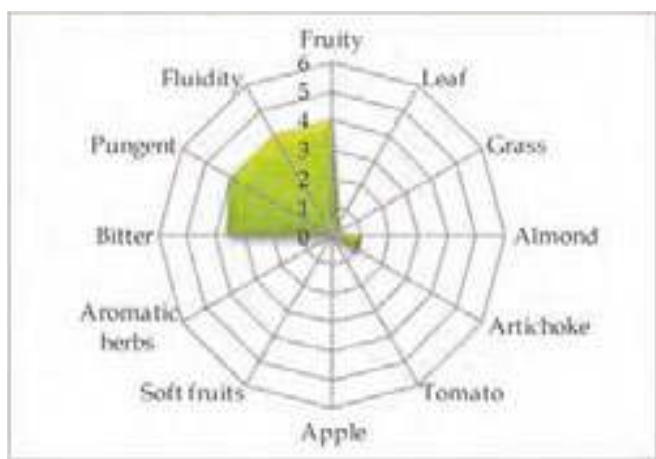

\section{Molecular Markers}

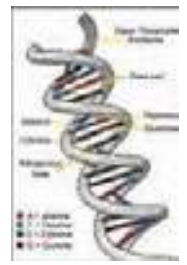

Table 2. Microsatellites (SSR) loci analyzed.

For each locus the allele size (expressed in base pairs) is reported.

\begin{tabular}{|c|c|c|c|c|c|}
\hline DCA09 & $D C A 18$ & GAPU59 & GAPU71A & GUPA71B & GAPU103A \\
\hline $162-198$ & $179-179$ & $214-214$ & $214-224$ & $126-144$ & $170-184$ \\
\hline UDO01 & UDO03 & UDO12 & UDO28 & UDO39 & \\
\hline $150-150$ & $143-143$ & $166-193$ & $182-182$ & $200-200$ & \\
\hline
\end{tabular}

\section{References:}

1 - Cimato A., Cantini C., Sani G., In: Atti Convegno Nazionale sulla Biodiversità, Ed Delfino (2000), pp. 497-500. 2 - Muzzalupo I., Lombardo N., Salimonti A., et al. Adv. Hort. Sci. (2008), 22(2): pp. 142-148. 


\section{“Nera di Cantinella "}

Areal distribution or origin area: Calabria

Flesh/pit weight ratio: low $(4,05 \pm 0,36)$

Oil content (\%): medium $(\mathbf{1 4 , 1 2} \pm \mathbf{1 , 2 6})$

Purpose: dual purpose

\section{Morphological characters}

Tree characters

Vigour: medium-weak

Growth habit: spreading

Canopy-density: dense

\section{Leaf characters}

Blade length $(\mathrm{cm})$ : medium $(5,24 \pm 0,60)$

Blade width (cm): broad $(\mathbf{1}, 69 \pm 0,29)$

Shape (length/width): elliptic

\section{Inflorescence characters}

Inflorescence length $(\mathrm{cm})$ : medium $(3,12 \pm 0,57)$

Number of flowers: medium $(20,55 \pm 3,64)$

\section{Fruit characters}

Fresh weight of 100 fruits $(\mathrm{g})$ : medium $(3,12 \pm 0,24)$

Shape (length/width): ovoid

Symmetry: slightly symmetric

Position of maximum transverse diameter:

central

Apex: rounded

Base: truncate

Niplle: tenuous

Lenticels: many and small

\section{Pit characters}

Weight of 100 pits (g): medium $(\mathbf{0 , 4 8} \pm \mathbf{0 , 1 1})$

Shape (length/width): elliptic

Mucron: obvious

Symmetry: slightly symmetric

Position of maximum transverse diameter:

central

Apex: rounded

Base: rounded

Surface: rugose

Number of grooves: medium
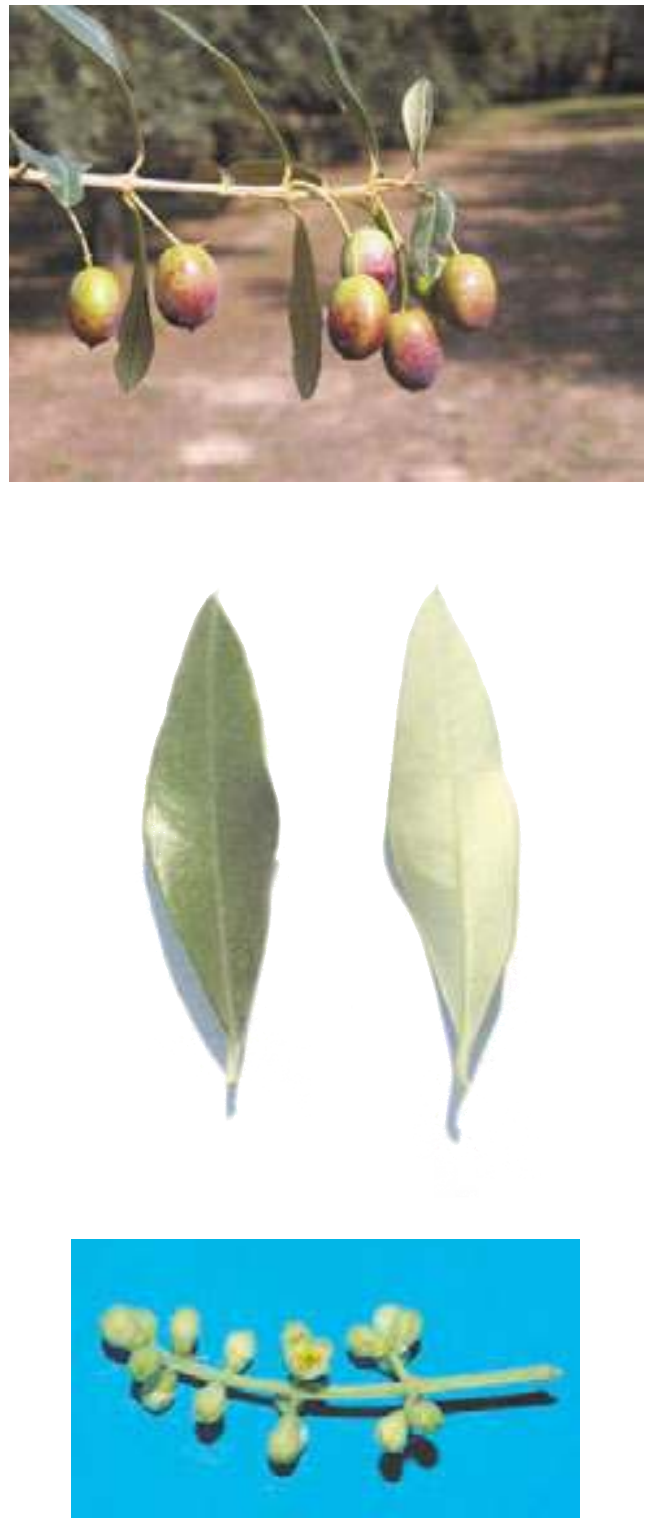


\section{Biochemical Characters}

\section{Fatty Acid Composition}

Table 1. Average values (express in $\% \pm$ standard deviations) of the fatty acids methyl esters and nutritional ratios obtained from single cultivar olive oils.

$\begin{array}{llllll}\text { Myristic acid } & \mathbf{0 , 0 1} \pm \mathbf{0 , 0 0} & \text { Linoleic acid }(\omega 6) & \mathbf{7 , 0 1} \pm \mathbf{0 , 6 3} & \text { Lignoceric acid } & \mathbf{0 , 0 5} \pm \mathbf{0 , 0 3} \\ \text { Palmitic acid } & \mathbf{1 1 , 4 0 \pm 0 , 8 9} & \text { Linolenic acid }(\omega 3) & \mathbf{0 , 7 6} \pm \mathbf{0 , 0 6} & & \\ \text { Palmitoleic acid } & \mathbf{0 , 8 7} \pm \mathbf{0 , 1 5} & \text { Arachic acid } & \mathbf{0 , 2 9} \pm \mathbf{0 , 0 3} & \text { Unsat./satured } & \mathbf{6 , 2 1} \pm \mathbf{0 , 5 4} \\ \text { Stearic acid } & \mathbf{2 , 1 6} \pm \mathbf{2 , 2 4} & \text { Eicosenoic acid } & \mathbf{0 , 0 9} \pm \mathbf{0 , 1 1} & \omega 6 / \omega 3 & \mathbf{9 , 1 8} \pm \mathbf{0 , 6 1} \\ \text { Oleic acid } & \mathbf{7 6 , 3 3} \pm \mathbf{1 , 5 9} & \text { Behenic acid } & \mathbf{0 , 0 8} \pm \mathbf{0 , 0 2} & & \end{array}$

\section{Organoleptic oil values}

Sensory Analysis (Panel test)

Comment: fruity medium, with hints of almond and tomato, read sensations of artichoke. Balanced taste sensation with a medium-light bitter and spicy. Medium fluidity.

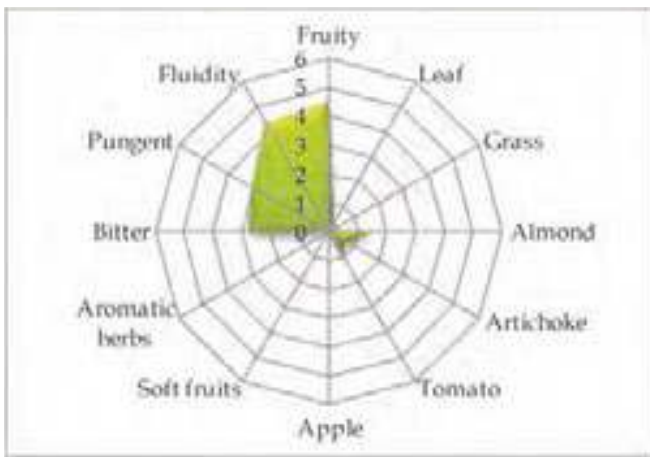

\section{Molecular Markers}

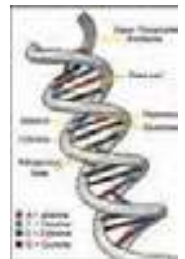

Table 2. Microsatellites (SSR) loci analyzed. For each locus the allele size (expressed in base pairs) is reported.

$\begin{array}{cccccc}D C A 09 & \text { DCA18 } & \text { GAPU59 } & \text { GAPU71A } & \text { GUPA71B } & \text { GAPU103A } \\ \mathbf{1 6 2} \mathbf{- 1 9 8} & \mathbf{1 7 7 - \mathbf { 1 7 9 }} & \mathbf{2 1 2 - \mathbf { 2 2 2 }} & \mathbf{2 1 2} \mathbf{- 2 1 2} & \mathbf{1 2 6 - \mathbf { 1 7 4 }} & \mathbf{1 3 6 - \mathbf { 1 5 0 }} \\ \text { UDO01 } & \text { UDO03 } & \text { UDO12 } & \text { UDO28 } & \text { UDO39 } & \\ \mathbf{1 4 4 - \mathbf { 1 4 4 }} & \mathbf{1 4 3 - \mathbf { 1 4 3 }} & \mathbf{1 6 6 - 1 9 3} & \mathbf{1 4 3 - \mathbf { 1 4 3 }} & \mathbf{2 0 0 - \mathbf { 2 0 0 }} & \end{array}$

\section{References:}

1 - Lombardo N., Perri E., Muzzalupo I., et al. In: Il germoplasma olivicolo Calabrese, Ist. Sper. Olivic. (2003), pp: 22.

2 - Lombardo N., Alessandrino.M., Belfiore T., et al. In: Germoplasma olivicolo e tipicità dell'olio (2004), pp: 64-70.

3 - Muzzalupo I., Stefanizzi F., Perri E. HortScience (2009), 44: pp. 582-588. 


\section{“Nera di Gonnos"}

(synonymy: Majorca di Dorgali, Niedda di Gonnos, Olia tonda, Tonda di Cagliari, etc.)

Areal distribution or origin area: Sardegna

Flesh/pit weight ratio: medium $(6,17 \pm 1,36)$

Oil content (\%): medium $(45,98 \pm 0,35)$

Purpose: dual purpose

\section{Morphological characters}

Tree characters

Vigour: medium-weak

Growth habit: spreading-erect

Canopy-density: medium

\section{Leaf characters}

Blade length $(\mathrm{cm})$ : medium $(6,38 \pm 0,59)$

Blade width $(\mathrm{cm})$ : medium $(\mathbf{1 , 3 4} \pm \mathbf{0 , 1 3})$

Shape (length/width): elliptic-lanceolate

\section{Inflorescence characters}

Inflorescence length (cm): medium $(2,30 \pm 1,93)$

Number of flowers: low $(17,90 \pm 2,47)$

\section{Fruit characters}

Fresh weight of 100 fruits (g): high $(\mathbf{5 , 2 3} \pm \mathbf{1 , 4 3})$

Shape (length/width): spherical

Symmetry: slightly asymmetric

Position of maximum transverse diameter:

central

Apex: rounded

Base: rounded

Niplle: absent

Lenticels: few and large

\section{Pit characters}

Weight of 100 pits (g): high $(0,63 \pm \mathbf{0 , 2 0})$

Shape (length/width): ovoid

Mucron: obvious

Symmetry: symmetric

Position of maximum transverse diameter:

towards apex

Apex: rounded

Base: rounded

Surface: scabrous

Number of grooves: medium
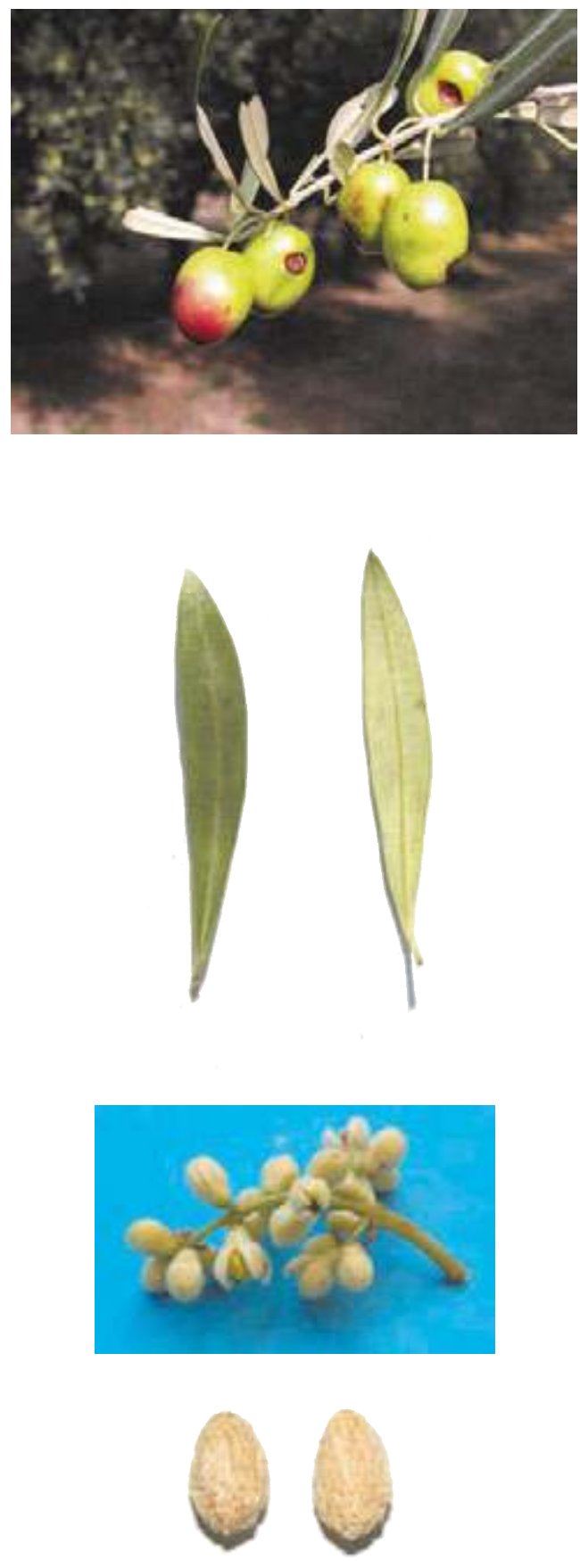


\section{Biochemical Characters}

\section{Fatty Acid Composition}

Table 1. Average values (express in $\% \pm$ standard deviations) of the fatty acids methyl esters and nutritional ratios obtained from single cultivar olive oils.

$\begin{array}{llllll}\text { Myristic acid } & \mathbf{0 , 0 1} \pm \mathbf{0 , 0 1} & \text { Linoleic acid }(\omega 6) & \mathbf{1 0 , 2 0} \pm \mathbf{1 , 7 1} & \text { Lignoceric acid } & \mathbf{0 , 0 3} \pm \mathbf{0 , 0 0} \\ \text { Palmitic acid } & \mathbf{9 , 5 7} \pm \mathbf{0 , 8 2} & \text { Linolenic acid }(\omega 3) & \mathbf{0 , 7 8} \pm \mathbf{0 , 1 2} & & \\ \text { Palmitoleic acid } & \mathbf{1 , 4 2} \pm \mathbf{0 , 6 2} & \text { Arachic acid } & \mathbf{0 , 1 6} \pm \mathbf{0 , 0 0} & \text { Unsat./satured } & \mathbf{7 , 9 1} \pm \mathbf{0 , 7 8} \\ \text { Stearic acid } & \mathbf{1 , 3 3} \pm \mathbf{0 , 0 0} & \text { Eicosenoic acid } & \mathbf{0 , 0 4} \pm \mathbf{0 , 0 1} & \omega 6 / \omega 3 & \mathbf{1 3 , 0 0 \pm 0 , 2 3} \\ \text { Oleic acid } & \mathbf{7 4 , 7 8 \pm \mathbf { 1 , 4 6 }} & \text { Behenic acid } & \mathbf{0 , 0 6} \pm \mathbf{0 , 0 1} & & \end{array}$

\section{Organoleptic oil values}

Sensory Analysis (Panel test)

Comment: fruity medium, with hints of almond and read sensations of grass, leaves and artichoke. Balanced taste sensation with a medium bitter and spicy. Medium fluidity.

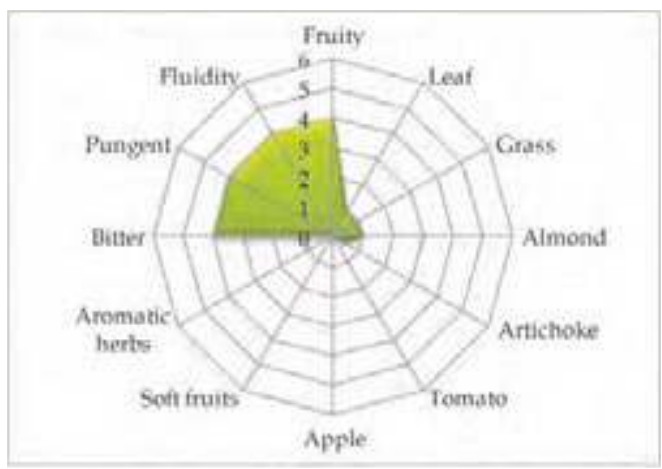

\section{Molecular Markers}

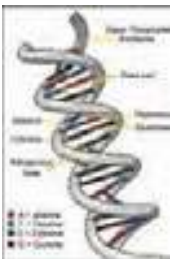

Table 2. Microsatellites (SSR) loci analyzed.

For each locus the allele size (expressed in base pairs) is reported.

\begin{tabular}{|c|c|c|c|c|c|}
\hline DCA09 & DCA18 & GAPU59 & GAPU71A & GUPA71B & GAPU103 \\
\hline $162-172$ & 185 - 185 & $212-212$ & $214-221$ & $126-144$ & $170-203$ \\
\hline UDO01 & UDO03 & UDO12 & UDO28 & UDO39 & \\
\hline $150-150$ & $135-135$ & $166-193$ & $143-143$ & $213-220$ & \\
\hline
\end{tabular}

\section{References:}

1 - Mulas M., Agabbio M., Chessa I., In: L'Olivo: le vecchie varietà della Sardegna, Ed. Delfino (1994), pp. 310-338.

2 - Muzzalupo I., Stefanizzi F., Perri E. HortScience (2009), 44: pp. 582-588. 


\section{“Nocellara del Belice"}

(synonymy: Bariddara, Bianculidda, Nuciddara, Oliva da salari, Tunna, etc.)

Areal distribution or origin area: Sicilia

Flesh/ pit weight ratio: high $(\mathbf{9}, \mathbf{1 3} \pm \mathbf{1 , 0 7 )}$

Oil content (\%): medium $(43,82 \pm 1,04)$

Purpose: table

\section{Morphological characters}

Tree characters

Vigour: medium-weak

Growth habit: spreading

Canopy-density: medium-dense

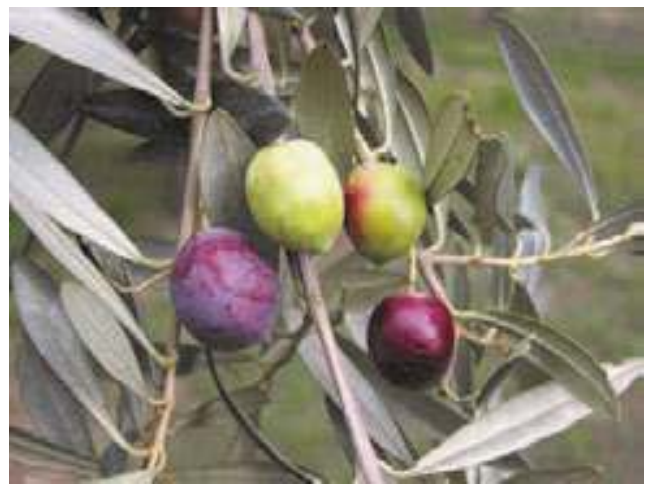

\section{Leaf characters}

Blade length $(\mathrm{cm})$ : medium $(6,39 \pm 0,62)$

Blade width (cm): broad $(\mathbf{1}, 55 \pm 0,16)$

Shape (length/width): elliptic-lanceolate

\section{Inflorescence characters}

Inflorescence length $(\mathrm{cm})$ : medium $(2,80 \pm 1,55)$

Number of flowers: low $(\mathbf{1 5 , 5 0 \pm 3 , 9 2 )}$

\section{Fruit characters}

Fresh weight of 100 fruits $(\mathrm{g})$ : high $(\mathbf{4}, 58 \pm 0,84)$

Shape (length/width): spherical

Symmetry: slightly asymmetric

Position of maximum transverse diameter:

central

Apex: rounded

Base: truncate

Niplle: absent

Lenticels: many and small

\section{Pit characters}

Weight of 100 pits (g): high $(0,53 \pm 0,09)$

Shape (length/width): ovoid

Mucron: obvious

Symmetry: symmetric

Position of maximum transverse diameter:

central

Apex: rounded

Base: rounded

Surface: rugose

Number of grooves: medium
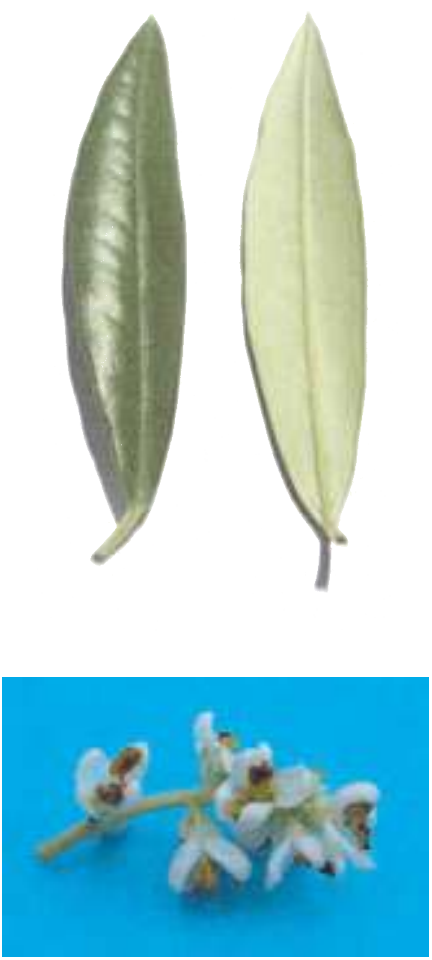


\section{Biochemical Characters}

\section{Fatty Acid Composition}

Table 1. Average values (express in $\% \pm$ standard deviations) of the fatty acids methyl esters and nutritional ratios obtained from single cultivar olive oils.

$\begin{array}{llllll}\text { Myristic acid } & \mathbf{0 , 0 1} \pm \mathbf{0 , 0 1} & \text { Linoleic acid }(\omega 6) & \mathbf{8 , 8 2} \pm 3,35 & \text { Lignoceric acid } & \mathbf{0 , 0 4 \pm 0 , 0 1} \\ \text { Palmitic acid } & \mathbf{1 0 , 5 0 \pm 0 , 4 2} & \text { Linolenic acid }(\omega 3) \mathbf{0 , 9 2} \pm \mathbf{0 , 0 6} & & \\ \text { Palmitoleic acid } & \mathbf{0 , 7 0 \pm 0 , 1 8} & \text { Arachic acid } & \mathbf{0 , 3 7} \pm \mathbf{0 , 0 8} & \text { Unsat./satured } & \mathbf{6 , 3 8} \pm \mathbf{0 , 0 3} \\ \text { Stearic acid } & \mathbf{2 , 7 0 \pm 0 , 4 8} & \text { Eicosenoic acid } & \mathbf{0 , 0 2} \pm \mathbf{0 , 0 1} & \omega 6 / \omega 3 & \mathbf{9 , 5 0} \pm 3,04 \\ \text { Oleic acid } & \mathbf{7 5 , 1 6 \pm 3 , 2 6} & \text { Behenic acid } & \mathbf{0 , 0 8} \pm \mathbf{0 , 0 1} & & \end{array}$

\section{Organoleptic oil values}

Sensory Analysis (Panel test)

Comment: fruity medium-high, with taste of artichoke, tomato, and grass-leaves. Balanced taste sensation with strong bitter and spicy. Medium-high fluidity.

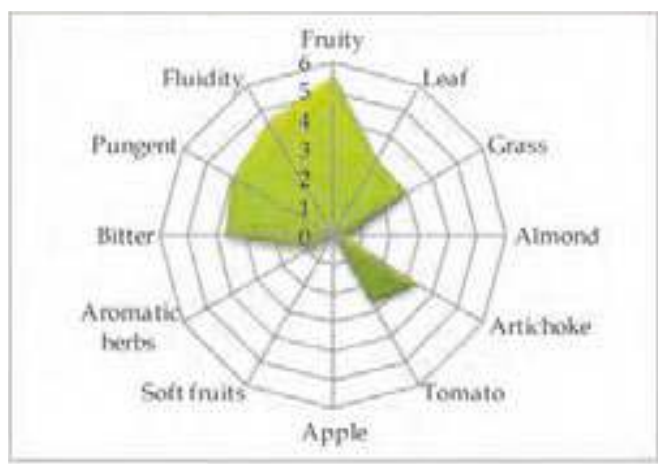

\section{Molecular Markers}

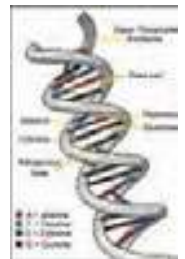

Table 2. Microsatellites (SSR) loci analyzed. For each locus the allele size (expressed in base pairs) is reported.

$\begin{array}{cccccc}D C A 09 & D C A 18 & \text { GAPU59 } & \text { GAPU71A } & \text { GUPA71B } & \text { GAPU103A } \\ \mathbf{1 6 2} \mathbf{- 1 7 2} & \mathbf{1 7 7 - \mathbf { 1 8 1 }} & \mathbf{2 1 2} \mathbf{- 2 1 2} & \mathbf{2 1 4} \mathbf{- 2 1 4} & \mathbf{1 4 4 - \mathbf { 1 4 4 }} & \mathbf{1 5 0} \mathbf{- 1 8 4} \\ \text { UDO01 } & \text { UDOO3 } & \text { UDO12 } & \text { UDO28 } & \text { UDO39 } & \\ \mathbf{1 4 0 - \mathbf { 1 4 0 }} & \mathbf{1 6 6 - \mathbf { 1 6 6 }} & \mathbf{1 6 6 - \mathbf { 1 9 3 }} & \mathbf{1 8 2} \mathbf{- 1 8 2} & \mathbf{1 0 8 - \mathbf { 2 0 5 }} & \end{array}$

\section{References:}

1 - Caruso T., Cartabellotta D., Motisi A., et all. In: Cultivar di olivo siciliane, Università degli Studi di Palermo (2007), pp. 48-52.

2 - Muzzalupo I., Stefanizzi F., Perri E. HortScience (2009), 44: pp. 582-588. 


\section{“Nocellara etnea"}

(synonymy: Augghialora, Nuciddara, Paturnisa, Pizzuta, Virdisa, etc.)

Areal distribution or origin area: Sicilia

Flesh/pit weight ratio: high $(\mathbf{8}, \mathbf{7 1} \pm \mathbf{1 , 0 1})$

Oil content (\%): high $(\mathbf{5 1 , 5 9 \pm 0 , 9 3 )}$

Purpose: table

\section{Morphological characters}

Tree characters

Vigour: medium

Growth habit: spreading

Canopy-density: medium-dense

\section{Leaf characters}

Blade length $(\mathrm{cm})$ : medium $(5,62 \pm 0,63)$

Blade width $(\mathrm{cm})$ : medium $(\mathbf{1 , 1 5} \pm 0,15)$

Shape (length/width): elliptic-lanceolate

\section{Inflorescence characters}

Inflorescence length $(\mathrm{cm})$ : short $(2,05 \pm 0,43)$

Number of flowers: low $(\mathbf{1 4 , 2 7} \pm \mathbf{2 , 5 3})$

\section{Fruit characters}

Fresh weight of 100 fruits $(\mathrm{g})$ : high $(\mathbf{4 , 4 5} \pm \mathbf{1 , 2 5})$

Shape (length/width): ovoid

Symmetry: slightly asymmetric

Position of maximum transverse diameter:

central

Apex: rounded

Base: rounded

Niplle: tenuous

Lenticels: few and large

\section{Pit characters}

Weight of 100 pits (g): high $(0,47 \pm 0,09)$

Shape (length/width): elliptic

Mucron: obvious

Symmetry: slightly asymmetric

Position of maximum transverse diameter:

central

Apex: pointed

Base: rounded

Surface: rugose

Number of grooves: medium
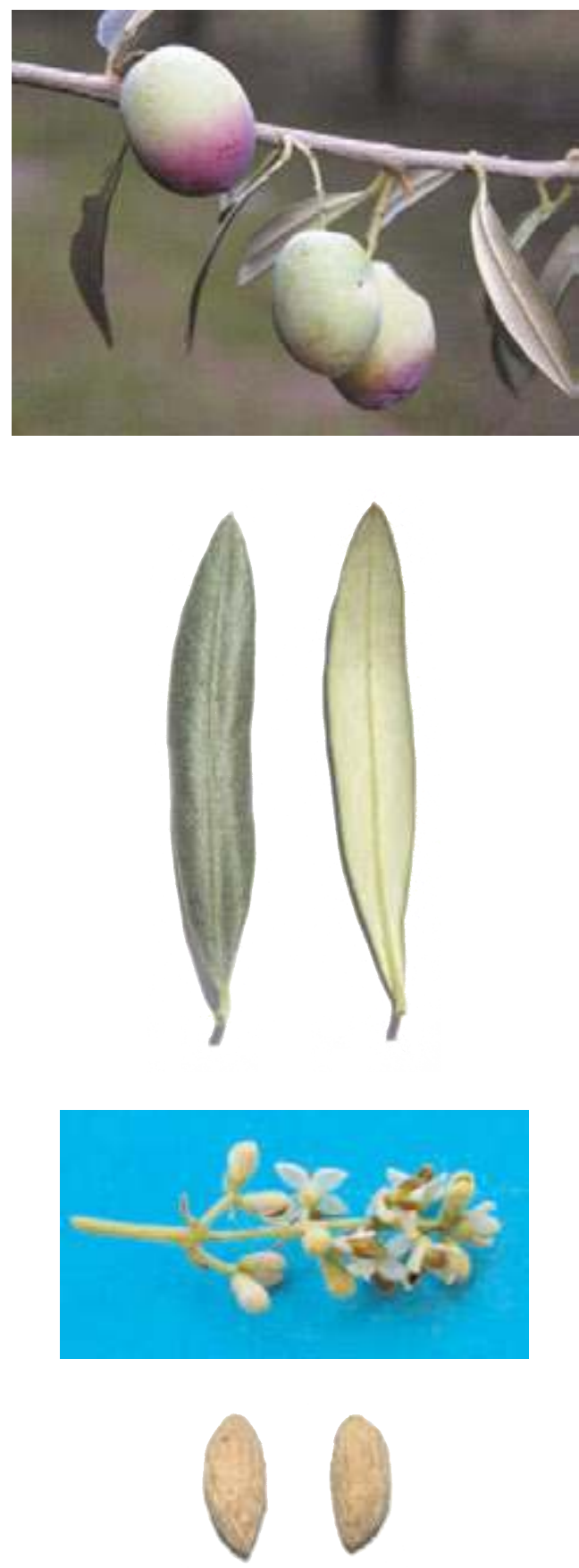


\section{Biochemical Characters}

\section{Fatty Acid Composition}

Table 1. Average values (express in $\% \pm$ standard deviations) of the fatty acids methyl esters and nutritional ratios obtained from single cultivar olive oils.

$\begin{array}{llllll}\text { Myristic acid } & \mathbf{0 , 0 1} \pm \mathbf{0 , 0 0} & \text { Linoleic acid }(\omega 6) & \mathbf{1 1 , 8 4} \pm \mathbf{1 , 8 3} & \text { Lignoceric acid } & \mathbf{0 , 0 6} \pm \mathbf{0 , 0 4} \\ \text { Palmitic acid } & \mathbf{1 2 , 7 0 \pm \mathbf { 1 , 3 2 }} & \text { Linolenic acid }(\omega 3) \mathbf{0 , 9 1} \pm \mathbf{0 , 0 7} & & \\ \text { Palmitoleic acid } & \mathbf{0 , 7 6 \pm 0 , 1 6} & \text { Arachic acid } & \mathbf{0 , 3 9} \pm \mathbf{0 , 1 1} & \text { Unsat./satured } & \mathbf{5 , 3 9} \pm \mathbf{0 , 5 7} \\ \text { Stearic acid } & \mathbf{2 , 5 7 \pm 0 , 3 4} & \text { Eicosenoic acid } & \mathbf{0 , 1 4} \pm \mathbf{0 , 2 1} & \omega 6 / \omega 3 & \mathbf{1 2 , 9 9 \pm 1 , 6 1} \\ \text { Oleic acid } & \mathbf{6 9 , 5 4 \pm 0 , 4 4} & \text { Behenic acid } & \mathbf{0 , 1 1} \pm \mathbf{0 , 0 4} & & \end{array}$

\section{Organoleptic oil values}

Sensory Analysis (Panel test)

Comment: fruity medium-high, with taste of almond and artichoke, read sensations of tomato, grass and leaves. Balanced taste sensation with medium bitter and spicy. Medium-high fluidity.

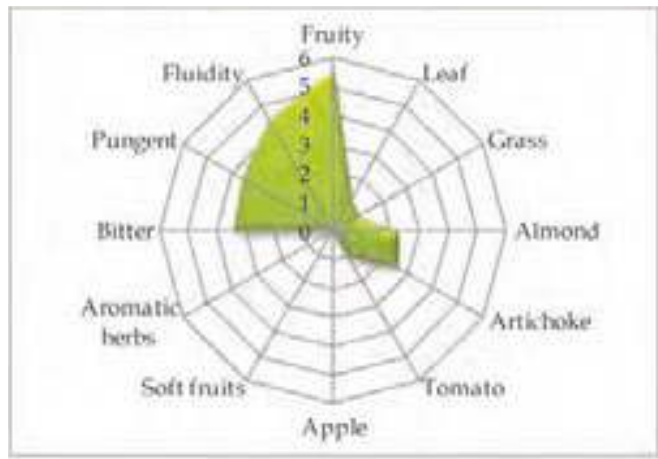

\section{Molecular Markers}

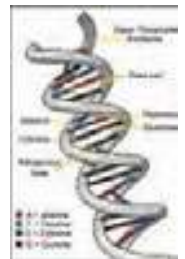

Table 2. Microsatellites (SSR) loci analyzed. For each locus the allele size (expressed in base pairs) is reported.

$\begin{array}{cccccc}D C A 09 & D C A 18 & \text { GAPU59 } & \text { GAPU71A } & \text { GUPA71B } & \text { GAPU103A } \\ \mathbf{1 7 2} \mathbf{- 1 8 2} & \mathbf{1 8 1} \mathbf{- 1 8 1} & \mathbf{2 1 2} \mathbf{- 2 1 2} & \mathbf{2 1 0 - \mathbf { 2 1 0 }} & \mathbf{1 2 4} \mathbf{- 1 4 4} & \mathbf{1 5 9} \mathbf{- 1 8 4} \\ \text { UDO01 } & \text { UDOO3 } & \text { UDO12 } & \text { UDO28 } & \text { UDO39 } & \\ \mathbf{1 4 4 - \mathbf { 1 4 4 }} & \mathbf{1 5 0 - \mathbf { 1 5 0 }} & \mathbf{1 6 6 - \mathbf { 1 9 3 }} & \mathbf{1 4 3 - \mathbf { 1 8 2 }} & \mathbf{2 2 0} \mathbf{- 2 2 0} & \end{array}$

\section{References:}

1 - Caruso T., Cartabellotta D., Motisi A., et all. In: Cultivar di olivo siciliane, Università degli Studi di Palermo (2007), pp. 54-58.

2 - Muzzalupo I., Stefanizzi F., Perri E. HortScience (2009), 44: pp. 582-588. 


\section{“Nocellara messinese "}

(synonymy: Nuciddara, Verdella.)

Areal distribution or origin area: Sicilia

Flesh/pit weight ratio: high $(7,99 \pm 0,61)$

Oil content (\%): medium $(47,17 \pm 0,74)$

Purpose: dual purpose

\section{Morphological characters}

Tree characters

Vigour: medium

Growth habit: spreading-erect

Canopy-density: medium

\section{Leaf characters}

Blade length (cm): medium $(6,49 \pm 0,66)$

Blade width $(\mathrm{cm})$ : medium $(\mathbf{1 , 1 9} \pm 0,14)$

Shape (length/width): elliptic-lanceolate

\section{Inflorescence characters}

Inflorescence length $(\mathrm{cm})$ : short $(2,49 \pm 1,38)$

Number of flowers: low $(17,81 \pm 2,45)$

\section{Fruit characters}

Fresh weight of 100 fruits (g): high $(\mathbf{5 , 2 0} \pm \mathbf{0 , 6 8})$

Shape (length/width): ovoid

Symmetry: slightly asymmetric

Position of maximum transverse diameter:

central

Apex: rounded

Base: rounded

Niplle: absent

Lenticels: many and large

\section{Pit characters}

Weight of 100 pits (g): high $(\mathbf{0 , 6 2} \pm \mathbf{1 , 7 5})$

Shape (length/width): ovoid

Mucron: absent

Symmetry: symmetric

Position of maximum transverse diameter:

central

Apex: rounded

Base: rounded

Surface: rugose

Number of grooves: medium
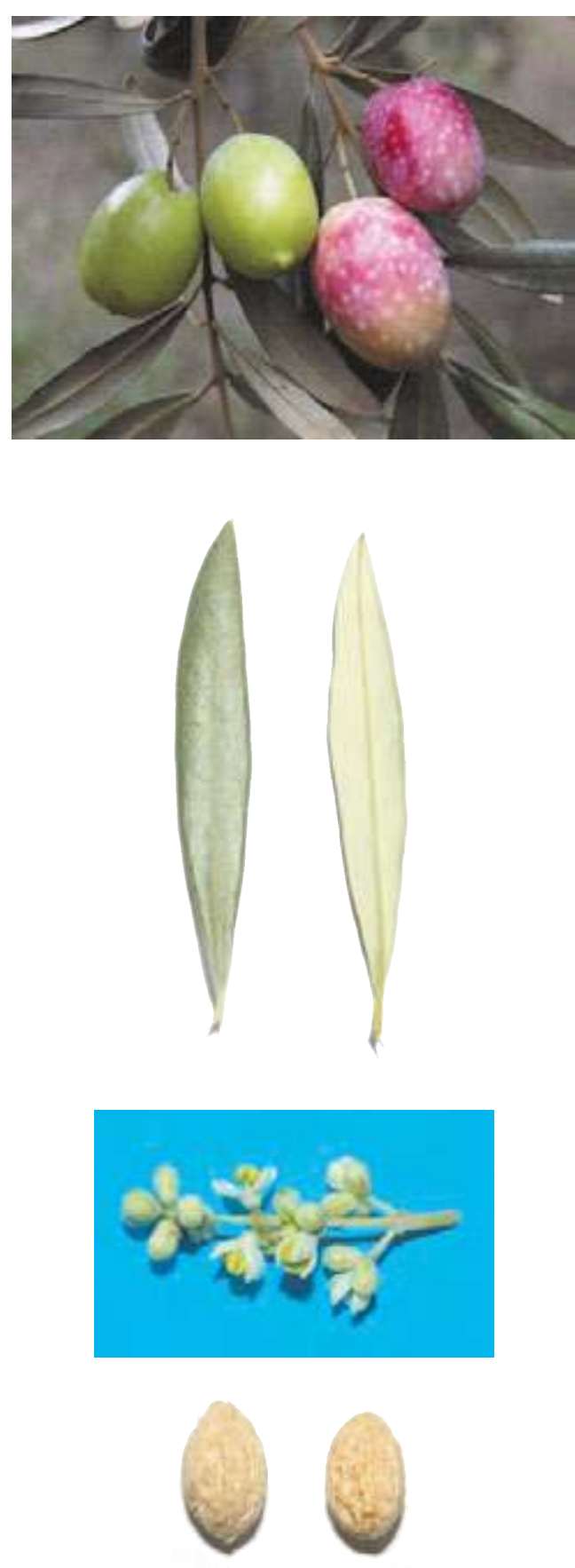


\section{Biochemical Characters}

\section{Fatty Acid Composition}

Table 1. Average values (express in $\% \pm$ standard deviations) of the fatty acids methyl esters and nutritional ratios obtained from single cultivar olive oils.

$\begin{array}{llllll}\text { Myristic acid } & \mathbf{0 , 0 1} \pm \mathbf{0 , 0 0} & \text { Linoleic acid }(\omega 6) & \mathbf{5 , 0 7} \pm \mathbf{0 , 6 3} & \text { Lignoceric acid } & \mathbf{0 , 0 3} \pm \mathbf{0 , 0 1} \\ \text { Palmitic acid } & \mathbf{9 , 4 2} \pm \mathbf{1 , 7 8} & \text { Linolenic acid }(\omega 3) & \mathbf{0 , 9 3} \pm \mathbf{0 , 1 0} & & \\ \text { Palmitoleic acid } & \mathbf{0 , 3 2} \pm \mathbf{0 , 0 4} & \text { Arachic acid } & \mathbf{0 , 2 9} \pm \mathbf{0 , 0 2} & \text { Unsat./satured } & \mathbf{7 , 5 7 \pm \mathbf { 1 , 2 9 }} \\ \text { Stearic acid } & \mathbf{2 , 1 7} \pm \mathbf{0 , 0 2} & \text { Eicosenoic acid } & \mathbf{0 , 0 4} \pm \mathbf{0 , 0 1} & \omega 6 / \omega 3 & \mathbf{5 , 5 0 \pm \mathbf { 1 , 2 7 }} \\ \text { Oleic acid } & \mathbf{8 1 , 5 3 \pm \mathbf { 1 , 1 6 }} & \text { Behenic acid } & \mathbf{0 , 0 7} \pm \mathbf{0 , 0 2} & & \end{array}$

\section{Organoleptic oil values}

Sensory Analysis (Panel test)

Comment: fruity medium-high, with taste of almond and tomato. Balanced taste sensation with medium bitter and spicy. Medium-high fluidity.

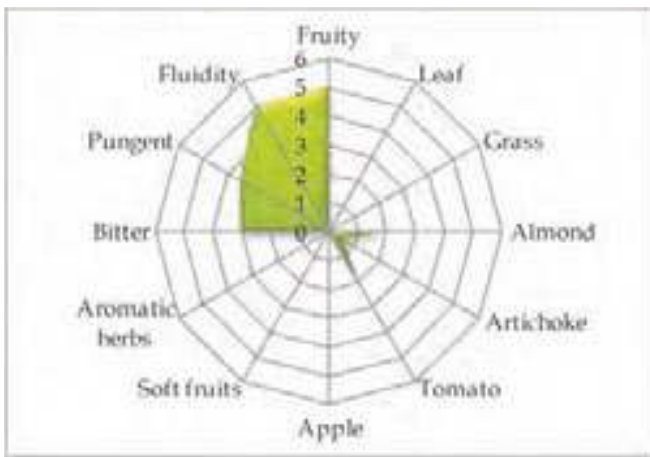

\section{Molecular Markers}

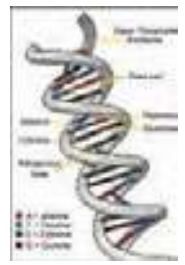

Table 2. Microsatellites (SSR) loci analyzed.

For each locus the allele size (expressed in base pairs) is reported.

\begin{tabular}{|c|c|c|c|c|c|}
\hline$D C A 09$ & $D C A 18$ & GAPU59 & GAPU71A & GUPA71B & GAPU103A \\
\hline $162-176$ & $179-179$ & $208-212$ & $214-214$ & $124-144$ & $159-184$ \\
\hline UDO01 & UDO03 & UDO12 & UDO28 & UDO39 & \\
\hline $144-144$ & $150-150$ & $166-193$ & $143-143$ & $213-232$ & \\
\hline
\end{tabular}

\section{References:}

1 - Caruso T., Cartabellotta D., Motisi A., et all. In: Cultivar di olivo siciliane, Università degli Studi di Palermo (2007), pp. 112-116.

2 - Muzzalupo I., Stefanizzi F., Perri E. HortScience (2009), 44: pp. 582-588. 


\section{“Nociara"}

(synonymy: Fra' Michele)

Areal distribution or origin area: Puglia

Flesh/pit weight ratio: medium $(6,27 \pm 1,51)$

Oil content (\%): medium $(46,65 \pm 0,95)$

Purpose: dual purpose

\section{Morphological characters}

Tree characters

Vigour: medium

Growth habit: spreading-erect

Canopy-density: medium

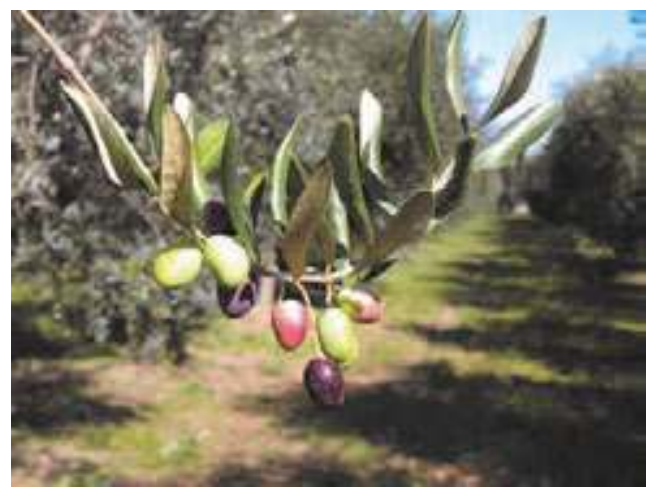

\section{Leaf characters}

Blade length (cm): medium $(5,86 \pm 0,54)$

Blade width (cm): broad $(\mathbf{1}, \mathbf{9 1} \pm \mathbf{0 , 3 0})$

Shape (length/width): elliptic

\section{Inflorescence characters}

Inflorescence length $(\mathrm{cm})$ : long $(3,67 \pm 0,70)$

Number of flowers: medium $(\mathbf{1 8 , 7 5} \pm \mathbf{2 , 8 2})$

\section{Fruit characters}

Fresh weight of 100 fruits (g): medium $(2,64 \pm 0,68)$

Shape (length/width): ovoid

Symmetry: asymmetric

Position of maximum transverse diameter:

central

Apex: rounded

Base: rounded

Niplle: absent

Lenticels: many and small

\section{Pit characters}

Weight of 100 pits (g): medium $(0,45 \pm 0,06)$

Shape (length/width): elliptic

Mucron: absent

Symmetry: asymmetric

Position of maximum transverse diameter:

\section{towards base}

Apex: pointed

Base: rounded

Surface: rugose

Number of grooves: high
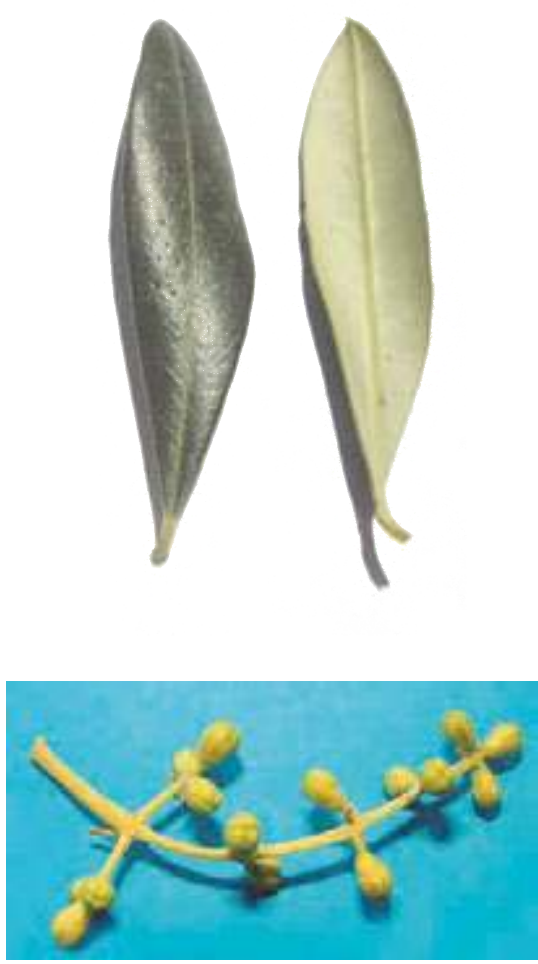


\section{Biochemical Characters}

\section{Fatty Acid Composition}

Table 1. Average values (express in $\% \pm$ standard deviations) of the fatty acids methyl esters and nutritional ratios obtained from single cultivar olive oils.

$\begin{array}{llllll}\text { Myristic acid } & \mathbf{0 , 0 1} \pm \mathbf{0 , 0 1} & \text { Linoleic acid }(\omega 6) & \mathbf{8 , 0 9} \pm \mathbf{1 , 3 2} & \text { Lignoceric acid } & \mathbf{0 , 0 5} \pm \mathbf{0 , 0 2} \\ \text { Palmitic acid } & \mathbf{1 2 , 7 3 \pm \mathbf { 1 , 5 8 }} & \text { Linolenic acid }(\omega 3) & \mathbf{0 , 8 4} \pm \mathbf{0 , 0 5} & & \\ \text { Palmitoleic acid } & \mathbf{1 , 0 3} \pm \mathbf{0 , 2 6} & \text { Arachic acid } & \mathbf{0 , 3 6} \pm \mathbf{0 , 0 5} & \text { Unsat./satured } & \mathbf{5 , 3 9 \pm 0 , 5 4} \\ \text { Stearic acid } & \mathbf{2 , 5 8} \pm \mathbf{0 , 2 2} & \text { Eicosenoic acid } & \mathbf{0 , 1 2} \pm \mathbf{0 , 1 4} & \omega 6 / \omega 3 & \mathbf{9 , 7 0} \pm \mathbf{1 , 7 1} \\ \text { Oleic acid } & \mathbf{7 3 , 2 3} \pm \mathbf{2 , 1 9} & \text { Behenic acid } & \mathbf{0 , 1 1} \pm \mathbf{0 , 0 2} & & \end{array}$

\section{Organoleptic oil values}

Sensory Analysis (Panel test)

Comment: Fruity medium, with read sensations of aromatic herb. Balanced taste sensation with medium bitter and spicy. Medium fluidity.

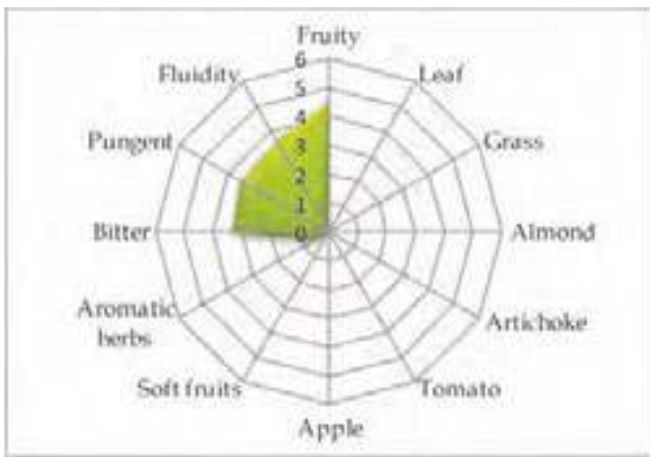

\section{Molecular Markers}

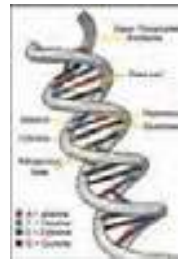

Table 2. Microsatellites (SSR) loci analyzed.

For each locus the allele size (expressed in base pairs) is reported.

\begin{tabular}{|c|c|c|c|c|c|}
\hline DCA09 & DCA18 & GAPU59 & GAPU71A & $G U P A 71 B$ & GAPU103A \\
\hline $162-162$ & $179-185$ & $212-222$ & $210-214$ & $124-126$ & $170-170$ \\
\hline UDO01 & UDO03 & UDO12 & UDO28 & UDO39 & \\
\hline $140-140$ & $143-143$ & $166-193$ & $182-182$ & $164-232$ & \\
\hline
\end{tabular}

\section{References:}

1 - Lombardo N., Perri E., Muzzalupo I., et al. In: Contributo alla caratterizzazione del germoplasma olivicolo pugliese, Istituto Sperimentale per l'Olivicoltura (2004), pp. 61-64.

2 - Muzzalupo I., Stefanizzi F., Perri E. HortScience (2009), 44: pp. 582-588. 


\section{“Nolca"}

(synonymy: Amele, Anolca, Dolce, Dolce Mele, Mele, Noce, etc.)

Areal distribution or origin area: Puglia

Flesh/pit weight ratio: medium $(7,39 \pm 1,91)$

Oil content (\%): medium $(41,06 \pm 1,56)$

Purpose: table

\section{Morphological characters}

Tree characters

Vigour: medium

Growth habit: spreading

Canopy-density: medium

\section{Leaf characters}

Blade length ( $\mathrm{cm})$ : medium $(5,84 \pm 0,76)$

Blade width $(\mathrm{cm})$ : broad $(\mathbf{1}, 67 \pm 0,35)$

Shape (length/width): elliptic

\section{Inflorescence characters}

Inflorescence length (cm): short $(2,45 \pm 0,44)$

Number of flowers: medium $(16,84 \pm 1,98)$

\section{Fruit characters}

Fresh weight of 100 fruits $(\mathrm{g})$ : medium $(7,73 \pm 0,75)$

Shape (length/width): spherical

Symmetry: asymmetric

Position of maximum transverse diameter:

central

Apex: rounded

Base: truncate

Niplle: absent

Lenticels: many and small

\section{Pit characters}

Weight of 100 pits (g): high $(0,53 \pm 0,06)$

Shape (length/width): ovoid

Mucron: obvious

Symmetry: symmetric

Position of maximum transverse diameter:

towards apex

Apex: rounded

Base: rounded

Surface: rugose

Number of grooves: medium
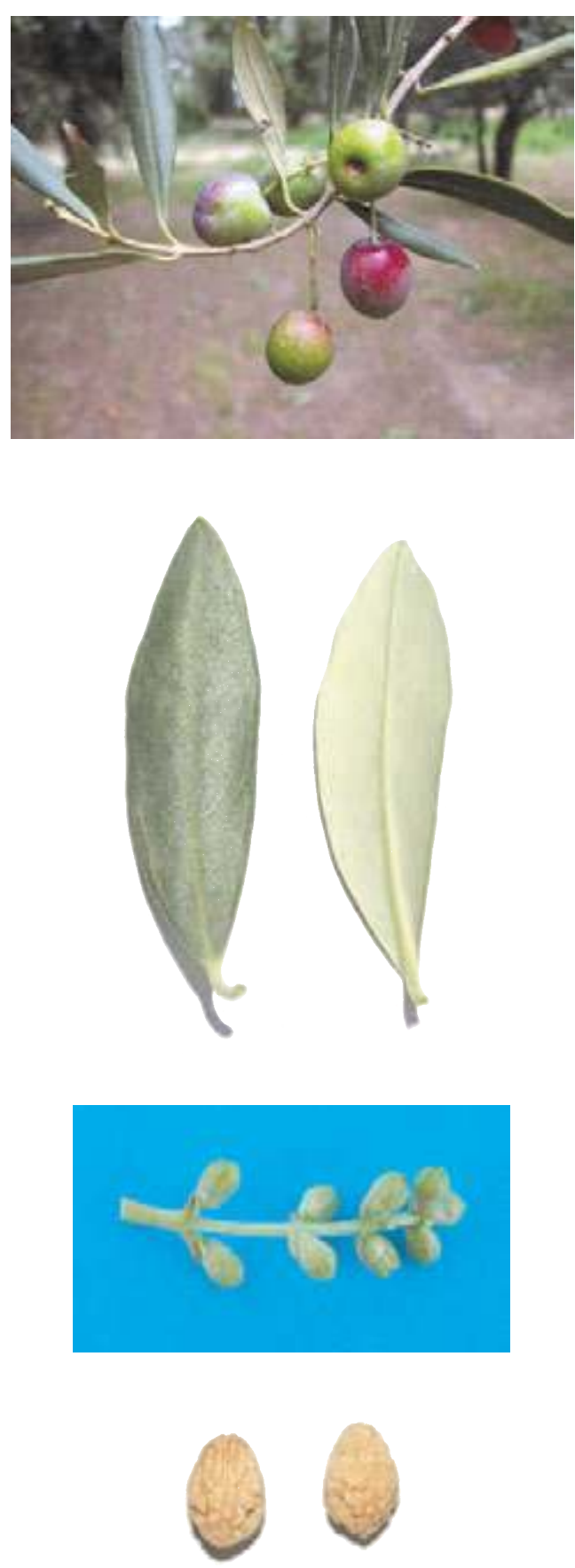


\section{Biochemical Characters}

\section{Fatty Acid Composition}

Table 1. Average values (express in $\% \pm$ standard deviations) of the fatty acids methyl esters and nutritional ratios obtained from single cultivar olive oils.

$\begin{array}{lllll}\text { Myristic acid } & \mathbf{0 , 0 1} \pm \mathbf{0 , 0 0} \quad \text { Linoleic acid }(\omega 6) & \mathbf{1 1 , 0 5} \pm \mathbf{0 , 2 1} \quad \text { Lignoceric acid } \quad 0,02 \pm 0,01\end{array}$

Palmitic acid $11,09 \pm 0,17 \quad$ Linolenic acid $(\omega 3)$ 0,87 $\pm 0,16$

Palmitoleic acid

$$
1,00 \pm 0,04
$$

Arachic acid

$0,22 \pm 0,01$

Unsat./satured

$6,62 \pm 0,35$

Stearic acid

$1,74 \pm 0,07$

Eicosenoic acid

$0,02 \pm 0,01$

Oleic acid

$72,90 \pm 3,65$

Behenic acid

$0,06 \pm 0,01$

\section{Organoleptic oil values}

Sensory Analysis (Panel test)

Comment: fruity medium - light, with hints of artichoke and almond. Balanced in flavours, with hints of bitter and spicy medium-light intensity. Medium fluidity.

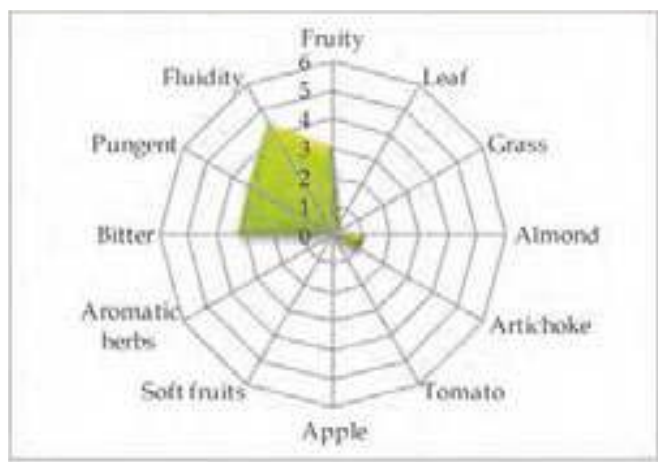

\section{Molecular Markers}

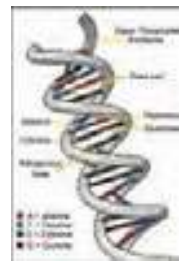

Table 2. Microsatellites (SSR) loci analyzed.

For each locus the allele size (expressed in base pairs) is reported.

\begin{tabular}{|c|c|c|c|c|c|}
\hline DCA09 & DCA18 & GAPU59 & GAPU71A & GUPA71B & GAPU103A \\
\hline $162-182$ & $177-185$ & $208-208$ & $214-214$ & $124-144$ & $150-157$ \\
\hline UDO01 & UDO03 & UDO12 & UDO28 & UDO39 & \\
\hline $144-144$ & $153-153$ & $166-177$ & $150-182$ & $108-170$ & \\
\hline
\end{tabular}

\section{References:}

1 - Lombardo N., Perri E., Muzzalupo I., et al. In: Contributo alla caratterizzazione del germoplasma olivicolo pugliese, Ist. Sper. Olivic. (2004), pp. 57-60.

2 - Muzzalupo I., Stefanizzi F., Perri E. HortScience (2009), 44: pp. 582-588. 


\section{“Nostrale di Fiano Romano"}

(synonymy: Nostrale, Nostrale di Lauro, etc.)

Areal distribution or origin area: Lazio

Flesh/pit weight ratio: medium $(5,43 \pm 0,64)$

Oil content (\%): medium $(45,78 \pm 0,47)$

Purpose: oil

\section{Morphological characters}

Tree characters

Vigour: medium-strong

Growth habit: spreading

Canopy-density: medium-dense

\section{Leaf characters}

Blade length $(\mathrm{cm})$ : medium $(5,92 \pm 0,81)$

Blade width $(\mathrm{cm})$ : broad $(\mathbf{1}, 63 \pm \mathbf{0 , 1 4})$

Shape (length/width): elliptic

\section{Inflorescence characters}

Inflorescence length (cm): long $(3,62 \pm 0,29)$

Number of flowers: low $(\mathbf{1 6 , 8 8} \pm \mathbf{2 , 1 1})$

\section{Fruit characters}

Fresh weight of 100 fruits $(\mathrm{g})$ : medium $(2,47 \pm 0,19)$

Shape (length/width): ovoid

Symmetry: slightly asymmetric

Position of maximum transverse diameter:

central

Apex: rounded

Base: rounded

Niplle: absent

Lenticels: many and large

\section{Pit characters}

Weight of 100 pits (g): high $(\mathbf{0 , 4 9} \pm \mathbf{0 , 1 3})$

Shape (length/width): elliptic

Mucron: obvious

Symmetry: symmetric

Position of maximum transverse diameter:

central

Apex: rounded

Base: rounded

Surface: rugose

Number of grooves: high
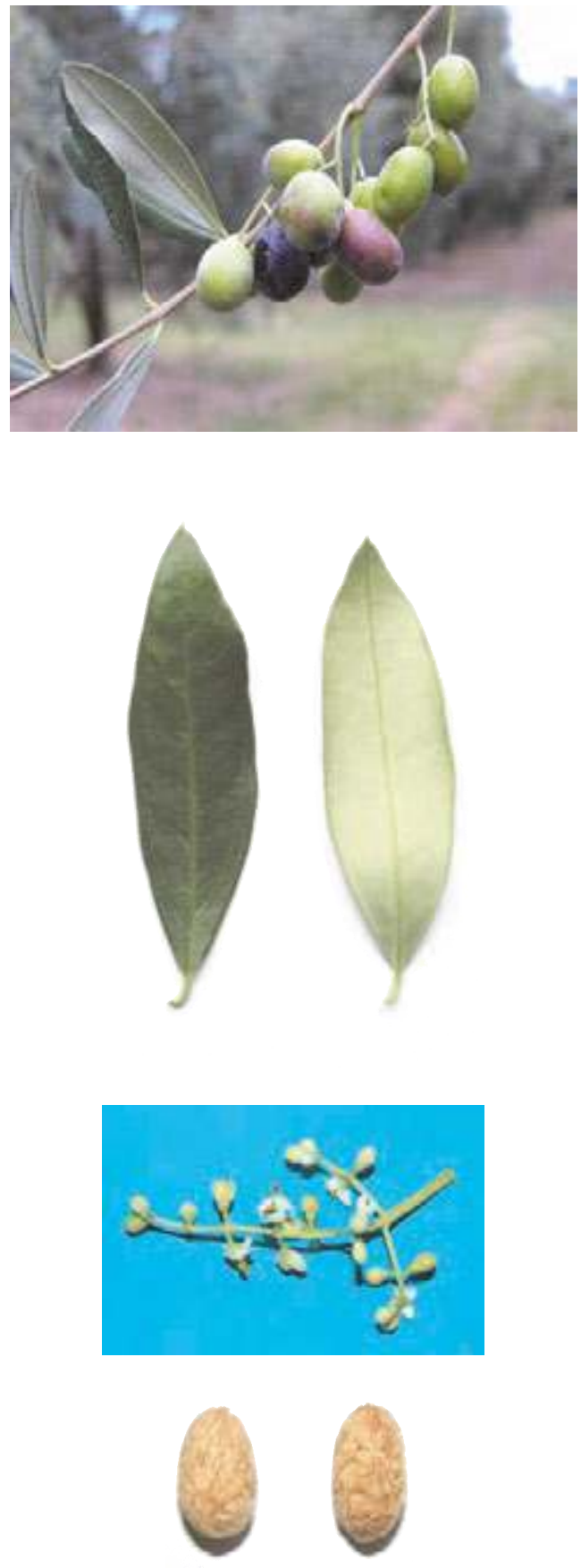


\section{Biochemical Characters}

\section{Fatty Acid Composition}

Table 1. Average values (express in $\% \pm$ standard deviations) of the fatty acids methyl esters and nutritional ratios obtained from single cultivar olive oils.

$\begin{array}{llllll}\text { Myristic acid } & \mathbf{0 , 0 1} \pm \mathbf{0 , 0 0} & \text { Linoleic acid }(\omega 6) & \mathbf{6 , 1 9} \pm \mathbf{1 , 1 2} & \text { Lignoceric acid } & \mathbf{0 , 0 2} \pm \mathbf{0 , 0 1} \\ \text { Palmitic acid } & \mathbf{1 5 , 0 8} \pm \mathbf{1 , 2 6} & \text { Linolenic acid }(\omega 3) & \mathbf{0 , 8 2} \pm \mathbf{0 , 0 4} & & \\ \text { Palmitoleic acid } & \mathbf{0 , 7 5} \pm \mathbf{0 , 0 1} & \text { Arachic acid } & \mathbf{0 , 2 4} \pm \mathbf{0 , 0 3} & \text { Unsat./satured } & \mathbf{4 , 8 9} \pm \mathbf{0 , 4 2} \\ \text { Stearic acid } & \mathbf{1 , 6 4} \pm \mathbf{0 , 0 8} & \text { Eicosenoic acid } & \mathbf{0 , 0 1} \pm \mathbf{0 , 0 0} & \omega 6 / \omega 3 & \mathbf{7 , 5 2} \pm \mathbf{0 , 9 7} \\ \text { Oleic acid } & \mathbf{7 4 , 6 4 \pm \mathbf { 0 , 1 1 }} & \text { Behenic acid } & \mathbf{0 , 0 7} \pm \mathbf{0 , 0 2} & & \end{array}$

\section{Organoleptic oil values}

Sensory Analysis (Panel test)

Comment: fruity medium, with hints of artichoke, grass and almond. Balanced in flavours, with hints of bitter and spicy medium intensity, medium fluidity.

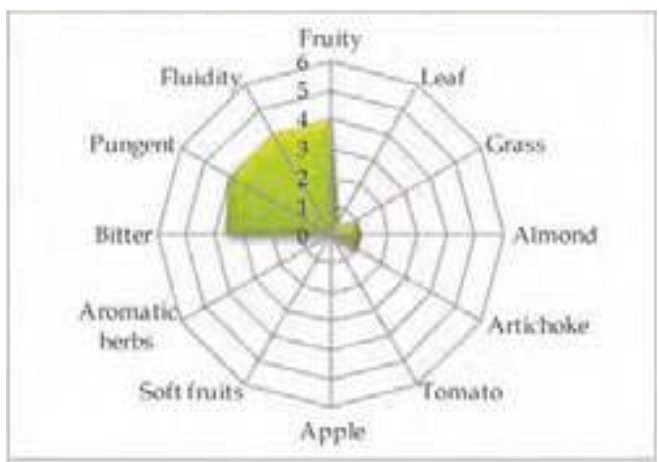

\section{Molecular Markers}

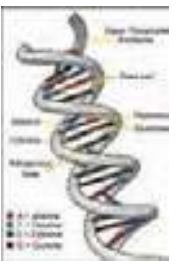

Table 2. Microsatellites (SSR) loci analyzed.

For each locus the allele size (expressed in base pairs) is reported.

\begin{tabular}{|c|c|c|c|c|c|}
\hline$D C A 09$ & $D C A 18$ & GAPU59 & GAPU71A & GUPA71B & GAPU103A \\
\hline $182-206$ & $179-181$ & $212-222$ & $214-224$ & $126-144$ & $159-170$ \\
\hline UDO01 & UDO03 & UDO12 & UDO28 & UDO39 & \\
\hline $144-144$ & $143-143$ & $177-182$ & $182-210$ & $185-185$ & \\
\hline
\end{tabular}

\section{References:}

1 - Parlati M. V., Pandolfi S., In: Olivo e Olio, (2001), 4: pp. 67-72.

2 - Muzzalupo I., Stefanizzi F., Bucci C., et al. In Acta Italus Hortus, (2011), 1: 138 -140. 


\section{“Nostrale di Rigali ”}

(synonymy: Borciona, Limona, Nostrale, Oliva, etc.)

Areal distribution or origin area: Umbria

Flesh/ pit weight ratio: high $(7,97 \pm 1,39)$

Oil content (\%): high $\mathbf{( 5 1 , 0 2 \pm \mathbf { 1 , 5 7 } )}$

Purpose: oil

\section{Morphological characters}

Tree characters

Vigour: medium-strong

Growth habit: spreading

Canopy-density: dense

\section{Leaf characters}

Blade length (cm): medium $(6,60 \pm 0,63)$

Blade width (cm): medium $(\mathbf{1}, 21 \pm 0,17)$

Shape (length/width): elliptic-lanceolate

\section{Inflorescence characters}

Inflorescence length (cm): medium $(2,88 \pm 0,15)$

Number of flowers: low $(16,21 \pm 1,62)$

\section{Fruit characters}

Fresh weight of 100 fruits $(\mathrm{g})$ : high $(\mathbf{4 , 5 2} \pm \mathbf{0 , 1 1})$

Shape (length/width): ovoid

Symmetry: slightly asymmetric

Position of maximum transverse diameter:

central

Apex: rounded

Base: truncate

Niplle: absent

Lenticels: many and small

\section{Pit characters}

Weight of 100 pits (g): high $(0,52 \pm \mathbf{0 , 0 6})$

Shape (length/width): elliptic

Mucron: absent

Symmetry: slightly asymmetric

Position of maximum transverse diameter:

central

Apex: rounded

Base: rounded

Surface: scabrous

Number of grooves: low
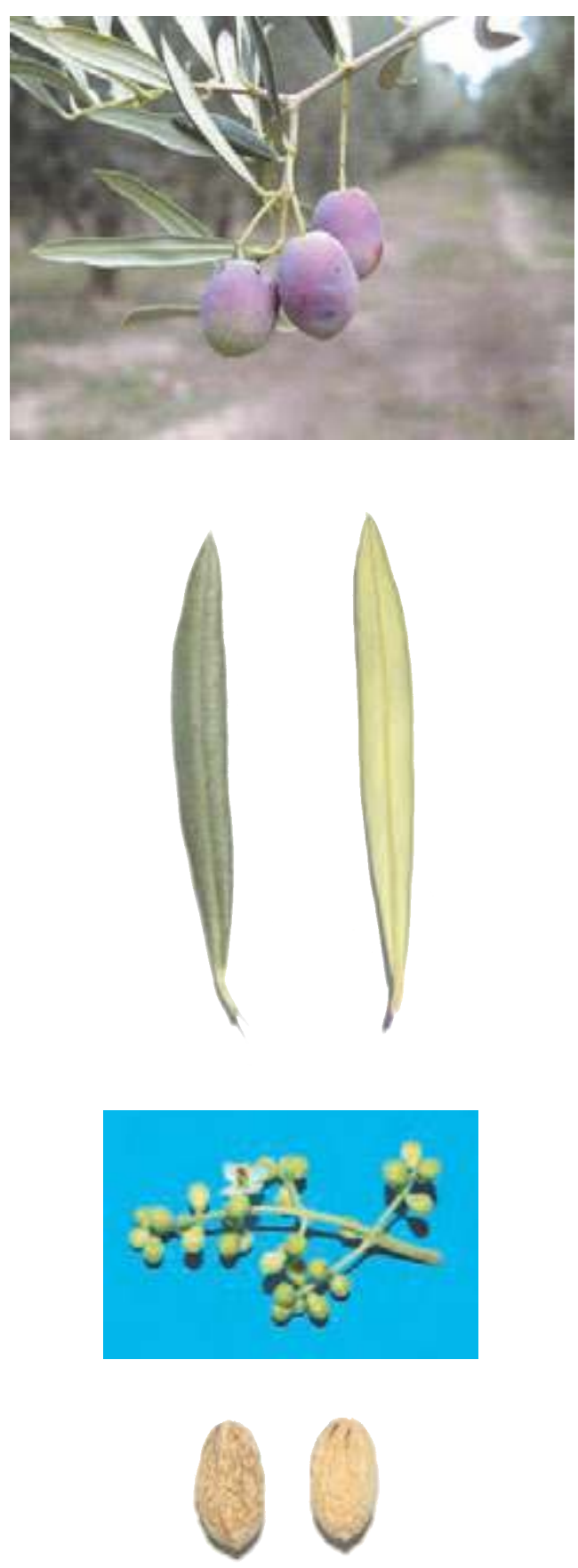


\section{Biochemical Characters}

\section{Fatty Acid Composition}

Table 1. Average values (express in $\% \pm$ standard deviations) of the fatty acids methyl esters and nutritional ratios obtained from single cultivar olive oils.

$\begin{array}{llllll}\text { Myristic acid } & \mathbf{0 , 0 1} \pm \mathbf{0 , 0 0} & \text { Linoleic acid }(\omega 6) & \mathbf{9 , 5 1} \pm \mathbf{1 , 3 1} & \text { Lignoceric acid } & \mathbf{0 , 0 5} \pm \mathbf{0 , 0 3} \\ \text { Palmitic acid } & \mathbf{1 2 , 1 9} \pm \mathbf{1 , 5 0} & \text { Linolenic acid }(\omega 3) & \mathbf{0 , 8 7} \pm \mathbf{0 , 1 1} & & \\ \text { Palmitoleic acid } & \mathbf{0 , 8 4} \pm \mathbf{0 , 0 6} & \text { Arachic acid } & \mathbf{0 , 4 3} \pm \mathbf{0 , 1 7} & \text { Unsat./satured } & \mathbf{4 , 9 9} \pm \mathbf{0 , 4 5} \\ \text { Stearic acid } & \mathbf{4 , 1 4} \pm \mathbf{1 , 6 9} & \text { Eicosenoic acid } & \mathbf{0 , 1 2} \pm \mathbf{0 , 1 7} & \omega 6 / \omega 3 & \mathbf{1 1 , 1 2} \pm \mathbf{2 , 8 4} \\ \text { Oleic acid } & \mathbf{7 1 , 0 6} \pm \mathbf{2 , 5 1} & \text { Behenic acid } & \mathbf{0 , 1 0} \pm \mathbf{0 , 0 3} & & \end{array}$

\section{Organoleptic oil values}

Sensory Analysis (Panel test)

Comment: fruity medium, with taste of grass, leaves, and almond, read sensations of soft fruits. Balanced taste sensation with medium bitter and spicy. Medium fluidity.

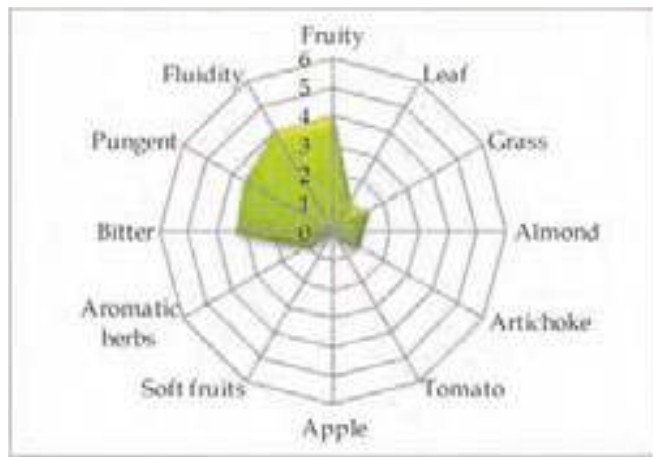

\section{Molecular Markers}

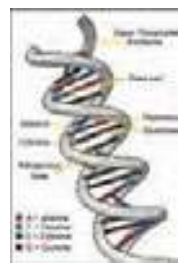

Table 2. Microsatellites (SSR) loci analyzed.

For each locus the allele size (expressed in base pairs) is reported.

\begin{tabular}{|c|c|c|c|c|c|}
\hline$D C A 09$ & $D C A 18$ & GAPU59 & GAPU71A & GUPA71B & GAPU103A \\
\hline $162-172$ & $179-181$ & $214-218$ & $218-224$ & $124-130$ & $184-197$ \\
\hline UDO01 & UDO03 & UDO12 & UDO28 & UDO39 & \\
\hline $144-144$ & $135-143$ & $166-182$ & $150-161$ & $205-220$ & \\
\hline
\end{tabular}

\section{References:}

1 - Pannelli G., Alfei B., D’ Ambrosio A., et al. In: Varietà di olivo in Umbria, Ed. Pliniana (2000), pp. 49-54.

2 - Various authors. In: Catalogo Nazionale delle Varietà di Olivo, University of Bari (in press), ISBN 978-88-8879397-9. 


\section{“Nostrana di Brisighella"}

(synonymy: Nostrale di Brisighella, Nostrana.)

Areal distribution or origin area: Emilia

Flesh/pit weight ratio: medium $(6,82 \pm 0,07)$

Oil content (\%): medium $(40,34 \pm 1,28)$

Purpose: oil

\section{Morphological characters}

Tree characters

Vigour: medium-weak

Growth habit: spreading

Canopy-density: medium-dense

\section{Leaf characters}

Blade length $(\mathrm{cm})$ : medium $(6,13 \pm 0,50)$

Blade width $(\mathrm{cm})$ : medium $(\mathbf{1 , 1 1} \pm \mathbf{0 , 1 8})$

Shape (length/width): elliptic-lanceolate

\section{Inflorescence characters}

Inflorescence length (cm): medium $(2,91 \pm 1,87)$

Number of flowers: medium $(\mathbf{3 1}, \mathbf{2 9} \pm \mathbf{2 , 1 8})$

\section{Fruit characters}

Fresh weight of 100 fruits (g): medium $(\mathbf{3 , 1 2} \pm \mathbf{0 , 1 8})$

Shape (length/width): spherical

Symmetry: slightly asymmetric

Position of maximum transverse diameter:

towards base

Apex: rounded

Base: truncate

Niplle: tenuous

Lenticels: many and small

\section{Pit characters}

Weight of 100 pits (g): medium $(\mathbf{0 , 4 8} \pm \mathbf{0 , 0 2})$

Shape (length/width): ovoid

Mucron: absent

Symmetry: slightly asymmetric

Position of maximum transverse diameter:

towards base

Apex: pointed

Base: rounded

Surface: rugose

Number of grooves: medium
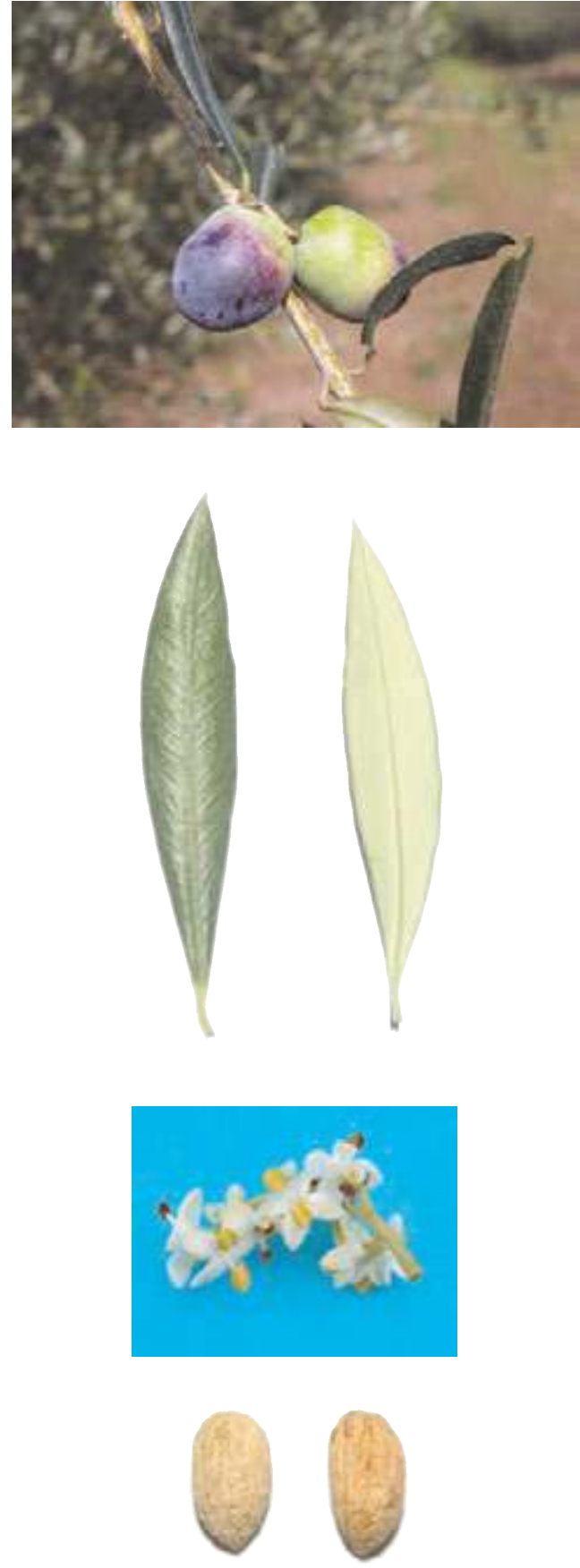


\section{Biochemical Characters}

\section{Fatty Acid Composition}

Table 1. Average values (express in $\% \pm$ standard deviations) of the fatty acids methyl esters and nutritional ratios obtained from single cultivar olive oils.

$\begin{array}{llllll}\text { Myristic acid } & \mathbf{0 , 0 1} \pm \mathbf{0 , 0 1} & \text { Linoleic acid }(\omega 6) & \mathbf{8 , 2 9} \pm \mathbf{1 , 0 1} & \text { Lignoceric acid } & \mathbf{0 , 0 5} \pm \mathbf{0 , 0 3} \\ \text { Palmitic acid } & \mathbf{1 4 , 0 9} \pm \mathbf{0 , 5 9} & \text { Linolenic acid }(\omega 3) \mathbf{0 , 9 0} \pm \mathbf{0 , 1 4} & & \\ \text { Palmitoleic acid } & \mathbf{2 , 1 0} \pm \mathbf{0 , 2 7} & \text { Arachic acid } & \mathbf{0 , 3 0} \pm \mathbf{0 , 0 4} & \text { Unsat./satured } & \mathbf{5 , 0 7 \pm 0 , 2 6} \\ \text { Stearic acid } & \mathbf{1 , 8 5} \pm \mathbf{0 , 0 5} & \text { Eicosenoic acid } & \mathbf{0 , 1 0} \pm \mathbf{0 , 1 4} & \omega 6 / \omega 3 & \mathbf{9 , 5 1} \pm \mathbf{2 , 8 5} \\ \text { Oleic acid } & \mathbf{7 0 , 4 7 \pm 2 , 1 2} & \text { Behenic acid } & \mathbf{0 , 0 9} \pm \mathbf{0 , 0 3} & & \end{array}$

\section{Organoleptic oil values}

Sensory Analysis (Panel test)

Comment: fruity medium-high, with good sensation of grass, artichoke and tomato. Balanced in flavours, with hints of bitter and spicy medium-high intensity. Medium-high fluidity.

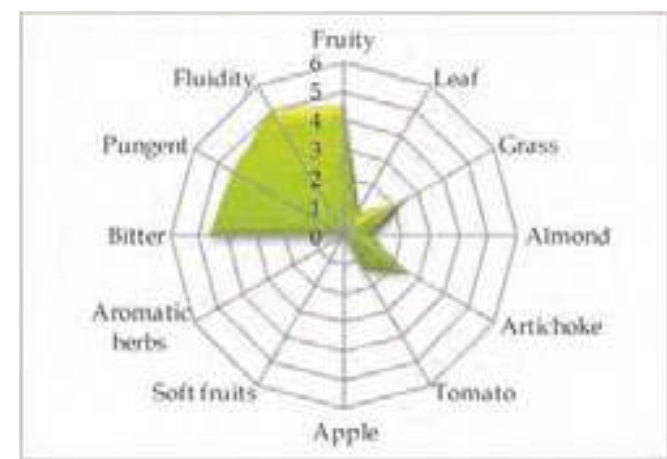

\section{Molecular Markers}

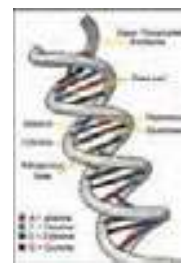

Table 2. Microsatellites (SSR) loci analyzed.

For each locus the allele size (expressed in base pairs) is reported.

$\begin{array}{cccccc}D C A 09 & \text { DCA18 } & \text { GAPU59 } & \text { GAPU71A } & \text { GUPA71B } & \text { GAPU103A } \\ \mathbf{1 9 8} \mathbf{- 1 9 8} & \mathbf{1 7 7 - \mathbf { 1 7 9 }} & \mathbf{2 1 4} \mathbf{- 2 1 4} & \mathbf{2 1 4} \mathbf{- 2 2 4} & \mathbf{1 2 6 - \mathbf { 1 4 4 }} & \mathbf{1 3 6 - \mathbf { 1 4 4 }} \\ \text { UDOO1 } & \text { UDOO3 } & \text { UDO12 } & \text { UDO28 } & \text { UDO39 } & \\ \mathbf{1 4 4 - \mathbf { 1 4 4 }} & \mathbf{1 3 5 - \mathbf { 1 3 5 }} & \mathbf{1 6 6 - \mathbf { 1 6 6 }} & \mathbf{1 5 0 - \mathbf { 1 6 1 }} & \mathbf{2 1 3 - \mathbf { 2 1 3 }} & \end{array}$

\section{References:}

1 - Rotondi A., Babini A.R. In: Olivo e Olio, (2001), 4: pp. 94-95.

2 - Cristoferi G., Rotondi A., Magli M. In Il germoplasma dell'olivo in Emilia Romagna, ISTEA_CNR (1997)

3 - Muzzalupo I., Stefanizzi F., Bucci C., et al. In Acta Italus Hortus, (2011), 1: 138 -140. 


\section{“Ogliara”}

(synonymy: Ogliarola)

Areal distribution or origin area: Campania Flesh/pit weight ratio: high $(8,06 \pm 0,34)$ Oil content (\%): medium $(48,05 \pm 3,44)$

Purpose: oil

\section{Morphological characters}

Tree characters

Vigour: medium-strong

Growth habit: spreading

Canopy-density: medium

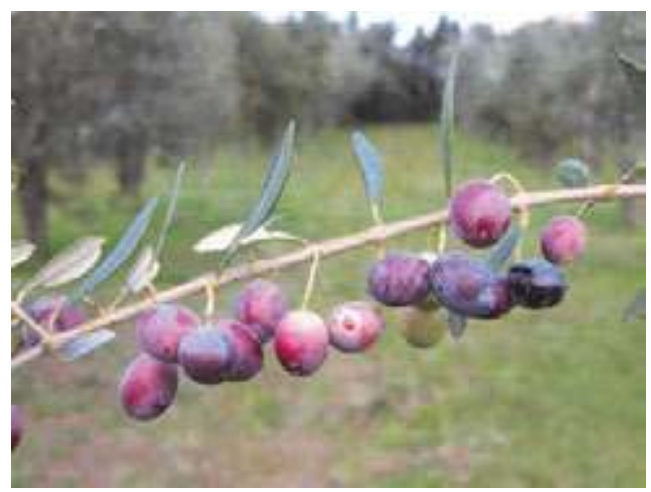

\section{Leaf characters}

Blade length (cm): medium $(5,71 \pm 0,53)$

Blade width $(\mathrm{cm})$ : medium $(\mathbf{1 , 3 8} \pm 0,13)$

Shape (length/width): elliptic-lanceolate

\section{Inflorescence characters}

Inflorescence length (cm): medium $(2,98 \pm 0,51)$

Number of flowers: low $(16,43 \pm 1,87)$

\section{Fruit characters}

Fresh weight of 100 fruits $(\mathrm{g})$ : medium $(2,42 \pm 0,02)$

Shape (length/width): ovoid

Symmetry: symmetric

Position of maximum transverse diameter:

central

Apex: rounded

Base: truncate

Niplle: tenuous

Lenticels: many and small

\section{Pit characters}

Weight of 100 pits (g): low $(0,27 \pm 0,01)$

Shape (length/width): elliptic

Mucron: obvious

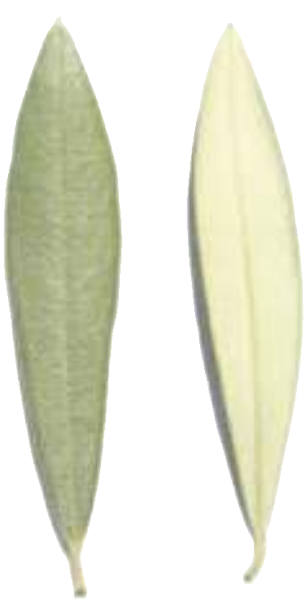

Symmetry: slightly asymmetric

Position of maximum transverse diameter:

towards apex

Apex: rounded

Base: pointed

Surface: smooth

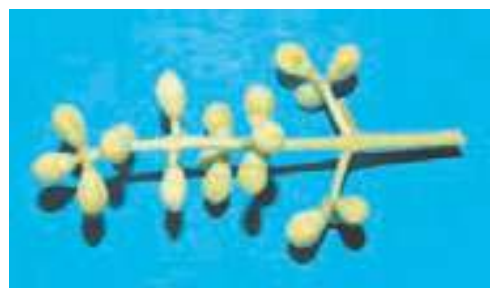

Number of grooves: medium 


\section{Biochemical Characters}

\section{Fatty Acid Composition}

Table 1. Average values (express in $\% \pm$ standard deviations) of the fatty acids methyl esters and nutritional ratios obtained from single cultivar olive oils.

$\begin{array}{llllll}\text { Myristic acid } & \mathbf{0 , 0 1} \pm \mathbf{0 , 0 1} & \text { Linoleic acid }(\omega 6) & \mathbf{1 0 , 2 7} \pm \mathbf{0 , 2 5} & \text { Lignoceric acid } & \mathbf{0 , 0 5} \pm \mathbf{0 , 0 4} \\ \text { Palmitic acid } & \mathbf{1 3 , 8 0 \pm 0 , 1 5} & \text { Linolenic acid }(\omega 3) & \mathbf{0 , 7 7} \pm \mathbf{0 , 0 3} & & \\ \text { Palmitoleic acid } & \mathbf{1 , 2 6} \pm \mathbf{0 , 1 9} & \text { Arachic acid } & \mathbf{0 , 3 7} \pm \mathbf{0 , 0 8} & \text { Unsat./satured } & \mathbf{4 , 8 9} \pm \mathbf{0 , 1 7} \\ \text { Stearic acid } & \mathbf{2 , 6 9} \pm \mathbf{0 , 4 7} & \text { Eicosenoic acid } & \mathbf{0 , 0 9} \pm \mathbf{0 , 1 7} & \omega 6 / \omega 3 & \mathbf{1 3 , 2 6} \pm \mathbf{0 , 2 3} \\ \text { Oleic acid } & \mathbf{6 9 , 4 7 \pm 0 , 7 9} & \text { Behenic acid } & \mathbf{0 , 0 9} \pm \mathbf{0 , 0 3} & & \end{array}$

\section{Organoleptic oil values}

Sensory Analysis (Panel test)

Comment: fruity medium-high, with hints of grass and almond, read sensations of artichoke and tomato. Balanced taste sensation with a medium-high bitter and spicy. Medium-high fluidity.

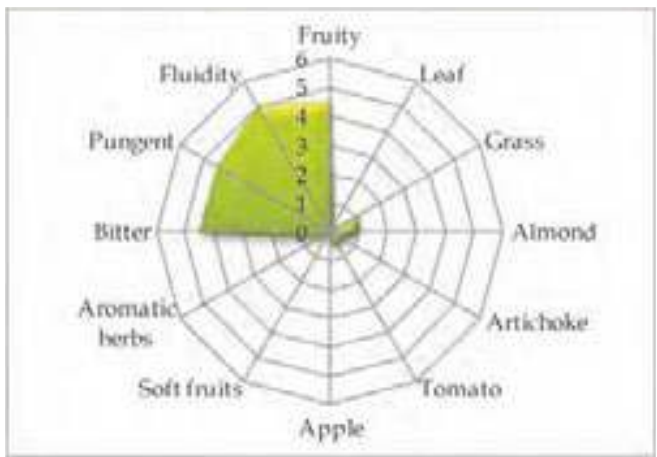

\section{Molecular Markers}

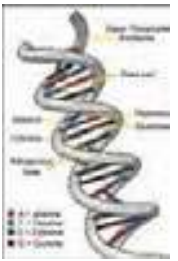

Table 2. Microsatellites (SSR) loci analyzed.

For each locus the allele size (expressed in base pairs) is reported.

$\begin{array}{cccccc}D C A 09 & \text { DCA18 } & \text { GAPU59 } & \text { GAPU71A } & \text { GUPA71B } & \text { GAPU103A } \\ \mathbf{1 7 2} \mathbf{- 1 9 8} & \mathbf{1 7 9 - \mathbf { 1 8 5 }} & \mathbf{2 1 4 - \mathbf { 2 2 2 }} & \mathbf{2 1 4} \mathbf{- 2 1 4} & \mathbf{1 2 4} \mathbf{- 1 2 6} & \mathbf{1 5 0 - \mathbf { 1 5 7 }} \\ \text { UDOO1 } & \text { UDOO3 } & \text { UDO12 } & \text { UDO28 } & \text { UDO39 } & \\ \mathbf{1 4 0 - \mathbf { 1 4 0 }} & \mathbf{1 4 3 - \mathbf { 1 4 3 }} & \mathbf{1 6 4 - \mathbf { 1 9 3 }} & \mathbf{1 6 1 - \mathbf { 1 6 1 }} & \mathbf{2 1 3 - \mathbf { 2 3 2 }} & \end{array}$

\section{References:}

1 - Pugliano G., Flaminio G., Pugliano M.L., et al. In: La risorsa genetica dell'olivo in Campania, SE. S.I.R.C.A. Ed. Napoli, (2000).

2 - Muzzalupo I., Stefanizzi F., Perri E. HortScience (2009), 44: pp. 582-588. 


\section{“Ogliarola barese"}

(synonymy: Cima di Bitonto, Baresana, Marinese, Nostrana, Paesana, etc.)

Areal distribution or origin area: Puglia

Flesh/pit weight ratio: low $(4,40 \pm 0,30)$

Oil content (\%): medium $(42,69 \pm 1,85)$

Purpose: oil

\section{Morphological characters}

Tree characters

Vigour: medium-strong

Growth habit: spreading-erect

Canopy-density: medium-dense

\section{Leaf characters}

Blade length $(\mathrm{cm})$ : medium $(5,55 \pm 0,70)$

Blade width $(\mathrm{cm})$ : medium $(1,46 \pm 0,18)$

Shape (length/width): elliptic

\section{Inflorescence characters}

Inflorescence length (cm): long $(3,74 \pm 0,66)$

Number of flowers: medium $(19,89 \pm 2,52)$

\section{Fruit characters}

Fresh weight of 100 fruits $(\mathrm{g})$ : medium $(2,54 \pm 0,45)$

Shape (length/width): ovoid

Symmetry: slightly asymmetric

Position of maximum transverse diameter:

central

Apex: rounded

Base: rounded

Niplle: tenuous

Lenticels: many and large

\section{Pit characters}

Weight of 100 pits (g): high $(0,49 \pm 0,06)$

Shape (length/width): elliptic

Mucron: obvious

Symmetry: slightly asymmetric

Position of maximum transverse diameter:

towards apex

Apex: rounded

Base: pointed

Surface: smooth

Number of grooves: medium
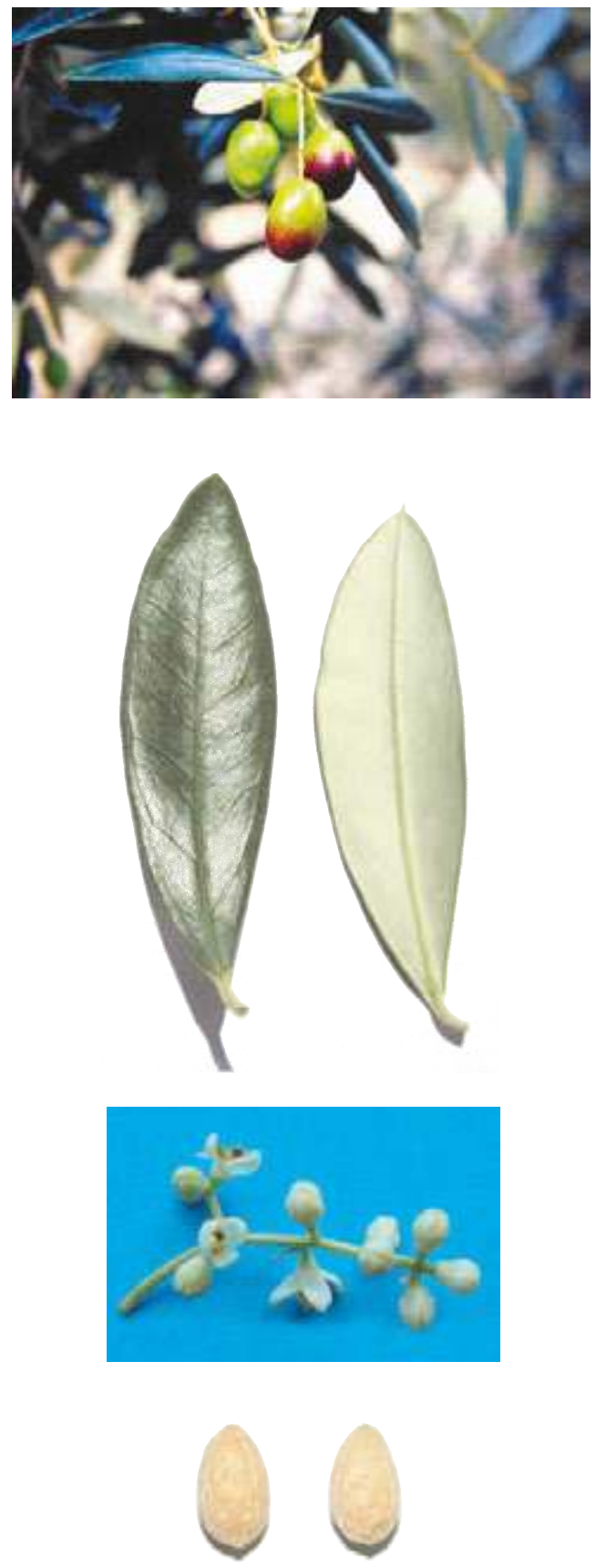


\section{Biochemical Characters}

\section{Fatty Acid Composition}

Table 1. Average values (express in $\% \pm$ standard deviations) of the fatty acids methyl esters and nutritional ratios obtained from single cultivar olive oils.

$\begin{array}{llllll}\text { Myristic acid } & \mathbf{0 , 0 1} \pm \mathbf{0 , 0 0} & \text { Linoleic acid }(\omega 6) & \mathbf{1 0 , 2 5} \pm \mathbf{2 , 5 2} & \text { Lignoceric acid } & \mathbf{0 , 0 5} \pm \mathbf{0 , 0 3} \\ \text { Palmitic acid } & \mathbf{1 2 , 1 9 \pm \mathbf { 1 } 4 5} & \text { Linolenic acid }(\omega 3) \mathbf{0 , 9 0} \mathbf{0 , 1 1} & & \\ \text { Palmitoleic acid } & \mathbf{0 , 9 5 \pm 0 , 4 4} & \text { Arachic acid } & \mathbf{0 , 3 1} \pm \mathbf{0 , 0 7} & \text { Unsat./satured } & \mathbf{5 , 9 1} \pm \mathbf{0 , 6 8} \\ \text { Stearic acid } & \mathbf{1 , 9 9} \pm \mathbf{0 , 2 7} & \text { Eicosenoic acid } & \mathbf{0 , 1 2} \pm \mathbf{0 , 0 9} & \omega 6 / \omega 3 & \mathbf{1 1 , 3 8} \pm \mathbf{1 , 8 5} \\ \text { Oleic acid } & \mathbf{7 7 , 3 2} \pm \mathbf{2 , 8 7} & \text { Behenic acid } & \mathbf{0 , 0 9} \pm \mathbf{0 , 0 4} & & \end{array}$

\section{Organoleptic oil values}

Sensory Analysis (Panel test)

Comment: fruity medium-high, with hints of almond and tomato, read sensations of grass and artichoke. Balanced taste sensation with a medium-high bitter and spicy. Mediumhigh fluidity.

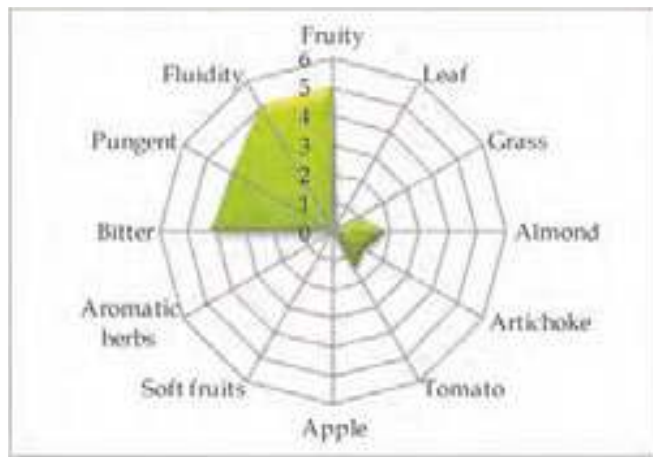

\section{Molecular Markers}

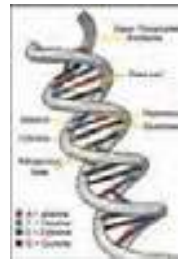

Table 2. Microsatellites (SSR) loci analyzed.

For each locus the allele size (expressed in base pairs) is reported.

$\begin{array}{cccccc}D C A 09 & \text { DCA } 18 & \text { GAPU59 } & \text { GAPU71A } & \text { GUPA71B } & \text { GAPU103A } \\ \mathbf{1 7 2} \mathbf{- 1 9 4} & \mathbf{1 7 9 - \mathbf { 1 8 1 }} & \mathbf{2 0 8 - \mathbf { 2 1 2 }} & \mathbf{2 1 4 - \mathbf { 2 2 4 }} & \mathbf{1 2 4 - \mathbf { 1 4 4 }} & \mathbf{1 5 9} \mathbf{- 1 7 0} \\ \text { UDO01 } & \text { UDO03 } & \text { UDO12 } & \text { UDO28 } & \text { UDO39 } & \\ \mathbf{1 4 4} \mathbf{- 1 4 4} & \mathbf{1 4 3} \mathbf{- 1 4 3} & \mathbf{1 7 7} \mathbf{- 1 8 2} & \mathbf{1 8 2} \mathbf{- 2 0 5} & \mathbf{2 0 5 - \mathbf { 2 0 5 }} & \end{array}$

\section{References:}

1 - Lombardo N., Perri E., Muzzalupo I., et al. In: Contributo alla caratterizzazione del germoplasma olivicolo pugliese, Ist. Sper. Olivic. (2004), pp. 65-68.

2 - Muzzalupo I., Stefanizzi F., Perri E. HortScience (2009), 44: pp. 582-588. 


\section{"Ogliarola del Vulture"}

(synonymy: Nostrale, Ogliarola di Melfi, Rapolese, Ripolese, etc.)

Areal distribution or origin area: Basilicata

Flesh/pit weight ratio: medium $(6,22 \pm 0,59)$

Oil content (\%): medium $(44,23 \pm 2,07)$

Purpose: dual purpose

\section{Morphological characters}

Tree characters

Vigour: medium-strong

Growth habit: spreading

Canopy-density: medium-dense

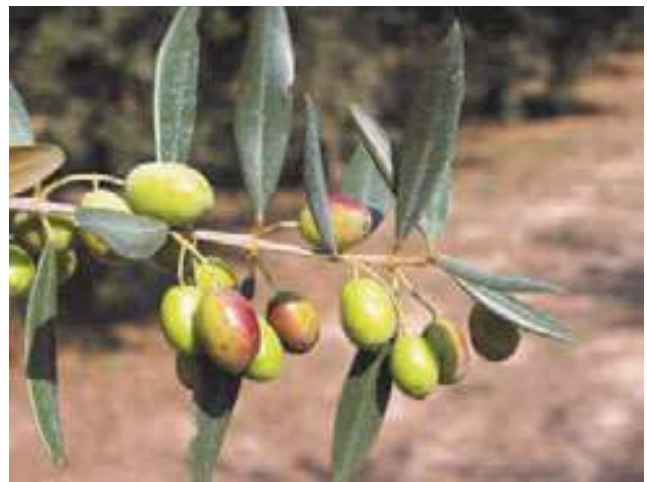

\section{Leaf characters}

Blade length $(\mathrm{cm})$ : medium $(6,14 \pm 0,57)$

Blade width (cm): broad $(\mathbf{1}, 53 \pm 0,24)$

Shape (length/width): elliptic-lanceolate

\section{Inflorescence characters}

Inflorescence length $(\mathrm{cm})$ : medium $(3,29 \pm 0,30)$

Number of flowers: low $(16,19 \pm 2,67)$

\section{Fruit characters}

Fresh weight of 100 fruits $(\mathrm{g})$ : low $(\mathbf{1}, \mathbf{9 4} \pm \mathbf{0 , 1 5})$

Shape (length/width): ovoid

Symmetry: asymmetric

Position of maximum transverse diameter:

central

Apex: rounded

Base: truncate

Niplle: obvious

Lenticels: many and small

\section{Pit characters}

Weight of 100 pits $(\mathrm{g})$ : low $(0,27 \pm \mathbf{0 , 0 3})$

Shape (length/width): elliptic

Mucron: obvious

Symmetry: slightly asymmetric

Position of maximum transverse diameter:

\section{towards apex}

Apex: rounded

Base: rounded

Surface: rugose

Number of grooves: medium
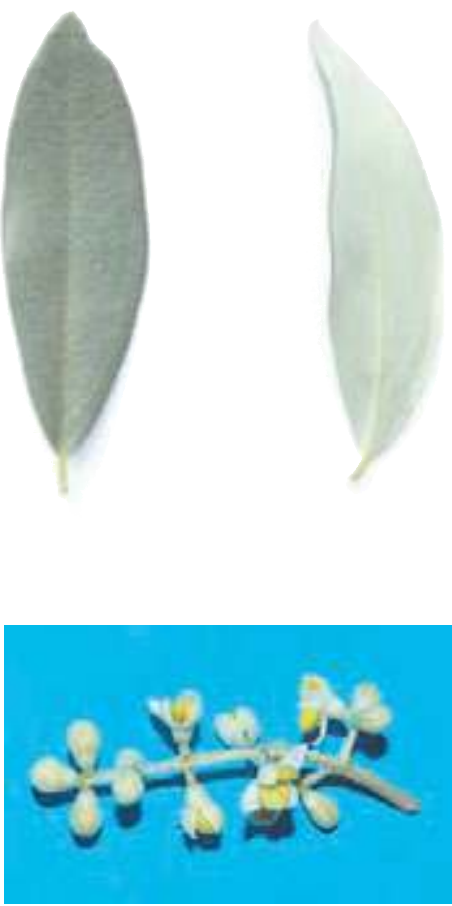


\section{Biochemical Characters}

\section{Fatty Acid Composition}

Table 1. Average values (express in $\% \pm$ standard deviations) of the fatty acids methyl esters and nutritional ratios obtained from single cultivar olive oils.

$\begin{array}{llllll}\text { Myristic acid } & \mathbf{0 , 0 1} \pm \mathbf{0 , 0 1} & \text { Linoleic acid }(\omega 6) & \mathbf{9 , 7 9} \pm \mathbf{0 , 6 3} & \text { Lignoceric acid } & \mathbf{0 , 0 6} \pm \mathbf{0 , 0 4} \\ \text { Palmitic acid } & \mathbf{1 3 , 6 2} \pm \mathbf{0 , 2 5} & \text { Linolenic acid }(\omega 3) \mathbf{0 , 8 4} \pm \mathbf{0 , 1 1} & & \\ \text { Palmitoleic acid } & \mathbf{1 , 0 9} \pm \mathbf{0 , 0 4} & \text { Arachic acid } & \mathbf{0 , 4 7} \pm \mathbf{0 , 0 8} & \text { Unsat./satured } & \mathbf{4 , 7 7} \pm \mathbf{0 , 0 6} \\ \text { Stearic acid } & \mathbf{3 , 1 5} \pm \mathbf{0 , 2 2} & \text { Eicosenoic acid } & \mathbf{0 , 1 5} \pm \mathbf{0 , 1 6} & \omega 6 / \omega 3 & \mathbf{1 1 , 8 2} \pm \mathbf{1 , 1 1} \\ \text { Oleic acid } & \mathbf{6 9 , 6 8} \pm \mathbf{0 , 7 7} & \text { Behenic acid } & \mathbf{0 , 1 3} \pm \mathbf{0 , 0 5} & & \end{array}$

\section{Organoleptic oil values}

Sensory Analysis (Panel test)

Comment: fruity medium-high, with hints of almond, grass, and artichoke, read sensations of leaf. Balanced taste sensation with a medium-high bitter and spicy. Medium-high fluidity.

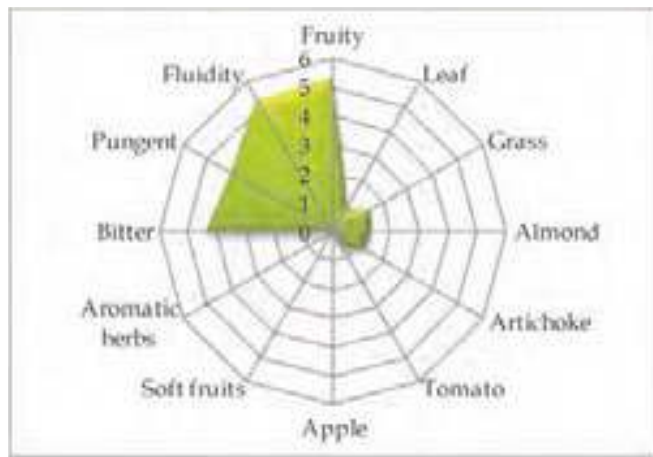

\section{Molecular Markers}

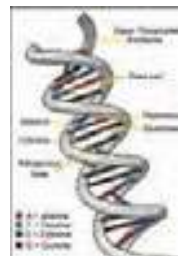

Table 2. Microsatellites (SSR) loci analyzed. For each locus the allele size (expressed in base pairs) is reported.

$\begin{array}{cccccc}D C A 09 & \text { DCA18 } & \text { GAPU59 } & \text { GAPU71A } & \text { GUPA71B } & \text { GAPU103A } \\ \mathbf{1 7 2} \mathbf{- 1 8 2} & \mathbf{1 7 9 - \mathbf { 1 8 1 }} & \mathbf{2 1 2} \mathbf{- 2 1 8} & \mathbf{2 1 4} \mathbf{- 2 1 4} & \mathbf{1 2 4 - \mathbf { 1 2 4 }} & \mathbf{1 5 0 - \mathbf { 1 8 4 }} \\ \text { UDOO1 } & \text { UDOO3 } & \text { UDO12 } & \text { UDO28 } & \text { UDO39 } & \\ \mathbf{1 4 4 - \mathbf { 1 5 0 }} & \mathbf{1 4 3 - \mathbf { 1 4 3 }} & \mathbf{1 6 6 - \mathbf { 1 9 3 }} & \mathbf{1 8 2} \mathbf{- 1 8 2} & \mathbf{2 1 3 - \mathbf { 2 1 3 }} & \end{array}$

\section{References:}

1 - Rotundo A., Marone E. In: Il germoplasma olivicolo lucano, Olita - Potenza (2002), pp. 115-118.

2 - Rotundo A., Perri E.,Muzzalupo I., et al. In: Il germoplasma olivicolo meridionale, (2012 in press).

3 - Muzzalupo I., Stefanizzi F., Perri E. HortScience (2009), 44: pp. 582-588. 


\section{"Ogliarola messinese"}

(synonymy: Aliva grossa, Castriciana, Marcellese, Nostrale, Ogliara, etc.)

Areal distribution or origin area: Sicilia

Flesh/pit weight ratio: high $(\mathbf{9 , 7 5} \pm \mathbf{1 , 4 8})$

Oil content (\%): high $(\mathbf{5 3 , 2 2} \pm \mathbf{0 , 6 0})$

Purpose: oil

\section{Morphological characters}

Tree characters

Vigour: medium-strong

Growth habit: spreading-erect

Canopy-density: medium-dense

\section{Leaf characters}

Blade length $(\mathrm{cm})$ : medium $(6,32 \pm 0,57)$

Blade width $(\mathrm{cm})$ : medium $(1,43 \pm 0,16)$

Shape (length/width): elliptic - lanceolate

\section{Inflorescence characters}

Inflorescence length $(\mathrm{cm})$ : short $(2,07 \pm 0,52)$

Number of flowers: low $(\mathbf{1 4 , 5 7} \pm \mathbf{2 , 1 6})$

\section{Fruit characters}

Fresh weight of 100 fruits (g): high $(5,56 \pm 0,62)$

Shape (length/width): ovoid

Symmetry: slightly asymmetric

Position of maximum transverse diameter:

central

Apex: rounded

Base: truncate

Niplle: obvoius

Lenticels: many and small

\section{Pit characters}

Weight of 100 pits (g): high $(\mathbf{0 , 6 0} \pm \mathbf{0 , 1 8})$

Shape (length/width): elliptic

Mucron: obvious

Symmetry: symmetric

Position of maximum transverse diameter:

central

Apex: pointed

Base: pointed

Surface: rugose

Number of grooves: medium
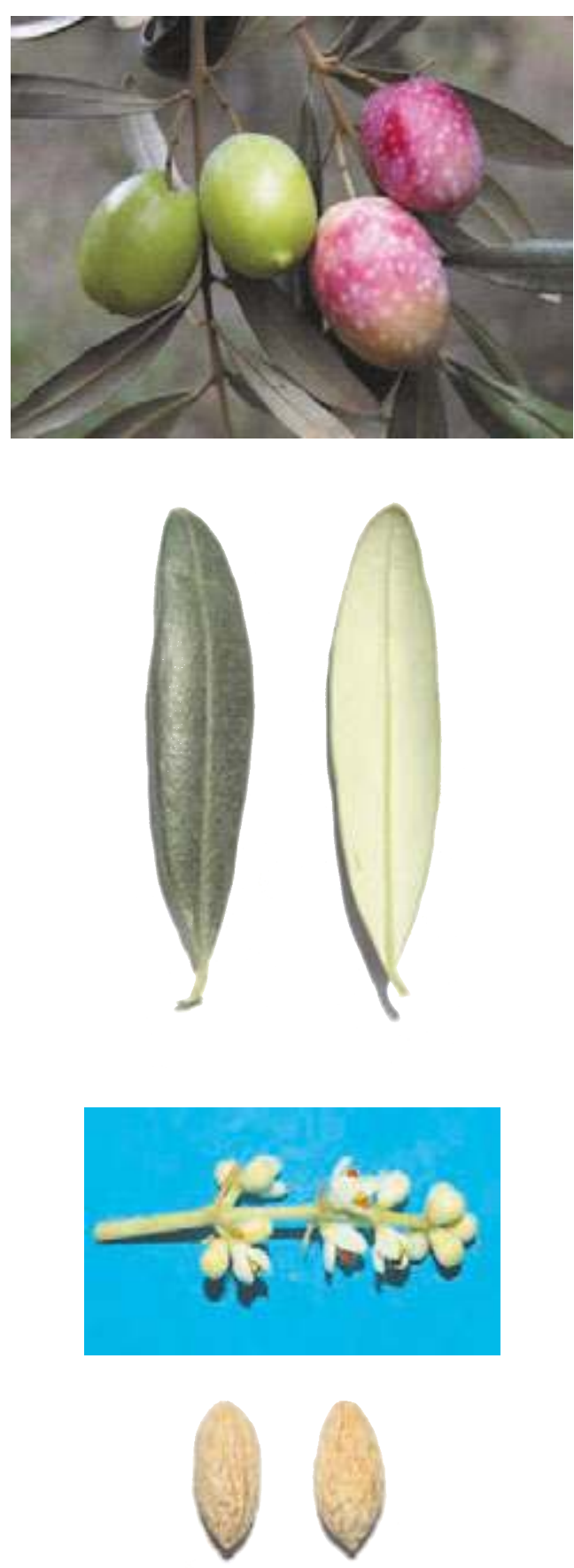


\section{Biochemical Characters}

\section{Fatty Acid Composition}

Table 1. Average values (express in $\% \pm$ standard deviations) of the fatty acids methyl esters and nutritional ratios obtained from single cultivar olive oils.

$\begin{array}{llllll}\text { Myristic acid } & \mathbf{0 , 0 1} \pm \mathbf{0 , 0 1} & \text { Linoleic acid }(\omega 6) & \mathbf{4 , 0 8} \pm \mathbf{0 , 4 4} & \text { Lignoceric acid } & \mathbf{0 , 0 9} \pm \mathbf{0 , 0 7} \\ \text { Palmitic acid } & \mathbf{1 0 , 0 0 \pm \mathbf { 1 , 1 3 }} & \text { Linolenic acid }(\omega 3) \mathbf{0 , 7 2} \pm \mathbf{0 , 3 0} & & \\ \text { Palmitoleic acid } & \mathbf{0 , 5 5} \pm \mathbf{0 , 0 4} & \text { Arachic acid } & \mathbf{0 , 4 5} \pm \mathbf{0 , 0 4} & \text { Unsat./satured } & \mathbf{6 , 7 6} \pm \mathbf{0 , 6 2} \\ \text { Stearic acid } & \mathbf{2 , 4 1} \pm \mathbf{0 , 1 7} & \text { Eicosenoic acid } & \mathbf{0 , 1 9} \pm \mathbf{0 , 2 5} & \omega 6 / \omega 3 & \mathbf{6 , 3 8} \pm \mathbf{3 , 2 6} \\ \text { Oleic acid } & \mathbf{8 0 , 2 3} \pm \mathbf{1 , 0 9} & \text { Behenic acid } & \mathbf{0 , 1 1} \pm \mathbf{0 , 0 2} & & \end{array}$

\section{Organoleptic oil values}

Sensory Analysis (Panel test)

Comment: fruity medium, with taste of artichoke and tomato, read sensations of almond. Balanced taste sensation with medium-light bitter and spicy. Medium fluidity.

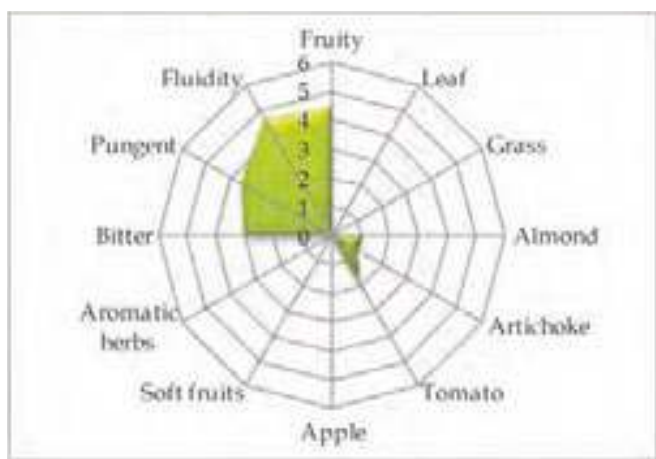

\section{Molecular Markers}

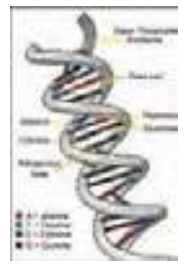

Table 2. Microsatellites (SSR) loci analyzed.

For each locus the allele size (expressed in base pairs) is reported.

$\begin{array}{cccccc}D C A 09 & D C A 18 & \text { GAPU59 } & \text { GAPU71A } & \text { GUPA71B } & \text { GAPU103A } \\ \mathbf{1 6 2}-\mathbf{1 6 2} & \mathbf{1 6 9 - \mathbf { 1 8 1 }} & \mathbf{2 0 8 - \mathbf { 2 1 2 }} & \mathbf{2 1 4} \mathbf{- 2 1 4} & \mathbf{1 2 4} \mathbf{- 1 4 4} & \mathbf{1 5 9} \mathbf{- 1 8 4} \\ \text { UDOO1 } & \text { UDO03 } & \text { UDO12 } & \text { UDO28 } & \text { UDO39 } & \\ \mathbf{1 4 4 - \mathbf { 1 4 4 }} & \mathbf{1 6 6 - \mathbf { 1 8 2 }} & \mathbf{1 6 6 - \mathbf { 1 9 3 }} & \mathbf{1 8 2} \mathbf{- 1 8 2} & \mathbf{1 0 8 - \mathbf { 2 0 5 }} & \end{array}$

\section{References:}

1 - Caruso T., Cartabellotta D., Motisi A., et al. In: Cultivar di olivo siciliane, Università degli Studi di Palermo (2007), pp. 60-64.

2 - Muzzalupo I., Stefanizzi F., Perri E. HortScience (2009), 44: pp.582-588. 


\section{"Ogliastro grande"}

(synonymy: Ogliastro di Torre Orsaia)

Areal distribution or origin area: Campania Flesh/pit weight ratio: high $(\mathbf{9}, 29 \pm \mathbf{1}, \mathbf{7 4})$ Oil content (\%): medium $(46,83 \pm 1,22)$

Purpose: oil

\section{Morphological characters}

Tree characters

Vigour: medium

Growth habit: erect

Canopy-density: medium-dense

\section{Leaf characters}

Blade length $(\mathrm{cm})$ : medium $(6,56 \pm 0,54)$

Blade width (cm): medium $(\mathbf{1 , 1 4} \pm 0,14)$

Shape (length/width): elliptic-lanceolate

\section{Inflorescence characters}

Inflorescence length $(\mathrm{cm})$ : medium $(2,80 \pm 0,75)$

Number of flowers: low $(16,43 \pm 1,87)$

\section{Fruit characters}

Fresh weight of 100 fruits $(\mathrm{g})$ : high $(\mathbf{4 , 1 5} \pm \mathbf{0 , 3 3})$

Shape (length/width): ovoid

Symmetry: slightly asymmetric

Position of maximum transverse diameter: central

Apex: rounded

Base: rounded

Niplle: absent

Lenticels: many and small

\section{Pit characters}

Weight of 100 pits (g): medium $(\mathbf{0 , 4 1} \pm \mathbf{0 , 0 4})$

Shape (length/width): elongated

Mucron: obvious

Symmetry: slightly asymmetric

Position of maximum transverse diameter: towards apex

Apex: rounded

Base: pointed

Surface: smooth

Number of grooves: low
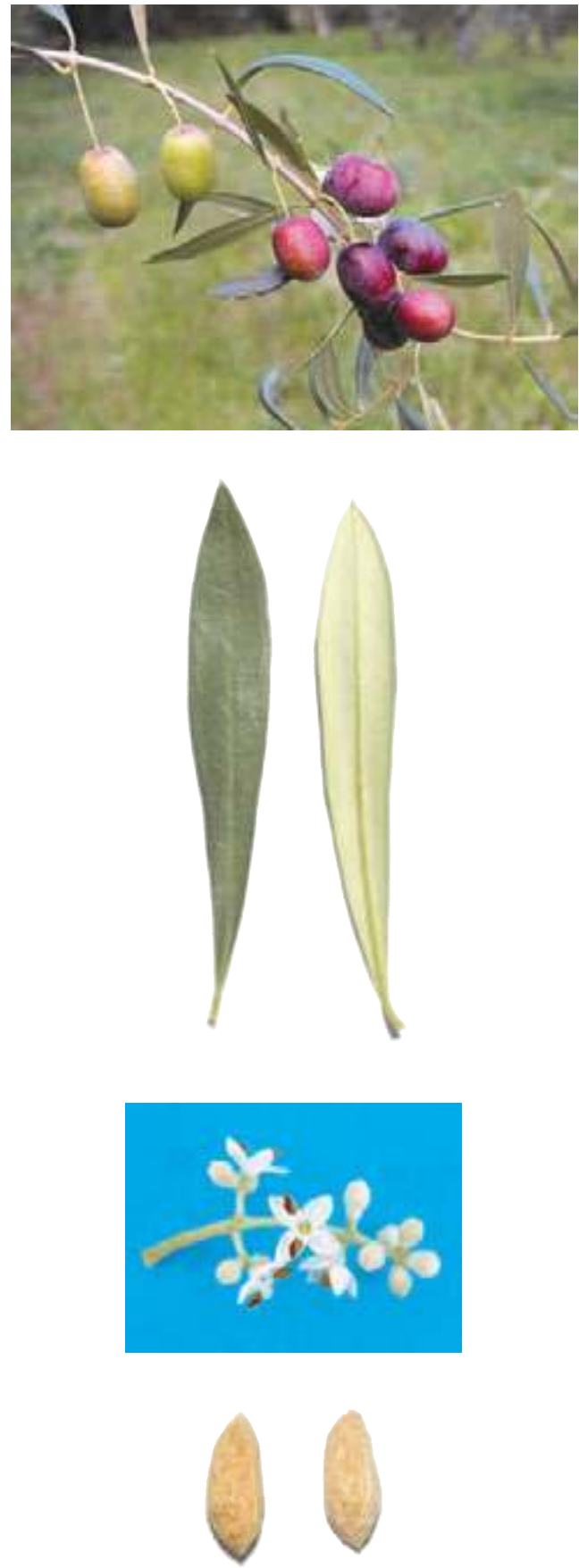


\section{Biochemical Characters}

\section{Fatty Acid Composition}

Table 1. Average values (express in $\% \pm$ standard deviations) of the fatty acids methyl esters and nutritional ratios obtained from single cultivar olive oils.

$\begin{array}{llllll}\text { Myristic acid } & \mathbf{0 , 0 0 \pm 0 , 0 0} & \text { Linoleic acid }(\omega 6) & \mathbf{6 , 1 0} \pm \mathbf{0 , 1 4} & \text { Lignoceric acid } & \mathbf{0 , 0 5} \pm \mathbf{0 , 0 2} \\ \text { Palmitic acid } & \mathbf{1 1 , 7 9 \pm 0 , 1 9} & \text { Linolenic acid }(\omega 3) & \mathbf{0 , 6 8} \pm \mathbf{0 , 0 1} & & \\ \text { Palmitoleic acid } & \mathbf{1 , 9 1} \pm \mathbf{0 , 3 5} & \text { Arachic acid } & \mathbf{0 , 3 1} \pm \mathbf{0 , 1 6} & \text { Unsat./satured } & \mathbf{6 , 1 1} \pm \mathbf{0 , 2 2} \\ \text { Stearic acid } & \mathbf{1 , 9 0 \pm 0 , 3 6} & \text { Eicosenoic acid } & \mathbf{0 , 0 2} \pm \mathbf{0 , 0 1} & \omega 6 / \omega 3 & \mathbf{8 , 9 5} \pm \mathbf{0 , 0 5} \\ \text { Oleic acid } & \mathbf{7 5 , 5 6 \pm 0 , 3 9} & \text { Behenic acid } & \mathbf{0 , 0 3} \pm \mathbf{0 , 0 2} & & \end{array}$

\section{Organoleptic oil values}

Sensory Analysis (Panel test)

Comment: fruity medium, with hints of almond and read sensations of leaves, artichoke and grass. Balanced taste sensation with a medium-high bitter and spicy. Medium-high fluidity.

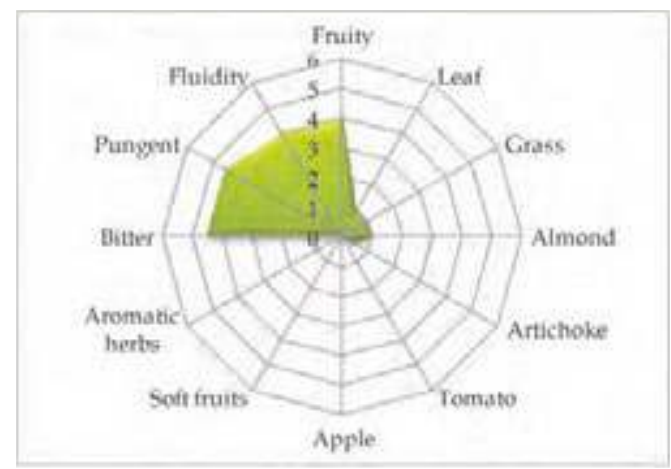

\section{Molecular Markers}

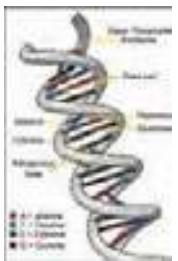

Table 2. Microsatellites (SSR) loci analyzed.

For each locus the allele size (expressed in base pairs) is reported.

$\begin{array}{cccccc}D C A 09 & \text { DCA18 } & \text { GAPU59 } & \text { GAPU71A } & \text { GUPA71B } & \text { GAPU103A } \\ \mathbf{1 8 2} \mathbf{- 2 0 6} & \mathbf{1 8 1} \mathbf{- 1 8 5} & \mathbf{2 1 4 - \mathbf { 2 1 8 }} & \mathbf{2 1 8 - \mathbf { 2 1 8 }} & \mathbf{1 2 6} \mathbf{- 1 2 6} & \mathbf{1 5 9 - \mathbf { 1 5 9 }} \\ \text { UDOO1 } & \text { UDOO3 } & \text { UDO12 } & \text { UDO28 } & \text { UDO39 } & \\ \mathbf{1 4 4 - \mathbf { 1 4 4 }} & \mathbf{1 5 0 - \mathbf { 1 5 0 }} & \mathbf{1 6 4 - \mathbf { 1 6 4 }} & \mathbf{1 4 3 - \mathbf { 1 4 3 }} & \mathbf{2 0 0 - \mathbf { 2 2 0 }} & \end{array}$

\section{References:}

1 - Pugliano G., Flaminio G., Pugliano M.L., et al. In: La risorsa genetica dell'olivo in Campania, SE. S.I.R.C.A. Ed. Napoli, (2000).

2 - Muzzalupo I., Stefanizzi F., Perri E. HortScience (2009), 44: pp. 582-588. 


\section{“Olivago"}

\section{(synonymy: Selvatico)}

Areal distribution or origin area: Umbria

Flesh/pit weight ratio: low $(2,51 \pm 0,06)$

Oil content (\%): medium $(46,27 \pm 0,08)$

Purpose: oil

\section{Morphological characters}

Tree characters

Vigour: medium-strong

Growth habit: spreading-erect

Canopy-density: medium

\section{Leaf characters}

Blade length (cm): medium $(5,47 \pm 0,42)$

Blade width $(\mathrm{cm})$ : medium $(\mathbf{1}, 25 \pm 0,12)$

Shape (length/width): elliptic-lanceolate

\section{Inflorescence characters}

Inflorescence length $(\mathrm{cm})$ : medium $(2,50 \pm 1,06)$

Number of flowers: low $(\mathbf{1 7}, \mathbf{8 4} \pm \mathbf{1}, \mathbf{0 7})$

\section{Fruit characters}

Fresh weight of 100 fruits $(\mathrm{g})$ : low $(0,93 \pm \mathbf{0 , 1 2})$

Shape (length/width): elongated

Symmetry: symmetric

Position of maximum transverse diameter:

towards apex

Apex: rounded

Base: rounded

Niplle: tenuous

Lenticels: many and small

\section{Pit characters}

Weight of 100 pits (g): low $(0,27 \pm 0,03)$

Shape (length/width): elliptic

Mucron: obvious

Symmetry: symmetric

Position of maximum transverse diameter:

towards apex

Apex: rounded

Base: rounded

Surface: rugose

Number of grooves: medium
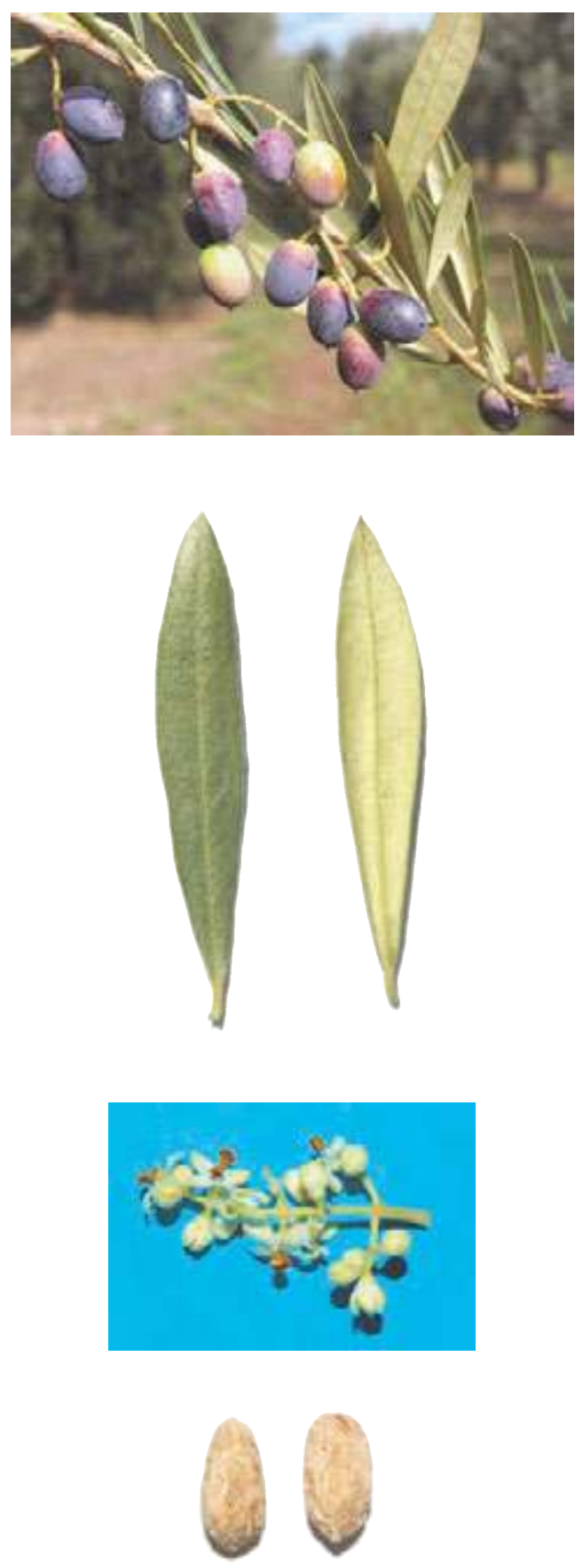


\section{Biochemical Characters}

\section{Fatty Acid Composition}

Table 1. Average values (express in $\% \pm$ standard deviations) of the fatty acids methyl esters and nutritional ratios obtained from single cultivar olive oils.

$\begin{array}{llllll}\text { Myristic acid } & \mathbf{0 , 0 1} \pm \mathbf{0 , 0 1} & \text { Linoleic acid }(\omega 6) & \mathbf{1 0 , 3 5} \pm \mathbf{0 , 8 7} & \text { Lignoceric acid } & \mathbf{0 , 0 9} \pm \mathbf{0 , 0 9} \\ \text { Palmitic acid } & \mathbf{1 4 , 5 5} \pm \mathbf{0 , 1 1} & \text { Linolenic acid }(\omega 3) & \mathbf{0 , 7 8} \pm \mathbf{0 , 0 4} & & \\ \text { Palmitoleic acid } & \mathbf{3 , 0 5} \pm \mathbf{0 , 0 7} & \text { Arachic acid } & \mathbf{0 , 2 5} \pm \mathbf{0 , 0 8} & \text { Unsat./satured } & \mathbf{4 , 9 2} \pm \mathbf{0 , 3 1} \\ \text { Stearic acid } & \mathbf{1 , 6 1} \pm \mathbf{0 , 1 8} & \text { Eicosenoic acid } & \mathbf{0 , 1 8} \pm \mathbf{0 , 2 1} & \omega 6 / \omega 3 & \mathbf{1 3 , 2 8} \pm \mathbf{0 , 4 6} \\ \text { Oleic acid } & \mathbf{6 6 , 1 8} \pm \mathbf{0 , 5 6} & \text { Behenic acid } & \mathbf{0 , 0 7} \pm \mathbf{0 , 0 4} & & \end{array}$

\section{Organoleptic oil values}

Sensory Analysis (Panel test)

Comment: fruity medium, with taste of almond and aromatic herbs, read sensations of artichoke and grass. Balanced taste sensation with medium-light bitter and spicy. Medium -high fluidity.

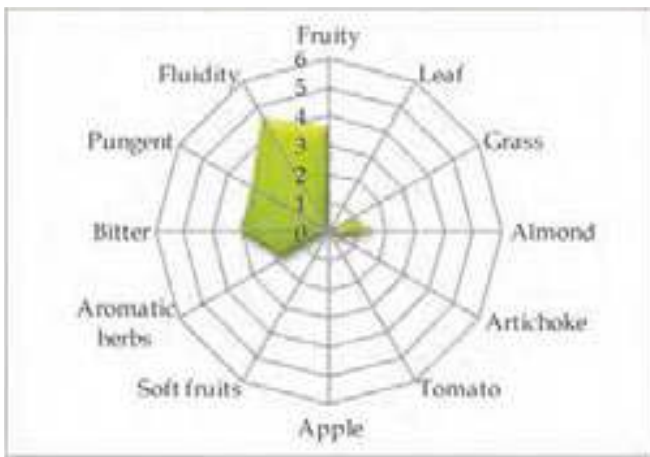

\section{Molecular Markers}

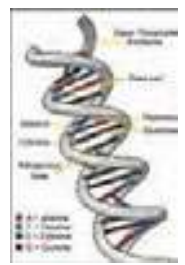

Table 2. Microsatellites (SSR) loci analyzed.

For each locus the allele size (expressed in base pairs) is reported.

$\begin{array}{cccccc}D C A 09 & \text { DCA18 } & \text { GAPU59 } & \text { GAPU71A } & \text { GUPA71B } & \text { GAPU103A } \\ \mathbf{1 7 2} \mathbf{- 1 9 8} & \mathbf{1 7 7 - \mathbf { 1 8 1 }} & \mathbf{2 1 2} \mathbf{- 2 1 2} & \mathbf{2 1 2} \mathbf{- 2 1 2} & \mathbf{1 2 6 - \mathbf { 1 4 4 }} & \mathbf{1 5 0 - \mathbf { 1 5 7 }} \\ \text { UDOO1 } & \text { UDO03 } & \text { UDO12 } & \text { UDO28 } & \text { UDO39 } & \\ \mathbf{1 4 4 - \mathbf { 1 4 4 }} & \mathbf{1 3 5 - \mathbf { 1 3 5 }} & \mathbf{1 6 6 - \mathbf { 1 6 6 }} & \mathbf{1 6 1 - \mathbf { 2 1 0 }} & \mathbf{1 8 5} \mathbf{- 1 8 5} & \end{array}$

\section{References:}

1 - Preziosi P., Tini M. In: Acta Horticulturae, (1990), 286 pp. 85-88.

2 - Various authors. In: Catalogo Nazionale delle Varietà di Olivo, University of Bari (in press), ISBN 978-88-8879397-9. 


\section{“Olivastra seggianese"}

(synonymy: Giogliaia, Olivastra di Montalcino, Olivastra di Seggiano, etc.)

Areal distribution or origin area: Toscana

Flesh/ pit weight ratio: high $(7,94 \pm 0,68)$

Oil content (\%): medium $(47,20 \pm 2,17)$

Purpose: oil

\section{Morphological characters}

Tree characters

Vigour: medium-weak

Growth habit: spreading

Canopy-density: medium

\section{Leaf characters}

Blade length $(\mathrm{cm})$ : medium $(5,97 \pm 0,49)$

Blade width $(\mathrm{cm})$ : medium $(\mathbf{1 , 2 7} \pm 0,12)$

Shape (length/width): elliptic-lanceolate

\section{Inflorescence characters}

Inflorescence length (cm): long $(3,71 \pm 0,67)$

Number of flowers: medium $(\mathbf{1 8 , 2 5} \pm \mathbf{1 , 5 8})$

\section{Fruit characters}

Fresh weight of 100 fruits (g): medium $(2,73 \pm 0,62)$

Shape (length/width): spherical

Symmetry: symmetric

Position of maximum transverse diameter:

central

Apex: rounded

Base: truncate

Niplle: absent

Lenticels: many and small

\section{Pit characters}

Weight of 100 pits (g): medium $(0,38 \pm 0,03$ )

Shape (length/width): spherical

Mucron: tenuous

Symmetry: symmetric

Position of maximum transverse diameter:

central

Apex: rounded

Base: rounded

Surface: rugose

Number of grooves: medium
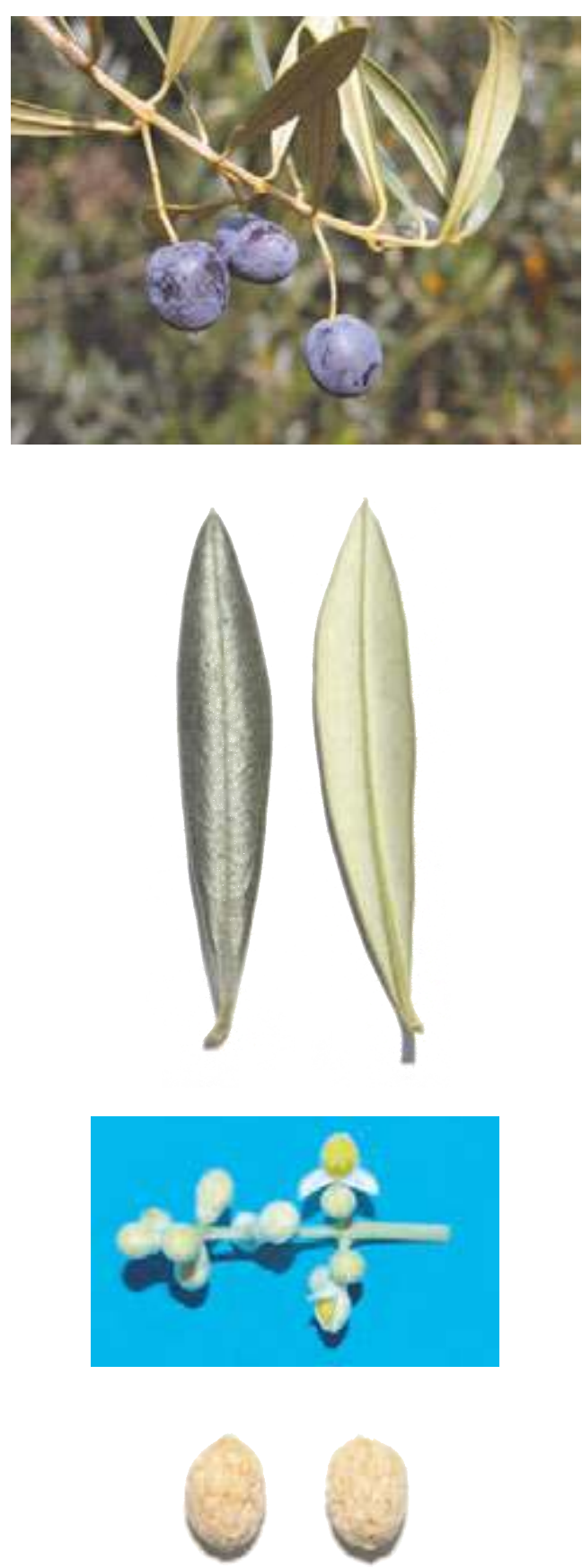


\section{Biochemical Characters}

\section{Fatty Acid Composition}

Table 1. Average values (express in $\% \pm$ standard deviations) of the fatty acids methyl esters and nutritional ratios obtained from single cultivar olive oils.

$\begin{array}{llllll}\text { Myristic acid } & \mathbf{0 , 0 1} \pm \mathbf{0 , 0 0} & \text { Linoleic acid }(\omega 6) & \mathbf{8 , 6 7} \pm \mathbf{0 , 4 1} & \text { Lignoceric acid } & \mathbf{0 , 0 2} \pm \mathbf{0 , 0 0} \\ \text { Palmitic acid } & \mathbf{1 3 , 8 2} \pm \mathbf{0 , 2 5} & \text { Linolenic acid }(\omega 3) & \mathbf{0 , 7 7} \pm \mathbf{0 , 0 2} & & \\ \text { Palmitoleic acid } & \mathbf{1 , 5 0 \pm 0 , 0 2} & \text { Arachic acid } & \mathbf{0 , 2 9} \pm \mathbf{0 , 0 3} & \text { Unsat./satured } & \mathbf{5 , 1 7} \pm \mathbf{0 , 1 2} \\ \text { Stearic acid } & \mathbf{2 , 0 0 \pm 0 , 0 3} & \text { Eicosenoic acid } & \mathbf{0 , 0 1} \pm \mathbf{0 0 , 0 0} & \omega 6 / \omega 3 & \mathbf{1 1 , 2 8} \pm \mathbf{0 , 3 0} \\ \text { Oleic acid } & \mathbf{7 1 , 7 1} \pm \mathbf{0 , 0 3} & \text { Behenic acid } & \mathbf{0 , 0 7} \pm \mathbf{0 , 0 1} & & \end{array}$

\section{Organoleptic oil values}

Sensory Analysis (Panel test)

Comment: fruity medium-high, with hints of almond and read sensations of grass and artichoke. Balanced taste sensation with a medium-high bitter and spicy. Medium-high fluidity.

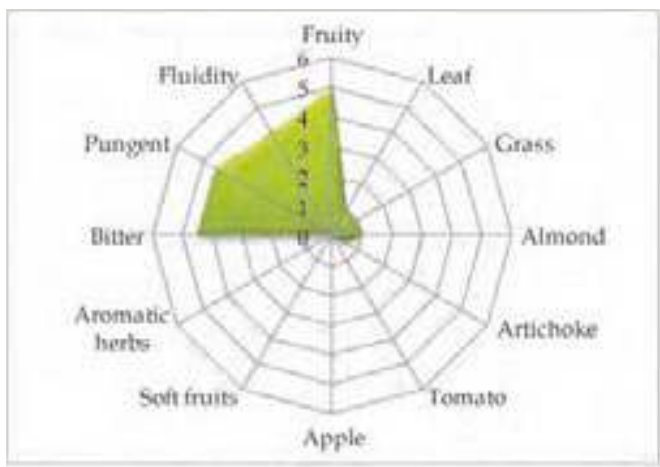

\section{Molecular Markers}

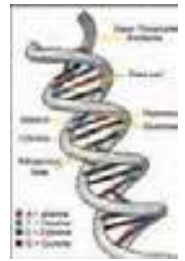

Table 2. Microsatellites (SSR) loci analyzed.

For each locus the allele size (expressed in base pairs) is reported.

$\begin{array}{cccccc}D C A 09 & \text { DCA } 18 & \text { GAPU59 } & \text { GAPU71A } & \text { GUPA71B } & \text { GAPU103A } \\ \mathbf{1 8 2} \mathbf{- 2 0 6} & \mathbf{1 7 9 - \mathbf { 1 8 5 }} & \mathbf{2 1 2} \mathbf{- 2 1 8} & \mathbf{2 1 0 - \mathbf { 2 1 2 }} & \mathbf{1 2 6 - \mathbf { 1 4 4 }} & \mathbf{1 5 0} \mathbf{- 1 5 9} \\ \text { UDO01 } & \text { UDO03 } & \text { UDO12 } & \text { UDO28 } & \text { UDO39 } & \\ \mathbf{1 5 0} \mathbf{- 1 5 0} & \mathbf{1 5 0} \mathbf{- 1 5 0} & \mathbf{1 5 6 - \mathbf { 1 6 4 }} & \mathbf{1 5 6 - \mathbf { 1 8 2 }} & \mathbf{2 0 5 - \mathbf { 2 0 5 }} & \end{array}$

\section{References:}

1 - Cimato A., Cantini C., Sani G., In: L'olivo in Toscana: il germoplasma autoctono, Ed. ARSIA (2001).

2 - Various authors. In: Catalogo Nazionale delle Varietà di Olivo, University of Bari (in press), ISBN 978-88-88793-

97-9. 


\section{"Olivastro di Bucchianico"}

(synonymy: Olivastro)

Areal distribution or origin area: Abruzzo

Flesh/pit weight ratio: medium $(7,39 \pm 0,12)$

Oil content (\%): high $(\mathbf{5 3}, \mathbf{1 1} \pm \mathbf{1 , 7 5})$

Purpose: oil

\section{Morphological characters}

Tree characters

Vigour: medium-weak

Growth habit: erect

Canopy-density: medium

\section{Leaf characters}

Blade length (cm): short $(4,37 \pm 0,43)$

Blade width $(\mathrm{cm})$ : medium $(\mathbf{1}, 23 \pm \mathbf{0 , 1 5})$

Shape (length/width): elliptic

\section{Inflorescence characters}

Inflorescence length (cm): long $(3,55 \pm 0,59)$

Number of flowers: medium $(18,63 \pm 0,34)$

\section{Fruit characters}

Fresh weight of 100 fruits (g): medium $(2,48 \pm 0,37)$

Shape (length/width): spherical

Symmetry: symmetric

Position of maximum transverse diameter:

central

Apex: rounded

Base: truncate

Niplle: absent

Lenticels: many and small

\section{Pit characters}

Weight of 100 pits (g): medium $(0,30 \pm 0,03)$

Shape (length/width): elliptic

Mucron: obvious

Symmetry: slightly asymmetric

Position of maximum transverse diameter:

towards apex

Apex: rounded

Base: rounded

Surface: smooth

Number of grooves: medium
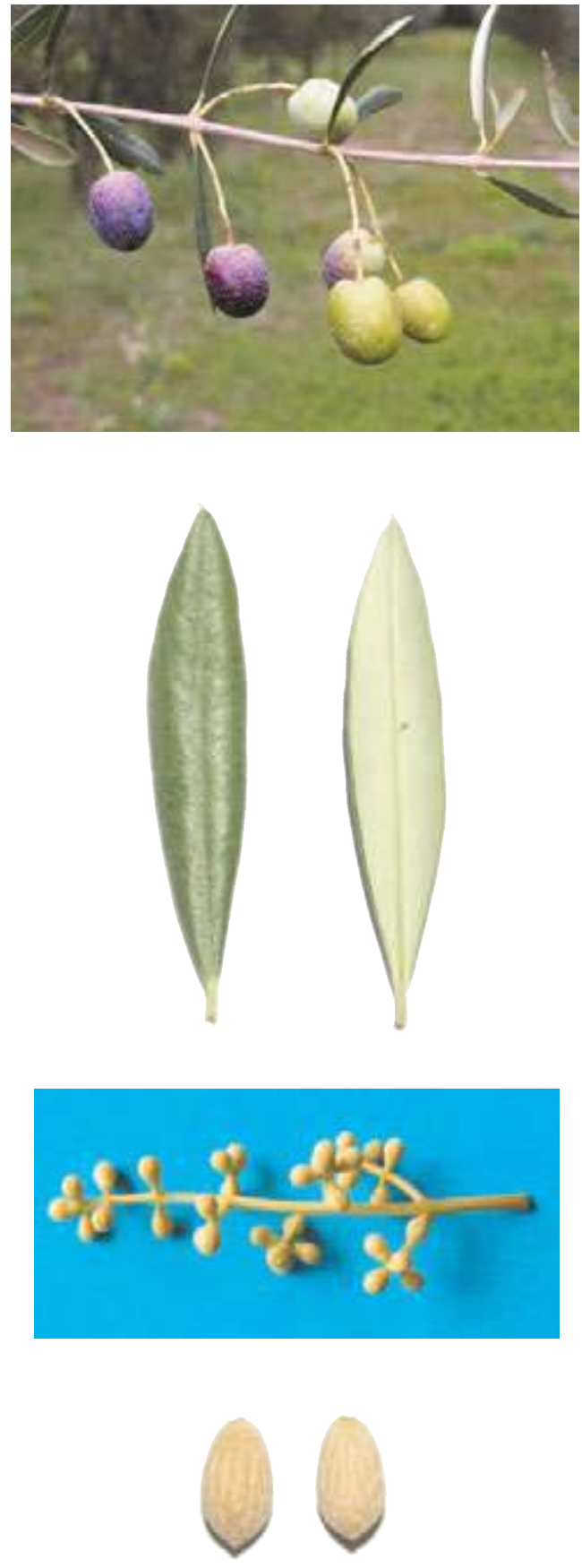


\section{Biochemical Characters}

\section{Fatty Acid Composition}

Table 1. Average values (express in $\% \pm$ standard deviations) of the fatty acids methyl esters and nutritional ratios obtained from single cultivar olive oils.

$\begin{array}{llllll}\text { Myristic acid } & \mathbf{0 , 0 1} \pm \mathbf{0 , 0 0} & \text { Linoleic acid }(\omega 6) & \mathbf{9 , 9 7} \pm \mathbf{0 , 2 0} & \text { Lignoceric acid } & \mathbf{0 , 1 0} \pm \mathbf{0 , 0 1} \\ \text { Palmitic acid } & \mathbf{1 1 , 5 0 \pm 0 , 2 0} & \text { Linolenic acid }(\omega 3) \mathbf{0 , 4 1} \pm \mathbf{0 , 0 2} & & \\ \text { Palmitoleic acid } & \mathbf{0 , 5 0 \pm 0 , 0 1} & \text { Arachic acid } & \mathbf{0 , 4 9} \pm \mathbf{0 , 1 3} & \text { Unsat./satured } & \mathbf{5 , 4 8} \pm \mathbf{0 , 0 7} \\ \text { Stearic acid } & \mathbf{3 , 3 9} \pm \mathbf{0 , 3 5} & \text { Eicosenoic acid } & \mathbf{0 , 2 5} \pm \mathbf{0 , 0 5} & \omega 6 / \omega 3 & \mathbf{2 4 , 1 4} \pm \mathbf{0 , 8 9} \\ \text { Oleic acid } & \mathbf{7 2 , 7 3 \pm 0 , 0 6} & \text { Behenic acid } & \mathbf{0 , 1 5} \pm \mathbf{0 , 0 1} & & \end{array}$

\section{Organoleptic oil values}

Sensory Analysis (Panel test)

Comment: fruity medium, with hints of grass and leaves, read sensations of almond and aromatic herbs. Balanced taste sensation with a medium bitter and medium-high spicy. Medium fluidity.

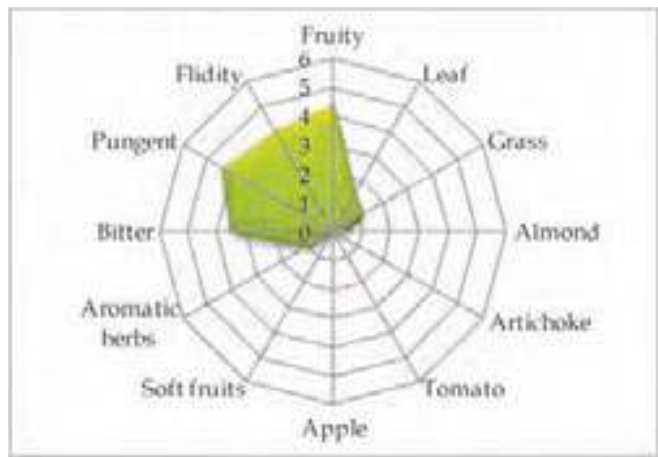

\section{Molecular Markers}

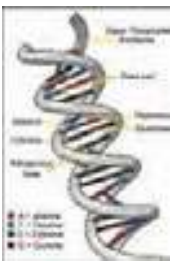

Table 2. Microsatellites (SSR) loci analyzed.

For each locus the allele size (expressed in base pairs) is reported.

$\begin{array}{cccccc}D C A 09 & \text { DCA18 } & \text { GAPU59 } & \text { GAPU71A } & \text { GUPA71B } & \text { GAPU103A } \\ \mathbf{1 9 8} \mathbf{- 2 1 0} & \mathbf{1 7 9 - \mathbf { 1 8 1 }} & \mathbf{2 0 8 - \mathbf { 2 2 2 }} & \mathbf{2 1 0 - \mathbf { 2 1 4 }} & \mathbf{1 2 4 - \mathbf { 1 2 4 }} & \mathbf{1 5 9 - \mathbf { 1 7 0 }} \\ \text { UDOO1 } & \text { UDO03 } & \text { UDO12 } & \text { UDO28 } & \text { UDO39 } & \\ \mathbf{1 4 0 - \mathbf { 1 4 0 }} & \mathbf{1 8 2} \mathbf{- 1 8 2} & \mathbf{1 7 7 - \mathbf { 1 9 3 }} & \mathbf{1 5 4 - \mathbf { 2 1 0 }} & \mathbf{2 1 3 - \mathbf { 2 1 3 }} & \end{array}$

\section{References:}

1 - Pietrangeli E., Russo A., In: Olivi D'Abruzzo, Grafiche di Prinzio (1997), pp. 50-51.

2 - Muzzalupo I., Salimonti A., Caravita M. A., et al. Adv. Hort. Sci. (2008), 22(2): pp. 129-135. 


\section{"Olivastro frentano"}

Areal distribution or origin area: Abruzzo

Flesh/pit weight ratio: low $(\mathbf{4 , 9 8} \pm \mathbf{0 , 1 2})$

Oil content (\%): medium $(42,40 \pm 1,56)$

Purpose: oil

\section{Morphological characters}

Tree characters

Vigour: medium-strong

Growth habit: erect

Canopy-density: medium

\section{Leaf characters}

Blade length $(\mathrm{cm})$ : medium $(5,22 \pm 0,37)$

Blade width $(\mathrm{cm})$ : medium $(\mathbf{1}, 45 \pm 0,16)$

Shape (length/width): elliptic

\section{Inflorescence characters}

Inflorescence length $(\mathrm{cm})$ : medium $(3,40 \pm 0,55)$

Number of flowers: medium $(\mathbf{1 8 , 4 8} \pm \mathbf{2 , 7 8})$

\section{Fruit characters}

Fresh weight of 100 fruits $(\mathrm{g})$ : low $(\mathbf{1}, \mathbf{6 7} \pm \mathbf{0 , 0 1})$

Shape (length/width): ovoid

Symmetry: symmetric

Position of maximum transverse diameter:

central

Apex: rounded

Base: truncate

Niplle: absent

Lenticels: few and small

\section{Pit characters}

Weight of 100 pits (g): medium $(0,31 \pm 0,03$ )

Shape (length/width): elliptic

Mucron: obvious

Symmetry: slightly asymmetric

Position of maximum transverse diameter:

towards apex

Apex: pointed

Base: rounded

Surface: smooth

Number of grooves: low
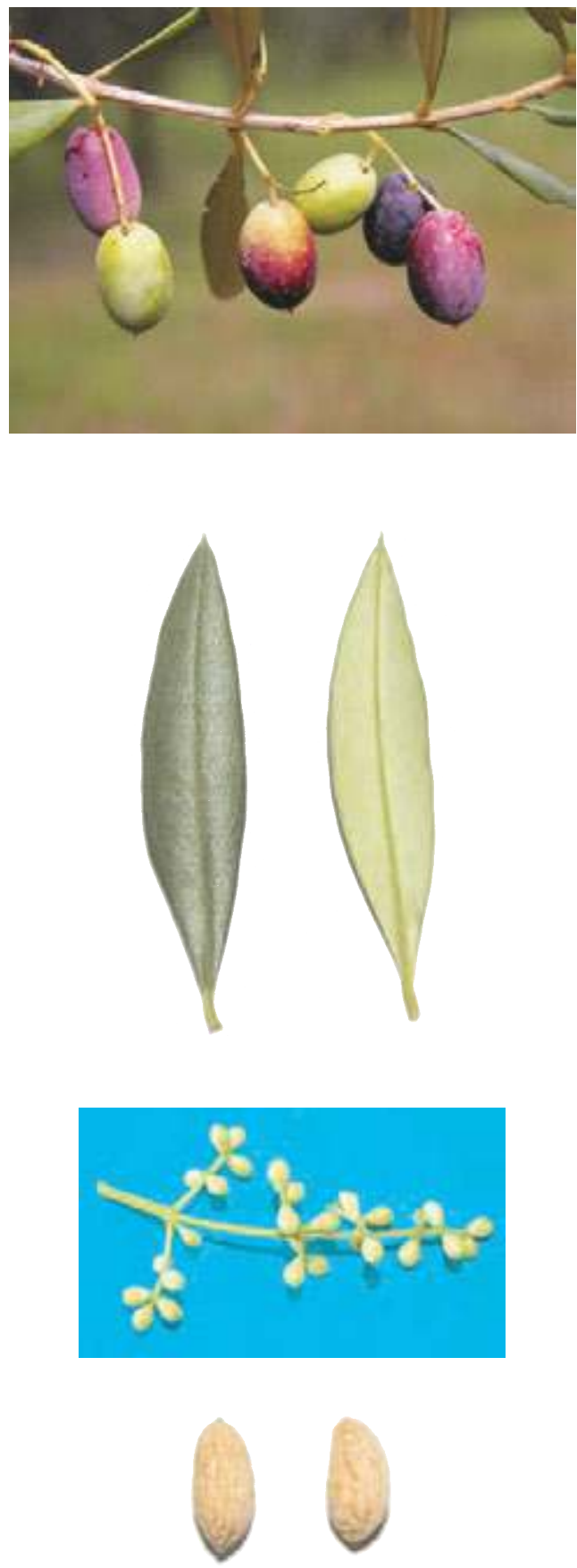


\section{Biochemical Characters}

\section{Fatty Acid Composition}

Table 1. Average values (express in $\% \pm$ standard deviations) of the fatty acids methyl esters and nutritional ratios obtained from single cultivar olive oils.

$\begin{array}{llllll}\text { Myristic acid } & \mathbf{0 , 0 2} \pm \mathbf{0 , 0 1} & \text { Linoleic acid }(\omega 6) & \mathbf{6 , 6 8} \pm \mathbf{0 , 4 3} & \text { Lignoceric acid } & \mathbf{0 , 0 6} \pm \mathbf{0 , 0 6} \\ \text { Palmitic acid } & \mathbf{1 1 , 5 4} \pm \mathbf{0 , 4 7} & \text { Linolenic acid }(\omega 3) & \mathbf{0 , 7 0} \pm \mathbf{0 , 0 7} & & \\ \text { Palmitoleic acid } & \mathbf{1 , 4 3} \pm \mathbf{0 , 3 2} & \text { Arachic acid } & \mathbf{0 , 2 7} \pm \mathbf{0 , 0 1} & \text { Unsat./satured } & \mathbf{6 , 1 2} \pm \mathbf{0 , 0 3} \\ \text { Stearic acid } & \mathbf{1 , 9 4} \pm \mathbf{0 , 2 4} & \text { Eicosenoic acid } & \mathbf{0 , 0 2} \pm \mathbf{0 , 0 1} & \omega 6 / \omega 3 & \mathbf{9 , 6 7} \pm \mathbf{1 , 5 3} \\ \text { Oleic acid } & \mathbf{7 5 , 7 3 \pm 0 , 9 0} & \text { Behenic acid } & \mathbf{0 , 1 3} \pm \mathbf{0 , 0 9} & & \end{array}$

\section{Organoleptic oil values}

Sensory Analysis (Panel test)

Comment: fruity medium, with hints of grass, read sensations of almond and artichoke.

Balanced taste sensation with a medium bitter and spicy. Medium fluidity.

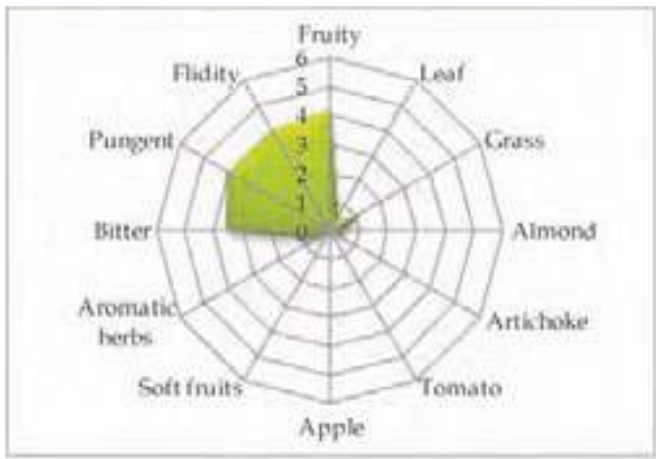

\section{Molecular Markers}

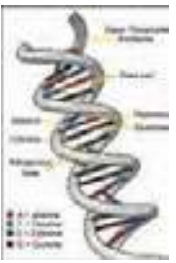

Table 2. Microsatellites (SSR) loci analyzed.

For each locus the allele size (expressed in base pairs) is reported.

$\begin{array}{cccccc}D C A 09 & \text { DCA } 18 & \text { GAPU59 } & \text { GAPU71A } & \text { GUPA71B } & \text { GAPU103A } \\ \mathbf{1 8 2} \mathbf{- 2 0 6} & \mathbf{1 7 7 - \mathbf { 1 8 1 }} & \mathbf{2 0 8 - \mathbf { 2 2 2 }} & \mathbf{2 1 0 - \mathbf { 2 1 4 }} & \mathbf{1 2 6 - \mathbf { 1 4 4 }} & \mathbf{1 7 0 - \mathbf { 1 7 0 }} \\ \text { UDO01 } & \text { UDO03 } & \text { UDO12 } & \text { UDO28 } & \text { UDO39 } & \\ \mathbf{1 4 0} \mathbf{- 1 4 0} & \mathbf{1 8 2} \mathbf{- 1 8 2} & \mathbf{1 7 7} \mathbf{- 1 7 7} & \mathbf{1 5 4} \mathbf{- 2 1 0} & \mathbf{2 0 5 - \mathbf { 2 3 2 }} & \end{array}$

\section{References:}

1 - Pietrangeli E., Russo A., In: Olivi D’Abruzzo, Grafiche di Prinzio (1997), pp. 52-53.

2 - Muzzalupo I., Salimonti A., Caravita M. A., et al. Adv. Hort. Sci. (2008), 22(2): pp. 129-135. 


\section{"Olivo da olio"}

(synonymy: Cicinella, Minucciolo, Oliva a pasta gialla, Sessanella, etc.)

Areal distribution or origin area: Campania

Flesh/pit weight ratio: medium $(6,91 \pm 0,22)$

Oil content (\%): medium $(46,08 \pm 0,81)$

Purpose: oil

\section{Morphological characters}

Tree characters

Vigour: medium-strong

Growth habit: spreading

Canopy-density: medium

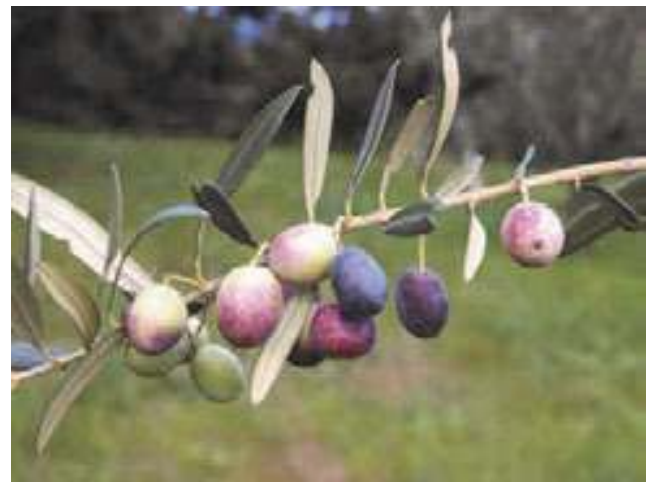

\section{Leaf characters}

Blade length $(\mathrm{cm})$ : medium $(5,07 \pm 0,44)$

Blade width $(\mathrm{cm})$ : medium $(\mathbf{1 , 2 4} \pm \mathbf{0 , 1 2})$

Shape (length/width): elliptic-lanceolate

\section{Inflorescence characters}

Inflorescence length (cm): medium $(2,86 \pm 1,81)$

Number of flowers: low $(\mathbf{1 4}, \mathbf{9 1} \pm 0,95)$

\section{Fruit characters}

Fresh weight of 100 fruits $(\mathrm{g})$ : medium $(2,19 \pm 0,07)$

Shape (length/width): ovoid

Symmetry: symmetric

Position of maximum transverse diameter:

central

Apex: rounded

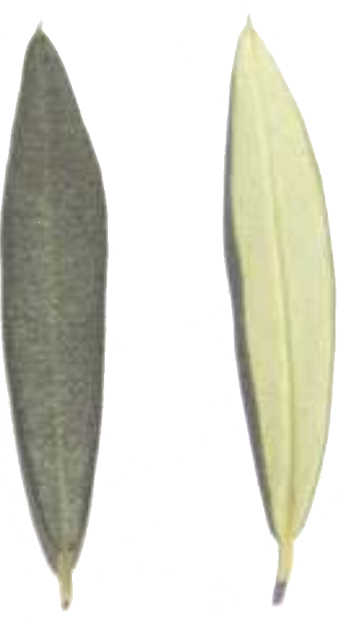

Base: truncate

Niplle: tenuous

Lenticels: many and small

\section{Pit characters}

Weight of 100 pits (g): low $(0,28 \pm \mathbf{0 , 0 2})$

Shape (length/width): elliptic

Mucron: obvious

Symmetry: slightly asymmetric

Position of maximum transverse diameter:

towards apex

Apex: rounded

Base: pointed

Surface: smooth

Number of grooves: medium
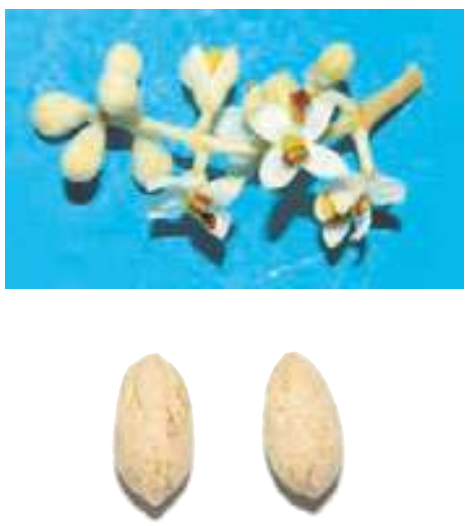


\section{Biochemical Characters}

\section{Fatty Acid Composition}

Table 1. Average values (express in $\% \pm$ standard deviations) of the fatty acids methyl esters and nutritional ratios obtained from single cultivar olive oils.

$\begin{array}{llllll}\text { Myristic acid } & \mathbf{0 , 0 1} \pm \mathbf{0 , 0 0} & \text { Linoleic acid }(\omega 6) & \mathbf{9 , 9 9} \pm \mathbf{0 , 5 9} & \text { Lignoceric acid } & \mathbf{0 , 0 3} \pm \mathbf{0 , 0 1} \\ \text { Palmitic acid } & \mathbf{1 3 , 9 1} \pm \mathbf{0 , 3 5} & \text { Linolenic acid }(\omega 3) \mathbf{0 , 7 7} \pm \mathbf{0 , 0 3} & & \\ \text { Palmitoleic acid } & \mathbf{1 , 2 2} \pm \mathbf{0 , 1 5} & \text { Arachic acid } & \mathbf{0 , 3 0} \pm \mathbf{0 , 1 0} & \text { Unsat./satured } & \mathbf{4 , 9 2} \pm \mathbf{0 , 0 6} \\ \text { Stearic acid } & \mathbf{2 , 6 4} \pm \mathbf{0 , 0 6} & \text { Eicosenoic acid } & \mathbf{0 , 0 2} \pm \mathbf{0 , 0 1} & \omega 6 / \omega 3 & \mathbf{1 2 , 9 8} \pm \mathbf{1 , 2 6} \\ \text { Oleic acid } & \mathbf{7 0 , 1 9} \pm \mathbf{0 , 5 5} & \text { Behenic acid } & \mathbf{0 , 0 7} \pm \mathbf{0 , 0 2} & & \end{array}$

\section{Organoleptic oil values}

Sensory Analysis (Panel test)

Comment: fruity medium-high, with hints of leaves, grass, almond and artichoke. Balanced taste sensation with a medium-high bitter and spicy. Medium-high fluidity.

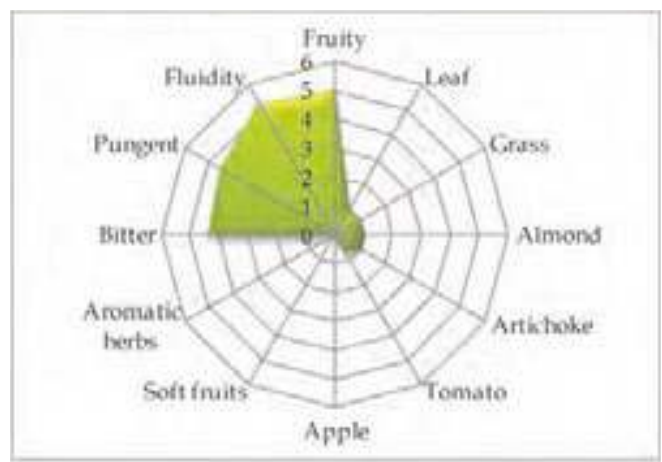

\section{Molecular Markers}

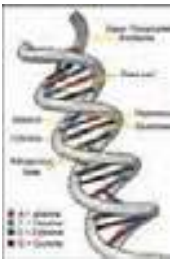

Table 2. Microsatellites (SSR) loci analyzed.

For each locus the allele size (expressed in base pairs) is reported.

$\begin{array}{cccccc}D C A 09 & \text { DCA18 } & \text { GAPU59 } & \text { GAPU71A } & \text { GUPA71B } & \text { GAPU103A } \\ \mathbf{1 7 2} \mathbf{- 1 9 8} & \mathbf{1 7 9 - \mathbf { 1 8 5 }} & \mathbf{2 1 4 - \mathbf { 2 1 4 }} & \mathbf{2 1 8 - \mathbf { 2 1 8 }} & \mathbf{1 2 4} \mathbf{- 1 3 0} & \mathbf{1 5 0} \mathbf{- 1 5 7} \\ \text { UDOO1 } & \text { UDOO3 } & \text { UDO12 } & \text { UDO28 } & \text { UDO39 } & \\ \mathbf{1 4 0 - \mathbf { 1 5 4 }} & \mathbf{1 4 3 - \mathbf { 1 5 0 }} & \mathbf{1 6 4 - \mathbf { 1 7 7 }} & \mathbf{1 8 2} \mathbf{- 1 8 2} & \mathbf{2 1 3 - \mathbf { 2 1 3 }} & \end{array}$

\section{References:}

1 - Pugliano G., Flaminio G., Pugliano M.L., et al. In: La risorsa genetica dell'olivo in Campania, SE. S.I.R.C.A. Ed. Napoli, (2000).

2 - Muzzalupo I., Stefanizzi F., Perri E. HortScience (2009), 44: pp. 582-588. 


\section{"Olivo da salare"}

(synonymy: Gaetana)

Areal distribution or origin area: Campania

Flesh/pit weight ratio: medium $(5,95 \pm 0,50)$

Oil content (\%): medium $(46,43 \pm 1,84)$

Purpose: dual purpose

\section{Morphological characters}

Tree characters

Vigour: weak

Growth habit: spreading

Canopy-density: medium-sparse

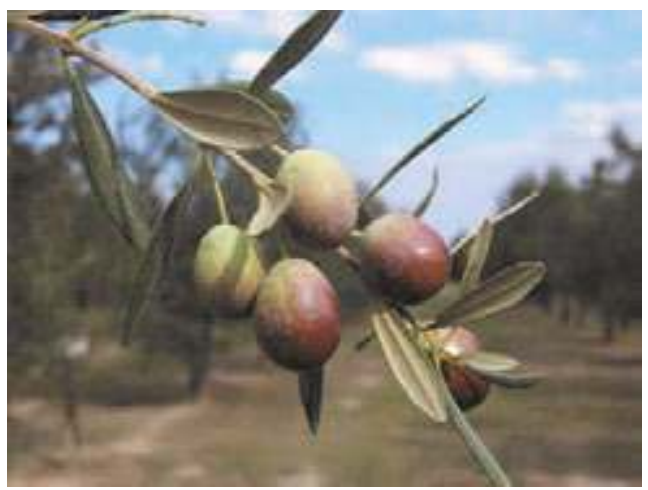

\section{Leaf characters}

Blade length $(\mathrm{cm})$ : medium $(5,86 \pm 0,36)$

Blade width (cm): medium $(\mathbf{1}, \mathbf{4 1} \pm \mathbf{0 , 1 4})$

Shape (length/width): elliptic-lanceolate

\section{Inflorescence characters}

Inflorescence length $(\mathrm{cm})$ : medium $(2,69 \pm 0,55)$

Number of flowers: low $(13,12 \pm 1,40)$

\section{Fruit characters}

Fresh weight of 100 fruits (g): medium $(3,48 \pm 0,16)$

Shape (length/width): spherical

Symmetry: symmetric

Position of maximum transverse diameter:

central

Apex: rounded

Base: rounded

Niplle: absent

Lenticels: many and small

\section{Pit characters}

Weight of 100 pits (g): high $(0,51 \pm \mathbf{0 , 0 6})$

Shape (length/width): ovoid

Mucron: absent

Symmetry: symmetric

Position of maximum transverse diameter:

towards apex

Apex: rounded

Base: rounded

Surface: rugose

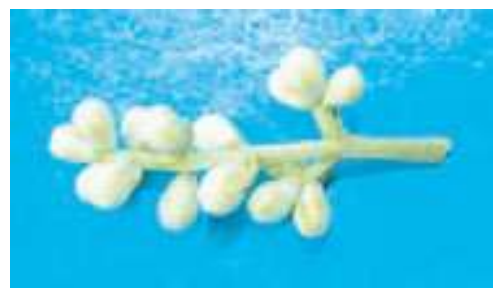

Number of grooves: medium 


\section{Biochemical Characters}

\section{Fatty Acid Composition}

Table 1. Average values (express in $\% \pm$ standard deviations) of the fatty acids methyl esters and nutritional ratios obtained from single cultivar olive oils.

$\begin{array}{lllll}\text { Myristic acid } \quad 0,01 \pm 0,00 & \text { Linoleic acid }(\omega 6) & \mathbf{1 2 , 0 1} \pm \mathbf{1 , 2 4} \quad \text { Lignoceric acid } \quad 0,03 \pm 0,01\end{array}$

Palmitic acid $12,39 \pm 0,79 \quad$ Linolenic acid $(\omega 3) 0,60 \pm 0,00$

Palmitoleic acid

$1,92 \pm 0,17$

Arachic acid

$0,31 \pm 0,08$

Unsat./satured

$5,70 \pm 0,39$

Stearic acid

$2,08 \pm 0,81$

Eicosenoic acid

$0,03 \pm 0,00$

$\omega 6 / \omega 3$

$20,13 \pm 1,91$

Oleic acid

$68,81 \pm 0,14$

Behenic acid

$0,08 \pm 0,01$

\section{Organoleptic oil values}

Sensory Analysis (Panel test)

Comment: fruity medium-high, with hints of almond and read sensations of leaves, grass, artichoke and tomato. Balanced taste sensation with a medium-high bitter and spicy. Medium-high fluidity.

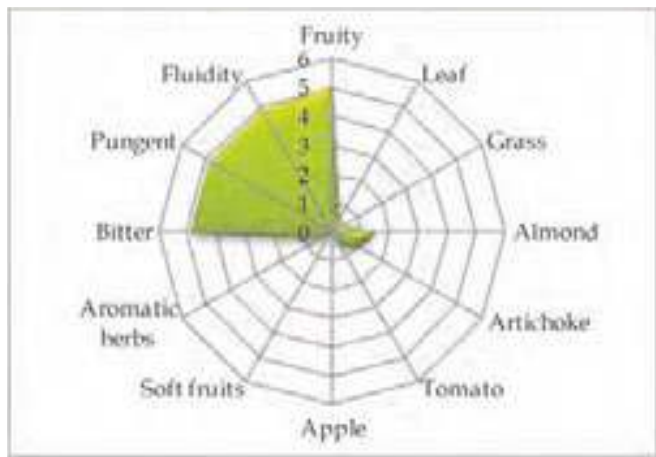

\section{Molecular Markers}

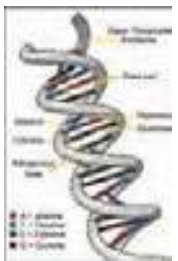

Table 2. Microsatellites (SSR) loci analyzed.

For each locus the allele size (expressed in base pairs) is reported.

$\begin{array}{cccccc}D C A 09 & \text { DCA18 } & \text { GAPU59 } & \text { GAPU71A } & \text { GUPA71B } & \text { GAPU103A } \\ \mathbf{1 6 2} \mathbf{- 1 9 4} & \mathbf{1 7 9 - \mathbf { 1 8 1 }} & \mathbf{2 1 4 - \mathbf { 2 2 2 }} & \mathbf{2 1 4} \mathbf{- 2 1 4} & \mathbf{1 2 6 - \mathbf { 1 3 0 }} & \mathbf{1 5 0 - \mathbf { 1 5 7 }} \\ \text { UDOO1 } & \text { UDOO3 } & \text { UDO12 } & \text { UDO28 } & \text { UDO39 } & \\ \mathbf{1 4 0 - \mathbf { 1 4 0 }} & \mathbf{1 3 5 - \mathbf { 1 3 5 }} & \mathbf{1 2 3 - \mathbf { 1 2 3 }} & \mathbf{1 6 6 - \mathbf { 1 7 7 }} & \mathbf{2 0 0 - \mathbf { 2 0 0 }} & \end{array}$

\section{References:}

1 - Pugliano G., Flaminio G., Pugliano M.L., et al. In: La risorsa genetica dell'olivo in Campania, SE. S.I.R.C.A. Ed. Napoli, (2000).

2 - Muzzalupo I., Stefanizzi F., Perri E. HortScience (2009), 44: pp. 582-588. 


\section{“Olivo della Madonna "}

Areal distribution or origin area: Toscana

Flesh/pit weight ratio: low $(3,87 \pm 0,44)$

Oil content (\%): low $(39,38 \pm 0,08)$

Purpose: oil

\section{Morphological characters}

Tree characters

Vigour: medium

Growth habit: erect-sprading

Canopy-density: medium-sparse

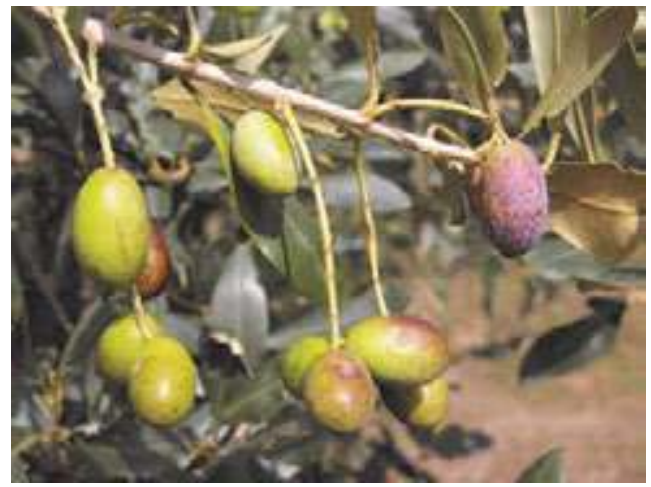

\section{Leaf characters}

Blade length $(\mathrm{cm})$ : medium $(6,13 \pm 0,49)$

Blade width $(\mathrm{cm})$ : broad $(2,08 \pm \mathbf{0 , 2 6})$

Shape (length/width): elliptic

\section{Inflorescence characters}

Inflorescence length $(\mathrm{cm})$ : medium $(2,92 \pm 0,49)$

Number of flowers: medium $(24,73 \pm 1,31)$

\section{Fruit characters}

Fresh weight of 100 fruits $(\mathrm{g})$ : medium $(2,34 \pm 0,03)$

Shape (length/width): elongated

Symmetry: slightly asymmetric

Position of maximum transverse diameter:

central

Apex: rounded

Base: truncate

Niplle: absent

Lenticels: many and small

\section{Pit characters}

Weight of 100 pits (g): high $(0,49 \pm 0,03)$

Shape (length/width): elliptic

Mucron: obvious

Symmetry: asymmetric

Position of maximum transverse diameter:

towards apex

Apex: pointed

Base: rounded

Surface: scabrous

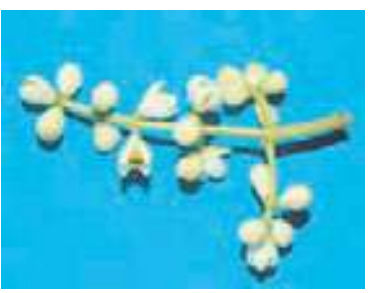

Number of grooves: low 


\section{Biochemical Characters}

\section{Fatty Acid Composition}

Table 1. Average values (express in $\% \pm$ standard deviations) of the fatty acids methyl esters and nutritional ratios obtained from single cultivar olive oils.

$\begin{array}{llllll}\text { Myristic acid } & \mathbf{0 , 0 1} \pm \mathbf{0 , 0 1} & \text { Linoleic acid }(\omega 6) & \mathbf{7 , 0 6} \pm \mathbf{0 , 1 3} & \text { Lignoceric acid } & \mathbf{0 , 0 3} \pm \mathbf{0 , 0 1} \\ \text { Palmitic acid } & \mathbf{1 2 , 3 3} \pm \mathbf{1 , 1 6} & \text { Linolenic acid }(\omega 3) \mathbf{0 , 6 9} \pm \mathbf{0 , 0 4} & & \\ \text { Palmitoleic acid } & \mathbf{0 , 7 2} \pm \mathbf{0 , 0 9} & \text { Arachic acid } & \mathbf{0 , 2 3} \pm \mathbf{0 , 0 5} & \text { Unsat./satured } & \mathbf{5 , 8 0} \pm \mathbf{0 , 2 4} \\ \text { Stearic acid } & \mathbf{2 , 1 2} \pm \mathbf{0 , 7 9} & \text { Eicosenoic acid } & \mathbf{0 , 0 2} \pm \mathbf{0 , 0 0} & \omega 6 / \omega 3 & \mathbf{1 0 , 1 7} \pm \mathbf{0 , 3 4} \\ \text { Oleic acid } & \mathbf{7 6 , 1 3} \pm \mathbf{1 , 2 3} & \text { Behenic acid } & \mathbf{0 , 0 5} \pm \mathbf{0 , 0 1} & & \end{array}$

\section{Organoleptic oil values}

Sensory Analysis (Panel test)

Comment: fruity medium-high, with hints of almond and read sensations of artichoke and grass. Balanced taste sensation with a medium-high bitter and spicy. Medium-high fluidity.

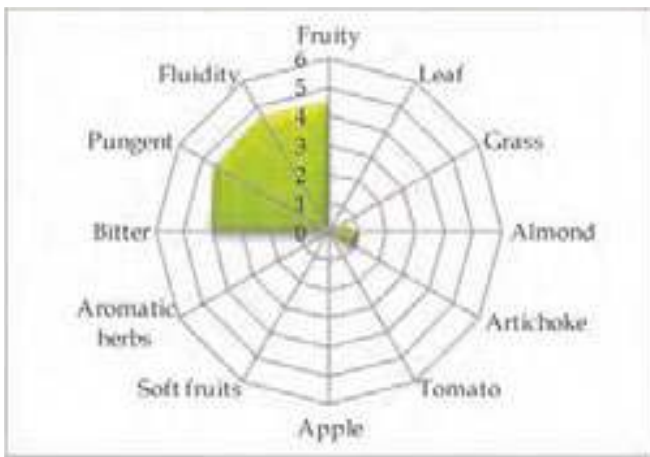

\section{Molecular Markers}

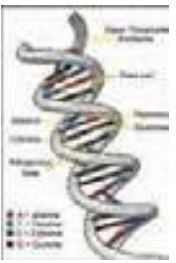

Table 2. Microsatellites (SSR) loci analyzed.

For each locus the allele size (expressed in base pairs) is reported.

\begin{tabular}{|c|c|c|c|c|c|}
\hline$D C A 09$ & $D C A 18$ & GAPU59 & GAPU71A & GUPA71B & GAPU103A \\
\hline $162-206$ & $177-177$ & $212-222$ & $214-214$ & $124-144$ & $170-170$ \\
\hline UDO01 & UDO03 & UDO12 & UDO28 & UDO39 & \\
\hline $140-140$ & $150-150$ & $177-177$ & $182-210$ & $205-205$ & \\
\hline
\end{tabular}

\section{References:}

1 - Basso M. In: Le cultivar coltivate sui monti pisani, Ann. Fac. Agr. Pisa (1958), 12 pp. 14-54.

2 - Muzzalupo I., Lombardo N., Salimonti A., et al. Adv. Hort. Sci. (2008), 22(2): pp. 142-148. 


\section{"Orbetana"}

(synonymy: Marchigiana, Oliva bastarda, Oliva di San Francesco, Sarga, etc.)

Areal distribution or origin area: Marche

Flesh/pit weight ratio: medium $(5,79 \pm 0,31)$

Oil content (\%): medium $(43,10 \pm 1,65)$

Purpose: dual purpose

\section{Morphological characters}

Tree characters

Vigour: medium-weak

Growth habit: spreading

Canopy-density: medium

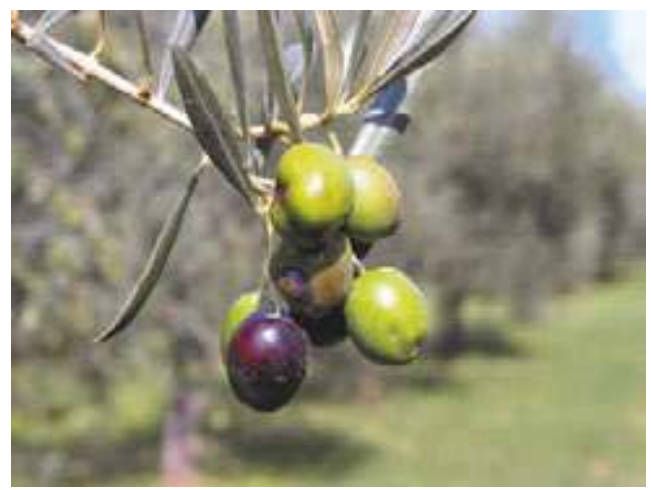

\section{Leaf characters}

Blade length (cm): long $(7,06 \pm 0,66)$

Blade width $(\mathrm{cm})$ : medium $(\mathbf{1}, 23 \pm \mathbf{0 , 1 2})$

Shape (length/width): elliptic - lanceolate

\section{Inflorescence characters}

Inflorescence length $(\mathrm{cm})$ : medium $(2,98 \pm 0,46)$

Number of flowers: medium $(\mathbf{2 3 , 1 5} \pm 0,94)$

\section{Fruit characters}

Fresh weight of 100 fruits (g): high $(\mathbf{4}, 29 \pm 0,72)$

Shape (length/width): spherical

Symmetry: asymmetric

Position of maximum transverse diameter:

central

Apex: rounded

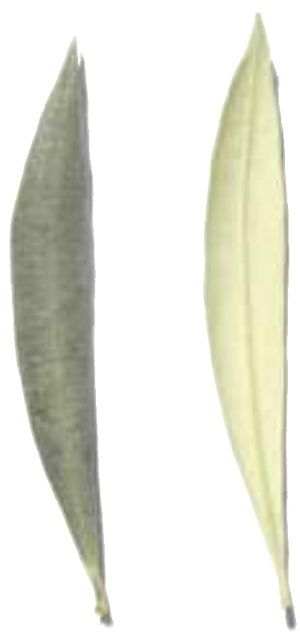

Base: truncate

Niplle: tenuous

Lenticels: many and small

\section{Pit characters}

Weight of 100 pits (g): high $(0,58 \pm 0,03)$

Shape (length/width): ovoid

Mucron: obvious

Symmetry: slightly asymmetric

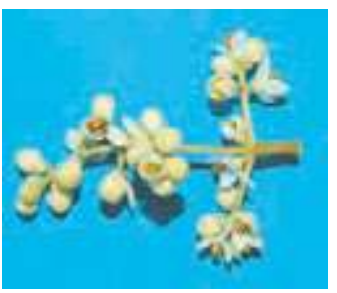

Position of maximum transverse diameter:

central

Apex: pointed

Base: rounded

Surface: rugose

Number of grooves: medium

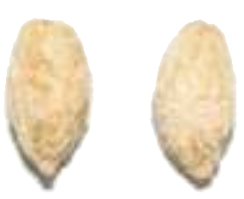




\section{Biochemical Characters}

\section{Fatty Acid Composition}

Table 1. Average values (express in $\% \pm$ standard deviations) of the fatty acids methyl esters and nutritional ratios obtained from single cultivar olive oils.

$\begin{array}{llllll}\text { Myristic acid } & \mathbf{0 , 0 1} \pm \mathbf{0 , 0 1} & \text { Linoleic acid }(\omega 6) & \mathbf{1 6 , 4 6} \pm \mathbf{1 , 5 0} & \text { Lignoceric acid } & \mathbf{0 , 0 4} \pm \mathbf{0 , 0 2} \\ \text { Palmitic acid } & \mathbf{1 5 , 2 4} \pm \mathbf{1 , 4 7} & \text { Linolenic acid }(\omega 3) & \mathbf{0 , 3 1} \pm \mathbf{0 , 0 2} & & \\ \text { Palmitoleic acid } & \mathbf{1 , 5 8} \pm \mathbf{0 , 0 5} & \text { Arachic acid } & \mathbf{0 , 8 1} \pm \mathbf{0 , 1 5} & \text { Unsat./satured } & \mathbf{4 , 6 4 \pm 0 , 4 6} \\ \text { Stearic acid } & \mathbf{1 , 8 7} \pm \mathbf{0 , 1 0} & \text { Eicosenoic acid } & \mathbf{0 , 2 6} \pm \mathbf{0 , 0 9} & \omega 6 / \omega 3 & \mathbf{5 3 , 8 9} \pm \mathbf{0 , 5 6} \\ \text { Oleic acid } & \mathbf{6 1 , 5 0 \pm 0 , 6 8} & \text { Behenic acid } & \mathbf{0 , 1 0} \pm \mathbf{0 , 0 2} & & \end{array}$

\section{Organoleptic oil values}

Sensory Analysis (Panel test)

Comment: fruity medium - light, with hints of artichoke and almond. Balanced in flavours, with hints of bitter and spicy medium intensity, medium fluidity.

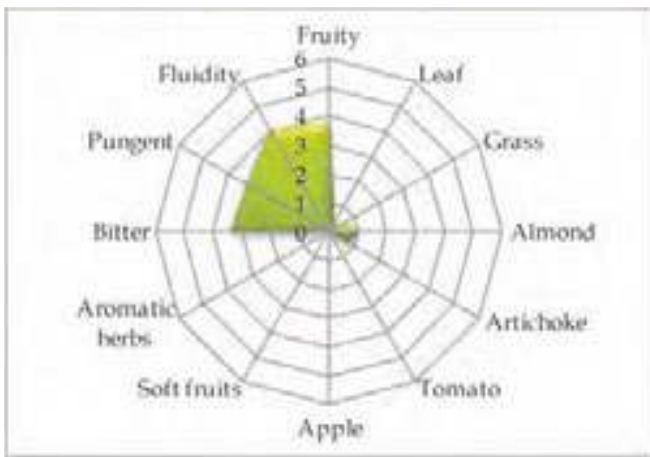

\section{Molecular Markers}

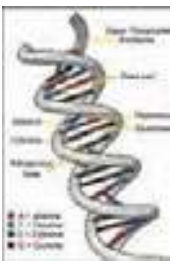

Table 2. Microsatellites (SSR) loci analyzed.

For each locus the allele size (expressed in base pairs) is reported.

$\begin{array}{cccccc}D C A 09 & \text { DCA18 } & \text { GAPU59 } & \text { GAPU71A } & \text { GUPA71B } & \text { GAPU103A } \\ \mathbf{1 6 2} \mathbf{- 1 8 2} & \mathbf{1 7 7 - \mathbf { 1 8 9 }} & \mathbf{2 1 4 - \mathbf { 2 1 8 }} & \mathbf{2 1 8 - \mathbf { 2 2 4 }} & \mathbf{1 2 4 - \mathbf { 1 4 4 }} & \mathbf{1 8 4} \mathbf{- 1 9 7} \\ \text { UDOO1 } & \text { UDO03 } & \text { UDO12 } & \text { UDO28 } & \text { UDO39 } & \\ \mathbf{1 4 0 - \mathbf { 1 3 5 }} & \mathbf{1 3 5 - \mathbf { 1 3 5 }} & \mathbf{1 6 6 - \mathbf { 1 7 7 }} & \mathbf{1 8 2} \mathbf{- 2 1 0} & \mathbf{2 0 0 - \mathbf { 2 0 0 }} & \end{array}$

\section{References:}

1 - Pannelli G., Alfei B., Santinelli A. In: Varietà di olivo nelle Marche, (2001), ASSAM pp. 85-88.

2 - Muzzalupo I., Stefanizzi F., Bucci C., et al. In Acta Italus Hortus, (2011), 1: 138 -140. 


\section{"Ortice "}

(synonymy: Iessana, Olivastro, Oliviello, Olivona, Testicolo di Gallo, etc.)

Areal distribution or origin area: Campania

Flesh/pit weight ratio: medium $(5,43 \pm 0,33)$

Oil content (\%): medium $(43,38 \pm 0,38)$

Purpose: dual purpose

\section{Morphological characters}

Tree characters

Vigour: medium-strong

Growth habit: erect-spreading

Canopy-density: dense

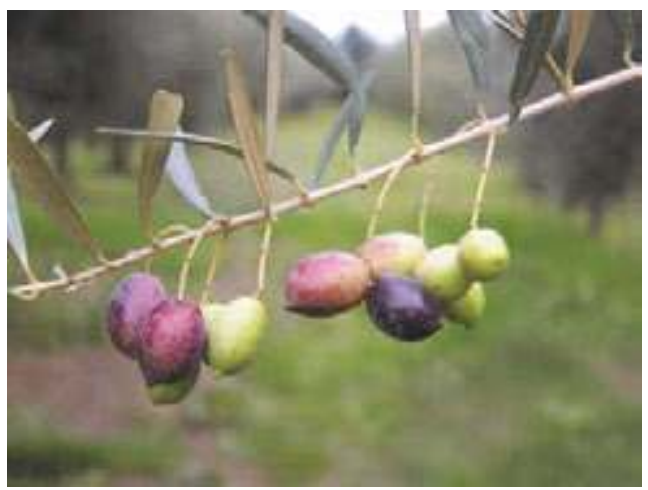

\section{Leaf characters}

Blade length $(\mathrm{cm})$ : medium $(6,44 \pm 0,56)$

Blade width $(\mathrm{cm})$ : medium $(\mathbf{1}, \mathbf{1 1} \pm \mathbf{0 , 1 3})$

Shape (length/width): elliptic-lanceolate

\section{Inflorescence characters}

Inflorescence length $(\mathrm{cm})$ : medium $(3,45 \pm 0,50)$

Number of flowers: medium $(22,84 \pm 1,62)$

\section{Fruit characters}

Fresh weight of 100 fruits $(\mathrm{g})$ : medium $(\mathbf{2 , 2 8} \pm \mathbf{0 , 2 2})$

Shape (length/width): elongated

Symmetry: slightly asymmetric

Position of maximum transverse diameter:

towards apex

Apex: rounded

Base: rounded

Niplle: obvious

Lenticels: many and small

\section{Pit characters}

Weight of 100 pits $(\mathrm{g})$ : medium $(0,37 \pm \mathbf{0 , 0 2})$

Shape (length/width): elliptic

Mucron: obvious
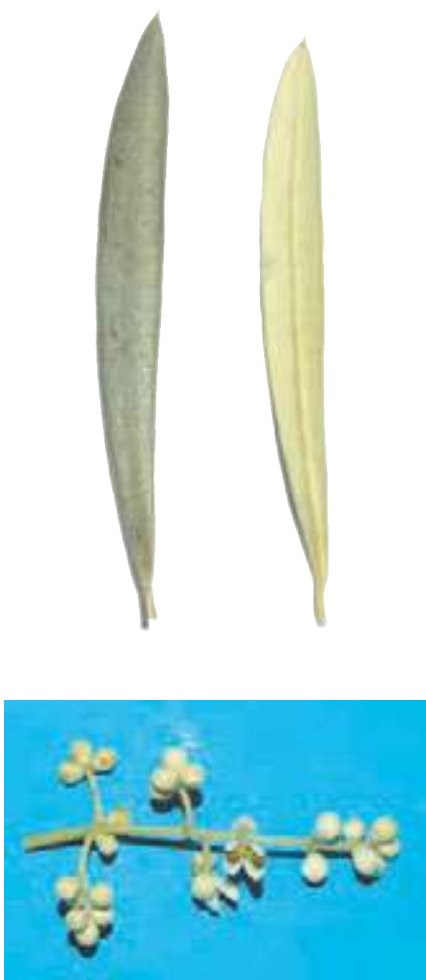

Symmetry: slightly asymmetric

Position of maximum transverse diameter:

towards apex

Apex: rounded

Base: pointed

Surface: smooth

Number of grooves: low 


\section{Biochemical Characters}

\section{Fatty Acid Composition}

Table 1. Average values (express in $\% \pm$ standard deviations) of the fatty acids methyl esters and nutritional ratios obtained from single cultivar olive oils.

$\begin{array}{llllll}\text { Myristic acid } & \mathbf{0 , 0 0} \pm \mathbf{0 , 0 0} & \text { Linoleic acid }(\omega 6) & \mathbf{8 , 2 0} \pm \mathbf{0 , 1 9} & \text { Lignoceric acid } & \mathbf{0 , 0 5} \pm \mathbf{0 , 0 2} \\ \text { Palmitic acid } & \mathbf{1 3 , 5 4 \pm 0 , 0 7} & \text { Linolenic acid }(\omega 3) & \mathbf{0 , 8 9} \pm \mathbf{0 , 0 2} & & \\ \text { Palmitoleic acid } & \mathbf{0 , 7 2} \pm \mathbf{0 , 1 2} & \text { Arachic acid } & \mathbf{0 , 4 1} \pm \mathbf{0 , 0 7} & \text { Unsat./satured } & \mathbf{4 , 9 8} \pm \mathbf{0 , 1 7} \\ \text { Stearic acid } & \mathbf{2 , 7 9} \pm \mathbf{0 , 3 1} & \text { Eicosenoic acid } & \mathbf{0 , 2 0} \pm \mathbf{0 , 1 5} & \omega 6 / \omega 3 & \mathbf{9 , 2 1} \pm \mathbf{0 , 3 7} \\ \text { Oleic acid } & \mathbf{7 2 , 5 5 \pm 0 , 7 2} & \text { Behenic acid } & \mathbf{0 , 1 0} \pm \mathbf{0 , 0 3} & & \end{array}$

\section{Organoleptic oil values}

Sensory Analysis (Panel test)

Comment: medium fruity, with good sensation of grass, less almond and artichoke. Balanced taste sensation, with bitter and spicy medium. Medium fluidity.

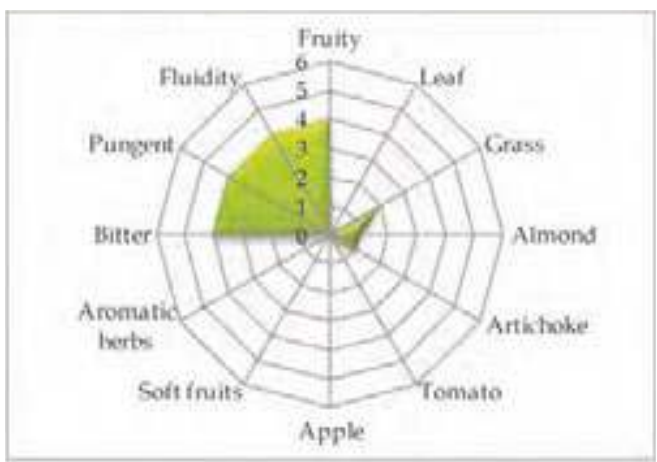

\section{Molecular Markers}

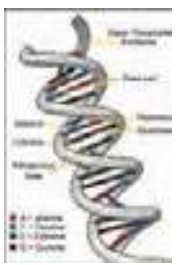

Table 2. Microsatellites (SSR) loci analyzed.

For each locus the allele size (expressed in base pairs) is reported.

\begin{tabular}{|c|c|c|c|c|c|}
\hline DCA09 & $D C A 18$ & GAPU59 & GAPU71A & $G U P A 71 B$ & GAPU103A \\
\hline $194-198$ & $177-185$ & $218-218$ & $214-224$ & $126-144$ & $150-157$ \\
\hline UDO01 & UDO03 & UDO12 & UDO28 & UDO39 & \\
\hline $140-140$ & $135-166$ & $166-182$ & $161-205$ & $164-164$ & \\
\hline
\end{tabular}

\section{References:}

1 - Pugliano G., Flaminio G., Pugliano M.L., et al. In: La risorsa genetica dell'olivo in Campania, SE. S.I.R.C.A. Ed. Napoli, (2000).

2 - Muzzalupo I., Stefanizzi F., Perri E. HortScience (2009), 44: pp. 582-588. 


\section{"Ortolana "}

(synonymy: Bella di San Lorenzo, Melella, etc.)

Areal distribution or origin area: Campania

Flesh/pit weight ratio: medium $(6,46 \pm 1,50)$

Oil content (\%): medium $(45,28 \pm 0,86)$

Purpose: dual purpose

\section{Morphological characters}

Tree characters

Vigour: medium-strong

Growth habit: spreading-erect

Canopy-density: medium-dense

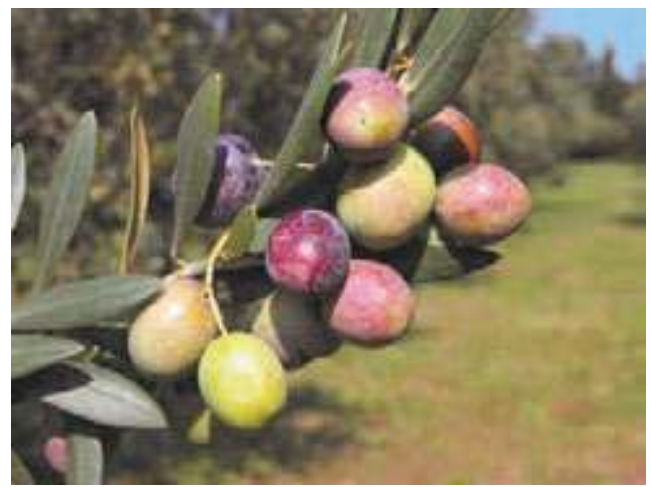

\section{Leaf characters}

Blade length $(\mathrm{cm})$ : medium $(5,59 \pm 0,51)$

Blade width $(\mathrm{cm})$ : broad $(1,63 \pm 0,22)$

Shape (length/width): elliptic-lanceolate

\section{Inflorescence characters}

Inflorescence length ( $\mathrm{cm})$ : medium $(3,28 \pm 2,87)$

Number of flowers: medium $(21,91 \pm 1,51)$

\section{Fruit characters}

Fresh weight of 100 fruits (g): high $(4,37 \pm 0,45)$

Shape (length/width): ovoid

Symmetry: slightly asymmetric

Position of maximum transverse diameter:

central

Apex: rounded

Base: rounded

Niplle: absent

Lenticels: many and small

\section{Pit characters}

Weight of 100 pits (g): high $(\mathbf{0 , 6 0} \pm \mathbf{0 , 0 6})$

Shape (length/width): elliptic

Mucron: absent

Symmetry: asymmetric

Position of maximum transverse diameter:

towards apex

Apex: rounded

Base: rounded

Surface: scabrous
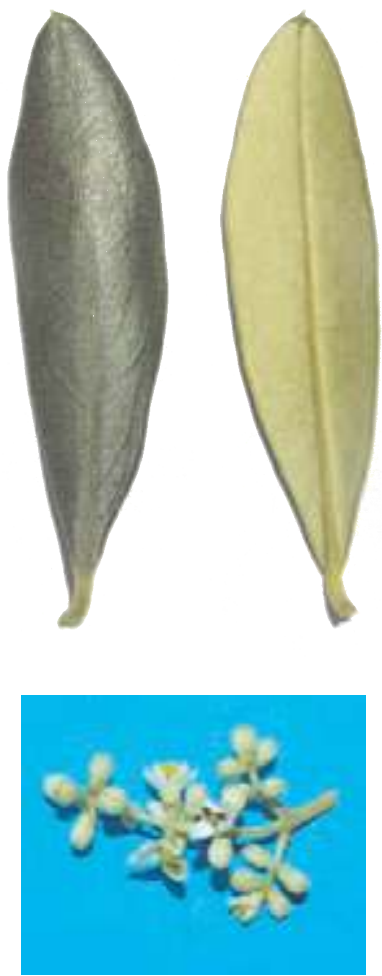

Number of grooves: medium 


\section{Biochemical Characters}

\section{Fatty Acid Composition}

Table 1. Average values (express in $\% \pm$ standard deviations) of the fatty acids methyl esters and nutritional ratios obtained from single cultivar olive oils.

$\begin{array}{llllll}\text { Myristic acid } & \mathbf{0 , 0 1} \pm \mathbf{0 , 0 0} & \text { Linoleic acid }(\omega 6) & \mathbf{9 , 3 0} \pm \mathbf{0 , 8 4} & \text { Lignoceric acid } & \mathbf{0 , 0 3} \pm \mathbf{0 , 0 1} \\ \text { Palmitic acid } & \mathbf{1 4 , 1 9 \pm 0 , 2 8} & \text { Linolenic acid }(\omega 3) \mathbf{0 , 7 0 \pm 0 , 1 2} & & \\ \text { Palmitoleic acid } & \mathbf{0 , 7 5} \pm \mathbf{0 , 4 4} & \text { Arachic acid } & \mathbf{0 , 2 6} \pm \mathbf{0 , 0 6} & \text { Unsat./satured } & \mathbf{5 , 3 0 \pm 0 , 2 2} \\ \text { Stearic acid } & \mathbf{1 , 3 8} \pm \mathbf{0 , 3 4} & \text { Eicosenoic acid } & \mathbf{0 , 0 2} \pm \mathbf{0 , 0 1} & \omega 6 / \omega 3 & \mathbf{1 3 , 3 9 \pm 1 , 0 1} \\ \text { Oleic acid } & \mathbf{7 2 , 5 9 \pm 0 , 6 4} & \text { Behenic acid } & \mathbf{0 , 0 6} \pm \mathbf{0 , 0 1} & & \end{array}$

\section{Organoleptic oil values}

Sensory Analysis (Panel test)

Comment: fruity medium-high, with taste of almond and artichoke, with read sensations of aromatic herbs. Balanced taste sensation with medium bitter and spicy. Medium-high fluidity.

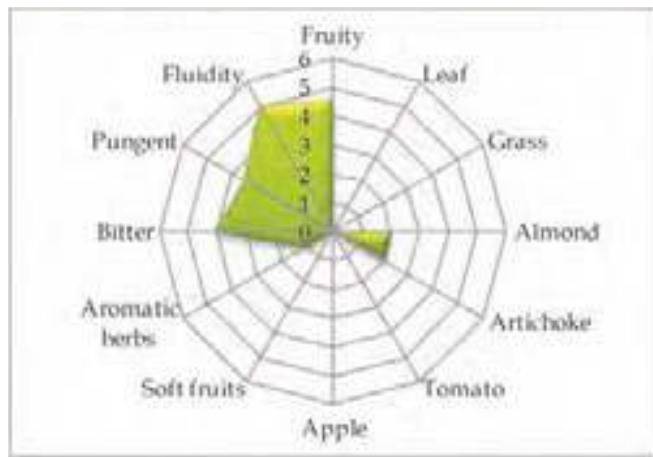

\section{Molecular Markers}

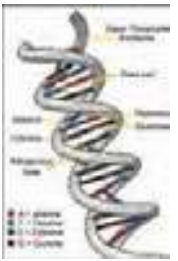

Table 2. Microsatellites (SSR) loci analyzed.

For each locus the allele size (expressed in base pairs) is reported.

$\begin{array}{cccccc}D C A 09 & \text { DCA18 } & \text { GAPU59 } & \text { GAPU71A } & \text { GUPA71B } & \text { GAPU103A } \\ \mathbf{1 6 2} \mathbf{- 1 9 4} & \mathbf{1 7 7 - \mathbf { 1 8 1 }} & \mathbf{2 1 4 - \mathbf { 2 1 8 }} & \mathbf{2 1 8 - \mathbf { 2 1 8 }} & \mathbf{2 1 2} \mathbf{- 1 2 4} & \mathbf{1 7 0 - \mathbf { 1 8 4 }} \\ \text { UDOO1 } & \text { UDOO3 } & \text { UDO12 } & \text { UDO28 } & \text { UDO39 } & \\ \mathbf{1 4 0 - \mathbf { 1 4 0 }} & \mathbf{1 4 3 - \mathbf { 1 4 3 }} & \mathbf{1 6 4 - \mathbf { 1 6 4 }} & \mathbf{1 6 1 - \mathbf { 1 6 1 }} & \mathbf{2 0 5 - \mathbf { 2 1 3 }} & \end{array}$

\section{References:}

1 - Pugliano G., Flaminio G., Pugliano M.L., et al. In: La risorsa genetica dell'olivo in Campania, SE. S.I.R.C.A. Ed. Napoli, (2000).

2 - Muzzalupo I., Stefanizzi F., Perri E. HortScience (2009), 44: pp. 582-588. 


\section{"Ottobratica"}

(synonymy: Dedarico, Dolce, Mirtoleo, Ottobratico, Perciasacchi, etc.)

Areal distribution or origin area: Calabria

Flesh/pit weight ratio: low $(4,80 \pm 2,06)$

Oil content (\%): medium $(44,33 \pm 2,49)$

Purpose: oil

\section{Morphological characters}

Tree characters

Vigour: medium-strong

Growth habit: spreading

Canopy-density: medium-dense

\section{Leaf characters}

Blade length ( $\mathrm{cm})$ : medium $(5,56 \pm 0,61)$

Blade width (cm): broad $(\mathbf{1}, 53 \pm \mathbf{0 , 1 8})$

Shape (length/width): elliptic

\section{Inflorescence characters}

Inflorescence length $(\mathrm{cm})$ : medium $(3,00 \pm 1,05)$

Number of flowers: low $(\mathbf{1 7}, \mathbf{2 4} \pm \mathbf{2 , 7 8})$

\section{Fruit characters}

Fresh weight of 100 fruits $(\mathrm{g})$ : low $(\mathbf{1}, 74 \pm \mathbf{0 , 0 4})$

Shape (length/width): elongated

Symmetry: asymmetric

Position of maximum transverse diameter:

central

Apex: pointed

Base: rounded

Niplle: tenuous

Lenticels: few and small

\section{Pit characters}

Weight of 100 pits (g): medium $(0,31 \pm 0,06)$

Shape (length/width): elongated

Mucron: obvious

Symmetry: slightly asymmetric

Position of maximum transverse diameter:

central

Apex: pointed

Base: pointed

Surface: rugose

Number of grooves: medium
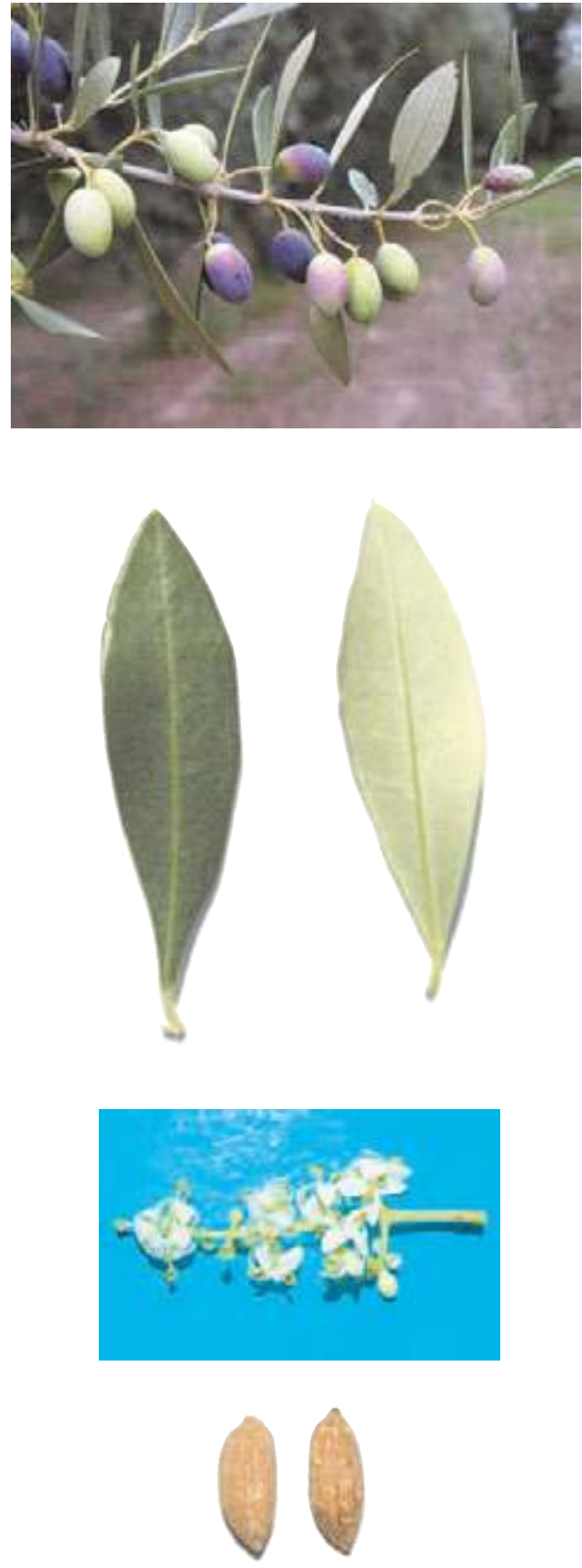


\section{Biochemical Characters}

\section{Fatty Acid Composition}

Table 1. Average values (express in $\% \pm$ standard deviations) of the fatty acids methyl esters and nutritional ratios obtained from single cultivar olive oils.

$\begin{array}{llllll}\text { Myristic acid } & \mathbf{0 , 0 1} \pm \mathbf{0 , 0 1} & \text { Linoleic acid }(\omega 6) & \mathbf{9 , 7 9} \pm \mathbf{0 , 8 5} & \text { Lignoceric acid } & \mathbf{0 , 0 8} \pm \mathbf{0 , 0 6} \\ \text { Palmitic acid } & \mathbf{1 4 , 1 9 \pm \mathbf { 1 } 1 8} & \text { Linolenic acid }(\omega 3) \mathbf{0 , 6 5} \pm \mathbf{0 , 1 8} & & \\ \text { Palmitoleic acid } & \mathbf{1 , 1 2} \pm \mathbf{0 , 2 1} & \text { Arachic acid } & \mathbf{0 , 3 2} \pm \mathbf{0 , 0 9} & \text { Unsat./satured } & \mathbf{4 , 9 4} \pm \mathbf{0 , 5 2} \\ \text { Stearic acid } & \mathbf{2 , 2 3} \pm \mathbf{0 , 3 6} & \text { Eicosenoic acid } & \mathbf{0 , 1 5} \pm \mathbf{0 , 1 0} & \omega 6 / \omega 3 & \mathbf{1 6 , 0 7 \pm 4 , 2 7} \\ \text { Oleic acid } & \mathbf{7 0 , 5 2} \pm \mathbf{2 , 0 2} & \text { Behenic acid } & \mathbf{0 , 1 4} \pm \mathbf{0 , 0 6} & & \end{array}$

\section{Organoleptic oil values}

Sensory Analysis (Panel test)

Comment: fruity medium-high, with hints of almond and read sensations of grass and artichoke. Balanced taste sensation with a medium-high bitter and medium spicy. Medium-high fluidity.

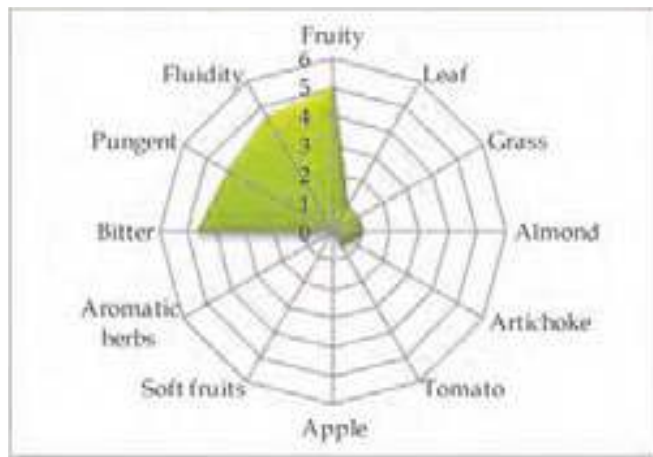

\section{Molecular Markers}

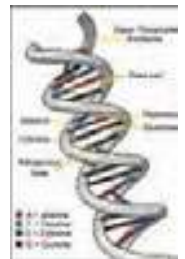

Table 2. Microsatellites (SSR) loci analyzed. For each locus the allele size (expressed in base pairs) is reported.

$\begin{array}{cccccc}D C A 09 & D C A 18 & \text { GAPU59 } & \text { GAPU71A } & \text { GUPA71B } & \text { GAPU103A } \\ \mathbf{1 8 2} \mathbf{- 2 0 6} & \mathbf{1 7 7 - \mathbf { 1 8 5 }} & \mathbf{2 1 2} \mathbf{- 2 2 2} & \mathbf{2 1 2} \mathbf{- 2 1 2} & \mathbf{1 2 4} \mathbf{- 1 2 4} & \mathbf{1 5 7 - \mathbf { 1 7 0 }} \\ \text { UDO01 } & \text { UDOO3 } & \text { UDO12 } & \text { UDO28 } & \text { UDO39 } & \\ \mathbf{1 4 0 - \mathbf { 1 5 0 }} & \mathbf{1 4 3 - \mathbf { 1 4 3 }} & \mathbf{1 7 7 - \mathbf { 1 9 3 }} & \mathbf{1 4 3 - \mathbf { 1 5 4 }} & \mathbf{1 7 0 - \mathbf { 2 0 5 }} & \end{array}$

\section{References:}

1 - Lombardo N., Perri E., Muzzalupo I., et al. In: Il germoplasma olivicolo calabrese, Ist. Sper. Olivic.(2003), pp: 11.

2 - Perri E., Mazzotti F., Muzzalupo I., et al. In: Relazione attività, CO.R.ASS.OL. (2003).

3 - Muzzalupo I., Chiappetta A., Benincasa C., et al Sci Hortic-Amsterdam, (2010), 126: pp. 324-329. 


\section{"Ottobrina"}

Areal distribution or origin area: Liguria

Flesh/pit weight ratio: medium $(5,54 \pm 1,02)$

Oil content (\%): medium $(46,48 \pm 0,52)$

Purpose: oil

\section{Morphological characters}

Tree characters

Vigour: medium

Growth habit: spreading

Canopy-density: dense

\section{Leaf characters}

Blade length ( $\mathrm{cm})$ : medium $(5,07 \pm 0,32)$

Blade width $(\mathrm{cm})$ : medium $(1,31 \pm 0,19)$

Shape (length/width): elliptic

\section{Inflorescence characters}

Inflorescence length ( $\mathrm{cm})$ : long $(3,82 \pm 1,56)$

Number of flowers: medium $(21,17 \pm 2,68)$

\section{Fruit characters}

Fresh weight of 100 fruits $(\mathrm{g})$ : medium $(2,74 \pm 0,29)$

Shape (length/width): ovoid

Symmetry: slightly asymmetric

Position of maximum transverse diameter:

central

Apex: rounded

Base: rounded

Niplle: tenuous

Lenticels: many and small

\section{Pit characters}

Weight of 100 pits (g): high $(\mathbf{0 , 4 6} \pm \mathbf{0 , 0 6})$

Shape (length/width): elliptic

Mucron: obvious

Symmetry: slightly asymmetric

Position of maximum transverse diameter:

towards apex

Apex: pointed

Base: rounded

Surface: smooth

Number of grooves: low
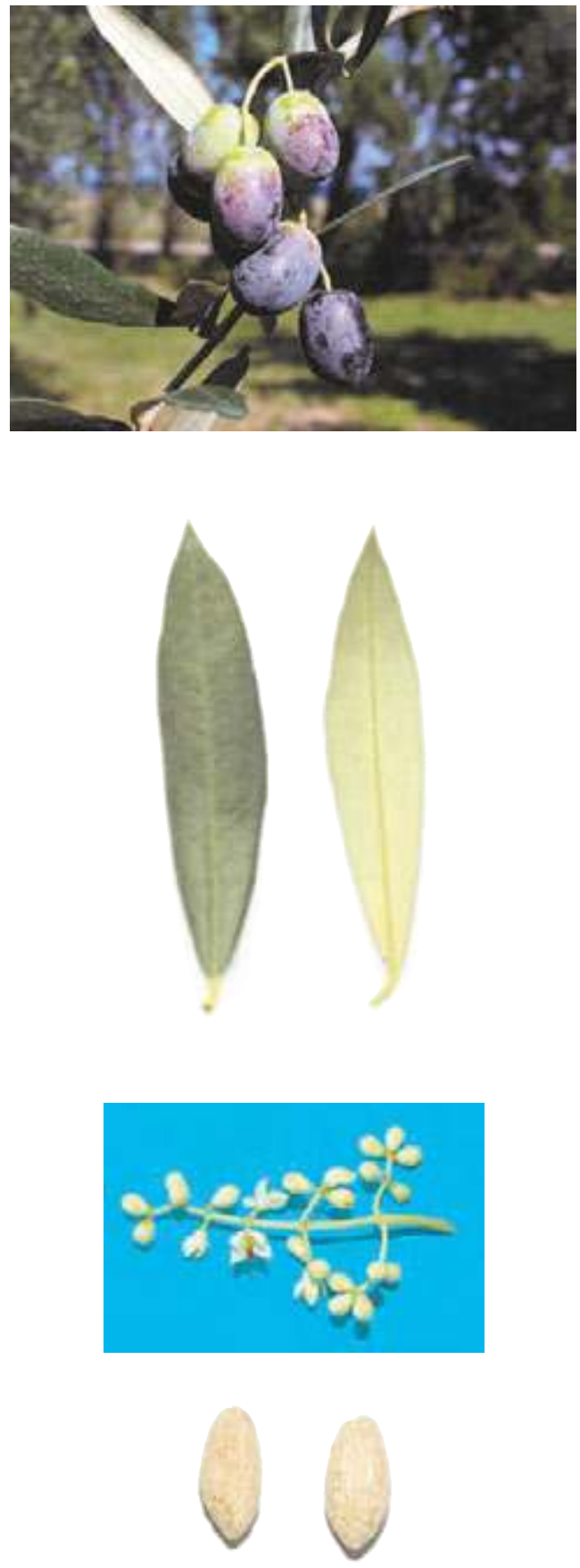


\section{Biochemical Characters}

\section{Fatty Acid Composition}

Table 1. Average values (express in $\% \pm$ standard deviations) of the fatty acids methyl esters and nutritional ratios obtained from single cultivar olive oils.

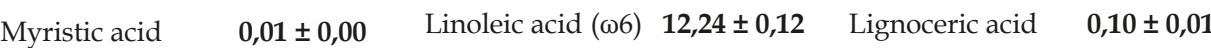

Palmitic acid $14,92 \pm 0,46 \quad$ Linolenic acid $(\omega 3) 0,59 \pm 0,01$

Palmitoleic acid

$1,95 \pm 0,08$

Arachic acid

$0,30 \pm 0,02$

Stearic acid

$1,78 \pm 0,05$

Eicosenoic acid

$0,30 \pm 0,02$

Unsat./satured

$4,78 \pm 0,15$

Oleic acid

$65,97 \pm 0,49$

Behenic acid

$0,08 \pm 0,00$

\section{Organoleptic oil values}

Sensory Analysis (Panel test)

Comment: fruity medium, with hints of almond and read sensations of grass and artichoke. Balanced taste sensation with a medium bitter and spicy. Medium fluidity.

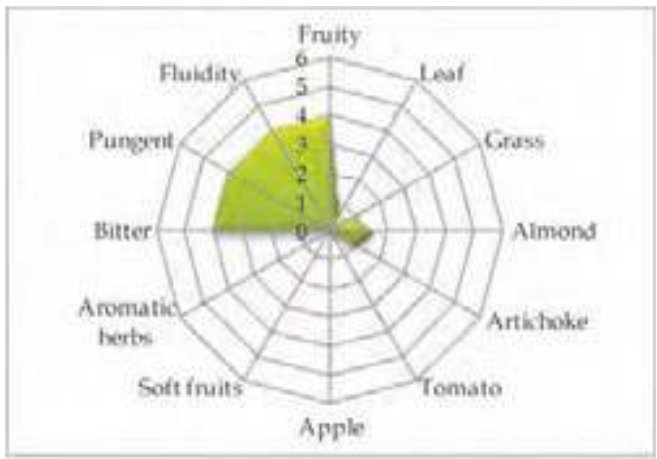

\section{Molecular Markers}

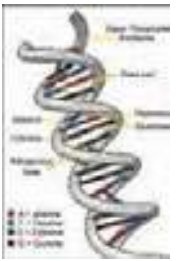

Table 2. Microsatellites (SSR) loci analyzed.

For each locus the allele size (expressed in base pairs) is reported.

$\begin{array}{cccccc}D C A 09 & \text { DCA18 } & \text { GAPU59 } & \text { GAPU71A } & \text { GUPA71B } & \text { GAPU103A } \\ \mathbf{1 6 2} \mathbf{- 1 6 6} & \mathbf{1 6 3 - \mathbf { 1 7 7 }} & \mathbf{2 0 8 - \mathbf { 2 1 2 }} & \mathbf{2 1 4} \mathbf{- 2 2 4} & \mathbf{1 2 4} \mathbf{- 1 4 4} & \mathbf{1 2 9} \mathbf{- 1 8 4} \\ \text { UDOO1 } & \text { UDOO3 } & \text { UDO12 } & \text { UDO28 } & \text { UDO39 } & \\ \mathbf{1 4 4 - \mathbf { 1 4 4 }} & \mathbf{1 5 0 - \mathbf { 1 5 0 }} & \mathbf{1 7 7 - \mathbf { 1 7 7 }} & \mathbf{1 8 2} \mathbf{- 2 1 0} & \mathbf{2 0 5 - \mathbf { 2 0 5 }} & \end{array}$

\section{References:}

1 - Various authors. In: Catalogo Nazionale delle Varietà di Olivo, University of Bari (in press), ISBN 978-88-8879397-9.

2 - Muzzalupo I., Salimonti A., Caravita M. A., et al. Adv. Hort. Sci. (2008), 22(2): pp. 129-135. 


\section{“Paesana bianca"}

(synonymy: Oliva bianca)

Areal distribution or origin area: Molise

Flesh/pit weight ratio: low $(\mathbf{4}, \mathbf{4 1} \pm \mathbf{0 , 1 4})$

Oil content (\%): medium $(45,54 \pm 0,49)$

Purpose: oil

\section{Morphological characters}

Tree characters

Vigour: medium-strong

Growth habit: spreading-erect

Canopy-density: medium-dense

\section{Leaf characters}

Blade length ( $\mathrm{cm})$ : medium $(5,82 \pm 0,58)$

Blade width (cm): broad $(\mathbf{1}, 64 \pm \mathbf{0 , 2 1})$

Shape (length/width): elliptic

\section{Inflorescence characters}

Inflorescence length (cm): long $(3,53 \pm 1,64)$

Number of flowers: low $(16,92 \pm 0,87)$

\section{Fruit characters}

Fresh weight of 100 fruits $(\mathrm{g})$ : medium $(2,49 \pm 0,14)$

Shape (length/width): ovoid

Symmetry: slightly asymmetric

Position of maximum transverse diameter:

central

Apex: rounded

Base: rounded

Niplle: absent

Lenticels: many and small

\section{Pit characters}

Weight of 100 pits (g): high $(\mathbf{0 , 4 6} \pm \mathbf{0 , 0 4})$

Shape (length/width): elliptic

Mucron: obvious

Symmetry: slightly asymmetric

Position of maximum transverse diameter:

towards apex

Apex: rounded

Base: rounded

Surface: smooth

Number of grooves: low
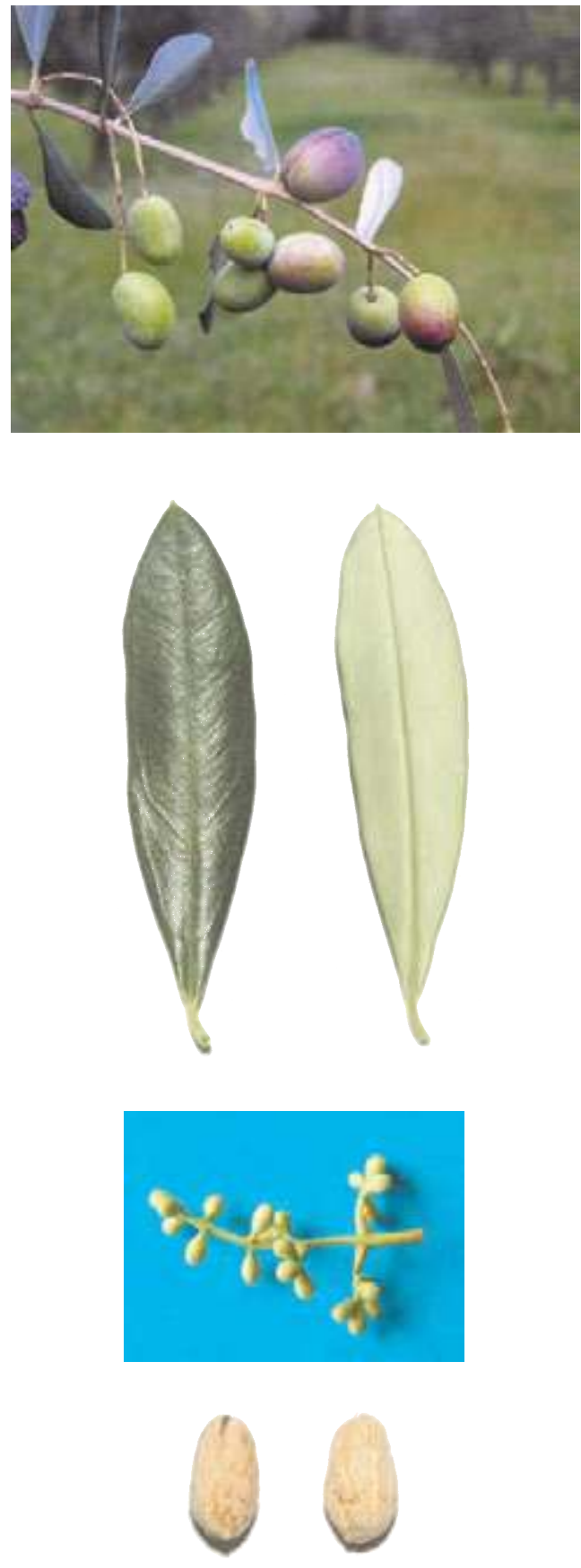


\section{Biochemical Characters}

\section{Fatty Acid Composition}

Table 1. Average values (express in $\% \pm$ standard deviations) of the fatty acids methyl esters and nutritional ratios obtained from single cultivar olive oils.

$\begin{array}{lllll}\text { Myristic acid } & \mathbf{0 , 0 1} \pm \mathbf{0 , 0 0} & \text { Linoleic acid }(\omega 6) & \mathbf{1 2 , 2 4} \pm \mathbf{0 , 1 2} \quad \text { Lignoceric acid } & \mathbf{0 , 1 0} \pm \mathbf{0 , 0 1}\end{array}$

Palmitic acid $14,92 \pm 0,46 \quad$ Linolenic acid $(\omega 3) 0,59 \pm 0,01$

Palmitoleic acid

$1,95 \pm 0,08$

Arachic acid

$0,30 \pm 0,02$

Stearic acid

$1,78 \pm 0,05$

Eicosenoic acid

$0,30 \pm 0,02$

Unsat./satured

$4,78 \pm 0,15$

Oleic acid

$65,97 \pm 0,49$

Behenic acid

$0,08 \pm 0,00$

\section{Organoleptic oil values}

Sensory Analysis (Panel test)

Comment: fruity medium, with hints of almond and read sensations of grass and artichoke. Balanced taste sensation with a medium bitter and spicy. Medium fluidity.

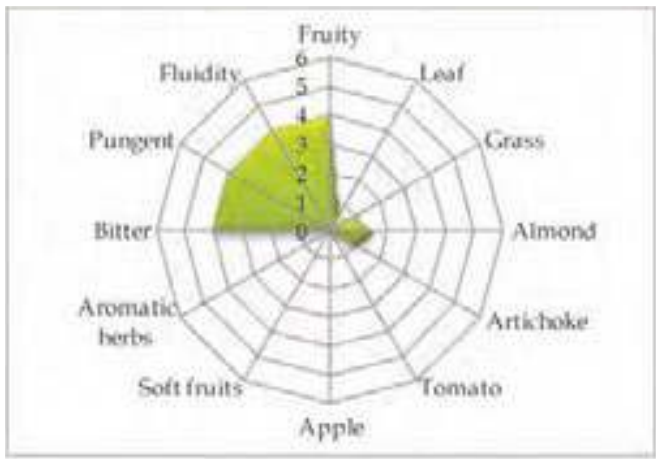

\section{Molecular Markers}

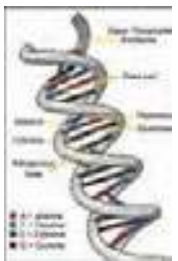

Table 2. Microsatellites (SSR) loci analyzed.

For each locus the allele size (expressed in base pairs) is reported.

$\begin{array}{cccccc}D C A 09 & \text { DCA18 } & \text { GAPU59 } & \text { GAPU71A } & \text { GUPA71B } & \text { GAPU103A } \\ \mathbf{1 6 2} \mathbf{- 1 6 6} & \mathbf{1 6 3 - \mathbf { 1 7 7 }} & \mathbf{2 0 8 - \mathbf { 2 1 2 }} & \mathbf{2 1 4} \mathbf{- 2 2 4} & \mathbf{1 2 4} \mathbf{- 1 4 4} & \mathbf{1 2 9} \mathbf{- 1 8 4} \\ \text { UDOO1 } & \text { UDOO3 } & \text { UDO12 } & \text { UDO28 } & \text { UDO39 } & \\ \mathbf{1 4 4 - \mathbf { 1 4 4 }} & \mathbf{1 5 0 - \mathbf { 1 5 0 }} & \mathbf{1 7 7 - \mathbf { 1 7 7 }} & \mathbf{1 8 2} \mathbf{- 2 1 0} & \mathbf{2 0 5 - \mathbf { 2 0 5 }} & \end{array}$

\section{References:}

1 - Various authors. In: Catalogo Nazionale delle Varietà di Olivo, University of Bari (in press), ISBN 978-88-8879397-9.

2 - Muzzalupo I., Salimonti A., Caravita M. A., et al. Adv. Hort. Sci. (2008), 22(2): pp. 129-135. 


\section{"Paesana nera"}

(synonymy: Ceciarola, Cicerola, Oliva nera, etc.)

Areal distribution or origin area: Molise

Flesh/pit weight ratio: medium $(\mathbf{6}, \mathbf{4 1} \pm \mathbf{0 , 0 1})$

Oil content (\%): medium $(47,93 \pm 0,06)$

Purpose: oil

\section{Morphological characters}

Tree characters

Vigour: medium-weak

Growth habit: spreading-erect

Canopy-density: medium

\section{Leaf characters}

Blade length (cm): medium $(5,68 \pm 0,46)$

Blade width $(\mathrm{cm})$ : medium $(1,35 \pm 0,18)$

Shape (length/width): elliptic-lanceolate

\section{Inflorescence characters}

Inflorescence length (cm): medium $(3,43 \pm 1,08)$

Number of flowers: medium $(23,18 \pm 1,07)$

\section{Fruit characters}

Fresh weight of 100 fruits (g): medium $(2,15 \pm 0,02)$

Shape (length/width): spherical

Symmetry: slightly asymmetric

Position of maximum transverse diameter:

central

Apex: rounded

Base: truncate

Niplle: absent

Lenticels: many and small

\section{Pit characters}

Weight of 100 pits (g): low $(0,29 \pm 0,04)$

Shape (length/width): ovoid

Mucron: obvious

Symmetry: slightly asymmetric

Position of maximum transverse diameter:

central

Apex: rounded

Base: rounded

Surface: rugose

Number of grooves: medium
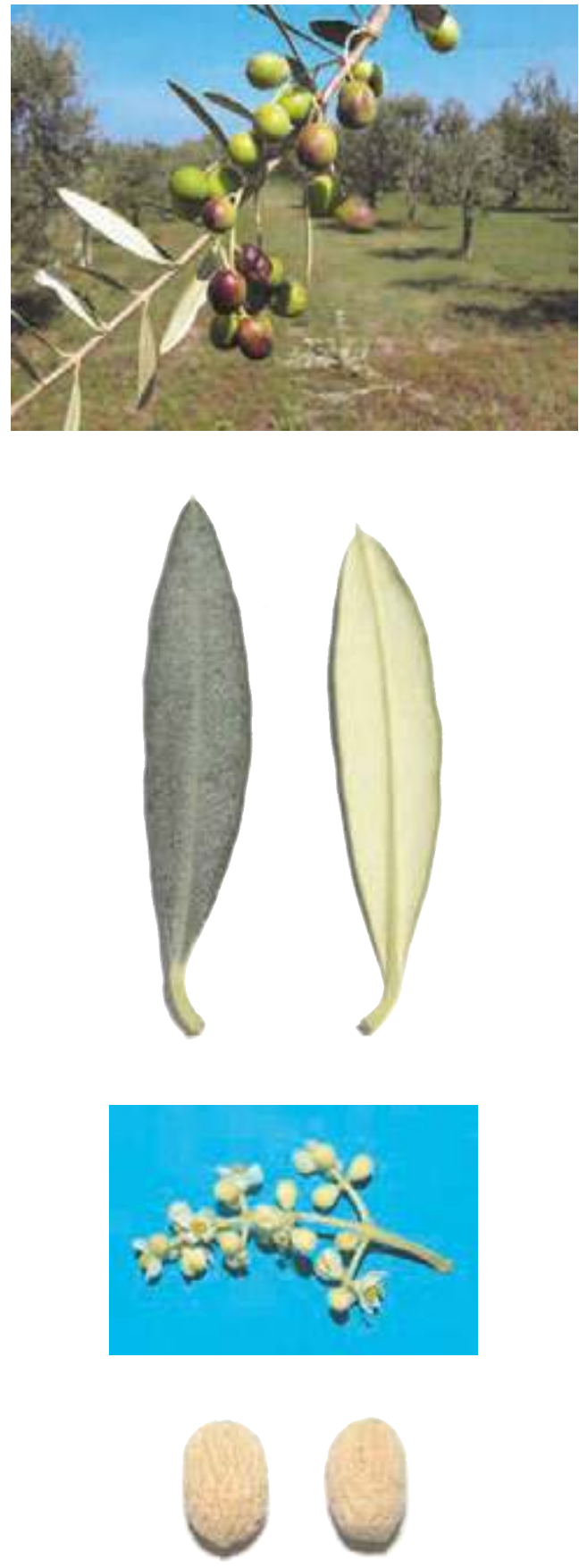


\section{Biochemical Characters}

\section{Fatty Acid Composition}

Table 1. Average values (express in $\% \pm$ standard deviations) of the fatty acids methyl esters and nutritional ratios obtained from single cultivar olive oils.

$\begin{array}{llllll}\text { Myristic acid } & \mathbf{0 , 0 1} \pm \mathbf{0 , 0 1} & \text { Linoleic acid }(\omega 6) & \mathbf{1 1 , 5 1} \pm \mathbf{0 , 2 6} & \text { Lignoceric acid } & \mathbf{0 , 0 6} \pm \mathbf{0 , 0 3} \\ \text { Palmitic acid } & \mathbf{1 4 , 6 6 \pm \mathbf { 1 , 5 2 }} & \text { Linolenic acid }(\omega 3) \mathbf{0 , 7 3} \pm \mathbf{0 , 0 2} & & \\ \text { Palmitoleic acid } & \mathbf{1 , 3 9} \pm \mathbf{0 , 1 1} & \text { Arachic acid } & \mathbf{0 , 3 0} \pm \mathbf{0 , 0 3} & \text { Unsat./satured } & \mathbf{4 , 6 8} \pm \mathbf{0 , 2 0} \\ \text { Stearic acid } & \mathbf{2 , 4 9 \pm \mathbf { 1 , 0 0 }} & \text { Eicosenoic acid } & \mathbf{0 , 0 3} \pm \mathbf{0 , 0 1} & \omega 6 / \omega 3 & \mathbf{1 5 , 8 7 \pm 0 , 0 9} \\ \text { Oleic acid } & \mathbf{6 7 , 5 8} \pm \mathbf{1 , 0 7} & \text { Behenic acid } & \mathbf{0 , 0 8} \pm \mathbf{0 , 0 3} & & \end{array}$

\section{Organoleptic oil values}

Sensory Analysis (Panel test)

Comment: fruity medium, with read sensations of artichoke, almond and grass. Balanced taste sensation with medium spicy and bitter. Medium fluidity.

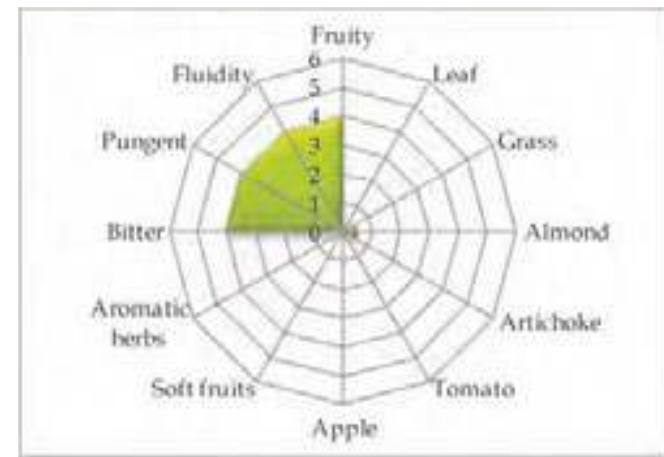

\section{Molecular Markers}

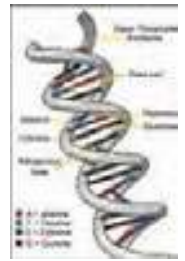

Table 2. Microsatellites (SSR) loci analyzed.

For each locus the allele size (expressed in base pairs) is reported.

$\begin{array}{cccccc}D C A 09 & \text { DCA18 } & \text { GAPU59 } & \text { GAPU71A } & \text { GUPA71B } & \text { GAPU103A } \\ \mathbf{1 6 2} \mathbf{- 2 0 4} & \mathbf{1 7 7 - \mathbf { 1 7 7 }} & \mathbf{2 1 2} \mathbf{- 2 2 2} & \mathbf{2 1 4 - \mathbf { 2 1 4 }} & \mathbf{1 2 4} \mathbf{- 1 3 0} & \mathbf{1 5 0 - \mathbf { 1 5 0 }} \\ \text { UDOO1 } & \text { UDOO3 } & \text { UDO12 } & \text { UDO28 } & \text { UDO39 } & \\ \mathbf{1 4 4 - \mathbf { 1 4 4 }} & \mathbf{1 5 0 - \mathbf { 1 8 2 }} & \mathbf{1 7 7 - \mathbf { 1 9 3 }} & \mathbf{1 5 4 - \mathbf { 2 0 5 }} & \mathbf{2 1 3 - \mathbf { 2 1 3 }} & \end{array}$

\section{References:}

1 - Ente regionale di sviluppo agricolo per il Molise. In: Il germoplasma dell'olivo nel Molise, Quaderno divulgativo dell'ERSA (2000), $\mathrm{n}^{\circ} 5$.

2 - Muzzalupo I., Salimonti A., Caravita M. A., et al. Adv. Hort. Sci. (2008), 22(2): pp. 129-135. 


\section{"Palmarola"}

(synonymy: Palmina, Palmina, Parmarola, Selvatica, etc.)

Areal distribution or origin area: Basilicata

Flesh/pit weight ratio: low $(3,65 \pm 0,24)$

Oil content (\%): medium $(47,56 \pm 0,90)$

Purpose: dual purpose

\section{Morphological characters}

Tree characters

Vigour: medium-strong

Growth habit: spreading-drooping

Canopy-density: medium

\section{Leaf characters}

Blade length $(\mathrm{cm})$ : medium $(5,64 \pm 0,56)$

Blade width (cm): broad $(\mathbf{1}, \mathbf{5 2} \pm \mathbf{0 , 2 3})$

Shape (length/width): elliptic

\section{Inflorescence characters}

Inflorescence length $(\mathrm{cm})$ : medium $(2,64 \pm 1,42)$

Number of flowers: low $(\mathbf{1 2 , 7 0} \pm \mathbf{2 , 3 7})$

\section{Fruit characters}

Fresh weight of 100 fruits (g): medium $(2,72 \pm 0,65)$

Shape (length/width): ovoid

Symmetry: slightly asymmetric

Position of maximum transverse diameter:

central

Apex: rounded

Base: rounded

Niplle: absent

Lenticels: few and small

\section{Pit characters}

Weight of 100 pits (g): high $(0,50 \pm 0,07)$

Shape (length/width): elliptic

Mucron: tenuous

Symmetry: slightly asymmetric

Position of maximum transverse diameter:

central

Apex: pointed

Base: rounded

Surface: rugose

Number of grooves: medium
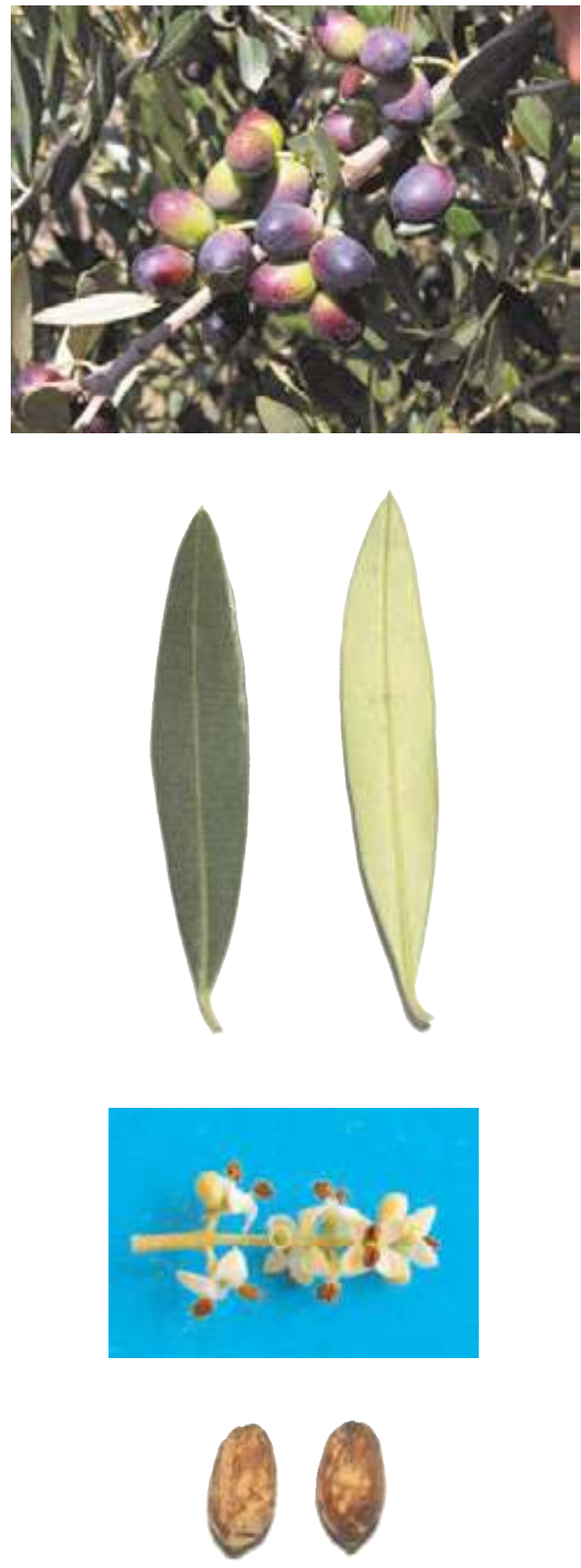


\section{Biochemical Characters}

\section{Fatty Acid Composition}

Table 1. Average values (express in $\% \pm$ standard deviations) of the fatty acids methyl esters and nutritional ratios obtained from single cultivar olive oils.

$\begin{array}{llllll}\text { Myristic acid } & \mathbf{0 , 0 1} \pm \mathbf{0 , 0 0} & \text { Linoleic acid }(\omega 6) & \mathbf{6 , 3 7} \pm \mathbf{1 , 9 4} & \text { Lignoceric acid } & \mathbf{0 , 0 4 \pm 0 , 0 1} \\ \text { Palmitic acid } & \mathbf{1 3 , 0 2} \pm \mathbf{0 , 9 5} & \text { Linolenic acid }(\omega 3) \mathbf{0 , 7 4} \pm \mathbf{0 , 1 0} & & \\ \text { Palmitoleic acid } & \mathbf{1 , 7 1} \pm \mathbf{0 , 2 2} & \text { Arachic acid } & \mathbf{0 , 2 5} \pm \mathbf{0 , 0 7} & \text { Unsat./satured } & \mathbf{5 , 6 1} \pm \mathbf{0 , 4 1} \\ \text { Stearic acid } & \mathbf{1 , 6 5} \pm \mathbf{0 , 1 3} & \text { Eicosenoic acid } & \mathbf{0 , 1 4} \pm \mathbf{0 , 1 4} & \omega 6 / \omega 3 & \mathbf{8 , 4 5} \pm \mathbf{1 , 6 2} \\ \text { Oleic acid } & \mathbf{7 4 , 1 1} \pm \mathbf{2 , 4 3} & \text { Behenic acid } & \mathbf{0 , 0 7} \pm \mathbf{0 , 0 2} & & \end{array}$

\section{Organoleptic oil values}

Sensory Analysis (Panel test)

Comment: fruity medium-high, with taste of almond and tomato, read sensations of grass, leaf, and aromatic herbs. Balanced taste sensation with medium-light bitter and medium spicy. Medium-high fluidity.

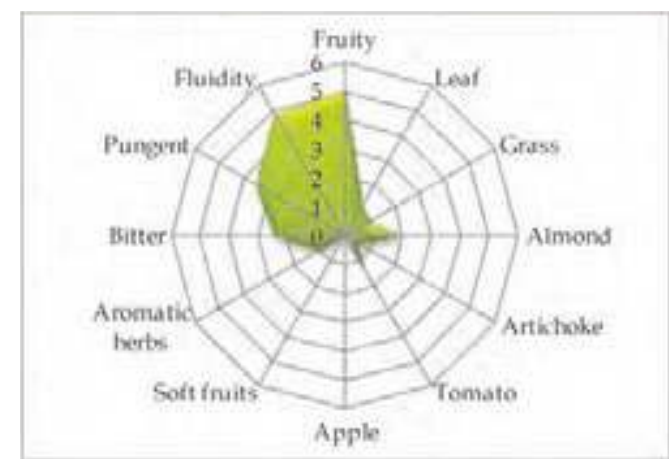

\section{Molecular Markers}

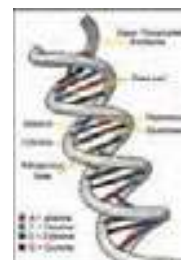

Table 2. Microsatellites (SSR) loci analyzed. For each locus the allele size (expressed in base pairs) is reported.

$\begin{array}{cccccc}D C A 09 & \text { DCA18 } & \text { GAPU59 } & \text { GAPU71A } & \text { GUPA71B } & \text { GAPU103A } \\ \mathbf{1 9 4 - \mathbf { 1 9 8 }} & \mathbf{1 7 7 - \mathbf { 1 7 9 }} & \mathbf{2 0 8 - \mathbf { 2 2 2 }} & \mathbf{2 1 4 - \mathbf { 2 2 4 }} & \mathbf{1 2 4 - \mathbf { 1 4 4 }} & \mathbf{1 5 0 - \mathbf { 1 5 7 }} \\ \text { UDOO1 } & \text { UDOO3 } & \text { UDO12 } & \text { UDO28 } & \text { UDO39 } & \\ \mathbf{1 4 4 - \mathbf { 1 4 4 }} & \mathbf{1 4 3 - \mathbf { 1 4 3 }} & \mathbf{1 6 6 - \mathbf { 1 6 6 }} & \mathbf{1 8 2} \mathbf{- 2 1 0} & \mathbf{2 0 0 - \mathbf { 2 0 0 }} & \end{array}$

\section{References:}

1 - Rotundo A., Marone E. In: Il germoplasma olivicolo lucano, Olita - Potenza (2002), pp. 127-130.

2 - Rotundo A., Perri E.,Muzzalupo I., et al. In: Il germoplasma olivicolo meridionale, (2012 in press).

3 - Muzzalupo I., Stefanizzi F., Perri E. HortScience (2009), 44: pp. 582-588. 


\section{"Pasola"}

(synonymy: Fasola,Passula.)

Areal distribution or origin area: Puglia

Flesh/pit weight ratio: medium $(5,36 \pm 0,69)$

Oil content (\%): medium $(44,38 \pm 0,69)$

Purpose: table

\section{Morphological characters}

Tree characters

Vigour: strong

Growth habit: spreading-erect

Canopy-density: medium

\section{Leaf characters}

Blade length $(\mathrm{cm})$ : medium $(5,38 \pm 0,77)$

Blade width $(\mathrm{cm})$ : medium $(\mathbf{1 , 4 8} \pm \mathbf{0 , 2 0})$

Shape (length/width): elliptic

\section{Inflorescence characters}

Inflorescence length (cm): medium $(2,75 \pm 0,44)$

Number of flowers: medium $(\mathbf{1 6 , 7 5} \pm 2,66)$

\section{Fruit characters}

Fresh weight of 100 fruits $(\mathrm{g})$ : medium $(3,63 \pm 0,38)$

Shape (length/width): spherical

Symmetry: slightly asymmetric

Position of maximum transverse diameter:

central

Apex: rounded

Base: truncate

Niplle: absent

Lenticels: many and small

\section{Pit characters}

Weight of 100 pits (g): medium $(0,53 \pm \mathbf{0 , 0 6})$

Shape (length/width): elliptic

Mucron: obvious

Symmetry: symmetric

Position of maximum transverse diameter:

towards apex

Apex: rounded

Base: pointed

Surface: rugose

Number of grooves: high
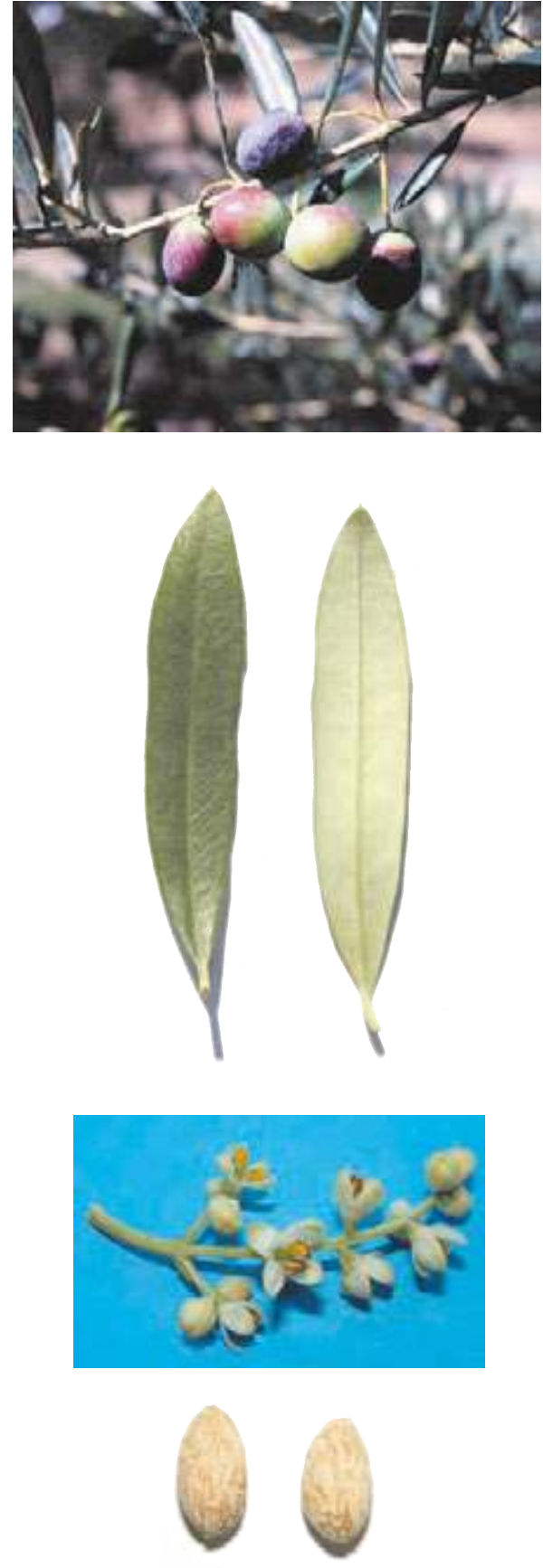


\section{Biochemical Characters}

\section{Fatty Acid Composition}

Table 1. Average values (express in $\% \pm$ standard deviations) of the fatty acids methyl esters and nutritional ratios obtained from single cultivar olive oils.

$\begin{array}{llllll}\text { Myristic acid } & \mathbf{0 , 0 1} \pm \mathbf{0 , 0 0} & \text { Linoleic acid }(\omega 6) & \mathbf{9 , 8 3} \pm \mathbf{0 , 7 6} & \text { Lignoceric acid } & \mathbf{0 , 0 3} \pm \mathbf{0 , 0 2} \\ \text { Palmitic acid } & \mathbf{1 0 , 8 9} \pm \mathbf{1 , 2 7} & \text { Linolenic acid }(\omega 3) \mathbf{0 , 8 8} \pm \mathbf{0 , 0 7} & & \\ \text { Palmitoleic acid } & \mathbf{0 , 8 6} \pm \mathbf{0 , 4 9} & \text { Arachic acid } & \mathbf{0 , 3 1} \pm \mathbf{0 , 0 5} & \text { Unsat./satured } & \mathbf{6 , 5 1} \pm \mathbf{0 , 4 6} \\ \text { Stearic acid } & \mathbf{2 , 1 2} \pm \mathbf{0 , 5 9} & \text { Eicosenoic acid } & \mathbf{0 , 1 1} \pm \mathbf{0 , 1 7} & \omega 6 / \omega 3 & \mathbf{1 1 , 1 4} \pm \mathbf{0 , 8 8} \\ \text { Oleic acid } & \mathbf{7 4 , 1 5 \pm \mathbf { 1 , 0 5 }} & \text { Behenic acid } & \mathbf{0 , 0 8} \pm \mathbf{0 , 0 2} & & \end{array}$

\section{Organoleptic oil values}

Sensory Analysis (Panel test)

Comment: fruity medium, with hints of artichoke and almond. Balanced in flavours, with hints of bitter and spicy medium-light intensity. Medium fluidity.

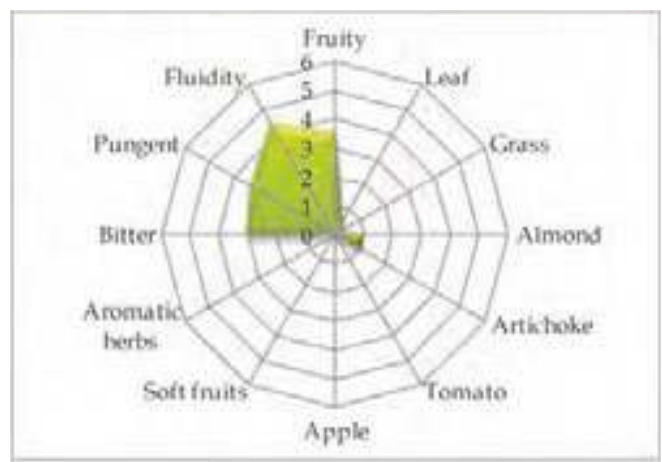

\section{Molecular Markers}

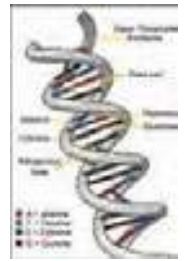

Table 2. Microsatellites (SSR) loci analyzed.

For each locus the allele size (expressed in base pairs) is reported.

$\begin{array}{cccccc}D C A 09 & \text { DCA18 } & \text { GAPU59 } & \text { GAPU71A } & \text { GUPA71B } & \text { GAPU103A } \\ \mathbf{1 9 8} \mathbf{- 2 0 6} & \mathbf{1 7 7 - \mathbf { 1 7 7 }} & \mathbf{2 0 8 - \mathbf { 2 1 8 }} & \mathbf{2 1 4} \mathbf{- 2 1 4} & \mathbf{1 2 4} \mathbf{- 1 4 4} & \mathbf{1 5 0 - \mathbf { 1 5 7 }} \\ \text { UDOO1 } & \text { UDOO3 } & \text { UDO12 } & \text { UDO28 } & \text { UDO39 } & \\ \mathbf{1 4 4 - \mathbf { 1 4 4 }} & \mathbf{1 4 3 - \mathbf { 1 4 3 }} & \mathbf{1 7 7 - \mathbf { 1 7 7 }} & \mathbf{1 5 0 - \mathbf { 1 8 2 }} & \mathbf{1 7 0 - \mathbf { 1 7 0 }} & \end{array}$

\section{References:}

1 - Lombardo N., Perri E., Muzzalupo I., et al. In: Contributo alla caratterizzazione del germoplasma olivicolo pugliese, Ist. Sper. Olivic. (2004), pp. 77-80.

2 - Muzzalupo I., Stefanizzi F., Perri E. HortScience (2009), 44: pp. 582-588. 


\section{"Passulunara"}

(synonymy: Palermitana, Passalunara, etc.)

Areal distribution or origin area: Sicilia

Flesh/pit weight ratio: high $(8,37 \pm 1,20)$

Oil content (\%): medium $(49,54 \pm 0,20)$

Purpose: dual purpose

\section{Morphological characters}

Tree characters

Vigour: weak

Growth habit: spreading

Canopy-density: medium

\section{Leaf characters}

Blade length $(\mathrm{cm})$ : medium $(5,65 \pm 0,56)$

Blade width $(\mathrm{cm})$ : medium $(\mathbf{1 , 2 9} \pm \mathbf{0 , 1 2})$

Shape (length/width): elliptic

\section{Inflorescence characters}

Inflorescence length (cm): medium $(3,20 \pm 1,60)$

Number of flowers: low $(\mathbf{1 1}, \mathbf{8 7} \pm \mathbf{2 , 0 3})$

\section{Fruit characters}

Fresh weight of 100 fruits (g): high $(5,08 \pm 0,49)$

Shape (length/width): spherical

Symmetry: slightly asymmetric

Position of maximum transverse diameter:

towards base

Apex: rounded

Base: truncate

Niplle: absent

Lenticels: few and large

\section{Pit characters}

Weight of 100 pits (g): high $(0,55 \pm \mathbf{0 , 0 2})$

Shape (length/width): ovoid

Mucron: tenuous

Symmetry: slightly asymmetric

Position of maximum transverse diameter:

towards apex

Apex: rounded

Base: rounded

Surface: smooth

Number of grooves: medium
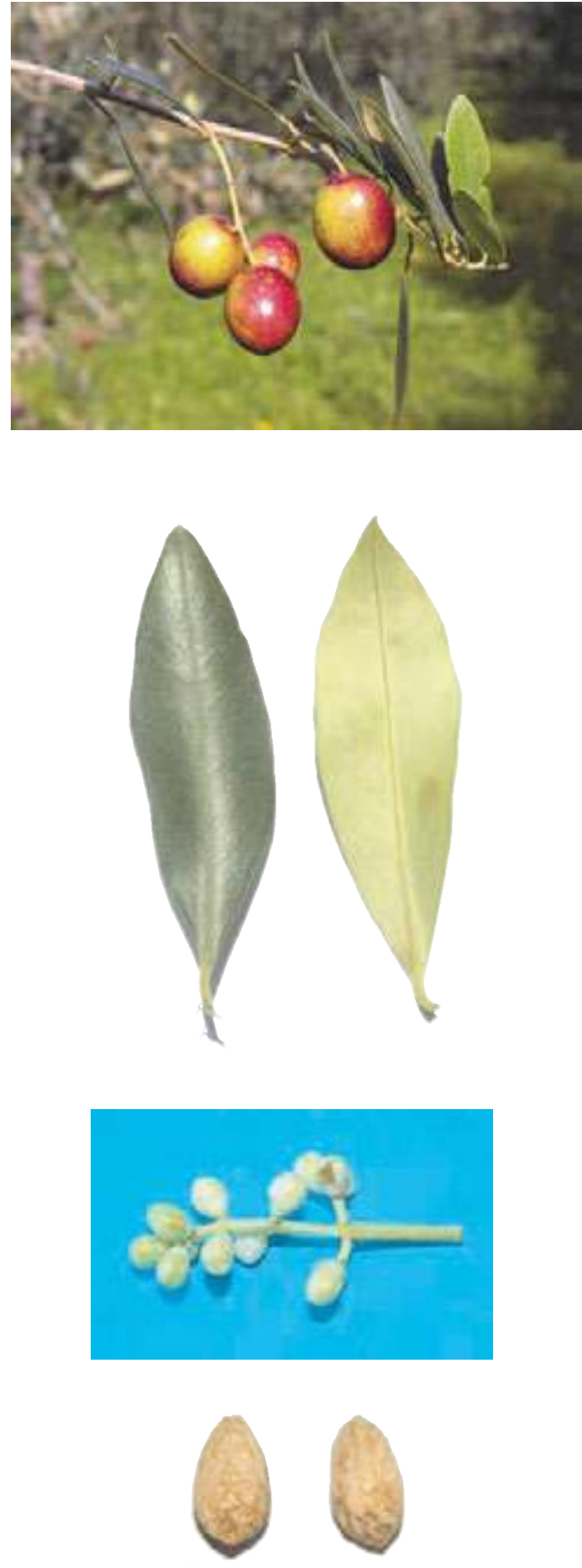


\section{Biochemical Characters}

\section{Fatty Acid Composition}

Table 1. Average values (express in $\% \pm$ standard deviations) of the fatty acids methyl esters and nutritional ratios obtained from single cultivar olive oils.

$\begin{array}{lllll}\text { Myristic acid } & \mathbf{0 , 0 1} \pm \mathbf{0 , 0 0} \quad \text { Linoleic acid }(\omega 6) & \mathbf{1 1 , 7 6} \pm \mathbf{0 , 2 9} \quad \text { Lignoceric acid } \quad 0,07 \pm 0,01\end{array}$

Palmitic acid $10,36 \pm 0,38 \quad$ Linolenic acid $(\omega 3) 0,33 \pm 0,02$

Palmitoleic acid

$0,58 \pm 0,03$

Arachic acid

$0,61 \pm 0,07$

Unsat./satured

$6,81 \pm 0,21$

Stearic acid

$2,05 \pm 0,03$

Eicosenoic acid

$0,40 \pm 0,02$

Oleic acid

$73,40 \pm 0,07$

Behenic acid

$0,11 \pm 0,01$

\section{Organoleptic oil values}

Sensory Analysis (Panel test)

Comment: fruity medium, with taste of artichoke and tomato, read sensations of almond. Balanced taste sensation with medium-light bitter and spicy. Medium fluidity.

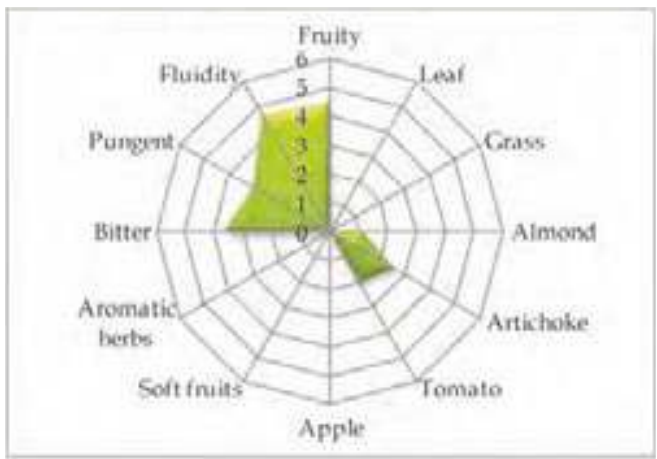

\section{Molecular Markers}

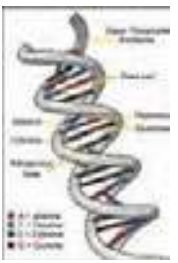

Table 2. Microsatellites (SSR) loci analyzed.

For each locus the allele size (expressed in base pairs) is reported.

$\begin{array}{cccccc}D C A 09 & D C A 18 & \text { GAPU59 } & \text { GAPU71A } & \text { GUPA71B } & \text { GAPU103A } \\ \mathbf{1 7 6 - 1 8 2} & \mathbf{1 7 9 - \mathbf { 1 7 9 }} & \mathbf{2 1 2} \mathbf{- 2 2 2} & \mathbf{2 1 0 - \mathbf { 2 1 0 }} & \mathbf{1 2 4} \mathbf{- 1 4 4} & \mathbf{1 5 9} \mathbf{- 1 8 4} \\ \text { UDOO1 } & \text { UDOO3 } & \text { UDO12 } & \text { UDO28 } & \text { UDO39 } & \\ \mathbf{1 4 4 - \mathbf { 1 4 4 }} & \mathbf{1 5 0 - \mathbf { 1 5 0 }} & \mathbf{1 6 6 - \mathbf { 1 9 3 }} & \mathbf{1 8 2} \mathbf{- 1 8 2} & \mathbf{1 0 8 - \mathbf { 2 0 5 }} & \end{array}$

\section{References:}

1 - Caruso T., Cartabellotta D., Motisi A., et all. In: Cultivar di olivo siciliane, Università degli Studi di Palermo (2007), pp. 59-64.

2 - Muzzalupo I., Stefanizzi F., Perri E. HortScience (2009), 44: pp. 582-588. 


\section{"Pennulara"}

(synonymy: Caccuri, Nostrale di Caccuri.)

Areal distribution or origin area: Calabria

Flesh/ pit weight ratio: high $(8,07 \pm 0,80)$

Oil content (\%): medium $(49,73 \pm 1,98)$

Purpose: oil

\section{Morphological characters}

Tree characters

Vigour: medium-weak

Growth habit: spreading

Canopy-density: medium

\section{Leaf characters}

Blade length (cm): medium $(6,08 \pm 0,98)$

Blade width $(\mathrm{cm})$ : medium $(1,30 \pm 0,09)$

Shape (length/width): elliptic-lanceolate

\section{Inflorescence characters}

Inflorescence length (cm): short $(2,43 \pm 1,30)$

Number of flowers: low $(10,64 \pm 1,03)$

\section{Fruit characters}

Fresh weight of 100 fruits (g): high $(5,66 \pm 1,47)$

Shape (length/width): spherical

Symmetry: slightly asymmetric

Position of maximum transverse diameter:

\section{towards base}

Apex: pointed

Base: truncate

Niplle: absent

Lenticels: many and small

\section{Pit characters}

Weight of 100 pits (g): high $(\mathbf{0 , 6 4} \pm \mathbf{0 , 2 2})$

Shape (length/width): ovoid

Mucron: obvious

Symmetry: slightly asymmetric

Position of maximum transverse diameter:

towards base

Apex: pointed

Base: rounded

Surface: scabrous

Number of grooves: low
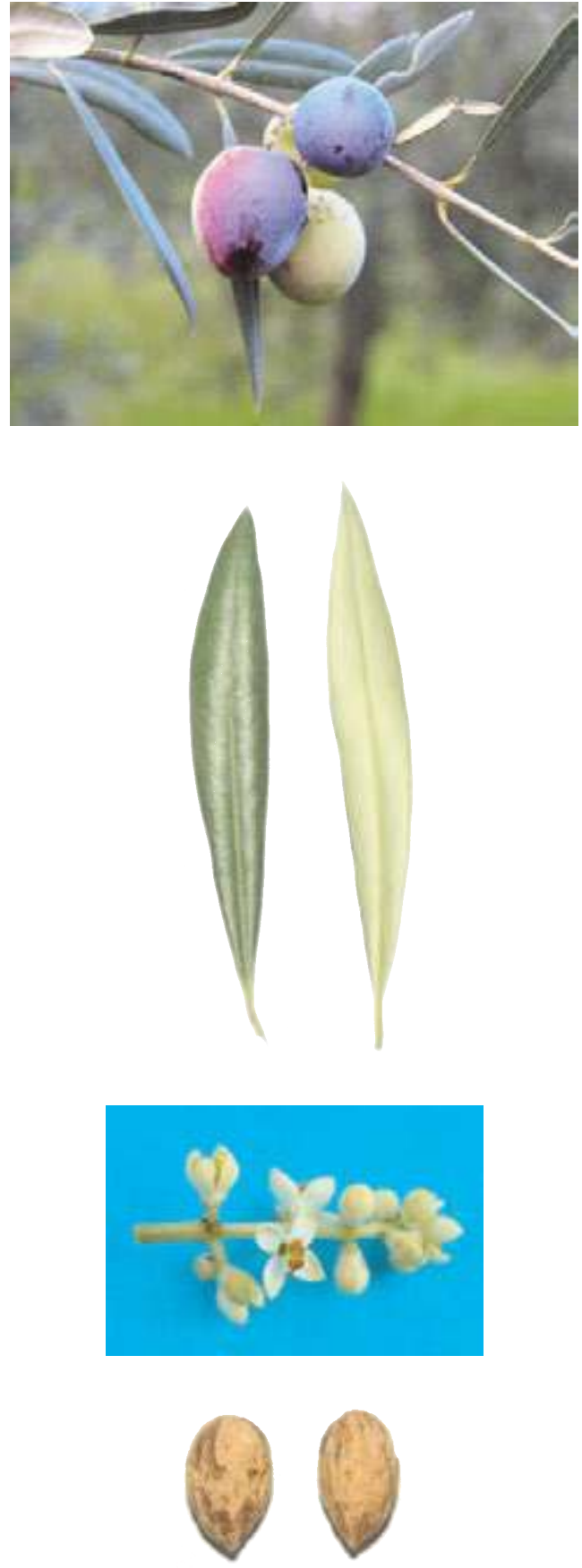


\section{Biochemical Characters}

\section{Fatty Acid Composition}

Table 1. Average values (express in $\% \pm$ standard deviations) of the fatty acids methyl esters and nutritional ratios obtained from single cultivar olive oils.

$\begin{array}{llllll}\text { Myristic acid } & \mathbf{0 , 0 1} \pm \mathbf{0 , 0 1} & \text { Linoleic acid }(\omega 6) & \mathbf{8 , 4 8} \pm \mathbf{0 , 7 3} & \text { Lignoceric acid } & \mathbf{0 , 0 6} \pm \mathbf{0 , 0 1} \\ \text { Palmitic acid } & \mathbf{8 , 5 7} \pm \mathbf{0 , 6 6} & \text { Linolenic acid }(\omega 3) \mathbf{0 , 6 9} \pm \mathbf{0 , 1 8} & & \\ \text { Palmitoleic acid } & \mathbf{0 , 4 4} \pm \mathbf{0 , 0 3} & \text { Arachic acid } & \mathbf{0 , 3 2} \pm \mathbf{0 , 0 1} & \text { Unsat./satured } & \mathbf{7 , 8 7} \pm \mathbf{0 , 4 8} \\ \text { Stearic acid } & \mathbf{2 , 4 2} \pm \mathbf{0 , 0 3} & \text { Eicosenoic acid } & \mathbf{0 , 0 3} \pm \mathbf{0 , 0 0} & \omega 6 / \omega 3 & \mathbf{1 2 , 4 9} \pm \mathbf{2 , 1 3} \\ \text { Oleic acid } & \mathbf{7 8 , 5 5} \pm \mathbf{1 , 0 5} & \text { Behenic acid } & \mathbf{0 , 1 0} \pm \mathbf{0 , 0 3} & & \end{array}$

\section{Organoleptic oil values}

Sensory Analysis (Panel test)

Comment: fruity medium, with taste of grass, read sensations of almond, artichoke and leaves. Balanced taste sensation with medium bitter and spicy. Medium fluidity.

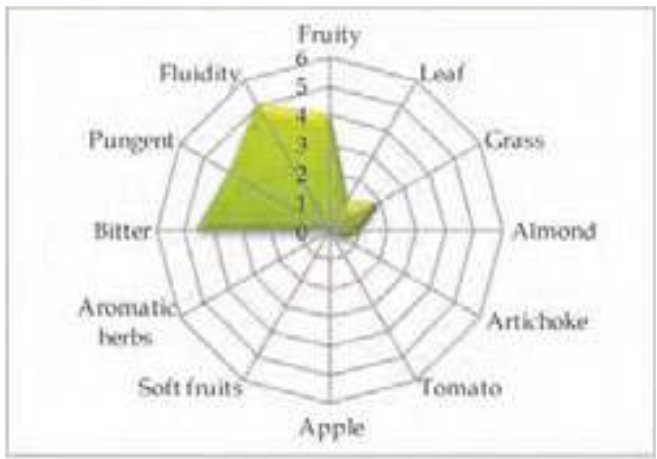

\section{Molecular Markers}

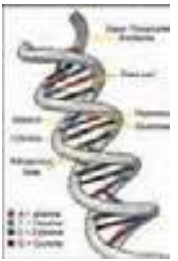

Table 2. Microsatellites (SSR) loci analyzed.

For each locus the allele size (expressed in base pairs) is reported.

$\begin{array}{cccccc}D C A 09 & \text { DCA18 } & \text { GAPU59 } & \text { GAPU71A } & \text { GUPA71B } & \text { GAPU103A } \\ \mathbf{1 9 8} \mathbf{- 2 0 6} & \mathbf{1 7 9 - \mathbf { 1 7 9 }} & \mathbf{2 1 2} \mathbf{- 2 2 2} & \mathbf{2 1 4} \mathbf{- 2 1 4} & \mathbf{1 3 0 - \mathbf { 1 4 4 }} & \mathbf{1 5 0 - \mathbf { 1 5 9 }} \\ \text { UDOO1 } & \text { UDOO3 } & \text { UDO12 } & \text { UDO28 } & \text { UDO39 } & \\ \mathbf{1 4 0 - \mathbf { 1 5 0 }} & \mathbf{1 4 3 - \mathbf { 1 4 3 }} & \mathbf{1 7 7 - \mathbf { 1 9 3 }} & \mathbf{1 4 3 - \mathbf { 1 8 2 }} & \mathbf{2 0 9 - \mathbf { 2 2 0 }} & \end{array}$

\section{References:}

1 - Lombardo N., Perri E., Muzzalupo I., et al. In: Il germoplasma olivicolo calabrese, Ist. Sper. Olivic.(2003), pp: 25.

2 - Perri E., Mazzotti F., Muzzalupo I., et al. In: Relazione attività, CO.R.ASS.OL. (2003).

3 - Muzzalupo I., Chiappetta A., Benincasa C., et al Sci Hortic-Amsterdam, (2010), 126: pp. 324-329. 


\section{"Peranzana "}

(synonymy: Francese, Provenzale, Provenzana, Tondina, etc.)

Areal distribution or origin area: Puglia

Flesh/pit weight ratio: medium $(4,86 \pm 1,08)$

Oil content (\%): low $(40,17 \pm 2,01)$

Purpose: dual purpose

\section{Morphological characters}

Tree characters

Vigour: weak

Growth habit: spreading

Canopy-density: medium

\section{Leaf characters}

Blade length (cm): medium $(5,51 \pm 0,60)$

Blade width $(\mathrm{cm})$ : medium $(1,46 \pm 0,13)$

Shape (length/width): elliptic-lanceolate

\section{Inflorescence characters}

Inflorescence length (cm): $\operatorname{shotr}(2,41 \pm 0,49)$

Number of flowers: medium $(17,09 \pm 1,33)$

\section{Fruit characters}

Fresh weight of 100 fruits $(\mathrm{g})$ : medium $(3,10 \pm 0,36)$

Shape (length/width): spherical

Symmetry: symmetric

Position of maximum transverse diameter:

central

Apex: rounded

Base: rounded

Niplle: absent

Lenticels: many and small

\section{Pit characters}

Weight of 100 pits (g): high $(0,55 \pm 0,07)$

Shape (length/width): ovoid

Mucron: obvious

Symmetry: symmetric

Position of maximum transverse diameter:

towards apex

Apex: rounded

Base: rounded

Surface: rugose

Number of grooves: medium
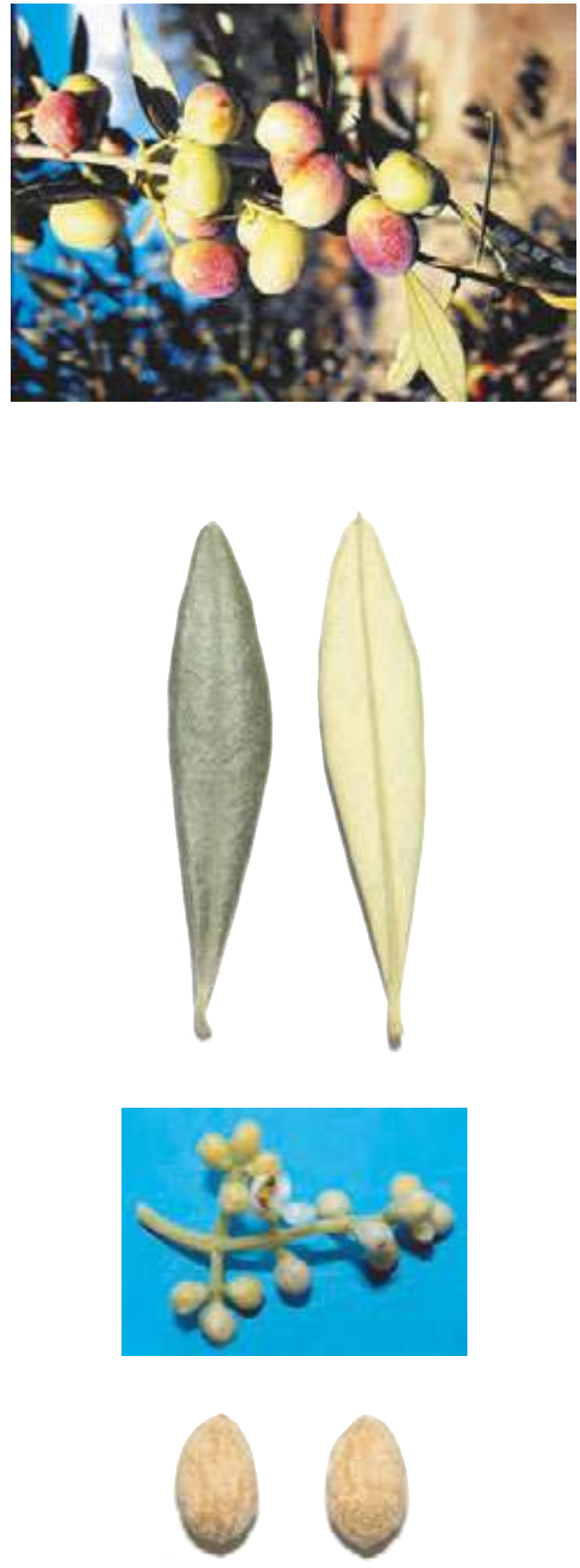


\section{Biochemical Characters}

\section{Fatty Acid Composition}

Table 1. Average values (express in $\% \pm$ standard deviations) of the fatty acids methyl esters and nutritional ratios obtained from single cultivar olive oils.

$\begin{array}{llllll}\text { Myristic acid } & \mathbf{0 , 0 1} \pm \mathbf{0 , 0 0} & \text { Linoleic acid }(\omega 6) & \mathbf{5 , 4 4} \pm \mathbf{0 , 3 7} & \text { Lignoceric acid } & \mathbf{0 , 0 3} \pm \mathbf{0 , 0 1} \\ \text { Palmitic acid } & \mathbf{1 1 , 4 7} \pm \mathbf{0 , 8 9} & \text { Linolenic acid }(\omega 3) & \mathbf{0 , 9 5} \pm \mathbf{0 , 0 5} & & \\ \text { Palmitoleic acid } & \mathbf{0 , 6 1} \pm \mathbf{0 , 2 4} & \text { Arachic acid } & \mathbf{0 , 2 9} \pm \mathbf{0 , 0 7} & \text { Unsat./satured } & \mathbf{6 , 3 1} \pm \mathbf{0 , 4 2} \\ \text { Stearic acid } & \mathbf{1 , 9 4} \pm \mathbf{0 , 2 4} & \text { Eicosenoic acid } & \mathbf{0 , 0 2} \pm \mathbf{0 , 0 2} & \omega 6 / \omega 3 & \mathbf{5 , 7 4} \pm \mathbf{0 , 1 6} \\ \text { Oleic acid } & \mathbf{7 8 , 6 6 \pm \mathbf { 1 , 2 0 }} & \text { Behenic acid } & \mathbf{0 , 0 8} \pm \mathbf{0 , 0 3} & & \end{array}$

\section{Organoleptic oil values}

Sensory Analysis (Panel test)

Comment: medium fruity, with good sensation of grass, less almond and artichoke. Balanced taste sensation, with bitter and spicy medium. Medium fluidity.

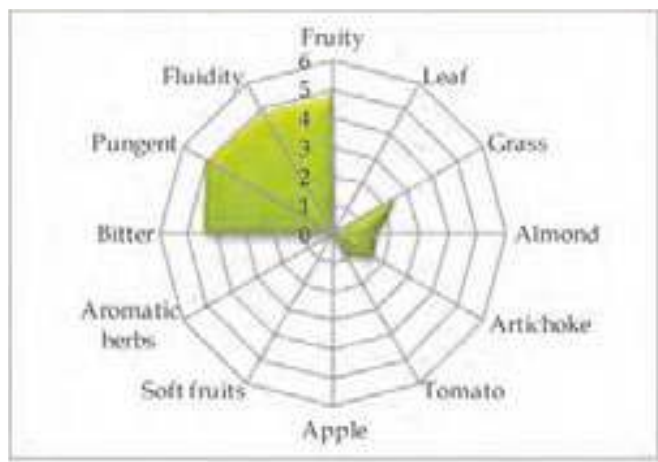

\section{Molecular Markers}

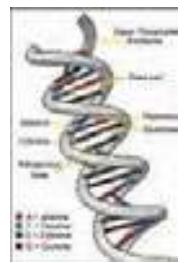

Table 2. Microsatellites (SSR) loci analyzed.

For each locus the allele size (expressed in base pairs) is reported.

\begin{tabular}{|c|c|c|c|c|c|}
\hline$D C A 09$ & DCA18 & GAPU59 & GAPU71A & $G U P A 71 B$ & GAPU103A \\
\hline $162-172$ & $181-181$ & $212-222$ & $212-214$ & 130 - 144 & $170-170$ \\
\hline UDO01 & UDO03 & UDO12 & UDO28 & UDO39 & \\
\hline $144-144$ & $182-182$ & $166-193$ & $143-205$ & $108-205$ & \\
\hline
\end{tabular}

\section{References:}

1 - Lombardo N., Perri E., Muzzalupo I., et al. In: Contributo alla caratterizzazione del germoplasma olivicolo pugliese, Ist. Sper. Olivic. (2004), pp. 81-84.

2 - Muzzalupo I., Stefanizzi F., Perri E. HortScience (2009), 44: pp. 582-588. 


\section{"Perciasacchi "}

Areal distribution or origin area: Campania Flesh/pit weight ratio: low $(4,01 \pm 0,03)$ Oil content (\%): medium $(40,27 \pm 0,55)$

Purpose: oil

\section{Morphological characters}

Tree characters

Vigour: medium-weak

Growth habit: spreading

Canopy-density: medium

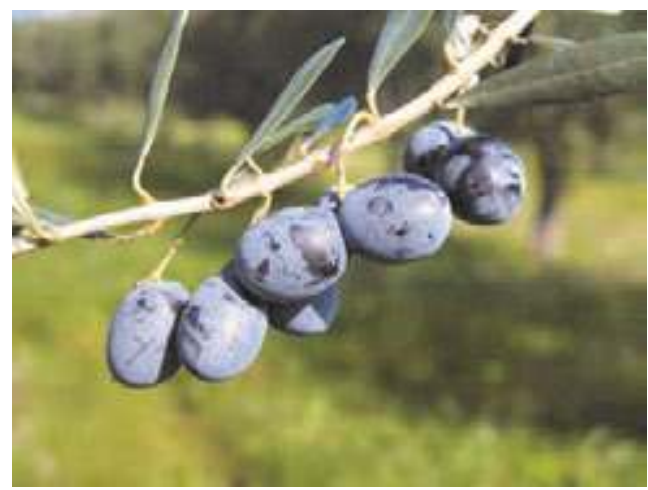

\section{Leaf characters}

Blade length (cm): medium $(6,70 \pm 0,63)$

Blade width (cm): medium $(1,43 \pm 0,16)$

Shape (length/width): elliptic-lanceolate

\section{Inflorescence characters}

Inflorescence length $(\mathrm{cm})$ : medium $(2,70 \pm 0,46)$

Number of flowers: medium $(20,97 \pm 1,29)$

\section{Fruit characters}

Fresh weight of 100 fruits (g): medium $(2,74 \pm 0,11)$

Shape (length/width): elongated

Symmetry: asymmetric

Position of maximum transverse diameter:

towards apex

Apex: rounded

Base: rounded

Niplle: absent

Lenticels: many and small

\section{Pit characters}

Weight of 100 pits (g): high $(0,55 \pm \mathbf{0 , 0 2})$

Shape (length/width): elongated

Mucron: obvious

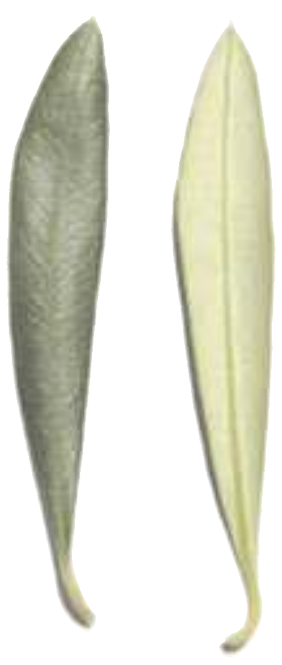

Symmetry: slightly asymmetric

Position of maximum transverse diameter:

towards apex

Apex: rounded

Base: pointed

Surface: scabrous

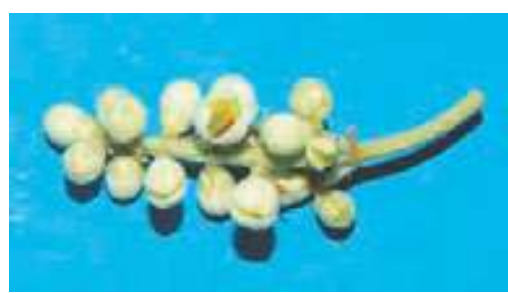

Number of grooves: medium 


\section{Biochemical Characters}

\section{Fatty Acid Composition}

Table 1. Average values (express in $\% \pm$ standard deviations) of the fatty acids methyl esters and nutritional ratios obtained from single cultivar olive oils.

\begin{tabular}{|c|c|c|c|c|c|}
\hline Myristic acid & $0,01 \pm 0,01$ & Linoleic acid $(\omega 6)$ & $4,79 \pm 1,33$ & Lignoceric acid & $0,04 \pm 0,02$ \\
\hline Palmitic acid & $7,40 \pm 0,28$ & Linolenic acid $(\omega 3)$ & $0,79 \pm 0,01$ & & \\
\hline Palmitoleic acid & $2,61 \pm 0,22$ & Arachic acid & $0,27 \pm 0,05$ & Unsat./satured & $9,26 \pm 0,08$ \\
\hline Stear & $1,89 \pm 0,45$ & Eicosenoic acid & $0,01 \pm 0,01$ & $\omega 6 / \omega 3$ & $6,04 \pm 1,57$ \\
\hline Dleic acid & $79,69 \pm 0,35$ & Behenic acid & $0,08 \pm 0,02$ & & \\
\hline
\end{tabular}

\section{Organoleptic oil values}

Sensory Analysis (Panel test)

Comment: fruity medium, with read sensations of artichoke, almond and grass. Balanced taste sensation with medium spicy and bitter. Medium fluidity.

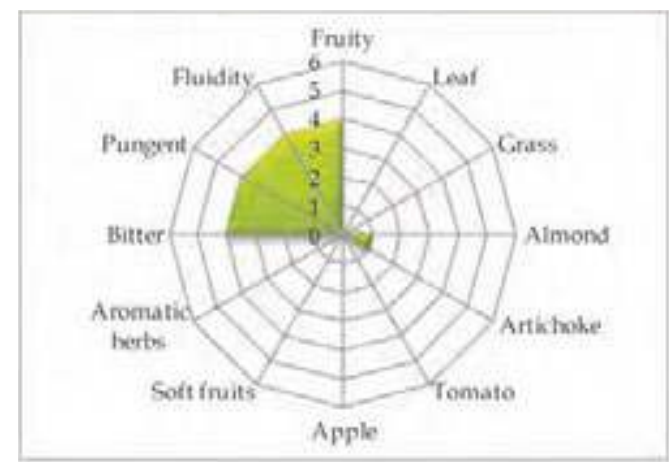

\section{Molecular Markers}

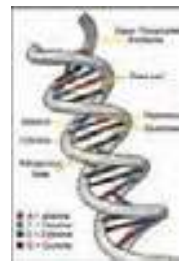

Table 2. Microsatellites (SSR) loci analyzed.

For each locus the allele size (expressed in base pairs) is reported.

\begin{tabular}{|c|c|c|c|c|c|}
\hline DCA09 & DCA18 & GAPU59 & GAPU71A & $G U P A 71 B$ & GAPU103A \\
\hline $162-198$ & $181-181$ & $212-214$ & $214-224$ & $124-144$ & $136-136$ \\
\hline UDO01 & UDO03 & UDO12 & UDO28 & UDO39 & \\
\hline $150-150$ & $143-143$ & $164-177$ & $161-182$ & $213-220$ & \\
\hline
\end{tabular}

\section{References:}

1 - Pugliano G., Flaminio G., Pugliano M.L., et al. In: La risorsa genetica dell'olivo in Campania, SE. S.I.R.C.A. Ed. Napoli, (2000).

2 - Muzzalupo I., Stefanizzi F., Perri E. HortScience (2009), 44: pp. 582-588. 


\section{“Piangente"}

(synonymy: Alloro, Leccino pendulo, Leccino piangente, etc.)

Areal distribution or origin area: Toscana

Flesh/pit weight ratio: medium $(5,71 \pm 1,27)$

Oil content $(\%)$ : low $(39,31 \pm 0,07)$

Purpose: oil

\section{Morphological characters}

Tree characters

Vigour: strong

Growth habit: spreading

Canopy-density: dense

\section{Leaf characters}

Blade length $(\mathrm{cm})$ : medium $(5,90 \pm 0,36)$

Blade width $(\mathrm{cm})$ : medium $(\mathbf{1 , 3 3} \pm \mathbf{0 , 1 0})$

Shape (length/width): elliptic

\section{Inflorescence characters}

Inflorescence length $(\mathrm{cm})$ : medium $(3,27 \pm 1,09)$

Number of flowers: low $(\mathbf{1 7 , 7 4} \pm 0, \mathbf{2 8})$

\section{Fruit characters}

Fresh weight of 100 fruits (g): medium $(2,48 \pm 0,47)$

Shape (length/width): ovoid

Symmetry: slightly asymmetric

Position of maximum transverse diameter:

towards apex

Apex: rounded

Base: truncate

Niplle: absent

Lenticels: many and small

\section{Pit characters}

Weight of 100 pits (g): medium $(0,37 \pm 0,07)$

Shape (length/width): elliptic

Mucron: obvious

Symmetry: asymmetric

Position of maximum transverse diameter:

towards apex

Apex: rounded

Base: rounded

Surface: smooth

Number of grooves: medium
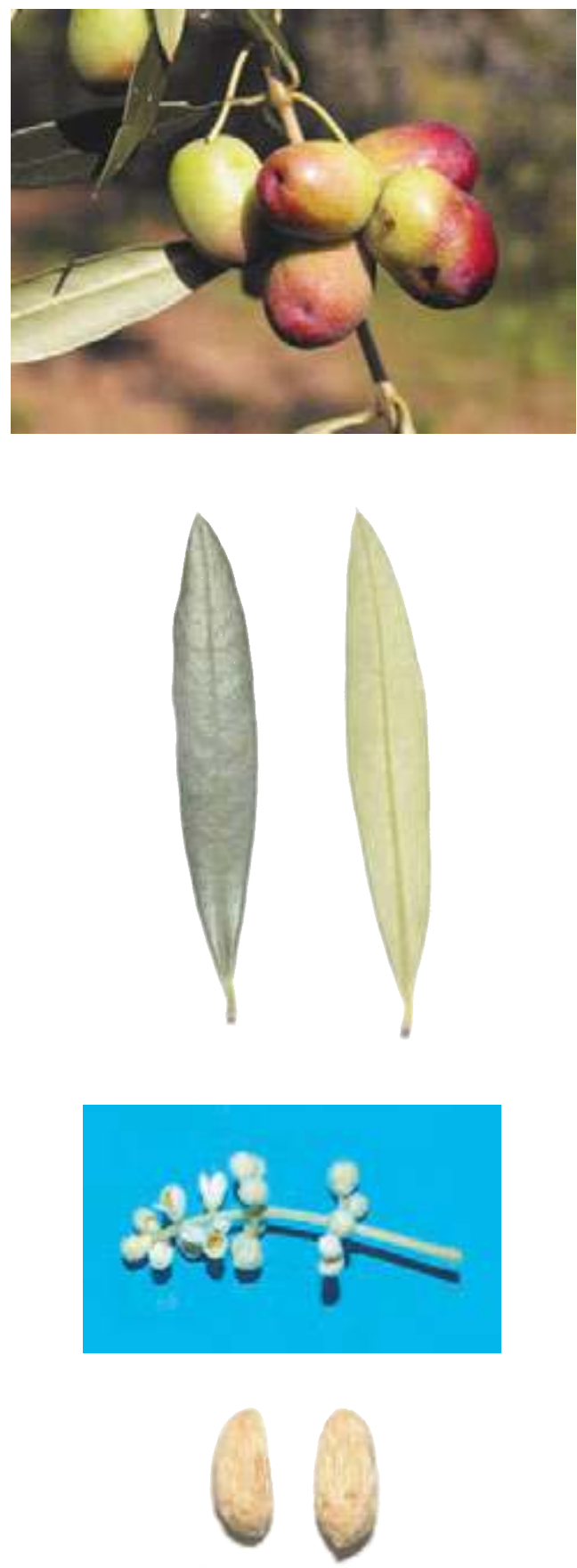


\section{Biochemical Characters}

\section{Fatty Acid Composition}

Table 1. Average values (express in $\% \pm$ standard deviations) of the fatty acids methyl esters and nutritional ratios obtained from single cultivar olive oils.

$\begin{array}{llllll}\text { Myristic acid } & \mathbf{0 , 0 2} \pm \mathbf{0 , 0 1} & \text { Linoleic acid }(\omega 6) & \mathbf{1 0 , 1 1} \pm \mathbf{0 , 1 3} & \text { Lignoceric acid } & \mathbf{0 , 0 5} \pm \mathbf{0 , 0 2} \\ \text { Palmitic acid } & \mathbf{1 0 , 3 1} \pm \mathbf{0 , 3 7} & \text { Linolenic acid }(\omega 3) & \mathbf{0 , 9 3} \pm \mathbf{0 , 0 6} & & \\ \text { Palmitoleic acid } & \mathbf{0 , 4 0 \pm 0 , 1 0} & \text { Arachic acid } & \mathbf{0 , 2 8} \pm \mathbf{0 , 0 6} & \text { Unsat./satured } & \mathbf{7 , 2 5} \pm \mathbf{0 , 9 8} \\ \text { Stearic acid } & \mathbf{1 , 8 5} \pm \mathbf{0 , 4 1} & \text { Eicosenoic acid } & \mathbf{0 , 0 6} \pm \mathbf{0 , 0 6} & \omega 6 / \omega 3 & \mathbf{1 0 , 8 9 \pm 0 , 8 0} \\ \text { Oleic acid } & \mathbf{7 7 , 7 9 \pm \mathbf { 1 , 3 3 }} & \text { Behenic acid } & \mathbf{0 , 1 1} \pm \mathbf{0 , 0 4} & & \end{array}$

\section{Organoleptic oil values}

Sensory Analysis (Panel test)

Comment: fruity medium - light, with hints of almond and read sensations of grass and artichoke. Balanced taste sensation with a medium bitter and spicy. Medium fluidity.

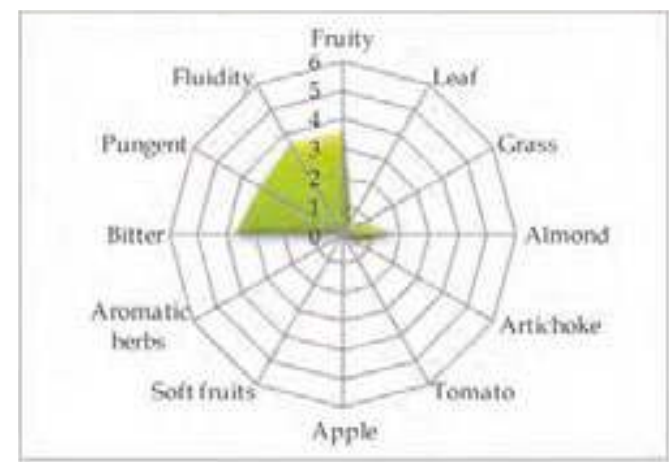

\section{Molecular Markers}

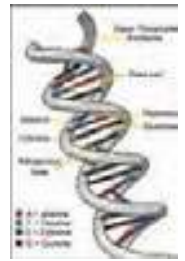

Table 2. Microsatellites (SSR) loci analyzed.

For each locus the allele size (expressed in base pairs) is reported.

$\begin{array}{cccccc}D C A 09 & \text { DCA18 } & \text { GAPU59 } & \text { GAPU71A } & \text { GUPA71B } & \text { GAPU103A } \\ \mathbf{1 6 2} \mathbf{- 2 0 6} & \mathbf{1 8 1 - \mathbf { 1 8 1 }} & \mathbf{2 0 8 - \mathbf { 2 1 2 }} & \mathbf{2 1 4} \mathbf{- 2 1 4} & \mathbf{1 2 6} \mathbf{- 1 2 6} & \mathbf{1 5 7 - \mathbf { 1 8 4 }} \\ \text { UDOO1 } & \text { UDOO3 } & \text { UDO12 } & \text { UDO28 } & \text { UDO39 } & \\ \mathbf{1 4 4 - \mathbf { 1 4 4 }} & \mathbf{1 5 0 - \mathbf { 1 5 0 }} & \mathbf{1 6 6 - \mathbf { 1 9 3 }} & \mathbf{1 5 4 - \mathbf { 1 5 4 }} & \mathbf{2 0 5 - \mathbf { 2 0 5 }} & \end{array}$

\section{References:}

1 - Cimato A., Cantini C., Sani G., In: L'olivo in Toscana: il germoplasma autoctono, Ed. ARSIA (2001).

2 - Various authors. In: Catalogo Nazionale delle Varietà di Olivo, University of Bari (in press), ISBN 978-88-88793-

97-9. 


\section{"Piantone di Falerone"}

(synonymy: Piantone)

Areal distribution or origin area: Marche

Flesh/pit weight ratio: medium $(5,35 \pm 0,25)$

Oil content (\%): medium $(46,75 \pm 1,14)$

Purpose: oil

\section{Morphological characters}

Tree characters

Vigour: medium

Growth habit: spreading-erect

Canopy-density: medium-dense

\section{Leaf characters}

Blade length (cm): short $(4,16 \pm 0,33)$

Blade width $(\mathrm{cm})$ : narrow $(0,99 \pm 0,08)$

Shape (length/width): elliptic - lanceolate

\section{Inflorescence characters}

Inflorescence length (cm): medium $(2,54 \pm 0,78)$

Number of flowers: low $(\mathbf{1 5 , 9 1} \pm 0,87)$

\section{Fruit characters}

Fresh weight of 100 fruits (g): medium $(2,42 \pm 0,48)$

Shape (length/width): ovoid

Symmetry: slightly asymmetric

Position of maximum transverse diameter:

central

Apex: rounded

Base: truncate

Niplle: absent

Lenticels: many and small

\section{Pit characters}

Weight of 100 pits (g): medium $(0,36 \pm 0,04)$

Shape (length/width): elliptic

Mucron: obvious

Symmetry: slightly asymmetric

Position of maximum transverse diameter:

towards apex

Apex: rounded

Base: pointed

Surface: smooth

Number of grooves: medium
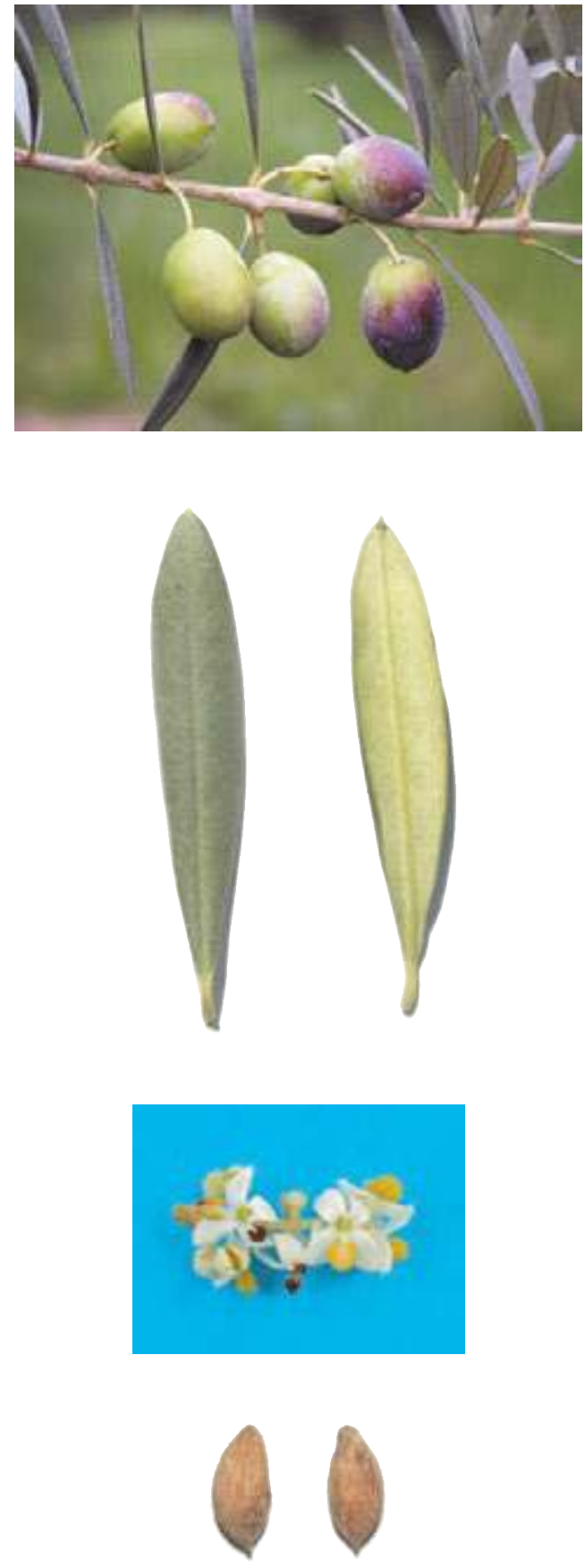


\section{Biochemical Characters}

\section{Fatty Acid Composition}

Table 1. Average values (express in $\% \pm$ standard deviations) of the fatty acids methyl esters and nutritional ratios obtained from single cultivar olive oils.

$\begin{array}{llllll}\text { Myristic acid } & \mathbf{0 , 0 1} \pm \mathbf{0 , 0 1} & \text { Linoleic acid }(\omega 6) & \mathbf{1 2 , 4 5} \pm 0,50 & \text { Lignoceric acid } & \mathbf{0 , 0 6} \pm \mathbf{0 , 0 2} \\ \text { Palmitic acid } & \mathbf{1 1 , 8 9} \pm \mathbf{0 , 3 6} & \text { Linolenic acid }(\omega 3) & \mathbf{0 , 3 1} \pm \mathbf{0 , 0 3} & & \\ \text { Palmitoleic acid } & \mathbf{1 , 3 6} \pm \mathbf{0 , 0 3} & \text { Arachic acid } & \mathbf{0 , 5 7} \pm \mathbf{0 , 0 8} & \text { Unsat./satured } & \mathbf{6 , 0 4} \pm \mathbf{0 , 2 3} \\ \text { Stearic acid } & \mathbf{1 , 7 7} \pm \mathbf{0 , 1 2} & \text { Eicosenoic acid } & \mathbf{0 , 3 0} \pm \mathbf{0 , 1 3} & \omega 6 / \omega 3 & \mathbf{4 0 , 3 9 \pm 1 , 8 9} \\ \text { Oleic acid } & \mathbf{7 0 , 0 3 \pm 0 , 2 4} & \text { Behenic acid } & \mathbf{0 , 1 4} \pm \mathbf{0 , 0 0} & & \end{array}$

\section{Organoleptic oil values}

Sensory Analysis (Panel test)

Comment: fruity medium - light, with hints of almond and read sensations of grass and artichoke. Balanced taste sensation with a medium - light bitter and spicy. Medium fluidity.

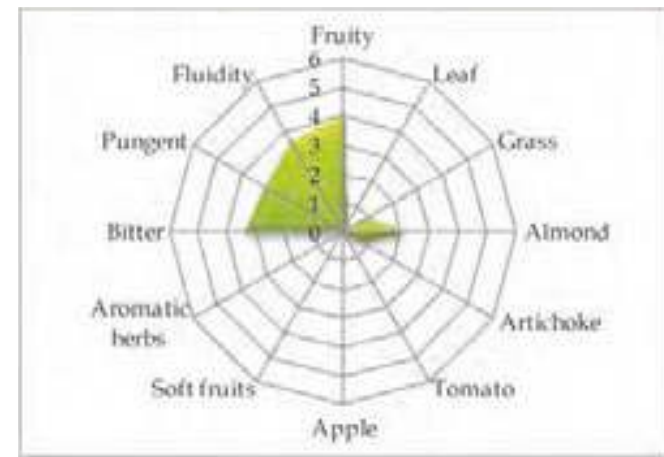

\section{Molecular Markers}

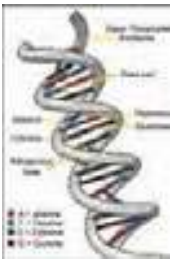

Table 2. Microsatellites (SSR) loci analyzed.

For each locus the allele size (expressed in base pairs) is reported.

$\begin{array}{cccccc}D C A 09 & \text { DCA18 } & \text { GAPU59 } & \text { GAPU71A } & \text { GUPA71B } & \text { GAPU103A } \\ \mathbf{1 8 2} \mathbf{- 1 8 2} & \mathbf{1 7 7 - \mathbf { 1 8 5 }} & \mathbf{2 1 8 - \mathbf { 2 2 2 }} & \mathbf{2 1 8 - \mathbf { 2 1 8 }} & \mathbf{1 2 4} \mathbf{- 1 3 0} & \mathbf{1 5 0 - \mathbf { 1 5 7 }} \\ \text { UDO01 } & \text { UDO03 } & \text { UDO12 } & \text { UDO28 } & \text { UDO39 } & \\ \mathbf{1 4 4 - \mathbf { 1 4 4 }} & \mathbf{1 3 5 - \mathbf { 1 3 5 }} & \mathbf{1 6 6 - \mathbf { 1 7 7 }} & \mathbf{2 1 0 - \mathbf { 2 1 0 }} & \mathbf{2 0 5 - \mathbf { 2 3 2 }} & \end{array}$

\section{References:}

1 - Pannelli G., Alfei B., Santinelli A. In: Varietà di olivo nelle Marche, (2001), ASSAM pp. 93-96.

2 - Various authors. In: Catalogo Nazionale delle Varietà di Olivo . University of Bari (in press), ISBN 978-88-8879397-9. 


\section{“Piantone di Mogliano"}

(synonymy: Limoncella, Oliva riccia, Piantone, Raggiola, Rosciola, etc.)

Areal distribution or origin area: Marche

Flesh/pit weight ratio: medium $(5,92 \pm 0,23)$

Oil content (\%): medium $(42,17 \pm 1,36)$

Purpose: dual purpose

\section{Morphological characters}

Tree characters

Vigour: medium

Growth habit: spreading-erect

Canopy-density: dense

\section{Leaf characters}

Blade length (cm): short $(4,92 \pm 0,34)$

Blade width $(\mathrm{cm})$ : medium $(1,08 \pm 0,09)$

Shape (length/width): elliptic-lanceolate

\section{Inflorescence characters}

Inflorescence length (cm): long $(3,56 \pm 1,20)$

Number of flowers: medium $(\mathbf{2 1}, \mathbf{0 8} \pm \mathbf{1}, \mathbf{8 7})$

\section{Fruit characters}

Fresh weight of 100 fruits $(\mathrm{g})$ : medium $(\mathbf{2}, 85 \pm 0,75)$

Shape (length/width): ovoid

Symmetry: slightly asymmetric

Position of maximum transverse diameter:

central

Apex: rounded

Base: truncate

Niplle: obvious

Lenticels: many and small

\section{Pit characters}

Weight of 100 pits (g): medium $(0,36 \pm 0,05)$

Shape (length/width): ovoid

Mucron: absent

Symmetry: symmetric

Position of maximum transverse diameter:

towards apex

Apex: rounded

Base: rounded

Surface: rugose

Number of grooves: medium
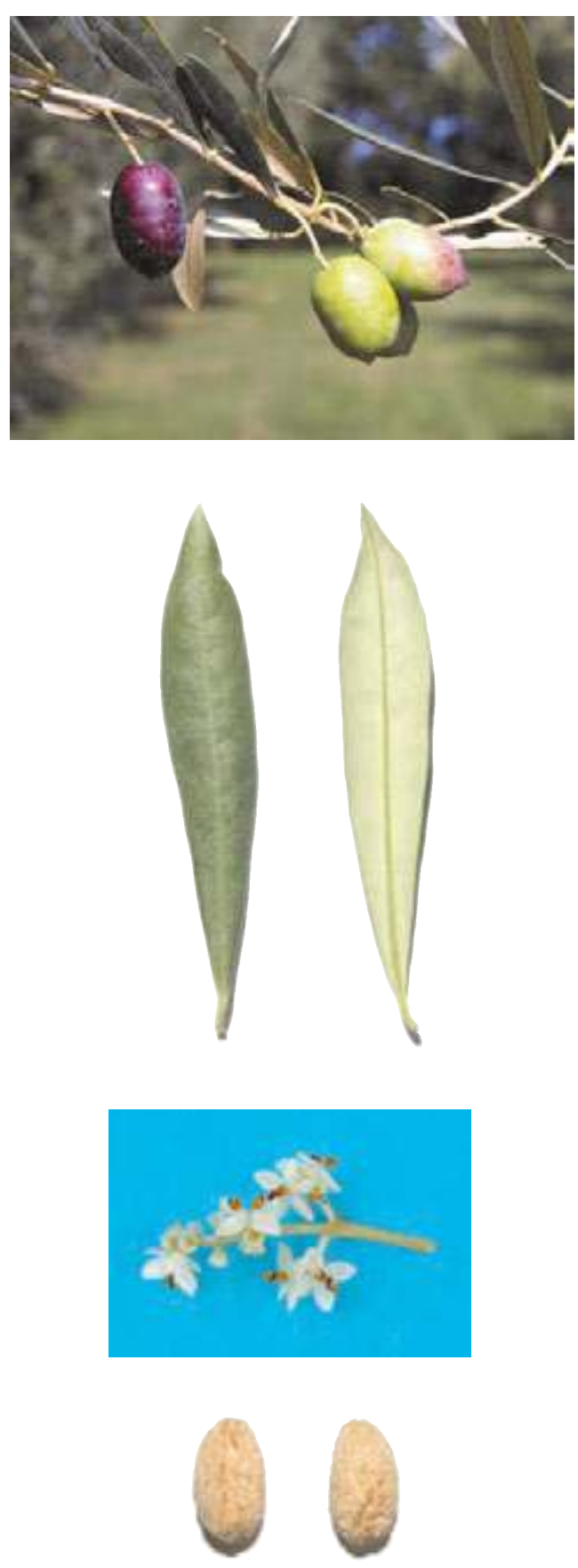


\section{Biochemical Characters}

\section{Fatty Acid Composition}

Table 1. Average values (express in $\% \pm$ standard deviations) of the fatty acids methyl esters and nutritional ratios obtained from single cultivar olive oils.

\begin{tabular}{|c|c|c|c|c|c|}
\hline Myristic acid & $0,01 \pm 0,01$ & Linoleic acid $(\omega 6)$ & $12,45 \pm 0,15$ & Lignoceric acid & $0,08 \pm 0,01$ \\
\hline Palmitic acid & $11,39 \pm 0,15$ & Linolenic acid $(\omega 3)$ & $0,39 \pm 0,02$ & & \\
\hline Palmitoleic acid & $0,74 \pm 0,05$ & Arachic acid & $0,67 \pm 0,10$ & Unsat./satured & $6,07 \pm 0,27$ \\
\hline ea & $2,36 \pm 0,29$ & Eicosenoic acid & $0,44 \pm 0,06$ & $\omega 6 / \omega 3$ & $31,69 \pm 1,10$ \\
\hline leic acid & $70,75 \pm 0,39$ & Behenic acid & $0,08 \pm 0,08$ & & \\
\hline
\end{tabular}

\section{Organoleptic oil values}

Sensory Analysis (Panel test)

Comment: fruity medium-high, with hints of almond and read sensations of grass and artichoke. Balanced taste sensation with a medium-high bitter and spicy. Medium-high fluidity.

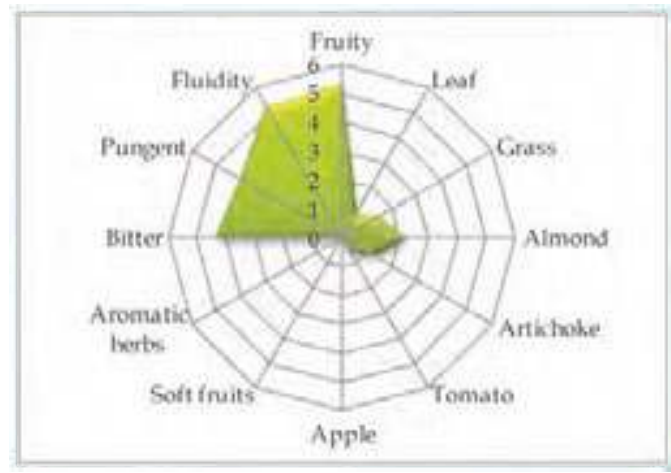

\section{Molecular Markers}

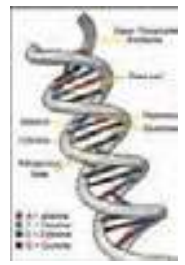

Table 2. Microsatellites (SSR) loci analyzed.

For each locus the allele size (expressed in base pairs) is reported.

\begin{tabular}{|c|c|c|c|c|c|}
\hline DCA09 & DCA18 & GAPU59 & GAPU71A & $G U P A 71 B$ & GAPU103A \\
\hline $198-206$ & $181-181$ & $214-214$ & $218-224$ & $124-130$ & $159-159$ \\
\hline UDO01 & UDO03 & UDO12 & UDO28 & UDO39 & \\
\hline $144-144$ & $135-135$ & $166-166$ & $182-210$ & $200-232$ & \\
\hline
\end{tabular}

\section{References:}

1 - Pannelli G., Alfei B., Santinelli A. In: Varietà di olivo nelle Marche. ASSAM (2001), pp. 97-100.

2 - Various authors. In: Catalogo Nazionale delle Varietà di Olivo . University of Bari (in press), ISBN 978-88-8879397-9. 


\section{"Pidicuddara "}

(synonymy: Minutedda, Ogliara, Oliva Nera, Piricuddara, Ugghiara, etc.)

Areal distribution or origin area: Sicilia

Flesh/pit weight ratio: medium $(7,00 \pm 0,57)$

Oil content (\%): medium $(45,70 \pm 1,58)$

Purpose: oil

\section{Morphological characters}

Tree characters

Vigour: medium weak

Growth habit: erect-spreading

Canopy-density: medium

\section{Leaf characters}

Blade length $(\mathrm{cm})$ : medium $(6,38 \pm 0,75)$

Blade width (cm): broad $(\mathbf{1}, 54 \pm \mathbf{0 , 1 8})$

Shape (length/width): elliptic-lanceolate

\section{Inflorescence characters}

Inflorescence length (cm): medium $(3,40 \pm 1,21)$

Number of flowers: medium $(22,34 \pm 2,75)$

\section{Fruit characters}

Fresh weight of 100 fruits $(\mathrm{g})$ : medium $(3,00 \pm 0,24)$

Shape (length/width): ovoid

Symmetry: symmetric

Position of maximum transverse diameter:

central

Apex: rounded

Base: rounded

Niplle: absent

Lenticels: few and small

\section{Pit characters}

Weight of 100 pits (g): medium $(0,38 \pm 0,06)$

Shape (length/width): elliptic

Mucron: obvious

Symmetry: asymmetric

Position of maximum transverse diameter:

towards apex

Apex: rounded

Base: rounded

Surface: rugose

Number of grooves: low
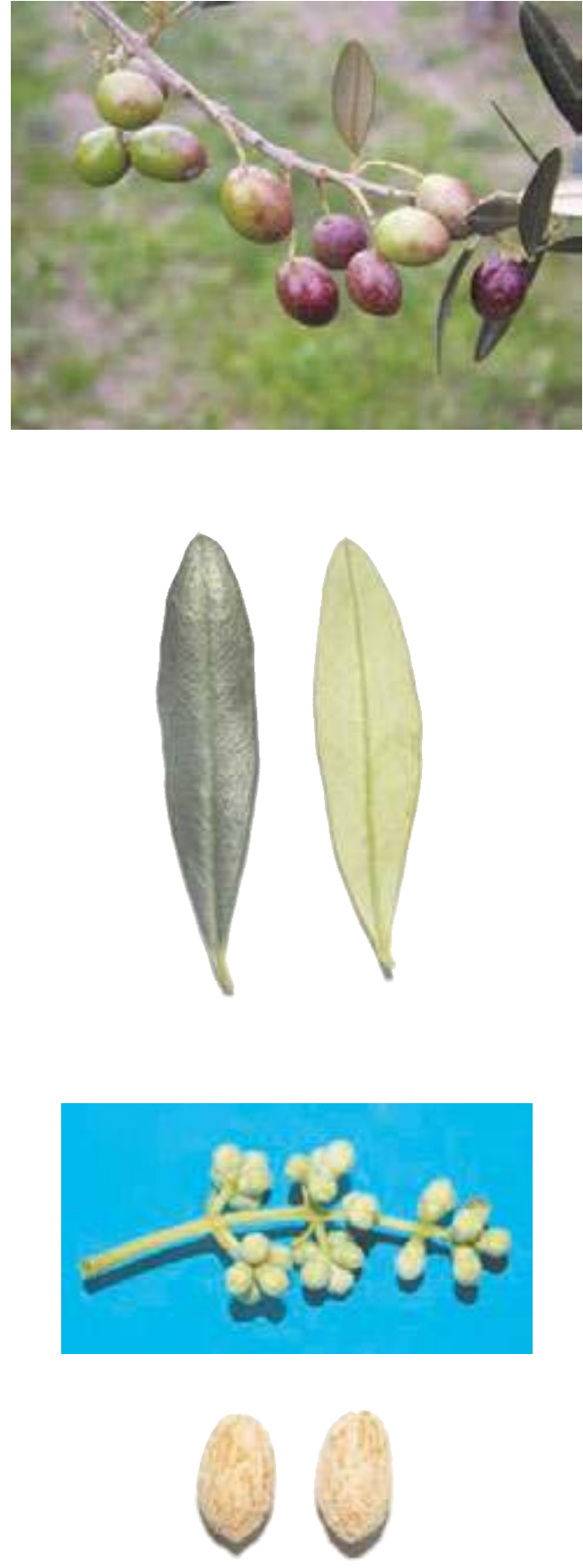


\section{Biochemical Characters}

\section{Fatty Acid Composition}

Table 1. Average values (express in $\% \pm$ standard deviations) of the fatty acids methyl esters and nutritional ratios obtained from single cultivar olive oils.

$\begin{array}{llllll}\text { Myristic acid } & \mathbf{0 , 0 0 \pm 0 , 0 0} & \text { Linoleic acid }(\omega 6) & \mathbf{8 , 5 5} \pm \mathbf{0 , 5 2} & \text { Lignoceric acid } & \mathbf{0 , 0 7 \pm 0 , 0 3} \\ \text { Palmitic acid } & \mathbf{1 1 , 4 6 \pm \mathbf { 1 , 3 4 }} & \text { Linolenic acid }(\omega 3) & \mathbf{0 , 8 8} \pm \mathbf{0 , 0 9} & & \\ \text { Palmitoleic acid } & \mathbf{0 , 6 4} \pm \mathbf{0 , 0 6} & \text { Arachic acid } & \mathbf{0 , 4 7} \pm \mathbf{0 , 5 1} & \text { Unsat./satured } & \mathbf{6 , 2 1} \pm \mathbf{0 , 7 5} \\ \text { Stearic acid } & \mathbf{2 , 0 3} \pm \mathbf{0 , 3 4} & \text { Eicosenoic acid } & \mathbf{0 , 2 9} \pm \mathbf{0 , 2 4} & \omega 6 / \omega 3 & \mathbf{9 , 8 0} \pm \mathbf{1 , 5 5} \\ \text { Oleic acid } & \mathbf{7 5 , 0 4 \pm \mathbf { 0 , 2 6 }} & \text { Behenic acid } & \mathbf{0 , 1 5} \pm \mathbf{0 , 0 5} & & \end{array}$

\section{Organoleptic oil values}

Sensory Analysis (Panel test)

Comment: fruity medium-high, with taste of almond, read sensations of tomato, grass, leaf and aromatic herbs. Balanced taste sensation with medium bitter and spicy. Medium-high fluidity.

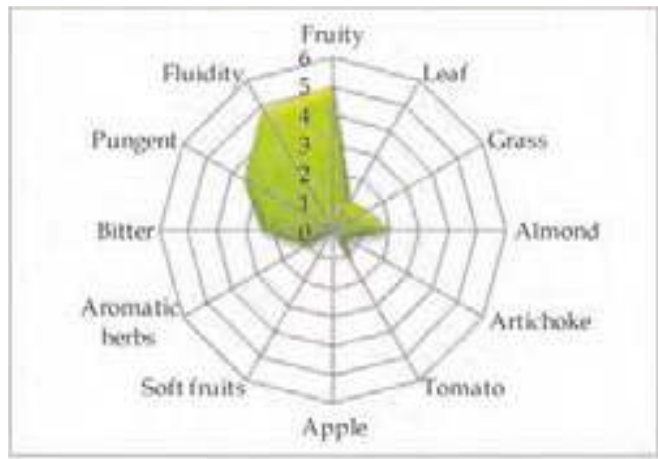

\section{Molecular Markers}

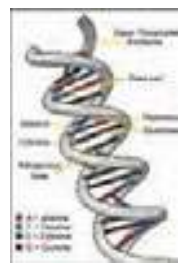

Table 2. Microsatellites (SSR) loci analyzed.

For each locus the allele size (expressed in base pairs) is reported.

$\begin{array}{cccccc}D C A 09 & \text { DCA18 } & \text { GAPU59 } & \text { GAPU71A } & \text { GUPA71B } & \text { GAPU103A } \\ \mathbf{1 6 2} \mathbf{- 1 8 2} & \mathbf{1 7 7 - \mathbf { 1 7 9 }} & \mathbf{2 1 2} \mathbf{- 2 1 2} & \mathbf{2 1 0 - \mathbf { 2 1 0 }} & \mathbf{1 2 4} \mathbf{- 1 4 4} & \mathbf{1 5 0 - \mathbf { 1 8 4 }} \\ \text { UDOO1 } & \text { UDO03 } & \text { UDO12 } & \text { UDO28 } & \text { UDO39 } & \\ \mathbf{1 4 4 - \mathbf { 1 4 4 }} & \mathbf{1 5 0 - \mathbf { 1 5 0 }} & \mathbf{1 6 6 - \mathbf { 1 6 6 }} & \mathbf{1 8 2} \mathbf{- 1 8 2} & \mathbf{2 0 5 - \mathbf { 2 0 5 }} & \end{array}$

\section{References:}

1 - Caruso T., Cartabellotta D., Motisi A., et all. In: Cultivar di olivo siciliane, Università degli Studi di Palermo (2007), pp. 118-122.

2 - Muzzalupo I., Stefanizzi F., Perri E. HortScience (2009), 44: pp. 582-588. 


\section{“Pignola "}

(synonymy: Crovia, Merletta, Negretta, Pignola doppia, Pinola, etc.)

Areal distribution or origin area: Liguria

Flesh/pit weight ratio: medium $(6,26 \pm 0,33)$

Oil content (\%): medium $(40,20 \pm 0,07)$

Purpose: oil

\section{Morphological characters}

Tree characters

Vigour: medium-weak

Growth habit: spreading-erect

Canopy-density: medium-rade

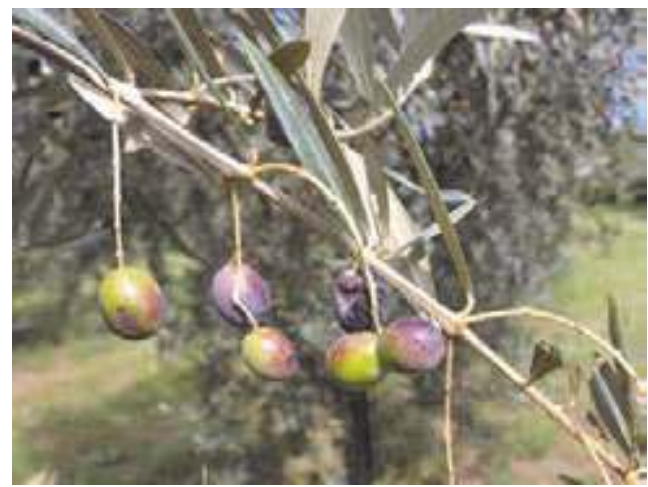

\section{Leaf characters}

Blade length $(\mathrm{cm})$ : medium $(5,35 \pm 0,42)$

Blade width (cm): medium $(\mathbf{1}, 21 \pm 0,12)$

Shape (length/width): elliptic - lanceolate

\section{Inflorescence characters}

Inflorescence length (cm): long $(3,83 \pm 0,29)$

Number of flowers: high $(\mathbf{2 9 , 7 2} \pm \mathbf{2 , 8 0})$

\section{Fruit characters}

Fresh weight of 100 fruits (g): medium $(2,35 \pm 0,04)$

Shape (length/width): spherical

Symmetry: slightly asymmetric

Position of maximum transverse diameter:

central

Apex: rounded

Base: rounded

Niplle: absent

Lenticels: many and small

\section{Pit characters}

Weight of 100 pits (g): medium $(\mathbf{0 , 3 3} \pm \mathbf{0 , 0 2})$

Shape (length/width): ovoid

Mucron: absent
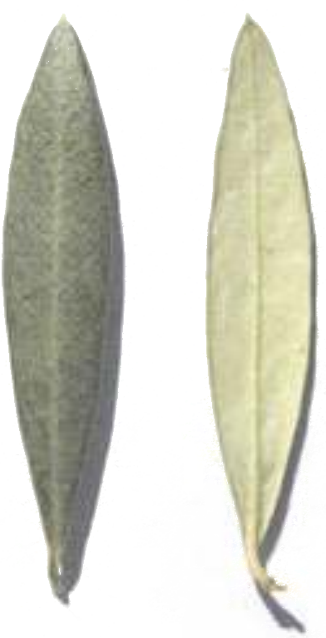

Symmetry: symmetric

Position of maximum transverse diameter: central

Apex: rounded

Base: rounded

Surface: rugose

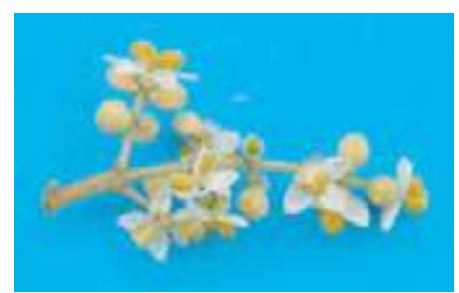

Number of grooves: low 


\section{Biochemical Characters}

\section{Fatty Acid Composition}

Table 1. Average values (express in $\% \pm$ standard deviations) of the fatty acids methyl esters and nutritional ratios obtained from single cultivar olive oils.

\begin{tabular}{|c|c|c|c|c|c|}
\hline Myristic acid & $0,01 \pm 0,01$ & Linoleic acid $(\omega 6)$ & $10,27 \pm 0,04$ & Lignoceric acid & $0,05 \pm 0,00$ \\
\hline Palmitic acid & $13,81 \pm 0,24$ & Linolenic acid $(\omega 3)$ & $0,31 \pm 0,01$ & & \\
\hline Palmitoleic acid & $1,19 \pm 0,01$ & Arachic acid & $0,48 \pm 0,26$ & Unsat./satured & $5,28 \pm 0,02$ \\
\hline teari & $1,62 \pm 0,08$ & Eicosenoic acid & $0,38 \pm 0,02$ & $\omega 6 / \omega 3$ & $33,66 \pm 0,68$ \\
\hline leic acid & $70,76 \pm 0,31$ & Behenic acid & $0,11 \pm 0,01$ & & \\
\hline
\end{tabular}

\section{Organoleptic oil values}

Sensory Analysis (Panel test)

Comment: fruity medium - light, with hints of artichoke and almond. Balanced in flavours, with hints of bitter and spicy medium -light intensity, medium fluidity.

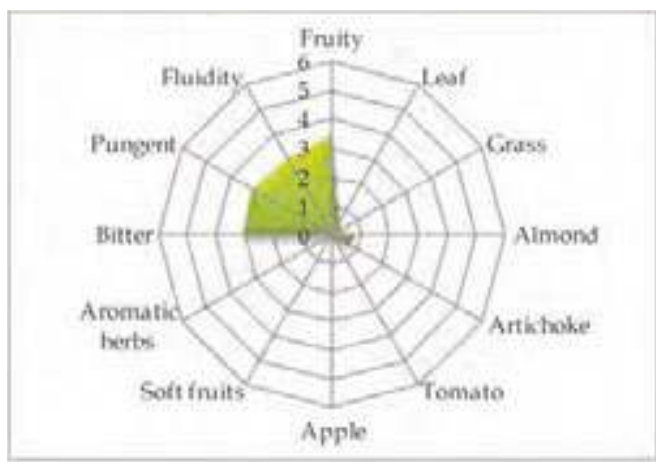

\section{Molecular Markers}

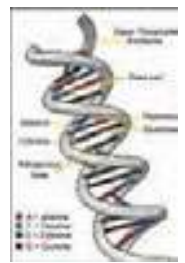

Table 2. Microsatellites (SSR) loci analyzed.

For each locus the allele size (expressed in base pairs) is reported.

$\begin{array}{cccccc}D C A 09 & \text { DCA18 } & \text { GAPU59 } & \text { GAPU71A } & \text { GUPA71B } & \text { GAPU103A } \\ \mathbf{1 8 2} \mathbf{- 2 0 6} & \mathbf{1 8 1} \mathbf{- 1 8 5} & \mathbf{2 1 4 - \mathbf { 2 2 2 }} & \mathbf{2 1 4} \mathbf{- 2 1 4} & \mathbf{1 2 6} \mathbf{- 1 3 0} & \mathbf{1 5 0 - \mathbf { 1 5 7 }} \\ \text { UDOO1 } & \text { UDOO3 } & \text { UDO12 } & \text { UDO28 } & \text { UDO39 } & \\ \mathbf{1 5 0 - \mathbf { 1 5 0 }} & \mathbf{1 4 3 - \mathbf { 1 4 3 }} & \mathbf{1 7 7 - \mathbf { 1 9 3 }} & \mathbf{1 5 4} \mathbf{- 1 8 2} & \mathbf{2 0 5 - \mathbf { 2 0 5 }} & \end{array}$

\section{References:}

1 - Various authors. In: Catalogo Nazionale delle Varietà di Olivo . University of Bari (in press), ISBN 978-88-8879397-9.

2 - Muzzalupo I., Lombardo N., Salimonti A., et al. Adv. Hort. Sci. (2008), 22(2): pp. 142-148. 


\section{"Pisciottana"}

(synonymy: Ogliastrina, Olivo dell'ascea.)

Areal distribution or origin area: Campania

Flesh/pit weight ratio: medium $(6,83 \pm 1,47)$

Oil content (\%): medium $(40,12 \pm 0,85)$

Purpose: oil

\section{Morphological characters}

Tree characters

Vigour: medium-strong

Growth habit: spreading

Canopy-density: dense

\section{Leaf characters}

Blade length $(\mathrm{cm})$ : medium $(6,67 \pm 0,54)$

Blade width $(\mathrm{cm})$ : medium $(1,68 \pm 0,16)$

Shape (length/width): elliptic

\section{Inflorescence characters}

Inflorescence length (cm): medium $(3,12 \pm 1,35)$

Number of flowers: low $(13,39 \pm 0,29)$

\section{Fruit characters}

Fresh weight of 100 fruits $(\mathrm{g})$ : low $(\mathbf{1}, \mathbf{8 3} \pm \mathbf{0 , 3 1})$

Shape (length/width): ovoid

Symmetry: slightly asymmetric

Position of maximum transverse diameter: central

Apex: rounded

Base: truncate

Niplle: tenuous

Lenticels: many and small

\section{Pit characters}

Weight of 100 pits (g): low $(0,29 \pm 0,06)$

Shape (length/width): elliptic

Mucron: obvious

Symmetry: slightly asymmetric

Position of maximum transverse diameter:

towards apex

Apex: rounded

Base: pointed

Surface: rugose

Number of grooves: medium
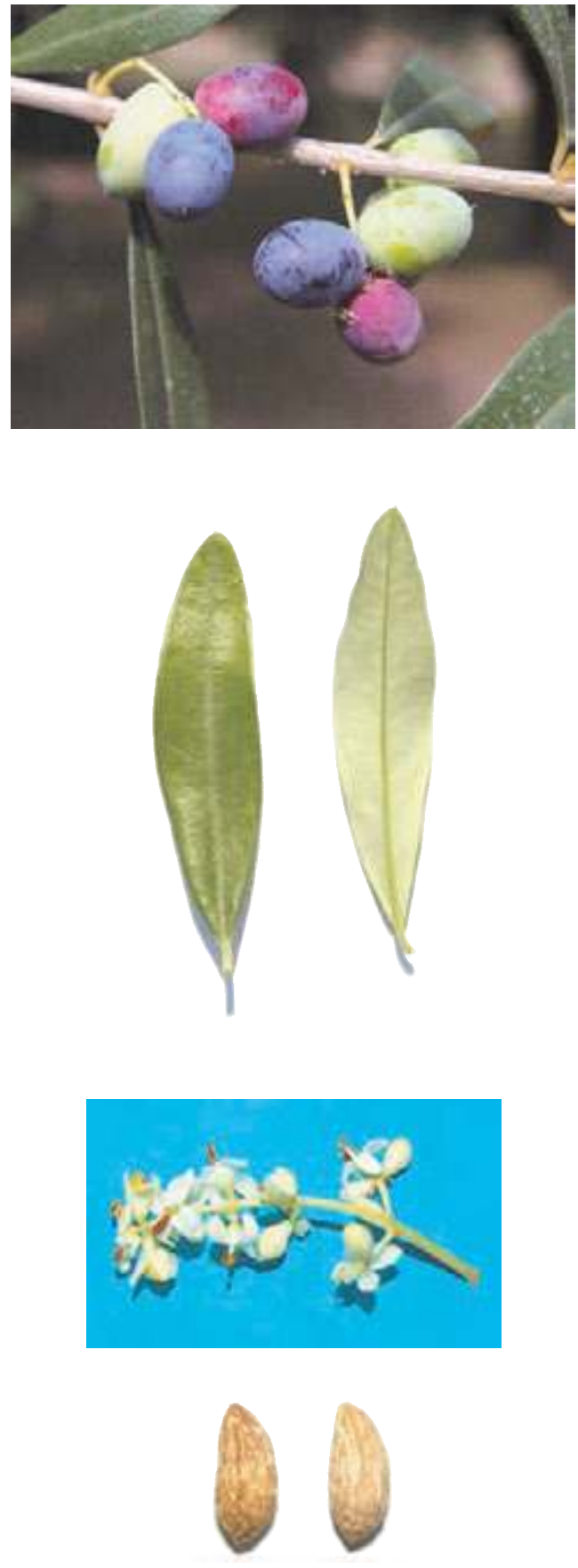


\section{Biochemical Characters}

\section{Fatty Acid Composition}

Table 1. Average values (express in $\% \pm$ standard deviations) of the fatty acids methyl esters and nutritional ratios obtained from single cultivar olive oils.

$\begin{array}{llllll}\text { Myristic acid } & \mathbf{0 , 0 1} \pm \mathbf{0 , 0 1} & \text { Linoleic acid }(\omega 6) & \mathbf{3 , 9 3} \pm \mathbf{0 , 8 0} & \text { Lignoceric acid } & \mathbf{0 , 0 3} \pm \mathbf{0 , 0 1} \\ \text { Palmitic acid } & \mathbf{1 1 , 2 0} \pm \mathbf{0 , 0 7} & \text { Linolenic acid }(\omega 3) & \mathbf{0 , 7 5} \pm \mathbf{0 , 0 5} & & \\ \text { Palmitoleic acid } & \mathbf{1 , 1 8} \pm \mathbf{0 , 5 3} & \text { Arachic acid } & \mathbf{0 , 2 4} \pm \mathbf{0 , 0 6} & \text { Unsat./satured } & \mathbf{6 , 4 2} \pm \mathbf{0 , 4 2} \\ \text { Stearic acid } & \mathbf{2 , 0 0 \pm 0 , 7 2} & \text { Eicosenoic acid } & \mathbf{0 , 0 2} \pm \mathbf{0 , 0 1} & \omega 6 / \omega 3 & \mathbf{5 , 1 9} \pm \mathbf{0 , 8 3} \\ \text { Oleic acid } & \mathbf{7 9 , 7 2 \pm \mathbf { 1 , 2 2 }} & \text { Behenic acid } & \mathbf{0 , 0 6} \pm \mathbf{0 , 0 1} & & \end{array}$

\section{Organoleptic oil values}

Sensory Analysis (Panel test)

Comment: fruity medium-high, with good sensation of grass, artichoke and almond. Balanced in flavours, with hints of bitter and spicy medium intensity. Medium-high fluidity.

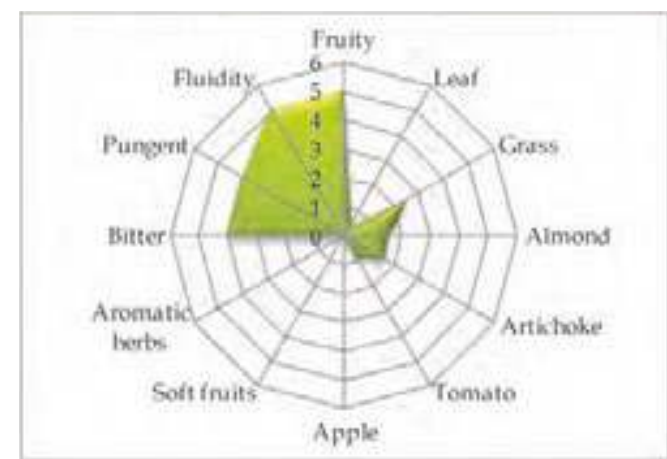

\section{Molecular Markers}

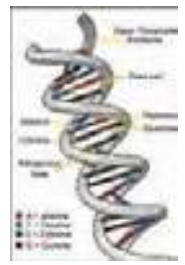

Table 2. Microsatellites (SSR) loci analyzed.

For each locus the allele size (expressed in base pairs) is reported.

\begin{tabular}{|c|c|c|c|c|c|}
\hline DCA09 & DCA18 & GAPU59 & GAPU71A & GUPA71B & GAPU103A \\
\hline $172-182$ & $177-181$ & $214-222$ & $218-218$ & $121-124$ & $136-150$ \\
\hline UDO01 & UDO03 & UDO12 & UDO28 & UDO39 & \\
\hline $140-140$ & $143-143$ & $177-182$ & $150-161$ & $213-232$ & \\
\hline
\end{tabular}

\section{References:}

1 - Various authors. In: Catalogo Nazionale delle Varietà di Olivo . University of Bari (in press), ISBN 978-88-8879397-9.

2 - Muzzalupo I., Stefanizzi F., Perri E. HortScience (2009), 44: pp. 582-588. 


\section{“Pizz'è carroga "}

Areal distribution or origin area: Sardegna

Flesh/pit weight ratio: medium $(7,24 \pm 0,35)$

Oil content (\%): medium $(46,53 \pm 1,18)$

Purpose: dual purpose

\section{Morphological characters}

Tree characters

Vigour: medium-weak

Growth habit: spreading

Canopy-density: medium

\section{Leaf characters}

Blade length $(\mathrm{cm})$ : medium $(5,17 \pm 0,51)$

Blade width $(\mathrm{cm})$ : medium $(\mathbf{1}, 24 \pm 0,16)$

Shape (length/width): elliptic - lanceolate

\section{Inflorescence characters}

Inflorescence length (cm): short $(2,18 \pm 0,97)$

Number of flowers: low $(12,68 \pm 2,59)$

\section{Fruit characters}

Fresh weight of 100 fruits $(\mathrm{g})$ : high $(5,86 \pm 0,59)$

Shape (length/width): ovoid

Symmetry: asymmetric

Position of maximum transverse diameter:

central

Apex: rounded

Base: truncate

Niplle: obvious

Lenticels: many and small

\section{Pit characters}

Weight of 100 pits (g): high $(\mathbf{0 , 6 3} \pm \mathbf{0 , 1 6})$

Shape (length/width): elliptic

Mucron: obvious

Symmetry: asymmetric

Position of maximum transverse diameter:

central

Apex: pointed

Base: pointed

Surface: rugose

Number of grooves: medium
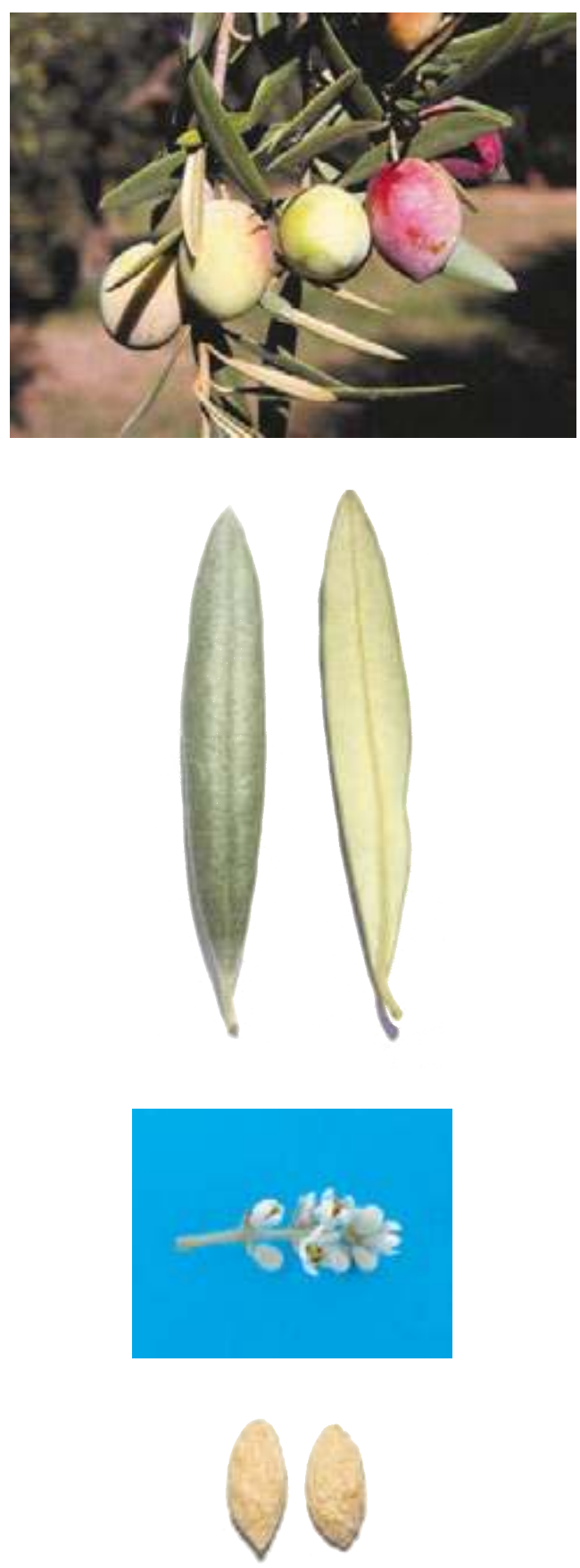


\section{Biochemical Characters}

\section{Fatty Acid Composition}

Table 1. Average values (express in $\% \pm$ standard deviations) of the fatty acids methyl esters and nutritional ratios obtained from single cultivar olive oils.

$\begin{array}{llllll}\text { Myristic acid } & \mathbf{0 , 0 1} \pm \mathbf{0 , 0 0} & \text { Linoleic acid }(\omega 6) & \mathbf{1 0 , 6 9} \pm \mathbf{0 , 6 8} & \text { Lignoceric acid } & \mathbf{0 , 0 4} \pm \mathbf{0 , 0 3} \\ \text { Palmitic acid } & \mathbf{1 5 , 0 0 \pm \mathbf { 1 , 0 7 }} & \text { Linolenic acid }(\omega 3) & \mathbf{0 , 8 2} \pm \mathbf{0 , 1 6} & & \\ \text { Palmitoleic acid } & \mathbf{1 , 4 9} \pm \mathbf{0 , 3 0} & \text { Arachic acid } & \mathbf{0 , 2 5} \pm \mathbf{0 , 0 5} & \text { Unsat./ satured } & \mathbf{4 , 7 3} \pm \mathbf{0 , 3 5} \\ \text { Stearic acid } & \mathbf{2 , 0 0 \pm 0 , 2 6} & \text { Eicosenoic acid } & \mathbf{0 , 0 3} \pm 0,02 & \omega 6 / \omega 3 & \mathbf{1 3 , 4 4} \pm \mathbf{2 , 8 4} \\ \text { Oleic acid } & \mathbf{6 8 , 0 1 \pm \mathbf { 1 , 6 1 }} & \text { Behenic acid } & \mathbf{0 , 0 8} \pm \mathbf{0 , 0 3} & & \end{array}$

\section{Organoleptic oil values}

Sensory Analysis (Panel test)

Comment: medium-high fruity, with good sensation of grass, less almond and artichoke. Balanced taste sensation, with bitter and spicy medium-high. Medium-high fluidity.

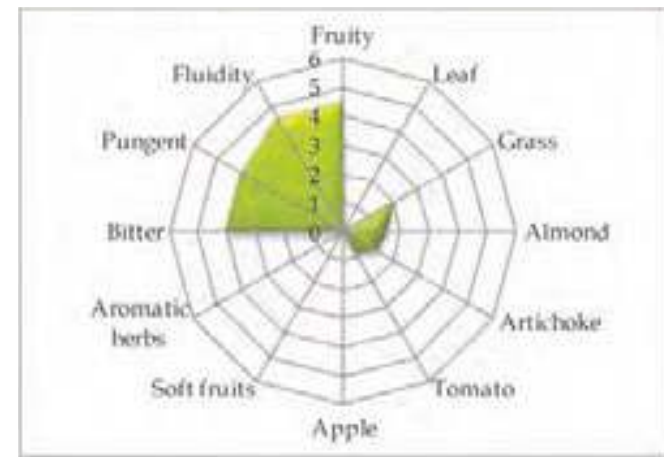

\section{Molecular Markers}

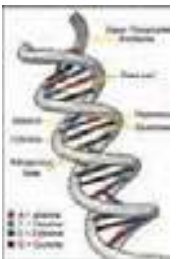

Table 2. Microsatellites (SSR) loci analyzed.

For each locus the allele size (expressed in base pairs) is reported.

$\begin{array}{cccccc}D C A 09 & \text { DCA } 18 & \text { GAPU59 } & \text { GAPU71A } & \text { GUPA71B } & \text { GAPU103A } \\ \mathbf{1 6 2} \mathbf{- 1 7 2} & \mathbf{1 7 9 - \mathbf { 1 8 5 }} & \mathbf{2 1 2} \mathbf{- 2 2 2} & \mathbf{2 1 8 - \mathbf { 2 1 8 }} & \mathbf{1 2 6 - \mathbf { 1 4 4 }} & \mathbf{1 7 0 - \mathbf { 1 8 4 }} \\ \text { UDO01 } & \text { UDO03 } & \text { UDO12 } & \text { UDO28 } & \text { UDO39 } & \\ \mathbf{1 4 0} \mathbf{- 1 4 0} & \mathbf{1 3 5} \mathbf{- 1 3 5} & \mathbf{1 6 4} \mathbf{- 1 9 3} & \mathbf{1 5 0} \mathbf{- 1 5 0} & \mathbf{1 2 5} \mathbf{- 1 2 5} & \end{array}$

\section{References:}

1 - Mulas M., Agabbio M., Chessa I., In: L'Olivo: le vecchie varietà della Sardegna, Ed. Delfino (1994), pp. 310-338.

2 - Muzzalupo I., Stefanizzi F., Perri E. HortScience (2009), 44: pp. 582-588. 


\section{"Pizzutella"}

(synonymy: Pizziricò, Pizzutedda, etc.)

Areal distribution or origin area: Sicilia

Flesh/pit weight ratio: medium $(5,32 \pm 0,39)$

Oil content (\%): medium $(44,06 \pm 2,06)$

Purpose: oil

\section{Morphological characters}

Tree characters

Vigour: medium-strong

Growth habit: spreading

Canopy-density: medium

\section{Leaf characters}

Blade length (cm): short $(4,90 \pm 0,47)$

Blade width (cm): medium $(\mathbf{1 , 2 4} \pm \mathbf{0 , 1 5})$

Shape (length/width): elliptic

\section{Inflorescence characters}

Inflorescence length $(\mathrm{cm})$ : short $(2,12 \pm 0,89)$

Number of flowers: medium $(24,41 \pm 1,86)$

\section{Fruit characters}

Fresh weight of 100 fruits (g): medium $(3,34 \pm 0,44)$

Shape (length/width): ovoid

Symmetry: slightly asymmetric

Position of maximum transverse diameter:

towards base

Apex: pointed

Base: rounded

Niplle: tenuous

Lenticels: many and large

\section{Pit characters}

Weight of 100 pits (g): medium $(0,53 \pm \mathbf{0 , 0 6})$

Shape (length/width): elliptic

Mucron: absent

Symmetry: slightly asymmetric

Position of maximum transverse diameter:

central

Apex: pointed

Base: pointed

Surface: rugose

Number of grooves: low
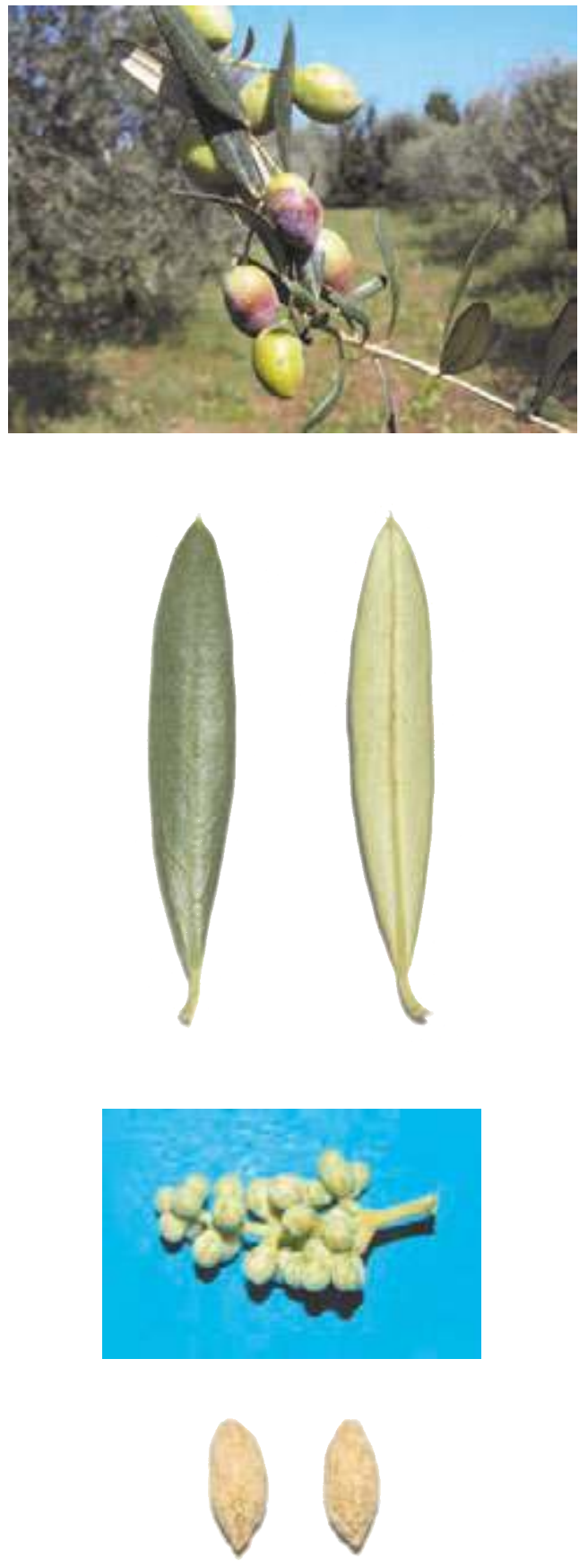


\section{Biochemical Characters}

\section{Fatty Acid Composition}

Table 1. Average values (express in $\% \pm$ standard deviations) of the fatty acids methyl esters and nutritional ratios obtained from single cultivar olive oils.

\begin{tabular}{|c|c|c|c|c|c|}
\hline Myristic acid & $0,01 \pm 0,00$ & Linoleic acid $(\omega 6)$ & $11,86 \pm 1,07$ & Lignoceric acid & $0,03 \pm 0,00$ \\
\hline Palmitic acid & $14,67 \pm 0,76$ & Linolenic acid $(\omega 3)$ & $0,96 \pm 0,01$ & & \\
\hline Palmitoleic acid & $1,61 \pm 0,06$ & Arachic acid & $0,33 \pm 0,01$ & Unsat./satured & $4,75 \pm 0,19$ \\
\hline teari & $2,19 \pm 0,17$ & Eicosenoic acid & $0,02 \pm 0,00$ & $\omega 6 / \omega 3$ & $12,37 \pm 1,27$ \\
\hline leic acid & $66,63 \pm 0,65$ & Behenic acid & $0,08 \pm 0,01$ & & \\
\hline
\end{tabular}

\section{Organoleptic oil values}

Sensory Analysis (Panel test)

Comment: fruity medium-high, with hints of almond and artichoke, read sensations of grass and tomato. Balanced taste sensation with a medium bitter and spicy. Medium fluidity.

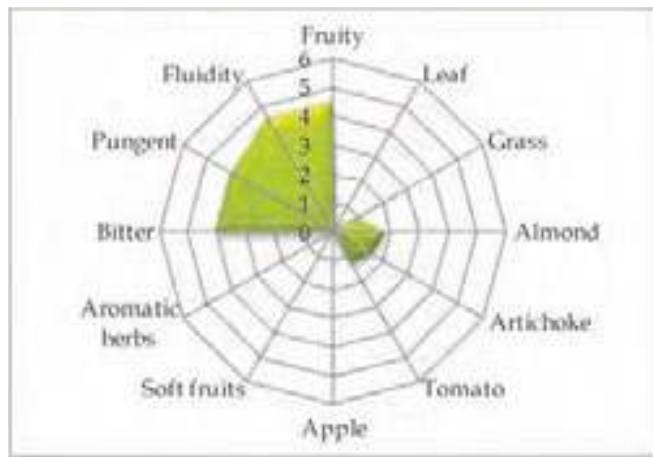

\section{Molecular Markers}

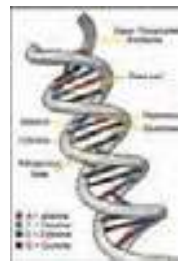

Table 2. Microsatellites (SSR) loci analyzed.

For each locus the allele size (expressed in base pairs) is reported.

\begin{tabular}{|c|c|c|c|c|c|}
\hline$D C A 09$ & $D C A 18$ & GAPU59 & GAPU71A & GUPA71B & GAPU103A \\
\hline $176-182$ & $179-181$ & $208-222$ & $210-214$ & $124-124$ & $157-170$ \\
\hline UDO01 & UDO03 & UDO12 & UDO28 & UDO39 & \\
\hline $144-144$ & $150-150$ & $166-166$ & $143-154$ & $164-164$ & \\
\hline
\end{tabular}

\section{References:}

1 - Bottari V., Spina P. In: Le varietà di olivo coltivate in Sicilia, Ann. Sper. Agr. (1953), 7: pp. 937-1004.

2 - Muzzalupo I., Stefanizzi F., Perri E. HortScience (2009), 44: pp. 582-588. 


\section{"Posola"}

(synonymy: D'Alannese, Pennese, Pizzutella, etc.)

Areal distribution or origin area: Abruzzo

Flesh/pit weight ratio: medium $(7,46 \pm 0,10)$

Oil content (\%): medium $(41,04 \pm 2,48)$

Purpose: oil

\section{Morphological characters}

Tree characters

Vigour: medium-weak

Growth habit: spreading-erect

Canopy-density: medium-sparse

\section{Leaf characters}

Blade length $(\mathrm{cm})$ : medium $(5,32 \pm 0,40)$

Blade width $(\mathrm{cm})$ : medium $(1,06 \pm 0,12)$

Shape (length/width): elliptic-lanceolate

\section{Inflorescence characters}

Inflorescence length (cm): short $(2,25 \pm 0,70)$

Number of flowers: medium $(\mathbf{1 5 , 5 1} \pm \mathbf{1 , 3 2})$

\section{Fruit characters}

Fresh weight of 100 fruits $(\mathrm{g})$ : medium $(3,81 \pm 0,02)$

Shape (length/width): ovoid

Symmetry: slightly asymmetric

Position of maximum transverse diameter:

central

Apex: rounded

Base: rounded

Niplle: absent

Lenticels: few and large

\section{Pit characters}

Weight of 100 pits (g): medium $(\mathbf{0 , 4 5} \pm \mathbf{0 , 0 1})$

Shape (length/width): elliptic

Mucron: obvious

Symmetry: slightly asymmetric

Position of maximum transverse diameter:

central

Apex: pointed

Base: rounded

Surface: rugose

Number of grooves: low
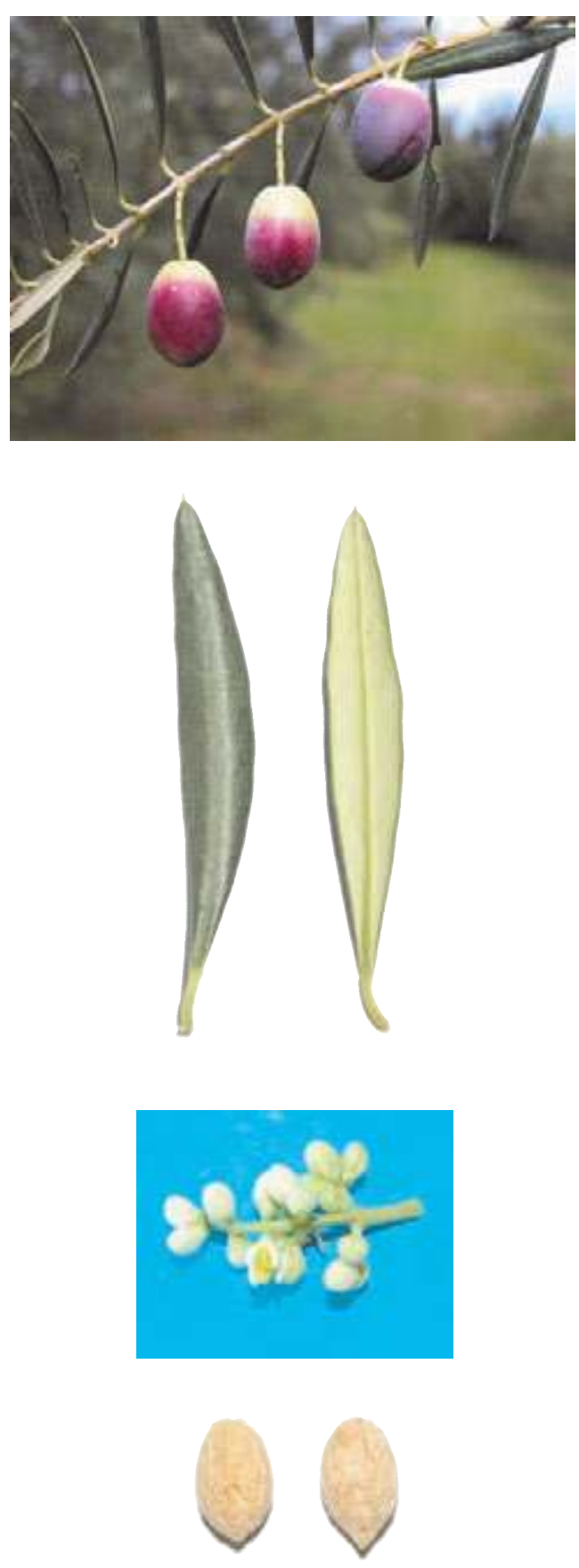


\section{Biochemical Characters}

\section{Fatty Acid Composition}

Table 1. Average values (express in $\% \pm$ standard deviations) of the fatty acids methyl esters and nutritional ratios obtained from single cultivar olive oils.

\begin{tabular}{|c|c|c|c|c|c|}
\hline Myristic acid & $0,02 \pm 0,01$ & Linoleic acid $(\omega 6)$ & $20,78 \pm 0,14$ & Lignoceric acid & $0,03 \pm 0,01$ \\
\hline Palmitic acid & $14,63 \pm 0,22$ & Linolenic acid $(\omega 3)$ & $0,85 \pm 0,06$ & & \\
\hline Palmitoleic acid & $5,26 \pm 0,30$ & Arachic acid & $0,23 \pm 0,07$ & Unsat./satured & $4,90 \pm 0,11$ \\
\hline tearic & $1,22 \pm 0,18$ & Eicosenoic acid & $0,02 \pm 0,01$ & $\omega 6 / \omega 3$ & $24,60 \pm 1,74$ \\
\hline leic acid & $51,47 \pm 0,29$ & Behenic acid & $0,04 \pm 0,00$ & & \\
\hline
\end{tabular}

\section{Organoleptic oil values}

Sensory Analysis (Panel test)

Comment: medium fruity, with good sensation of almond and aromatic herbs, less grass and artichoke. Balanced taste sensation, with bitter and spicy medium. Medium-high fluidity.

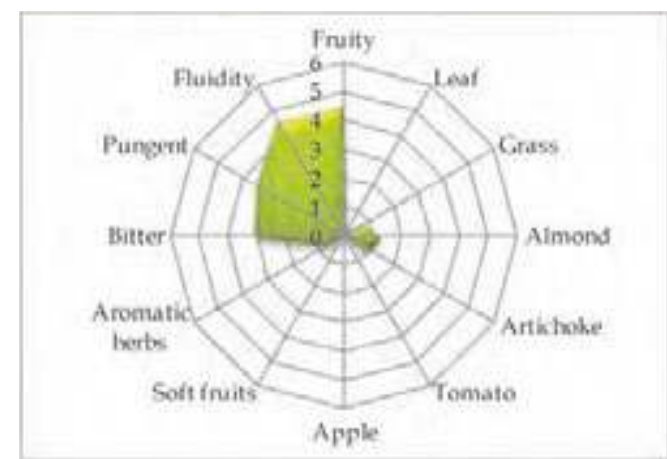

\section{Molecular Markers}

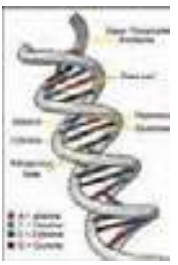

Table 2. Microsatellites (SSR) loci analyzed.

For each locus the allele size (expressed in base pairs) is reported.

$\begin{array}{cccccc}D C A 09 & \text { DCA18 } & \text { GAPU59 } & \text { GAPU71A } & \text { GUPA71B } & \text { GAPU103A } \\ \mathbf{1 8 2} \mathbf{- 2 0 6} & \mathbf{1 7 3 - \mathbf { 1 8 1 }} & \mathbf{2 1 2} \mathbf{- 2 1 8} & \mathbf{2 1 4} \mathbf{- 2 1 4} & \mathbf{1 2 4} \mathbf{- 1 4 4} & \mathbf{1 7 0 - \mathbf { 1 8 4 }} \\ \text { UDOO1 } & \text { UDO03 } & \text { UDO12 } & \text { UDO28 } & \text { UDO39 } & \\ \mathbf{1 4 4 - \mathbf { 1 4 4 }} & \mathbf{1 3 5 - \mathbf { 1 6 6 }} & \mathbf{1 6 6 - \mathbf { 1 9 3 }} & \mathbf{1 5 4 - \mathbf { 2 0 5 }} & \mathbf{2 1 3 - \mathbf { 2 1 3 }} & \end{array}$

\section{References:}

1 - Pietrangeli E., Russo A., In: Olivi D'Abruzzo, Grafiche di Prinzio (1997), pp. 54-55.

2 - Muzzalupo I., Salimonti A., Caravita M. A., et al. Adv. Hort. Sci. (2008), 22(2): pp. 129-135. 


\section{“Posolella"}

(synonymy: Gentile, Precoce.)

Areal distribution or origin area: Abruzzo

Flesh/pit weight ratio: medium $(5,11 \pm \mathbf{0 , 2 1})$

Oil content (\%): medium $(43,01 \pm 2,05)$

Purpose: oil

\section{Morphological characters}

Tree characters

Vigour: medium

Growth habit: spreading-erect

Canopy-density: medium-sparse

\section{Leaf characters}

Blade length (cm): medium $(5,50 \pm 0,38)$

Blade width $(\mathrm{cm})$ : medium $(\mathbf{1}, 00 \pm 0,12)$

Shape (length/width): elliptic - lanceolate

\section{Inflorescence characters}

Inflorescence length (cm): long $(3,53 \pm 1,28)$

Number of flowers: medium $(18,79 \pm 1,69)$

\section{Fruit characters}

Fresh weight of 100 fruits (g): low $(\mathbf{1}, \mathbf{6 0} \pm \mathbf{0 , 0 7})$

Shape (length/width): ovoid

Symmetry: slightly asymmetric

Position of maximum transverse diameter:

central

Apex: rounded

Base: rounded

Niplle: absent

Lenticels: many and small

\section{Pit characters}

Weight of 100 pits (g): low $(\mathbf{0 , 2 6} \pm \mathbf{0 , 0 1})$

Shape (length/width): ovoid

Mucron: obvious

Symmetry: slightly asymmetric

Position of maximum transverse diameter:

towards apex

Apex: rounded

Base: rounded

Surface: smooth

Number of grooves: medium
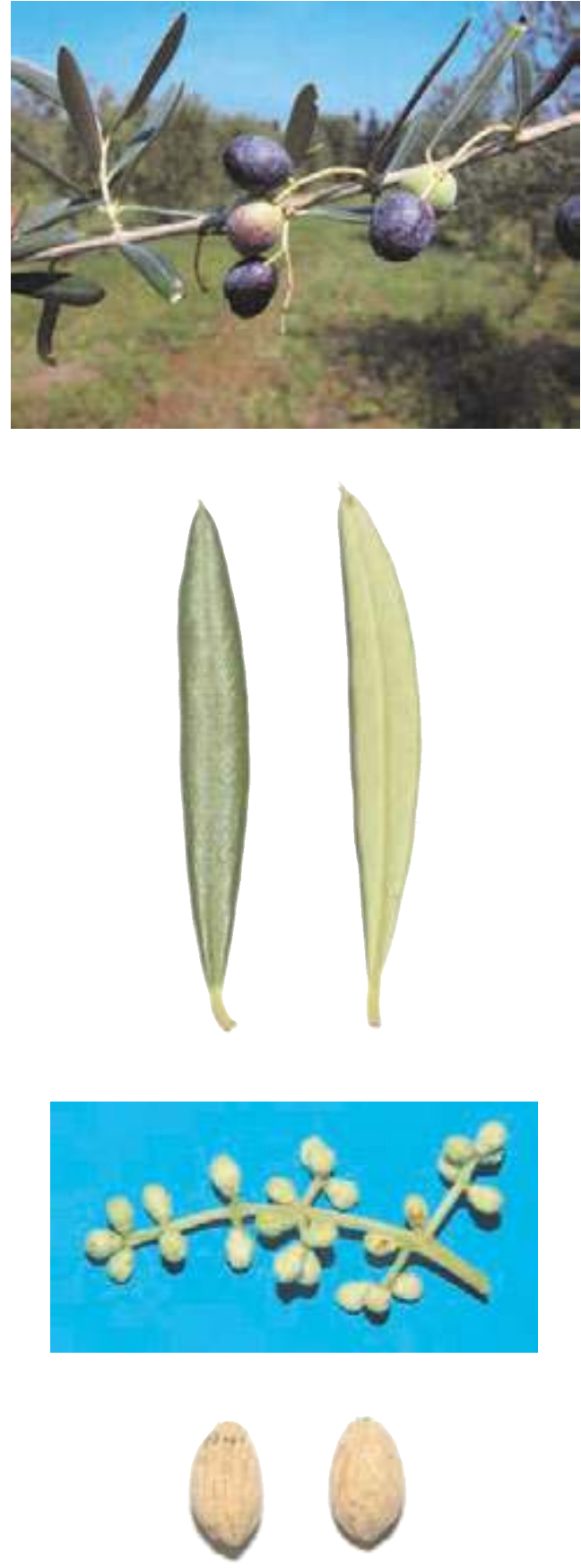


\section{Biochemical Characters}

\section{Fatty Acid Composition}

Table 1. Average values (express in $\% \pm$ standard deviations) of the fatty acids methyl esters and nutritional ratios obtained from single cultivar olive oils.

$\begin{array}{llllll}\text { Myristic acid } & \mathbf{0 , 0 1} \pm \mathbf{0 , 0 0} & \text { Linoleic acid }(\omega 6) & \mathbf{9 , 4 7} \pm \mathbf{0 , 2 1} & \text { Lignoceric acid } & \mathbf{0 , 0 4} \pm \mathbf{0 , 0 3} \\ \text { Palmitic acid } & \mathbf{1 2 , 4 4} \pm \mathbf{0 , 8 5} & \text { Linolenic acid }(\omega 3) \mathbf{0 , 8 2} \pm \mathbf{0 , 0 6} & & \\ \text { Palmitoleic acid } & \mathbf{0 , 9 6} \pm \mathbf{0 , 1 8} & \text { Arachic acid } & \mathbf{0 , 2 7} \pm \mathbf{0 , 0 5} & \text { Unsat./satured } & \mathbf{5 , 8 8} \pm \mathbf{0 , 4 9} \\ \text { Stearic acid } & \mathbf{1 , 8 1} \pm \mathbf{0 , 1 5} & \text { Eicosenoic acid } & \mathbf{0 , 0 2} \pm \mathbf{0 , 0 0} & \omega 6 / \omega 3 & \mathbf{1 1 , 6 4 \pm 0 , 8 0} \\ \text { Oleic acid } & \mathbf{7 3 , 1 5} \pm \mathbf{1 , 5 5} & \text { Behenic acid } & \mathbf{0 , 0 5} \pm \mathbf{0 , 0 1} & & \end{array}$

\section{Organoleptic oil values}

Sensory Analysis (Panel test)

Comment: medium-high fruity, with good sensation of almond, less grass, tomato and artichoke. Balanced taste sensation, with bitter and spicy medium. Medium-high fluidity.

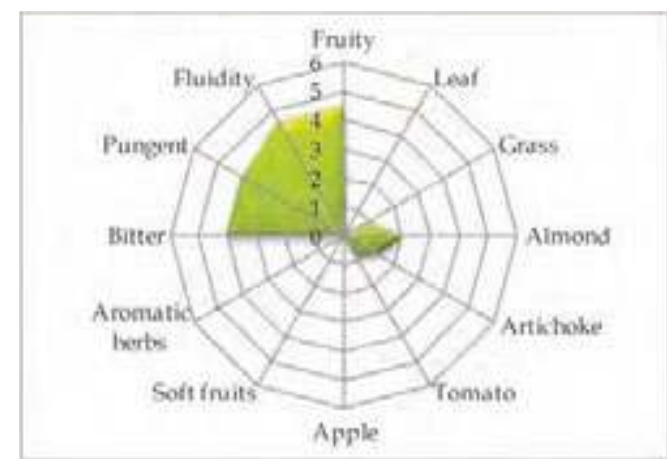

\section{Molecular Markers}

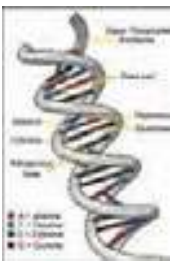

Table 2. Microsatellites (SSR) loci analyzed.

For each locus the allele size (expressed in base pairs) is reported.

$\begin{array}{cccccc}D C A 09 & D C A 18 & \text { GAPU59 } & \text { GAPU71A } & \text { GUPA71B } & \text { GAPU103A } \\ \mathbf{1 9 4 - \mathbf { 2 0 6 }} & \mathbf{1 7 3 - \mathbf { 1 8 5 }} & \mathbf{2 1 4 - \mathbf { 2 1 8 }} & \mathbf{2 1 4} \mathbf{- 2 1 4} & \mathbf{1 2 6 - \mathbf { 1 4 4 }} & \mathbf{1 5 9 - \mathbf { 1 5 9 }} \\ \text { UDO01 } & \text { UDOO3 } & \text { UDO12 } & \text { UDO28 } & \text { UDO39 } & \\ \mathbf{1 4 4 - \mathbf { 1 4 4 }} & \mathbf{1 5 0 - \mathbf { 1 5 0 }} & \mathbf{1 7 7 - \mathbf { 1 9 3 }} & \mathbf{1 5 4 - \mathbf { 2 0 5 }} & \mathbf{2 1 3 - \mathbf { 2 1 3 }} & \end{array}$

\section{References:}

1 - Pietrangeli E., Russo A., In: Olivi D'Abruzzo, Grafiche di Prinzio (1997), pp. 56-57.

2 - Muzzalupo I., Salimonti A., Caravita M. A., et al. Adv. Hort. Sci. (2008), 22(2): pp. 129-135. 


\section{"Provenzale"}

(synonymy: Olivastro, Olivone, etc.)

Areal distribution or origin area: Campania

Flesh/pit weight ratio: medium $(7,05 \pm 1,00)$

Oil content (\%): medium $(41,78 \pm 0,43)$

Purpose: dual purpose

\section{Morphological characters}

Tree characters

Vigour: strong

Growth habit: spreading-erect

Canopy-density: dense

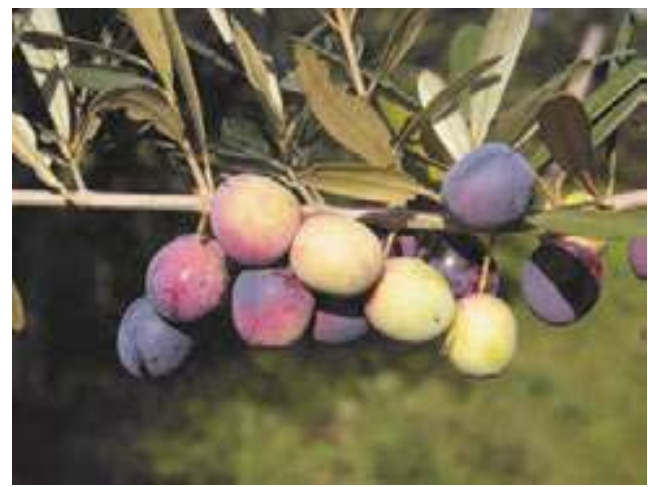

\section{Leaf characters}

Blade length $(\mathrm{cm})$ : medium $(5,44 \pm 0,46)$

Blade width $(\mathrm{cm})$ : medium $(1,06 \pm 0,10)$

Shape (length/width): elliptic-lanceolate

\section{Inflorescence characters}

Inflorescence length $(\mathrm{cm})$ : medium $(2,60 \pm 0,86)$

Number of flowers: low $(13,49 \pm 1,59)$

\section{Fruit characters}

Fresh weight of 100 fruits (g): medium $(2,83 \pm 0,08)$

Shape (length/width): spherical

Symmetry: symmetric

Position of maximum transverse diameter:

central

Apex: rounded

Base: truncate

Niplle: absent

Lenticels: many and small

\section{Pit characters}

Weight of 100 pits (g): medium $(0,36 \pm \mathbf{0 , 0 4})$

Shape (length/width): ovoid

Mucron: tenuous

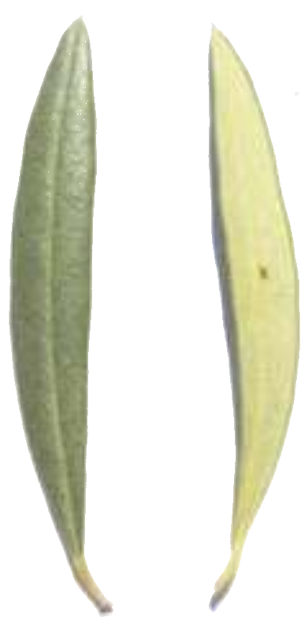

Symmetry: slightly asymmetric

Position of maximum transverse diameter:

towards apex

Apex: rounded

Base: pointed

Surface: rugose

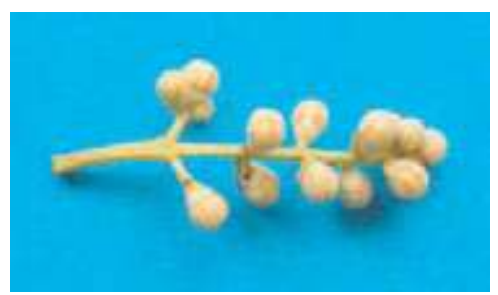

Number of grooves: medium 


\section{Biochemical Characters}

\section{Fatty Acid Composition}

Table 1. Average values (express in $\% \pm$ standard deviations) of the fatty acids methyl esters and nutritional ratios obtained from single cultivar olive oils.

$\begin{array}{llllll}\text { Myristic acid } & \mathbf{0 , 0 1} \pm \mathbf{0 , 0 0} & \text { Linoleic acid }(\omega 6) & \mathbf{3 , 6 3} \pm \mathbf{0 , 7 1} & \text { Lignoceric acid } & \mathbf{0 , 0 4} \pm \mathbf{0 , 0 2} \\ \text { Palmitic acid } & \mathbf{1 2 , 6 3 \pm \mathbf { 0 , 4 3 }} & \text { Linolenic acid }(\omega 3) \mathbf{0 , 8 9} \pm \mathbf{0 , 1 0} & & \\ \text { Palmitoleic acid } & \mathbf{2 , 2 8} \pm \mathbf{0 , 8 2} & \text { Arachic acid } & \mathbf{0 , 2 3} \pm \mathbf{0 , 0 3} & \text { Unsat./satured } & \mathbf{5 , 8 3} \pm \mathbf{0 , 2 5} \\ \text { Stearic acid } & \mathbf{1 , 5 1} \pm \mathbf{0 , 2 7} & \text { Eicosenoic acid } & \mathbf{0 , 0 3} \pm \mathbf{0 , 0 1} & \omega 6 / \omega 3 & \mathbf{4 , 0 8} \pm \mathbf{0 , 4 5} \\ \text { Oleic acid } & \mathbf{7 6 , 6 3 \pm \mathbf { 1 , 9 7 }} & \text { Behenic acid } & \mathbf{0 , 0 7} \pm \mathbf{0 , 0 3} & & \end{array}$

\section{Organoleptic oil values}

Sensory Analysis (Panel test)

Comment: fruity medium, with taste of artichoke and tomato, read sensations of almond. Balanced taste sensation with medium bitter and spicy. Medium fluidity.

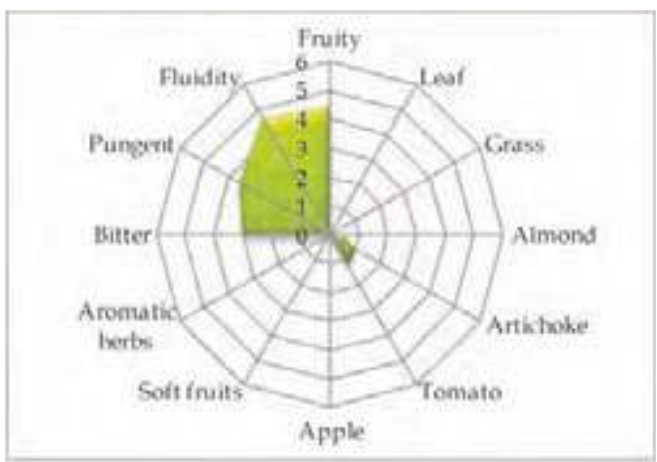

\section{Molecular Markers}

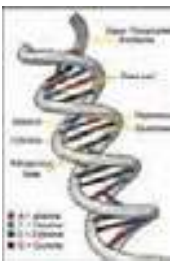

Table 2. Microsatellites (SSR) loci analyzed.

For each locus the allele size (expressed in base pairs) is reported.

$\begin{array}{cccccc}D C A 09 & \text { DCA18 } & \text { GAPU59 } & \text { GAPU71A } & \text { GUPA71B } & \text { GAPU103A } \\ \mathbf{1 9 4 - \mathbf { 2 0 6 }} & \mathbf{1 7 9 - \mathbf { 1 8 1 }} & \mathbf{2 1 8 - \mathbf { 2 1 8 }} & \mathbf{2 1 8 - \mathbf { 2 1 8 }} & \mathbf{1 2 6 - \mathbf { 1 4 4 }} & \mathbf{1 5 7 - \mathbf { 1 5 9 }} \\ \text { UDOO1 } & \text { UDO03 } & \text { UDO12 } & \text { UDO28 } & \text { UDO39 } & \\ \mathbf{1 4 4 - \mathbf { 1 5 0 }} & \mathbf{1 4 3 - \mathbf { 1 5 0 }} & \mathbf{1 6 4 - \mathbf { 1 6 4 }} & \mathbf{1 4 3 - \mathbf { 2 1 0 }} & \mathbf{2 0 0 - \mathbf { 2 2 0 }} & \end{array}$

\section{References:}

1 - Cimato A., Cantini C., Sani G., In: L'olivo in Toscana: il germoplasma autoctono, Ed. ARSIA (2001).

2 - Muzzalupo I., Stefanizzi F., Perri E. HortScience (2009), 44: pp. 582-588. 


\section{“Puntella"}

Areal distribution or origin area: Abruzzo

Flesh/pit weight ratio: medium $(5,08 \pm 0,31)$

Oil content (\%): medium $(45,09 \pm 0,66)$

Purpose: oil

\section{Morphological characters}

Tree characters

Vigour: medium-strong

Growth habit: erect

Canopy-density: medium-dense

\section{Leaf characters}

Blade length ( $\mathrm{cm})$ : medium $(5,78 \pm 0,45)$

Blade width (cm): broad $(\mathbf{1}, \mathbf{5 3} \pm \mathbf{0 , 2 2})$

Shape (length/width): elliptic

\section{Inflorescence characters}

Inflorescence length (cm): medium $(3,13 \pm 0,36)$

Number of flowers: medium $(21,00 \pm 0,87)$

\section{Fruit characters}

Fresh weight of 100 fruits $(\mathrm{g})$ : low $(\mathbf{1}, \mathbf{6 6} \pm \mathbf{0 , 1 7})$

Shape (length/width): spherical

Symmetry: asymmetric

Position of maximum transverse diameter:

central

Apex: rounded

Base: truncate

Niplle: tenuous

Lenticels: many and small

\section{Pit characters}

Weight of 100 pits (g): low $(0,28 \pm \mathbf{0 , 0 3})$

Shape (length/width): ovoid

Mucron: obvious

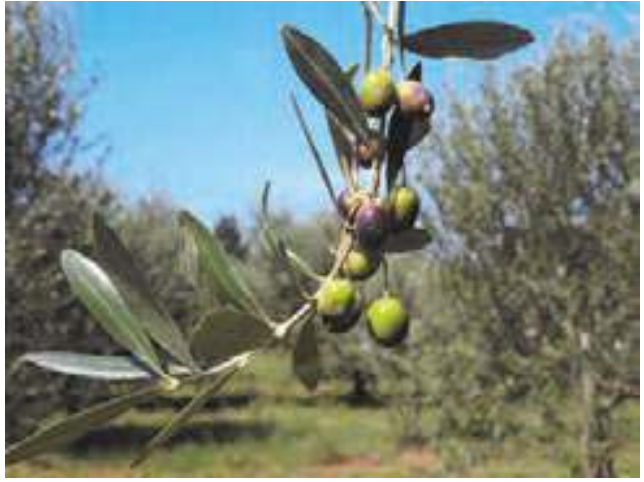

Symmetry: slightly asymmetric

Position of maximum transverse diameter:

central

Apex: rounded

Base: rounded

Surface: scabrous
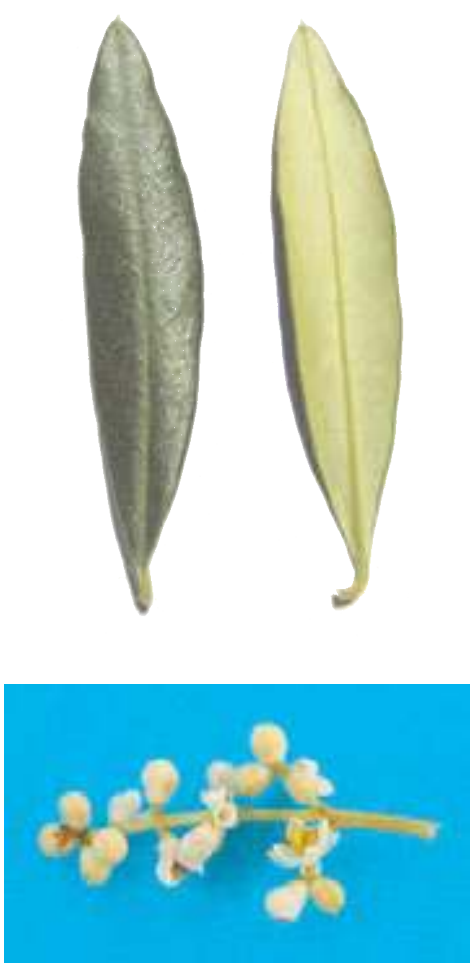

Number of grooves: medium 


\section{Biochemical Characters}

\section{Fatty Acid Composition}

Table 1. Average values (express in $\% \pm$ standard deviations) of the fatty acids methyl esters and nutritional ratios obtained from single cultivar olive oils.

$\begin{array}{llll}\text { Myristic acid } \quad 0,01 \pm 0,00 & \text { Linoleic acid }(\omega 6) & \mathbf{1 5}, 44 \pm 0,65 & \text { Lignoceric acid } \quad 0,05 \pm 0,04\end{array}$

Palmitic acid $\quad 16,71 \pm 0,35$ Linolenic acid $(\omega 3) 0,96 \pm 0,02$

Palmitoleic acid

$2,93 \pm 0,52 \quad$ Arachic acid $\quad 0,29 \pm 0,05$

Unsat./satured

$4,20 \pm 2,10$

Stearic acid

$1,64 \pm 0,07$

Eicosenoic acid

$0,02 \pm 0,00$

$\omega 6 / \omega 3$

$16,15 \pm 1,01$

Oleic acid

$58,86 \pm 0,22$

Behenic acid

$0,10 \pm 0,04$

\section{Organoleptic oil values}

Sensory Analysis (Panel test)

Comment: fruity medium, with read sensa-

tions of artichoke and almond. Balanced taste sensation with medium spicy and bitter. Medium fluidity.

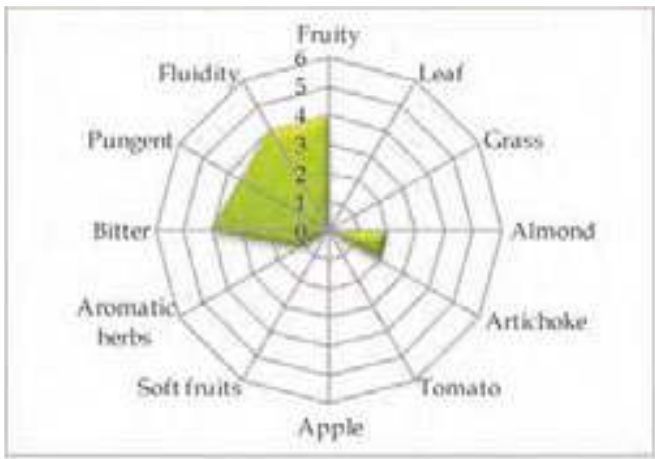

\section{Molecular Markers}

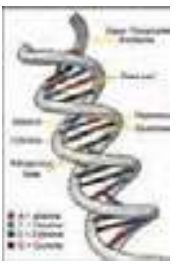

Table 2. Microsatellites (SSR) loci analyzed.

For each locus the allele size (expressed in base pairs) is reported.

$\begin{array}{cccccc}D C A 09 & \text { DCA18 } & \text { GAPU59 } & \text { GAPU71A } & \text { GUPA71B } & \text { GAPU103A } \\ \mathbf{1 9 4 - \mathbf { 1 9 8 }} & \mathbf{1 7 7 - \mathbf { 1 8 5 }} & \mathbf{2 2 2} \mathbf{- 2 1 4} & \mathbf{2 1 4} \mathbf{- 2 1 4} & \mathbf{1 2 4} \mathbf{- 1 3 0} & \mathbf{1 5 9 - \mathbf { 1 5 9 }} \\ \text { UDOO1 } & \text { UDO03 } & \text { UDO12 } & \text { UDO28 } & \text { UDO39 } & \\ \mathbf{1 4 4 - \mathbf { 1 4 4 }} & \mathbf{2 0 2 - \mathbf { 2 0 2 }} & \mathbf{1 6 6 - \mathbf { 1 9 3 }} & \mathbf{1 5 4 - \mathbf { 2 0 5 }} & \mathbf{2 1 3 - \mathbf { 2 1 3 }} & \end{array}$

\section{References:}

1 - Castorina S. In: Le varietà di olivo coltivate in Abruzzo, Ann. Sper. Agr. (1954), 9 (6) pp. 11-53.

2 - Muzzalupo I., Salimonti A., Caravita M. A., et al. Adv. Hort. Sci. (2008), 22(2): pp. 129-135. 


\section{“Racioppa "}

(synonymy: Cioppella, Racioppella, Racioppa di Rivello, etc.)

Areal distribution or origin area: Basilicata

Flesh/pit weight ratio: medium $(5,37 \pm 1,26)$

Oil content (\%): medium $(46,38 \pm 2,38)$

Purpose: oil

\section{Morphological characters}

Tree characters

Vigour: medium-strong

Growth habit: erect-sreading

Canopy-density: medium-dense

\section{Leaf characters}

Blade length (cm): medium $(6,59 \pm 1,09)$

Blade width $(\mathrm{cm})$ : medium $(1,48 \pm 0,38)$

Shape (length/width): elliptic-lanceolate

\section{Inflorescence characters}

Inflorescence length (cm): short $(2,48 \pm 0,31)$

Number of flowers: medium $(19,58 \pm 1,78)$

\section{Fruit characters}

Fresh weight of 100 fruits (g): medium $(2,23 \pm 0,32)$

Shape (length/width): spherical

Symmetry: symmetric

Position of maximum transverse diameter:

central

Apex: rounded

Base: rounded

Niplle: absent

Lenticels: many and small

\section{Pit characters}

Weight of 100 pits (g): medium $(0,36 \pm 0,03)$

Shape (length/width): ovoid

Mucron: obvious

Symmetry: slightly asymmetric

Position of maximum transverse diameter:

towards apex

Apex: rounded

Base: rounded

Surface: smooth

Number of grooves: medium
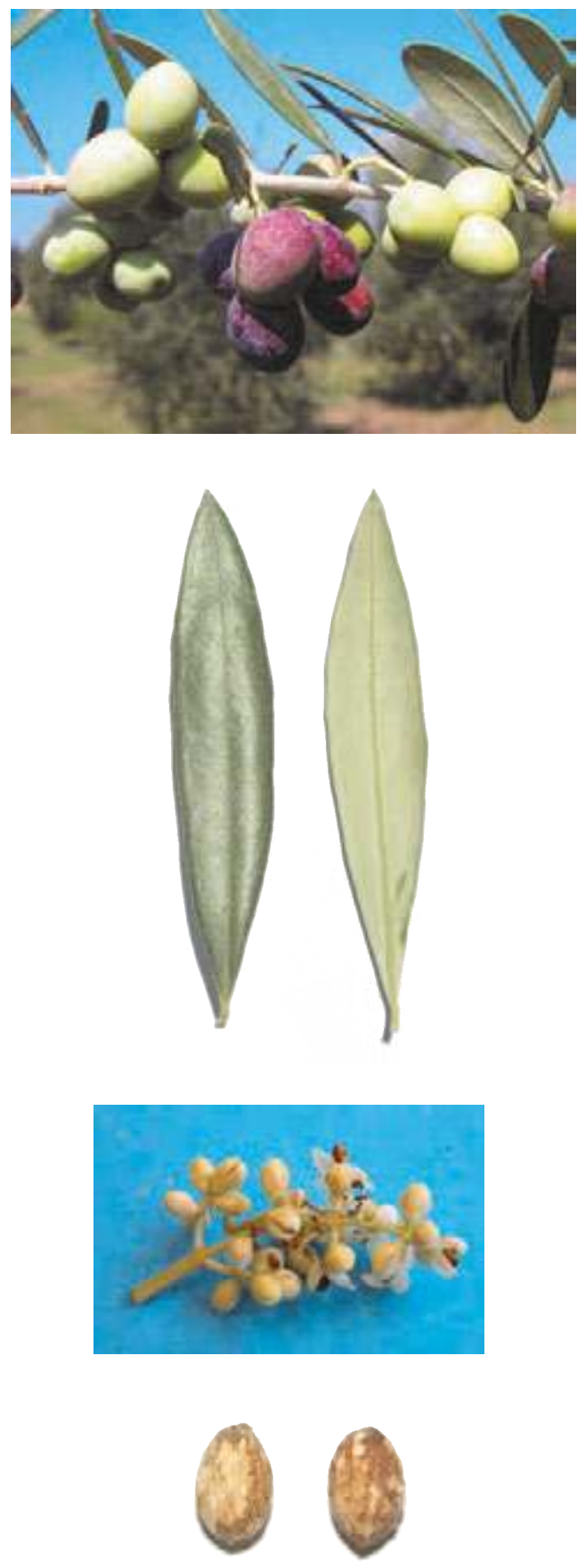


\section{Biochemical Characters}

\section{Fatty Acid Composition}

Table 1. Average values (express in $\% \pm$ standard deviations) of the fatty acids methyl esters and nutritional ratios obtained from single cultivar olive oils.

$\begin{array}{llllll}\text { Myristic acid } & \mathbf{0 , 0 1} \pm \mathbf{0 , 0 1} & \text { Linoleic acid }(\omega 6) & \mathbf{7 , 0 0} \pm \mathbf{0 , 3 8} & \text { Lignoceric acid } & \mathbf{0 , 0 7 \pm 0 , 0 5} \\ \text { Palmitic acid } & \mathbf{1 3 , 2 1} \pm \mathbf{2 , 3 3} & \text { Linolenic acid }(\omega 3) \mathbf{0 , 6 2} \pm \mathbf{0 , 0 7} & & \\ \text { Palmitoleic acid } & \mathbf{2 , 0 3} \pm \mathbf{0 , 4 7} & \text { Arachic acid } & \mathbf{0 , 2 7} \pm \mathbf{0 , 0 8} & \text { Unsat./satured } & \mathbf{5 , 5 4} \pm \mathbf{0 , 9 8} \\ \text { Stearic acid } & \mathbf{1 , 7 3} \pm \mathbf{0 , 1 3} & \text { Eicosenoic acid } & \mathbf{0 , 1 5} \pm \mathbf{0 , 1 2} & \omega 6 / \omega 3 & \mathbf{1 1 , 2 7 \pm 0 , 8 9} \\ \text { Oleic acid } & \mathbf{7 2 , 9 2 \pm 2 , 9 8} & \text { Behenic acid } & \mathbf{0 , 0 8} \pm 0,04 & & \end{array}$

\section{Organoleptic oil values}

Sensory Analysis (Panel test)

Comment: fruity medium, with taste of almond and artichoke, with read sensations of aromatic herbs. Balanced taste sensation with medium-high bitter and spicy. Medium fluidity.

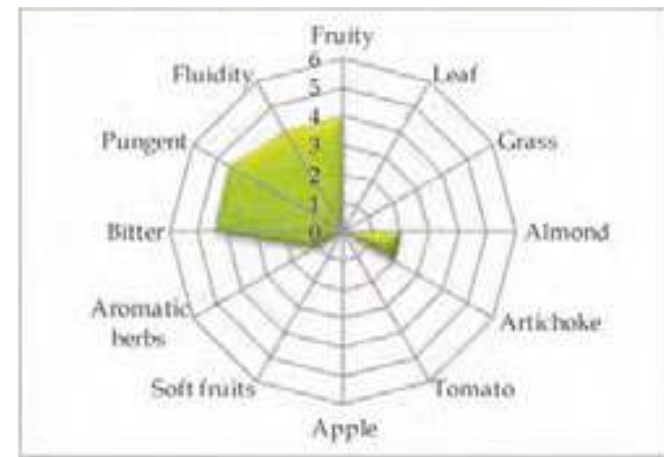

\section{Molecular Markers}

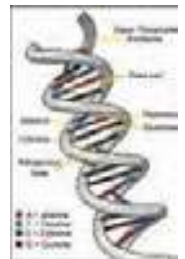

Table 2. Microsatellites (SSR) loci analyzed. For each locus the allele size (expressed in base pairs) is reported.

$\begin{array}{cccccc}D C A 09 & D C A 18 & \text { GAPU59 } & \text { GAPU71A } & \text { GUPA71B } & \text { GAPU103A } \\ \mathbf{1 7 2} \mathbf{- 1 8 2} & \mathbf{1 7 9 - \mathbf { 1 8 5 }} & \mathbf{2 0 8 - \mathbf { 2 0 8 }} & \mathbf{2 1 2} \mathbf{- 2 1 4} & \mathbf{1 2 4} \mathbf{- 1 3 0} & \mathbf{1 5 9 - \mathbf { 1 7 0 }} \\ \text { UDOO1 } & \text { UDOO3 } & \text { UDO12 } & \text { UDO28 } & \text { UDO39 } & \\ \mathbf{1 4 4 - \mathbf { 1 4 4 }} & \mathbf{1 4 3 - \mathbf { 1 4 3 }} & \mathbf{1 6 6 - 1 9 3} & \mathbf{1 6 1 - 2 0 5} & \mathbf{2 2 0} \mathbf{- 2 3 2} & \end{array}$

\section{References:}

1 - Rotundo A., Marone E. In: Il germoplasma olivicolo lucano, Olita-Potenza (2002), pp. 135-138.

2 - Rotundo A., Perri E.,Muzzalupo I., et al. In: Il germoplasma olivicolo meridionale, (2012 in press).

3 - Muzzalupo I., Stefanizzi F., Perri E. HortScience (2009), 44: pp. 582-588. 


\section{“Racioppella"}

(synonymy: Cacaccella, Grappetella, Olivo a racioppella etc.)

Areal distribution or origin area: Campania

Flesh/pit weight ratio: medium $(5,63 \pm 1,39)$

Oil content (\%): medium $(43,34 \pm 0,97)$

Purpose: oil

\section{Morphological characters}

Tree characters

Vigour: medium-weak

Growth habit: erect-spreading

Canopy-density: medium-sparse

\section{Leaf characters}

Blade length $(\mathrm{cm})$ : medium $(5,49 \pm 0,45)$

Blade width $(\mathrm{cm})$ : broad $(\mathbf{1}, \mathbf{5 1} \pm \mathbf{0 , 1 2})$

Shape (length/width): elliptic

\section{Inflorescence characters}

Inflorescence length $(\mathrm{cm})$ : long $(3,71 \pm 0,72)$

Number of flowers: medium $(\mathbf{1 4 , 1 2} \pm \mathbf{1 , 2 7})$

\section{Fruit characters}

Fresh weight of 100 fruits $(\mathrm{g})$ : medium $(2,40 \pm 0,09)$

Shape (length/width): ovoid

Symmetry: asymmetric

Position of maximum transverse diameter:

central

Apex: rounded

Base: truncate

Niplle: absent

Lenticels: many and small

\section{Pit characters}

Weight of 100 pits (g): medium $(0,38 \pm 0,07)$

Shape (length/width): ovoid

Mucron: obvious

Symmetry: slightly asymmetric

Position of maximum transverse diameter:

towards apex

Apex: rounded

Base: rounded

Surface: scabrous

Number of grooves: high
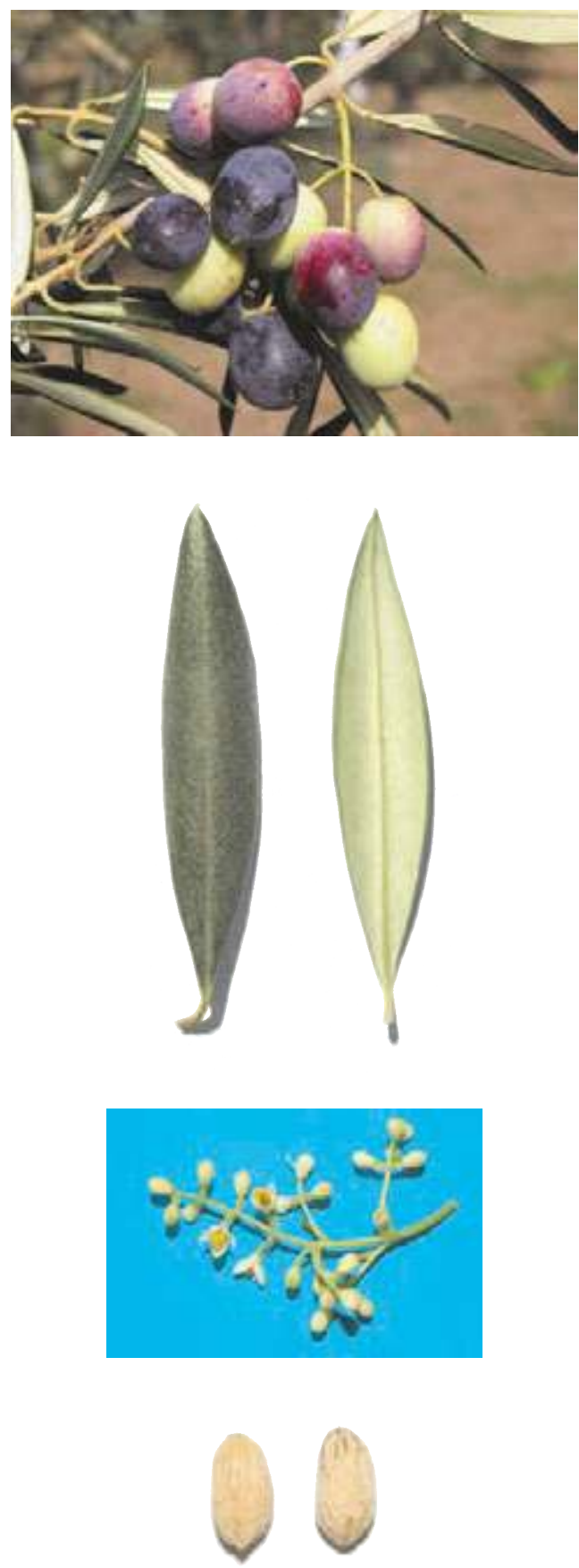


\section{Biochemical Characters}

\section{Fatty Acid Composition}

Table 1. Average values (express in $\% \pm$ standard deviations) of the fatty acids methyl esters and nutritional ratios obtained from single cultivar olive oils.

$\begin{array}{llllll}\text { Myristic acid } & \mathbf{0 , 0 1} \pm \mathbf{0 , 0 1} & \text { Linoleic acid }(\omega 6) & \mathbf{1 1 , 8 0} \pm \mathbf{0 , 8 5} & \text { Lignoceric acid } & \mathbf{0 , 0 5} \pm \mathbf{0 , 0 2} \\ \text { Palmitic acid } & \mathbf{1 0 , 2 6} \pm \mathbf{0 , 3 9} & \text { Linolenic acid }(\omega 3) & \mathbf{0 , 8 1} \pm \mathbf{0 , 0 2} & & \\ \text { Palmitoleic acid } & \mathbf{1 , 1 7} \pm \mathbf{0 , 7 3} & \text { Arachic acid } & \mathbf{0 , 6 1} \pm \mathbf{0 , 4 8} & \text { Unsat./satured } & \mathbf{6 , 5 9} \pm \mathbf{0 , 1 5} \\ \text { Stearic acid } & \mathbf{2 , 4 4} \pm \mathbf{0 , 0 3} & \text { Eicosenoic acid } & \mathbf{0 , 1 5} \pm \mathbf{0 , 1 7} & \omega 6 / \omega 3 & \mathbf{1 4 , 5 4} \pm \mathbf{1 , 4 8} \\ \text { Oleic acid } & \mathbf{7 1 , 2 4} \pm \mathbf{0 , 3 6} & \text { Behenic acid } & \mathbf{0 , 0 9} \pm \mathbf{0 , 0 3} & & \end{array}$

\section{Organoleptic oil values}

Sensory Analysis (Panel test)

Comment: fruity medium, with read sensations of almond and leaves. Balanced taste sensation with a medium bitter and mediumhigh spicy. Medium fluidity.

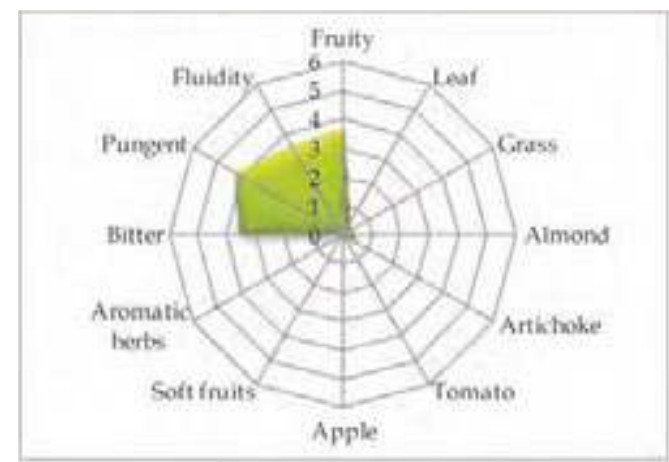

\section{Molecular Markers}

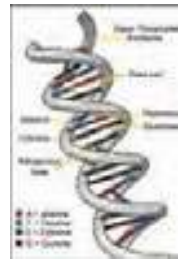

Table 2. Microsatellites (SSR) loci analyzed.

For each locus the allele size (expressed in base pairs) is reported.

$\begin{array}{cccccc}D C A 09 & \text { DCA18 } & \text { GAPU59 } & \text { GAPU71A } & \text { GUPA71B } & \text { GAPU103A } \\ \mathbf{1 9 4 - \mathbf { 1 9 8 }} & \mathbf{1 7 7 - \mathbf { 1 7 9 }} & \mathbf{2 1 8 - \mathbf { 2 2 2 }} & \mathbf{2 1 2} \mathbf{- 2 2 4} & \mathbf{1 2 4 - \mathbf { 1 3 0 }} & \mathbf{1 7 0 - \mathbf { 1 8 4 }} \\ \text { UDOO1 } & \text { UDO03 } & \text { UDO12 } & \text { UDO28 } & \text { UDO39 } & \\ \mathbf{1 4 4 - \mathbf { 1 4 4 }} & \mathbf{1 3 5 - \mathbf { 1 3 5 }} & \mathbf{1 6 4 - \mathbf { 1 8 2 }} & \mathbf{1 4 3 - \mathbf { 1 4 3 }} & \mathbf{2 0 5 - \mathbf { 2 2 0 }} & \end{array}$

\section{References:}

1 - Various authors. In: Catalogo Nazionale delle Varietà di Olivo . University of Bari (in press), ISBN 978-88-8879397-9.

2 - Muzzalupo I., Stefanizzi F., Perri E. HortScience (2009), 44: pp. 582-588. 


\section{“Raggiola"}

(synonymy: Corgiola, Correggiolo, Ragiola, Vergiola, etc.)

Areal distribution or origin area: Marche

Flesh/pit weight ratio: low $(3,53 \pm 0,61)$

Oil content (\%): medium $(43,76 \pm 1,15)$

Purpose: oil

\section{Morphological characters}

Tree characters

Vigour: medium

Growth habit: spreading-erect

Canopy-density: dense

\section{Leaf characters}

Blade length $(\mathrm{cm})$ : medium $(5,32 \pm 0,37)$

Blade width (cm): medium $(\mathbf{1 , 3 8} \pm 0,16)$

Shape (length/width): elliptic

\section{Inflorescence characters}

Inflorescence length $(\mathrm{cm})$ : long $(3,98 \pm 0,38)$

Number of flowers: medium $(19,08 \pm 1,21)$

\section{Fruit characters}

Fresh weight of 100 fruits (g): medium $(2,40 \pm 0,10)$

Shape (length/width): ovoid

Symmetry: slightly asymmetric

Position of maximum transverse diameter:

central

Apex: rounded

Base: rounded

Niplle: tenuous

Lenticels: many and small

\section{Pit characters}

Weight of 100 pits (g): high $(0,54 \pm 0,09)$

Shape (length/width): elliptic

Mucron: tenuous

Symmetry: slightly asymmetric

Position of maximum transverse diameter:

\section{towards apex}

Apex: rounded

Base: rounded

Surface: rugose

Number of grooves: low
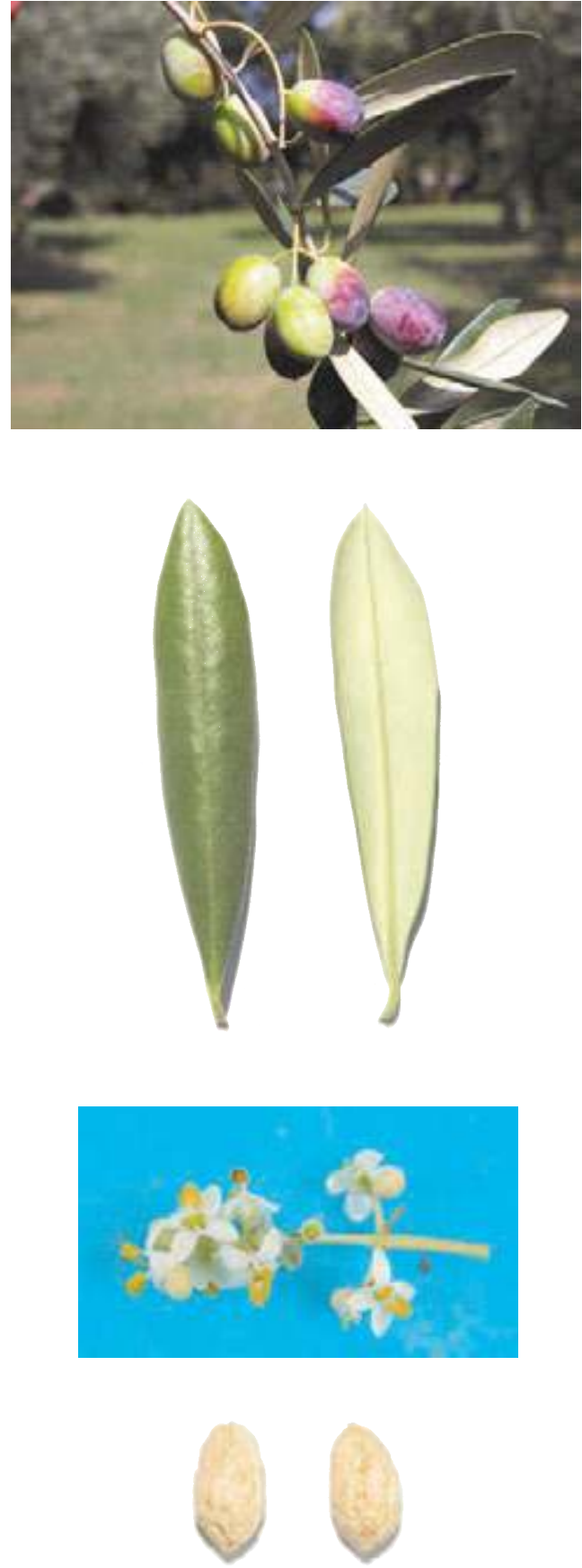


\section{Biochemical Characters}

\section{Fatty Acid Composition}

Table 1. Average values (express in $\% \pm$ standard deviations) of the fatty acids methyl esters and nutritional ratios obtained from single cultivar olive oils.

\begin{tabular}{|c|c|c|c|c|c|}
\hline Myristic acid & $0,01 \pm 0,01$ & Linoleic acid $(\omega 6)$ & $10,81 \pm 0,22$ & Lignoceric acid & $0,06 \pm 0,01$ \\
\hline Palmitic acid & $13,35 \pm 0,14$ & Linolenic acid $(\omega 3)$ & $0,87 \pm 0,03$ & & \\
\hline Palmitoleic acid & $1,64 \pm 0,03$ & Arachic acid & $0,49 \pm 0,12$ & Unsat./satured & $5,27 \pm 0,12$ \\
\hline tear & $2,05 \pm 0,08$ & Eicosenoic acid & $0,39 \pm 0,01$ & $\omega 6 / \omega 3$ & $12,46 \pm 0,66$ \\
\hline leic acid & $68,96 \pm 0,26$ & Behenic acid & $0,11 \pm 0,01$ & & \\
\hline
\end{tabular}

\section{Organoleptic oil values}

Sensory Analysis (Panel test)

Comment: fruity medium - light, with hints of artichoke and almond. Balanced in flavours, with hints of bitter and spicy medium intensity, medium fluidity.

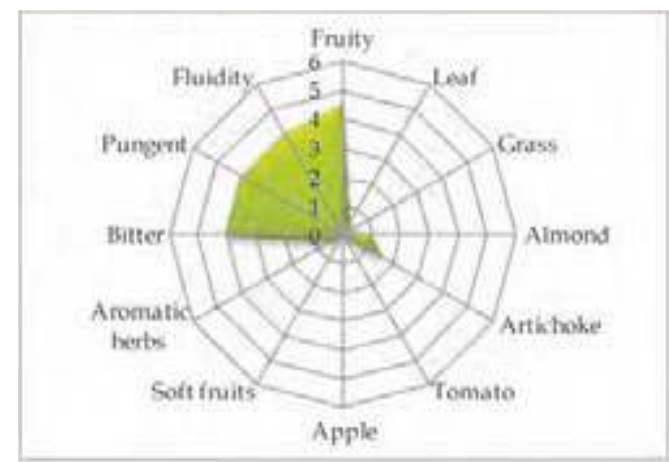

\section{Molecular Markers}

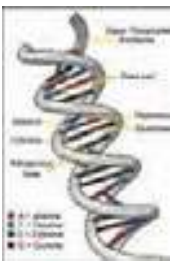

Table 2. Microsatellites (SSR) loci analyzed.

For each locus the allele size (expressed in base pairs) is reported.

$\begin{array}{cccccc}D C A 09 & D C A 18 & \text { GAPU59 } & \text { GAPU71A } & \text { GUPA71B } & \text { GAPU103A } \\ \mathbf{1 8 2} \mathbf{- 2 0 6} & \mathbf{1 7 9 - \mathbf { 1 8 1 }} & \mathbf{2 1 2} \mathbf{- 2 2 2} & \mathbf{2 1 8 - \mathbf { 2 2 4 }} & \mathbf{1 2 6 - \mathbf { 1 4 4 }} & \mathbf{1 5 7 - \mathbf { 1 7 0 }} \\ \text { UDO01 } & \text { UDOO3 } & \text { UDO12 } & \text { UDO28 } & \text { UDO39 } & \\ \mathbf{1 4 4 - \mathbf { 1 4 4 }} & \mathbf{1 5 0 - \mathbf { 1 5 0 }} & \mathbf{1 7 7 - \mathbf { 1 8 2 }} & \mathbf{1 6 1 - \mathbf { 1 8 2 }} & \mathbf{1 8 5 - \mathbf { 1 8 5 }} & \end{array}$

\section{References:}

1 - Pannelli G., Alfei B., Santinelli A. In: Varietà di olivo nelle Marche. ASSAM (2001), pp. 105-108.

2 - Muzzalupo I., Stefanizzi F., Bucci C., et al. In Acta Italus Hortus, (2011), 1: 138-140. 


\section{"Raja sabina"}

Areal distribution or origin area: Lazio

Flesh/pit weight ratio: low $(\mathbf{4 , 2 2} \pm \mathbf{0 , 7 1})$

Oil content (\%): low $(\mathbf{4 2 , 1 8} \pm 0,79)$

Purpose: oil

\section{Morphological characters}

Tree characters

Vigour: medium-strong

Growth habit: spreading

Canopy-density: medium-dense

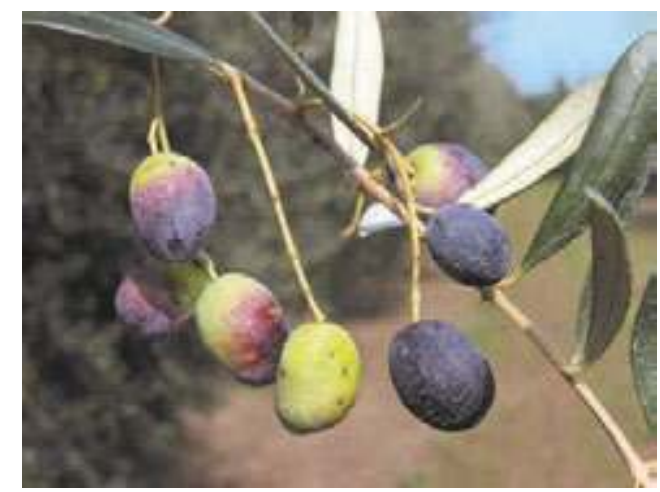

\section{Leaf characters}

Blade length $(\mathrm{cm})$ : medium $(5,87 \pm 0,47)$

Blade width $(\mathrm{cm})$ : broad $(\mathbf{1}, \mathbf{5 3} \pm \mathbf{0 , 1 7})$

Shape (length/width): elliptic

\section{Inflorescence characters}

Inflorescence length $(\mathrm{cm})$ : long $(3,70 \pm 1,43)$

Number of flowers: low $(17,08 \pm 3,62)$

\section{Fruit characters}

Fresh weight of 100 fruits $(\mathrm{g})$ : medium $(\mathbf{2 , 6 8} \pm \mathbf{0 , 3 6})$

Shape (length/width): ovoid

Symmetry: slightly asymmetric

Position of maximum transverse diameter:

towards apex

Apex: rounded

Base: rounded

Niplle: absent

Lenticels: many and large

\section{Pit characters}

Weight of 100 pits (g): high $(\mathbf{0 , 5 2} \pm \mathbf{0 , 0 8})$

Shape (length/width): elliptic

Mucron: obvious
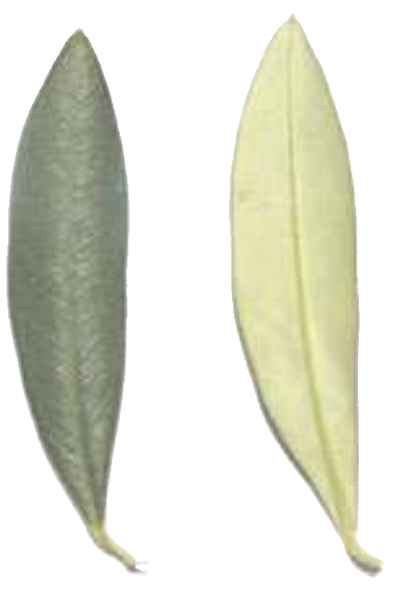

Symmetry: slighltly asymmetric

Position of maximum transverse diameter:

towards apex

Apex: rounded

Base: rounded

Surface: smooth

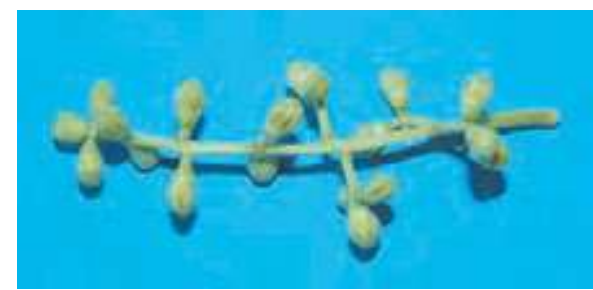

Number of grooves: low 


\section{Biochemical Characters}

\section{Fatty Acid Composition}

Table 1. Average values (express in $\% \pm$ standard deviations) of the fatty acids methyl esters and nutritional ratios obtained from single cultivar olive oils.

$\begin{array}{llllll}\text { Myristic acid } & \mathbf{0 , 0 1} \pm \mathbf{0 , 0 1} & \text { Linoleic acid }(\omega 6) & \mathbf{9 , 2 3} \pm \mathbf{1 , 3 0} & \text { Lignoceric acid } & \mathbf{0 , 0 5} \pm \mathbf{0 , 0 3} \\ \text { Palmitic acid } & \mathbf{1 3 , 2 8} \pm \mathbf{1 , 0 1} & \text { Linolenic acid }(\omega 3) \mathbf{0 , 7 6} \pm \mathbf{0 , 0 6} & & \\ \text { Palmitoleic acid } & \mathbf{1 , 4 4} \pm \mathbf{0 , 0 6} & \text { Arachic acid } & \mathbf{0 , 2 2} \pm \mathbf{0 , 0 7} & \text { Unsat./satured } & \mathbf{5 , 5 0 \pm 0 , 4 9} \\ \text { Stearic acid } & \mathbf{1 , 7 0} \pm \mathbf{0 , 0 7} & \text { Eicosenoic acid } & \mathbf{0 . 0 3} \pm \mathbf{0 , 0 1} & \omega 6 / \omega 3 & \mathbf{1 2 , 2 3} \pm \mathbf{2 , 6 4} \\ \text { Oleic acid } & \mathbf{7 1 , 9 0 \pm 2 , 4 7} & \text { Behenic acid } & \mathbf{0 , 0 8} \pm \mathbf{0 , 0 1} & & \end{array}$

\section{Organoleptic oil values}

Sensory Analysis (Panel test)

Comment: fruity medium - light, with hints of artichoke and almond. Balanced in flavours, with hints of bitter and spicy medium intensity, medium fluidity.

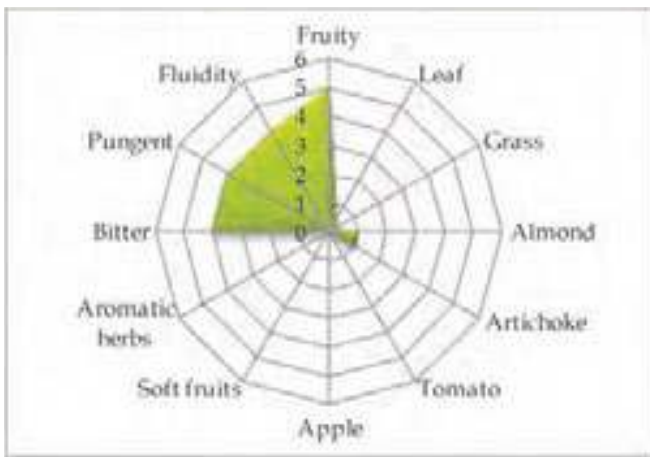

\section{Molecular Markers}

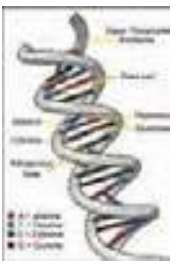

Table 2. Microsatellites (SSR) loci analyzed.

For each locus the allele size (expressed in base pairs) is reported.

$\begin{array}{cccccc}D C A 09 & \text { DCA18 } & \text { GAPU59 } & \text { GAPU71A } & \text { GUPA71B } & \text { GAPU103A } \\ \mathbf{1 7 6 - 1 9 8} & \mathbf{1 7 9 - \mathbf { 1 8 1 }} & \mathbf{2 1 2} \mathbf{- 2 2 2} & \mathbf{2 1 8 - \mathbf { 2 2 4 }} & \mathbf{1 2 4} \mathbf{- 1 4 4} & \mathbf{1 5 7 - \mathbf { 1 5 9 }} \\ \text { UDOO1 } & \text { UDOO3 } & \text { UDO12 } & \text { UDO28 } & \text { UDO39 } & \\ \mathbf{1 4 4 - \mathbf { 1 4 4 }} & \mathbf{1 5 0 - \mathbf { 1 5 0 }} & \mathbf{1 7 7 - \mathbf { 1 8 2 }} & \mathbf{1 8 2} \mathbf{- 2 1 0} & \mathbf{1 8 5 - \mathbf { 1 8 5 }} & \end{array}$

\section{References:}

1 - Lombardo N. In: Le risorse genetiche vegetali presso gli IRSA, MIPAF (2001), 1: pp. 361-405.

2 - Muzzalupo I., Stefanizzi F., Bucci C., et al. In Acta Italus Hortus, (2011), 1: 138 -140. 


\section{"Ravece"}

(synonymy: Curatona, Olivona, Ravaiola.)

Areal distribution or origin area: Campania Flesh/ pit weight ratio: low $(3,19 \pm 0,20)$ Oil content (\%): medium $(41,11 \pm 1,67)$ Purpose: oil

\section{Morphological characters}

Tree characters

Vigour: medium-weak

Growth habit: spreading-erect

Canopy-density: medium-sparse

\section{Leaf characters}

Blade length (cm): medium $(5,98 \pm 1,19)$

Blade width $(\mathrm{cm})$ : medium $(1,22 \pm 0,27)$

Shape (length/width): elliptic-lanceolate

\section{Inflorescence characters}

Inflorescence length $(\mathrm{cm})$ : medium $(2,57 \pm 2,38)$

Number of flowers: low $(\mathbf{1 4}, 76 \pm 0, \mathbf{5 7})$

\section{Fruit characters}

Fresh weight of 100 fruits $(\mathrm{g})$ : medium $(2,86 \pm 0,10)$

Shape (length/width): ovoid

Symmetry: slightly asymmetric

Position of maximum transverse diameter:

central

Apex: pointed

Base: rounded

Niplle: absent

Lenticels: many and small

\section{Pit characters}

Weight of 100 pits (g): high $(\mathbf{0 , 6 8} \pm \mathbf{0 , 0 3})$

Shape (length/width): elliptic

Mucron: obvious

Symmetry: slightly asymmetric

Position of maximum transverse diameter:

towards apex

Apex: rounded

Base: pointed

Surface: scabrous

Number of grooves: high
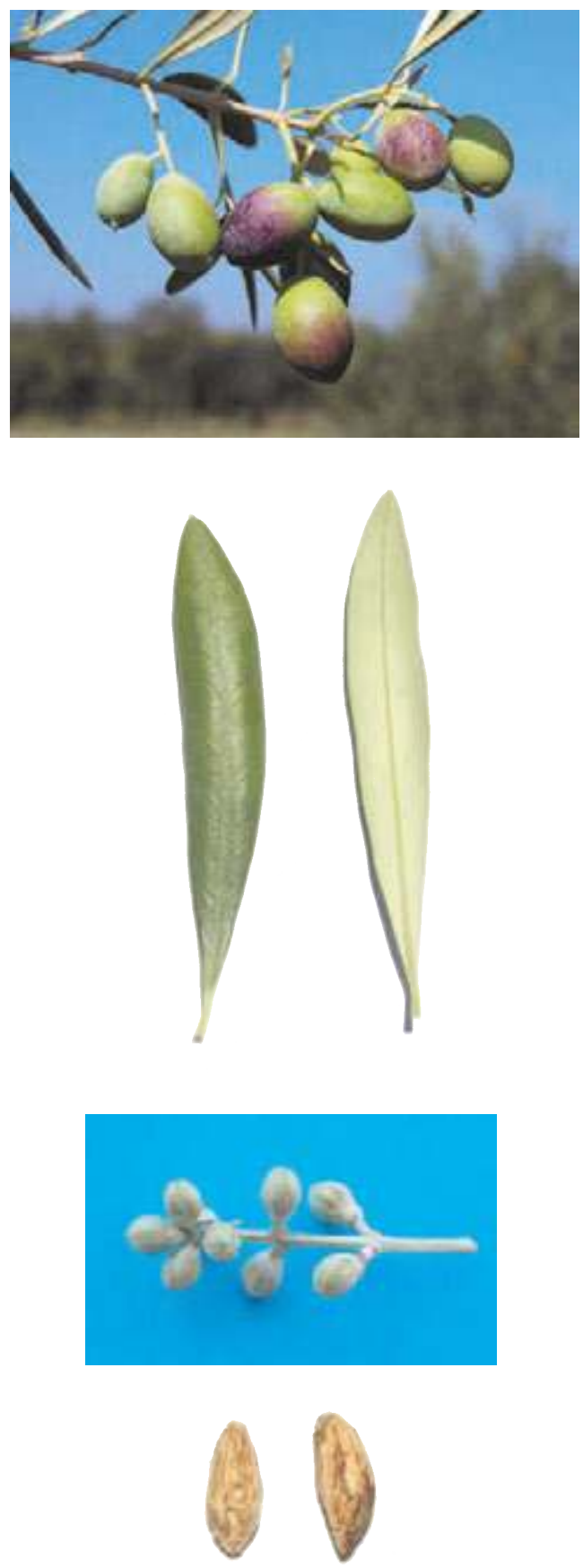


\section{Biochemical Characters}

\section{Fatty Acid Composition}

Table 1. Average values (express in $\% \pm$ standard deviations) of the fatty acids methyl esters and nutritional ratios obtained from single cultivar olive oils.

$\begin{array}{llllll}\text { Myristic acid } & \mathbf{0 , 0 1} \pm \mathbf{0 , 0 0} & \text { Linoleic acid }(\omega 6) & \mathbf{4 , 0 2} \pm \mathbf{0 , 4 0} & \text { Lignoceric acid } & \mathbf{0 , 0 8} \pm \mathbf{0 , 0 2} \\ \text { Palmitic acid } & \mathbf{1 2 , 4 3} \pm \mathbf{0 , 1 8} & \text { Linolenic acid }(\omega 3) & \mathbf{0 , 8 5} \pm \mathbf{0 , 0 7} & & \\ \text { Palmitoleic acid } & \mathbf{2 , 2 0} \pm \mathbf{0 , 5 7} & \text { Arachic acid } & \mathbf{0 , 2 7} \pm \mathbf{0 , 0 4} & \text { Unsat./satured } & \mathbf{5 , 8 2} \pm \mathbf{0 , 0 3} \\ \text { Stearic acid } & \mathbf{1 , 6 1} \pm \mathbf{0 , 2 8} & \text { Eicosenoic acid } & \mathbf{0 , 3 2} \pm \mathbf{0 , 0 2} & \omega 6 / \omega 3 & \mathbf{4 , 7 6 \pm 0 , 8 7} \\ \text { Oleic acid } & \mathbf{7 6 , 1 2} \pm \mathbf{0 , 8 2} & \text { Behenic acid } & \mathbf{0 , 1 2} \pm \mathbf{0 , 0 2} & & \end{array}$

\section{Organoleptic oil values}

Sensory Analysis (Panel test)

Comment: fruity medium-high, with hints of grass and tomato, read sensations of almond and artichoke. Balanced taste sensation with a medium-high bitter and spicy. Mediumhigh fluidity.

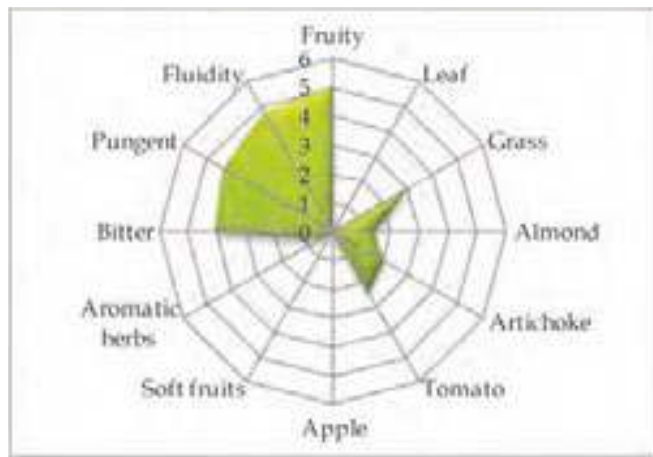

\section{Molecular Markers}

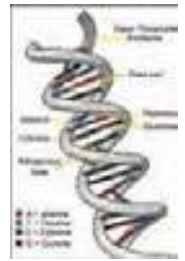

Table 2. Microsatellites (SSR) loci analyzed.

For each locus the allele size (expressed in base pairs) is reported.

$\begin{array}{cccccc}D C A 09 & \text { DCA18 } & \text { GAPU59 } & \text { GAPU71A } & \text { GUPA71B } & \text { GAPU103A } \\ \mathbf{1 6 2} \mathbf{- 1 9 4} & \mathbf{1 8 1} \mathbf{- 1 8 5} & \mathbf{2 1 4 - \mathbf { 2 1 8 }} & \mathbf{2 1 2} \mathbf{- 2 2 4} & \mathbf{1 2 4} \mathbf{- 1 4 4} & \mathbf{1 3 6} \mathbf{- 1 3 6} \\ \text { UDOO1 } & \text { UDOO3 } & \text { UDO12 } & \text { UDO28 } & \text { UDO39 } & \\ \mathbf{1 4 4 - \mathbf { 1 5 0 }} & \mathbf{1 3 5 - \mathbf { 1 3 5 }} & \mathbf{1 6 4 - \mathbf { 1 7 7 }} & \mathbf{1 4 3 - \mathbf { 1 5 0 }} & \mathbf{2 0 5 - \mathbf { 2 0 5 }} & \end{array}$

\section{References:}

1 - Various authors. In: Catalogo Nazionale delle Varietà di Olivo . University of Bari (in press), ISBN 978-88-8879397-9.

2 - Muzzalupo I., Stefanizzi F., Perri E. HortScience (2009), 44: pp. 582-588. 


\section{“Raza "}

(synonymy: Bezzana, Olivo selvatico, Rezzana, etc.)

Areal distribution or origin area: Veneto

Flesh/pit weight ratio: low $(3,29 \pm 0,20)$

Oil content (\%): medium $(45,49 \pm 1,11)$

Purpose: oil

\section{Morphological characters}

Tree characters

Vigour: medium-strong

Growth habit: spreading

Canopy-density: medium-dense

\section{Leaf characters}

Blade length (cm): short $(4,85 \pm 0,44)$

Blade width $(\mathrm{cm})$ : medium $(\mathbf{1 , 3 0} \pm \mathbf{0 , 1 4})$

Shape (length/width): elliptic

\section{Inflorescence characters}

Inflorescence length (cm): medium $(3,33 \pm 1,43)$

Number of flowers: low $(13,61 \pm 0,94)$

\section{Fruit characters}

Fresh weight of 100 fruits $(\mathrm{g})$ : medium $(2,17 \pm 0,04)$

Shape (length/width): ovoid

Symmetry: slightly asymmetric

Position of maximum transverse diameter:

towards apex

Apex: rounded

Base: rounded

Niplle: absent

Lenticels: many and small

\section{Pit characters}

Weight of 100 pits (g): high $(\mathbf{0 , 5 1} \pm \mathbf{0 , 0 2})$

Shape (length/width): ovoid

Mucron: tenuous

Symmetry: slightly asymmetrc

Position of maximum transverse diameter:

towards apex

Apex: rounded

Base: rounded

Surface: smooth

Number of grooves: low
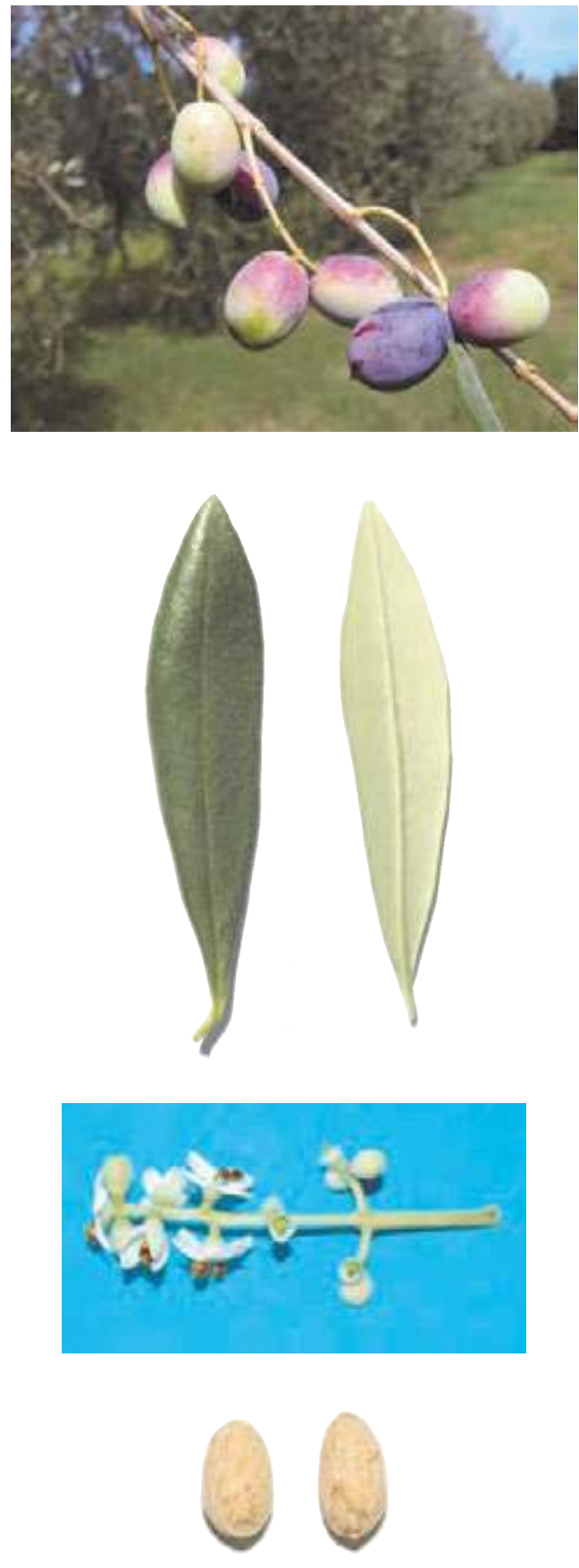


\section{Biochemical Characters}

\section{Fatty Acid Composition}

Table 1. Average values (express in $\% \pm$ standard deviations) of the fatty acids methyl esters and nutritional ratios obtained from single cultivar olive oils.

$\begin{array}{llllll}\text { Myristic acid } & \mathbf{0 , 0 1} \pm \mathbf{0 , 0 0} & \text { Linoleic acid }(\omega 6) & \mathbf{8 , 8 5} \pm \mathbf{0 , 1 3} & \text { Lignoceric acid } & \mathbf{0 , 0 3} \pm \mathbf{0 , 0 1} \\ \text { Palmitic acid } & \mathbf{1 3 , 7 4 \pm 0 , 3 1} & \text { Linolenic acid }(\omega 3) & \mathbf{0 , 7 1} \pm \mathbf{0 , 0 4} & & \\ \text { Palmitoleic acid } & \mathbf{2 , 1 9} \pm \mathbf{0 , 0 3} & \text { Arachic acid } & \mathbf{0 , 2 5} \pm \mathbf{0 , 0 6} & \text { Unsat./satured } & \mathbf{5 , 2 4} \pm \mathbf{0 , 2 0} \\ \text { Stearic acid } & \mathbf{1 , 8 1} \pm \mathbf{0 , 1 0} & \text { Eicosenoic acid } & \mathbf{0 , 0 1} \pm \mathbf{0 , 0 1} & \omega 6 / \omega 3 & \mathbf{1 2 , 4 1} \pm \mathbf{0 , 4 7} \\ \text { Oleic acid } & \mathbf{7 0 , 4 9 \pm 0 , 6 5} & \text { Behenic acid } & \mathbf{0 , 0 7} \pm \mathbf{0 , 0 1} & & \end{array}$

\section{Organoleptic oil values}

Sensory Analysis (Panel test)

Comment: fruity medium, with good sensation of leaves, grass, and artichoke. Balanced in flavours, with hints of bitter and spicy medium-light intensity. Medium fluidity.

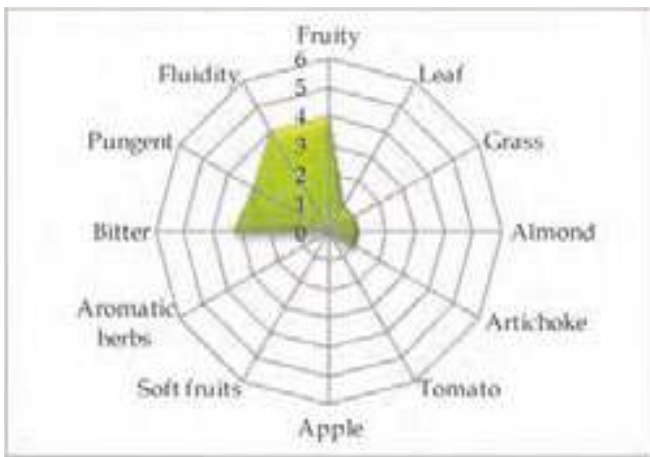

\section{Molecular Markers}

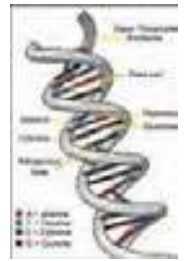

Table 2. Microsatellites (SSR) loci analyzed.

For each locus the allele size (expressed in base pairs) is reported.

$\begin{array}{cccccc}D C A 09 & \text { DCA18 } & \text { GAPU59 } & \text { GAPU71A } & \text { GUPA71B } & \text { GAPU103A } \\ \mathbf{1 8 2} \mathbf{- 2 0 6} & \mathbf{1 7 9 - \mathbf { 1 7 9 }} & \mathbf{2 1 2} \mathbf{- 2 2 2} & \mathbf{2 1 4} \mathbf{- 2 2 4} & \mathbf{1 2 6} \mathbf{- 1 4 4} & \mathbf{1 5 9 - \mathbf { 1 7 0 }} \\ \text { UDOO1 } & \text { UDOO3 } & \text { UDO12 } & \text { UDO28 } & \text { UDO39 } & \\ \mathbf{1 5 0 - \mathbf { 1 5 0 }} & \mathbf{1 4 3 - \mathbf { 1 4 3 }} & \mathbf{1 7 7 - \mathbf { 1 8 2 }} & \mathbf{1 8 2} \mathbf{- 2 2 0} & \mathbf{1 8 5} \mathbf{- 1 8 5} & \end{array}$

\section{References:}

1 - Carocci Buzi C. In: Annali Istituto Sperimentale per l'Olivicoltura e l'Oleificio, Ed. Bracco Imperia (1965), pp. 1-31

2 - Muzzalupo I., Lombardo N., Salimonti A., et al. Adv. Hort. Sci. (2008), 22(2): pp. 142-148. 


\section{“Razzo"}

(synonymy: Grossaio, Pendaglio, Razzarolo, etc.)

Areal distribution or origin area: Toscana

Flesh/pit weight ratio: medium $(5,79 \pm 1,07)$

Oil content (\%): medium $(43,83 \pm 0,25)$

Purpose: oil

\section{Morphological characters}

Tree characters

Vigour: medium-weak

Growth habit: spreading

Canopy-density: medium

\section{Leaf characters}

Blade length (cm): short $(4,94 \pm 0,37)$

Blade width $(\mathrm{cm})$ : medium $(1,06 \pm 0,13)$

Shape (length/width): elliptic-lanceolate

\section{Inflorescence characters}

Inflorescence length (cm): medium $(2,61 \pm 1,29)$

Number of flowers: low $(\mathbf{1 6 , 6 1} \pm 0,85)$

\section{Fruit characters}

Fresh weight of 100 fruits $(\mathrm{g})$ : medium $(2,35 \pm 0,08)$

Shape (length/width): ovoid

Symmetry: slightly asymmetric

Position of maximum transverse diameter:

central

Apex: rounded

Base: rounded

Niplle: absent

Lenticels: many and large

\section{Pit characters}

Weight of 100 pits (g): medium $(0,36 \pm 0,05)$

Shape (length/width): elliptic

Mucron: tenuous

Symmetry: slightly asymmetric

Position of maximum transverse diameter:

towards apex

Apex: rounded

Base: pointed

Surface: smooth

Number of grooves: low
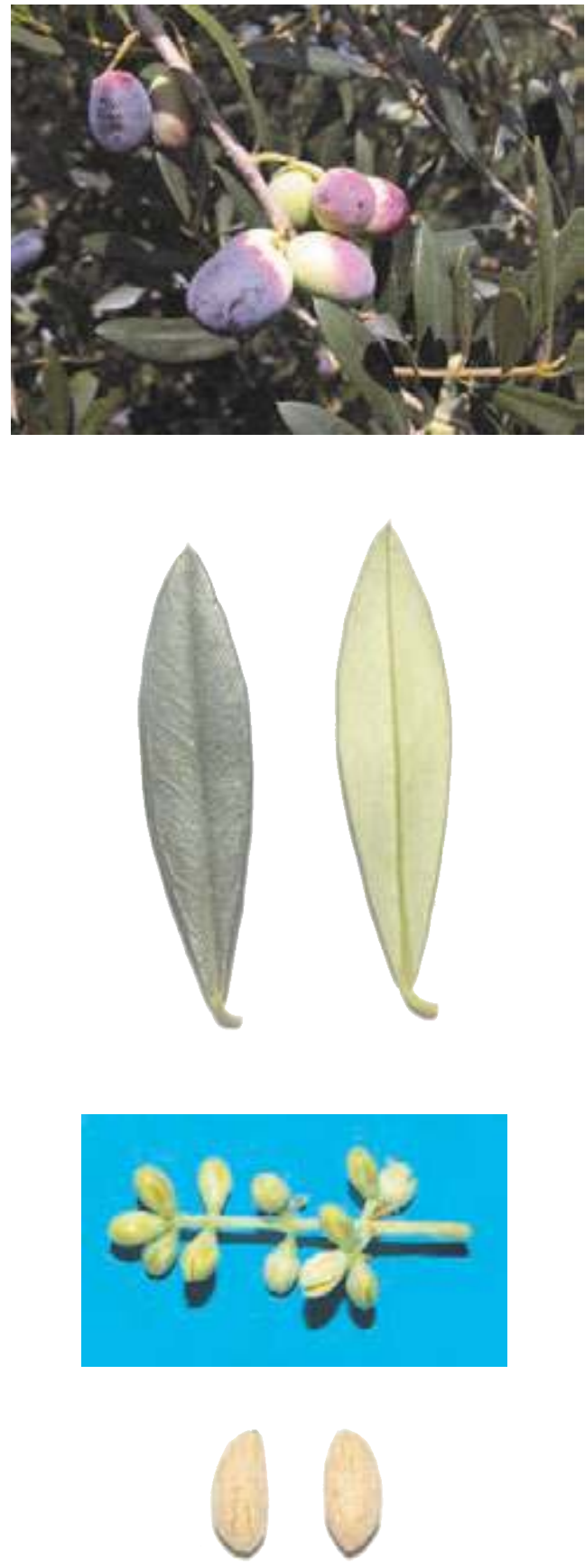


\section{Biochemical Characters}

\section{Fatty Acid Composition}

Table 1. Average values (express in $\% \pm$ standard deviations) of the fatty acids methyl esters and nutritional ratios obtained from single cultivar olive oils.

$\begin{array}{llllll}\text { Myristic acid } & \mathbf{0 , 0 1} \pm \mathbf{0 , 0 0} & \text { Linoleic acid }(\omega 6) & \mathbf{1 0 , 6 3} \pm \mathbf{0 , 5 0} & \text { Lignoceric acid } & \mathbf{0 , 0 2} \pm \mathbf{0 , 0 1} \\ \text { Palmitic acid } & \mathbf{1 4 , 2 0} \pm \mathbf{0 , 1 4} & \text { Linolenic acid }(\omega 3) & \mathbf{0 , 7 3} \pm \mathbf{0 , 0 5} & & \\ \text { Palmitoleic acid } & \mathbf{1 , 3 1} \pm \mathbf{0 , 0 4} & \text { Arachic acid } & \mathbf{0 , 2 4} \pm \mathbf{0 , 0 5} & \text { Unsat./satured } & \mathbf{4 , 9 5} \pm \mathbf{0 , 1 1} \\ \text { Stearic acid } & \mathbf{2 , 2 4} \pm \mathbf{0 , 1 4} & \text { Eicosenoic acid } & \mathbf{0 , 0 2} \pm \mathbf{0 , 0 1} & \omega 6 / \omega 3 & \mathbf{1 4 , 6 1} \pm \mathbf{0 , 2 3} \\ \text { Oleic acid } & \mathbf{6 9 , 4 2} \pm \mathbf{0 , 2 4} & \text { Behenic acid } & \mathbf{0 , 0 7} \pm \mathbf{0 , 0 1} & & \end{array}$

\section{Organoleptic oil values}

Sensory Analysis (Panel test)

Comment: fruity medium - light, with hints of almond and read sensations of grass and artichoke. Balanced taste sensation with a medium - light bitter and spicy. Medium fluidity.

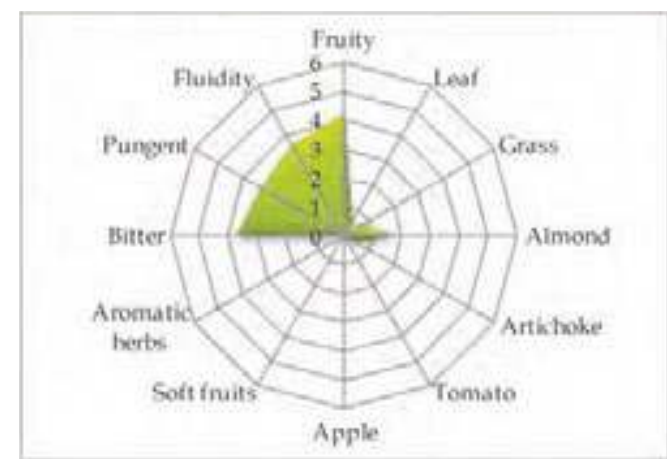

\section{Molecular Markers}

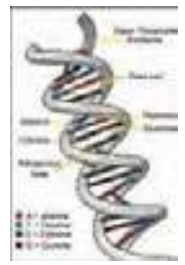

Table 2. Microsatellites (SSR) loci analyzed.

For each locus the allele size (expressed in base pairs) is reported.

$\begin{array}{cccccc}D C A 09 & \text { DCA18 } & \text { GAPU59 } & \text { GAPU71A } & \text { GUPA71B } & \text { GAPU103A } \\ \mathbf{1 8 2} \mathbf{- 2 0 6} & \mathbf{1 8 1} \mathbf{- 1 8 5} & \mathbf{2 0 8 - \mathbf { 2 1 2 }} & \mathbf{2 1 0 - \mathbf { 2 1 4 }} & \mathbf{1 2 4} \mathbf{- 1 3 0} & \mathbf{1 5 0 - \mathbf { 1 7 0 }} \\ \text { UDOO1 } & \text { UDOO3 } & \text { UDO12 } & \text { UDO28 } & \text { UDO39 } & \\ \mathbf{1 4 0 - \mathbf { 1 4 4 }} & \mathbf{1 5 0 - \mathbf { 1 5 0 }} & \mathbf{1 7 7 - \mathbf { 1 7 7 }} & \mathbf{1 5 4 - \mathbf { 2 0 5 }} & \mathbf{2 0 5 - \mathbf { 2 0 5 }} & \end{array}$

\section{References:}

1 - Cimato A., Cantini C., Sani G., In: L'olivo in Toscana: il germoplasma autoctono, Ed. ARSIA (2001).

2 - Muzzalupo I., Stefanizzi F., Bucci C., et al. In Acta Italus Hortus, (2011), 1: 138-140. 


\section{“Razzola"}

(synonymy: Corno maurino, Fischiettara, Martina a cornetto, Olivo femmina, Razzuola, etc.)

Areal distribution or origin area: Liguria

Flesh/pit weight ratio: low $(4,35 \pm 0,15)$

Oil content (\%): medium $(46,14 \pm 1,96)$

Purpose: oil

\section{Morphological characters}

Tree characters

Vigour: medium-strong

Growth habit: spreading-erect

Canopy-density: medium-dense

\section{Leaf characters}

Blade length $(\mathrm{cm})$ : medium $(5,79 \pm 0,77)$

Blade width $(\mathrm{cm})$ : broad $(\mathbf{1}, 63 \pm \mathbf{0 , 2 3})$

Shape (length/width): elliptic

\section{Inflorescence characters}

Inflorescence length (cm): medium $(3,24 \pm 2,11)$

Number of flowers: medium $(19,03 \pm 1,88)$

\section{Fruit characters}

Fresh weight of 100 fruits (g): medium $(\mathbf{2 , 3 8} \pm \mathbf{0 , 1 2})$

Shape (length/width): ovoid

Symmetry: slightly asymmetric

Position of maximum transverse diameter:

central

Apex: rounded

Base: rounded

Niplle: absent

Lenticels: many and small

\section{Pit characters}

Weight of 100 pits (g): high $(0,47 \pm 0,06)$

Shape (length/width): elliptic

Mucron: absent

Symmetry: symmetric

Position of maximum transverse diameter:

central

Apex: rounded

Base: rounded

Surface: rugose

Number of grooves: medium
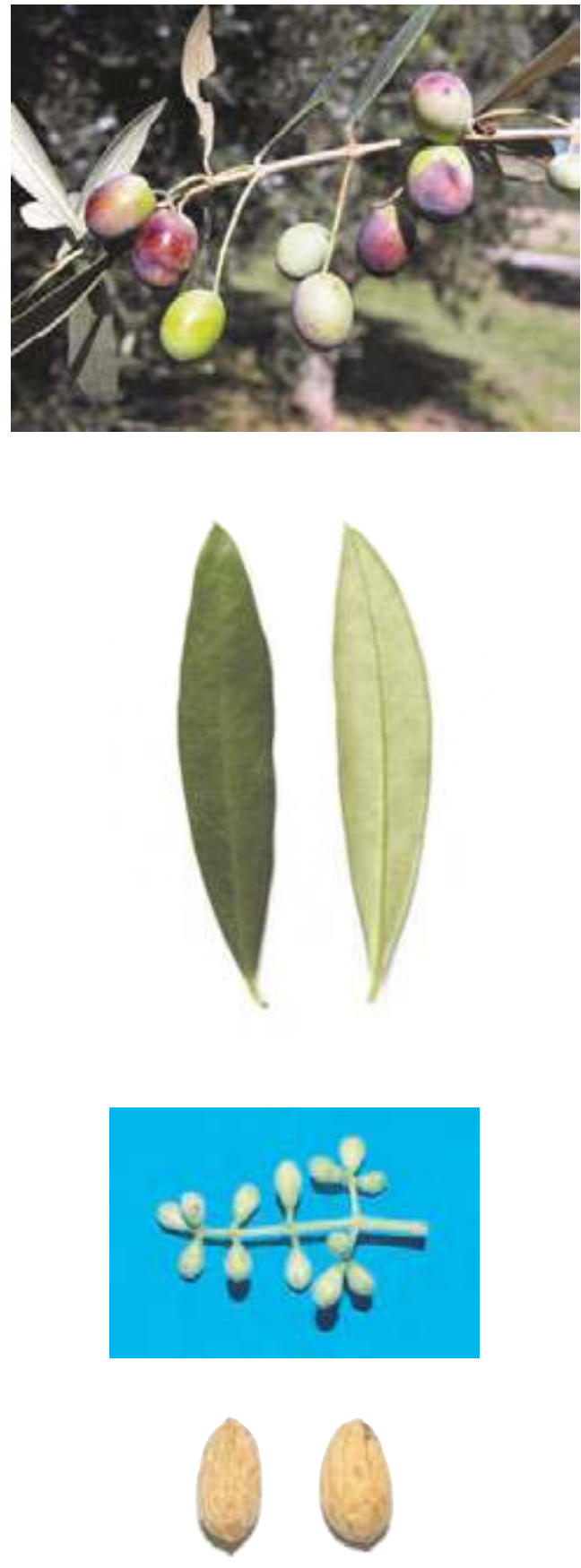


\section{Biochemical Characters}

\section{Fatty Acid Composition}

Table 1. Average values (express in $\% \pm$ standard deviations) of the fatty acids methyl esters and nutritional ratios obtained from single cultivar olive oils.

$\begin{array}{llllll}\text { Myristic acid } & \mathbf{0 , 0 1} \pm \mathbf{0 , 0 0} & \text { Linoleic acid }(\omega 6) & \mathbf{6 , 9 9} \pm \mathbf{0 , 8 0} & \text { Lignoceric acid } & \mathbf{0 , 0 3} \pm \mathbf{0 , 0 1} \\ \text { Palmitic acid } & \mathbf{6 , 5 2} \pm \mathbf{0 , 8 1} & \text { Linolenic acid }(\omega 3) \mathbf{0 , 9 2} \pm \mathbf{0 , 0 2} & & \\ \text { Palmitoleic acid } & \mathbf{0 , 9 7 \pm 0 , 0 8} & \text { Arachic acid } & \mathbf{0 , 2 9} \pm \mathbf{0 , 0 2} & \text { Unsat./satured } & \mathbf{1 0 , 7 2 \pm 1 , 0 0} \\ \text { Stearic acid } & \mathbf{1 , 7 7} \pm \mathbf{0 , 1 8} & \text { Eicosenoic acid } & \mathbf{0 , 0 1} \pm \mathbf{0 , 0 0} & \omega 6 / \omega 3 & \mathbf{7 , 6 5} \pm \mathbf{1 , 0 5} \\ \text { Oleic acid } & \mathbf{8 1 , 4 9 \pm 0 , 1 1} & \text { Behenic acid } & \mathbf{0 , 0 7} \pm \mathbf{0 , 0 3} & & \end{array}$

\section{Organoleptic oil values}

Sensory Analysis (Panel test)

Comment: medium fruity, with good sensation of almond and aromatic herbs, less grass and artichoke. Balanced taste sensation, with bitter and spicy medium. Medium-high fluidity.

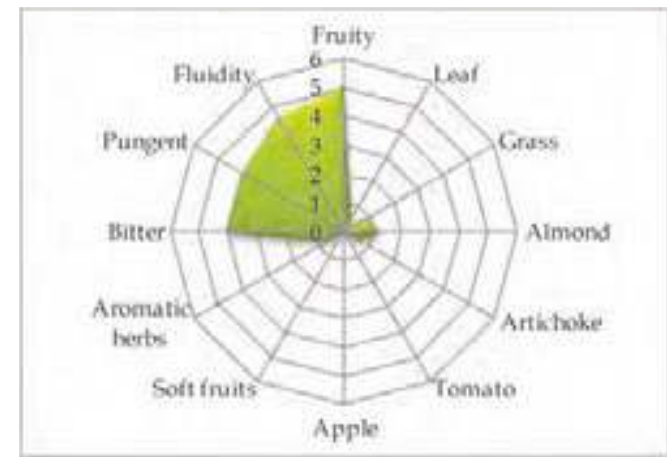

\section{Molecular Markers}

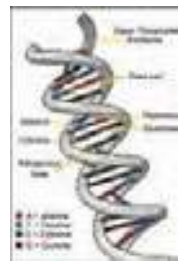

Table 2. Microsatellites (SSR) loci analyzed.

For each locus the allele size (expressed in base pairs) is reported.

$\begin{array}{cccccc}D C A 09 & \text { DCA18 } & \text { GAPU59 } & \text { GAPU71A } & \text { GUPA71B } & \text { GAPU103A } \\ \mathbf{1 7 6 - \mathbf { 2 0 6 }} & \mathbf{1 8 1} \mathbf{- 1 8 1} & \mathbf{2 1 4 - \mathbf { 2 2 2 }} & \mathbf{2 1 8 - \mathbf { 2 2 4 }} & \mathbf{1 2 6} \mathbf{- 1 4 4} & \mathbf{1 5 9} \mathbf{- 1 7 0} \\ \text { UDOO1 } & \text { UDOO3 } & \text { UDO12 } & \text { UDO28 } & \text { UDO39 } & \\ \mathbf{1 5 0 - \mathbf { 1 5 0 }} & \mathbf{1 5 0 - \mathbf { 1 5 0 }} & \mathbf{1 7 7 - \mathbf { 1 8 2 }} & \mathbf{1 8 2} \mathbf{- 2 0 5} & \mathbf{2 0 0 - \mathbf { 2 0 0 }} & \end{array}$

\section{References:}

1 - Various authors. In: Catalogo Nazionale delle Varietà di Olivo . University of Bari (in press), ISBN 978-88-8879397-9.

2 - Muzzalupo I., Lombardo N., Salimonti A., et al. Adv. Hort. Sci. (2008), 22(2): pp. 142-148. 


\section{"Riminino"}

Areal distribution or origin area: Lazio

Flesh/pit weight ratio: medium $(6,84 \pm 0,63)$

Oil content (\%): low $(38,45 \pm 1,02)$

Purpose: dual purpose

\section{Morphological characters}

Tree characters

Vigour: strong

Growth habit: spreading-erect

Canopy-density: dense

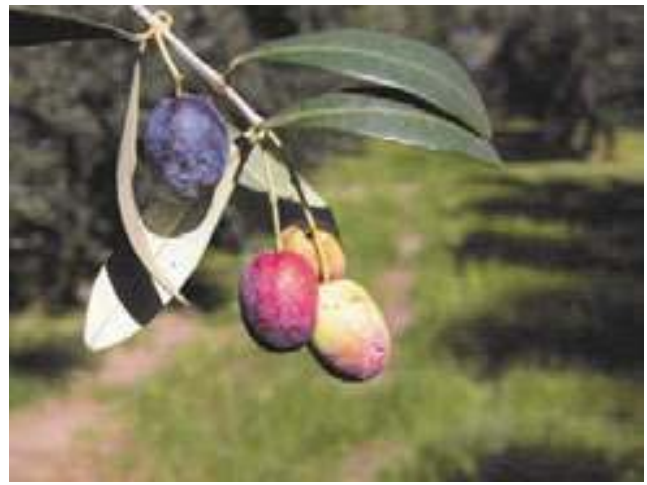

\section{Leaf characters}

Blade length $(\mathrm{cm})$ : medium $(5,29 \pm 0,52)$

Blade width (cm): broad $(\mathbf{1}, 63 \pm \mathbf{0 , 1 4})$

Shape (length/width): elliptic

\section{Inflorescence characters}

Inflorescence length (cm): long $(4,67 \pm 0,65)$

Number of flowers: high $(28,59 \pm 2,63)$

\section{Fruit characters}

Fresh weight of 100 fruits $(\mathrm{g})$ : medium $(3,61 \pm 0,27)$

Shape (length/width): ovoid

Symmetry: slightly asymmetric

Position of maximum transverse diameter:

central

Apex: rounded

Base: truncate

Niplle: absent

Lenticels: many and small

\section{Pit characters}

Weight of 100 pits (g): medium $(0,45 \pm \mathbf{0 , 0 1})$

Shape (length/width): elliptic

Mucron: absent

Symmetry: symmetric

Position of maximum transverse diameter:

towards apex

Apex: rounded

Base: pointed

Surface: rugose

Number of grooves: medium
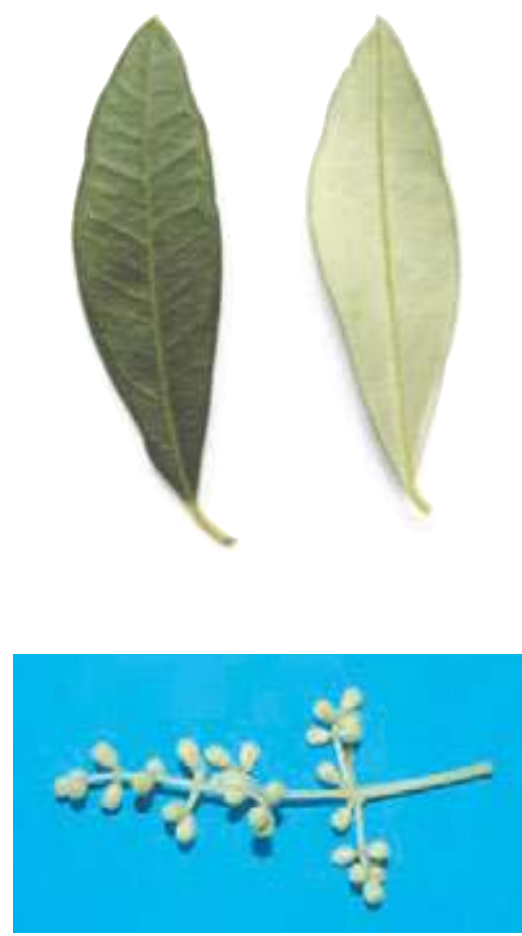


\section{Biochemical Characters}

\section{Fatty Acid Composition}

Table 1. Average values (express in $\% \pm$ standard deviations) of the fatty acids methyl esters and nutritional ratios obtained from single cultivar olive oils.

$\begin{array}{llllll}\text { Myristic acid } & \mathbf{0 , 0 1} \pm \mathbf{0 , 0 0} & \text { Linoleic acid }(\omega 6) & \mathbf{6 , 7 6} \pm \mathbf{0 , 1 5} & \text { Lignoceric acid } & \mathbf{0 , 0 2} \pm \mathbf{0 , 0 0} \\ \text { Palmitic acid } & \mathbf{1 2 , 8 2} \pm \mathbf{0 , 2 3} & \text { Linolenic acid }(\omega 3) & \mathbf{0 , 7 1} \pm \mathbf{0 , 1 5} & & \\ \text { Palmitoleic acid } & \mathbf{1 , 2 8} \pm \mathbf{0 , 5 3} & \text { Arachic acid } & \mathbf{0 , 2 9} \pm \mathbf{0 , 0 5} & \text { Unsat./satured } & \mathbf{5 , 4 4} \pm \mathbf{0 , 2 7} \\ \text { Stearic acid } & \mathbf{2 , 3 4} \pm \mathbf{0 , 2 5} & \text { Eicosenoic acid } & \mathbf{0 , 0 1} \pm \mathbf{0 , 0 0} & \omega 6 / \omega 3 & \mathbf{9 , 7 4} \pm \mathbf{2 , 3 3} \\ \text { Oleic acid } & \mathbf{7 4 , 4 9 \pm \mathbf { 1 , 9 2 }} & \text { Behenic acid } & \mathbf{0 , 0 6} \pm \mathbf{0 , 0 0} & & \end{array}$

\section{Organoleptic oil values}

Sensory Analysis (Panel test)

Comment: fruity medium, with good sensation of almond, grass, and artichoke. Balanced in flavours, with hints of bitter and spicy medium intensity. Medium fluidity.

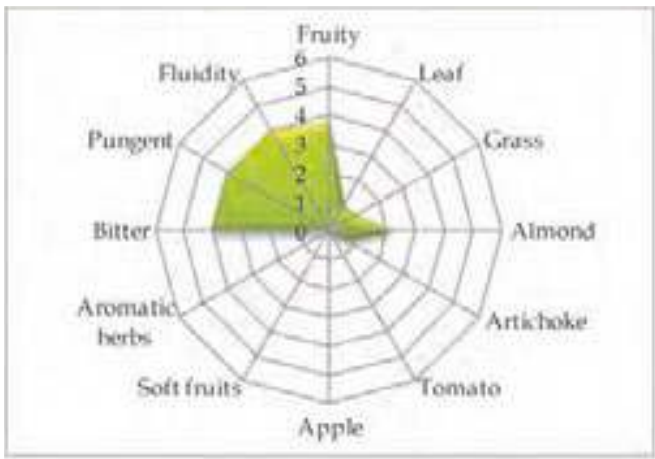

\section{Molecular Markers}

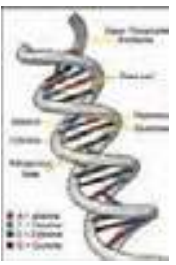

Table 2. Microsatellites (SSR) loci analyzed.

For each locus the allele size (expressed in base pairs) is reported.

\begin{tabular}{|c|c|c|c|c|c|}
\hline DCA09 & DCA18 & GAPU59 & GAPU71A & GUPA71B & GAPU103A \\
\hline $176-198$ & 177 - 181 & $214-222$ & $214-224$ & $126-144$ & $157-157$ \\
\hline UDO01 & UDO03 & UDO12 & UDO28 & UDO39 & \\
\hline $144-144$ & $143-182$ & $166-177$ & $143-182$ & $205-205$ & \\
\hline
\end{tabular}

\section{References:}

1 - Lombardo N. In: Le risorse genetiche vegetali presso gli IRSA, MIPAF (2001), 1: pp. 361-405.

2 - Muzzalupo I., Stefanizzi F., Bucci C., et al. In Acta Italus Hortus, (2011), 1: 138 -140. 


\section{“Ritonnella"}

(synonymy: Marinella, Romanella, Tonnella, etc.)

Areal distribution or origin area: Campania

Flesh/pit weight ratio: medium $(7,24 \pm 0,16)$

Oil content (\%): medium $(45,36 \pm 2,24)$

Purpose: oil

\section{Morphological characters}

Tree characters

Vigour: weak

Growth habit: spreading

Canopy-density: medium

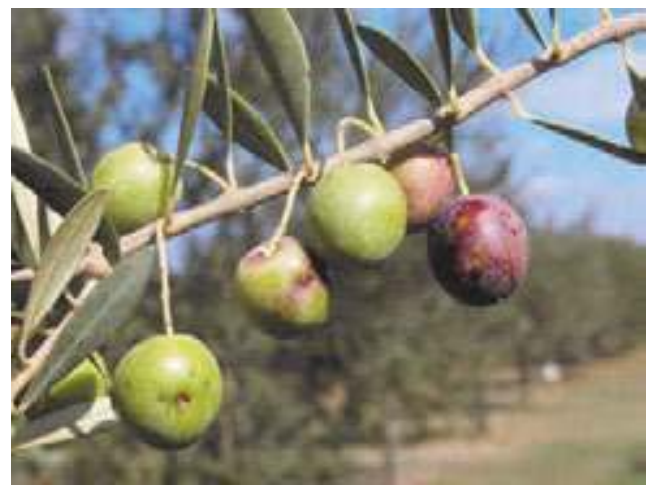

\section{Leaf characters}

Blade length (cm): short $(4,81 \pm 0,41)$

Blade width $(\mathrm{cm})$ : medium $(\mathbf{1 , 3 2} \pm \mathbf{0 , 1 4})$

Shape (length/width): elliptic

\section{Inflorescence characters}

Inflorescence length $(\mathrm{cm})$ : medium $(2,73 \pm 0,62)$

Number of flowers: medium $(21,19 \pm 1,77)$

\section{Fruit characters}

Fresh weight of 100 fruits $(\mathrm{g})$ : medium $(3,74 \pm 0,18)$

Shape (length/width): spherical

Symmetry: symmetric

Position of maximum transverse diameter:

central

Apex: rounded

Base: truncate

Niplle: absent

Lenticels: many and small

\section{Pit characters}

Weight of 100 pits (g): high $(\mathbf{0 , 4 6} \pm \mathbf{0 , 0 2})$

Shape (length/width): ovoid

Mucron: tenuous

Symmetry: symmetric

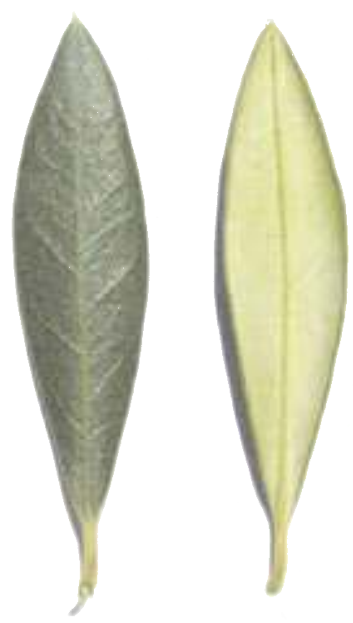

Position of maximum transverse diameter:

towards apex

Apex: rounded

Base: pointed

Surface: rugose

Number of grooves: medium
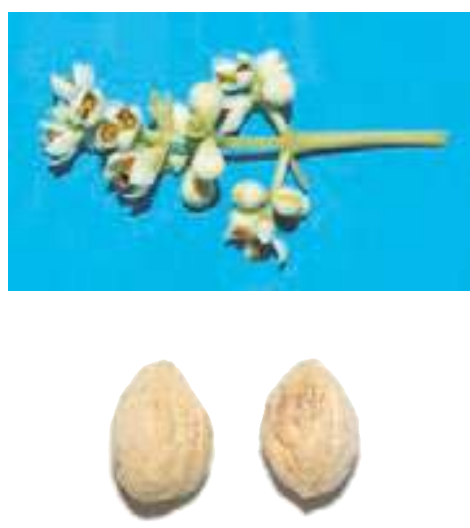


\section{Biochemical Characters}

\section{Fatty Acid Composition}

Table 1. Average values (express in $\% \pm$ standard deviations) of the fatty acids methyl esters and nutritional ratios obtained from single cultivar olive oils.

$\begin{array}{llllll}\text { Myristic acid } & \mathbf{0 , 0 1} \pm \mathbf{0 , 0 1} & \text { Linoleic acid }(\omega 6) & \mathbf{8 , 7 2} \pm \mathbf{0 , 3 9} & \text { Lignoceric acid } & \mathbf{0 , 0 5} \pm \mathbf{0 , 0 3} \\ \text { Palmitic acid } & \mathbf{1 0 , 3 1} \pm \mathbf{0 , 9 7} & \text { Linolenic acid }(\omega 3) & \mathbf{0 , 8 7} \pm \mathbf{0 , 0 2} & & \\ \text { Palmitoleic acid } & \mathbf{0 , 7 1} \pm \mathbf{0 , 0 6} & \text { Arachic acid } & \mathbf{0 , 3 2} \pm \mathbf{0 , 0 4} & \text { Unsat./satured } & \mathbf{4 , 3 6} \pm \mathbf{0 , 1 4} \\ \text { Stearic acid } & \mathbf{6 , 1 8} \pm \mathbf{0 , 6 0} & \text { Eicosenoic acid } & \mathbf{0 , 0 3} \pm \mathbf{0 , 0 1} & \omega 6 / \omega 3 & \mathbf{9 , 9 8} \pm \mathbf{0 , 1 8} \\ \text { Oleic acid } & \mathbf{7 2 , 1 7} \pm \mathbf{0 , 0 9} & \text { Behenic acid } & \mathbf{0 , 0 5} \pm \mathbf{0 , 0 1} & & \end{array}$

\section{Organoleptic oil values}

Sensory Analysis (Panel test)

Comment: fruity medium, with taste of almond, grass and leaves, read sensations of soft fruits. Balanced taste sensation with medium bitter and spicy. Medium-high fluidity.

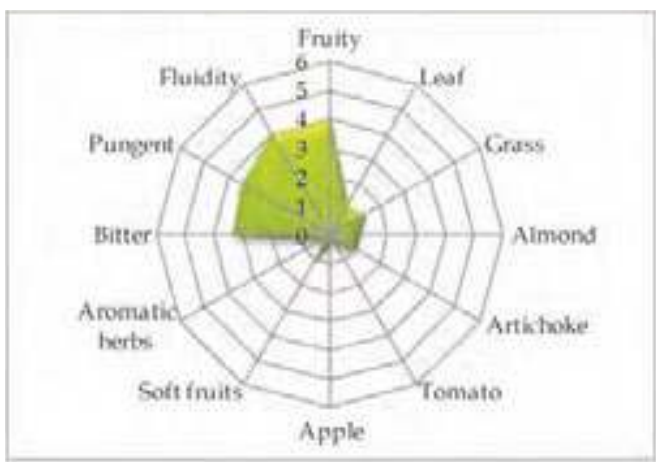

\section{Molecular Markers}

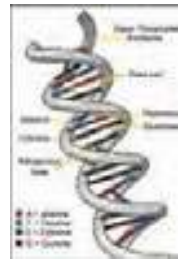

Table 2. Microsatellites (SSR) loci analyzed.

For each locus the allele size (expressed in base pairs) is reported.

$\begin{array}{cccccc}D C A 09 & \text { DCA18 } & \text { GAPU59 } & \text { GAPU71A } & \text { GUPA71B } & \text { GAPU103A } \\ \mathbf{1 9 8} \mathbf{- 2 0 6} & \mathbf{1 7 7 - \mathbf { 1 8 1 }} & \mathbf{2 1 4 - \mathbf { 2 1 8 }} & \mathbf{2 1 2} \mathbf{- 2 1 8} & \mathbf{1 2 6} \mathbf{- 1 4 4} & \mathbf{1 5 0 - \mathbf { 1 5 7 }} \\ \text { UDOO1 } & \text { UDOO3 } & \text { UDO12 } & \text { UDO28 } & \text { UDO39 } & \\ \mathbf{1 4 0 - \mathbf { 1 5 0 }} & \mathbf{1 4 3 - \mathbf { 1 4 3 }} & \mathbf{1 9 3 - 1 9 3} & \mathbf{1 5 4} \mathbf{- 1 6 1} & \mathbf{2 1 3 - \mathbf { 2 2 0 }} & \end{array}$

\section{References:}

1 - Various authors. In: Catalogo Nazionale delle Varietà di Olivo . University of Bari (in press), ISBN 978-88-8879397-9.

2 - Muzzalupo I., Stefanizzi F., Perri E. HortScience (2009), 44: pp. 582-588. 


\section{"Rizzitella"}

(synonymy: Rizzinella)

Areal distribution or origin area: Campania Flesh/pit weight ratio: low $(3,93 \pm 0,56)$ Oil content (\%): medium $(41,49 \pm 1,79)$

Purpose: oil

\section{Morphological characters}

Tree characters

Vigour: weak

Growth habit: spreading

Canopy-density: medium-sparse

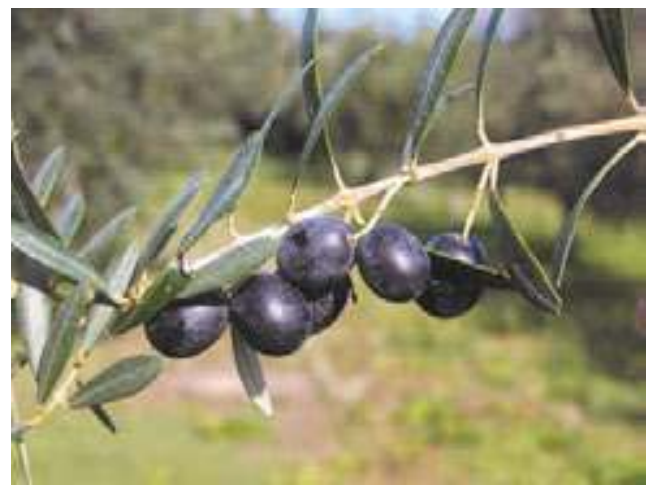

\section{Leaf characters}

Blade length (cm): short $(4,28 \pm 0,31)$

Blade width $(\mathrm{cm})$ : medium $(1,04 \pm 0,09)$

Shape (length/width): elliptic-lanceolate

\section{Inflorescence characters}

Inflorescence length $(\mathrm{cm})$ : short $(2,41 \pm 0,50)$

Number of flowers: low $(17,66 \pm 0,88)$

\section{Fruit characters}

Fresh weight of 100 fruits (g): medium $(2,35 \pm 0,27)$

Shape (length/width): spherical

Symmetry: symmetric

Position of maximum transverse diameter: central

Apex: rounded

Base: rounded

Niplle: absent

Lenticels: many and small

\section{Pit characters}

Weight of 100 pits (g): medium $(0,40 \pm 0,03)$

Shape (length/width): ovoid

Mucron: obvious

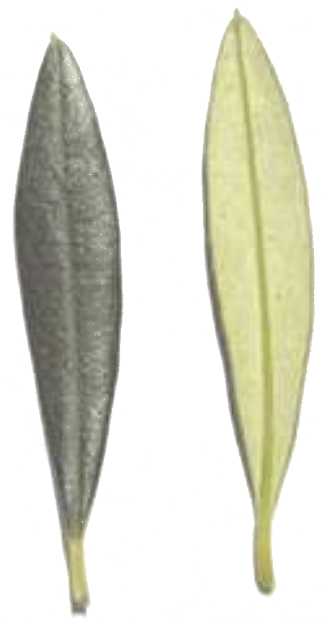

Symmetry: slightly asymmetric

Position of maximum transverse diameter:

\section{towards apex}

Apex: rounded

Base: rounded

Surface: rugose

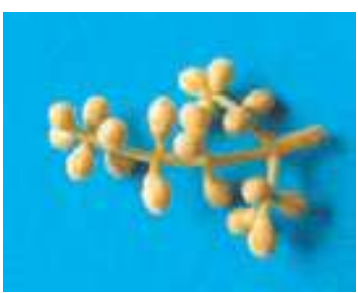

Number of grooves: medium 


\section{Biochemical Characters}

\section{Fatty Acid Composition}

Table 1. Average values (express in $\% \pm$ standard deviations) of the fatty acids methyl esters and nutritional ratios obtained from single cultivar olive oils.

$\begin{array}{llllll}\text { Myristic acid } & \mathbf{0 , 0 1} \pm 0,01 & \text { Linoleic acid }(\omega 6) & \mathbf{8 , 7 5} \pm 0,05 & \text { Lignoceric acid } & \mathbf{0 , 0 5} \pm \mathbf{0 , 0 4} \\ \text { Palmitic acid } & \mathbf{1 2 , 7 7 \pm 0 , 5 0} & \text { Linolenic acid }(\omega 3) \mathbf{0 , 6 9} \pm \mathbf{0 , 0 2} & & \\ \text { Palmitoleic acid } & \mathbf{2 , 0 3} \pm 0,03 & \text { Arachic acid } & \mathbf{0 , 2 4} \pm \mathbf{0 , 0 5} & \text { Unsat./satured } & \mathbf{5 , 4 5} \pm \mathbf{0 , 1 5} \\ \text { Stearic acid } & \mathbf{2 , 1 6} \pm 0,05 & \text { Eicosenoic acid } & \mathbf{0 , 1 7} \pm \mathbf{0 , 2 1} & \omega 6 / \omega 3 & \mathbf{1 2 , 7 1} \pm \mathbf{0 , 3 6} \\ \text { Oleic acid } & \mathbf{7 0 , 9 4 \pm 0 , 0 3} & \text { Behenic acid } & \mathbf{0 , 0 9} \pm 0,04 & & \end{array}$

\section{Organoleptic oil values}

Sensory Analysis (Panel test)

Comment: fruity medium, with taste of aromatic herbs and leaves. Balanced taste sensation with medium bitter and spicy. Medium fluidity.

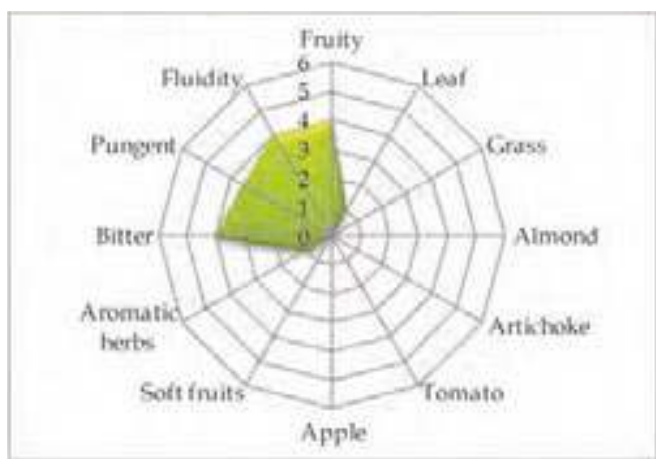

\section{Molecular Markers}

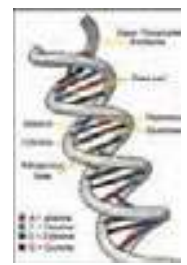

Table 2. Microsatellites (SSR) loci analyzed.

For each locus the allele size (expressed in base pairs) is reported.

$\begin{array}{cccccc}D C A 09 & \text { DCA18 } & \text { GAPU59 } & \text { GAPU71A } & \text { GUPA71B } & \text { GAPU103A } \\ \mathbf{1 7 2} \mathbf{- 1 9 4} & \mathbf{1 7 7 - \mathbf { 1 7 9 }} & \mathbf{2 1 4} \mathbf{- 2 1 8} & \mathbf{2 1 4} \mathbf{- 2 1 4} & \mathbf{1 2 4} \mathbf{- 1 4 4} & \mathbf{1 5 0 - \mathbf { 1 5 7 }} \\ \text { UDOO1 } & \text { UDOO3 } & \text { UDO12 } & \text { UDO28 } & \text { UDO39 } & \\ \mathbf{1 4 0 - \mathbf { 1 4 0 }} & \mathbf{1 4 3 - \mathbf { 1 4 3 }} & \mathbf{1 7 7 - \mathbf { 1 7 7 }} & \mathbf{1 6 1 - \mathbf { 1 6 1 }} & \mathbf{2 1 3 - \mathbf { 2 3 2 }} & \end{array}$

\section{References:}

1 - Pugliano G., Flaminio G., Pugliano M.L., et al. In: La risorsa genetica dell'olivo in Campania, SE. S.I.R.C.A. Ed. Napoli, (2000).

2 - Muzzalupo I., Stefanizzi F., Perri E. HortScience (2009), 44: pp. 582-588. 


\section{“Romanella molisana "}

Areal distribution or origin area: Molise

Flesh/pit weight ratio: low $(4,85 \pm 0,76)$

Oil content (\%): medium $(47,97 \pm 0,86)$

Purpose: oil

\section{Morphological characters}

Tree characters

Vigour: medium-weak

Growth habit: spreading

Canopy-density: medium-sparse

\section{Leaf characters}

Blade length (cm): medium $(5,66 \pm 0,53)$

Blade width $(\mathrm{cm})$ : medium $(\mathbf{1}, \mathbf{4 3} \pm \mathbf{0 , 1 3})$

Shape (length/width): elliptic

\section{Inflorescence characters}

Inflorescence length (cm): medium $(3,23 \pm 0,52)$

Number of flowers: medium $(22,17 \pm 1,62)$

\section{Fruit characters}

Fresh weight of 100 fruits (g): low $(1,95 \pm 0,04)$

Shape (length/width): spherical

Symmetry: symmetric

Position of maximum transverse diameter:

central

Apex: rounded

Base: truncate

Niplle: absent

Lenticels: many and small

\section{Pit characters}

Weight of 100 pits (g): medium $(0,34 \pm \mathbf{0 , 0 4})$

Shape (length/width): ovoid

Mucron: absent

Symmetry: slightly asymmetric

Position of maximum transverse diameter:

central

Apex: rounded

Base: rounded

Surface: rugose

Number of grooves: medium
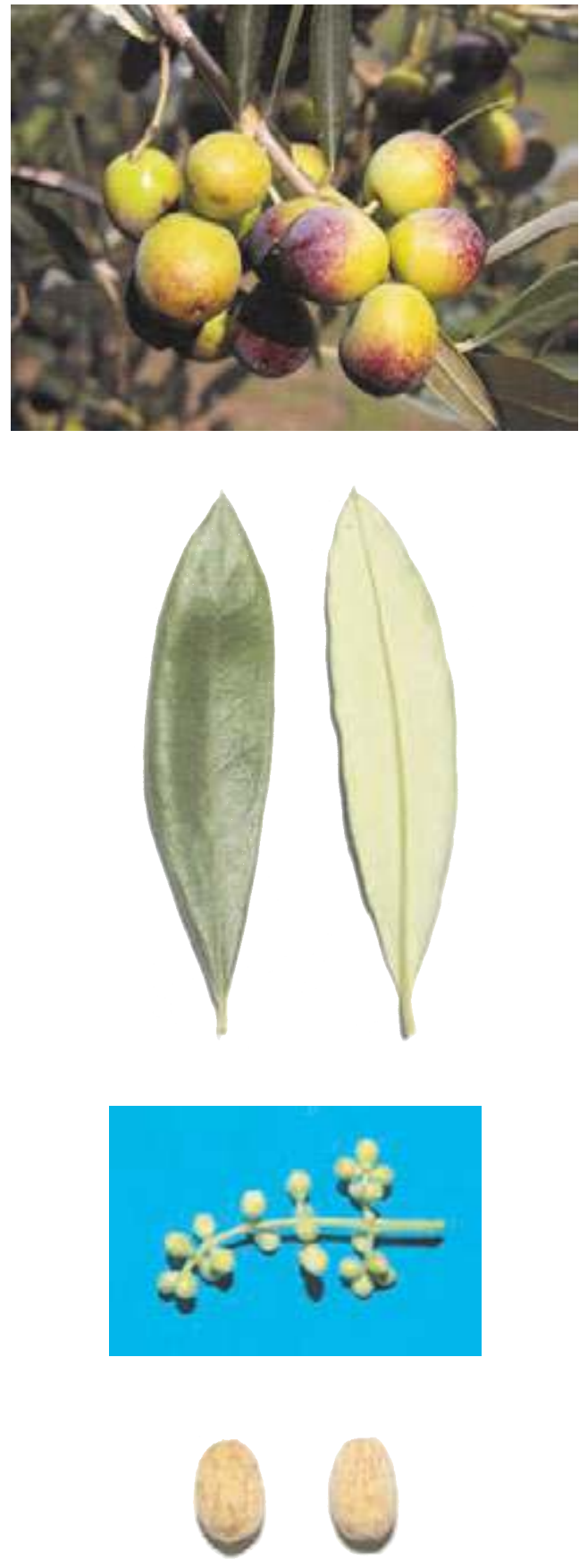


\section{Biochemical Characters}

\section{Fatty Acid Composition}

Table 1. Average values (express in $\% \pm$ standard deviations) of the fatty acids methyl esters and nutritional ratios obtained from single cultivar olive oils.

$\begin{array}{llllll}\text { Myristic acid } & \mathbf{0 , 0 1} \pm \mathbf{0 , 0 0} & \text { Linoleic acid }(\omega 6) & \mathbf{6 , 4 9} \pm \mathbf{0 , 5 7} & \text { Lignoceric acid } & \mathbf{0 , 0 2} \pm \mathbf{0 , 0 1} \\ \text { Palmitic acid } & \mathbf{1 3 , 4 0 \pm 0 , 3 6} & \text { Linolenic acid }(\omega 3) & \mathbf{0 , 7 5} \pm \mathbf{0 , 0 4} & & \\ \text { Palmitoleic acid } & \mathbf{1 , 4 7} \pm \mathbf{0 , 0 4} & \text { Arachic acid } & \mathbf{0 , 2 1} \pm \mathbf{0 , 0 3} & \text { Unsat./satured } & \mathbf{5 , 3 7 \pm 0 , 1 8} \\ \text { Stearic acid } & \mathbf{1 , 9 7} \pm \mathbf{0 , 0 2} & \text { Eicosenoic acid } & \mathbf{0 , 0 2} \pm \mathbf{0 , 0 0} & \omega 6 / \omega 3 & \mathbf{8 , 6 2} \pm \mathbf{0 , 3 0} \\ \text { Oleic acid } & \mathbf{7 4 , 4 1 \pm 0 , 1 6} & \text { Behenic acid } & \mathbf{0 , 0 5} \pm \mathbf{0 , 0 1} & & \end{array}$

\section{Organoleptic oil values}

Sensory Analysis (Panel test)

Comment: fruity medium-high, with hints of almond and read sensations of grass, tomato and artichoke. Balanced taste sensation with a medium bitter and spicy. Medium-high fluidity.

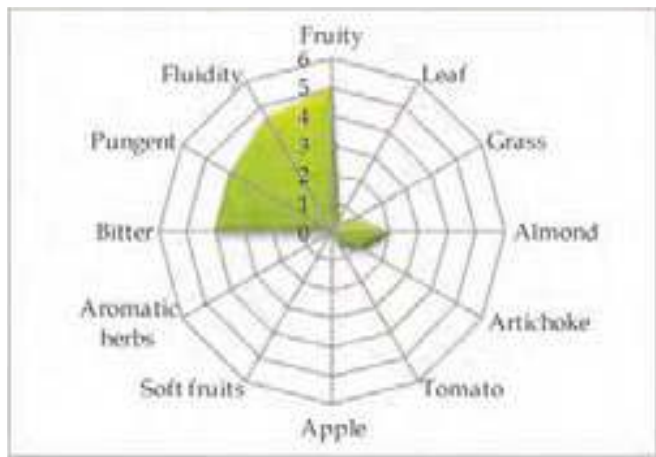

\section{Molecular Markers}

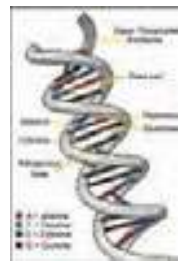

Table 2. Microsatellites (SSR) loci analyzed.

For each locus the allele size (expressed in base pairs) is reported.

$\begin{array}{cccccc}D C A 09 & \text { DCA18 } & \text { GAPU59 } & \text { GAPU71A } & \text { GUPA71B } & \text { GAPU103A } \\ \mathbf{1 9 8} \mathbf{- 2 1 0} & \mathbf{1 8 1} \mathbf{- 1 8 1} & \mathbf{2 1 2} \mathbf{- 2 2 2} & \mathbf{2 1 4} \mathbf{- 2 1 4} & \mathbf{1 3 0 - \mathbf { 1 2 6 }} & \mathbf{1 5 0 - \mathbf { 1 5 0 }} \\ \text { UDOO1 } & \text { UDOO3 } & \text { UDO12 } & \text { UDO28 } & \text { UDO39 } & \\ \mathbf{1 4 0 - \mathbf { 1 4 0 }} & \mathbf{1 4 3 - \mathbf { 1 4 3 }} & \mathbf{1 7 7 - \mathbf { 1 9 3 }} & \mathbf{1 5 4 - \mathbf { 2 0 5 }} & \mathbf{2 1 3 - \mathbf { 2 4 3 }} & \end{array}$

\section{References:}

1 - Ente regionale di sviluppo agricolo per il Molise. In: Il germoplasma dell'olivo nel Molise, Quaderno divulgativo dell'ERSA (2000), $\mathrm{n}^{\circ} 5$.

2 - Muzzalupo I., Salimonti A., Caravita M. A., et al. Adv. Hort. Sci. (2008), 22(2): pp. 129-135. 


\section{“Rosciola Coltodino"}

Areal distribution or origin area: Lazio

Flesh/pit weight ratio: medium $(5,53 \pm 1,00)$

Oil content (\%): medium $(45,60 \pm 1,66)$

Purpose: oil

\section{Morphological characters}

Tree characters

Vigour: weak

Growth habit: spreading

Canopy-density: medium-dense

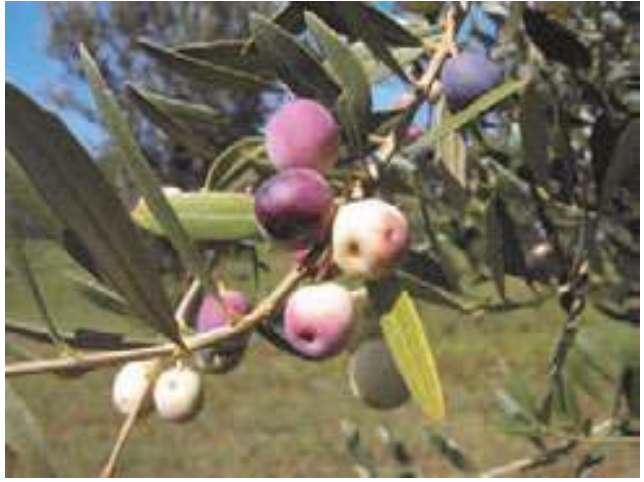

\section{Leaf characters}

Blade length $(\mathrm{cm})$ : medium $(5,49 \pm 0,43)$

Blade width $(\mathrm{cm})$ : medium $(\mathbf{1}, 23 \pm 0,11)$

Shape (length/width): elliptic-lanceolate

\section{Inflorescence characters}

Inflorescence length $(\mathrm{cm})$ : medium $(3,65 \pm 0,49)$

Number of flowers: medium $(24,04 \pm 2,31)$

\section{Fruit characters}

Fresh weight of 100 fruits (g): low $(\mathbf{1}, 65 \pm 0,08)$

Shape (length/width): spherical

Symmetry: symmetric

Position of maximum transverse diameter:

central

Apex: rounded

Base: truncate

Niplle: absent

Lenticels: few and small

\section{Pit characters}

Weight of 100 pits $(\mathrm{g})$ : low $(\mathbf{0 , 2 6} \pm \mathbf{0 , 0 5})$

Shape (length/width): ovoid

Mucron: obvious

Symmetry: slightly asymmetric

Position of maximum transverse diameter:

central

Apex: rounded

Base: rounded

Surface: rugose

Number of grooves: medium
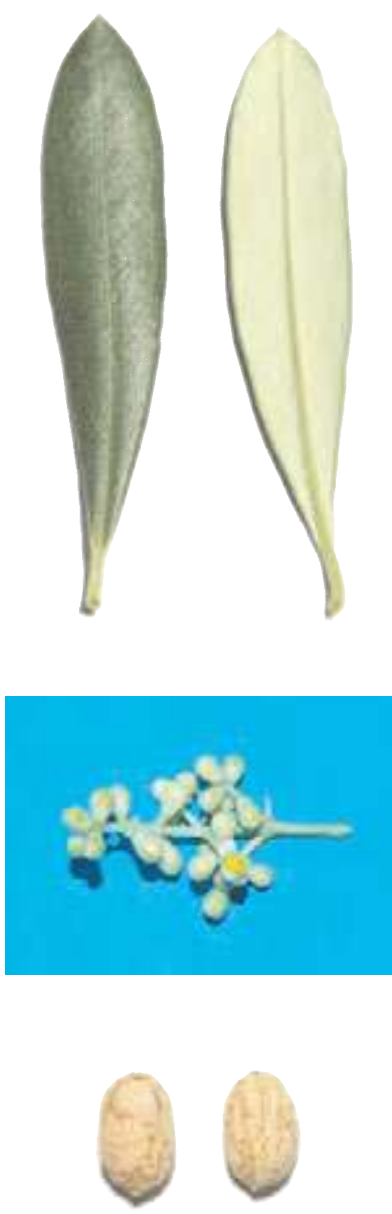


\section{Biochemical Characters}

\section{Fatty Acid Composition}

Table 1. Average values (express in $\% \pm$ standard deviations) of the fatty acids methyl esters and nutritional ratios obtained from single cultivar olive oils.

$\begin{array}{lllll}\text { Myristic acid } \quad 0,02 \pm 0,01 & \text { Linoleic acid }(\omega 6) & \mathbf{1 2 , 2 2} \pm \mathbf{1 , 4 7} & \text { Lignoceric acid } & \mathbf{0 , 0 2} \pm \mathbf{0 , 0 0}\end{array}$

Palmitic acid $15,89 \pm 0,12 \quad$ Linolenic acid $(\omega 3)$ 0,78 $\pm 0,03$

Palmitoleic acid

$1,97 \pm 0,07$

Arachic acid

$0,16 \pm 0,02$

Unsat./satured

$4,54 \pm 0,03$

Stearic acid

$1,66 \pm 0,01$

Eicosenoic acid

$0,02 \pm 0,01$

$\omega 6 / \omega 3$

$15,71 \pm 2,46$

Oleic acid

$65,22 \pm 1,24$

Behenic acid

$0,04 \pm 0,02$

\section{Organoleptic oil values}

Sensory Analysis (Panel test)

Comment: fruity medium, with hints of almond and read sensations of grass and artichoke. Balanced taste sensation with a medium bitter and spicy. Medium fluidity.

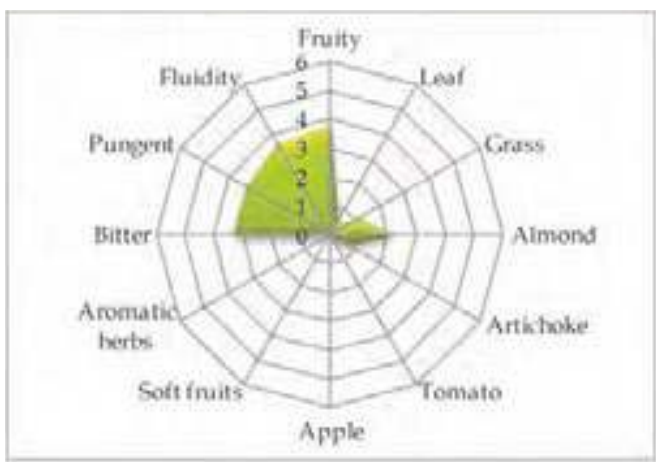

\section{Molecular Markers}

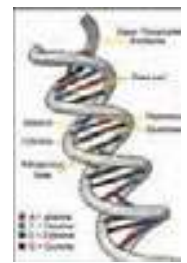

Table 2. Microsatellites (SSR) loci analyzed.

For each locus the allele size (expressed in base pairs) is reported.

$\begin{array}{cccccc}D C A 09 & \text { DCA18 } & \text { GAPU59 } & \text { GAPU71A } & \text { GUPA71B } & \text { GAPU103A } \\ \mathbf{1 8 2} \mathbf{- 2 0 6} & \mathbf{1 7 9 - \mathbf { 1 8 1 }} & \mathbf{2 0 8 - \mathbf { 2 1 8 }} & \mathbf{2 1 2} \mathbf{- 2 1 2} & \mathbf{1 2 6 - \mathbf { 1 4 4 }} & \mathbf{1 8 4} \mathbf{- 1 8 4} \\ \text { UDOO1 } & \text { UDO03 } & \text { UDO12 } & \text { UDO28 } & \text { UDO39 } & \\ \mathbf{1 5 0 - \mathbf { 1 5 0 }} & \mathbf{1 8 2} \mathbf{- 2 0 2} & \mathbf{1 7 7 - \mathbf { 1 8 2 }} & \mathbf{1 5 6 - \mathbf { 1 6 1 }} & \mathbf{2 0 0 - \mathbf { 2 0 0 }} & \end{array}$

\section{References:}

1 - Various authors. In: Catalogo Nazionale delle Varietà di Olivo, University of Bari (in press), ISBN 978-88-8879397-9.

2 - Muzzalupo I., Stefanizzi F., Bucci C., et al. In Acta Italus Hortus, (2011), 1: 138 -140. 


\section{"Rosciola di Rotello"}

(synonymy: Rusciola)

Areal distribution or origin area: Molise

Flesh/pit weight ratio: low $(3,84 \pm 0,34)$

Oil content $(\%)$ : medium $(48,10 \pm 0,08)$

Purpose: oil

\section{Morphological characters}

Tree characters

Vigour: medium-strong

Growth habit: erect

Canopy-density: medium-dense

\section{Leaf characters}

Blade length $(\mathrm{cm})$ : medium $(5,38 \pm 0,43)$

Blade width $(\mathrm{cm})$ : medium $(\mathbf{1}, 46 \pm 0,21)$

Shape (length/width): elliptic

\section{Inflorescence characters}

Inflorescence length (cm): medium $(3,16 \pm 0,73)$

Number of flowers: low $(13,55 \pm 1,46)$

\section{Fruit characters}

Fresh weight of 100 fruits $(\mathrm{g})$ : medium $(\mathbf{2 , 1 4} \pm \mathbf{0 , 1 8})$

Shape (length/width): ovoid

Symmetry: asymmetric

Position of maximum transverse diameter:

towards apex

Apex: rounded

Base: rounded

Niplle: absent

Lenticels: many and small

\section{Pit characters}

Weight of 100 pits (g): medium $(0,45 \pm 0,01)$

Shape (length/width): ovoid

Mucron: obvious

Symmetry: slightly asymmetric

Position of maximum transverse diameter:

\section{towards apex}

Apex: rounded

Base: rounded

Surface: rugose

Number of grooves: low
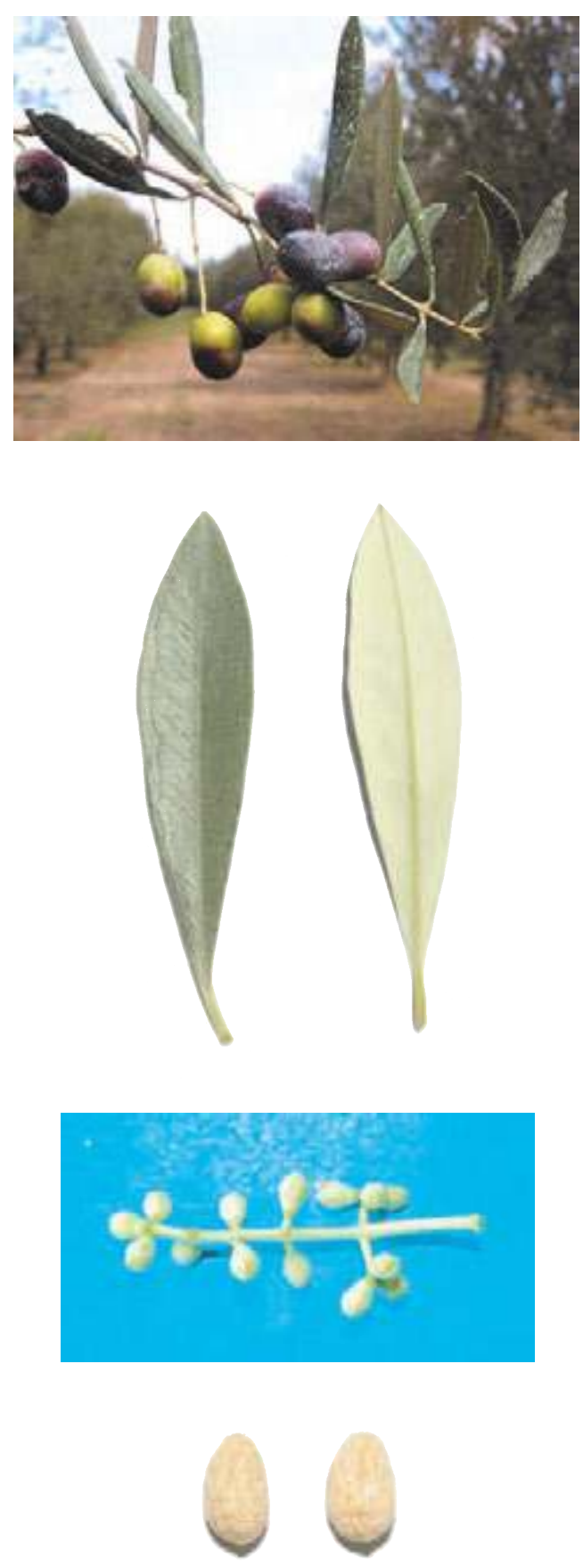


\section{Biochemical Characters}

\section{Fatty Acid Composition}

Table 1. Average values (express in $\% \pm$ standard deviations) of the fatty acids methyl esters and nutritional ratios obtained from single cultivar olive oils.

$\begin{array}{llllll}\text { Myristic acid } & \mathbf{0 , 0 1} \pm \mathbf{0 , 0 1} & \text { Linoleic acid }(\omega 6) & \mathbf{8 , 8 7} \pm \mathbf{0 , 9 5} & \text { Lignoceric acid } & \mathbf{0 , 0 3} \pm \mathbf{0 , 0 2} \\ \text { Palmitic acid } & \mathbf{1 0 , 6 3} \pm \mathbf{2 , 0 9} & \text { Linolenic acid }(\omega 3) \mathbf{0 , 8 9} \pm \mathbf{0 , 0 2} & & \\ \text { Palmitoleic acid } & \mathbf{1 , 9 9} \pm \mathbf{1 , 0 1} & \text { Arachic acid } & \mathbf{0 , 2 3} \pm \mathbf{0 , 0 8} & \text { Unsat./satured } & \mathbf{7 , 0 6} \pm \mathbf{1 , 5 4} \\ \text { Stearic acid } & \mathbf{1 , 6 1} \pm \mathbf{0 , 2 4} & \text { Eicosenoic acid } & \mathbf{0 , 0 2} \pm \mathbf{0 , 0 0} & \omega 6 / \omega 3 & \mathbf{9 , 9 9} \pm \mathbf{0 , 8 6} \\ \text { Oleic acid } & \mathbf{7 4 , 0 2 \pm 0 , 0 6} & \text { Behenic acid } & \mathbf{0 , 0 7} \pm \mathbf{0 , 0 5} & & \end{array}$

\section{Organoleptic oil values}

Sensory Analysis (Panel test)

Comment: fruity medium, with hints of almond and artichoke. Balanced taste sensation with a medium-light bitter and medium spicy. Medium fluidity.

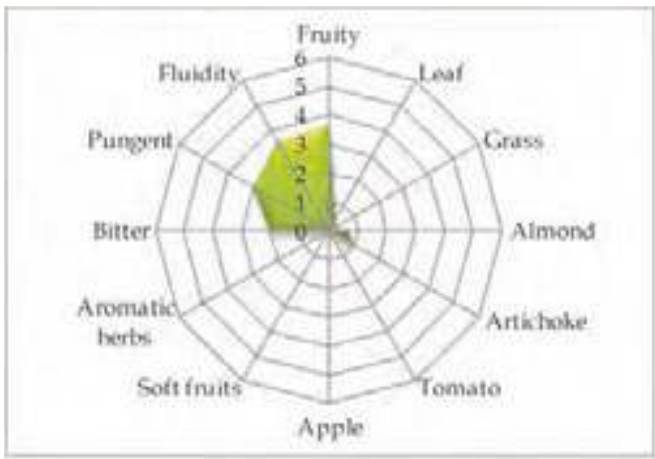

\section{Molecular Markers}

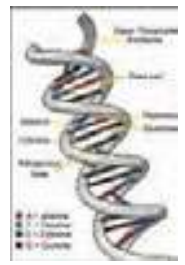

Table 2. Microsatellites (SSR) loci analyzed.

For each locus the allele size (expressed in base pairs) is reported.

$\begin{array}{cccccc}D C A 09 & \text { DCA18 } & \text { GAPU59 } & \text { GAPU71A } & \text { GUPA71B } & \text { GAPU103A } \\ \mathbf{1 6 2} \mathbf{- 1 6 6} & \mathbf{1 6 3 - \mathbf { 1 7 7 }} & \mathbf{2 0 8 - \mathbf { 2 1 2 }} & \mathbf{2 1 4} \mathbf{- 2 2 4} & \mathbf{1 2 4} \mathbf{- 1 4 4} & \mathbf{1 5 9} \mathbf{- 1 8 4} \\ \text { UDOO1 } & \text { UDOO3 } & \text { UDO12 } & \text { UDO28 } & \text { UDO39 } & \\ \mathbf{1 4 4 - \mathbf { 1 4 4 }} & \mathbf{1 5 0 - \mathbf { 1 5 0 }} & \mathbf{1 7 7 - \mathbf { 1 7 7 }} & \mathbf{1 8 2} \mathbf{- 1 8 2} & \mathbf{2 0 5 - \mathbf { 2 0 5 }} & \end{array}$

\section{References:}

1 - Various authors. In: Catalogo Nazionale delle Varietà di Olivo, University of Bari (in press), ISBN 978-88-8879397-9.

2 - Muzzalupo I., Salimonti A., Caravita M. A., et al. Adv. Hort. Sci. (2008), 22(2): pp. 129-135. 


\section{“Rosciola di Venafro"}

Areal distribution or origin area: Molise

Flesh/pit weight ratio: low $(4,90 \pm 1,13)$

Oil content (\%): medium $(45,42 \pm 1,19)$

Purpose: oil

\section{Morphological characters}

Tree characters

Vigour: medium-weak

Growth habit: spreading

Canopy-density: medium

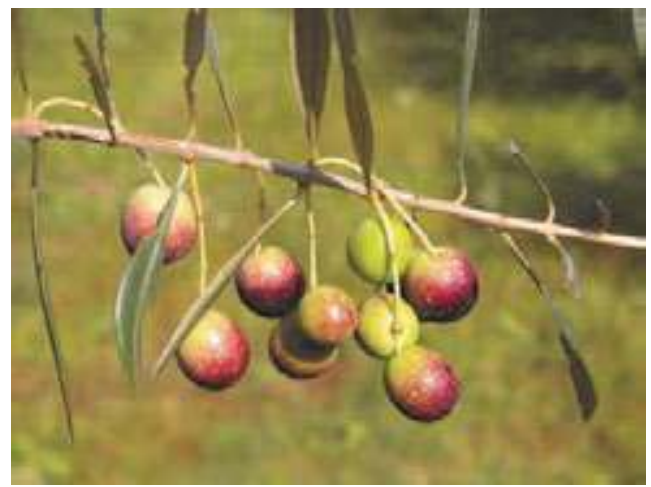

\section{Leaf characters}

Blade length (cm): medium $(8,83 \pm 0,54)$

Blade width $(\mathrm{cm})$ : medium $(1,45 \pm 0,19)$

Shape (length/width): elliptic-lanceolate

\section{Inflorescence characters}

Inflorescence length (cm): long $(3,54 \pm 2,92)$

Number of flowers: medium $(19,99 \pm 1,78)$

\section{Fruit characters}

Fresh weight of 100 fruits (g): medium $(2,38 \pm \mathbf{0 , 2 2})$

Shape (length/width): spherical

Symmetry: asymmetric

Position of maximum transverse diameter:

central

Apex: rounded

Base: truncate

Niplle: tenuous

Lenticels: few and large

\section{Pit characters}

Weight of 100 pits (g): medium $(\mathbf{0 , 4 3} \pm \mathbf{0 , 1 1})$

Shape (length/width): ovoid

Mucron: obvious

Symmetry: slightly asymmetric

Position of maximum transverse diameter:

towards apex

Apex: rounded

Base: rounded

Surface: rugose

Number of grooves: medium
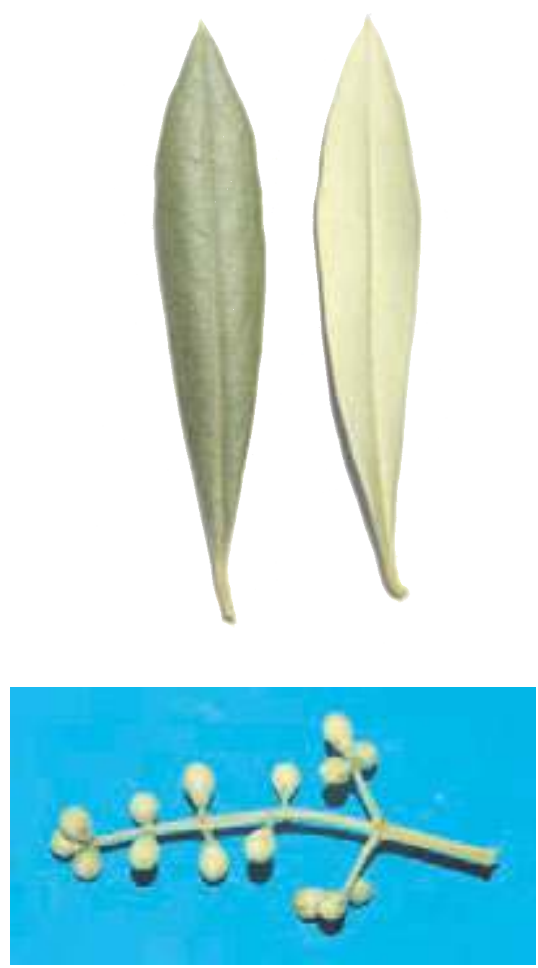


\section{Biochemical Characters}

\section{Fatty Acid Composition}

Table 1. Average values (express in $\% \pm$ standard deviations) of the fatty acids methyl esters and nutritional ratios obtained from single cultivar olive oils.

$\begin{array}{llllll}\text { Myristic acid } & \mathbf{0 , 0 1} \pm \mathbf{0 , 0 0} & \text { Linoleic acid }(\omega 6) & \mathbf{1 0 , 6 4} \pm \mathbf{0 , 8 7} & \text { Lignoceric acid } & \mathbf{0 , 0 3} \pm \mathbf{0 , 0 1} \\ \text { Palmitic acid } & \mathbf{1 4 , 1 3} \pm \mathbf{0 , 0 4} & \text { Linolenic acid }(\omega 3) \mathbf{0 , 7 9} \pm \mathbf{0 , 0 0} & & \\ \text { Palmitoleic acid } & \mathbf{1 , 8 8} \pm \mathbf{0 , 1 6} & \text { Arachic acid } & \mathbf{0 , 2 6} \pm \mathbf{0 , 0 1} & \text { Unsat./satured } & \mathbf{5 , 0 2} \pm \mathbf{0 , 0 3} \\ \text { Stearic acid } & \mathbf{2 , 0 2} \pm \mathbf{0 , 0 8} & \text { Eicosenoic acid } & \mathbf{0 , 0 2} \pm \mathbf{0 , 0 0} & \omega 6 / \omega 3 & \mathbf{1 3 , 4 4} \pm \mathbf{1 , 0 5} \\ \text { Oleic acid } & \mathbf{6 8 , 5 0 \pm 0 , 7 9} & \text { Behenic acid } & \mathbf{0 , 0 6} \pm \mathbf{0 , 0 0} & & \end{array}$

\section{Organoleptic oil values}

Sensory Analysis (Panel test)

Comment: fruity medium - light, with hints of aromatic herbs, artichoke and almond. Balanced in flavours, with hints of bitter and spicy medium intensity, medium fluidity.

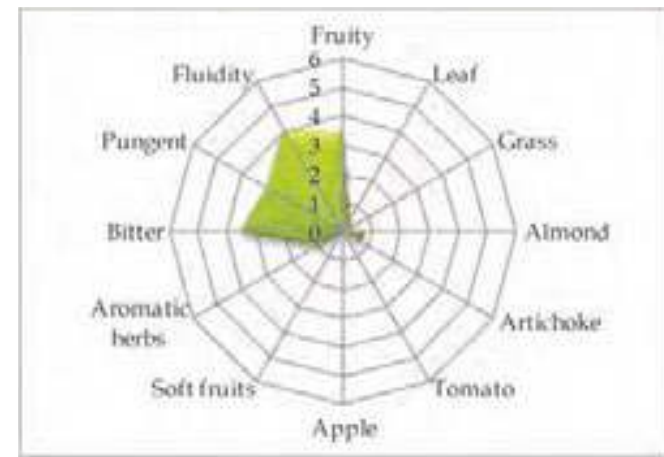

\section{Molecular Markers}

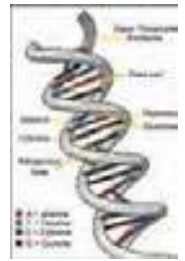

Table 2. Microsatellites (SSR) loci analyzed.

For each locus the allele size (expressed in base pairs) is reported.

$\begin{array}{cccccc}D C A 09 & \text { DCA18 } & \text { GAPU59 } & \text { GAPU71A } & \text { GUPA71B } & \text { GAPU103A } \\ \mathbf{1 7 6 - \mathbf { 2 0 6 }} & \mathbf{1 7 7 - \mathbf { 1 8 1 }} & \mathbf{2 0 8 - \mathbf { 2 2 2 }} & \mathbf{2 1 4} \mathbf{- 2 1 4} & \mathbf{1 2 6 - \mathbf { 1 4 4 }} & \mathbf{1 3 6} \mathbf{- 1 7 0} \\ \text { UDOO1 } & \text { UDOO3 } & \text { UDO12 } & \text { UDO28 } & \text { UDO39 } & \\ \mathbf{1 4 0 - \mathbf { 1 4 0 }} & \mathbf{1 4 3 - \mathbf { 1 4 3 }} & \mathbf{1 7 7 - \mathbf { 1 9 3 }} & \mathbf{1 4 3 - \mathbf { 1 5 4 }} & \mathbf{2 1 3 - \mathbf { 2 1 3 }} & \end{array}$

\section{References:}

1 - Ente regionale di sviluppo agricolo per il Molise. In: Il germoplasma dell'olivo nel Molise, Quaderno divulgativo dell'ERSA (2000), $\mathrm{n}^{\circ} 5$.

2 - Muzzalupo I., Salimonti A., Caravita M. A., et al. Adv. Hort. Sci. (2008), 22(2): pp. 129-135. 


\section{“Rosciola "}

(synonymy: Raggiola, Rossetta.)

Areal distribution or origin area: Marche

Flesh/pit weight ratio: medium $(6,79 \pm 0,31)$

Oil content (\%): medium $(44,55 \pm 1,90)$

Purpose: oil

\section{Morphological characters}

Tree characters

Vigour: medium-weak

Growth habit: spreading

Canopy-density: medium-dense

\section{Leaf characters}

Blade length $(\mathrm{cm})$ : medium $(5,43 \pm 0,37)$

Blade width $(\mathrm{cm})$ : medium $(1,22 \pm 0,09)$

Shape (length/width): elliptic-lanceolate

\section{Inflorescence characters}

Inflorescence length (cm): medium $(2,98 \pm 0,55)$

Number of flowers: medium $(20,02 \pm 0,60)$

\section{Fruit characters}

Fresh weight of 100 fruits (g): low $(\mathbf{1}, 79 \pm \mathbf{0 , 1 2})$

Shape (length/width): spherical

Symmetry: slightly asymmetric

Position of maximum transverse diameter: central

Apex: rounded

Base: rounded

Niplle: absent

Lenticels: many and small

\section{Pit characters}

Weight of 100 pits (g): low $(\mathbf{0 , 2 4} \pm \mathbf{0 , 0 2})$

Shape (length/width): ovoid

Mucron: obvious

Symmetry: symmetric

Position of maximum transverse diameter:

towards apex

Apex: rounded

Base: pointed

Surface: rugose

Number of grooves: medium
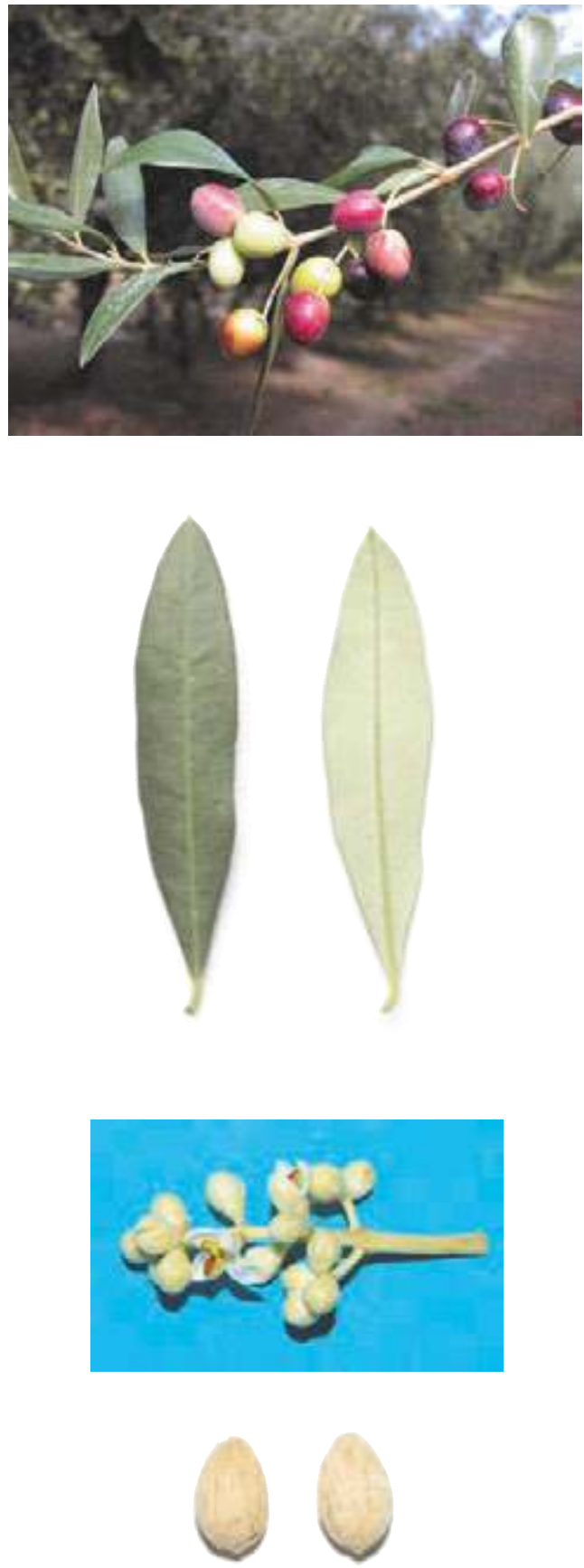


\section{Biochemical Characters}

\section{Fatty Acid Composition}

Table 1. Average values (express in $\% \pm$ standard deviations) of the fatty acids methyl esters and nutritional ratios obtained from single cultivar olive oils.

$\begin{array}{llllll}\text { Myristic acid } & \mathbf{0 , 0 1} \pm \mathbf{0 , 0 1} & \text { Linoleic acid }(\omega 6) & \mathbf{1 3 , 7 4} \pm \mathbf{0 , 7 0} & \text { Lignoceric acid } & \mathbf{0 , 0 3} \pm \mathbf{0 , 0 1} \\ \text { Palmitic acid } & \mathbf{1 2 , 4 5} \pm \mathbf{0 , 2 9} & \text { Linolenic acid }(\omega 3) & \mathbf{0 , 3 8} \pm \mathbf{0 , 0 2} & & \\ \text { Palmitoleic acid } & \mathbf{1 , 3 6} \pm \mathbf{0 , 0 1} & \text { Arachic acid } & \mathbf{0 , 2 4} \pm \mathbf{0 , 0 4} & \text { Unsat./satured } & \mathbf{5 , 7 6 \pm 0 , 1 1} \\ \text { Stearic acid } & \mathbf{1 , 9 1} \pm \mathbf{0 , 1 0} & \text { Eicosenoic acid } & \mathbf{0 , 2 6} \pm \mathbf{0 , 0 2} & \omega 6 / \omega 3 & \mathbf{3 6 , 7 4 \pm 0 , 9 5} \\ \text { Oleic acid } & \mathbf{6 8 , 2 5 \pm \mathbf { 1 , 0 4 }} & \text { Behenic acid } & \mathbf{0 , 0 9} \pm \mathbf{0 , 0 1} & & \end{array}$

\section{Organoleptic oil values}

Sensory Analysis (Panel test)

Comment: fruity medium, with hints of artichoke, read sensations of almond, aromatic herbs and grass. Balanced taste sensation with a medium bitter and spicy. Medium fluidity.

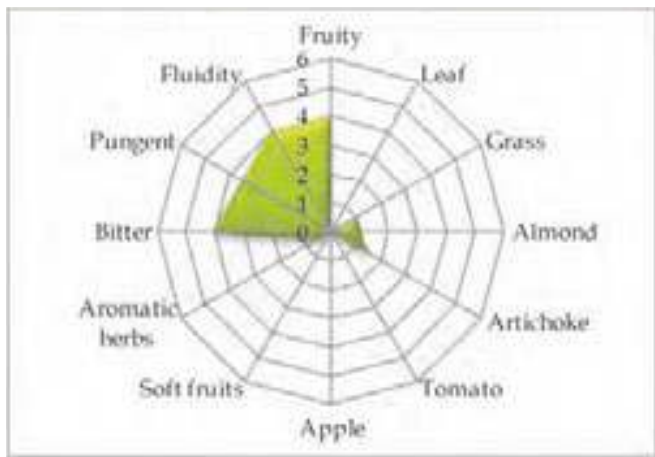

\section{Molecular Markers}

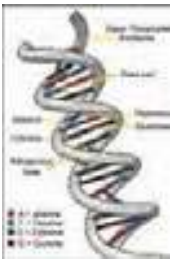

Table 2. Microsatellites (SSR) loci analyzed.

For each locus the allele size (expressed in base pairs) is reported.

$\begin{array}{cccccc}D C A 09 & \text { DCA } 18 & \text { GAPU59 } & \text { GAPU71A } & \text { GUPA71B } & \text { GAPU103A } \\ \mathbf{1 9 8} \mathbf{- 2 0 6} & \mathbf{1 7 7 - \mathbf { 1 8 9 }} & \mathbf{2 1 4} \mathbf{- 2 2 2} & \mathbf{2 1 8 - \mathbf { 2 1 8 }} & \mathbf{1 2 6 - \mathbf { 1 4 4 }} & \mathbf{1 8 4} \mathbf{- 1 9 7} \\ \text { UDO01 } & \text { UDO03 } & \text { UDO12 } & \text { UDO28 } & \text { UDO39 } & \\ \mathbf{1 4 4 - \mathbf { 1 5 0 }} & \mathbf{1 4 3} \mathbf{- 1 4 3} & \mathbf{1 7 7} \mathbf{- 1 8 2} & \mathbf{1 5 4} \mathbf{- 2 0 5} & \mathbf{2 0 5 - \mathbf { 2 0 5 }} & \end{array}$

\section{References:}

1 - Pannelli G., Alfei B., Santinelli A. In: Varietà di olivo nelle Marche, (2001), ASSAM pp. 109-112.

1 - Various authors. In: Catalogo Nazionale delle Varietà di Olivo, University of Bari (in press), ISBN 978-88-8879397-9. 


\section{“Rotondella di Sanza"}

Areal distribution or origin area: Campania

Flesh/pit weight ratio: medium $(6,39 \pm 0,42)$

Oil content (\%): medium $(46,51 \pm 2,52)$

Purpose: oil

\section{Morphological characters}

Tree characters

Vigour: medium

Growth habit: erect-spreading

Canopy-density: medium-dense

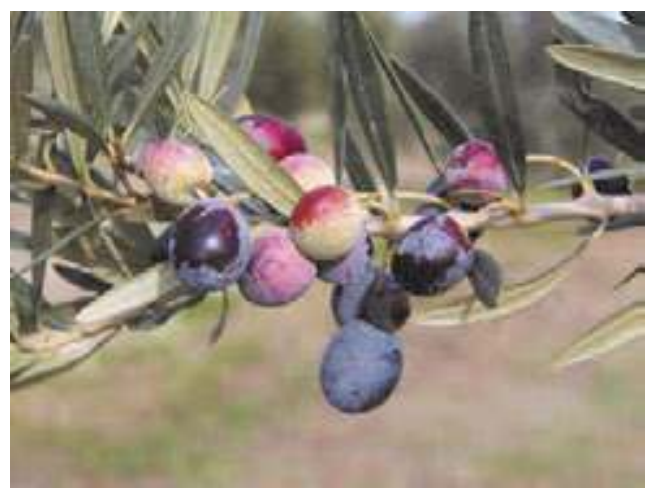

\section{Leaf characters}

Blade length (cm): medium $(5,58 \pm 0,79)$

Blade width $(\mathrm{cm})$ : narrow $(\mathbf{1}, 00 \pm \mathbf{0 , 1 9})$

Shape (length/width): elliptic-lanceolate

\section{Inflorescence characters}

Inflorescence length (cm): medium $(2,74 \pm 1,20)$

Number of flowers: low $(\mathbf{1 5 , 5 2} \pm 0,72)$

\section{Fruit characters}

Fresh weight of 100 fruits (g): medium $(2,87 \pm 0,19)$

Shape (length/width): spherical

Symmetry: symmetric

Position of maximum transverse diameter:

towards base

Apex: pointed

Base: truncate

Niplle: absent

Lenticels: many and small

\section{Pit characters}

Weight of 100 pits (g): medium $(0,39 \pm 0,05)$

Shape (length/width): ovoid

Mucron: absent

Symmetry: symmetric

Position of maximum transverse diameter:

towards base

Apex: rounded

Base: truncate

Surface: scabrous

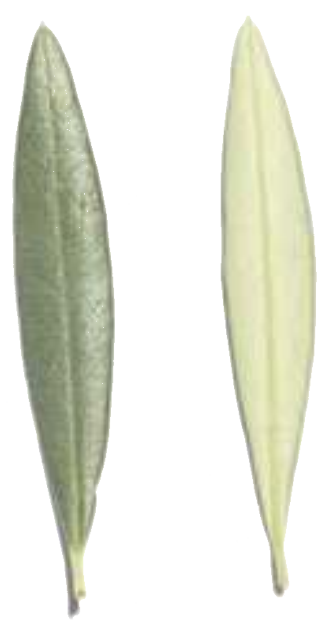

Number of grooves: medium
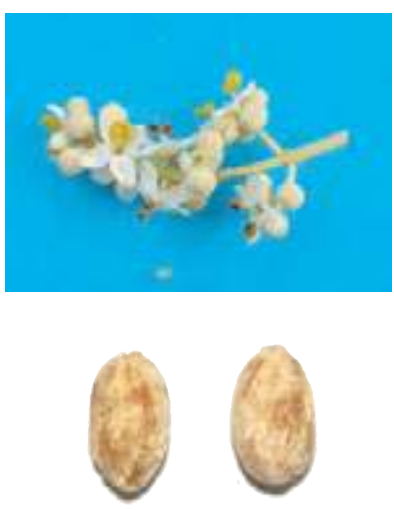


\section{Biochemical Characters}

\section{Fatty Acid Composition}

Table 1. Average values (express in $\% \pm$ standard deviations) of the fatty acids methyl esters and nutritional ratios obtained from single cultivar olive oils.

$\begin{array}{llllll}\text { Myristic acid } & \mathbf{0 , 0 1} \pm \mathbf{0 , 0 1} & \text { Linoleic acid }(\omega 6) & \mathbf{3 , 0 8} \pm \mathbf{0 , 3 0} & \text { Lignoceric acid } & \mathbf{0 , 0 6} \pm \mathbf{0 , 0 4} \\ \text { Palmitic acid } & \mathbf{1 2 , 0 8} \pm \mathbf{1 , 0 0} & \text { Linolenic acid }(\omega 3) & \mathbf{0 , 7 4} \pm \mathbf{0 , 1 2} & & \\ \text { Palmitoleic acid } & \mathbf{1 , 8 1} \pm \mathbf{0 , 2 6} & \text { Arachic acid } & \mathbf{0 , 2 5} \pm \mathbf{0 , 0 5} & \text { Unsat./satured } & \mathbf{6 , 1 4} \pm \mathbf{0 , 5 4} \\ \text { Stearic acid } & \mathbf{1 , 4 7} \pm \mathbf{0 , 1 5} & \text { Eicosenoic acid } & \mathbf{0 , 1 9} \pm \mathbf{0 , 1 4} & \omega 6 / \omega 3 & \mathbf{4 , 2 9} \pm \mathbf{0 , 9 8} \\ \text { Oleic acid } & \mathbf{7 8 , 5 0 \pm \mathbf { 1 , 4 6 }} & \text { Behenic acid } & \mathbf{0 , 0 9} \pm \mathbf{0 , 0 4} & & \end{array}$

\section{Organoleptic oil values}

Sensory Analysis (Panel test)

Comment: fruity medium-high, with hints of almond and read sensations of grass and artichoke. Balanced taste sensation with a medium-high bitter and spicy. Medium-high fluidity.

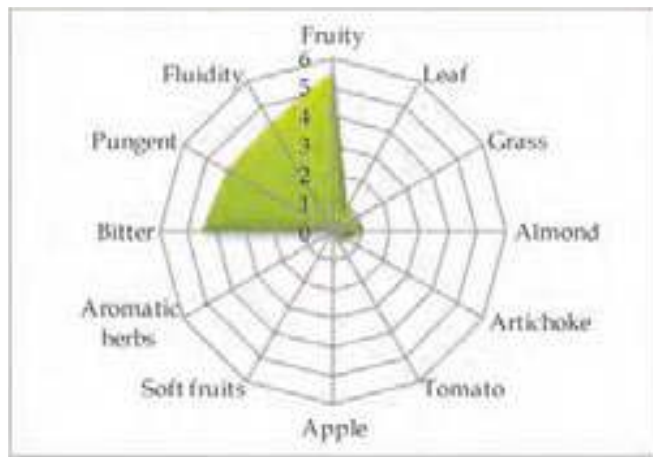

\section{Molecular Markers}

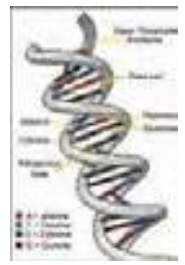

Table 2. Microsatellites (SSR) loci analyzed.

For each locus the allele size (expressed in base pairs) is reported.

$\begin{array}{cccccc}D C A 09 & \text { DCA18 } & \text { GAPU59 } & \text { GAPU71A } & \text { GUPA71B } & \text { GAPU103A } \\ \mathbf{1 9 8} \mathbf{- 2 0 6} & \mathbf{1 7 9 - \mathbf { 1 8 1 }} & \mathbf{2 1 2} \mathbf{- 2 1 3} & \mathbf{2 1 8 - \mathbf { 2 1 8 }} & \mathbf{1 2 6 - \mathbf { 1 4 4 }} & \mathbf{1 5 7 - \mathbf { 1 5 9 }} \\ \text { UDOO1 } & \text { UDOO3 } & \text { UDO12 } & \text { UDO28 } & \text { UDO39 } & \\ \mathbf{1 4 0 - \mathbf { 1 4 0 }} & \mathbf{1 4 3 - \mathbf { 1 5 0 }} & \mathbf{1 6 4 - \mathbf { 1 8 2 }} & \mathbf{1 5 0 - \mathbf { 2 0 5 }} & \mathbf{2 0 5 - \mathbf { 2 3 2 }} & \end{array}$

\section{References:}

1 - Various authors. In: Catalogo Nazionale delle Varietà di Olivo, University of Bari (in press), ISBN 978-88-8879397-9.

2 - Muzzalupo I., Stefanizzi F., Perri E. HortScience, (2009), 44: pp. 582-588. 


\section{"Salvia"}

(synonymy: Salvia Montelibretti)

Areal distribution or origin area: Lazio

Flesh/pit weight ratio: medium $(6,59 \pm 0,72)$

Oil content (\%): medium $(\mathbf{4 1 , 1 1} \pm \mathbf{0 , 8 4})$

Purpose: oil

\section{Morphological characters}

Tree characters

Vigour: medium-strong

Growth habit: spreading-erect

Canopy-density: medium-dense

\section{Leaf characters}

Blade length (cm): short $(4,89 \pm 0,42)$

Blade width (cm): medium $(\mathbf{1}, \mathbf{2 3} \pm \mathbf{0 , 1 3})$

Shape (length/width): elliptic

\section{Inflorescence characters}

Inflorescence length $(\mathrm{cm})$ : short $(2,04 \pm 0,57)$

Number of flowers: low $(\mathbf{1 7}, \mathbf{3 2} \pm \mathbf{2 , 2 9})$

\section{Fruit characters}

Fresh weight of 100 fruits $(\mathrm{g})$ : low $(\mathbf{1}, \mathbf{6 0} \pm \mathbf{0 , 1 4})$

Shape (length/width): ovoid

Symmetry: symmetric

Position of maximum transverse diameter:

central

Apex: rounded

Base: truncate

Niplle: absent

Lenticels: many and small

\section{Pit characters}

Weight of 100 pits (g): low $(\mathbf{0 , 2 6} \pm \mathbf{0 , 0 7})$

Shape (length/width): ovoid

Mucron: absent

Symmetry: symmetric

Position of maximum transverse diameter:

\section{towards apex}

Apex: rounded

Base: rounded

Surface: rugose

Number of grooves: medium
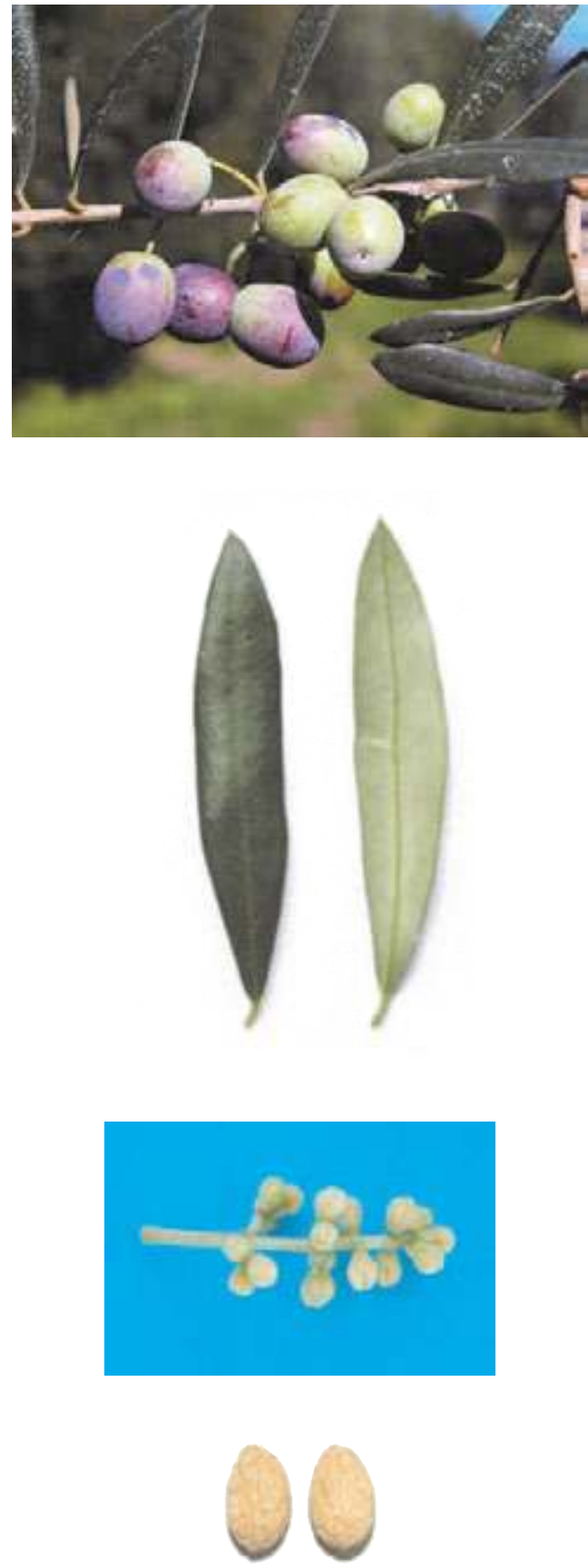


\section{Biochemical Characters}

\section{Fatty Acid Composition}

Table 1. Average values (express in $\% \pm$ standard deviations) of the fatty acids methyl esters and nutritional ratios obtained from single cultivar olive oils.

$\begin{array}{llllll}\text { Myristic acid } & \mathbf{0 , 0 1} \pm \mathbf{0 , 0 0} & \text { Linoleic acid }(\omega 6) & \mathbf{7 , 4 1} \pm \mathbf{0 , 8 0} & \text { Lignoceric acid } & \mathbf{0 , 0 7 \pm 0 , 0 1} \\ \text { Palmitic acid } & \mathbf{1 0 , 9 0 \pm \mathbf { 1 , 0 6 }} & \text { Linolenic acid }(\omega 3) \mathbf{0 , 8 4} \pm \mathbf{0 , 0 8} & & \\ \text { Palmitoleic acid } & \mathbf{1 , 3 4} \pm \mathbf{0 , 0 5} & \text { Arachic acid } & \mathbf{0 , 3 8} \pm \mathbf{0 , 0 3} & \text { Unsat./satured } & \mathbf{6 , 7 1} \pm \mathbf{0 , 6 3} \\ \text { Stearic acid } & \mathbf{1 , 6 7} \pm \mathbf{0 , 0 4} & \text { Eicosenoic acid } & \mathbf{0 , 3 4} \pm \mathbf{0 , 0 4} & \omega 6 / \omega 3 & \mathbf{8 , 8 7} \pm \mathbf{0 , 1 4} \\ \text { Oleic acid } & \mathbf{7 6 , 0 0 \pm 0 , 0 8} & \text { Behenic acid } & \mathbf{0 , 1 0} \pm \mathbf{0 , 0 2} & & \end{array}$

\section{Organoleptic oil values}

Sensory Analysis (Panel test)

Comment: fruity medium-high, with read sensations of aromatic herbs, almond, and artichoke. Balanced taste sensation with a medium-high bitter and spicy. Medium-high fluidity.

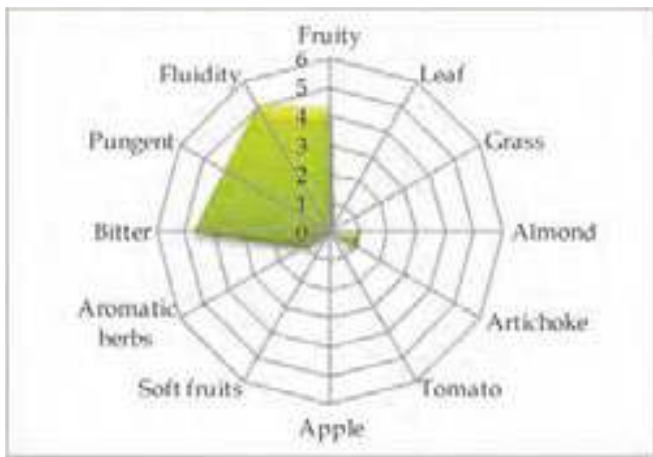

\section{Molecular Markers}

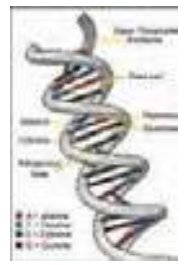

Table 2. Microsatellites (SSR) loci analyzed.

For each locus the allele size (expressed in base pairs) is reported.

\begin{tabular}{|c|c|c|c|c|c|}
\hline DCA09 & DCA18 & GAPU59 & GAPU71A & GUPA71B & GAPU103A \\
\hline $172-182$ & $179-181$ & $218-222$ & $218-224$ & $126-144$ & $150-159$ \\
\hline UDO01 & UDO03 & UDO12 & UDO28 & UDO39 & \\
\hline $144-150$ & $143-143$ & $177-193$ & $154-210$ & $185-185$ & \\
\hline
\end{tabular}

\section{References:}

1 - Parlati M. V., Pandolfi S. In: Olivo e Olio, (2001), 4: pp. 67-72.

2 - Various authors. In: Catalogo Nazionale delle Varietà di Olivo, University of Bari (in press), ISBN 978-88-8879397-9. 


\section{“Sammartinara "}

(synonymy: San Martinara)

Areal distribution or origin area: Sicilia

Flesh/pit weight ratio: high $(7,87 \pm 1,09)$

Oil content (\%): medium $(47,43 \pm 2,06)$

Purpose: oil

\section{Morphological characters}

Tree characters

Vigour: medium

Growth habit: erect

Canopy-density: medium

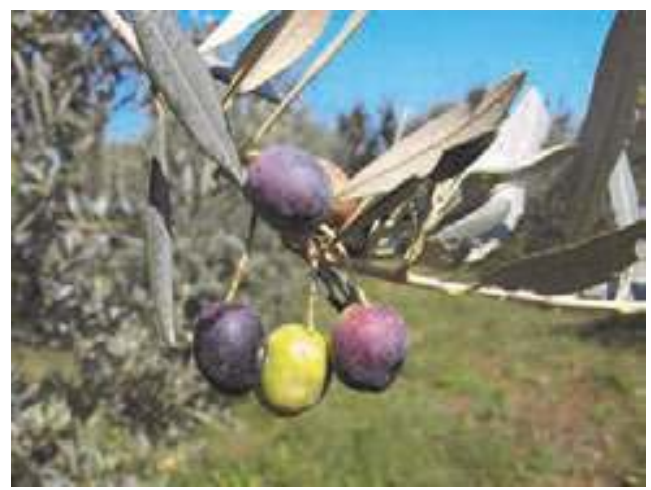

\section{Leaf characters}

Blade length ( $\mathrm{cm})$ : medium $(6,27 \pm 0,50)$

Blade width $(\mathrm{cm})$ : medium $(1,47 \pm 0,15)$

Shape (length/width): elliptic-lanceolate

\section{Inflorescence characters}

Inflorescence length (cm): medium $(3,03 \pm 2,45)$

Number of flowers: low $(\mathbf{1 4 , 4 6 \pm 0 , 6 0 )}$

\section{Fruit characters}

Fresh weight of 100 fruits (g): high $(\mathbf{4 , 0 4} \pm \mathbf{0 , 3 7})$

Shape (length/width): spherical

Symmetry: slightly asymmetric

Position of maximum transverse diameter:

central

Apex: rounded

Base: truncate

Niplle: absent

Lenticels: many and large

\section{Pit characters}

Weight of 100 pits (g): high $(0,46 \pm 0,06)$

Shape (length/width): elliptic

Mucron: absent

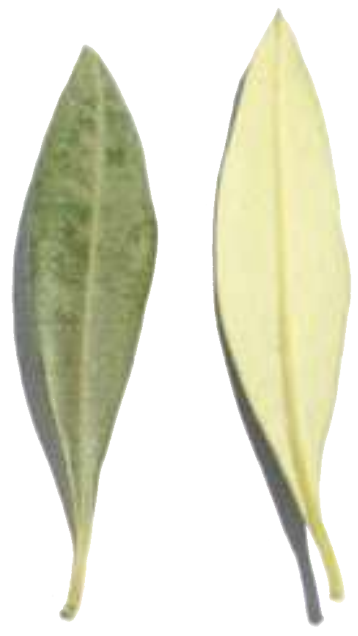

Symmetry: slightly asymmetric

Position of maximum transverse diameter:

towards apex

Apex: rounded

Base: rounded

Surface: rugose

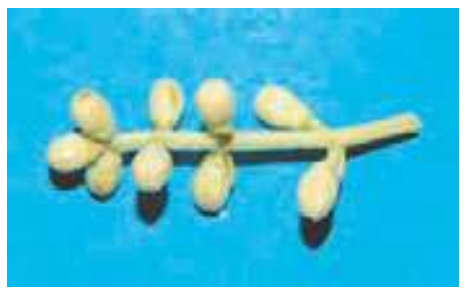

Number of grooves: medium 


\section{Biochemical Characters}

\section{Fatty Acid Composition}

Table 1. Average values (express in $\% \pm$ standard deviations) of the fatty acids methyl esters and nutritional ratios obtained from single cultivar olive oils.

$\begin{array}{llllll}\text { Myristic acid } & \mathbf{0 , 0 2} \pm \mathbf{0 , 0 0} & \text { Linoleic acid }(\omega 6) & \mathbf{1 3 , 1 3} \pm \mathbf{0 , 2 1} & \text { Lignoceric acid } & \mathbf{0 , 0 6} \pm \mathbf{0 , 0 5} \\ \text { Palmitic acid } & \mathbf{1 2 , 8 3} \pm \mathbf{1 , 0 3} & \text { Linolenic acid }(\omega 3) \mathbf{0 , 8 5} \pm \mathbf{0 , 0 2} & & \\ \text { Palmitoleic acid } & \mathbf{0 , 9 0 \pm 0 , 0 3} & \text { Arachic acid } & \mathbf{0 , 2 3} \pm \mathbf{0 , 0 1} & \text { Unsat./satured } & \mathbf{5 , 1 3} \pm \mathbf{0 , 1 0} \\ \text { Stearic acid } & \mathbf{2 , 9 9} \pm \mathbf{1 , 2 8} & \text { Eicosenoic acid } & \mathbf{0 , 0 2} \pm \mathbf{0 , 0 0} & \omega 6 / \omega 3 & \mathbf{1 5 , 4 9} \pm \mathbf{0 , 5 7} \\ \text { Oleic acid } & \mathbf{6 8 , 0 1} \pm \mathbf{0 , 1 6} & \text { Behenic acid } & \mathbf{0 , 1 4} \pm \mathbf{0 , 0 2} & & \end{array}$

\section{Organoleptic oil values}

Sensory Analysis (Panel test)

Comment: medium-high fruity, with good sensation of almond and artichoke, less grass and aromatic herbs. Balanced taste sensation, with bitter and spicy medium. Medium-high fluidity.

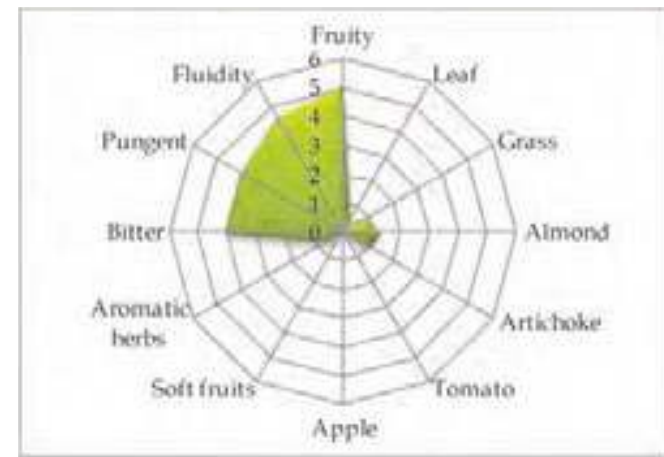

\section{Molecular Markers}

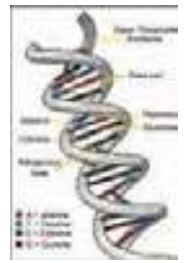

Table 2. Microsatellites (SSR) loci analyzed.

For each locus the allele size (expressed in base pairs) is reported.

$\begin{array}{cccccc}D C A 09 & D C A 18 & \text { GAPU59 } & \text { GAPU71A } & \text { GUPA71B } & \text { GAPU103A } \\ \mathbf{1 6 2} \mathbf{- 1 6 2} & \mathbf{1 7 9 - \mathbf { 1 8 1 }} & \mathbf{2 1 2} \mathbf{- 2 1 2} & \mathbf{2 1 4} \mathbf{- 2 1 4} & \mathbf{1 2 6 - \mathbf { 1 4 4 }} & \mathbf{1 5 9} \mathbf{- 1 5 9} \\ \text { UDO01 } & \text { UDOO3 } & \text { UDO12 } & \text { UDO28 } & \text { UDO39 } & \\ \mathbf{1 4 4 - \mathbf { 1 4 4 }} & \mathbf{1 5 0 - \mathbf { 1 5 0 }} & \mathbf{1 7 7 - \mathbf { 1 7 7 }} & \mathbf{1 8 2} \mathbf{- 1 8 2} & \mathbf{2 0 5 - \mathbf { 2 0 5 }} & \end{array}$

\section{References:}

1 - Sottile I. In: Giornate di studio sulla propagazione delle specie legnose, Ist. Colt. Arb. Pisa (1964), pp. 21-26.

2 - Muzzalupo I., Stefanizzi F., Perri E. HortScience (2009), 44: pp. 582-588. 


\section{“Sammartinenga "}

Areal distribution or origin area: Basilicata

Flesh/pit weight ratio: medium $(6,77 \pm 2,80)$

Oil content (\%): medium $(41,74 \pm 1,68)$

Purpose: oil

\section{Morphological characters}

Tree characters

Vigour: medium-weak

Growth habit: spreading

Canopy-density: medium

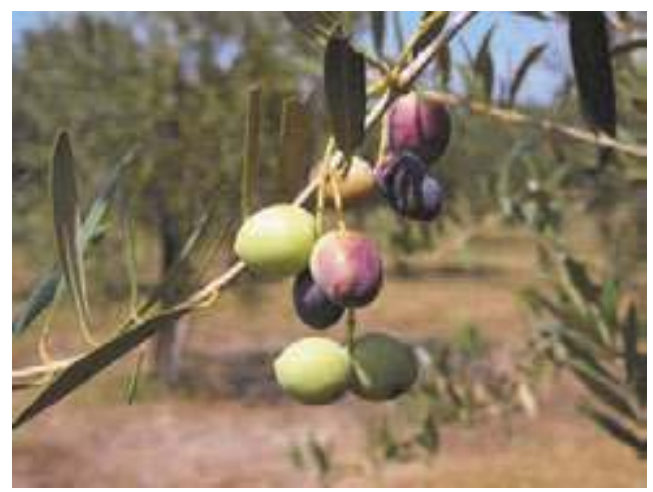

\section{Leaf characters}

Blade length ( $\mathrm{cm})$ : medium $(5,83 \pm 0,58)$

Blade width $(\mathrm{cm})$ : medium $(\mathbf{1}, 04 \pm 0,14)$

Shape (length/width): elliptic-lanceolate

\section{Inflorescence characters}

Inflorescence length (cm): medium $(2,95 \pm 1,25)$

Number of flowers: medium $(22,99 \pm 3,41)$

\section{Fruit characters}

Fresh weight of 100 fruits $(\mathrm{g})$ : medium $(2,12 \pm 0,36)$

Shape (length/width): spherical

Symmetry: slightly asymmetric

Position of maximum transverse diameter:

towards base

Apex: rounded

Base: truncate

Niplle: tenuous

Lenticels: many and small

\section{Pit characters}

Weight of 100 pits (g): medium $(0,28 \pm \mathbf{0 , 0 7})$

Shape (length/width): ovoid

Mucron: obvious

Symmetry: symmetric

Position of maximum transverse diameter:

towards base

Apex: pointed

Base: rounded

Surface: smooth

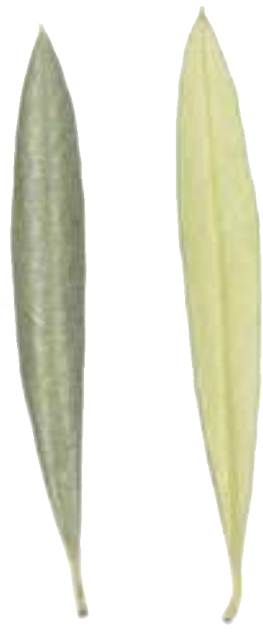

Number of grooves: medium
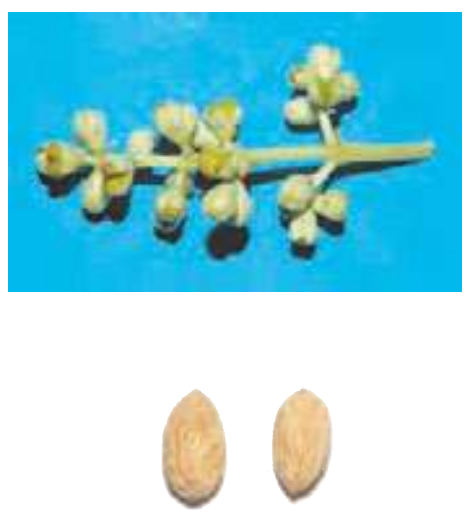


\section{Biochemical Characters}

\section{Fatty Acid Composition}

Table 1. Average values (express in $\% \pm$ standard deviations) of the fatty acids methyl esters and nutritional ratios obtained from single cultivar olive oils.

$\begin{array}{llllll}\text { Myristic acid } & \mathbf{0 , 0 1} \pm \mathbf{0 , 0 1} & \text { Linoleic acid }(\omega 6) & \mathbf{6 , 7 6} \pm \mathbf{0 , 3 2} & \text { Lignoceric acid } & \mathbf{0 , 0 6} \pm \mathbf{0 , 0 5} \\ \text { Palmitic acid } & \mathbf{1 1 , 6 9} \pm \mathbf{0 , 6 3} & \text { Linolenic acid }(\omega 3) & \mathbf{0 , 7 7} \pm \mathbf{0 , 2 0} & & \\ \text { Palmitoleic acid } & \mathbf{1 , 1 6} \pm \mathbf{0 , 1 8} & \text { Arachic acid } & \mathbf{0 , 3 8} \pm \mathbf{0 , 1 1} & \text { Unsat./satured } & \mathbf{5 , 8 6} \pm \mathbf{0 , 2 0} \\ \text { Stearic acid } & \mathbf{2 , 3 7 \pm 0 , 0 7} & \text { Eicosenoic acid } & \mathbf{0 , 1 7} \pm \mathbf{0 , 2 1} & \omega 6 / \omega 3 & \mathbf{9 , 1 8} \pm \mathbf{2 , 8 3} \\ \text { Oleic acid } & \mathbf{7 5 , 0 7 \pm \mathbf { 0 , 1 4 }} & \text { Behenic acid } & 0,10 \pm \mathbf{0 , 0 4} & & \end{array}$

\section{Organoleptic oil values}

Sensory Analysis (Panel test)

Comment: fruity medium-high, with hints of almond and read sensations of grass and artichoke. Balanced taste sensation with a medium-high bitter and spicy. Medium-high fluidity.

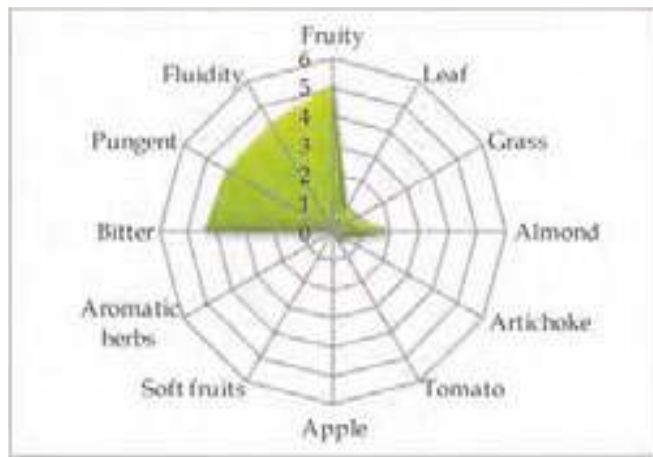

\section{Molecular Markers}

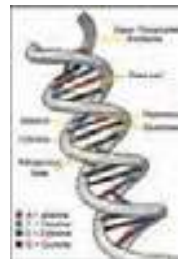

Table 2. Microsatellites (SSR) loci analyzed. For each locus the allele size (expressed in base pairs) is reported.

$\begin{array}{cccccc}D C A 09 & \text { DCA18 } & \text { GAPU59 } & \text { GAPU71A } & \text { GUPA71B } & \text { GAPU103A } \\ \mathbf{1 6 2} \mathbf{- 1 7 6} & \mathbf{1 7 9 - \mathbf { 1 8 1 }} & \mathbf{2 0 8 - \mathbf { 2 2 2 }} & \mathbf{2 1 4} \mathbf{- 2 1 4} & \mathbf{1 2 4} \mathbf{- 1 4 4} & \mathbf{1 3 6} \mathbf{- 1 5 9} \\ \text { UDOO1 } & \text { UDOO3 } & \text { UDO12 } & \text { UDO28 } & \text { UDO39 } & \\ \mathbf{1 4 4 - \mathbf { 1 4 4 }} & \mathbf{1 4 3 - \mathbf { 1 4 3 }} & \mathbf{1 6 6 - \mathbf { 1 7 7 }} & \mathbf{1 4 3 - \mathbf { 1 8 2 }} & \mathbf{2 1 3 - \mathbf { 2 1 3 }} & \end{array}$

\section{References:}

1 - Rotundo A., Marone E. In: Il germoplasma olivicolo lucano, Olita - Potenza (2002), pp. 85-88.

2 - Rotundo A., Perri E.,Muzzalupo I., et al. In: Il germoplasma olivicolo meridionale, (2012 in press).

3 - Muzzalupo I., Stefanizzi F., Perri E. HortScience (2009), 44: pp. 582-588. 


\section{"San Benedetto"}

Areal distribution or origin area: Puglia

Flesh/pit weight ratio: medium $(5,02 \pm 0,47)$

Oil content (\%): medium $(43,30 \pm 1,20)$

Purpose: dual purpose

\section{Morphological characters}

Tree characters

Vigour: medium

Growth habit: erect-spreading

Canopy-density: medium

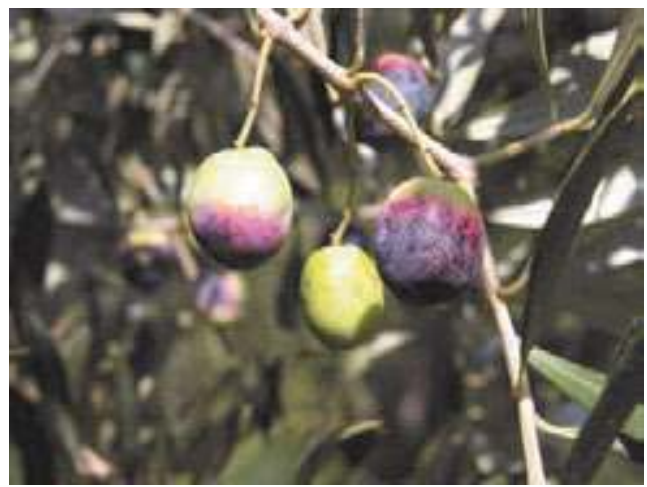

\section{Leaf characters}

Blade length $(\mathrm{cm})$ : medium $(5,70 \pm 0,44)$

Blade width $(\mathrm{cm})$ : medium $(\mathbf{1 , 1 2} \pm \mathbf{0 , 1 0})$

Shape (length/width): elliptic - lanceolate

\section{Inflorescence characters}

Inflorescence length $(\mathrm{cm})$ : medium $(2,86 \pm 0,77)$

Number of flowers: medium $(\mathbf{1 5 , 6 5} \pm \mathbf{1 , 0 6})$

\section{Fruit characters}

Fresh weight of 100 fruits $(\mathrm{g})$ : medium $(2,98 \pm 0,15)$

Shape (length/width): ovoid

Symmetry: slightly asymmetric

Position of maximum transverse diameter:

towards apex

Apex: rounded

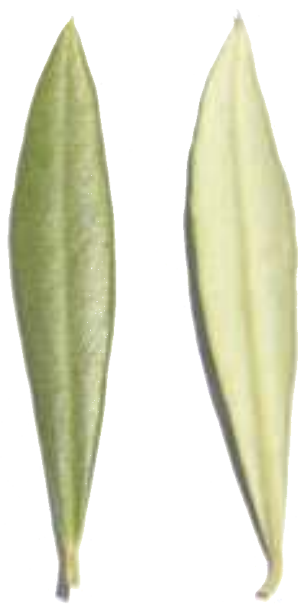

Base: rounded

Niplle: tenuous

Lenticels: many and small

\section{Pit characters}

Weight of 100 pits (g): high $(0,49 \pm 0,07)$

Shape (length/width): symmetric

Mucron: absent

Symmetry: slightly asymmetric

Position of maximum transverse diameter:

\section{towards apex}

Apex: rounded

Base: pointed

Surface: rugose

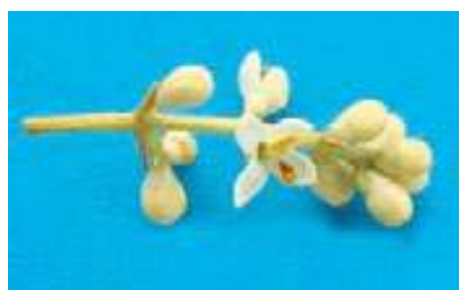

Number of grooves: medium 


\section{Biochemical Characters}

\section{Fatty Acid Composition}

Table 1. Average values (express in $\% \pm$ standard deviations) of the fatty acids methyl esters and nutritional ratios obtained from single cultivar olive oils.

\begin{tabular}{|c|c|c|c|c|c|}
\hline Myristic acid & $0,01 \pm 0,01$ & Linoleic acid $(\omega 6)$ & $12,69 \pm 1,47$ & Lignoceric acid & $0,04 \pm 0,01$ \\
\hline Palmitic acid & $14,55 \pm 0,43$ & Linolenic acid $(\omega 3)$ & $0,87 \pm 0,08$ & & \\
\hline Palmitoleic acid & $3,74 \pm 0,66$ & Arachic acid & $0,36 \pm 0,18$ & Unsat./satured & $4,85 \pm 0,17$ \\
\hline ea & $1,69 \pm 0,11$ & Eicosenoic acid & $0,11 \pm 0,15$ & $\omega 6 / \omega 3$ & $14,56 \pm 2,35$ \\
\hline leic acid & $62,33 \pm 0,69$ & Behenic acid & $0,09 \pm 0,02$ & & \\
\hline
\end{tabular}

\section{Organoleptic oil values}

Sensory Analysis (Panel test)

Comment: fruity medium, with taste of almond, read sensations of grass and leaves.

Balanced taste sensation with medium light bitter and spicy. Medium fluidity.

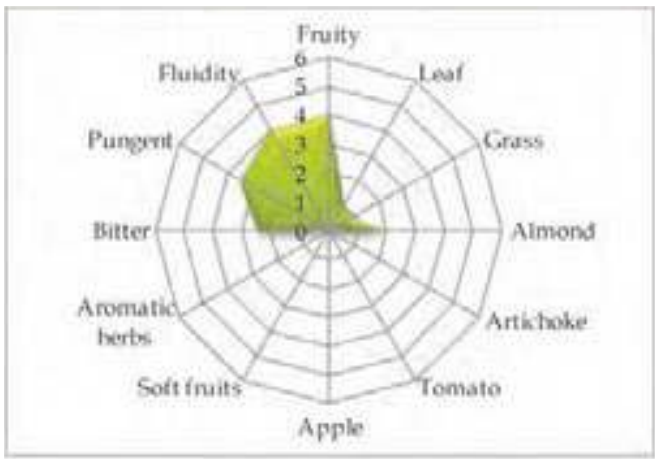

\section{Molecular Markers}

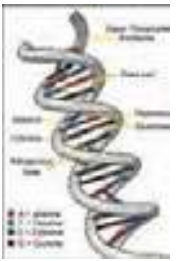

Table 2. Microsatellites (SSR) loci analyzed.

For each locus the allele size (expressed in base pairs) is reported.

\begin{tabular}{|c|c|c|c|c|c|}
\hline DCA09 & $D C A 18$ & GAPU59 & GAPU71A & $G U P A 71 B$ & GAPU103A \\
\hline $162-198$ & $179-185$ & $208-212$ & $214-214$ & $124-144$ & $157-157$ \\
\hline UDO01 & UDO03 & UDO12 & UDO28 & UDO39 & \\
\hline $144-144$ & $135-143$ & $166-193$ & $154-182$ & $205-205$ & \\
\hline
\end{tabular}

\section{References:}

1 - bellomo F. In: Seminario olive da tavola nell'olivicoltura, Acc. Naz. Olivo Cerignola (1969), pp. 9-18.

2 - Muzzalupo I., Lombardo N., Salimonti A., et al. Adv. Hort. Sci. (2008), 22(2): pp. 142-148. 


\section{"San Francesco"}

Areal distribution or origin area: Toscana

Flesh/pit weight ratio: medium $(5,00 \pm 0,01)$

Oil content (\%): low $(35,88 \pm 1,78)$

Purpose: dual purpose

\section{Morphological characters}

Tree characters

Vigour: medium

Growth habit: spreading

Canopy-density: medium-sparse

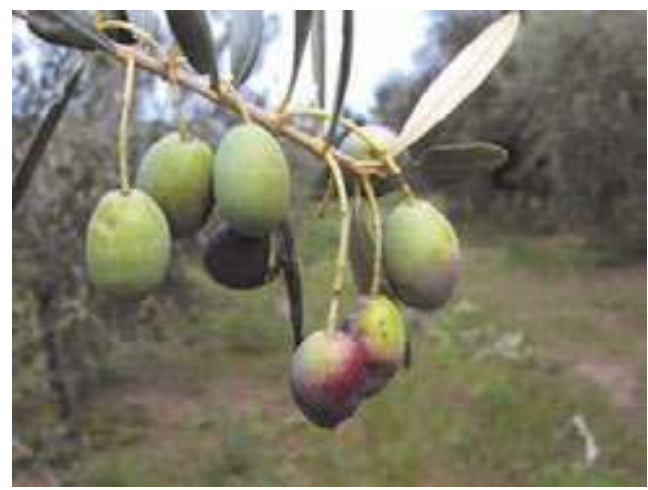

\section{Leaf characters}

Blade length $(\mathrm{cm})$ : medium $(5,34 \pm 0,44)$

Blade width $(\mathrm{cm})$ : broad $(5,00 \pm 0,11)$

Shape (length/width): elliptic-lanceolate

\section{Inflorescence characters}

Inflorescence length (cm): long $(3,82 \pm 1,44)$

Number of flowers: high $(\mathbf{2 5 , 5 8} \pm \mathbf{1 , 4 0})$

\section{Fruit characters}

Fresh weight of 100 fruits $(\mathrm{g})$ : medium $(3,52 \pm 0,11)$

Shape (length/width): ovoid

Symmetry: asymmetric

Position of maximum transverse diameter:

central

Apex: rounded

Base: truncate

Niplle: absent

Lenticels: many and small

\section{Pit characters}

Weight of 100 pits (g): high $(\mathbf{0 , 5 9} \pm \mathbf{0 , 0 2})$

Shape (length/width): elongated

Mucron: obvious
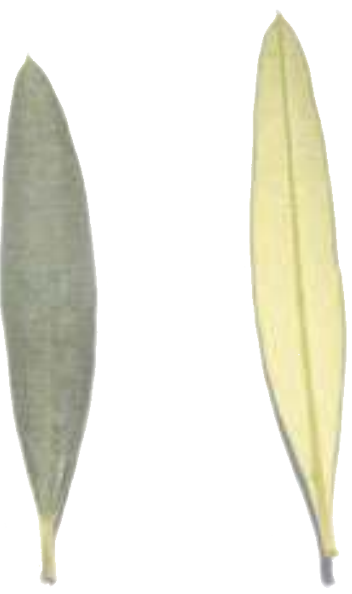

Symmetry: slightly asymmetric

Position of maximum transverse diameter:

towards apex

Apex: pointed

Base: pointed

Surface: rugose

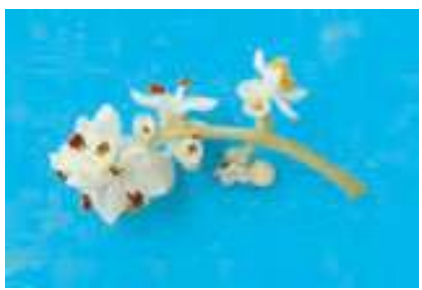

Number of grooves: low 


\section{Biochemical Characters}

\section{Fatty Acid Composition}

Table 1. Average values (express in $\% \pm$ standard deviations) of the fatty acids methyl esters and nutritional ratios obtained from single cultivar olive oils.

$\begin{array}{llllll}\text { Myristic acid } & \mathbf{0 , 0 1} \pm \mathbf{0 , 0 0} & \text { Linoleic acid }(\omega 6) & \mathbf{4 , 7 9} \pm \mathbf{0 , 4 4} & \text { Lignoceric acid } & \mathbf{0 , 0 3} \pm \mathbf{0 , 0 0} \\ \text { Palmitic acid } & \mathbf{1 0 , 6 8} \pm \mathbf{0 , 6 7} & \text { Linolenic acid }(\omega 3) & \mathbf{0 , 9 1} \pm \mathbf{0 , 0 9} & & \\ \text { Palmitoleic acid } & \mathbf{0 , 9 8} \pm \mathbf{0 , 0 1} & \text { Arachic acid } & \mathbf{0 , 2 0} \pm \mathbf{0 , 0 1} & \text { Unsat./satured } & \mathbf{7 , 1 3} \pm \mathbf{0 , 5 6} \\ \text { Stearic acid } & \mathbf{1 , 4 1} \pm \mathbf{0 , 1 1} & \text { Eicosenoic acid } & \mathbf{0 , 0 3} \pm \mathbf{0 , 0 1} & \omega 6 / \omega 3 & \mathbf{5 , 3 2} \pm \mathbf{1 , 0 3} \\ \text { Oleic acid } & \mathbf{8 0 , 1 0 \pm \mathbf { 1 , 5 4 }} & \text { Behenic acid } & \mathbf{0 , 0 4} \pm \mathbf{0 , 0 1} & & \end{array}$

\section{Organoleptic oil values}

Sensory Analysis (Panel test)

Comment: medium fruity, with good sensation of almond and aromatic herbs, less grass and artichoke. Balanced taste sensation, with bitter and spicy medium. Medium-high fluidity.

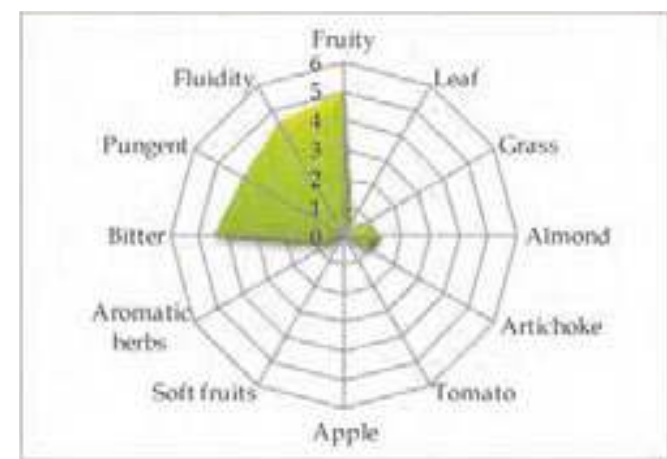

\section{Molecular Markers}

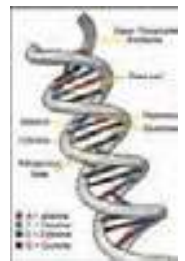

Table 2. Microsatellites (SSR) loci analyzed.

For each locus the allele size (expressed in base pairs) is reported.

$\begin{array}{cccccc}D C A 09 & \text { DCA18 } & \text { GAPU59 } & \text { GAPU71A } & \text { GUPA71B } & \text { GAPU103A } \\ \mathbf{1 8 2} \mathbf{- 2 0 6} & \mathbf{1 7 9 - \mathbf { 1 8 1 }} & \mathbf{2 0 8 - \mathbf { 2 0 8 }} & \mathbf{2 1 0 - \mathbf { 2 1 0 }} & \mathbf{1 2 6 - \mathbf { 1 3 0 }} & \mathbf{1 7 0 - \mathbf { 1 8 4 }} \\ \text { UDOO1 } & \text { UDOO3 } & \text { UDO12 } & \text { UDO28 } & \text { UDO39 } & \\ \mathbf{1 4 0 - \mathbf { 1 4 0 }} & \mathbf{1 5 0 - \mathbf { 1 5 0 }} & \mathbf{1 5 6 - \mathbf { 1 8 2 }} & \mathbf{1 8 2} \mathbf{- 1 8 2} & \mathbf{2 1 3 - 2 1 3} & \end{array}$

\section{References:}

1 - Cimato A., Cantini C., Sani G., et al. In: Atti convegno tecniche, norme e qualità in olivicoltura, Uni. Bas.(1993).

2 - Various authors. In: Catalogo Nazionale delle Varietà di Olivo. University of Bari (in press), ISBN 978-88-8879397-9. 


\section{"Santa Caterina"}

(synonymy: Oliva da indolcire, Oliva di San Biagio, Oliva lucchese, etc.)

Areal distribution or origin area: Toscana

Flesh/ pit weight ratio: very high $(\mathbf{1 0 , 1 3} \pm 0,98)$

Oil content (\%): medium $(41,40 \pm 0,59)$

Purpose: table

\section{Morphological characters}

Tree characters

Vigour: medium

Growth habit: spreading-erect

Canopy-density: medium-dense

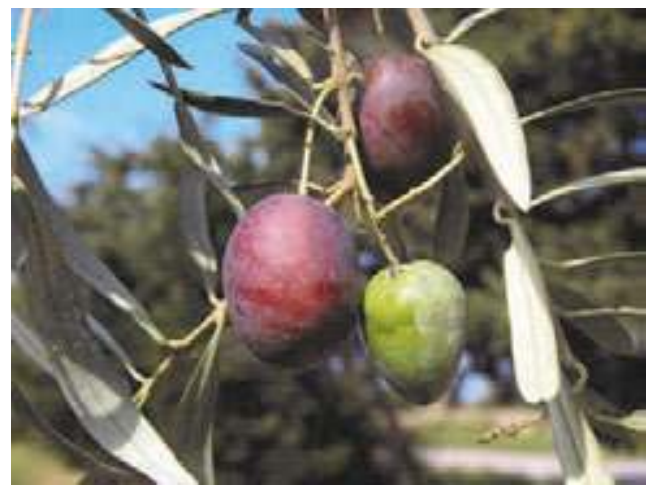

\section{Leaf characters}

Blade length $(\mathrm{cm})$ : long $(7,14 \pm 0,64)$

Blade width (cm): medium $(\mathbf{1}, \mathbf{2 6} \pm \mathbf{0 , 1 3})$

Shape (length/width): elliptic-lanceolate

\section{Inflorescence characters}

Inflorescence length $(\mathrm{cm})$ : medium $(2,76 \pm 0,51)$

Number of flowers: low $(16,70 \pm 2,59)$

\section{Fruit characters}

Fresh weight of 100 fruits $(\mathrm{g})$ : very high $(7,70 \pm 0,52)$

Shape (length/width): spherical

Symmetry: slightly asymmetric

Position of maximum transverse diameter:

\section{towards base}

Apex: rounded

Base: truncate

Niplle: absent

Lenticels: many and small

\section{Pit characters}

Weight of 100 pits (g): very high $(\mathbf{0 , 7 2} \pm \mathbf{0 , 1 0})$

Shape (length/width): elliptic

Mucron: obvious

Symmetry: slightly asymmetric

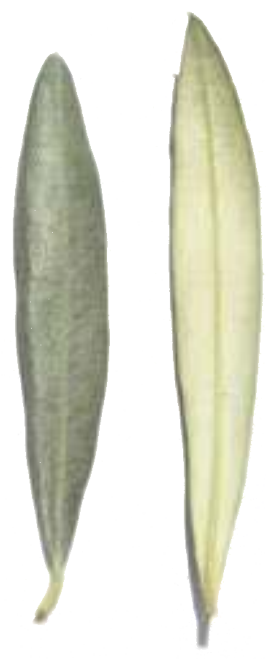

Position of maximum transverse diameter:

\section{towards base}

Apex: pointed

Base: truncate

Surface: rugose

Number of grooves: medium
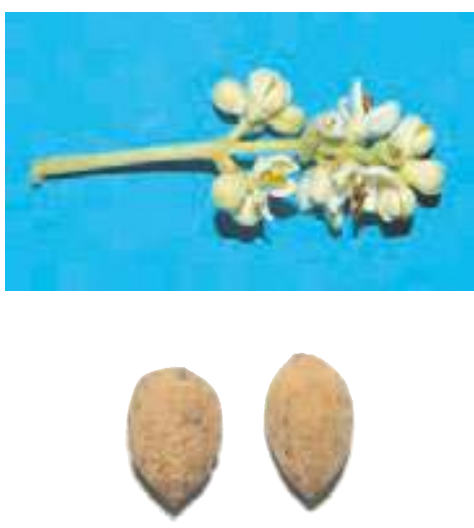


\section{Biochemical Characters}

\section{Fatty Acid Composition}

Table 1. Average values (express in $\% \pm$ standard deviations) of the fatty acids methyl esters and nutritional ratios obtained from single cultivar olive oils.

$\begin{array}{lllll}\text { Myristic acid } & \mathbf{0 , 0 1} \pm 0,00 & \text { Linoleic acid }(\omega 6) & \mathbf{1 1 , 1 7} \pm \mathbf{1 , 4 4} \quad \text { Lignoceric acid } \quad 0,02 \pm 0,01\end{array}$

Palmitic acid $12,06 \pm 1,31 \quad$ Linolenic acid $(\omega 3) 0,97 \pm 0,02$

Palmitoleic acid

$0,50 \pm 0,02$

Arachic acid

$0,17 \pm 0,04$

Unsat./satured

$6,23 \pm 0,70$

Stearic acid

$1,56 \pm 0,13$

Eicosenoic acid

$0,01 \pm 0,00$

$\omega 6 / \omega 3$

$11,56 \pm 1,24$

Oleic acid

$72,56 \pm 0,61$

Behenic acid

$0,05 \pm 0,02$

\section{Organoleptic oil values}

Sensory Analysis (Panel test)

Comment: fruity medium, with hints of artichoke and almond. Balanced in flavours, with hints of bitter and spicy medium-light intensity. Medium fluidity.

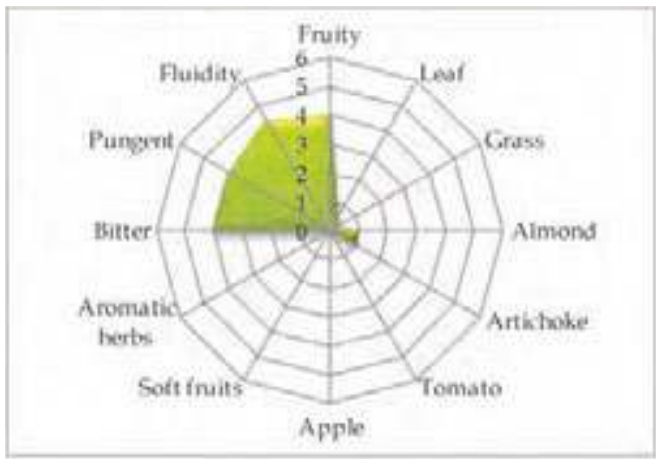

\section{Molecular Markers}

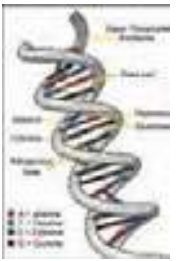

Table 2. Microsatellites (SSR) loci analyzed.

For each locus the allele size (expressed in base pairs) is reported.

$\begin{array}{cccccc}D C A 09 & D C A 18 & \text { GAPU59 } & \text { GAPU71A } & \text { GUPA71B } & \text { GAPU103A } \\ \mathbf{1 6 2} \mathbf{- 1 9 4} & \mathbf{1 8 5 - \mathbf { 1 8 5 }} & \mathbf{2 0 8 - \mathbf { 2 0 8 }} & \mathbf{2 1 0 - \mathbf { 2 1 2 }} & \mathbf{1 2 4 - \mathbf { 1 4 4 }} & \mathbf{1 3 6} \mathbf{- 1 9 7} \\ \text { UDOO1 } & \text { UDOO3 } & \text { UDO12 } & \text { UDO28 } & \text { UDO39 } & \\ \mathbf{1 5 0 - \mathbf { 1 5 0 }} & \mathbf{1 4 3 - \mathbf { 1 4 3 }} & \mathbf{1 5 6 - \mathbf { 1 6 4 }} & \mathbf{1 5 0 - \mathbf { 1 8 2 }} & \mathbf{2 2 0 - \mathbf { 2 2 0 }} & \end{array}$

\section{References:}

1 - Cimato A., Cantini C., Sani G., In: L'olivo in Toscana: il germoplasma autoctono, Ed. ARSIA (2001).

2 - Muzzalupo I., Stefanizzi F., Bucci C., et al. In Acta Italus Hortus, (2011), 1: 138 -140. 


\section{“Santa Maria"}

Areal distribution or origin area: Campania

Flesh/pit weight ratio: medium $(6,18 \pm 0,46)$

Oil content (\%): medium $(48,21 \pm 0,21)$

Purpose: oil

\section{Morphological characters}

Tree characters

Vigour: strong

Growth habit: spreading-erect

Canopy-density: medium-dense

\section{Leaf characters}

Blade length $(\mathrm{cm})$ : medium $(6,27 \pm 0,52)$

Blade width (cm): broad $(\mathbf{1}, 62 \pm \mathbf{0 , 1 4})$

Shape (length/width): elliptic

\section{Inflorescence characters}

Inflorescence length (cm): long $(3,72 \pm 2,03)$

Number of flowers: medium $(\mathbf{1 5}, \mathbf{5 8} \pm \mathbf{2 , 4 9})$

\section{Fruit characters}

Fresh weight of 100 fruits (g): low $(\mathbf{1}, \mathbf{4 2} \pm \mathbf{0 , 1 0})$

Shape (length/width): ovoid

Symmetry: slightly asymmetric

Position of maximum transverse diameter:

central

Apex: rounded

Base: truncate

Niplle: tenuous

Lenticels: many and small

\section{Pit characters}

Weight of 100 pits (g): low $(\mathbf{0 , 2 0} \pm \mathbf{0 , 0 0})$

Shape (length/width): ovoid

Mucron: obvious

Symmetry: slightly asymmetric

Position of maximum transverse diameter:

central

Apex: rounded

Base: pointed

Surface: smooth

Number of grooves: medium
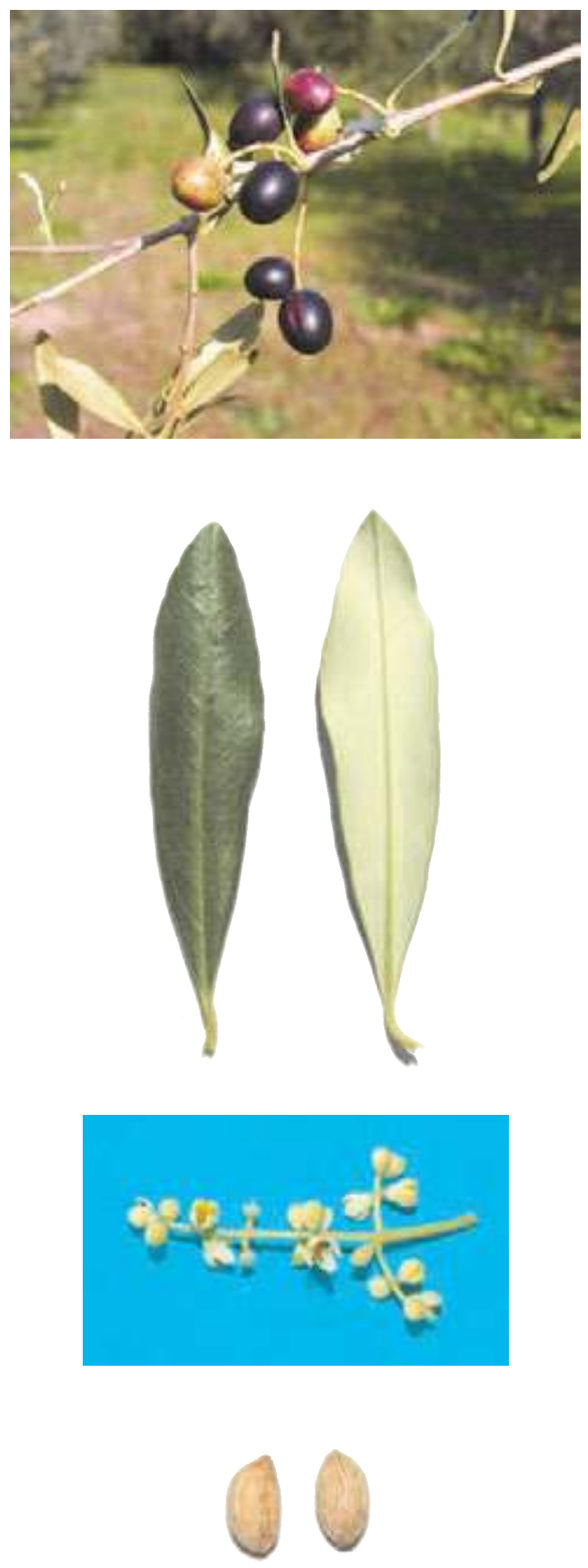


\section{Biochemical Characters}

\section{Fatty Acid Composition}

Table 1. Average values (express in $\% \pm$ standard deviations) of the fatty acids methyl esters and nutritional ratios obtained from single cultivar olive oils.

$\begin{array}{lllll}\text { Myristic acid } \quad 0,02 \pm 0,01 & \text { Linoleic acid }(\omega 6) & \mathbf{1 0}, \mathbf{4 1} \pm \mathbf{1 , 1 0} \quad \text { Lignoceric acid } \quad 0,08 \pm 0,08\end{array}$

Palmitic acid

$11,21 \pm 1,42 \quad$ Linolenic acid $(\omega 3)$ 0,72 $\pm 0,01$

Palmitoleic acid

$$
2,45 \pm 0,48
$$

Arachic acid

$0,28 \pm 0,03$

Unsat./satured

$6,51 \pm 0,87$

Stearic acid

$1,56 \pm 0,12$

Eicosenoic acid

$0,09 \pm 0,11$

$\omega 6 / \omega 3$

$14,55 \pm 1,40$

Oleic acid

$70,82 \pm 0,02$

Behenic acid

$0,11 \pm 0,06$

\section{Organoleptic oil values}

Sensory Analysis (Panel test)

Comment: fruity medium-high, with taste of almond, tomato and artichoke. Balanced taste sensation with medium bitter and spicy. Medium fluidity.

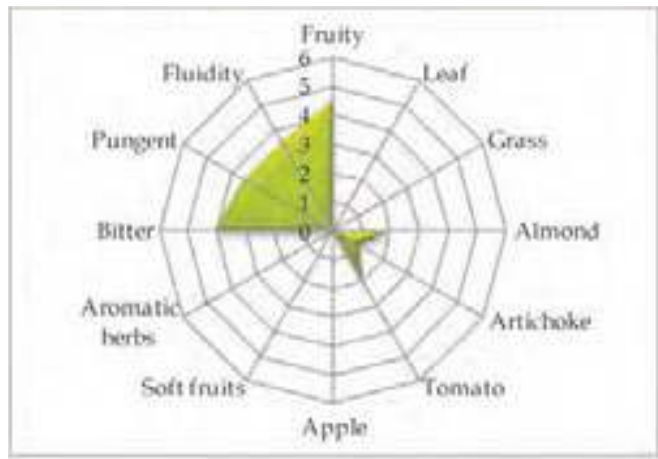

\section{Molecular Markers}

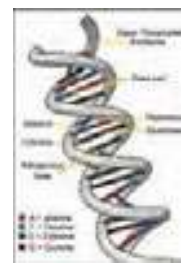

Table 2. Microsatellites (SSR) loci analyzed.

For each locus the allele size (expressed in base pairs) is reported.

$\begin{array}{cccccc}D C A 09 & \text { DCA18 } & \text { GAPU59 } & \text { GAPU71A } & \text { GUPA71B } & \text { GAPU103A } \\ \mathbf{1 7 2} \mathbf{- 1 7 2} & \mathbf{1 7 3 - 1 7 9} & \mathbf{2 1 4 - \mathbf { 2 1 8 }} & \mathbf{2 1 4 - \mathbf { 2 1 8 }} & \mathbf{1 2 4 - \mathbf { 1 4 4 }} & \mathbf{1 5 7 - \mathbf { 1 5 9 }} \\ \text { UDOO1 } & \text { UDO03 } & \text { UDO12 } & \text { UDO28 } & \text { UDO39 } & \\ \mathbf{1 4 0 - \mathbf { 1 5 0 }} & \mathbf{1 4 3 - \mathbf { 1 4 3 }} & \mathbf{1 6 4 - \mathbf { 1 7 7 }} & \mathbf{1 5 4 - \mathbf { 2 0 5 }} & \mathbf{2 0 5 - 2 0 5} & \end{array}$

\section{References:}

1 - Pugliano G., Flaminio G., Pugliano M.L., et al. In: La risorsa genetica dell'olivo in Campania, SE. S.I.R.C.A. Ed. Napoli, (2000).

2 - Muzzalupo I., Stefanizzi F., Perri E. HortScience (2009), 44: pp. 582-588. 


\section{"Sant'Agatese "}

(synonymy: Aliva d'oggi, Comune, Ogliara, Palermitana, Ugghiara, etc.)

Areal distribution or origin area: Sicilia

Flesh/pit weight ratio: high $(7,77 \pm 0,61)$

Oil content (\%): medium $(47,29 \pm 0,20)$

Purpose: oil

\section{Morphological characters}

Tree characters

Vigour: medium

Growth habit: erect-spreading

Canopy-density: medium

\section{Leaf characters}

Blade length $(\mathrm{cm})$ : medium $(5,50 \pm 0,33)$

Blade width $(\mathrm{cm})$ : medium $(\mathbf{1 , 2 7} \pm 0,10)$

Shape (length/width): elliptic-lanceolate

\section{Inflorescence characters}

Inflorescence length (cm): medium $(2,66 \pm 1,44)$

Number of flowers: low $(\mathbf{1 5 , 8 1} \pm \mathbf{0 , 5 2})$

\section{Fruit characters}

Fresh weight of 100 fruits (g): high $(\mathbf{4 , 4 0} \pm \mathbf{1 , 4 1})$

Shape (length/width): ovoid

Symmetry: slightly asymmetric

Position of maximum transverse diameter:

central

Apex: rounded

Base: rounded

Niplle: absent

Lenticels: few and small

\section{Pit characters}

Weight of 100 pits (g): high $(\mathbf{0 , 5 2} \pm \mathbf{0 , 1 8})$

Shape (length/width): elliptic

Mucron: absent

Symmetry: symmetric

Position of maximum transverse diameter:

towards apex

Apex: rounded

Base: rounded

Surface: rugose

Number of grooves: medium
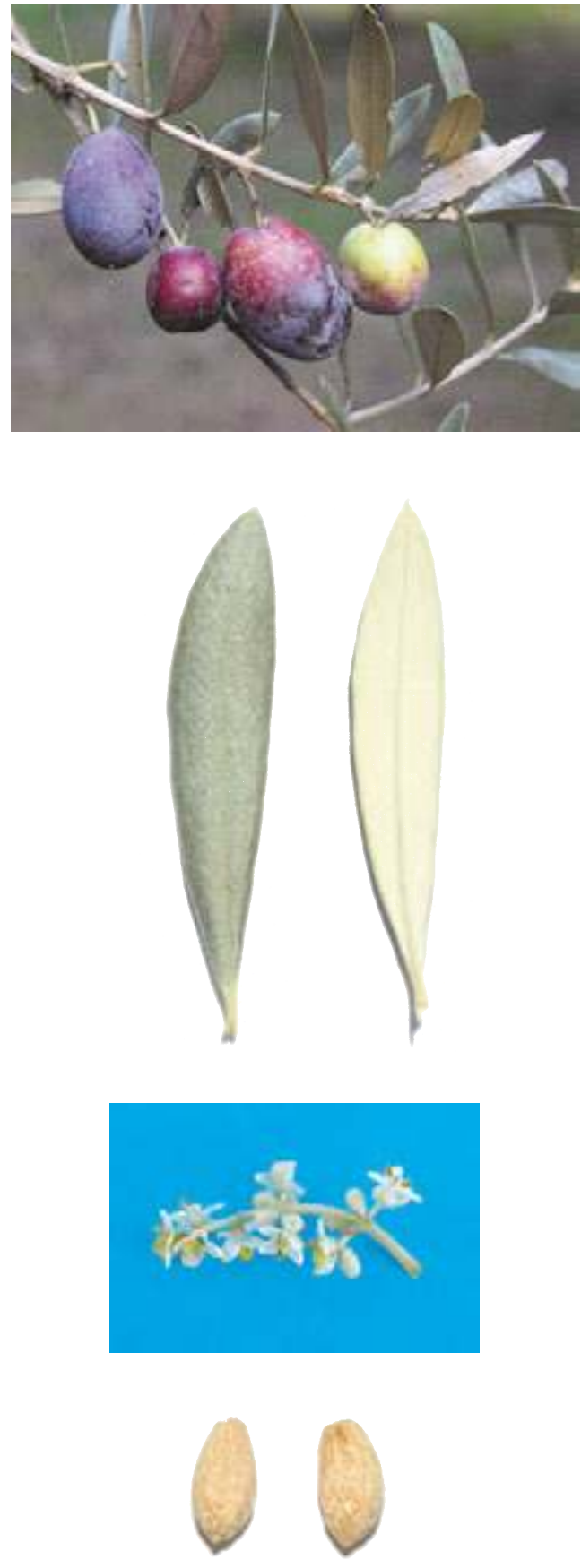


\section{Biochemical Characters}

\section{Fatty Acid Composition}

Table 1. Average values (express in $\% \pm$ standard deviations) of the fatty acids methyl esters and nutritional ratios obtained from single cultivar olive oils.

$\begin{array}{llllll}\text { Myristic acid } & \mathbf{0 , 0 2} \pm \mathbf{0 , 0 1} & \text { Linoleic acid }(\omega 6) & \mathbf{8 , 5 4} \pm \mathbf{0 , 8 0} & \text { Lignoceric acid } & \mathbf{0 , 0 5} \pm \mathbf{0 , 0 4} \\ \text { Palmitic acid } & \mathbf{1 4 , 5 8} \pm \mathbf{0 , 2 5} & \text { Linolenic acid }(\omega 3) \mathbf{0 , 7 1} \pm \mathbf{0 , 0 6} & & \\ \text { Palmitoleic acid } & \mathbf{0 , 9 9 \pm 0 , 4 2} & \text { Arachic acid } & \mathbf{0 , 2 6} \pm \mathbf{0 , 0 2} & \text { Unsat./satured } & 4,85 \pm \mathbf{0 , 0 5} \\ \text { Stearic acid } & \mathbf{2 , 0 1} \pm \mathbf{0 , 1 9} & \text { Eicosenoic acid } & \mathbf{0 , 0 3} \pm \mathbf{0 , 0 1} & \omega 6 / \omega 3 & 12,05 \pm \mathbf{0 , 7 9} \\ \text { Oleic acid } & \mathbf{7 1 , 4 7 \pm \mathbf { 0 , 4 5 }} & \text { Behenic acid } & \mathbf{0 , 0 9} \pm \mathbf{0 , 0 3} & & \end{array}$

\section{Organoleptic oil values}

Sensory Analysis (Panel test)

Comment: fruity medium-light, with read sensations of artichoke, almond and grass. Balanced taste sensation with medium-light spicy and bitter. Medium-light fluidity.

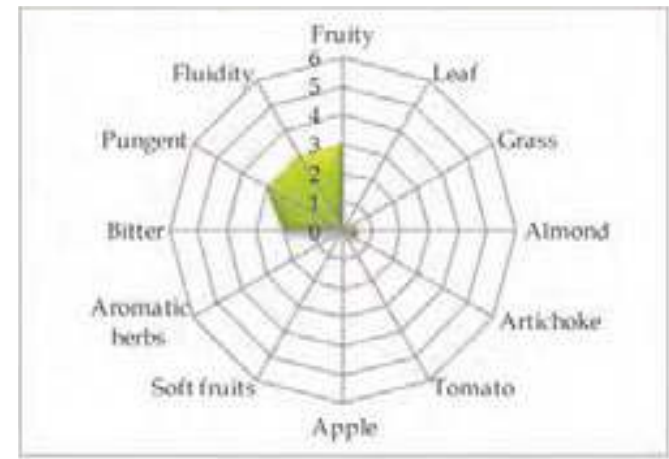

\section{Molecular Markers}

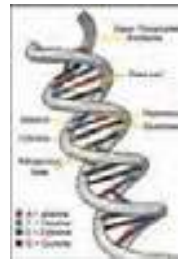

Table 2. Microsatellites (SSR) loci analyzed.

For each locus the allele size (expressed in base pairs) is reported.

$\begin{array}{cccccc}D C A 09 & D C A 18 & \text { GAPU59 } & \text { GAPU71A } & \text { GUPA71B } & \text { GAPU103A } \\ \mathbf{1 7 2} \mathbf{- 1 8 2} & \mathbf{1 7 7 - \mathbf { 1 8 9 }} & \mathbf{2 0 8 - \mathbf { 2 2 2 }} & \mathbf{2 1 4} \mathbf{- 2 1 4} & \mathbf{1 2 4 - \mathbf { 1 2 6 }} & \mathbf{1 5 0 - \mathbf { 1 8 4 }} \\ \text { UDOO1 } & \text { UDOO3 } & \text { UDO12 } & \text { UDO28 } & \text { UDO39 } & \\ \mathbf{1 4 4 - \mathbf { 1 4 4 }} & \mathbf{1 5 0 - \mathbf { 1 5 0 }} & \mathbf{1 7 7 - \mathbf { 1 7 7 }} & \mathbf{1 8 2} \mathbf{- 1 8 2} & \mathbf{2 1 3 - \mathbf { 2 1 3 }} & \end{array}$

\section{References:}

1 - Caruso T., Cartabellotta D., Motisi A., et all. In: Cultivar di olivo siciliane, Università degli Studi di Palermo (2007), pp. 66-70.

2 - Muzzalupo I., Stefanizzi F., Perri E. HortScience (2009), 44: pp. 582-588. 


\section{“Sant'Agostino"}

(synonymy: Grossa di Andria, Oliva di Andria, Oliva grossa, etc.)

Areal distribution or origin area: Puglia

Flesh/pit weight ratio: high $(8,73 \pm 0,21)$

Oil content (\%): medium $(48,14 \pm 2,33)$

Purpose: table

\section{Morphological characters}

Tree characters

Vigour: medium

Growth habit: spreading

Canopy-density: medium

\section{Leaf characters}

Blade length $(\mathrm{cm})$ : medium $(6,32 \pm 0,77)$

Blade width (cm): broad $(\mathbf{1}, 56 \pm 0,21)$

Shape (length/width): elliptic - lanceolate

\section{Inflorescence characters}

Inflorescence length $(\mathrm{cm})$ : medium $(2,80 \pm 0,62)$

Number of flowers: low $(\mathbf{1 4 , 5 5} \pm \mathbf{1 , 3 8})$

\section{Fruit characters}

Fresh weight of 100 fruits (g): very high $(6,71 \pm 1,50)$

Shape (length/width): spherical

Symmetry: slightly asymmetric

Position of maximum transverse diameter:

central

Apex: rounded

Base: truncate

Niplle: absent

Lenticels: many and small

\section{Pit characters}

Weight of 100 pits $(\mathrm{g})$ : very high $(0,70 \pm 0,09)$

Shape (length/width): elliptic

Mucron: obvious

Symmetry: slightly asymmetric

Position of maximum transverse diameter:

\section{towards base}

Apex: pointed

Base: rounded

Surface: scabrous

Number of grooves: medium
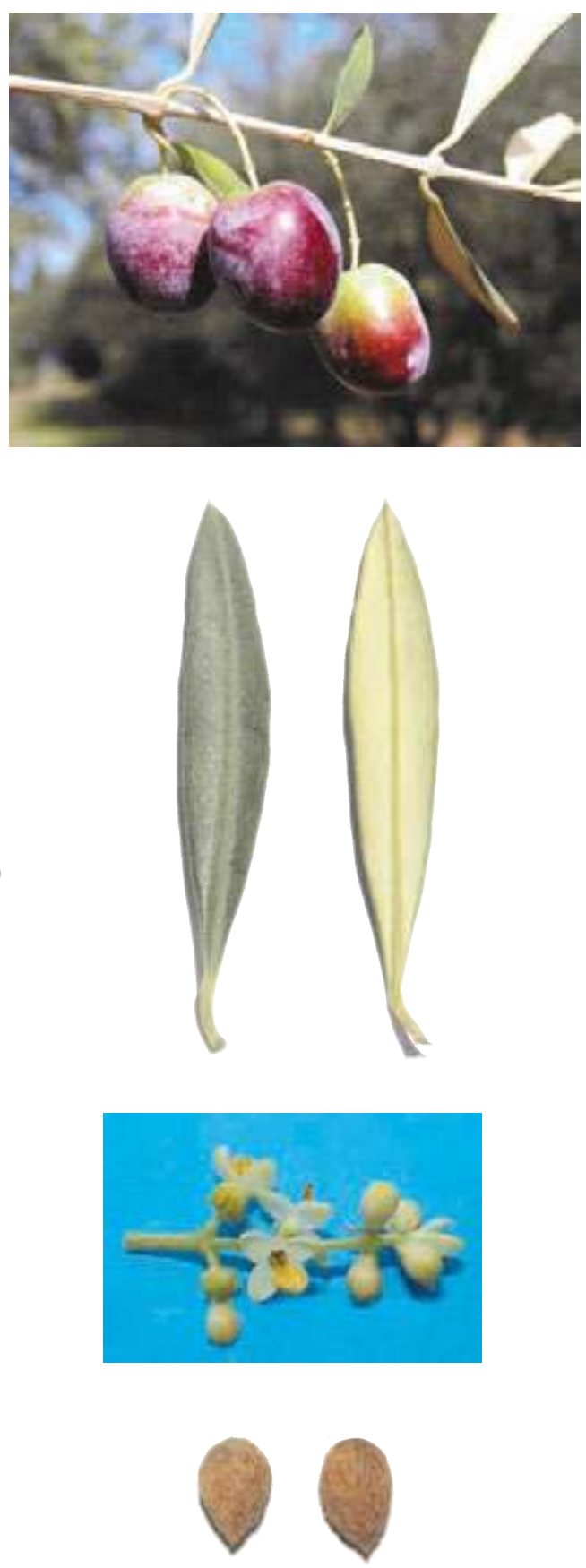


\section{Biochemical Characters}

\section{Fatty Acid Composition}

Table 1. Average values (express in $\% \pm$ standard deviations) of the fatty acids methyl esters and nutritional ratios obtained from single cultivar olive oils.

$\begin{array}{llllll}\text { Myristic acid } & \mathbf{0 , 0 1} \pm \mathbf{0 , 0 0} & \text { Linoleic acid }(\omega 6) & \mathbf{9 , 4 1} \pm \mathbf{0 , 1 5} & \text { Lignoceric acid } & \mathbf{0 , 0 6} \pm \mathbf{0 , 0 4} \\ \text { Palmitic acid } & \mathbf{1 2 , 6 9 \pm \mathbf { 1 } 4 0} & \text { Linolenic acid }(\omega 3) & \mathbf{0 , 9 2} \pm \mathbf{0 , 0 9} & & \\ \text { Palmitoleic acid } & \mathbf{0 , 7 3} \pm \mathbf{0 , 0 2} & \text { Arachic acid } & \mathbf{0 , 3 1} \pm \mathbf{0 , 2 0} & \text { Unsat./satured } & \mathbf{5 , 6 7 \pm 0 , 2 8} \\ \text { Stearic acid } & \mathbf{1 , 9 2} \pm \mathbf{0 , 5 5} & \text { Eicosenoic acid } & \mathbf{0 , 1 9} \pm \mathbf{0 , 2 4} & \omega 6 / \omega 3 & \mathbf{1 0 , 2 8} \pm \mathbf{0 , 8 0} \\ \text { Oleic acid } & \mathbf{7 3 , 0 9 \pm 0 , 1 5} & \text { Behenic acid } & \mathbf{0 , 1 1} \pm \mathbf{0 , 0 7} & & \end{array}$

\section{Organoleptic oil values}

Sensory Analysis (Panel test)

Comment: fruity medium, with hints of almond, artichoke and tomato. Balanced taste sensation with a medium bitter and spicy. Medium fluidity.

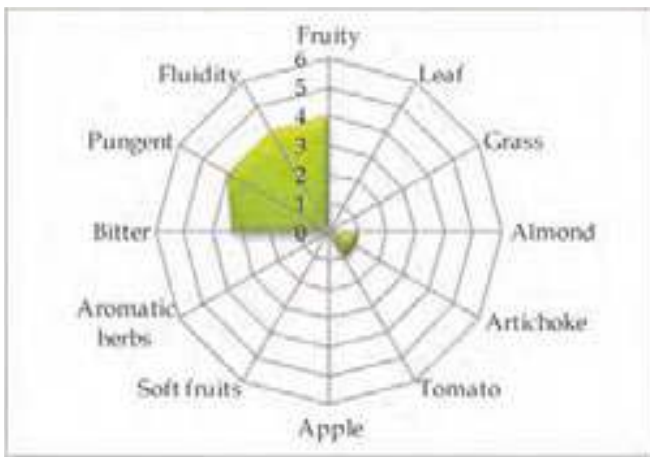

\section{Molecular Markers}

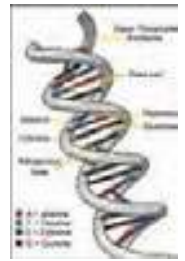

Table 2. Microsatellites (SSR) loci analyzed.

For each locus the allele size (expressed in base pairs) is reported.

\begin{tabular}{|c|c|c|c|c|c|}
\hline DCA09 & DCA18 & GAPU59 & GAPU71A & $G U P A 71 B$ & GAPU103A \\
\hline $172-198$ & $177-181$ & $212-212$ & $210-214$ & $124-144$ & $170-170$ \\
\hline UDO01 & UDO03 & UDO12 & UDO28 & UDO39 & \\
\hline $144-144$ & $143-143$ & $166-166$ & $154-154$ & $108-108$ & \\
\hline
\end{tabular}

\section{References:}

1 - Lombardo N., Perri E., Muzzalupo I., et al. In: Contributo alla caratterizzazione del germoplasma olivicolo pugliese, Ist. Sper. Olivic. (2004), pp. 85-88.

2 - Muzzalupo I., Stefanizzi F., Perri E. HortScience (2009), 44: pp. 582-588. 


\section{"Santomauro"}

(synonymy: Verace.)

Areal distribution or origin area: Calabria

Flesh/pit weight ratio: medium $(7,10 \pm 2,96)$

Oil content (\%): medium $(42,22 \pm 3,15)$

Purpose: oil

\section{Morphological characters}

Tree characters

Vigour: medium-strong

Growth habit: spreading-erect

Canopy-density: medium-dense

\section{Leaf characters}

Blade length $(\mathrm{cm})$ : medium $(5,26 \pm 0,39)$

Blade width $(\mathrm{cm})$ : medium $(1,05 \pm 0,09)$

Shape (length/width): elliptic-lanceolate

\section{Inflorescence characters}

Inflorescence length $(\mathrm{cm})$ : medium $(2,80 \pm 0,28)$

Number of flowers: low $(\mathbf{1 7 , 2 3} \pm \mathbf{2 , 9 5})$

\section{Fruit characters}

Fresh weight of 100 fruits $(\mathrm{g})$ : medium $(3,39 \pm 0,66)$

Shape (length/width): spherical

Symmetry: symmetric

Position of maximum transverse diameter:

towards base

Apex: rounded

Base: truncate

Niplle: absent

Lenticels: many and small

\section{Pit characters}

Weight of 100 pits (g): medium $(\mathbf{0 , 4 4} \pm \mathbf{0 , 6 1})$

Shape (length/width): ovoid

Mucron: absent

Symmetry: symmetric

Position of maximum transverse diameter:

\section{towards base}

Apex: rounded

Base: rounded

Surface: rugose

Number of grooves: low
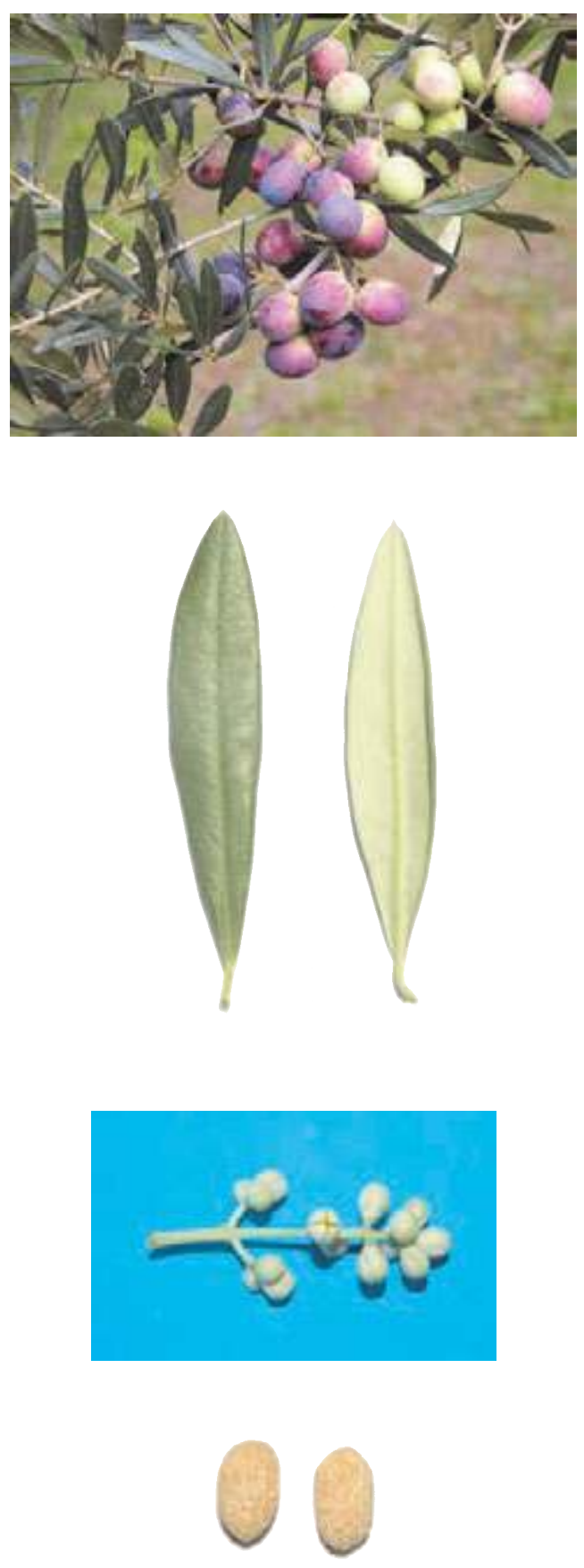


\section{Biochemical Characters}

\section{Fatty Acid Composition}

Table 1. Average values (express in $\% \pm$ standard deviations) of the fatty acids methyl esters and nutritional ratios obtained from single cultivar olive oils.

$\begin{array}{llllll}\text { Myristic acid } & \mathbf{0 , 0 1} \pm \mathbf{0 , 0 1} & \text { Linoleic acid }(\omega 6) & \mathbf{2 , 5 2} \pm \mathbf{0 , 3 2} & \text { Lignoceric acid } & \mathbf{0 , 0 4} \pm \mathbf{0 , 0 3} \\ \text { Palmitic acid } & \mathbf{1 2 , 2 2} \pm \mathbf{0 , 7 0} & \text { Linolenic acid }(\omega 3) \mathbf{0 , 9 3} \pm \mathbf{0 , 1 1} & & \\ \text { Palmitoleic acid } & \mathbf{1 , 8 0} \pm \mathbf{0 , 0 6} & \text { Arachic acid } & \mathbf{0 , 2 6} \pm \mathbf{0 , 0 4} & \text { Unsat./satured } & \mathbf{6 , 0 8} \pm \mathbf{0 , 4 7} \\ \text { Stearic acid } & \mathbf{1 , 4 3} \pm \mathbf{0 , 2 0} & \text { Eicosenoic acid } & \mathbf{0 , 1 5} \pm \mathbf{0 , 2 1} & \omega 6 / \omega 3 & \mathbf{2 , 7 8} \pm \mathbf{0 , 7 2} \\ \text { Oleic acid } & \mathbf{7 8 , 5 4 \pm \mathbf { 1 , 3 1 }} & \text { Behenic acid } & \mathbf{0 , 0 7} \pm \mathbf{0 , 0 2} & & \end{array}$

\section{Organoleptic oil values}

Sensory Analysis (Panel test)

Comment: fruity medium, with read sensations of almond, grass and artichoke. Balanced taste sensation with a medium-high bitter and spicy. Medium-high fluidity.

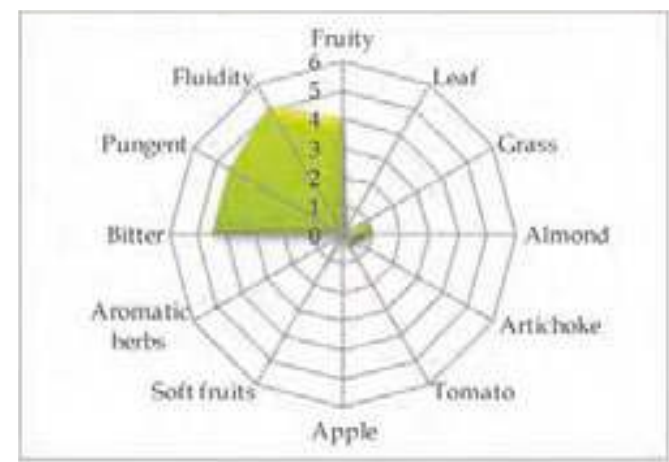

\section{Molecular Markers}

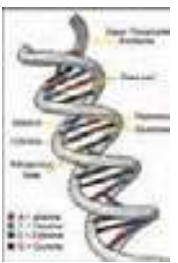

Table 2. Microsatellites (SSR) loci analyzed.

For each locus the allele size (expressed in base pairs) is reported.

$\begin{array}{cccccc}D C A 09 & \text { DCA18 } & \text { GAPU59 } & \text { GAPU71A } & \text { GUPA71B } & \text { GAPU103A } \\ \mathbf{1 8 2} \mathbf{- 1 9 8} & \mathbf{1 7 7 - \mathbf { 1 7 9 }} & \mathbf{2 0 8 - \mathbf { 2 2 2 }} & \mathbf{2 1 4} \mathbf{- 2 1 2 4} & \mathbf{1 2 4 - \mathbf { 1 4 4 }} & \mathbf{1 5 7 - \mathbf { 1 8 4 }} \\ \text { UDOO1 } & \text { UDO03 } & \text { UDO12 } & \text { UDO28 } & \text { UDO39 } & \\ \mathbf{1 4 4 - \mathbf { 1 5 0 }} & \mathbf{1 4 3 - \mathbf { 1 4 3 }} & \mathbf{1 6 6 - \mathbf { 1 8 2 }} & \mathbf{1 4 3 - \mathbf { 2 0 5 }} & \mathbf{2 0 5 - \mathbf { 2 3 2 }} & \end{array}$

\section{References:}

1 - Lombardo N., Perri E., Muzzalupo I., et al. In: Il germoplasma olivicolo calabrese, Ist. Sper. Olivic.(2003), pp: 25.

2 - Perri E., Mazzotti F., Muzzalupo I., et al. In: Relazione attività, CO.R.ASS.OL. (2003).

3 - Muzzalupo I., Chiappetta A., Benincasa C., et al Sci Hortic-Amsterdam, (2010), 126: pp. 324-329. 


\section{"Sargano di Fermo"}

(synonymy: Rosciola di Sirolo, Sargana, Sargano, etc.)

Areal distribution or origin area: Marche

Flesh/pit weight ratio: low $(4,85 \pm 0,61)$

Oil content (\%): medium $(47,96 \pm 0,78)$

Purpose: oil

\section{Morphological characters}

Tree characters

Vigour: medium-strong

Growth habit: spreading-erect

Canopy-density: medium

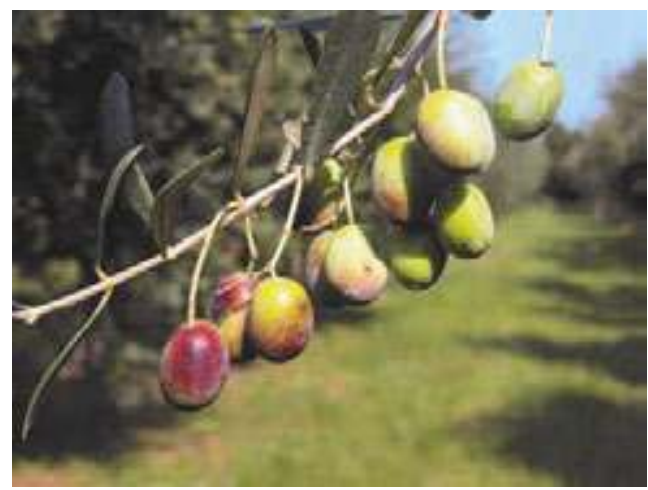

\section{Leaf characters}

Blade length (cm): medium $(5,30 \pm 0,38)$

Blade width (cm): medium $(\mathbf{1}, 38 \pm 0,12)$

Shape (length/width): elliptic

\section{Inflorescence characters}

Inflorescence length $(\mathrm{cm})$ : long $(3,92 \pm 0,81)$

Number of flowers: low $(17,77 \pm 1,33)$

\section{Fruit characters}

Fresh weight of 100 fruits (g): medium $(2,61 \pm 0,11)$

Shape (length/width): ovoid

Symmetry: slightly asymmetric

Position of maximum transverse diameter:

central

Apex: rounded

Base: rounded

Niplle: absent

Lenticels: many and small

\section{Pit characters}

Weight of 100 pits (g): medium $(0,45 \pm \mathbf{0 , 0 3})$

Shape (length/width): ovoid

Mucron: obvious

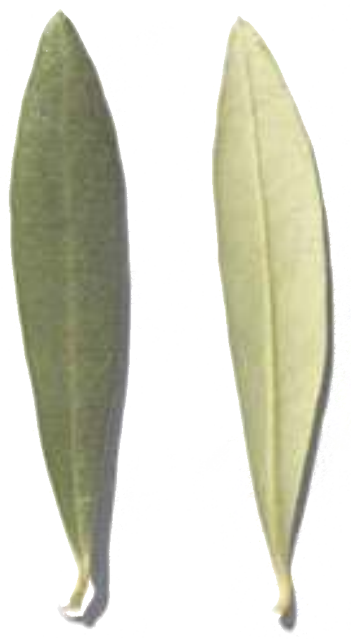

Symmetry: slightly asymmetric

Position of maximum transverse diameter:

\section{towards apex}

Apex: rounded

Base: pointed

Surface: rugose

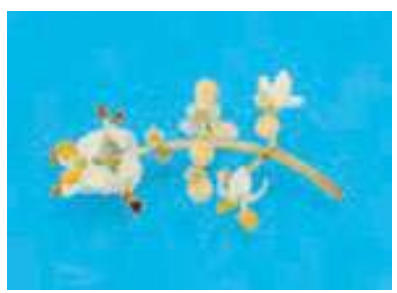

Number of grooves: low 


\section{Biochemical Characters}

\section{Fatty Acid Composition}

Table 1. Average values (express in $\% \pm$ standard deviations) of the fatty acids methyl esters and nutritional ratios obtained from single cultivar olive oils.

$\begin{array}{llllll}\text { Myristic acid } & \mathbf{0 , 0 1} \pm \mathbf{0 , 0 0} & \text { Linoleic acid }(\omega 6) & \mathbf{6 , 9 0} \pm \mathbf{0 , 4 9} & \text { Lignoceric acid } & \mathbf{0 , 0 3} \pm \mathbf{0 , 0 0} \\ \text { Palmitic acid } & \mathbf{9 , 0 3} \pm \mathbf{0 , 1 0} & \text { Linolenic acid }(\omega 3) & \mathbf{0 , 9 3} \pm \mathbf{0 , 0 3} & & \\ \text { Palmitoleic acid } & \mathbf{0 , 7 1} \pm \mathbf{0 , 0 4} & \text { Arachic acid } & \mathbf{0 , 3 3} \pm \mathbf{0 , 0 2} & \text { Unsat./satured } & \mathbf{8 , 2 4} \pm \mathbf{0 , 1 5} \\ \text { Stearic acid } & \mathbf{1 , 5 1} \pm \mathbf{0 , 0 3} & \text { Eicosenoic acid } & \mathbf{0 , 0 1} \pm \mathbf{0 , 0 0} & \omega 6 / \omega 3 & \mathbf{7 , 2 9} \pm \mathbf{0 , 2 6} \\ \text { Oleic acid } & \mathbf{8 0 , 0 9 \pm 0 , 2 1} & \text { Behenic acid } & \mathbf{0 , 0 9} \pm \mathbf{0 , 0 1} & & \end{array}$

\section{Organoleptic oil values}

Sensory Analysis (Panel test)

Comment: fruity medium-high, with hints of almond and read sensations of grass and artichoke. Balanced taste sensation with a medium-high bitter and spicy. Medium-high fluidity.

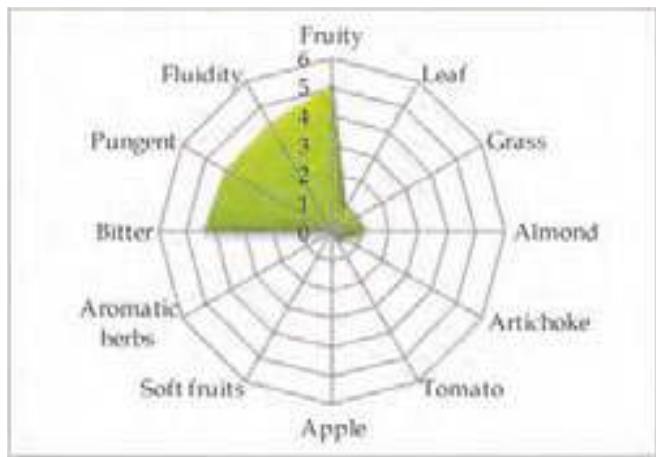

\section{Molecular Markers}

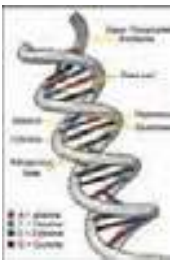

Table 2. Microsatellites (SSR) loci analyzed.

For each locus the allele size (expressed in base pairs) is reported.

$\begin{array}{cccccc}D C A 09 & D C A 18 & \text { GAPU59 } & \text { GAPU71A } & \text { GUPA71B } & \text { GAPU103A } \\ \mathbf{1 8 2} \mathbf{- 1 9 8} & \mathbf{1 7 9 - \mathbf { 1 8 1 }} & \mathbf{2 1 4} \mathbf{- 2 2 2} & \mathbf{2 1 8 - \mathbf { 2 2 4 }} & \mathbf{1 2 4} \mathbf{- 1 4 4} & \mathbf{1 5 9} \mathbf{- 1 8 4} \\ \text { UDOO1 } & \text { UDO03 } & \text { UDO12 } & \text { UDO28 } & \text { UDO39 } & \\ \mathbf{1 4 4 - \mathbf { 1 4 4 }} & \mathbf{1 4 3 - \mathbf { 1 4 3 }} & \mathbf{1 7 7 - \mathbf { 1 8 2 }} & \mathbf{1 8 2} \mathbf{- 2 1 0} & \mathbf{1 8 5} \mathbf{- 1 8 5} & \end{array}$

\section{References:}

1 - Pannelli G., Alfei B., Santinelli A. In: Varietà di olivo nelle Marche, (2001), ASSAM pp. 117-120.

2 - Muzzalupo I., Stefanizzi F., Bucci C., et al. In Acta Italus Hortus, (2011), 1: 138-140. 


\section{"Sargano di San Benedetto"}

(synonymy: Sargano)

Areal distribution or origin area: Marche

Flesh/pit weight ratio: low $(\mathbf{4 , 2 1} \pm \mathbf{0 , 7 6})$

Oil content (\%): medium $(40,13 \pm 0,18)$

Purpose: oil

\section{Morphological characters}

Tree characters

Vigour: medium-strong

Growth habit: erect

Canopy-density: medium-dense

\section{Leaf characters}

Blade length (cm): short $(4,83 \pm 0,41)$

Blade width $(\mathrm{cm})$ : narrow $(0,97 \pm 0,13)$

Shape (length/width): elliptic - lanceolate

\section{Inflorescence characters}

Inflorescence length (cm): long $(4,35 \pm 2,98)$

Number of flowers: high $(26,25 \pm 0,09)$

\section{Fruit characters}

Fresh weight of 100 fruits (g): low $(\mathbf{1 , 5 8} \pm \mathbf{0 , 1 2})$

Shape (length/width): ovoid

Symmetry: slightly asymmetric

Position of maximum transverse diameter:

central

Apex: rounded

Base: truncate

Niplle: absent

Lenticels: many and small

\section{Pit characters}

Weight of 100 pits (g): medium $(0,30 \pm 0,02)$

Shape (length/width): ovoid

Mucron: obvious

Symmetry: slightly asymmetric

Position of maximum transverse diameter:

central

Apex: rounded

Base: rounded

Surface: smooth

Number of grooves: low
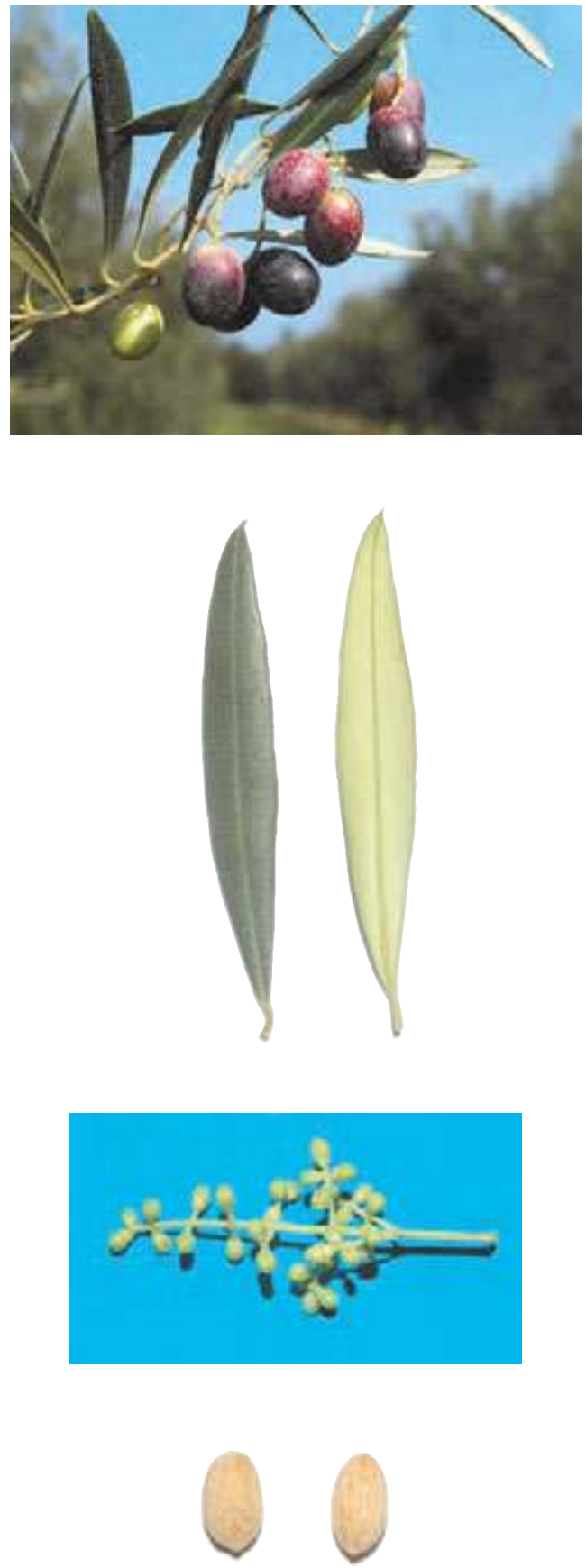


\section{Biochemical Characters}

\section{Fatty Acid Composition}

Table 1. Average values (express in $\% \pm$ standard deviations) of the fatty acids methyl esters and nutritional ratios obtained from single cultivar olive oils.

$\begin{array}{lllll}\text { Myristic acid } & \mathbf{0 , 0 1} \pm \mathbf{0 , 0 0} \quad \text { Linoleic acid }(\omega 6) & \mathbf{1 2 , 5 5} \pm \mathbf{1 , 5 0} \quad \text { Lignoceric acid } \quad \mathbf{0 , 0 2} \pm \mathbf{0 , 0 0}\end{array}$

Palmitic acid $16,62 \pm 0,75 \quad$ Linolenic acid $(\omega 3) 0,76 \pm 0,03$

Palmitoleic acid

$$
2,61 \pm 0,03
$$

Arachic acid

$0,27 \pm 0,04$

Unsat./satured

$4,25 \pm 0,24$

Stearic acid

$1,79 \pm 0,06$

Eicosenoic acid

$0,02 \pm 0,01$

Oleic acid

$62,99 \pm 0,62$

Behenic acid

$0,08 \pm 0,01$

\section{Organoleptic oil values}

Sensory Analysis (Panel test)

Comment: fruity medium, with hints of almond, grass, and artichoke. Balanced taste sensation with a medium-light bitter and spicy. Medium fluidity.

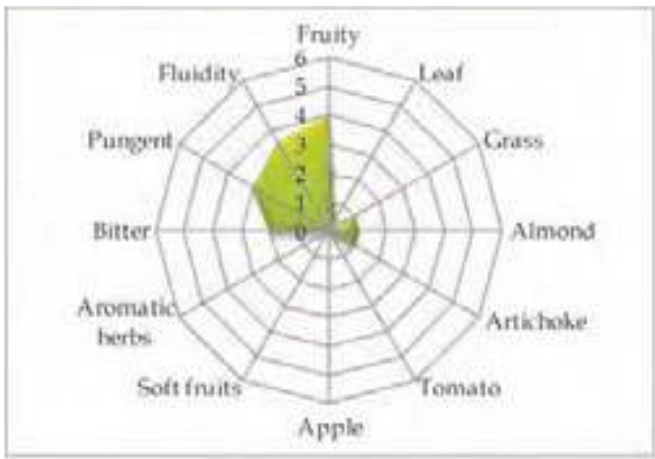

\section{Molecular Markers}

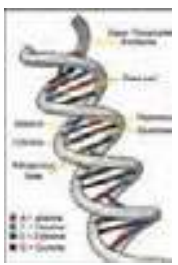

Table 2. Microsatellites (SSR) loci analyzed.

For each locus the allele size (expressed in base pairs) is reported.

$\begin{array}{cccccc}D C A 09 & \text { DCA18 } & \text { GAPU59 } & \text { GAPU71A } & \text { GUPA71B } & \text { GAPU103A } \\ \mathbf{1 7 2} \mathbf{- 1 8 2} & \mathbf{1 7 7 - \mathbf { 1 7 7 }} & \mathbf{2 1 8 - \mathbf { 2 2 2 }} & \mathbf{2 1 4 - \mathbf { 2 1 4 }} & \mathbf{1 3 0 - \mathbf { 1 4 4 }} & \mathbf{1 5 0 - \mathbf { 1 5 7 }} \\ \text { UDOO1 } & \text { UDO03 } & \text { UDO12 } & \text { UDO28 } & \text { UDO39 } & \\ \mathbf{1 5 0 - \mathbf { 1 5 0 }} & \mathbf{1 3 5 - \mathbf { 1 3 5 }} & \mathbf{1 6 6 - \mathbf { 1 6 7 }} & \mathbf{1 5 4 - \mathbf { 2 0 5 }} & \mathbf{2 0 0 - \mathbf { 2 0 5 }} & \end{array}$

\section{References:}

1 - Pannelli G., Alfei B., Santinelli A. In: Varietà di olivo nelle Marche, (2001), ASSAM pp. 121-124

2 - Muzzalupo I., Stefanizzi F., Bucci C., et al. In Acta Italus Hortus, (2011), 1: 138-140. 


\section{“Scarpetta"}

Areal distribution or origin area: Basilicata Flesh/pit weight ratio: low $(\mathbf{4 , 4 2} \pm \mathbf{0 , 8 4})$ Oil content (\%): medium $(48,82 \pm 1,96)$ Purpose: dual purpose

\section{Morphological characters}

Tree characters

Vigour: medium-strong

Growth habit: spreading-erect

Canopy-density: medium-dense

\section{Leaf characters}

Blade length ( $\mathrm{cm})$ : medium $(6,86 \pm 1,24)$

Blade width $(\mathrm{cm})$ : broad $(1,63 \pm 0,33)$

Shape (length/width): elliptic - lanceolate

\section{Inflorescence characters}

Inflorescence length $(\mathrm{cm})$ : medium $(2,93 \pm 1,23)$

Number of flowers: low $(15,98 \pm 2,46)$

\section{Fruit characters}

Fresh weight of 100 fruits $(\mathrm{g})$ : low $(\mathbf{1}, \mathbf{9 7} \pm \mathbf{0 , 2 7})$

Shape (length/width): ovoid

Symmetry: asymmetric

Position of maximum transverse diameter: central

Apex: pointed

Base: rounded

Niplle: absent

Lenticels: many and small

\section{Pit characters}

Weight of 100 pits (g): medium $(0,34 \pm \mathbf{0 , 0 3})$

Shape (length/width): elliptic

Mucron: obvious

Symmetry: asymmetric

Position of maximum transverse diameter: central

Apex: pointed

Base: rounded

Surface: rugose

Number of grooves: medium
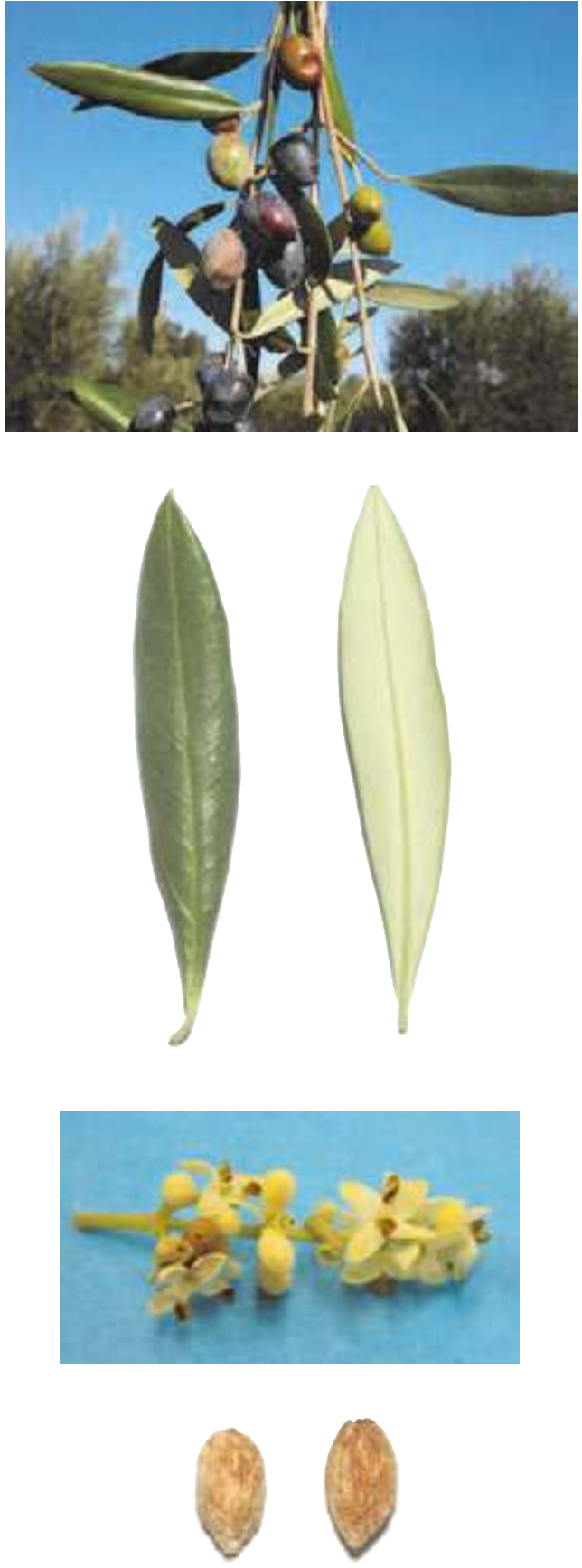


\section{Biochemical Characters}

\section{Fatty Acid Composition}

Table 1. Average values (express in $\% \pm$ standard deviations) of the fatty acids methyl esters and nutritional ratios obtained from single cultivar olive oils.

$\begin{array}{llllll}\text { Myristic acid } & \mathbf{0 , 0 2} \pm \mathbf{0 , 0 1} & \text { Linoleic acid }(\omega 6) & \mathbf{9 , 5 0} \pm \mathbf{1 , 3 4} & \text { Lignoceric acid } & \mathbf{0 , 0 6} \pm \mathbf{0 , 0 4} \\ \text { Palmitic acid } & \mathbf{9 , 7 8} \pm \mathbf{0 , 9 1} & \text { Linolenic acid }(\omega 3) \mathbf{0 , 9 5} \pm \mathbf{0 , 0 4} & & \\ \text { Palmitoleic acid } & \mathbf{0 , 3 9} \pm \mathbf{0 , 0 2} & \text { Arachic acid } & \mathbf{0 , 4 8} \pm \mathbf{0 , 1 0} & \text { Unsat./satured } & \mathbf{6 , 2 2} \pm \mathbf{0 , 2 8} \\ \text { Stearic acid } & \mathbf{3 , 6 6} \pm \mathbf{0 , 4 8} & \text { Eicosenoic acid } & \mathbf{0 , 1 5} \pm \mathbf{0 , 1 1} & \omega 6 / \omega 3 & \mathbf{9 , 9 4} \pm \mathbf{1 , 1 1} \\ \text { Oleic acid } & \mathbf{7 4 , 4 9} \pm \mathbf{2 , 0 9} & \text { Behenic acid } & \mathbf{0 , 1 2} \pm \mathbf{0 , 0 5} & & \end{array}$

\section{Organoleptic oil values}

Sensory Analysis (Panel test)

Comment: fruity medium-high, with hints of almond and artichoke, read sensations of grass and tomato. Balanced taste sensation with a medium bitter and spicy. Medium fluidity.

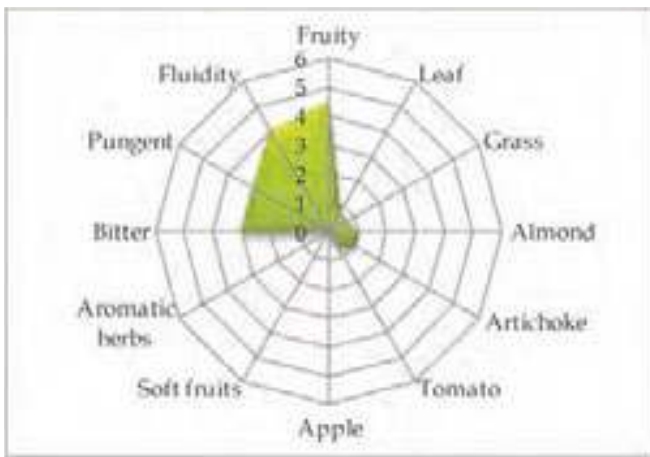

\section{Molecular Markers}

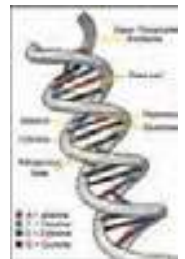

Table 2. Microsatellites (SSR) loci analyzed. For each locus the allele size (expressed in base pairs) is reported.

$\begin{array}{cccccc}D C A 09 & D C A 18 & \text { GAPU59 } & \text { GAPU71A } & \text { GUPA71B } & \text { GAPU103A } \\ \mathbf{1 7 2} \mathbf{- 1 9 8} & \mathbf{1 7 9 - \mathbf { 1 8 1 }} & \mathbf{2 0 8 - \mathbf { 2 1 8 }} & \mathbf{2 1 2} \mathbf{- 2 2 1} & \mathbf{1 2 4} \mathbf{- 1 2 4} & \mathbf{1 5 0 - \mathbf { 1 5 9 }} \\ \text { UDO01 } & \text { UDOO3 } & \text { UDO12 } & \text { UDO28 } & \text { UDO39 } & \\ \mathbf{1 4 4 - \mathbf { 1 4 4 }} & \mathbf{1 5 0 - \mathbf { 1 5 0 }} & \mathbf{1 7 7 - \mathbf { 1 8 2 }} & \mathbf{1 4 3 - \mathbf { 1 4 3 }} & \mathbf{2 0 0 - \mathbf { 2 0 0 }} & \end{array}$

\section{References:}

1 - Rotundo A., Marone E. In: Il germoplasma olivicolo lucano, Olita - Potenza (2002), pp. 151-154.

2 - Rotundo A., Perri E.,Muzzalupo I., et al. In: Il germoplasma olivicolo meridionale, (2012 in press).

3 - Muzzalupo I., Stefanizzi F., Perri E. HortScience (2009), 44: pp. 582-588. 


\section{"Semidana"}

(synonymy: Olia di Riola, Olieddu terzu, Terza, etc.)

Areal distribution or origin area: Sardegna

Flesh/pit weight ratio: medium $(5,05 \pm 0,37)$

Oil content (\%): medium $(44,46 \pm 2,71)$

Purpose: dual purpose

\section{Morphological characters}

Tree characters

Vigour: weak

Growth habit: spreading

Canopy-density: medium-sparse

\section{Leaf characters}

Blade length $(\mathrm{cm})$ : medium $(6,73 \pm 0,70)$

Blade width $(\mathrm{cm})$ : medium $(\mathbf{1 , 2 8} \pm \mathbf{0 , 1 7})$

Shape (length/width): elliptic-lanceolate

\section{Inflorescence characters}

Inflorescence length (cm): medium $(2,86 \pm 1,51)$

Number of flowers: medium $(18,57 \pm 1,48)$

\section{Fruit characters}

Fresh weight of 100 fruits $(\mathrm{g})$ : high $(\mathbf{4 , 4 5} \pm \mathbf{0 , 3 7})$

Shape (length/width): ovoid

Symmetry: slightly asymmetric

Position of maximum transverse diameter:

central

Apex: rounded

Base: rounded

Niplle: obvious

Lenticels: few and large

\section{Pit characters}

Weight of 100 pits (g): high $(\mathbf{0 , 6 0} \pm \mathbf{0 , 0 1})$

Shape (length/width): ovoid

Mucron: obvious

Symmetry: slightly asymmetric

Position of maximum transverse diameter:

central

Apex: rounded

Base: rounded

Surface: rugose

Number of grooves: low
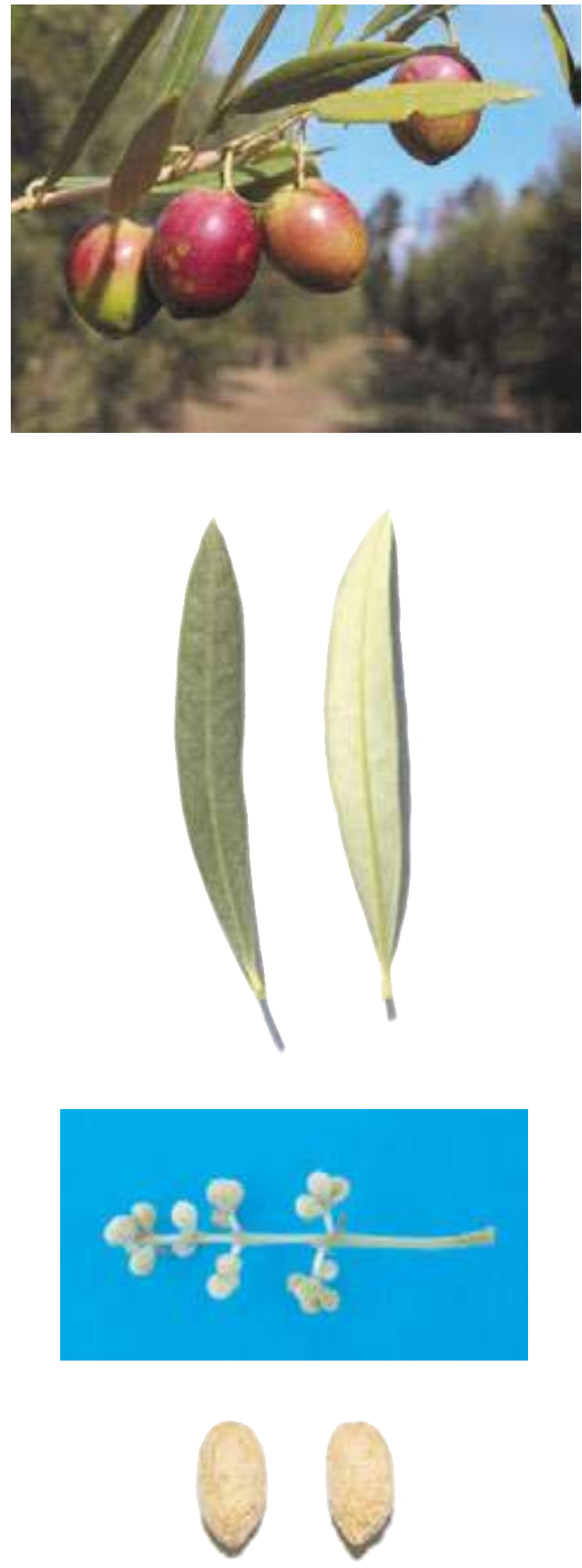


\section{Biochemical Characters}

\section{Fatty Acid Composition}

Table 1. Average values (express in $\% \pm$ standard deviations) of the fatty acids methyl esters and nutritional ratios obtained from single cultivar olive oils.

$\begin{array}{llllll}\text { Myristic acid } & \mathbf{0 , 0 1} \pm \mathbf{0 , 0 1} & \text { Linoleic acid }(\omega 6) & \mathbf{1 0 , 6 9} \pm \mathbf{1 , 8 5} & \text { Lignoceric acid } & \mathbf{0 , 0 4} \pm \mathbf{0 , 0 0} \\ \text { Palmitic acid } & \mathbf{1 2 , 1 5} \pm \mathbf{1 , 3 4} & \text { Linolenic acid }(\omega 3) & \mathbf{0 , 8 1} \pm \mathbf{0 , 2 3} & & \\ \text { Palmitoleic acid } & \mathbf{1 , 0 3} \pm \mathbf{0 , 3 6} & \text { Arachic acid } & \mathbf{0 , 2 7} \pm \mathbf{0 , 0 8} & \text { Unsat./satured } & \mathbf{5 , 8 7 \pm 0 , 4 5} \\ \text { Stearic acid } & \mathbf{2 , 0 6} \pm \mathbf{0 , 4 5} & \text { Eicosenoic acid } & \mathbf{0 , 0 3} \pm \mathbf{0 , 0 1} & \omega 6 / \omega 3 & \mathbf{1 3 , 5 2} \pm \mathbf{1 , 6 4} \\ \text { Oleic acid } & \mathbf{7 1 , 9 3 \pm 0 , 0 5} & \text { Behenic acid } & \mathbf{0 , 0 8} \pm \mathbf{0 , 0 2} & & \end{array}$

\section{Organoleptic oil values}

Sensory Analysis (Panel test)

Comment: fruity medium, with read sensations of almond, grass and artichoke. Balanced taste sensation with a medium-high bitter and spicy. Medium-high fluidity.

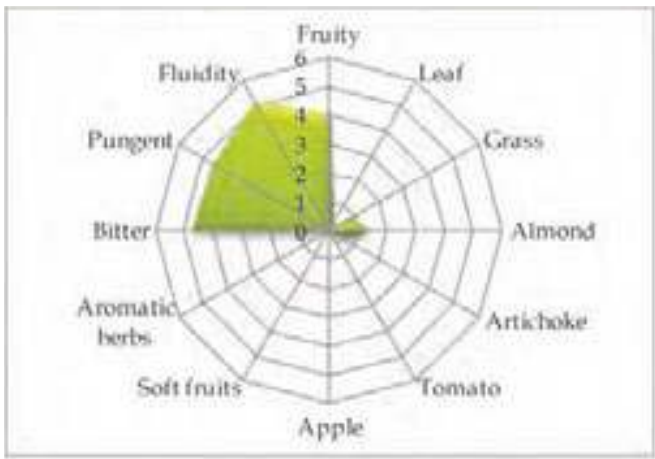

\section{Molecular Markers}

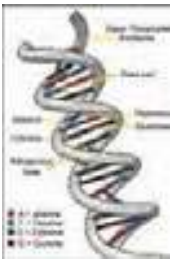

Table 2. Microsatellites (SSR) loci analyzed.

For each locus the allele size (expressed in base pairs) is reported.

$\begin{array}{cccccc}D C A 09 & \text { DCA18 } & \text { GAPU59 } & \text { GAPU71A } & \text { GUPA71B } & \text { GAPU103A } \\ \mathbf{1 6 2} \mathbf{- 1 7 2} & \mathbf{1 8 5} \mathbf{- 1 8 5} & \mathbf{2 1 4 - \mathbf { 2 1 4 }} & \mathbf{2 1 4} \mathbf{- 2 1 8} & \mathbf{1 3 0 - \mathbf { 1 4 4 }} & \mathbf{1 3 6} \mathbf{- 1 8 4} \\ \text { UDOO1 } & \text { UDOO3 } & \text { UDO12 } & \text { UDO28 } & \text { UDO39 } & \\ \mathbf{1 4 4 - \mathbf { 1 5 0 }} & \mathbf{1 3 5 - \mathbf { 1 3 5 }} & \mathbf{1 6 4 - \mathbf { 1 9 3 }} & \mathbf{1 5 0 - \mathbf { 1 6 1 }} & \mathbf{2 0 0 - \mathbf { 2 0 0 }} & \end{array}$

\section{References:}

1 - Various authors. In: Catalogo Nazionale delle Varietà di Olivo. University of Bari (in press), ISBN 978-88-8879397-9.

2 - Muzzalupo I., Stefanizzi F., Perri E. HortScience (2009), 44: pp. 582-588. 


\section{"Sessana "}

(synonymy: Cicinella, Minucciolo, etc.)

Areal distribution or origin area: Campania

Flesh/pit weight ratio: medium $(6,18 \pm 0,25)$

Oil content (\%): medium $(46,05 \pm 0,75)$

Purpose: oil

\section{Morphological characters}

Tree characters

Vigour: medium-strong

Growth habit: spreading

Canopy-density: medium sparse

\section{Leaf characters}

Blade length $(\mathrm{cm})$ : medium $(5,92 \pm 0,45)$

Blade width $(\mathrm{cm})$ : medium $(1,46 \pm 0,18)$

Shape (length/width): elliptic-lanceolate

\section{Inflorescence characters}

Inflorescence length (cm): medium $(2,65 \pm 1,06)$

Number of flowers: low $(17,71 \pm 1,66)$

\section{Fruit characters}

Fresh weight of 100 fruits $(\mathrm{g})$ : low $(\mathbf{1}, \mathbf{9 9} \pm \mathbf{0 , 1 8})$

Shape (length/width): spherical

Symmetry: symmetric

Position of maximum transverse diameter:

central

Apex: rounded

Base: truncate

Niplle: absent

Lenticels: few and small

\section{Pit characters}

Weight of 100 pits (g): low $(\mathbf{0 , 2 8} \pm \mathbf{0 , 0 3})$

Shape (length/width): ovoid

Mucron: absent

Symmetry: symmetric

Position of maximum transverse diameter:

central

Apex: rounded

Base: rounded

Surface: rugose

Number of grooves: medium
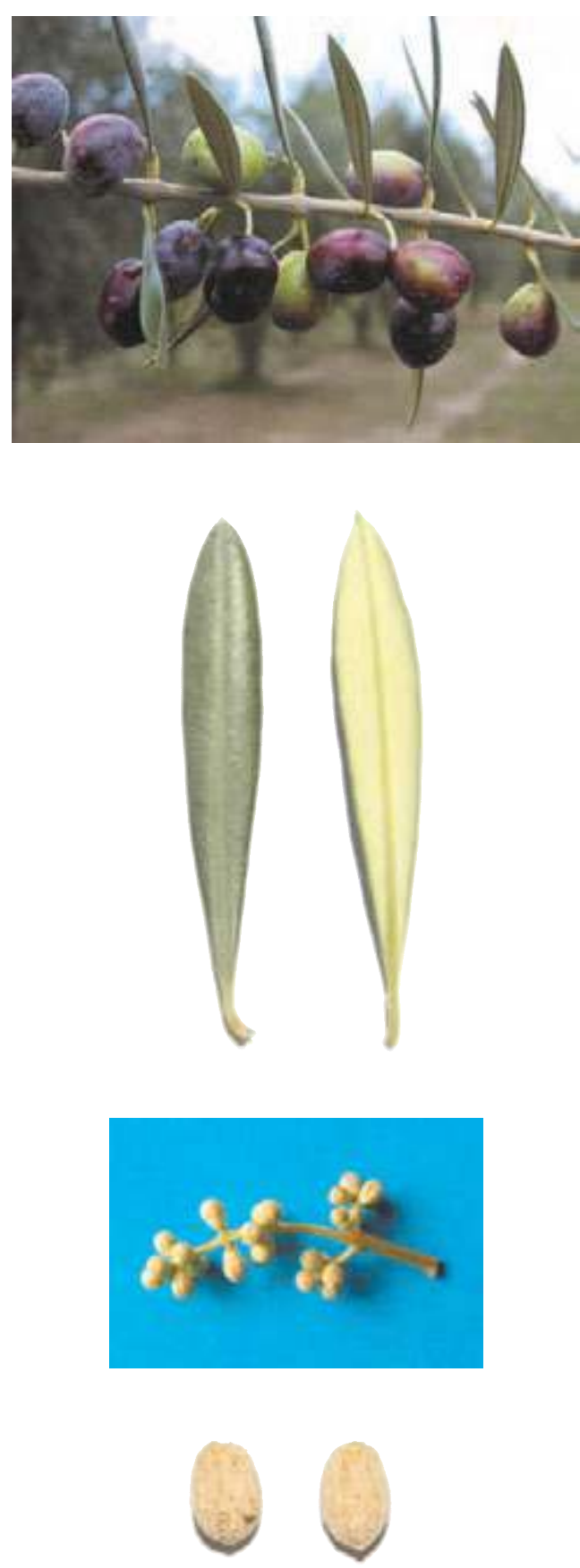


\section{Biochemical Characters}

\section{Fatty Acid Composition}

Table 1. Average values (express in $\% \pm$ standard deviations) of the fatty acids methyl esters and nutritional ratios obtained from single cultivar olive oils.

$\begin{array}{llllll}\text { Myristic acid } & \mathbf{0 , 0 1} \pm \mathbf{0 , 0 1} & \text { Linoleic acid }(\omega 6) & \mathbf{6 , 5 8} \pm \mathbf{0 , 9 9} & \text { Lignoceric acid } & \mathbf{0 , 0 9} \pm \mathbf{0 , 0 8} \\ \text { Palmitic acid } & \mathbf{1 1 , 4 6 \pm 0 , 4 5} & \text { Linolenic acid }(\omega 3) & \mathbf{0 , 9 7} \pm \mathbf{0 , 0 2} & & \\ \text { Palmitoleic acid } & \mathbf{1 , 1 8} \pm \mathbf{0 , 2 6} & \text { Arachic acid } & \mathbf{0 , 2 9} \pm \mathbf{0 , 0 5} & \text { Unsat./satured } & \mathbf{6 , 3 2} \pm \mathbf{0 , 1 6} \\ \text { Stearic acid } & \mathbf{1 , 7 5} \pm \mathbf{0 , 2 4} & \text { Eicosenoic acid } & \mathbf{0 , 2 4} \pm \mathbf{0 , 1 9} & \omega 6 / \omega 3 & \mathbf{6 , 7 8} \pm \mathbf{0 , 8 7} \\ \text { Oleic acid } & \mathbf{7 6 , 2 9} \pm \mathbf{0 , 6 3} & \text { Behenic acid } & \mathbf{0 , 0 9} \pm \mathbf{0 , 0 5} & & \end{array}$

\section{Organoleptic oil values}

Sensory Analysis (Panel test)

Comment: fruity medium-high, with hints of almond and read sensations of grass, tomato and artichoke. Balanced taste sensation with a medium-high bitter and spicy. Mediumhigh fluidity.

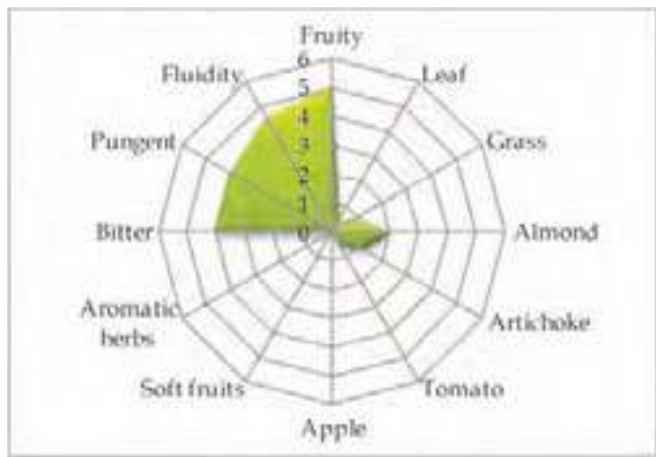

\section{Molecular Markers}

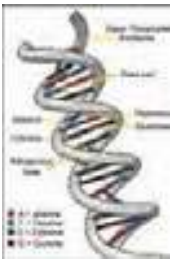

Table 2. Microsatellites (SSR) loci analyzed.

For each locus the allele size (expressed in base pairs) is reported.

$\begin{array}{cccccc}D C A 09 & D C A 18 & \text { GAPU59 } & \text { GAPU71A } & \text { GUPA71B } & \text { GAPU103A } \\ \mathbf{1 8 2} \mathbf{- 2 0 6} & \mathbf{1 8 1} \mathbf{- 1 8 5} & \mathbf{2 1 4} \mathbf{- 2 1 4} & \mathbf{2 1 4} \mathbf{- 2 2 4} & \mathbf{1 2 6 - \mathbf { 1 3 0 }} & \mathbf{1 7 0 - \mathbf { 1 8 4 }} \\ \text { UDOO1 } & \text { UDOO3 } & \text { UDO12 } & \text { UDO28 } & \text { UDO39 } & \\ \mathbf{1 4 4 - \mathbf { 1 4 4 }} & \mathbf{1 4 3 - \mathbf { 1 4 3 }} & \mathbf{1 6 6 - \mathbf { 1 7 7 }} & \mathbf{1 8 2} \mathbf{- 2 0 5} & \mathbf{2 1 3 - 2 1 3} & \end{array}$

\section{References:}

1 - Pugliano G., Flaminio G., Pugliano M.L., et al. In: La risorsa genetica dell'olivo in Campania, SE. S.I.R.C.A. Ed. Napoli, (2000).

2 - Muzzalupo I., Stefanizzi F., Perri E. HortScience (2009), 44: pp. 582-588. 


\section{"Simona"}

(synonymy: Simone)

Areal distribution or origin area: Puglia

Flesh/pit weight ratio: low $(3,96 \pm 0,18)$

Oil content (\%): medium $(40,63 \pm 3,23)$

Purpose: oil

\section{Morphological characters}

Tree characters

Vigour: strong

Growth habit: spreading-erect

Canopy-density: medium

\section{Leaf characters}

Blade length (cm): medium $(5,94 \pm 0,52)$

Blade width $(\mathrm{cm})$ : medium $(1,35 \pm 0,19)$

Shape (length/width): elliptic - lanceolate

\section{Inflorescence characters}

Inflorescence length $(\mathrm{cm})$ : long $(3,91 \pm 0,87)$

Number of flowers: high $(20,16 \pm 2,42)$

\section{Fruit characters}

Fresh weight of 100 fruits $(\mathrm{g})$ : low $(\mathbf{1}, \mathbf{6 9} \pm \mathbf{0 , 2 8})$

Shape (length/width): elongated

Symmetry: asymmetric

Position of maximum transverse diameter:

central

Apex: pointed

Base: rounded

Niplle: absent

Lenticels: may and small

\section{Pit characters}

Weight of 100 pits (g): medium $(0,35 \pm 0,05)$

Shape (length/width): elongated

Mucron: obvious

Symmetry: asymmetric

Position of maximum transverse diameter:

central

Apex: pointed

Base: pointed

Surface: smooth

Number of grooves: medium
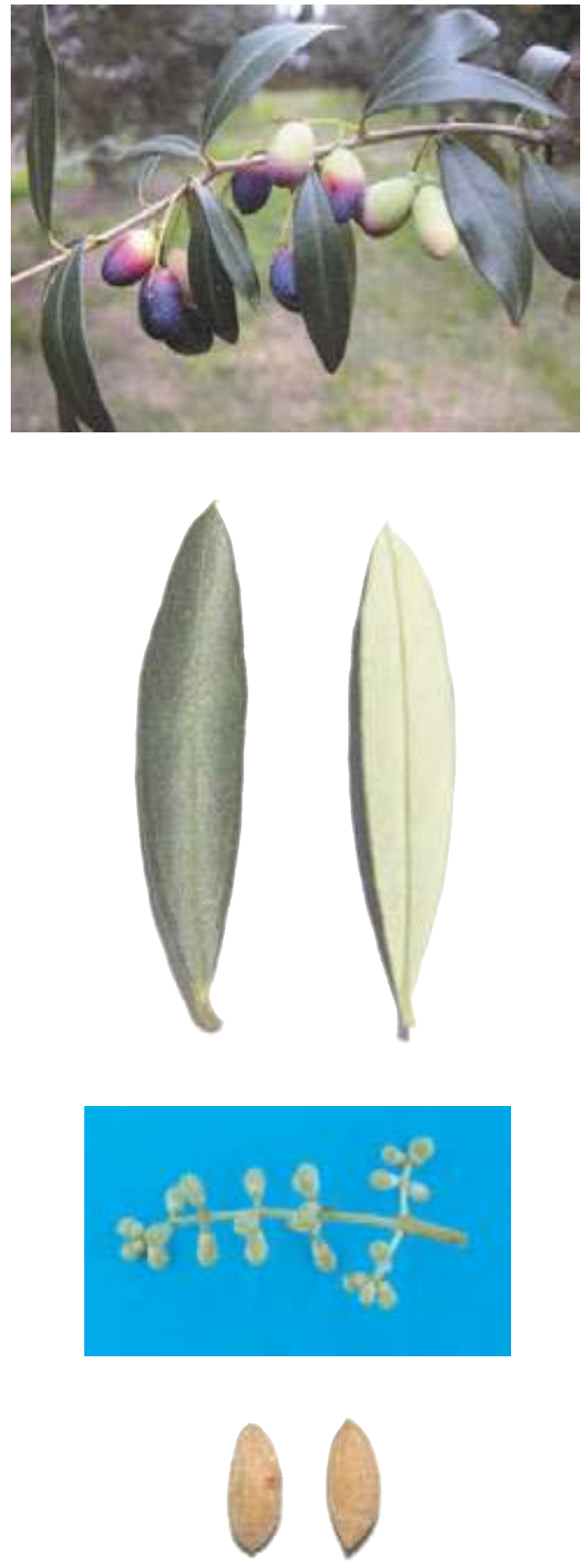


\section{Biochemical Characters}

\section{Fatty Acid Composition}

Table 1. Average values (express in $\% \pm$ standard deviations) of the fatty acids methyl esters and nutritional ratios obtained from single cultivar olive oils.

$\begin{array}{llllll}\text { Myristic acid } & \mathbf{0 , 0 1} \pm \mathbf{0 , 0 1} & \text { Linoleic acid }(\omega 6) & \mathbf{3 , 3 1} \pm \mathbf{0 , 5 4} & \text { Lignoceric acid } & \mathbf{0 , 1 0 \pm 0 , 0 5} \\ \text { Palmitic acid } & \mathbf{1 0 , 3 0 \pm 0 , 9 8} & \text { Linolenic acid }(\omega 3) & \mathbf{0 , 7 1} \pm \mathbf{0 , 0 6} & & \\ \text { Palmitoleic acid } & \mathbf{0 , 8 9} \pm \mathbf{0 , 2 0} & \text { Arachic acid } & \mathbf{0 , 4 2} \pm \mathbf{0 , 0 6} & \text { Unsat./satured } & \mathbf{6 , 3 6} \pm \mathbf{0 , 5 5} \\ \text { Stearic acid } & \mathbf{2 , 8 5} \pm \mathbf{0 , 1 5} & \text { Eicosenoic acid } & \mathbf{0 , 2 2} \pm \mathbf{0 , 1 4} & \omega 6 / \omega 3 & \mathbf{4 , 6 5} \pm \mathbf{0 , 6 9} \\ \text { Oleic acid } & \mathbf{8 0 , 4 1 \pm \mathbf { 1 , 7 9 }} & \text { Behenic acid } & \mathbf{0 , 1 3} \pm \mathbf{0 , 0 4} & & \end{array}$

\section{Organoleptic oil values}

Sensory Analysis (Panel test)

Comment: fruity medium-high, with taste of almond, artichoke and tomato. Balanced taste sensation with medium bitter and spicy. Medium fluidity.

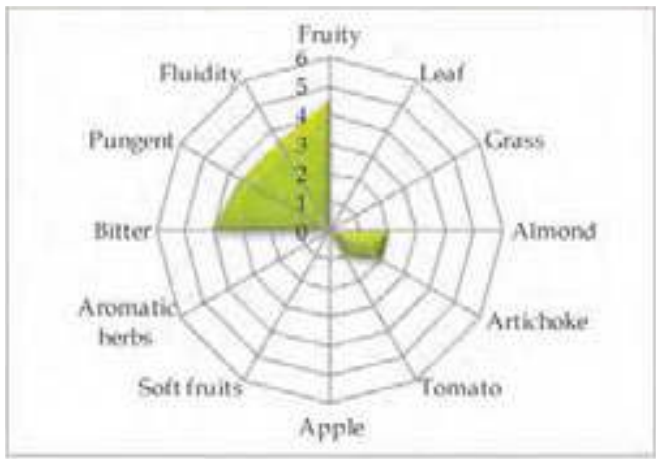

\section{Molecular Markers}

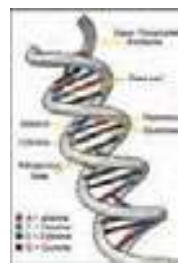

Table 2. Microsatellites (SSR) loci analyzed.

For each locus the allele size (expressed in base pairs) is reported.

\begin{tabular}{|c|c|c|c|c|c|}
\hline$D C A 09$ & $D C A 18$ & GAPU59 & GAPU71A & GUPA71B & GAPU103A \\
\hline $182-182$ & $175-181$ & $212-222$ & $210-210$ & $124-126$ & $159-159$ \\
\hline UDO01 & UDO03 & UDO12 & UDO28 & UDO39 & \\
\hline $144-144$ & $166-166$ & $166-177$ & $154-154$ & $108-170$ & \\
\hline
\end{tabular}

\section{References:}

1 - Lombardo N., Perri E., Muzzalupo I., et al. In: Contributo alla caratterizzazione del germoplasma olivicolo pugliese, Istituto Sperimentale per l'Olivicoltura (2004), pp. 89-82.

2 - Muzzalupo I., Stefanizzi F., Perri E. HortScience (2009), 44: pp. 582-588. 


\section{“Sinopolese"}

(synonymy: Calabrese, Chianota, Coccitano, Pizzocorno, Seminarota, etc.)

Areal distribution or origin area: Calabria

Flesh/pit weight ratio: high $(3,58 \pm 0,32)$

Oil content (\%): medium $(42,82 \pm 4,12)$

Purpose: oil

\section{Morphological characters}

Tree characters

Vigour: medium-strong

Growth habit: erect

Canopy-density: dense

\section{Leaf characters}

Blade length $(\mathrm{cm})$ : medium $(5,81 \pm 0,82)$

Blade width (cm): medium $(1,50 \pm 0,27)$

Shape (length/width): elliptic

\section{Inflorescence characters}

Inflorescence length $(\mathrm{cm})$ : medium $(3,07 \pm 1,74)$

Number of flowers: xmedium $(\mathbf{2 2 , 1 7} \pm \mathbf{1}, \mathbf{8 2})$

\section{Fruit characters}

Fresh weight of 100 fruits (g): low $(\mathbf{1}, \mathbf{4 6} \pm \mathbf{0 , 1 1})$

Shape (length/width): elongated

Symmetry: slightly asymmetric

Position of maximum transverse diameter:

central

Apex: pointed

Base: rounded

Niplle: tenuous

Lenticels: many and small

\section{Pit characters}

Weight of 100 pits (g): medium $(0,34 \pm 0,07)$

Shape (length/width): elliptic

Mucron: obvious

Symmetry: slightly asymmetric

Position of maximum transverse diameter:

towards apex

Apex: pointed

Base: pointed

Surface: smooth

Number of grooves: medium
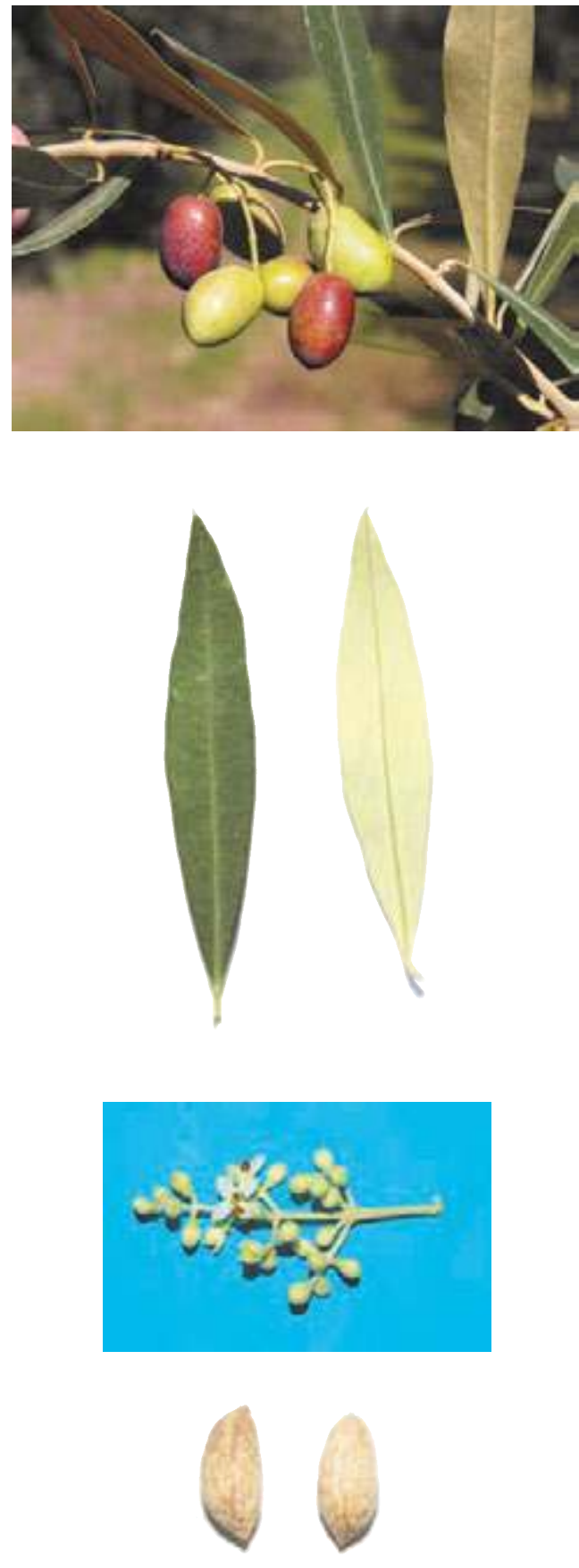


\section{Biochemical Characters}

\section{Fatty Acid Composition}

Table 1. Average values (express in $\% \pm$ standard deviations) of the fatty acids methyl esters and nutritional ratios obtained from single cultivar olive oils.

$\begin{array}{llllll}\text { Myristic acid } & \mathbf{0 , 0 1} \pm \mathbf{0 , 0 0} & \text { Linoleic acid }(\omega 6) & \mathbf{6 , 4 6} \pm \mathbf{2 , 2 0} & \text { Lignoceric acid } & \mathbf{0 , 0 4} \pm \mathbf{0 , 0 2} \\ \text { Palmitic acid } & \mathbf{1 2 , 2 5} \pm \mathbf{0 , 8 3} & \text { Linolenic acid }(\omega 3) & \mathbf{0 , 7 0} \pm \mathbf{0 , 1 5} & & \\ \text { Palmitoleic acid } & \mathbf{0 , 9 6} \pm \mathbf{0 , 3 0} & \text { Arachic acid } & \mathbf{0 , 3 6} \pm \mathbf{0 , 1 6} & \text { Unsat./satured } & \mathbf{5 , 6 6} \pm \mathbf{0 , 8 6} \\ \text { Stearic acid } & \mathbf{2 , 4 9} \pm \mathbf{0 , 8 8} & \text { Eicosenoic acid } & \mathbf{0 , 1 0} \pm \mathbf{0 , 1 6} & \omega 6 / \omega 3 & \mathbf{9 , 4 2} \pm \mathbf{2 , 9 7} \\ \text { Oleic acid } & \mathbf{7 5 , 5 2 \pm \mathbf { 1 , 2 8 }} & \text { Behenic acid } & \mathbf{0 , 1 1} \pm \mathbf{0 , 0 7} & & \end{array}$

\section{Organoleptic oil values}

Sensory Analysis (Panel test)

Comment: fruity medium, with hints of almond, artichoke and tomato. Balanced taste sensation with a medium-high bitter and medium-light spicy. Medium fluidity.

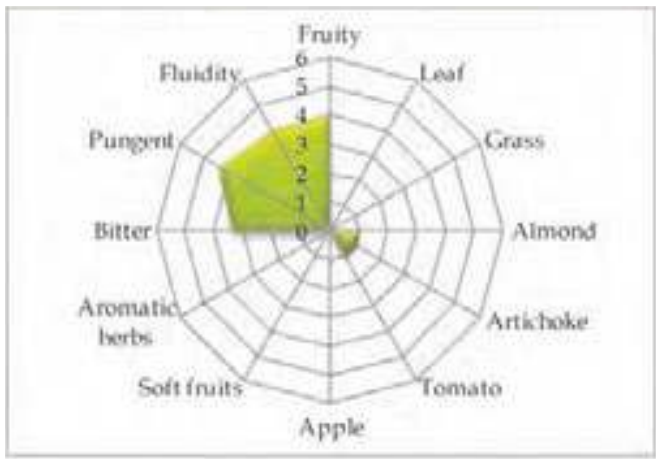

\section{Molecular Markers}

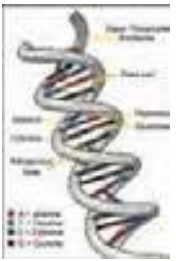

Table 2. Microsatellites (SSR) loci analyzed.

For each locus the allele size (expressed in base pairs) is reported.

\begin{tabular}{|c|c|c|c|c|c|}
\hline DCA09 & $D C A 18$ & GAPU59 & GAPU71A & $G U P A 71 B$ & GAPU103A \\
\hline $162-198$ & $177-179$ & $212-214$ & $212-212$ & $124-130$ & $170-184$ \\
\hline UDO01 & UDO03 & UDO12 & UDO28 & UDO39 & \\
\hline $144-144$ & $143-143$ & $177-193$ & $154-210$ & $164-205$ & \\
\hline
\end{tabular}

\section{References:}

1 - Lombardo N., Perri E., Muzzalupo I., et al. In: Il germoplasma olivicolo calabrese, Ist. Sper. Olivic.(2003), pp: 13.

2 - Perri E., Mazzotti F., Muzzalupo I., et al. In: Relazione attività, CO.R.ASS.OL. (2003).

3 - Muzzalupo I., Chiappetta A., Benincasa C., et al Sci Hortic-Amsterdam, (2010), 126: pp. 324-329. 


\section{"Sirole"}

(synonymy: Sirole, Sirole Soratte, Sirole Soratte 1, etc.)

Areal distribution or origin area: Lazio

Flesh/pit weight ratio: medium $(\mathbf{7 , 2 7} \pm \mathbf{0 , 4 1})$

Oil content (\%): medium $(44,80 \pm 0,19)$

Purpose: oil

\section{Morphological characters}

Tree characters

Vigour: medium-strong

Growth habit: spreading

Canopy-density: medium-dense

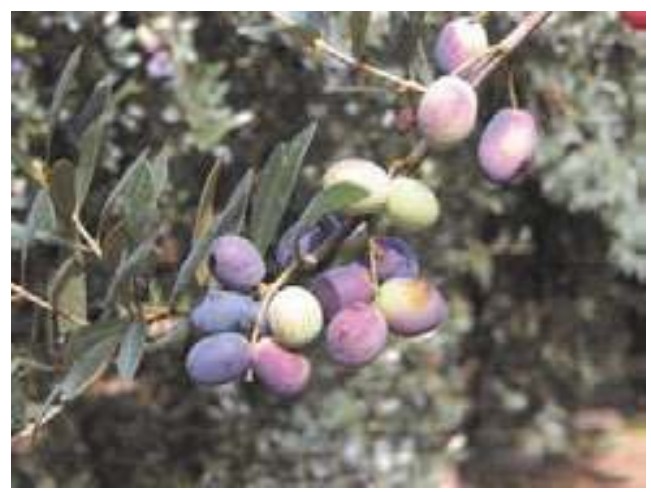

\section{Leaf characters}

Blade length $(\mathrm{cm})$ : short $(4,57 \pm 0,39)$

Blade width (cm): medium $(\mathbf{1 , 1 2} \pm \mathbf{0 , 1 8})$

Shape (length/width): elliptic-lanceolate

\section{Inflorescence characters}

Inflorescence length (cm): short $(2,45 \pm 0,10)$

Number of flowers: low $(\mathbf{1 4 , 7 3} \pm \mathbf{1 , 8 0})$

\section{Fruit characters}

Fresh weight of 100 fruits $(\mathrm{g})$ : medium $(2,34 \pm 0,37)$

Shape (length/width): elongated

Symmetry: slightly asymmetric

Position of maximum transverse diameter:

central

Apex: rounded

Base: truncate

Niplle: absent

Lenticels: few and large

\section{Pit characters}

Weight of 100 pits (g): medium $(0,33 \pm 0,09)$

Shape (length/width): elongated

Mucron: absent

Symmetry: slightly asymmetric

Position of maximum transverse diameter:

towards apex

Apex: pointed

Base: pointed

Surface: smooth

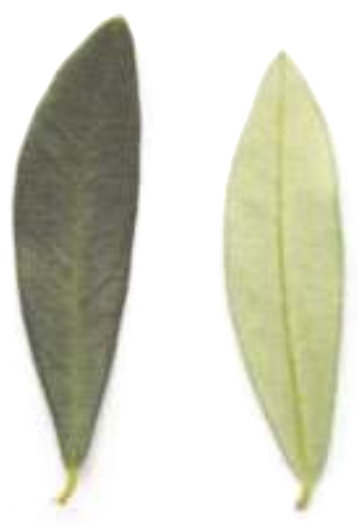

Number of grooves: medium 


\section{Biochemical Characters}

\section{Fatty Acid Composition}

Table 1. Average values (express in $\% \pm$ standard deviations) of the fatty acids methyl esters and nutritional ratios obtained from single cultivar olive oils.

$\begin{array}{llllll}\text { Myristic acid } & \mathbf{0 , 0 1} \pm \mathbf{0 , 0 1} & \text { Linoleic acid }(\omega 6) & \mathbf{1 0 , 9 3} \pm \mathbf{0 , 4 2} & \text { Lignoceric acid } & \mathbf{0 , 0 6} \pm \mathbf{0 , 0 0} \\ \text { Palmitic acid } & \mathbf{1 4 , 0 6} \pm \mathbf{1 , 2 8} & \text { Linolenic acid }(\omega 3) \mathbf{0 , 9 2} \pm \mathbf{0 , 0 8} & & \\ \text { Palmitoleic acid } & \mathbf{1 , 3 0} \pm \mathbf{0 , 0 8} & \text { Arachic acid } & \mathbf{0 , 2 9} \pm \mathbf{0 , 1 2} & \text { Unsat./satured } & \mathbf{4 , 7 3} \pm \mathbf{0 , 5 6} \\ \text { Stearic acid } & \mathbf{2 , 9 2} \pm \mathbf{0 , 4 7} & \text { Eicosenoic acid } & \mathbf{0 , 3 4} \pm \mathbf{0 , 0 7} & \omega 6 / \omega 3 & \mathbf{1 2 , 0 0} \pm \mathbf{1 , 4 8} \\ \text { Oleic acid } & \mathbf{6 7 , 9 3 \pm \mathbf { 1 , 1 1 }} & \text { Behenic acid } & \mathbf{0 , 1 3} \pm \mathbf{0 , 0 2} & & \end{array}$

\section{Organoleptic oil values}

Sensory Analysis (Panel test)

Comment: fruity medium-high, with hints of almond and read sensations of grass, leaves, and artichoke. Balanced taste sensation with a medium-high bitter and spicy. Mediumhigh fluidity.

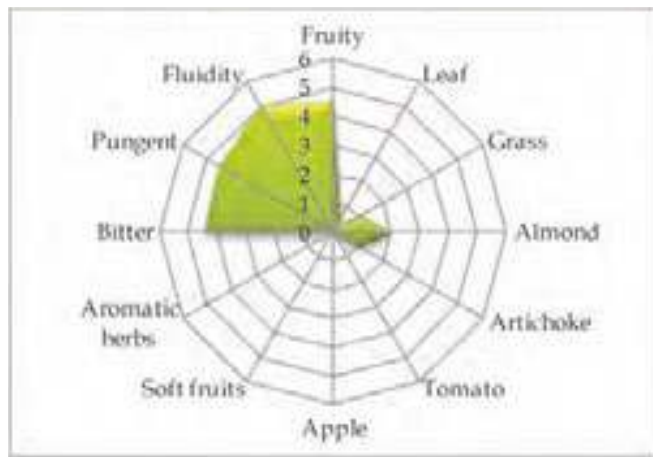

\section{Molecular Markers}

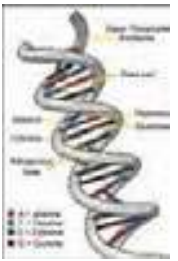

Table 2. Microsatellites (SSR) loci analyzed.

For each locus the allele size (expressed in base pairs) is reported.

$\begin{array}{cccccc}D C A 09 & \text { DCA } 18 & \text { GAPU59 } & \text { GAPU71A } & \text { GUPA71B } & \text { GAPU103A } \\ \mathbf{1 7 6 - \mathbf { 2 0 6 }} & \mathbf{1 8 1} \mathbf{- 1 8 5} & \mathbf{2 1 4} \mathbf{- 2 2 2} & \mathbf{2 1 8 - \mathbf { 2 2 4 }} & \mathbf{1 3 0 - \mathbf { 1 4 4 }} & \mathbf{1 5 0} \mathbf{- 1 5 7} \\ \text { UDO01 } & \text { UDO03 } & \text { UDO12 } & \text { UDO28 } & \text { UDO39 } & \\ \mathbf{1 4 0} \mathbf{- 1 4 0} & \mathbf{1 3 5} \mathbf{- 1 3 5} & \mathbf{1 7 7} \mathbf{- 1 8 2} & \mathbf{1 8 2} \mathbf{- 2 1 0} & \mathbf{1 8 5} \mathbf{- 2 2 0} & \end{array}$

\section{References:}

1 - Parlati M. V., Pandolfi S. In: Olivo e Olio, (2001), 4: pp. 67-72.

2 - Various authors. In: Catalogo Nazionale delle Varietà di Olivo . University of Bari (in press), ISBN 978-88-8879397-9. 


\section{"Sperone di gallo"}

(synonymy: Oliva torta, Olivona, Tortarella, Zampa di gallo, etc.)

Areal distribution or origin area: Molise

Flesh/ pit weight ratio: high $(\mathbf{9 , 8 2} \pm \mathbf{1 , 0 1})$

Oil content (\%): medium $(48,86 \pm 0,46)$

Purpose: dual purpose

\section{Morphological characters}

Tree characters

Vigour: medium

Growth habit: erect

Canopy-density: medium-sparse

\section{Leaf characters}

Blade length $(\mathrm{cm})$ : long $(7,48 \pm 0,98)$

Blade width $(\mathrm{cm})$ : broad $(2,06 \pm 0,35)$

Shape (length/width): elliptic

\section{Inflorescence characters}

Inflorescence length $(\mathrm{cm})$ : medium $(2,58 \pm 0,92)$

Number of flowers: medium $(19,85 \pm 2,32)$

\section{Fruit characters}

Fresh weight of 100 fruits (g): high $(\mathbf{4}, 21 \pm 007)$

Shape (length/width): ovoid

Symmetry: slightly asymmetric

Position of maximum transverse diameter:

central

Apex: rounded

Base: rounded

Niplle: absent

Lenticels: few and large

\section{Pit characters}

Weight of 100 pits $(\mathrm{g})$ : medium $(0,39 \pm 0,03)$

Shape (length/width): elliptic

Mucron: obvious

Symmetry: slightly asymmetric

Position of maximum transverse diameter:

\section{towards apex}

Apex: pointed

Base: pointed

Surface: smooth

Number of grooves: medium
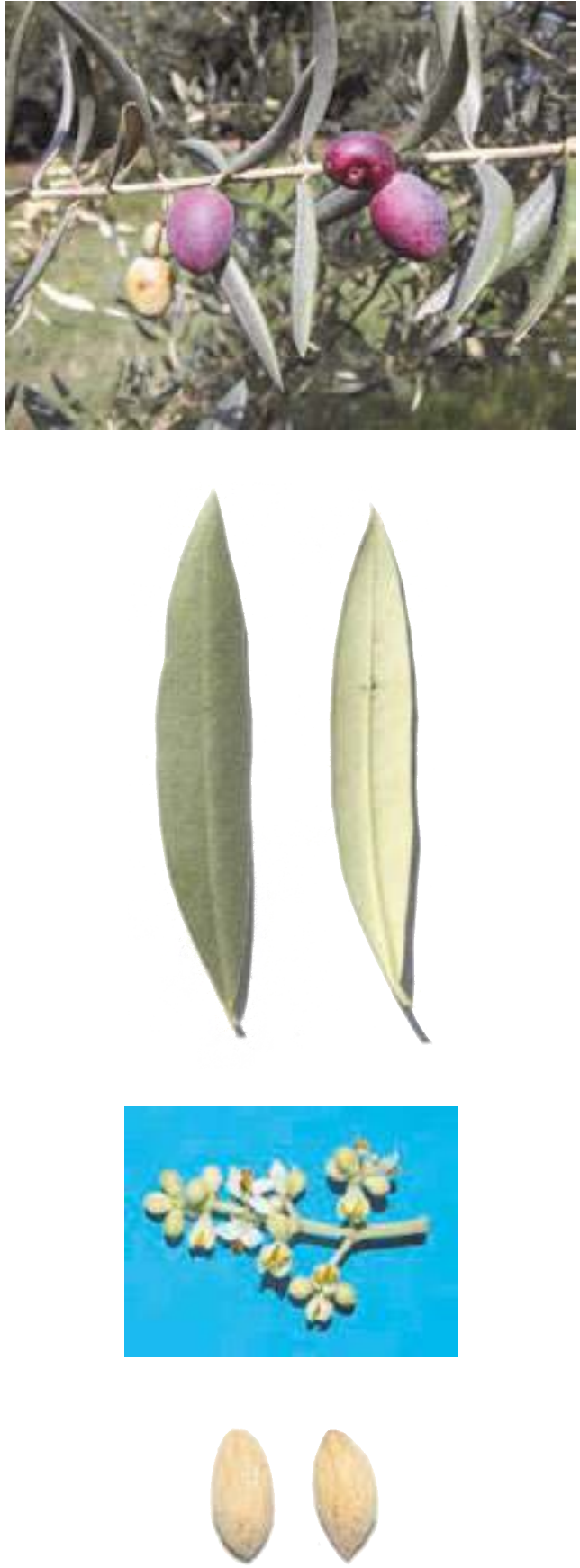


\section{Biochemical Characters}

\section{Fatty Acid Composition}

Table 1. Average values (express in $\% \pm$ standard deviations) of the fatty acids methyl esters and nutritional ratios obtained from single cultivar olive oils.

$\begin{array}{llllll}\text { Myristic acid } & \mathbf{0 , 0 2} \pm \mathbf{0 , 0 1} & \text { Linoleic acid }(\omega 6) & \mathbf{1 5 , 6 4 \pm \mathbf { 1 , 0 4 }} & \text { Lignoceric acid } & \mathbf{0 , 0 5} \pm \mathbf{0 , 0 4} \\ \text { Palmitic acid } & \mathbf{1 6 , 1 9 \pm \mathbf { 1 } 5 0} & \text { Linolenic acid }(\omega 3) \mathbf{0 , 7 2} \pm \mathbf{0 , 0 2} & & \\ \text { Palmitoleic acid } & \mathbf{2 , 0 1} \pm \mathbf{0 , 1 9} & \text { Arachic acid } & \mathbf{0 , 4 3} \pm \mathbf{0 , 1 7} & \text { Unsat./ satured } & \mathbf{4 , 0 5} \pm \mathbf{0 , 3 8} \\ \text { Stearic acid } & \mathbf{3 , 0 0} \pm \mathbf{0 , 3 7} & \text { Eicosenoic acid } & \mathbf{0 , 0 2} \pm \mathbf{0 , 0 0} & \omega 6 / \omega 3 & \mathbf{2 1 , 6 5} \pm \mathbf{1 , 5 5} \\ \text { Oleic acid } & \mathbf{6 0 , 0 1 \pm 0 , 2 6} & \text { Behenic acid } & \mathbf{0 , 1 0} \pm \mathbf{0 , 0 5} & & \end{array}$

\section{Organoleptic oil values}

Sensory Analysis (Panel test)

Comment: fruity medium, with taste of aromatic herbs. Balanced taste sensation with medium bitter and spicy. Medium-high fluidity.

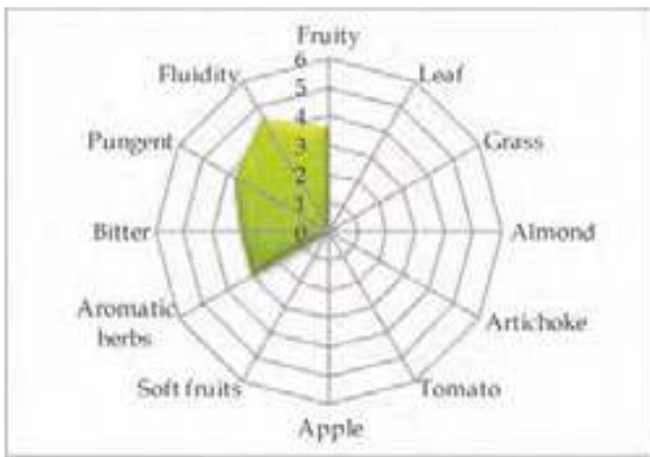

\section{Molecular Markers}

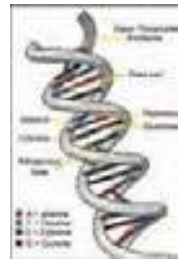

Table 2. Microsatellites (SSR) loci analyzed.

For each locus the allele size (expressed in base pairs) is reported.

$\begin{array}{cccccc}D C A 09 & \text { DCA18 } & \text { GAPU59 } & \text { GAPU71A } & \text { GUPA71B } & \text { GAPU103A } \\ \mathbf{1 6 2} \mathbf{- 1 7 2} & \mathbf{1 6 9 - \mathbf { 1 7 9 }} & \mathbf{2 1 2} \mathbf{- 2 1 2} & \mathbf{2 1 4} \mathbf{- 2 1 4} & \mathbf{1 3 0 - \mathbf { 1 3 0 }} & \mathbf{1 7 0 - \mathbf { 1 7 0 }} \\ \text { UDOO1 } & \text { UDOO3 } & \text { UDO12 } & \text { UDO28 } & \text { UDO39 } & \\ \mathbf{1 4 0 - \mathbf { 1 4 0 }} & \mathbf{1 4 3 - \mathbf { 1 4 3 }} & \mathbf{1 6 6 - \mathbf { 1 9 3 }} & \mathbf{1 8 2} \mathbf{- 1 8 2} & \mathbf{2 1 3 - \mathbf { 2 1 3 }} & \end{array}$

\section{References:}

1 - Various authors. In: Catalogo Nazionale delle Varietà di Olivo. University of Bari (in press), ISBN 978-88-8879397-9.

2 - Muzzalupo I., Salimonti A., Caravita M. A., et al. Adv. Hort. Sci. (2008), 22(2): pp. 129-135. 


\section{“Termite di Bitetto"}

(synonymy: Cima di Bitetto, Mele di Bitetto, Oliva mele, Termite, etc.)

Areal distribution or origin area: Puglia

Flesh/pit weight ratio: medium $(7,07 \pm 0,76)$

Oil content (\%): medium $(47,26 \pm 0,21)$

Purpose: dual purpose

\section{Morphological characters}

Tree characters

Vigour: medium

Growth habit: spreading

Canopy-density: medium-dense

\section{Leaf characters}

Blade length $(\mathrm{cm})$ : medium $(5,38 \pm 0,68)$

Blade width (cm): board $(\mathbf{1}, 58 \pm \mathbf{0 , 2 2})$

Shape (length/width): elliptic

\section{Inflorescence characters}

Inflorescence length $(\mathrm{cm})$ : medium $(2,80 \pm 0,53)$

Number of flowers: low $(\mathbf{1 4}, 46 \pm \mathbf{2 , 6 8})$

\section{Fruit characters}

Fresh weight of 100 fruits (g): high $(\mathbf{4 , 2 2} \pm \mathbf{0 , 6 8})$

Shape (length/width): spherical

Symmetry: slightly asymmetric

Position of maximum transverse diameter:

central

Apex: rounded

Base: truncate

Niplle: absent

Lenticels: many and small

\section{Pit characters}

Weight of 100 pits (g): high $(0,56 \pm \mathbf{0 , 0 7})$

Shape (length/width): ovoid

Mucron: obvious

Symmetry: symmetric

Position of maximum transverse diameter:

central

Apex: rounded

Base: rounded

Surface: rugose

Number of grooves: medium
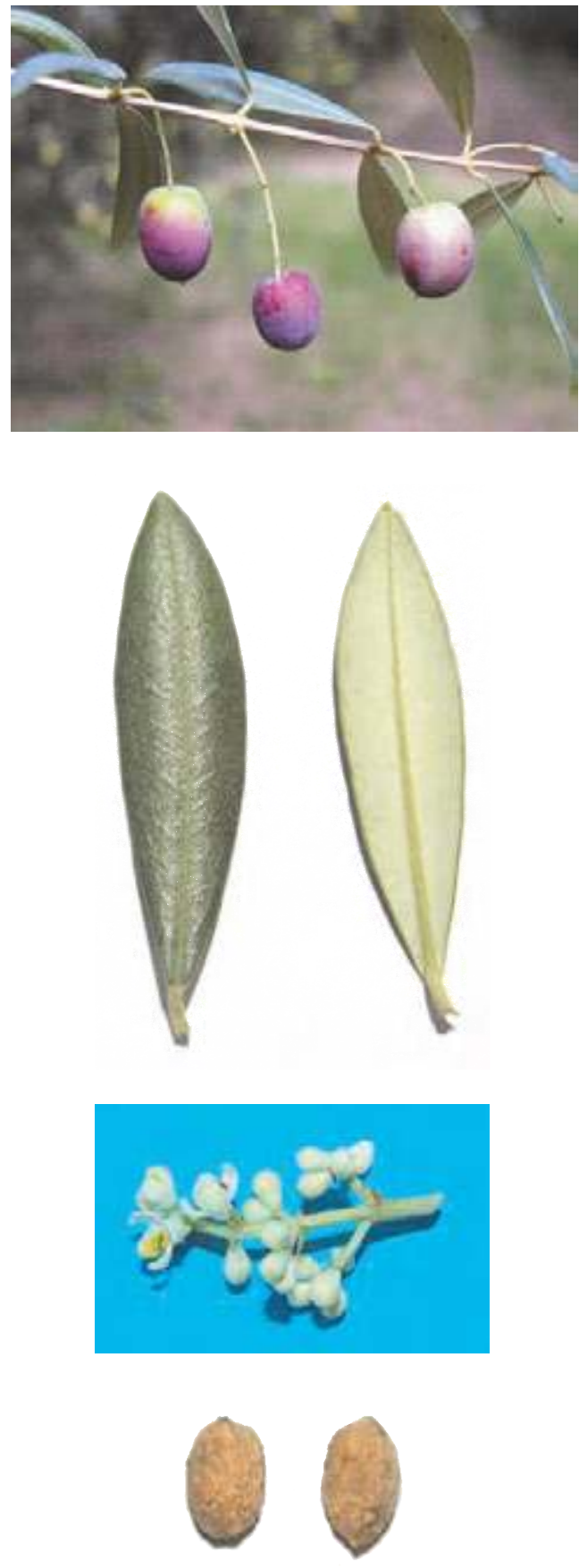


\section{Biochemical Characters}

\section{Fatty Acid Composition}

Table 1. Average values (express in $\% \pm$ standard deviations) of the fatty acids methyl esters and nutritional ratios obtained from single cultivar olive oils.

$\begin{array}{lllll}\text { Myristic acid } \quad 0,01 \pm 0,00 & \text { Linoleic acid }(\omega 6) & \mathbf{1 0}, 92 \pm 1,37 \quad \text { Lignoceric acid } \quad 0,02 \pm 0,01\end{array}$

Palmitic acid $16,04 \pm 2.74 \quad$ Linolenic acid $(\omega 3) 0,86 \pm 0,04$

Palmitoleic acid

$2,87 \pm 1,94$

Arachic acid

$0,17 \pm 0,03$

Unsat./satured

$4,62 \pm 0,78$

Stearic acid

$1,28 \pm 0,02$

Eicosenoic acid

$0,02 \pm 0,00$

$\omega 6 / \omega 3$

$12,69 \pm 0,99$

Oleic acid

$64,92 \pm 0,59$

Behenic acid

$0,06 \pm 0,01$

\section{Organoleptic oil values}

Sensory Analysis (Panel test)

Comment: fruity medium, with read sensations of artichoke and soft fruits. Balanced in flavours, with hints of bitter and spicy medium-light intensity. Medium fluidity.

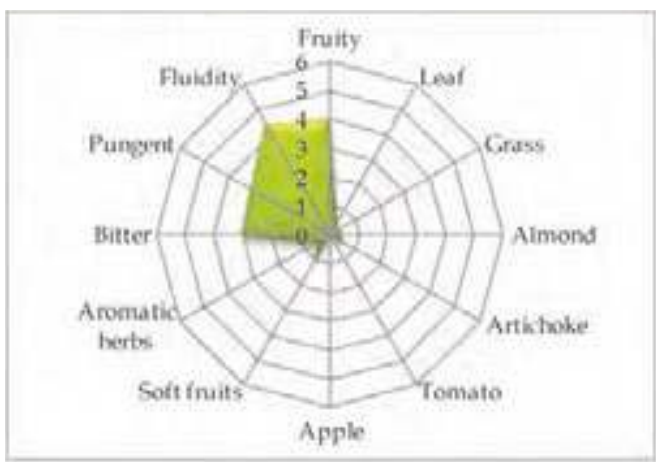

\section{Molecular Markers}

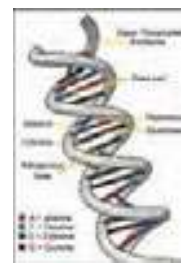

Table 2. Microsatellites (SSR) loci analyzed.

For each locus the allele size (expressed in base pairs) is reported.

$\begin{array}{cccccc}D C A 09 & \text { DCA } 18 & \text { GAPU59 } & \text { GAPU71A } & \text { GUPA71B } & \text { GAPU103A } \\ \mathbf{1 7 2} \mathbf{- 1 9 4} & \mathbf{1 7 9 - \mathbf { 1 7 9 }} & \mathbf{2 0 8 - \mathbf { 2 1 2 }} & \mathbf{2 1 4 - \mathbf { 2 1 4 }} & \mathbf{1 2 4 - \mathbf { 1 4 4 }} & \mathbf{1 5 0} \mathbf{- 1 5 9} \\ \text { UDO01 } & \text { UDO03 } & \text { UDO12 } & \text { UDO28 } & \text { UDO39 } & \\ \mathbf{1 4 4} \mathbf{- 1 4 4} & \mathbf{1 4 3} \mathbf{- 1 4 3} & \mathbf{1 7 7} \mathbf{- 1 8 2} & \mathbf{1 4 3} \mathbf{- 2 0 5} & \mathbf{1 0 8 - \mathbf { 1 7 0 }} & \end{array}$

\section{References:}

1 - Lombardo N., Perri E., Muzzalupo I., et al. In: Contributo alla caratterizzazione del germoplasma olivicolo pugliese, Ist. Sper. Olivic. (2004), pp. 93-96.

2 - Muzzalupo I., Stefanizzi F., Perri E. HortScience (2009), 44: pp. 582-588. 


\section{“Terza Grande”}

(synonymy: Di Bosa, Di Cuglieri, Olia Terza, Terza, etc.)

Areal distribution or origin area: Sardegna

Flesh/pit weight ratio: medium $(5,65 \pm 0,10)$

Oil content (\%): medium $(42,46 \pm 0,57)$

Purpose: dual purpose

\section{Morphological characters}

Tree characters

Vigour: medium-strong

Growth habit: spreading

Canopy-density: dense

\section{Leaf characters}

Blade length (cm): medium $(5,11 \pm 0,34)$

Blade width $(\mathrm{cm})$ : medium $(\mathbf{1}, 21 \pm \mathbf{0 , 1 1})$

Shape (length/width): elliptic-lanceolate

\section{Inflorescence characters}

Inflorescence length $(\mathrm{cm})$ : medium $(2,60 \pm 0,67)$

Number of flowers: low $(\mathbf{1 5 , 3 1} \pm \mathbf{1}, \mathbf{5 2})$

\section{Fruit characters}

Fresh weight of 100 fruits (g): high $(\mathbf{4 , 1 3} \pm \mathbf{1 , 0 3})$

Shape (length/width): spherical

Symmetry: slightly asymmetric

Position of maximum transverse diameter:

central

Apex: rounded

Base: truncate

Niplle: obvious

Lenticels: many and large

\section{Pit characters}

Weight of 100 pits (g): high $(\mathbf{0 , 6 0} \pm \mathbf{0 , 0 6})$

Shape (length/width): ovoid

Mucron: absent

Symmetry: slightly asymmetric

Position of maximum transverse diameter:

towards apex

Apex: rounded

Base: rounded

Surface: scabrous

Number of grooves: medium
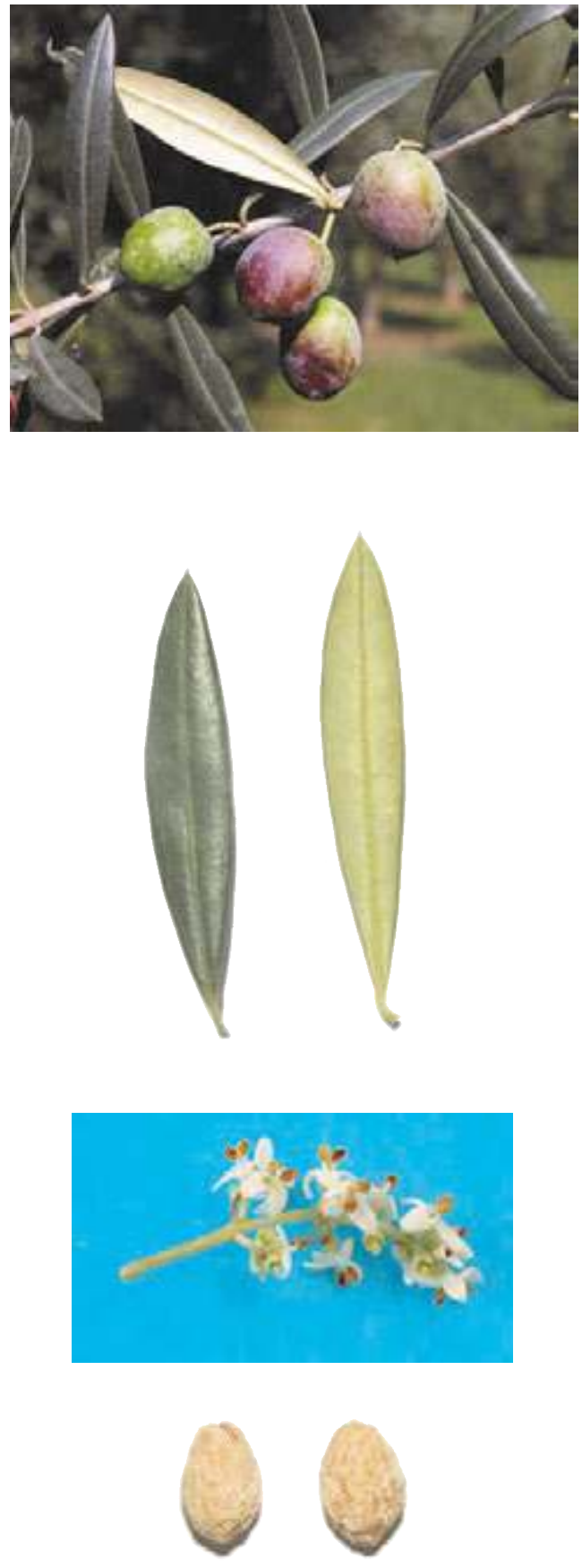


\section{Biochemical Characters}

\section{Fatty Acid Composition}

Table 1. Average values (express in $\% \pm$ standard deviations) of the fatty acids methyl esters and nutritional ratios obtained from single cultivar olive oils.

$\begin{array}{llllll}\text { Myristic acid } & \mathbf{0 , 0 1} \pm \mathbf{0 , 0 1} & \text { Linoleic acid }(\omega 6) & \mathbf{x 8 , 0 0 x} \pm \mathbf{1 , 5 7} & \text { Lignoceric acid } & \mathbf{0 , 0 6} \pm \mathbf{0 , 0 5} \\ \text { Palmitic acid } & \mathbf{1 2 , 1 3} \pm \mathbf{0 , 8 1} & \text { Linolenic acid }(\omega 3) & \mathbf{0 , 8 3} \pm \mathbf{0 , 0 8} & & \\ \text { Palmitoleic acid } & \mathbf{1 , 2 9} \pm \mathbf{0 , 1 7} & \text { Arachic acid } & \mathbf{0 , 3 1} \pm \mathbf{0 , 1 0} & \text { Unsat./satured } & \mathbf{6 , 1 2} \pm \mathbf{0 , 6 6} \\ \text { Stearic acid } & \mathbf{1 , 5 6} \pm \mathbf{0 , 3 5} & \text { Eicosenoic acid } & \mathbf{0 , 1 9} \pm \mathbf{0 , 2 4} & \omega 6 / \omega 3 & \mathbf{9 , 7 7} \pm \mathbf{2 , 8 7} \\ \text { Oleic acid } & \mathbf{7 4 , 4 2} \pm \mathbf{3 , 0 4} & \text { Behenic acid } & \mathbf{0 , 0 8} \pm \mathbf{0 , 0 3} & & \end{array}$

\section{Organoleptic oil values}

Sensory Analysis (Panel test)

Comment: fruity medium-high, with hints of almond and read sensations of grass, tomato, and artichoke. Balanced taste sensation with a medium-high bitter and medium spicy. Medium-high fluidity.

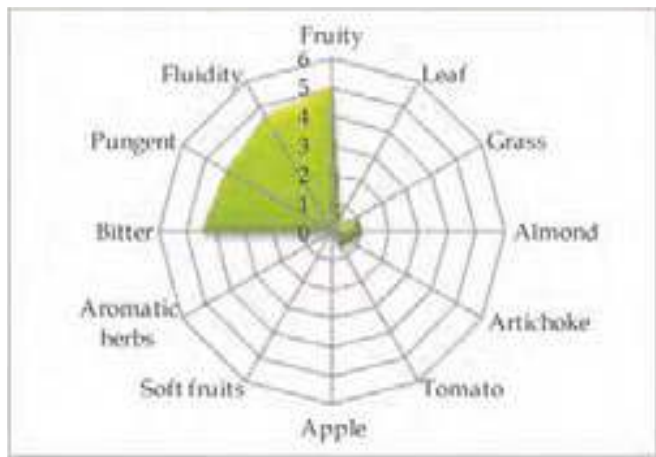

\section{Molecular Markers}

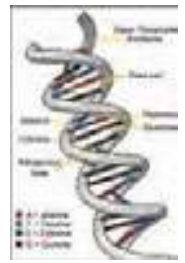

Table 2. Microsatellites (SSR) loci analyzed.

For each locus the allele size (expressed in base pairs) is reported.

\begin{tabular}{|c|c|c|c|c|c|}
\hline$D C A 09$ & $D C A 18$ & GAPU59 & GAPU71A & GUPA71B & GAPU103A \\
\hline $182-198$ & $179-181$ & $214-222$ & $214-221$ & $126-144$ & $157-159$ \\
\hline UDO01 & UDO03 & UDO12 & UDO28 & UDO39 & \\
\hline $140-150$ & $143-143$ & $164-177$ & $150-161$ & $213-213$ & \\
\hline
\end{tabular}

\section{References:}

1 - Various authors. In: Catalogo Nazionale delle Varietà di Olivo . University of Bari (in press), ISBN 978-88-8879397-9.

2 - Muzzalupo I., Stefanizzi F., Perri E. HortScience, (2009), 44: pp. 582-588. 


\section{“Tombarello"}

Areal distribution or origin area: Calabria

Flesh/pit weight ratio: medium $(5,14 \pm 1,44)$

Oil content (\%): medium $(44,95 \pm 4,80)$

Purpose: oil

\section{Morphological characters}

Tree characters

Vigour: medium-weak

Growth habit: spreading-erect

Canopy-density: medium-dense

\section{Leaf characters}

Blade length (cm): short $(4,36 \pm 0,30)$

Blade width (cm): medium $(\mathbf{1}, 27 \pm 0,16)$

Shape (length/width): elliptic

\section{Inflorescence characters}

Inflorescence length $(\mathrm{cm})$ : medium $(2,74 \pm 0,55)$

Number of flowers: medium $(19,34 \pm 1,60)$

\section{Fruit characters}

Fresh weight of 100 fruits $(\mathrm{g})$ : low $(\mathbf{1}, \mathbf{4 9} \pm \mathbf{0 , 1 4})$

Shape (length/width): spherical

Symmetry: symmetric

Position of maximum transverse diameter:

central

Apex: rounded

Base: truncate

Niplle: absent

Lenticels: many and small

\section{Pit characters}

Weight of 100 pits (g): low $(0,25 \pm \mathbf{0 , 0 4})$

Shape (length/width): ovoid

Mucron: absent

Symmetry: symmetric

Position of maximum transverse diameter:

\section{towards apex}

Apex: rounded

Base: rounded

Surface: smooth

Number of grooves: low
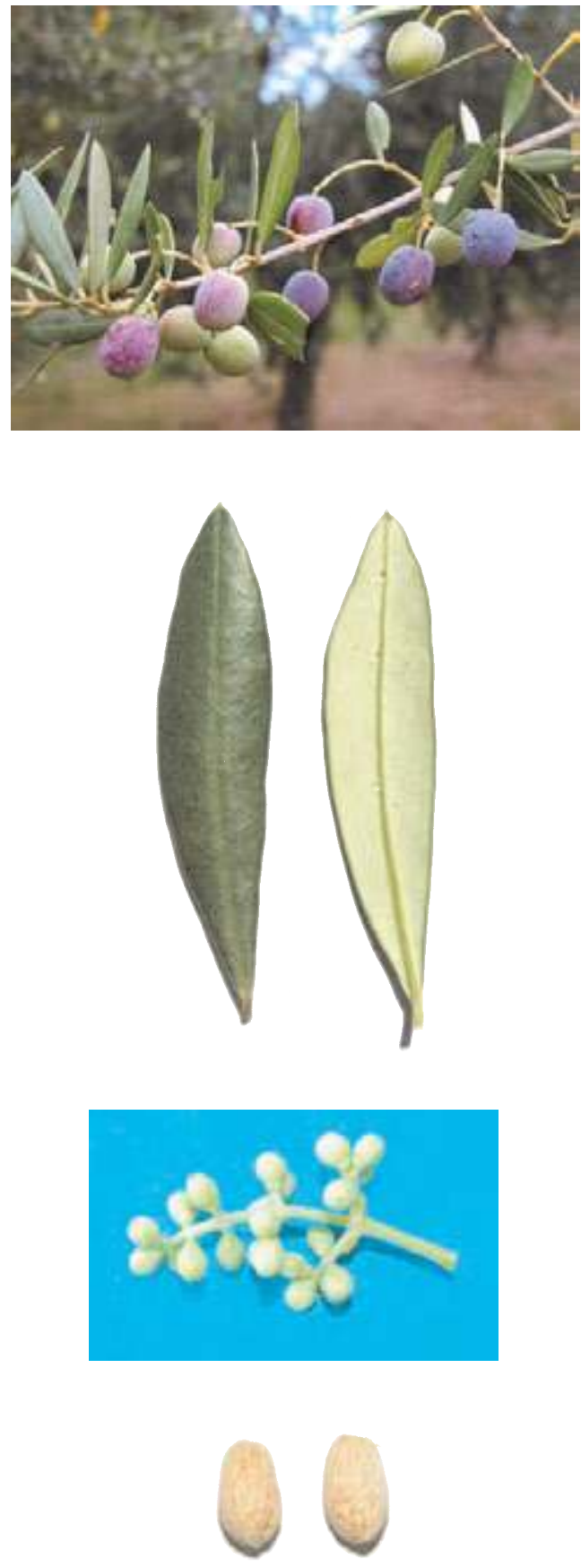


\section{Biochemical Characters}

\section{Fatty Acid Composition}

Table 1. Average values (express in $\% \pm$ standard deviations) of the fatty acids methyl esters and nutritional ratios obtained from single cultivar olive oils.

$\begin{array}{llllll}\text { Myristic acid } & \mathbf{0 , 0 1} \pm \mathbf{0 , 0 1} & \text { Linoleic acid }(\omega 6) & \mathbf{7 , 4 1} \pm \mathbf{1 , 1 4} & \text { Lignoceric acid } & \mathbf{0 , 0 5} \pm \mathbf{0 , 0 3} \\ \text { Palmitic acid } & \mathbf{1 3 , 7 0 \pm 0 , 9 1} & \text { Linolenic acid }(\omega 3) & \mathbf{0 , 6 7} \pm \mathbf{0 , 0 3} & & \\ \text { Palmitoleic acid } & \mathbf{1 , 7 2} \pm \mathbf{0 , 2 0} & \text { Arachic acid } & \mathbf{0 , 3 5} \pm \mathbf{0 , 0 9} & \text { Unsat./satured } & \mathbf{5 , 0 7 \pm 0 , 4 1} \\ \text { Stearic acid } & \mathbf{2 , 2 9} \pm \mathbf{0 , 1 6} & \text { Eicosenoic acid } & \mathbf{0 , 0 8} \pm \mathbf{0 , 1 2} & \omega 6 / \omega 3 & \mathbf{1 0 , 9 8} \pm \mathbf{1 , 4 9} \\ \text { Oleic acid } & \mathbf{7 1 , 9 1 \pm 2 , 0 4} & \text { Behenic acid } & \mathbf{0 , 0 8} \pm \mathbf{0 , 1 2} & & \end{array}$

\section{Organoleptic oil values}

Sensory Analysis (Panel test)

Comment: fruity medium-high, with hints of almond and read sensations of grass and artichoke. Balanced taste sensation with a medium-high bitter and spicy. Medium-high fluidity.

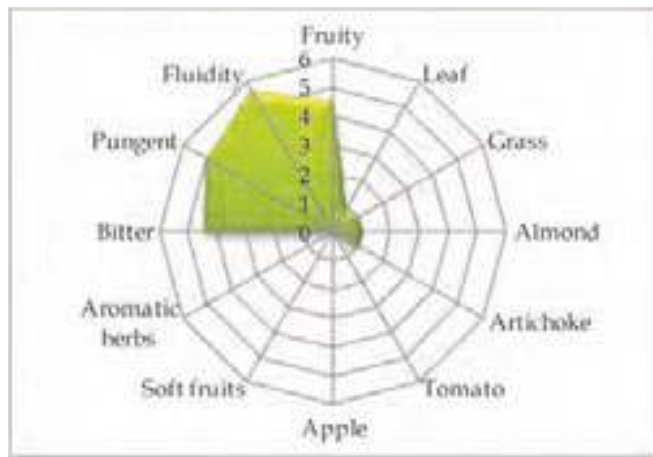

\section{Molecular Markers}

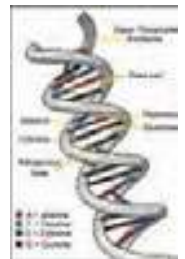

Table 2. Microsatellites (SSR) loci analyzed. For each locus the allele size (expressed in base pairs) is reported.

$\begin{array}{cccccc}D C A 09 & D C A 18 & \text { GAPU59 } & \text { GAPU71A } & \text { GUPA71B } & \text { GAPU103A } \\ \mathbf{1 7 2} \mathbf{- 2 0 6} & \mathbf{1 7 7 - \mathbf { 1 7 7 }} & \mathbf{2 1 2} \mathbf{- 2 1 2} & \mathbf{2 1 2} \mathbf{- 2 1 2} & \mathbf{1 3 0} \mathbf{- 1 4 4} & \mathbf{1 5 0 - \mathbf { 1 5 9 }} \\ \text { UDOO1 } & \text { UDO03 } & \text { UDO12 } & \text { UDO28 } & \text { UDO39 } & \\ \mathbf{1 5 0} \mathbf{- 1 5 0} & \mathbf{1 4 3 - \mathbf { 1 4 3 }} & \mathbf{1 7 7 - 1 9 3} & \mathbf{1 4 3 - \mathbf { 1 8 2 }} & \mathbf{2 0 5 - \mathbf { 2 0 5 }} & \end{array}$

\section{References:}

1 - Lombardo N., Perri E., Muzzalupo I., et al. In: Il germoplasma olivicolo calabrese, Ist. Sper. Olivic.(2003), pp: 25.

2 - Perri E., Mazzotti F., Muzzalupo I., et al. In: Relazione attività, CO.R.ASS.OL. (2003).

3 - Muzzalupo I., Chiappetta A., Benincasa C., et al Sci Hortic-Amsterdam, (2010), 126: pp. 324-329. 


\section{“Tonda di Alife"}

Areal distribution or origin area: Campania Flesh/pit weight ratio: medium $(5,86 \pm 0,29)$ Oil content (\%): medium $(42,05 \pm 1,79)$

Purpose: oil

\section{Morphological characters}

Tree characters

Vigour: medium

Growth habit: spreading

Canopy-density: medium-sparse

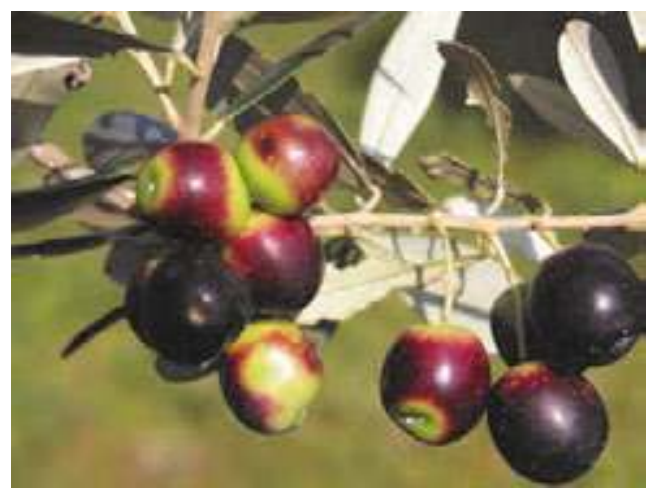

\section{Leaf characters}

Blade length (cm): medium $(5,62 \pm 0,37)$

Blade width $(\mathrm{cm})$ : medium $(\mathbf{1 , 3 6} \pm \mathbf{0 , 1 2})$

Shape (length/width): elliptic-lanceolate

\section{Inflorescence characters}

Inflorescence length $(\mathrm{cm})$ : medium $(3,12 \pm 0,45)$

Number of flowers: medium $(22,13 \pm 1,72)$

\section{Fruit characters}

Fresh weight of 100 fruits (g): medium $(2,29 \pm 0,07)$

Shape (length/width): spherical

Symmetry: symmetric

Position of maximum transverse diameter:

central

Apex: rounded

Base: truncate

Niplle: absent

Lenticels: many and small

\section{Pit characters}

Weight of 100 pits (g): medium $(\mathbf{0 , 3 4} \pm \mathbf{0 , 0 1})$

Shape (length/width): ovoid

Mucron: absent

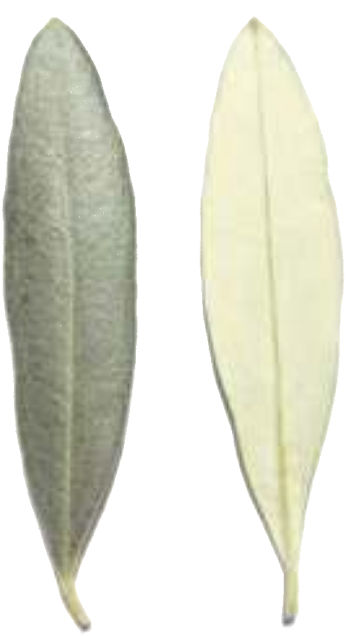

Symmetry: asymmetric

Position of maximum transverse diameter:

towards apex

Apex: rounded

Base: pointed

Surface: rugose

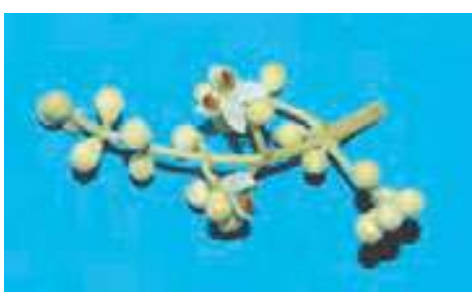

Number of grooves: medium 


\section{Biochemical Characters}

\section{Fatty Acid Composition}

Table 1. Average values (express in $\% \pm$ standard deviations) of the fatty acids methyl esters and nutritional ratios obtained from single cultivar olive oils.

$\begin{array}{llllll}\text { Myristic acid } & \mathbf{0 , 0 1} \pm \mathbf{0 , 0 0} & \text { Linoleic acid }(\omega 6) & \mathbf{1 0 , 1 3} \pm \mathbf{0 , 8 7} & \text { Lignoceric acid } & \mathbf{0 , 0 2} \pm \mathbf{0 , 0 0} \\ \text { Palmitic acid } & \mathbf{1 5 , 6 5} \pm \mathbf{0 , 3 5} & \text { Linolenic acid }(\omega 3) & \mathbf{0 , 9 6} \pm \mathbf{0 , 0 2} & & \\ \text { Palmitoleic acid } & \mathbf{1 , 4 2} \pm \mathbf{0 , 1 0} & \text { Arachic acid } & \mathbf{0 , 2 4} \pm \mathbf{0 , 0 2} & \text { Unsat./satured } & \mathbf{4 , 6 8} \pm \mathbf{0 , 1 7} \\ \text { Stearic acid } & \mathbf{1 , 5 9} \pm \mathbf{0 , 1 6} & \text { Eicosenoic acid } & \mathbf{0 , 0 2} \pm \mathbf{0 , 0 1} & \omega 6 / \omega 3 & \mathbf{1 0 , 5 1} \pm \mathbf{0 , 6 5} \\ \text { Oleic acid } & \mathbf{6 8 , 7 9 \pm \mathbf { 1 , 5 0 }} & \text { Behenic acid } & \mathbf{0 , 0 7} \pm \mathbf{0 , 0 0} & & \end{array}$

\section{Organoleptic oil values}

Sensory Analysis (Panel test)

Comment: fruity medium-high, with taste of tomato, read sensations of almond. Balanced taste sensation with medium-light spicy and bitter. Medium-high fluidity.

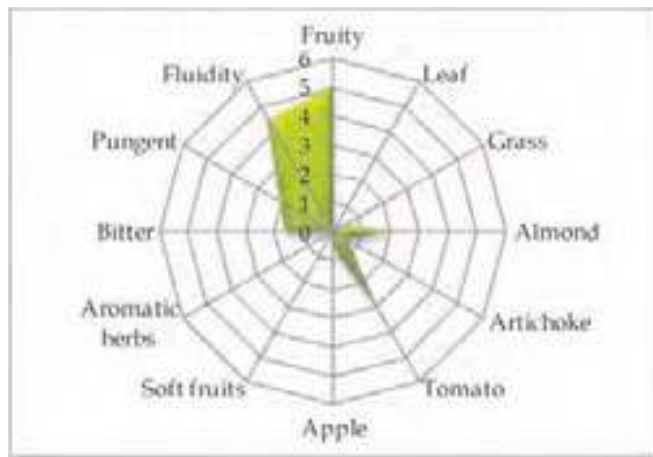

\section{Molecular Markers}

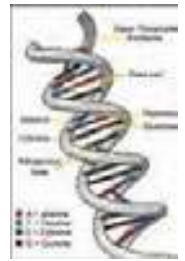

Table 2. Microsatellites (SSR) loci analyzed.

For each locus the allele size (expressed in base pairs) is reported.

$\begin{array}{cccccc}D C A 09 & \text { DCA18 } & \text { GAPU59 } & \text { GAPU71A } & \text { GUPA71B } & \text { GAPU103A } \\ \mathbf{1 8 2} \mathbf{- 2 0 6} & \mathbf{1 8 1 - \mathbf { 1 8 5 }} & \mathbf{2 1 4 - \mathbf { 2 1 4 }} & \mathbf{2 1 4} \mathbf{- 2 1 4} & \mathbf{1 2 4} \mathbf{- 1 2 6} & \mathbf{1 5 0 - \mathbf { 1 5 7 }} \\ \text { UDOO1 } & \text { UDOO3 } & \text { UDO12 } & \text { UDO28 } & \text { UDO39 } & \\ \mathbf{1 5 0} \mathbf{- 1 5 0} & \mathbf{1 6 6 - \mathbf { 1 6 6 }} & \mathbf{1 6 4 - \mathbf { 1 9 3 }} & \mathbf{1 5 0 - \mathbf { 1 5 4 }} & \mathbf{2 0 5 - \mathbf { 2 0 5 }} & \end{array}$

\section{References:}

1 - Various authors. In: Catalogo Nazionale delle Varietà di Olivo. University of Bari (in press), ISBN 978-88-8879397-9.

2 - Muzzalupo I., Stefanizzi F., Perri E. HortScience (2009), 44: pp. 582-588. 


\section{“Tonda di Filadelfia"}

(synonymy: Oliva di Pentone, Strongolese, Tonda di Filogaso, Tonda dolce, etc.)

Areal distribution or origin area: Calabria

Flesh/pit weight ratio: high $(\mathbf{7 , 9 0 \pm 1 , 7 9 )}$

Oil content (\%): high $(\mathbf{5 1 , 4 5 \pm 5 , 1 4 )}$

Purpose: dual purpose

\section{Morphological characters}

Tree characters

Vigour: medium-strong

Growth habit: spreading

Canopy-density: medium-dense

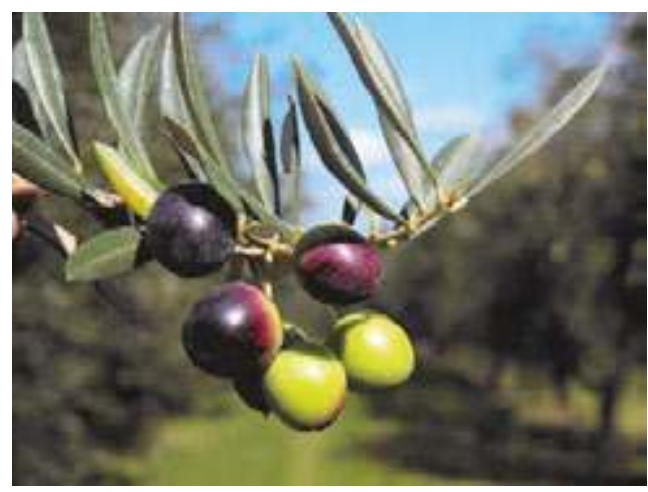

\section{Leaf characters}

Blade length $(\mathrm{cm})$ : medium $(5,95 \pm 0,74)$

Blade width (cm): broad $(\mathbf{1}, \mathbf{5 1} \pm \mathbf{0 , 1 1})$

Shape (length/width): elliptic

\section{Inflorescence characters}

Inflorescence length (cm): short $(2,40 \pm 1,21)$

Number of flowers: low $(\mathbf{1 4 , 0 0 \pm 1 , 4 6 )}$

\section{Fruit characters}

Fresh weight of 100 fruits $(\mathrm{g})$ : medium $(3,22 \pm 0,64)$

Shape (length/width): spherical

Symmetry: slightly asymmetric

Position of maximum transverse diameter:

central

Apex: rounded

Base: truncate

Niplle: absent

Lenticels: many and small

\section{Pit characters}

Weight of 100 pits (g): medium $(0,36 \pm 0,03)$

Shape (length/width): ovoid

Mucron: obvious

Symmetry: slightly asymmetric

Position of maximum transverse diameter:

central

Apex: rounded

Base: rounded

Surface: rugose

Number of grooves: medium
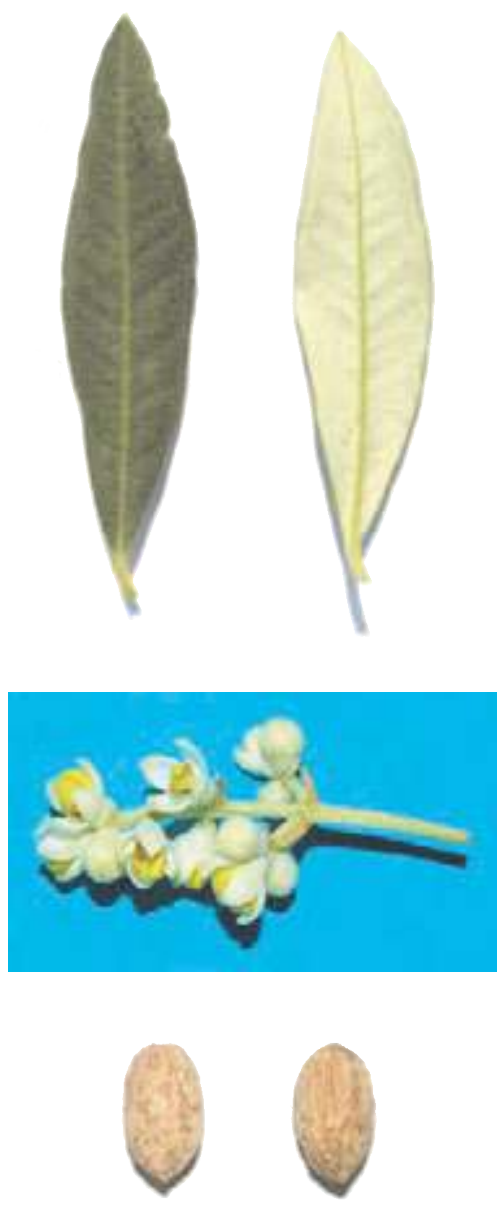


\section{Biochemical Characters}

\section{Fatty Acid Composition}

Table 1. Average values (express in $\% \pm$ standard deviations) of the fatty acids methyl esters and nutritional ratios obtained from single cultivar olive oils.

$\begin{array}{llllll}\text { Myristic acid } & \mathbf{0 , 0 1} \pm \mathbf{0 , 0 0} & \text { Linoleic acid }(\omega 6) & \mathbf{7 , 5 5} \pm \mathbf{1 , 0 0} & \text { Lignoceric acid } & \mathbf{0 , 0 2} \pm \mathbf{0 , 0 0} \\ \text { Palmitic acid } & \mathbf{1 1 , 1 0} \pm \mathbf{0 , 0 1} & \text { Linolenic acid }(\omega 3) & \mathbf{0 , 7 9} \pm \mathbf{0 , 0 2} & & \\ \text { Palmitoleic acid } & \mathbf{1 , 0 9} \pm \mathbf{0 , 3 5} & \text { Arachic acid } & \mathbf{0 , 2 5} \pm \mathbf{0 , 0 4} & \text { Unsat./satured } & \mathbf{6 , 5 3} \pm \mathbf{0 , 1 4} \\ \text { Stearic acid } & \mathbf{1 , 8 9} \pm \mathbf{0 , 1 9} & \text { Eicosenoic acid } & \mathbf{0 , 0 2} \pm \mathbf{0 , 0 0} & \omega 6 / \omega 3 & \mathbf{9 , 5 2} \pm \mathbf{1 , 0 0} \\ \text { Oleic acid } & \mathbf{7 6 , 1 3} \pm \mathbf{0 , 0 4} & \text { Behenic acid } & \mathbf{0 , 0 5} \pm \mathbf{0 , 0 1} & & \end{array}$

\section{Organoleptic oil values}

Sensory Analysis (Panel test)

Comment: fruity medium-high, with read sensations of artichoke, almond, and leaves. Balanced taste sensation with a medium bitter and spicy. Medium-high fluidity.

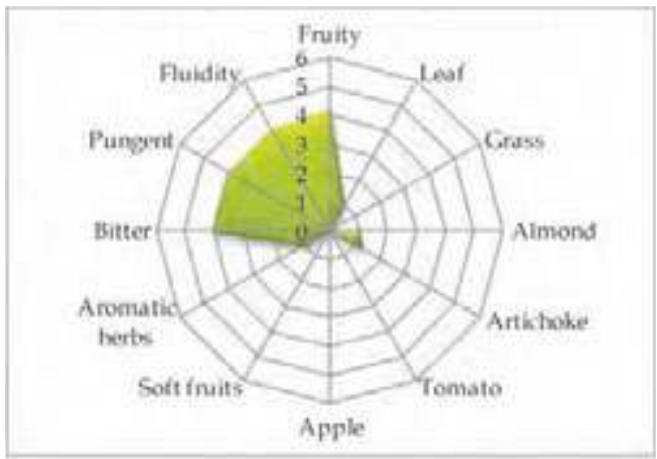

\section{Molecular Markers}

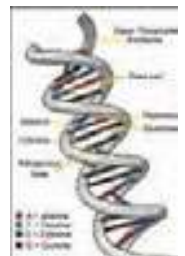

Table 2. Microsatellites (SSR) loci analyzed. For each locus the allele size (expressed in base pairs) is reported.

$\begin{array}{cccccc}D C A 09 & \text { DCA18 } & \text { GAPU59 } & \text { GAPU71A } & \text { GUPA71B } & \text { GAPU103A } \\ \mathbf{1 6 2} \mathbf{- 1 9 8} & \mathbf{1 7 7 - \mathbf { 1 8 5 }} & \mathbf{2 1 2} \mathbf{- 2 2 2} & \mathbf{2 1 2} \mathbf{- 2 1 8} & \mathbf{1 2 6 - \mathbf { 1 4 4 }} & \mathbf{1 5 0 - \mathbf { 1 5 7 }} \\ \text { UDO01 } & \text { UDOO3 } & \text { UDO12 } & \text { UDO28 } & \text { UDO39 } & \\ \mathbf{1 4 4 - \mathbf { 1 4 4 }} & \mathbf{1 4 3 - \mathbf { 1 4 3 }} & \mathbf{1 6 6 - \mathbf { 1 9 3 }} & \mathbf{1 4 3 - \mathbf { 2 0 5 }} & \mathbf{2 0 5 - \mathbf { 2 3 2 }} & \end{array}$

\section{References:}

1 - Lombardo N., Perri E., Muzzalupo I., et al. In: Il germoplasma olivicolo calabrese, Ist. Sper. Olivic.(2003), pp: 23.

2 - Perri E., Mazzotti F., Muzzalupo I., et al. In: Relazione attività, CO.R.ASS.OL. (2003).

3 - Muzzalupo I., Chiappetta A., Benincasa C., et al Sci Hortic-Amsterdam, (2010), 126: pp. 324-329. 


\section{“Tonda di Filogaso"}

(synonymy: Oliva di Pentone, Strongolese, Tonda di Filadelfia, Tonda Dolce, etc.)

Areal distribution or origin area: Calabria

Flesh/pit weight ratio: medium $(6,24 \pm 1,73)$

Oil content (\%): medium $(46,58 \pm 2,19)$

Purpose: dual purpose

\section{Morphological characters}

Tree characters

Vigour: medium-strong

Growth habit: spreading

Canopy-density: medium

\section{Leaf characters}

Blade length $(\mathrm{cm})$ : medium $(5,26 \pm 0,57)$

Blade width (cm): broad $(\mathbf{1}, 59 \pm 0,18)$

Shape (length/width): elliptic

\section{Inflorescence characters}

Inflorescence length (cm): long $(3,53 \pm 0,18)$

Number of flowers: medium $(23,75 \pm 2,97)$

\section{Fruit characters}

Fresh weight of 100 fruits $(\mathrm{g})$ : low $(1,90 \pm 0,33)$

Shape (length/width): ovoid

Symmetry: slightly asymmetric

Position of maximum transverse diameter:

central

Apex: rounded

Base: rounded

Niplle: absent

Lenticels: many and small

\section{Pit characters}

Weight of 100 pits (g): medium $(0,30 \pm 0,06)$

Shape (length/width): elliptic

Mucron: absent

Symmetry: asymmetric

Position of maximum transverse diameter:

towards apex

Apex: rounded

Base: pointed

Surface: smooth

Number of grooves: medium
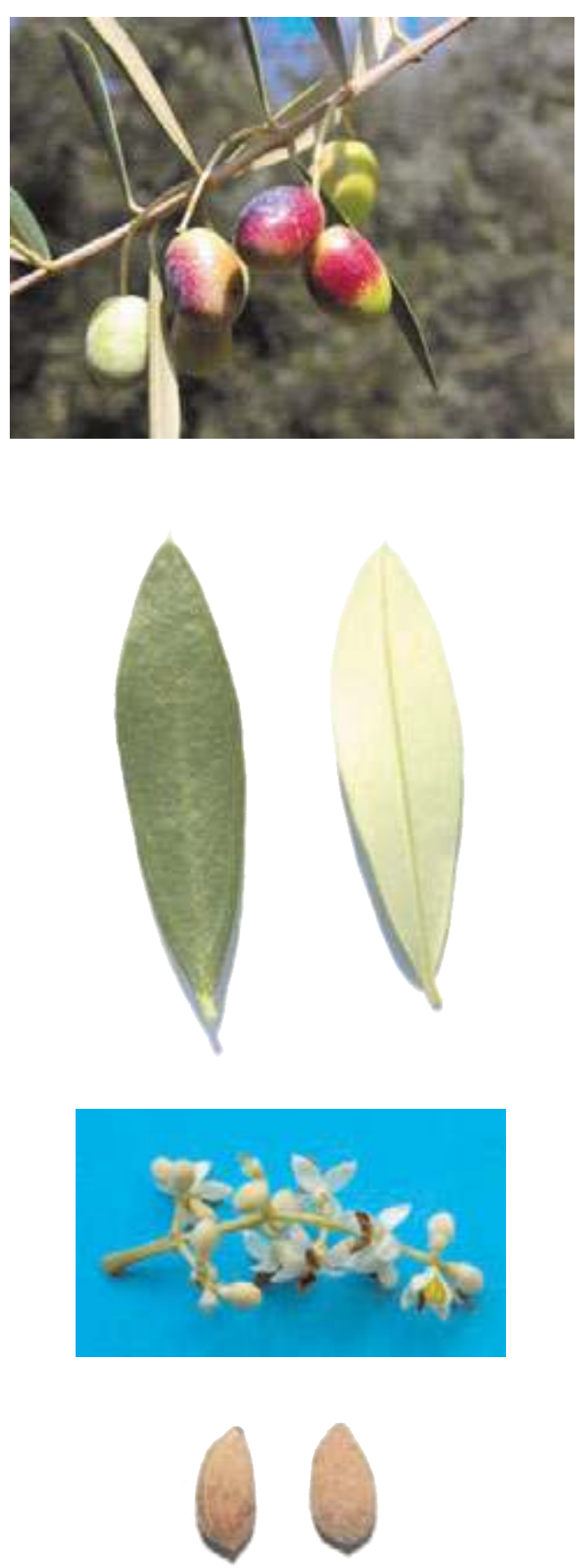


\section{Biochemical Characters}

\section{Fatty Acid Composition}

Table 1. Average values (express in $\% \pm$ standard deviations) of the fatty acids methyl esters and nutritional ratios obtained from single cultivar olive oils.

$\begin{array}{llllll}\text { Myristic acid } & \mathbf{0 , 0 2} \pm \mathbf{0 , 0 2} & \text { Linoleic acid }(\omega 6) & \mathbf{6 , 6 9} \pm \mathbf{0 , 2 6} & \text { Lignoceric acid } & \mathbf{0 , 0 4} \pm \mathbf{0 , 0 3} \\ \text { Palmitic acid } & \mathbf{1 1 , 4 9} \pm \mathbf{0 , 7 2} & \text { Linolenic acid }(\omega 3) & \mathbf{0 , 7 2} \pm \mathbf{0 , 0 4} & & \\ \text { Palmitoleic acid } & \mathbf{1 , 0 8} \pm \mathbf{0 , 3 0} & \text { Arachic acid } & \mathbf{0 , 2 7} \pm \mathbf{0 , 0 3} & \text { Unsat./satured } & \mathbf{6 , 1 7} \pm \mathbf{0 , 3 8} \\ \text { Stearic acid } & \mathbf{2 , 1 1} \pm \mathbf{0 , 0 7} & \text { Eicosenoic acid } & \mathbf{0 , 0 3} \pm \mathbf{0 , 0 3} & \omega 6 / \omega 3 & \mathbf{9 , 2 4} \pm \mathbf{0 , 3 9} \\ \text { Oleic acid } & \mathbf{7 6 , 4 6 \pm 0 , 6 9} & \text { Behenic acid } & \mathbf{0 , 0 7} \pm \mathbf{0 , 0 1} & & \end{array}$

\section{Organoleptic oil values}

Sensory Analysis (Panel test)

Comment: medium fruity, with good sensation of almond and aromatic herbs, less grass and artichoke. Balanced taste sensation, with bitter and spicy medium. Medium-high fluidity.

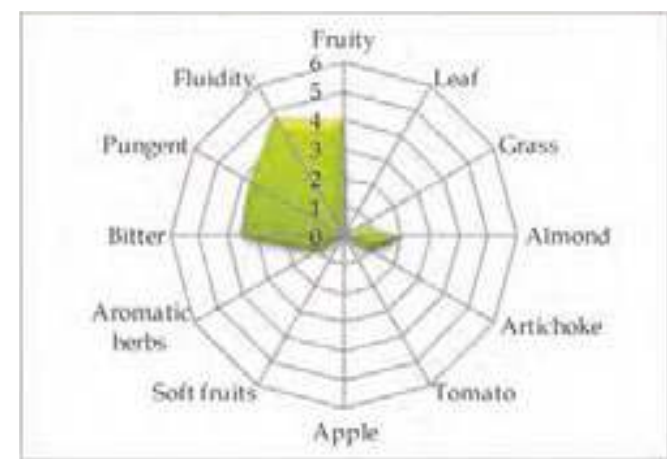

\section{Molecular Markers}

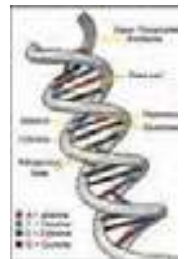

Table 2. Microsatellites (SSR) loci analyzed. For each locus the allele size (expressed in base pairs) is reported.

$\begin{array}{cccccc}D C A 09 & \text { DCA18 } & \text { GAPU59 } & \text { GAPU71A } & \text { GUPA71B } & \text { GAPU103A } \\ \mathbf{1 9 8} \mathbf{- 2 0 6} & \mathbf{1 7 7 - \mathbf { 1 7 9 }} & \mathbf{2 1 2} \mathbf{- 2 1 2} & \mathbf{2 1 2} \mathbf{- 2 5 0} & \mathbf{1 2 4 - \mathbf { 1 2 4 }} & \mathbf{1 5 7 - \mathbf { 1 7 0 }} \\ \text { UDOO1 } & \text { UDOO3 } & \text { UDO12 } & \text { UDO28 } & \text { UDO39 } & \\ \mathbf{1 5 0 - \mathbf { 1 5 0 }} & \mathbf{1 4 3 - \mathbf { 1 4 3 }} & \mathbf{1 6 6 - \mathbf { 1 6 6 }} & \mathbf{1 4 3 - \mathbf { 1 8 2 }} & \mathbf{2 0 5 - \mathbf { 2 0 5 }} & \end{array}$

\section{References:}

1 - Lombardo N., Perri E., Muzzalupo I., et al. In: Il germoplasma olivicolo calabrese, Ist. Sper. Olivic.(2003), pp: 24.

2 - Perri E., Mazzotti F., Muzzalupo I., et al. In: Relazione attività, CO.R.ASS.OL. (2003).

3 - Muzzalupo I., Chiappetta A., Benincasa C., et al Sci Hortic-Amsterdam, (2010), 126: pp. 324-329. 


\section{“Tonda di Strongoli "}

(synonymy: Olivo di Pentone, Strongolese, Tonda di Filadelfia, Tonda di Filogaso, etc.)

Areal distribution or origin area: Calabria

Flesh/ pit weight ratio: high $(8,58 \pm 0,60)$

Oil content (\%): medium $(\mathbf{5 1}, 88 \pm \mathbf{2 , 9 1 )}$

Purpose: dual purpose

\section{Morphological characters}

Tree characters

Vigour: medium

Growth habit: spreading

Canopy-density: medium

\section{Leaf characters}

Blade length $(\mathrm{cm})$ : medium $(6,19 \pm 0,61)$

Blade width $(\mathrm{cm})$ : medium $(\mathbf{1}, 01 \pm 0,14)$

Shape (length/width): lanceolate

\section{Inflorescence characters}

Inflorescence length (cm): short $(2,44 \pm 1,0)$

Number of flowers: low $(17,08 \pm 1,13)$

\section{Fruit characters}

Fresh weight of 100 fruits $(\mathrm{g})$ : high $(\mathbf{5 , 1 0} \pm \mathbf{1 , 4 9})$

Shape (length/width): ovoid

Symmetry: asymmetric

Position of maximum transverse diameter:

central

Apex: rounded

Base: truncate

Niplle: tenuous

Lenticels: few and large

\section{Pit characters}

Weight of 100 pits (g): high $(\mathbf{0 , 5 3} \pm \mathbf{0 , 1 3})$

Shape (length/width): ovoid

Mucron: absent

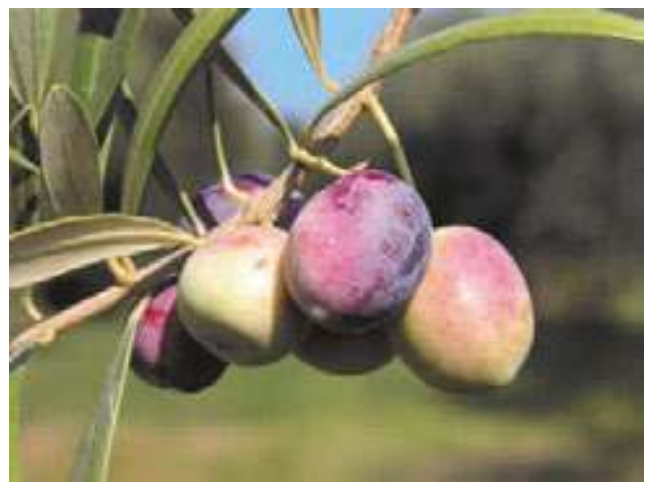

Symmetry: slightly asymmetric

Position of maximum transverse diameter:

towards apex

Apex: rounded

Base: rounded

Surface: rugose

Number of grooves: low
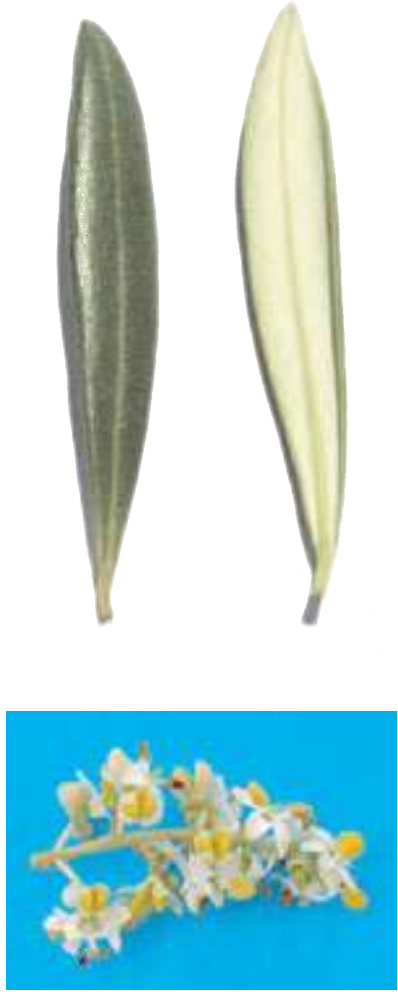


\section{Biochemical Characters}

\section{Fatty Acid Composition}

Table 1. Average values (express in $\% \pm$ standard deviations) of the fatty acids methyl esters and nutritional ratios obtained from single cultivar olive oils.

$\begin{array}{llllll}\text { Myristic acid } & \mathbf{0 , 0 3} \pm \mathbf{0 , 0 3} & \text { Linoleic acid }(\omega 6) & \mathbf{7 , 9 5} \pm \mathbf{1 , 5 6} & \text { Lignoceric acid } & \mathbf{0 , 0 3} \pm \mathbf{0 , 0 0} \\ \text { Palmitic acid } & \mathbf{1 3 , 0 4 \pm 0 , 6 7} & \text { Linolenic acid }(\omega 3) & \mathbf{0 , 8 5} \pm \mathbf{0 , 0 5} & & \\ \text { Palmitoleic acid } & \mathbf{1 , 4 6} \pm \mathbf{0 , 4 5} & \text { Arachic acid } & \mathbf{0 , 2 3} \pm \mathbf{0 , 0 5} & \text { Unsat./satured } & \mathbf{5 , 5 2} \pm \mathbf{0 , 2 6} \\ \text { Stearic acid } & \mathbf{1 , 8 8} \pm \mathbf{0 , 0 7} & \text { Eicosenoic acid } & \mathbf{0 , 0 4} \pm \mathbf{0 , 0 2} & \omega 6 / \omega 3 & \mathbf{9 , 2 9} \pm \mathbf{1 , 3 1} \\ \text { Oleic acid } & \mathbf{7 2 , 9 3 \pm \mathbf { 1 , 1 4 }} & \text { Behenic acid } & \mathbf{0 , 0 6} \pm \mathbf{0 , 0 1} & & \end{array}$

\section{Organoleptic oil values}

Sensory Analysis (Panel test)

Comment: fruity medium-high, with hints of almond and read sensations of grass and artichoke. Balanced taste sensation with a medium bitter and spicy. Medium fluidity.

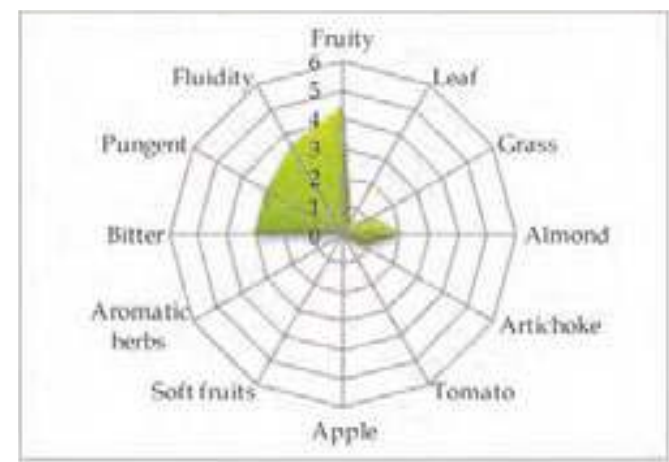

\section{Molecular Markers}

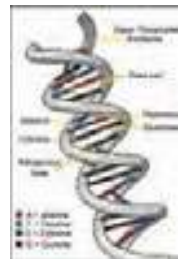

Table 2. Microsatellites (SSR) loci analyzed. For each locus the allele size (expressed in base pairs) is reported.

$\begin{array}{cccccc}D C A 09 & D C A 18 & \text { GAPU59 } & \text { GAPU71A } & \text { GUPA71B } & \text { GAPU103A } \\ \mathbf{1 6 2} \mathbf{- 1 9 8} & \mathbf{1 7 7 - \mathbf { 1 8 5 }} & \mathbf{2 1 2} \mathbf{- 2 2 2} & \mathbf{2 1 2} \mathbf{- 2 1 8} & \mathbf{1 2 4} \mathbf{- 1 2 6} & \mathbf{1 3 6} \mathbf{- 1 5 0} \\ \text { UDOO1 } & \text { UDO03 } & \text { UDO12 } & \text { UDO28 } & \text { UDO39 } & \\ \mathbf{1 4 4 - \mathbf { 1 4 4 }} & \mathbf{1 4 3 - \mathbf { 1 4 3 }} & \mathbf{1 6 6 - \mathbf { 1 9 3 }} & \mathbf{1 4 3 - \mathbf { 1 8 2 }} & \mathbf{2 0 5 - \mathbf { 2 2 0 }} & \end{array}$

\section{References:}

1 - Lombardo N., Perri E., Muzzalupo I., et al. In: Il germoplasma olivicolo calabrese, Ist. Sper. Olivic.(2003), pp: 15.

2 - Perri E., Mazzotti F., Muzzalupo I., et al. In: Relazione attività, CO.R.ASS.OL. (2003).

3 - Muzzalupo I., Chiappetta A., Benincasa C., et al Sci Hortic-Amsterdam, (2010), 126: pp. 324-329. 


\section{“Tonda dolce di Partanna"}

Areal distribution or origin area: Sicilia

Flesh/pit weight ratio: high $(8,87 \pm 0,57)$

Oil content (\%): medium $(48,55 \pm 0,03)$

Purpose: dual purpose

\section{Morphological characters}

Tree characters

Vigour: medium-weak

Growth habit: spreading

Canopy-density: medium

\section{Leaf characters}

Blade length $(\mathrm{cm})$ : medium $(6,39 \pm 0,68)$

Blade width $(\mathrm{cm})$ : broad $(\mathbf{1}, \mathbf{5 3} \pm \mathbf{0 , 1 7})$

Shape (length/width): elliptic-lanceolate

\section{Inflorescence characters}

Inflorescence length $(\mathrm{cm})$ : medium $(2,61 \pm 0,66)$

Number of flowers: low $(\mathbf{1 4}, \mathbf{4 5} \pm \mathbf{2 , 1 7})$

\section{Fruit characters}

Fresh weight of 100 fruits (g): high $(5,30 \pm 0,71)$

Shape (length/width): spherical

Symmetry: symmetric

Position of maximum transverse diameter:

towards base

Apex: rounded

Base: truncate

Niplle: obvious

Lenticels: many and small

\section{Pit characters}

Weight of 100 pits (g): high $(\mathbf{0 , 5 4} \pm \mathbf{0 , 0 4})$

Shape (length/width): ovoid

Mucron: obvious

Symmetry: slightly asymmetric

Position of maximum transverse diameter:

towards base

Apex: rounded

Base: truncate

Surface: scabrous

Number of grooves: low
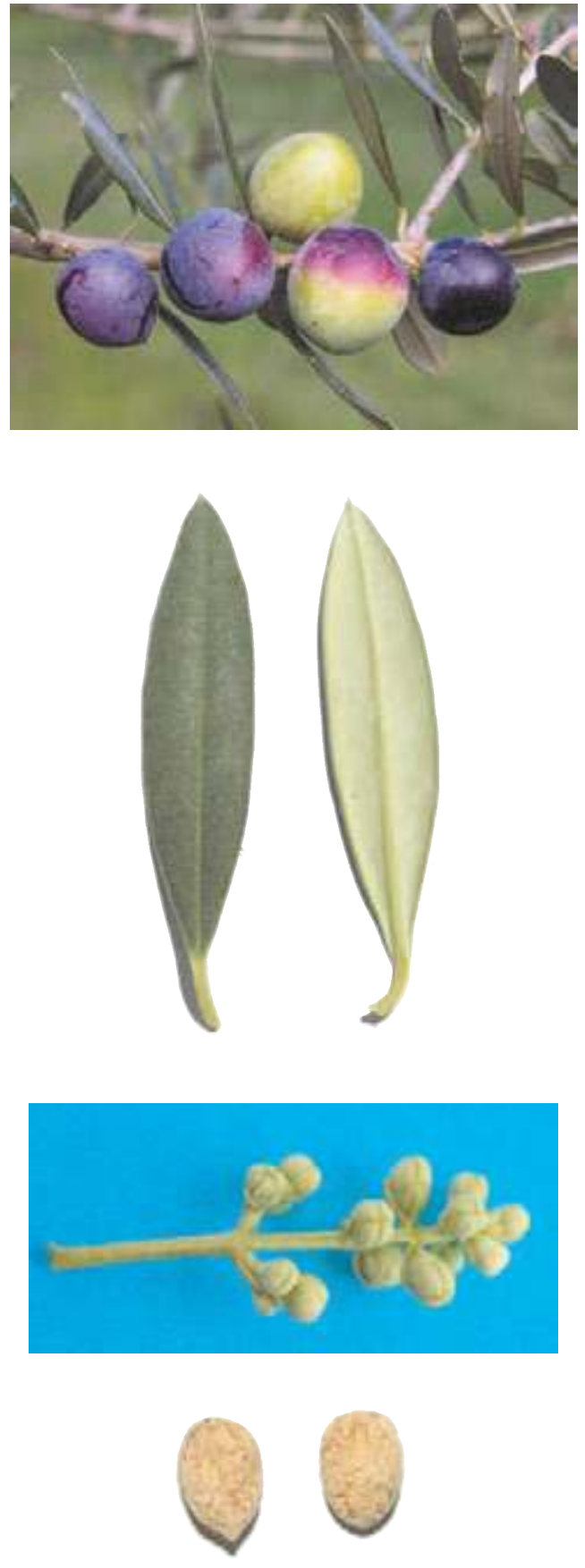


\section{Biochemical Characters}

\section{Fatty Acid Composition}

Table 1. Average values (express in $\% \pm$ standard deviations) of the fatty acids methyl esters and nutritional ratios obtained from single cultivar olive oils.

$\begin{array}{llllll}\text { Myristic acid } & \mathbf{0 , 0 1} \pm \mathbf{0 , 0 1} & \text { Linoleic acid }(\omega 6) & \mathbf{9 , 7 3} \pm \mathbf{0 , 0 8} & \text { Lignoceric acid } & \mathbf{0 , 0 4} \pm \mathbf{0 , 0 1} \\ \text { Palmitic acid } & \mathbf{1 0 , 1 8} \pm \mathbf{0 , 1 1} & \text { Linolenic acid }(\omega 3) \mathbf{0 , 8 9} \pm \mathbf{0 , 1 3} & & \\ \text { Palmitoleic acid } & \mathbf{0 , 8 1} \pm \mathbf{0 , 0 3} & \text { Arachic acid } & \mathbf{0 , 2 6} \pm \mathbf{0 , 0 1} & \text { Unsat./satured } & \mathbf{6 , 9 0 \pm 0 , 0 4} \\ \text { Stearic acid } & \mathbf{2 , 2 3} \pm \mathbf{0 , 0 3} & \text { Eicosenoic acid } & \mathbf{0 , 0 2} \pm \mathbf{0 , 0 1} & \omega 6 / \omega 3 & \mathbf{1 1 , 0 1} \pm \mathbf{1 , 4 6} \\ \text { Oleic acid } & \mathbf{7 5 , 2 2} \pm \mathbf{0 , 2 9} & \text { Behenic acid } & \mathbf{0 , 0 5} \pm \mathbf{0 , 0 1} & & \end{array}$

\section{Organoleptic oil values}

Sensory Analysis (Panel test)

Comment: fruity medium, with hints of artichoke and almond. Balanced in flavours, with hints of bitter and spicy medium - light intensity, medium fluidity.

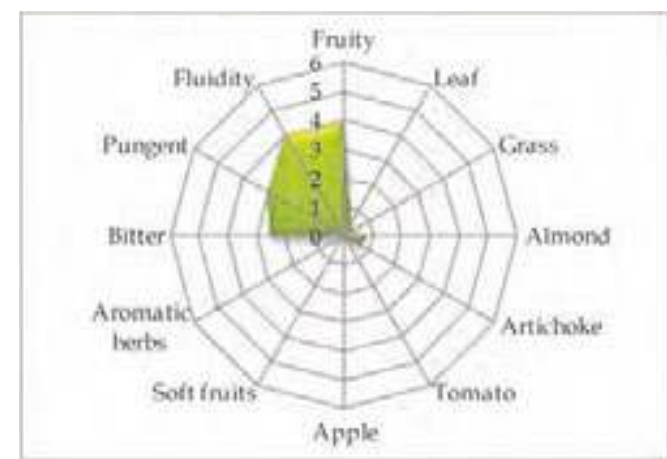

\section{Molecular Markers}

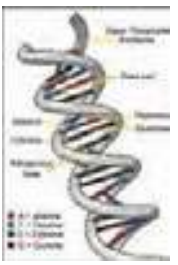

Table 2. Microsatellites (SSR) loci analyzed.

For each locus the allele size (expressed in base pairs) is reported.

$\begin{array}{cccccc}D C A 09 & \text { DCA18 } & \text { GAPU59 } & \text { GAPU71A } & \text { GUPA71B } & \text { GAPU103A } \\ \mathbf{1 8 2} \mathbf{- 1 8 2} & \mathbf{1 7 7 - \mathbf { 1 8 1 }} & \mathbf{2 1 2} \mathbf{- 2 1 2} & \mathbf{2 1 4} \mathbf{- 2 1 4} & \mathbf{1 4 4 - \mathbf { 1 4 4 }} & \mathbf{1 5 7 - \mathbf { 1 8 4 }} \\ \text { UDOO1 } & \text { UDOO3 } & \text { UDO12 } & \text { UDO28 } & \text { UDO39 } & \\ \mathbf{1 4 0 - \mathbf { 1 4 0 }} & \mathbf{1 5 0 - \mathbf { 1 5 0 }} & \mathbf{1 6 6 - \mathbf { 1 9 3 }} & \mathbf{1 8 2} \mathbf{- 1 8 2} & \mathbf{2 0 5 - \mathbf { 2 0 5 }} & \end{array}$

\section{References:}

1 - Mulè R., Fodale A. S., Parlati M. V.,, et al. In: Frutticoltura, (1992), 11 pp. 25-29.

2 - Muzzalupo I., Stefanizzi F., Perri E. HortScience (2009), 44: pp. 582-588. 


\section{“Tonda Iblea"}

(synonymy: Alimena, Cetrala, Ferlisa, Nuciddara, Tunna, etc.)

Areal distribution or origin area: Sicilia

Flesh/pit weight ratio: medium $(7,17 \pm 0,65)$

Oil content (\%): high $(\mathbf{5 4 , 2 3} \pm \mathbf{0 , 5 8})$

Purpose: table

\section{Morphological characters}

Tree characters

Vigour: medium-weak

Growth habit: spreading-erect

Canopy-density: medium

\section{Leaf characters}

Blade length $(\mathrm{cm})$ : medium $(5,15 \pm 0,30)$

Blade width $(\mathrm{cm})$ : medium $(\mathbf{1 , 1 5} \pm \mathbf{0 , 1 3})$

Shape (length/width): elliptic-lanceolate

\section{Inflorescence characters}

Inflorescence length (cm): short $(1,86 \pm 0,37)$

Number of flowers: low $(\mathbf{1 2 , 9 3} \pm 3,70)$

\section{Fruit characters}

Fresh weight of 100 fruits (g): high $(\mathbf{4 , 8 4} \pm \mathbf{1 , 4 8})$

Shape (length/width): spherical

Symmetry: asymmetric

Position of maximum transverse diameter:

central

Apex: rounded

Base: truncate

Niplle: absent

Lenticels: many and small

\section{Pit characters}

Weight of 100 pits (g): high $(\mathbf{0 , 6 5} \pm \mathbf{0 , 0 7})$

Shape (length/width): ovoid

Mucron: absent

Symmetry: symmetric

Position of maximum transverse diameter:

central

Apex: rounded

Base: rounded

Surface: scabrous

Number of grooves: medium
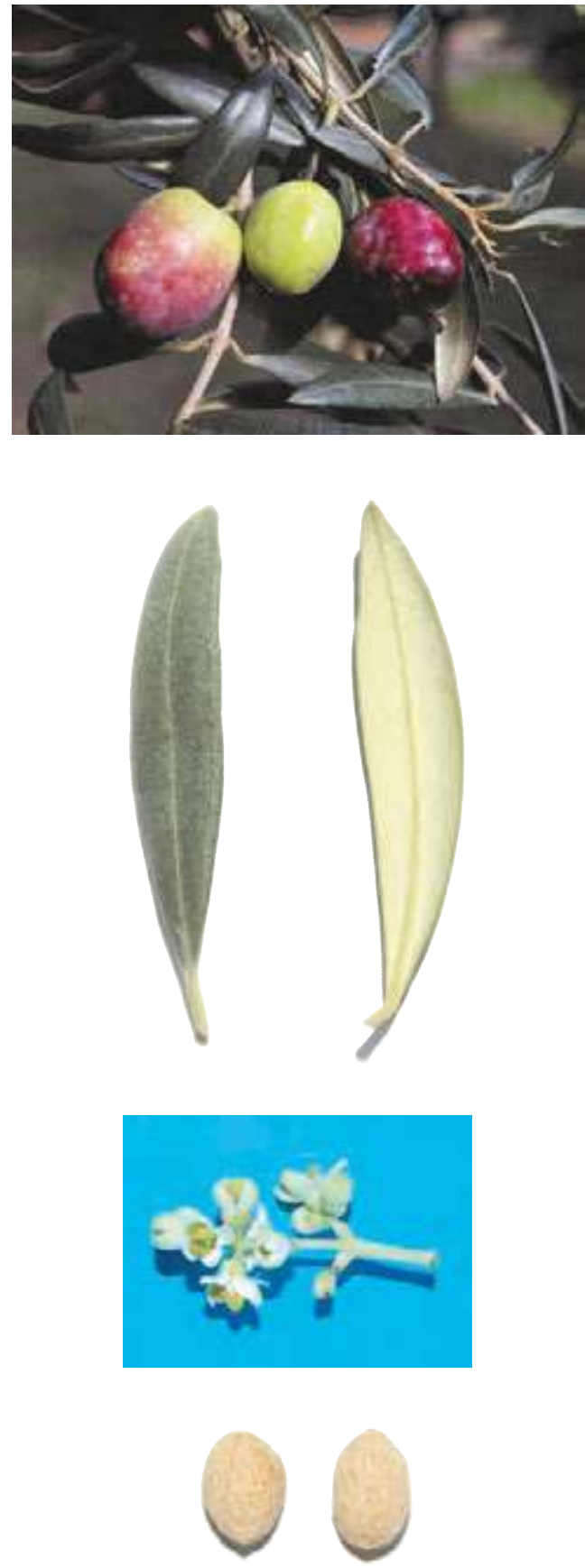


\section{Biochemical Characters}

\section{Fatty Acid Composition}

Table 1. Average values (express in $\% \pm$ standard deviations) of the fatty acids methyl esters and nutritional ratios obtained from single cultivar olive oils.

$\begin{array}{llllll}\text { Myristic acid } & \mathbf{0 , 0 3} \pm \mathbf{0 , 0 3} & \text { Linoleic acid }(\omega 6) & \mathbf{8 , 8 2} \pm \mathbf{0 , 6 1} & \text { Lignoceric acid } & \mathbf{0 , 0 4} \pm \mathbf{0 , 0 2} \\ \text { Palmitic acid } & \mathbf{1 3 , 4 4} \pm \mathbf{0 , 5 7} & \text { Linolenic acid }(\omega 3) \mathbf{0 , 8 5} \pm \mathbf{0 , 0 3} & & \\ \text { Palmitoleic acid } & \mathbf{1 , 2 3} \pm \mathbf{0 , 2 3} & \text { Arachic acid } & \mathbf{0 , 2 8} \pm \mathbf{0 , 0 4} & \text { Unsat./satured } & \mathbf{5 , 3 4} \pm \mathbf{0 , 2 9} \\ \text { Stearic acid } & \mathbf{1 , 9 4} \pm \mathbf{0 , 1 4} & \text { Eicosenoic acid } & \mathbf{0 , 1 0} \pm \mathbf{0 , 1 4} & \omega 6 / \omega 3 & \mathbf{1 0 , 3 7 \pm 0 , 8 3} \\ \text { Oleic acid } & \mathbf{7 2 , 1 1} \pm \mathbf{1 , 1 7} & \text { Behenic acid } & \mathbf{0 , 0 7} \pm \mathbf{0 , 0 1} & & \end{array}$

\section{Organoleptic oil values}

Sensory Analysis (Panel test)

Comment: fruity medium-high, with hints of grass and tomato, read sensations of almond and artichoke. Balanced taste sensation with a medium- bitter and spicy. Medium-high fluidity.

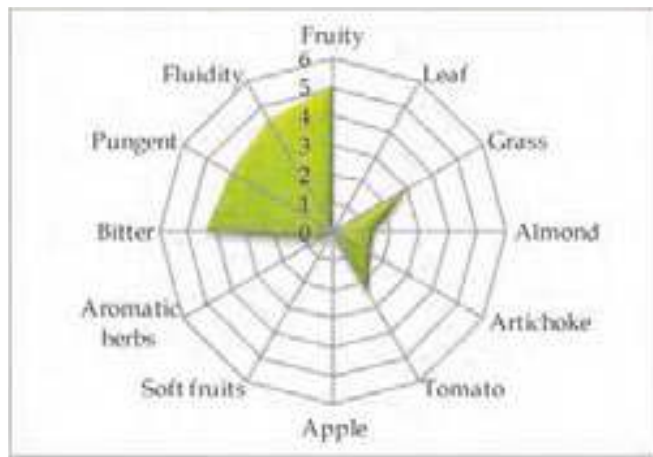

\section{Molecular Markers}

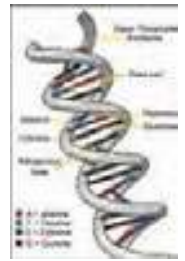

Table 2. Microsatellites (SSR) loci analyzed.

For each locus the allele size (expressed in base pairs) is reported.

\begin{tabular}{|c|c|c|c|c|c|}
\hline DCA09 & DCA18 & GAPU59 & GAPU71A & $G U P A 71 B$ & GAPU103A \\
\hline $162-198$ & $169-177$ & $208-212$ & $210-214$ & $124-144$ & $136-159$ \\
\hline UDO01 & UDO03 & UDO12 & UDO28 & UDO39 & \\
\hline $144-144$ & $150-150$ & $166-193$ & $154-154$ & $108-205$ & \\
\hline
\end{tabular}

\section{References:}

1 - Caruso T., Cartabellotta D., Motisi A., et all. In: Cultivar di olivo siciliane, Università degli Studi di Palermo (2007), pp. 72-76.

2 - Muzzalupo I., Stefanizzi F., Perri E. HortScience (2009), 44: pp. 582-588. 


\section{“Tondina"}

(synonymy: Amaro, Roggianella, Roggianese, Spezzanota, Vernile, etc.)

Areal distribution or origin area: Calabria

Flesh/ pit weight ratio: high $(7,54 \pm 0,92)$

Oil content (\%): low $(39,70 \pm 0,88)$

Purpose: oil

\section{Morphological characters}

Tree characters

Vigour: strong

Growth habit: spreading-erect

Canopy-density: dense

\section{Leaf characters}

Blade length $(\mathrm{cm})$ : medium $(5,23 \pm 0,44)$

Blade width $(\mathrm{cm})$ : medium $(\mathbf{1 , 1 3} \pm \mathbf{0 , 1 2})$

Shape (length/width): elliptic - lanceolate

\section{Inflorescence characters}

Inflorescence length (cm): medium $(3,02 \pm 1,34)$

Number of flowers: medium $(\mathbf{1 9 , 3 8} \pm \mathbf{2 , 5 7})$

\section{Fruit characters}

Fresh weight of 100 fruits (g): medium $(2,60 \pm 0,26)$

Shape (length/width): spherical

Symmetry: symmetric

Position of maximum transverse diameter:

central

Apex: rounded

Base: rounded

Niplle: absent

Lenticels: many and small

\section{Pit characters}

Weight of 100 pits (g): medium $(0,37 \pm 0,05)$

Shape (length/width): ovoid

Mucron: obvious

Symmetry: slightly asymmetric

Position of maximum transverse diameter:

central

Apex: rounded

Base: pointed

Surface: rugose

Number of grooves: medium
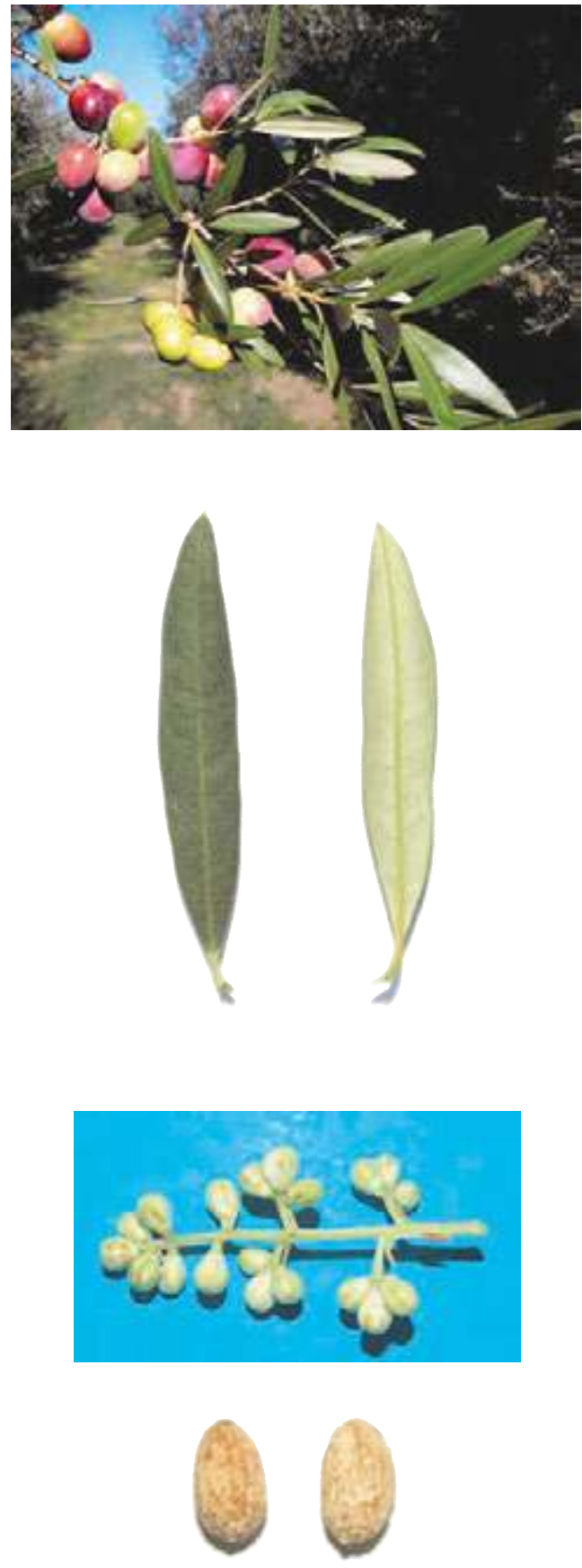


\section{Biochemical Characters}

\section{Fatty Acid Composition}

Table 1. Average values (express in $\% \pm$ standard deviations) of the fatty acids methyl esters and nutritional ratios obtained from single cultivar olive oils.

$\begin{array}{llllll}\text { Myristic acid } & 0,01 \pm \mathbf{0 , 0 1} & \text { Linoleic acid }(\omega 6) & \mathbf{4 , 7 5} \pm \mathbf{3 , 0 9} & \text { Lignoceric acid } & \mathbf{0 , 0 7 \pm 0 , 0 6} \\ \text { Palmitic acid } & \mathbf{1 2 , 4 0 \pm 0 , 7 8} & \text { Linolenic acid }(\omega 3) & \mathbf{0 , 8 9} \pm \mathbf{0 , 1 8} & & \\ \text { Palmitoleic acid } & \mathbf{1 , 3 1} \pm \mathbf{0 , 2 7} & \text { Arachic acid } & \mathbf{0 , 2 9} \pm \mathbf{0 , 1 6} & \text { Unsat./satured } & \mathbf{5 , 9 3} \pm \mathbf{0 , 3 8} \\ \text { Stearic acid } & \mathbf{1 , 5 9} \pm \mathbf{0 , 3 4} & \text { Eicosenoic acid } & \mathbf{0 , 1 9} \mathbf{0 , 1 4} & \omega 6 / \omega 3 & \mathbf{5 , 1 1} \pm \mathbf{2 , 4 1} \\ \text { Oleic acid } & \mathbf{7 7 , \mathbf { 1 1 } \pm 3 , 3 1} & \text { Behenic acid } & \mathbf{0 , 0 8} \pm \mathbf{0 , 0 3} & & \end{array}$

\section{Organoleptic oil values}

Sensory Analysis (Panel test)

Comment: fruity medium, with read sensations of almond, grass and artichoke. Balanced taste sensation with a medium-high bitter and spicy. Medium-high fluidity.

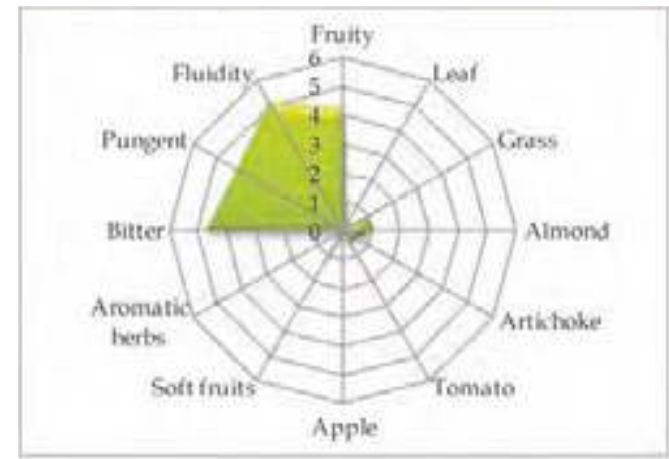

\section{Molecular Markers}

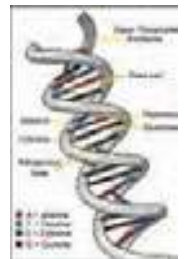

Table 2. Microsatellites (SSR) loci analyzed. For each locus the allele size (expressed in base pairs) is reported.

$\begin{array}{cccccc}D C A 09 & \text { DCA18 } & \text { GAPU59 } & \text { GAPU71A } & \text { GUPA71B } & \text { GAPU103A } \\ \mathbf{1 9 8} \mathbf{- 2 0 6} & \mathbf{1 7 7 - \mathbf { 1 7 9 }} & \mathbf{2 0 8 - \mathbf { 2 2 2 }} & \mathbf{2 1 4} \mathbf{- 2 1 4} & \mathbf{1 2 6 - \mathbf { 1 4 4 }} & \mathbf{1 5 9 - \mathbf { 1 5 9 }} \\ \text { UDO01 } & \text { UDOO3 } & \text { UDO12 } & \text { UDO28 } & \text { UDO39 } & \\ \mathbf{1 5 0 - \mathbf { 1 5 0 }} & \mathbf{1 4 3 - \mathbf { 1 4 3 }} & \mathbf{1 6 6 - \mathbf { 1 8 2 }} & \mathbf{1 4 3 - \mathbf { 1 8 2 }} & \mathbf{2 0 5 - \mathbf { 2 3 2 }} & \end{array}$

\section{References:}

1 - Lombardo N., Perri E., Muzzalupo I., et al. In: Il germoplasma olivicolo calabrese, Ist. Sper. Olivic.(2003), pp:17.

2 - Perri E., Mazzotti F., Muzzalupo I., et al. In: Relazione attività, CO.R.ASS.OL. (2003).

3 - Muzzalupo I., Chiappetta A., Benincasa C., et al Sci Hortic-Amsterdam, (2010), 126: pp. 324-329. 


\section{“Tortiglione"}

Areal distribution or origin area: Abruzzo Flesh/ pit weight ratio: high $(9,47 \pm 0,37)$ Oil content (\%): medium $(41,86 \pm 2,07)$ Purpose: oil

\section{Morphological characters}

Tree characters

Vigour: medium

Growth habit: erect-spreading

Canopy-density: medium

\section{Leaf characters}

Blade length $(\mathrm{cm})$ : medium $(4,87 \pm 0,65)$

Blade width $(\mathrm{cm})$ : medium $(\mathbf{1 , 2 1} \pm 0,15)$

Shape (length/width): elliptic - lanceolate

\section{Inflorescence characters}

Inflorescence length (cm): medium $(2,66 \pm 2,81)$

Number of flowers: low $(17,39 \pm 2,58)$

\section{Fruit characters}

Fresh weight of 100 fruits $(\mathrm{g})$ : low $(\mathbf{1}, \mathbf{6 9} \pm \mathbf{0 , 2 7})$

Shape (length/width): spherical

Symmetry: slightly asymmetric

Position of maximum transverse diameter: central

Apex: rounded

Base: rounded

Niplle: absent

Lenticels: many and small

\section{Pit characters}

Weight of 100 pits (g): low $(\mathbf{0 , 2 0} \pm \mathbf{0 , 0 4})$

Shape (length/width): ovoid

Mucron: obvious

Symmetry: slightly asymmetric

Position of maximum transverse diameter: central

Apex: rounded

Base: rounded

Surface: rugose

Number of grooves: medium
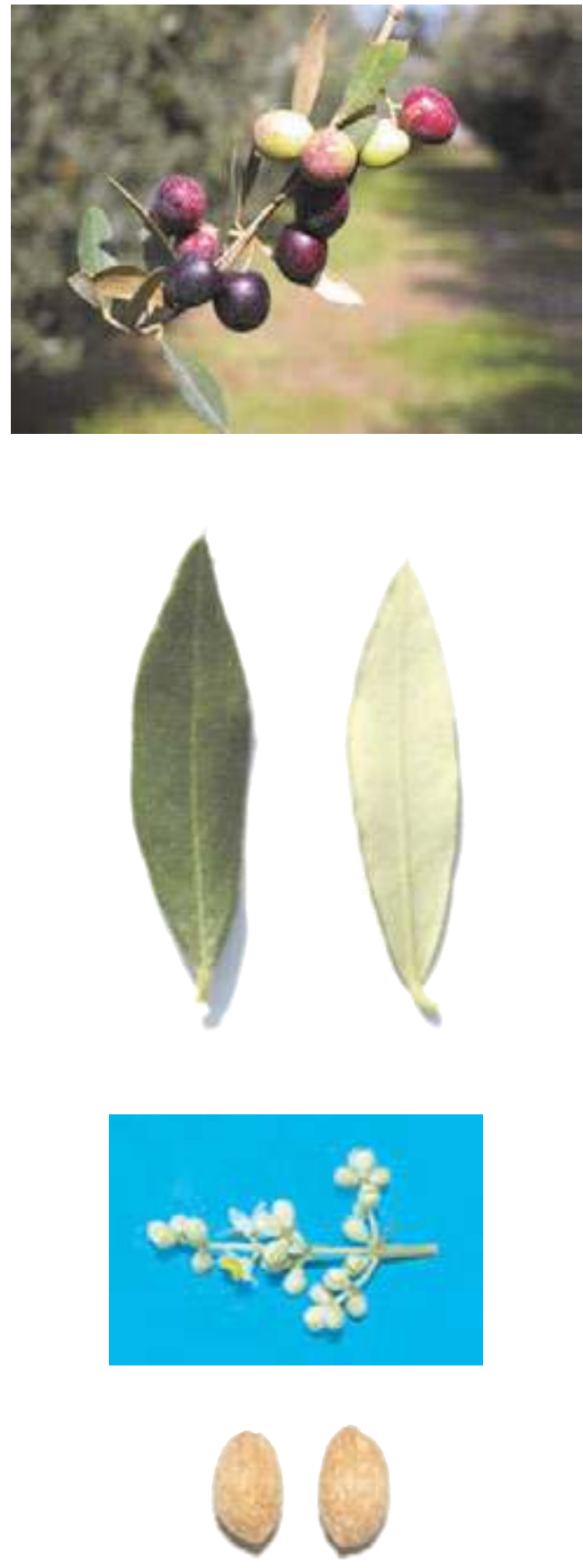


\section{Biochemical Characters}

\section{Fatty Acid Composition}

Table 1. Average values (express in $\% \pm$ standard deviations) of the fatty acids methyl esters and nutritional ratios obtained from single cultivar olive oils.

$\begin{array}{llllll}\text { Myristic acid } & \mathbf{0 , 0 1} \pm \mathbf{0 , 0 1} & \text { Linoleic acid }(\omega 6) & \mathbf{9 , 4 5} \pm \mathbf{0 , 5 0} & \text { Lignoceric acid } & \mathbf{0 , 0 4} \pm \mathbf{0 , 0 1} \\ \text { Palmitic acid } & \mathbf{1 0 , 9 0 \pm 0 , 8 7} & \text { Linolenic acid }(\omega 3) \mathbf{0 , 8 8} \pm \mathbf{0 , 0 1} & & \\ \text { Palmitoleic acid } & \mathbf{0 , 8 5} \pm \mathbf{0 , 2 1} & \text { Arachic acid } & \mathbf{0 , 4 3} \pm \mathbf{0 , 1 1} & \text { Unsat./satured } & \mathbf{6 , 4 7} \pm \mathbf{0 , 7 9} \\ \text { Stearic acid } & \mathbf{2 , 1 9} \pm \mathbf{0 , 4 0} & \text { Eicosenoic acid } & \mathbf{0 , 1 6} \pm \mathbf{0 , 1 9} & \omega 6 / \omega 3 & \mathbf{1 0 , 7 3} \pm \mathbf{0 , 7 2} \\ \text { Oleic acid } & \mathbf{7 4 , 4 6 \pm \mathbf { 1 , 9 3 }} & \text { Behenic acid } & \mathbf{0 , 1 0} \pm \mathbf{0 , 0 2} & & \end{array}$

\section{Organoleptic oil values}

Sensory Analysis (Panel test)

Comment: fruity medium, with taste of aromatic herbs. Balanced taste sensation with medium bitter and spicy. Medium-high fluidity.

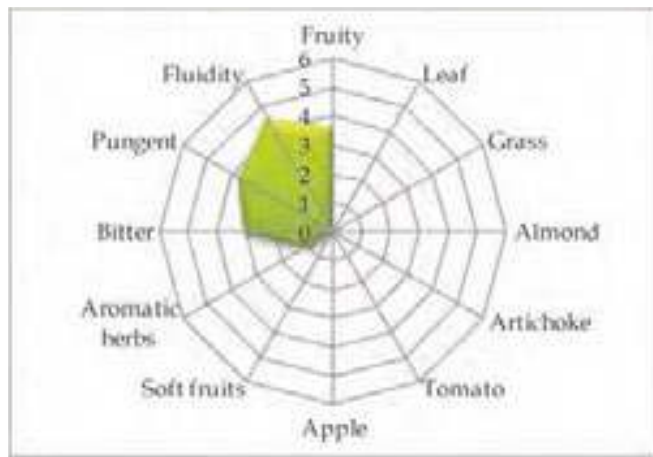

\section{Molecular Markers}

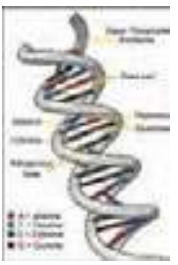

Table 2. Microsatellites (SSR) loci analyzed.

For each locus the allele size (expressed in base pairs) is reported.

$\begin{array}{cccccc}D C A 09 & \text { DCA18 } & \text { GAPU59 } & \text { GAPU71A } & \text { GUPA71B } & \text { GAPU103A } \\ \mathbf{1 9 8} \mathbf{- 2 0 6} & \mathbf{1 7 9 - \mathbf { 1 8 1 }} & \mathbf{2 0 8 - \mathbf { 2 1 2 }} & \mathbf{2 1 4} \mathbf{- 2 2 4} & \mathbf{1 2 4} \mathbf{- 1 2 6} & \mathbf{1 5 9 - \mathbf { 1 7 0 }} \\ \text { UDOO1 } & \text { UDOO3 } & \text { UDO12 } & \text { UDO28 } & \text { UDO39 } & \\ \mathbf{1 4 4 - \mathbf { 1 4 4 }} & \mathbf{1 6 6 - \mathbf { 1 6 6 }} & \mathbf{1 7 7 - \mathbf { 1 7 7 }} & \mathbf{1 5 4 - \mathbf { 2 1 0 }} & \mathbf{2 2 0 - \mathbf { 2 4 3 }} & \end{array}$

\section{References:}

1 - Pietrangeli E., Russo A., In: Olivi D’Abruzzo, Grafiche di Prinzio (1997), pp. 32-33.

2 - Muzzalupo I., Salimonti A., Caravita M. A., et al. Adv. Hort. Sci. (2008), 22(2): pp. 129-135. 


\section{“Toscanina"}

(synonymy: Oliva a grappa)

Areal distribution or origin area: Puglia

Flesh/pit weight ratio: low $(4,51 \pm 0,61)$

Oil content (\%): medium $(39,79 \pm 0,02)$

Purpose: oil

\section{Morphological characters}

Tree characters

Vigour: medium

Growth habit: spreading

Canopy-density: medium-dense

\section{Leaf characters}

Blade length $(\mathrm{cm})$ : medium $(5,44 \pm 0,80)$

Blade width $(\mathrm{cm})$ : medium $(1,47 \pm 0,27)$

Shape (length/width): elliptic

\section{Inflorescence characters}

Inflorescence length (cm): medium $(3,14 \pm 0,58)$

Number of flowers: medium $(16,62 \pm 1,87)$

\section{Fruit characters}

Fresh weight of 100 fruits $(\mathrm{g})$ : low $(\mathbf{1}, 78 \pm \mathbf{0 , 2 7})$

Shape (length/width): spherical

Symmetry: slightly asymmetric

Position of maximum transverse diameter:

central

Apex: rounded

Base: truncate

Niplle: tenuous

Lenticels: many and small

\section{Pit characters}

Weight of 100 pits (g): medium $(\mathbf{0 , 3 0} \pm \mathbf{0 , 0 4})$

Shape (length/width): ovoid

Mucron: absent

Symmetry: symmetric

Position of maximum transverse diameter:

central

Apex: pointed

Base: rounded

Surface: rugose

Number of grooves: medium
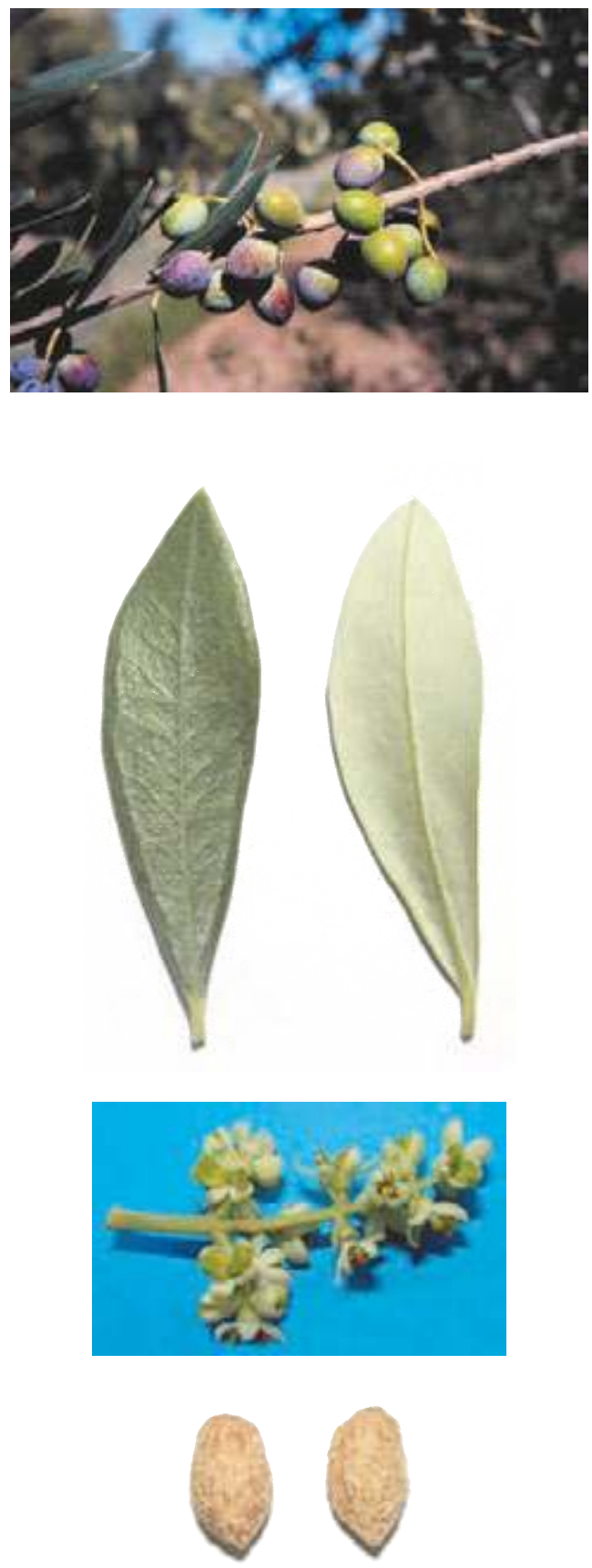


\section{Biochemical Characters}

\section{Fatty Acid Composition}

Table 1. Average values (express in $\% \pm$ standard deviations) of the fatty acids methyl esters and nutritional ratios obtained from single cultivar olive oils.

$\begin{array}{llllll}\text { Myristic acid } & \mathbf{0 , 0 1} \pm \mathbf{0 , 0 0} & \text { Linoleic acid }(\omega 6) & \mathbf{3 , 5 3} \pm \mathbf{0 , 7 0} & \text { Lignoceric acid } & \mathbf{0 , 0 4} \pm \mathbf{0 , 0 3} \\ \text { Palmitic acid } & \mathbf{1 1 , 0 2} \pm \mathbf{0 , 5 6} & \text { Linolenic acid }(\omega 3) & \mathbf{0 , 7 6} \pm \mathbf{0 , 0 2} & & \\ \text { Palmitoleic acid } & \mathbf{1 , 1 7} \pm \mathbf{0 , 1 2} & \text { Arachic acid } & \mathbf{0 , 3 2} \pm \mathbf{0 , 1 2} & \text { Unsat./satured } & \mathbf{6 , 7 4 \pm 0 , 3 0} \\ \text { Stearic acid } & \mathbf{1 , 5 1} \pm \mathbf{0 , 1 4} & \text { Eicosenoic acid } & \mathbf{0 , 1 7} \pm \mathbf{0 , 2 6} & \omega 6 / \omega 3 & \mathbf{4 , 6 3 \pm 0 , 7 8} \\ \text { Oleic acid } & \mathbf{8 , 2 4} \pm \mathbf{1 , 2 7} & \text { Behenic acid } & \mathbf{0 , 0 9} \pm \mathbf{0 , 0 2} & & \end{array}$

\section{Organoleptic oil values}

Sensory Analysis (Panel test)

Comment: fruity medium, with hints of almond and grass, read sensations of soft fruits. Balanced taste sensation with a medium-high bitter and spicy. Medium-high fluidity.

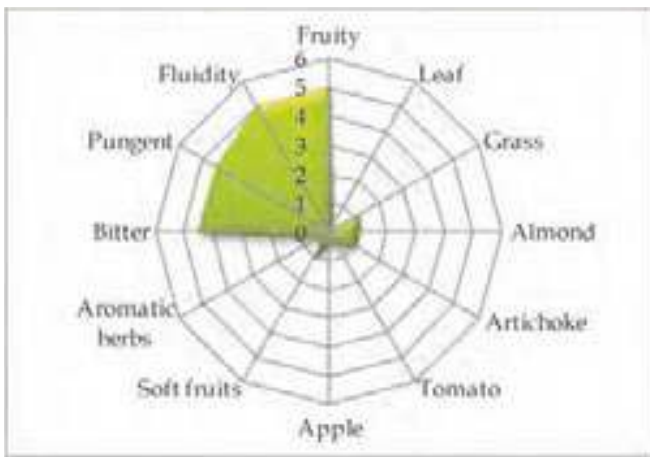

\section{Molecular Markers}

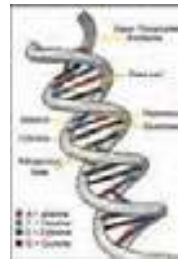

Table 2. Microsatellites (SSR) loci analyzed.

For each locus the allele size (expressed in base pairs) is reported.

\begin{tabular}{|c|c|c|c|c|c|}
\hline DCA09 & $D C A 18$ & GAPU59 & GAPU71A & GUPA71B & GAPU103A \\
\hline $172-198$ & $177-181$ & $218-218$ & $210-214$ & $124-144$ & $150-159$ \\
\hline UDO01 & UDO03 & UDO12 & UDO28 & UDO39 & \\
\hline $144-144$ & $143-143$ & $177-177$ & $161-182$ & $164-164$ & \\
\hline
\end{tabular}

\section{References:}

1 - Lombardo N., Perri E., Muzzalupo I., et al. In: Contributo alla caratterizzazione del germoplasma olivicolo pugliese, Ist. Sper. Olivic. (2004), pp. 97-100.

2 - Muzzalupo I., Stefanizzi F., Perri E. HortScience (2009), 44: pp. 582-588. 


\section{“Tunnulidda"}

(synonymy: Marsalesa, Marsalese, Marsalisa, etc.)

Areal distribution or origin area: Sicilia

Flesh/ pit weight ratio: medium $(5,93 \pm 1,02)$

Oil content (\%): medium $(49,72 \pm 1,28)$

Purpose: oil

\section{Morphological characters}

Tree characters

Vigour: medium-strong

Growth habit: erect

Canopy-density: medium-dense

\section{Leaf characters}

Blade length $(\mathrm{cm})$ : medium $(5,52 \pm 0,44)$

Blade width $(\mathrm{cm})$ : medium $(1,40 \pm 0,19)$

Shape (length/width): elliptic

\section{Inflorescence characters}

Inflorescence length (cm): medium $(2,72 \pm 0,11)$

Number of flowers: low $(\mathbf{1 5 , 7 3 \pm 2 , 5 7 )}$

\section{Fruit characters}

Fresh weight of 100 fruits $(\mathrm{g})$ : medium $(3,72 \pm 0,29)$

Shape (length/width): spherical

Symmetry: symmetric

Position of maximum transverse diameter:

central

Apex: rounded

Base: rounded

Niplle: absent

Lenticels: few and large

\section{Pit characters}

Weight of 100 pits (g): high $(0,54 \pm \mathbf{0 , 0 5})$

Shape (length/width): ovoid

Mucron: abvious

Symmetry: symmetric

Position of maximum transverse diameter:

towards apex

Apex: rounded

Base: rounded

Surface: smooth

Number of grooves: medium
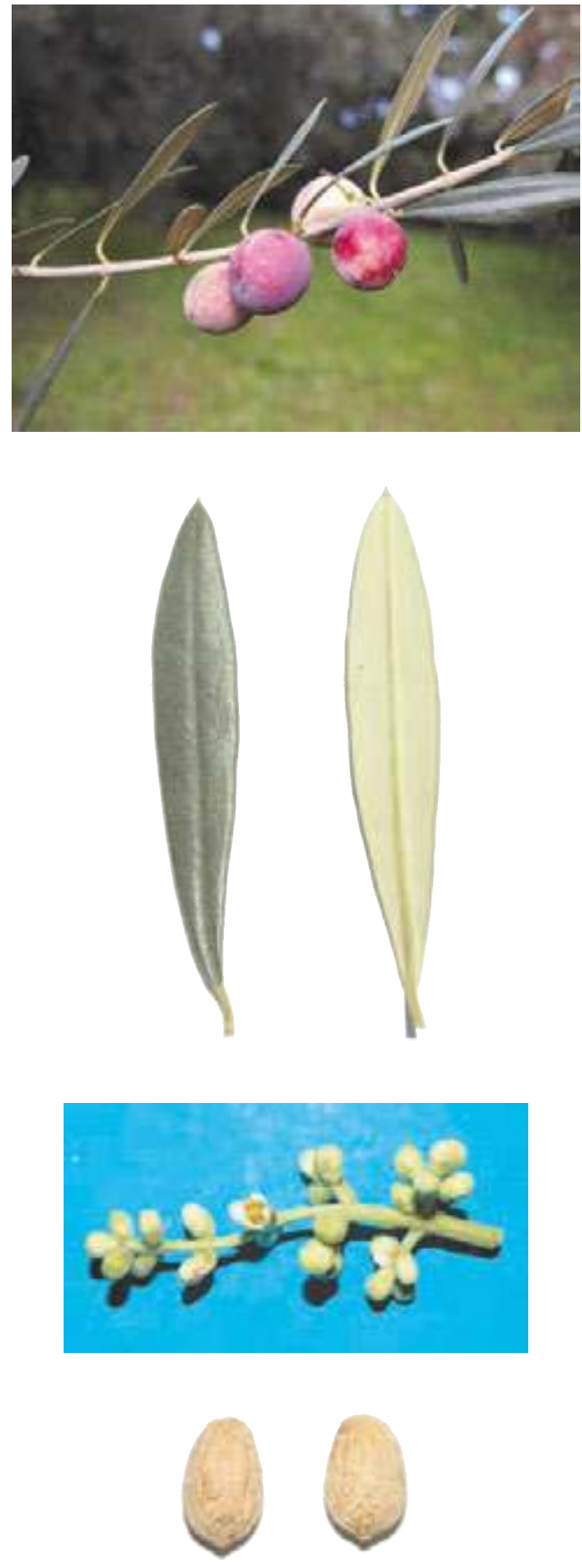


\section{Biochemical Characters}

\section{Fatty Acid Composition}

Table 1. Average values (express in $\% \pm$ standard deviations) of the fatty acids methyl esters and nutritional ratios obtained from single cultivar olive oils.

$\begin{array}{llllll}\text { Myristic acid } & \mathbf{0 , 0 1} \pm \mathbf{0 , 0 0} & \text { Linoleic acid }(\omega 6) & \mathbf{9 , 4 7} \pm \mathbf{0 , 8 1} & \text { Lignoceric acid } & \mathbf{0 , 0 3} \pm \mathbf{0 , 0 1} \\ \text { Palmitic acid } & \mathbf{7 , 1 9} \pm \mathbf{1 , 2 3} & \text { Linolenic acid }(\omega 3) \mathbf{0 , 8 6} \pm \mathbf{0 , 0 4} & & \\ \text { Palmitoleic acid } & \mathbf{0 , 9 6} \pm \mathbf{0 , 7 2} & \text { Arachic acid } & \mathbf{0 , 2 5} \pm \mathbf{0 , 0 5} & \text { Unsat./satured } & \mathbf{9 , 2 1} \pm \mathbf{0 , 0 6} \\ \text { Stearic acid } & \mathbf{2 , 3 5} \pm \mathbf{1 , 1 6} & \text { Eicosenoic acid } & \mathbf{0 , 0 3} \pm \mathbf{0 , 0 0} & \omega 6 / \omega 3 & \mathbf{1 1 , 0 7} \pm \mathbf{1 , 4 4} \\ \text { Oleic acid } & \mathbf{7 7 , 8 6} \pm \mathbf{2 , 4 9} & \text { Behenic acid } & \mathbf{0 , 0 6} \pm \mathbf{0 , 0 1} & & \end{array}$

\section{Organoleptic oil values}

Sensory Analysis (Panel test)

Comment: fruity medium, with hints of almond and read sensations of grass and artichoke. Balanced taste sensation with a medium bitter and spicy. Medium-high fluidity.

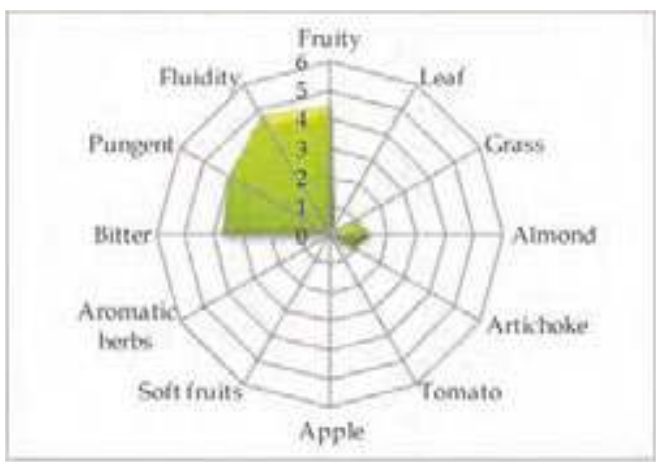

\section{Molecular Markers}

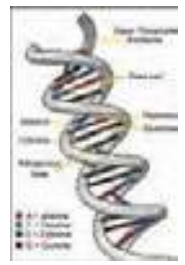

Table 2. Microsatellites (SSR) loci analyzed.

For each locus the allele size (expressed in base pairs) is reported.

$\begin{array}{cccccc}D C A 09 & D C A 18 & \text { GAPU59 } & \text { GAPU71A } & \text { GUPA71B } & \text { GAPU103A } \\ \mathbf{1 7 2} \mathbf{- 1 8 2} & \mathbf{1 6 9 - \mathbf { 1 8 1 }} & \mathbf{2 0 8 - \mathbf { 2 1 2 }} & \mathbf{2 1 4 - \mathbf { 2 1 4 }} & \mathbf{1 2 6 - \mathbf { 1 4 4 }} & \mathbf{1 5 9 - \mathbf { 1 8 4 }} \\ \text { UDOO1 } & \text { UDOO3 } & \text { UDO12 } & \text { UDO28 } & \text { UDO39 } & \\ \mathbf{1 4 4 - \mathbf { 1 4 4 }} & \mathbf{1 5 0 - \mathbf { 1 5 0 }} & \mathbf{1 6 6 - \mathbf { 1 9 3 }} & \mathbf{1 8 2} \mathbf{- 1 8 2} & \mathbf{2 0 5 - \mathbf { 2 0 5 }} & \end{array}$

\section{References:}

1 - Bottari V., Spina P. In: Le varietà di olivo coltivate in Sicilia, Ann. Sper. Agr. (1953), 7: pp. 937-1004.

2 - Muzzalupo I., Stefanizzi F., Perri E. HortScience (2009), 44: pp. 582-588. 


\section{“Vaddara"}

(synonymy: Agghiarica, Gaddarica, Gallarichi, Vaddarica, etc.)

Areal distribution or origin area: Sicilia

Flesh/ pit weight ratio: medium $(5,42 \pm 1,11)$

Oil content (\%): medium $(40,84 \pm 2,84)$

Purpose: table

\section{Morphological characters}

Tree characters

Vigour: medium-strong

Growth habit: erect-spreading

Canopy-density: medium-dense

\section{Leaf characters}

Blade length $(\mathrm{cm})$ : medium $(5,02 \pm 1,06)$

Blade width $(\mathrm{cm})$ : medium $(1,31 \pm 0,25)$

Shape (length/width): elliptic

\section{Inflorescence characters}

Inflorescence length (cm): short $(2,14 \pm 0,49)$

Number of flowers: medium $(\mathbf{1 9 , 4 4} \pm 3,18)$

\section{Fruit characters}

Fresh weight of 100 fruits $(\mathrm{g})$ : medium $(3,37 \pm 0,54)$

Shape (length/width): ovoid

Symmetry: symmetric

Position of maximum transverse diameter:

\section{towards base}

Apex: pointed

Base: truncate

Niplle: tenuous

Lenticels: many and large

\section{Pit characters}

Weight of 100 pits (g): high $(0,53 \pm 0,09)$

Shape (length/width): elliptic

Mucron: obvious

Symmetry: slightly asymmetric

Position of maximum transverse diameter:

towards apex

Apex: rounded

Base: pointed

Surface: rugose

Number of grooves: low
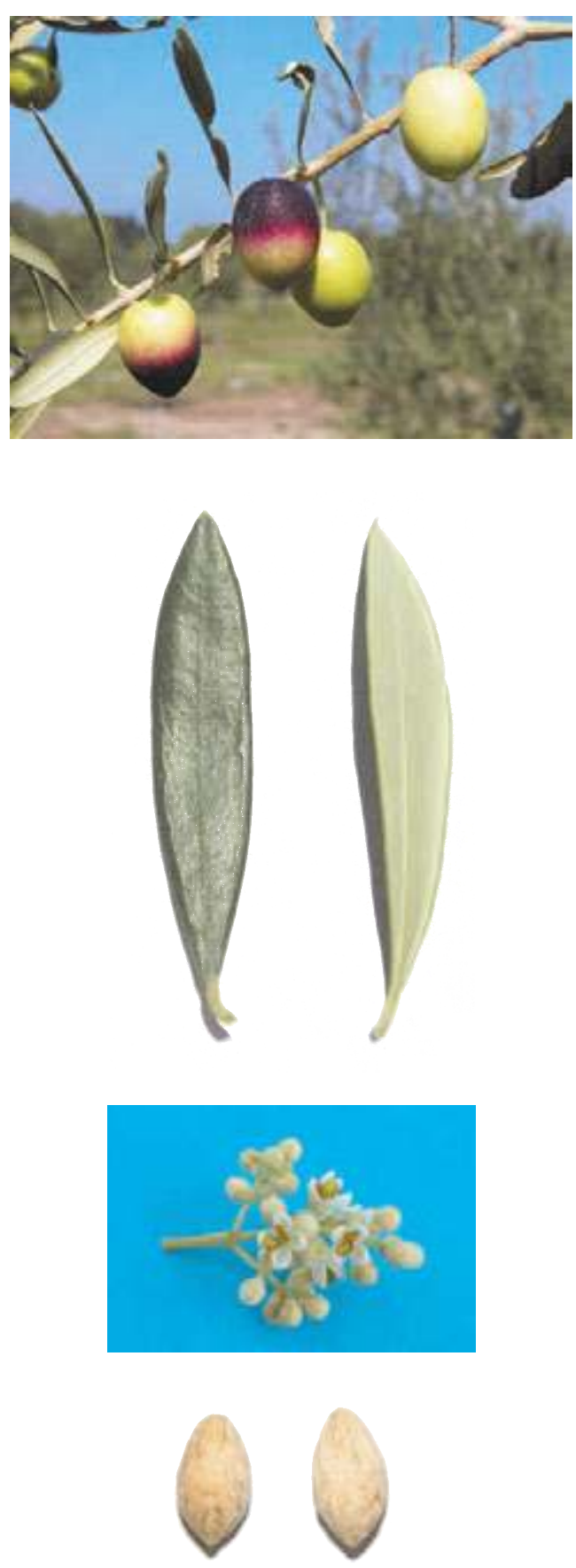


\section{Biochemical Characters}

\section{Fatty Acid Composition}

Table 1. Average values (express in $\% \pm$ standard deviations) of the fatty acids methyl esters and nutritional ratios obtained from single cultivar olive oils.

\begin{tabular}{|c|c|c|c|c|c|}
\hline Myristic acid & $0,01 \pm 0,01$ & Linoleic acid $(\omega 6)$ & $19,14 \pm 5,05$ & Lignoceric acid & $0,08 \pm 0,01$ \\
\hline Palmitic acid & $15,41 \pm 2,44$ & Linolenic acid $(\omega 3)$ & $0,74 \pm 0,06$ & & \\
\hline lmitoleic acid & $3,17 \pm 1,27$ & Arachic acid & $0,32 \pm 0,01$ & Unsat./satured & $4,57 \pm 0,64$ \\
\hline eari & $1,73 \pm 0,12$ & Eicosenoic acid & $0,25 \pm 0,07$ & $\omega 6 / \omega 3$ & $25,84 \pm 4,63$ \\
\hline leic acid & $55,68 \pm 4,84$ & Behenic acid & $0,11 \pm 0,02$ & & \\
\hline
\end{tabular}

\section{Organoleptic oil values}

Sensory Analysis (Panel test)

Comment: fruity medium, with taste of aromatic herbs. Balanced taste sensation with medium bitter and spicy. Medium fluidity.

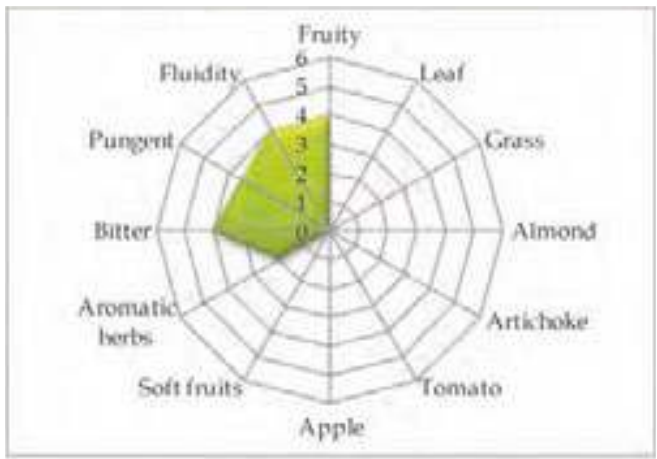

\section{Molecular Markers}

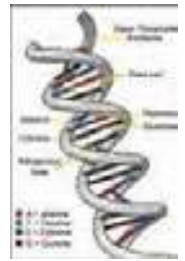

Table 2. Microsatellites (SSR) loci analyzed.

For each locus the allele size (expressed in base pairs) is reported.

$\begin{array}{cccccc}D C A 09 & \text { DCA18 } & \text { GAPU59 } & \text { GAPU71A } & \text { GUPA71B } & \text { GAPU103A } \\ \mathbf{1 9 8} \mathbf{- 1 9 8} & \mathbf{1 7 9 - \mathbf { 1 7 9 }} & \mathbf{2 0 8 - \mathbf { 2 1 2 }} & \mathbf{2 1 0 - \mathbf { 2 1 4 }} & \mathbf{1 2 4 - \mathbf { 1 2 4 }} & \mathbf{1 5 7 - \mathbf { 1 5 9 }} \\ \text { UDOO1 } & \text { UDO03 } & \text { UDO12 } & \text { UDO28 } & \text { UDO39 } & \\ \mathbf{1 4 4 - \mathbf { 1 4 4 }} & \mathbf{1 5 0 - \mathbf { 1 5 0 }} & \mathbf{1 7 7 - \mathbf { 1 9 3 }} & \mathbf{1 5 4} \mathbf{- 1 8 2} & \mathbf{1 6 4 - \mathbf { 2 0 5 }} & \end{array}$

\section{References:}

1 - Caruso T., Cartabellotta D., Motisi A., et all. In: Cultivar di olivo siciliane, Università degli Studi di Palermo (2007), pp. 182-186.

2 - Muzzalupo I., Stefanizzi F., Perri E. HortScience (2009), 44: pp. 582-588. 


\section{“Vallanella"}

(synonymy: Vallanella Ravebianca

Areal distribution or origin area: Lazio

Flesh/pit weight ratio: medium $(6,11 \pm 2,83)$

Oil content (\%): medium $(44,29 \pm 1,80)$

Purpose: dual purpose

\section{Morphological characters}

Tree characters

Vigour: medium

Growth habit: spreading

Canopy-density: medium-dense

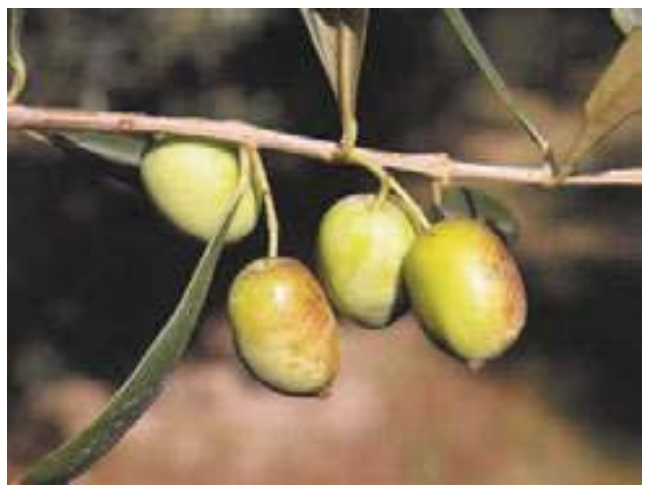

\section{Leaf characters}

Blade length (cm): long $(7,02 \pm 0,42)$

Blade width $(\mathrm{cm})$ : medium $(\mathbf{1}, 49 \pm 0,15)$

Shape (length/width): elliptic-lanceolata

\section{Inflorescence characters}

Inflorescence length $(\mathrm{cm})$ : medium $(2,76 \pm 1,63)$

Number of flowers: low $(15,50 \pm 4,27)$

\section{Fruit characters}

Fresh weight of 100 fruits $(\mathrm{g})$ : medium $(3,93 \pm 1,99)$

Shape (length/width): ovoid

Symmetry: asymmetric

Position of maximum transverse diameter:

central

Apex: rounded

Base: truncate

Niplle: obvious

Lenticels: many and small

\section{Pit characters}

Weight of 100 pits (g): high $(\mathbf{0 , 5 7} \pm \mathbf{0 , 0 8})$

Shape (length/width): elliptic

Mucron: obvious

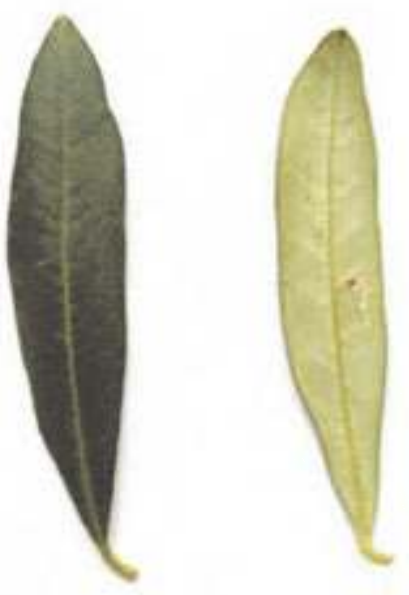

Symmetry: slightly asymmetric

Position of maximum transverse diameter:

central

Apex: rounded

Base: rounded

Surface: rugose

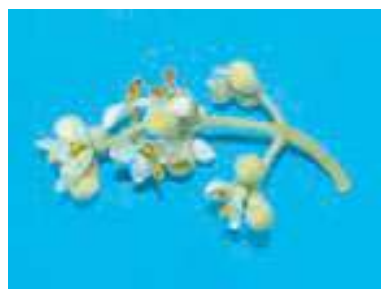

Number of grooves: medium 


\section{Biochemical Characters}

\section{Fatty Acid Composition}

Table 1. Average values (express in $\% \pm$ standard deviations) of the fatty acids methyl esters and nutritional ratios obtained from single cultivar olive oils.

$\begin{array}{llllll}\text { Myristic acid } & \mathbf{0 , 0 1} \pm \mathbf{0 , 0 1} & \text { Linoleic acid }(\omega 6) & \mathbf{8 , 4 8} \pm \mathbf{2 , 1 2} & \text { Lignoceric acid } & \mathbf{0 , 0 3} \pm \mathbf{0 , 0 1} \\ \text { Palmitic acid } & \mathbf{1 0 , 3 5 \pm 0 , 6 8} & \text { Linolenic acid }(\omega 3) & \mathbf{0 , 9 8} \pm \mathbf{0 , 1 2} & & \\ \text { Palmitoleic acid } & \mathbf{0 , 4 2} \pm \mathbf{0 , 0 5} & \text { Arachic acid } & \mathbf{0 , 4 8} \pm \mathbf{0 , 1 5} & \text { Unsat./satured } & \mathbf{6 , 7 7} \pm \mathbf{0 , 8 9} \\ \text { Stearic acid } & \mathbf{2 , 3 2} \pm \mathbf{0 , 8 3} & \text { Eicosenoic acid } & \mathbf{0 , 2 8} \pm \mathbf{0 , 2 5} & \omega 6 / \omega 3 & \mathbf{8 , 9 0 \pm 3 , 2 7} \\ \text { Oleic acid } & \mathbf{7 6 , 6 0 \pm 0 , 8 9} & \text { Behenic acid } & \mathbf{0 , 1 0} \pm \mathbf{0 , 0 1} & & \end{array}$

\section{Organoleptic oil values}

Sensory Analysis (Panel test)

Comment: fruity medium - light, with hints of almond and artichoke. Balanced in flavours, with hints of bitter and spicy medium intensity, medium fluidity.

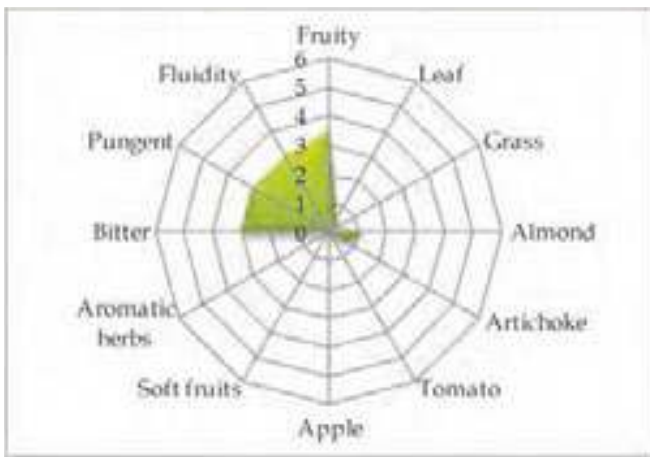

\section{Molecular Markers}

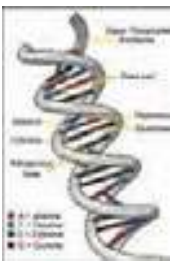

Table 2. Microsatellites (SSR) loci analyzed.

For each locus the allele size (expressed in base pairs) is reported.

\begin{tabular}{|c|c|c|c|c|c|}
\hline$D C A 09$ & $D C A 18$ & GAPU59 & GAPU71A & GUPA71B & GAPU103A \\
\hline $162-206$ & $173-175$ & $208-212$ & $214-224$ & $130-144$ & $170-170$ \\
\hline UDO01 & UDO03 & UDO12 & UDO28 & UDO39 & \\
\hline $144-144$ & $150-150$ & $166-193$ & $143-210$ & $213-232$ & \\
\hline
\end{tabular}

\section{References:}

1 - Parlati M. V., Pandolfi S. In: Olivo e Olio, (2001), 4: pp. 67-72.

2 - Muzzalupo I., Stefanizzi F., Bucci C., et al. In Acta Italus Hortus, (2011), 1: 138 -140. 


\section{“Verdello"}

Areal distribution or origin area: Sicilia

Flesh/pit weight ratio: medium $(6,03 \pm 0,08)$

Oil content (\%): medium $(50,35 \pm 0,26)$

Purpose: oil

\section{Morphological characters}

Tree characters

Vigour: medium

Growth habit: spreading-erect

Canopy-density: medium

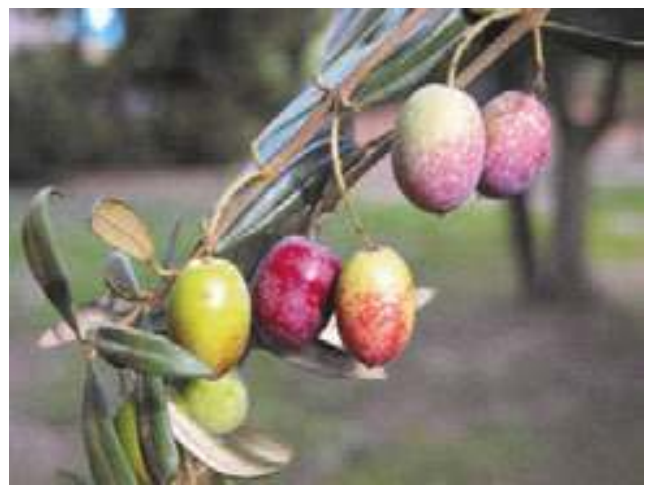

\section{Leaf characters}

Blade length (cm): short $(4,51 \pm 0,44)$

Blade width $(\mathrm{cm})$ : narrow $(0,95 \pm 0,12)$

Shape (length/width): elliptic - lanceolate

\section{Inflorescence characters}

Inflorescence length $(\mathrm{cm})$ : medium $(2,97 \pm 1,56)$

Number of flowers: medium $(21,78 \pm 3,48)$

\section{Fruit characters}

Fresh weight of 100 fruits (g): medium $(2,39 \pm 0,45)$

Shape (length/width): ovoid

Symmetry: slightly asymmetric

Position of maximum transverse diameter: central

Apex: rounded

Base: truncate

Niplle: tenuous

Lenticels: many and small

\section{Pit characters}

Weight of 100 pits (g): medium $(\mathbf{0 , 3 0} \pm \mathbf{0 , 0 1})$

Shape (length/width): elliptic

Mucron: absent

Symmetry: slightly asymmetric

Position of maximum transverse diameter:

central

Apex: pointed

Base: pointed

Surface: rugose

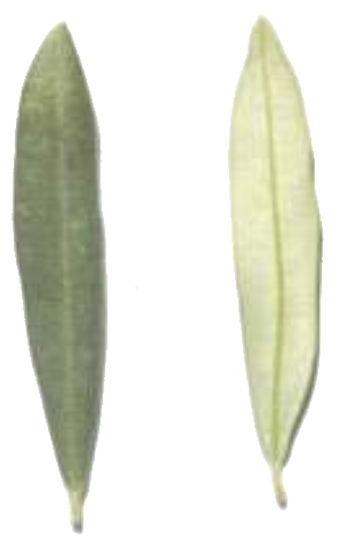

Number of grooves: medium
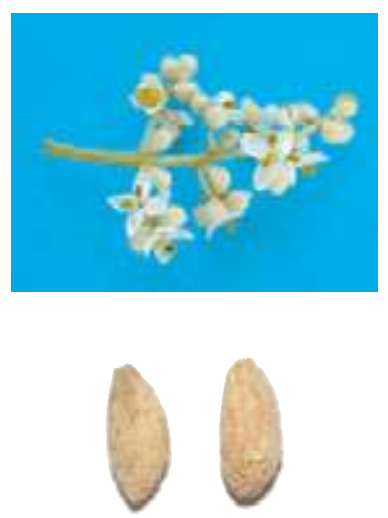


\section{Biochemical Characters}

\section{Fatty Acid Composition}

Table 1. Average values (express in $\% \pm$ standard deviations) of the fatty acids methyl esters and nutritional ratios obtained from single cultivar olive oils.

\begin{tabular}{|c|c|c|c|c|c|}
\hline Myristic acid & $0,01 \pm 0,00$ & Linoleic acid $(\omega 6)$ & $13,05 \pm 1,38$ & Lignoceric acid & $0,04 \pm 0,02$ \\
\hline Palmitic acid & $13,12 \pm 0,67$ & Linolenic acid $(\omega 3)$ & $0,91 \pm 0,07$ & & \\
\hline Palmitoleic acid & $0,88 \pm 0,17$ & Arachic acid & $0,30 \pm 0,03$ & Unsat./satured & $5,58 \pm 0,44$ \\
\hline earic acid & $1,78 \pm 0,34$ & Eicosenoic acid & $0,16 \pm 0,18$ & $\omega 6 / \omega 3$ & $14,40 \pm 1,86$ \\
\hline leic acid & $68,98 \pm 1,88$ & Behenic acid & $0,09 \pm 0,02$ & & \\
\hline
\end{tabular}

\section{Organoleptic oil values}

Sensory Analysis (Panel test)

Comment: fruity medium-high, with taste of artichoke and almond, read sensations of grass and leaves. Balanced taste sensation with medium bitter and spicy. Medium-high fluidity.

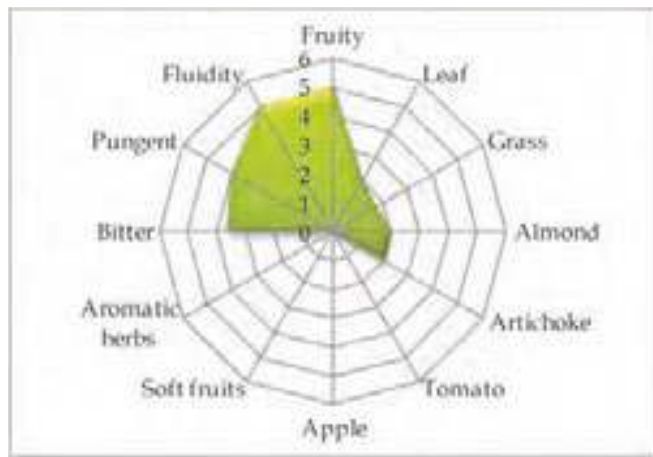

\section{Molecular Markers}

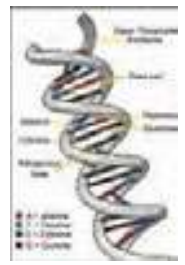

Table 2. Microsatellites (SSR) loci analyzed.

For each locus the allele size (expressed in base pairs) is reported.

$\begin{array}{cccccc}D C A 09 & D C A 18 & \text { GAPU59 } & \text { GAPU71A } & \text { GUPA71B } & \text { GAPU103A } \\ \mathbf{1 8 2} \mathbf{- 2 0 6} & \mathbf{1 8 1} \mathbf{- 1 8 1} & \mathbf{2 1 2} \mathbf{- 2 2 2} & \mathbf{2 1 0 - \mathbf { 2 1 4 }} & \mathbf{1 2 4} \mathbf{- 1 2 6} & \mathbf{1 5 9 - \mathbf { 1 7 0 }} \\ \text { UDOO1 } & \text { UDOO3 } & \text { UDO12 } & \text { UDO28 } & \text { UDO39 } & \\ \mathbf{1 4 4 - \mathbf { 1 4 4 }} & \mathbf{1 5 0 - \mathbf { 1 5 0 }} & \mathbf{1 6 6 - \mathbf { 1 6 6 }} & \mathbf{1 4 3} \mathbf{- 1 4 3} & \mathbf{1 7 0 - \mathbf { 2 2 0 }} & \end{array}$

\section{References:}

1 - Caruso T., Cartabellotta D., Motisi A., et all. In: Cultivar di olivo siciliane, Università degli Studi di Palermo (2007), pp. 124-128.

2 - Muzzalupo I., Stefanizzi F., Perri E. HortScience (2009), 44: pp. 582-588. 


\section{"Vocio"}

(synonymy: Corniolo da curare, Corniolo dolce, Olivo da pasto, Olivoce, Voce, etc.)

Areal distribution or origin area: Umbria

Flesh/pit weight ratio: high $(7,76 \pm 0,49)$

Oil content (\%): medium $(45,82 \pm 0,08)$

Purpose: dual purpose

\section{Morphological characters}

Tree characters

Vigour: medium-weak

Growth habit: erect-spreading

Canopy-density: medium

\section{Leaf characters}

Blade length (cm): medium $(5,45 \pm 0,47)$

Blade width $(\mathrm{cm})$ : medium $(\mathbf{1 , 2 0} \pm \mathbf{0 , 1 7})$

Shape (length/width): elliptic-lanceolate

\section{Inflorescence characters}

Inflorescence length (cm): medium $(2,55 \pm 1,07)$

Number of flowers: low $(\mathbf{1 5 , 1 7} \pm \mathbf{1}, \mathbf{5 4})$

\section{Fruit characters}

Fresh weight of 100 fruits $(\mathrm{g})$ : medium $(3,86 \pm 0,29)$

Shape (length/width): spherical

Symmetry: symmetric

Position of maximum transverse diameter:

central

Apex: rounded

Base: truncate

Niplle: absent

Lenticels: many and small

\section{Pit characters}

Weight of 100 pits (g): medium $(\mathbf{0 , 4 4} \pm \mathbf{0 , 0 1})$

Shape (length/width): ovoid

Mucron: obvious

Symmetry: slightly asymmetric

Position of maximum transverse diameter:

towards apex

Apex: rounded

Base: truncate

Surface: rugose

Number of grooves: low
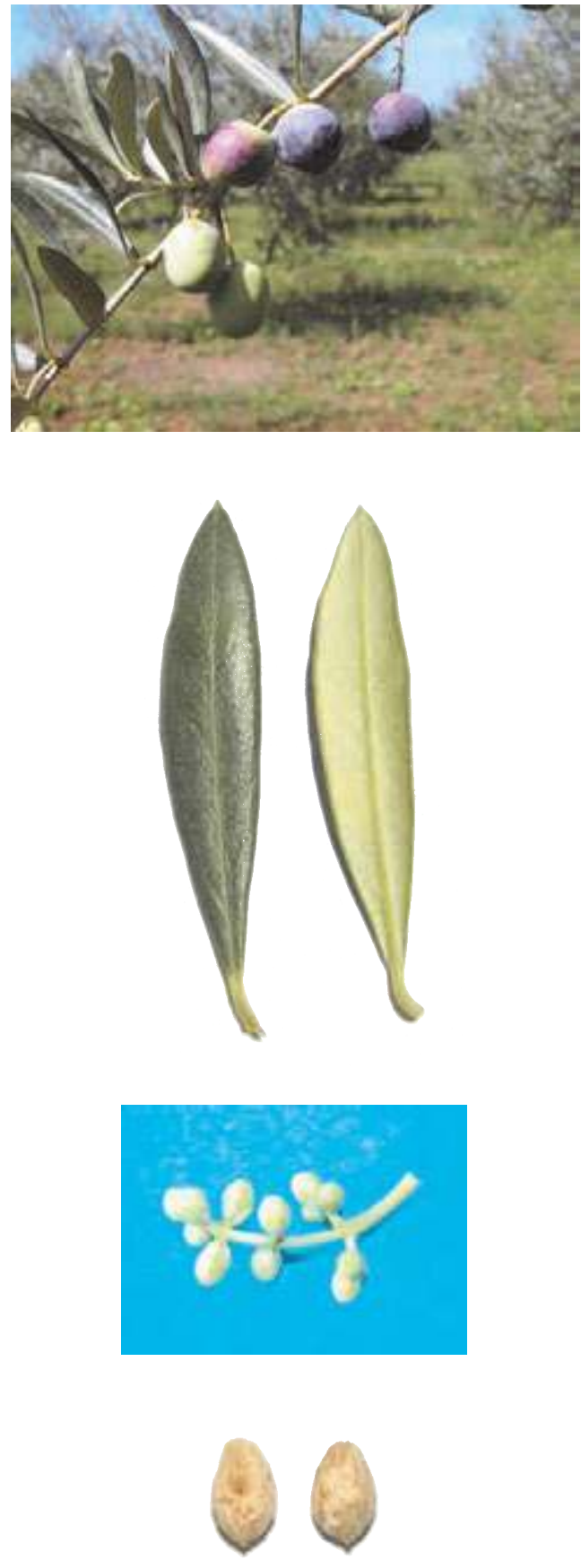


\section{Biochemical Characters}

\section{Fatty Acid Composition}

Table 1. Average values (express in $\% \pm$ standard deviations) of the fatty acids methyl esters and nutritional ratios obtained from single cultivar olive oils.

\begin{tabular}{|c|c|c|c|c|c|}
\hline Myristic acid & $0,01 \pm 0,00$ & Linoleic acid $(\omega 6)$ & $18,38 \pm 0,56$ & Lignoceric acid & $0,08 \pm 0,01$ \\
\hline Palmitic acid & $14,42 \pm 0,49$ & Linolenic acid $(\omega 3)$ & $0,66 \pm 0,02$ & & \\
\hline Palmitoleic acid & $1,23 \pm 0,04$ & Arachic acid & $0,39 \pm 0,03$ & Unsat./satured & $4,81 \pm 0,14$ \\
\hline teari & $2,13 \pm 0,04$ & Eicosenoic acid & $0,35 \pm 0,04$ & $\omega 6 / \omega 3$ & $28,05 \pm 0,06$ \\
\hline leic acid & $60,80 \pm 0,07$ & Behenic acid & $0,15 \pm 0,02$ & & \\
\hline
\end{tabular}

\section{Organoleptic oil values}

Sensory Analysis (Panel test)

Comment: fruity medium-high, with hints of almond, grass and artichoke, read sensations of leaves. Balanced taste sensation with a medium bitter and spicy. Medium-high fluidity.

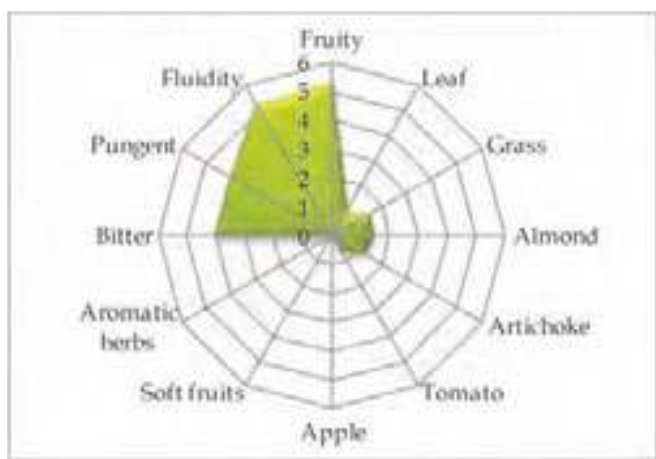

\section{Molecular Markers}

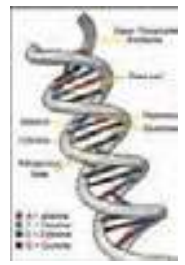

Table 2. Microsatellites (SSR) loci analyzed.

For each locus the allele size (expressed in base pairs) is reported.

\begin{tabular}{|c|c|c|c|c|c|}
\hline DCA09 & DCA18 & GAPU59 & GAPU71A & $G U P A 71 B$ & GAPU103A \\
\hline $198-206$ & $177-181$ & $214-214$ & $214-214$ & $130-144$ & $150-157$ \\
\hline UDO01 & UDO03 & UDO12 & UDO28 & UDO39 & \\
\hline $140-140$ & $143-143$ & $166-177$ & $161-205$ & $205-213$ & \\
\hline
\end{tabular}

\section{References:}

1 - Pannelli G., Alfei B., D’ Ambrosio A., et al. In: Varietà di olivo in Umbria, Ed. Pliniana (2000), pp. 1-6.

2 - Muzzalupo I., Stefanizzi F., Bucci C., et al. In Acta Italus Hortus, (2011), 1: 138 -140. 


\section{“Zaituna"}

(synonymy: Saracena, Saracinesca, Siracusana, Verdisi, Zaituni, etc.)

Areal distribution or origin area: Sicilia

Flesh/pit weight ratio: medium $(\mathbf{6 , 2 4} \pm \mathbf{1 , 6 0})$

Oil content (\%): medium $(49,05 \pm 1,59)$

Purpose: table

\section{Morphological characters}

Tree characters

Vigour: medium

Growth habit: erect

Canopy-density: sparse

\section{Leaf characters}

Blade length $(\mathrm{cm})$ : medium $(5,56 \pm 0,45)$

Blade width $(\mathrm{cm})$ : medium $(\mathbf{1 , 2 7} \pm \mathbf{0 , 1 1})$

Shape (length/width): elliptic-lanceolate

\section{Inflorescence characters}

Inflorescence length (cm): short $(2,35 \pm 1,50)$

Number of flowers: low $(\mathbf{1 2 , 5 8} \pm \mathbf{2 , 7 0})$

\section{Fruit characters}

Fresh weight of 100 fruits $(\mathrm{g})$ : high $(\mathbf{4 , 0 0} \pm \mathbf{1 , 9 9})$

Shape (length/width): ovoid

Symmetry: slightly asymmetric

Position of maximum transverse diameter:

central

Apex: rounded

Base: rounded

Niplle: tenuous

Lenticels: few and small

\section{Pit characters}

Weight of 100 pits (g): high $(\mathbf{0 , 5 4} \pm \mathbf{0 , 1 8})$

Shape (length/width): ovoid

Mucron: absent

Symmetry: slightly asymmetric

Position of maximum transverse diameter:

towards apex

Apex: rounded

Base: pointed

Surface: rugose

Number of grooves: medium
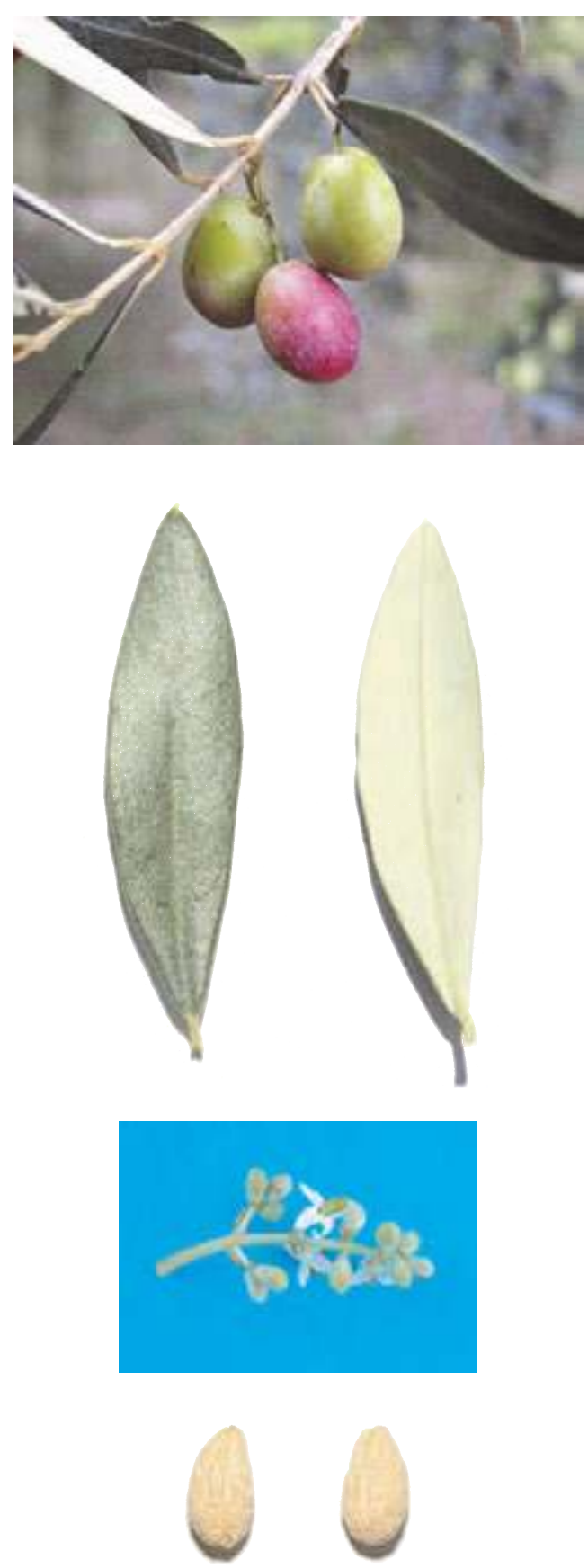


\section{Biochemical Characters}

\section{Fatty Acid Composition}

Table 1. Average values (express in $\% \pm$ standard deviations) of the fatty acids methyl esters and nutritional ratios obtained from single cultivar olive oils.

$\begin{array}{llllll}\text { Myristic acid } & \mathbf{0 , 0 3} \pm \mathbf{0 , 0 1} & \text { Linoleic acid }(\omega 6) & \mathbf{3 , 9 2} \pm \mathbf{1 , 3 7} & \text { Lignoceric acid } & \mathbf{0 , 0 6} \pm \mathbf{0 , 0 1} \\ \text { Palmitic acid } & \mathbf{1 1 , 4 4} \pm \mathbf{0 , 7 7} & \text { Linolenic acid }(\omega 3) \mathbf{0 , 8 4} \pm \mathbf{0 , 1 3} & & \\ \text { Palmitoleic acid } & \mathbf{0 , 6 8} \pm \mathbf{0 , 1 3} & \text { Arachic acid } & \mathbf{0 , 2 4} \pm \mathbf{0 , 0 5} & \text { Unsat./satured } & \mathbf{6 , 1 9} \pm \mathbf{0 , 2 5} \\ \text { Stearic acid } & \mathbf{2 , 0 5} \pm \mathbf{0 , 5 0} & \text { Eicosenoic acid } & \mathbf{0 , 0 4} \pm \mathbf{0 , 0 2} & \omega 6 / \omega 3 & \mathbf{4 , 6 0 \pm 0 , 9 1} \\ \text { Oleic acid } & \mathbf{7 9 , 7 7 \pm 0 , 7 9} & \text { Behenic acid } & \mathbf{0 , 1 1} \pm \mathbf{0 , 0 8} & & \end{array}$

\section{Organoleptic oil values}

Sensory Analysis (Panel test)

Comment: fruity medium-high, with good sensation of grass, artichoke, almond and tomato. Balanced in flavours, with hints of bitter and spicy medium-high intensity. Medium-high fluidity.

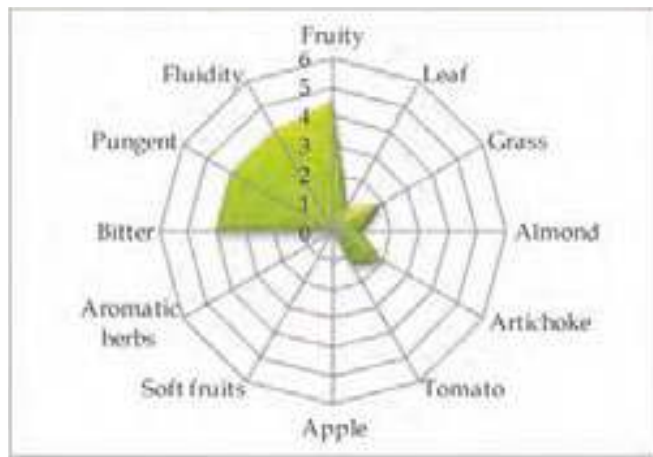

\section{Molecular Markers}

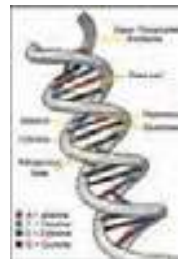

Table 2. Microsatellites (SSR) loci analyzed. For each locus the allele size (expressed in base pairs) is reported.

$\begin{array}{cccccc}D C A 09 & \text { DCA18 } & \text { GAPU59 } & \text { GAPU71A } & \text { GUPA71B } & \text { GAPU103A } \\ \mathbf{1 8 2} \mathbf{- 2 0 6} & \mathbf{1 7 7 - \mathbf { 1 7 7 }} & \mathbf{2 1 2} \mathbf{- 2 1 2} & \mathbf{2 1 4} \mathbf{- 2 1 4} & \mathbf{1 2 4} \mathbf{- 1 4 4} & \mathbf{1 5 0 - \mathbf { 1 7 0 }} \\ \text { UDOO1 } & \text { UDOO3 } & \text { UDO12 } & \text { UDO28 } & \text { UDO39 } & \\ \mathbf{1 4 0 - \mathbf { 1 4 0 }} & \mathbf{1 5 0 - \mathbf { 1 5 0 }} & \mathbf{1 6 6 - \mathbf { 1 9 3 }} & \mathbf{1 8 2} \mathbf{- 1 8 2} & \mathbf{1 0 8} \mathbf{- 1 0 8} & \end{array}$

\section{References:}

1 - Bottari V., Spina P. In: Le varietà di olivo coltivate in Sicilia, Ann. Sper. Agr. (1953), 7: pp. 937-1004.

2 - Muzzalupo I., Stefanizzi F., Perri E. HortScience, (2009), 44: pp. 582-588. 


\section{“Zimbimbo"}

(synonymy: Nzimbibolo)

Areal distribution or origin area: Puglia

Flesh/pit weight ratio: medium $(5,66 \pm 1,34)$

Oil content (\%): medium $(39,72 \pm 1,98)$

Purpose: oil

\section{Morphological characters}

Tree characters

Vigour: strong

Growth habit: spreading-erect

Canopy-density: dense

\section{Leaf characters}

Blade length (cm): medium $(5,54 \pm 0,36)$

Blade width (cm): broad $(\mathbf{1}, 62 \pm \mathbf{0 , 1 6})$

Shape (length/width): elliptic

\section{Inflorescence characters}

Inflorescence length (cm): short $(2,13 \pm 0,38)$

Number of flowers: low $(13,92 \pm 0,96)$

\section{Fruit characters}

Fresh weight of 100 fruits (g): low $(2,00 \pm 0,32)$

Shape (length/width): ovoid

Symmetry: slightly asymmetric

Position of maximum transverse diameter:

central

Apex: rounded

Base: rounded

Niplle: tenuous

Lenticels: many and small

\section{Pit characters}

Weight of 100 pits (g): medium $(0,35 \pm 0,04)$

Shape (length/width): elliptic

Mucron: obvious

Symmetry: asymmetric

Position of maximum transverse diameter:

central

Apex: pointed

Base: pointed

Surface: rugose

Number of grooves: low
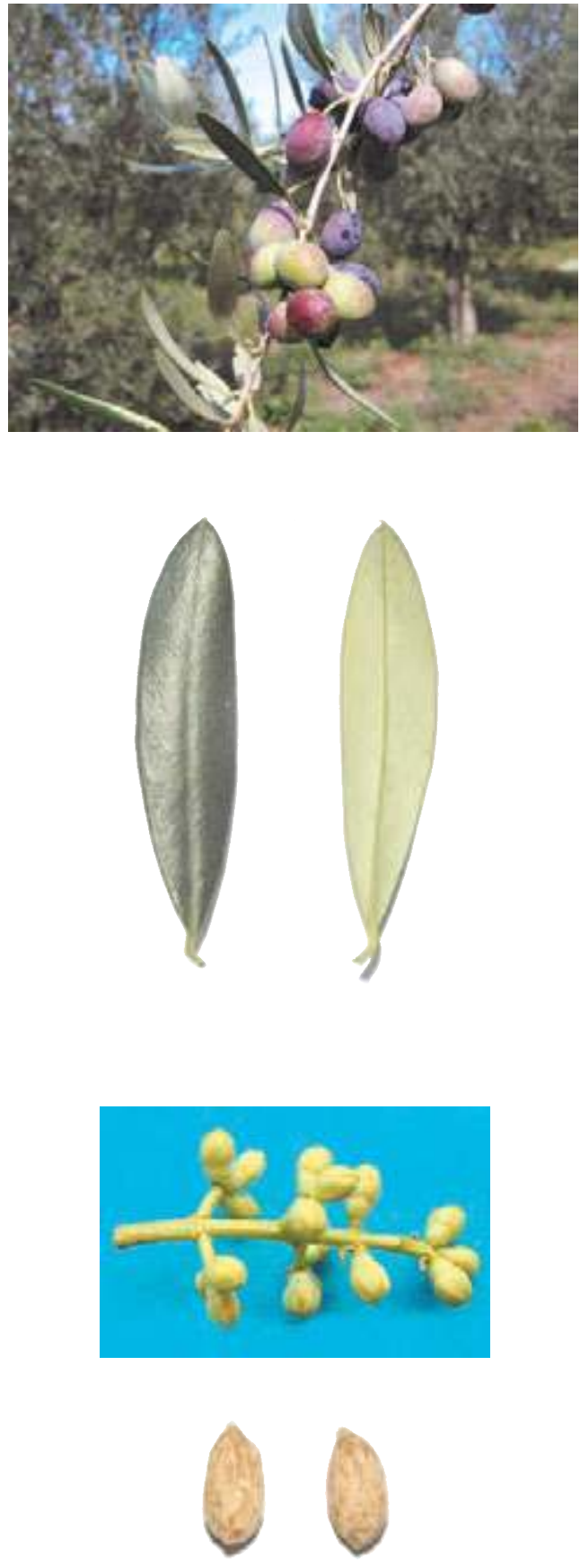


\section{Biochemical Characters}

\section{Fatty Acid Composition}

Table 1. Average values (express in $\% \pm$ standard deviations) of the fatty acids methyl esters and nutritional ratios obtained from single cultivar olive oils.

\begin{tabular}{|c|c|c|c|c|c|}
\hline Myristic acid & $0,01 \pm 0,01$ & Linoleic acid $(\omega 6)$ & $10,88 \pm 1,12$ & Lignoceric acid & $0,07 \pm 0,00$ \\
\hline Palmitic acid & $12,68 \pm 1,28$ & Linolenic acid $(\omega 3$ & $0,53 \pm 0,06$ & & \\
\hline Palmitoleic acid & $2,41 \pm 0,54$ & Arachic acid & $0,24 \pm 0,04$ & Unsat./satured & $5,97 \pm 0,55$ \\
\hline tearic & $1,12 \pm 0,06$ & Eicosenoic acid & $0,29 \pm 0,02$ & $\omega 6 / \omega 3$ & $20,61 \pm 4,53$ \\
\hline leic acid & $69,35 \pm 1,10$ & Behenic acid & $0,07 \pm 0,02$ & & \\
\hline
\end{tabular}

\section{Organoleptic oil values}

Sensory Analysis (Panel test)

Comment: fruity medium-high, with hints of artichoke, read sensations of grass, tomato and almond. Balanced taste sensation with a medium bitter and spicy. Medium fluidity.

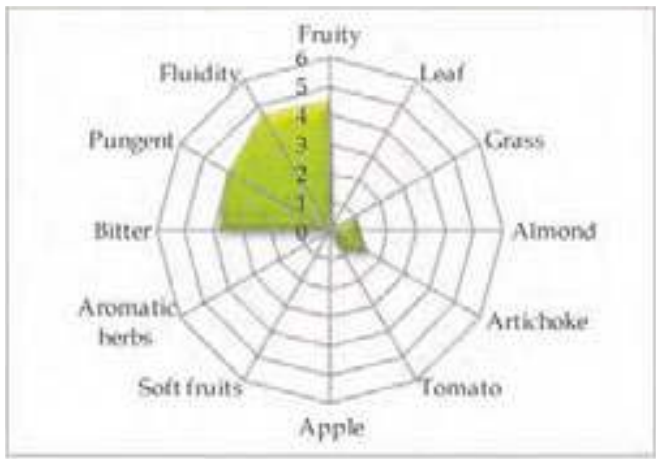

\section{Molecular Markers}

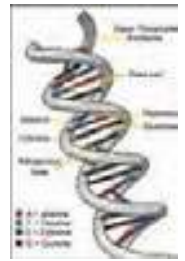

Table 2. Microsatellites (SSR) loci analyzed.

For each locus the allele size (expressed in base pairs) is reported.

$\begin{array}{cccccc}D C A 09 & \text { DCA18 } & \text { GAPU59 } & \text { GAPU71A } & \text { GUPA71B } & \text { GAPU103A } \\ \mathbf{1 9 8} \mathbf{- 1 9 8} & \mathbf{1 7 7 - \mathbf { 1 7 9 }} & \mathbf{2 0 8 - \mathbf { 2 1 8 }} & \mathbf{2 1 4} \mathbf{- 2 2 4} & \mathbf{1 2 4} \mathbf{- 1 4 4} & \mathbf{1 5 7 - \mathbf { 1 7 0 }} \\ \text { UDOO1 } & \text { UDO03 } & \text { UDO12 } & \text { UDO28 } & \text { UDO39 } & \\ \mathbf{1 4 4 - \mathbf { 1 4 4 }} & \mathbf{1 3 5 - \mathbf { 1 3 5 }} & \mathbf{1 7 7 - \mathbf { 1 8 2 }} & \mathbf{1 8 2} \mathbf{- 1 8 2} & \mathbf{1 0 8 - 1 0 8} & \end{array}$

\section{References:}

1 - Lombardo N., Perri E., Muzzalupo I., et al. In: Contributo alla caratterizzazione del germoplasma olivicolo pugliese, Ist. Sper. Olivic. (2004), pp. 101-104.

2 - Muzzalupo I., Stefanizzi F., Perri E. HortScience (2009), 44: pp. 582-588. 


\section{“Zinzifarica"}

(synonymy: Zinzolarica, Zuzufarica.)

Areal distribution or origin area: Calabria

Flesh/ pit weight ratio: high $(7,74 \pm 2,23)$

Oil content (\%): medium $(48,22 \pm 0,18)$

Purpose: oil

\section{Morphological characters}

Tree characters

Vigour: medium

Growth habit: spreading

Canopy-density: medium-sparse

\section{Leaf characters}

Blade length (cm): medium $(5,25 \pm 0,63)$

Blade width $(\mathrm{cm})$ : medium $(\mathbf{1 , 1 3} \pm 0,07)$

Shape (length/width): elliptic-lanceolate

\section{Inflorescence characters}

Inflorescence length ( $\mathrm{cm})$ : short $(2,37 \pm 1,26)$

Number of flowers: low $(16,21 \pm 1,88)$

\section{Fruit characters}

Fresh weight of 100 fruits $(\mathrm{g})$ : medium $(3,92 \pm 0,08)$

Shape (length/width): spherical

Symmetry: symmetric

Position of maximum transverse diameter:

central

Apex: rounded

Base: truncate

Niplle: absent

Lenticels: few and small

\section{Pit characters}

Weight of 100 pits (g): high $(\mathbf{0 , 4 7} \pm \mathbf{0 , 1 1})$

Shape (length/width): ovoid

Mucron: tenuous

Symmetry: symmetric

Position of maximum transverse diameter:

towards apex

Apex: rounded

Base: rounded

Surface: scabrous

Number of grooves: medium
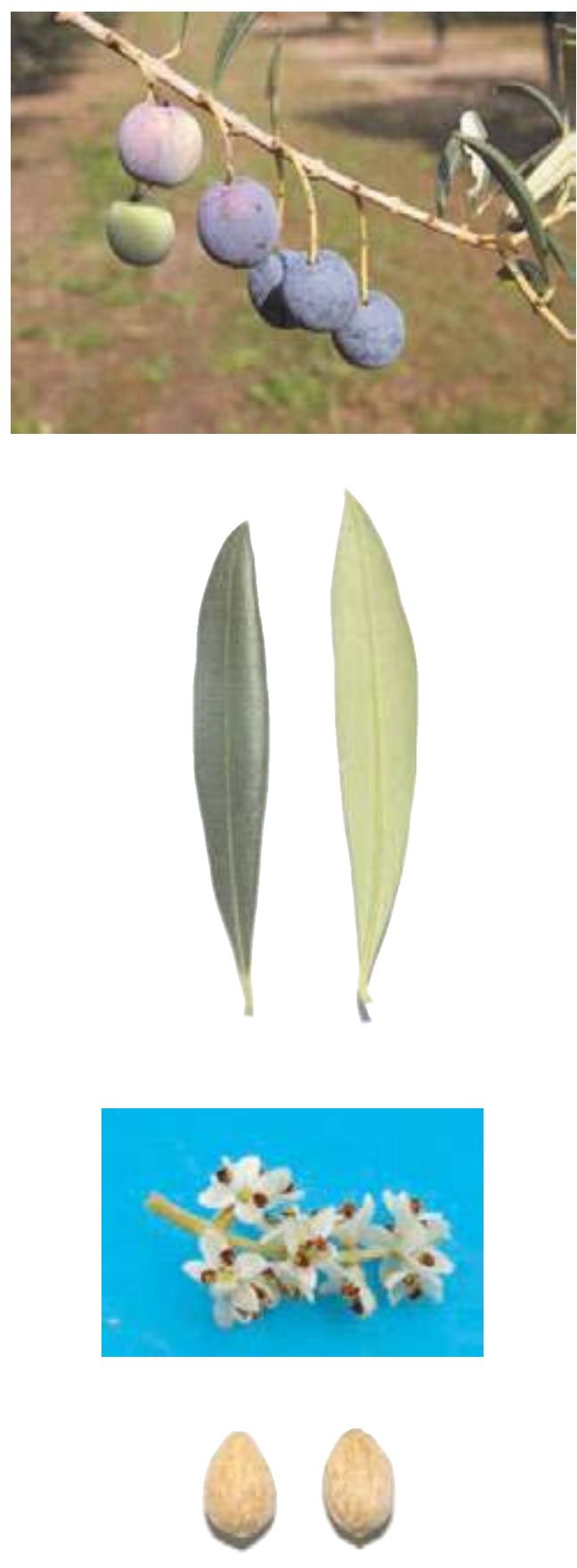


\section{Biochemical Characters}

\section{Fatty Acid Composition}

Table 1. Average values (express in $\% \pm$ standard deviations) of the fatty acids methyl esters and nutritional ratios obtained from single cultivar olive oils.

\begin{tabular}{|c|c|c|c|c|c|}
\hline Myristic acid & $0,01 \pm 0,00$ & Linoleic acid $(\omega 6)$ & $14,66 \pm 1,55$ & Lignoceric acid & $0,05 \pm 0,03$ \\
\hline Palmitic acid & $12,60 \pm 0,50$ & Linolenic acid $(\omega 3)$ & $0,75 \pm 0,03$ & & \\
\hline almitoleic acid & $0,11 \pm 0,01$ & Arachic acid & $0,32 \pm 0,06$ & Unsat./satured & $5,77 \pm 0,25$ \\
\hline earic acid & $1,84 \pm 0,12$ & Eicosenoic acid & $0,11 \pm 0,18$ & $\omega 6 / \omega 3$ & $19,63 \pm 2,54$ \\
\hline leic acid & $68,34 \pm 1,98$ & Behenic acid & $0,10 \pm 0,04$ & & \\
\hline
\end{tabular}

\section{Organoleptic oil values}

Sensory Analysis (Panel test)

Comment: fruity medium, with read sensations of aromatic herb and leaves. Balanced taste sensation with medium bitter and spicy. Medium fluidity.

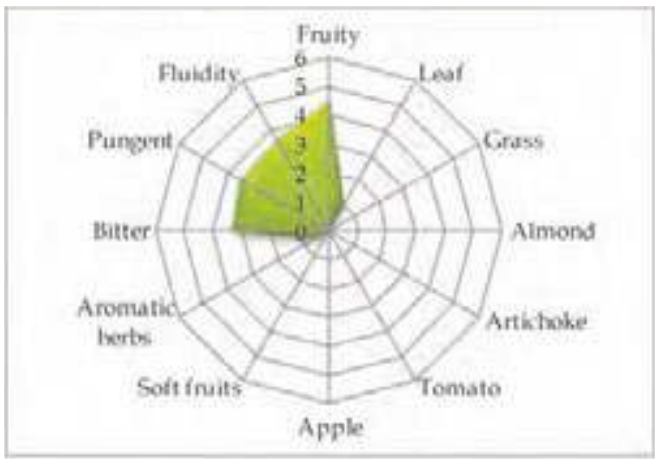

\section{Molecular Markers}

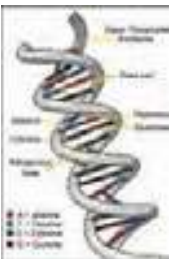

Table 2. Microsatellites (SSR) loci analyzed.

For each locus the allele size (expressed in base pairs) is reported.

\begin{tabular}{|c|c|c|c|c|c|}
\hline$D C A 09$ & $D C A 18$ & GAPU59 & GAPU71A & GUPA71B & GAPU103A \\
\hline $162-198$ & $177-179$ & $212-222$ & $214-214$ & $126-144$ & $150-159$ \\
\hline UDO01 & UDO03 & UDO12 & UDO28 & UDO39 & \\
\hline $144-144$ & $143-143$ & $177-177$ & $143-182$ & $205-205$ & \\
\hline
\end{tabular}

\section{References:}

1 - Lombardo N., Perri E., Muzzalupo I., et al. In: Il germoplasma olivicolo calabrese, Ist. Sper. Olivic.(2003), pp: 24.

2 - Perri E., Mazzotti F., Muzzalupo I., et al. In: Relazione attività, CO.R.ASS.OL. (2003).

3 - Muzzalupo I., Chiappetta A., Benincasa C., et al Sci Hortic-Amsterdam, (2010), 126: pp. 324-329. 